\title{
Enthalpies of Vaporization of Organic and Organometallic Compounds, 1880-2002
}

\author{
James S. Chickos ${ }^{\text {a) }}$ \\ Department of Chemistry, University of Missouri-St. Louis, St. Louis, Missouri 63121 \\ William E. Acree, Jr. ${ }^{\text {b) }}$ \\ Department of Chemistry, University of North Texas, Denton, Texas 76203
}

(Received 17 June 2002; accepted 17 October 2002; published 21 April 2003)

\begin{abstract}
A compendium of vaporization enthalpies published within the period 1910-2002 is reported. A brief review of temperature adjustments of vaporization enthalpies from temperature of measurement to the standard reference temperature, $298.15 \mathrm{~K}$, is included as are recently suggested reference materials. Vaporization enthalpies are included for organic, organo-metallic, and a few inorganic compounds. This compendium is the third in a series focusing on phase change enthalpies. Previous compendia focused on fusion and sublimation enthalpies. Sufficient data are presently available for many compounds that thermodynamic cycles can be constructed to evaluate the reliability of the measurements. A protocol for doing so is described. (c) 2003 American Institute of Physics. [DOI: $10.1063 / 1.1529214]$
\end{abstract}

Key words: compendium; enthalpies of condensation; evaporation; organic compounds; vaporization enthalpy.

\section{Contents}

1. Introduction

2. Reference Materials for Vaporization Enthalpy Measurements. .................... 520

3. Heat Capacity Adjustments. ............ 520

4. Group Additivity Values for $C_{p_{l}}(298.15 \mathrm{~K})$ Estimations. . . . . . . . . . . . . . . . 521

5. A Thermochemical Cycle: Sublimation, Vaporization, and Fusion Enthalpies. ........ 523

6. Estimation of Vaporization Enthalpies. ....... 525

7. Vaporization Enthalpy Compendium......... 525

8. Acknowledgment. . . . . . . . . . . . . . 878

9. References for Introductory Material. . . . . . . . 878

\section{List of Tables}

1. Recommended reference standards for vaporization enthalpy measurements.........

2. Group values $\Gamma_{1}$ and $\Gamma_{\mathrm{c}}$ for estimating $C_{p_{l}}(298.15 \mathrm{~K})$ and $C_{p_{c}}(298.15 \mathrm{~K})$.

. Some estimations of $C_{p_{l}}(298.15 \mathrm{~K})$ and $C_{p_{c}}(298.15 \mathrm{~K})$ using group values...........

4. A comparison of experimental sublimation enthalpies and those calculated using Eq. (6).....

5. A list of acronyms used in Tables 6 and $7 \ldots \ldots$.

6. Enthalpies of vaporization of organic compounds, 1880-2002. . . . . . . . . . . . . . . . .

7. Enthalpies of vaporization of organometallic and

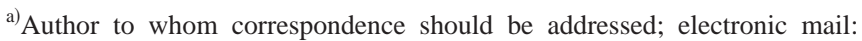
jsc@umsl.edu

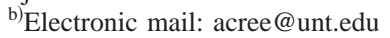

(C) 2003 American Institute of Physics.
}

inorganic compounds, $1880-2002 \ldots \ldots \ldots \ldots . \ldots 20$

8. References to Tables 6 and $7 \ldots \ldots \ldots \ldots \ldots . \ldots 53$

\section{List of Figures}

1. A thermodynamic cycle for adjusting vaporization enthalpies to $T=298.15 \mathrm{~K} \ldots \ldots \ldots \ldots \ldots \ldots$

2. A hypothetical molecule illustrating the different hydrocarbon groups in estimating $C_{p} \ldots \ldots \ldots \ldots$

\section{Introduction}

Vaporization enthalpies are important thermodynamic properties of the condensed phase. Vaporization enthalpies are used frequently in adjusting enthalpies of formation of liquids to the standard state and in evaluating environmental transport properties. ${ }^{1,2}$ To the chemical engineer, the magnitude of this property needs to be taken into consideration in designing equipment for chemical processing and synthesis. Thus vaporization enthalpy data are of interest at a variety of temperatures. As a consequence, there is a wealth of information in the literature that covers measurements over a broad range of temperatures. The data covered by this compendium include as much of the spectrum as was available to us.

Our interest in vaporization enthalpies goes back nearly two decades. Our primary focus in measuring vaporization enthalpies was as a means of adjusting enthalpies of formation of liquids to the standard state and in conjunction with fusion enthalpies to adjust solids in a similar fashion. ${ }^{3}$ Since then we have focused our attention on their estimation, ${ }^{4}$ and assessment. ${ }^{5}$ In parallel studies, compilations of available sublimation and fusion enthalpies were also initiated. ${ }^{6,7} \mathrm{~A}$ 
TABLE 1. Recommended reference standards for vaporization enthalpy measurements

\begin{tabular}{|c|c|c|c|c|c|}
\hline & Substance & $T / \mathrm{K}$ range & Vapor pressure range/Pa & $\Delta_{\mathrm{vap}} H_{m}(298.15 \mathrm{~K}) \mathrm{kJ} \mathrm{mol}^{-1}$ & Classification \\
\hline $\mathrm{C}_{3} \mathrm{H}_{8} \mathrm{O}$ & 1-propanol ${ }^{\mathrm{a}, \mathrm{d}}$ & $333-378$ & $20 E+3-135 E+3$ & $47.45 \pm 0.1$ & Primary \\
\hline $\mathrm{C}_{5} \mathrm{H}_{12}$ & pentane $e^{b, d}$ & $269-315$ & $2.0 \mathrm{E}+4-1.2 \mathrm{E}+5$ & $26.42 \pm 0.1$ & Primary \\
\hline $\mathrm{C}_{6} \mathrm{~F}_{6}$ & hexafluorobenzene $\mathrm{e}^{\mathrm{a}, \mathrm{d}}$ & $290-377$ & $7.5 \mathrm{E}+3-2.1 \mathrm{E}+5$ & $35.71 \pm 0.07$ & Primary \\
\hline $\mathrm{C}_{6} \mathrm{H}_{6}$ & benzene $\mathrm{e}^{\mathrm{a}, \mathrm{c}, \mathrm{d}}$ & $286-383$ & $7.0 \mathrm{E}+3-2.3 \mathrm{E}+5$ & $33.83 \pm 0.07$ & Primary \\
\hline $\mathrm{C}_{6} \mathrm{H}_{14}$ & hexane $e^{b, d}$ & $286-343$ & $1.2 \mathrm{E}+4-1.0 \mathrm{E}+5$ & $31.52 \pm 0.13$ & Primary \\
\hline $\mathrm{C}_{7} \mathrm{H}_{16}$ & heptane $e^{b, d}$ & $299-372$ & $6.4 \mathrm{E}+3-1.0 \mathrm{E}+5$ & $36.57 \pm 0.15$ & Primary \\
\hline $\mathrm{C}_{8} \mathrm{H}_{18}$ & octane $^{\mathrm{b}, \mathrm{d}}$ & $326-400$ & $7.7 \mathrm{E}+3-1.0 \mathrm{E}+5$ & $41.56 \pm 0.17$ & Primary \\
\hline $\mathrm{C}_{9} \mathrm{H}_{20}$ & nonane $e^{b, d}$ & $344-425$ & $6.4 \mathrm{E}+3-1.0 \mathrm{E}+5$ & $46.55 \pm 0.19$ & Primary \\
\hline $\mathrm{C}_{10} \mathrm{H}_{8}$ & naphthalene $\mathrm{d}^{\mathrm{d}}$ & $353-434$ & $1.0 \mathrm{E}+3-2.3 \mathrm{E}+4$ & $55.65 \pm 2.8$ & Secondary \\
\hline $\mathrm{C}_{10} \mathrm{H}_{22}$ & decane $e^{b, d}$ & $268-348$ & $1.7 \mathrm{E}+1-3.2 \mathrm{E}+3$ & $51.42 \pm 0.21$ & Primary \\
\hline $\mathrm{C}_{11} \mathrm{H}_{24}$ & undecane $^{\mathrm{b}, \mathrm{d}}$ & $294-382$ & $4.1 \mathrm{E}+1-6.4 \mathrm{E}+3$ & $56.58 \pm 0.57$ & Primary \\
\hline $\mathrm{C}_{12} \mathrm{H}_{26}$ & dodecane $e^{b, d}$ & $313-403$ & $5.8 \mathrm{E}+1-7.3 \mathrm{E}+3$ & $61.52 \pm 0.62$ & Primary \\
\hline $\mathrm{C}_{13} \mathrm{H}_{28}$ & tridecane $e^{\mathrm{b}, \mathrm{d}}$ & $323-402$ & $4.7 \mathrm{E}+1-3.7 \mathrm{E}+3$ & $66.68 \pm 0.67$ & Primary \\
\hline $\mathrm{C}_{14} \mathrm{H}_{30}$ & tetradecane $\mathrm{b}^{\mathrm{b}, \mathrm{d}}$ & $344-422$ & $7.6 \mathrm{E}+1-4.4 \mathrm{E}+3$ & $71.73 \pm 0.72$ & Primary \\
\hline $\mathrm{C}_{16} \mathrm{H}_{32}$ & hexadecane $\mathrm{e}^{\mathrm{b}, \mathrm{d}}$ & $364-452$ & $5.5 E+1-4.7 E+3$ & $81.35 \pm 0.81$ & Primary \\
\hline $\mathrm{C}_{18} \mathrm{H}_{38}$ & octadecane $^{\mathrm{b}, \mathrm{d}}$ & $312-590$ & $1.0 \mathrm{E}-1-1.0 \mathrm{E}+5$ & $91.44 \pm 1.83$ & Primary \\
\hline $\mathrm{C}_{20} \mathrm{H}_{42}$ & eicosane $e^{b, d}$ & $344-380$ & $4.1 \mathrm{E}-1-9.1 \mathrm{E}+0$ & $101.81 \pm 2.0$ & Primary \\
\hline $\mathrm{H}_{2} \mathrm{O}$ & water $^{\mathrm{d}}$ & $273-373$ & $6.1 \mathrm{E}+1-1.0 \mathrm{E}+5$ & $43.99 \pm 0.07$ & Primary \\
\hline
\end{tabular}

${ }^{\mathrm{a}}$ Reference 12 .

beference 13 .

${ }^{\mathrm{c}}$ Cancer suspect agent.

${ }^{\mathrm{d}}$ Reference 5 .

reasonably exhaustive version of these databases covering the literature to the present has recently been published and is also available on line at http://webbook.nist.gov/ chemistry/. Combined with fusion and sublimation enthalpies appropriately adjusted to $T=298.15 \mathrm{~K}$, vaporization enthalpies $\Delta_{\text {vap }} H_{m}(298.15 \mathrm{~K})$, complete a thermochemical cycle that can be used to assess the quality of the available data. Application of this thermochemical cycle is illustrated below. A goal of the present contribution has been to provide an exhaustive coverage of the literature from about 1880 to 2002; regrettably however, this compilation is probably still incomplete.

\section{Reference Materials for Vaporization Enthalpy Measurements}

Calibration is a fundamental requirement in every thermochemical measurement of vaporization enthalpy. Regardless of which technique is used, the measurement ultimately depends either directly or indirectly on vapor pressure. Vapor pressures of liquids vary over many orders of magnitude. An experimental technique calibrated with a standard in one pressure or temperature regime does not in itself guarantee the same accuracy in another. Substantial variations in vaporization enthalpy are revealed in the tables that follow. This variance clearly establishes the importance of documenting the accuracy of the measurements through the use of appropriate reference materials that approximate the temperature and pressure regimes of the measurements of interest.

A series of compounds have been recently proposed as reference materials for vaporization enthalpy. ${ }^{5}$ These have been classified as primary, secondary, or tertiary reference materials, on the basis of various criteria. The materials classified as primary and secondary reference materials are listed in Table 1. The temperature range, the corresponding vapor pressures, and the recommended molar vaporization enthalpies at $T=298.15 \mathrm{~K}$ are also included in the table. Some of these reference materials are solids at $T=298.15 \mathrm{~K}$ and therefore the vaporization enthalpies are hypothetical values. The reader should consult the literature cited at the bottom of the table to obtain the vaporization enthalpy at the temperature of interest.

\section{Heat Capacity Adjustments}

Vaporization enthalpies, like their sublimation counterparts, are measurements based on mass transport and as such are directly or indirectly dependent upon vapor pressure. The vapor pressure of different liquids at a given temperature can vary many orders of magnitude. In order to obtain a reasonable amount of mass transport, it is frequently necessary to conduct these measurements at temperatures that differ substantially from the standard reference temperature, $298.15 \mathrm{~K}$. The actual temperature of measurement depends on the sensitivity of the instrument or apparatus and the properties of the substance of interest. Vaporization enthalpy measurements are often conducted as a function of temperature.

The magnitude of the vaporization enthalpy is dependent on temperature. Figure 1 and Eqs. (1) and (2) illustrate the origin of this temperature dependence in terms of a thermodynamic cycle. If the heat capacities of the liquid and gas phase are known, $C_{p_{l}}$ and $C_{p_{g}}$, respectively, then the vaporization enthalpy at $T=298.15 \mathrm{~K}$ can be related to the experimental measurements by using Eq. (1). This equation, generally referred to as Kirchhoff's equation, can be used to adjust sublimation enthalpy measurements to any reference temperature. The term $T_{m}$ represents either the temperature 


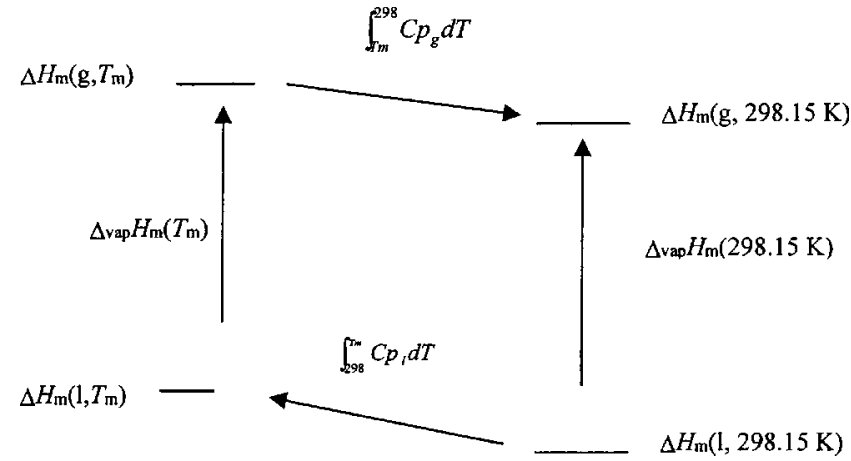

FIG. 1. A thermodynamic cycle for adjusting vaporization enthalpies to $T$ $=298.15 \mathrm{~K}$.

of measurement for calorimetric measurements or the mean temperature of measurement for experiments conducted

$$
\begin{array}{r}
\Delta_{\text {vap }} H_{m}(298.15 \mathrm{~K})=\Delta_{\text {vap }} H_{m}\left(T_{m}\right) \\
+\int_{298.15}^{T_{m}}\left(C_{p_{l}}-C_{p_{g}}\right) d T \\
\Delta_{\text {vap }} H_{m}(298.15 \mathrm{~K}) \approx \Delta_{\text {vap }} H_{m}\left(T_{m}\right) \\
+\left(C_{p_{l}}-C_{p_{g}}\right)\left(T_{m}-298.15\right)
\end{array}
$$

over narrow ranges of temperature. Treating the heat capacities of the two phases as independent of temperature and integrating Eq. (1) results in Eq. (2). Since the magnitude of the heat capacity of the gas phase is usually smaller than that of the liquid phase $(l)$, vaporization enthalpies increase with decreasing temperature.

Experimental heat capacities for many liquids at $T$ $=298.15 \mathrm{~K}$ are available ${ }^{8}$ Experimental gas phase heat capacities for compounds that are liquids at $T=298.15 \mathrm{~K}$ are unavailable and generally need to be estimated. Gas phase heat capacities can be calculated from statistical mechanics or estimated by group additivity methods. A number of group additivity methods have been developed to estimate gas phase heat capacities. ${ }^{9,10}$ However, group values for some functional groups are not available. Equation (3) is an example of an attempt to circumvent the lack of sufficient group values. ${ }^{11}$ The term $C_{p_{l}}(298.15 \mathrm{~K})$ refers to the heat capacity of the liquid at $T=298.15 \mathrm{~K}$. This term may either be estimated or experimental values may be used if available. The uncertainty associated with the term in brackets in Eq. (3) is $\pm 30 \mathrm{~J} \mathrm{~mol}^{-1}( \pm 2 \sigma)$

$$
\begin{aligned}
\left(C_{p_{l}}\right. & \left.-C_{p_{g}}\right)\left\{T_{m}-298.15\right\} \\
& =\left[10.58+0.26 C_{p_{l}}(298.15 \mathrm{~K})\right]\left(T_{m}-298.15\right) .
\end{aligned}
$$

The vaporization enthalpies reported in this compendium have not been adjusted to $T=298.15 \mathrm{~K}$ unless done so by the authors reporting the measurements. The vaporization enthalpies are reported at the mean temperature of measurement wherever possible. This allows the reader to verify the reported data and to adjust for temperature in a consistent manner. Authors have applied different methods to adjust for temperature. In some cases experimental data have been used for $C_{p_{l}}$ and in other cases both $C_{p_{l}}$ and $C_{p_{g}}$ have been estimated. The reader is encouraged to refer to the original literature for details. In an effort to provide some assistance to the reader in this regard, a brief discussion of one of the group additivity methods that are available for estimating the heat capacity of liquids is included below. This is followed by an illustration of how this value can be used in conjunction with Eq. (3) to provide temperature adjustments.

\section{Group Additivity Values for $C_{p_{1}}(298.15 \mathrm{~K})$ Estimations}

Tables 2A and 2B lists a set of group values that can be used in estimations of $C_{p_{l}}(298.15 \mathrm{~K})$. The groups and their corresponding values are identified by italics. A hypothetical molecule is given in Fig. 2 that identifies each hydrocarbon group. The functional groups listed in Table 2B are self explanatory. The $R$ terms in this table represent variable groups and are not included in the value. Values in brackets should be considered as tentative assignments. The use of these group values is illustrated with the examples in Table 3. Estimations of $C_{p_{c}}(298.15 \mathrm{~K})$ for these compounds follow the same protocol.

Groups are defined on the basis of their substitution and hybridization patterns. These groups are further subdivided into cyclic, acyclic and aromatic categories. The estimation of $C_{p_{l}}(298.15 \mathrm{~K})$ for propionic acid illustrates how directly $C_{p}$ values can be estimated. Identification of the appropriate group is important. Although not shown in Table 3, the same protocol is used for estimating $C_{p_{c}}(298.15 \mathrm{~K})$. Estimation of the heat capacity of piperadine is similar. In this case it is important to use the appropriate cyclic group. Once the proper groups are identified, the estimation is direct. Di- $t$ butyl ether is estimated in a similar fashion. Benzothiazole illustrates the estimation of an aromatic molecule containing a heterocyclic ring. The aromatic ring carbons are selected based on their substitution pattern; the sulfur and nitrogen atoms of the thiazole ring are identified as cyclic atoms; the nitrogen atom is identified on the basis of its hybridization and substitution pattern. 2-Methylcyclohexanone is another example of an estimation of a cyclic molecule. This estimation includes the contributions of a cyclic carbonyl, four cyclic methylene groups, a methyl, and a cyclic tertiary methine group. The estimation of limonene illustrate an example of a molecule that contains both cyclic and acyclic double bonds. Additional examples can be found in the literature. ${ }^{4,11}$

Heat capacities calculated according to the protocol just described have been used when necessary in conjunction with Eqs. (4)-(6) to adjust vaporization, sublimation, and fusion enthalpies to $298.15 \mathrm{~K}$. These temperature adjustments are necessary in illustrating the thermochemical cycle described below. Equations (4)-(6) have been used in those cases where the measurements were referenced to some other temperature 
TABLE 2. Group values $\Gamma_{l}$ and $\Gamma_{c}$ for estimating $C_{p_{l}}(298.15 \mathrm{~K})$ and $C_{p_{c}}(298.15 \mathrm{~K})$

(A) Group values for estimating the $C_{p_{l}}(298.15 \mathrm{~K})$ and $C_{p_{c}}(298.15 \mathrm{~K})$ of hydrocarbons. ${ }^{\mathrm{a}}$

Hydrocarbon groups

\begin{tabular}{|c|c|c|c|c|c|c|c|}
\hline \multicolumn{4}{|c|}{ Aliphatic groups } & \multicolumn{4}{|c|}{ Cyclic aliphatic and olefinic groups } \\
\hline \multirow[b]{2}{*}{ Description of group } & \multirow[b]{2}{*}{ Formula } & \multicolumn{2}{|c|}{$\mathrm{J} \mathrm{mol}^{-1} \mathrm{~K}^{-1}$} & \multirow[b]{2}{*}{ Description of group } & \multirow[b]{2}{*}{ Formula } & \multicolumn{2}{|c|}{$\mathrm{J} \mathrm{mol}^{-1} \mathrm{~K}^{-1}$} \\
\hline & & $\Gamma_{l}$ & $\Gamma_{c}$ & & & $\Gamma_{l}$ & $\Gamma_{c}$ \\
\hline $\begin{array}{l}\text { primary } s p^{3} \mathrm{C} \\
\text { secondary } s p^{3} \mathrm{C} \\
\text { tertiary } s p^{3} \mathrm{C} \\
\text { quaternary } s p^{3} \mathrm{C}\end{array}$ & $\begin{array}{l}-\mathrm{CH}_{3} \\
-\mathrm{CH}_{2}- \\
-\mathrm{CHR}- \\
-\mathrm{CR}_{3}\end{array}$ & $\begin{array}{l}34.9 \\
31.9 \\
22.4 \\
14.0\end{array}$ & $\begin{array}{c}36.6 \\
26.9 \\
9 \\
-5\end{array}$ & $\begin{array}{l}\text { cyclic secondary } s p^{3} \mathrm{C} \\
\text { cyclic tertiary } s p^{3} \mathrm{C} \\
\text { cyclic quaternary } s p^{3} \mathrm{C} \\
\text { cyclic tertiary } s p^{2} \mathrm{C} \\
\text { cyclic quaternary } s p^{2} \mathrm{C}\end{array}$ & $\begin{array}{l}-\mathrm{C}_{c} \mathrm{H}_{2}- \\
-\mathrm{C}_{c} \mathrm{HR}- \\
-\mathrm{C}_{c} \mathrm{R}_{2}- \\
-\mathrm{C}_{c} \mathrm{HR}- \\
-\mathrm{C}_{c} \mathrm{R}_{2}-\end{array}$ & $\begin{array}{l}25.9 \\
20.6 \\
18.0 \\
21.8 \\
21.2\end{array}$ & $\begin{array}{r}24.6 \\
11.7 \\
6.1 \\
15.9 \\
{[4.7]}\end{array}$ \\
\hline \multicolumn{4}{|c|}{ Olefinic and acetylenic groups } & \multicolumn{4}{|c|}{ Aromatic groups } \\
\hline & & \multicolumn{2}{|c|}{$\mathrm{J} \mathrm{mol}^{-1} \mathrm{~K}^{-1}$} & & & \multicolumn{2}{|c|}{$\mathrm{J} \mathrm{mol}^{-1} \mathrm{~K}^{-1}$} \\
\hline Description of group & Formula & $\Gamma_{l}$ & $\Gamma_{c}$ & Description of group & Formula & $\Gamma_{l}$ & $\Gamma_{c}$ \\
\hline $\begin{array}{l}\text { secondary } s p^{2} \mathrm{C} \\
\text { tertiary } s p^{2} \mathrm{C} \\
\text { quaternary } s p^{2} \mathrm{C} \\
\text { tertiary } s p \mathrm{C} \\
\text { quaternary } s p \mathrm{C}\end{array}$ & $\begin{array}{l}=\mathrm{CH}_{2} \\
=\mathrm{CH}- \\
=\mathrm{C}- \\
\equiv \mathrm{C}-\mathrm{H} \\
\equiv \mathrm{C}-\end{array}$ & $\begin{array}{l}25.8 \\
27.8 \\
21.7 \\
34.3 \\
28.9\end{array}$ & $\begin{array}{r}46 \\
21.4 \\
6.9 \\
37.1 \\
15.5\end{array}$ & $\begin{array}{l}\text { tertiary aromatic } s p^{2} \mathrm{C} \\
\text { quaternary aromatic } s p^{2} \mathrm{C} \\
\text { internal quaternary aromatic } \mathrm{C}\end{array}$ & $\begin{array}{l}=\mathrm{C}_{a} \mathrm{H}- \\
=\mathrm{C}_{a} \mathrm{R}- \\
=\mathrm{C}_{a} \mathrm{R}-\end{array}$ & $\begin{array}{l}21.8 \\
15.3 \\
{[16]}\end{array}$ & $\begin{array}{r}17.5 \\
8.5 \\
{[9.1]}\end{array}$ \\
\hline
\end{tabular}

(B) Group values $\Gamma_{l}$ and $\Gamma_{c}$ of various functional groups. ${ }^{\text {b }}$

\begin{tabular}{|c|c|c|c|c|c|c|c|}
\hline \multicolumn{4}{|c|}{ Monodentate functional groups } & \multicolumn{4}{|c|}{ Acyclic bidentate functional groups } \\
\hline \multirow[b]{2}{*}{ Description of group } & \multirow[b]{2}{*}{ Formula } & \multicolumn{2}{|c|}{$\mathrm{J} \mathrm{mol}^{-1} \mathrm{~K}^{-1}$} & \multirow[b]{2}{*}{ Description of group } & \multirow[b]{2}{*}{ Formula } & \multicolumn{2}{|c|}{$\mathrm{J} \mathrm{mol}^{-1} \mathrm{~K}^{-1}$} \\
\hline & & $\Gamma_{l}$ & $\Gamma_{c}$ & & & $\Gamma_{l}$ & $\Gamma_{c}$ \\
\hline alcohols, phenols & $-\mathrm{OH}$ & 53.1 & 23.5 & ketones & $-\mathrm{CO}-$ & 51.5 & 28 \\
\hline fluorine & $-\mathrm{F}$ & 16.2 & {$[24.8]$} & ester & $-\mathrm{CO}_{2} \mathrm{R}$ & 63.2 & 40.3 \\
\hline chlorine & $-\mathrm{Cl}$ & 30.8 & 28.7 & ether & $-\mathrm{O}-$ & 29.8 & 49.8 \\
\hline bromine & $-\mathrm{Br}$ & 34.6 & 32.4 & secondary $s p^{3} \mathrm{~N}$ & $-\mathrm{NH}-$ & {$[51]$} & -0.3 \\
\hline iodines & $-\mathrm{I}$ & 39.1 & {$[27.9]$} & secondary amide & $-\mathrm{CONH}-$ & 79.9 & 44.4 \\
\hline nitrile & $-\mathrm{CN}$ & 47.7 & 42.3 & carbamates & $-\mathrm{OCONH}-$ & & 76.1 \\
\hline carboxylic acid & $-\mathrm{CO}_{2} \mathrm{H}$ & 87.4 & 53.1 & sulfides & $-\mathrm{S}-$ & 40.3 & [116] \\
\hline acid chloride & $-\mathrm{COCl}$ & {$[62.8]$} & {$[60.2]$} & disulfides & $-\mathrm{S}-\mathrm{S}-$ & {$[74.5]$} & 41 \\
\hline aldehyde & $-(\mathrm{C}=\mathrm{O}) \mathrm{H}$ & 57.7 & {$[84.5]$} & sulfoxides & $-\mathrm{SO}-$ & {$[83.7]$} & 47.7 \\
\hline isocyanate & $-\mathrm{NCO}$ & {$[58.2]$} & {$[52.7]$} & sulfones & $-\mathrm{SO}_{2}-$ & & 88.7 \\
\hline nitro group & $-\mathrm{NO}_{2}$ & 58.6 & 56.1 & & & & \\
\hline secondary $s p^{3}$ nitrogen & $-\mathrm{NH}_{2}$ & 51.0 & 21.6 & & & & \\
\hline primary amides & $-\mathrm{CONH}_{2}$ & 54.4 & 54.4 & & & & \\
\hline thiols & $-\mathrm{SH}$ & 49.0 & {$[51.9]$} & & & & \\
\hline sulfonamide & $-\mathrm{SO}_{2} \mathrm{NH}_{2}$ & 104 & 104 & & & & \\
\hline substituted urea & $-\mathrm{NHCONH}_{2}$ & 82.8 & 82.8 & & & & \\
\hline \multicolumn{4}{|c|}{ Acyclic tridentate functional groups } & \multicolumn{4}{|c|}{ Acyclic tetradentate functional groups } \\
\hline & & \multicolumn{2}{|c|}{$\mathrm{J} \mathrm{mol}^{-1} \mathrm{~K}^{-1}$} & & & \multicolumn{2}{|c|}{$\mathrm{J} \mathrm{mol}^{-1} \mathrm{~K}^{-1}$} \\
\hline Description of group & Formula & $\Gamma_{l}$ & $\Gamma_{c}$ & Description of group & Formula & $\Gamma_{l}$ & $\Gamma_{c}$ \\
\hline tertiary $s p^{3} \mathrm{~N}$ & $-\mathrm{NR}_{2}$ & 22.0 & [31.5] & quaternary silicon & $-\mathrm{SiR}_{2}-$ & 30.9 & 32.4 \\
\hline tertiary $s p^{2} \mathrm{~N}$ & $=\mathrm{N}-$ & {$[44.4]$} & 10.7 & quaternary tin & $-\mathrm{SnR}_{2}-$ & {$[58.6]$} & {$[77.2]$} \\
\hline phosphine oxide & $-(\mathrm{PO}) \mathrm{R}-$ & & 28.5 & quaternary germanium & $\mathrm{Ge}$ & {$[48.1]$} & [18.9] \\
\hline
\end{tabular}

Cyclic functional groups

\begin{tabular}{lccc}
\hline & & \multicolumn{2}{c}{$\mathrm{J} \mathrm{mol}^{-1} \mathrm{~K}^{-1}$} \\
\cline { 3 - 4 } Description of group & Formula & $\Gamma_{l}$ & $\Gamma_{c}$ \\
\hline cyclic ketones & $-(\mathrm{CO})_{c}-$ & {$[46.4]$} & 34.3 \\
lactones & $-\mathrm{CO}_{2}-$ & {$[67.4]$} & 45.2 \\
cyclic carbonates & $-\mathrm{OCO}_{2}-$ & {$[92]$} & {$[68.2]$} \\
cyclic anhydrides & $-\mathrm{CO}_{2} \mathrm{CO}-$ & & 80.3 \\
cyclic ether & $-\mathrm{Oc}-$ & 24.6 & 9.7 \\
cyclic sec. $s p^{3} \mathrm{~N}$ & $-\mathrm{N}_{c} \mathrm{H}-$ & 46.0 & 23.9
\end{tabular}


TABLE 2. Group values $\Gamma_{l}$ and $\Gamma_{c}$ for estimating $C_{p_{l}}(298.15 \mathrm{~K})$ and $C_{p_{c}}(298.15 \mathrm{~K})-$ Continued

\begin{tabular}{lccc}
\hline \hline & Cyclic functional groups & \\
\hline & & \multicolumn{2}{c}{$\mathrm{J} \mathrm{mol}^{-1} \mathrm{~K}^{-1}$} \\
\cline { 3 - 4 } Description of group & Formula & $\Gamma_{l}$ & $\Gamma_{c}$ \\
\hline cyclic tertiary $s p^{3} \mathrm{~N}$ & $-\mathrm{N}_{c} \mathrm{R}-$ & 28.6 & 1.2 \\
cyclic tertiary $s p^{2} \mathrm{~N}$ & $=\mathrm{N}_{c}-$ & 20.7 & 13.9 \\
cyclic urea & $-\mathrm{NHCONH}-$ & & 63.6 \\
cyclic sec. amide & $-\mathrm{CONH}-$ & {$[92]$} & 46.4 \\
cyclic tertiary amide & $-\mathrm{CONR}-$ & {$[170]$} & 52.7 \\
cyclic imide & $-\mathrm{CONHCO}-$ & & 74.1 \\
cyclic sulfides & $-\mathrm{S}_{c}-$ & 33.8 & 20.3 \\
\hline
\end{tabular}

${ }^{\mathrm{a}}$ See Ref. 19.

${ }^{\mathrm{b}}$ Values reported in brackets are tentative assignments.

$$
\begin{aligned}
& \Delta_{\text {vap }} H_{m}(298.15 \mathrm{~K}) / \mathrm{J} \mathrm{mol} \mathrm{mol}^{-1} \\
& \approx \Delta_{\text {vap }} H_{m}\left(T_{m}\right)+\left[10.58+0.26 C_{p_{l}}(298.15 \mathrm{~K})\right] \\
& \quad \times\left(T_{m}-298.15\right), \\
& \Delta_{\text {sub }} H_{m}(298.15 \mathrm{~K}) / \mathrm{J} \mathrm{mol}^{-1} \\
& \approx \Delta_{\text {sub }} H_{m}\left(T_{m}\right)+\left[0.75+0.15 C_{p_{c}}(298.15 \mathrm{~K})\right] \\
& \quad \times\left(T_{m}-298.15\right), \\
& \Delta_{\text {fus }} H_{m}(298.15 \mathrm{~K}) / \mathrm{J} \mathrm{mol}-1 \\
& \approx \Delta_{\text {fus }} H_{m}\left(T_{\text {fus }}\right)+\left\{\left[0.75+0.15 C_{p_{c}}(298.15 \mathrm{~K})\right]\right. \\
& \quad \times\left(T_{m}-298.15\right)-\left[10.58+0.26 C_{p_{l}}(298.15 \mathrm{~K})\right] \\
& \quad \times\left(T_{m}-298.15\right) .
\end{aligned}
$$

An uncertainty $( \pm 2 \sigma)$ of one third the temperature adjustment has been associated with the use of Eqs. (5) and (6) in Table 4. The uncertainty has been assigned arbitrarily.

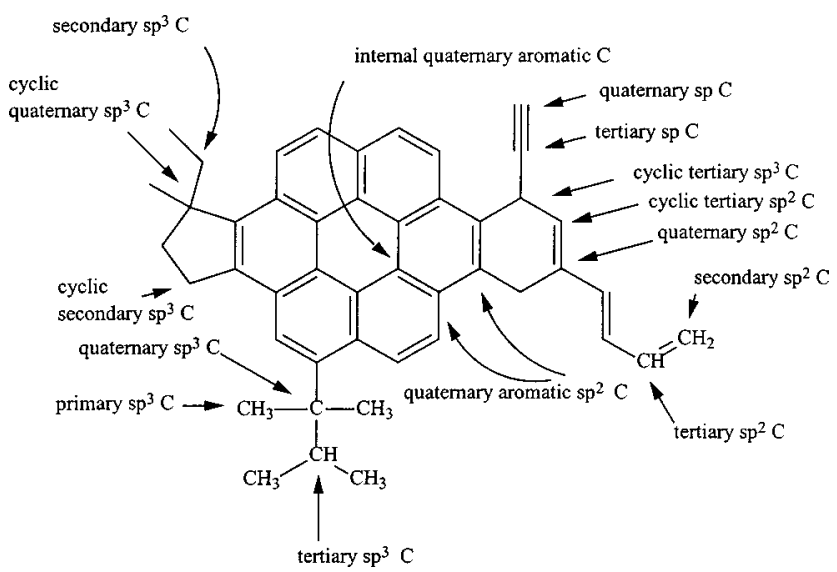

FIG. 2. A hypothetical molecule illustrating the different hydrocarbon groups in estimating $C_{p}$.

\section{A Thermochemical Cycle: Sublimation, Vaporization, and Fusion Enthalpies}

The use of a thermochemical cycle is an extremely effective manner of evaluating the quality of enthalpy data associated with phase changes. It should be emphasized at the start that internal consistency within a cycle does not guarantee that all the data are necessarily of high quality. Sublimation enthalpy is related to the sum of the fusion and vaporization enthalpies, Eq. (7), provided all are referenced to the same temperature. Vaporization enthalpy is the larger of

$$
\begin{aligned}
\Delta_{\text {sub }} H_{m}(298.15 \mathrm{~K})= & \Delta_{\text {vap }} H_{m}(298.15 \mathrm{~K}) \\
& +\Delta_{\text {fus }} H_{m}(298.15 \mathrm{~K})
\end{aligned}
$$

the two enthalpies on the right hand side of the equation. Since fusion enthalpies of solids usually decrease when adjusted to $298.15 \mathrm{~K}$, the latter contributes a smaller amount to the sublimation enthalpy, particularly if the melting point of the material is high. Thus an accurate vaporization enthalpy can tolerate a less accurately determined fusion enthalpy. This is fortunate since as discussed below, there is a physical basis for variance in fusion enthalpy.

Many solids exhibit polymorphism or undergo solid-solid phase transitions. These may occur unnoticed. Solid-solid phase transitions occurring below $T=298.15 \mathrm{~K}$ generally do not pose a problem if the vaporization and sublimation enthalpy measurements are conducted at temperatures above the phase change. Enthalpic differences between polymorphic forms generally tend to be small in relation to solid-gas, liquid-gas transitions. Since the smaller $\Delta_{\text {fus }} H_{m}(298.15 \mathrm{~K})$ term in Eq. (7) is the one affected by polymorphism, this phenomena should not have a large effect on the quality of the thermochemical cycle, even if the sublimation and fusion enthalpy measurements are conducted on different polymorphic forms. In many cases the sublimation enthalpy measured directly should compare favorably with the sum of the fusion and vaporization enthalpy. Yet often this is not the case.

The results given in Table 4 illustrate some of the situations that can arise when constructing a thermochemical 
TABLE 3. Some estimations of $C_{p_{l}}(298.15 \mathrm{~K})$ and $C_{p_{c}}(298.15 \mathrm{~K})$ using group values

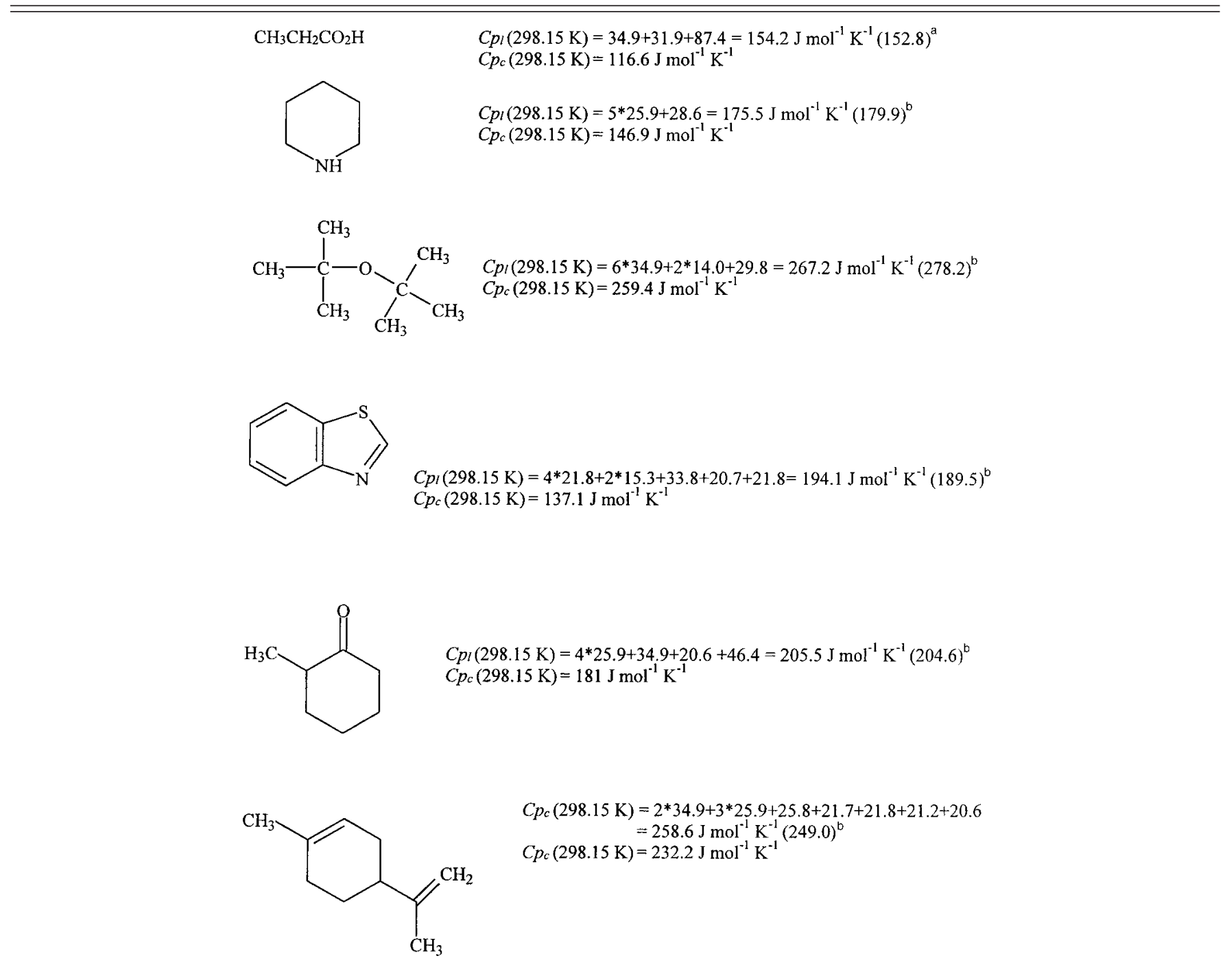

${ }^{a}$ See Ref. 8.

${ }^{\mathrm{b}}$ See Ref. 14.

cycle based on phase changes. The sublimation enthalpy of $p$-nitroaniline has been measured frequently and reproducibly. The value of $(100.9 \pm 1.0) \mathrm{kJ} \mathrm{mol}^{-1}$ is the mean value of nine measurements. Two measurements of the fusion and vaporization enthalpy are also available. Both fusion enthalpies are in good agreement with each other; the mean value is reported in Table 4. Comparison of the vaporization enthalpies at $T=298.15 \mathrm{~K}$ results in values with a $12 \mathrm{~kJ} \mathrm{~mol}^{-1}$ discrepancy. In this instance the experimental sublimation enthalpy falls halfway between the two values calculated by Eq. (7). The experimental data suggest a vaporization enthalpy of $85.5 \mathrm{~kJ} \mathrm{~mol}^{-1}$. This is an instance where the vaporization enthalpies are in disagreement; disagreement between sublimation enthalpies is the more common occurrence.

Data for 1,6-hexanedioic acid represents results that are more typical. The fusion and vaporization enthalpies have been measured once while the sublimation enthalpy has been reported three times. In this instance good agreement is ob- served between three of the four sublimation values.

Acetanilide provides a different but similar example. The sublimation enthalpies calculated using Eq. (6) are slightly larger than the two direct measurements. The data suggest a vaporization enthalpy of 73.9 and a sublimation enthalpy of $90 \mathrm{~kJ} \mathrm{~mol}^{-1}$ at $T=298.15 \mathrm{~K}$.

The vaporization and sublimation enthalpies of 4-chlorobiphenyl have been reported a number of times. One set of sublimation enthalpies cluster around $83 \mathrm{~kJ} \mathrm{~mol}^{-1}$ and the other around $75 \mathrm{~kJ} \mathrm{~mol}^{-1}$. A distinction between these two possibilities is possible by examining the vaporization enthalpies of the isomeric 2- and 3-chlorobiphenyls (see Table 6). The vaporization enthalpies of these isomeric materials would be expected to be similar. On this basis, a vaporization of around $72 \mathrm{~kJ} \mathrm{~mol}^{-1}$ and a sublimation enthalpy of approximately $83 \mathrm{~kJ} \mathrm{~mol}^{-1}$ for 4-chlorobiphenyl at $T$ $=298.15 \mathrm{~K}$ is selected for 4-chlorobiphenyl.

Benzil is a case where all the available data appear to be 
TABLE 4. A comparison of experimental sublimation enthalpies and those calculated using Eq. (6); enthalpies in $\mathrm{kJ}^{\mathrm{mol}}{ }^{-1}$; $C_{p}$ in $\mathrm{J}_{\mathrm{mol}}^{-1} \mathrm{~K}^{-1}$ a

\begin{tabular}{|c|c|c|c|c|c|c|c|c|c|c|c|c|c|}
\hline & & $\begin{array}{c}\Delta_{\text {fus }} H_{m} \\
\left(T_{\text {fus }}\right) \\
\end{array}$ & $T_{\text {fus }} / \mathrm{K}$ & $\begin{array}{l}\Delta_{\mathrm{vap}} H_{m} \\
\left(T_{m} / \mathrm{K}\right) \\
\end{array}$ & $T_{m} / \mathrm{K}$ & $\begin{array}{c}\Delta_{\mathrm{sub}} H_{m} \\
\left(T_{m}\right)\end{array}$ & $T_{m} / \mathrm{K}$ & $C_{p_{l}}$ & $C_{p_{c}}$ & $\begin{array}{c}\Delta_{\text {fus }} H_{m} \\
(298 \mathrm{~K})\end{array}$ & $\begin{array}{c}\Delta_{\text {vap }} H_{m} \\
(298 \mathrm{~K})\end{array}$ & $\begin{array}{c}\Delta_{\text {sub }} H_{m} \\
(298 \mathrm{~K}) \\
{[\text { Eq. (6)] }}\end{array}$ & $\begin{array}{c}\Delta_{\mathrm{sub}} H_{m} \\
(298 \mathrm{~K})^{\mathrm{a}}\end{array}$ \\
\hline $\mathrm{C}_{6} \mathrm{H}_{6} \mathrm{~N}_{2} \mathrm{O}_{2}$ & 4-nitroaniline & 21.1 & 420.7 & $\begin{array}{l}77.9 \\
70\end{array}$ & $\begin{array}{l}488 \\
430\end{array}$ & 100.7 & 298 & 235.8 & 164.7 & $15.4 \pm 1.9$ & $\begin{array}{l}91.5 \pm 4.5 \\
79.5 \pm 3.2\end{array}$ & $\begin{array}{r}106.9 \pm 4.9 \\
94.9 \pm 3.7\end{array}$ & 100.9 \\
\hline $\mathrm{C}_{6} \mathrm{H}_{10} \mathrm{O}_{4}$ & 1,6-hexanedioic acid & 34.85 & 426.4 & 92 & 447 & $\begin{array}{l}133.6 \\
129.3 \\
140\end{array}$ & $\begin{array}{l}298 \\
383 \\
306.5\end{array}$ & 302.4 & 213.8 & $27.6 \pm 2.4$ & $105.3 \pm 3.5$ & $132.9 \pm 4.3$ & $\begin{array}{l}133.6 \\
132.1 \pm 0.9 \\
140.3 \pm 0.1\end{array}$ \\
\hline $\mathrm{C}_{8} \mathrm{H}_{9} \mathrm{NO}$ & acetanilide & 21.65 & 387.5 & $\begin{array}{l}64.8 \\
66.3\end{array}$ & $\begin{array}{l}488 \\
402\end{array}$ & $\begin{array}{l}80.6 \\
87.2\end{array}$ & $\begin{array}{l}313.5 \\
326.5\end{array}$ & 239.1 & 177.0 & $17.6 \pm 1.3$ & $\begin{array}{l}78.6 \pm 4.6 \\
73.9 \pm 2.4\end{array}$ & $\begin{array}{l}96.2 \pm 4.7 \\
91.4 \pm 2.8\end{array}$ & $\begin{array}{l}81.0 \pm 0.1 \\
88.0 \pm 0.3\end{array}$ \\
\hline $\mathrm{C}_{12} \mathrm{H}_{9} \mathrm{Cl}$ & 4-chlorobiphenyl & 13.32 & 348.6 & $\begin{array}{l}71.6 \\
66.8 \\
59 \\
65.9\end{array}$ & $\begin{array}{l}298 \\
368 \\
384 \\
466\end{array}$ & $\begin{array}{l}86 \\
73.7\end{array}$ & $\begin{array}{l}278 \\
326\end{array}$ & 272.9 & 211.7 & $10.8 \pm 0.8$ & $\begin{array}{l}71.6 \\
72.5 \pm 1.7 \\
66.0 \pm 2.1 \\
79.6 \pm 4.0\end{array}$ & $\begin{array}{l}82.4 \pm 0.8 \\
83.3 \pm 1.9 \\
76.8 \pm 2.2 \\
90.4 \pm 4.1\end{array}$ & $\begin{array}{l}85.3 \pm 0.2 \\
74.6 \pm 0.3\end{array}$ \\
\hline $\mathrm{C}_{14} \mathrm{H}_{10} \mathrm{O}_{2}$ & benzil & 23.1 & 368 & 69.2 & 416 & 98.4 & 329 & 351.6 & 248.0 & $18.6 \pm 1.5$ & $81.2 \pm 2.8$ & $99.8 \pm 2.2$ & 99.62 .0 \\
\hline $\mathrm{C}_{14} \mathrm{H}_{22} \mathrm{O}$ & 2,6-di-t-butylphenol & 16.57 & 310.7 & 60.4 & 401 & $\begin{array}{r}84.6 \\
81.5 \\
110.9\end{array}$ & $\begin{array}{l}298 \\
298 \\
298\end{array}$ & 401.8 & 311.1 & $15.7 \pm 0.3$ & $72.2 \pm 2.4$ & $88.0 \pm 2.5$ & $\begin{array}{r}84.6 \\
81.5 \\
110.9\end{array}$ \\
\hline $\mathrm{C}_{16} \mathrm{H}_{10}$ & fluoranthene & 18.8 & 383.3 & $\begin{array}{l}62.3 \\
86.2 \\
77.4\end{array}$ & $\begin{array}{l}518 \\
495 \\
398\end{array}$ & $\begin{array}{r}98.3 \\
84.6 \\
99.2 \\
102.1 \\
102.6\end{array}$ & $\begin{array}{l}298 \\
303 \\
298 \\
340 \\
328\end{array}$ & 310.5 & 226.6 & $14.0 \pm 1.6$ & $\begin{array}{r}82.4 \pm 5.3 \\
104.2 \pm 4.7 \\
86.5 \pm 2.4\end{array}$ & $\begin{array}{r}96.4 \pm 5.5 \\
118.2 \pm 5.0 \\
100.5 \pm 2.9\end{array}$ & $\begin{array}{l}98.3 \\
84.8 \pm 0.1 \\
99.2 \\
103.6 \pm 0.5 \\
103.6 \pm 0.3\end{array}$ \\
\hline $\mathrm{C}_{17} \mathrm{H}_{34} \mathrm{O}_{2}$ & methylhexadecanoate & 55.35 & 305.2 & $\begin{array}{l}69.6 \\
82.4 \\
82.6 \\
71.4\end{array}$ & $\begin{array}{l}302 \\
426 \\
393 \\
437\end{array}$ & 152.3 & 296 & 579.6 & 490.1 & $\begin{array}{l}67.4 \pm 0.3 \\
54.7 \pm 0.2\end{array}$ & $\begin{array}{r}70.2 \pm 0.1 \\
103.0 \pm 3.1 \\
97.9 \pm 2.3 \\
93.8 \pm 3.3\end{array}$ & $\begin{array}{l}137.6 \pm 0.3 \\
170.6 \pm 3.1 \\
165.3 \pm 2.3 \\
161.2 \pm 3.3 \\
125.0 \pm 0.2 \\
157.8 \pm 3.1 \\
152.6 \pm 2.3 \\
148.5 \pm 3.3\end{array}$ & $152 . \pm 0.1$ \\
\hline
\end{tabular}

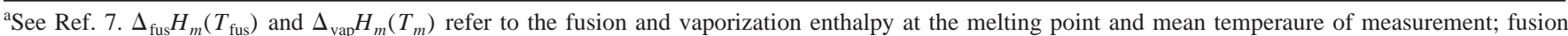
enthalpies are from Chickos et al. ${ }^{6}$ and Domalski and Hearing ${ }^{14}$; sublimation enthalpies from Chickos and Acree; ${ }^{7}$ vaporization enthalpies from Table 6 ; uncertainties in fusion and vaporization enthalpies $( \pm 2 \sigma)$ are those associated with the temperature adjustment only.

internally consistent. The fusion enthalpy reported is the average of two literature values. ${ }^{14}$ Similarly with 2,6 -di-tbutylphenol and fluoranthene. The sublimation enthalpies measured directly and those calculated by Eq. (7) agree within $\pm 4.0 \mathrm{~kJ} \mathrm{~mol}^{-1}$, which is the accuracy typical of many experimental sublimation and vaporization enthalpy measurements.

The scatter of data obtained for methyl hexadecanoate illustrate another common situation that can arise when data from the literature are examined. Two fusion enthalpies and several vaporization enthalpies have been reported which are not in particularly good agreement. Using the value of the sublimation enthalpy as a guide, consistent results are obtained if the fusion enthalpy of $54.7 \mathrm{~kJ} \mathrm{~mol}^{-1}(298.15 \mathrm{~K})$ is combined with the mean of the last three vaporization enthalpies, $98.3 \pm 5.3 \mathrm{~kJ} \mathrm{~mol}^{-1}$, resulting in a sublimation enthalpy of $152.9 \pm 5.4 \mathrm{~kJ} \mathrm{~mol}^{-1}$.

\section{Estimation of Vaporization Enthalpies}

Vaporization enthalpy is a property that is successfully modeled by various computational methods. Many estimation methods in the chemical engineering literature have been developed to provide vaporization enthalpies near or at the boiling point and are accurate to a few $\%$. Some require critical constants and other parameters that may have to be estimated themselves. A summary of some of the existing methods and their applications can be found in the book edited by Lyman et al. ${ }^{10}$ (see also Ref. 15). The estimation of vaporization enthalpy at or near the boiling point continues to be a topic of recurring interest. ${ }^{16-23}$

A number of methods and equations for the estimation of vaporization enthalpies at $T=298.15 \mathrm{~K}$ have also been developed. Some model vaporization enthalpies on concepts of group additivity while some are based on other thermodynamic principles. ${ }^{24-35}$ Details on the application of some of these methods in estimating vaporization enthalpies are available. $^{4,10,25}$

\section{Vaporization Enthalpy Compendium}

Vaporization enthalpies reported from the 1880s to 2002 are included in Tables 6 and 7. Table 5 contains a listing of the acronyms and abbreviations that are used in both tables. Table 6 contains vaporization enthalpy data for organic compounds; Table 7 contains similar information for a selected number of organometallic and inorganic substances. Information in the tables is organized as described below. 
TABLE 5. A list of acronyms and abbreviations used in Tables 6 and 7

\begin{tabular}{ll}
\hline \hline A & $\begin{array}{l}\text { calculated from the vapor pressure data reported by the } \\
\text { method of least squares } \\
\text { calculated from the difference of the enthalpies of } \\
\text { B }\end{array}$ \\
& poblimation at temperature T, and fusion at the melting \\
BG & Bourdon gauge \\
C & calorimetric determination \\
GC & gas chromatography \\
GCC & gas chromatography-calorimetry \\
CGC & correlation gas chromatography \\
DM & diaphram manometer \\
DSC & differential scanning calorimeter \\
EB & ebullometry \\
EST & estimated value \\
GS & gas saturation, transpiration \\
HG & Heise gauge \\
HSA & head space analysis \\
I & isoteniscope \\
IPM & inclined piston manometry \\
ME & mass effusion-Knudsen effusion \\
MG & McLeod gauge \\
MM & mercury manometer \\
NA & not available at the time of publication \\
OM & oil manometer \\
RG & Rodebush gauge \\
SG & spoon gauge \\
STG & strain gauge \\
T & tensimeter \\
TE & ultraviolet absorption \\
UV & \\
\hline &
\end{tabular}

Compounds are arranged according to molecular formula. The compound's name, occasionally a synonym, and the CAS registry number are included on the first line. The range of temperatures studied is the next entry in the table. For measurements performed at a constant temperature or when not specified, this entry is left blank. The vaporization enthalpy at the mean temperature of measurement, $\Delta_{\text {vap }} H_{m}\left(T_{m}\right)$, is the next entry followed by the mean temperature $\left(T_{m} / \mathrm{K}\right)$, an acronym or abbreviation briefly describing the type of measurement and the reference to the original work. In some cases the type of measurement was not available, or recorded. In these instances this entry was left blank. If the authors of the work have adjusted their results to $298.15 \mathrm{~K}$, then this information along with the reference is entered on the third line. This information is repeated for multiple measurements. The measurements are arranged in reverse chronological order. Entries for compounds with deuterium substitution are listed after the unlabeled parent.

The authors have made an effort to present the data accurately and without error. Much of the earlier data have been retrieved with the aid of existing compendia that have been published over the years. Most of these compendia include references to the original literature. A notable exception is the very useful reference Handbook of the Thermodynamics of Organic Compounds by Stephenson and Malanowski published in 1987 by Elsevier Science Publishing Co. Some of the data included in this compendium, notably for the higher $n$-alkanes and haloalkanes, appears to be data that have been estimated. $^{36,37}$ Estimated vaporization enthalpies are generally not included in Table 5 unless the data are reported in the Stephenson and Malanowski compendium or the source is identified. The reader should exercise some caution when using data that are not referenced to the primary literature. Additionally, some of the information has originated from non-English language journals with translations occasionally provided by the author's students. These tables, having been complied over a period of twenty years, have gone through numerous revisions. Some errors have been corrected; however it is unlikely that all of them have been detected. The reader is encouraged to consult the original literature when using this data.

Several sources of data contains vapor pressure data represented in the form of the Antoine equation [Eq. (8)], where $P$ represents the vapor pressure, $T$ is the temperature in $\mathrm{K}$, and $A, B$, and $C$ are the Antoine constants obtained from treatment of the vapor pressure-temperature data

$$
\log _{10} P=A-B /(C+T) \text {. }
$$

Vaporization enthalpies were calculated from the Antoine constants in one of two ways. A Lotus macro was written to calculate vapor pressures using the Antoine constants over a $30 \mathrm{~K}$ temperature range; the resulting vapor pressuretemperature data were reformulated in terms of the integrated form of the Clausius-Clapeyron equation. $A \ln P$ versus $1 / T$ linear regression analysis provided $\Delta_{\mathrm{vap}} H_{m}\left(T_{m}\right) / R$ at the mean temperature, $T_{m}$, of the $30 \mathrm{~K}$ range. The $30 \mathrm{~K}$ temperature range was chosen closest to $T=298.15 \mathrm{~K}$ but within the temperature range specified. Alternatively, vaporization enthalpy was calculated using the Antoine constants from the following equation:

$$
\Delta_{\text {vap }} H_{m}\left(T_{m}\right)=2.303 R B\left[T_{m} /\left(T_{m}+\mathrm{C}\right)\right]^{2} .
$$

Temperatures were chosen to lie with the range of measurement, often at the mean temperature. In a number of instances, the authors only provide vapor pressuretemperature data. In these instances, a vaporization enthalpy was calculated from the data as described above.

An examination of data in Tables 6 and 7 reveals that many compounds have been measured repeatedly. All the data here are treated equally; there has been no attempt to recommend or identify recommended values. A critical evaluation of the data is beyond the scope of this compendium. However, the reader should be aware of a number of critical reviews that are available. The IUPAC monograph, "Enthalpies of Vaporization of Organic Compounds by Majer and Svoboda ${ }^{12}$ is among the most extensive evaluations available. Others are included in the references associated with Tables 6 and 7. The reader is encouraged to consult these references before deciding on the best value to use. 
TABLE 6. Enthalpies of vaporization of organic compounds, 1880-2002

\begin{tabular}{|c|c|c|c|c|c|}
\hline Molecular formula & $\begin{array}{c}\text { Compound } \\
\text { (Temperature range/K) }\end{array}$ & $\begin{array}{c}\Delta_{\mathrm{vap}} H_{m} \\
\left(\mathrm{~kJ} \mathrm{~mol}^{-1}\right)\end{array}$ & $\begin{array}{c}\text { Mean temperature } \\
\qquad\left(T_{m} / \mathrm{K}\right)\end{array}$ & Method & $\begin{array}{c}\text { CAS registry number } \\
\text { Reference }\end{array}$ \\
\hline \multirow[t]{7}{*}{$\mathrm{CBrClF}_{2}$} & bromochlorodifluoromethane & & & & {$[353-59-3]$} \\
\hline & $(268-324)$ & 23.0 & $(283)$ & A & {$[87 / 5]$} \\
\hline & $(194-287)$ & 23.1 & $(272)$ & $\mathrm{A}$ & {$[87 / 5]$} \\
\hline & $(321-403)$ & 22.4 & (336) & A & {$[87 / 5]$} \\
\hline & $(403-427)$ & 23.1 & (415) & A & {$[87 / 5]$} \\
\hline & $(178-283)$ & 26.0 & (193) & & [79/12] \\
\hline & $(178-283)$ & 18.7 & $(268)$ & & {$[60 / 16][84 / 9]$} \\
\hline \multirow[t]{3}{*}{$\mathrm{CBrCl}_{3}$} & bromotrichloromethane & & & & {$[75-62-7]$} \\
\hline & $(273-387)$ & 35.0 & (288) & & [79/12] \\
\hline & $(294-443)$ & 36.1 & (309) & A & {$[87 / 5][70 / 16]$} \\
\hline \multirow[t]{4}{*}{$\mathrm{CBrF}_{3}$} & bromotrifluoromethane & & & & {$[75-63-8]$} \\
\hline & $(276-340)$ & 17.8 & (291) & A & {$[87 / 5]$} \\
\hline & $(160-267)$ & 17.7 & $(252)$ & A & {$[87 / 5]$} \\
\hline & $(165-216)$ & 19.1 & $(180)$ & & [79/12] \\
\hline \multirow[t]{2}{*}{$\mathrm{CBrFO}$} & carbonic bromide fluoride & & & & {$[753-56-0]$} \\
\hline & $(197-256)$ & 22.9 & $(241)$ & A & {$[87 / 5]$} \\
\hline \multirow[t]{2}{*}{$\mathrm{CBrN}$} & cyanogen bromide & & & & [506-68-3] \\
\hline & $(273-313)$ & 45.9 & (288) & & {$[54 / 14][84 / 9]$} \\
\hline \multirow[t]{2}{*}{$\mathrm{CBrN}_{3} \mathrm{O}_{6}$} & bromotrinitromethane & & & & {$[560-95-2]$} \\
\hline & $(318-335)$ & 47.8 & $(326)$ & A & {$[87 / 5][70 / 10]$} \\
\hline \multirow[t]{4}{*}{$\mathrm{CBr}_{2} \mathrm{~F}_{2}$} & dibromodifluoromethane & & & & {$[75-61-6]$} \\
\hline & $(247-297)$ & 26.1 & $(282)$ & A & {$[87 / 5][59 / 1]$} \\
\hline & & & & & {$[79 / 12][70 / 16]$} \\
\hline & $(156-218)$ & 18.6 & (203) & & {$[48 / 7]$} \\
\hline \multirow[t]{2}{*}{$\mathrm{CBr}_{3} \mathrm{~F}$} & tribromofluoromethane & & & & {$[353-54-8]$} \\
\hline & $(315-380)$ & 34.4 & $(330)$ & A & {$[87 / 5][48 / 7]$} \\
\hline \multirow[t]{3}{*}{$\mathrm{CBr}_{4}$} & carbon tetrabromide & & & & {$[558-13-4]$} \\
\hline & $(375-463)$ & 48.3 & $(390)$ & & {$[79 / 12]$} \\
\hline & $(369-463)$ & 48.2 & $(384)$ & A & {$[87 / 5][47 / 5]$} \\
\hline \multirow[t]{3}{*}{$\mathrm{CClFO}$} & carbonic chloride fluoride & & & & {$[353-49-1]$} \\
\hline & $(165-211)$ & 22.7 & (196) & A & {$[87 / 5][64 / 3]$} \\
\hline & $(157-227)$ & 22.0 & (192) & & {$[48 / 17]$} \\
\hline \multirow{2}{*}{$\mathrm{CClF}_{2} \mathrm{NO}$} & difluorocarbamoyl chloride & & & & [16847-30-6] \\
\hline & $(189-234)$ & 25.8 & (219) & A & {$[87 / 5]$} \\
\hline \multirow[t]{8}{*}{$\mathrm{CClF}_{3}$} & chlorotrifluoromethane & & & & [75-72-9] \\
\hline & $(268-302)$ & 16.0 & (283) & A & {$[87 / 5]$} \\
\hline & $(133-185)$ & 17.0 & $(170)$ & A & {$[87 / 5]$} \\
\hline & $(184-246)$ & 15.7 & $(231)$ & A & {$[87 / 5]$} \\
\hline & $(243-271)$ & 15.7 & $(257)$ & A & {$[87 / 5]$} \\
\hline & $(145-192)$ & 16.8 & $(177)$ & A & {$[87 / 5][79 / 12]$} \\
\hline & $(124-191)$ & 17.1 & $(177)$ & A & {$[47 / 5]$} \\
\hline & $(134-298)$ & NA & & & [41/13] \\
\hline \multirow{3}{*}{$\mathrm{CClF}_{3} \mathrm{O}$} & trifluoromethyl hypochlorite & & & & [22082-78-6] \\
\hline & $(160-226)$ & 21.2 & $(211)$ & A & {$[87 / 5]$} \\
\hline & $(142-219)$ & 19.6 & (204) & A & {$[87 / 5]$} \\
\hline \multirow[t]{2}{*}{$\mathrm{CClF}_{3} \mathrm{O}_{2}$} & peroxyhypochlorous acid, tri & thyl ester & & & [32755-26-3] \\
\hline & $(163-296)$ & 23.4 & $(281)$ & A & {$[87 / 5]$} \\
\hline $\mathrm{CClF}_{3} \mathrm{O}_{3} \mathrm{~S}$ & fluorosulfuric acid, chlorodif & hyl ester & & & [6069-31-4] \\
\hline & $(227-309)$ & 32.1 & $(243)$ & & [99/16] \\
\hline & $(228-310)$ & 34.6 & $(243)$ & A & {$[87 / 5][66 / 15]$} \\
\hline $\mathrm{CClF}_{3} \mathrm{~S}$ & trifluoromethanesulfenyl chlc & & & & [421-17-0] \\
\hline & $(247-272)$ & 24.5 & $(260)$ & A & {$[87 / 5][99 / 16]$} \\
\hline $\mathrm{CClF}_{4} \mathrm{~N}$ & difluoro(difluorochloromethy & & & & [13880-71-2] \\
\hline & $(209-277)$ & 26.6 & $(262)$ & A & {$[87 / 5]$} \\
\hline $\mathrm{CClF}_{4} \mathrm{NO}_{2} \mathrm{~S}$ & chloro(trifluoromethyl) sulfa & oride & & & [19419-95-5] \\
\hline & $(253-288)$ & 28.8 & (273) & A & {$[87 / 5][99 / 16]$} \\
\hline $\mathrm{CClF}_{4} \mathrm{NO}_{12} \mathrm{~S}_{4}$ & fluorosulfuric acid, bis $[$ [(flur & 1)oxy]amino] & nethylene ester & & [53684-03-0] \\
\hline & & 42.6 & $(424)$ & & {$[75 / 21]$} \\
\hline $\mathrm{CClF}_{7} \mathrm{~S}$ & chlorotetrafluoro (trifluorome & fur & & & [42179-04-4] \\
\hline & $(293-353)$ & 25.9 & $(323)$ & & [99/16] \\
\hline $\mathrm{CClN}$ & cyanogen chloride & & & & {$[506-77-4]$} \\
\hline & $(196-286)$ & 32.2 & $(271)$ & & {$[47 / 5]$} \\
\hline $\mathrm{CCl}_{2} \mathrm{FNO}$ & dichlorocarbamic fluoride & & & & [32751-02-3] \\
\hline & & 40.7 & & & {$[72 / 40]$} \\
\hline
\end{tabular}


TABLE 6. Enthalpies of vaporization of organic compounds, 1880-2002-Continued

\begin{tabular}{|c|c|c|c|c|c|}
\hline Molecular formula & $\begin{array}{c}\text { Compound } \\
\text { (Temperature range/K) }\end{array}$ & $\begin{array}{c}\Delta_{\mathrm{vap}} H_{m} \\
\left(\mathrm{~kJ} \mathrm{~mol}^{-1}\right)\end{array}$ & $\begin{array}{l}\text { Mean temperature } \\
\qquad\left(T_{m} / \mathrm{K}\right)\end{array}$ & Method & $\begin{array}{c}\text { CAS registry number } \\
\text { Reference }\end{array}$ \\
\hline \multirow[t]{8}{*}{$\mathrm{CCl}_{2} \mathrm{~F}_{2}$} & \multicolumn{4}{|l|}{ dichlorodifluoromethane } & {$[75-71-8]$} \\
\hline & $(282-345)$ & 20.0 & $(297)$ & A & {$[87 / 5]$} \\
\hline & $(173-244)$ & 21.4 & (229) & A & {$[87 / 5]$} \\
\hline & $(173-240)$ & 21.6 & $(225)$ & A & {$[87 / 5]$} \\
\hline & $(236-285)$ & 20.4 & $(270)$ & A & {$[87 / 5]$} \\
\hline & $(341-385)$ & 20.5 & $(356)$ & A & {$[87 / 5]$} \\
\hline & $(172-279)$ & 22.9 & $(187)$ & & {$[79 / 12]$} \\
\hline & $(154-243)$ & 21.5 & $(228)$ & & {$[47 / 5]$} \\
\hline \multirow[t]{2}{*}{$\mathrm{CCl}_{2} \mathrm{~F}_{3} \mathrm{~N}$} & \multicolumn{5}{|c|}{$\mathrm{N}, \mathrm{N}$-difluoro-1,1-dichloro-1-fluoromethylamine } \\
\hline & $(209-277)$ & 27.0 & $(262)$ & I & {$[70 / 15]$} \\
\hline \multirow[t]{2}{*}{$\mathrm{CCl}_{2} \mathrm{~F}_{3} \mathrm{~N}$} & \multicolumn{4}{|c|}{$\mathrm{N}, \mathrm{N}$-dichloro-1,1,1-trifluoromethylamine } & {$[13880-73-4]$} \\
\hline & $(226-291)$ & 25.8 & $(276)$ & A & {$[87 / 5]$} \\
\hline \multirow[t]{2}{*}{$\mathrm{CCl}_{2} \mathrm{~F}_{3} \mathrm{~N}$} & \multicolumn{5}{|c|}{$\mathrm{N}, 1$-dichloro-N,1,1-trifluoromethylamine } \\
\hline & $(226-291)$ & 26.4 & $(258)$ & & {$[71 / 17]$} \\
\hline \multirow[t]{3}{*}{$\mathrm{CCl}_{2} \mathrm{~F}_{3} \mathrm{NS}$} & \multicolumn{4}{|c|}{ (trifluoromethyl)imidosulfurous dichloride } & [10564-47-3] \\
\hline & $(284-344)$ & 35.4 & $(298)$ & & {$[99 / 16]$} \\
\hline & $(283-362)$ & 33.7 & $(298)$ & A & {$[87 / 5]$} \\
\hline \multirow{2}{*}{$\mathrm{CCl}_{2} \mathrm{~F}_{3} \mathrm{P}$} & \multicolumn{2}{|c|}{ (trifluoromethyl)dichlorophosphine } & & & {$[421-58-9]$} \\
\hline & $(208-276)$ & 29.2 & (260) & & {$[64 / 4]$} \\
\hline \multirow[t]{2}{*}{$\mathrm{CCl}_{2} \mathrm{~F}_{3} \mathrm{PS}$} & \multicolumn{2}{|c|}{ dichloro(trifluoromethylthio)phosphine } & & & {$[18799-78-5]$} \\
\hline & $(293-363)$ & 31.7 & $(308)$ & A & $\begin{array}{c}{[87 / 5][99 / 16]} \\
{[60 / 25]}\end{array}$ \\
\hline $\mathrm{CCl}_{2} \mathrm{O}$ & phosgene & & & & {$[75-44-5]$} \\
\hline & $(280-341)$ & 24.5 & $(295)$ & A & {$[87 / 5]$} \\
\hline & $(240-281)$ & 25.7 & $(266)$ & A & {$[87 / 5]$} \\
\hline & $(338-410)$ & 24.5 & $(353)$ & A & {$[87 / 5]$} \\
\hline & $(406-455)$ & 24.4 & $(421)$ & A & {$[87 / 5]$} \\
\hline & $(215-248)$ & 27.0 & (233) & & {$[48 / 2]$} \\
\hline & $(180-273)$ & 25.8 & $(258)$ & & {$[47 / 5]$} \\
\hline $\mathrm{CCl}_{3} \mathrm{~F}$ & trichlorofluoromethane & & & & {$[75-69-4]$} \\
\hline & $(213-301)$ & 28.5 & $(228)$ & $\mathrm{A}$ & {$[87 / 5]$} \\
\hline & $(213-249)$ & 28.2 & $(234)$ & $\mathrm{A}$ & {$[87 / 5]$} \\
\hline & $(295-363)$ & 25.6 & $(310)$ & A & {$[87 / 5]$} \\
\hline & $(357-429)$ & 24.7 & $(372)$ & $\mathrm{A}$ & {$[87 / 5]$} \\
\hline & $(424-468)$ & 25.1 & (439) & $\mathrm{A}$ & {$[87 / 5]$} \\
\hline & $(237-293)$ & 27.3 & $(251)$ & & {$[79 / 12]$} \\
\hline & $(237-293)$ & 27.1 & $(276)$ & & {$[41 / 4]$} \\
\hline & & 25.2 & $(290)$ & $\mathrm{C}$ & {$[41 / 4]$} \\
\hline & $(244-334)$ & 26.4 & $(259)$ & & {$[40 / 1]$} \\
\hline $\mathrm{CCl}_{3} \mathrm{~F}_{2} \mathrm{~N}$ & $\mathrm{~N}, \mathrm{~N}$-difluoro-1,1,1-trichlor & nine & & & \\
\hline & $(252-325)$ & 33.4 & $(267)$ & I & {$[87 / 5][70 / 15]$} \\
\hline $\mathrm{CCl}_{3} \mathrm{~F}_{2} \mathrm{~N}$ & $\mathrm{~N}, 1,1$-trichloro-N,1-difluor & nine & & & \\
\hline & $(273-319)$ & 27.8 & (296) & & [71/17] \\
\hline $\mathrm{CCl}_{3} \mathrm{~F}_{2} \mathrm{P}$ & difluoro(trichloromethyl)ph & & & & [1112-03-4] \\
\hline & $(289-313)$ & 32.5 & $(301)$ & A & {$[87 / 5]$} \\
\hline $\mathrm{CCl}_{3} \mathrm{NO}$ & trichloronitrosomethane & & & & [3711-49-7] \\
\hline & $(253-333)$ & 32.4 & $(268)$ & A & {$[87 / 5]$} \\
\hline $\mathrm{CCl}_{3} \mathrm{NO}_{2}$ & trichloronitromethane & & & & [76-06-2] \\
\hline & $(273-333)$ & 39.3 & $(288)$ & $\mathrm{A}$ & {$[87 / 5]$} \\
\hline & $(301-449)$ & 38.5 & (316) & $\mathrm{A}$ & {$[87 / 5][70 / 16]$} \\
\hline & $(247-385)$ & 40.0 & $(262)$ & & {$[47 / 5]$} \\
\hline $\mathrm{CCl}_{4}$ & carbon tetrachloride & & & & {$[56-23-5]$} \\
\hline & $(349-416)$ & 30.4 & $(364)$ & A & {$[87 / 5]$} \\
\hline & $(412-497)$ & 29.2 & $(427)$ & A & {$[87 / 5]$} \\
\hline & $(494-555)$ & 30.6 & (509) & A & {$[87 / 5]$} \\
\hline & & 32.4 & (298) & $\mathrm{C}$ & {$[80 / 1]$} \\
\hline & $(262-349)$ & 33.7 & (277) & $\mathrm{A}, \mathrm{EB}$ & {$[87 / 5][72 / 6]$} \\
\hline & $(293-351)$ & 32.3 & (308) & & {$[59 / 2]$} \\
\hline & $(313-338)$ & 31.7 & $(325)$ & & {$[53 / 1]$} \\
\hline CFIO & carbonyl fluoride iodide & & & & \\
\hline & $(230-292)$ & 26.1 & $(277)$ & $\mathrm{A}$ & {$[87 / 5]$} \\
\hline $\mathrm{CFN}$ & cyanogen fluoride & & & & {$[1495-50-7]$} \\
\hline & $(201-227)$ & 22.4 & $(214)$ & $\mathrm{A}$ & $\begin{array}{c}{[87 / 5][64 / 25]} \\
{[70 / 16]}\end{array}$ \\
\hline
\end{tabular}


TABLE 6. Enthalpies of vaporization of organic compounds, 1880-2002-Continued

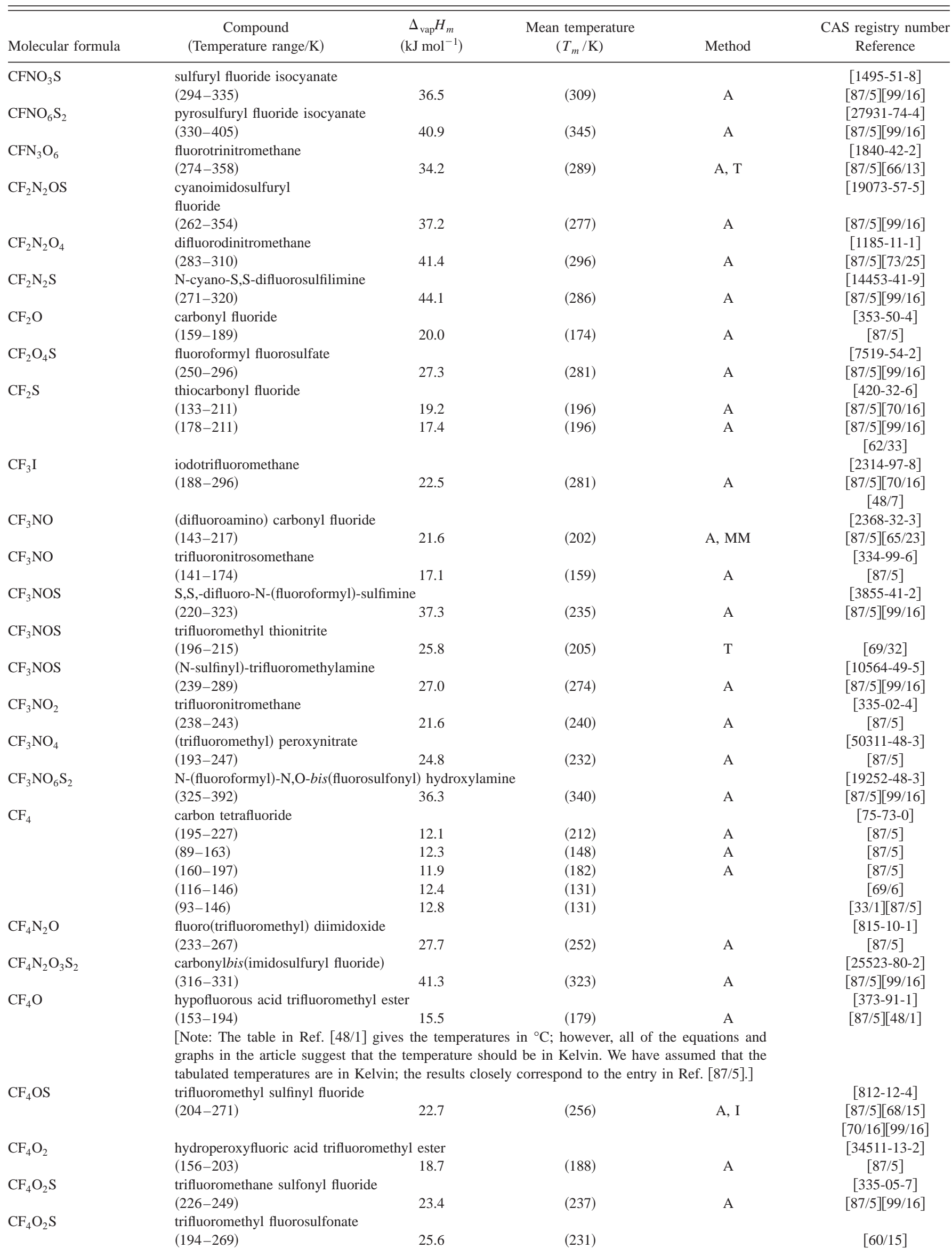


TABLE 6. Enthalpies of vaporization of organic compounds, 1880-2002-Continued

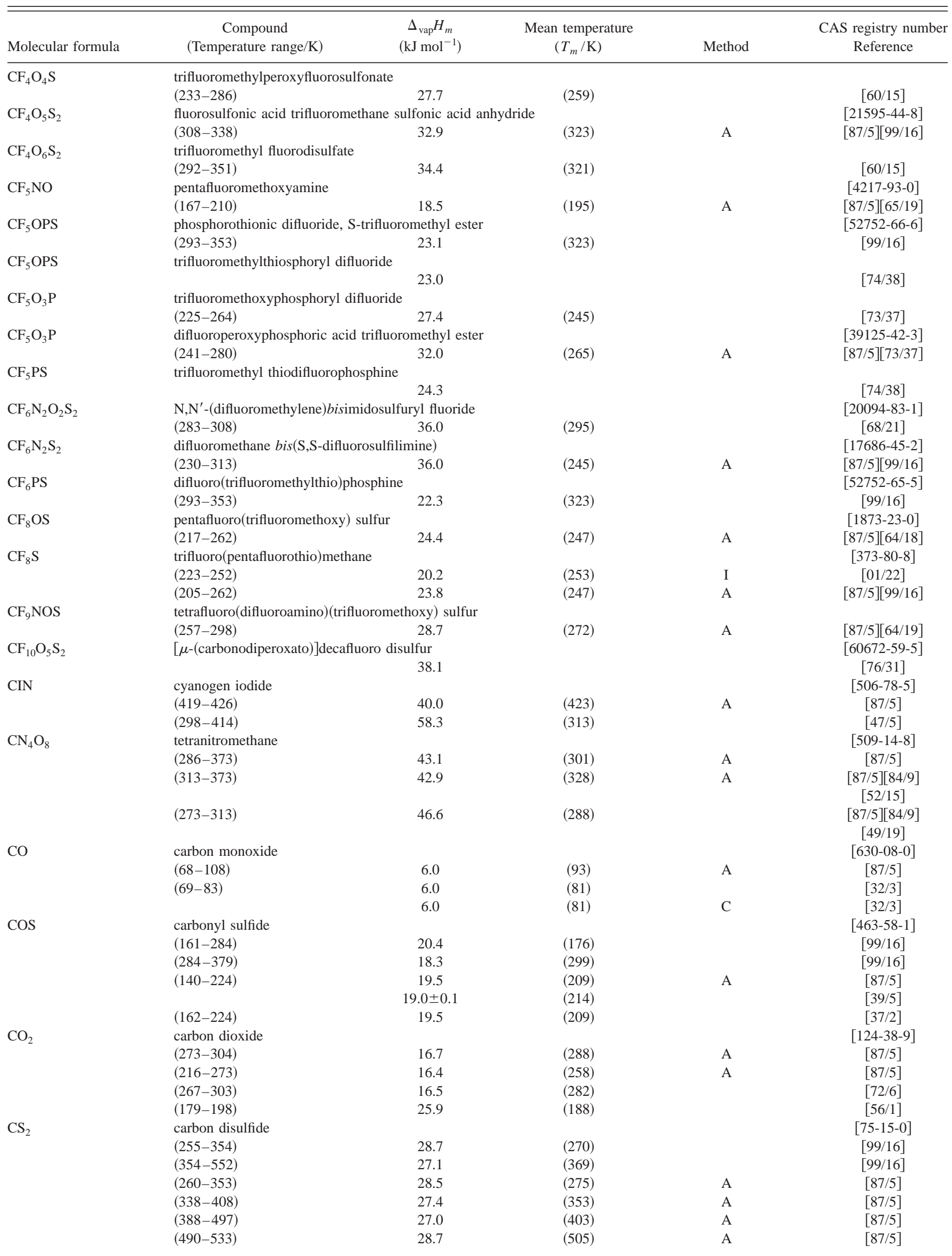


TABLE 6. Enthalpies of vaporization of organic compounds, 1880-2002-Continued

\begin{tabular}{|c|c|c|c|c|c|}
\hline Molecular formula & $\begin{array}{c}\text { Compound } \\
\text { (Temperature range/K) }\end{array}$ & $\begin{array}{c}\Delta_{\mathrm{vap}} H_{m} \\
\left(\mathrm{~kJ} \mathrm{~mol}^{-1}\right)\end{array}$ & $\begin{array}{l}\text { Mean temperature } \\
\qquad\left(T_{m} / \mathrm{K}\right)\end{array}$ & Method & $\begin{array}{c}\text { CAS registry number } \\
\text { Reference }\end{array}$ \\
\hline & $(255-318)$ & 28.7 & $(270)$ & $\mathrm{EB}$ & {$[72 / 6][87 / 5]$} \\
\hline & $(277-353)$ & 28.1 & $(292)$ & $\mathrm{EB}$ & {$[62 / 2]$} \\
\hline & & $28.1 \pm 0.1$ & $(282)$ & $\mathrm{C}$ & {$[62 / 2]$} \\
\hline & & $27.5 \pm 0.1$ & $(298)$ & $\mathrm{C}$ & {$[62 / 2]$} \\
\hline & & $26.7 \pm 0.1$ & (319) & $\mathrm{C}$ & {$[62 / 2]$} \\
\hline & & 27.7 & $(298)$ & & {$[61 / 25]$} \\
\hline & $(303-358)$ & 27.6 & $(318)$ & & {$[46 / 4]$} \\
\hline \multirow[t]{3}{*}{$\mathrm{CHBrF}_{2}$} & bromodifluoromethane & & & & {$[1511-62-2]$} \\
\hline & $(194-259)$ & 24.0 & $(244)$ & A & {$[87 / 5]$} \\
\hline & $(194-288)$ & 24.7 & $(209)$ & & {$[79 / 12]$} \\
\hline \multirow[t]{4}{*}{$\mathrm{CHBr}_{3}$} & tribromomethane & & & & {$[75-25-2]$} \\
\hline & & $46.1 \pm 0.1$ & $(298)$ & $\mathrm{C}$ & {$[72 / 41]$} \\
\hline & $(320-412)$ & 42.3 & $(335)$ & EB & {$[72 / 6][79 / 12]$} \\
\hline & $(303-373)$ & 44.0 & $(318)$ & & {$[41 / 7][84 / 9]$} \\
\hline \multirow[t]{8}{*}{$\mathrm{CHClF}_{2}$} & chlorodifluoromethane & & & & {$[75-45-6]$} \\
\hline & $(275-327)$ & 20.0 & $(290)$ & A & {$[87 / 5]$} \\
\hline & $(170-233)$ & 21.3 & $(218)$ & A & {$[87 / 5]$} \\
\hline & $(230-275)$ & 20.4 & $(260)$ & A & {$[87 / 5]$} \\
\hline & $(324-366)$ & 20.1 & (339) & A & {$[87 / 5]$} \\
\hline & $(194-310)$ & 21.8 & $(209)$ & & {$[79 / 12]$} \\
\hline & $(229-236)$ & 21.0 & $(232)$ & & {$[64 / 2]$} \\
\hline & & 20.2 & $(232)$ & $\mathrm{C}$ & {$[57 / 18]$} \\
\hline \multirow[t]{8}{*}{$\mathrm{CHCl}_{2} \mathrm{~F}$} & dichlorofluoromethane & & & & {$[75-43-4]$} \\
\hline & $(225-282)$ & 26.1 & $(267)$ & A & {$[87 / 5]$} \\
\hline & $(279-344)$ & 25.3 & $(294)$ & A & {$[87 / 5]$} \\
\hline & $(341-399)$ & 24.2 & $(356)$ & A & {$[87 / 5]$} \\
\hline & $(397-450)$ & 24.1 & $(412)$ & A & {$[87 / 5]$} \\
\hline & $(229-236)$ & U20.9 & $(233)$ & & {$[64 / 2]$} \\
\hline & $(181-282)$ & 26.2 & $(267)$ & & {$[47 / 5]$} \\
\hline & $(244-317)$ & 36.7 & $(259)$ & & {$[40 / 1]$} \\
\hline \multirow[t]{2}{*}{$\mathrm{CHCl}_{2} \mathrm{FO}_{3} \mathrm{~S}$} & fluorosulfuric acid, dichlo & ster & & & [42016-50-2] \\
\hline & $(275-293)$ & 36.2 & $(284)$ & A & {$[87 / 5][99 / 16]$} \\
\hline \multirow[t]{10}{*}{$\mathrm{CHCl}_{3}$} & chloroform & & & & [67-66-3] \\
\hline & $(306-427)$ & 30.8 & $(321)$ & & {$[95 / 16]$} \\
\hline & $(227-269)$ & 31.8 & $(254)$ & A & {$[87 / 5]$} \\
\hline & $(333-416)$ & 30.4 & $(348)$ & A & {$[87 / 5]$} \\
\hline & $(410-481)$ & 28.9 & $(425)$ & A & {$[87 / 5]$} \\
\hline & $(479-523)$ & 30.1 & $(494)$ & A & {$[87 / 5]$} \\
\hline & & 31.1 & $(298)$ & $\mathrm{C}$ & {$[80 / 1]$} \\
\hline & $(260-333)$ & 32.5 & $(275)$ & EB & {$[72 / 6]$} \\
\hline & $(215-334)$ & 35.0 & $(230)$ & & {$[47 / 5]$} \\
\hline & $(308-333)$ & 30.9 & $(320)$ & & {$[38 / 5]$} \\
\hline \multirow[t]{2}{*}{$\mathrm{CHFI}_{2}$} & diiodofluoromethane & & & & {$[1493-01-2]$} \\
\hline & $(299-332)$ & 32.9 & $(314)$ & A & $\begin{array}{c}{[87 / 5][79 / 12]} \\
{[70 / 16]}\end{array}$ \\
\hline \multirow[t]{2}{*}{$\mathrm{CHFN}_{2} \mathrm{O}_{4}$} & fluorodinitromethane & & & & [7182-87-8] \\
\hline & $(298-338)$ & 43.6 & $(313)$ & A & {$[87 / 5]$} \\
\hline \multirow[t]{2}{*}{$\mathrm{CHFO}$} & formyl fluoride & & & & [1493-02-3] \\
\hline & $(178-235)$ & 24.4 & $(220)$ & A & $\begin{array}{c}{[87 / 5][64 / 3]} \\
{[70 / 16]}\end{array}$ \\
\hline \multirow[t]{2}{*}{$\mathrm{CHF}_{2} \mathrm{I}$} & difluoroiodomethane & & & & [1493-03-4] \\
\hline & $(227-287)$ & 26.0 & $(272)$ & A & $\begin{array}{c}{[87 / 5][79 / 12]} \\
{[70 / 16]}\end{array}$ \\
\hline \multirow[t]{4}{*}{$\mathrm{CHF}_{3}$} & trifluoromethane & & & & {$[75-46-7]$} \\
\hline & $(138-190)$ & 18.1 & $(175)$ & A & {$[87 / 5]$} \\
\hline & $(198-298)$ & 16.8 & $(213)$ & A & {$[87 / 5]$} \\
\hline & $(146-192)$ & 18.0 & $(177)$ & & {$[62 / 1]$} \\
\hline \multirow[t]{2}{*}{$\mathrm{CHF}_{3} \mathrm{O}_{2}$} & trifluoromethyl hydropero & & & & [16156-36-8] \\
\hline & $(248-285)$ & 30.9 & $(270)$ & A & {$[87 / 5]$} \\
\hline \multirow[t]{2}{*}{$\mathrm{CHF}_{3} \mathrm{O}_{3} \mathrm{~S}$} & $\begin{array}{l}\text { trifluoromethylsulfonic } \\
\text { acid }\end{array}$ & & & & {$[1493-13-6]$} \\
\hline & $(354-435)$ & 47.7 & $(369)$ & A & {$[87 / 5][99 / 16]$} \\
\hline \multirow[t]{2}{*}{$\mathrm{CHF}_{3} \mathrm{~S}$} & trifluoromethanethiol & & & & {$[1493-15-8]$} \\
\hline & $(167-236)$ & 21.8 & $(183)$ & & [99/16] \\
\hline
\end{tabular}


TABLE 6. Enthalpies of vaporization of organic compounds, 1880-2002-Continued

\begin{tabular}{|c|c|c|c|c|c|}
\hline Molecular formula & $\begin{array}{c}\text { Compound } \\
\text { (Temperature range/K) }\end{array}$ & $\begin{array}{c}\Delta_{\mathrm{vap}} H_{m} \\
\left(\mathrm{~kJ} \mathrm{~mol}^{-1}\right)\end{array}$ & $\begin{array}{l}\text { Mean temperature } \\
\qquad\left(T_{m} / \mathrm{K}\right)\end{array}$ & Method & $\begin{array}{l}\text { CAS registry number } \\
\text { Reference }\end{array}$ \\
\hline & $(167-236)$ & 21.0 & $(221)$ & A & {$[87 / 5][99 / 16]$} \\
\hline \multirow[t]{3}{*}{$\mathrm{CHF}_{7} \mathrm{~S}$} & (difluoromethyl) sulfur pentafluoride & & & & {$[420-67-7]$} \\
\hline & $(221-292)$ & 27.5 & $(237)$ & & {$[99 / 16]$} \\
\hline & $(221-293)$ & 25.6 & $(278)$ & A & {$[87 / 5][99 / 16]$} \\
\hline \multirow[t]{8}{*}{$\mathrm{CHN}$} & hydrogen cyanide & & & & {$[74-90-8]$} \\
\hline & $(259-299)$ & 28.1 & $(274)$ & A & {$[87 / 5]$} \\
\hline & $(298-457)$ & 27.8 & $(313)$ & A & {$[87 / 5]$} \\
\hline & $(257-315)$ & 28.1 & $(272)$ & & {$[75 / 10]$} \\
\hline & $(259-294)$ & 28.0 & $(277)$ & & {$[34 / 5]$} \\
\hline & $(257-319)$ & 28.1 & $(272)$ & & {$[26 / 1]$} \\
\hline & $(265-300)$ & 27.8 & $(282)$ & & {$[26 / 8]$} \\
\hline & $(256-319)$ & 27.2 & $(303)$ & MM & {$[26 / 9]$} \\
\hline \multirow[t]{3}{*}{$\mathrm{CDN}$} & deuterium cyanide & & & & {$[3017-23-0]$} \\
\hline & $(182-282)$ & 26.2 & $(267)$ & & {$[47 / 5]$} \\
\hline & $(265-293)$ & 27.6 & $(279)$ & & {$[34 / 5]$} \\
\hline \multirow[t]{3}{*}{$\mathrm{CHNO}$} & cyanic acid & & & & {$[420-05-3]$} \\
\hline & $(233-268)$ & 30.7 & $(253)$ & A & {$[87 / 5]$} \\
\hline & $(197-267)$ & NA & & & {$[38 / 2]$} \\
\hline \multirow[t]{2}{*}{ CHNS } & thiocyanic acid & & & & [463-56-9] \\
\hline & $(278-396)$ & 28.0 & $(293)$ & A & {$[87 / 5]$} \\
\hline \multirow[t]{2}{*}{$\mathrm{CHN}_{3} \mathrm{O}_{6}$} & trinitromethane & & & & {$[517-25-9]$} \\
\hline & $(290-317)$ & 32.6 & (303) & A & {$[87 / 5][67 / 25]$} \\
\hline \multirow[t]{3}{*}{$\mathrm{CH}_{2} \mathrm{BrCl}$} & bromochloromethane & & & & {$[74-95-7]$} \\
\hline & $(226-341)$ & 42.0 & $(241)$ & A & {$[87 / 5]$} \\
\hline & $(289-341)$ & 33.5 & $(304)$ & & {$[59 / 1][79 / 12]$} \\
\hline \multirow[t]{5}{*}{$\mathrm{CH}_{2} \mathrm{Br}_{2}$} & dibromomethane & & & & {$[74-95-3]$} \\
\hline & & $37.0 \pm 0.1$ & $(298)$ & $\mathrm{C}$ & {$[72 / 41]$} \\
\hline & $(273-373)$ & 36.5 & $(288)$ & & {$[79 / 12]$} \\
\hline & $(290-409)$ & 37.2 & $(305)$ & A, EST & $\begin{array}{c}{[87 / 5][56 / 16]} \\
{[70 / 16]}\end{array}$ \\
\hline & $(238-371)$ & 37.8 & $(253)$ & & {$[47 / 5]$} \\
\hline \multirow[t]{2}{*}{$\mathrm{CH}_{2} \mathrm{ClF}$} & chlorofluoromethane & & & & [593-70-4] \\
\hline & $(140-264)$ & 23.3 & $(249)$ & A & {$[87 / 5][70 / 16]$} \\
\hline \multirow[t]{9}{*}{$\mathrm{CH}_{2} \mathrm{Cl}_{2}$} & dichloromethane & & & & [75-09-2] \\
\hline & & $30.6 \pm 0.1$ & $(298)$ & $\mathrm{C}$ & {$[89 / 10]$} \\
\hline & $(311-383)$ & 29.0 & $(326)$ & A & {$[87 / 5]$} \\
\hline & & 28.8 & $(298)$ & $\mathrm{C}$ & {$[80 / 1]$} \\
\hline & $(264-311)$ & 30.3 & $(279)$ & $\mathrm{EB}$ & {$[72 / 6]$} \\
\hline & $(303-313)$ & 29.2 & $(308)$ & & {$[60 / 3]$} \\
\hline & $(233-313)$ & 30.2 & $(248)$ & & {$[48 / 5]$} \\
\hline & & NA & & & {$[46 / 17]$} \\
\hline & $(186-312)$ & 29.4 & & & {$[27 / 2]$} \\
\hline \multirow[t]{7}{*}{$\mathrm{CH}_{2} \mathrm{~F}_{2}$} & difluoromethane & & & & {$[75-10-5]$} \\
\hline & $(256-321)$ & 19.9 & $(271)$ & A & {$[87 / 5]$} \\
\hline & $(191-222)$ & 21.2 & $(207)$ & A & {$[87 / 5]$} \\
\hline & $(191-258)$ & 20.3 & $(243)$ & A & {$[87 / 5]$} \\
\hline & $(316-351)$ & 20.3 & $(331)$ & A & {$[87 / 5]$} \\
\hline & $(191-221)$ & 21.2 & $(206)$ & & {$[68 / 5]$} \\
\hline & $(191-242)$ & 20.6 & $(227)$ & & {$[68 / 5]$} \\
\hline \multirow[t]{2}{*}{$\mathrm{CH}_{2} \mathrm{~F}_{3} \mathrm{NS}$} & 1,1,1-trifluoromethanesulfenamide & & & & {$[1512-33-0]$} \\
\hline & $(218-291)$ & 34.1 & $(276)$ & A & $\begin{array}{c}{[87 / 5][99 / 16]} \\
{[60 / 24]}\end{array}$ \\
\hline \multirow[t]{5}{*}{$\mathrm{CH}_{2} \mathrm{I}_{2}$} & diiodomethane & & & & {$[75-11-6]$} \\
\hline & & 45.6 & (298) & $\mathrm{GC}$ & [94/19] \\
\hline & & 49.0 & $(298)$ & $\mathrm{C}$ & {$[87 / 4]$} \\
\hline & $(293-455)$ & 48.8 & $(307)$ & & [79/12] \\
\hline & $(356-505)$ & 45.4 & $(371)$ & A & {$[87 / 5][70 / 16]$} \\
\hline \multirow[t]{3}{*}{$\mathrm{CH}_{2} \mathrm{O}$} & formaldehyde & & & & {$[50-00-0]$} \\
\hline & $(184-251)$ & 24.3 & $(236)$ & A & [87/5] \\
\hline & $(173-251)$ & 24.2 & $(236)$ & & {$[35 / 1][87 / 5]$} \\
\hline \multirow[t]{3}{*}{$\mathrm{CH}_{2} \mathrm{O}_{2}$} & formic acid & & & & [64-18-6] \\
\hline & $(300-392)$ & 35.2 & $(315)$ & $\mathrm{EB}$ & [87/9] \\
\hline & $(283-384)$ & 36.0 & (298) & A & {$[87 / 5]$} \\
\hline monomer & & $20.1 \pm 0.1$ & $(298)$ & $\mathrm{C}$ & {$[70 / 8]$} \\
\hline
\end{tabular}


TABLE 6. Enthalpies of vaporization of organic compounds, 1880-2002-Continued

\begin{tabular}{|c|c|c|c|c|c|}
\hline Molecular formula & $\begin{array}{c}\text { Compound } \\
\text { (Temperature range/K) }\end{array}$ & $\begin{array}{c}\Delta_{\mathrm{vap}} H_{m} \\
\left(\mathrm{~kJ} \mathrm{~mol}^{-1}\right)\end{array}$ & $\begin{array}{l}\text { Mean temperature } \\
\qquad\left(T_{m} / \mathrm{K}\right)\end{array}$ & Method & $\begin{array}{c}\text { CAS registry number } \\
\text { Reference }\end{array}$ \\
\hline & & $46.3 \pm 0.5$ & $(298)$ & $\mathrm{C}$ & {$[70 / 8]$} \\
\hline & $(310-374)$ & 35.2 & $(325)$ & & {$[49 / 1]$} \\
\hline & & 19.9 & $(298)$ & & {$[41 / 10]$} \\
\hline & & 29.6 & (303) & & {$[34 / 8]$} \\
\hline & $(273-373)$ & 20.3 & $(315)$ & & {$[30 / 8]$} \\
\hline & $(273-373)$ & 20.9 & (338) & & {$[30 / 8]$} \\
\hline & & 20.4 & $(315)$ & $\mathrm{C}$ & {$[30 / 8]$} \\
\hline & & 21.1 & (338) & $\mathrm{C}$ & {$[30 / 8]$} \\
\hline & $(273-307)$ & 36.8 & $(288)$ & & {$[1894 / 1]$} \\
\hline & $(295-374)$ & 47.7 & (374) & & {$[1883 / 1]$} \\
\hline \multirow[t]{5}{*}{$\mathrm{CH}_{3} \mathrm{Br}$} & methyl bromide & & & & {$[74-83-9]$} \\
\hline & $(223-278)$ & 25.8 & $(238)$ & & {$[79 / 12]$} \\
\hline & $(201-296)$ & 24.6 & $(281)$ & $\mathrm{A}, \mathrm{EST}$ & {$[87 / 5][61 / 13]$} \\
\hline & $(203-277)$ & 25.2 & $(262)$ & & {$[47 / 1]$} \\
\hline & $(203-278)$ & 25.3 & $(263)$ & & {$[38 / 3]$} \\
\hline \multirow[t]{9}{*}{$\mathrm{CH}_{3} \mathrm{Cl}$} & methyl chloride & & & & {$[74-87-31]$} \\
\hline & $(247-310)$ & 22.0 & $(262)$ & A & {$[87 / 5]$} \\
\hline & $(368-416)$ & 21.8 & (383) & $\mathrm{A}$ & {$[87 / 5]$} \\
\hline & $(308-373)$ & 21.0 & (323) & A & {$[87 / 5]$} \\
\hline & $(198-278)$ & 22.0 & (263) & & {$[48 / 5]$} \\
\hline & $(183-250)$ & 22.7 & $(235)$ & & {$[47 / 1]$} \\
\hline & $(191-249)$ & 23.5 & (206) & & {$[46 / 4]$} \\
\hline & $(192-249)$ & 22.6 & $(234)$ & & {$[40 / 2]$} \\
\hline & & 20.1 & $(293)$ & $\mathrm{C}$ & {$[26 / 6]$} \\
\hline \multirow[t]{2}{*}{$\mathrm{CH}_{3} \mathrm{Cl}_{2} \mathrm{P}$} & dichloromethyl phosphine & & & & {$[676-83-5]$} \\
\hline & $(229-297)$ & 35.5 & $(282)$ & A & {$[87 / 5][63 / 29]$} \\
\hline \multirow[t]{6}{*}{$\mathrm{CH}_{3} \mathrm{~F}$} & methyl fluoride & & & & {$[593-53-3]$} \\
\hline & $(205-242)$ & 16.9 & $(227)$ & A & {$[87 / 5]$} \\
\hline & $(240-288)$ & 16.9 & $(273)$ & A & {$[87 / 5]$} \\
\hline & $(141-208)$ & 17.1 & (193) & $\mathrm{A}, \mathrm{EST}$ & $\begin{array}{c}{[87 / 5][61 / 13]} \\
{[70 / 16]}\end{array}$ \\
\hline & $(165-217)$ & 16.4 & $(202)$ & & $\begin{array}{c}{[87 / 5][48 / 15]} \\
{[84 / 9]}\end{array}$ \\
\hline & $(170-197)$ & 17.7 & $(183)$ & A & $\begin{array}{c}{[87 / 5][19 / 1]} \\
{[84 / 9]}\end{array}$ \\
\hline \multirow[t]{3}{*}{$\mathrm{CH}_{3} \mathrm{~F}_{2} \mathrm{~N}$} & $\mathrm{~N}, \mathrm{~N}$-difluoromethylamine & & & & {$[753-58-2]$} \\
\hline & $(203-257)$ & 23.5 & $(242)$ & A & {$[87 / 5]$} \\
\hline & & 22.9 & $(257)$ & & {$[60 / 19]$} \\
\hline \multirow[t]{3}{*}{$\mathrm{CH}_{3} \mathrm{~F}_{2} \mathrm{NS}$} & methylimidosulfurous diflu & & & & {$[758-20-3]$} \\
\hline & $(194-258)$ & 28.7 & $(226)$ & & [99/16] \\
\hline & $(194-258)$ & 28.6 & $(243)$ & A & {$[87 / 5][99 / 16]$} \\
\hline \multirow[t]{2}{*}{$\mathrm{CH}_{3} \mathrm{~F}_{2} \mathrm{P}$} & difluoromethyl phosphine & & & & {$[753-59-3]$} \\
\hline & $(174-236)$ & 23.4 & $(221)$ & A & {$[87 / 5]$} \\
\hline \multirow[t]{2}{*}{$\mathrm{CH}_{3} \mathrm{~F}_{2} \mathrm{OPS}$} & difluorothiophosphoric, S-1 & & & & {$[25237-37-0]$} \\
\hline & $(236-298)$ & 31.2 & $(251)$ & A & {$[87 / 5][99 / 16]$} \\
\hline \multirow[t]{2}{*}{$\mathrm{CH}_{3} \mathrm{~F}_{2} \mathrm{PS}_{2}$} & difluorodithiophosphoric a & 1 ester & & & {$[21348-13-0]$} \\
\hline & $(253-298)$ & 39.0 & $(268)$ & A & {$[87 / 5][99 / 16]$} \\
\hline \multirow[t]{2}{*}{$\mathrm{CH}_{3} \mathrm{~F}_{4} \mathrm{NP}_{2} \mathrm{~S}_{2}$} & $\mathrm{~N}, \mathrm{~N}-$ bis (difluorothiophospl & hethylamine & & & {$[25741-62-2]$} \\
\hline & $(273-325)$ & 38.7 & $(288)$ & A & {$[87 / 5][99 / 16]$} \\
\hline \multirow[t]{7}{*}{$\mathrm{CH}_{3} \mathrm{I}$} & methyl iodide & & & & [74-88-4] \\
\hline & $(228-337)$ & 30.4 & $(243)$ & A & {$[87 / 5]$} \\
\hline & $(315-502)$ & 26.5 & $(330)$ & A & {$[87 / 5]$} \\
\hline & $(208-227)$ & 31.1 & $(217)$ & & {$[82 / 17]$} \\
\hline & $(259-314)$ & 29.2 & $(274)$ & $\mathrm{EB}$ & {$[72 / 6][79 / 12]$} \\
\hline & $(218-315)$ & 30.4 & $(233)$ & & {$[47 / 5]$} \\
\hline & $(273-307)$ & 28.2 & $(288)$ & & {$[36 / 2]$} \\
\hline \multirow[t]{5}{*}{$\mathrm{CH}_{3} \mathrm{NO}$} & formamide & & & & {$[75-12-7]$} \\
\hline & $(293-377)$ & 70.8 & $(308)$ & A & {$[87 / 5]$} \\
\hline & $(415-466)$ & 61.2 & $(430)$ & A & {$[87 / 5]$} \\
\hline & & 60.2 & $(298)$ & A & {$[85 / 7][85 / 6]$} \\
\hline & $(343-483)$ & 64.0 & $(358)$ & & {$[47 / 5]$} \\
\hline \multirow[t]{2}{*}{$\mathrm{CH}_{3} \mathrm{NOS}$} & N-sulfinyl methanamine & & & & {$[4291-05-8]$} \\
\hline & $(252-277)$ & 31.8 & $(264)$ & A & {$[87 / 5][99 / 16]$} \\
\hline $\mathrm{CH}_{3} \mathrm{NO}_{2}$ & methyl nitrite & & & & [624-91-9] \\
\hline
\end{tabular}


TABLE 6. Enthalpies of vaporization of organic compounds, 1880-2002-Continued

\begin{tabular}{|c|c|c|c|c|c|}
\hline Molecular formula & $\begin{array}{c}\text { Compound } \\
\text { (Temperature range/K) }\end{array}$ & $\begin{array}{c}\Delta_{\mathrm{vap}} H_{m} \\
\left(\mathrm{~kJ} \mathrm{~mol}^{-1}\right)\end{array}$ & $\begin{array}{l}\text { Mean temperature } \\
\qquad\left(T_{m} / \mathrm{K}\right)\end{array}$ & Method & $\begin{array}{c}\text { CAS registry number } \\
\text { Reference }\end{array}$ \\
\hline & $(218-273)$ & 22.1 & $(258)$ & A & {$[87 / 5]$} \\
\hline & $(154-225)$ & 26.2 & $(190)$ & & {$[82 / 2]$} \\
\hline & & $22.6 \pm 0.2$ & & & {$[58 / 19]$} \\
\hline \multirow[t]{9}{*}{$\mathrm{CH}_{3} \mathrm{NO}_{2}$} & nitromethane & & & & {$[75-52-2]$} \\
\hline & $(313-353)$ & 37.2 & $(298)$ & CGC & {$[95 / 21]$} \\
\hline & $(405-476)$ & 35.2 & $(420)$ & A & {$[87 / 5][67 / 12]$} \\
\hline & $(328-410)$ & 36.8 & $(343)$ & A & {$[87 / 5][54 / 2]$} \\
\hline & & $37.2 \pm 0.1$ & $(318)$ & $\mathrm{C}$ & {$[54 / 2]$} \\
\hline & & $36.3 \pm 0.1$ & $(335)$ & $\mathrm{C}$ & {$[54 / 2]$} \\
\hline & & $35.2 \pm 0.1$ & $(353)$ & $\mathrm{C}$ & {$[54 / 2]$} \\
\hline & & $34.0 \pm 0.1$ & $(374)$ & $\mathrm{C}$ & {$[54 / 2]$} \\
\hline & & $38.3 \pm 0.1$ & (298) & $\mathrm{C}$ & {$[47 / 11]$} \\
\hline \multirow[t]{2}{*}{$\mathrm{CH}_{3} \mathrm{NO}_{3}$} & methyl nitrate & & & & {$[598-58-3]$} \\
\hline & $(273-303)$ & 34.8 & $(288)$ & A & {$[87 / 5]$} \\
\hline \multirow[t]{15}{*}{$\mathrm{CH}_{4}$} & methane & & & & {$[74-82-8]$} \\
\hline & $(90-120)$ & 8.6 & $(105)$ & A & {$[87 / 5]$} \\
\hline & $(115-149)$ & 8.4 & (134) & A & {$[87 / 5]$} \\
\hline & $(148-189)$ & 8.7 & (174) & A & {$[87 / 5]$} \\
\hline & $(91-127)$ & 8.6 & $(112)$ & & {$[72 / 5][84 / 9]$} \\
\hline & $(91-190)$ & 8.5 & $(175)$ & & {$[72 / 5]$} \\
\hline & & 8.1 & (137) & & {$[71 / 28]$} \\
\hline & $(100-190)$ & 8.6 & $(175)$ & & {$[70 / 4]$} \\
\hline & & 8.2 & (112) & $\mathrm{C}$ & {$[61 / 31]$} \\
\hline & & 7.5 & $(130)$ & $\mathrm{C}$ & {$[61 / 31]$} \\
\hline & & 5.9 & $(160)$ & $\mathrm{C}$ & {$[61 / 31]$} \\
\hline & & 4.0 & $(180)$ & $\mathrm{C}$ & {$[61 / 31]$} \\
\hline & $(109-189)$ & 8.5 & (149) & & {$[61 / 31]$} \\
\hline & & $8.5 \pm 0.1$ & $(99)$ & & {$[39 / 5]$} \\
\hline & $(92-110)$ & 8.6 & $(101)$ & & {$[21 / 1][84 / 9]$} \\
\hline \multirow[t]{2}{*}{$\mathrm{CH}_{4} \mathrm{~F}_{2} \mathrm{NPS}$} & difluorothiophosphoric aci & ylamide & & & [31411-30-0] \\
\hline & $(273-325)$ & 39.1 & $(288)$ & A & {$[87 / 5][99 / 16]$} \\
\hline \multirow[t]{2}{*}{$\mathrm{CH}_{4} \mathrm{~N}_{2}$} & ammonium cyanide & & & & [12211-52-8] \\
\hline & $(222-305)$ & 47.1 & $(237)$ & & {$[47 / 5]$} \\
\hline \multirow[t]{2}{*}{$\mathrm{CH}_{4} \mathrm{~N}_{2}$} & methyl diazene & & & & [26981-93-1] \\
\hline & $(195-236)$ & 27.5 & $(221)$ & A & {$[87 / 5]$} \\
\hline \multirow[t]{2}{*}{$\mathrm{CH}_{4} \mathrm{~N}_{2} \mathrm{O}_{2}$} & ammonium carbamate & & & & \\
\hline & $(247-331)$ & 54.1 & $(262)$ & & {$[47 / 5]$} \\
\hline \multirow[t]{27}{*}{$\mathrm{CH}_{4} \mathrm{O}$} & methanol & & & & {$[67-56-1]$} \\
\hline & & 34.3 & & & {$[99 / 32]$} \\
\hline & $(175-273)$ & 39.2 & $(258)$ & A & {$[87 / 5]$} \\
\hline & $(338-487)$ & 36.9 & $(353)$ & A & {$[87 / 5]$} \\
\hline & $(188-228)$ & 43.7 & $(213)$ & A & {$[87 / 5]$} \\
\hline & $(224-290)$ & 38.9 & $(275)$ & A & {$[87 / 5]$} \\
\hline & $(285-345)$ & 38.3 & $(300)$ & A & {$[87 / 5]$} \\
\hline & $(335-376)$ & 37.0 & $(350)$ & A & {$[87 / 5]$} \\
\hline & $(373-458)$ & 36.1 & $(388)$ & A & {$[87 / 5]$} \\
\hline & $(453-513)$ & 35.1 & $(468)$ & A & {$[87 / 5]$} \\
\hline & & 32.7 & $(373)$ & $\mathrm{C}$ & {$[86 / 17]$} \\
\hline & & 28.1 & $(423)$ & $\mathrm{C}$ & {$[86 / 17]$} \\
\hline & & 20.6 & $(473)$ & $\mathrm{C}$ & {$[86 / 17]$} \\
\hline & & 7.4 & $(510)$ & $\mathrm{C}$ & {$[86 / 17]$} \\
\hline & $(316-336)$ & 37.5 & $(331)$ & EB & {$[84 / 26]$} \\
\hline & $(243-303)$ & 37.8 & (298) & & [83/14] \\
\hline & $(288-337)$ & 38.3 & (303) & & {$[74 / 9][84 / 9]$} \\
\hline & $(337-383)$ & 37.0 & $(352)$ & & {$[73 / 26]$} \\
\hline & & $37.4 \pm 0.1$ & $(298)$ & $\mathrm{C}$ & {$[73 / 13]$} \\
\hline & & $36.7 \pm 0.1$ & (313) & $\mathrm{C}$ & {$[73 / 13]$} \\
\hline & & $36.2 \pm 0.1$ & (323) & $\mathrm{C}$ & {$[73 / 13]$} \\
\hline & & $35.6 \pm 0.1$ & (333) & $\mathrm{C}$ & {$[73 / 13]$} \\
\hline & & $35.3 \pm 0.1$ & (338) & $\mathrm{C}$ & [73/13] \\
\hline & & $34.7 \pm 0.1$ & (343) & $\mathrm{C}$ & {$[73 / 13]$} \\
\hline & & $35.2 \pm 0.1$ & (338) & $\mathrm{C}$ & {$[73 / 3]$} \\
\hline & & $35.6 \pm 0.1$ & $(331)$ & $\mathrm{C}$ & {$[73 / 3]$} \\
\hline & & $36.2 \pm 0.1$ & $(321)$ & $\mathrm{C}$ & {$[73 / 3]$} \\
\hline
\end{tabular}


TABLE 6. Enthalpies of vaporization of organic compounds, 1880-2002-Continued

\begin{tabular}{|c|c|c|c|c|c|}
\hline Molecular formula & $\begin{array}{c}\text { Compound } \\
\text { (Temperature range/K) }\end{array}$ & $\begin{array}{c}\Delta_{\mathrm{vap}} H_{m} \\
\left(\mathrm{~kJ} \mathrm{~mol}^{-1}\right)\end{array}$ & $\begin{array}{l}\text { Mean temperature } \\
\qquad\left(T_{m} / \mathrm{K}\right)\end{array}$ & Method & $\begin{array}{c}\text { CAS registry number } \\
\text { Reference }\end{array}$ \\
\hline & \multirow{3}{*}{$(275-336)$} & $37.0 \pm 0.1$ & $(306)$ & $\mathrm{C}$ & {$[73 / 3]$} \\
\hline & & 38.7 & $(290)$ & $\mathrm{EB}$ & {$[72 / 6][87 / 5]$} \\
\hline & & $37.43 \pm 0.02$ & $(298)$ & $\mathrm{C}$ & {$[71 / 27]$} \\
\hline & $(288-357)$ & 38.3 & (303) & $\mathrm{EB}$ & {$[70 / 2]$} \\
\hline & \multirow[t]{3}{*}{$(353-483)$} & 36.3 & $(368)$ & & {$[67 / 38]$} \\
\hline & & $37.3 \pm 0.1$ & $(298)$ & $\mathrm{C}$ & {$[66 / 2]$} \\
\hline & & $37.7 \pm 0.1$ & $(298)$ & $\mathrm{C}$ & {$[63 / 2]$} \\
\hline & $(278-323)$ & 38.4 & $(293)$ & & {$[60 / 5]$} \\
\hline \multirow[t]{2}{*}{$\mathrm{CH}_{4} \mathrm{O}_{2}$} & methyl hydroperoxide & & & & {$[3031-73-0]$} \\
\hline & $(253-313)$ & 37.7 & $(268)$ & $\mathrm{A}$ & {$[87 / 5][51 / 10]$} \\
\hline \multirow{2}{*}{$\mathrm{CH}_{4} \mathrm{O}_{3} \mathrm{~S}$} & methanesulfonic acid & & & & {$[75-75-2]$} \\
\hline & $(395-440)$ & 73.9 & $(410)$ & A & {$[87 / 5][99 / 16]$} \\
\hline \multirow[t]{8}{*}{$\mathrm{CH}_{4} \mathrm{~S}$} & methyl mercaptan & & & & {$[74-93-1]$} \\
\hline & $(208-298)$ & 27.2 & $(223)$ & & {$[99 / 16]$} \\
\hline & $(267-359)$ & 25.2 & $(359)$ & A & {$[87 / 5]$} \\
\hline & $(221-283)$ & 25.7 & $(268)$ & A & {$[87 / 5]$} \\
\hline & $(345-424)$ & 23.7 & $(360)$ & A & {$[87 / 5]$} \\
\hline & $(414-470)$ & 24.2 & $(429)$ & A & {$[87 / 5]$} \\
\hline & & 23.8 & $(298)$ & & {$[71 / 28]$} \\
\hline & $(222-279)$ & 25.8 & $(264)$ & & {$[87 / 5][42 / 1]$} \\
\hline \multirow[t]{6}{*}{$\mathrm{CH}_{5} \mathrm{~N}$} & methylamine & & & & [74-89-5] \\
\hline & $(319-381)$ & 24.8 & $(334)$ & A & {$[87 / 5]$} \\
\hline & $(373-430)$ & 23.5 & $(388)$ & A & {$[87 / 5]$} \\
\hline & $(263-329)$ & 26.1 & $(278)$ & A & {$[87 / 5]$} \\
\hline & $(223-273)$ & 27.2 & $(258)$ & A & {$[87 / 5][70 / 16]$} \\
\hline & $(190-267)$ & 27.4 & $(252)$ & & {$[37 / 10][84 / 9]$} \\
\hline \multirow[t]{3}{*}{$\mathrm{CH}_{5} \mathrm{NO}$} & N-methylhydroxylamine & & & & {$[593-77-1]$} \\
\hline & $(293-338)$ & 49.7 & $(308)$ & A & {$[87 / 5][70 / 16]$} \\
\hline & $(313-338)$ & 49.3 & $(325)$ & A & $\begin{array}{c}{[87 / 5][57 / 4]} \\
{[84 / 9]}\end{array}$ \\
\hline \multirow[t]{3}{*}{$\mathrm{CH}_{5} \mathrm{NO}$} & O-methylhydroxylamine & & & & [67-62-9] \\
\hline & $(228-322)$ & 36.9 & $(243)$ & A & {$[87 / 5]$} \\
\hline & $(210-321)$ & 38.0 & $(225)$ & & {$[57 / 4][84 / 9]$} \\
\hline \multirow[t]{2}{*}{$\mathrm{CH}_{6} \mathrm{ClN}$} & methylamine hydrochloride & & & & {$[593-51-1]$} \\
\hline & $(518-593)$ & 114.5 & $(533)$ & A & {$[87 / 5]$} \\
\hline \multirow[t]{2}{*}{$\mathrm{CH}_{6} \mathrm{~N}_{2}$} & methylhydrazine & & & & {$[60-33-4]$} \\
\hline & $(274-299)$ & 41.8 & $(286)$ & A & {$[87 / 5][51 / 1]$} \\
\hline \multirow[t]{2}{*}{$\mathrm{C}_{2} \mathrm{BrCl}_{2} \mathrm{~F}_{3} \mathrm{O}_{4}$} & perchloric acid, 1,2,2-trifluo & oro-2-bromoe & & & [38217-36-6] \\
\hline & $(273-294)$ & 42.5 & $(283)$ & A & {$[87 / 5][73 / 19]$} \\
\hline \multirow[t]{2}{*}{$\mathrm{C}_{2} \mathrm{BrCl}_{3} \mathrm{O}$} & trichloroacetyl bromide & & & & [34069-94-8] \\
\hline & $(265-416)$ & 42.6 & $(280)$ & A & {$[87 / 5][47 / 5]$} \\
\hline \multirow{2}{*}{$\mathrm{C}_{2} \mathrm{BrF}_{3}$} & bromotrifluoroethylene & & & & {$[598-73-2]$} \\
\hline & $(260-340)$ & 25.0 & $(275)$ & A & {$[87 / 5]$} \\
\hline $\mathrm{C}_{2} \mathrm{BrF}_{5} \mathrm{O}_{3} \mathrm{~S}$ & 2-bromotetrafluoroethyl fluo & & & & \\
\hline & $(273-298)$ & 33.2 & $(285)$ & & {$[63 / 17]$} \\
\hline $\mathrm{C}_{2} \mathrm{BrF}_{9} \mathrm{~S}$ & pentafluoro(1-bromo-1,2,2,2 & oroethyl) sulf & & & {$[63011-81-4]$} \\
\hline & $(294-330)$ & 30.7 & $(309)$ & A & {$[87 / 5][99 / 16]$} \\
\hline $\mathrm{C}_{2} \mathrm{Br}_{2} \mathrm{ClF}_{3}$ & 2-chloro-1,2-dibromo-1,1,2- & ethane & & & {$[354-51-8]$} \\
\hline & $(343-428)$ & 31.4 & $(358)$ & A & {$[87 / 5]$} \\
\hline & & $35.0 \pm 0.1$ & $(298)$ & $\mathrm{C}$ & {$[81 / 13]$} \\
\hline & & $34.2 \pm 0.1$ & $(313)$ & $\mathrm{C}$ & {$[81 / 13]$} \\
\hline & & $33.5 \pm 0.1$ & $(328)$ & $\mathrm{C}$ & {$[81 / 13]$} \\
\hline & & $32.6 \pm 0.1$ & $(343)$ & $\mathrm{C}$ & {$[81 / 13]$} \\
\hline & & $31.6 \pm 0.1$ & $(358)$ & $\mathrm{C}$ & {$[81 / 13]$} \\
\hline $\mathrm{C}_{2} \mathrm{Br}_{2} \mathrm{~F}_{4}$ & 1,2-dibromotetrafluoroethan & & & & [124-73-2] \\
\hline & $(283-357)$ & 28.5 & $(298)$ & A & {$[87 / 5]$} \\
\hline & $(354-443)$ & 26.9 & $(369)$ & A & {$[87 / 5]$} \\
\hline & $(440-488)$ & 27.1 & $(455)$ & A & {$[87 / 5]$} \\
\hline & & $28.4 \pm 0.1$ & $(298)$ & $\mathrm{C}$ & {$[81 / 13]$} \\
\hline & & $27.5 \pm 0.1$ & $(313)$ & $\mathrm{C}$ & {$[81 / 13]$} \\
\hline & & $26.5 \pm 0.1$ & $(328)$ & $\mathrm{C}$ & {$[81 / 13]$} \\
\hline & $(246-295)$ & 30.0 & $(280)$ & & {$[87 / 5][70 / 16]$} \\
\hline $\mathrm{C}_{2} \mathrm{ClFN}_{2}$ & cis chloro(fluoroimino)aceto & & & & [30915-40-3] \\
\hline & $(254-320)$ & 31.7 & $(269)$ & A & {$[87 / 5][71 / 19]$} \\
\hline
\end{tabular}


TABLE 6. Enthalpies of vaporization of organic compounds, 1880-2002-Continued

\begin{tabular}{|c|c|c|c|c|c|}
\hline Molecular formula & $\begin{array}{c}\text { Compound } \\
\text { (Temperature range/K) }\end{array}$ & $\begin{array}{c}\Delta_{\mathrm{vap}} H_{m} \\
\left(\mathrm{~kJ} \mathrm{~mol}^{-1}\right)\end{array}$ & $\begin{array}{l}\text { Mean temperature } \\
\qquad\left(T_{m} / \mathrm{K}\right)\end{array}$ & Method & $\begin{array}{c}\text { CAS registry number } \\
\text { Reference }\end{array}$ \\
\hline $\mathrm{C}_{2} \mathrm{ClFN}_{2}$ & $\begin{array}{l}\text { trans chloro(fluoroimino)a } \\
(257-320)\end{array}$ & 32.7 & $(272)$ & A & $\begin{array}{l}{[30915-39-0]} \\
{[87 / 5][71 / 19]}\end{array}$ \\
\hline $\mathrm{C}_{2} \mathrm{ClF}_{2} \mathrm{NO}_{2}$ & chloro(fluorocarbonyl)carb & $\begin{array}{l}\text { ride } \\
36.8\end{array}$ & $(376)$ & & $\begin{array}{c}{[42016-33-1]} \\
{[73 / 21]}\end{array}$ \\
\hline $\mathrm{C}_{2} \mathrm{ClF}_{3}$ & $\begin{array}{l}\text { chlorotrifluoroethylene } \\
(206-262) \\
(298-379) \\
(206-263) \\
(195-250)\end{array}$ & $\begin{array}{l}21.8 \\
20.2 \\
21.7 \\
21.9\end{array}$ & $\begin{array}{l}(247) \\
(313) \\
(248) \\
(235)\end{array}$ & $\begin{array}{l}\text { A } \\
\text { A }\end{array}$ & $\begin{array}{c}{[79-38-9]} \\
{[87 / 5]} \\
{[87 / 5]} \\
{[51 / 2]} \\
{[33 / 2]}\end{array}$ \\
\hline $\mathrm{C}_{2} \mathrm{ClF}_{3} \mathrm{O}_{2}$ & $\begin{array}{l}\text { chloroformic acid, trifluor } \\
(195-273)\end{array}$ & 24.1 & $(258)$ & A & $\begin{array}{c}{[23213-83-4]} \\
{[87 / 5]}\end{array}$ \\
\hline $\mathrm{C}_{2} \mathrm{ClF}_{3} \mathrm{O}_{4} \mathrm{~S}$ & $\begin{array}{l}\text { difluorochloroacetic acid, } \\
(265-352)\end{array}$ & $\begin{array}{l}\text { uric acid anh } \\
39.8\end{array}$ & $(280)$ & A & $\begin{array}{c}{[6069-32-5]} \\
{[87 / 5][66 / 15]} \\
{[99 / 16]}\end{array}$ \\
\hline $\mathrm{C}_{2} \mathrm{ClF}_{4} \mathrm{NO}$ & chloro(trifluoromethyl)car & $\begin{array}{l}\text { ride } \\
28.9\end{array}$ & $(310)$ & & $\begin{array}{c}{[42016-31-9]} \\
{[73 / 21]}\end{array}$ \\
\hline $\mathrm{C}_{2} \mathrm{ClF}_{4} \mathrm{NO}_{4} \mathrm{~S}$ & fluorosulfuric acid, chloro & $\begin{array}{c}\text { ethyl)carban } \\
28.5\end{array}$ & $\begin{array}{l}\text { anhydride } \\
\qquad(398)\end{array}$ & & $\begin{array}{c}{[42016-34-2]} \\
{[73 / 21]}\end{array}$ \\
\hline $\mathrm{C}_{2} \mathrm{ClF}_{5}$ & $\begin{array}{l}\text { chloropentafluoroethane } \\
(262-317) \\
(234-265) \\
(312-353) \\
(178-234) \\
(176-235)\end{array}$ & $\begin{array}{c}19.7 \\
20.1 \\
19.7 \\
20.9 \\
20.9 \\
19.4 \pm 0.1\end{array}$ & $\begin{array}{l}(277) \\
(250) \\
(327) \\
(219) \\
(220) \\
(234)\end{array}$ & $\begin{array}{l}\mathrm{A} \\
\mathrm{A} \\
\mathrm{A}\end{array}$ & $\begin{array}{c}{[76-15-3]} \\
{[87 / 5]} \\
{[87 / 5]} \\
{[87 / 5]} \\
{[66 / 4]} \\
{[87 / 5][55 / 1]} \\
{[55 / 1]}\end{array}$ \\
\hline $\mathrm{C}_{2} \mathrm{ClF}_{5} \mathrm{O}$ & $\begin{array}{l}\text { hypochlorous acid, pentafl } \\
(193-248)\end{array}$ & $\begin{array}{l}\text { ester } \\
\qquad 25.0\end{array}$ & $(233)$ & A & $\begin{array}{l}{[22675-67-8]} \\
{[87 / 5][73 / 22]}\end{array}$ \\
\hline $\mathrm{C}_{2} \mathrm{ClF}_{5} \mathrm{OS}$ & $\begin{array}{l}\text { pentafluoroethanesulfinyl } \\
(273-338)\end{array}$ & 32.7 & $(288)$ & A & $\begin{array}{c}{[39937-08-1]} \\
{[87 / 5][64 / 22]} \\
{[99 / 16]}\end{array}$ \\
\hline $\mathrm{C}_{2} \mathrm{ClF}_{5} \mathrm{O}_{3} \mathrm{~S}$ & $\begin{array}{l}\text { 2-chlorotetrafluoroethyl flu } \\
(248-330)\end{array}$ & 32.9 & $(289)$ & & {$[63 / 17]$} \\
\hline $\mathrm{C}_{2} \mathrm{ClF}_{5} \mathrm{O}_{6} \mathrm{~S}_{2}$ & $\begin{array}{l}\text { 1,2,2-trifluoro-1-chloro-1, } \\
(308-406)\end{array}$ & $\begin{array}{l}\text { ol bis(fluoros } \\
53.2\end{array}$ & $(323)$ & A & $\begin{array}{c}{[1957-17-1]} \\
{[87 / 5][99 / 16]}\end{array}$ \\
\hline $\mathrm{C}_{2} \mathrm{ClF}_{6} \mathrm{NOS}$ & (pentafluoroethyl)imidosul & $\begin{array}{l}\text { oride fluorid } \\
35.6\end{array}$ & $(326)$ & I & $\begin{array}{c}{[74366-11-3]} \\
{[80 / 10]}\end{array}$ \\
\hline $\mathrm{C}_{2} \mathrm{ClF}_{6} \mathrm{P}$ & $\begin{array}{l}\text { bis(trifluoromethyl) chloro } \\
(193-273)\end{array}$ & 27.8 & $(258)$ & & $\begin{array}{c}{[650-52-2]} \\
{[64 / 4][84 / 9]}\end{array}$ \\
\hline $\mathrm{C}_{2} \mathrm{ClF}_{6} \mathrm{PS}_{2}$ & $\begin{array}{l}\text { chloro bis(trifluoromethylt } \\
(293-373)\end{array}$ & $\begin{array}{l}\text { hine } \\
\qquad 33.0\end{array}$ & (333) & & {$[60 / 25]$} \\
\hline $\mathrm{C}_{2} \mathrm{ClF}_{9} \mathrm{NP}$ & $\begin{array}{l}\text { [bis(trifluoromethyl)amino } \\
(223-273)\end{array}$ & $\begin{array}{c}\text { hlorophosph } \\
26.4\end{array}$ & $(248)$ & & {$[66 / 23]$} \\
\hline $\mathrm{C}_{2} \mathrm{ClF}_{9} \mathrm{~S}$ & 2-chlorotetrafluoroethylsul & $\begin{array}{l}\text { luoride } \\
28.3\end{array}$ & $(320)$ & & $\begin{array}{c}{[646-63-9]} \\
{[61 / 21][99 / 16]}\end{array}$ \\
\hline $\mathrm{C}_{2} \mathrm{Cl}_{2} \mathrm{~F}_{2}$ & $\begin{array}{l}\text { 1,2-dichloro-1,2-difluoroet } \\
(191-294) \\
(240-294)\end{array}$ & $\begin{array}{l}27.9 \\
27.2\end{array}$ & $\begin{array}{l}(279) \\
(279)\end{array}$ & A & $\begin{array}{c}{[598-88-9]} \\
{[87 / 5]} \\
{[33 / 3]}\end{array}$ \\
\hline $\mathrm{C}_{2} \mathrm{Cl}_{2} \mathrm{~F}_{2} \mathrm{~N}_{2}$ & $\begin{array}{l}\text { dichloro(difluoroamino)ac } \\
(238-341)\end{array}$ & 26.8 & $(253)$ & $\mathrm{A}$ & $\begin{array}{l}{[30913-21-4]} \\
{[87 / 5][71 / 19]}\end{array}$ \\
\hline $\mathrm{C}_{2} \mathrm{Cl}_{2} \mathrm{~F}_{2} \mathrm{O}$ & $\begin{array}{l}\text { fluorodichloroacetyl fluori } \\
(208-273)\end{array}$ & 21.8 & $(258)$ & A & $\begin{array}{c}{[354-18-7]} \\
{[87 / 5]}\end{array}$ \\
\hline $\mathrm{C}_{2} \mathrm{Cl}_{2} \mathrm{~F}_{3} \mathrm{NO}$ & $\mathrm{N}, \mathrm{N}^{\prime}$-dichloro-2,2,2-trifluo & 40.9 & & & $\begin{array}{c}{[32751-03-4]} \\
{[72 / 40]}\end{array}$ \\
\hline $\mathrm{C}_{2} \mathrm{Cl}_{2} \mathrm{~F}_{3} \mathrm{NOS}$ & $\begin{array}{l}\text { S,S-dichloro-N-(trifluoroad } \\
(306-333)\end{array}$ & $\begin{array}{l}\text { limine } \\
44.2\end{array}$ & $(319)$ & A & $\begin{array}{c}{[24433-67-8]} \\
{[87 / 5][69 / 22]} \\
{[99 / 16]}\end{array}$ \\
\hline $\mathrm{C}_{2} \mathrm{Cl}_{2} \mathrm{~F}_{3} \mathrm{NO}_{2} \mathrm{~S}$ & $\begin{array}{l}\text { (trifluoromethyl)sulfonyl c } \\
(312-405)\end{array}$ & $\begin{array}{l}\text { lic dichloride } \\
44.1\end{array}$ & $(327)$ & A & $\begin{array}{l}{[51587-33-8]} \\
{[87 / 5][99 / 16]}\end{array}$ \\
\hline $\mathrm{C}_{2} \mathrm{Cl}_{2} \mathrm{~F}_{4}$ & $\begin{array}{l}\text { 1,1-dichloro-1,2,2,2-tetrafl } \\
(231-373)\end{array}$ & $\begin{array}{l}23.5 \\
23.2 \\
22.5 \\
20.8 \\
17.7\end{array}$ & $\begin{array}{l}(246) \\
(233) \\
(273) \\
(313) \\
(353)\end{array}$ & $\begin{array}{c}\text { A } \\
\text { BG } \\
\text { BG } \\
\text { BG } \\
\text { BG }\end{array}$ & $\begin{array}{c}{[374-07-2]} \\
{[87 / 5][70 / 16]} \\
{[55 / 20]} \\
{[55 / 20]} \\
{[55 / 20]} \\
{[55 / 20]}\end{array}$ \\
\hline
\end{tabular}


TABLE 6. Enthalpies of vaporization of organic compounds, 1880-2002-Continued

\begin{tabular}{|c|c|c|c|c|c|}
\hline Molecular formula & $\begin{array}{c}\text { Compound } \\
\text { (Temperature range/K) }\end{array}$ & $\begin{array}{c}\Delta_{\mathrm{vap}} H_{m} \\
\left(\mathrm{~kJ} \mathrm{~mol}^{-1}\right)\end{array}$ & $\begin{array}{l}\text { Mean temperature } \\
\qquad\left(T_{m} / \mathrm{K}\right)\end{array}$ & Method & $\begin{array}{c}\text { CAS registry number } \\
\text { Reference }\end{array}$ \\
\hline \multirow[t]{4}{*}{$\mathrm{C}_{2} \mathrm{Cl}_{2} \mathrm{~F}_{4}$} & \multicolumn{4}{|c|}{ 1,2-dichloro-1,1,2,2-tetrafluoroethane } & {$[76-14-2]$} \\
\hline & $(277-391)$ & 24.3 & $(292)$ & A & {$[87 / 5]$} \\
\hline & $(210-277)$ & 25.1 & $(262)$ & A & {$[87 / 5]$} \\
\hline & $(178-277)$ & 25.3 & $(261)$ & & {$[47 / 5]$} \\
\hline \multirow[t]{2}{*}{$\mathrm{C}_{2} \mathrm{Cl}_{2} \mathrm{~F}_{4} \mathrm{O}_{4}$} & \multicolumn{4}{|c|}{ perchloric acid, 1,1,2,2-tetrafluoro-2-chloroethyl ester } & {$[38126-28-2]$} \\
\hline & $(249-294)$ & 32.6 & $(279)$ & A & {$[87 / 5][73 / 19]$} \\
\hline \multirow[t]{2}{*}{$\mathrm{C}_{2} \mathrm{Cl}_{2} \mathrm{~F}_{5} \mathrm{NS}$} & \multicolumn{4}{|c|}{$\mathrm{S}, \mathrm{S}$-dichloro-N-(pentafluoroethyl) sulfilimine } & {$[10564-48-4]$} \\
\hline & $(297-375)$ & 37.4 & $(312)$ & A & {$[87 / 5][99 / 16]$} \\
\hline \multirow[t]{2}{*}{$\mathrm{C}_{2} \mathrm{Cl}_{2} \mathrm{~F}_{8} \mathrm{NP}$} & \multicolumn{5}{|c|}{ [bis(trifluoromethyl)amino]difluorodichlorophosphorous (V) } \\
\hline & $(262-305)$ & 32.9 & $(293)$ & & {$[66 / 33]$} \\
\hline \multirow[t]{6}{*}{$\mathrm{C}_{2} \mathrm{Cl}_{3} \mathrm{~F}_{3}$} & \multicolumn{3}{|c|}{ 1,1,1-trichloro-2,2,2-trifluoroethane } & & {$[354-58-5]$} \\
\hline & & $28.1 \pm 0.1$ & $(298)$ & $\mathrm{C}$ & {$[80 / 9]$} \\
\hline & & $27.2 \pm 0.1$ & $(313)$ & $\mathrm{C}$ & {$[80 / 9]$} \\
\hline & & $26.3 \pm 0.1$ & $(328)$ & $\mathrm{C}$ & {$[80 / 9]$} \\
\hline & $(297-319)$ & 28.9 & $(308)$ & & {$[80 / 9]$} \\
\hline & $(286-310)$ & 29.2 & $(298)$ & A & {$[87 / 5][63 / 6]$} \\
\hline \multirow{12}{*}{$\mathrm{C}_{2} \mathrm{Cl}_{3} \mathrm{~F}_{3}$} & \multicolumn{4}{|c|}{ 1,1,2-trichloro-1,2,2-trifluoroethane } & {$[76-13-1]$} \\
\hline & $(273-319)$ & 28.3 & $(288)$ & A & {$[87 / 5]$} \\
\hline & $(238-364)$ & 30.9 & $(253)$ & A & {$[87 / 5]$} \\
\hline & $(360-473)$ & 26.9 & $(375)$ & A & {$[87 / 5]$} \\
\hline & \multirow[t]{5}{*}{$(297-317)$} & 28.8 & $(307)$ & A & {$[87 / 5]$} \\
\hline & & $28.4 \pm 0.1$ & $(298)$ & $\mathrm{C}$ & {$[80 / 9]$} \\
\hline & & $27.5 \pm 0.1$ & (313) & $\mathrm{C}$ & {$[80 / 9]$} \\
\hline & & $26.6 \pm 0.1$ & $(328)$ & $\mathrm{C}$ & {$[80 / 9]$} \\
\hline & & $28.2 \pm 0.4$ & $(298)$ & & {$[74 / 5]$} \\
\hline & $(273-318)$ & 28.2 & $(288)$ & & {$[63 / 6]$} \\
\hline & $(248-356)$ & 30.8 & $(263)$ & & {$[40 / 1]$} \\
\hline & $(243-353)$ & NA & & & {$[39 / 7]$} \\
\hline \multirow[t]{2}{*}{$\mathrm{C}_{2} \mathrm{Cl}_{3} \mathrm{~F}_{3}$} & trichlorotrifluoroethane & & & & \\
\hline & $(248-352)$ & 30.5 & $(263)$ & & {$[38 / 4]$} \\
\hline $\mathrm{C}_{2} \mathrm{Cl}_{3} \mathrm{~F}_{3} \mathrm{O}_{4}$ & perchloric acid, 1,2,2-trifl & chloroethyl & & & {$[38126-27-1]$} \\
\hline & $(273-296)$ & 26.9 & $(284)$ & A & {$[87 / 5]$} \\
\hline $\mathrm{C}_{2} \mathrm{Cl}_{3} \mathrm{~N}$ & trichloroacetonitrile & & & & [545-06-2] \\
\hline & $(289-357)$ & 35.1 & $(304)$ & A & {$[87 / 5][70 / 16]$} \\
\hline & $(289-356)$ & 34.7 & $(304)$ & & {$[54 / 4]$} \\
\hline $\mathrm{C}_{2} \mathrm{Cl}_{4}$ & tetrachloroethylene & & & & {$[127-18-4]$} \\
\hline & $(307-393)$ & 38.4 & $(322)$ & & {$[95 / 13]$} \\
\hline & $(310-393)$ & 38.7 & $(325)$ & A & {$[87 / 5][72 / 6]$} \\
\hline & $(300-380)$ & 38.9 & $(315)$ & & {$[70 / 3][84 / 9]$} \\
\hline & $(333-373)$ & 37.6 & $(348)$ & & {$[67 / 30]$} \\
\hline & & $39.6 \pm 0.1$ & $(298)$ & $\mathrm{C}$ & {$[60 / 1]$} \\
\hline $\mathrm{C}_{2} \mathrm{Cl}_{4} \mathrm{~F}_{2}$ & 1,2-difluorotetrachloroeth & & & & {$[76-12-0]$} \\
\hline & & $34.6 \pm 0.1$ & $(308)$ & $\mathrm{C}$ & [92/11] \\
\hline & & $34.1 \pm 0.1$ & $(315)$ & $\mathrm{C}$ & {$[92 / 11]$} \\
\hline & & $33.6 \pm 0.1$ & $(323)$ & $\mathrm{C}$ & {$[92 / 11]$} \\
\hline & & $33.1 \pm 0.1$ & $(330)$ & $\mathrm{C}$ & {$[92 / 11]$} \\
\hline & & $32.6 \pm 0.1$ & $(338)$ & $\mathrm{C}$ & {$[92 / 11]$} \\
\hline & $(301-365)$ & 36.6 & (316) & A & {$[87 / 5]$} \\
\hline & $(235-293)$ & 36.4 & $(278)$ & A & {$[87 / 5]$} \\
\hline & $(312-362)$ & 34.0 & $(327)$ & A & {$[87 / 5]$} \\
\hline & $(283-364)$ & 32.7 & $(298)$ & & {$[33 / 4]$} \\
\hline $\mathrm{C}_{2} \mathrm{Cl}_{4} \mathrm{~F}_{2} \mathrm{O}_{3} \mathrm{~S}$ & 2-fluorotetrachloroethyl fl & & & & \\
\hline & $(311-437)$ & 42.0 & $(329)$ & & {$[63 / 17]$} \\
\hline $\mathrm{C}_{2} \mathrm{Cl}_{4} \mathrm{~F}_{2} \mathrm{O}_{4}$ & perchloric acid, 1,2-difluo & chloroethyl & & & [38126-29-3] \\
\hline & $(273-294)$ & 30.2 & $(283)$ & A & {$[87 / 5][73 / 19]$} \\
\hline $\mathrm{C}_{2} \mathrm{Cl}_{4} \mathrm{~F}_{4} \mathrm{~N}_{2}$ & 1,2-bis(dichloroamino)tetr & ane & & & \\
\hline & & 43.1 & & & {$[72 / 40]$} \\
\hline $\mathrm{C}_{2} \mathrm{Cl}_{4} \mathrm{~F}_{6} \mathrm{OS}$ & pentafluoro(2-fluoro-1,1,2 & roethoxy)sul & & & {$[762-90-3]$} \\
\hline & $(314-418)$ & 42.8 & (329) & A & {$[87 / 5][62 / 19]$} \\
\hline $\mathrm{C}_{2} \mathrm{Cl}_{4} \mathrm{O}$ & tetrachloroethylene oxide & & & & {$[16650-10-5]$} \\
\hline & $(308-348)$ & 36.9 & (323) & A & {$[87 / 5]$} \\
\hline $\mathrm{C}_{2} \mathrm{Cl}_{4} \mathrm{O}$ & trichloroacetyl chloride & & & & {$[76-02-8]$} \\
\hline & $(305-393)$ & 38.3 & $(320)$ & A & $\begin{array}{c}{[87 / 5][59 / 1]} \\
{[70 / 16]}\end{array}$ \\
\hline
\end{tabular}


TABLE 6. Enthalpies of vaporization of organic compounds, 1880-2002-Continued

\begin{tabular}{|c|c|c|c|c|c|}
\hline Molecular formula & $\begin{array}{c}\text { Compound } \\
\text { (Temperature range/K) }\end{array}$ & $\begin{array}{c}\Delta_{\mathrm{vap}} H_{m} \\
\left(\mathrm{~kJ} \mathrm{~mol}^{-1}\right)\end{array}$ & $\begin{array}{l}\text { Mean temperature } \\
\qquad\left(T_{m} / \mathrm{K}\right)\end{array}$ & Method & $\begin{array}{c}\text { CAS registry number } \\
\text { Reference }\end{array}$ \\
\hline \multirow[t]{4}{*}{$\mathrm{C}_{2} \mathrm{Cl}_{6}$} & hexachloroethane & & & & {$[67-12-11]$} \\
\hline & $(460-513)$ & 40.3 & $(475)$ & A & {$[87 / 5]$} \\
\hline & $(345-460)$ & 51.2 & $(360)$ & A & {$[87 / 5][70 / 16]$} \\
\hline & $(305-458)$ & 53.7 & $(320)$ & & {$[47 / 5]$} \\
\hline \multirow[t]{2}{*}{$\mathrm{C}_{2} \mathrm{D}_{4} \mathrm{O}$} & ethylene oxide $-\mathrm{d}_{4}$ oxide & & & & {$[6552-57-4]$} \\
\hline & $(230-273)$ & 27.6 & $(258)$ & & {$[52 / 2][84 / 9]$} \\
\hline \multirow[t]{2}{*}{$\mathrm{C}_{2} \mathrm{FNO}_{2}$} & fluorocarbonyl isocyanate & & & & [15435-14-0] \\
\hline & $(228-264)$ & 33.5 & $(249)$ & A & {$[87 / 5][67 / 31]$} \\
\hline \multirow[t]{2}{*}{$\mathrm{C}_{2} \mathrm{~F}_{2} \mathrm{~N}_{2} \mathrm{O}$} & difluorocarboncyamidic amide & & & & [32837-63-1] \\
\hline & & 29.7 & (383) & & {$[73 / 23]$} \\
\hline \multirow[t]{2}{*}{$\mathrm{C}_{2} \mathrm{~F}_{2} \mathrm{~N}_{2} \mathrm{O}_{2}$} & difluorocarbonisocyantidic amide & & & & [32837-64-2] \\
\hline & & 33.9 & $(327)$ & & {$[73 / 23]$} \\
\hline \multirow[t]{2}{*}{$\mathrm{C}_{2} \mathrm{~F}_{2} \mathrm{~N}_{4} \mathrm{O}_{8}$} & 1,2-difluoro-1,1,2,2-tetranitroethane & & & & [20165-39-3] \\
\hline & $(297-323)$ & 62.8 & $(310)$ & A & {$[87 / 5][73 / 25]$} \\
\hline \multirow[t]{2}{*}{$\mathrm{C}_{2} \mathrm{~F}_{2} \mathrm{O}_{2}$} & oxalyl fluoride & & & & {$[359-40-0]$} \\
\hline & $(264-272)$ & 29.7 & $(268)$ & & {$[87 / 5]$} \\
\hline \multirow[t]{2}{*}{$\mathrm{C}_{2} \mathrm{~F}_{2} \mathrm{O}_{4}$} & bis(fluorocarbonyl)peroxide & & & & {$[692-74-0]$} \\
\hline & $(226-266)$ & 30.6 & $(251)$ & & {$[62 / 3][84 / 9]$} \\
\hline \multirow[t]{7}{*}{$\mathrm{C}_{2} \mathrm{~F}_{3} \mathrm{~N}$} & trifluoroacetonitrile & & & & {$[353-85-5]$} \\
\hline & $(151-206)$ & 19.3 & $(191)$ & A & {$[87 / 5]$} \\
\hline & $(141-203)$ & 19.2 & (188) & A & {$[87 / 5]$} \\
\hline & $(197-241)$ & 18.5 & $(226)$ & A & {$[87 / 5]$} \\
\hline & $(282-336)$ & 17.4 & $(309)$ & $\mathrm{A}$ & {$[87 / 5]$} \\
\hline & $(272-311)$ & 17.4 & (287) & A & {$[87 / 5]$} \\
\hline & $(142-206)$ & 19.2 & $(191)$ & & {$[61 / 2]$} \\
\hline \multirow[t]{2}{*}{$\mathrm{C}_{2} \mathrm{~F}_{3} \mathrm{NO}$} & trifluoromethyl isocyanate & & & & {$[460-49-1]$} \\
\hline & $(195-228)$ & 22.5 & $(213)$ & A & {$[87 / 5]$} \\
\hline \multirow[t]{2}{*}{$\mathrm{C}_{2} \mathrm{~F}_{3} \mathrm{NO}$} & trifluoronitrosoethylene & & & & [2713-04-4] \\
\hline & $(247-250)$ & 25.7 & $(248)$ & $\mathrm{A}$ & {$[87 / 5]$} \\
\hline \multirow[t]{2}{*}{$\mathrm{C}_{2} \mathrm{~F}_{3} \mathrm{NOS}$} & trifluoromethanesulfinyl cyanide & & & & [61951-27-7] \\
\hline & & 40.2 & $(352)$ & I & {$[77 / 17]$} \\
\hline \multirow[t]{2}{*}{$\mathrm{C}_{2} \mathrm{~F}_{3} \mathrm{NOS}$} & trifluoromethylsulfenyl isocyanate & & & & [691-03-2] \\
\hline & $(231-293)$ & 27.9 & (278) & A & $\begin{array}{c}{[87 / 5][99 / 16]} \\
{[63 / 42]}\end{array}$ \\
\hline \multirow[t]{2}{*}{$\mathrm{C}_{2} \mathrm{~F}_{3} \mathrm{NO}_{2} \mathrm{~S}$} & 2,2,2-trifluoro-N-sulfinylacetamide & & & & [26454-68-2] \\
\hline & $(267-302)$ & 36.4 & $(282)$ & A & {$[87 / 5][99 / 16]$} \\
\hline \multirow[t]{2}{*}{$\mathrm{C}_{2} \mathrm{~F}_{3} \mathrm{NO}_{2} \mathrm{~S}_{2}$} & trifluoromethanesulfonyl isothiocyanate & & & & [51587-30-5] \\
\hline & $(297-385)$ & 41.0 & $(312)$ & A & {$[87 / 5][99 / 16]$} \\
\hline $\mathrm{C}_{2} \mathrm{~F}_{3} \mathrm{NO}_{3} \mathrm{~S}$ & trifluoromethanesulfonyl isocyanate & & & & [30227-06-6] \\
\hline & $(275-345)$ & 36.9 & (290) & A & {$[87 / 5][99 / 16]$} \\
\hline $\mathrm{C}_{2} \mathrm{~F}_{3} \mathrm{NS}$ & thiocyanic acid, trifluoromethyl ester & & & & [690-24-4] \\
\hline & $(226-294)$ & 32.6 & (279) & A & $\begin{array}{c}{[87 / 5][99 / 16]} \\
{[63 / 42]}\end{array}$ \\
\hline $\mathrm{C}_{2} \mathrm{~F}_{3} \mathrm{~N}_{3} \mathrm{O}_{6}$ & 1,1,2-trifluoro-1,2,2-trinitroethane & & & & [20165-38-2] \\
\hline & $(313-353)$ & 57.7 & $(328)$ & A & {$[87 / 5][73 / 25]$} \\
\hline $\mathrm{C}_{2} \mathrm{~F}_{4}$ & tetrafluoroethylene & & & & [116-14-3] \\
\hline & $(197-273)$ & 16.8 & $(258)$ & A & {$[87 / 5]$} \\
\hline & $(273-306)$ & 16.6 & (288) & $\mathrm{A}$ & {$[87 / 5]$} \\
\hline & $(142-208)$ & 18.6 & (193) & A & $\begin{array}{c}{[87 / 5][53 / 2]} \\
{[84 / 9]}\end{array}$ \\
\hline $\mathrm{C}_{2} \mathrm{~F}_{4} \mathrm{~N}_{2}$ & tetrafluoroaminoacetic, nitrile & & & & [5131-88-4] \\
\hline & $(193-238)$ & 23.9 & (223) & A & {$[87 / 5]$} \\
\hline $\mathrm{C}_{2} \mathrm{~F}_{4} \mathrm{~N}_{2} \mathrm{O}_{3}$ & 1,1,2,2-tetrafluoro-1-nitro-2-nitrosoetha & tane & & & {$[679-08-3]$} \\
\hline & $(233-293)$ & 28.8 & (278) & A & {$[87 / 5]$} \\
\hline $\mathrm{C}_{2} \mathrm{~F}_{4} \mathrm{~N}_{2} \mathrm{O}_{4}$ & 1,1,2,2-tetrafluoro-1,2-dinitroethane & & & & [356-16-1] \\
\hline & $(303-343)$ & 67.8 & (323) & & {$[73 / 25]$} \\
\hline & $(259-333)$ & 34.7 & (274) & $\mathrm{A}, \mathrm{I}$ & {$[87 / 5][57 / 19]$} \\
\hline $\mathrm{C}_{2} \mathrm{~F}_{4} \mathrm{~N}_{2} \mathrm{O}_{6} \mathrm{~S}_{2}$ & 1,2-bis(fluoroformyl)-1,2-bis(fluorosulf & lfonyl)hydrazine & & & {$[19252-50-7]$} \\
\hline & $(273-296)$ & 49.8 & $(284)$ & A & {$[87 / 5][99 / 16]$} \\
\hline $\mathrm{C}_{2} \mathrm{~F}_{4} \mathrm{O}$ & trifluoroacetyl fluoride & & & & [354-34-7] \\
\hline & $(161-215)$ & 20.9 & $(200)$ & A & {$[87 / 5][72 / 2]$} \\
\hline $\mathrm{C}_{2} \mathrm{~F}_{4} \mathrm{O}_{2} \mathrm{~S}$ & trifluoroethylene sulfonyl fluoride & & & & [684-106] \\
\hline & $(270-313)$ & 27.0 & (285) & A & {$[87 / 5][99 / 16]$} \\
\hline $\mathrm{C}_{2} \mathrm{~F}_{4} \mathrm{O}_{3}$ & fluoroperoxyformic acid, trifluorometh & hyl ester & & & [16118-40-4] \\
\hline
\end{tabular}


TABLE 6. Enthalpies of vaporization of organic compounds, 1880-2002-Continued

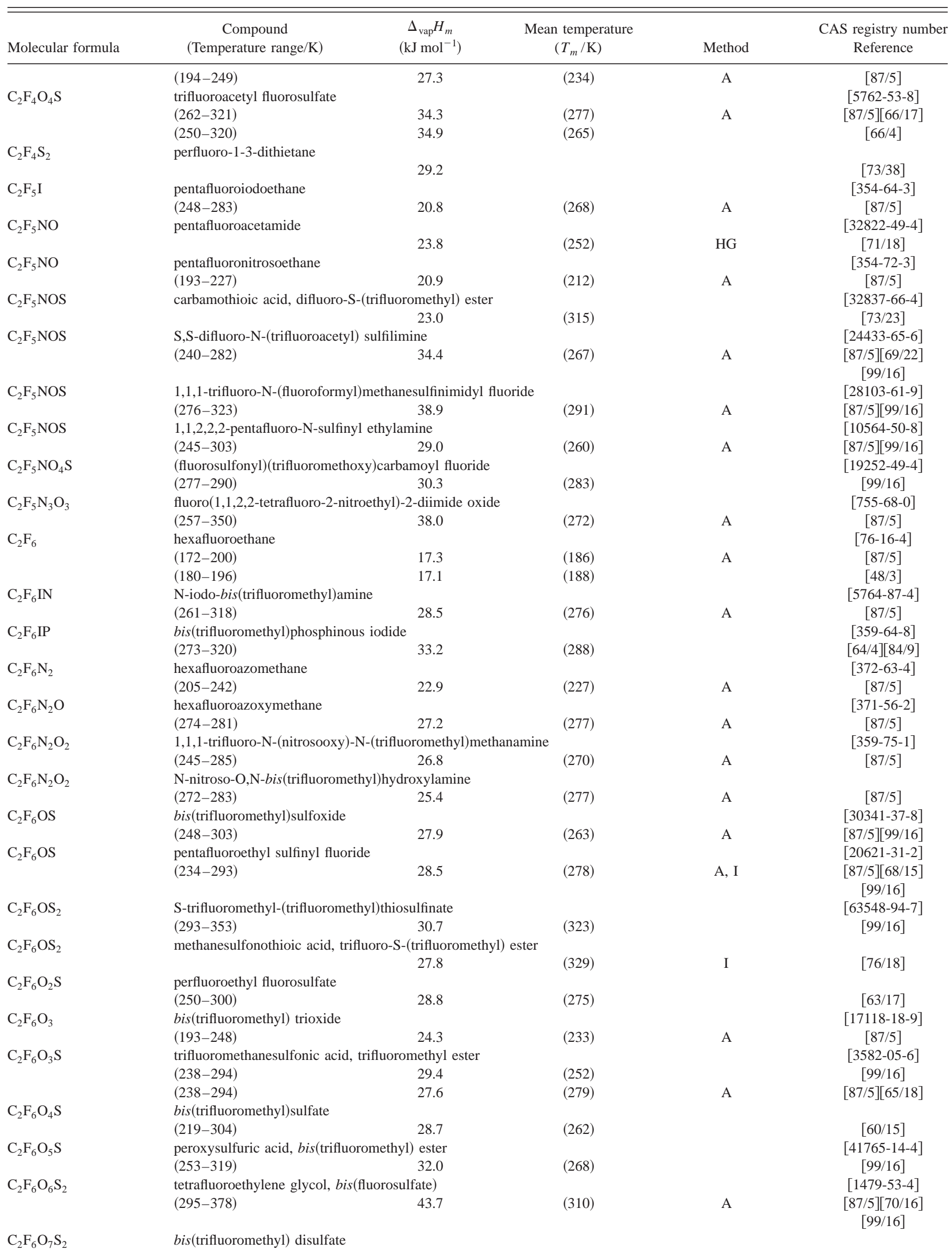


TABLE 6. Enthalpies of vaporization of organic compounds, 1880-2002-Continued

\begin{tabular}{|c|c|c|c|c|c|}
\hline Molecular formula & $\begin{array}{c}\text { Compound } \\
\text { (Temperature range/K) }\end{array}$ & $\begin{array}{c}\Delta_{\mathrm{vap}} H_{m} \\
\left(\mathrm{~kJ} \mathrm{~mol}^{-1}\right)\end{array}$ & $\begin{array}{l}\text { Mean temperature } \\
\qquad\left(T_{m} / \mathrm{K}\right)\end{array}$ & Method & $\begin{array}{l}\text { CAS registry number } \\
\text { Reference }\end{array}$ \\
\hline & $(328-357)$ & 38.3 & $(342)$ & & {$[60 / 15]$} \\
\hline \multirow[t]{2}{*}{$\mathrm{C}_{2} \mathrm{~F}_{6} \mathrm{~S}$} & bis(trifluoromethyl) sulfide & & & & {$[371-78-8]$} \\
\hline & & 23.6 & & & {$[52 / 22]$} \\
\hline \multirow{2}{*}{$\mathrm{C}_{2} \mathrm{~F}_{6} \mathrm{~S}_{2}$} & bis(trifluoromethyl) disulfide & & & & [372-64-5] \\
\hline & & 28.8 & & & {$[52 / 22]$} \\
\hline \multirow[t]{3}{*}{$\mathrm{C}_{2} \mathrm{~F}_{7} \mathrm{~N}$} & perfluorodimethylamine & & & & {$[359-62-6]$} \\
\hline & $(199-230)$ & 21.4 & $(215)$ & $\mathrm{A}$ & {$[87 / 5]$} \\
\hline & $(203-233)$ & 18.6 & $(218)$ & A & {$[87 / 5][49 / 27]$} \\
\hline \multirow[t]{2}{*}{$\mathrm{C}_{2} \mathrm{~F}_{7} \mathrm{~N}$} & perfluoroethylamine & & & & [354-80-3] \\
\hline & $(171-236)$ & 20.8 & $(221)$ & A & {$[87 / 5][70 / 16]$} \\
\hline \multirow[t]{2}{*}{$\mathrm{C}_{2} \mathrm{~F}_{7} \mathrm{NOS}$} & (pentafluoroethyl)imidosulfu & ide & & & {$[59617-28-6]$} \\
\hline & & 30.7 & & & {$[76 / 29]$} \\
\hline \multirow{2}{*}{$\mathrm{C}_{2} \mathrm{~F}_{7} \mathrm{NO}_{3} \mathrm{~S}$} & fluorosulfuric acid, 1,1,2,2-t & o-2-(difluoro & thyl ester & & {$[4188-34-5]$} \\
\hline & $(276-326)$ & 31.1 & $(291)$ & A & {$[87 / 5][99 / 16]$} \\
\hline $\mathrm{C}_{2} \mathrm{~F}_{7} \mathrm{NO}_{12} \mathrm{~S}_{4}$ & fluorosulfuric acid, 1-[bis $[$ (fl & $\begin{array}{c}\text { onyl)oxo]am } \\
43.4\end{array}$ & $\begin{array}{l}\text {,2-trifluoro-ethyliden } \\
\text { (418) }\end{array}$ & & $\begin{array}{c}{[53684-02-9]} \\
{[75 / 21]}\end{array}$ \\
\hline \multirow[t]{2}{*}{$\mathrm{C}_{2} \mathrm{~F}_{8} \mathrm{NOP}$} & [bis(difluoromethyl)amino] & hosphine ox & & & \\
\hline & $(233-278)$ & 30.4 & $(255)$ & & {$[66 / 33]$} \\
\hline \multirow[t]{2}{*}{$\mathrm{C}_{2} \mathrm{~F}_{8} \mathrm{NOP}$} & phosphorous bis(trifluorome & oxide difluor & & & \\
\hline & & 28.0 & $(288)$ & & {$[73 / 24]$} \\
\hline \multirow[t]{3}{*}{$\mathrm{C}_{2} \mathrm{~F}_{8} \mathrm{OS}$} & difluorooxo-bis-(trifluoromet & & & & {$[33716-15-3]$} \\
\hline & $(239-299)$ & 22.4 & $(254)$ & A & {$[87 / 5][99 / 16]$} \\
\hline & & 28.4 & & & {$[71 / 34]$} \\
\hline \multirow[t]{2}{*}{$\mathrm{C}_{2} \mathrm{~F}_{8} \mathrm{OS}$} & pentafluoro(trifluoroacetyl) s & & & & {$[82390-51-0]$} \\
\hline & $(162-290)$ & 26.6 & $(177)$ & & {$[99 / 16]$} \\
\hline \multirow{2}{*}{$\mathrm{C}_{2} \mathrm{~F}_{8} \mathrm{O}_{3} \mathrm{~S}$} & pentafluoro (trifluoroethanep & sulfur & & & {$[60672-61-9]$} \\
\hline & & 28.0 & & & {$[76 / 31]$} \\
\hline \multirow[t]{2}{*}{$\mathrm{C}_{2} \mathrm{~F}_{8} \mathrm{~S}$} & trifluorovinyl sulfur pentaflu & & & & {$[1186-51-2]$} \\
\hline & & 25.1 & $(292)$ & & {$[61 / 21]$} \\
\hline \multirow[t]{2}{*}{$\mathrm{C}_{2} \mathrm{~F}_{8} \mathrm{~S}$} & difluoro bis(trifluoromethyl) & & & & {$[30341-38-9]$} \\
\hline & & 28.8 & & & {$[71 / 34]$} \\
\hline \multirow[t]{2}{*}{$\mathrm{C}_{2} \mathrm{~F}_{10} \mathrm{OS}$} & pentafluoro(pentafluoroethox & & & & \\
\hline & $(245-287)$ & 27.6 & $(272)$ & A & {$[87 / 5][62 / 19]$} \\
\hline $\mathrm{C}_{2} \mathrm{~F}_{10} \mathrm{O}_{2} \mathrm{~S}$ & tetrafluorobis(trifluorometho & & & & {$[2004-38-8]$} \\
\hline & $(246-302)$ & 29.9 & $(261)$ & A & {$[87 / 5][64 / 18]$} \\
\hline $\mathrm{C}_{2} \mathrm{~F}_{10} \mathrm{O}_{3} \mathrm{~S}$ & (trifluoromethoxy) $[$ (trifluoror & lioxy] sulfur & ride & & [41938-43-6] \\
\hline & $(255-317)$ & 32.5 & $(270)$ & & {$[99 / 16]$} \\
\hline $\mathrm{C}_{2} \mathrm{~F}_{10} \mathrm{O}_{3} \mathrm{~S}_{2}$ & pentafluoro[1,2,2,2-tetrafluor & lorosulfonyl) & l] sulfur & & {$[68010-32-2]$} \\
\hline & & 34.8 & & & {$[78 / 23]$} \\
\hline $\mathrm{C}_{2} \mathrm{~F}_{10} \mathrm{~S}$ & trans tetrafluorobis(trifluoror & sulfur & & & {$[42179-02-2]$} \\
\hline & $(233-293)$ & 23.3 & $(278)$ & A & {$[87 / 5][99 / 16]$} \\
\hline $\mathrm{C}_{2} \mathrm{~F}_{11} \mathrm{NS}$ & [bis(trifluoromethyl)amino] & ntafluoride & & & [13888-13-6] \\
\hline & $(233-306)$ & 29.3 & $(248)$ & A & $\begin{array}{c}{[87 / 5][66 / 21]} \\
{[99 / 16]}\end{array}$ \\
\hline $\mathrm{C}_{2} \mathrm{~F}_{12} \mathrm{~S}_{2}$ & perfluoro-1,3-dithietane octa & & & & \\
\hline & & 35.6 & & & {$[73 / 38]$} \\
\hline $\mathrm{C}_{2} \mathrm{~N}_{2}$ & cyanogen & & & & {$[460-19-5]$} \\
\hline & $(240-253)$ & 24.5 & $(246)$ & A & {$[87 / 5]$} \\
\hline & $(246-273)$ & 23.9 & $(257)$ & & {$[25 / 1]$} \\
\hline & $(246-273)$ & 23.5 & $(267)$ & & {$[25 / 1]$} \\
\hline $\mathrm{C}_{2} \mathrm{HBr}$ & bromoacetylene & & & & {$[593-61-3]$} \\
\hline & $(214-273)$ & 25.6 & $(273)$ & A & {$[87 / 5]$} \\
\hline $\mathrm{C}_{2} \mathrm{HBrClF}_{3}$ & 2-bromo-2-chloro-1,1,1-triflu & & & & {$[151-67-7]$} \\
\hline & $(298-323)$ & 30.0 & $(310)$ & A & {$[87 / 5]$} \\
\hline & & $29.6 \pm 0.3$ & $(298)$ & & {$[81 / 5]$} \\
\hline & & $29.6 \pm 0.1$ & $(298)$ & $\mathrm{C}$ & {$[80 / 9]$} \\
\hline & & $28.7 \pm 0.1$ & $(313)$ & $\mathrm{C}$ & {$[80 / 9]$} \\
\hline & & $27.8 \pm 0.1$ & $(328)$ & $\mathrm{C}$ & {$[80 / 9]$} \\
\hline & & $26.8 \pm 0.1$ & $(343)$ & $\mathrm{C}$ & {$[80 / 9]$} \\
\hline & $(227-318)$ & 34.3 & $(242)$ & & {$[65 / 6]$} \\
\hline & $(222-329)$ & 33.2 & $(237)$ & A & {$[87 / 5][63 / 7]$} \\
\hline & & & & & {$[70 / 16]$} \\
\hline $\mathrm{C}_{2} \mathrm{HBrClF}_{3}$ & 1-bromo-2-chloro-1,1,2-triflu & & & & {$[354-06-3]$} \\
\hline & & $30.0 \pm 0.1$ & $(298)$ & $\mathrm{C}$ & {$[81 / 13]$} \\
\hline
\end{tabular}


TABLE 6. Enthalpies of vaporization of organic compounds, 1880-2002-Continued

\begin{tabular}{|c|c|c|c|c|c|}
\hline Molecular formula & $\begin{array}{c}\text { Compound } \\
\text { (Temperature range/K) }\end{array}$ & $\begin{array}{c}\Delta_{\mathrm{vap}} H_{m} \\
\left(\mathrm{~kJ} \mathrm{~mol}^{-1}\right)\end{array}$ & $\begin{array}{l}\text { Mean temperature } \\
\qquad\left(T_{m} / \mathrm{K}\right)\end{array}$ & Method & $\begin{array}{c}\text { CAS registry number } \\
\text { Reference }\end{array}$ \\
\hline & & $29.0 \pm 0.1$ & $(313)$ & $\mathrm{C}$ & {$[81 / 13]$} \\
\hline & & $28.1 \pm 0.1$ & $(328)$ & $\mathrm{C}$ & {$[81 / 13]$} \\
\hline & & $27.2 \pm 0.1$ & $(343)$ & $\mathrm{C}$ & {$[81 / 13]$} \\
\hline \multirow[t]{2}{*}{$\mathrm{C}_{2} \mathrm{HBrF}_{8} \mathrm{~S}$} & (1-bromo-2,2,2-trifluoroet & pentafluoride & & & [82390-50-9] \\
\hline & & 32.0 & & & [82/19] \\
\hline \multirow[t]{2}{*}{$\mathrm{C}_{2} \mathrm{HBr}_{2} \mathrm{FO}_{2}$} & dibromofluoroacetic acid & & & & [353-99-1] \\
\hline & $(403-468)$ & 60.2 & $(418)$ & A & {$[87 / 5]$} \\
\hline \multirow[t]{2}{*}{$\mathrm{C}_{2} \mathrm{HBr}_{3} \mathrm{O}$} & tribromoacetaldehyde & & & & {$[115-17-3]$} \\
\hline & $(291-447)$ & 47.8 & $(306)$ & A & {$[87 / 5][47 / 5]$} \\
\hline \multirow[t]{2}{*}{$\mathrm{C}_{2} \mathrm{HCl}$} & chloroacetylene & & & & [593-63-5] \\
\hline & $(204-238)$ & 21.8 & $(223)$ & A & {$[87 / 5]$} \\
\hline \multirow[t]{5}{*}{$\mathrm{C}_{2} \mathrm{HClF}_{2}$} & 1,1-difluoro-2-chloroethen & & & & [359-10-4] \\
\hline & & 23.5 & $(233)$ & BG & {$[55 / 20]$} \\
\hline & & 21.4 & $(273)$ & BG & {$[55 / 20]$} \\
\hline & & 18.5 & $(313)$ & BG & {$[55 / 20]$} \\
\hline & & 14.1 & $(353)$ & BG & {$[55 / 20]$} \\
\hline \multirow[t]{2}{*}{$\mathrm{C}_{2} \mathrm{HClF}_{6} \mathrm{OS}$} & trans [(2-chloro-2-fluorov & sulfur pentaflu & & & {$[20407-78-7]$} \\
\hline & & 36.8 & & & {$[68 / 17]$} \\
\hline \multirow[t]{2}{*}{$\mathrm{C}_{2} \mathrm{HClF}_{6} \mathrm{OS}$} & cis [(2-chloro-2-fluoroviny & fur pentafluor & & & {$[20407-79-8]$} \\
\hline & & 34.3 & & & {$[68 / 17]$} \\
\hline \multirow[t]{2}{*}{$\mathrm{C}_{2} \mathrm{HClF}_{8} \mathrm{OS}$} & (2-chloro-1,2,2-trifluoroetl & ur pentafluoric & & & [20334-47-8] \\
\hline & & 33.3 & & & {$[68 / 17]$} \\
\hline \multirow[t]{2}{*}{$\mathrm{C}_{2} \mathrm{HClF}_{12} \mathrm{O}_{2} \mathrm{~S}$} & [(2-chloro-2,2-difluoroethy & oxy]bis (pentat & ulfur & & [20563-90-0] \\
\hline & & 39.0 & & & {$[68 / 17]$} \\
\hline \multirow[t]{2}{*}{$\mathrm{C}_{2} \mathrm{HClF}_{8} \mathrm{~S}$} & (1,1,2-trifluoro-2-chloroetl & pentafluoride & & & {$[22756-13-4]$} \\
\hline & $(279-323)$ & 30.2 & $(294)$ & A & {$[87 / 5][99 / 16]$} \\
\hline \multirow[t]{3}{*}{$\mathrm{C}_{2} \mathrm{HCl}_{2} \mathrm{~F}_{3}$} & 1,1,1-trifluoro-2,2-dichlor & & & & [306-83-2] \\
\hline & & $26.6 \pm 0.3$ & $(298)$ & & {$[02 / 1]$} \\
\hline & $(243-448)$ & 28.7 & $(258)$ & MM & {$[92 / 19]$} \\
\hline \multirow[t]{2}{*}{$\mathrm{C}_{2} \mathrm{HCl}_{2} \mathrm{~F}_{3}$} & 1,1,2-trifluoro-1,2-dichlor & & & & {$[354-23-4]$} \\
\hline & & $26.8 \pm 0.3$ & $(298)$ & & {$[02 / 1]$} \\
\hline \multirow[t]{6}{*}{$\mathrm{C}_{2} \mathrm{HCl}_{3}$} & trichloroethylene & & & & {$[79-01-6]$} \\
\hline & $(297-360)$ & 34.2 & (313) & & {$[95 / 14]$} \\
\hline & & $34.5 \pm 0.1$ & $(298)$ & $\mathrm{C}$ & {$[80 / 1]$} \\
\hline & $(280-428)$ & 34.6 & $(295)$ & & {$[87 / 5][70 / 16]$} \\
\hline & $(290-359)$ & 36.2 & $(305)$ & & {$[44 / 1]$} \\
\hline & $(298-360)$ & 35.6 & (313) & & {$[12 / 1][84 / 9]$} \\
\hline \multirow[t]{2}{*}{$\mathrm{C}_{2} \mathrm{HCl}_{3} \mathrm{~F}_{2} \mathrm{O}_{3} \mathrm{~S}$} & fluorosulfuric acid, 2-fluo & ichloroethyl es & & & [42087-88-7] \\
\hline & $(317-353)$ & 36.6 & $(332)$ & A & {$[87 / 5][99 / 16]$} \\
\hline $\mathrm{C}_{2} \mathrm{HCl}_{3} \mathrm{O}$ & trichloroacetaldehyde & & & & {$[75-87-6]$} \\
\hline & $(235-371)$ & 36.6 & $(250)$ & A & {$[87 / 5][47 / 5]$} \\
\hline $\mathrm{C}_{2} \mathrm{HCl}_{3} \mathrm{O}_{2}$ & trichloroacetic acid & & & & [76-03-9] \\
\hline & $(326-473)$ & 65.0 & $(341)$ & A & {$[87 / 5][70 / 16]$} \\
\hline & $(385-470)$ & 57.2 & $(400)$ & & {$[59 / 1]$} \\
\hline $\mathrm{C}_{2} \mathrm{HCl}_{4} \mathrm{FS}$ & (dichloromethyl)(fluorodic & nyl) sulfide & & & \\
\hline & $(322-352)$ & 46.5 & $(337)$ & A & {$[87 / 5][99 / 16]$} \\
\hline $\mathrm{C}_{2} \mathrm{HCl}_{5}$ & pentachloroethane & & & & {$[76-01-7]$} \\
\hline & $(274-434)$ & 40.9 & $(289)$ & A & {$[87 / 5]$} \\
\hline & $(298-435)$ & 45.5 & (313) & & {$[30 / 1]$} \\
\hline $\mathrm{C}_{2} \mathrm{HF}_{3} \mathrm{O}_{2}$ & trifluoroacetic acid & & & & [76-05-1] \\
\hline & $(285-345)$ & 35.9 & $(300)$ & A & {$[87 / 5][62 / 4]$} \\
\hline & & & & & {$[70 / 16][84 / 9]$} \\
\hline $\mathrm{C}_{2} \mathrm{HF}_{5}$ & pentafluoroethane & & & & [354-33-6] \\
\hline & & 22.8 & $(175)$ & $\mathrm{C}$ & {$[99 / 26]$} \\
\hline & & 21.9 & $(190)$ & $\mathrm{C}$ & {$[99 / 26]$} \\
\hline & & 20.9 & $(205)$ & $\mathrm{C}$ & {$[99 / 26]$} \\
\hline & & 20.3 & $(215)$ & $\mathrm{C}$ & {$[99 / 26]$} \\
\hline $\mathrm{C}_{2} \mathrm{HF}_{5} \mathrm{O}$ & pentafluorodimethyl ether & & & & [3822-68-2] \\
\hline & $(216-238)$ & 22.3 & $(239)$ & $\mathrm{I}$ & {$[01 / 22]$} \\
\hline & $(229-331)$ & 19.3 & $(260)$ & $\mathrm{EB}$ & {$[96 / 6]$} \\
\hline & $(229-331)$ & 17.6 & $(280)$ & $\mathrm{EB}$ & {$[96 / 6]$} \\
\hline & $(229-331)$ & 15.6 & $(300)$ & EB & {$[96 / 6]$} \\
\hline & $(240-313)$ & 20.4 & $(255)$ & A & {$[92 / 12]$} \\
\hline $\mathrm{C}_{2} \mathrm{HF}_{6} \mathrm{NOS}$ & $\mathrm{S}, \mathrm{S}-$ bis(trifluoromethyl)su & & & & {$[34556-22-4]$} \\
\hline
\end{tabular}


TABLE 6. Enthalpies of vaporization of organic compounds, 1880-2002-Continued

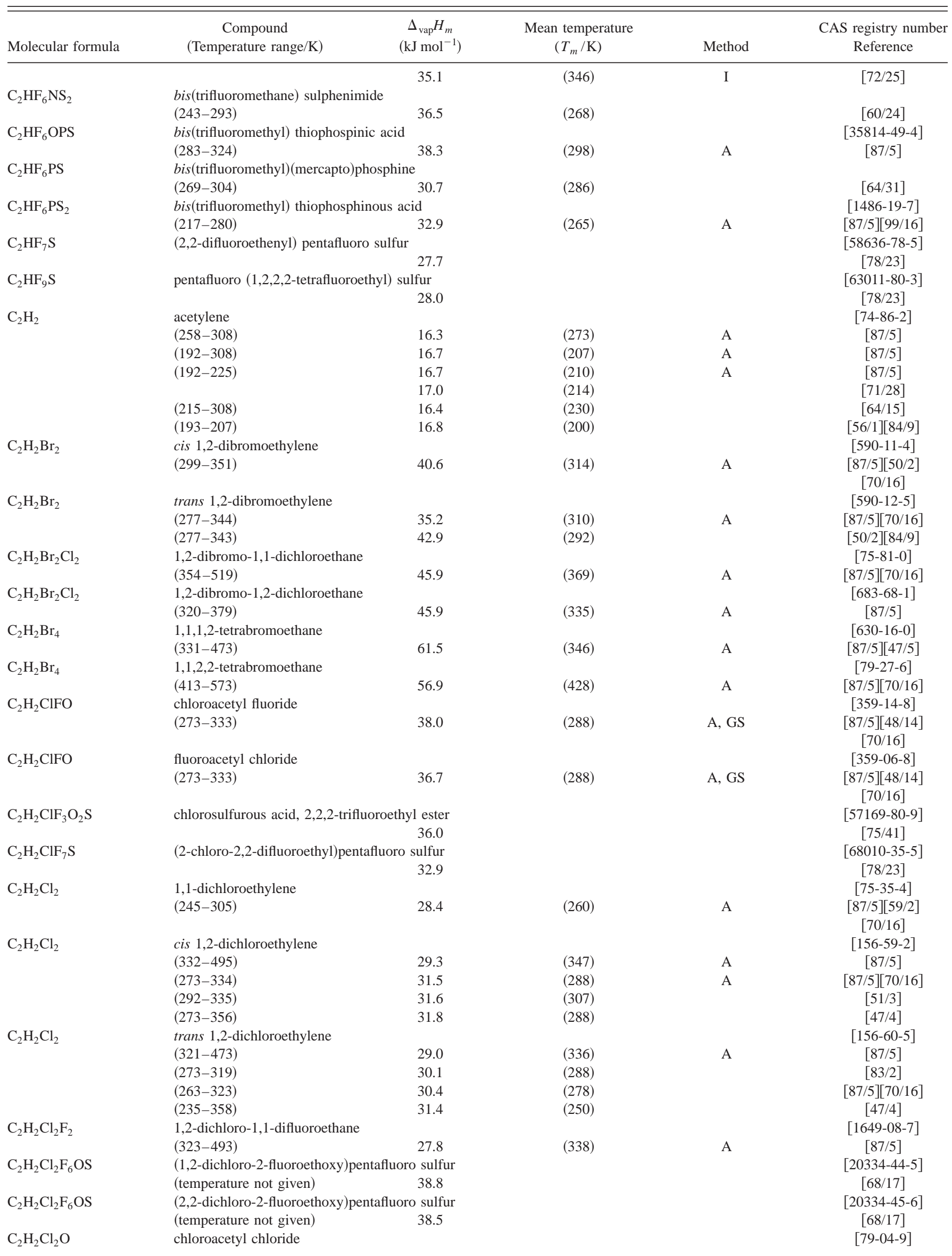


TABLE 6. Enthalpies of vaporization of organic compounds, 1880-2002-Continued

\begin{tabular}{|c|c|c|c|c|c|}
\hline Molecular formula & $\begin{array}{c}\text { Compound } \\
\text { (Temperature range/K) }\end{array}$ & $\begin{array}{c}\Delta_{\mathrm{vap}} H_{m} \\
\left(\mathrm{~kJ} \mathrm{~mol}^{-1}\right)\end{array}$ & $\begin{array}{l}\text { Mean temperature } \\
\qquad\left(T_{m} / \mathrm{K}\right)\end{array}$ & Method & $\begin{array}{c}\text { CAS registry number } \\
\text { Reference }\end{array}$ \\
\hline & $(253-379)$ & 45.0 & $(268)$ & A & {$[87 / 5][70 / 16]$} \\
\hline & $(301-380)$ & 40.8 & $(316)$ & & {$[59 / 1]$} \\
\hline & $(290-373)$ & 44.1 & $(305)$ & & {$[35 / 5]$} \\
\hline \multirow[t]{2}{*}{$\mathrm{C}_{2} \mathrm{H}_{2} \mathrm{Cl}_{2} \mathrm{O}_{2}$} & dichloroacetic acid & & & & {$[79-43-6]$} \\
\hline & $(317-468)$ & 55.7 & $(332)$ & A & {$[87 / 5][47 / 5]$} \\
\hline \multirow[t]{4}{*}{$\mathrm{C}_{2} \mathrm{H}_{2} \mathrm{Cl}_{4}$} & 1,1,1,2-tetrachloroethane & & & & {$[630-20-6]$} \\
\hline & & $45.7 \pm 0.1$ & $(298)$ & $\mathrm{C}$ & {$[80 / 1]$} \\
\hline & $(316-447)$ & 40.1 & $(331)$ & $\mathrm{A}$ & {$[87 / 5][70 / 16]$} \\
\hline & $(332-403)$ & 39.2 & $(347)$ & & {$[49 / 1]$} \\
\hline \multirow[t]{9}{*}{$\mathrm{C}_{2} \mathrm{H}_{2} \mathrm{Cl}_{4}$} & 1,1,2,2-tetrachloroethane & & & & {$[79-34-5]$} \\
\hline & $(377-419)$ & 40.4 & $(392)$ & A & {$[87 / 5]$} \\
\hline & $(371-419)$ & 40.8 & (394) & & {$[84 / 20]$} \\
\hline & $(377-418)$ & 40.1 & $(398)$ & & {$[78 / 21]$} \\
\hline & & 39.0 & $(415)$ & & {$[77 / 30]$} \\
\hline & & $45.8 \pm 0.2$ & $(298)$ & $\mathrm{C}$ & {$[72 / 41]$} \\
\hline & $(328-464)$ & 41.9 & $(343)$ & A & {$[87 / 5][70 / 16]$} \\
\hline & $(298-403)$ & 47.7 & $(313)$ & & {$[50 / 1]$} \\
\hline & $(304-419)$ & 45.7 & (319) & & {$[30 / 1]$} \\
\hline \multirow[t]{2}{*}{$\mathrm{C}_{2} \mathrm{H}_{2} \mathrm{Cl}_{4} \mathrm{~S}$} & bis(dichloromethyl) sulfide & & & & {$[51174-93-7]$} \\
\hline & $(355-462)$ & 47.6 & $(370)$ & A & {$[87 / 5]$} \\
\hline \multirow[t]{2}{*}{$\mathrm{C}_{2} \mathrm{H}_{2} \mathrm{FN}$} & fluoroacetonitrile & & & & [503-20-8] \\
\hline & $(273-333)$ & 38.1 & $(288)$ & $\mathrm{A}, \mathrm{GS}$ & $\begin{array}{c}{[87 / 5][48 / 14]} \\
{[70 / 16]}\end{array}$ \\
\hline \multirow[t]{3}{*}{$\mathrm{C}_{2} \mathrm{H}_{2} \mathrm{~F}_{2}$} & 1,1-difluoroethene & & & & {$[75-38-7]$} \\
\hline & & 13.2 & $(233)$ & BG & {$[55 / 20]$} \\
\hline & & 9.5 & $(273)$ & BG & {$[55 / 20]$} \\
\hline \multirow[t]{7}{*}{$\mathrm{C}_{2} \mathrm{H}_{2} \mathrm{~F}_{4}$} & 1,1,1,2-tetrafluoroethane & & & & {$[811-97-2]$} \\
\hline & $(221-246)$ & 23.7 & $(249)$ & $\mathrm{I}$ & {$[01 / 22]$} \\
\hline & & 26.4 & $(180)$ & & {$[98 / 24]$} \\
\hline & & 25.0 & $(200)$ & & {$[98 / 24]$} \\
\hline & & 23.8 & $(220)$ & & {$[98 / 24]$} \\
\hline & & 22.7 & $(240)$ & & {$[98 / 24]$} \\
\hline & $(279-363)$ & 22.0 & $(294)$ & & {$[92 / 16]$} \\
\hline \multirow[t]{2}{*}{$\mathrm{C}_{2} \mathrm{H}_{2} \mathrm{~F}_{4} \mathrm{O}_{2} \mathrm{~S}$} & fluorosulfurous acid, 2,2,2-t & thyl ester & & & \\
\hline & & 33.6 & & & {$[75 / 41]$} \\
\hline \multirow[t]{2}{*}{$\mathrm{C}_{2} \mathrm{H}_{2} \mathrm{~F}_{6} \mathrm{P}_{2}$} & 1,2-bis-(trifluoromethyl) dip & & & & {$[462-57-7]$} \\
\hline & $(233-292)$ & 33.8 & $(277)$ & $\mathrm{A}, \mathrm{SG}$ & {$[87 / 5][58 / 10]$} \\
\hline \multirow[t]{2}{*}{$\mathrm{C}_{2} \mathrm{H}_{2} \mathrm{~F}_{8} \mathrm{~S}$} & pentafluoro (2,2,2-trifluoroet & & & & {$[65227-29-4]$} \\
\hline & & 29.3 & & & {$[78 / 23]$} \\
\hline \multirow[t]{3}{*}{$\mathrm{C}_{2} \mathrm{H}_{2} \mathrm{I}_{2}$} & cis 1,2-diiodoethylene & & & & [590-26-1] \\
\hline & $(302-425)$ & 46.5 & $(317)$ & A & {$[87 / 5][70 / 16]$} \\
\hline & $(302-424)$ & 47.3 & $(317)$ & & {$[50 / 2]$} \\
\hline \multirow[t]{3}{*}{$\mathrm{C}_{2} \mathrm{H}_{2} \mathrm{I}_{2}$} & trans 1,2-diiodoethylene & & & & {$[590-27-2]$} \\
\hline & $(350-403)$ & 42.3 & $(365)$ & A & {$[87 / 5][70 / 16]$} \\
\hline & $(350-403)$ & 43.8 & $(365)$ & & {$[50 / 2]$} \\
\hline \multirow[t]{2}{*}{$\mathrm{C}_{2} \mathrm{H}_{2} \mathrm{O}$} & ketene & & & & {$[463-51-4]$} \\
\hline & $(159-224)$ & $20.4 \pm 0.1$ & $(209)$ & $\mathrm{A}, \mathrm{MM}$ & {$[87 / 5][69 / 7]$} \\
\hline \multirow[t]{4}{*}{$\mathrm{C}_{2} \mathrm{H}_{3} \mathrm{Br}$} & vinyl bromide & & & & {$[593-60-2]$} \\
\hline & $(224-319)$ & 27.3 & $(239)$ & A & {$[87 / 5][70 / 16]$} \\
\hline & $(186-289)$ & 24.8 & $(274)$ & & {$[37 / 4][84 / 9]$} \\
\hline & $(207-285)$ & 26.9 & $(270)$ & & {$[34 / 1][84 / 9]$} \\
\hline \multirow[t]{3}{*}{$\mathrm{C}_{2} \mathrm{H}_{3} \mathrm{BrO}$} & acetyl bromide & & & & {$[506-96-7]$} \\
\hline & $(289-334)$ & 29.5 & $(304)$ & A & {$[87 / 5]$} \\
\hline & $(275-333)$ & 31.4 & $(290)$ & & {$[69 / 3]$} \\
\hline $\mathrm{C}_{2} \mathrm{H}_{3} \mathrm{BrO}_{2}$ & bromoacetic acid & & & & [79-08-3] \\
\hline & $(327-481)$ & 57.2 & $(342)$ & A & {$[87 / 5]$} \\
\hline $\mathrm{C}_{2} \mathrm{H}_{3} \mathrm{Br}_{3}$ & 1,1,2-tribromoethane & & & & [78-74-0] \\
\hline & $(368-511)$ & 50.5 & $(383)$ & A & {$[87 / 5][70 / 16]$} \\
\hline & $(305-461)$ & 52.9 & $(321)$ & & {$[47 / 5]$} \\
\hline $\mathrm{C}_{2} \mathrm{H}_{3} \mathrm{Cl}$ & vinyl chloride & & & & {$[75-01-4]$} \\
\hline & $(243-288)$ & 22.7 & $(265)$ & & {$[67 / 35]$} \\
\hline & $(213-273)$ & 22.9 & $(258)$ & & {$[67 / 11]$} \\
\hline & $(209-260)$ & 23.3 & $(245)$ & A & $\begin{array}{c}{[87 / 5][59 / 1]} \\
{[70 / 16]}\end{array}$ \\
\hline
\end{tabular}


TABLE 6. Enthalpies of vaporization of organic compounds, 1880-2002-Continued

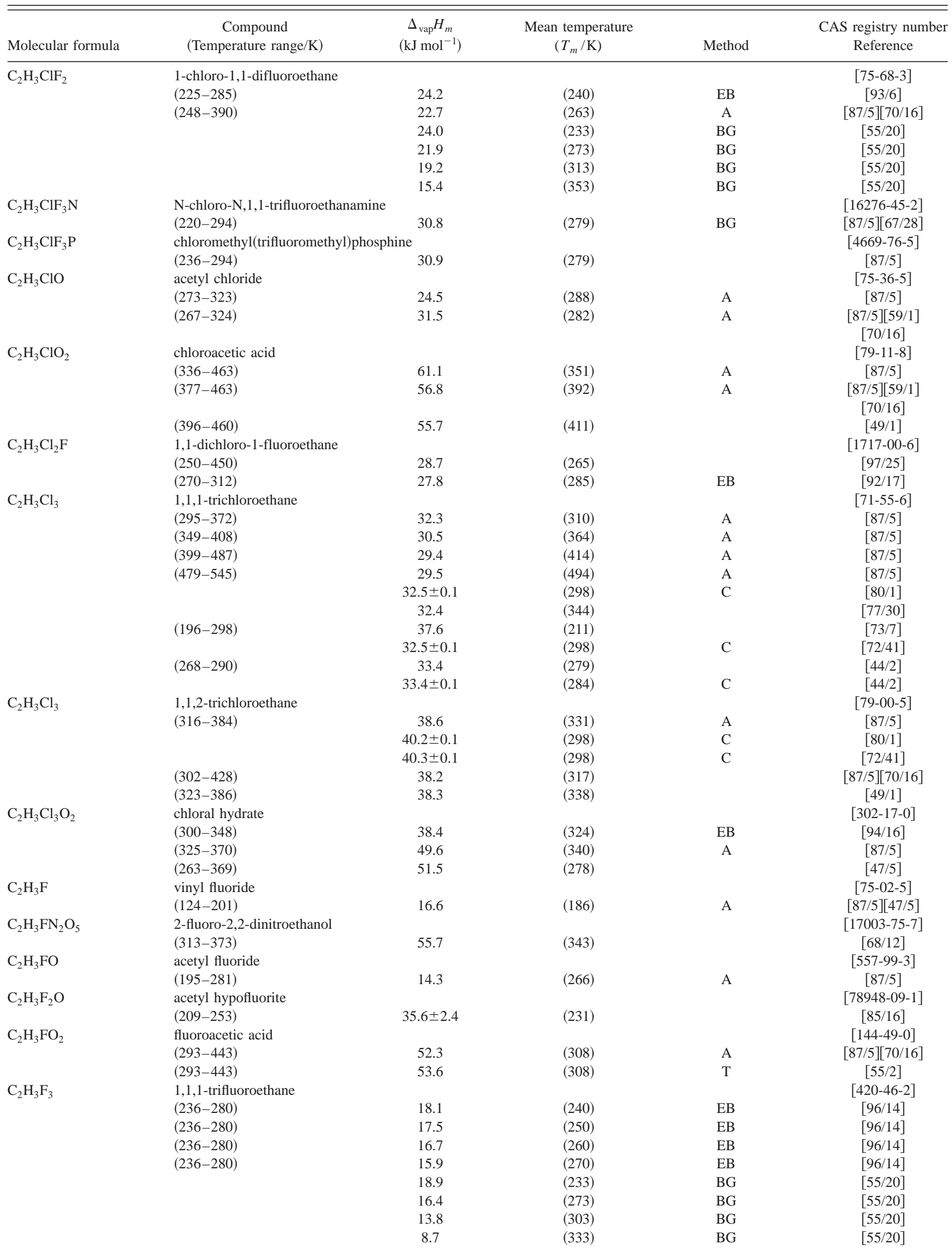


TABLE 6. Enthalpies of vaporization of organic compounds, 1880-2002-Continued

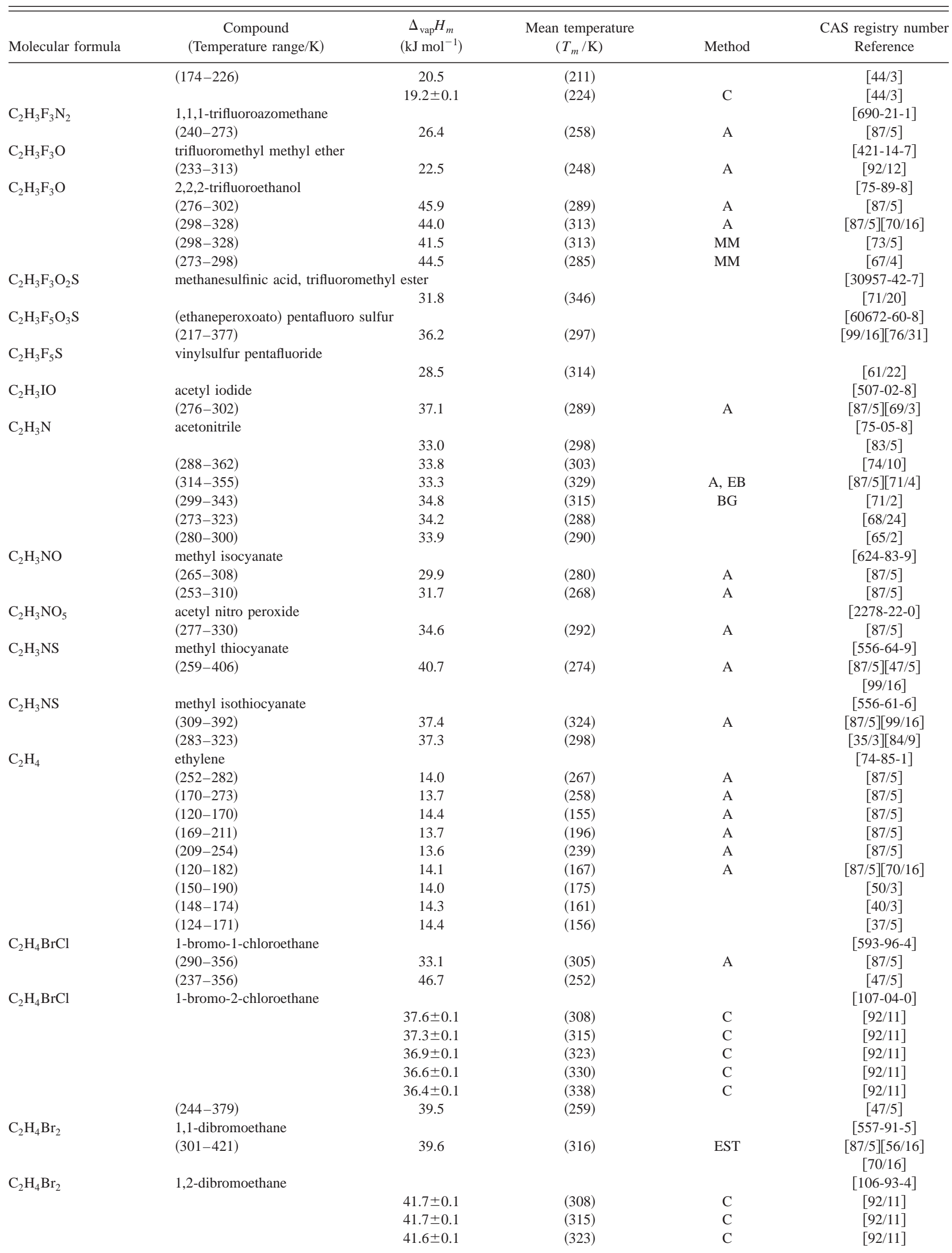


TABLE 6. Enthalpies of vaporization of organic compounds, 1880-2002-Continued

\begin{tabular}{|c|c|c|c|c|c|}
\hline Molecular formula & $\begin{array}{c}\text { Compound } \\
\text { (Temperature range/K) }\end{array}$ & $\begin{array}{c}\Delta_{\mathrm{vap}} H_{m} \\
\left(\mathrm{~kJ} \mathrm{~mol}^{-1}\right)\end{array}$ & $\begin{array}{l}\text { Mean temperature } \\
\qquad\left(T_{m} / \mathrm{K}\right)\end{array}$ & Method & $\begin{array}{c}\text { CAS registry number } \\
\text { Reference }\end{array}$ \\
\hline & & $41.5 \pm 0.1$ & $(330)$ & $\mathrm{C}$ & {$[92 / 11]$} \\
\hline & & $41.4 \pm 0.1$ & (338) & $\mathrm{C}$ & {$[92 / 11]$} \\
\hline & $(283-317)$ & 41.8 & $(298)$ & A & {$[87 / 5]$} \\
\hline & $(316-488)$ & 40.0 & $(331)$ & A & {$[87 / 5]$} \\
\hline & $(404-578)$ & 37.4 & $(419)$ & $\mathrm{A}$ & {$[87 / 5]$} \\
\hline & $(285-298)$ & 49.6 & $(291)$ & MM, A & {$[57 / 1]$} \\
\hline & $(325-404)$ & 39.6 & $(340)$ & & {$[49 / 1]$} \\
\hline & $(246-404)$ & 31.1 & $(261)$ & & {$[47 / 5]$} \\
\hline \multirow[t]{2}{*}{$\mathrm{C}_{2} \mathrm{H}_{4} \mathrm{ClF}$} & 1-chloro-2-fluoroethane & & & & {$[762-50-5]$} \\
\hline & $(288-327)$ & 32.1 & $(303)$ & A & {$[87 / 5]$} \\
\hline \multirow[t]{2}{*}{$\mathrm{C}_{2} \mathrm{H}_{4} \mathrm{ClN}_{3}$} & 1-chloro-2-azidoethane & & & & {$[53422-48-3]$} \\
\hline & $(273-333)$ & 43.8 & $(288)$ & A & {$[87 / 5]$} \\
\hline \multirow[t]{8}{*}{$\mathrm{C}_{2} \mathrm{H}_{4} \mathrm{Cl}_{2}$} & 1,1-dichloroethane & & & & {$[75-34-3]$} \\
\hline & $(326-345)$ & 33.5 & $(336)$ & & {$[87 / 15]$} \\
\hline & $(323-535)$ & 29.2 & (338) & A & {$[87 / 5]$} \\
\hline & $(363-535)$ & 28.2 & $(378)$ & A & {$[87 / 5]$} \\
\hline & & $30.6 \pm 0.1$ & $(298)$ & $\mathrm{C}$ & {$[72 / 41]$} \\
\hline & $(234-290)$ & 31.9 & $(275)$ & & {$[56 / 2]$} \\
\hline & $(213-330)$ & 34.4 & $(228)$ & & {$[47 / 5]$} \\
\hline & $(258-365)$ & 32.2 & $(273)$ & EST & $\begin{array}{c}{[87 / 5][56 / 16]} \\
{[70 / 16]}\end{array}$ \\
\hline \multirow[t]{12}{*}{$\mathrm{C}_{2} \mathrm{H}_{4} \mathrm{Cl}_{2}$} & 1,2-dichloroethane & & & & [107-06-2] \\
\hline & & 34.4 & $(298)$ & GC & [94/19] \\
\hline & & $35.1 \pm 0.1$ & $(298)$ & $\mathrm{C}$ & {$[89 / 10]$} \\
\hline & $(356-558)$ & 31.1 & $(371)$ & A & {$[87 / 5]$} \\
\hline & $(279-374)$ & 34.8 & $(294)$ & A & {$[87 / 5]$} \\
\hline & $(368-524)$ & 31.1 & $(383)$ & A & {$[87 / 5]$} \\
\hline & $(523-561)$ & 40.8 & $(538)$ & A & {$[87 / 5]$} \\
\hline & $(301-357)$ & 34.7 & $(316)$ & & {$[82 / 7]$} \\
\hline & & $35.2 \pm 0.1$ & $(298)$ & $\mathrm{C}$ & {$[80 / 1]$} \\
\hline & $(279-434)$ & 34.8 & $(294)$ & & {$[87 / 5][70 / 16]$} \\
\hline & & 34.7 & & & {$[56 / 25][38 / 12]$} \\
\hline & $(243-372)$ & 37.5 & $(258)$ & & {$[29 / 1]$} \\
\hline \multirow[t]{2}{*}{$\mathrm{C}_{2} \mathrm{H}_{4} \mathrm{Cl}_{2} \mathrm{~S}$} & bis(chloromethyl)sulfide & & & & [3592-44-7] \\
\hline & $(320-430)$ & 45.1 & $(335)$ & A & {$[87 / 5][99 / 16]$} \\
\hline \multirow[t]{2}{*}{$\mathrm{C}_{2} \mathrm{H}_{4} \mathrm{FNO}_{3}$} & 2-fluoroethyl nitrate & & & & [763-97-3] \\
\hline & $(273-333)$ & 38.3 & $(288)$ & GS & $\begin{array}{c}{[87 / 5][48 / 14]} \\
{[70 / 16]}\end{array}$ \\
\hline \multirow[t]{11}{*}{$\mathrm{C}_{2} \mathrm{H}_{4} \mathrm{~F}_{2}$} & 1,1-difluoroethane & & & & {$[75-37-6]$} \\
\hline & $(218-248)$ & 22.7 & $(249)$ & $\mathrm{I}$ & {$[01 / 22]$} \\
\hline & $(303-333)$ & 22.1 & $(318)$ & & {$[99 / 29]$} \\
\hline & $(219-273)$ & 23.3 & $(234)$ & EB & {$[93 / 6]$} \\
\hline & $(250-386)$ & 21.8 & $(265)$ & A & {$[87 / 5]$} \\
\hline & $(193-275)$ & 22.1 & $(260)$ & A, EST & $\begin{array}{c}{[87 / 5][56 / 16]} \\
{[70 / 16]}\end{array}$ \\
\hline & & 21.8 & $(233)$ & BG & {$[55 / 20]$} \\
\hline & & 20.4 & $(273)$ & BG & {$[55 / 20]$} \\
\hline & & 17.8 & $(313)$ & BG & {$[55 / 20]$} \\
\hline & & 12.9 & $(353)$ & BG & {$[55 / 20]$} \\
\hline & $(161-247)$ & 23.8 & $(232)$ & & {$[47 / 5]$} \\
\hline \multirow[t]{2}{*}{$\mathrm{C}_{2} \mathrm{H}_{4} \mathrm{~F}_{3} \mathrm{NS}$} & 1,1,1-trifluoro- $\mathrm{N}$-methyl $\mathrm{n}$ & enamide & & & [62067-12-3] \\
\hline & $(223-294)$ & 33.6 & $(279)$ & $\mathrm{A}$ & {$[87 / 5][60 / 24]$} \\
\hline \multirow[t]{2}{*}{$\mathrm{C}_{2} \mathrm{H}_{4} \mathrm{~F}_{3} \mathrm{OP}$} & (trifluoromethyl)phosphin & lethyl ester & & & {$[6395-71-7]$} \\
\hline & $(194-291)$ & 29.4 & $(276)$ & A & {$[87 / 5]$} \\
\hline \multirow[t]{2}{*}{$\mathrm{C}_{2} \mathrm{H}_{4} \mathrm{~F}_{3} \mathrm{OP}$} & methyl(trifluoromethyl)ph & ide & & & [26348-89-0] \\
\hline & $(305-322)$ & 50.7 & $(313)$ & & {$[70 / 26]$} \\
\hline \multirow[t]{2}{*}{$\mathrm{C}_{2} \mathrm{H}_{4} \mathrm{~F}_{6} \mathrm{OS}$} & pentafluoro(2-fluoroethoxy & & & & \\
\hline & $(290-364)$ & 39.3 & $(305)$ & A & $\begin{array}{c}{[87 / 5][62 / 19]} \\
{[99 / 16]}\end{array}$ \\
\hline \multirow[t]{3}{*}{$\mathrm{C}_{2} \mathrm{H}_{4} \mathrm{I}_{2}$} & 1,2-diiodoethane & & & & {$[624-73-7]$} \\
\hline & & 49.8 & $(298)$ & GC & [94/19] \\
\hline & $(371-526)$ & 47.7 & $(386)$ & A & {$[87 / 5][70 / 16]$} \\
\hline \multirow[t]{2}{*}{$\mathrm{C}_{2} \mathrm{H}_{4} \mathrm{~N}_{2} \mathrm{O}_{4}$} & 1,1-dinitroethane & & & & {$[600-40-8]$} \\
\hline & $(303-363)$ & 51.0 & $(318)$ & $\mathrm{A}$ & {$[87 / 5]$} \\
\hline
\end{tabular}


TABLE 6. Enthalpies of vaporization of organic compounds, 1880-2002-Continued

\begin{tabular}{|c|c|c|c|c|c|}
\hline Molecular formula & $\begin{array}{c}\text { Compound } \\
\text { (Temperature range/K) }\end{array}$ & $\begin{array}{c}\Delta_{\mathrm{vap}} H_{m} \\
\left(\mathrm{~kJ} \mathrm{~mol}^{-1}\right)\end{array}$ & $\begin{array}{c}\text { Mean temperature } \\
\qquad\left(T_{m} / \mathrm{K}\right)\end{array}$ & Method & $\begin{array}{l}\text { CAS registry number } \\
\text { Reference }\end{array}$ \\
\hline \multirow[t]{6}{*}{$\mathrm{C}_{2} \mathrm{H}_{4} \mathrm{~N}_{2} \mathrm{O}_{6}$} & ethylene glycol dinitrate & & & & [628-96-6] \\
\hline & $(283-535)$ & 70.5 & $(298)$ & A & {$[87 / 5]$} \\
\hline & $(343-465)$ & 55.3 & (358) & $\mathrm{A}$ & {$[87 / 5]$} \\
\hline & $(240-298)$ & 68.3 & $(255)$ & & {$[87 / 5][77 / 6]$} \\
\hline & $(278-390)$ & $62.3 \pm 0.4$ & & & [41/1] \\
\hline & $(293-323)$ & $68.6 \pm 0.4$ & & & {$[38 / 1]$} \\
\hline \multirow[t]{5}{*}{$\mathrm{C}_{2} \mathrm{H}_{4} \mathrm{O}$} & acetaldehyde & & & & {$[75-07-0]$} \\
\hline & $(293-377)$ & 26.0 & $(308)$ & A & {$[87 / 5]$} \\
\hline & $(293-345)$ & 26.3 & (308) & & {$[77 / 24]$} \\
\hline & $(272-294)$ & 27.6 & $(283)$ & A & {$[87 / 5][70 / 16]$} \\
\hline & $(273-307)$ & 27.0 & $(307)$ & & {$[50 / 4]$} \\
\hline \multirow[t]{5}{*}{$\mathrm{C}_{2} \mathrm{H}_{4} \mathrm{O}$} & ethylene oxide (oxirane) & & & & {$[75-21-8]$} \\
\hline & $(283-385)$ & 25.9 & (298) & $\mathrm{A}$ & {$[87 / 5]$} \\
\hline & $(239-284)$ & 26.8 & (269) & A & $\begin{array}{c}{[87 / 5][59 / 1]} \\
{[70 / 16]}\end{array}$ \\
\hline & $(223-284)$ & 26.8 & (269) & $\mathrm{A}$ & {$[87 / 5][49 / 4]$} \\
\hline & $(268-313)$ & 26.9 & $(290)$ & & {$[37 / 7]$} \\
\hline \multirow[t]{2}{*}{$\mathrm{C}_{2} \mathrm{D}_{4} \mathrm{O}$} & ethylene oxide- $\mathrm{d}_{4}$ oxide & & & & [6552-57-4] \\
\hline & $(230-273)$ & 27.6 & $(258)$ & & {$[52 / 2][84 / 9]$} \\
\hline \multirow[t]{2}{*}{$\mathrm{C}_{2} \mathrm{H}_{4} \mathrm{OS}$} & thioacetic acid & & & & {$[507-09-5]$} \\
\hline & $(307-360)$ & 35.2 & (333) & & {$[99 / 16]$} \\
\hline \multirow{9}{*}{$\mathrm{C}_{2} \mathrm{H}_{4} \mathrm{O}_{2}$} & acetic acid & & & & [64-19-7] \\
\hline & $(345-383)$ & 39.1 & $(360)$ & $\mathrm{EB}$ & {$[01 / 15]$} \\
\hline & $(391-550)$ & 37.9 & (406) & A & {$[87 / 5]$} \\
\hline & $(290-396)$ & 42.0 & (305) & A & {$[87 / 5]$} \\
\hline & $(391-447)$ & 38.7 & $(406)$ & A & {$[87 / 5]$} \\
\hline & $(437-535)$ & 38.1 & $(452)$ & A & {$[87 / 5]$} \\
\hline & $(525-593)$ & 38.8 & $(540)$ & $\mathrm{A}$ & {$[87 / 5]$} \\
\hline & & 43.0 & $(308)$ & & [83/13] \\
\hline & $(289-392)$ & 41.6 & (304) & A & {$[87 / 5][70 / 16]$} \\
\hline \multirow[t]{4}{*}{ monomer } & & $23.3 \pm 0.1$ & (298) & $\mathrm{C}$ & {$[70 / 8]$} \\
\hline & & $51.6 \pm 1.6$ & (298) & $\mathrm{C}$ & {$[70 / 8]$} \\
\hline & $(325-391)$ & 40.3 & $(340)$ & & {$[59 / 1]$} \\
\hline & $(303-399)$ & 41.6 & (318) & MM & {$[54 / 3]$} \\
\hline \multirow{8}{*}{$\mathrm{C}_{2} \mathrm{H}_{4} \mathrm{O}_{2}$} & methyl formate & & & & [107-31-3] \\
\hline & $(279-305)$ & 29.6 & $(292)$ & A & {$[87 / 5]$} \\
\hline & $(305-443)$ & 28.4 & $(320)$ & $\mathrm{A}$ & {$[87 / 5]$} \\
\hline & & $28.7 \pm 0.1$ & $(293)$ & $\mathrm{C}$ & {$[76 / 14]$} \\
\hline & & $27.9 \pm 0.1$ & $(305)$ & $\mathrm{C}$ & {$[76 / 14]$} \\
\hline & & $27.4 \pm 0.1$ & (313) & $\mathrm{C}$ & {$[76 / 14]$} \\
\hline & $(261-305)$ & 30.1 & (283) & BG & {$[71 / 2]$} \\
\hline & $(294-304)$ & 52.7 & (299) & & {$[28 / 1][84 / 9]$} \\
\hline \multirow{2}{*}{$\mathrm{C}_{2} \mathrm{H}_{4} \mathrm{O}_{2} \mathrm{~S}$} & mercaptoacetic acid & & & & [68-11-1] \\
\hline & $(333-427)$ & 56.8 & $(348)$ & A & {$[87 / 5][99 / 16]$} \\
\hline \multirow[t]{2}{*}{$\mathrm{C}_{2} \mathrm{H}_{4} \mathrm{O}_{3}$} & ethylene ozonide & & & & \\
\hline & $(273-289)$ & 34.8 & $(281)$ & $\mathrm{A}$ & {$[87 / 5][56 / 18]$} \\
\hline \multirow[t]{2}{*}{$\mathrm{C}_{2} \mathrm{H}_{4} \mathrm{O}_{3}$} & hydroxyacetic acid & & & & [79-14-1] \\
\hline & $(350-375)$ & 51.8 & $(362)$ & A & {$[87 / 5]$} \\
\hline \multirow[t]{2}{*}{$\mathrm{C}_{2} \mathrm{H}_{4} \mathrm{O}_{3}$} & peroxyacetic acid & & & & [79-21-0] \\
\hline & $(273-383)$ & 44.2 & (288) & A & {$[87 / 5][70 / 16]$} \\
\hline \multirow[t]{4}{*}{$\mathrm{C}_{2} \mathrm{H}_{4} \mathrm{~S}$} & ethylene sulfide & & & & {$[420-12-2]$} \\
\hline & $(291-361)$ & 30.5 & $(306)$ & $\mathrm{A}$ & {$[87 / 5][52 / 3]$} \\
\hline & & & & & [99/16] \\
\hline & & 30.3 & (298) & & {$[71 / 28]$} \\
\hline \multirow[t]{10}{*}{$\mathrm{C}_{2} \mathrm{H}_{5} \mathrm{Br}$} & ethyl bromide & & & & [74-96-4] \\
\hline & $(334-504)$ & 26.9 & (349) & A & {$[87 / 5]$} \\
\hline & $(326-454)$ & 26.6 & $(341)$ & $\mathrm{A}$ & {$[87 / 5]$} \\
\hline & $(452-503)$ & 31.0 & (467) & A & {$[87 / 5]$} \\
\hline & & $27.6 \pm 0.1$ & $(305)$ & $\mathrm{C}$ & {$[77 / 8]$} \\
\hline & & $27.0 \pm 0.1$ & $(312)$ & $\mathrm{C}$ & {$[77 / 8]$} \\
\hline & & $26.2 \pm 0.1$ & (323) & $\mathrm{C}$ & {$[77 / 8]$} \\
\hline & $(225-333)$ & 30.6 & $(240)$ & EST & {$[87 / 5][61 / 13]$} \\
\hline & & & & & {$[70 / 16]$} \\
\hline & $(301-348)$ & 27.9 & (316) & & {$[30 / 3][84 / 9]$} \\
\hline
\end{tabular}


TABLE 6. Enthalpies of vaporization of organic compounds, 1880-2002-Continued

\begin{tabular}{|c|c|c|c|c|c|}
\hline Molecular formula & $\begin{array}{c}\text { Compound } \\
\text { (Temperature range/K) }\end{array}$ & $\begin{array}{c}\Delta_{\mathrm{vap}} H_{m} \\
\left(\mathrm{~kJ} \mathrm{~mol}^{-1}\right)\end{array}$ & $\begin{array}{l}\text { Mean temperature } \\
\qquad\left(T_{m} / \mathrm{K}\right)\end{array}$ & Method & $\begin{array}{c}\text { CAS registry number } \\
\text { Reference }\end{array}$ \\
\hline \multirow[t]{7}{*}{$\mathrm{C}_{2} \mathrm{H}_{5} \mathrm{Cl}$} & ethyl chloride & & & & {$[75-00-3]$} \\
\hline & $(285-344)$ & 25.1 & $(300)$ & A & {$[87 / 5]$} \\
\hline & $(334-413)$ & 24.4 & (349) & $\mathrm{A}$ & {$[87 / 5]$} \\
\hline & $(403-460)$ & 24.4 & (418) & A & [87/5] \\
\hline & $(207-305)$ & 27.8 & $(222)$ & A, EST & $\begin{array}{c}{[87 / 5][61 / 13]} \\
{[70 / 16]}\end{array}$ \\
\hline & $(218-285)$ & 25.9 & $(270)$ & & {$[48 / 6]$} \\
\hline & & 24.8 & $(294)$ & $\mathrm{C}$ & {$[26 / 6]$} \\
\hline \multirow[t]{5}{*}{$\mathrm{C}_{2} \mathrm{H}_{5} \mathrm{ClO}$} & 2-chloroethanol & & & & {$[107-07-3]$} \\
\hline & $(328-401)$ & 43.3 & $(343)$ & A & {$[87 / 5]$} \\
\hline & $(323-363)$ & 46.9 & $(338)$ & & {$[73 / 9]$} \\
\hline & $(363-403)$ & 39.1 & (378) & & {$[73 / 9]$} \\
\hline & $(269-401)$ & 45.7 & $(284)$ & & {$[47 / 5]$} \\
\hline \multirow[t]{2}{*}{$\mathrm{C}_{2} \mathrm{H}_{5} \mathrm{ClO}$} & methyl(chloromethyl)ether & & & & {$[107-30-2]$} \\
\hline & $(290-332)$ & 32.2 & $(305)$ & A & {$[87 / 5]$} \\
\hline \multirow[t]{3}{*}{$\mathrm{C}_{2} \mathrm{H}_{5} \mathrm{ClO}_{2} \mathrm{~S}$} & ethane sulfonyl chloride & & & & [594-44-5] \\
\hline & $(349-449)$ & 47.7 & $(364)$ & & [99/16] \\
\hline & $(233-263)$ & 56.4 & $(248)$ & A & {$[87 / 5][99 / 16]$} \\
\hline \multirow{2}{*}{$\mathrm{C}_{2} \mathrm{H}_{5} \mathrm{Cl}_{2} \mathrm{P}$} & dichloroethyl phosphine & & & & [1498-40-4] \\
\hline & $(313-385)$ & 36.8 & $(328)$ & A & {$[87 / 5]$} \\
\hline \multirow[t]{2}{*}{$\mathrm{C}_{2} \mathrm{H}_{5} \mathrm{Cl}_{2} \mathrm{OP}$} & ethylphosphonic dichloride & & & & \\
\hline & & $42.7 \pm 4.2$ & & & {$[56 / 23][82 / 15]$} \\
\hline \multirow[t]{8}{*}{$\mathrm{C}_{2} \mathrm{H}_{5} \mathrm{~F}$} & ethyl fluoride & & & & {$[353-36-6]$} \\
\hline & $(200-235)$ & 20.7 & $(236)$ & $\mathrm{I}$ & {$[01 / 22]$} \\
\hline & $(275-353)$ & 20.2 & $(290)$ & A & {$[87 / 5]$} \\
\hline & $(235-280)$ & 20.5 & $(265)$ & $\mathrm{A}$ & {$[87 / 5]$} \\
\hline & $(343-375)$ & 20.7 & $(358)$ & A & {$[87 / 5]$} \\
\hline & $(170-255)$ & 4.2 & $(240)$ & & {$[75 / 10]$} \\
\hline & $(173-251)$ & 20.8 & (236) & EST & $\begin{array}{c}{[87 / 5][61 / 13]} \\
{[70 / 16]}\end{array}$ \\
\hline & $(156-241)$ & 22.0 & $(226)$ & & {$[47 / 5]$} \\
\hline \multirow[t]{2}{*}{$\mathrm{C}_{2} \mathrm{H}_{5} \mathrm{FO}$} & 2-fluoroethanol & & & & [371-62-0] \\
\hline & $(273-333)$ & 44.1 & $(288)$ & GS & $\begin{array}{c}{[87 / 5][48 / 14]} \\
{[70 / 16]}\end{array}$ \\
\hline \multirow[t]{2}{*}{$\mathrm{C}_{2} \mathrm{H}_{5} \mathrm{FO}_{3} \mathrm{~S}$} & ethyl fluorosulfonate & & & & [371-69-7] \\
\hline & $(273-333)$ & 38.5 & $(288)$ & GS & $\begin{array}{c}{[87 / 5][48 / 14]} \\
{[70 / 16]}\end{array}$ \\
\hline \multirow[t]{3}{*}{$\mathrm{C}_{2} \mathrm{H}_{5} \mathrm{~F}_{2} \mathrm{~N}$} & $\mathrm{~N}, \mathrm{~N}$-difluoroethylamine & & & & [758-18-9] \\
\hline & $(241-259)$ & 27.3 & $(250)$ & A & {$[87 / 5]$} \\
\hline & & 25.7 & $(288)$ & & {$[60 / 19]$} \\
\hline \multirow[t]{2}{*}{$\mathrm{C}_{2} \mathrm{H}_{5} \mathrm{~F}_{3} \mathrm{NP}$} & methyl(trifluoromethyl)phos & cid amide & & & {$[4669-74-3]$} \\
\hline & $(238-294)$ & 36.8 & (279) & & {$[87 / 5]$} \\
\hline \multirow[t]{7}{*}{$\mathrm{C}_{2} \mathrm{H}_{5} \mathrm{I}$} & ethyl iodide & & & & {$[75-03-6]$} \\
\hline & $(313-353)$ & 31.7 & $(298)$ & CGC & {$[95 / 21]$} \\
\hline & & $31.9 \pm 0.1$ & (298) & $\mathrm{C}$ & {$[68 / 1]$} \\
\hline & $(249-369)$ & 33.6 & (264) & EST & {$[87 / 5][61 / 13]$} \\
\hline & $(219-345)$ & 34.7 & $(234)$ & & {$[47 / 5]$} \\
\hline & $(254-293)$ & 32.0 & $(278)$ & & {$[44 / 4]$} \\
\hline & $(303-333)$ & 31.7 & (318) & & [29/2] \\
\hline \multirow[t]{2}{*}{$\mathrm{C}_{2} \mathrm{H}_{5} \mathrm{~N}$} & aziridine & & & & [151-56-4] \\
\hline & $(274-303)$ & 34.9 & $(288)$ & $\mathrm{A}$ & {$[87 / 5]$} \\
\hline \multirow[t]{2}{*}{$\mathrm{C}_{2} \mathrm{H}_{5} \mathrm{NO}$} & acetaldehyde oxime & & & & [107-29-9] \\
\hline & $(288-388)$ & 48.0 & (303) & A & {$[87 / 5]$} \\
\hline \multirow[t]{3}{*}{$\mathrm{C}_{2} \mathrm{H}_{5} \mathrm{NO}$} & acetamide & & & & [60-35-5] \\
\hline & $(381-492)$ & 63.8 & (396) & A & {$[87 / 5]$} \\
\hline & $(338-495)$ & 60.9 & $(353)$ & & {$[47 / 5]$} \\
\hline \multirow[t]{6}{*}{$\mathrm{C}_{2} \mathrm{H}_{5} \mathrm{NO}$} & $\mathrm{N}$-methylformamide & & & & [123-39-7] \\
\hline & $(340-440)$ & 53.8 & (355) & & {$[96 / 28]$} \\
\hline & $(340-440)$ & $54.4 \pm 1.3$ & (298) & & {$[96 / 28]$} \\
\hline & $(310-391)$ & 54.5 & $(325)$ & A & {$[87 / 5]$} \\
\hline & & 56.2 & (298) & A & {$[85 / 7][86 / 5]$} \\
\hline & $(369-472)$ & 53.4 & (384) & A & $\begin{array}{c}{[87 / 5][61 / 3]} \\
{[70 / 16]}\end{array}$ \\
\hline $\mathrm{C}_{2} \mathrm{H}_{5} \mathrm{NO}_{2}$ & ethyl nitrite & & & & [109-95-5] \\
\hline
\end{tabular}


TABLE 6. Enthalpies of vaporization of organic compounds, 1880-2002-Continued

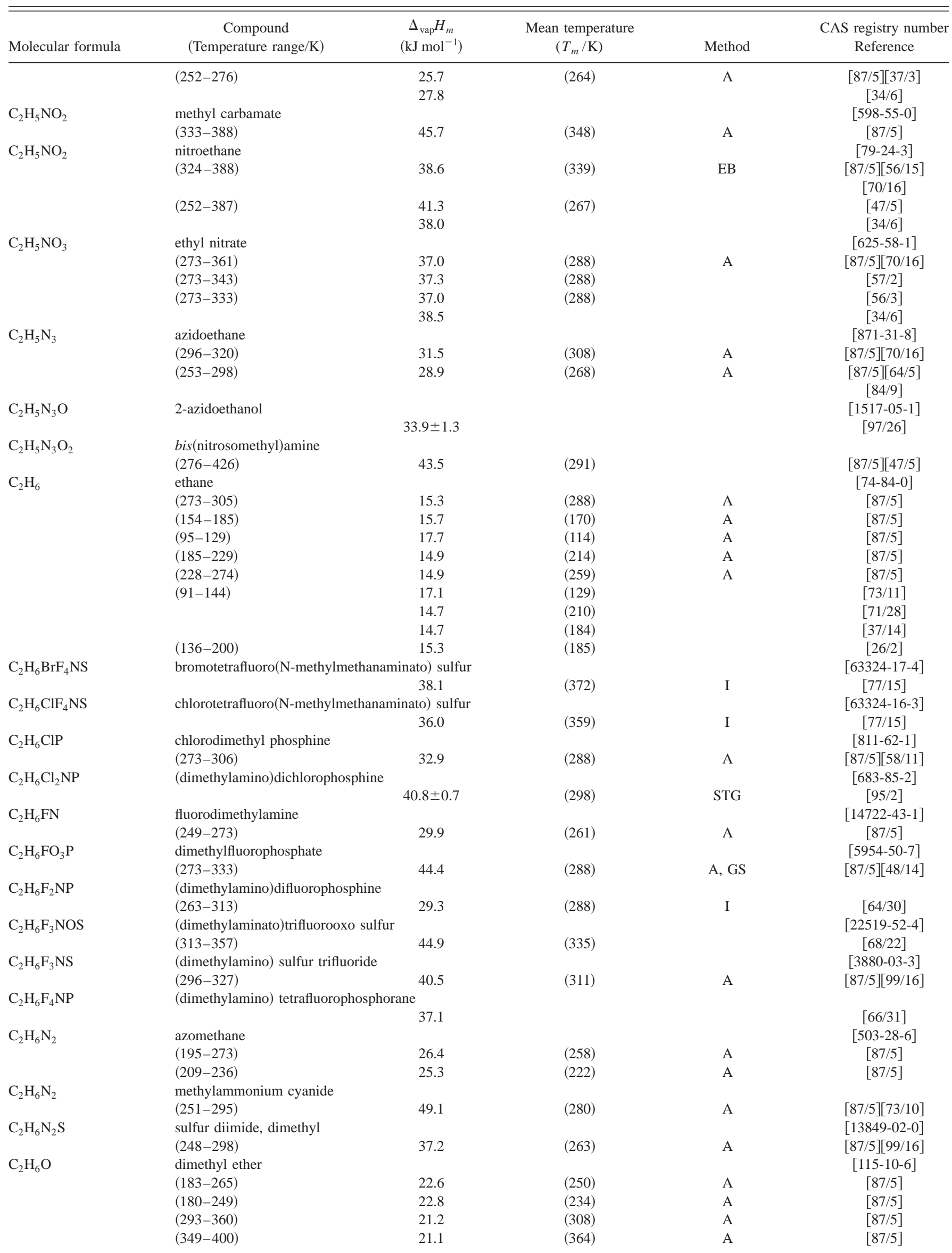


TABLE 6. Enthalpies of vaporization of organic compounds, 1880-2002-Continued

\begin{tabular}{|c|c|c|c|c|c|}
\hline Molecular formula & $\begin{array}{c}\text { Compound } \\
\text { (Temperature range/K) }\end{array}$ & $\begin{array}{c}\Delta_{\mathrm{vap}} H_{m} \\
\left(\mathrm{~kJ} \mathrm{~mol}^{-1}\right)\end{array}$ & $\begin{array}{l}\text { Mean temperature } \\
\qquad\left(T_{m} / \mathrm{K}\right)\end{array}$ & Method & $\begin{array}{c}\text { CAS registry number } \\
\text { Reference }\end{array}$ \\
\hline & $(241-303)$ & 22.2 & $(256)$ & A & {$[87 / 5]$} \\
\hline & $(171-248)$ & 18.5 & $(298)$ & & {$[76 / 2]$} \\
\hline & $(171-248)$ & 21.4 & $(248)$ & & {$[76 / 2]$} \\
\hline & $(195-248)$ & 22.7 & (233) & & {$[41 / 3]$} \\
\hline & & $21.5 \pm 0.1$ & $(248)$ & $\mathrm{C}$ & [41/3] \\
\hline \multirow[t]{38}{*}{$\mathrm{C}_{2} \mathrm{H}_{6} \mathrm{O}$} & ethanol & & & & {$[64-17-5]$} \\
\hline & & 38.9 & & & {$[99 / 32]$} \\
\hline & $(323-357)$ & 39.3 & (338) & & {$[99 / 30]$} \\
\hline & $(309-343)$ & 40.7 & $(321)$ & EB & [95/9] \\
\hline & $(309-343)$ & 42.4 & (298) & EB & [95/9] \\
\hline & $(342-357)$ & 40.5 & $(357)$ & & {$[90 / 10]$} \\
\hline & & 35.2 & (393) & $\mathrm{C}$ & {$[89 / 15]$} \\
\hline & & 30.6 & $(423)$ & $\mathrm{C}$ & {$[89 / 15]$} \\
\hline & & 25.7 & (453) & $\mathrm{C}$ & {$[89 / 15]$} \\
\hline & & 21.8 & $(473)$ & $\mathrm{C}$ & {$[89 / 15]$} \\
\hline & & 17.3 & (493) & $\mathrm{C}$ & {$[89 / 15]$} \\
\hline & & 14.2 & $(503)$ & $\mathrm{C}$ & {$[89 / 15]$} \\
\hline & & 40.9 & $(320)$ & $\mathrm{C}$ & [88/19] \\
\hline & & 40.4 & (328) & $\mathrm{C}$ & {$[88 / 19]$} \\
\hline & & 40.2 & $(335)$ & $\mathrm{C}$ & [88/19] \\
\hline & & 39.4 & $(344)$ & $\mathrm{C}$ & [88/19] \\
\hline & & 38.8 & $(351)$ & $\mathrm{C}$ & [88/19] \\
\hline & $(320-359)$ & 41.3 & $(335)$ & $\mathrm{A}$ & {$[87 / 5]$} \\
\hline & $(210-271)$ & 45.6 & $(256)$ & A & {$[87 / 5]$} \\
\hline & $(193-223)$ & 44.0 & (208) & A & {$[87 / 5]$} \\
\hline & $(320-359)$ & 41.3 & $(335)$ & A & {$[87 / 5]$} \\
\hline & $(349-374)$ & 40.1 & $(361)$ & A & {$[87 / 5]$} \\
\hline & $(370-464)$ & 39.1 & $(385)$ & A & {$[87 / 5]$} \\
\hline & $(459-514)$ & 36.1 & $(474)$ & $\mathrm{A}$ & {$[87 / 5]$} \\
\hline & $(292-353)$ & 42.5 & $(307)$ & A & {$[87 / 5]$} \\
\hline & $(243-303)$ & 42.3 & (298) & & {$[83 / 14]$} \\
\hline & $(271-373)$ & 42.9 & $(286)$ & & {$[73 / 26]$} \\
\hline & & $42.26 \pm 0.02$ & (298) & $\mathrm{C}$ & {$[71 / 27]$} \\
\hline & & $41.0 \pm 0.1$ & $(320)$ & $\mathrm{C}$ & {$[70 / 20]$} \\
\hline & & $40.0 \pm 0.1$ & $(335)$ & $\mathrm{C}$ & {$[70 / 20]$} \\
\hline & & $38.7 \pm 0.1$ & $(351)$ & $\mathrm{C}$ & {$[70 / 20]$} \\
\hline & $(293-366)$ & 42.5 & $(308)$ & A, EB & {$[87 / 5][70 / 2]$} \\
\hline & $(288-348)$ & 42.4 & $(303)$ & & {$[67 / 10]$} \\
\hline & & $42.3 \pm 0.1$ & $(298)$ & $\mathrm{C}$ & {$[66 / 2]$} \\
\hline & & $42.2 \pm 0.1$ & $(298)$ & $\mathrm{C}$ & {$[63 / 2]$} \\
\hline & $(298-351)$ & 42.2 & (313) & & {$[49 / 5]$} \\
\hline & & 40.0 & $(351)$ & & {$[34 / 7]$} \\
\hline & $(286-351)$ & 54.1 & $(301)$ & & {$[1883 / 1]$} \\
\hline \multirow[t]{8}{*}{$\mathrm{C}_{2} \mathrm{H}_{6} \mathrm{OS}$} & dimethyl sulfoxide & & & & {$[67-68-5]$} \\
\hline & $(377-483)$ & 48.6 & $(392)$ & & {$[99 / 16]$} \\
\hline & $(353-383)$ & 48.1 & $(368)$ & TGA & {$[87 / 18]$} \\
\hline & $(305-464)$ & 51.7 & $(320)$ & A & {$[87 / 5]$} \\
\hline & $(298-318)$ & 52.3 & $(308)$ & & {$[74 / 36]$} \\
\hline & $(325-442)$ & 50.6 & $(340)$ & MM & {$[72 / 8][84 / 9]$} \\
\hline & $(303-423)$ & 52.1 & $(318)$ & & {$[72 / 35]$} \\
\hline & $(293-323)$ & 52.5 & $(308)$ & & {$[69 / 25]$} \\
\hline \multirow[t]{2}{*}{$\mathrm{C}_{2} \mathrm{H}_{6} \mathrm{OS}$} & 2-mercaptoethanol & & & & [60-24-2] \\
\hline & $(293-440)$ & 54.1 & $(308)$ & A & $\begin{array}{c}{[87 / 5][70 / 16]} \\
{[99 / 16]}\end{array}$ \\
\hline \multirow[t]{9}{*}{$\mathrm{C}_{2} \mathrm{H}_{6} \mathrm{O}_{2}$} & ethylene glycol & & & & {$[107-21-1]$} \\
\hline & & $65.6 \pm 0.3$ & $(298)$ & $\mathrm{C}$ & {$[88 / 14]$} \\
\hline & $(363-408)$ & 57.4 & $(385)$ & TGA & {$[87 / 18]$} \\
\hline & $(323-473)$ & 65.2 & (338) & A & {$[87 / 5]$} \\
\hline & $(363-418)$ & 62.5 & $(378)$ & A & {$[87 / 5]$} \\
\hline & $(323-473)$ & 64.0 & $(338)$ & & {$[52 / 5]$} \\
\hline & $(363-403)$ & 61.1 & (383) & & {$[35 / 4]$} \\
\hline & $(403-470)$ & 57.3 & $(436)$ & & {$[35 / 4]$} \\
\hline & $(395-459)$ & 63.5 & $(410)$ & & [1901/1] \\
\hline $\mathrm{C}_{2} \mathrm{H}_{6} \mathrm{O}_{2}$ & ethyl hydroperoxide & & & & {$[3031-74-1]$} \\
\hline
\end{tabular}


TABLE 6. Enthalpies of vaporization of organic compounds, 1880-2002-Continued

\begin{tabular}{|c|c|c|c|c|c|}
\hline Molecular formula & $\begin{array}{c}\text { Compound } \\
\text { (Temperature range/K) }\end{array}$ & $\begin{array}{c}\Delta_{\mathrm{vap}} H_{m} \\
\left(\mathrm{~kJ} \mathrm{~mol}^{-1}\right)\end{array}$ & $\begin{array}{l}\text { Mean temperature } \\
\qquad\left(T_{m} / \mathrm{K}\right)\end{array}$ & Method & $\begin{array}{c}\text { CAS registry number } \\
\text { Reference }\end{array}$ \\
\hline & $(253-363)$ & 64.0 & $(268)$ & A & $\begin{array}{c}{[87 / 5][51 / 10]} \\
{[70 / 16]}\end{array}$ \\
\hline \multirow[t]{2}{*}{$\mathrm{C}_{2} \mathrm{H}_{6} \mathrm{O}_{2} \mathrm{~S}$} & dimethyl sulfone & & & & [67-71-0] \\
\hline & $(387-523)$ & 56.0 & $(404)$ & A & $\begin{array}{c}{[87 / 5][70 / 16]} \\
{[99 / 16]}\end{array}$ \\
\hline \multirow[t]{2}{*}{$\mathrm{C}_{2} \mathrm{H}_{6} \mathrm{O}_{4} \mathrm{~S}$} & dimethyl sulfate & & & & [77-78-1] \\
\hline & $(340-470)$ & 46.7 & $(355)$ & A & {$[87 / 5][99 / 16]$} \\
\hline \multirow[t]{15}{*}{$\mathrm{C}_{2} \mathrm{H}_{6} \mathrm{~S}$} & dimethyl sulfide & & & & {$[75-18-3]$} \\
\hline & & $28.5 \pm 0.1$ & & & {$[97 / 30]$} \\
\hline & & $27.9 \pm 0.6$ & $(298)$ & $\mathrm{C}$ & {$[89 / 12]$} \\
\hline & $(268-319)$ & 28.9 & $(283)$ & A & {$[87 / 5]$} \\
\hline & $(307-379)$ & 27.7 & $(322)$ & A & {$[87 / 5]$} \\
\hline & $(372-453)$ & 26.6 & $(387)$ & A & {$[87 / 5]$} \\
\hline & $(447-503)$ & 26.7 & $(462)$ & A & {$[87 / 5]$} \\
\hline & & 27.5 & (298) & & {$[81 / 12]$} \\
\hline & & 27.7 & $(298)$ & & {$[71 / 28]$} \\
\hline & & $28.8 \pm 0.1$ & $(276)$ & $\mathrm{C}$ & {$[57 / 10]$} \\
\hline & & $27.9 \pm 0.1$ & $(292)$ & $\mathrm{C}$ & {$[57 / 10]$} \\
\hline & & $27.0 \pm 0.1$ & $(310)$ & $\mathrm{C}$ & {$[57 / 10]$} \\
\hline & $(287-318)$ & 28.2 & $(302)$ & $\mathrm{EB}$ & {$[52 / 9]$} \\
\hline & $(251-293)$ & 28.9 & $(278)$ & & {$[42 / 2]$} \\
\hline & & 28.9 & $(310)$ & & {$[35 / 2]$} \\
\hline \multirow[t]{9}{*}{$\mathrm{C}_{2} \mathrm{H}_{6} \mathrm{~S}$} & ethyl mercaptan (ethanethiol) & & & & {$[75-08-1]$} \\
\hline & $(273-313)$ & 28.4 & $(288)$ & A & {$[87 / 5]$} \\
\hline & $(303-375)$ & 27.5 & $(318)$ & A & {$[87 / 5]$} \\
\hline & $(265-448)$ & 26.3 & $(380)$ & A & {$[87 / 5]$} \\
\hline & $(442-499)$ & 26.6 & $(457)$ & A & {$[87 / 5]$} \\
\hline & & 27.3 & $(298)$ & & {$[71 / 28]$} \\
\hline & $(273-339)$ & 28.4 & $(288)$ & & {$[66 / 5]$} \\
\hline & $(273-339)$ & 28.4 & $(288)$ & $\mathrm{A}, \mathrm{EB}$ & $\begin{array}{c}{[87 / 5][52 / 6]} \\
{[66 / 5]}\end{array}$ \\
\hline & & 28.7 & $(306)$ & & {$[35 / 2]$} \\
\hline \multirow[t]{2}{*}{$\mathrm{C}_{2} \mathrm{H}_{6} \mathrm{~S}_{2}$} & 1,2-ethanedithiol & & & & [540-63-6] \\
\hline & & 44.7 & $(298)$ & & {$[62 / 11]$} \\
\hline \multirow[t]{12}{*}{$\mathrm{C}_{2} \mathrm{H}_{6} \mathrm{~S}_{2}$} & dimethyl disulfide & & & & [624-92-0] \\
\hline & & $38.5 \pm 0.6$ & $(298)$ & $\mathrm{C}$ & [89/12] \\
\hline & $(297-402)$ & 37.8 & (312) & A & {$[87 / 5]$} \\
\hline & & $37.8 \pm 0.1$ & $(298)$ & $\mathrm{C}$ & {$[85 / 2]$} \\
\hline & & 37.8 & (298) & & [81/12] \\
\hline & & 38.4 & $(298)$ & & {$[71 / 28]$} \\
\hline & & $36.0 \pm 0.1$ & $(341)$ & $\mathrm{C}$ & {$[58 / 8]$} \\
\hline & & $34.9 \pm 0.1$ & $(360)$ & $\mathrm{C}$ & {$[58 / 8]$} \\
\hline & & $33.7 \pm 0.1$ & (383) & $\mathrm{C}$ & {$[58 / 8]$} \\
\hline & $(321-388)$ & 36.7 & (336) & $\mathrm{EB}$ & {$[52 / 9]$} \\
\hline & $(334-401)$ & 36.2 & (349) & & {$[50 / 5]$} \\
\hline & $(288-333)$ & 38.2 & (303) & & {$[50 / 5]$} \\
\hline \multirow[t]{4}{*}{$\mathrm{C}_{2} \mathrm{H}_{7} \mathrm{~N}$} & dimethylamine & & & & [124-40-3] \\
\hline & $(277-360)$ & 27.0 & (292) & A & [87/5] \\
\hline & $(358-438)$ & 23.8 & (373) & A & {$[87 / 5]$} \\
\hline & $(202-279)$ & 28.4 & $(264)$ & A & $\begin{array}{c}{[87 / 5][39 / 1]} \\
{[84 / 9]}\end{array}$ \\
\hline \multirow[t]{7}{*}{$\mathrm{C}_{2} \mathrm{H}_{7} \mathrm{~N}$} & ethyl amine & & & & [75-04-7] \\
\hline & $(213-297)$ & 29.0 & $(282)$ & A & {$[87 / 5]$} \\
\hline & $(290-449)$ & 27.2 & $(305)$ & A & {$[87 / 5]$} \\
\hline & $(291-387)$ & 27.6 & $(306)$ & A & {$[87 / 5]$} \\
\hline & $(377-456)$ & 25.9 & $(392)$ & A & {$[87 / 5]$} \\
\hline & $(275-288)$ & 29.1 & $(281)$ & & {$[62 / 5]$} \\
\hline & $(190-290)$ & 28.9 & $(275)$ & & {$[47 / 5]$} \\
\hline \multirow[t]{2}{*}{$\mathrm{C}_{2} \mathrm{H}_{7} \mathrm{NO}$} & N,N-dimethylhydroxyl amine & & & & {$[5725-96-2]$} \\
\hline & $(290-363)$ & 45.7 & $(305)$ & A & $\begin{array}{c}{[87 / 5][57 / 4]} \\
{[84 / 9]}\end{array}$ \\
\hline \multirow[t]{2}{*}{$\mathrm{C}_{2} \mathrm{H}_{7} \mathrm{NO}$} & N,O-dimethylhydroxyl amine & & & & {$[1117-97-1]$} \\
\hline & $(228-316)$ & 34.3 & $(243)$ & A & $\begin{array}{c}{[87 / 5][57 / 4]} \\
{[84 / 9]}\end{array}$ \\
\hline
\end{tabular}


TABLE 6. Enthalpies of vaporization of organic compounds, 1880-2002-Continued

\begin{tabular}{|c|c|c|c|c|c|}
\hline Molecular formula & $\begin{array}{c}\text { Compound } \\
\text { (Temperature range/K) }\end{array}$ & $\begin{array}{c}\Delta_{\mathrm{vap}} H_{m} \\
\left(\mathrm{~kJ} \mathrm{~mol}^{-1}\right)\end{array}$ & $\begin{array}{c}\text { Mean temperature } \\
\left(T_{m} / \mathrm{K}\right)\end{array}$ & Method & $\begin{array}{c}\text { CAS registry number } \\
\text { Reference }\end{array}$ \\
\hline \multirow[t]{5}{*}{$\mathrm{C}_{2} \mathrm{H}_{7} \mathrm{NO}$} & 2-aminoethanol & & & & {$[141-43-5]$} \\
\hline & $(310-444)$ & 61.7 & $(325)$ & A & {$[87 / 5]$} \\
\hline & $(379-443)$ & 54.7 & (394) & & {$[59 / 1]$} \\
\hline & $(273-301)$ & U50.8 & (287) & A, GS & {$[57 / 25]$} \\
\hline & $(338-443)$ & 58.9 & $(353)$ & & {$[50 / 1][84 / 9]$} \\
\hline \multirow[t]{3}{*}{$\mathrm{C}_{2} \mathrm{H}_{7} \mathrm{O}_{3} \mathrm{P}$} & dimethyl phosphite & & & & {$[868-85-9]$} \\
\hline & & 39.5 & & & {$[93 / 20]$} \\
\hline & $(346-456)$ & 38.7 & $(361)$ & A & {$[87 / 5][70 / 16]$} \\
\hline \multirow[t]{3}{*}{$\mathrm{C}_{2} \mathrm{H}_{8} \mathrm{ClN}$} & dimethylammonium chloride & & & & [506-59-2] \\
\hline & $(429-533)$ & 95.6 & (444) & A & {$[87 / 5]$} \\
\hline & $(533-569)$ & 143.9 & (548) & $\mathrm{A}$ & {$[87 / 5]$} \\
\hline \multirow{2}{*}{$\mathrm{C}_{2} \mathrm{H}_{8} \mathrm{ClN}$} & ethylammonium chloride & & & & [557-66-4] \\
\hline & $(382-480)$ & 34.3 & (397) & A & {$[87 / 5]$} \\
\hline \multirow[t]{3}{*}{$\mathrm{C}_{2} \mathrm{H}_{8} \mathrm{~N}_{2}$} & 1,1-dimethylhydrazine & & & & {$[57-14-7]$} \\
\hline & $(267-303)$ & 34.1 & (284) & & {$[00 / 18]$} \\
\hline & $(238-292)$ & 36.5 & (277) & $\mathrm{A}$ & $\begin{array}{c}{[87 / 5][53 / 3]} \\
{[84 / 9]}\end{array}$ \\
\hline \multirow[t]{2}{*}{$\mathrm{C}_{2} \mathrm{H}_{8} \mathrm{~N}_{2}$} & 1,2-dimethylhydrazine & & & & [540-73-8] \\
\hline & $(274-297)$ & 41.0 & (286) & $\mathrm{A}$ & $\begin{array}{c}{[87 / 5][51 / 15]} \\
{[84 / 9]}\end{array}$ \\
\hline \multirow[t]{6}{*}{$\mathrm{C}_{2} \mathrm{H}_{8} \mathrm{~N}_{2}$} & ethylenediamine & & & & [107-15-3] \\
\hline & $(303-391)$ & 43.9 & (318) & A & {$[87 / 5]$} \\
\hline & $(284-419)$ & 45.9 & (299) & A, IPM & {$[87 / 5][75 / 4]$} \\
\hline & & $45.0 \pm 0.1$ & $(298)$ & $\mathrm{C}$ & {$[69 / 2]$} \\
\hline & & $46.0 \pm 0.2$ & (298) & IPM & {$[65 / 8][70 / 11]$} \\
\hline & $(299-390)$ & 45.6 & $(314)$ & & {$[34 / 2][84 / 9]$} \\
\hline \multirow[t]{2}{*}{$\mathrm{C}_{3} \mathrm{BrClF}_{6} \mathrm{O}_{4}$} & perchloric acid, 1,1,2,3,3,3-he & ro-2-bromopi & & & [38126-26-0] \\
\hline & $(273-293)$ & 38.1 & (283) & A & {$[87 / 5][73 / 19]$} \\
\hline \multirow[t]{2}{*}{$\mathrm{C}_{3} \mathrm{BrF}_{5} \mathrm{O}$} & 2-bromo-2,3,3,3-tetrafluoroprc & fluoride & & & {$[6129-62-0]$} \\
\hline & $(224-282)$ & 30.2 & (267) & A & {$[87 / 5]$} \\
\hline \multirow[t]{2}{*}{$\mathrm{C}_{3} \mathrm{BrF}_{6} \mathrm{NO}$} & $\mathrm{N}, \mathrm{N}$-bis(trifluoromethyl) carb & bromide & & & \\
\hline & $(233-293)$ & 30.7 & (278) & A & {$[87 / 5]$} \\
\hline \multirow[t]{2}{*}{$\mathrm{C}_{3} \mathrm{BrF}_{9} \mathrm{~N}_{2}$} & N-bromo-tris(trifluoromethyl) & & & & \\
\hline & $(283-333)$ & 36.8 & (308) & & {$[66 / 31]$} \\
\hline \multirow[t]{2}{*}{$\mathrm{C}_{3} \mathrm{BrF}_{10} \mathrm{NS}$} & bromotrifluoro[1,1,1,2,3,3,3-h & oro-2-propan & $o(2-)]$ sulfur & & {$[62977-73-5]$} \\
\hline & & 35.1 & (394) & I & {$[77 / 15]$} \\
\hline \multirow[t]{2}{*}{$\mathrm{C}_{3} \mathrm{Br}_{2} \mathrm{~F}_{6} \mathrm{O}$} & (trifluoromethyl)(1,2-dibromo & trifluoroethyl & & & {$[2356-57-2]$} \\
\hline & $(299-335)$ & 34.6 & (314) & A & {$[87 / 5]$} \\
\hline \multirow[t]{2}{*}{$\mathrm{C}_{3} \mathrm{Br}_{3} \mathrm{~F}_{6} \mathrm{NO}$} & $1,1,1,1^{\prime}, 1^{\prime}, 1^{\prime}$-hexafluoro-N-(tr & omethoxy)dil & nine & & [29528-78-7] \\
\hline & $(297-338)$ & 28.9 & $(312)$ & A & {$[87 / 5]$} \\
\hline \multirow[t]{2}{*}{$\mathrm{C}_{3} \mathrm{ClF}_{4} \mathrm{NO}_{2}$} & chloro(trifluoroacetyl)carbami & & & & [42016-32-0] \\
\hline & & 39.3 & $(371)$ & & {$[73 / 21]$} \\
\hline $\mathrm{C}_{3} \mathrm{ClF}_{5} \mathrm{O}$ & chloropentafluoroacetone & & & & {$[79-53-8]$} \\
\hline & $(232-303)$ & 27.3 & (247) & A & $\begin{array}{c}{[87 / 5][64 / 6]} \\
{[84 / 9]}\end{array}$ \\
\hline $\mathrm{C}_{3} \mathrm{ClF}_{5} \mathrm{O}$ & 2-chloro-2,3,3,3-tetrafluoropro & fluoride & & & {$[28627-00-1]$} \\
\hline & $(195-273)$ & 23.9 & $(258)$ & A & {$[87 / 5]$} \\
\hline $\mathrm{C}_{3} \mathrm{ClF}_{6} \mathrm{NO}_{2}$ & O-(chloroformyl)-N,N-bis(trif & ethyl)hydrox & & & [15496-01-2] \\
\hline & $(227-286)$ & 34.5 & (271) & A & {$[87 / 5]$} \\
\hline $\mathrm{C}_{3} \mathrm{ClF}_{6} \mathrm{NS}$ & chloro(hexafluoroisopylidenim & ulfur & & & \\
\hline & & 37.7 & $(368)$ & I & {$[72 / 22]$} \\
\hline $\mathrm{C}_{3} \mathrm{ClF}_{7} \mathrm{O}$ & heptafluoroisopropyl hypocho & & & & [22675-68-9] \\
\hline & $(196-287)$ & 26.7 & (272) & A & {$[87 / 5]$} \\
\hline & $(194-273)$ & 22.7 & (258) & $\mathrm{A}$ & {$[87 / 5]$} \\
\hline $\mathrm{C}_{3} \mathrm{ClF}_{8} \mathrm{~N}$ & $\mathrm{~N}$-chloro-N-1,2,2,2-pentafluor & ifluoromethy & nine & & [33757-13-0] \\
\hline & $(240-311)$ & 28.8 & $(255)$ & A & {$[87 / 5][71 / 17]$} \\
\hline $\mathrm{C}_{3} \mathrm{ClF}_{8} \mathrm{NOS}$ & (heptafluoropropyl)imidosulfu & oride fluorid & & & [74366-14-6] \\
\hline & & 26.7 & (346) & I & {$[80 / 10]$} \\
\hline $\mathrm{C}_{3} \mathrm{ClF}_{10} \mathrm{NS}$ & chlorotrifluoro[1,1,1,2,3,3,3-h & oro-2-propan & o(2-)] sulfur & & {$[62977-71-3]$} \\
\hline & & 33.5 & $(391)$ & I & {$[77 / 15]$} \\
\hline $\mathrm{C}_{3} \mathrm{Cl}_{2} \mathrm{~F}_{5} \mathrm{~N}$ & 2,2-difluoro-1,2-dichloro- $\mathrm{N}$-(t & methyl)ethy & & & \\
\hline & $(283-318)$ & 31.2 & (298) & A & [87/5] \\
\hline $\mathrm{C}_{3} \mathrm{Cl}_{2} \mathrm{~F}_{6}$ & 1,2-dichlorohexafluoropropan & & & & {$[661-97-2]$} \\
\hline & $(296-307)$ & 28.1 & $(301)$ & & {$[80 / 9]$} \\
\hline
\end{tabular}


TABLE 6. Enthalpies of vaporization of organic compounds, 1880-2002-Continued

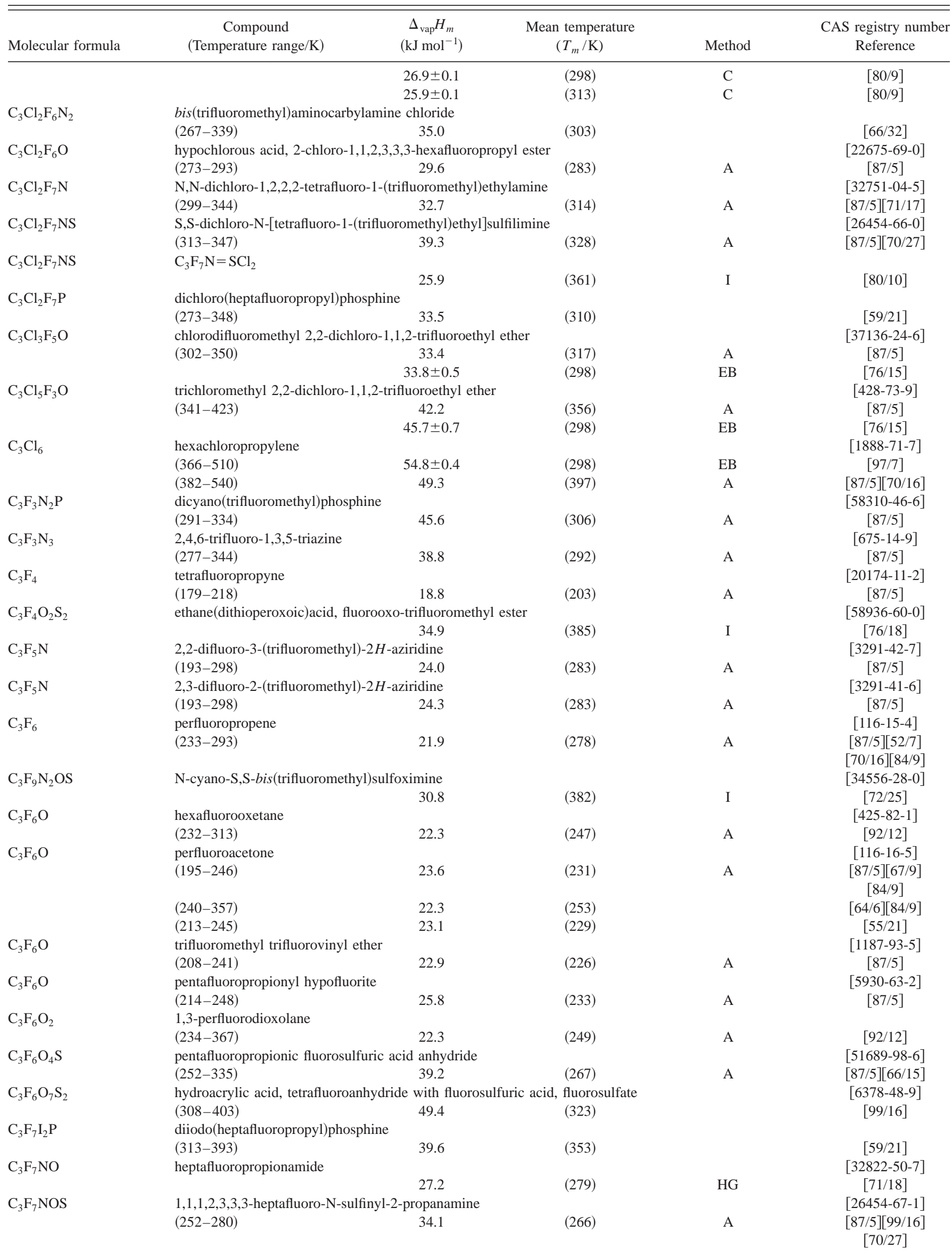


TABLE 6. Enthalpies of vaporization of organic compounds, 1880-2002-Continued

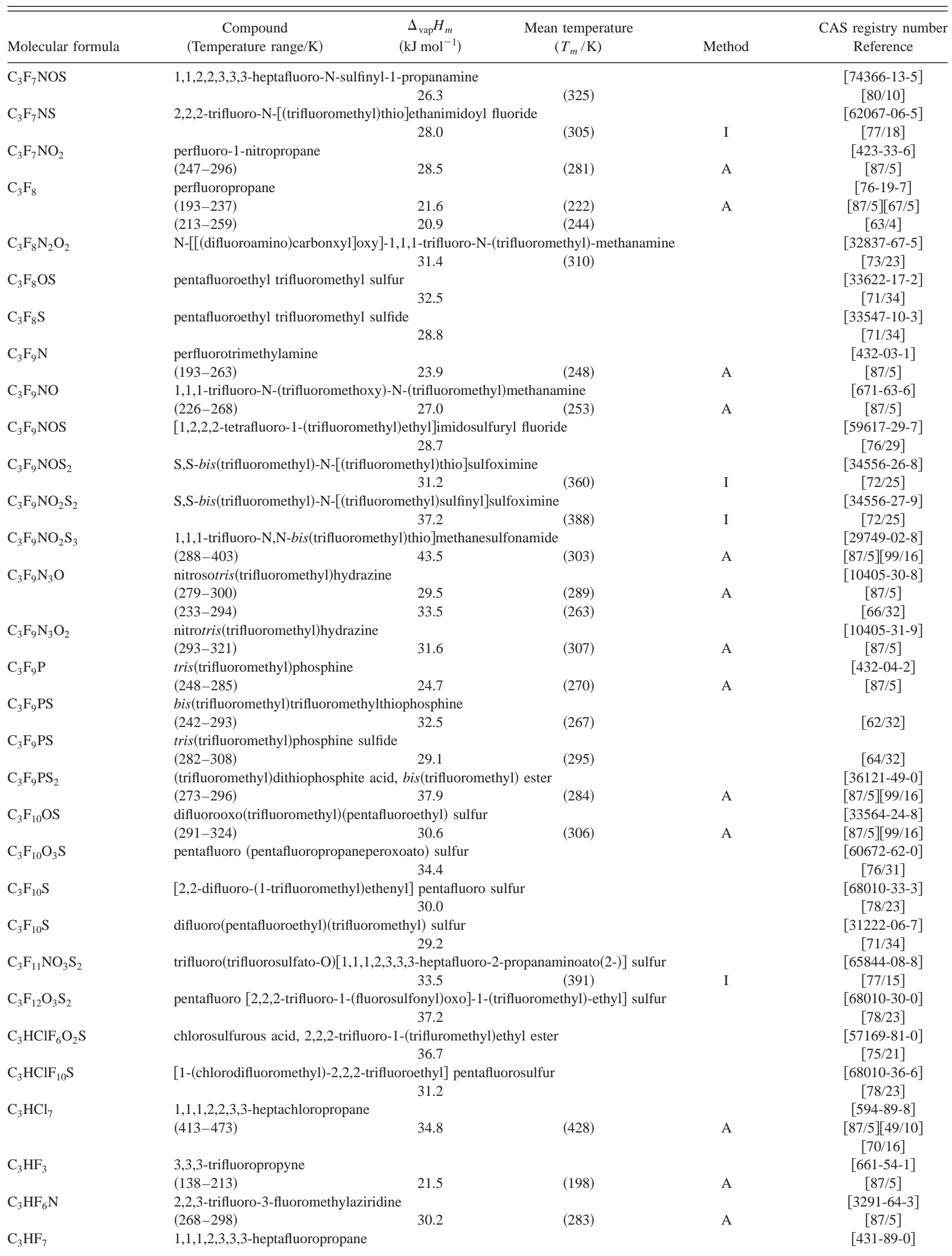


TABLE 6. Enthalpies of vaporization of organic compounds, 1880-2002-Continued

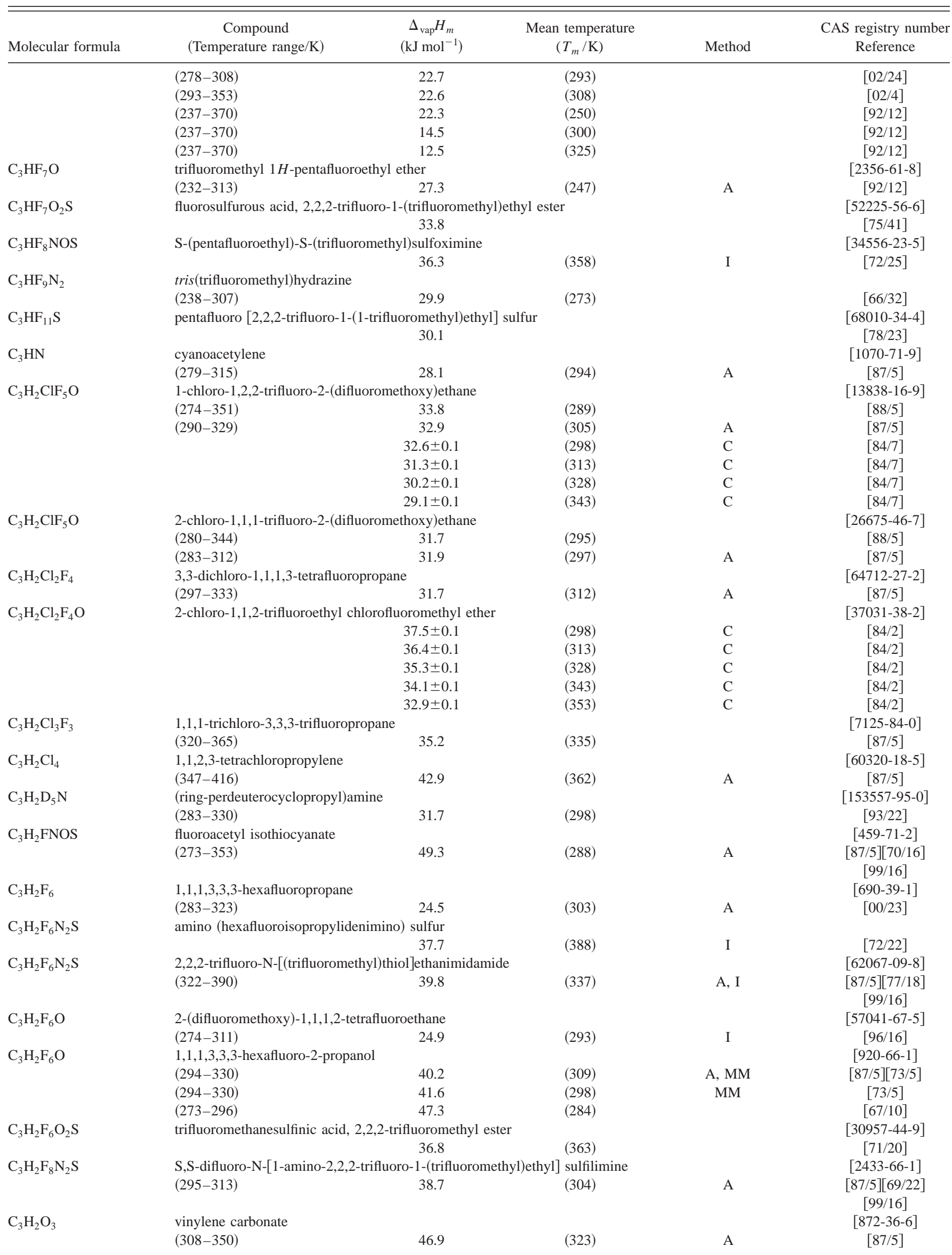


TABLE 6. Enthalpies of vaporization of organic compounds, 1880-2002-Continued

\begin{tabular}{|c|c|c|c|c|c|}
\hline Molecular formula & $\begin{array}{c}\text { Compound } \\
\text { (Temperature range/K) }\end{array}$ & $\begin{array}{c}\Delta_{\mathrm{vap}} H_{m} \\
\left(\mathrm{~kJ} \mathrm{~mol}^{-1}\right)\end{array}$ & $\begin{array}{l}\text { Mean temperature } \\
\qquad\left(T_{m} / \mathrm{K}\right)\end{array}$ & Method & $\begin{array}{c}\text { CAS registry number } \\
\text { Reference }\end{array}$ \\
\hline & $(308-400)$ & 41.3 & & MM & {$[71 / 1]$} \\
\hline \multirow[t]{2}{*}{$\mathrm{C}_{3} \mathrm{H}_{3} \mathrm{Cl}$} & 1-chloro-1-propyne & & & & {$[7747-84-4]$} \\
\hline & $(200-289)$ & 28.3 & $(274)$ & A & {$[87 / 5]$} \\
\hline \multirow[t]{2}{*}{$\mathrm{C}_{3} \mathrm{H}_{3} \mathrm{Cl}_{2} \mathrm{~F}_{3}$} & 1,1-dichloro-3,3,3-trifluoropropane & & & & {$[460-69-5]$} \\
\hline & $(301-342)$ & 33.7 & $(316)$ & A & {$[87 / 5]$} \\
\hline \multirow{6}{*}{$\mathrm{C}_{3} \mathrm{H}_{3} \mathrm{Cl}_{2} \mathrm{~F}_{3} \mathrm{O}$} & 2-chloro-1,1,2-trifluoroethyl chloron & nethyl ether & & & {$[428-92-2]$} \\
\hline & & $42.4 \pm 0.1$ & (298) & $\mathrm{C}$ & {$[84 / 7]$} \\
\hline & & $41.2 \pm 0.1$ & (313) & $\mathrm{C}$ & {$[84 / 7]$} \\
\hline & & $40.1 \pm 0.1$ & (328) & $\mathrm{C}$ & {$[84 / 7]$} \\
\hline & & $39.0 \pm 0.1$ & (343) & $\mathrm{C}$ & {$[84 / 7]$} \\
\hline & & $37.8 \pm 0.1$ & (358) & $\mathrm{C}$ & {$[84 / 7]$} \\
\hline \multirow[t]{2}{*}{$\mathrm{C}_{3} \mathrm{H}_{3} \mathrm{Cl}_{3} \mathrm{O}_{2}$} & methyl trichloroacetate & & & & \\
\hline & & $48.3 \pm 0.1$ & $(298)$ & $\mathrm{C}$ & {$[72 / 41]$} \\
\hline \multirow[t]{2}{*}{$\mathrm{C}_{3} \mathrm{H}_{3} \mathrm{Cl}_{5}$} & 1,1,2,2,3-pentachloropropane & & & & [16714-68-4] \\
\hline & $(365-447)$ & 46.3 & $(380)$ & A & {$[87 / 5][70 / 16]$} \\
\hline \multirow{2}{*}{$\mathrm{C}_{3} \mathrm{H}_{3} \mathrm{~F}_{3}$} & 3,3,3-trifluoro-1-propene & & & & {$[677-21-4]$} \\
\hline & $(283-363)$ & 22.0 & $(298)$ & $\mathrm{A}$ & {$[87 / 5]$} \\
\hline \multirow[t]{2}{*}{$\mathrm{C}_{3} \mathrm{H}_{3} \mathrm{~F}_{4} \mathrm{I}$} & 1,1,1,2-tetrafluoro-3-iodopropane & & & & {$[1737-76-4]$} \\
\hline & $(295-356)$ & 28.4 & $(310)$ & A & {$[87 / 5]$} \\
\hline \multirow[t]{2}{*}{$\mathrm{C}_{3} \mathrm{H}_{3} \mathrm{~F}_{4} \mathrm{I}$} & 1,1,1,3-tetrafluoro-3-iodopropane & & & & {$[460-74-2]$} \\
\hline & $(301-356)$ & 31.2 & $(316)$ & A & {$[87 / 5]$} \\
\hline \multirow[t]{2}{*}{$\mathrm{C}_{3} \mathrm{H}_{3} \mathrm{~F}_{4} \mathrm{NO}_{2}$} & methoxy (trifluoromethyl)carbamic & luoride & & & \\
\hline & & 27.8 & & & {$[79 / 28]$} \\
\hline \multirow[t]{2}{*}{$\mathrm{C}_{3} \mathrm{H}_{3} \mathrm{~F}_{5}$} & 1,1,2,2,3-pentafluoropropane & & & & [679-86-7] \\
\hline & $(258-353)$ & 30.2 & $(273)$ & A & {$[02 / 25]$} \\
\hline \multirow[t]{3}{*}{$\mathrm{C}_{3} \mathrm{H}_{3} \mathrm{~F}_{5}$} & 1,1,1,2,2-pentafluoropropane & & & & [1814-88-6] \\
\hline & $(232-283)$ & 22.9 & (268) & A & {$[87 / 5][70 / 16]$} \\
\hline & $(233-379)$ & 23.0 & $(248)$ & & {$[67 / 7]$} \\
\hline \multirow{4}{*}{$\mathrm{C}_{3} \mathrm{H}_{3} \mathrm{~F}_{5} \mathrm{O}$} & 2,2,3,3,3-pentafluoro-1-propanol & & & & {$[422-05-9]$} \\
\hline & $(273-297)$ & 47.0 & $(285)$ & $\mathrm{A}, \mathrm{MM}$ & $\begin{array}{c}{[87 / 5][67 / 4]} \\
{[84 / 9]}\end{array}$ \\
\hline & & 44.4 & (298) & MM & {$[73 / 5][67 / 4]$} \\
\hline & & 41.3 & $(298)$ & & {$[67 / 3]$} \\
\hline \multirow[t]{2}{*}{$\mathrm{C}_{3} \mathrm{H}_{3} \mathrm{~F}_{5} \mathrm{O}$} & 1,1,2,2-tetrafluoro-1-(fluoromethoxy & ethane & & & [37031-31-5] \\
\hline & $(288-317)$ & 33.5 & $(303)$ & I & {$[02 / 19]$} \\
\hline \multirow[t]{2}{*}{$\mathrm{C}_{3} \mathrm{H}_{3} \mathrm{~F}_{6} \mathrm{NOS}$} & N-methyl-S,S-bis(trifluoromethyl)su & foximine & & & [34556-25-7] \\
\hline & & 30.7 & $(338)$ & I & {$[72 / 25]$} \\
\hline $\mathrm{C}_{3} \mathrm{H}_{3} \mathrm{~F}_{6} \mathrm{NS}$ & $\mathrm{N}, \mathrm{N}-$ bis(trifluoromethyl)methanesul & enamide & & & \\
\hline & $(269-309)$ & 31.1 & $(284)$ & A & {$[87 / 5]$} \\
\hline $\mathrm{C}_{3} \mathrm{H}_{3} \mathrm{~F}_{6} \mathrm{O}_{2} \mathrm{P}$ & bis(trifluromethyl)phosphinic acid, & nethyl ester & & & [25439-11-6] \\
\hline & $(258-313)$ & 40.5 & (273) & $\mathrm{A}$ & {$[87 / 5]$} \\
\hline $\mathrm{C}_{3} \mathrm{H}_{3} \mathrm{~F}_{6} \mathrm{PS}$ & bis(trifluoromethyl) methylthiophos & hine & & & \\
\hline & $(273-321)$ & 36.9 & $(297)$ & $\mathrm{T}$ & {$[64 / 31]$} \\
\hline $\mathrm{C}_{3} \mathrm{H}_{3} \mathrm{~F}_{6} \mathrm{PS}_{2}$ & bis(trifluromethyl)dithiophosphinic & icid, methyl ester & & & {$[18799-79-6]$} \\
\hline & $(273-344)$ & 41.5 & $(288)$ & A & {$[87 / 5][99 / 16]$} \\
\hline $\mathrm{C}_{3} \mathrm{H}_{3} \mathrm{~N}$ & acrylonitrile & & & & {$[107-13-1]$} \\
\hline & $(257-352)$ & 33.6 & $(272)$ & $\mathrm{A}$ & {$[87 / 5]$} \\
\hline & $(283-343)$ & 31.6 & $(298)$ & A & {$[87 / 5]$} \\
\hline & $(222-351)$ & 35.5 & $(237)$ & & {$[64 / 9]$} \\
\hline & $(293-343)$ & 32.9 & $(308)$ & & {$[64 / 7]$} \\
\hline & $(273-353)$ & 32.6 & & & {$[45 / 6]$} \\
\hline $\mathrm{C}_{3} \mathrm{H}_{3} \mathrm{NO}$ & oxazole & & & & {$[288-42-6]$} \\
\hline & $(293-344)$ & 34.6 & $(308)$ & A & {$[87 / 5]$} \\
\hline & & $32.5 \pm 0.1$ & (298) & $\mathrm{C}$ & {$[78 / 4]$} \\
\hline $\mathrm{C}_{3} \mathrm{H}_{3} \mathrm{NO}$ & isoxazole & & & & [288-14-2] \\
\hline & $(314-404)$ & $37.2 \pm 0.2$ & $(298)$ & EB & {$[96 / 4]$} \\
\hline & & $36.5 \pm 0.1$ & $(298)$ & $\mathrm{C}$ & {$[78 / 4]$} \\
\hline $\mathrm{C}_{3} \mathrm{H}_{3} \mathrm{NO}_{2}$ & cyanoformic acid, methyl ester & & & & {$[17640-15-2]$} \\
\hline & $(273-333)$ & 39.3 & $(288)$ & A & {$[87 / 5][70 / 16]$} \\
\hline $\mathrm{C}_{3} \mathrm{H}_{3} \mathrm{NS}$ & thiazole & & & & {$[288-47-1]$} \\
\hline & $(333-393)$ & 39.7 & $(348)$ & A & {$[87 / 5]$} \\
\hline & $(336-391)$ & 38.9 & $(351)$ & A & {$[87 / 5][69 / 4]$} \\
\hline $\mathrm{C}_{3} \mathrm{H}_{4}$ & allene & & & & {$[463-49-0]$} \\
\hline & $(136-274)$ & 22.6 & $(259)$ & A & {$[87 / 5]$} \\
\hline
\end{tabular}


TABLE 6. Enthalpies of vaporization of organic compounds, 1880-2002-Continued

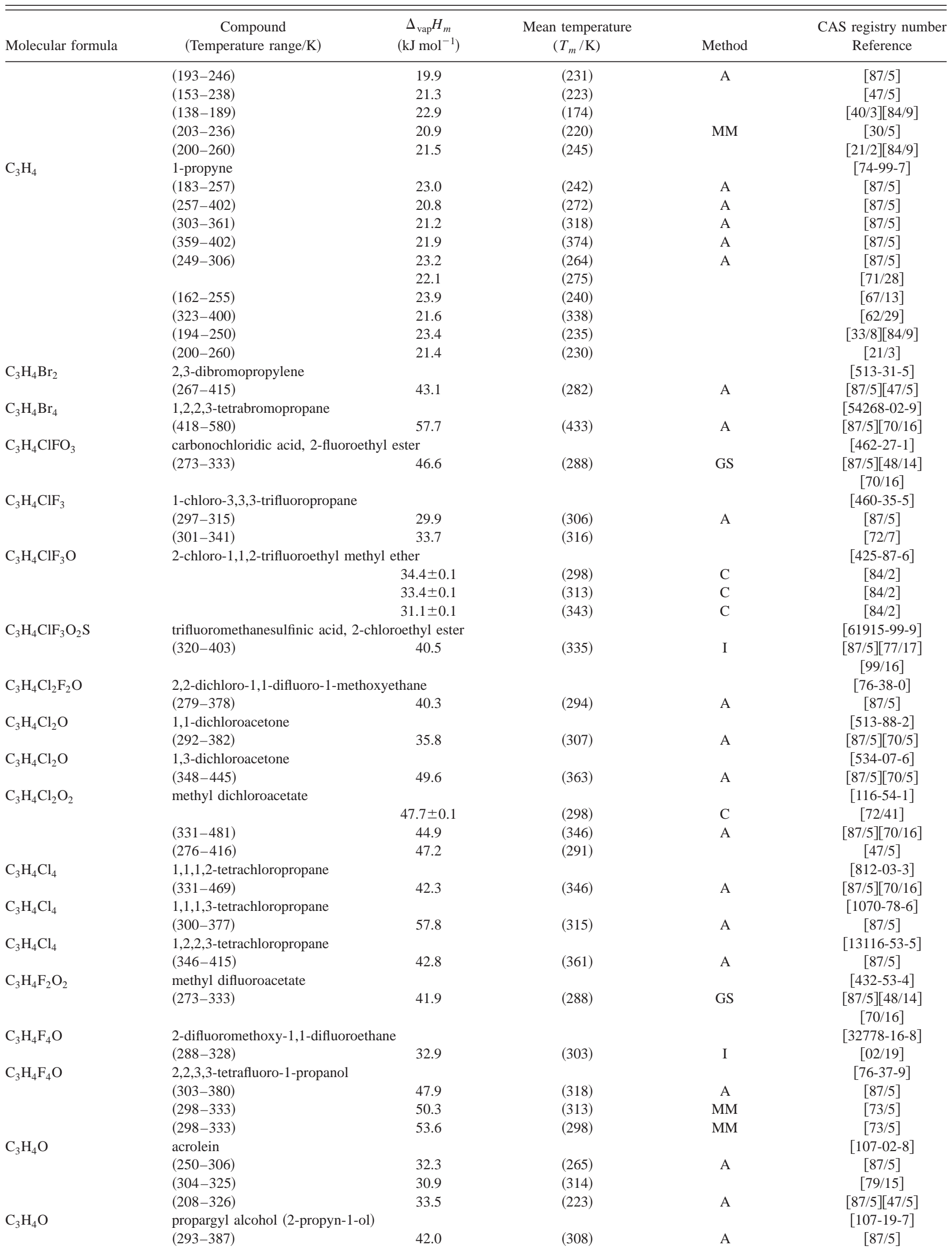


TABLE 6. Enthalpies of vaporization of organic compounds, 1880-2002-Continued

\begin{tabular}{|c|c|c|c|c|c|}
\hline Molecular formula & $\begin{array}{c}\text { Compound } \\
\text { (Temperature range/K) }\end{array}$ & $\begin{array}{c}\Delta_{\mathrm{vap}} H_{m} \\
\left(\mathrm{~kJ} \mathrm{~mol}^{-1}\right)\end{array}$ & $\begin{array}{l}\text { Mean temperature } \\
\qquad\left(T_{m} / \mathrm{K}\right)\end{array}$ & Method & $\begin{array}{c}\text { CAS registry number } \\
\text { Reference }\end{array}$ \\
\hline \multirow[t]{4}{*}{$\mathrm{C}_{3} \mathrm{H}_{4} \mathrm{O}_{2}$} & acrylic acid & & & & {$[79-10-7]$} \\
\hline & & $53.1 \pm 4.2$ & $(298)$ & $\mathrm{C}$ & {$[96 / 8]$} \\
\hline & $(341-414)$ & 45.3 & $(356)$ & $\mathrm{A}$ & {$[87 / 5][73 / 14]$} \\
\hline & $(293-343)$ & 32.7 & $(308)$ & & {$[64 / 7]$} \\
\hline \multirow[t]{3}{*}{$\mathrm{C}_{3} \mathrm{H}_{4} \mathrm{O}_{2}$} & $\beta$-propiolactone (2-oxetan & & & & {$[127-17-3]$} \\
\hline & $(324-435)$ & 46.4 & (339) & $\mathrm{A}$ & {$[87 / 5]$} \\
\hline & & $47.0 \pm 0.1$ & (298) & $\mathrm{C}$ & {$[66 / 19]$} \\
\hline \multirow[t]{5}{*}{$\mathrm{C}_{3} \mathrm{H}_{4} \mathrm{O}_{3}$} & ethylene carbonate & & & & [96-49-1] \\
\hline & $(381-437)$ & 59.6 & $(396)$ & A & {$[87 / 5]$} \\
\hline & $(368-449)$ & 60.3 & (383) & EB & {$[82 / 8]$} \\
\hline & $(368-449)$ & 56.3 & (423) & EB & {$[82 / 8]$} \\
\hline & $(368-433)$ & 55.0 & (433) & $\mathrm{EB}$ & {$[82 / 8]$} \\
\hline \multirow[t]{2}{*}{$\mathrm{C}_{3} \mathrm{H}_{4} \mathrm{O}_{3}$} & pyruvic acid & & & & {$[127-17-3]$} \\
\hline & $(294-438)$ & 51.4 & $(309)$ & $\mathrm{A}$ & {$[87 / 5][47 / 5]$} \\
\hline \multirow[t]{2}{*}{$\mathrm{C}_{3} \mathrm{H}_{4} \mathrm{~S}_{3}$} & trithiocarbonic acid, cycli & ester & & & {$[822-38-8]$} \\
\hline & $(294-303)$ & 82.9 & (298) & & [99/16] \\
\hline \multirow[t]{5}{*}{$\mathrm{C}_{3} \mathrm{H}_{5} \mathrm{Br}$} & allyl bromide & & & & {$[106-95-6]$} \\
\hline & $(297-338)$ & 32.2 & (312) & $\mathrm{A}, \mathrm{EB}$ & {$[87 / 5][77 / 8]$} \\
\hline & & $31.7 \pm 0.1$ & (318) & $\mathrm{C}$ & {$[77 / 8]$} \\
\hline & & $31.0 \pm 0.1$ & $(330)$ & $\mathrm{C}$ & {$[77 / 8]$} \\
\hline & & $30.4 \pm 0.1$ & $(341)$ & $\mathrm{C}$ & {$[77 / 8]$} \\
\hline \multirow[t]{2}{*}{$\mathrm{C}_{3} \mathrm{H}_{5} \mathrm{Br}$} & cis 1-bromopropylene & & & & [590-14-7] \\
\hline & $(257-366)$ & 32.0 & (272) & $\mathrm{A}$ & {$[87 / 5][70 / 16]$} \\
\hline \multirow[t]{2}{*}{$\mathrm{C}_{3} \mathrm{H}_{5} \mathrm{Br}$} & trans 1-bromopropylene & & & & {$[590-14-7]$} \\
\hline & $(262-372)$ & 32.5 & (277) & A & {$[87 / 5][70 / 16]$} \\
\hline \multirow[t]{3}{*}{$\mathrm{C}_{3} \mathrm{H}_{5} \mathrm{Br}_{3}$} & 1,2,3-tribromopropane & & & & [96-11-7] \\
\hline & $(390-595)$ & 50.8 & $(405)$ & A & {$[87 / 5]$} \\
\hline & $(400-478)$ & 50.2 & $(415)$ & & {$[49 / 1][84 / 9]$} \\
\hline \multirow[t]{3}{*}{$\mathrm{C}_{3} \mathrm{H}_{5} \mathrm{Cl}$} & allyl chloride & & & & {$[107-05-1]$} \\
\hline & $(203-318)$ & 33.1 & (218) & A & {$[87 / 5]$} \\
\hline & $(286-317)$ & 30.0 & (301) & & {$[44 / 5][84 / 9]$} \\
\hline \multirow{2}{*}{$\mathrm{C}_{3} \mathrm{H}_{5} \mathrm{Cl}$} & 1-chloropropene & & & & [590-21-6] \\
\hline & $(191-310)$ & 29.5 & $(206)$ & & {$[47 / 5]$} \\
\hline \multirow[t]{3}{*}{$\mathrm{C}_{3} \mathrm{H}_{5} \mathrm{Cl}$} & cis 1-chloropropene & & & & [16136-84-8] \\
\hline & $(276-332)$ & 27.9 & $(291)$ & & [01/19] \\
\hline & $(237-338)$ & 29.2 & $(252)$ & A & {$[87 / 5][70 / 16]$} \\
\hline \multirow[t]{3}{*}{$\mathrm{C}_{3} \mathrm{H}_{5} \mathrm{Cl}$} & trans 1-chloropropene & & & & [16136-85-9] \\
\hline & $(277-340)$ & 28.5 & (292) & & [01/19] \\
\hline & $(241-343)$ & 29.7 & $(256)$ & A & {$[87 / 5][70 / 16]$} \\
\hline \multirow[t]{2}{*}{$\mathrm{C}_{3} \mathrm{H}_{5} \mathrm{Cl}$} & 2-chloropropene & & & & [557-98-2] \\
\hline & $(229-327)$ & 28.0 & (244) & A & {$[87 / 5][70 / 16]$} \\
\hline \multirow[t]{2}{*}{$\mathrm{C}_{3} \mathrm{H}_{5} \mathrm{ClO}$} & epichlorohydrin & & & & [106-89-8] \\
\hline & $(256-391)$ & 42.9 & (272) & & {$[47 / 5]$} \\
\hline $\mathrm{C}_{3} \mathrm{H}_{5} \mathrm{ClO}$ & chloroacetone & & & & {$[78-95-5]$} \\
\hline & $(316-392)$ & 40.1 & $(331)$ & A & {$[87 / 5]$} \\
\hline $\mathrm{C}_{3} \mathrm{H}_{5} \mathrm{ClO}_{2}$ & methyl chloroacetate & & & & [96-34-4] \\
\hline & & $46.7 \pm 0.1$ & (298) & $\mathrm{C}$ & {$[72 / 41]$} \\
\hline & $(318-402)$ & 45.5 & (333) & A & $\begin{array}{c}{[87 / 5][67 / 6]} \\
{[84 / 9]}\end{array}$ \\
\hline & $(298-403)$ & 46.7 & (313) & & {$[28 / 2][84 / 9]$} \\
\hline $\mathrm{C}_{3} \mathrm{H}_{5} \mathrm{ClO}_{2}$ & ethylchloroformate & & & & {$[541-41-3]$} \\
\hline & $(281-286)$ & $38.7 \pm 0.2$ & (283) & BG & {$[80 / 2]$} \\
\hline & $(281-286)$ & $37.8 \pm 0.2$ & (298) & BG & [80/2] \\
\hline $\mathrm{C}_{3} \mathrm{H}_{5} \mathrm{Cl}_{3}$ & 1,1,1-trichloropropane & & & & [7789-89-1] \\
\hline & $(244-382)$ & 38.8 & (259) & $\mathrm{A}$ & {$[87 / 5][47 / 5]$} \\
\hline $\mathrm{C}_{3} \mathrm{H}_{5} \mathrm{Cl}_{3}$ & 1,1,3-trichloropropane & & & & [20395-25-9] \\
\hline & $(328-464)$ & 41.8 & (343) & A & {$[87 / 5][70 / 16]$} \\
\hline $\mathrm{C}_{3} \mathrm{H}_{5} \mathrm{Cl}_{3}$ & 1,2,3-trichloropropane & & & & [96-18-4] \\
\hline & & $47.8 \pm 0.1$ & $(298)$ & $\mathrm{C}$ & {$[89 / 10]$} \\
\hline & $(337-477)$ & 43.0 & $(352)$ & $\mathrm{A}$ & {$[87 / 5][70 / 16]$} \\
\hline & $(282-431)$ & 46.8 & (297) & & {$[47 / 5]$} \\
\hline $\mathrm{C}_{3} \mathrm{H}_{5} \mathrm{FO}$ & 1,2-epoxy-3-fluoropropan & & & & [503-09-3] \\
\hline & $(273-333)$ & 39.9 & (288) & A, GS & $\begin{array}{c}{[87 / 5][48 / 14]} \\
{[70 / 16]}\end{array}$ \\
\hline
\end{tabular}


TABLE 6. Enthalpies of vaporization of organic compounds, 1880-2002-Continued

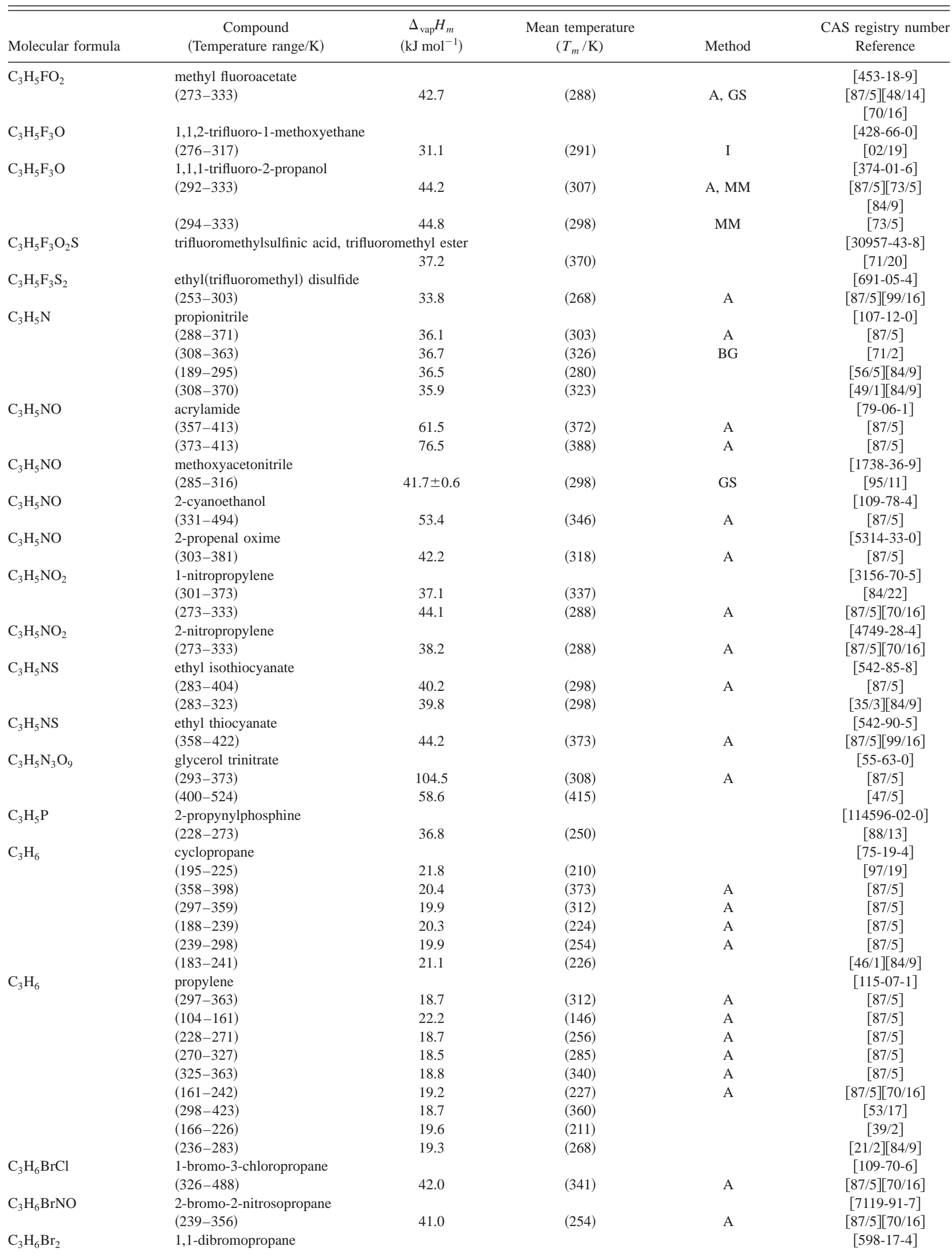


TABLE 6. Enthalpies of vaporization of organic compounds, 1880-2002-Continued

\begin{tabular}{|c|c|c|c|c|c|}
\hline Molecular formula & $\begin{array}{c}\text { Compound } \\
\text { (Temperature range/K) }\end{array}$ & $\begin{array}{c}\Delta_{\mathrm{vap}} H_{m} \\
\left(\mathrm{~kJ} \mathrm{~mol}^{-1}\right)\end{array}$ & $\begin{array}{l}\text { Mean temperature } \\
\qquad\left(T_{m} / \mathrm{K}\right)\end{array}$ & Method & $\begin{array}{c}\text { CAS registry number } \\
\text { Reference }\end{array}$ \\
\hline & $(322-449)$ & 42.5 & $(337)$ & A, EST & $\begin{array}{c}{[87 / 5][56 / 16]} \\
{[70 / 16]}\end{array}$ \\
\hline \multirow[t]{6}{*}{$\mathrm{C}_{3} \mathrm{H}_{6} \mathrm{Br}_{2}$} & 1,2-dibromopropane & & & & [78-75-1] \\
\hline & $(312-403)$ & 41.4 & (327) & $\mathrm{A}$ & {$[87 / 5]$} \\
\hline & $(310-400)$ & 42.2 & (298) & & {$[91 / 2][75 / 16]$} \\
\hline & & $42.3 \pm 0.7$ & (298) & EB & {$[75 / 15]$} \\
\hline & $(329-456)$ & 44.6 & $(344)$ & A & {$[87 / 5][70 / 16]$} \\
\hline & $(266-415)$ & 42.8 & $(281)$ & & {$[47 / 5]$} \\
\hline \multirow{10}{*}{$\mathrm{C}_{3} \mathrm{H}_{6} \mathrm{Br}_{2}$} & 1,3-dibromopropane & & & & [109-64-8] \\
\hline & & 47.6 & $(298)$ & GC & [94/19] \\
\hline & & $47.3 \pm 0.1$ & $(308)$ & $\mathrm{C}$ & {$[92 / 11]$} \\
\hline & & $46.7 \pm 0.1$ & $(315)$ & $\mathrm{C}$ & [92/11] \\
\hline & & $46.1 \pm 0.1$ & (323) & $\mathrm{C}$ & [92/11] \\
\hline & & $45.5 \pm 0.1$ & $(330)$ & $\mathrm{C}$ & [92/11] \\
\hline & & $44.8 \pm 0.1$ & (338) & $\mathrm{C}$ & {$[92 / 11]$} \\
\hline & $(307-437)$ & 46.6 & $(322)$ & A & {$[87 / 5]$} \\
\hline & $(351-487)$ & 47.8 & (366) & A & {$[87 / 5][70 / 16]$} \\
\hline & $(283-440)$ & 45.3 & (298) & & {$[47 / 5]$} \\
\hline \multirow[t]{2}{*}{$\mathrm{C}_{3} \mathrm{H}_{6} \mathrm{Br}_{2} \mathrm{O}$} & 2,3-dibromo-1-propanol & & & & {$[96-13-9]$} \\
\hline & $(330-492)$ & 57.3 & $(345)$ & A & {$[87 / 5][47 / 5]$} \\
\hline \multirow[t]{3}{*}{$\mathrm{C}_{3} \mathrm{H}_{6} \mathrm{Cl}_{2}$} & 1,1-dichloropropane & & & & [78-99-9] \\
\hline & $(310-360)$ & 35.2 & (298) & & {$[67 / 11][91 / 2]$} \\
\hline & $(282-399)$ & 35.5 & (297) & A, EST & $\begin{array}{c}{[87 / 5][56 / 16]} \\
{[70 / 16]}\end{array}$ \\
\hline \multirow[t]{7}{*}{$\mathrm{C}_{3} \mathrm{H}_{6} \mathrm{Cl}_{2}$} & 1,2-dichloropropane & & & & [78-87-5] \\
\hline & $(294-406)$ & $38.4 \pm 0.3$ & $(298)$ & $\mathrm{EB}$ & {$[97 / 8]$} \\
\hline & $(300-370)$ & 36.3 & $(298)$ & & {$[91 / 2]$} \\
\hline & & $36.1 \pm 0.1$ & (298) & $\mathrm{C}$ & {$[89 / 10]$} \\
\hline & $(239-373)$ & 39.4 & $(254)$ & A & {$[87 / 5]$} \\
\hline & $(321-369)$ & 34.7 & $(336)$ & & {$[49 / 1]$} \\
\hline & $(288-373)$ & 34.3 & (303) & & {$[33 / 5]$} \\
\hline \multirow{5}{*}{$\mathrm{C}_{3} \mathrm{H}_{6} \mathrm{Cl}_{2}$} & 1,3-dichloropropane & & & & [142-28-9] \\
\hline & & 41.0 & $(298)$ & GC & [94/19] \\
\hline & $(330-400)$ & 41.0 & (298) & & {$[87 / 11][91 / 2]$} \\
\hline & & $40.6 \pm 0.1$ & (298) & $\mathrm{C}$ & [89/10] \\
\hline & $(307-435)$ & 39.0 & $(322)$ & $\mathrm{A}$ & {$[87 / 5][70 / 16]$} \\
\hline \multirow[t]{3}{*}{$\mathrm{C}_{3} \mathrm{H}_{6} \mathrm{Cl}_{2}$} & 2,2-dichloropropane & & & & [594-20-7] \\
\hline & $(295-340)$ & 32.1 & (298) & $\mathrm{A}$ & {$[87 / 11][91 / 2]$} \\
\hline & $(267-378)$ & 33.2 & $(282)$ & A & {$[87 / 5][70 / 16]$} \\
\hline \multirow[t]{2}{*}{$\mathrm{C}_{3} \mathrm{H}_{6} \mathrm{Cl}_{2} \mathrm{O}$} & 2,3-dichloro-1-propanol & & & & [616-23-9] \\
\hline & $(384-419)$ & 48.5 & (399) & A & {$[87 / 5]$} \\
\hline \multirow[t]{2}{*}{$\mathrm{C}_{3} \mathrm{H}_{6} \mathrm{Cl}_{2} \mathrm{O}$} & 1,3-dichloro-2-propanol & & & & {$[96-23-1]$} \\
\hline & $(301-448)$ & 50.4 & (316) & A & {$[87 / 5][47 / 5]$} \\
\hline \multirow[t]{2}{*}{$\mathrm{C}_{3} \mathrm{H}_{6} \mathrm{~F}_{2}$} & 1,1-difluoropropane & & & & {$[430-81-5]$} \\
\hline & $(219-311)$ & 27.2 & (234) & A, EST & $\begin{array}{c}{[87 / 5][56 / 16]} \\
{[70 / 16]}\end{array}$ \\
\hline \multirow[t]{2}{*}{$\mathrm{C}_{3} \mathrm{H}_{6} \mathrm{~F}_{2}$} & 2,2-difluoropropane & & & & {$[420-45-1]$} \\
\hline & $(211-302)$ & 25.6 & (226) & A & {$[87 / 5][70 / 16]$} \\
\hline \multirow[t]{2}{*}{$\mathrm{C}_{3} \mathrm{H}_{6} \mathrm{~F}_{2} \mathrm{O}$} & 1,1-difluoro-2-methoxyethan & & & & {$[461-57-4]$} \\
\hline & $(288-322)$ & 31.8 & (303) & I & {$[02 / 19]$} \\
\hline \multirow[t]{2}{*}{$\mathrm{C}_{3} \mathrm{H}_{6} \mathrm{~F}_{3} \mathrm{NS}$} & $\mathrm{N}, \mathrm{N}$-dimethyl-trifluorometha & henamide & & & \\
\hline & $(223-295)$ & 30.2 & (259) & & {$[60 / 24]$} \\
\hline \multirow[t]{2}{*}{$\mathrm{C}_{3} \mathrm{H}_{6} \mathrm{~F}_{3} \mathrm{NS}$} & dimethyl(trifluoromethylthio) & & & & [62067-13-4] \\
\hline & $(273-329)$ & 31.1 & (288) & A & {$[87 / 5][99 / 16]$} \\
\hline \multirow[t]{2}{*}{$\mathrm{C}_{3} \mathrm{H}_{6} \mathrm{~F}_{3} \mathrm{OP}$} & methyl(trifluoromethyl)phosp & acid, methyl & & & [26348-84-5] \\
\hline & $(232-285)$ & 33.9 & $(258)$ & & {$[70 / 26]$} \\
\hline $\mathrm{C}_{3} \mathrm{H}_{6} \mathrm{~F}_{3} \mathrm{OP}$ & dimethyl(trifluoromethyl)pho & oxide & & & [26348-91-4] \\
\hline & $(347-360)$ & 52.4 & (353) & & {$[70 / 26]$} \\
\hline $\mathrm{C}_{3} \mathrm{H}_{6} \mathrm{~F}_{3} \mathrm{O}_{2} \mathrm{P}$ & (trifluoromethyl)phosphonic & methyl ester & & & [684-56-0] \\
\hline & $(237-318)$ & 37.4 & $(252)$ & A & {$[87 / 5][61 / 14]$} \\
\hline $\mathrm{C}_{3} \mathrm{H}_{6} \mathrm{~F}_{3} \mathrm{PS}$ & methyl(trifluoromethyl)phosp & ous acid, me & & & [26348-86-7] \\
\hline & $(273-313)$ & 38.4 & (293) & & {$[70 / 26]$} \\
\hline $\mathrm{C}_{3} \mathrm{H}_{6} \mathrm{~F}_{3} \mathrm{PS}$ & dimethyl(trifluoromethyl)pho & sulfide & & & [26348-92-5] \\
\hline & $(323-357)$ & 47.2 & $(340)$ & & {$[70 / 26]$} \\
\hline
\end{tabular}


TABLE 6. Enthalpies of vaporization of organic compounds, 1880-2002-Continued

\begin{tabular}{|c|c|c|c|c|c|}
\hline Molecular formula & $\begin{array}{c}\text { Compound } \\
\text { (Temperature range/K) }\end{array}$ & $\begin{array}{c}\Delta_{\mathrm{vap}} H_{m} \\
\left(\mathrm{~kJ} \mathrm{~mol}^{-1}\right)\end{array}$ & $\begin{array}{l}\text { Mean temperature } \\
\left(T_{m} / \mathrm{K}\right)\end{array}$ & Method & $\begin{array}{c}\text { CAS registry number } \\
\text { Reference }\end{array}$ \\
\hline \multirow[t]{2}{*}{$\mathrm{C}_{3} \mathrm{H}_{6} \mathrm{I}_{2}$} & 1,3-diiodopropane & & & & {$[627-31-6]$} \\
\hline & & 54.1 & $(298)$ & GC & [94/19] \\
\hline \multirow[t]{2}{*}{$\mathrm{C}_{3} \mathrm{H}_{6} \mathrm{~N}_{2} \mathrm{O}_{4}$} & 1,1-dinitropropane & & & & {$[601-76-3]$} \\
\hline & $(323-383)$ & 57.9 & $(338)$ & A & {$[87 / 5]$} \\
\hline \multirow[t]{2}{*}{$\mathrm{C}_{3} \mathrm{H}_{6} \mathrm{~N}_{2} \mathrm{O}_{4}$} & 2,2-dinitropropane & & & & {$[595-49-3]$} \\
\hline & $(363-553)$ & 46.3 & $(378)$ & A & {$[87 / 5]$} \\
\hline \multirow[t]{2}{*}{$\mathrm{C}_{3} \mathrm{H}_{6} \mathrm{~N}_{2} \mathrm{O}_{6}$} & 1,2-propanediol dinitrate & & & & {$[6423-43-4]$} \\
\hline & $(288-328)$ & 63.8 & $(303)$ & A & {$[87 / 5][70 / 16]$} \\
\hline \multirow[t]{2}{*}{$\mathrm{C}_{3} \mathrm{H}_{6} \mathrm{~N}_{2} \mathrm{O}_{6}$} & 1,3-propanediol dinitrate & & & & {$[3457-90-7]$} \\
\hline & $(293-313)$ & $74.3 \pm 4.6$ & $(303)$ & $\mathrm{A}, \mathrm{GS}$ & {$[87 / 5][57 / 5]$} \\
\hline \multirow{2}{*}{$\mathrm{C}_{3} \mathrm{H}_{6} \mathrm{~N}_{6} \mathrm{O}_{6}$} & hexahydro-1,3,5-trinitro-1,3,5-triazine & & & & {$[121-82-4]$} \\
\hline & $(503-523)$ & 84.4 & $(513)$ & A & {$[87 / 5]$} \\
\hline \multirow[t]{2}{*}{$\mathrm{C}_{3} \mathrm{H}_{6} \mathrm{O}$} & 2-propen-1-ol & & & & {$[107-18-6]$} \\
\hline & $(323-373)$ & 47.3 & $(298)$ & CGC & {$[95 / 21]$} \\
\hline \multirow[t]{2}{*}{$\mathrm{C}_{3} \mathrm{H}_{6} \mathrm{O}$} & oxetane & & & & {$[503-30-0]$} \\
\hline & & 29.8 & $(298)$ & $\mathrm{C}$ & {$[81 / 8]$} \\
\hline \multirow[t]{22}{*}{$\mathrm{C}_{3} \mathrm{H}_{6} \mathrm{O}$} & acetone & & & & {$[67-64-1]$} \\
\hline & $(329-488)$ & 29.9 & $(344)$ & A & {$[87 / 5]$} \\
\hline & $(178-243)$ & 32.9 & $(228)$ & A & {$[87 / 5]$} \\
\hline & $(203-269)$ & 33.8 & $(254)$ & A & {$[87 / 5]$} \\
\hline & $(323-379)$ & 30.6 & $(338)$ & A & {$[87 / 5]$} \\
\hline & $(374-464)$ & 29.5 & (389) & A & {$[87 / 5]$} \\
\hline & $(457-508)$ & 29.7 & $(472)$ & A & {$[87 / 5]$} \\
\hline & & 26.1 & (373) & $\mathrm{C}$ & {$[86 / 18]$} \\
\hline & & 21.7 & $(423)$ & $\mathrm{C}$ & {$[86 / 18]$} \\
\hline & & 15.3 & $(473)$ & $\mathrm{C}$ & [86/18] \\
\hline & & 9.2 & (498) & $\mathrm{C}$ & {$[86 / 18]$} \\
\hline & $(285-329)$ & 31.9 & $(300)$ & $\mathrm{EB}$ & {$[86 / 10]$} \\
\hline & $(305-333)$ & 31.8 & (319) & & {$[84 / 23]$} \\
\hline & $(259-351)$ & 32.8 & $(274)$ & A & {$[87 / 5][74 / 6]$} \\
\hline & & & & & {$[75 / 8]$} \\
\hline & & 31.3 & $(298)$ & & {$[75 / 8]$} \\
\hline & $(261-328)$ & 32.7 & $(276)$ & $\mathrm{A}, \mathrm{EB}$ & {$[87 / 5][72 / 6]$} \\
\hline & $(278-293)$ & 32.6 & $(285)$ & & {$[63 / 25]$} \\
\hline & $(310-329)$ & 31.1 & (319) & & {$[57 / 3]$} \\
\hline & $(204-339)$ & 35.0 & $(253)$ & MG & {$[26 / 7]$} \\
\hline & $(204-339)$ & 32.1 & $(293)$ & MG & {$[26 / 7]$} \\
\hline & $(204-339)$ & 30.7 & $(313)$ & MG & {$[26 / 7]$} \\
\hline \multirow[t]{5}{*}{$\mathrm{C}_{3} \mathrm{H}_{6} \mathrm{O}$} & allyl alcohol & & & & [107-18-6] \\
\hline & $(310-340)$ & 44.6 & $(325)$ & & {$[02 / 45]$} \\
\hline & $(253-370)$ & 46.7 & $(268)$ & A & {$[87 / 5]$} \\
\hline & & NA & & & {$[36 / 2]$} \\
\hline & $(283-313)$ & 44.8 & $(298)$ & & {$[35 / 3]$} \\
\hline \multirow[t]{2}{*}{$\mathrm{C}_{3} \mathrm{H}_{6} \mathrm{O}$} & methyl vinyl ether & & & & {$[107-25-5]$} \\
\hline & $(278-412)$ & 23.4 & $(293)$ & A & {$[87 / 5]$} \\
\hline \multirow[t]{9}{*}{$\mathrm{C}_{3} \mathrm{H}_{6} \mathrm{O}$} & propanal & & & & {$[123-38-6]$} \\
\hline & $(263-373)$ & 31.5 & $(278)$ & & [77/24] \\
\hline & $(286-321)$ & NA & $(301)$ & & {$[74 / 8]$} \\
\hline & & 28.3 & $(321)$ & & {$[72 / 4]$} \\
\hline & & 29.4 & (303) & & {$[72 / 4]$} \\
\hline & & 30.3 & $(286)$ & & {$[72 / 4]$} \\
\hline & & 29.6 & $(298)$ & & {$[72 / 4]$} \\
\hline & $(290-322)$ & 30.3 & $(305)$ & A & {$[87 / 5][70 / 16]$} \\
\hline & $(250-330)$ & 31.9 & $(265)$ & $\mathrm{EB}$ & {$[87 / 5][51 / 8]$} \\
\hline \multirow[t]{7}{*}{$\mathrm{C}_{3} \mathrm{H}_{6} \mathrm{O}$} & propylene oxide & & & & [75-56-9] \\
\hline & $(225-308)$ & 31.6 & $(240)$ & A & {$[87 / 5][70 / 16]$} \\
\hline & $(292-345)$ & 28.5 & $(307)$ & & {$[66 / 3]$} \\
\hline & & 27.9 & $(298)$ & $\mathrm{C}$ & {$[62 / 28]$} \\
\hline & $(249-308)$ & 30.1 & $(264)$ & & {$[59 / 1]$} \\
\hline & $(285-322)$ & 28.2 & (303) & & {$[37 / 7]$} \\
\hline & $(243-306)$ & 32.9 & $(273)$ & & {$[35 / 5]$} \\
\hline \multirow[t]{3}{*}{$\mathrm{C}_{3} \mathrm{H}_{6} \mathrm{O}_{2}$} & 1,3-dioxolane & & & & {$[646-06-0]$} \\
\hline & $(305-347)$ & 34.6 & $(326)$ & & [89/3] \\
\hline & $(280-323)$ & 35.8 & $(295)$ & A & {$[87 / 5]$} \\
\hline
\end{tabular}


TABLE 6. Enthalpies of vaporization of organic compounds, 1880-2002-Continued

\begin{tabular}{|c|c|c|c|c|c|}
\hline Molecular formula & $\begin{array}{c}\text { Compound } \\
\text { (Temperature range/K) }\end{array}$ & $\begin{array}{c}\Delta_{\mathrm{vap}} H_{m} \\
\left(\mathrm{~kJ} \mathrm{~mol}^{-1}\right)\end{array}$ & $\begin{array}{l}\text { Mean temperature } \\
\qquad\left(T_{m} / \mathrm{K}\right)\end{array}$ & Method & $\begin{array}{c}\text { CAS registry number } \\
\text { Reference }\end{array}$ \\
\hline & $(321-357)$ & 33.7 & (339) & & {$[82 / 3]$} \\
\hline & $(306-346)$ & 33.7 & $(326)$ & & {$[80 / 18]$} \\
\hline & $(280-355)$ & 34.1 & $(296)$ & & {$[68 / 2][84 / 9]$} \\
\hline & & $35.6 \pm 0.4$ & & & {$[59 / 23]$} \\
\hline \multirow[t]{7}{*}{$\mathrm{C}_{3} \mathrm{H}_{6} \mathrm{O}_{2}$} & ethyl formate & & & & [109-94-4] \\
\hline & $(300-326)$ & 31.4 & (313) & & [93/8] \\
\hline & $(327-498)$ & 29.9 & $(342)$ & A & {$[87 / 5]$} \\
\hline & & $31.6 \pm 0.1$ & (304) & $\mathrm{C}$ & {$[76 / 14]$} \\
\hline & & $30.9 \pm 0.1$ & (313) & $\mathrm{C}$ & {$[76 / 14]$} \\
\hline & & $29.8 \pm 0.1$ & (328) & $\mathrm{C}$ & {$[76 / 14]$} \\
\hline & $(213-336)$ & 35.8 & $(228)$ & A & {$[87 / 5][70 / 16]$} \\
\hline \multirow{14}{*}{$\mathrm{C}_{3} \mathrm{H}_{6} \mathrm{O}_{2}$} & methyl acetate & & & & [79-20-9] \\
\hline & $(260-351)$ & 34.1 & $(275)$ & A & [87/5] \\
\hline & & $32.3 \pm 0.1$ & (298) & $\mathrm{C}$ & {$[80 / 13]$} \\
\hline & & $29.5 \pm 0.1$ & $(343)$ & $\mathrm{C}$ & {$[80 / 13]$} \\
\hline & $(308-338)$ & 31.8 & (323) & DTA & {$[80 / 8]$} \\
\hline & & $32.6 \pm 0.1$ & $(298)$ & $\mathrm{C}$ & {$[79 / 1]$} \\
\hline & & $32.2 \pm 0.1$ & (304) & $\mathrm{C}$ & {$[77 / 12]$} \\
\hline & & $31.6 \pm 0.1$ & (313) & $\mathrm{C}$ & {$[77 / 12]$} \\
\hline & & $30.5 \pm 0.1$ & (328) & $\mathrm{C}$ & {$[77 / 12]$} \\
\hline & & $30.3 \pm 0.1$ & $(331)$ & $\mathrm{C}$ & {$[77 / 12]$} \\
\hline & & 32.5 & $(295)$ & & {$[76 / 8]$} \\
\hline & & 30.2 & $(330)$ & & {$[76 / 8]$} \\
\hline & $(273-318)$ & 34.5 & $(296)$ & BG & {$[71 / 2]$} \\
\hline & $(274-329)$ & 33.4 & (289) & $\mathrm{A}$ & $\begin{array}{c}{[87 / 5][65 / 3]} \\
{[70 / 16]}\end{array}$ \\
\hline \multirow[t]{6}{*}{$\mathrm{C}_{3} \mathrm{H}_{6} \mathrm{O}_{2}$} & propionic acid & & & & [79-09-4] \\
\hline & $(353-393)$ & 54.9 & (298) & CGC & {$[95 / 21]$} \\
\hline & $(343-419)$ & 47.0 & $(358)$ & A & {$[87 / 5]$} \\
\hline & $(414-511)$ & 60.6 & $(429)$ & A & {$[87 / 5]$} \\
\hline & $(345-401)$ & 46.4 & $(360)$ & $\mathrm{A}$ & {$[87 / 5]$} \\
\hline & & 56.0 & $(303)$ & & {$[83 / 13]$} \\
\hline \multirow[t]{3}{*}{ monomer } & & $31.1 \pm 0.1$ & $(298)$ & $\mathrm{C}$ & {$[70 / 8]$} \\
\hline & & $55 \pm 2$ & $(298)$ & $\mathrm{C}$ & {$[70 / 8]$} \\
\hline & $(328-437)$ & 48.3 & $(343)$ & & [81/11] \\
\hline \multirow[t]{2}{*}{$\mathrm{C}_{3} \mathrm{H}_{6} \mathrm{O}_{3}$} & 2-methoxyacetic acid & & & & {$[625-45-6]$} \\
\hline & $(325-477)$ & 54.5 & $(340)$ & A & {$[87 / 5][47 / 5]$} \\
\hline \multirow[t]{3}{*}{$\mathrm{C}_{3} \mathrm{H}_{6} \mathrm{O}_{3}$} & methyl glycolate & & & & {$[96-35-5]$} \\
\hline & $(326-381)$ & $52.5 \pm 6.3$ & (298) & $\mathrm{EB}$ & {$[96 / 5]$} \\
\hline & $(282-425)$ & 47.4 & $(297)$ & $\mathrm{A}$ & {$[87 / 5][47 / 4]$} \\
\hline \multirow[t]{3}{*}{$\mathrm{C}_{3} \mathrm{H}_{6} \mathrm{O}_{3}$} & dimethylcarbonate & & & & {$[616-38-6]$} \\
\hline & $(326-411)$ & 36.4 & $(341)$ & & {$[02 / 23]$} \\
\hline & $(311-397)$ & $37.7 \pm 0.2$ & $(298)$ & $\mathrm{EB}$ & {$[97 / 7][97 / 6]$} \\
\hline \multirow[t]{2}{*}{$\mathrm{C}_{3} \mathrm{H}_{6} \mathrm{O}_{3}$} & peroxypropionic acid & & & & {$[4212-43-5]$} \\
\hline & $(273-393)$ & 43.2 & $(288)$ & A & $\begin{array}{c}{[87 / 5][51 / 10]} \\
{[70 / 16]}\end{array}$ \\
\hline \multirow[t]{2}{*}{$\mathrm{C}_{3} \mathrm{H}_{6} \mathrm{O}_{3}$} & propylene ozonide & & & & \\
\hline & $(261-296)$ & 36.9 & $(281)$ & A & {$[87 / 5][56 / 18]$} \\
\hline \multirow[t]{2}{*}{$\mathrm{C}_{3} \mathrm{H}_{6} \mathrm{O}_{3}$} & 1,3,5-trioxane & & & & [110-88-3] \\
\hline & $(329-386)$ & 40.0 & $(344)$ & A & {$[87 / 5][65 / 4]$} \\
\hline \multirow[t]{2}{*}{$\mathrm{C}_{3} \mathrm{H}_{6} \mathrm{~S}$} & 2-methylthiirane & & & & {$[1072-43-1]$} \\
\hline & $(272-423)$ & 34.6 & $(287)$ & & $\begin{array}{c}{[87 / 5][70 / 16]} \\
{[99 / 16]}\end{array}$ \\
\hline \multirow[t]{4}{*}{$\mathrm{C}_{3} \mathrm{H}_{6} \mathrm{~S}$} & thiacyclobutane (thietane) & & & & {$[287-27-4]$} \\
\hline & $(275-393)$ & 36.5 & $(290)$ & & [99/16] \\
\hline & & 35.8 & $(298)$ & & [71/28] \\
\hline & $(321-404)$ & 34.6 & $(336)$ & $\mathrm{A}, \mathrm{EB}$ & $\begin{array}{c}{[87 / 5][53 / 8]} \\
{[66 / 5]}\end{array}$ \\
\hline \multirow[t]{6}{*}{$\mathrm{C}_{3} \mathrm{H}_{7} \mathrm{Br}$} & 1-bromopropane & & & & {$[106-94-5]$} \\
\hline & $(301-344)$ & 31.8 & $(316)$ & $\mathrm{A}, \mathrm{EB}$ & {$[87 / 5][77 / 8]$} \\
\hline & & $31.1 \pm 0.1$ & $(322)$ & C & {$[77 / 8]$} \\
\hline & & $30.5 \pm 0.1$ & $(332)$ & $\mathrm{C}$ & {$[77 / 8]$} \\
\hline & & $30.1 \pm 0.1$ & (339) & $\mathrm{C}$ & {$[77 / 8]$} \\
\hline & & $29.3 \pm 0.1$ & $(352)$ & $\mathrm{C}$ & [77/8] \\
\hline
\end{tabular}


TABLE 6. Enthalpies of vaporization of organic compounds, 1880-2002-Continued

\begin{tabular}{|c|c|c|c|c|c|}
\hline Molecular formula & $\begin{array}{c}\text { Compound } \\
\text { (Temperature range/K) }\end{array}$ & $\begin{array}{c}\Delta_{\mathrm{vap}} H_{m} \\
\left(\mathrm{~kJ} \mathrm{~mol}^{-1}\right)\end{array}$ & $\begin{array}{l}\text { Mean temperature } \\
\qquad\left(T_{m} / \mathrm{K}\right)\end{array}$ & Method & $\begin{array}{c}\text { CAS registry number } \\
\text { Reference }\end{array}$ \\
\hline & & $31.9 \pm 0.1$ & (298) & C & {$[66 / 2]$} \\
\hline & $(250-368)$ & 34.1 & $(265)$ & A, EST & {$[87 / 5][61 / 13]$} \\
\hline & & & & & {$[70 / 16]$} \\
\hline & $(220-344)$ & 35.5 & (235) & & {$[47 / 5]$} \\
\hline & $(273-303)$ & 32.6 & (288) & & {$[06 / 1][84 / 9]$} \\
\hline \multirow[t]{11}{*}{$\mathrm{C}_{3} \mathrm{H}_{7} \mathrm{Br}$} & 2-bromopropane & & & & {$[75-26-3]$} \\
\hline & $(323-363)$ & 30.6 & $(298)$ & CGC & {$[95 / 21]$} \\
\hline & $(236-328)$ & 32.1 & (251) & A & {$[87 / 5]$} \\
\hline & $(299-332)$ & 30.1 & (314) & $\mathrm{EB}$ & {$[87 / 5][77 / 8]$} \\
\hline & & $29.8 \pm 0.1$ & (305) & $\mathrm{C}$ & {$[77 / 8]$} \\
\hline & & $29.2 \pm 0.1$ & (318) & $\mathrm{C}$ & {$[77 / 8]$} \\
\hline & & $28.5 \pm 0.1$ & $(330)$ & $\mathrm{C}$ & {$[77 / 8]$} \\
\hline & & $28.0 \pm 0.1$ & (338) & $\mathrm{C}$ & [77/8] \\
\hline & & $30.2 \pm 0.1$ & (298) & $\mathrm{C}$ & {$[66 / 2]$} \\
\hline & $(211-333)$ & 33.4 & (226) & & {$[47 / 5]$} \\
\hline & $(273-303)$ & 30.9 & (288) & & {$[06 / 1][84 / 9]$} \\
\hline \multirow[t]{6}{*}{$\mathrm{C}_{3} \mathrm{H}_{7} \mathrm{Cl}$} & 1-chloropropane & & & & {$[540-54-5]$} \\
\hline & $(250-320)$ & 29.0 & (298) & & {$[84 / 9][91 / 2]$} \\
\hline & & $28.5 \pm 0.2$ & (298) & $\mathrm{C}$ & {$[77 / 1]$} \\
\hline & $(248-320)$ & 31.0 & (263) & $\mathrm{A}$ & {$[87 / 5][69 / 5]$} \\
\hline & & & & & {$[70 / 16]$} \\
\hline & $(205-319)$ & 33.1 & (219) & & {$[47 / 5]$} \\
\hline \multirow[t]{4}{*}{$\mathrm{C}_{3} \mathrm{H}_{7} \mathrm{Cl}$} & 2-chloropropane & & & & [75-29-6] \\
\hline & $(239-310)$ & 30.2 & $(254)$ & A & {$[87 / 5]$} \\
\hline & $(194-309)$ & 30.6 & (209) & & [47/5] \\
\hline & $(273-303)$ & 27.3 & (288) & & {$[06 / 1][84 / 9]$} \\
\hline \multirow[t]{3}{*}{$\mathrm{C}_{3} \mathrm{H}_{7} \mathrm{ClO}$} & 1-chloro-2-propanol & & & & {$[127-00-4]$} \\
\hline & $(308-399)$ & $45.0 \pm 2.2$ & $(340)$ & EB & {$[02 / 14]$} \\
\hline & $(308-399)$ & $42.2 \pm 1.9$ & $(380)$ & EB & {$[02 / 14]$} \\
\hline \multirow[t]{2}{*}{$\mathrm{C}_{3} \mathrm{H}_{7} \mathrm{ClO}$} & 2-chloro-1-propanol & & & & {$[78-89-7]$} \\
\hline & $(316-399)$ & 45.0 & $(331)$ & $\mathrm{A}$ & {$[87 / 5]$} \\
\hline \multirow[t]{2}{*}{$\mathrm{C}_{3} \mathrm{H}_{7} \mathrm{ClO}_{2}$} & 3-chloro-1,2-propanediol & & & & [96-24-2] \\
\hline & $(343-409)$ & 66.6 & $(358)$ & & {$[96 / 13]$} \\
\hline \multirow[t]{4}{*}{$\mathrm{C}_{3} \mathrm{H}_{7} \mathrm{ClO}_{2} \mathrm{~S}$} & 1-propanesulfonyl chloride & & & & [10147-36-1] \\
\hline & $(273-362)$ & 52.3 & $(288)$ & & [99/16] \\
\hline & $(362-464)$ & 49.9 & (377) & & {$[99 / 16]$} \\
\hline & $(243-273)$ & 60.1 & $(258)$ & A & {$[87 / 5][99 / 16]$} \\
\hline \multirow[t]{2}{*}{$\mathrm{C}_{3} \mathrm{H}_{7} \mathrm{ClS}$} & methyl(2-chloroethyl) sulfide & & & & \\
\hline & $(293-333)$ & 42.4 & $(308)$ & A, GS & $\begin{array}{c}{[87 / 5][48 / 9]} \\
{[70 / 16]}\end{array}$ \\
\hline \multirow[t]{2}{*}{$\mathrm{C}_{3} \mathrm{H}_{7} \mathrm{~F}$} & 1-fluoropropane & & & & [460-13-9] \\
\hline & $(196-289)$ & 24.0 & $(274)$ & A, EST & $\begin{array}{c}{[87 / 5][61 / 13]} \\
{[70 / 16]}\end{array}$ \\
\hline \multirow[t]{2}{*}{$\mathrm{C}_{3} \mathrm{H}_{7} \mathrm{~F}$} & 2-fluoropropane & & & & [420-26-8] \\
\hline & $(190-264)$ & 23.7 & $(249)$ & A & {$[87 / 5]$} \\
\hline \multirow[t]{6}{*}{$\mathrm{C}_{3} \mathrm{H}_{7} \mathrm{I}$} & 1-iodopropane & & & & {$[107-08-4]$} \\
\hline & $(171-271)$ & 37.8 & $(256)$ & A & {$[87 / 5]$} \\
\hline & & $36.3 \pm 0.1$ & (298) & $\mathrm{C}$ & [68/1] \\
\hline & $(271-402)$ & 36.8 & $(286)$ & A, EST & {$[87 / 5][61 / 13]$} \\
\hline & & & & & [70/16] \\
\hline & $(237-375)$ & 37.0 & $(252)$ & & [47/5] \\
\hline \multirow[t]{5}{*}{$\mathrm{C}_{3} \mathrm{H}_{7} \mathrm{I}$} & 2-iodopropane & & & & [75-30-9] \\
\hline & $(313-353)$ & 34.0 & $(298)$ & CGC & {$[95 / 21]$} \\
\hline & $(173-262)$ & 36.7 & (247) & A & {$[87 / 5]$} \\
\hline & & $34.1 \pm 0.1$ & (298) & $\mathrm{C}$ & {$[68 / 1]$} \\
\hline & $(230-363)$ & 36.3 & (244) & & {$[47 / 5]$} \\
\hline \multirow[t]{2}{*}{$\mathrm{C}_{3} \mathrm{H}_{7} \mathrm{~N}$} & cyclopropylamine & & & & [765-30-0] \\
\hline & & $31.3 \pm 0.4$ & & & {$[71 / 36]$} \\
\hline \multirow[t]{3}{*}{$\mathrm{C}_{3} \mathrm{H}_{7} \mathrm{~N}$} & allylamine & & & & [107-11-9] \\
\hline & $(273-303)$ & 33.0 & (288) & A & {$[87 / 5]$} \\
\hline & $(273-324)$ & 32.6 & (288) & A & [87/5] \\
\hline \multirow[t]{2}{*}{$\mathrm{C}_{3} \mathrm{H}_{7} \mathrm{~N}$} & azetidine & & & & [503-29-7] \\
\hline & $(273-303)$ & 32.6 & $(288)$ & A & [87/5] \\
\hline $\mathrm{C}_{3} \mathrm{H}_{7} \mathrm{NO}$ & acetone oxime & & & & [127-06-0] \\
\hline
\end{tabular}


TABLE 6. Enthalpies of vaporization of organic compounds, 1880-2002-Continued

\begin{tabular}{|c|c|c|c|c|c|}
\hline Molecular formula & $\begin{array}{c}\text { Compound } \\
\text { (Temperature range/K) }\end{array}$ & $\begin{array}{c}\Delta_{\mathrm{vap}} H_{m} \\
\left(\mathrm{~kJ} \mathrm{~mol}^{-1}\right)\end{array}$ & $\begin{array}{l}\text { Mean temperature } \\
\qquad\left(T_{m} / \mathrm{K}\right)\end{array}$ & Method & $\begin{array}{c}\text { CAS registry number } \\
\text { Reference }\end{array}$ \\
\hline & $(338-352)$ & 51.4 & $(345)$ & A & {$[87 / 5]$} \\
\hline \multirow[t]{2}{*}{$\mathrm{C}_{3} \mathrm{H}_{7} \mathrm{NO}$} & propionamide & & & & [79-05-0] \\
\hline & $(338-486)$ & 60.3 & $(353)$ & & {$[47 / 5]$} \\
\hline \multirow[t]{6}{*}{$\mathrm{C}_{3} \mathrm{H}_{7} \mathrm{NO}$} & $\mathrm{N}, \mathrm{N}$-dimethyl formamide & & & & {$[68-12-2]$} \\
\hline & $(301-426)$ & 49.2 & (316) & A & {$[87 / 5]$} \\
\hline & & 46.9 & (298) & $\mathrm{A}$ & {$[85 / 7][85 / 6]$} \\
\hline & $(318-423)$ & 42.5 & (370) & & {$[79 / 21]$} \\
\hline & $(331-425)$ & 56.7 & (346) & & {$[74 / 7][84 / 9]$} \\
\hline & $(303-363)$ & 46.7 & $(318)$ & & {$[68 / 3]$} \\
\hline \multirow[t]{2}{*}{$\mathrm{C}_{3} \mathrm{H}_{7} \mathrm{NO}$} & N-ethylformamide & & & & {$[627-45-2]$} \\
\hline & & 58.4 & $(298)$ & $\mathrm{A}$ & {$[85 / 7][85 / 6]$} \\
\hline \multirow{5}{*}{$\mathrm{C}_{3} \mathrm{H}_{7} \mathrm{NO}$} & $\mathrm{N}$-methylacetamide & & & & {$[79-16-3]$} \\
\hline & $(353-428)$ & 62.0 & $(368)$ & & {$[93 / 2]$} \\
\hline & $(333-443)$ & 59.6 & (348) & A & {$[87 / 5]$} \\
\hline & $(353-479)$ & 53.5 & $(368)$ & A & {$[87 / 5]$} \\
\hline & & NA & & & {$[68 / 3]$} \\
\hline \multirow[t]{2}{*}{$\mathrm{C}_{3} \mathrm{H}_{7} \mathrm{NO}$} & propionaldehyde oxime & & & & {$[627-39-4]$} \\
\hline & $(313-339)$ & 51.2 & $(326)$ & A & {$[87 / 5]$} \\
\hline \multirow[t]{2}{*}{$\mathrm{C}_{3} \mathrm{H}_{7} \mathrm{NO}_{2}$} & ethyl carbamate & & & & {$[51-79-6]$} \\
\hline & $(338-457)$ & 56.6 & $(353)$ & A & {$[87 / 5][47 / 5]$} \\
\hline \multirow{2}{*}{$\mathrm{C}_{3} \mathrm{H}_{7} \mathrm{NO}_{2}$} & isopropyl nitrite & & & & {$[541-42-4]$} \\
\hline & $(253-268)$ & 26.0 & (260) & A & {$[87 / 5][37 / 3]$} \\
\hline \multirow[t]{5}{*}{$\mathrm{C}_{3} \mathrm{H}_{7} \mathrm{NO}_{2}$} & 1-nitropropane & & & & [108-03-2] \\
\hline & $(313-353)$ & 43.9 & (298) & CGC & {$[95 / 21]$} \\
\hline & $(293-405)$ & 42.6 & $(308)$ & A, EB & {$[87 / 5][56 / 15]$} \\
\hline & & & & & {$[70 / 16]$} \\
\hline & $(331-404)$ & 40.6 & $(346)$ & & {$[49 / 1]$} \\
\hline \multirow[t]{4}{*}{$\mathrm{C}_{3} \mathrm{H}_{7} \mathrm{NO}_{2}$} & 2-nitropropane & & & & [79-46-9] \\
\hline & $(313-353)$ & 43.9 & $(298)$ & CGC & {$[75 / 21]$} \\
\hline & $(284-394)$ & 40.9 & (299) & $\mathrm{A}, \mathrm{EB}$ & {$[87 / 5][47 / 5]$} \\
\hline & & & & & {$[56 / 15][70 / 16]$} \\
\hline \multirow{2}{*}{$\mathrm{C}_{3} \mathrm{H}_{7} \mathrm{NO}_{2}$} & propyl nitrite & & & & [543-67-9] \\
\hline & $(253-268)$ & 28.3 & (260) & A & {$[87 / 5][37 / 3]$} \\
\hline \multirow[t]{4}{*}{$\mathrm{C}_{3} \mathrm{H}_{7} \mathrm{NO}_{3}$} & isopropyl nitrate & & & & [1712-64-7] \\
\hline & & $35.3 \pm 0.1$ & & DSC & [99/12] \\
\hline & $(273-343)$ & 39.7 & $(288)$ & A & {$[87 / 5][57 / 2]$} \\
\hline & & & & & {$[70 / 16]$} \\
\hline \multirow[t]{3}{*}{$\mathrm{C}_{3} \mathrm{H}_{7} \mathrm{NO}_{3}$} & propyl nitrate & & & & {$[627-13-4]$} \\
\hline & $(273-343)$ & 41.7 & $(288)$ & A & {$[87 / 5][57 / 2]$} \\
\hline & & & & & {$[70 / 16]$} \\
\hline \multirow[t]{2}{*}{$\mathrm{C}_{3} \mathrm{H}_{7} \mathrm{~N}_{3}$} & 1-azidopropane & & & & [22293-25-0] \\
\hline & $(253-298)$ & 31.1 & $(268)$ & A & $\begin{array}{c}{[87 / 5][64 / 5]} \\
{[84 / 9]}\end{array}$ \\
\hline \multirow[t]{3}{*}{$\mathrm{C}_{3} \mathrm{H}_{7} \mathrm{~N}_{3}$} & 2-azidopropane & & & & [691-57-6] \\
\hline & $(253-298)$ & 33.2 & $(268)$ & A & {$[87 / 5][64 / 5]$} \\
\hline & & & & & {$[84 / 9]$} \\
\hline $\mathrm{C}_{3} \mathrm{H}_{7} \mathrm{P}$ & 2-propenylphosphine & & & & [81637-99-2] \\
\hline & $(210-273)$ & 32.7 & $(241)$ & & {$[88 / 13]$} \\
\hline $\mathrm{C}_{3} \mathrm{H}_{8}$ & propane & & & & [74-98-6] \\
\hline & $(278-332)$ & 18.8 & (293) & A & {$[87 / 5]$} \\
\hline & $(165-248)$ & 19.5 & (233) & $\mathrm{A}$ & {$[87 / 5]$} \\
\hline & $(104-165)$ & 22.1 & (150) & A & {$[87 / 5]$} \\
\hline & $(231-281)$ & 19.0 & (266) & A & {$[87 / 5]$} \\
\hline & $(329-369)$ & 19.2 & (344) & $\mathrm{A}$ & {$[87 / 5]$} \\
\hline & $(312-367)$ & 18.9 & $(327)$ & & {$[80 / 1]$} \\
\hline & & 18.77 & $(256)$ & & {$[71 / 28]$} \\
\hline & $(166-231)$ & 20.0 & (216) & & {$[38 / 4]$} \\
\hline $\mathrm{C}_{3} \mathrm{H}_{8} \mathrm{~N}_{2}$ & dimethyl ammonium cyanide & & & & \\
\hline & $(251-295)$ & 49.0 & $(280)$ & & {$[87 / 5][73 / 10]$} \\
\hline $\mathrm{C}_{3} \mathrm{H}_{8} \mathrm{~N}_{2} \mathrm{~S}$ & 1,3-dimethylthiourea & & & & \\
\hline & $(342-375)$ & $93 \pm 4.0$ & $(359)$ & ME, TE & [94/21] \\
\hline $\mathrm{C}_{3} \mathrm{H}_{8} \mathrm{O}$ & methyl ethyl ether & & & & {$[540-67-0]$} \\
\hline & $(281-433)$ & 30.1 & (296) & A & {$[87 / 5]$} \\
\hline & $(216-299)$ & 37.0 & $(231)$ & A & {$[87 / 5]$} \\
\hline
\end{tabular}


TABLE 6. Enthalpies of vaporization of organic compounds, 1880-2002-Continued

\begin{tabular}{|c|c|c|c|c|c|}
\hline Molecular formula & $\begin{array}{c}\text { Compound } \\
\text { (Temperature range/K) }\end{array}$ & $\begin{array}{c}\Delta_{\mathrm{vap}} H_{m} \\
\left(\mathrm{~kJ} \mathrm{~mol}^{-1}\right)\end{array}$ & $\begin{array}{l}\text { Mean temperature } \\
\qquad\left(T_{m} / \mathrm{K}\right)\end{array}$ & Method & $\begin{array}{c}\text { CAS registry number } \\
\text { Reference }\end{array}$ \\
\hline \multirow{41}{*}{$\mathrm{C}_{3} \mathrm{H}_{8} \mathrm{O}$} & $(281-438)$ & 37.1 & (296) & A & {$[87 / 5]$} \\
\hline & $(278-281)$ & NA & & & {$[67 / 7]$} \\
\hline & $(182-280)$ & 26.3 & $(265)$ & & {$[47 / 5]$} \\
\hline & 1-propanol & & & & [71-23-8] \\
\hline & & 41.2 & $(371)$ & & {$[00 / 24]$} \\
\hline & & 35.2 & $(423)$ & & {$[00 / 24]$} \\
\hline & & 29.4 & $(453)$ & & {$[00 / 24]$} \\
\hline & & 21.0 & (498) & & {$[00 / 24]$} \\
\hline & & 11.4 & $(528)$ & & {$[00 / 24]$} \\
\hline & $(323-373)$ & 49.2 & (298) & CGC & {$[95 / 21]$} \\
\hline & $(303-370)$ & 47.0 & (318) & & {$[95 / 14]$} \\
\hline & $(360-377)$ & 42.9 & $(375)$ & & {$[90 / 10]$} \\
\hline & $(200-228)$ & 48.0 & (214) & A & {$[87 / 5]$} \\
\hline & $(356-376)$ & 43.5 & $(366)$ & A & {$[87 / 5]$} \\
\hline & $(369-407)$ & 42.3 & (384) & $\mathrm{A}$ & {$[87 / 5]$} \\
\hline & $(401-482)$ & 40.1 & (416) & A & {$[87 / 5]$} \\
\hline & $(478-507)$ & 36.5 & (492) & A & {$[87 / 5]$} \\
\hline & $(243-303)$ & 46.3 & (298) & & {$[83 / 14]$} \\
\hline & $(275-373)$ & 49.3 & $(290)$ & & {$[73 / 26]$} \\
\hline & & $46.4 \pm 0.1$ & (313) & $\mathrm{C}$ & {$[73 / 13]$} \\
\hline & & $45.7 \pm 0.1$ & (323) & $\mathrm{C}$ & {$[73 / 13]$} \\
\hline & & $44.9 \pm 0.1$ & (333) & $\mathrm{C}$ & {$[73 / 13]$} \\
\hline & & $44.0 \pm 0.1$ & (343) & $\mathrm{C}$ & {$[73 / 13]$} \\
\hline & & $43.2 \pm 0.1$ & (353) & $\mathrm{C}$ & {$[73 / 13]$} \\
\hline & & $42.4 \pm 0.1$ & (363) & $\mathrm{C}$ & {$[73 / 13]$} \\
\hline & & $47.49 \pm 0.02$ & (298) & $\mathrm{C}$ & [71/27] \\
\hline & $(333-377)$ & 44.7 & (348) & $\mathrm{EB}$ & {$[70 / 2][87 / 5]$} \\
\hline & $(292-370)$ & 46.9 & $(307)$ & DTA & {$[69 / 5]$} \\
\hline & $(288-348)$ & 46.7 & (303) & & {$[67 / 10]$} \\
\hline & & $47.3 \pm 0.1$ & (298) & $\mathrm{C}$ & {$[66 / 2]$} \\
\hline & $(338-378)$ & 44.3 & (353) & $\mathrm{EB}$ & {$[63 / 8]$} \\
\hline & & 46.6 & (298) & $\mathrm{C}$ & {$[63 / 2]$} \\
\hline & $(405-537)$ & 40.7 & $(420)$ & & {$[63 / 20]$} \\
\hline & $(343-385)$ & 44.1 & $(358)$ & & {$[61 / 18]$} \\
\hline & & $43.9 \pm 0.1$ & (343) & $\mathrm{C}$ & [61/18] \\
\hline & & $42.3 \pm 0.1$ & $(360)$ & $\mathrm{C}$ & {$[61 / 18]$} \\
\hline & & $41.2 \pm 0.1$ & $(370)$ & $\mathrm{C}$ & {$[61 / 18]$} \\
\hline & & $40.3 \pm 0.1$ & $(378)$ & $\mathrm{C}$ & [61/18] \\
\hline & & $39.7 \pm 0.1$ & (384) & $\mathrm{C}$ & {$[61 / 18]$} \\
\hline & $(321-367)$ & 45.5 & $(366)$ & & {$[59 / 3]$} \\
\hline & & 43.2 & $(354)$ & & {$[57 / 21]$} \\
\hline \multirow[t]{23}{*}{$\mathrm{C}_{3} \mathrm{H}_{8} \mathrm{O}$} & 2-propanol & & & & [67-63-0] \\
\hline & $(322-355)$ & 43.2 & (337) & & {$[02 / 26]$} \\
\hline & & 39.8 & $(355)$ & & {$[00 / 25]$} \\
\hline & & 29.7 & $(423)$ & & {$[00 / 25]$} \\
\hline & & 23.7 & (453) & & {$[00 / 25]$} \\
\hline & & 16.5 & $(483)$ & & {$[00 / 25]$} \\
\hline & & 10.5 & $(503)$ & & {$[00 / 25]$} \\
\hline & & 40.4 & & & [99/32] \\
\hline & $(300-355)$ & 44.8 & $(315)$ & & {$[95 / 14]$} \\
\hline & $(195-228)$ & 50.3 & (213) & A & {$[87 / 5]$} \\
\hline & $(347-368)$ & 42.0 & $(355)$ & A & {$[87 / 5]$} \\
\hline & $(350-383)$ & 41.3 & $(365)$ & A & {$[87 / 5]$} \\
\hline & $(379-461)$ & 39.2 & (394) & A & {$[87 / 5]$} \\
\hline & $(453-508)$ & 35.3 & $(468)$ & A & {$[87 / 5]$} \\
\hline & $(273-374)$ & 45.7 & $(288)$ & & [73/26] \\
\hline & $(325-362)$ & 43.1 & $(340)$ & $\mathrm{A}, \mathrm{EB}$ & {$[87 / 5][70 / 2]$} \\
\hline & & $45.34 \pm 0.02$ & (298) & C & [71/27] \\
\hline & $(288-348)$ & 45.5 & (303) & & {$[67 / 10]$} \\
\hline & & $45.2 \pm 0.1$ & (298) & $\mathrm{C}$ & {$[66 / 2]$} \\
\hline & & $42.7 \pm 0.1$ & $(330)$ & $\mathrm{C}$ & {$[64 / 23]$} \\
\hline & & $41.0 \pm 0.1$ & $(346)$ & $\mathrm{C}$ & {$[64 / 23]$} \\
\hline & & $39.8 \pm 0.1$ & $(355)$ & $\mathrm{C}$ & {$[64 / 23]$} \\
\hline & & $38.9 \pm 0.1$ & (363) & $\mathrm{C}$ & {$[64 / 23]$} \\
\hline
\end{tabular}


TABLE 6. Enthalpies of vaporization of organic compounds, 1880-2002-Continued

\begin{tabular}{|c|c|c|c|c|c|}
\hline Molecular formula & $\begin{array}{c}\text { Compound } \\
\text { (Temperature range/K) }\end{array}$ & $\begin{array}{c}\Delta_{\mathrm{vap}} H_{m} \\
\left(\mathrm{~kJ} \mathrm{~mol}^{-1}\right)\end{array}$ & $\begin{array}{l}\text { Mean temperature } \\
\qquad\left(T_{m} / \mathrm{K}\right)\end{array}$ & Method & $\begin{array}{c}\text { CAS registry number } \\
\text { Reference }\end{array}$ \\
\hline & $(329-363)$ & 42.8 & $(344)$ & $\mathrm{EB}$ & {$[63 / 8]$} \\
\hline & & 44.0 & $(298)$ & $\mathrm{C}$ & {$[63 / 2]$} \\
\hline & $(395-508)$ & 39.1 & $(410)$ & & {$[63 / 20]$} \\
\hline & & 43.2 & $(324)$ & $\mathrm{C}$ & {$[63 / 28]$} \\
\hline & & 41.7 & $(339)$ & $\mathrm{C}$ & {$[63 / 28]$} \\
\hline & & 39.8 & $(355)$ & $\mathrm{C}$ & {$[63 / 28]$} \\
\hline & $(354-420)$ & 41.1 & $(369)$ & & {$[55 / 4]$} \\
\hline & $(273-363)$ & 44.4 & $(298)$ & & {$[28 / 5]$} \\
\hline \multirow[t]{2}{*}{$\mathrm{C}_{3} \mathrm{H}_{8} \mathrm{OS}_{2}$} & 2,3-dimercaptopropanol & & & & [59-52-9] \\
\hline & $(353-413)$ & 61.2 & $(382)$ & & {$[99 / 16][87 / 5]$} \\
\hline \multirow[t]{4}{*}{$\mathrm{C}_{3} \mathrm{H}_{8} \mathrm{O}_{2}$} & 2-methoxyethanol & & & & [109-86-4] \\
\hline & $(333-423)$ & 42.8 & $(348)$ & A & {$[87 / 5]$} \\
\hline & & $45.2 \pm 0.2$ & $(298)$ & $\mathrm{C}$ & {$[71 / 5]$} \\
\hline & $(329-396)$ & 42.9 & $(344)$ & & {$[56 / 6]$} \\
\hline \multirow[t]{5}{*}{$\mathrm{C}_{3} \mathrm{H}_{8} \mathrm{O}_{2}$} & dimethoxymethane & & & & [109-87-5] \\
\hline & $(273-316)$ & 31.2 & $(288)$ & A & {$[87 / 5]$} \\
\hline & $(273-318)$ & 29.8 & $(288)$ & A & {$[87 / 5]$} \\
\hline & $(296-314)$ & 30.3 & $(305)$ & & {$[76 / 20]$} \\
\hline & $(273-308)$ & 30.1 & $(288)$ & & {$[49 / 2]$} \\
\hline \multirow[t]{8}{*}{$\mathrm{C}_{3} \mathrm{H}_{8} \mathrm{O}_{2}$} & 1,2-propanediol & & & & {$[57-55-6]$} \\
\hline & $(365-496)$ & $60.0 \pm 0.3$ & $(380)$ & EB & {$[02 / 17]$} \\
\hline & $(365-496)$ & $56.2 \pm 0.2$ & $(420)$ & $\mathrm{EB}$ & {$[02 / 17]$} \\
\hline & $(365-496)$ & $52.0 \pm 0.3$ & $(460)$ & $\mathrm{EB}$ & {$[02 / 17]$} \\
\hline & $(365-496)$ & $47.5 \pm 0.6$ & $(500)$ & $\mathrm{EB}$ & {$[02 / 17]$} \\
\hline & $(318-461)$ & 58.6 & (333) & $\mathrm{A}$ & {$[87 / 5][47 / 5]$} \\
\hline & $(353-403)$ & 58.2 & $(378)$ & & {$[35 / 4]$} \\
\hline & $(403-460)$ & 56.0 & $(431)$ & & {$[35 / 4]$} \\
\hline \multirow[t]{5}{*}{$\mathrm{C}_{3} \mathrm{H}_{8} \mathrm{O}_{2}$} & 1,3-propanediol & & & & {$[504-63-2]$} \\
\hline & & $72.4 \pm 0.3$ & $(298)$ & $\mathrm{C}$ & {$[88 / 14]$} \\
\hline & $(332-448)$ & 57.2 & $(347)$ & A & {$[87 / 5][47 / 5]$} \\
\hline & $(383-433)$ & 63.3 & $(408)$ & & {$[35 / 4]$} \\
\hline & $(433-488)$ & 60.4 & $(460)$ & & {$[35 / 4]$} \\
\hline \multirow[t]{2}{*}{$\mathrm{C}_{3} \mathrm{H}_{8} \mathrm{O}_{2}$} & propylene glycol & & & & \\
\hline & $(373-408)$ & 66.5 & $(413)$ & TGA & {$[87 / 18]$} \\
\hline \multirow[t]{11}{*}{$\mathrm{C}_{3} \mathrm{H}_{8} \mathrm{O}_{3}$} & glycerol & & & & {$[56-81-5]$} \\
\hline & & $91.7 \pm 0.9$ & $(298)$ & $\mathrm{C}$ & {$[88 / 10]$} \\
\hline & $(469-563)$ & 78.5 & $(484)$ & A & {$[87 / 5]$} \\
\hline & $(291-341)$ & 86.8 & $(316)$ & ME & {$[77 / 11]$} \\
\hline & & 67.5 & $(343)$ & GC & {$[77 / 34]$} \\
\hline & & 66.8 & $(353)$ & $\mathrm{GC}$ & {$[77 / 34]$} \\
\hline & & 66.2 & $(363)$ & GC & {$[77 / 34]$} \\
\hline & & 65.5 & $(373)$ & $\mathrm{GC}$ & {$[77 / 34]$} \\
\hline & & 64.8 & $(383)$ & GC & [77/34] \\
\hline & $(293-343)$ & 85.8 & $(308)$ & $\mathrm{ME}$ & $\begin{array}{c}{[87 / 5][62 / 18]} \\
{[70 / 16]}\end{array}$ \\
\hline & $(456-553)$ & 86.0 & $(471)$ & & {$[1886 / 1]$} \\
\hline \multirow[t]{7}{*}{$\mathrm{C}_{3} \mathrm{H}_{8} \mathrm{~S}$} & ethyl methyl sulfide & & & & {$[624-89-5]$} \\
\hline & $(253-363)$ & 33.7 & $(268)$ & & [99/16] \\
\hline & & 31.5 & $(298)$ & & {$[81 / 12]$} \\
\hline & $(296-373)$ & 31.8 & $(311)$ & $\mathrm{A}, \mathrm{EB}$ & {$[87 / 5][51 / 4]$} \\
\hline & & 31.8 & $(298)$ & & {$[71 / 28]$} \\
\hline & & & & & {$[66 / 5][54 / 11]$} \\
\hline & & 30.3 & $(338)$ & & {$[35 / 2]$} \\
\hline \multirow[t]{9}{*}{$\mathrm{C}_{3} \mathrm{H}_{8} \mathrm{~S}$} & 1-propanethiol & & & & {$[107-03-9]$} \\
\hline & $(254-364)$ & 33.7 & $(269)$ & & [99/16] \\
\hline & & 31.9 & $(298)$ & & {$[71 / 28]$} \\
\hline & $(297-375)$ & 31.8 & $(312)$ & $\mathrm{A}, \mathrm{EB}$ & {$[87 / 5][56 / 7]$} \\
\hline & & & & & {$[66 / 5][54 / 11]$} \\
\hline & & $31.6 \pm 0.1$ & $(303)$ & $\mathrm{C}$ & {$[56 / 7]$} \\
\hline & & $30.7 \pm 0.1$ & $(320)$ & $\mathrm{C}$ & {$[56 / 7]$} \\
\hline & & $29.5 \pm 0.1$ & $(341)$ & $\mathrm{C}$ & {$[56 / 7]$} \\
\hline & $(284-340)$ & 31.5 & $(312)$ & & {$[33 / 7]$} \\
\hline \multirow[t]{2}{*}{$\mathrm{C}_{3} \mathrm{H}_{8} \mathrm{~S}$} & 2-propanethiol & & & & {$[75-33-2]$} \\
\hline & $(242-348)$ & 31.9 & $(257)$ & & {$[99 / 16]$} \\
\hline
\end{tabular}


TABLE 6. Enthalpies of vaporization of organic compounds, 1880-2002-Continued

\begin{tabular}{|c|c|c|c|c|c|}
\hline Molecular formula & $\begin{array}{c}\text { Compound } \\
\text { (Temperature range/K) }\end{array}$ & $\begin{array}{c}\Delta_{\mathrm{vap}} H_{m} \\
\left(\mathrm{~kJ} \mathrm{~mol}^{-1}\right)\end{array}$ & $\begin{array}{l}\text { Mean temperature } \\
\qquad\left(T_{m} / \mathrm{K}\right)\end{array}$ & Method & $\begin{array}{c}\text { CAS registry number } \\
\text { Reference }\end{array}$ \\
\hline & & 29.5 & $(298)$ & & {$[71 / 28]$} \\
\hline & $(283-358)$ & 30.1 & $(298)$ & $\mathrm{A}, \mathrm{EB}$ & {$[87 / 5][54 / 2]$} \\
\hline & & & & & {$[66 / 5][54 / 11]$} \\
\hline \multirow[t]{4}{*}{$\mathrm{C}_{3} \mathrm{H}_{8} \mathrm{~S}_{2}$} & 1,3-propanedithiol & & & & {$[109-80-8]$} \\
\hline & $(338-446)$ & 50.9 & $(353)$ & & {$[99 / 16]$} \\
\hline & $(377-446)$ & 41.6 & (398) & A & {$[87 / 5]$} \\
\hline & & 49.7 & $(298)$ & & {$[62 / 11]$} \\
\hline \multirow[t]{5}{*}{$\mathrm{C}_{3} \mathrm{H}_{9} \mathrm{~N}$} & isopropylamine & & & & {$[75-31-0]$} \\
\hline & & 28.4 & $(298)$ & & {$[79 / 9]$} \\
\hline & & 27.2 & (313) & & {$[79 / 9]$} \\
\hline & $(277-334)$ & 29.7 & $(292)$ & A, EB, IPM & {$[87 / 5][68 / 4]$} \\
\hline & & & & & {$[70 / 16]$} \\
\hline \multirow[t]{6}{*}{$\mathrm{C}_{3} \mathrm{H}_{9} \mathrm{~N}$} & propylamine & & & & {$[107-10-8]$} \\
\hline & & 31.3 & $(298)$ & & {$[79 / 9]$} \\
\hline & & 30.1 & $(313)$ & & {$[79 / 9]$} \\
\hline & & 28.9 & $(328)$ & & {$[79 / 9]$} \\
\hline & $(296-350)$ & 31.3 & $(311)$ & $\mathrm{A}, \mathrm{EB}, \mathrm{IPM}$ & {$[87 / 5][68 / 4]$} \\
\hline & & & & & {$[70 / 16]$} \\
\hline \multirow[t]{5}{*}{$\mathrm{C}_{3} \mathrm{H}_{9} \mathrm{~N}$} & trimethylamine & & & & {$[75-50-3]$} \\
\hline & $(333-403)$ & 23.0 & $(368)$ & & {$[50 / 9]$} \\
\hline & $(273-313)$ & 24.1 & $(288)$ & & {$[45 / 1]$} \\
\hline & $(193-276)$ & 24.6 & $(261)$ & A & {$[87 / 5][44 / 6]$} \\
\hline & & 24.5 & $(250)$ & $\mathrm{C}$ & {$[44 / 6]$} \\
\hline \multirow[t]{2}{*}{$\mathrm{C}_{3} \mathrm{H}_{9} \mathrm{NO}$} & 2-(methylamino)ethanol & & & & {$[109-83-1]$} \\
\hline & $(340-461)$ & $57.0 \pm 0.5$ & $(298)$ & $\mathrm{EB}$ & {$[97 / 8]$} \\
\hline \multirow[t]{2}{*}{$\mathrm{C}_{3} \mathrm{H}_{9} \mathrm{NO}$} & 1-amino-2-propanol & & & & {$[78-96-6]$} \\
\hline & $(306-431)$ & 51.6 & $(321)$ & A & {$[87 / 5]$} \\
\hline \multirow[t]{2}{*}{$\mathrm{C}_{3} \mathrm{H}_{9} \mathrm{NO}$} & N-methoxy dimethyl amin & ylhydroxylam & & & {$[5669-39-6]$} \\
\hline & $(194-297)$ & 28.0 & $(282)$ & $\mathrm{A}$ & {$[87 / 5][57 / 4]$} \\
\hline \multirow[t]{2}{*}{$\mathrm{C}_{3} \mathrm{H}_{9} \mathrm{NO}$} & 2-methoxyethyl amine & & & & [109-85-3] \\
\hline & $(278-318)$ & 38.8 & $(293)$ & A & {$[87 / 5]$} \\
\hline \multirow[t]{2}{*}{$\mathrm{C}_{3} \mathrm{H}_{9} \mathrm{O}_{3} \mathrm{P}$} & methylphosphonic acid, di & & & & {$[756-79-6]$} \\
\hline & $(336-408)$ & 64.0 & $(351)$ & A & {$[87 / 5][55 / 5]$} \\
\hline \multirow[t]{3}{*}{$\mathrm{C}_{3} \mathrm{H}_{9} \mathrm{O}_{3} \mathrm{P}$} & trimethyl phosphite & & & & {$[121-45-9]$} \\
\hline & $(302-342)$ & 42.5 & $(317)$ & EB & {$[90 / 9]$} \\
\hline & $(422-494)$ & 32.8 & $(437)$ & A & {$[87 / 5]$} \\
\hline \multirow[t]{2}{*}{$\mathrm{C}_{3} \mathrm{H}_{9} \mathrm{O}_{4} \mathrm{P}$} & trimethyl phosphate & & & & {$[512-56-1]$} \\
\hline & $(296-466)$ & 48.8 & $(311)$ & A & {$[87 / 5][47 / 5]$} \\
\hline \multirow[t]{3}{*}{$\mathrm{C}_{3} \mathrm{H}_{9} \mathrm{P}$} & trimethyl phosphine & & & & [594-09-2] \\
\hline & $(248-310)$ & 28.9 & $(263)$ & A & {$[87 / 5]$} \\
\hline & & $28.0 \pm 2.1$ & & & {$[57 / 23][62 / 15]$} \\
\hline \multirow[t]{2}{*}{$\mathrm{C}_{3} \mathrm{H}_{10} \mathrm{~N}_{2}$} & 1,3-diaminopropane & & & & [109-76-2] \\
\hline & & $50.2 \pm 0.1$ & $(298)$ & $\mathrm{C}$ & {$[69 / 2]$} \\
\hline \multirow[t]{5}{*}{$\mathrm{C}_{3} \mathrm{H}_{10} \mathrm{~N}_{2}$} & ( $d l$ ) 1,2-propanediamine & & & & {$[78-90-0]$} \\
\hline & $(293-393)$ & 42.2 & $(308)$ & A & {$[87 / 5]$} \\
\hline & $(242-293)$ & 47.2 & $(278)$ & A, IPM & {$[87 / 5][75 / 4]$} \\
\hline & $(242-293)$ & $43.9 \pm 0.2$ & $(298)$ & IPM & {$[75 / 4]$} \\
\hline & & $44.2 \pm 0.2$ & $(298)$ & IPM & {$[65 / 8][70 / 11]$} \\
\hline $\mathrm{C}_{3} \mathrm{H}_{10} \mathrm{~N}_{2}$ & trimethylhydrazine & & & & {$[1741-01-1]$} \\
\hline & $(257-287)$ & 34.6 & $(272)$ & & {$[55 / 6]$} \\
\hline & & $33.4 \pm 0.1$ & $(292)$ & $\mathrm{C}$ & {$[55 / 6]$} \\
\hline $\mathrm{C}_{3} \mathrm{~N}_{2} \mathrm{O}$ & carbonyl cyanide & & & & {$[1115-12-4]$} \\
\hline & $(250-291)$ & 37.5 & $(276)$ & A & {$[87 / 5][48 / 16]$} \\
\hline $\mathrm{C}_{3} \mathrm{O}_{2}$ & carbon suboxide & & & & [504-64-3] \\
\hline & $(161-249)$ & 26.2 & $(234)$ & A & {$[87 / 5][65 / 5]$} \\
\hline $\mathrm{C}_{3} \mathrm{~S}_{2}$ & carbon subsulfide & & & & [627-34-9] \\
\hline & $(287-383)$ & 45.1 & $(302)$ & A & {$[87 / 5]$} \\
\hline $\mathrm{C}_{4} \mathrm{BrClF}_{9} \mathrm{~N}$ & 1,1,2-trifluoro-2-chloro-2-1 & $\mathrm{J}-$ bis (trifluorc & ethylamine & & \\
\hline & $(329-364)$ & 33.1 & (344) & A & {$[87 / 5]$} \\
\hline $\mathrm{C}_{4} \mathrm{BrF}_{6} \mathrm{~N}$ & 2-bromo-N,N-bis(trifluoro & ynylamine & & & {$[22130-38-7]$} \\
\hline & $(311-329)$ & 30.4 & $(320)$ & A & {$[87 / 5]$} \\
\hline $\mathrm{C}_{4} \mathrm{BrF}_{8} \mathrm{~N}$ & $\mathrm{~N}, \mathrm{~N}-$ bis(trifluoromethyl)-2 & -1-bromovin & & & {$[17727-57-4]$} \\
\hline & $(293-320)$ & 31.2 & $(320)$ & A & {$[87 / 5]$} \\
\hline $\mathrm{C}_{4} \mathrm{BrF}_{9} \mathrm{O}_{3} \mathrm{~S}$ & 2-bromo-2-fluorosulfatooc & tane & & & \\
\hline
\end{tabular}


TABLE 6. Enthalpies of vaporization of organic compounds, 1880-2002-Continued

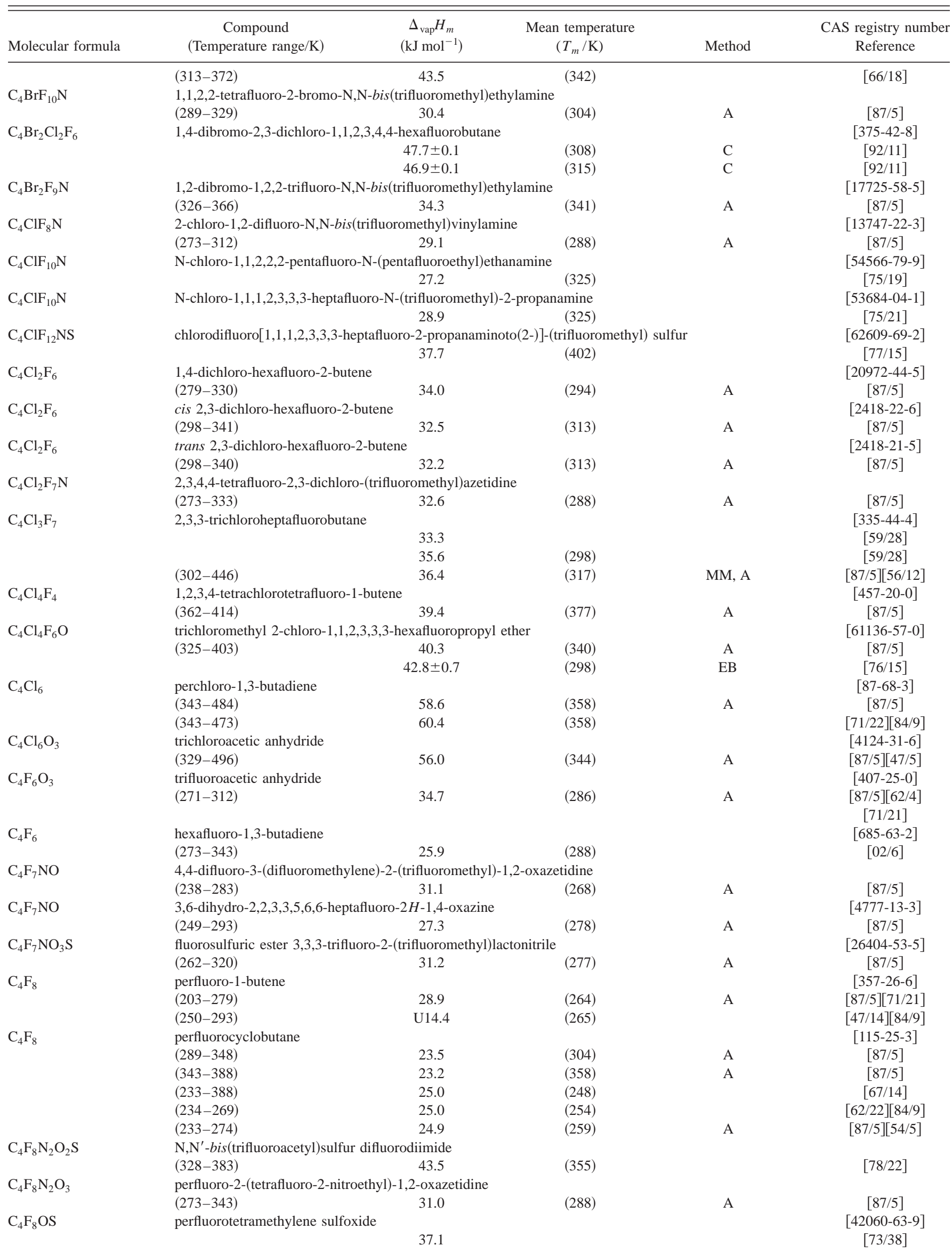


TABLE 6. Enthalpies of vaporization of organic compounds, 1880-2002-Continued

\begin{tabular}{|c|c|c|c|c|c|}
\hline Molecular formula & $\begin{array}{l}\text { Compound } \\
\text { (Temperature range/K) }\end{array}$ & $\begin{array}{c}\Delta_{\mathrm{vap}} H_{m} \\
\left(\mathrm{~kJ} \mathrm{~mol}^{-1}\right)\end{array}$ & $\begin{array}{l}\text { Mean temperature } \\
\qquad\left(T_{m} / \mathrm{K}\right)\end{array}$ & Method & $\begin{array}{l}\text { CAS registry number } \\
\text { Reference }\end{array}$ \\
\hline $\mathrm{C}_{4} \mathrm{~F}_{8} \mathrm{O}_{2} \mathrm{~S}$ & \multicolumn{4}{|c|}{ perfluorotetramethylene sulfone } & $\begin{array}{c}{[42060-64-0]} \\
{[73 / 38]}\end{array}$ \\
\hline $\mathrm{C}_{4} \mathrm{~F}_{8} \mathrm{O}_{4} \mathrm{~S}$ & \multicolumn{4}{|c|}{ heptafluorobutyric acid and fluorosulfuric acid anhydride } & $\begin{array}{c}{[6069-35-8]} \\
{[87 / 5][66 / 15]}\end{array}$ \\
\hline $\mathrm{C}_{4} \mathrm{~F}_{8} \mathrm{~S}$ & \multicolumn{4}{|c|}{ perfluorotetramethylene sulfide } & $\begin{array}{c}{[706-76-3]} \\
{[73 / 38]}\end{array}$ \\
\hline $\mathrm{C}_{4} \mathrm{~F}_{8} \mathrm{~S}_{2}$ & \multicolumn{4}{|l|}{ perfluoro-1,4-dithiane } & $\begin{array}{l}{[710-65-6]} \\
{[73 / 38]}\end{array}$ \\
\hline $\mathrm{C}_{4} \mathrm{~F}_{9} \mathrm{~N}$ & \multicolumn{4}{|c|}{ 1,1,1-trifluoro-N-[2,2,2-trifluoro-1-(trifluoromethyl)ethylidene]-methanamine } & $\begin{array}{l}{[453-22-5]} \\
{[75 / 21]}\end{array}$ \\
\hline $\mathrm{C}_{4} \mathrm{~F}_{9} \mathrm{~N}$ & \multicolumn{3}{|c|}{ perfluoro[N,N-dimethyl(vinylamine)] } & A & $\begin{array}{c}{[13821-49-3]} \\
{[87 / 5]}\end{array}$ \\
\hline $\mathrm{C}_{4} \mathrm{~F}_{9} \mathrm{~N}$ & $\begin{array}{l}\text { perfluoro[N-methyl(propy } \\
(245-280)\end{array}$ & 26.6 & $(265)$ & A & {$[87 / 5]$} \\
\hline $\mathrm{C}_{4} \mathrm{~F}_{9} \mathrm{~N}$ & \multicolumn{3}{|c|}{ perfluoro[N-propyl(methylenenamine)] } & A & {$[87 / 5]$} \\
\hline $\mathrm{C}_{4} \mathrm{~F}_{9} \mathrm{NO}$ & \multicolumn{4}{|l|}{ nonafluorobutyramide } & $\begin{array}{l}{[32822-51-8]} \\
{[71 / 18]}\end{array}$ \\
\hline $\mathrm{C}_{4} \mathrm{~F}_{9} \mathrm{NO}$ & \multicolumn{5}{|c|}{ 2,2,4,4,5,5-hexafluoro-3-(trifluoromethyl)oxazolidine } \\
\hline $\mathrm{C}_{4} \mathrm{~F}_{9} \mathrm{NO}$ & \multicolumn{5}{|c|}{ perfluoro[2,4-bis(trifluoromethyl)-1,2-oxazetidine] } \\
\hline $\mathrm{C}_{4} \mathrm{~F}_{9} \mathrm{NOS}$ & \multicolumn{3}{|c|}{ 1,1,1-trifluoro-N-[2,2,2-trifluoro-1-(trifluoromethyl)ethylidene]-methanesulfinamide } & I & $\begin{array}{c}{[31340-35-9]} \\
{[72 / 24]}\end{array}$ \\
\hline $\mathrm{C}_{4} \mathrm{~F}_{9} \mathrm{NO}_{2}$ & \multicolumn{3}{|c|}{ O-(trifluoroacetyl)-N,N-bis(trifluoromethyl)hydroxylamine } & A & $\begin{array}{c}{[15496-02-3]} \\
{[87 / 5]}\end{array}$ \\
\hline $\mathrm{C}_{4} \mathrm{~F}_{9} \mathrm{NO}_{2} \mathrm{~S}$ & \multicolumn{4}{|c|}{$\mathrm{N}$-(trifluoroacetyl)-S,S-bis(trifluoromethyl)sulfoximine } & $\begin{array}{c}{[34556-29-1]} \\
{[72 / 25]}\end{array}$ \\
\hline $\mathrm{C}_{4} \mathrm{~F}_{9} \mathrm{NS}$ & \multicolumn{4}{|c|}{ 1,1,1-trifluoro-N-[2,2,2-trifluoro-1-(trifluoromethyl)ethylidene]-methanesulfenamide } & $\begin{array}{c}{[31340-34-8]} \\
{[72 / 24]}\end{array}$ \\
\hline $\mathrm{C}_{4} \mathrm{~F}_{10}$ & $\begin{array}{l}\text { perfluorobutane } \\
(272-327) \\
(233-273) \\
(323-386) \\
(233-383) \\
(233-383) \\
(233-260)\end{array}$ & $\begin{array}{l}24.2 \\
24.2 \\
23.1 \\
21.0 \\
17.1 \\
25.8\end{array}$ & $\begin{array}{l}(287) \\
(258) \\
(338) \\
(293) \\
(333) \\
(247)\end{array}$ & $\begin{array}{l}\text { A } \\
\text { A } \\
\text { A }\end{array}$ & $\begin{array}{c}{[355-25-9]} \\
{[87 / 5]} \\
{[87 / 5]} \\
{[87 / 5]} \\
{[58 / 4]} \\
{[58 / 4]} \\
{[52 / 16][84 / 9]}\end{array}$ \\
\hline $\mathrm{C}_{4} \mathrm{~F}_{10} \mathrm{OS}$ & \multicolumn{4}{|c|}{ heptafluoropropyl trifluoromethyl sulfoxide } & $\begin{array}{c}{[33622-18-3]} \\
{[71 / 34]}\end{array}$ \\
\hline $\mathrm{C}_{4} \mathrm{~F}_{10} \mathrm{OS}$ & \multicolumn{4}{|c|}{ bis(pentafluoroethyl) sulfoxide } & $\begin{array}{c}{[33622-19-4]} \\
{[71 / 34]}\end{array}$ \\
\hline $\mathrm{C}_{4} \mathrm{~F}_{10} \mathrm{O}_{3} \mathrm{~S}$ & \multicolumn{4}{|c|}{ fluorosulfuric acid, perfluoro(1-methylpropyl) ester } & $\begin{array}{c}{[5762-52-7]} \\
{[87 / 5]}\end{array}$ \\
\hline $\mathrm{C}_{4} \mathrm{~F}_{10} \mathrm{O}_{6} \mathrm{~S}_{2}$ & $\begin{array}{l}1,1,1,2,3,4,4,4 \text {-octafluoro- } \\
(316-393) \\
(392-411)\end{array}$ & $\begin{array}{l}\text { orosulfato)but } \\
\quad 30.1 \\
27.1\end{array}$ & $\begin{array}{l}(331) \\
(401)\end{array}$ & $\begin{array}{l}\mathrm{A} \\
\mathrm{A}\end{array}$ & $\begin{array}{l}{[2261-44-1]} \\
{[87 / 5][64 / 22]} \\
{[87 / 5][64 / 22]}\end{array}$ \\
\hline $\mathrm{C}_{4} \mathrm{~F}_{10} \mathrm{~S}$ & \multicolumn{4}{|c|}{$\begin{array}{l}(392-411) \\
\text { perfluoroetramethylene sulfur difluoride }\end{array}$} & $\begin{array}{c}{[42069-60-6]} \\
{[73 / 38]}\end{array}$ \\
\hline $\mathrm{C}_{4} \mathrm{~F}_{10} \mathrm{~S}$ & \multicolumn{4}{|c|}{ heptafluoropropyl trifluoromethyl sulfide } & $\begin{array}{c}{[33547-11-4]} \\
{[71 / 34]}\end{array}$ \\
\hline $\mathrm{C}_{4} \mathrm{~F}_{11} \mathrm{NOS}$ & \multicolumn{4}{|c|}{$\begin{array}{c}27.7 \\
\text { difluoro(1,1,1,3,3,3-hexafluoro-2-propaniminato)oxo(trifluoromethyl) sulfur }\end{array}$} & $\begin{array}{c}{[62609-62-5]} \\
{[77 / 19]}\end{array}$ \\
\hline $\mathrm{C}_{4} \mathrm{~F}_{11} \mathrm{NS}$ & \multicolumn{4}{|c|}{ fluoro(trifluoromethyl)[2,2,2,1-tetrafluoro-1-(trifluoromethyl)ethyl]-imino sulfur } & $\begin{array}{l}{[37826-43-0]} \\
{[87 / 5][72 / 21]}\end{array}$ \\
\hline $\mathrm{C}_{4} \mathrm{~F}_{12} \mathrm{~N}_{2} \mathrm{O}$ & \multicolumn{5}{|c|}{ perfluoro(2,3-dimethyl)-4-oxo-diazepentane } \\
\hline $\mathrm{C}_{4} \mathrm{~F}_{12} \mathrm{~N}_{2} \mathrm{O}$ & $\begin{array}{l}\text { perfluoro(2,4-dimethyl)-4- } \\
(288-318)\end{array}$ & pentane & $(303)$ & A & {$[87 / 5][71 / 21]$} \\
\hline $\mathrm{C}_{4} \mathrm{~F}_{12} \mathrm{~N}_{2} \mathrm{~S}$ & \multicolumn{4}{|c|}{ difluorobis[1,1,2,2,2-pentafluorothananaminato] sulfur } & $\begin{array}{c}{[4101-59-1]} \\
{[76 / 29]}\end{array}$ \\
\hline $\mathrm{C}_{4} \mathrm{~F}_{12} \mathrm{OS}$ & \multicolumn{4}{|c|}{ difluorooxobis(pentafluoroethyl) sulfur } & $\begin{array}{c}{[33564-25-9]} \\
{[99 / 16]}\end{array}$ \\
\hline $\mathrm{C}_{4} \mathrm{~F}_{12} \mathrm{O}_{2} \mathrm{~S}$ & \multicolumn{2}{|c|}{$\begin{array}{l}(284-341) \\
\text { bis(trifluoromethyl) bis(trifluoromethoxy) sulfur }\end{array}$} & $(288)$ & A, I & $\begin{array}{l}{[63465-11-2]} \\
{[87 / 5][77 / 16]}\end{array}$ \\
\hline
\end{tabular}


TABLE 6. Enthalpies of vaporization of organic compounds, 1880-2002-Continued

\begin{tabular}{|c|c|c|c|c|c|}
\hline Molecular formula & $\begin{array}{c}\text { Compound } \\
\text { (Temperature range/K) }\end{array}$ & $\begin{array}{c}\Delta_{\mathrm{vap}} H_{m} \\
\left(\mathrm{~kJ} \mathrm{~mol}^{-1}\right)\end{array}$ & $\begin{array}{l}\text { Mean temperature } \\
\qquad\left(T_{m} / \mathrm{K}\right)\end{array}$ & Method & $\begin{array}{c}\text { CAS registry number } \\
\text { Reference }\end{array}$ \\
\hline & & & & & {$[78 / 14]$} \\
\hline \multirow[t]{2}{*}{$\mathrm{C}_{4} \mathrm{~F}_{12} \mathrm{O}_{3} \mathrm{~S}$} & oxobis(trifluoromethyl)bis & ethoxy) sulfur & & & {$[66632-46-0]$} \\
\hline & $(273-335)$ & 33.4 & $(288)$ & $\mathrm{A}, \mathrm{I}$ & {$[87 / 5][78 / 14]$} \\
\hline \multirow[t]{2}{*}{$\mathrm{C}_{4} \mathrm{~F}_{12} \mathrm{P}_{2} \mathrm{~S}$} & di[bis(trifluoromethyl)phos & fide & & & \\
\hline & $(273-335)$ & 42.2 & $(304)$ & $\mathrm{T}$ & {$[64 / 31]$} \\
\hline \multirow[t]{2}{*}{$\mathrm{C}_{4} \mathrm{~F}_{12} \mathrm{P}_{4}$} & 1,2,3,4-tetrakis(trifluorome & hosphetane & & & [393-02-2] \\
\hline & $(313-375)$ & 43.2 & $(328)$ & A, SG & {$[87 / 5][58 / 10]$} \\
\hline \multirow[t]{3}{*}{$\mathrm{C}_{4} \mathrm{~F}_{12} \mathrm{~S}$} & difluorobis (pentafluoroethy & & & & {$[33622-15-0]$} \\
\hline & $(284-341)$ & 34.0 & (299) & A & {$[87 / 5]$} \\
\hline & & 32.2 & & & {$[71 / 34]$} \\
\hline \multirow{2}{*}{$\mathrm{C}_{4} \mathrm{~F}_{12} \mathrm{~S}$} & difluoro(heptafluoropropyl & methyl) sulfur & & & [31206-31-2] \\
\hline & & 32.8 & & & {$[71 / 34]$} \\
\hline \multirow[t]{2}{*}{$\mathrm{C}_{4} \mathrm{~F}_{13} \mathrm{NOS}$} & trifluoro[1,1,1,2,3,3,3-hept & ropanaminoat & (trifluoromethanolato & & [65844-09-9] \\
\hline & & 33.9 & $(389)$ & $\mathrm{I}$ & {$[78 / 14]$} \\
\hline \multirow[t]{2}{*}{$\mathrm{C}_{4} \mathrm{~F}_{15} \mathrm{~N}_{2} \mathrm{O}_{2} \mathrm{P}$} & phosphorous bis [bis(trifluo & nitroxide] diflu & & & \\
\hline & $(303-370)$ & 37.6 & $(336)$ & & {$[73 / 24]$} \\
\hline \multirow[t]{2}{*}{$\mathrm{C}_{4} \mathrm{~F}_{16} \mathrm{~S}_{2}$} & hexadecafluoro-octahydro- & & & & [4556-31-4] \\
\hline & $(323-408)$ & 40.5 & $(365)$ & & {$[99 / 16][73 / 38]$} \\
\hline \multirow[t]{2}{*}{$\mathrm{C}_{4} \mathrm{~N}_{4}$} & dicyanoacetylene & & & & [1071-98-3] \\
\hline & $(295-350)$ & 27.3 & $(310)$ & A & {$[87 / 5]$} \\
\hline \multirow[t]{2}{*}{$\mathrm{C}_{4} \mathrm{HBrF}_{7} \mathrm{~N}$} & 1-bromo-2-fluoro-N,N-bis & ethyl)vinylami & & & [25273-49-8] \\
\hline & $(321-342)$ & 29.8 & $(331)$ & A & {$[87 / 5]$} \\
\hline \multirow{2}{*}{$\mathrm{C}_{4} \mathrm{HBrF}_{9} \mathrm{~N}$} & 2-bromo-1,1,2-trifluoro-N, & loromethyl)eth & & & \\
\hline & $(308-342)$ & 31.9 & $(323)$ & A & {$[87 / 5]$} \\
\hline \multirow[t]{2}{*}{$\mathrm{C}_{4} \mathrm{HBrF}_{9} \mathrm{~N}$} & 2-bromo-1,2,2-trifluoro-N, & loromethyl)eth & & & [4905-96-8] \\
\hline & $(301-332)$ & 33.8 & $(316)$ & A & {$[87 / 5]$} \\
\hline \multirow[t]{2}{*}{$\mathrm{C}_{4} \mathrm{HBr}_{2} \mathrm{~F}_{6} \mathrm{~N}$} & trans 1,2-dibromo-N,N-bis & nethyl)vinylam & & & \\
\hline & $(355-382)$ & 33.4 & $(369)$ & A & {$[87 / 5]$} \\
\hline \multirow[t]{2}{*}{$\mathrm{C}_{4} \mathrm{HCl}_{2} \mathrm{~F}_{5} \mathrm{O}_{2}$} & 3,4-dichloro-2,2,3,4,4-pent & yric acid & & & {$[375-07-5]$} \\
\hline & $(373-456)$ & 54.8 & $(388)$ & A & {$[87 / 5][57 / 17]$} \\
\hline $\mathrm{C}_{4} \mathrm{HF}_{5}$ & 3,3,4,4,4-pentafluoro-1-but & & & & {$[7096-51-7]$} \\
\hline & $(203-261)$ & 23.6 & $(246)$ & A & {$[87 / 5]$} \\
\hline $\mathrm{C}_{4} \mathrm{HF}_{6} \mathrm{~N}$ & $\mathrm{~N}, \mathrm{~N}-$ bis(trifluoromethyl)et & & & & {$[13747-21-2]$} \\
\hline & $(229-271)$ & 26.0 & $(256)$ & A & {$[87 / 5]$} \\
\hline $\mathrm{C}_{4} \mathrm{HF}_{7} \mathrm{O}_{2}$ & perfluorobutyric acid & & & & {$[375-22-4]$} \\
\hline & $(310-426)$ & $50.1 \pm 0.2$ & $(320)$ & $\mathrm{EB}$ & {$[02 / 21]$} \\
\hline & $(310-426)$ & $45.9 \pm 0.2$ & $(360)$ & $\mathrm{EB}$ & {$[02 / 21]$} \\
\hline & $(310-426)$ & $41.0 \pm 0.5$ & $(400)$ & EB & {$[02 / 21]$} \\
\hline & $(329-493)$ & 47.8 & $(344)$ & A & {$[87 / 5]$} \\
\hline & $(353-393)$ & 47.3 & $(368)$ & A & {$[87 / 5]$} \\
\hline $\mathrm{C}_{4} \mathrm{HF}_{8} \mathrm{~N}$ & $\mathrm{~N}, \mathrm{~N}-$ bis (trifluoromethyl)-1 & vinylamine & & & [13747-24-5] \\
\hline & $(276-296)$ & 28.8 & $(286)$ & A & {$[87 / 5]$} \\
\hline $\mathrm{C}_{4} \mathrm{HF}_{8} \mathrm{~N}$ & $\mathrm{~N}, \mathrm{~N}$-bis(trifluoromethyl)-2 & vinylamine & & & {$[13747-23-4]$} \\
\hline & $(274-291)$ & 27.7 & $(282)$ & A & {$[87 / 5]$} \\
\hline $\mathrm{C}_{4} \mathrm{HF}_{8} \mathrm{NO}$ & $2,2,3,3,5,5,6,6$-octafluorom & & & & {$[375-17-7]$} \\
\hline & $(273-323)$ & 32.7 & $(288)$ & A & {$[87 / 5]$} \\
\hline $\mathrm{C}_{4} \mathrm{HF}_{8} \mathrm{NOS}$ & $2,2,3,3,4,4,5,5$-octafluoro- 1 & hexahydro-1-i & iophene-1-oxide & & {$[77589-47-0]$} \\
\hline & & 28.0 & $(397)$ & & {$[81 / 15]$} \\
\hline $\mathrm{C}_{4} \mathrm{HF}_{9} \mathrm{~N}_{2} \mathrm{OS}$ & 1,1,1-trifluoro-N-[2,2,2-trif & ifluoromethyl) & dene]-methanesulfoni & & [62609-65-8] \\
\hline & & 37.2 & $(388)$ & I & {$[77 / 19]$} \\
\hline $\mathrm{C}_{4} \mathrm{HF}_{9} \mathrm{O}_{2} \mathrm{~S}$ & trifluoromethanesulfinic ac & rifluoro-1-(trifl & ethyl)ethyl ester & & {$[52225-50-0]$} \\
\hline & & 39.3 & $(362)$ & $\mathrm{HG}$ & {$[74 / 25]$} \\
\hline $\mathrm{C}_{4} \mathrm{HF}_{10} \mathrm{~N}$ & $1,1,1,2,3,3,3$-heptafluoro-N & oroethyl)ethan & & & {$[54566-81-3]$} \\
\hline & & 26.4 & $(306)$ & & {$[75 / 19]$} \\
\hline $\mathrm{C}_{4} \mathrm{HF}_{10} \mathrm{~N}$ & $1,1,1,2,3,3,3$-heptafluoro-N & methyl)-2-prol & & & [53684-05-2] \\
\hline & & 28.1 & $(309)$ & & {$[75 / 21]$} \\
\hline $\mathrm{C}_{4} \mathrm{HF}_{10} \mathrm{NOS}$ & S,S-bis(pentafluoroethyl)su & & & & [34556-24-6] \\
\hline & & 35.6 & $(366)$ & I & {$[72 / 25]$} \\
\hline $\mathrm{C}_{4} \mathrm{H}_{2}$ & 1,3-butadiyne & & & & [460-12-8] \\
\hline & $(237-283)$ & 26.1 & $(268)$ & A & {$[87 / 5][71 / 21]$} \\
\hline & $(191-282)$ & 26.4 & (267) & & {$[47 / 5]$} \\
\hline & $(188-234)$ & 33.4 & (219) & & {$[33 / 10][84 / 9]$} \\
\hline & $(195-273)$ & 25.4 & $(258)$ & & {$[26 / 3][84 / 9]$} \\
\hline $\mathrm{C}_{4} \mathrm{H}_{2} \mathrm{BrF}_{6} \mathrm{~N}$ & 1-bromo-N,N-bis(trifluoror & ethyl)vinylamine & & & {$[19451-87-7]$} \\
\hline
\end{tabular}


TABLE 6. Enthalpies of vaporization of organic compounds, 1880-2002-Continued

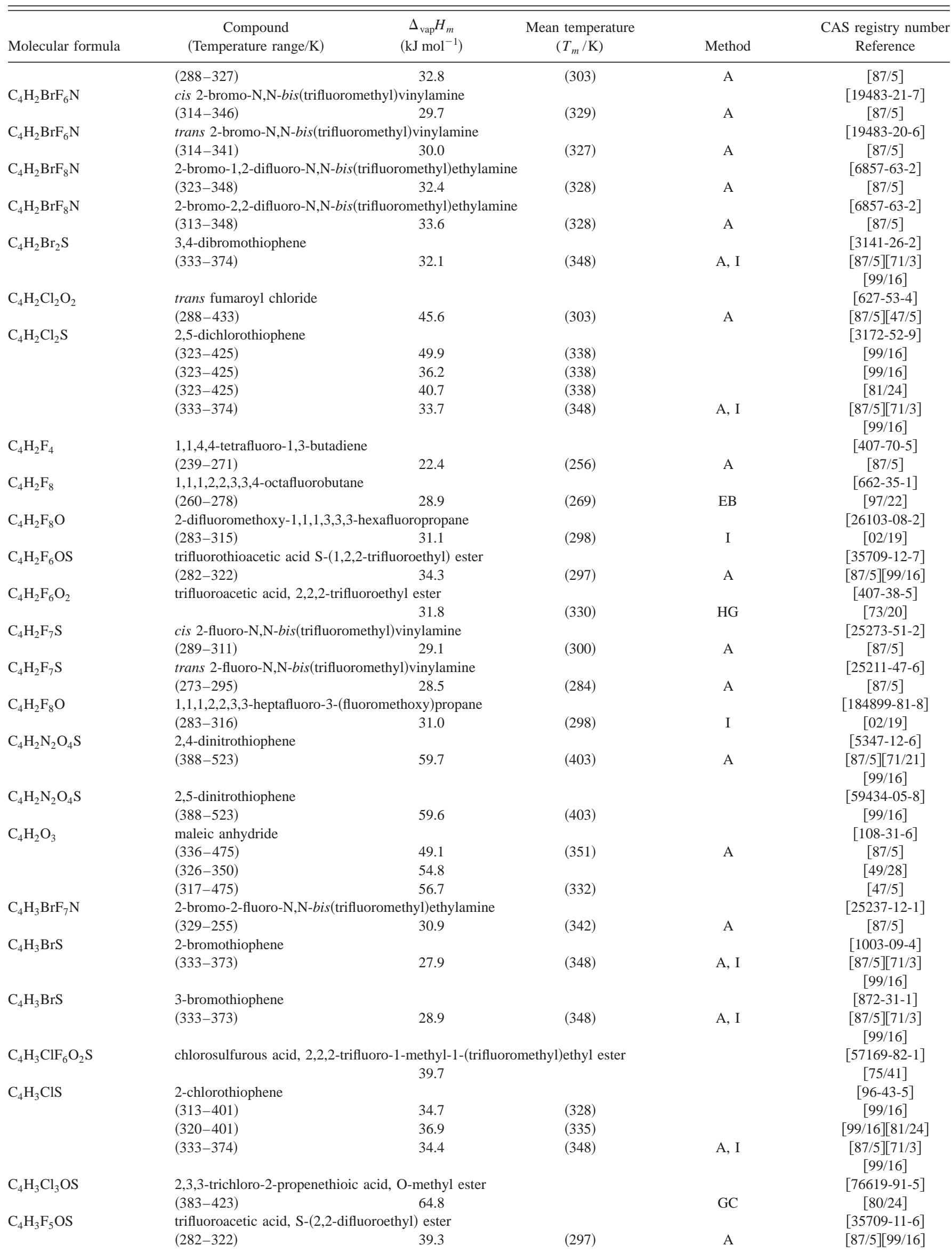


TABLE 6. Enthalpies of vaporization of organic compounds, 1880-2002-Continued

\begin{tabular}{|c|c|c|c|c|c|}
\hline Molecular formula & $\begin{array}{c}\text { Compound } \\
\text { (Temperature range/K) }\end{array}$ & $\begin{array}{c}\Delta_{\mathrm{vap}} H_{m} \\
\left(\mathrm{~kJ} \mathrm{~mol}^{-1}\right)\end{array}$ & $\begin{array}{l}\text { Mean temperature } \\
\qquad\left(T_{m} / \mathrm{K}\right)\end{array}$ & Method & $\begin{array}{l}\text { CAS registry number } \\
\text { Reference }\end{array}$ \\
\hline \multirow[t]{2}{*}{$\mathrm{C}_{4} \mathrm{H}_{3} \mathrm{~F}_{6} \mathrm{NO}_{2}$} & \multicolumn{4}{|c|}{ N,N-bis(trifluoromethyl)acetamide-N-oxide } & {$[22743-78-8]$} \\
\hline & $(268-336)$ & 40.6 & $(283)$ & A & {$[87 / 5]$} \\
\hline \multirow[t]{2}{*}{$\mathrm{C}_{4} \mathrm{H}_{3} \mathrm{~F}_{6} \mathrm{O}_{2} \mathrm{P}$} & \multicolumn{5}{|c|}{ bis(trifluoromethyl)acetoxyphosphine } \\
\hline & $(273-313)$ & 41.0 & $(288)$ & & {$[64 / 4][84 / 9]$} \\
\hline \multirow[t]{2}{*}{$\mathrm{C}_{4} \mathrm{H}_{3} \mathrm{~F}_{7} \mathrm{O}$} & \multicolumn{4}{|c|}{ 2,2,3,3,4,4,4-heptafluoro-1-butanol } & {$[375-01-9]$} \\
\hline & $(273-298)$ & 43.6 & $(286)$ & $\mathrm{A}, \mathrm{MM}$ & $\begin{array}{c}{[87 / 5][71 / 21]} \\
{[67 / 4]}\end{array}$ \\
\hline \multirow[t]{2}{*}{$\mathrm{C}_{4} \mathrm{H}_{3} \mathrm{~F}_{7} \mathrm{O}$} & \multicolumn{4}{|c|}{ 1-(2,2-difluoroethoxy)-1,1,2,2,2-pentafluoroethane } & [171182-95-9] \\
\hline & $(288-318)$ & 31.5 & $(303)$ & $\mathrm{I}$ & {$[02 / 19]$} \\
\hline \multirow[t]{2}{*}{$\mathrm{C}_{4} \mathrm{H}_{3} \mathrm{~F}_{7} \mathrm{O}$} & \multicolumn{4}{|c|}{ 1,1,2,2-tetrafluoro-1-(2,2,2-trifluoroethoxy)ethane } & {$[306-78-0]$} \\
\hline & $(283-329)$ & 34.0 & $(298)$ & $\mathrm{I}$ & {$[02 / 19]$} \\
\hline \multirow[t]{2}{*}{$\mathrm{C}_{4} \mathrm{H}_{3} \mathrm{~F}_{7} \mathrm{O}$} & \multicolumn{4}{|c|}{ 1,1,2,2-tetrafluoro-3-(trifluoromethoxy)propane } & {$[1683-81-4]$} \\
\hline & $(288-319)$ & 31.3 & $(303)$ & $\mathrm{I}$ & {$[02 / 19]$} \\
\hline \multirow[t]{2}{*}{$\mathrm{C}_{4} \mathrm{H}_{3} \mathrm{~F}_{7} \mathrm{O}$} & \multicolumn{4}{|c|}{ 1,1,1,3,3,3-hexafluoro-2-fluoromethoxypropane } & {$[28523-86-6]$} \\
\hline & $(288-331)$ & 34.1 & $(303)$ & $\mathrm{I}$ & {$[02 / 19]$} \\
\hline \multirow[t]{2}{*}{$\mathrm{C}_{4} \mathrm{H}_{3} \mathrm{~F}_{7} \mathrm{O}$} & \multicolumn{4}{|c|}{ 3-(difluoromethoxy)-1,1,1,2,2-pentafluoropropane } & {$[56860-81-2]$} \\
\hline & $(283-319)$ & 31.2 & $(298)$ & $\mathrm{I}$ & [02/19] \\
\hline $\mathrm{C}_{4} \mathrm{H}_{3} \mathrm{~F}_{7} \mathrm{O}_{2} \mathrm{~S}$ & fluorosulfurous acid, 2,2,2 & $\begin{array}{l}\text { 1-methyl-1-(tri } \\
36.4\end{array}$ & nethyl)ethyl ester & & $\begin{array}{c}{[57169-83-2]} \\
{[75 / 41]}\end{array}$ \\
\hline $\mathrm{C}_{4} \mathrm{H}_{3} \mathrm{IS}$ & 2-iodothiophene & & & & {$[3437-95-4]$} \\
\hline & $(333-374)$ & 29.0 & $(348)$ & $\mathrm{A}, \mathrm{I}$ & $\begin{array}{c}{[87 / 5][71 / 3]} \\
{[99 / 16]}\end{array}$ \\
\hline $\mathrm{C}_{4} \mathrm{H}_{3} \mathrm{NO}_{2} \mathrm{~S}$ & 2-nitrothiophene & & & & {$[609-40-5]$} \\
\hline & $(378-443)$ & 48.6 & (393) & & [99/16] \\
\hline & $(321-498)$ & 50.4 & $(336)$ & A & {$[87 / 5]$} \\
\hline $\mathrm{C}_{4} \mathrm{H}_{4}$ & 1-butene-3-yne & & & & [689-97-4] \\
\hline & $(180-278)$ & 26.0 & $(236)$ & A & {$[87 / 5][47 / 5]$} \\
\hline $\mathrm{C}_{4} \mathrm{H}_{4} \mathrm{BrF}_{6} \mathrm{~N}$ & 2-bromo-N,N-bis(trifluoro & ylamine & & & [1683-83-6] \\
\hline & $(323-356)$ & 31.0 & $(338)$ & A & {$[87 / 5]$} \\
\hline $\mathrm{C}_{4} \mathrm{H}_{4} \mathrm{Cl}_{2}$ & 1,2-dichloro-1,3-butadiene & & & & {$[3574-40-1]$} \\
\hline & $(260-308)$ & 33.3 & $(275)$ & A & {$[87 / 5]$} \\
\hline $\mathrm{C}_{4} \mathrm{H}_{4} \mathrm{Cl}_{2}$ & 2,3-dichloro-1,3-butadiene & & & & [1653-19-6] \\
\hline & $(299-368)$ & 33.8 & $(314)$ & A & {$[87 / 5]$} \\
\hline $\mathrm{C}_{4} \mathrm{H}_{4} \mathrm{Cl}_{2} \mathrm{O}_{2}$ & succinyl chloride & & & & {$[543-20-4]$} \\
\hline & $(312-466)$ & 54.7 & $(327)$ & A & {$[87 / 5][47 / 5]$} \\
\hline $\mathrm{C}_{4} \mathrm{H}_{4} \mathrm{Cl}_{2} \mathrm{O}_{3}$ & chloroacetic acid anhydrid & & & & {$[541-88-8]$} \\
\hline & $(340-490)$ & 61.8 & $(355)$ & A & {$[87 / 5][47 / 5]$} \\
\hline $\mathrm{C}_{4} \mathrm{H}_{4} \mathrm{Cl}_{4} \mathrm{O}_{2} \mathrm{~S}$ & 3,3,4,4-tetrachlorotetrahyd & e-1,1-dioxide & & & {$[3737-41-5]$} \\
\hline & $(303-348)$ & 88.6 & (318) & A & {$[87 / 5][99 / 16]$} \\
\hline $\mathrm{C}_{4} \mathrm{H}_{4} \mathrm{~F}_{4} \mathrm{OS}$ & trifluorothioacetic acid, S- & hyl) ester & & & [35709-10-5] \\
\hline & $(282-322)$ & 41.4 & $(297)$ & A & {$[87 / 5][99 / 16]$} \\
\hline $\mathrm{C}_{4} \mathrm{H}_{4} \mathrm{~F}_{6} \mathrm{~N}_{2} \mathrm{~S}$ & 2,2,2-trifluoro-N-methyl-N & omethyl)thio] & nidamide & & [62067-10-1] \\
\hline & $(339-387)$ & 34.9 & $(354)$ & $\mathrm{A}, \mathrm{I}$ & $\begin{array}{c}{[87 / 5][77 / 18]} \\
{[99 / 16]}\end{array}$ \\
\hline $\mathrm{C}_{4} \mathrm{H}_{4} \mathrm{~F}_{6} \mathrm{O}$ & 3-difluoromethoxy-1,1,2,2 & propane & & & {$[35042-99-0]$} \\
\hline & $(283-349)$ & 35.9 & $(298)$ & $\mathrm{I}$ & [02/19] \\
\hline $\mathrm{C}_{4} \mathrm{H}_{4} \mathrm{~F}_{6} \mathrm{O}$ & 1,1,1,3,3,3-hexafluoro-2-m & pane & & & {$[13171-18-1]$} \\
\hline & $(283-324)$ & 32.6 & $(298)$ & I & {$[02 / 19]$} \\
\hline $\mathrm{C}_{4} \mathrm{H}_{4} \mathrm{~F}_{6} \mathrm{O}$ & 1,1,1-trifluoro-2-(1,1,2-trif & $y)$ ethane & & & [25449-61-0] \\
\hline & $(283-338)$ & 35.4 & $(298)$ & $\mathrm{I}$ & {$[02 / 19]$} \\
\hline $\mathrm{C}_{4} \mathrm{H}_{4} \mathrm{~F}_{6} \mathrm{O}$ & 1,1,1-trifluoro-2-(2,2,2-trif & y)ethane & & & {$[333-36-8]$} \\
\hline & $(283-337)$ & 35.0 & $(298)$ & $\mathrm{I}$ & {$[02 / 19]$} \\
\hline $\mathrm{C}_{4} \mathrm{H}_{4} \mathrm{~F}_{6} \mathrm{O}$ & 1-(1,1-difluoroethoxy)-1,1, & uoroethane & & & {$[50807-77-7]$} \\
\hline & $(288-352)$ & 38.1 & (303) & $\mathrm{I}$ & {$[02 / 19]$} \\
\hline $\mathrm{C}_{4} \mathrm{H}_{4} \mathrm{~F}_{6} \mathrm{O}$ & 1,1,1,2,3,3-hexafluoro-3-m & pane & & & {$[382-34-3]$} \\
\hline & $(288-327)$ & 32.4 & $(303)$ & $\mathrm{I}$ & {$[02 / 19]$} \\
\hline $\mathrm{C}_{4} \mathrm{H}_{4} \mathrm{~F}_{6} \mathrm{O}$ & 1,1,2,2,3,3-hexafluoro-1-m & pane & & & [160620-20-2] \\
\hline & $(288-341)$ & 34.5 & $(303)$ & I & {$[02 / 19]$} \\
\hline $\mathrm{C}_{4} \mathrm{H}_{4} \mathrm{~F}_{6} \mathrm{O}_{2} \mathrm{~S}$ & trifluoromethanesulfinic ac & rifluoro-1-metl & 1 ester & & {$[52225-48-6]$} \\
\hline & & 36.8 & $(375)$ & $\mathrm{HG}$ & {$[74 / 25]$} \\
\hline $\mathrm{C}_{4} \mathrm{H}_{4} \mathrm{~N}_{2}$ & pyrazine & & & & {$[290-37-9]$} \\
\hline & $(354-426)$ & $36.5 \pm 0.2$ & $(380)$ & $\mathrm{EB}$ & [02/17] \\
\hline & $(354-426)$ & $34.1 \pm 0.4$ & $(420)$ & $\mathrm{EB}$ & {$[02 / 17]$} \\
\hline & $(332-373)$ & 37.9 & $(352)$ & & {$[95 / 4]$} \\
\hline
\end{tabular}


TABLE 6. Enthalpies of vaporization of organic compounds, 1880-2002-Continued

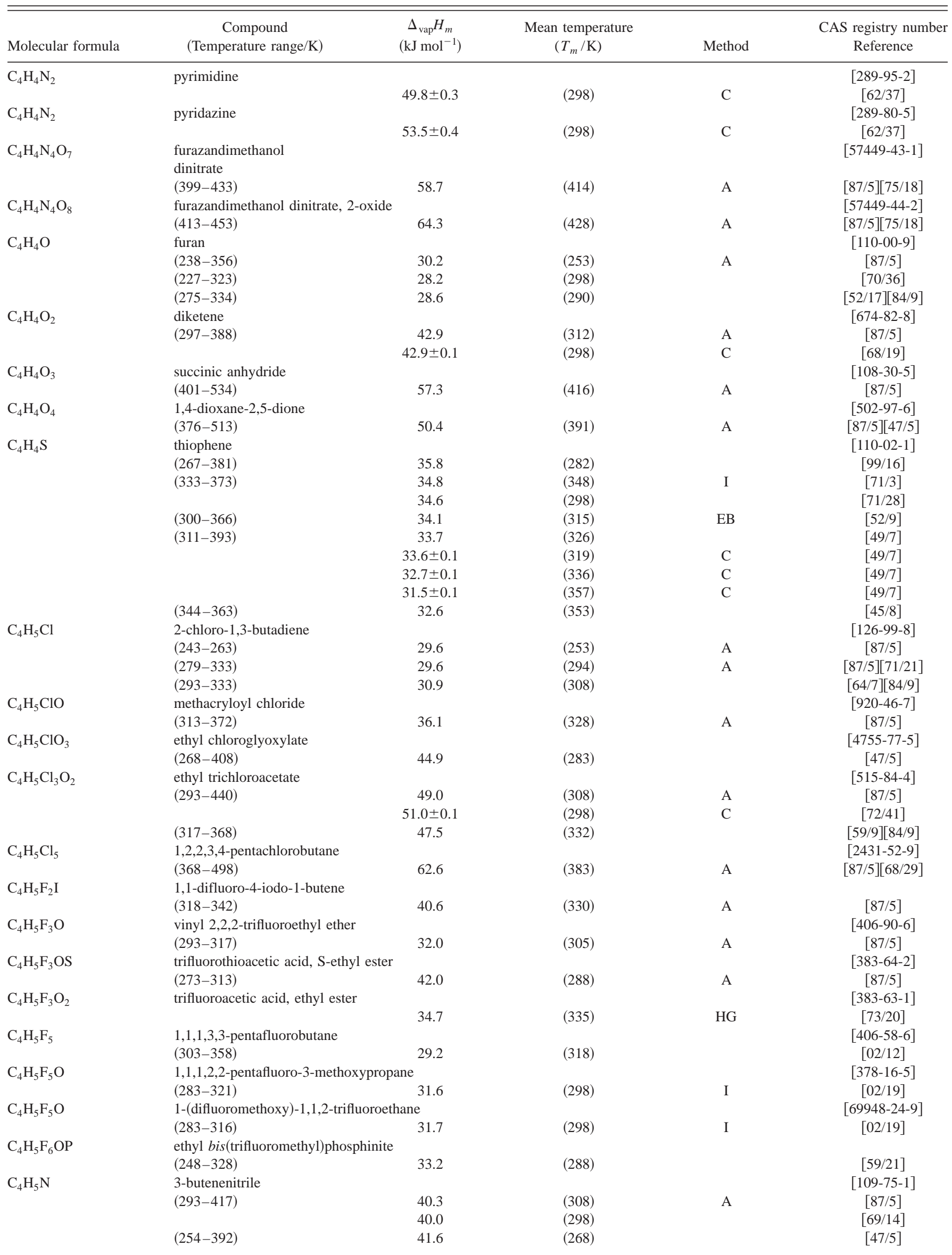


TABLE 6. Enthalpies of vaporization of organic compounds, 1880-2002-Continued

\begin{tabular}{|c|c|c|c|c|c|}
\hline Molecular formula & $\begin{array}{c}\text { Compound } \\
\text { (Temperature range/K) }\end{array}$ & $\begin{array}{c}\Delta_{\mathrm{vap}} H_{m} \\
\left(\mathrm{~kJ} \mathrm{~mol}^{-1}\right)\end{array}$ & $\begin{array}{l}\text { Mean temperature } \\
\qquad\left(T_{m} / \mathrm{K}\right)\end{array}$ & Method & $\begin{array}{c}\text { CAS registry number } \\
\text { Reference }\end{array}$ \\
\hline \multirow[t]{2}{*}{$\mathrm{C}_{4} \mathrm{H}_{5} \mathrm{~N}$} & (E) 2-butenenitrile & & & & [627-26-9] \\
\hline & & 40.0 & $(298)$ & & {$[69 / 14]$} \\
\hline \multirow[t]{2}{*}{$\mathrm{C}_{4} \mathrm{H}_{5} \mathrm{~N}$} & (Z) 2-butenenitrile & & & & [1190-76-7] \\
\hline & & 38.9 & $(298)$ & & {$[69 / 14]$} \\
\hline \multirow[t]{3}{*}{$\mathrm{C}_{4} \mathrm{H}_{5} \mathrm{~N}$} & cis crotonitrile & & & & [1190-76-7] \\
\hline & $(297-405)$ & 37.1 & $(312)$ & $\mathrm{A}$ & {$[87 / 5]$} \\
\hline & $(244-381)$ & 39.0 & (259) & & {$[47 / 5]$} \\
\hline \multirow[t]{3}{*}{$\mathrm{C}_{4} \mathrm{H}_{5} \mathrm{~N}$} & trans crotonitrile & & & & [627-26-7] \\
\hline & $(292-420)$ & 39.7 & (307) & A & {$[87 / 5]$} \\
\hline & $(254-395)$ & 40.5 & $(268)$ & & [47/5] \\
\hline \multirow[t]{3}{*}{$\mathrm{C}_{4} \mathrm{H}_{5} \mathrm{~N}$} & methacrylonitrile & & & & [126-98-7] \\
\hline & $(273-373)$ & 36.5 & $(288)$ & $\mathrm{A}$ & {$[87 / 5]$} \\
\hline & $(229-363)$ & 35.4 & (243) & & {$[47 / 5]$} \\
\hline \multirow[t]{4}{*}{$\mathrm{C}_{4} \mathrm{H}_{5} \mathrm{~N}$} & cyclopropylcyanide & & & & [5500-21-0] \\
\hline & & $41.9 \pm 0.1$ & (298) & $\mathrm{C}$ & {$[82 / 5]$} \\
\hline & $(310-391)$ & 39.4 & $(325)$ & BG & {$[71 / 2]$} \\
\hline & $(310-391)$ & $39.8 \pm 0.4$ & (298) & BG & {$[71 / 2]$} \\
\hline \multirow[t]{6}{*}{$\mathrm{C}_{4} \mathrm{H}_{5} \mathrm{~N}$} & pyrrole & & & & [109-97-7] \\
\hline & $(285-329)$ & 42.5 & $(300)$ & & [92/18] \\
\hline & $(313-373)$ & 41.9 & (328) & I & {$[71 / 3]$} \\
\hline & $(338-440)$ & 42.5 & (353) & $\mathrm{A}, \mathrm{EB}, \mathrm{IPM}$ & $\begin{array}{c}{[87 / 5][68 / 4]} \\
{[67 / 10]}\end{array}$ \\
\hline & & 41.8 & & & {$[61 / 26]$} \\
\hline & $(333-373)$ & 41.9 & $(348)$ & & {$[47 / 5]$} \\
\hline \multirow[t]{2}{*}{$\mathrm{C}_{4} \mathrm{DH}_{4} \mathrm{~N}$} & $\mathrm{~N}$-deutero pyrrole & & & & [10162-82-0] \\
\hline & $(285-329)$ & 42.9 & $(300)$ & & {$[92 / 18]$} \\
\hline \multirow[t]{2}{*}{$\mathrm{C}_{4} \mathrm{H}_{5} \mathrm{NO}$} & 3-methylisoxazole & & & & [30842-90-1] \\
\hline & & $39.8 \pm 0.2$ & $(298)$ & $\mathrm{C}$ & {$[78 / 9]$} \\
\hline \multirow[t]{2}{*}{$\mathrm{C}_{4} \mathrm{H}_{5} \mathrm{NO}$} & 5-methylisoxazole & & & & [5765-44-6] \\
\hline & & $39.7 \pm 0.2$ & $(298)$ & $\mathrm{C}$ & {$[78 / 9]$} \\
\hline \multirow[t]{3}{*}{$\mathrm{C}_{4} \mathrm{H}_{5} \mathrm{NO}_{2}$} & methyl cyanoacetate & & & & {$[105-34-0]$} \\
\hline & $(292-322)$ & $66.2 \pm 0.9$ & $(298)$ & GS & {$[95 / 11]$} \\
\hline & $(385-573)$ & 54.9 & $(400)$ & $\mathrm{A}$ & {$[87 / 5][71 / 21]$} \\
\hline \multirow[t]{3}{*}{$\mathrm{C}_{4} \mathrm{H}_{5} \mathrm{NO}_{2}$} & succinimide & & & & [123-56-8] \\
\hline & $(416-561)$ & 66.9 & $(431)$ & A & {$[87 / 5]$} \\
\hline & $(388-560)$ & 73.5 & $(403)$ & & {$[47 / 5]$} \\
\hline \multirow[t]{5}{*}{$\mathrm{C}_{4} \mathrm{H}_{5} \mathrm{NS}$} & allyl isothiocyanate & & & & {$[57-06-7]$} \\
\hline & $(277-323)$ & 47.6 & & GC & [97/27] \\
\hline & $(370-430)$ & 56.8 & $(385)$ & A & {$[87 / 5]$} \\
\hline & $(283-323)$ & 42.1 & (298) & & {$[35 / 3][84 / 9]$} \\
\hline & & & & & [99/16] \\
\hline \multirow[t]{3}{*}{$\mathrm{C}_{4} \mathrm{H}_{5} \mathrm{NS}$} & 2-methylthiazole & & & & [3581-87-1] \\
\hline & $(353-402)$ & 39.4 & $(368)$ & & {$[87 / 5][99 / 16]$} \\
\hline & $(342-404)$ & 40.0 & $(357)$ & A & {$[87 / 5][69 / 29]$} \\
\hline $\mathrm{C}_{4} \mathrm{H}_{5} \mathrm{NS}$ & 4-methylthiazole & & & & [693-95-8] \\
\hline & $(346-408)$ & 40.8 & $(361)$ & A & {$[87 / 5]$} \\
\hline & & $43.8 \pm 0.2$ & $(298)$ & $\mathrm{C}$ & {$[66 / 35]$} \\
\hline $\mathrm{C}_{4} \mathrm{H}_{5} \mathrm{~N}_{7} \mathrm{O}_{12}$ & 2,2,2-trinitro-N-(2,2,2-trin & thanamine & & & [34880-53-0] \\
\hline & $(337-349)$ & 80.8 & $(343)$ & $\mathrm{A}$ & {$[87 / 5]$} \\
\hline $\mathrm{C}_{4} \mathrm{H}_{6}$ & 1,2-butadiene & & & & [590-19-2] \\
\hline & $(243-291)$ & 25.3 & $(276)$ & $\mathrm{A}$ & {$[87 / 5]$} \\
\hline & $(204-243)$ & 26.4 & (228) & $\mathrm{A}$ & {$[87 / 5]$} \\
\hline & & 23.9 & (298) & & {$[71 / 28]$} \\
\hline & $(184-291)$ & 25.2 & (276) & & {$[47 / 5]$} \\
\hline & & $24.6 \pm 0.1$ & (273) & $\mathrm{C}$ & [47/13] \\
\hline $\mathrm{C}_{4} \mathrm{H}_{6}$ & 1,3-butadiene & & & & [106-99-0] \\
\hline & $(270-318)$ & 23.0 & $(285)$ & A & {$[87 / 5]$} \\
\hline & $(193-213)$ & 25.7 & (203) & A & {$[87 / 5]$} \\
\hline & $(213-276)$ & 23.6 & (261) & $\mathrm{A}$ & {$[87 / 5]$} \\
\hline & $(315-382)$ & 22.4 & $(330)$ & A & {$[87 / 5]$} \\
\hline & $(380-425)$ & 22.9 & (395) & A & {$[87 / 5]$} \\
\hline & & 21.1 & (298) & & [71/28] \\
\hline & $(198-271)$ & 23.7 & (256) & & {$[33 / 11][84 / 9]$} \\
\hline & $(191-249)$ & 24.7 & $(235)$ & & {$[32 / 1][84 / 9]$} \\
\hline
\end{tabular}


TABLE 6. Enthalpies of vaporization of organic compounds, 1880-2002-Continued

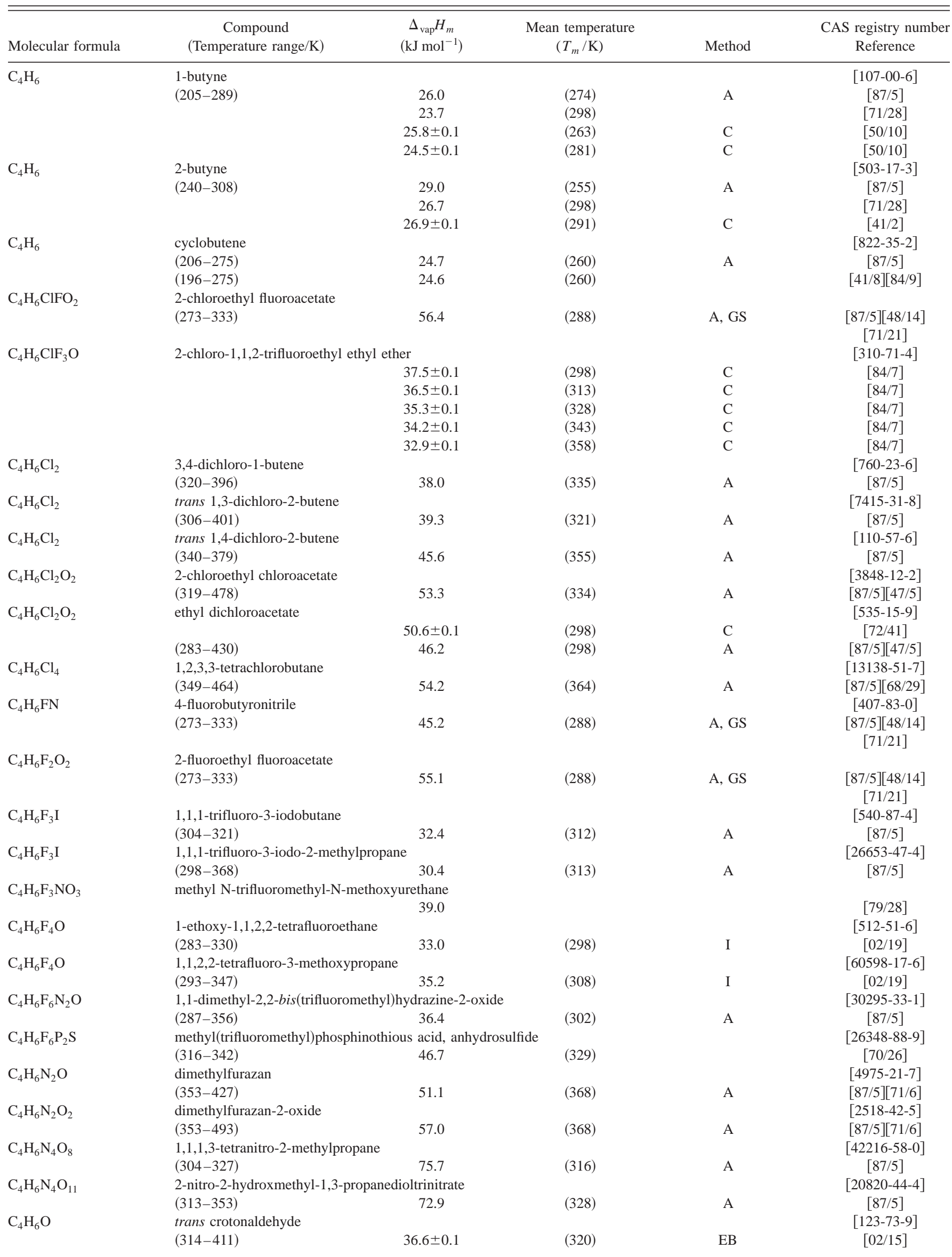


TABLE 6. Enthalpies of vaporization of organic compounds, 1880-2002-Continued

\begin{tabular}{|c|c|c|c|c|c|}
\hline Molecular formula & $\begin{array}{c}\text { Compound } \\
\text { (Temperature range/K) }\end{array}$ & $\begin{array}{c}\Delta_{\mathrm{vap}} H_{m} \\
\left(\mathrm{~kJ} \mathrm{~mol}^{-1}\right)\end{array}$ & $\begin{array}{l}\text { Mean temperature } \\
\qquad\left(T_{m} / \mathrm{K}\right)\end{array}$ & Method & $\begin{array}{c}\text { CAS registry number } \\
\text { Reference }\end{array}$ \\
\hline & $(314-411)$ & $34.5 \pm 0.2$ & $(360)$ & $\mathrm{EB}$ & {$[02 / 15]$} \\
\hline & $(314-411)$ & $32.1 \pm 0.5$ & $(400)$ & $\mathrm{EB}$ & {$[02 / 15]$} \\
\hline \multirow[t]{6}{*}{$\mathrm{C}_{4} \mathrm{H}_{6} \mathrm{O}$} & crotonaldehyde & & & & {$[4170-30-3]$} \\
\hline & & $37.3 \pm 0.4$ & $(298)$ & $\mathrm{C}$ & {$[96 / 8]$} \\
\hline & & 38.8 & $(325)$ & $\mathrm{EB}$ & [94/16] \\
\hline & $(288-376)$ & $35.1 \pm 0.5$ & (332) & & {$[88 / 4]$} \\
\hline & $(304-377)$ & 37.3 & $(319)$ & & {$[79 / 15]$} \\
\hline & $(306-376)$ & 36.8 & $(321)$ & A & $\begin{array}{c}{[87 / 5][73 / 23]} \\
{[84 / 9]}\end{array}$ \\
\hline \multirow[t]{5}{*}{$\mathrm{C}_{4} \mathrm{H}_{6} \mathrm{O}$} & cyclobutanone & & & & [1191-95-3] \\
\hline & $(301-344)$ & 37.7 & $(322)$ & $\mathrm{EB}$ & [94/16] \\
\hline & $(283-313)$ & 38.4 & $(298)$ & $\mathrm{A}$ & {$[87 / 5]$} \\
\hline & $(317-380)$ & 36.3 & $(332)$ & A, EB & {$[87 / 5][76 / 10]$} \\
\hline & $(249-298)$ & 38.5 & $(273)$ & & {$[42 / 7][84 / 9]$} \\
\hline \multirow[t]{3}{*}{$\mathrm{C}_{4} \mathrm{H}_{6} \mathrm{O}$} & divinyl ether & & & & [109-93-3] \\
\hline & $(253-323)$ & 29.2 & $(268)$ & A & {$[87 / 5]$} \\
\hline & $(253-323)$ & 26.1 & $(301)$ & $\mathrm{I}$ & {$[33 / 13]$} \\
\hline \multirow[t]{3}{*}{$\mathrm{C}_{4} \mathrm{H}_{6} \mathrm{O}$} & methyl vinyl ketone & & & & {$[78-94-4]$} \\
\hline & $(279-355)$ & 32.9 & $(294)$ & A & {$[87 / 5]$} \\
\hline & $(300-355)$ & 33.6 & $(315)$ & A & {$[87 / 5]$} \\
\hline \multirow[t]{3}{*}{$\mathrm{C}_{4} \mathrm{H}_{6} \mathrm{O}$} & 2,3-dihydrofuran & & & & [1191-99-7] \\
\hline & $(302-260)$ & $30.8 \pm 0.1$ & $(300)$ & $\mathrm{EB}$ & {$[02 / 21]$} \\
\hline & $(302-360)$ & $28.6 \pm 0.3$ & $(340)$ & $\mathrm{EB}$ & {$[02 / 21]$} \\
\hline \multirow[t]{2}{*}{$\mathrm{C}_{4} \mathrm{H}_{6} \mathrm{OS}$} & divinyl sulfoxide & & & & {$[1115-15-7]$} \\
\hline & & $51.2 \pm 0.9$ & $(298)$ & $\mathrm{C}$ & {$[89 / 12]$} \\
\hline \multirow[t]{5}{*}{$\mathrm{C}_{4} \mathrm{H}_{6} \mathrm{O}_{2}$} & cyclopropane carboxylic acid & & & & {$[1759-53-1]$} \\
\hline & $(357-473)$ & $58.9 \pm 0.3$ & $(340)$ & $\mathrm{EB}$ & {$[02 / 21]$} \\
\hline & $(357-473)$ & $55.7 \pm 0.2$ & $(380)$ & $\mathrm{EB}$ & {$[02 / 21]$} \\
\hline & $(357-473)$ & $52.4 \pm 0.2$ & $(420)$ & $\mathrm{EB}$ & {$[02 / 21]$} \\
\hline & $(357-473)$ & $48.8 \pm 0.4$ & $(460)$ & $\mathrm{EB}$ & {$[02 / 21]$} \\
\hline \multirow[t]{3}{*}{$\mathrm{C}_{4} \mathrm{H}_{6} \mathrm{O}_{2}$} & 2,3-butanedione (biacetyl) & & & & {$[431-03-8]$} \\
\hline & $(273-348)$ & 38.5 & $(288)$ & $\mathrm{A}, \mathrm{I}$ & {$[87 / 5][72 / 12]$} \\
\hline & $(273-293)$ & $39.6 \pm 0.2$ & $(283)$ & & {$[54 / 8]$} \\
\hline \multirow[t]{2}{*}{$\mathrm{C}_{4} \mathrm{H}_{6} \mathrm{O}_{2}$} & cis 2-butenoic acid & & & & {$[503-64-0]$} \\
\hline & $(306-445)$ & 55.8 & $(321)$ & A & {$[87 / 5][47 / 5]$} \\
\hline \multirow[t]{2}{*}{$\mathrm{C}_{4} \mathrm{H}_{6} \mathrm{O}_{2}$} & trans 2-butenoic acid & & & & {$[107-93-7]$} \\
\hline & $(353-458)$ & 56.7 & $(368)$ & A & {$[87 / 5][47 / 5]$} \\
\hline \multirow[t]{2}{*}{$\mathrm{C}_{4} \mathrm{H}_{6} \mathrm{O}_{2}$} & 2-butyne-1,4-diol & & & & {$[110-65-6]$} \\
\hline & $(418-520)$ & 69.0 & $(433)$ & A & $\begin{array}{c}{[87 / 5][66 / 14]} \\
{[71 / 21]}\end{array}$ \\
\hline \multirow[t]{7}{*}{$\mathrm{C}_{4} \mathrm{H}_{6} \mathrm{O}_{2}$} & $\gamma$-butyrolactone & & & & [96-48-0] \\
\hline & $(378-406)$ & $49.5 \pm 0.1$ & $(392)$ & $\mathrm{EB}$ & {$[91 / 7]$} \\
\hline & $(378-406)$ & $55.2 \pm 1.3$ & $(298)$ & EB & {$[91 / 7]$} \\
\hline & $(345-370)$ & $51.8 \pm 0.6$ & $(357)$ & MM & {$[91 / 7]$} \\
\hline & $(345-370)$ & $55.6 \pm 1.4$ & $(298)$ & $\mathrm{MM}$ & {$[91 / 7]$} \\
\hline & $(392-474)$ & 48.2 & $(407)$ & A & {$[87 / 5]$} \\
\hline & & $54.4 \pm 0.4$ & $(298)$ & $\mathrm{C}$ & {$[90 / 1]$} \\
\hline \multirow[t]{4}{*}{$\mathrm{C}_{4} \mathrm{H}_{6} \mathrm{O}_{2}$} & 2-methyl-2-propenoic acid & & & & {$[79-41-4]$} \\
\hline & & $47.5 \pm 0.4$ & $(298)$ & $\mathrm{C}$ & [96/8] \\
\hline & $(321-435)$ & 53.9 & $(336)$ & A & {$[87 / 5]$} \\
\hline & $(298-434)$ & 51.6 & (313) & A & {$[87 / 5][47 / 5]$} \\
\hline \multirow[t]{4}{*}{$\mathrm{C}_{4} \mathrm{H}_{6} \mathrm{O}_{2}$} & methyl acrylate & & & & [96-33-3] \\
\hline & $(316-354)$ & 34.2 & $(331)$ & $\mathrm{A}$ & {$[87 / 5]$} \\
\hline & $(299-337)$ & 28.8 & $(314)$ & $\mathrm{BG}$ & {$[71 / 2]$} \\
\hline & $(229-353)$ & 38.0 & $(244)$ & & {$[47 / 5]$} \\
\hline \multirow[t]{3}{*}{$\mathrm{C}_{4} \mathrm{H}_{6} \mathrm{O}_{2}$} & vinyl acetate & & & & [108-05-4] \\
\hline & $(294-346)$ & 34.4 & $(309)$ & A & {$[87 / 5][71 / 21]$} \\
\hline & & & & & {$[63 / 21][84 / 9]$} \\
\hline $\mathrm{C}_{4} \mathrm{H}_{6} \mathrm{O}_{2} \mathrm{~S}$ & diacetyl sulfide & & & & {$[3232-39-1]$} \\
\hline & $(325-355)$ & 54.2 & $(340)$ & & [99/16] \\
\hline & $(325-355)$ & 50.9 & $(340)$ & A & {$[87 / 5]$} \\
\hline $\mathrm{C}_{4} \mathrm{H}_{6} \mathrm{O}_{2} \mathrm{~S}$ & divinyl sulfone & & & & {$[77-77-0]$} \\
\hline & & $56.4 \pm 0.9$ & $(298)$ & $\mathrm{C}$ & {$[89 / 12]$} \\
\hline & & $56.5 \pm 0.8$ & $(298)$ & & {$[69 / 24]$} \\
\hline
\end{tabular}


TABLE 6. Enthalpies of vaporization of organic compounds, 1880-2002-Continued

\begin{tabular}{|c|c|c|c|c|c|}
\hline Molecular formula & $\begin{array}{c}\text { Compound } \\
\text { (Temperature range/K) }\end{array}$ & $\begin{array}{c}\Delta_{\mathrm{vap}} H_{m} \\
\left(\mathrm{~kJ} \mathrm{~mol}^{-1}\right)\end{array}$ & $\begin{array}{l}\text { Mean temperature } \\
\left(T_{m} / \mathrm{K}\right)\end{array}$ & Method & $\begin{array}{c}\text { CAS registry number } \\
\text { Reference }\end{array}$ \\
\hline \multirow[t]{5}{*}{$\mathrm{C}_{4} \mathrm{H}_{6} \mathrm{O}_{3}$} & acetic anhydride & & & & [108-24-7] \\
\hline & $(349-429)$ & 43.3 & $(364)$ & EB & {$[87 / 6]$} \\
\hline & $(413-526)$ & 47.6 & $(428)$ & A & {$[87 / 5]$} \\
\hline & $(320-413)$ & 45.5 & $(335)$ & A & {$[87 / 5][71 / 21]$} \\
\hline & $(336-412)$ & 44.2 & $(351)$ & & {$[59 / 1]$} \\
\hline \multirow[t]{7}{*}{$\mathrm{C}_{4} \mathrm{H}_{6} \mathrm{O}_{3}$} & propylene carbonate & & & & [108-32-7] \\
\hline & $(412-466)$ & 54.4 & $(427)$ & A & {$[87 / 5]$} \\
\hline & $(368-462)$ & 57.8 & (383) & $\mathrm{EB}$ & {$[82 / 8]$} \\
\hline & $(368-462)$ & 55.2 & $(423)$ & $\mathrm{EB}$ & {$[82 / 8]$} \\
\hline & $(368-462)$ & 53.0 & $(443)$ & $\mathrm{EB}$ & {$[82 / 8]$} \\
\hline & $(293-353)$ & 55.2 & (323) & & {$[72 / 37]$} \\
\hline & $(323-370)$ & 33.8 & (338) & $\mathrm{A}, \mathrm{MM}$ & {$[87 / 5][71 / 1]$} \\
\hline \multirow[t]{3}{*}{$\mathrm{C}_{4} \mathrm{H}_{6} \mathrm{O}_{4}$} & dimethyl oxalate & & & & {$[553-90-2]$} \\
\hline & $(347-485)$ & 44.7 & $(416)$ & $\mathrm{HG}, \mathrm{EB}$ & {$[88 / 3]$} \\
\hline & $(293-437)$ & 48.8 & $(308)$ & A & {$[87 / 5][47 / 5]$} \\
\hline \multirow[t]{2}{*}{$\mathrm{C}_{4} \mathrm{H}_{6} \mathrm{~S}$} & 2-vinylthiirane & & & & {$[5954-75-6]$} \\
\hline & $(273-335)$ & 38.7 & $(288)$ & A & {$[87 / 5][99 / 16]$} \\
\hline \multirow[t]{2}{*}{$\mathrm{C}_{4} \mathrm{H}_{6} \mathrm{~S}$} & divinyl sulfide & & & & {$[627-51-0]$} \\
\hline & & $38.3 \pm 0.7$ & $(298)$ & $\mathrm{C}$ & [89/12] \\
\hline \multirow[t]{3}{*}{$\mathrm{C}_{4} \mathrm{H}_{7} \mathrm{Br}$} & cis 1-bromo-1-butene & & & & [31849-78-2] \\
\hline & $(280-397)$ & 35.1 & $(295)$ & A & {$[87 / 5][71 / 21]$} \\
\hline & $(229-359)$ & 36.5 & $(244)$ & & {$[47 / 5]$} \\
\hline \multirow[t]{2}{*}{$\mathrm{C}_{4} \mathrm{H}_{7} \mathrm{Br}$} & trans 1-bromo-1-butene & & & & [32620-08-9] \\
\hline & $(234-368)$ & 36.1 & $(249)$ & A & {$[87 / 5][47 / 5]$} \\
\hline \multirow[t]{3}{*}{$\mathrm{C}_{4} \mathrm{H}_{7} \mathrm{Br}$} & 2-bromo-1-butene & & & & {$[23074-36-4]$} \\
\hline & $(276-391)$ & 34.5 & $(291)$ & A & {$[87 / 5][71 / 21]$} \\
\hline & $(226-354)$ & 36.1 & $(241)$ & & {$[47 / 5]$} \\
\hline \multirow[t]{2}{*}{$\mathrm{C}_{4} \mathrm{H}_{7} \mathrm{Br}$} & cis 2-bromo-2-butene & & & & {$[3017-71-8]$} \\
\hline & $(234-367)$ & 36.5 & $(249)$ & A & {$[87 / 5][47 / 5]$} \\
\hline \multirow[t]{2}{*}{$\mathrm{C}_{4} \mathrm{H}_{7} \mathrm{Br}$} & trans 2-bromo-2-butene & & & & [3017-68-3] \\
\hline & $(228-359)$ & 35.7 & $(243)$ & A & {$[87 / 5][47 / 5]$} \\
\hline \multirow[t]{3}{*}{$\mathrm{C}_{4} \mathrm{H}_{7} \mathrm{BrO}$} & 1-bromo-2-butanone & & & & [816-40-0] \\
\hline & $(322-428)$ & 49.9 & $(337)$ & A & {$[87 / 5]$} \\
\hline & $(279-420)$ & 47.7 & $(294)$ & & {$[47 / 5]$} \\
\hline \multirow[t]{2}{*}{$\mathrm{C}_{4} \mathrm{H}_{7} \mathrm{BrO}$} & 3-bromo-2-butanone & & & & {$[814-75-5]$} \\
\hline & $(306-409)$ & 46.4 & $(321)$ & A & {$[87 / 5]$} \\
\hline \multirow[t]{2}{*}{$\mathrm{C}_{4} \mathrm{H}_{7} \mathrm{BrO}$} & isobutyryl bromide & & & & {$[2736-37-0]$} \\
\hline & $(286-436)$ & 45.7 & $(301)$ & A & {$[87 / 5][47 / 5]$} \\
\hline \multirow[t]{2}{*}{$\mathrm{C}_{4} \mathrm{H}_{7} \mathrm{Br}_{3}$} & 1,3-dibromo-2-(bromomethyl)propane & & & & \\
\hline & $(475-660)$ & 66.1 & $(490)$ & A & {$[87 / 5]$} \\
\hline $\mathrm{C}_{4} \mathrm{H}_{7} \mathrm{Br}_{3}$ & 1,1,2-tribromobutane & & & & {$[3675-68-1]$} \\
\hline & $(361-490)$ & 49.4 & $(376)$ & A & {$[87 / 5]$} \\
\hline $\mathrm{C}_{4} \mathrm{H}_{7} \mathrm{Br}_{3}$ & 1,2,2-tribromobutane & & & & {$[3675-69-2]$} \\
\hline & $(356-487)$ & 48.4 & $(371)$ & A & {$[87 / 5]$} \\
\hline & $(314-486)$ & 50.7 & $(329)$ & & {$[47 / 5]$} \\
\hline $\mathrm{C}_{4} \mathrm{H}_{7} \mathrm{Br}_{3}$ & 1,2,3-tribromobutane & & & & {$[632-05-3]$} \\
\hline & $(394-546)$ & 54.1 & $(409)$ & A & {$[87 / 5][71 / 21]$} \\
\hline & $(318-489)$ & 51.3 & $(333)$ & & {$[47 / 5]$} \\
\hline $\mathrm{C}_{4} \mathrm{H}_{7} \mathrm{Br}_{3}$ & 1,2,4-tribromobutane & & & & [38300-67-3] \\
\hline & $(390-541)$ & 53.5 & $(405)$ & A & {$[87 / 5][71 / 21]$} \\
\hline $\mathrm{C}_{4} \mathrm{H}_{7} \mathrm{Br}_{3}$ & 2,2,3-tribromobutane & & & & {$[62127-47-3]$} \\
\hline & $(311-480)$ & 51.7 & $(326)$ & A & {$[87 / 5][47 / 5]$} \\
\hline $\mathrm{C}_{4} \mathrm{H}_{7} \mathrm{Cl}$ & 1-chloro-2-methyl-1-propene & & & & {$[513-37-1]$} \\
\hline & $(285-343)$ & 33.2 & $(300)$ & A & {$[87 / 5]$} \\
\hline $\mathrm{C}_{4} \mathrm{H}_{7} \mathrm{Cl}$ & 3-chloro-2-methyl-1-propene & & & & {$[563-47-3]$} \\
\hline & $(285-348)$ & 33.3 & $(300)$ & A & {$[87 / 5]$} \\
\hline $\mathrm{C}_{4} \mathrm{H}_{7} \mathrm{ClO}$ & 1-chloro-2-butanone & & & & {$[616-27-3]$} \\
\hline & $(307-411)$ & 49.2 & $(322)$ & A & {$[87 / 5][71 / 21]$} \\
\hline $\mathrm{C}_{4} \mathrm{H}_{7} \mathrm{ClO}$ & 3-chloro-2-butanone & & & & {$[4091-39-8]$} \\
\hline & $(313-389)$ & 38.8 & $(328)$ & A & {$[87 / 5]$} \\
\hline $\mathrm{C}_{4} \mathrm{H}_{7} \mathrm{ClO}$ & 3-chloro-2-butene-1-ol & & & & {$[40605-42-3]$} \\
\hline & $(345-437)$ & 50.0 & $(360)$ & A & {$[87 / 5]$} \\
\hline $\mathrm{C}_{4} \mathrm{H}_{7} \mathrm{ClO}_{2}$ & propyl chloroformate & & & & {$[109-61-5]$} \\
\hline & $(293-303)$ & $40.7 \pm 0.4$ & $(298)$ & & [90/12] \\
\hline
\end{tabular}


TABLE 6. Enthalpies of vaporization of organic compounds, 1880-2002-Continued

\begin{tabular}{|c|c|c|c|c|c|}
\hline Molecular formula & $\begin{array}{c}\text { Compound } \\
\text { (Temperature range/K) }\end{array}$ & $\begin{array}{c}\Delta_{\mathrm{vap}} H_{m} \\
\left(\mathrm{~kJ} \mathrm{~mol}^{-1}\right)\end{array}$ & $\begin{array}{l}\text { Mean temperature } \\
\qquad\left(T_{m} / \mathrm{K}\right)\end{array}$ & Method & $\begin{array}{c}\text { CAS registry number } \\
\text { Reference }\end{array}$ \\
\hline & & $40.7 \pm 0.4$ & (298) & $\mathrm{C}$ & [90/12] \\
\hline \multirow[t]{4}{*}{$\mathrm{C}_{4} \mathrm{H}_{7} \mathrm{ClO}_{2}$} & ethyl chloroacetate & & & & {$[105-39-5]$} \\
\hline & $(274-418)$ & 45.0 & $(289)$ & A & {$[87 / 5]$} \\
\hline & & $49.5 \pm 0.1$ & (298) & $\mathrm{C}$ & {$[72 / 41]$} \\
\hline & $(298-418)$ & 48.5 & $(313)$ & & {$[28 / 2][84 / 9]$} \\
\hline \multirow[t]{2}{*}{$\mathrm{C}_{4} \mathrm{H}_{7} \mathrm{ClS}$} & 2-butene-3-chloro-1-thiol & & & & \\
\hline & $(341-397)$ & 48.2 & $(356)$ & A & {$[87 / 5]$} \\
\hline \multirow{2}{*}{$\mathrm{C}_{4} \mathrm{H}_{7} \mathrm{Cl}_{2} \mathrm{O}_{4} \mathrm{P}$} & dimethyl-(2,2-dichlorovinyl) phosphate & & & & {$[62-73-7]$} \\
\hline & $(283-387)$ & 68.0 & $(298)$ & A & {$[87 / 5]$} \\
\hline \multirow[t]{2}{*}{$\mathrm{C}_{4} \mathrm{H}_{7} \mathrm{Cl}_{3}$} & 1,2,3-trichlorobutane & & & & {$[18338-40-4]$} \\
\hline & $(273-442)$ & 41.3 & (288) & A & {$[87 / 5][47 / 5]$} \\
\hline \multirow[t]{2}{*}{$\mathrm{C}_{4} \mathrm{H}_{7} \mathrm{FOS}$} & 2-fluoroethyl thioacetate & & & & {$[462-31-7]$} \\
\hline & $(273-333)$ & 44.7 & $(288)$ & A, GS & {$[87 / 5][48 / 14]$} \\
\hline \multirow[t]{2}{*}{$\mathrm{C}_{4} \mathrm{H}_{7} \mathrm{FO}_{2}$} & ethyl fluoroacetate & & & & {$[459-72-3]$} \\
\hline & $(273-333)$ & 41.9 & $(288)$ & A, GS & $\begin{array}{c}{[87 / 5][48 / 14]} \\
{[71 / 21]}\end{array}$ \\
\hline \multirow[t]{2}{*}{$\mathrm{C}_{4} \mathrm{H}_{7} \mathrm{~F}_{3}$} & 1,1,1-trifluorobutane & & & & [460-34-4] \\
\hline & $(226-320)$ & 28.1 & (241) & A & {$[87 / 5][71 / 21]$} \\
\hline \multirow[t]{2}{*}{$\mathrm{C}_{4} \mathrm{H}_{7} \mathrm{IO}_{2}$} & ethyl iodoacetate & & & & [623-48-3] \\
\hline & $(301-362)$ & 52.1 & $(316)$ & A & {$[87 / 5][47 / 3]$} \\
\hline \multirow[t]{3}{*}{$\mathrm{C}_{4} \mathrm{H}_{7} \mathrm{~N}$} & isobutyronitrile & & & & {$[78-82-0]$} \\
\hline & $(324-354)$ & 35.9 & (339) & & {$[79 / 18]$} \\
\hline & $(303-352)$ & 37.5 & $(321)$ & BG & {$[71 / 2]$} \\
\hline \multirow[t]{4}{*}{$\mathrm{C}_{4} \mathrm{H}_{7} \mathrm{~N}$} & butyronitrile & & & & [109-74-0] \\
\hline & & $39.2 \pm 0.1$ & $(298)$ & $\mathrm{C}$ & {$[82 / 5]$} \\
\hline & $(303-493)$ & 38.8 & $(318)$ & EB & {$[71 / 4]$} \\
\hline & $(332-401)$ & 37.7 & $(347)$ & $\mathrm{A}, \mathrm{EB}$ & $\begin{array}{c}{[87 / 5][47 / 5]} \\
{[73 / 12]}\end{array}$ \\
\hline \multirow[t]{2}{*}{$\mathrm{C}_{4} \mathrm{H}_{7} \mathrm{NO}$} & acetone cyanohydrin & & & & {$[75-86-5]$} \\
\hline & $(355-393)$ & 106.5 & $(370)$ & A & {$[87 / 5]$} \\
\hline \multirow[t]{2}{*}{$\mathrm{C}_{4} \mathrm{H}_{7} \mathrm{NO}$} & 2-hydroxybutyronitrile & & & & {$[4476-02-2]$} \\
\hline & $(314-452)$ & 57.9 & $(329)$ & $\mathrm{A}$ & {$[87 / 5][47 / 5]$} \\
\hline \multirow[t]{2}{*}{$\mathrm{C}_{4} \mathrm{H}_{7} \mathrm{NO}$} & 2-methyl-2-oxazoline & & & & [1120-64-5] \\
\hline & & $39.1 \pm 0.3$ & $(298)$ & $\mathrm{C}$ & {$[76 / 27]$} \\
\hline \multirow[t]{2}{*}{$\mathrm{C}_{4} \mathrm{H}_{7} \mathrm{NO}$} & methyacrylamide & & & & {$[79-39-0]$} \\
\hline & $(390-418)$ & 86.3 & $(404)$ & A & {$[87 / 5]$} \\
\hline \multirow[t]{2}{*}{$\mathrm{C}_{4} \mathrm{H}_{7} \mathrm{NO}$} & ethoxyacetonitrile & & & & [62957-60-2] \\
\hline & $(273-313)$ & $46.5 \pm 0.3$ & $(298)$ & GS & {$[95 / 11]$} \\
\hline $\mathrm{C}_{4} \mathrm{H}_{7} \mathrm{NO}$ & 3-methoxypropionitrile & & & & [33695-59-9] \\
\hline & $(328-438)$ & 47.6 & $(343)$ & $\mathrm{A}$ & {$[87 / 5]$} \\
\hline $\mathrm{C}_{4} \mathrm{H}_{7} \mathrm{NO}$ & 2-pyrrolidone & & & & {$[616-45-5]$} \\
\hline & & $73.6 \pm 1.3$ & (298) & $\mathrm{EB}, \mathrm{BG}$ & {$[98 / 14]$} \\
\hline & & $41.7 \pm 0.6$ & & A & [95/26] \\
\hline & & $69.1 \pm 0.5$ & (298) & $\mathrm{C}$ & {$[90 / 1]$} \\
\hline & $(395-518)$ & 60.0 & $(410)$ & A & {$[87 / 5]$} \\
\hline $\mathrm{C}_{4} \mathrm{H}_{7} \mathrm{NO}_{2}$ & diacetamide & & & & {$[625-77-4]$} \\
\hline & $(368-496)$ & 59.7 & $(383)$ & $\mathrm{A}$ & {$[87 / 5]$} \\
\hline & $(343-496)$ & 64.6 & $(358)$ & & {$[47 / 5]$} \\
\hline $\mathrm{C}_{4} \mathrm{H}_{7} \mathrm{NO}_{2}$ & 2-nitro-1-butene & & & & [2783-12-2] \\
\hline & $(273-333)$ & 44.0 & $(288)$ & $\mathrm{A}$ & {$[87 / 5][71 / 21]$} \\
\hline $\mathrm{C}_{4} \mathrm{H}_{7} \mathrm{~N}_{3} \mathrm{O}_{9}$ & 1,2,4-butanetriol trinitrate & & & & {$[6859-60-5]$} \\
\hline & $(293-313)$ & $60.0 \pm 11.3$ & $(303)$ & A, GS & {$[87 / 5][57 / 11]$} \\
\hline $\mathrm{C}_{4} \mathrm{H}_{8}$ & 1-butene & & & & [106-98-9] \\
\hline & $(200-274)$ & 23.3 & (259) & A & {$[87 / 5]$} \\
\hline & $(126-192)$ & 28.3 & (177) & A & {$[87 / 5]$} \\
\hline & $(267-345)$ & 22.8 & (282) & A & {$[87 / 5]$} \\
\hline & $(342-411)$ & 22.0 & $(357)$ & $\mathrm{A}$ & {$[87 / 5]$} \\
\hline & $(267-411)$ & 22.5 & $(282)$ & $\mathrm{A}$ & {$[87 / 5]$} \\
\hline & & 20.1 & (298) & & {$[71 / 28]$} \\
\hline & & 25.3 & (202) & & {$[46 / 7]$} \\
\hline & & 24.5 & (219) & & {$[46 / 7]$} \\
\hline & & 23.3 & (242) & & {$[46 / 7]$} \\
\hline & & 21.9 & (267) & & {$[46 / 7]$} \\
\hline & $(216-273)$ & 23.2 & (258) & & {$[40 / 3][84 / 9]$} \\
\hline
\end{tabular}


TABLE 6. Enthalpies of vaporization of organic compounds, 1880-2002-Continued

\begin{tabular}{|c|c|c|c|c|c|}
\hline Molecular formula & $\begin{array}{c}\text { Compound } \\
\text { (Temperature range/K) }\end{array}$ & $\begin{array}{c}\Delta_{\mathrm{vap}} H_{m} \\
\left(\mathrm{~kJ} \mathrm{~mol}^{-1}\right)\end{array}$ & $\begin{array}{l}\text { Mean temperature } \\
\qquad\left(T_{m} / \mathrm{K}\right)\end{array}$ & Method & $\begin{array}{c}\text { CAS registry number } \\
\text { Reference }\end{array}$ \\
\hline \multirow[t]{7}{*}{$\mathrm{C}_{4} \mathrm{H}_{8}$} & cis 2-butene & & & & {$[590-18-1]$} \\
\hline & $(221-290)$ & 24.4 & $(275)$ & A & {$[87 / 5]$} \\
\hline & $(276-325)$ & 24.0 & $(291)$ & A & {$[87 / 5]$} \\
\hline & $(324-386)$ & 23.6 & (339) & A & {$[87 / 5]$} \\
\hline & $(383-431)$ & 23.6 & (398) & A & {$[87 / 5]$} \\
\hline & & 22.1 & $(298)$ & & {$[71 / 28]$} \\
\hline & $(195-267)$ & 25.3 & $(252)$ & & {$[42 / 8][84 / 9]$} \\
\hline \multirow[t]{9}{*}{$\mathrm{C}_{4} \mathrm{H}_{8}$} & trans 2-butene & & & & [624-64-6] \\
\hline & $(205-287)$ & 23.9 & $(272)$ & A & {$[87 / 5]$} \\
\hline & $(273-315)$ & 23.6 & $(288)$ & A & {$[87 / 5]$} \\
\hline & $(313-385)$ & 23.3 & $(328)$ & A & {$[87 / 5]$} \\
\hline & $(382-428)$ & 23.2 & (397) & $\mathrm{A}$ & {$[87 / 5]$} \\
\hline & & 21.3 & $(298)$ & & {$[71 / 28]$} \\
\hline & & $22.8 \pm 0.1$ & $(274)$ & $\mathrm{C}$ & {$[45 / 3]$} \\
\hline & $(203-274)$ & 24.2 & $(259)$ & & {$[45 / 3][84 / 9]$} \\
\hline & $(205-283)$ & 23.9 & $(268)$ & & {$[40 / 3][84 / 9]$} \\
\hline \multirow[t]{3}{*}{$\mathrm{C}_{4} \mathrm{H}_{8}$} & cyclobutane & & & & [287-23-0] \\
\hline & $(198-287)$ & 25.2 & $(272)$ & A & {$[87 / 5]$} \\
\hline & $(217-285)$ & 25.2 & $(270)$ & & {$[53 / 12][84 / 9]$} \\
\hline \multirow[t]{2}{*}{$\mathrm{C}_{4} \mathrm{H}_{8}$} & methylcyclopropane & & & & [594-11-6] \\
\hline & $(177-278)$ & 24.8 & $(263)$ & A & {$[87 / 5][47 / 5]$} \\
\hline \multirow[t]{8}{*}{$\mathrm{C}_{4} \mathrm{H}_{8}$} & 2-methylpropene & & & & [115-11-7] \\
\hline & $(212-279)$ & 23.1 & $(264)$ & A & {$[87 / 5]$} \\
\hline & $(266-313)$ & 22.7 & $(281)$ & A & {$[87 / 5]$} \\
\hline & $(310-376)$ & 22.2 & $(325)$ & A & {$[87 / 5]$} \\
\hline & $(371-418)$ & 22.3 & $(386)$ & A & {$[87 / 5]$} \\
\hline & & 20.6 & $(298)$ & & {$[71 / 28]$} \\
\hline & $(303-398)$ & 22.2 & $(350)$ & & {$[42 / 6]$} \\
\hline & $(216-273)$ & 22.8 & $(258)$ & & {$[40 / 3][84 / 9]$} \\
\hline \multirow[t]{2}{*}{$\mathrm{C}_{4} \mathrm{H}_{8} \mathrm{BrClO}$} & 2-bromoethyl 2-chloroethyl ether & & & & {$[51070-66-7]$} \\
\hline & $(309-469)$ & 53.3 & $(324)$ & A & {$[87 / 5][47 / 5]$} \\
\hline \multirow[t]{2}{*}{$\mathrm{C}_{4} \mathrm{H}_{8} \mathrm{Br}_{2}$} & 1,1-dibromobutane & & & & {$[62168-25-6]$} \\
\hline & $(342-477)$ & 45.8 & $(357)$ & $\mathrm{A}, \mathrm{EST}$ & $\begin{array}{c}{[87 / 5][56 / 16]} \\
{[71 / 21]}\end{array}$ \\
\hline \multirow[t]{6}{*}{$\mathrm{C}_{4} \mathrm{H}_{8} \mathrm{Br}_{2}$} & 1,2-dibromobutane & & & & {$[533-98-2]$} \\
\hline & $(338-425)$ & 43.5 & $(353)$ & A & {$[87 / 5]$} \\
\hline & $(330-425)$ & 45.9 & $(298)$ & & {$[75 / 16][75 / 15]$} \\
\hline & & $45.6 \pm 0.7$ & $(298)$ & $\mathrm{EB}$ & {$[75 / 15]$} \\
\hline & $(281-439)$ & 42.8 & $(296)$ & A & {$[87 / 5][47 / 5]$} \\
\hline & $(273-333)$ & 45.1 & $(300)$ & & {$[41 / 6]$} \\
\hline \multirow[t]{2}{*}{$\mathrm{C}_{4} \mathrm{H}_{8} \mathrm{Br}_{2}$} & 1,3-dibromobutane & & & & {$[107-80-2]$} \\
\hline & $(351-450)$ & 44.7 & $(366)$ & A & {$[87 / 5]$} \\
\hline \multirow[t]{4}{*}{$\mathrm{C}_{4} \mathrm{H}_{8} \mathrm{Br}_{2}$} & 1,4-dibromobutane & & & & {$[110-52-1]$} \\
\hline & & 52.6 & $(298)$ & GC & [94/19] \\
\hline & $(375-520)$ & 51.4 & $(390)$ & A & {$[87 / 5][71 / 21]$} \\
\hline & $(305-470)$ & 49.4 & $(320)$ & & {$[47 / 5]$} \\
\hline \multirow[t]{2}{*}{$\mathrm{C}_{4} \mathrm{H}_{8} \mathrm{Br}_{2}$} & meso 2,3-dibromobutane & & & & {$[5780-13-2]$} \\
\hline & $(274-431)$ & 41.7 & $(289)$ & A & {$[87 / 5][47 / 5]$} \\
\hline \multirow[t]{2}{*}{$\mathrm{C}_{4} \mathrm{H}_{8} \mathrm{Br}_{2}$} & threo 2,3-dibromobutane & & & & {$[598-71-0]$} \\
\hline & $(278-434)$ & 40.9 & $(293)$ & A & {$[87 / 5]$} \\
\hline \multirow[t]{3}{*}{$\mathrm{C}_{4} \mathrm{H}_{8} \mathrm{Br}_{2}$} & 1,2-dibromo-2-methylpropane & & & & [594-34-3] \\
\hline & & $43.3 \pm 0.1$ & & $\mathrm{C}$ & {$[74 / 2]$} \\
\hline & $(244-422)$ & 33.3 & $(259)$ & A & {$[87 / 5][47 / 5]$} \\
\hline \multirow[t]{2}{*}{$\mathrm{C}_{4} \mathrm{H}_{8} \mathrm{Br}_{2}$} & 1,3-dibromo-2-methylpropane & & & & {$[28148-04-1]$} \\
\hline & $(287-448)$ & 45.1 & $(302)$ & A & {$[87 / 5][47 / 5]$} \\
\hline $\mathrm{C}_{4} \mathrm{H}_{8} \mathrm{Br}_{2} \mathrm{O}$ & bis (2-bromoethyl) ether & & & & {$[5414-19-7]$} \\
\hline & $(320-486)$ & 55.1 & $(335)$ & A & {$[87 / 5][47 / 5]$} \\
\hline $\mathrm{C}_{4} \mathrm{H}_{8} \mathrm{Cl}_{2}$ & 1,1-dichlorobutane & & & & {$[541-33-3]$} \\
\hline & $(310-390)$ & 39.5 & $(298)$ & & {$[91 / 2]$} \\
\hline & $(304-386)$ & 38.7 & (319) & A & {$[87 / 5]$} \\
\hline & & $39.4 \pm 0.6$ & $(298)$ & EB & {$[77 / 14]$} \\
\hline & $(303-428)$ & 38.8 & $(318)$ & EST & {$[87 / 5][56 / 16]$} \\
\hline $\mathrm{C}_{4} \mathrm{H}_{8} \mathrm{Cl}_{2}$ & 1,2-dichlorobutane & & & & [616-21-7] \\
\hline & & $40.1 \pm 0.1$ & $(298)$ & $\mathrm{C}$ & {$[92 / 7]$} \\
\hline
\end{tabular}


TABLE 6. Enthalpies of vaporization of organic compounds, 1880-2002-Continued

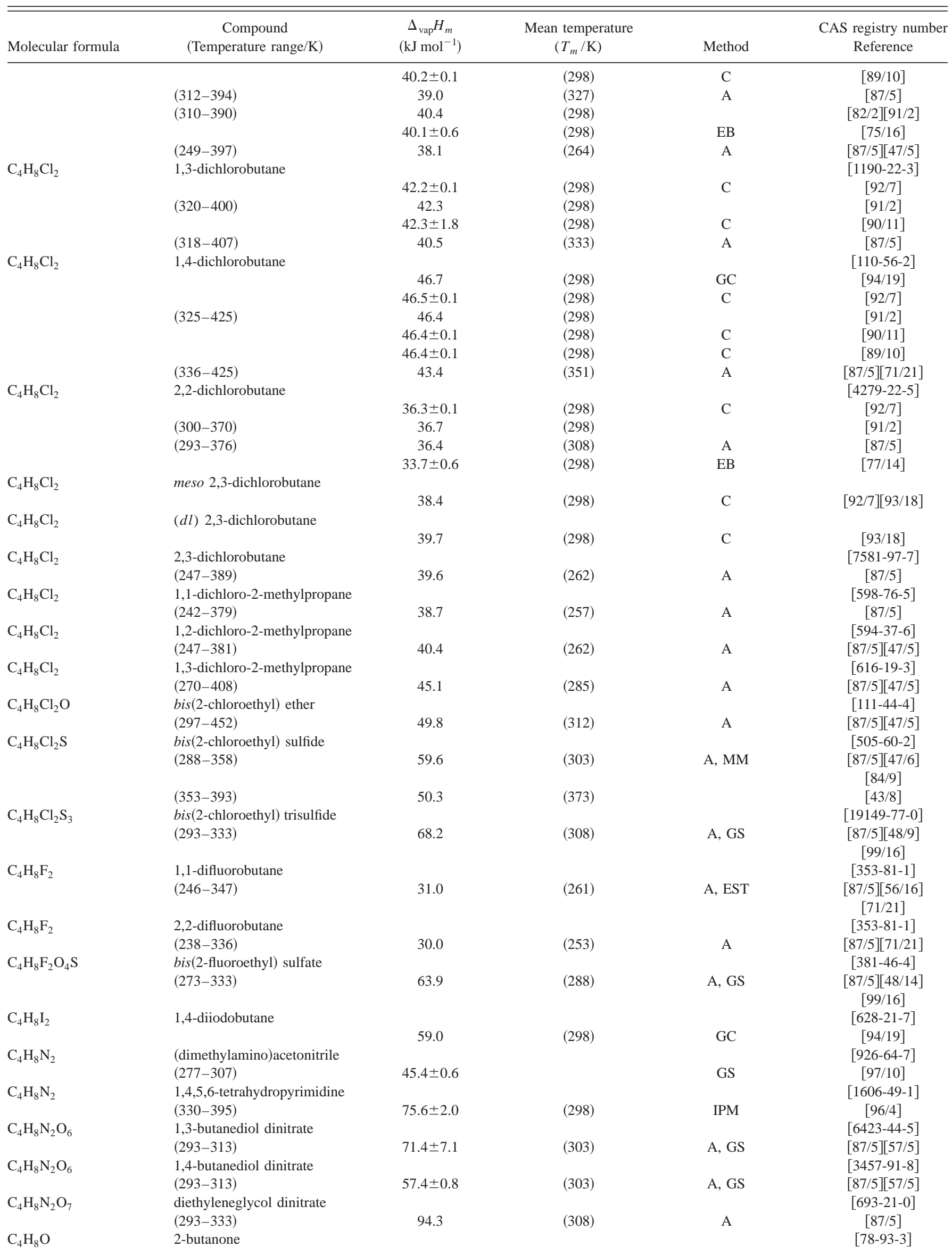


TABLE 6. Enthalpies of vaporization of organic compounds, 1880-2002-Continued

\begin{tabular}{|c|c|c|c|c|c|}
\hline Molecular formula & $\begin{array}{c}\text { Compound } \\
\text { (Temperature range/K) }\end{array}$ & $\begin{array}{c}\Delta_{\mathrm{vap}} H_{m} \\
\left(\mathrm{~kJ} \mathrm{~mol}^{-1}\right)\end{array}$ & $\begin{array}{l}\text { Mean temperature } \\
\qquad\left(T_{m} / \mathrm{K}\right)\end{array}$ & Method & $\begin{array}{l}\text { CAS registry number } \\
\text { Reference }\end{array}$ \\
\hline & $(294-342)$ & 34.6 & $(309)$ & A & {$[87 / 5]$} \\
\hline & $(353-403)$ & 32.5 & $(368)$ & A & {$[87 / 5]$} \\
\hline & $(397-479)$ & 31.6 & $(412)$ & A & {$[87 / 5]$} \\
\hline & $(473-537)$ & 31.1 & $(488)$ & A & {$[87 / 5]$} \\
\hline & & $34.8 \pm 0.1$ & $(298)$ & $\mathrm{C}$ & {$[83 / 3]$} \\
\hline & & $34.5 \pm 0.1$ & $(298)$ & $\mathrm{C}$ & {$[79 / 1]$} \\
\hline & $(258-362)$ & 35.6 & $(273)$ & & {$[78 / 20]$} \\
\hline & & 34.7 & $(298)$ & & {$[75 / 8]$} \\
\hline & $(315-363)$ & 33.9 & $(330)$ & $\mathrm{A}, \mathrm{EB}, \mathrm{GS}$ & $\begin{array}{c}{[87 / 5][75 / 8]} \\
{[65 / 7]}\end{array}$ \\
\hline & & $33.8 \pm 0.1$ & $(314)$ & $\mathrm{C}$ & {$[61 / 17]$} \\
\hline & & $32.3 \pm 0.1$ & $(338)$ & $\mathrm{C}$ & {$[61 / 17]$} \\
\hline & & $31.3 \pm 0.1$ & $(352)$ & $\mathrm{C}$ & {$[61 / 17]$} \\
\hline & & $30.5 \pm 0.1$ & $(363)$ & $\mathrm{C}$ & {$[61 / 17]$} \\
\hline & & $30.0 \pm 0.1$ & $(370)$ & $\mathrm{C}$ & {$[61 / 17]$} \\
\hline & $(314-370)$ & 33.9 & (329) & & {$[47 / 5]$} \\
\hline \multirow[t]{2}{*}{$\mathrm{C}_{4} \mathrm{H}_{8} \mathrm{O}$} & 2-methyl-2-propen-1-ol & & & & {$[513-42-8]$} \\
\hline & $(323-373)$ & 51.9 & $(298)$ & CGC & {$[95 / 21]$} \\
\hline \multirow[t]{4}{*}{$\mathrm{C}_{4} \mathrm{H}_{8} \mathrm{O}$} & 3-buten-1-ol & & & & {$[627-27-0]$} \\
\hline & & $50.9 \pm 0.1$ & (313) & $\mathrm{C}$ & {$[96 / 9]$} \\
\hline & & $48.8 \pm 0.1$ & $(328)$ & $\mathrm{C}$ & {$[96 / 9]$} \\
\hline & & $46.7 \pm 0.1$ & $(343)$ & $\mathrm{C}$ & [96/9] \\
\hline \multirow[t]{2}{*}{$\mathrm{C}_{4} \mathrm{H}_{8} \mathrm{O}$} & ( $d l$ ) 3-buten-2-ol & & & & [6118-14-5] \\
\hline & $(304-370)$ & 39.2 & (319) & A & {$[87 / 5]$} \\
\hline \multirow[t]{6}{*}{$\mathrm{C}_{4} \mathrm{H}_{8} \mathrm{O}$} & butyraldehyde & & & & {$[123-72-8]$} \\
\hline & $(313-353)$ & 33.2 & $(298)$ & CGC & {$[95 / 21]$} \\
\hline & $(293-349)$ & 34.2 & $(308)$ & A & {$[87 / 5]$} \\
\hline & $(330-348)$ & 32.9 & (339) & EB & {$[63 / 15]$} \\
\hline & $(304-347)$ & 33.3 & $(319)$ & & {$[59 / 5][84 / 9]$} \\
\hline & $(258-353)$ & 33.9 & $(306)$ & & {$[38 / 7]$} \\
\hline \multirow[t]{2}{*}{$\mathrm{C}_{4} \mathrm{H}_{8} \mathrm{O}$} & (dl) 1,2-epoxybutane & & & & [106-88-7] \\
\hline & $(254-347)$ & 24.7 & $(269)$ & A & {$[87 / 5]$} \\
\hline \multirow[t]{2}{*}{$\mathrm{C}_{4} \mathrm{H}_{8} \mathrm{O}$} & 1,2-epoxy-2-methylpropan & ethyloxirane) & & & {$[558-30-5]$} \\
\hline & $(204-329)$ & 30.6 & $(219)$ & A & {$[87 / 5][47 / 5]$} \\
\hline \multirow[t]{2}{*}{$\mathrm{C}_{4} \mathrm{H}_{8} \mathrm{O}$} & ethyl vinyl ether & & & & {$[109-92-2]$} \\
\hline & $(223-309)$ & 29.5 & $(238)$ & A & {$[87 / 5]$} \\
\hline \multirow[t]{6}{*}{$\mathrm{C}_{4} \mathrm{H}_{8} \mathrm{O}$} & isobutyraldehyde & & & & {$[78-84-2]$} \\
\hline & $(313-353)$ & 32.3 & $(298)$ & CGC & {$[95 / 21]$} \\
\hline & $(313-324)$ & 31.4 & $(318)$ & & {$[84 / 30]$} \\
\hline & $(309-337)$ & 31.8 & $(324)$ & & {$[76 / 20]$} \\
\hline & $(333-347)$ & 33.4 & $(340)$ & EB & {$[63 / 15]$} \\
\hline & $(283-337)$ & 32.8 & $(298)$ & A & $\begin{array}{c}{[87 / 5][59 / 5]} \\
{[64 / 21]}\end{array}$ \\
\hline \multirow[t]{2}{*}{$\mathrm{C}_{4} \mathrm{H}_{8} \mathrm{O}$} & 2-methoxy-1-propene & & & & [116-11-0] \\
\hline & $(281-309)$ & $28.3 \pm 0.1$ & $(295)$ & & {$[88 / 4]$} \\
\hline \multirow[t]{2}{*}{$\mathrm{C}_{4} \mathrm{H}_{8} \mathrm{O}$} & cis methyl propenyl ether & & & & [4188-68-5] \\
\hline & $(293-318)$ & 30.6 & $(305)$ & A & {$[87 / 5]$} \\
\hline \multirow[t]{2}{*}{$\mathrm{C}_{4} \mathrm{H}_{8} \mathrm{O}$} & trans methyl propenyl ethe & & & & [4188-69-6] \\
\hline & $(293-322)$ & 29.5 & $(307)$ & A & {$[87 / 5]$} \\
\hline \multirow[t]{10}{*}{$\mathrm{C}_{4} \mathrm{H}_{8} \mathrm{O}$} & tetrahydrofuran & & & & [109-99-9] \\
\hline & $(273-339)$ & 33.1 & $(288)$ & A & {$[87 / 5]$} \\
\hline & $(399-479)$ & 29.0 & $(414)$ & A & {$[87 / 5]$} \\
\hline & $(467-541)$ & 29.6 & $(482)$ & A & {$[87 / 5]$} \\
\hline & & 32.0 & $(298)$ & $\mathrm{C}$ & {$[81 / 8]$} \\
\hline & $(235-340)$ & $32.5 \pm 0.2$ & $(288)$ & & {$[76 / 16]$} \\
\hline & $(302-339)$ & 30.8 & $(320)$ & & {$[75 / 33]$} \\
\hline & $(273-308)$ & 32.8 & $(288)$ & & {$[70 / 22][84 / 9]$} \\
\hline & $(296-373)$ & 31.9 & $(311)$ & & {$[70 / 23][84 / 9]$} \\
\hline & $(224-360)$ & 32.9 & $(298)$ & & {$[70 / 36]$} \\
\hline \multirow[t]{3}{*}{$\mathrm{C}_{4} \mathrm{H}_{8} \mathrm{OS}$} & 1,4-oxathiane & & & & [15980-15-1] \\
\hline & $(342-411)$ & 42.1 & $(378)$ & & [99/16] \\
\hline & $(342-411)$ & 44.8 & $(357)$ & A & {$[87 / 5]$} \\
\hline \multirow[t]{2}{*}{$\mathrm{C}_{4} \mathrm{H}_{8} \mathrm{OS}$} & S-ethyl thiolacetate & & & & {$[625-60-5]$} \\
\hline & & $40.0 \pm 0.2$ & $(298)$ & $\mathrm{C}$ & {$[66 / 2]$} \\
\hline
\end{tabular}


TABLE 6. Enthalpies of vaporization of organic compounds, 1880-2002-Continued

\begin{tabular}{|c|c|c|c|c|c|}
\hline Molecular formula & $\begin{array}{c}\text { Compound } \\
\text { (Temperature range/K) }\end{array}$ & $\begin{array}{c}\Delta_{\mathrm{vap}} H_{m} \\
\left(\mathrm{~kJ} \mathrm{~mol}^{-1}\right)\end{array}$ & $\begin{array}{l}\text { Mean temperature } \\
\qquad\left(T_{m} / \mathrm{K}\right)\end{array}$ & Method & $\begin{array}{c}\text { CAS registry number } \\
\text { Reference }\end{array}$ \\
\hline \multirow[t]{2}{*}{$\mathrm{C}_{4} \mathrm{H}_{8} \mathrm{O}_{2}$} & 2-methyl-1,3-dioxolane & & & & [497-26-7] \\
\hline & $(270-308)$ & $43.0 \pm 0.6$ & & GS & {$[98 / 21][02 / 32]$} \\
\hline \multirow[t]{2}{*}{$\mathrm{C}_{4} \mathrm{H}_{8} \mathrm{O}_{2}$} & cis 2-butene-1,4-diol & & & & [6117-80-2] \\
\hline & $(373-508)$ & 74.7 & $(388)$ & A & {$[87 / 5]$} \\
\hline \multirow[t]{2}{*}{$\mathrm{C}_{4} \mathrm{H}_{8} \mathrm{O}_{2}$} & 1,1-dimethoxyethene & & & & \\
\hline & $(303-362)$ & 39.6 & $(333)$ & & {$[95 / 29]$} \\
\hline \multirow[t]{8}{*}{$\mathrm{C}_{4} \mathrm{H}_{8} \mathrm{O}_{2}$} & butanoic acid & & & & {$[107-92-6]$} \\
\hline & $(391-429)$ & 50.3 & $(406)$ & EB & {$[01 / 15]$} \\
\hline & $(278-308)$ & $58.5 \pm 0.3$ & (293) & GS & {$[00 / 6]$} \\
\hline & $(278-308)$ & $58.2 \pm 0.3$ & $(298)$ & GS & {$[00 / 6]$} \\
\hline & $(353-393)$ & 60.7 & $(298)$ & CGC & {$[95 / 21]$} \\
\hline & $(437-592)$ & 47.7 & $(452)$ & A & {$[87 / 5]$} \\
\hline & $(301-358)$ & 51.1 & $(316)$ & A & {$[87 / 5]$} \\
\hline & $(355-453)$ & 53.2 & $(370)$ & A & {$[87 / 5][71 / 21]$} \\
\hline \multirow[t]{3}{*}{ monomer } & & $40.5 \pm 0.1$ & $(298)$ & $\mathrm{C}$ & {$[70 / 8]$} \\
\hline & & $58 \pm 4$ & $(298)$ & $\mathrm{C}$ & {$[70 / 8]$} \\
\hline & $(363-436)$ & 52.0 & $(378)$ & & {$[49 / 1][84 / 9]$} \\
\hline \multirow[t]{3}{*}{$\mathrm{C}_{4} \mathrm{H}_{8} \mathrm{O}_{2}$} & 1,3-dioxane & & & & {$[505-22-6]$} \\
\hline & & $39.1 \pm 0.1$ & $(298)$ & $\mathrm{C}$ & {$[82 / 9]$} \\
\hline & & $35.6 \pm 0.4$ & & & {$[59 / 23]$} \\
\hline \multirow[t]{6}{*}{$\mathrm{C}_{4} \mathrm{H}_{8} \mathrm{O}_{2}$} & 1,4-dioxane & & & & [123-91-1] \\
\hline & $(285-375)$ & 38.0 & $(300)$ & A & {$[87 / 5]$} \\
\hline & $(329-372)$ & 36.5 & $(350)$ & & {$[84 / 20]$} \\
\hline & & $38.6 \pm 0.1$ & $(298)$ & $\mathrm{C}$ & {$[82 / 9]$} \\
\hline & $(293-398)$ & 37.3 & $(308)$ & & {$[63 / 22][84 / 9]$} \\
\hline & $(283-353)$ & 37.0 & $(318)$ & & {$[36 / 6]$} \\
\hline \multirow[t]{17}{*}{$\mathrm{C}_{4} \mathrm{H}_{8} \mathrm{O}_{2}$} & ethyl acetate & & & & {$[141-78-6]$} \\
\hline & $(300-390)$ & 34.1 & $(315)$ & & {$[97 / 11]$} \\
\hline & $(313-353)$ & 35.0 & $(298)$ & CGC & {$[95 / 21]$} \\
\hline & $(271-373)$ & 36.7 & $(286)$ & & {$[81 / 11][84 / 9]$} \\
\hline & & $35.6 \pm 0.1$ & $(298)$ & $\mathrm{C}$ & {$[80 / 13]$} \\
\hline & & $34.6 \pm 0.1$ & $(313)$ & $\mathrm{C}$ & {$[80 / 13]$} \\
\hline & & $31.4 \pm 0.1$ & $(343)$ & $\mathrm{C}$ & {$[80 / 13]$} \\
\hline & & $33.8 \pm 0.1$ & $(326)$ & $\mathrm{C}$ & {$[77 / 12]$} \\
\hline & & $33.4 \pm 0.1$ & $(331)$ & $\mathrm{C}$ & {$[77 / 12]$} \\
\hline & & $32.4 \pm 0.1$ & $(344)$ & $\mathrm{C}$ & {$[77 / 12]$} \\
\hline & & $31.9 \pm 0.1$ & $(351)$ & $\mathrm{C}$ & {$[77 / 12]$} \\
\hline & & $31.0 \pm 0.1$ & $(363)$ & $\mathrm{C}$ & {$[77 / 12]$} \\
\hline & & 34.0 & $(320)$ & & {$[76 / 8]$} \\
\hline & & 31.9 & $(350)$ & & {$[76 / 8]$} \\
\hline & & $35.1 \pm 0.2$ & $(298)$ & $\mathrm{C}$ & {$[66 / 2]$} \\
\hline & $(288-351)$ & 35.7 & $(303)$ & A & {$[87 / 5][65 / 3]$} \\
\hline & & & & & {$[71 / 21]$} \\
\hline \multirow[t]{2}{*}{$\mathrm{C}_{4} \mathrm{H}_{8} \mathrm{O}_{2}$} & 3-hydroxy-2-butanone & & & & {$[513-86-0]$} \\
\hline & $(273-418)$ & 38.4 & $(288)$ & A & {$[87 / 5]$} \\
\hline \multirow[t]{7}{*}{$\mathrm{C}_{4} \mathrm{H}_{8} \mathrm{O}_{2}$} & 2-methylpropanoic acid & & & & {$[79-31-2]$} \\
\hline & $(278-308)$ & $55.8 \pm 0.3$ & $(293)$ & GS & {$[00 / 6]$} \\
\hline & $(278-308)$ & $55.5 \pm 0.3$ & $(298)$ & GS & {$[00 / 6]$} \\
\hline & $(344-445)$ & 51.6 & $(359)$ & $\mathrm{EB}$ & [87/9] \\
\hline & $(288-428)$ & 50.9 & (303) & $\mathrm{A}$ & {$[87 / 5]$} \\
\hline & $(428-562)$ & 45.4 & $(443)$ & A & {$[87 / 5]$} \\
\hline & $(228-243)$ & $53.4 \pm 3$ & (398) & $\mathrm{TE}$ & {$[79 / 4]$} \\
\hline \multirow[t]{2}{*}{ monomer } & & $35.5 \pm 0.1$ & $(298)$ & $\mathrm{C}$ & {$[70 / 8]$} \\
\hline & & $53 \pm 4$ & $(298)$ & $\mathrm{C}$ & [70/8] \\
\hline \multirow[t]{2}{*}{$\mathrm{C}_{4} \mathrm{H}_{8} \mathrm{O}_{2}$} & isopropyl formate & & & & {$[625-55-8]$} \\
\hline & $(221-342)$ & 34.5 & $(236)$ & A & {$[87 / 5][47 / 5]$} \\
\hline \multirow[t]{8}{*}{$\mathrm{C}_{4} \mathrm{H}_{8} \mathrm{O}_{2}$} & methyl propionate & & & & [922-67-8] \\
\hline & $(313-363)$ & 28.9 & $(298)$ & CGC & {$[95 / 21]$} \\
\hline & & $35.6 \pm 0.4$ & $(298)$ & GC & {$[87 / 17]$} \\
\hline & $(231-353)$ & 39.1 & $(246)$ & A & {$[87 / 5]$} \\
\hline & $(353-486)$ & 32.8 & $(368)$ & A & {$[87 / 5]$} \\
\hline & & $35.9 \pm 0.1$ & (298) & $\mathrm{C}$ & {$[80 / 13]$} \\
\hline & & $34.9 \pm 0.1$ & (313) & $\mathrm{C}$ & {$[80 / 13]$} \\
\hline & & $36.3 \pm 0.3$ & $(298)$ & GCC & {$[80 / 5]$} \\
\hline
\end{tabular}


TABLE 6. Enthalpies of vaporization of organic compounds, 1880-2002-Continued

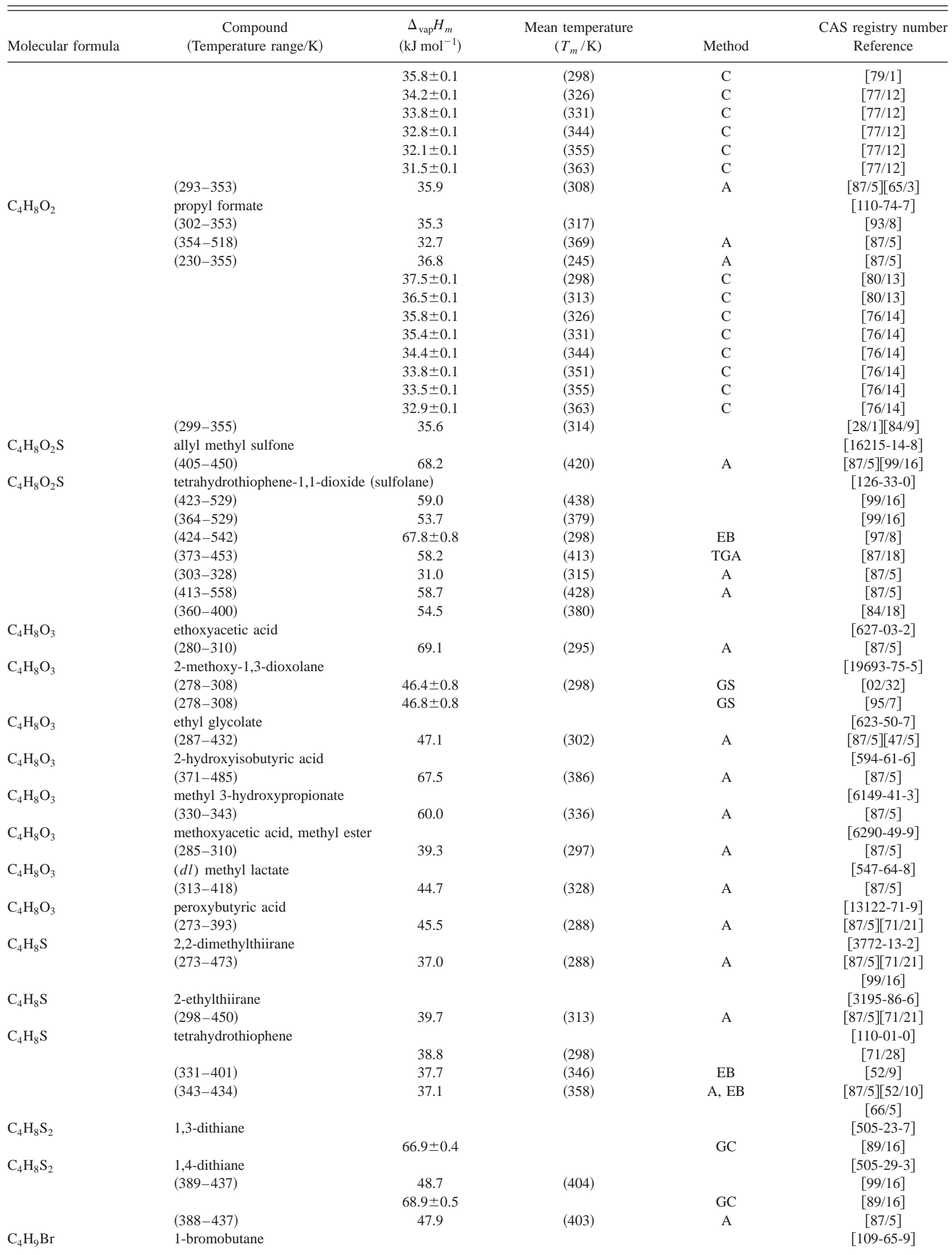


TABLE 6. Enthalpies of vaporization of organic compounds, 1880-2002-Continued

\begin{tabular}{|c|c|c|c|c|c|}
\hline Molecular formula & $\begin{array}{c}\text { Compound } \\
\text { (Temperature range/K) }\end{array}$ & $\begin{array}{c}\Delta_{\mathrm{vap}} H_{m} \\
\left(\mathrm{~kJ} \mathrm{~mol}^{-1}\right)\end{array}$ & $\begin{array}{l}\text { Mean temperature } \\
\qquad\left(T_{m} / \mathrm{K}\right)\end{array}$ & Method & $\begin{array}{c}\text { CAS registry number } \\
\text { Reference }\end{array}$ \\
\hline & $(323-363)$ & 36.4 & $(298)$ & CGC & {$[95 / 21]$} \\
\hline & $(340-370)$ & 36.4 & $(298)$ & & {$[91 / 2]$} \\
\hline & $(338-373)$ & 34.6 & $(353)$ & $\mathrm{A}, \mathrm{EB}$ & {$[87 / 5][77 / 8]$} \\
\hline & & $35.6 \pm 0.1$ & $(322)$ & $\mathrm{C}$ & {$[77 / 8]$} \\
\hline & & $34.9 \pm 0.1$ & $(332)$ & $\mathrm{C}$ & {$[77 / 8]$} \\
\hline & & $34.5 \pm 0.1$ & (339) & $\mathrm{C}$ & {$[77 / 8]$} \\
\hline & & $33.7 \pm 0.1$ & $(352)$ & $\mathrm{C}$ & {$[77 / 8]$} \\
\hline & & $33.0 \pm 0.1$ & $(366)$ & $\mathrm{C}$ & {$[77 / 8]$} \\
\hline & & $36.6 \pm 0.1$ & $(298)$ & $\mathrm{C}$ & {$[68 / 1]$} \\
\hline & & $36.7 \pm 0.1$ & $(298)$ & $\mathrm{C}$ & {$[66 / 2]$} \\
\hline & $(273-400)$ & 37.5 & $(288)$ & $\mathrm{A}, \mathrm{EST}$ & $\begin{array}{c}{[87 / 5][61 / 13]} \\
{[71 / 21]}\end{array}$ \\
\hline & $(293-343)$ & 33.5 & $(308)$ & & {$[29 / 2][84 / 9]$} \\
\hline \multirow[t]{4}{*}{$\mathrm{C}_{4} \mathrm{H}_{9} \mathrm{Br}$} & 2-bromobutane & & & & [78-76-2] \\
\hline & $(281-403)$ & 33.9 & $(296)$ & A & {$[87 / 5][71 / 21]$} \\
\hline & & $34.5 \pm 0.1$ & (298) & $\mathrm{C}$ & {$[68 / 1]$} \\
\hline & & $34.8 \pm 0.1$ & $(298)$ & $\mathrm{C}$ & {$[66 / 2]$} \\
\hline \multirow[t]{8}{*}{$\mathrm{C}_{4} \mathrm{H}_{9} \mathrm{Br}$} & 1-bromo-2-methylpropane & & & & {$[78-77-3]$} \\
\hline & $(305-363)$ & 34.1 & $(320)$ & $\mathrm{A}, \mathrm{EB}$ & {$[87 / 5][77 / 8]$} \\
\hline & & $33.1 \pm 0.1$ & $(330)$ & C & {$[77 / 8]$} \\
\hline & & $32.6 \pm 0.1$ & $(341)$ & $\mathrm{C}$ & {$[77 / 8]$} \\
\hline & & $32.0 \pm 0.1$ & $(353)$ & $\mathrm{C}$ & {$[77 / 8]$} \\
\hline & & $31.4 \pm 0.1$ & $(366)$ & $\mathrm{C}$ & {$[77 / 8]$} \\
\hline & $(281-404)$ & 34.0 & $(296)$ & A & {$[87 / 5][71 / 21]$} \\
\hline & & $34.9 \pm 0.1$ & $(298)$ & $\mathrm{C}$ & {$[68 / 1]$} \\
\hline \multirow[t]{6}{*}{$\mathrm{C}_{4} \mathrm{H}_{9} \mathrm{Br}$} & 2-bromo-2-methylpropane & & & & {$[507-19-7]$} \\
\hline & $(248-346)$ & 31.4 & $(263)$ & A & {$[87 / 5]$} \\
\hline & $(270-345)$ & 31.0 & $(298)$ & & {$[87 / 9][91 / 2]$} \\
\hline & $(298-323)$ & 31.5 & (313) & & {$[69 / 10]$} \\
\hline & & $31.8 \pm 0.1$ & $(298)$ & $\mathrm{C}$ & {$[68 / 1]$} \\
\hline & $(273-346)$ & 31.2 & $(288)$ & & {$[51 / 5][84 / 9]$} \\
\hline \multirow[t]{2}{*}{$\mathrm{C}_{4} \mathrm{H}_{9} \mathrm{BrO}$} & 1-bromo-2-butanol & & & & {$[2482-57-7]$} \\
\hline & $(296-418)$ & 58.4 & $(311)$ & A & {$[87 / 5][47 / 5]$} \\
\hline \multirow[t]{12}{*}{$\mathrm{C}_{4} \mathrm{H}_{9} \mathrm{Cl}$} & 1-chlorobutane & & & & [109-69-3] \\
\hline & $(260-350)$ & 33.5 & $(298)$ & & {$[84 / 9][91 / 2]$} \\
\hline & & $33.5 \pm 0.1$ & $(298)$ & $\mathrm{C}$ & {$[81 / 4]$} \\
\hline & & $32.7 \pm 0.1$ & (313) & $\mathrm{C}$ & {$[81 / 4]$} \\
\hline & & $31.8 \pm 0.1$ & $(328)$ & $\mathrm{C}$ & {$[81 / 4]$} \\
\hline & & $30.9 \pm 0.1$ & $(343)$ & $\mathrm{C}$ & {$[81 / 4]$} \\
\hline & & $30.0 \pm 0.1$ & $(358)$ & $\mathrm{C}$ & {$[81 / 4]$} \\
\hline & & $29.4 \pm 0.1$ & $(358)$ & $\mathrm{C}$ & {$[81 / 4]$} \\
\hline & $(256-352)$ & 35.6 & $(271)$ & DTA & {$[69 / 5]$} \\
\hline & & $33.5 \pm 0.1$ & $(298)$ & C & {$[68 / 1]$} \\
\hline & $(257-389)$ & 35.0 & $(272)$ & $\mathrm{A}, \mathrm{EST}$ & $\begin{array}{c}{[87 / 5][61 / 13]} \\
{[71 / 21]}\end{array}$ \\
\hline & $(293-343)$ & 37.2 & $(308)$ & & {$[29 / 2][84 / 9]$} \\
\hline \multirow[t]{10}{*}{$\mathrm{C}_{4} \mathrm{H}_{9} \mathrm{Cl}$} & 2-chlorobutane & & & & [78-86-4] \\
\hline & $(315-341)$ & 30.9 & $(328)$ & $\mathrm{EB}$ & {$[96 / 27]$} \\
\hline & $(266-377)$ & 33.1 & $(281)$ & A & {$[87 / 5]$} \\
\hline & & $31.5 \pm 0.1$ & $(298)$ & $\mathrm{C}$ & {$[81 / 4]$} \\
\hline & & $30.7 \pm 0.1$ & $(313)$ & $\mathrm{C}$ & {$[81 / 4]$} \\
\hline & & $29.9 \pm 0.1$ & $(328)$ & $\mathrm{C}$ & {$[81 / 4]$} \\
\hline & & $29.1 \pm 0.1$ & $(343)$ & $\mathrm{C}$ & {$[81 / 4]$} \\
\hline & & $28.2 \pm 0.1$ & $(358)$ & $\mathrm{C}$ & {$[81 / 4]$} \\
\hline & & $31.6 \pm 0.1$ & $(298)$ & $\mathrm{C}$ & {$[68 / 1]$} \\
\hline & $(273-312)$ & 31.8 & $(288)$ & & {$[28 / 3][84 / 9]$} \\
\hline \multirow[t]{3}{*}{$\mathrm{C}_{4} \mathrm{H}_{9} \mathrm{Cl}$} & 1-chloro-2-methylpropane & & & & {$[513-36-0]$} \\
\hline & $(219-342)$ & 36.1 & $(234)$ & A & {$[87 / 5][47 / 5]$} \\
\hline & & $31.7 \pm 0.1$ & $(298)$ & $\mathrm{C}$ & {$[68 / 1]$} \\
\hline \multirow[t]{5}{*}{$\mathrm{C}_{4} \mathrm{H}_{9} \mathrm{Cl}$} & 2-chloro-2-methylpropane & & & & {$[507-20-0]$} \\
\hline & $(313-353)$ & 28.6 & $(298)$ & CGC & {$[95 / 21]$} \\
\hline & $(253-358)$ & 32.3 & $(268)$ & A & {$[87 / 5][71 / 21]$} \\
\hline & $(295-323)$ & 27.8 & $(309)$ & A & {$[87 / 5][69 / 10]$} \\
\hline & $(295-323)$ & 27.0 & $(310)$ & & {$[69 / 10][84 / 9]$} \\
\hline
\end{tabular}


TABLE 6. Enthalpies of vaporization of organic compounds, 1880-2002-Continued

\begin{tabular}{|c|c|c|c|c|c|}
\hline Molecular formula & $\begin{array}{c}\text { Compound } \\
\text { (Temperature range/K) }\end{array}$ & $\begin{array}{c}\Delta_{\mathrm{vap}} H_{m} \\
\left(\mathrm{~kJ} \mathrm{~mol}^{-1}\right)\end{array}$ & $\begin{array}{l}\text { Mean temperature } \\
\qquad\left(T_{m} / \mathrm{K}\right)\end{array}$ & Method & $\begin{array}{c}\text { CAS registry number } \\
\text { Reference }\end{array}$ \\
\hline & & $29.0 \pm 0.1$ & $(298)$ & $\mathrm{C}$ & {$[68 / 1]$} \\
\hline & $(254-324)$ & 29.1 & $(269)$ & & {$[47 / 5]$} \\
\hline \multirow[t]{2}{*}{$\mathrm{C}_{4} \mathrm{H}_{9} \mathrm{ClO}_{2}$} & 2-(2-chloroethoxy)ethanol & & & & {$[628-89-7]$} \\
\hline & $(326-469)$ & 59.8 & $(341)$ & A & {$[87 / 5][47 / 5]$} \\
\hline \multirow[t]{4}{*}{$\mathrm{C}_{4} \mathrm{H}_{9} \mathrm{ClO}_{2} \mathrm{~S}$} & butyl sulfonyl chloride & & & & [2386-60-9] \\
\hline & $(283-373)$ & 55.7 & $(298)$ & & {$[99 / 16]$} \\
\hline & $(373-474)$ & 52.9 & $(388)$ & & {$[99 / 16]$} \\
\hline & $(253-283)$ & 60.2 & $(268)$ & $\mathrm{A}$ & {$[87 / 5][99 / 16]$} \\
\hline \multirow[t]{2}{*}{$\mathrm{C}_{4} \mathrm{H}_{9} \mathrm{ClS}$} & ethyl (2-chloroethyl) sulfide & & & & [693-07-2] \\
\hline & $(293-333)$ & 44.4 & $(308)$ & A, GS & $\begin{array}{c}{[87 / 5][48 / 9]} \\
{[71 / 21]}\end{array}$ \\
\hline \multirow[t]{2}{*}{$\mathrm{C}_{4} \mathrm{H}_{9} \mathrm{~F}$} & 1-fluorobutane & & & & {$[2366-52-1]$} \\
\hline & $(222-326)$ & 30.1 & $(237)$ & A, EST & $\begin{array}{c}{[87 / 5][61 / 13]} \\
{[71 / 21]}\end{array}$ \\
\hline \multirow[t]{2}{*}{$\mathrm{C}_{4} \mathrm{H}_{9} \mathrm{~F}$} & 2-fluorobutane & & & & [359-01-3] \\
\hline & $(233-329)$ & 29.2 & $(248)$ & A & {$[87 / 5][71 / 21]$} \\
\hline \multirow[t]{2}{*}{$\mathrm{C}_{4} \mathrm{H}_{9} \mathrm{~F}$} & 2-fluoro-2-methylpropane & & & & {$[353-61-7]$} \\
\hline & $(222-315)$ & 27.6 & $(237)$ & A & {$[87 / 5][71 / 21]$} \\
\hline \multirow[t]{2}{*}{$\mathrm{C}_{4} \mathrm{H}_{9} \mathrm{FO}$} & 4-fluoro-1-butanol & & & & {$[372-93-0]$} \\
\hline & $(323-343)$ & 64.0 & (333) & A & {$[87 / 5]$} \\
\hline \multirow[t]{5}{*}{$\mathrm{C}_{4} \mathrm{H}_{9} \mathrm{I}$} & 1-iodobutane & & & & {$[542-69-8]$} \\
\hline & $(313-353)$ & 40.3 & $(298)$ & CGC & {$[95 / 21]$} \\
\hline & $(313-353)$ & 39.7 & $(298)$ & CGC & {$[95 / 21]$} \\
\hline & & $40.6 \pm 0.1$ & (298) & $\mathrm{C}$ & {$[68 / 1]$} \\
\hline & $(292-431)$ & 39.9 & $(307)$ & $\mathrm{A}, \mathrm{EST}$ & $\begin{array}{c}{[87 / 5][61 / 13]} \\
{[71 / 21]}\end{array}$ \\
\hline \multirow[t]{4}{*}{$\mathrm{C}_{4} \mathrm{H}_{9} \mathrm{I}$} & 2-iodobutane & & & & {$[513-48-4]$} \\
\hline & $(313-353)$ & 37.9 & $(298)$ & CGC & {$[95 / 21]$} \\
\hline & $(313-353)$ & 38.8 & $(298)$ & CGC & {$[95 / 21]$} \\
\hline & & $38.5 \pm 0.1$ & $(298)$ & $\mathrm{C}$ & {$[68 / 1]$} \\
\hline \multirow[t]{3}{*}{$\mathrm{C}_{4} \mathrm{H}_{9} \mathrm{I}$} & 1-iodo-2-methylpropane & & & & {$[513-38-2]$} \\
\hline & $(256-393)$ & 41.1 & $(271)$ & $\mathrm{A}$ & {$[87 / 5][47 / 5]$} \\
\hline & & $38.8 \pm 0.1$ & $(298)$ & $\mathrm{C}$ & {$[68 / 1]$} \\
\hline \multirow[t]{4}{*}{$\mathrm{C}_{4} \mathrm{H}_{9} \mathrm{I}$} & 2-iodo-2-methylpropane & & & & {$[558-17-8]$} \\
\hline & $(313-353)$ & 37.0 & $(298)$ & CGC & {$[95 / 21]$} \\
\hline & $(236-294)$ & 34.8 & $(279)$ & A & {$[87 / 5][71 / 21]$} \\
\hline & & $35.4 \pm 0.1$ & $(298)$ & $\mathrm{C}$ & {$[68 / 1]$} \\
\hline \multirow[t]{7}{*}{$\mathrm{C}_{4} \mathrm{H}_{9} \mathrm{~N}$} & pyrrolidine & & & & {$[123-75-1]$} \\
\hline & $(273-313)$ & 38.4 & $(288)$ & A & {$[87 / 5]$} \\
\hline & $(316-394)$ & 35.8 & $(331)$ & $\mathrm{EB}, \mathrm{IPM}$ & $\begin{array}{c}{[87 / 5][59 / 4]} \\
{[68 / 4]}\end{array}$ \\
\hline & & $35.8 \pm 0.1$ & $(322)$ & $\mathrm{C}$ & {$[59 / 4]$} \\
\hline & & $34.5 \pm 0.1$ & $(340)$ & $\mathrm{C}$ & {$[59 / 4]$} \\
\hline & & $33.0 \pm 0.1$ & $(360)$ & $\mathrm{C}$ & {$[59 / 4]$} \\
\hline & $(294-360)$ & 37.3 & (309) & & {$[59 / 10][84 / 9]$} \\
\hline \multirow[t]{3}{*}{$\mathrm{C}_{4} \mathrm{H}_{9} \mathrm{NO}$} & 2-butanone oxime & & & & [96-29-7] \\
\hline & $(308-425)$ & 53.7 & $(323)$ & A & {$[87 / 5]$} \\
\hline & $(318-343)$ & 55.5 & $(330)$ & A & {$[87 / 5]$} \\
\hline \multirow[t]{2}{*}{$\mathrm{C}_{4} \mathrm{H}_{9} \mathrm{NO}$} & N-ethylacetamide & & & & {$[625-50-3]$} \\
\hline & & $64.9 \pm 0.2$ & $(298)$ & $\mathrm{C}$ & {$[84 / 6]$} \\
\hline \multirow[t]{2}{*}{$\mathrm{C}_{4} \mathrm{H}_{9} \mathrm{NO}$} & buyraldehyde oxime & & & & [110-69-0] \\
\hline & $(313-343)$ & 55.8 & $(328)$ & A & {$[87 / 5]$} \\
\hline \multirow[t]{2}{*}{$\mathrm{C}_{4} \mathrm{H}_{9} \mathrm{NO}$} & butyramide & & & & {$[541-35-5]$} \\
\hline & $(397-504)$ & 64.0 & $(412)$ & A & {$[87 / 5]$} \\
\hline $\mathrm{C}_{4} \mathrm{H}_{9} \mathrm{NO}$ & N,N-dimethylacetamide & & & & {$[127-19-5]$} \\
\hline & $(371-423)$ & 45.1 & $(386)$ & A & {$[87 / 5]$} \\
\hline & & 50.2 & $(298)$ & A & {$[85 / 7][85 / 6]$} \\
\hline & $(297-438)$ & 67.9 & (312) & & {$[74 / 7][84 / 9]$} \\
\hline & $(303-363)$ & 45.2 & $(318)$ & A & {$[87 / 5][68 / 3]$} \\
\hline $\mathrm{C}_{4} \mathrm{H}_{9} \mathrm{NO}$ & N-methylpropionamide & & & & {$[1187-58-2]$} \\
\hline & & $66.9 \pm 1.3$ & $(298)$ & $\mathrm{EB}, \mathrm{BG}$ & {$[98 / 14]$} \\
\hline & & $64.9 \pm 0.3$ & $(298)$ & C & {$[84 / 6]$} \\
\hline & $(303-363)$ & 54.4 & (318) & A & {$[87 / 5][68 / 3]$} \\
\hline $\mathrm{C}_{4} \mathrm{H}_{9} \mathrm{NO}$ & morpholine & & & & {$[110-91-8]$} \\
\hline
\end{tabular}


TABLE 6. Enthalpies of vaporization of organic compounds, 1880-2002-Continued

\begin{tabular}{|c|c|c|c|c|c|}
\hline Molecular formula & $\begin{array}{c}\text { Compound } \\
\text { (Temperature range/K) }\end{array}$ & $\begin{array}{c}\Delta_{\mathrm{vap}} H_{m} \\
\left(\mathrm{~kJ} \mathrm{~mol}^{-1}\right)\end{array}$ & $\begin{array}{l}\text { Mean temperature } \\
\qquad\left(T_{m} / \mathrm{K}\right)\end{array}$ & Method & $\begin{array}{c}\text { CAS registry number } \\
\text { Reference }\end{array}$ \\
\hline & $(274-303)$ & $45.6 \pm 0.4$ & $(288)$ & GS & {$[98 / 13]$} \\
\hline & $(274-303)$ & $45.0 \pm 0.4$ & $(298)$ & GS & {$[98 / 13]$} \\
\hline & $(313-343)$ & 44.3 & (328) & TGA & {$[87 / 18]$} \\
\hline & $(273-318)$ & 45.3 & (288) & A & {$[87 / 5]$} \\
\hline & $(317-443)$ & 42.3 & $(332)$ & A & {$[87 / 5]$} \\
\hline \multirow[t]{2}{*}{$\mathrm{C}_{4} \mathrm{H}_{9} \mathrm{NO}_{2}$} & ( $d l$ ) 2-aminobutyric acid & & & & {$[2835-81-6]$} \\
\hline & $(400-418)$ & 132.0 & $(409)$ & $\mathrm{A}$ & {$[87 / 5]$} \\
\hline \multirow{2}{*}{$\mathrm{C}_{4} \mathrm{H}_{9} \mathrm{NO}_{2}$} & S 2-aminobutyric acid & & & & {$[1492-24-6]$} \\
\hline & $(449-462)$ & 162.5 & $(455)$ & A & {$[87 / 5]$} \\
\hline \multirow[t]{2}{*}{$\mathrm{C}_{4} \mathrm{H}_{9} \mathrm{NO}_{2}$} & sec-butyl nitrite & & & & {$[924-43-6]$} \\
\hline & $(267-287)$ & 29.6 & $(277)$ & $\mathrm{A}$ & {$[87 / 5][37 / 3]$} \\
\hline \multirow[t]{2}{*}{$\mathrm{C}_{4} \mathrm{H}_{9} \mathrm{NO}_{2}$} & tert-butyl nitrite & & & & {$[540-80-7]$} \\
\hline & $(267-337)$ & 30.8 & $(282)$ & A & {$[87 / 5][37 / 3]$} \\
\hline $\mathrm{C}_{4} \mathrm{H}_{9} \mathrm{NO}_{2}$ & $\begin{array}{l}\text { lactic acid N-methyl amide } \\
(359-415)\end{array}$ & 72.7 & (374) & A & {$[87 / 5]$} \\
\hline \multirow[t]{2}{*}{$\mathrm{C}_{4} \mathrm{H}_{9} \mathrm{NO}_{2}$} & $\mathrm{~N}$-methyl carbamic acid, ethyl ester & & & & {$[105-40-8]$} \\
\hline & $(299-443)$ & 51.7 & $(314)$ & A & {$[87 / 5][47 / 5]$} \\
\hline \multirow[t]{2}{*}{$\mathrm{C}_{4} \mathrm{H}_{9} \mathrm{NO}_{2}$} & 2-methyl-1-nitropropane & & & & {$[625-74-1]$} \\
\hline & $(347-415)$ & 41.1 & $(362)$ & $\mathrm{A}, \mathrm{EB}$ & $\begin{array}{c}{[87 / 5][56 / 15]} \\
{[71 / 21]}\end{array}$ \\
\hline \multirow[t]{2}{*}{$\mathrm{C}_{4} \mathrm{H}_{9} \mathrm{NO}_{2}$} & 2-methyl-2-nitropropane & & & & {$[594-70-7]$} \\
\hline & $(334-401)$ & 39.1 & $(349)$ & EB & $\begin{array}{c}{[87 / 5][56 / 15]} \\
{[71 / 21]}\end{array}$ \\
\hline \multirow[t]{3}{*}{$\mathrm{C}_{4} \mathrm{H}_{9} \mathrm{NO}_{2}$} & 1-nitrobutane & & & & {$[627-05-4]$} \\
\hline & $(313-353)$ & 47.0 & $(298)$ & CGC & {$[95 / 21]$} \\
\hline & $(357-426)$ & 42.7 & $(372)$ & $\mathrm{A}, \mathrm{EB}$ & $\begin{array}{c}{[87 / 5][56 / 15]} \\
{[71 / 21]}\end{array}$ \\
\hline \multirow[t]{2}{*}{$\mathrm{C}_{4} \mathrm{H}_{9} \mathrm{NO}_{2}$} & $(d l)$ 2-nitrobutane & & & & [600-24-8] \\
\hline & $(345-413)$ & 40.3 & $(360)$ & $\mathrm{A}, \mathrm{EB}$ & $\begin{array}{c}{[87 / 5][56 / 15]} \\
{[71 / 21]}\end{array}$ \\
\hline \multirow[t]{2}{*}{$\mathrm{C}_{4} \mathrm{H}_{9} \mathrm{NO}_{2}$} & propyl carbamate & & & & {$[627-12-3]$} \\
\hline & $(325-468)$ & 61.6 & $(340)$ & A & {$[87 / 5][47 / 5]$} \\
\hline \multirow[t]{2}{*}{$\mathrm{C}_{4} \mathrm{H}_{9} \mathrm{NO}_{3}$} & butyl nitrate & & & & {$[928-45-0]$} \\
\hline & $(273-343)$ & 44.1 & $(288)$ & A & $\begin{array}{c}{[87 / 5][71 / 21]} \\
{[57 / 2]}\end{array}$ \\
\hline \multirow[t]{2}{*}{$\mathrm{C}_{4} \mathrm{H}_{9} \mathrm{NO}_{3}$} & isobutyl nitrate & & & & [543-29-3] \\
\hline & $(273-343)$ & 42.8 & $(288)$ & A & $\begin{array}{c}{[87 / 5][71 / 21]} \\
{[57 / 2]}\end{array}$ \\
\hline $\mathrm{C}_{4} \mathrm{H}_{9} \mathrm{~N}_{3} \mathrm{O}_{2}$ & bis(nitrosoethyl)amine & 464 & (306) & $\Delta$ & [87/5] \\
\hline \multirow[t]{2}{*}{$\mathrm{C}_{4} \mathrm{H}_{9} \mathrm{P}$} & allymethylphosphine & 46.4 & $(306)$ & A & {$[62778-93-2]$} \\
\hline & $(242-291)$ & 34.4 & $(276)$ & A & {$[87 / 5]$} \\
\hline \multirow[t]{2}{*}{$\mathrm{C}_{4} \mathrm{H}_{9} \mathrm{P}$} & 3-butenylphosphine & & & & [114596-01-9] \\
\hline & $(252-295)$ & 34.5 & $(273)$ & & {$[88 / 13]$} \\
\hline \multirow[t]{2}{*}{$\mathrm{C}_{4} \mathrm{H}_{9} \mathrm{P}$} & phospholane & & & & [3466-00-0] \\
\hline & $(257-347)$ & 37.4 & $(272)$ & A & {$[87 / 5]$} \\
\hline \multirow[t]{10}{*}{$\mathrm{C}_{4} \mathrm{H}_{10}$} & butane & & & & {$[106-97-8]$} \\
\hline & $(300-315)$ & 22.9 & $(308)$ & & {$[97 / 16]$} \\
\hline & $(195-292)$ & 23.4 & $(277)$ & A & {$[87 / 5]$} \\
\hline & $(273-321)$ & 23.2 & (288) & A & {$[87 / 5]$} \\
\hline & $(316-383)$ & 22.6 & (331) & $\mathrm{A}$ & {$[87 / 5]$} \\
\hline & $(375-425)$ & 22.8 & (390) & $\mathrm{A}$ & {$[87 / 5]$} \\
\hline & $(135-213)$ & 27.0 & (198) & $\mathrm{A}$ & {$[87 / 5][73 / 11]$} \\
\hline & & 22.4 & $(298)$ & & {$[71 / 28]$} \\
\hline & $(206-279)$ & 23.1 & (264) & & {$[45 / 4][84 / 9]$} \\
\hline & $(195-273)$ & 23.9 & $(258)$ & & {$[40 / 10][84 / 9]$} \\
\hline $\mathrm{C}_{4} \mathrm{H}_{10}$ & 2-methylpropene (isobutene) & & & & {$[75-28-5]$} \\
\hline & $(303-333)$ & 21.5 & $(318)$ & & [99/29] \\
\hline & $(186-280)$ & 22.4 & $(265)$ & $\mathrm{A}$ & {$[87 / 5]$} \\
\hline & $(121-187)$ & 26.9 & (172) & A & {$[87 / 5]$} \\
\hline & $(263-306)$ & 21.9 & (278) & A & {$[87 / 5]$} \\
\hline & $(301-366)$ & 21.4 & (316) & A & {$[87 / 5]$} \\
\hline & $(361-408)$ & 21.6 & (376) & A & {$[87 / 5]$} \\
\hline & $(277-344)$ & 21.6 & (292) & & {$[76 / 19][84 / 9]$} \\
\hline
\end{tabular}


TABLE 6. Enthalpies of vaporization of organic compounds, 1880-2002-Continued

\begin{tabular}{|c|c|c|c|c|c|}
\hline Molecular formula & $\begin{array}{c}\text { Compound } \\
\text { (Temperature range/K) }\end{array}$ & $\begin{array}{c}\Delta_{\mathrm{vap}} H_{m} \\
\left(\mathrm{~kJ} \mathrm{~mol}^{-1}\right)\end{array}$ & $\begin{array}{l}\text { Mean temperature } \\
\qquad\left(T_{m} / \mathrm{K}\right)\end{array}$ & Method & $\begin{array}{c}\text { CAS registry number } \\
\text { Reference }\end{array}$ \\
\hline & & 21.3 & $(286)$ & & {$[71 / 28]$} \\
\hline & $(188-262)$ & 22.6 & $(247)$ & & {$[40 / 11][84 / 9]$} \\
\hline \multirow[t]{2}{*}{$\mathrm{C}_{4} \mathrm{H}_{10} \mathrm{~F}_{3} \mathrm{NOS}$} & (diethylaminato)trifluoroox & & & & [26458-94-6] \\
\hline & $(329-354)$ & 49.5 & $(341)$ & A & {$[87 / 5][99 / 16]$} \\
\hline \multirow[t]{2}{*}{$\mathrm{C}_{4} \mathrm{H}_{10} \mathrm{~F}_{3} \mathrm{NS}$} & (N-ethylethaneaminato)trif & & & & [38078-09-0] \\
\hline & $(318-340)$ & 45.2 & $(329)$ & A & {$[87 / 5][99 / 16]$} \\
\hline \multirow[t]{2}{*}{$\mathrm{C}_{4} \mathrm{H}_{10} \mathrm{~N}_{2}$} & piperazine & & & & {$[275-02-5]$} \\
\hline & $(417-460)$ & $50.1 \pm 1.9$ & $(298)$ & EB & {$[97 / 7]$} \\
\hline \multirow[t]{2}{*}{$\mathrm{C}_{4} \mathrm{H}_{10} \mathrm{~N}_{2} \mathrm{O}_{2}$} & diethylnitramine & & & & [7119-92-8] \\
\hline & $(338-378)$ & 49.7 & $(358)$ & & {$[58 / 22]$} \\
\hline \multirow[t]{38}{*}{$\mathrm{C}_{4} \mathrm{H}_{10} \mathrm{O}$} & 1-butanol & & & & {$[71-36-3]$} \\
\hline & $(357-389)$ & 46.0 & $(372)$ & $\mathrm{EB}$ & {$[01 / 15]$} \\
\hline & & 38.2 & $(423)$ & & {$[00 / 20]$} \\
\hline & & 29.6 & $(473)$ & & {$[00 / 20]$} \\
\hline & & 20.8 & $(523)$ & & {$[00 / 20]$} \\
\hline & & 44.1 & & & {$[99 / 32]$} \\
\hline & $(323-373)$ & 52.5 & $(298)$ & CGC & {$[95 / 21]$} \\
\hline & $(315-390)$ & 49.9 & $(330)$ & & {$[95 / 13]$} \\
\hline & $(364-403)$ & 45.3 & $(379)$ & & {$[93 / 7]$} \\
\hline & $(283-323)$ & 55.2 & (298) & & {$[92 / 9]$} \\
\hline & $(376-399)$ & 45.3 & $(387)$ & A & {$[87 / 5]$} \\
\hline & $(323-413)$ & 50.1 & (338) & A & {$[87 / 5]$} \\
\hline & $(413-550)$ & 41.9 & $(428)$ & A & {$[87 / 5]$} \\
\hline & $(209-251)$ & 51.6 & $(236)$ & A & {$[87 / 5]$} \\
\hline & $(376-397)$ & 45.4 & (386) & A & {$[87 / 5]$} \\
\hline & $(391-429)$ & 43.8 & $(406)$ & A & {$[87 / 5]$} \\
\hline & $(415-501)$ & 41.9 & $(430)$ & A & {$[87 / 5]$} \\
\hline & $(497-563)$ & 37.4 & $(512)$ & A & {$[87 / 5]$} \\
\hline & $(243-303)$ & 51.7 & (298) & & {$[83 / 14]$} \\
\hline & $(329-391)$ & 49.0 & (344) & & [82/13] \\
\hline & & 52.1 & (298) & $\mathrm{C}$ & {$[82 / 6]$} \\
\hline & $(288-404)$ & 55.0 & (303) & & {$[73 / 26]$} \\
\hline & & $49.5 \pm 0.1$ & (333) & $\mathrm{C}$ & {$[73 / 13]$} \\
\hline & & $48.6 \pm 0.1$ & $(343)$ & $\mathrm{C}$ & [73/13] \\
\hline & & $47.5 \pm 0.1$ & (353) & $\mathrm{C}$ & {$[73 / 13]$} \\
\hline & & $46.4 \pm 0.1$ & $(363)$ & $\mathrm{C}$ & {$[73 / 13]$} \\
\hline & & $52.34 \pm 0.02$ & (298) & $\mathrm{C}$ & [71/27] \\
\hline & $(351-397)$ & 47.2 & $(366)$ & $\mathrm{EB}$ & {$[87 / 5][70 / 2]$} \\
\hline & $(295-391)$ & 53.0 & (310) & DTA & {$[69 / 5]$} \\
\hline & & $52.3 \pm 0.1$ & (298) & $\mathrm{C}$ & {$[66 / 2]$} \\
\hline & & $47.2 \pm 0.1$ & $(356)$ & $\mathrm{C}$ & {$[65 / 16]$} \\
\hline & & $45.4 \pm 0.1$ & $(381)$ & $\mathrm{C}$ & {$[65 / 16]$} \\
\hline & & $43.1 \pm 0.1$ & (391) & $\mathrm{C}$ & {$[65 / 16]$} \\
\hline & $(419-563)$ & 42.1 & (434) & & {$[63 / 20]$} \\
\hline & $(362-398)$ & 46.6 & (377) & $\mathrm{EB}$ & {$[63 / 8]$} \\
\hline & & $51.0 \pm 0.1$ & (298) & $\mathrm{C}$ & {$[63 / 2]$} \\
\hline & $(337-390)$ & 48.3 & $(352)$ & & {$[59 / 11][84 / 9]$} \\
\hline & $(314-390)$ & 48.3 & $(352)$ & & {$[1898][84 / 9]$} \\
\hline \multirow[t]{16}{*}{$\mathrm{C}_{4} \mathrm{H}_{10} \mathrm{O}$} & 2-butanol & & & & [78-92-2] \\
\hline & $(306-373)$ & 47.7 & $(321)$ & & [95/13] \\
\hline & $(303-403)$ & 49.3 & (318) & A & {$[87 / 5]$} \\
\hline & $(359-381)$ & 43.2 & $(370)$ & A & {$[87 / 5]$} \\
\hline & $(372-524)$ & 47.9 & $(387)$ & A & {$[87 / 5]$} \\
\hline & $(210-303)$ & 57.5 & $(225)$ & A & {$[87 / 5]$} \\
\hline & $(359-380)$ & 43.2 & $(369)$ & A & {$[87 / 5]$} \\
\hline & $(368-404)$ & 42.0 & $(383)$ & A & {$[87 / 5]$} \\
\hline & $(395-485)$ & 39.6 & $(410)$ & A & {$[87 / 5]$} \\
\hline & $(476-536)$ & 35.0 & $(491)$ & A & {$[87 / 5]$} \\
\hline & $(307-373)$ & 47.8 & $(322)$ & & {$[82 / 13]$} \\
\hline & $(293-380)$ & 53.2 & $(308)$ & & {$[78 / 20]$} \\
\hline & $(319-372)$ & 44.1 & (334) & & {$[75 / 23]$} \\
\hline & $(280-314)$ & 50.2 & $(295)$ & & {$[75 / 1]$} \\
\hline & $(298-393)$ & 48.1 & (313) & & {$[73 / 26]$} \\
\hline & & $49.74 \pm 0.02$ & (298) & $\mathrm{C}$ & {$[71 / 27]$} \\
\hline
\end{tabular}


TABLE 6. Enthalpies of vaporization of organic compounds, 1880-2002-Continued

\begin{tabular}{|c|c|c|c|c|c|}
\hline Molecular formula & $\begin{array}{c}\text { Compound } \\
\text { (Temperature range/K) }\end{array}$ & $\begin{array}{c}\Delta_{\mathrm{vap}} H_{m} \\
\left(\mathrm{~kJ} \mathrm{~mol}^{-1}\right)\end{array}$ & $\begin{array}{l}\text { Mean temperature } \\
\qquad\left(T_{m} / \mathrm{K}\right)\end{array}$ & Method & $\begin{array}{c}\text { CAS registry number } \\
\text { Reference }\end{array}$ \\
\hline & $(323-373)$ & 46.3 & $(338)$ & & {$[69 / 19][84 / 9]$} \\
\hline & & $49.7 \pm 0.1$ & (298) & $\mathrm{C}$ & {$[66 / 2]$} \\
\hline & $(422-538)$ & 38.4 & $(437)$ & & {$[63 / 20]$} \\
\hline & $(345-381)$ & 44.1 & $(360)$ & EB & {$[63 / 8]$} \\
\hline & & 48.5 & $(298)$ & $\mathrm{C}$ & {$[63 / 2]$} \\
\hline & $(340-379)$ & 44.7 & $(355)$ & $\mathrm{EB}$ & {$[87 / 5][62 / 15]$} \\
\hline & & & & & {$[70 / 2]$} \\
\hline & & $45.3 \pm 0.1$ & $(340)$ & $\mathrm{C}$ & {$[62 / 15]$} \\
\hline & & $43.3 \pm 0.1$ & $(355)$ & $\mathrm{C}$ & {$[62 / 15]$} \\
\hline & & $41.9 \pm 0.1$ & $(365)$ & $\mathrm{C}$ & {$[62 / 15]$} \\
\hline & & $40.8 \pm 0.1$ & $(372)$ & $\mathrm{C}$ & {$[62 / 15]$} \\
\hline \multirow[t]{25}{*}{$\mathrm{C}_{4} \mathrm{H}_{10} \mathrm{O}$} & 2-methyl-1-propanol & & & & {$[78-83-1]$} \\
\hline & $(350-400)$ & 45.4 & $(365)$ & $\mathrm{EB}$ & [93/5] \\
\hline & $(313-411)$ & 49.5 & (328) & A & {$[87 / 5]$} \\
\hline & $(381-524)$ & 46.0 & (396) & A & {$[87 / 5]$} \\
\hline & $(202-243)$ & 55.0 & $(228)$ & A & {$[87 / 5]$} \\
\hline & $(369-389)$ & 44.2 & $(379)$ & A & {$[87 / 5]$} \\
\hline & $(383-416)$ & 42.6 & (398) & A & {$[87 / 5]$} \\
\hline & $(401-493)$ & 41.1 & $(416)$ & A & {$[87 / 5]$} \\
\hline & $(483-548)$ & 36.2 & (498) & A & {$[87 / 5]$} \\
\hline & & $50.8 \pm 0.1$ & $(298)$ & $\mathrm{C}$ & {$[84 / 5]$} \\
\hline & & $49.7 \pm 0.1$ & (313) & $\mathrm{C}$ & {$[84 / 5]$} \\
\hline & & $48.3 \pm 0.1$ & $(328)$ & $\mathrm{C}$ & {$[84 / 5]$} \\
\hline & & $45.0 \pm 0.1$ & $(358)$ & $\mathrm{C}$ & {$[84 / 5]$} \\
\hline & $(320-382)$ & 48.1 & $(335)$ & & {$[82 / 13]$} \\
\hline & $(293-388)$ & 52.6 & $(308)$ & & {$[73 / 26]$} \\
\hline & & $50.79 \pm 0.02$ & (298) & $\mathrm{C}$ & {$[71 / 27]$} \\
\hline & & $46.2 \pm 0.1$ & $(347)$ & $\mathrm{C}$ & {$[70 / 20]$} \\
\hline & & $44.2 \pm 0.1$ & $(363)$ & $\mathrm{C}$ & {$[70 / 20]$} \\
\hline & & $41.9 \pm 0.1$ & $(381)$ & $\mathrm{C}$ & {$[70 / 20]$} \\
\hline & $(342-389)$ & 46.2 & $(357)$ & $\mathrm{A}, \mathrm{EB}$ & {$[87 / 5][70 / 2]$} \\
\hline & $(333-381)$ & 47.0 & $(348)$ & & {$[69 / 19][84 / 9]$} \\
\hline & & $50.8 \pm 0.1$ & (298) & $\mathrm{C}$ & {$[66 / 2]$} \\
\hline & $(423-548)$ & 40.1 & $(438)$ & & {$[63 / 20]$} \\
\hline & $(353-388)$ & 45.2 & $(368)$ & $\mathrm{EB}$ & {$[63 / 8]$} \\
\hline & & 49.8 & $(298)$ & $\mathrm{C}$ & {$[63 / 2]$} \\
\hline \multirow[t]{28}{*}{$\mathrm{C}_{4} \mathrm{H}_{10} \mathrm{O}$} & 2-methyl-2-propanol & & & & {$[75-65-0]$} \\
\hline & $(321-359)$ & 43.4 & $(336)$ & & {$[99 / 30]$} \\
\hline & $(323-373)$ & 45.4 & (298) & $\mathrm{CGC}$ & {$[95 / 21]$} \\
\hline & $(299-375)$ & 46.2 & $(314)$ & A & {$[87 / 5]$} \\
\hline & $(347-363)$ & 41.4 & $(355)$ & A & {$[87 / 5]$} \\
\hline & $(356-480)$ & 43.2 & $(371)$ & A & {$[87 / 5]$} \\
\hline & $(347-363)$ & 41.4 & $(355)$ & A & {$[87 / 5]$} \\
\hline & $(357-461)$ & 39.8 & $(372)$ & A & {$[87 / 5]$} \\
\hline & $(453-506)$ & 33.6 & $(468)$ & A & {$[87 / 5]$} \\
\hline & & $46.2 \pm 0.1$ & (303) & $\mathrm{C}$ & {$[84 / 5]$} \\
\hline & & $44.9 \pm 0.1$ & (313) & $\mathrm{C}$ & {$[84 / 5]$} \\
\hline & & $43.0 \pm 0.1$ & (328) & $\mathrm{C}$ & {$[84 / 5]$} \\
\hline & & $41.0 \pm 0.1$ & $(343)$ & $\mathrm{C}$ & {$[84 / 5]$} \\
\hline & & $37.2 \pm 0.1$ & $(368)$ & $\mathrm{C}$ & {$[84 / 5]$} \\
\hline & $(306-357)$ & 44.7 & $(321)$ & & [82/13] \\
\hline & $(293-376)$ & 46.5 & (308) & & {$[73 / 26]$} \\
\hline & & $46.94 \pm 0.02$ & (298) & $\mathrm{C}$ & {$[71 / 27]$} \\
\hline & $(313-355)$ & 44.2 & (328) & & {$[69 / 19][84 / 9]$} \\
\hline & & $46.6 \pm 0.1$ & (298) & $\mathrm{C}$ & {$[66 / 2]$} \\
\hline & $(333-363)$ & 42.1 & $(348)$ & $\mathrm{EB}$ & [63/18] \\
\hline & & $42.5 \pm 0.1$ & $(330)$ & $\mathrm{C}$ & {$[63 / 18]$} \\
\hline & & $41.3 \pm 0.1$ & $(340)$ & $\mathrm{C}$ & {$[63 / 18]$} \\
\hline & & $40.4 \pm 0.1$ & $(346)$ & $\mathrm{C}$ & {$[63 / 18]$} \\
\hline & & $40.0 \pm 0.1$ & (349) & $\mathrm{C}$ & {$[63 / 18]$} \\
\hline & & $39.0 \pm 0.1$ & $(356)$ & $\mathrm{C}$ & {$[63 / 18]$} \\
\hline & $(329-363)$ & 42.6 & $(344)$ & $\mathrm{EB}$ & {$[87 / 5][70 / 2]$} \\
\hline & & & & & [63/18] \\
\hline & & 44.9 & (298) & $\mathrm{C}$ & {$[63 / 2]$} \\
\hline
\end{tabular}


TABLE 6. Enthalpies of vaporization of organic compounds, 1880-2002-Continued

\begin{tabular}{|c|c|c|c|c|c|}
\hline Molecular formula & $\begin{array}{c}\text { Compound } \\
\text { (Temperature range/K) }\end{array}$ & $\begin{array}{c}\Delta_{\mathrm{vap}} H_{m} \\
\left(\mathrm{~kJ} \mathrm{~mol}^{-1}\right)\end{array}$ & $\begin{array}{l}\text { Mean temperature } \\
\qquad\left(T_{m} / \mathrm{K}\right)\end{array}$ & Method & $\begin{array}{c}\text { CAS registry number } \\
\text { Reference }\end{array}$ \\
\hline & $(373-506)$ & 38.7 & $(388)$ & & {$[63 / 20]$} \\
\hline & $(293-363)$ & 44.7 & $(323)$ & & {$[28 / 5]$} \\
\hline \multirow[t]{10}{*}{$\mathrm{C}_{4} \mathrm{H}_{10} \mathrm{O}$} & diethyl ether & & & & {$[60-29-7]$} \\
\hline & $(286-329)$ & 28.1 & $(301)$ & A & {$[87 / 5]$} \\
\hline & $(307-457)$ & 26.9 & $(322)$ & A & {$[87 / 5]$} \\
\hline & $(305-360)$ & 27.5 & $(320)$ & A & {$[87 / 5]$} \\
\hline & $(351-420)$ & 26.6 & $(366)$ & A & {$[87 / 5]$} \\
\hline & $(417-467)$ & 26.7 & $(432)$ & A & {$[87 / 5]$} \\
\hline & & $27.1 \pm 0.1$ & $(298)$ & $\mathrm{C}$ & {$[80 / 3]$} \\
\hline & $(250-329)$ & 27.2 & $(298)$ & & {$[76 / 2]$} \\
\hline & $(250-329)$ & 29.5 & $(265)$ & A & $\begin{array}{c}{[87 / 5][72 / 9]} \\
{[76 / 2]}\end{array}$ \\
\hline & $(213-293)$ & 28.4 & $(278)$ & & {$[22 / 2]$} \\
\hline \multirow[t]{5}{*}{$\mathrm{C}_{4} \mathrm{H}_{10} \mathrm{O}$} & isopropyl methyl ether & & & & {$[598-53-8]$} \\
\hline & $(250-325)$ & 28.8 & $(265)$ & A & {$[87 / 5]$} \\
\hline & & $26.4 \pm 0.1$ & $(298)$ & $\mathrm{C}$ & [80/3] \\
\hline & $(260-325)$ & 28.4 & $(275)$ & A & {$[87 / 5][76 / 2]$} \\
\hline & & 26.4 & $(298)$ & & {$[76 / 2]$} \\
\hline \multirow[t]{9}{*}{$\mathrm{C}_{4} \mathrm{H}_{10} \mathrm{O}$} & methyl propyl ether & & & & {$[557-17-5]$} \\
\hline & $(325-407)$ & 27.2 & $(340)$ & A & {$[87 / 5]$} \\
\hline & $(401-476)$ & 26.7 & $(416)$ & A & {$[87 / 5]$} \\
\hline & $(273-321)$ & 30.7 & $(288)$ & A & {$[87 / 5]$} \\
\hline & & $27.6 \pm 0.1$ & (298) & $\mathrm{C}$ & {$[80 / 3]$} \\
\hline & $(253-328)$ & 29.7 & $(268)$ & A & {$[87 / 5][76 / 2]$} \\
\hline & & 27.5 & $(298)$ & & {$[76 / 2]$} \\
\hline & & $27.9 \pm 0.2$ & $(298)$ & $\mathrm{C}$ & {$[75 / 3]$} \\
\hline & $(273-312)$ & 29.7 & $(288)$ & & {$[10 / 1][84 / 9]$} \\
\hline \multirow[t]{2}{*}{$\mathrm{C}_{4} \mathrm{H}_{10} \mathrm{O}_{2}$} & $( \pm)$ 1,2-butanediol & & & & [26171-83-5] \\
\hline & $(372-506)$ & $71.6 \pm 0.8$ & $(298)$ & $\mathrm{EB}$ & {$[96 / 3]$} \\
\hline \multirow[t]{5}{*}{$\mathrm{C}_{4} \mathrm{H}_{10} \mathrm{O}_{2}$} & $( \pm)$ 1,3-butanediol & & & & [107-88-0] \\
\hline & $(365-518)$ & $74.5 \pm 1.0$ & $(298)$ & EB & {$[96 / 3]$} \\
\hline & $(362-483)$ & 67.6 & $(377)$ & A & {$[87 / 5]$} \\
\hline & $(373-423)$ & 59.7 & (398) & & {$[35 / 4]$} \\
\hline & $(423-480)$ & 58.1 & $(451)$ & & [35/4] \\
\hline \multirow[t]{3}{*}{$\mathrm{C}_{4} \mathrm{H}_{10} \mathrm{O}_{2}$} & 1,4-butanediol & & & & {$[110-63-4]$} \\
\hline & & $79.3 \pm 0.5$ & $(298)$ & $\mathrm{C}$ & {$[88 / 14]$} \\
\hline & $(380-510)$ & 72.0 & $(395)$ & A & {$[87 / 5]$} \\
\hline \multirow[t]{5}{*}{$\mathrm{C}_{4} \mathrm{H}_{10} \mathrm{O}_{2}$} & (dl) 2,3-butanediol & & & & {$[513-85-9]$} \\
\hline & $(348-457)$ & 62.5 & $(363)$ & A & {$[87 / 5]$} \\
\hline & $(317-455)$ & 58.4 & $(332)$ & & {$[47 / 5]$} \\
\hline & $(353-403)$ & 57.9 & $(378)$ & & {$[35 / 4]$} \\
\hline & $(303-456)$ & 55.7 & $(380)$ & & {$[35 / 4]$} \\
\hline \multirow[t]{2}{*}{$\mathrm{C}_{4} \mathrm{H}_{10} \mathrm{O}_{2}$} & meso 2,3-butanediol & & & & \\
\hline & $(413-453)$ & 54.6 & $(433)$ & & {$[46 / 12]$} \\
\hline \multirow[t]{2}{*}{$\mathrm{C}_{4} \mathrm{H}_{10} \mathrm{O}_{2}$} & (l) 2,3-butanediol & & & & \\
\hline & $(413-453)$ & 52.6 & $(433)$ & & {$[46 / 12]$} \\
\hline \multirow[t]{2}{*}{$\mathrm{C}_{4} \mathrm{H}_{10} \mathrm{O}_{2}$} & diethylperoxide & & & & [628-37-5] \\
\hline & $(253-333)$ & 29.0 & $(268)$ & A & $\begin{array}{c}{[87 / 5][51 / 10]} \\
{[71 / 21]}\end{array}$ \\
\hline \multirow[t]{3}{*}{$\mathrm{C}_{4} \mathrm{H}_{10} \mathrm{O}_{2}$} & 1,1-dimethoxyethane & & & & {$[534-15-6]$} \\
\hline & & $36.4 \pm 0.1$ & $(298)$ & $\mathrm{C}$ & {$[70 / 17]$} \\
\hline & $(273-333)$ & 33.4 & $(288)$ & A & $\begin{array}{c}{[87 / 5][49 / 2]} \\
{[71 / 21]}\end{array}$ \\
\hline \multirow[t]{5}{*}{$\mathrm{C}_{4} \mathrm{H}_{10} \mathrm{O}_{2}$} & 1,2-dimethoxyethane & & & & {$[110-71-4]$} \\
\hline & $(305-392)$ & $36.8 \pm 0.2$ & $(298)$ & EB & {$[96 / 5]$} \\
\hline & $(238-298)$ & 39.4 & $(253)$ & A & {$[87 / 5]$} \\
\hline & $(238-363)$ & 39.1 & $(253)$ & A & {$[87 / 5]$} \\
\hline & $(225-366)$ & 33.9 & $(240)$ & & {$[47 / 5]$} \\
\hline \multirow[t]{5}{*}{$\mathrm{C}_{4} \mathrm{H}_{10} \mathrm{O}_{2}$} & 2-ethoxyethanol & & & & [110-80-5] \\
\hline & $(310-385)$ & 47.4 & $(325)$ & EB & {$[01 / 17]$} \\
\hline & $(323-353)$ & 45.9 & $(338)$ & TGA & {$[87 / 18]$} \\
\hline & & $48.2 \pm 0.1$ & $(298)$ & $\mathrm{C}$ & {$[71 / 5]$} \\
\hline & $(336-408)$ & 44.7 & $(351)$ & $\mathrm{A}$ & {$[87 / 5][56 / 6]$} \\
\hline $\mathrm{C}_{4} \mathrm{H}_{10} \mathrm{O}_{2} \mathrm{~S}$ & bis (2-hydroxyethyl) sulfide & & & & {$[111-48-8]$} \\
\hline
\end{tabular}


TABLE 6. Enthalpies of vaporization of organic compounds, 1880-2002-Continued

\begin{tabular}{|c|c|c|c|c|c|}
\hline Molecular formula & $\begin{array}{c}\text { Compound } \\
\text { (Temperature range/K) }\end{array}$ & $\begin{array}{c}\Delta_{\mathrm{vap}} H_{m} \\
\left(\mathrm{~kJ} \mathrm{~mol}^{-1}\right)\end{array}$ & $\begin{array}{l}\text { Mean temperature } \\
\qquad\left(T_{m} / \mathrm{K}\right)\end{array}$ & Method & $\begin{array}{c}\text { CAS registry number } \\
\text { Reference }\end{array}$ \\
\hline & $(368-483)$ & 27.1 & $(383)$ & A & {$[87 / 5]$} \\
\hline & $(315-558)$ & 28.3 & $(330)$ & & {$[47 / 5]$} \\
\hline \multirow[t]{9}{*}{$\mathrm{C}_{4} \mathrm{H}_{10} \mathrm{O}_{3}$} & diethylene glycol & & & & {$[111-46-6]$} \\
\hline & $(410-539)$ & $66.9 \pm 0.3$ & $(420)$ & $\mathrm{EB}$ & {$[02 / 15]$} \\
\hline & $(410-539)$ & $63.1 \pm 0.3$ & $(460)$ & $\mathrm{EB}$ & {$[02 / 15]$} \\
\hline & $(410-539)$ & $59.2 \pm 0.3$ & $(500)$ & $\mathrm{EB}$ & {$[02 / 15]$} \\
\hline & $(410-539)$ & $55.1 \pm 0.5$ & $(540)$ & $\mathrm{EB}$ & {$[02 / 15]$} \\
\hline & $(373-453)$ & 66.5 & $(413)$ & TGA & {$[87 / 18]$} \\
\hline & $(364-518)$ & 59.8 & $(379)$ & A & {$[87 / 5]$} \\
\hline & $(412-513)$ & 66.8 & $(427)$ & & {$[81 / 17][84 / 9]$} \\
\hline & $(403-513)$ & 69.2 & $(418)$ & & {$[27 / 1][84 / 9]$} \\
\hline \multirow{2}{*}{$\mathrm{C}_{4} \mathrm{H}_{10} \mathrm{O}_{3}$} & 1,2,3-butanetriol & & & & {$[4435-50-1]$} \\
\hline & $(375-537)$ & 68.1 & $(390)$ & & {$[47 / 5]$} \\
\hline \multirow[t]{3}{*}{$\mathrm{C}_{4} \mathrm{H}_{10} \mathrm{O}_{3}$} & orthoformic acid trimethyl ester & & & & {$[149-73-5]$} \\
\hline & $(273-358)$ & 39.0 & $(288)$ & A & {$[87 / 5]$} \\
\hline & & $38.1 \pm 0.8$ & $(298)$ & & {$[71 / 26]$} \\
\hline \multirow[t]{3}{*}{$\mathrm{C}_{4} \mathrm{H}_{10} \mathrm{O}_{3} \mathrm{~S}$} & diethyl sulfite & & & & {$[623-81-4]$} \\
\hline & & 44.7 & & & {$[75 / 43]$} \\
\hline & $(283-431)$ & 44.5 & $(298)$ & A & $\begin{array}{c}{[87 / 5][47 / 5]} \\
{[99 / 16]}\end{array}$ \\
\hline \multirow[t]{3}{*}{$\mathrm{C}_{4} \mathrm{H}_{10} \mathrm{O}_{4}$} & meso erythritol & & & & {$[149-32-6]$} \\
\hline & $(397-428)$ & $113.6 \pm 1.1$ & $(412)$ & $\mathrm{TE}$ & [90/16] \\
\hline & $(394-401)$ & 93.3 & $(397)$ & $\mathrm{A}$ & {$[87 / 5]$} \\
\hline \multirow[t]{3}{*}{$\mathrm{C}_{4} \mathrm{H}_{10} \mathrm{O}_{4} \mathrm{~S}$} & diethyl sulfate & & & & {$[64-67-5]$} \\
\hline & $(413-484)$ & 50.1 & $(428)$ & A & {$[87 / 5]$} \\
\hline & $(320-482)$ & 54.9 & $(335)$ & & {$[47 / 5][99 / 16]$} \\
\hline \multirow[t]{6}{*}{$\mathrm{C}_{4} \mathrm{H}_{10} \mathrm{~S}$} & 1-butanethiol & & & & {$[109-75-5]$} \\
\hline & & 36.5 & $(298)$ & & {$[71 / 28]$} \\
\hline & $(323-409)$ & 35.0 & $(338)$ & $\mathrm{A}, \mathrm{EB}$ & $\begin{array}{c}{[87 / 5][57 / 7]} \\
{[66 / 5]}\end{array}$ \\
\hline & & $34.7 \pm 0.1$ & $(330)$ & $\mathrm{C}$ & {$[57 / 7]$} \\
\hline & & $33.6 \pm 0.1$ & $(350)$ & $\mathrm{C}$ & {$[57 / 7]$} \\
\hline & & $32.2 \pm 0.1$ & $(371)$ & $\mathrm{C}$ & {$[57 / 7]$} \\
\hline \multirow[t]{7}{*}{$\mathrm{C}_{4} \mathrm{H}_{10} \mathrm{~S}$} & (dl) 2-butanethiol & & & & {$[513-53-1]$} \\
\hline & & 34.1 & $(298)$ & & {$[71 / 28]$} \\
\hline & $(310-395)$ & 33.2 & $(325)$ & $\mathrm{A}, \mathrm{EB}$ & $\begin{array}{c}{[87 / 5][58 / 7]} \\
{[66 / 5]}\end{array}$ \\
\hline & & $32.9 \pm 0.1$ & $(318)$ & $\mathrm{C}$ & {$[58 / 7]$} \\
\hline & & $32.3 \pm 0.1$ & $(329)$ & $\mathrm{C}$ & {$[58 / 7]$} \\
\hline & & $31.8 \pm 0.1$ & $(337)$ & $\mathrm{C}$ & {$[58 / 7]$} \\
\hline & & $30.6 \pm 0.1$ & $(358)$ & $\mathrm{C}$ & {$[58 / 7]$} \\
\hline \multirow[t]{6}{*}{$\mathrm{C}_{4} \mathrm{H}_{10} \mathrm{~S}$} & 2-methyl-1-propanethiol & & & & {$[513-44-0]$} \\
\hline & & 34.6 & $(298)$ & & {$[71 / 28]$} \\
\hline & $(314-399)$ & 33.6 & $(329)$ & $\mathrm{A}, \mathrm{EB}$ & $\begin{array}{c}{[87 / 5][58 / 6]} \\
{[66 / 5]}\end{array}$ \\
\hline & & $33.3 \pm 0.1$ & $(321)$ & $\mathrm{C}$ & {$[58 / 6]$} \\
\hline & & $32.3 \pm 0.1$ & $(340)$ & $\mathrm{C}$ & {$[58 / 6]$} \\
\hline & & $31.0 \pm 0.1$ & $(361)$ & $\mathrm{C}$ & {$[58 / 6]$} \\
\hline \multirow[t]{4}{*}{$\mathrm{C}_{4} \mathrm{H}_{10} \mathrm{~S}$} & tert-butyl mercaptan & & & & {$[75-66-1]$} \\
\hline & $(275-293)$ & 30.1 & $(284)$ & & {$[98 / 25]$} \\
\hline & & 30.8 & $(298)$ & & {$[71 / 28]$} \\
\hline & $(293-373)$ & 30.9 & $(308)$ & $\mathrm{A}, \mathrm{EB}$ & $\begin{array}{c}{[87 / 5][53 / 7]} \\
{[66 / 5]}\end{array}$ \\
\hline \multirow[t]{8}{*}{$\mathrm{C}_{4} \mathrm{H}_{10} \mathrm{~S}$} & diethyl sulfide & & & & [352-93-2] \\
\hline & & $35.8 \pm 0.7$ & $(298)$ & $\mathrm{C}$ & {$[89 / 12]$} \\
\hline & & 35.5 & $(298)$ & & {$[81 / 12]$} \\
\hline & & 35.8 & $(298)$ & & {$[71 / 28]$} \\
\hline & $(318-396)$ & 34.4 & $(333)$ & $\mathrm{A}, \mathrm{EB}$ & $\begin{array}{c}{[87 / 5][52 / 11]} \\
{[66 / 5]}\end{array}$ \\
\hline & $(309-371)$ & 34.8 & $(324)$ & $\mathrm{EB}$ & {$[52 / 9]$} \\
\hline & $(233-361)$ & 37.5 & $(248)$ & & {$[47 / 5]$} \\
\hline & & 33.5 & $(364)$ & & {$[35 / 2]$} \\
\hline \multirow[t]{2}{*}{$\mathrm{C}_{4} \mathrm{H}_{10} \mathrm{~S}$} & methyl isopropyl sulfide & & & & {$[1551-21-9]$} \\
\hline & & 34.1 & $(298)$ & & {$[71 / 28]$} \\
\hline
\end{tabular}


TABLE 6. Enthalpies of vaporization of organic compounds, 1880-2002-Continued

\begin{tabular}{|c|c|c|c|c|c|}
\hline Molecular formula & $\begin{array}{c}\text { Compound } \\
\text { (Temperature range/K) }\end{array}$ & $\begin{array}{c}\Delta_{\mathrm{vap}} H_{m} \\
\left(\mathrm{~kJ} \mathrm{~mol}^{-1}\right)\end{array}$ & $\begin{array}{l}\text { Mean temperature } \\
\qquad\left(T_{m} / \mathrm{K}\right)\end{array}$ & Method & $\begin{array}{c}\text { CAS registry number } \\
\text { Reference }\end{array}$ \\
\hline & & $33.0 \pm 0.1$ & (318) & $\mathrm{C}$ & {$[55 / 12]$} \\
\hline & & $32.0 \pm 0.1$ & $(336)$ & $\mathrm{C}$ & {$[55 / 12]$} \\
\hline & & $30.7 \pm 0.1$ & $(358)$ & $\mathrm{C}$ & [55/12] \\
\hline & $(298-368)$ & 33.8 & $(313)$ & $\mathrm{A}, \mathrm{EB}$ & {$[87 / 5][52 / 9]$} \\
\hline \multirow[t]{6}{*}{$\mathrm{C}_{4} \mathrm{H}_{10} \mathrm{~S}$} & methyl propyl sulfide & & & & {$[3877-15-4]$} \\
\hline & & 36.2 & (298) & & {$[71 / 28]$} \\
\hline & $(308-374)$ & 35.3 & $(323)$ & A, EB & {$[87 / 5][52 / 9]$} \\
\hline & & $34.5 \pm 0.1$ & $(328)$ & $\mathrm{C}$ & {$[57 / 7]$} \\
\hline & & $33.4 \pm 0.1$ & $(347)$ & $\mathrm{C}$ & {$[57 / 7]$} \\
\hline & & $32.1 \pm 0.1$ & (369) & $\mathrm{C}$ & {$[57 / 7]$} \\
\hline \multirow[t]{4}{*}{$\mathrm{C}_{4} \mathrm{H}_{10} \mathrm{~S}_{2}$} & 1,4-butanedithiol & & & & [1191-08-8] \\
\hline & $(347-469)$ & 50.9 & $(362)$ & A & {$[87 / 5][99 / 16]$} \\
\hline & & 55.3 & (298) & & {$[62 / 11]$} \\
\hline & & 54.9 & (298) & & {$[62 / 11]$} \\
\hline \multirow[t]{9}{*}{$\mathrm{C}_{4} \mathrm{H}_{10} \mathrm{~S}_{2}$} & diethyl disulfide & & & & {$[110-81-6]$} \\
\hline & $(383-423)$ & 44.8 & (298) & CGC & {$[95 / 21]$} \\
\hline & & $45.4 \pm 0.8$ & $(298)$ & $\mathrm{C}$ & [89/12] \\
\hline & $(287-434)$ & 45.7 & $(302)$ & A & {$[87 / 5]$} \\
\hline & & $45.2 \pm 0.1$ & $(298)$ & $\mathrm{C}$ & {$[85 / 2]$} \\
\hline & & 45.2 & (298) & & [81/12] \\
\hline & & 45.6 & $(298)$ & & {$[71 / 28]$} \\
\hline & $(373-431)$ & 40.9 & $(388)$ & EB & $\begin{array}{c}{[87 / 5][66 / 5]} \\
{[52 / 12]}\end{array}$ \\
\hline & $(359-433)$ & 41.5 & $(374)$ & EB & {$[52 / 9]$} \\
\hline \multirow[t]{11}{*}{$\mathrm{C}_{4} \mathrm{H}_{11} \mathrm{~N}$} & butyl amine & & & & [109-73-9] \\
\hline & $(323-373)$ & 35.6 & $(298)$ & $\mathrm{CGC}$ & [95/21] \\
\hline & $(313-350)$ & 34.7 & (328) & A & {$[87 / 5]$} \\
\hline & & $35.7 \pm 0.2$ & $(298)$ & $\mathrm{C}$ & {$[85 / 2]$} \\
\hline & $(296-349)$ & 35.5 & $(311)$ & EB & {$[79 / 9]$} \\
\hline & & $35.7 \pm 0.1$ & (298) & $\mathrm{C}$ & [79/9] \\
\hline & & $34.7 \pm 0.1$ & $(313)$ & $\mathrm{C}$ & [79/9] \\
\hline & & $33.5 \pm 0.1$ & $(323)$ & $\mathrm{C}$ & [79/9] \\
\hline & & $32.4 \pm 0.1$ & $(343)$ & $\mathrm{C}$ & {$[79 / 9]$} \\
\hline & & $31.1 \pm 0.1$ & $(358)$ & $\mathrm{C}$ & {$[79 / 9]$} \\
\hline & & $35.7 \pm 0.1$ & (298) & $\mathrm{C}$ & {$[69 / 2]$} \\
\hline \multirow[t]{2}{*}{$\mathrm{C}_{4} \mathrm{H}_{11} \mathrm{~N}$} & $(d l)$ 2-aminobutane & & & & [13952-84-6] \\
\hline & $(264-371)$ & 34.1 & $(279)$ & A & {$[87 / 5][71 / 21]$} \\
\hline \multirow[t]{7}{*}{$\mathrm{C}_{4} \mathrm{H}_{11} \mathrm{~N}$} & sec-butylamine & & & & [13952-84-6] \\
\hline & $(300-335)$ & 32.4 & $(315)$ & EB & {$[79 / 9]$} \\
\hline & & $32.7 \pm 0.1$ & (298) & $\mathrm{C}$ & {$[79 / 9]$} \\
\hline & & $31.6 \pm 0.1$ & $(313)$ & $\mathrm{C}$ & {$[79 / 9]$} \\
\hline & & $30.5 \pm 0.1$ & $(328)$ & $\mathrm{C}$ & {$[79 / 9]$} \\
\hline & & $29.4 \pm 0.1$ & $(343)$ & $\mathrm{C}$ & {$[79 / 9]$} \\
\hline & & $32.6 \pm 0.1$ & $(298)$ & $\mathrm{C}$ & {$[69 / 2]$} \\
\hline \multirow[t]{8}{*}{$\mathrm{C}_{4} \mathrm{H}_{11} \mathrm{~N}$} & isobutylamine & & & & {$[78-81-9]$} \\
\hline & $(248-347)$ & 37.6 & $(263)$ & & {$[87 / 5]$} \\
\hline & & $33.9 \pm 0.1$ & $(298)$ & $\mathrm{C}$ & {$[79 / 9]$} \\
\hline & & $32.7 \pm 0.1$ & $(313)$ & $\mathrm{C}$ & {$[79 / 9]$} \\
\hline & & $31.6 \pm 0.1$ & $(328)$ & $\mathrm{C}$ & [79/9] \\
\hline & $(297-340)$ & 33.5 & $(313)$ & $\mathrm{EB}$ & {$[79 / 9]$} \\
\hline & & $33.8 \pm 0.1$ & $(298)$ & $\mathrm{C}$ & {$[69 / 2]$} \\
\hline & & $33.9 \pm 0.2$ & $(298)$ & IPM & {$[65 / 8][70 / 11]$} \\
\hline \multirow[t]{3}{*}{$\mathrm{C}_{4} \mathrm{H}_{11} \mathrm{~N}$} & tert-butylamine & & & & [75-64-9] \\
\hline & & $29.6 \pm 0.1$ & $(298)$ & $\mathrm{C}$ & {$[69 / 2]$} \\
\hline & $(292-349)$ & 30.1 & $(307)$ & $\mathrm{A}, \mathrm{EB}, \mathrm{IPM}$ & {$[87 / 5][68 / 4]$} \\
\hline \multirow[t]{9}{*}{$\mathrm{C}_{4} \mathrm{H}_{11} \mathrm{~N}$} & diethylamine & & & & [109-89-7] \\
\hline & $(302-328)$ & 31.2 & $(315)$ & A & {$[87 / 5]$} \\
\hline & $(325-437)$ & 30.4 & $(340)$ & A & {$[87 / 5]$} \\
\hline & $(431-496)$ & 28.4 & $(446)$ & A & {$[87 / 5]$} \\
\hline & & $31.3 \pm 0.1$ & (298) & $\mathrm{C}$ & [79/9] \\
\hline & & $30.2 \pm 0.1$ & (313) & $\mathrm{C}$ & [79/9] \\
\hline & & $29.1 \pm 0.1$ & $(328)$ & $\mathrm{C}$ & [79/9] \\
\hline & & $28.0 \pm 0.1$ & $(343)$ & $\mathrm{C}$ & [79/9] \\
\hline & & $31.2 \pm 0.1$ & $(298)$ & $\mathrm{C}$ & {$[69 / 2]$} \\
\hline
\end{tabular}


TABLE 6. Enthalpies of vaporization of organic compounds, 1880-2002-Continued

\begin{tabular}{|c|c|c|c|c|c|}
\hline Molecular formula & $\begin{array}{c}\text { Compound } \\
\text { (Temperature range/K) }\end{array}$ & $\begin{array}{c}\Delta_{\mathrm{vap}} H_{m} \\
\left(\mathrm{~kJ} \mathrm{~mol}^{-1}\right)\end{array}$ & $\begin{array}{l}\text { Mean temperature } \\
\qquad\left(T_{m} / \mathrm{K}\right)\end{array}$ & Method & $\begin{array}{c}\text { CAS registry number } \\
\text { Reference }\end{array}$ \\
\hline & $(273-333)$ & $32.7 \pm 0.2$ & $(298)$ & $\mathrm{I}$ & {$[69 / 16]$} \\
\hline & $(292-313)$ & 31.8 & $(307)$ & & {$[65 / 24][84 / 9]$} \\
\hline & $(304-323)$ & 31.5 & $(319)$ & & {$[62 / 23][84 / 9]$} \\
\hline \multirow[t]{5}{*}{$\mathrm{C}_{4} \mathrm{H}_{11} \mathrm{~N}$} & N-methyl isopropyl amine & & & & {$[4747-21-1]$} \\
\hline & & $30.7 \pm 0.1$ & $(298)$ & $\mathrm{C}$ & {$[79 / 8]$} \\
\hline & & $29.5 \pm 0.1$ & (313) & $\mathrm{C}$ & {$[79 / 8]$} \\
\hline & & $27.1 \pm 0.1$ & $(343)$ & $\mathrm{C}$ & {$[79 / 8]$} \\
\hline & $(293-319)$ & 30.9 & $(306)$ & $\mathrm{EB}$ & {$[79 / 8]$} \\
\hline \multirow[t]{3}{*}{$\mathrm{C}_{4} \mathrm{H}_{11} \mathrm{NO}$} & 2-(dimethylamino)ethanol & & & & [108-01-0] \\
\hline & $(350-387)$ & 43.2 & $(365)$ & A & {$[87 / 5]$} \\
\hline & $(323-408)$ & 42.7 & $(338)$ & A & {$[87 / 5]$} \\
\hline \multirow{2}{*}{$\mathrm{C}_{4} \mathrm{H}_{11} \mathrm{NO}$} & 3-methoxypropylamine & & & & {$[5332-73-0]$} \\
\hline & $(278-390)$ & 44.5 & $(293)$ & A & {$[87 / 5]$} \\
\hline \multirow[t]{2}{*}{$\mathrm{C}_{4} \mathrm{H}_{11} \mathrm{NO}_{2}$} & 2,2'-iminodiethanol & & & & {$[111-42-2]$} \\
\hline & $(463-582)$ & 69.0 & $(478)$ & & {$[59 / 1]$} \\
\hline \multirow[t]{4}{*}{$\mathrm{C}_{4} \mathrm{H}_{11} \mathrm{NO}_{2}$} & diethanolamine & & & & {$[111-42-2]$} \\
\hline & $(423-542)$ & 74.4 & $(438)$ & A & {$[87 / 5]$} \\
\hline & $(376-454)$ & 77.0 & $(391)$ & & {$[69 / 20][84 / 9]$} \\
\hline & $(466-514)$ & 70.6 & $(481)$ & & {$[59 / 1][84 / 9]$} \\
\hline \multirow[t]{2}{*}{$\mathrm{C}_{4} \mathrm{H}_{11} \mathrm{NO}_{2} \mathrm{~S}$} & N,N-dimethylethanesulfonamide & & & & {$[6338-68-7]$} \\
\hline & $(384-517)$ & 54.3 & $(399)$ & A & {$[87 / 5]$} \\
\hline \multirow[t]{2}{*}{$\mathrm{C}_{4} \mathrm{H}_{11} \mathrm{O}_{3} \mathrm{P}$} & diethylphosphite & & & & {$[762-04-9]$} \\
\hline & $(338-471)$ & 38.1 & $(353)$ & A & {$[87 / 5]$} \\
\hline \multirow[t]{2}{*}{$\mathrm{C}_{4} \mathrm{H}_{11} \mathrm{O}_{3} \mathrm{P}$} & dimethyl ethylphosphonate & & & & \\
\hline & $(333-410)$ & 70.1 & $(348)$ & & $\begin{array}{c}{[87 / 5][55 / 5]} \\
{[84 / 9]}\end{array}$ \\
\hline \multirow[t]{2}{*}{$\mathrm{C}_{4} \mathrm{H}_{12} \mathrm{ClN}$} & butylammonium chloride & & & & {$[3858-78-4]$} \\
\hline & $(489-508)$ & 62.1 & $(498)$ & A & {$[87 / 5]$} \\
\hline \multirow[t]{2}{*}{$\mathrm{C}_{4} \mathrm{H}_{12} \mathrm{ClN}$} & diethylamine hydrochloride & & & & {$[660-68-4]$} \\
\hline & $(513-558)$ & 177.6 & $(528)$ & & {$[87 / 5]$} \\
\hline \multirow[t]{2}{*}{$\mathrm{C}_{4} \mathrm{H}_{12} \mathrm{ClN}_{2} \mathrm{P}$} & bis(dimethylamino)chlorophosphine & & & & \\
\hline & & $45.9 \pm 1.2$ & $(298)$ & STG & {$[95 / 2]$} \\
\hline \multirow[t]{2}{*}{$\mathrm{C}_{4} \mathrm{H}_{12} \mathrm{FN}_{2} \mathrm{OP}$} & bis(dimethylamido)fluorophosphate & & & & {$[115-26-4]$} \\
\hline & $(312-350)$ & 50.4 & $(327)$ & A & {$[87 / 5]$} \\
\hline \multirow[t]{2}{*}{$\mathrm{C}_{4} \mathrm{H}_{12} \mathrm{NP}$} & dimethyl(dimethylamino)phosphine & & & & {$[683-84-1]$} \\
\hline & $(264-372)$ & 36.8 & $(279)$ & A & {$[87 / 5]$} \\
\hline \multirow[t]{4}{*}{$\mathrm{C}_{4} \mathrm{H}_{12} \mathrm{~N}_{2}$} & (dl) 1,2-butanediamine & & & & {$[4426-48-6]$} \\
\hline & $(251-293)$ & 50.2 & $(278)$ & & {$[87 / 5][75 / 4]$} \\
\hline & $(251-293)$ & 46.9 & $(298)$ & IPM & {$[75 / 4]$} \\
\hline & & $46.3 \pm 0.2$ & $(298)$ & IPM & {$[65 / 8][70 / 11]$} \\
\hline $\mathrm{C}_{4} \mathrm{H}_{12} \mathrm{~N}_{2}$ & 2-methyl-1,2-propanediamine & & & & [811-93-8] \\
\hline & $(256-293)$ & 47.2 & $(278)$ & IPM & {$[87 / 5][75 / 4]$} \\
\hline & $(256-293)$ & $43.5 \pm 0.2$ & $(298)$ & IPM & {$[75 / 4]$} \\
\hline & & $43.6 \pm 0.2$ & $(298)$ & IPM & {$[65 / 8][70 / 11]$} \\
\hline $\mathrm{C}_{4} \mathrm{H}_{12} \mathrm{~N}_{2}$ & tetramethylhydrazine & & & & {$[6415-12-9]$} \\
\hline & $(290-346)$ & 32.9 & $(305)$ & $\mathrm{T}$ & {$[87 / 5][57 / 20]$} \\
\hline $\mathrm{C}_{4} \mathrm{H}_{12} \mathrm{~N}_{2} \mathrm{O}$ & $\mathrm{N}$-(2-hydroxyethyl)ethylenediamine & & & & {$[111-41-1]$} \\
\hline & $(383-517)$ & 62.8 & $(398)$ & A & {$[87 / 5]$} \\
\hline $\mathrm{C}_{4} \mathrm{H}_{12} \mathrm{~N}_{2} \mathrm{OS}$ & tetramethyl sulfurous diamide & & & & {$[3768-60-3]$} \\
\hline & $(320-351)$ & 41.9 & $(335)$ & A & {$[87 / 5][99 / 16]$} \\
\hline $\mathrm{C}_{4} \mathrm{H}_{12} \mathrm{~N}_{2} \mathrm{OS}$ & $\mathrm{N}, \mathrm{N}, \mathrm{N}^{\prime}, \mathrm{N}^{\prime}$-tetramethylsulfamide & & & & {$[3768-63-6]$} \\
\hline & $(358-495)$ & 53.2 & $(373)$ & A & {$[87 / 5][99 / 16]$} \\
\hline $\mathrm{C}_{4} \mathrm{H}_{12} \mathrm{~N}_{2} \mathrm{~S}$ & tetramethylsulfoxylic diamide & & & & {$[2129-20-6]$} \\
\hline & $(301-326)$ & 40.4 & $(313)$ & A & {$[87 / 5]$} \\
\hline $\mathrm{C}_{4} \mathrm{H}_{13} \mathrm{~N}_{3}$ & 2,2'-diaminodiethylamine & & & & {$[111-40-0]$} \\
\hline & $(371-521)$ & $63.4 \pm 0.7$ & $(298)$ & EB & {$[99 / 7]$} \\
\hline $\mathrm{C}_{4} \mathrm{H}_{13} \mathrm{~N}_{3}$ & dimethylene triamine & & & & {$[111-40-0]$} \\
\hline & $(371-441)$ & 54.8 & $(386)$ & A & {$[87 / 5]$} \\
\hline $\mathrm{C}_{5} \mathrm{BrF}_{12} \mathrm{~N}$ & 1,1,2,3,3,3-hexafluoro-2-bromo-N,N- & bis(trifluoron & propylamine & & \\
\hline & $(324-351)$ & 30.2 & (337) & A & {$[87 / 5]$} \\
\hline $\mathrm{C}_{5} \mathrm{ClF}_{5}$ & 1-chloro-2,3,4,5,5-pentafluoro-1,3-cy & clopentadien & & & {$[30221-57-9]$} \\
\hline & $(273-303)$ & 31.0 & $(288)$ & A & {$[87 / 5]$} \\
\hline $\mathrm{C}_{5} \mathrm{ClF}_{5}$ & 5-chloro-1,2,3,4,5-pentafluoro-1,3-cy & clopentadien & & & {$[30221-56-8]$} \\
\hline & $(283-323)$ & 28.7 & $(298)$ & A & {$[87 / 5]$} \\
\hline
\end{tabular}


TABLE 6. Enthalpies of vaporization of organic compounds, 1880-2002-Continued

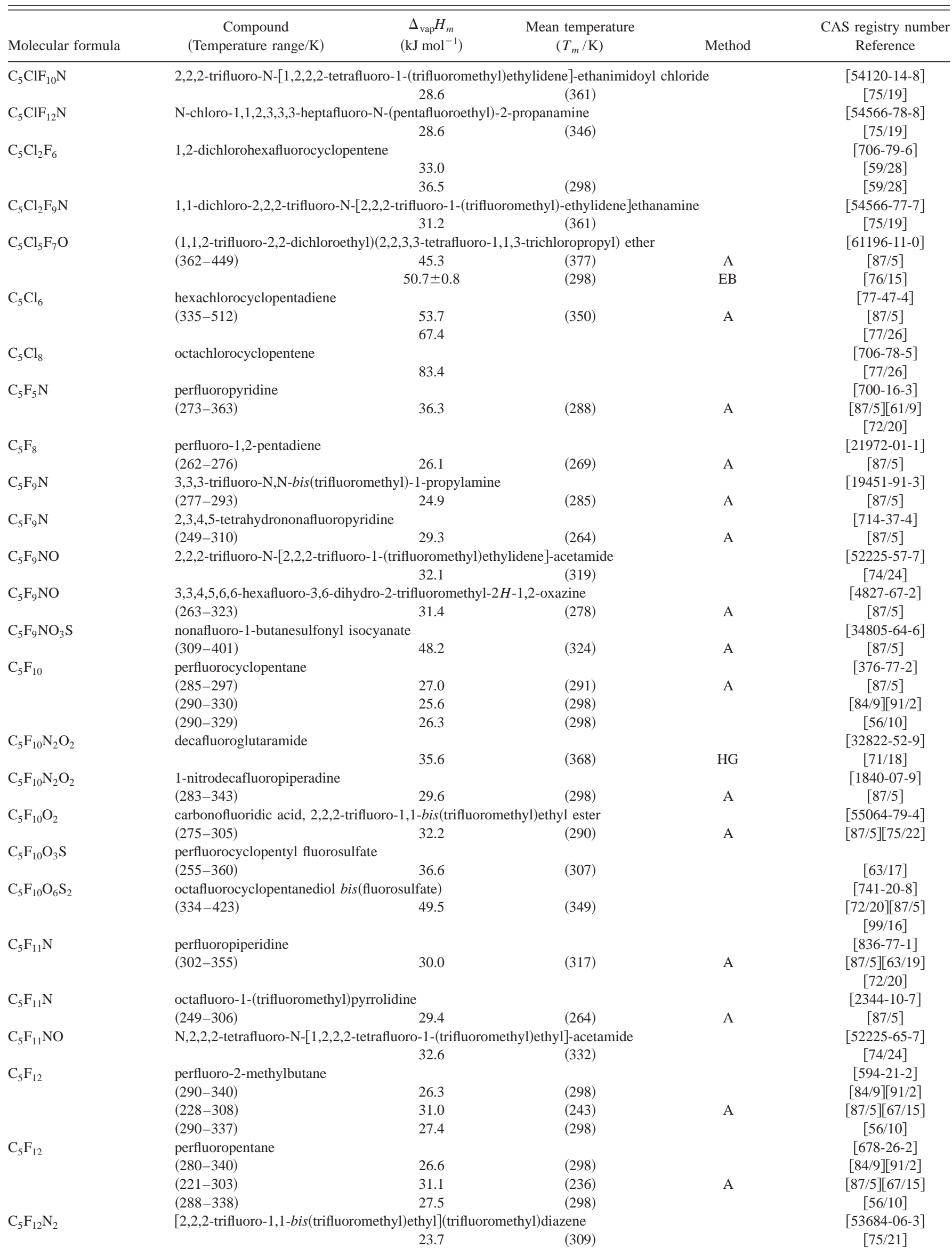


TABLE 6. Enthalpies of vaporization of organic compounds, 1880-2002-Continued

\begin{tabular}{|c|c|c|c|c|c|}
\hline Molecular formula & $\begin{array}{l}\text { Compound } \\
\text { (Temperature range/K) }\end{array}$ & $\begin{array}{c}\Delta_{\mathrm{vap}} H_{m} \\
\left(\mathrm{~kJ} \mathrm{~mol}^{-1}\right)\end{array}$ & $\begin{array}{l}\text { Mean temperature } \\
\qquad\left(T_{m} / \mathrm{K}\right)\end{array}$ & Method & $\begin{array}{l}\text { CAS registry number } \\
\text { Reference }\end{array}$ \\
\hline $\mathrm{C}_{5} \mathrm{~F}_{12} \mathrm{O}_{2}$ & \multicolumn{2}{|c|}{ bis(pentafluoroethoxy)difluoromethane } & $(261)$ & A & $\begin{array}{l}{[20822-11-1]} \\
\quad[87 / 5]\end{array}$ \\
\hline \multirow[t]{2}{*}{$\mathrm{C}_{5} \mathrm{~F}_{12} \mathrm{O}_{2} \mathrm{~S}$} & \multicolumn{4}{|c|}{$\begin{array}{l}\text { trifluoromethanesulfinic acid, 2,2,2-trifluoro-1,1-bis(trifluoromethyl) } \\
\text { ethyl ester }\end{array}$} & {$[52225-54-4]$} \\
\hline & & 37.7 & $(355)$ & $\mathrm{HG}$ & {$[74 / 25]$} \\
\hline $\mathrm{C}_{5} \mathrm{~F}_{12} \mathrm{O}_{4} \mathrm{~S}$ & \multicolumn{4}{|c|}{$\begin{array}{c}\text { pentafluoro(2,2,3,3,4,4,5-heptafluoro-5-oxopentaneperoxoato) sulfur } \\
39.2\end{array}$} & $\begin{array}{l}{[60672-63-1]} \\
{[76 / 31]}\end{array}$ \\
\hline $\mathrm{C}_{5} \mathrm{~F}_{13} \mathrm{~N}$ & $\begin{array}{l}\text { N-(trifluoromethyl) bis(pen } \\
(298-319)\end{array}$ & $\begin{array}{l}\text { hyl)amine } \\
30.2 \\
29.4 \pm 0.4\end{array}$ & $\begin{array}{l}(308) \\
(298)\end{array}$ & A & $\begin{array}{c}{[1481-55-6]} \\
{[87 / 5]} \\
{[77 / 13]}\end{array}$ \\
\hline $\mathrm{C}_{5} \mathrm{~F}_{13} \mathrm{NS}$ & \multicolumn{4}{|c|}{$\mathrm{N}$-[1,2,2,2-tetrafluoro-1-(trifluoromethyl)ethyl]-S,S-bis(trifluoro-methyl)sulfilimine } & $\begin{array}{l}{[37826-44-1]} \\
{[87 / 5][99 / 16]}\end{array}$ \\
\hline $\mathrm{C}_{5} \mathrm{~F}_{14} \mathrm{~N}_{2} \mathrm{O}$ & \multicolumn{4}{|c|}{ 1-[difluoro(trifluoromethoxy)methyl]-1,2,2-tris(trifluoromethyl)-hydrazine } & $\begin{array}{l}{[17636-89-4]} \\
{[87 / 5]}\end{array}$ \\
\hline $\mathrm{C}_{5} \mathrm{~F}_{14} \mathrm{~N}_{2} \mathrm{O}$ & \multicolumn{4}{|c|}{ 1,1-difluoro-N-(trifluoromethoxy)-N, $\mathrm{N}^{\prime}, \mathrm{N}^{\prime}$-tris(trifluoromethyl)-methanediamine } & $\begin{array}{l}{[17636-88-3]} \\
{[87 / 5]}\end{array}$ \\
\hline $\mathrm{C}_{5} \mathrm{~F}_{14} \mathrm{OS}$ & \multicolumn{4}{|c|}{ pentafluoro[(nonafluorocyclopentyl)oxyl] sulfur } & $\begin{array}{l}{[736-59-4]} \\
{[87 / 5][99 / 16]}\end{array}$ \\
\hline $\mathrm{C}_{5} \mathrm{~F}_{15} \mathrm{~N}$ & \multicolumn{4}{|c|}{$\mathrm{N}$-(trifluoromethyl)bis(pentafluoroethyl)amine } & $\begin{array}{c}{[758-48-5]} \\
{[77 / 20]}\end{array}$ \\
\hline $\mathrm{C}_{5} \mathrm{~F}_{15} \mathrm{NS}$ & \multicolumn{4}{|c|}{ difluoro[1,1,1,2,3,3,3-heptafluoro-2-propanaminoto(2-)]-bis(trifluoromethyl) sulfur } & $\begin{array}{c}{[65844-10-2]} \\
{[78 / 14]}\end{array}$ \\
\hline $\mathrm{C}_{5} \mathrm{~F}_{15} \mathrm{P}_{5}$ & \multicolumn{4}{|c|}{ 1,2,3,4,5-pentakis(trifluoromethyl)pentaphospholane } & $\begin{array}{c}{[745-23-3]} \\
{[87 / 5][58 / 10]}\end{array}$ \\
\hline $\mathrm{C}_{5} \mathrm{O}_{2}$ & \multicolumn{4}{|l|}{ pentacarbon dioxide } & $\begin{array}{c}{[51799-36-1]} \\
{[87 / 5][37 / 1]}\end{array}$ \\
\hline $\mathrm{C}_{5} \mathrm{HClF}_{8} \mathrm{O}_{2}$ & \multicolumn{4}{|c|}{ trifluoroacetic acid, 1-(chlorodifluoromethyl)-2,2,2-trifluoroethyl ester } & $\begin{array}{c}{[52225-55-5]} \\
{[74 / 25]}\end{array}$ \\
\hline $\mathrm{C}_{5} \mathrm{HF}_{10} \mathrm{NO}$ & \multicolumn{4}{|c|}{ 2,2,2-trifluoro-N-[1,2,2,2-tetrafluoro-1-(trifluoromethyl)ethyl]-acetamide } & {$[74 / 24]$} \\
\hline $\mathrm{C}_{5} \mathrm{HF}_{9}$ & $\begin{array}{l}\text { nonafluorocyclopentane } \\
(289-348) \\
(289-348)\end{array}$ & $\begin{array}{l}29.6 \\
29.4\end{array}$ & $\begin{array}{l}(304) \\
(298)\end{array}$ & A & $\begin{array}{l}{[376-65-8]} \\
{[87 / 5][56 / 10]} \\
{[56 / 10]}\end{array}$ \\
\hline $\mathrm{C}_{5} \mathrm{HF}_{9} \mathrm{IN}$ & $\begin{array}{l}\text { cis 3,3,3-trifluoro-1-iodo-1 } \\
(343-366)\end{array}$ & $\begin{array}{l}\text { fluoromethyl } \\
31.3\end{array}$ & ylamine & $\mathrm{A}$ & $\begin{array}{c}{[20257-34-5]} \\
{[87 / 5]}\end{array}$ \\
\hline $\mathrm{C}_{5} \mathrm{HF}_{9} \mathrm{IN}$ & $\begin{array}{l}\text { trans 3,3,3-trifluoro-1-iod } \\
(345-368)\end{array}$ & $\begin{array}{l}\text { (trifluorometl } \\
35.0\end{array}$ & $\begin{array}{l}\text { enylamine } \\
\qquad(356)\end{array}$ & A & $\begin{array}{c}{[20257-35-6]} \\
{[87 / 5]}\end{array}$ \\
\hline $\mathrm{C}_{5} \mathrm{HF}_{9} \mathrm{O}_{2}$ & trifluoroacetic acid, 2,2,2- & $\begin{array}{l}\text { omethyl)ethy } \\
28.5\end{array}$ & $(321)$ & $\mathrm{HG}$ & $\begin{array}{c}{[42031-15-2]} \\
{[73 / 20]}\end{array}$ \\
\hline $\mathrm{C}_{5} \mathrm{HF}_{10} \mathrm{~N}$ & $\begin{array}{l}2,2,3,3,4,4,5,5,6,6 \text {-decaflu } \\
(273-313)\end{array}$ & ine 32.7 & $(288)$ & A & $\begin{array}{c}{[559-31-9]} \\
{[87 / 5]}\end{array}$ \\
\hline $\mathrm{C}_{5} \mathrm{HF}_{12} \mathrm{~N}$ & 1,1,1,2,3,3,3-heptafluoro-I & $\begin{array}{l}\text { loroethyl)-2-1 } \\
29.8\end{array}$ & $(325)$ & & $\begin{array}{c}{[54566-80-2]} \\
{[75 / 19]}\end{array}$ \\
\hline $\mathrm{C}_{5} \mathrm{HN}_{3}$ & $\begin{array}{l}\text { ethylenetricarbonitrile } \\
(313-343)\end{array}$ & 66.0 & $(328)$ & $\mathrm{A}, \mathrm{MG}$ & $\begin{array}{c}{[997-76-2]} \\
{[87 / 5][63 / 1]}\end{array}$ \\
\hline $\mathrm{C}_{5} \mathrm{H}_{2} \mathrm{BrF}_{8} \mathrm{~N}$ & $\begin{array}{l}\text { 2-bromo-3,3-difluoro-N,N } \\
(336-367)\end{array}$ & $\begin{array}{c}\text { romethyl)all } \\
33.8\end{array}$ & $(351)$ & A & $\begin{array}{c}{[19451-93-5]} \\
{[87 / 5]}\end{array}$ \\
\hline $\mathrm{C}_{5} \mathrm{H}_{2} \mathrm{~F}_{6} \mathrm{O}_{2}$ & $\begin{array}{l}\text { 1,1,1,5,5,5-hexafluoropent } \\
(273-330)\end{array}$ & $\begin{array}{c}33.1 \\
30.6 \pm 0.1\end{array}$ & $\begin{array}{l}(301) \\
(298)\end{array}$ & GS & $\begin{array}{c}{[1522-22-1]} \\
{[98 / 5]} \\
{[97 / 2][75 / 2]} \\
{[78 / 18]}\end{array}$ \\
\hline $\mathrm{C}_{5} \mathrm{H}_{2} \mathrm{~F}_{9} \mathrm{~N}$ & $\begin{array}{l}\text { trans 3,3,3-trifluoro-N,N- } \\
(287-319)\end{array}$ & $\begin{array}{c}\text { omethyl)pror } \\
28.2\end{array}$ & $(302)$ & A & $\begin{array}{c}{[25273-42-1]} \\
{[87 / 5]}\end{array}$ \\
\hline $\mathrm{C}_{5} \mathrm{H}_{2} \mathrm{~F}_{9} \mathrm{NOS}$ & 2,2,2-trifluoro-N-[(trifluor & $\begin{array}{l}\text { hio]ethanimid } \\
35.8\end{array}$ & $\begin{array}{l}\text { 2,2,2-trifluoroethyl e } \\
\text { (373) }\end{array}$ & I & $\begin{array}{c}{[62067-07-6]} \\
{[77 / 18]}\end{array}$ \\
\hline $\mathrm{C}_{5} \mathrm{H}_{2} \mathrm{~F}_{9} \mathrm{NS}$ & 2,2,2-trifluoro-N-[2,2,2-tri & $\begin{array}{l}\text { rifluorometh } \\
36.9\end{array}$ & ethane-thioamide & & $\begin{array}{c}{[57682-29-8]} \\
{[75 / 42]}\end{array}$ \\
\hline $\mathrm{C}_{5} \mathrm{H}_{2} \mathrm{~F}_{10} \mathrm{O}$ & $\begin{array}{l}\text { 1,1,1,2,2,3,3-heptafluoro-3 } \\
(288-325)\end{array}$ & $\begin{array}{l}\text { fluoroethoxy } \\
31.5\end{array}$ & $(303)$ & I & $\begin{array}{c}{[142469-08-7]} \\
{[02 / 19]}\end{array}$ \\
\hline $\mathrm{C}_{5} \mathrm{H}_{2} \mathrm{~F}_{10} \mathrm{O}$ & $\begin{array}{l}1,1,1,2,4,4,4 \text {-heptafluoro- } \\
(288-323)\end{array}$ & $\begin{array}{c}\text { methoxy)but } \\
31.8\end{array}$ & $(303)$ & I & $\begin{array}{c}{[347148-74-7]} \\
{[02 / 19]}\end{array}$ \\
\hline $\mathrm{C}_{5} \mathrm{H}_{2} \mathrm{~F}_{10} \mathrm{O}$ & $\begin{array}{l}\text { 1,1,1,2,2-pentafluoro-3-(p } \\
(288-320)\end{array}$ & $\begin{array}{c}\text { thoxy)propa } \\
31.2\end{array}$ & $(303)$ & I & $\begin{array}{c}{[155653-44-4]} \\
{[02 / 19]}\end{array}$ \\
\hline $\mathrm{C}_{5} \mathrm{H}_{3} \mathrm{BrF}_{9} \mathrm{~N}$ & 2-bromo-3,3,3-trifluoro-N & uoromethyl) & ropylamine & & {$[19451-92-4]$} \\
\hline
\end{tabular}


TABLE 6. Enthalpies of vaporization of organic compounds, 1880-2002-Continued

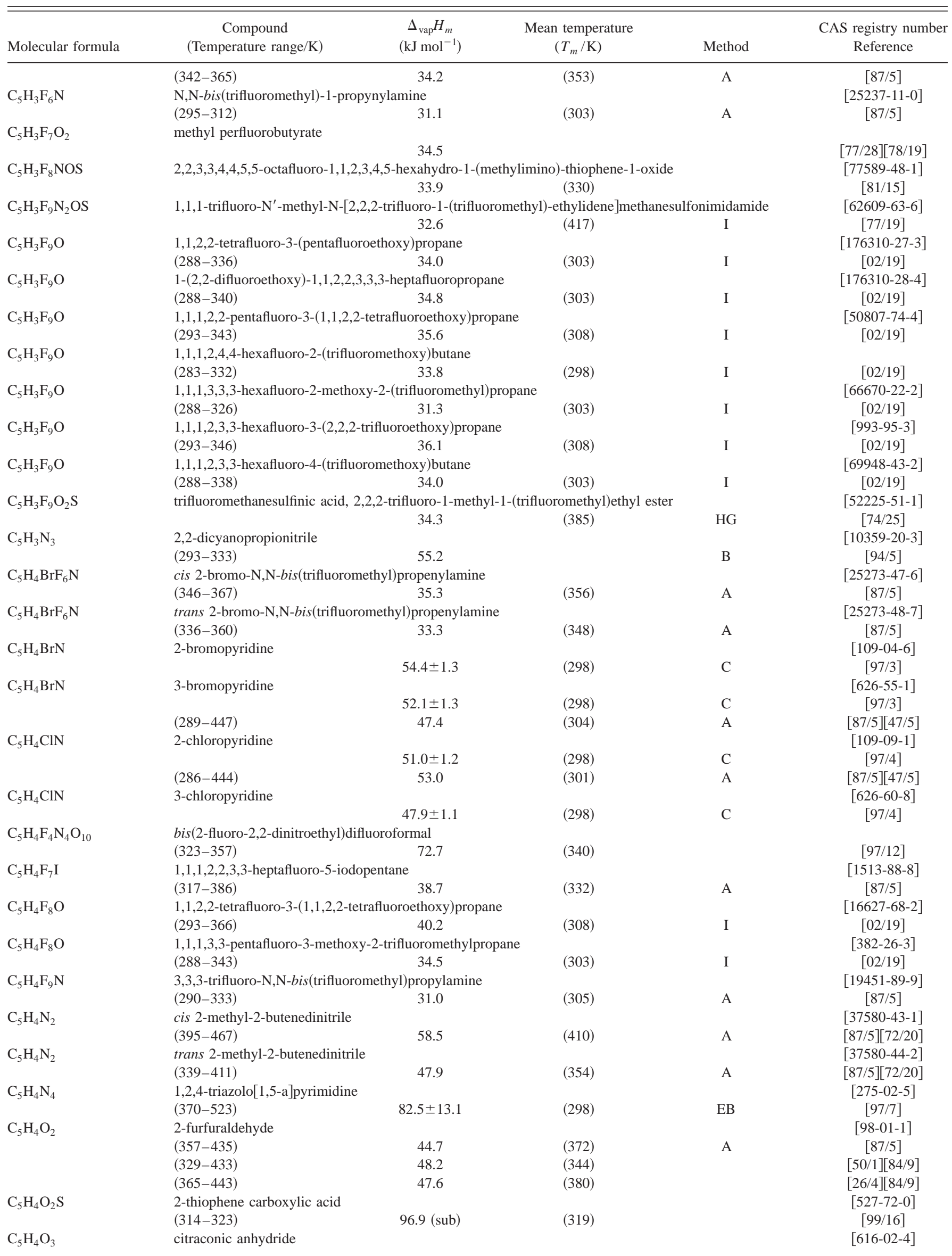


TABLE 6. Enthalpies of vaporization of organic compounds, 1880-2002-Continued

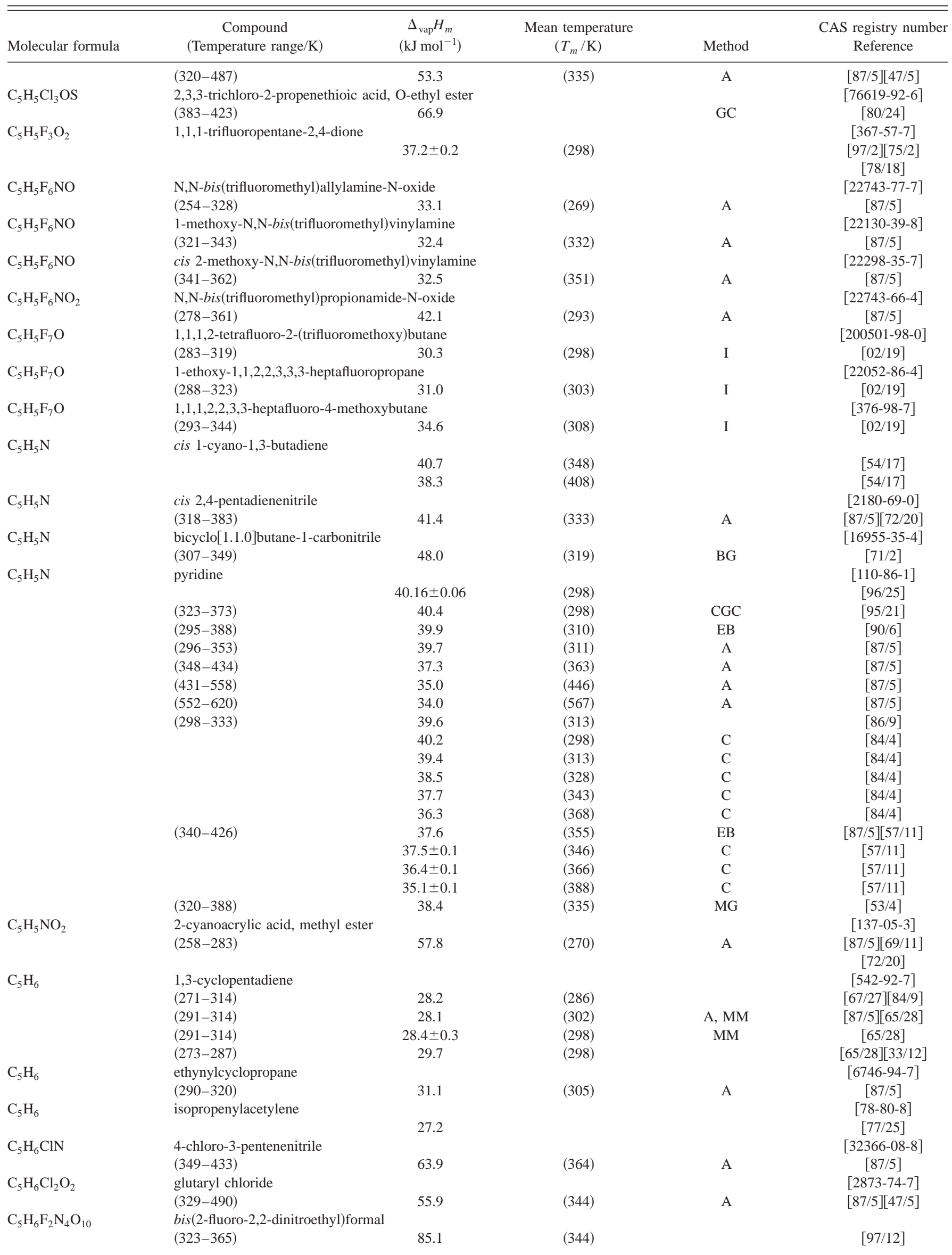


TABLE 6. Enthalpies of vaporization of organic compounds, 1880-2002-Continued

\begin{tabular}{|c|c|c|c|c|c|}
\hline Molecular formula & $\begin{array}{c}\text { Compound } \\
\text { (Temperature range/K) }\end{array}$ & $\begin{array}{c}\Delta_{\mathrm{vap}} H_{m} \\
\left(\mathrm{~kJ} \mathrm{~mol}^{-1}\right)\end{array}$ & $\begin{array}{l}\text { Mean temperature } \\
\qquad\left(T_{m} / \mathrm{K}\right)\end{array}$ & Method & $\begin{array}{c}\text { CAS registry number } \\
\text { Reference }\end{array}$ \\
\hline \multirow[t]{2}{*}{$\mathrm{C}_{5} \mathrm{H}_{6} \mathrm{~F}_{6} \mathrm{~N}_{2} \mathrm{~S}$} & \multicolumn{4}{|c|}{ 2,2,2-trifluoro-N,N-dimethyl-N'-[(trifluoromethyl)thio]-ethanimidamide } & {$[62067-11-2]$} \\
\hline & & 40.4 & $(400)$ & $\mathrm{I}$ & {$[77 / 18]$} \\
\hline \multirow[t]{2}{*}{$\mathrm{C}_{5} \mathrm{H}_{6} \mathrm{~F}_{6} \mathrm{O}$} & \multicolumn{4}{|c|}{ 1,1,1,2,3,3-hexafluoro-4-methoxybutane } & {$[58705-93-4]$} \\
\hline & $(293-360)$ & 37.0 & (308) & I & {$[02 / 19]$} \\
\hline \multirow[t]{2}{*}{$\mathrm{C}_{5} \mathrm{H}_{6} \mathrm{~F}_{6} \mathrm{~N}_{2} \mathrm{~S}$} & \multicolumn{5}{|c|}{ dimethylamino(hexafluoroisoproplidenimino) sulfur } \\
\hline & & 39.7 & $(383)$ & I & {$[72 / 22]$} \\
\hline \multirow[t]{2}{*}{$\mathrm{C}_{5} \mathrm{H}_{6} \mathrm{~F}_{6} \mathrm{O}_{2} \mathrm{~S}$} & \multicolumn{4}{|c|}{ trifluoromethanesulfinic acid, 2,2,2-trifluoro-1,1-dimethylethyl ester } & {$[52225-49-7]$} \\
\hline & \multicolumn{4}{|c|}{ 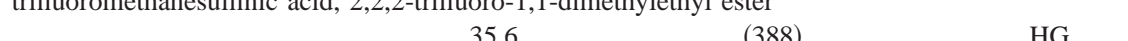 } & {$[74 / 25]$} \\
\hline \multirow[t]{2}{*}{$\mathrm{C}_{5} \mathrm{H}_{6} \mathrm{~F}_{6} \mathrm{O}_{5} \mathrm{~S}_{2}$} & \multicolumn{4}{|c|}{ 3,3-bis[(trifluoromethyl)sulfonyl]-1-propanol } & {$[61915-97-7]$} \\
\hline & $(333-418)$ & 32.8 & $(348)$ & A, I & $\begin{array}{c}{[87 / 5][77 / 17]} \\
{[99 / 16]}\end{array}$ \\
\hline \multirow[t]{2}{*}{$\mathrm{C}_{5} \mathrm{H}_{6} \mathrm{~N}_{2}$} & dimethylmalononitrile & & & & {$[7321-55-3]$} \\
\hline & $(322-413)$ & 47.5 & $(337)$ & A & {$[87 / 5][67 / 20]$} \\
\hline \multirow[t]{2}{*}{$\mathrm{C}_{5} \mathrm{H}_{6} \mathrm{~N}_{2}$} & 2-methylpyrazine & & & & [109-08-0] \\
\hline & $(288-392)$ & 42.4 & $(340)$ & & {$[95 / 4]$} \\
\hline \multirow[t]{3}{*}{$\mathrm{C}_{5} \mathrm{H}_{6} \mathrm{~N}_{2}$} & glutaronitrile & & & & [544-13-8] \\
\hline & $(364-560)$ & 60.1 & $(379)$ & A & {$[87 / 5]$} \\
\hline & $(277-303)$ & 66.8 & $(290)$ & A & {$[87 / 5][72 / 20]$} \\
\hline \multirow[t]{6}{*}{$\mathrm{C}_{5} \mathrm{H}_{6} \mathrm{O}$} & 2-methylfuran & & & & [534-22-5] \\
\hline & $(289-337)$ & 32.4 & (304) & & {$[02 / 30]$} \\
\hline & $(251-338)$ & 34.4 & (266) & A & {$[87 / 5]$} \\
\hline & $(288-303)$ & 32.5 & $(295)$ & & {$[72 / 20]$} \\
\hline & $(333-373)$ & 30.9 & (348) & & {$[71 / 3][84 / 9]$} \\
\hline & $(215-360)$ & 32.2 & (298) & & {$[70 / 36]$} \\
\hline $\mathrm{C}_{5} \mathrm{H}_{6} \mathrm{O}_{2}$ & 5-methyl-2(3H)-furanone & & & & {$[591-12-8]$} \\
\hline & $(324-442)$ & 40.3 & (339) & A & {$[87 / 5]$} \\
\hline $\mathrm{C}_{5} \mathrm{H}_{6} \mathrm{O}_{2}$ & (dl) 5-methyl-2(5H)-furan & & & & [591-11-7] \\
\hline & $(356-481)$ & 48.2 & $(371)$ & A & {$[87 / 5]$} \\
\hline $\mathrm{C}_{5} \mathrm{H}_{6} \mathrm{O}_{2}$ & furfuryl alcohol & & & & {$[98-00-0]$} \\
\hline & $(304-443)$ & 53.6 & $(319)$ & A & {$[87 / 5][47 / 4]$} \\
\hline $\mathrm{C}_{5} \mathrm{H}_{6} \mathrm{O}_{2}$ & 5-hydroxy-3-pentyn-2-one & & & & [15441-65-3] \\
\hline & $(273-333)$ & 64.4 & $(288)$ & $\mathrm{A}$ & {$[87 / 5][72 / 20]$} \\
\hline $\mathrm{C}_{5} \mathrm{H}_{6} \mathrm{O}_{3}$ & glutaric anhydride & & & & {$[108-55-4]$} \\
\hline & $(373-560)$ & 60.9 & $(388)$ & A & {$[87 / 5][47 / 5]$} \\
\hline $\mathrm{C}_{5} \mathrm{H}_{6} \mathrm{O}_{3}$ & $(d l)$ monomethylsuccinic & & & & {$[4100-80-5]$} \\
\hline & $(342-521)$ & 59.3 & $(357)$ & A & {$[87 / 5][47 / 5]$} \\
\hline $\mathrm{C}_{5} \mathrm{H}_{6} \mathrm{~S}$ & 2-methylthiophene & & & & [554-14-3] \\
\hline & $(333-373)$ & 36.8 & $(348)$ & I & {$[71 / 3][84 / 9]$} \\
\hline & & 38.7 & (298) & & {$[71 / 28]$} \\
\hline & $(324-391)$ & 37.2 & (339) & A, EB & {$[87 / 5][52 / 9]$} \\
\hline & & & & & [99/16] \\
\hline $\mathrm{C}_{5} \mathrm{H}_{6} \mathrm{~S}$ & 3-methylthiophene & & & & {$[616-44-4]$} \\
\hline & $(326-398)$ & 36.8 & (357) & & [99/16] \\
\hline & $(333-373)$ & 37.4 & $(348)$ & I & {$[71 / 3][84 / 9]$} \\
\hline & & 39.5 & (298) & & {$[71 / 28]$} \\
\hline & $(327-399)$ & 37.5 & $(342)$ & $\mathrm{A}, \mathrm{EB}$ & {$[87 / 5][52 / 9]$} \\
\hline $\mathrm{C}_{5} \mathrm{H}_{7} \mathrm{ClO}_{3}$ & acetic acid, chlorooxo, pro & & & & [54166-91-5] \\
\hline & $(282-396)$ & 52.7 & $(297)$ & $\mathrm{A}$ & {$[87 / 5][47 / 5]$} \\
\hline $\mathrm{C}_{5} \mathrm{H}_{7} \mathrm{FO}_{2}$ & allyl fluoroacetate & & & & \\
\hline & $(273-333)$ & 48.9 & $(288)$ & A, GS & $\begin{array}{c}{[87 / 5][48 / 14]} \\
{[72 / 20]}\end{array}$ \\
\hline $\mathrm{C}_{5} \mathrm{H}_{7} \mathrm{~N}$ & cyclobutanecarbonitrile & & & & [4426-11-3] \\
\hline & & 44.3 & $(298)$ & $\mathrm{C}$ & {$[83 / 6]$} \\
\hline & $(328-402)$ & 39.6 & $(347)$ & BG & {$[71 / 2]$} \\
\hline & & $40.0 \pm 0.4$ & (298) & BG & {$[71 / 2]$} \\
\hline $\mathrm{C}_{5} \mathrm{H}_{7} \mathrm{~N}$ & 2-ethylacrylonitrile & & & & \\
\hline & $(244-387)$ & 37.1 & (259) & & {$[87 / 5][47 / 5]$} \\
\hline $\mathrm{C}_{5} \mathrm{H}_{7} \mathrm{~N}$ & angelic acid, nitrile & & & & [20068-02-4] \\
\hline & $(265-413)$ & 42.8 & $(280)$ & A & {$[87 / 5][47 / 5]$} \\
\hline $\mathrm{C}_{5} \mathrm{H}_{7} \mathrm{~N}$ & 1-methylpyrrole & & & & {$[96-54-8]$} \\
\hline & $(333-373)$ & 38.0 & (343) & I & {$[71 / 3]$} \\
\hline & $(321-423)$ & 39.0 & (336) & A, EB, IPM & $\begin{array}{c}{[87 / 5][68 / 4]} \\
{[72 / 20]}\end{array}$ \\
\hline $\mathrm{C}_{5} \mathrm{H}_{7} \mathrm{~N}$ & (Z) 2-pentenenitrile & & & & [25899-50-7] \\
\hline
\end{tabular}


TABLE 6. Enthalpies of vaporization of organic compounds, 1880-2002-Continued

\begin{tabular}{|c|c|c|c|c|c|}
\hline Molecular formula & $\begin{array}{c}\text { Compound } \\
\text { (Temperature range/K) }\end{array}$ & $\begin{array}{c}\Delta_{\mathrm{vap}} H_{m} \\
\left(\mathrm{~kJ} \mathrm{~mol}^{-1}\right)\end{array}$ & $\begin{array}{c}\text { Mean temperature } \\
\left(T_{m} / \mathrm{K}\right)\end{array}$ & Method & $\begin{array}{l}\text { CAS registry number } \\
\text { Reference }\end{array}$ \\
\hline \multirow{3}{*}{$\mathrm{C}_{5} \mathrm{H}_{7} \mathrm{~N}$} & \multirow{3}{*}{ (E) 3-pentenenitrile } & 43.2 & $(298)$ & & {$[69 / 14]$} \\
\hline & & & & & [16529-66-1] \\
\hline & & 44.8 & $(298)$ & & {$[69 / 14]$} \\
\hline \multirow[t]{2}{*}{$\mathrm{C}_{5} \mathrm{H}_{7} \mathrm{~N}$} & \multirow[t]{2}{*}{ (E) 2-pentenenitrile } & & & & [26294-98-4] \\
\hline & & 44.9 & $(298)$ & & {$[69 / 14]$} \\
\hline \multirow[t]{2}{*}{$\mathrm{C}_{5} \mathrm{H}_{7} \mathrm{~N}$} & tiglic acid, nitrile & & & & [30574-97-1] \\
\hline & $(247-395)$ & 37.4 & $(262)$ & A & {$[87 / 5][47 / 5]$} \\
\hline \multirow[t]{2}{*}{$\mathrm{C}_{5} \mathrm{H}_{7} \mathrm{NO}$} & 4-oxo-pentanenitrile & & & & {$[927-56-0]$} \\
\hline & $(293-473)$ & 52.3 & $(308)$ & A & {$[87 / 5]$} \\
\hline \multirow{2}{*}{$\mathrm{C}_{5} \mathrm{H}_{7} \mathrm{NO}_{2}$} & ethyl cyanoacetate & & & & {$[105-56-6]$} \\
\hline & $(340-479)$ & 66.9 & $(355)$ & A & {$[87 / 5][47 / 5]$} \\
\hline \multirow[t]{2}{*}{$\mathrm{C}_{5} \mathrm{H}_{7} \mathrm{NS}$} & isothiocyanic acid, 3-butenyl ester & & & & [3386-97-8] \\
\hline & $(342-443)$ & 45.2 & $(357)$ & A & {$[87 / 5][99 / 16]$} \\
\hline \multirow[t]{2}{*}{$\mathrm{C}_{5} \mathrm{H}_{7} \mathrm{NS}$} & 2,4-dimethylthiazole & & & & {$[541-58-2]$} \\
\hline & $(357-421)$ & 42.0 & $(372)$ & A & {$[87 / 5]$} \\
\hline \multirow[t]{3}{*}{$\mathrm{C}_{5} \mathrm{H}_{8}$} & bicyclo[2.1.0]pentane & & & & [185-94-4] \\
\hline & & $28.0 \pm 0.5$ & $(298)$ & EB & {$[98 / 17][96 / 18]$} \\
\hline & $(296-315)$ & 28.6 & $(305)$ & A & {$[87 / 5]$} \\
\hline \multirow[t]{5}{*}{$\mathrm{C}_{5} \mathrm{H}_{8}$} & spiropentane & & & & {$[157-40-4]$} \\
\hline & $(276-344)$ & 28.6 & $(291)$ & A & {$[87 / 5][50 / 7]$} \\
\hline & & $28.3 \pm 0.1$ & $(283)$ & $\mathrm{C}$ & {$[50 / 7]$} \\
\hline & & $27.5 \pm 0.1$ & $(298)$ & $\mathrm{C}$ & {$[50 / 7]$} \\
\hline & & $26.7 \pm 0.1$ & $(312)$ & $\mathrm{C}$ & {$[50 / 7]$} \\
\hline \multirow{2}{*}{$\mathrm{C}_{5} \mathrm{H}_{8}$} & vinylcyclopropane & & & & [693-86-7] \\
\hline & $(289-310)$ & 28.9 & (299) & A & {$[87 / 5]$} \\
\hline \multirow[t]{4}{*}{$\mathrm{C}_{5} \mathrm{H}_{8}$} & cyclopentene & & & & {$[142-29-0]$} \\
\hline & $(249-318)$ & 29.9 & $(264)$ & A & {$[87 / 5]$} \\
\hline & $(289-318)$ & 24.8 & (299) & MM & {$[50 / 6]$} \\
\hline & $(230-293)$ & 28.4 & $(300)$ & & {$[41 / 6]$} \\
\hline \multirow[t]{4}{*}{$\mathrm{C}_{5} \mathrm{H}_{8}$} & methylenecyclobutane & & & & [1120-56-5] \\
\hline & $(290-316)$ & 26.1 & $(303)$ & A & {$[87 / 5]$} \\
\hline & $(292-306)$ & 29.1 & (299) & A & $\begin{array}{c}{[87 / 5][78 / 5]} \\
{[78 / 17]}\end{array}$ \\
\hline & & $27.7 \pm 0.4$ & $(298)$ & EB & {$[74 / 3]$} \\
\hline $\mathrm{C}_{5} \mathrm{H}_{8}$ & 3-methyl-1,2-butadiene & & & & {$[598-25-4]$} \\
\hline & $(227-253)$ & 31.0 & $(240)$ & $\mathrm{A}$ & {$[87 / 5]$} \\
\hline & $(252-323)$ & 29.9 & $(267)$ & $\mathrm{A}$ & {$[87 / 5]$} \\
\hline & & 28.0 & $(298)$ & & {$[71 / 28]$} \\
\hline & $(213-242)$ & 31.6 & $(230)$ & IPM & {$[69 / 12]$} \\
\hline & $(274-319)$ & 29.0 & (291) & EB & {$[69 / 12]$} \\
\hline $\mathrm{C}_{5} \mathrm{H}_{8}$ & 2-methyl-1,3-butadiene & & & & {$[78-79-5]$} \\
\hline & $(221-254)$ & 29.4 & (239) & A & {$[87 / 5]$} \\
\hline & $(254-316)$ & 28.3 & (269) & A & {$[87 / 5]$} \\
\hline & & 26.4 & (298) & & {$[71 / 28]$} \\
\hline & $(216-235)$ & 31.5 & $(225)$ & IPM & {$[69 / 12]$} \\
\hline & $(290-308)$ & 27.3 & (299) & MM & {$[50 / 6]$} \\
\hline & $(258-318)$ & 27.4 & $(288)$ & & {$[38 / 7]$} \\
\hline $\mathrm{C}_{5} \mathrm{H}_{8}$ & 3-methyl-1-butyne & & & & [598-23-2] \\
\hline & $(218-320)$ & 30.2 & $(233)$ & A & {$[87 / 5]$} \\
\hline & & 25.8 & $(298)$ & & {$[71 / 28]$} \\
\hline $\mathrm{C}_{5} \mathrm{H}_{8}$ & 1,2-pentadiene & & & & {$[591-95-7]$} \\
\hline & $(231-249)$ & 31.6 & $(240)$ & A & {$[87 / 5]$} \\
\hline & $(249-331)$ & 30.6 & (264) & A & {$[87 / 5]$} \\
\hline & & 28.7 & (298) & & {$[71 / 28]$} \\
\hline & $(213-245)$ & 32.2 & (231) & IPM & {$[69 / 12]$} \\
\hline & $(285-319)$ & 29.1 & $(300)$ & MM & {$[50 / 6]$} \\
\hline $\mathrm{C}_{5} \mathrm{H}_{8}$ & cis 1,3-pentadiene & & & & [1574-41-0] \\
\hline & $(255-326)$ & 30.1 & $(270)$ & $\mathrm{A}$ & {$[87 / 5]$} \\
\hline & $(230-255)$ & 31.2 & $(242)$ & A & {$[87 / 5]$} \\
\hline & & 28.3 & (298) & & {$[71 / 28]$} \\
\hline & $(213-242)$ & 31.9 & (230) & IPM & {$[69 / 12]$} \\
\hline & $(289-318)$ & 28.8 & (304) & MM & {$[50 / 6]$} \\
\hline $\mathrm{C}_{5} \mathrm{H}_{8}$ & trans 1,3 -pentadiene & & & & [2004-41-0] \\
\hline & $(228-256)$ & 30.7 & (242) & A & {$[87 / 5]$} \\
\hline
\end{tabular}


TABLE 6. Enthalpies of vaporization of organic compounds, 1880-2002-Continued

\begin{tabular}{|c|c|c|c|c|c|}
\hline Molecular formula & $\begin{array}{c}\text { Compound } \\
\text { (Temperature range/K) }\end{array}$ & $\begin{array}{c}\Delta_{\mathrm{vap}} H_{m} \\
\left(\mathrm{~kJ} \mathrm{~mol}^{-1}\right)\end{array}$ & $\begin{array}{c}\text { Mean temperature } \\
\left(T_{m} / \mathrm{K}\right)\end{array}$ & Method & $\begin{array}{l}\text { CAS registry number } \\
\text { Reference }\end{array}$ \\
\hline & $(256-324)$ & 29.5 & $(271)$ & A & {$[87 / 5]$} \\
\hline & & 27.8 & (298) & & {$[71 / 28]$} \\
\hline & $(213-242)$ & 31.3 & (230) & IPM & {$[69 / 12]$} \\
\hline & $(292-316)$ & 28.3 & (304) & MM & [50/6] \\
\hline \multirow[t]{7}{*}{$\mathrm{C}_{5} \mathrm{H}_{8}$} & 1,4-pentadiene & & & & [591-93-5] \\
\hline & $(216-236)$ & 29.1 & (226) & A & {$[87 / 5]$} \\
\hline & $(236-307)$ & 28.1 & (251) & $\mathrm{A}$ & {$[87 / 5]$} \\
\hline & & 25.2 & (298) & & {$[71 / 28]$} \\
\hline & $(213-230)$ & 29.3 & (221) & IPM & {$[69 / 12]$} \\
\hline & $(288-300)$ & 26.5 & (293) & MM & {$[50 / 6]$} \\
\hline & $(194-255)$ & 28.4 & $(240)$ & & {$[40 / 3]$} \\
\hline \multirow{6}{*}{$\mathrm{C}_{5} \mathrm{H}_{8}$} & 2,3-pentadiene & & & & [591-96-8] \\
\hline & $(234-258)$ & 32.3 & $(246)$ & $\mathrm{A}$ & {$[87 / 5]$} \\
\hline & $(258-330)$ & 31.1 & (273) & A & {$[87 / 5]$} \\
\hline & & 29.5 & (298) & & {$[71 / 28]$} \\
\hline & $(213-247)$ & 33.2 & (232) & IPM & [69/12] \\
\hline & $(298-322)$ & 29.6 & $(310)$ & MM & {$[50 / 6]$} \\
\hline \multirow[t]{3}{*}{$\mathrm{C}_{5} \mathrm{H}_{8}$} & 1-pentyne & & & & [627-19-0] \\
\hline & $(229-315)$ & 31.8 & $(244)$ & A & {$[87 / 5]$} \\
\hline & & 28.4 & (298) & & [71/28] \\
\hline \multirow[t]{3}{*}{$\mathrm{C}_{5} \mathrm{H}_{8}$} & 2-pentyne & & & & [627-21-4] \\
\hline & $(240-329)$ & 33.1 & (255) & A & {$[87 / 5]$} \\
\hline & & 30.8 & (298) & & {$[71 / 28]$} \\
\hline \multirow[t]{2}{*}{$\mathrm{C}_{5} \mathrm{H}_{8} \mathrm{Br}_{2}$} & trans 1,2-dibromocyclope & & & & [10230-26-9] \\
\hline & $(273-332)$ & 47.9 & $(288)$ & A & {$[87 / 5][41 / 6]$} \\
\hline \multirow[t]{2}{*}{$\mathrm{C}_{5} \mathrm{H}_{8} \mathrm{Br}_{4}$} & pentarythritol tetrabromid & & & & [3229-00-3] \\
\hline & $(439-466)$ & 61.0 & $(452)$ & A & {$[87 / 5]$} \\
\hline \multirow[t]{2}{*}{$\mathrm{C}_{5} \mathrm{H}_{8} \mathrm{ClFO}_{2}$} & 3-chloro-4-fluorobutyric a & 1 ester & & & \\
\hline & $(273-333)$ & 54.5 & $(288)$ & GS & $\begin{array}{c}{[87 / 5][48 / 14]} \\
{[72 / 20]}\end{array}$ \\
\hline \multirow[t]{4}{*}{$\mathrm{C}_{5} \mathrm{H}_{8} \mathrm{ClF}_{3} \mathrm{O}$} & 2-chloro-1,1,2-trifluoroeth & l ether & & & \\
\hline & & 39.2 & (298) & $\mathrm{C}$ & {$[84 / 2]$} \\
\hline & & 38.1 & (313) & $\mathrm{C}$ & {$[84 / 2]$} \\
\hline & & 37.0 & (324) & $\mathrm{C}$ & {$[84 / 2]$} \\
\hline \multirow[t]{6}{*}{$\mathrm{C}_{5} \mathrm{H}_{8} \mathrm{ClF}_{3} \mathrm{O}$} & 2-chloro-1,1,1-trifluoroeth & ther & & & \\
\hline & & 41.0 & (298) & $\mathrm{C}$ & {$[84 / 7]$} \\
\hline & & 39.9 & (313) & $\mathrm{C}$ & {$[84 / 7]$} \\
\hline & & 38.7 & (328) & $\mathrm{C}$ & {$[84 / 7]$} \\
\hline & & 37.5 & (343) & $\mathrm{C}$ & {$[84 / 7]$} \\
\hline & & 36.2 & $(358)$ & $\mathrm{C}$ & {$[84 / 7]$} \\
\hline \multirow[t]{2}{*}{$\mathrm{C}_{5} \mathrm{H}_{8} \mathrm{Cl}_{2} \mathrm{O}$} & 3,3-bis(chloromethyl)oxet & & & & {$[78-71-7]$} \\
\hline & & $56.0 \pm 0.4$ & $(298)$ & $\mathrm{C}$ & {$[71 / 25]$} \\
\hline \multirow[t]{2}{*}{$\mathrm{C}_{5} \mathrm{H}_{8} \mathrm{Cl}_{4}$} & 1,1,1,5-tetrachloropentane & & & & [2467-10-9] \\
\hline & $(340-432)$ & 61.7 & $(355)$ & A & {$[87 / 5]$} \\
\hline \multirow[t]{2}{*}{$\mathrm{C}_{5} \mathrm{H}_{8} \mathrm{~F}_{2} \mathrm{O}_{3}$} & bis(2-fluoroethyl)carbonat & & & & \\
\hline & $(273-333)$ & 61.5 & $(288)$ & GS & $\begin{array}{c}{[87 / 5][48 / 14]} \\
{[72 / 20]}\end{array}$ \\
\hline \multirow[t]{3}{*}{$\mathrm{C}_{5} \mathrm{H}_{8} \mathrm{~N}_{2}$} & 1,3-dimethyl-2-imidazolid & & & & [80-73-9] \\
\hline & $(355-498)$ & 54.3 & (375) & EB & {$[87 / 2]$} \\
\hline & $(355-498)$ & 48.5 & $(450)$ & EB & [87/2] \\
\hline \multirow[t]{2}{*}{$\mathrm{C}_{5} \mathrm{H}_{8} \mathrm{~N}_{2}$} & 1-ethylimidazole & & & & [7098-07-9] \\
\hline & & $66.0 \pm 3.9$ & $(298)$ & $\mathrm{C}$ & [99/1] \\
\hline \multirow[t]{2}{*}{$\mathrm{C}_{5} \mathrm{H}_{8} \mathrm{~N}_{2}$} & 1-ethylpyrazole & & & & {$[2817-71-2]$} \\
\hline & & $53.3 \pm 2.4$ & $(298)$ & $\mathrm{C}$ & [99/1] \\
\hline $\mathrm{C}_{5} \mathrm{H}_{8} \mathrm{O}$ & cyclopentanone & & & & [120-93-2] \\
\hline & & $42.1 \pm 0.2$ & (298) & & [91/17] \\
\hline & & $43.2 \pm 0.3$ & & $\mathrm{GC}$ & [89/16] \\
\hline & $(317-427)$ & 40.6 & (332) & & {$[87 / 7]$} \\
\hline & $(293-404)$ & 42.6 & (308) & A & {$[87 / 5]$} \\
\hline & $(338-416)$ & 39.6 & (353) & $\mathrm{A}, \mathrm{EB}$ & {$[87 / 5][76 / 10]$} \\
\hline & & $42.7 \pm 0.1$ & (298) & $\mathrm{C}$ & {$[68 / 17]$} \\
\hline & $(273-299)$ & 43.6 & $(286)$ & & {$[42 / 7]$} \\
\hline $\mathrm{C}_{5} \mathrm{H}_{8} \mathrm{O}$ & 2-ethylacrolein & & & & {$[922-63-4]$} \\
\hline & & $36.8 \pm 0.4$ & (298) & $\mathrm{C}$ & {$[96 / 8]$} \\
\hline
\end{tabular}


TABLE 6. Enthalpies of vaporization of organic compounds, 1880-2002-Continued

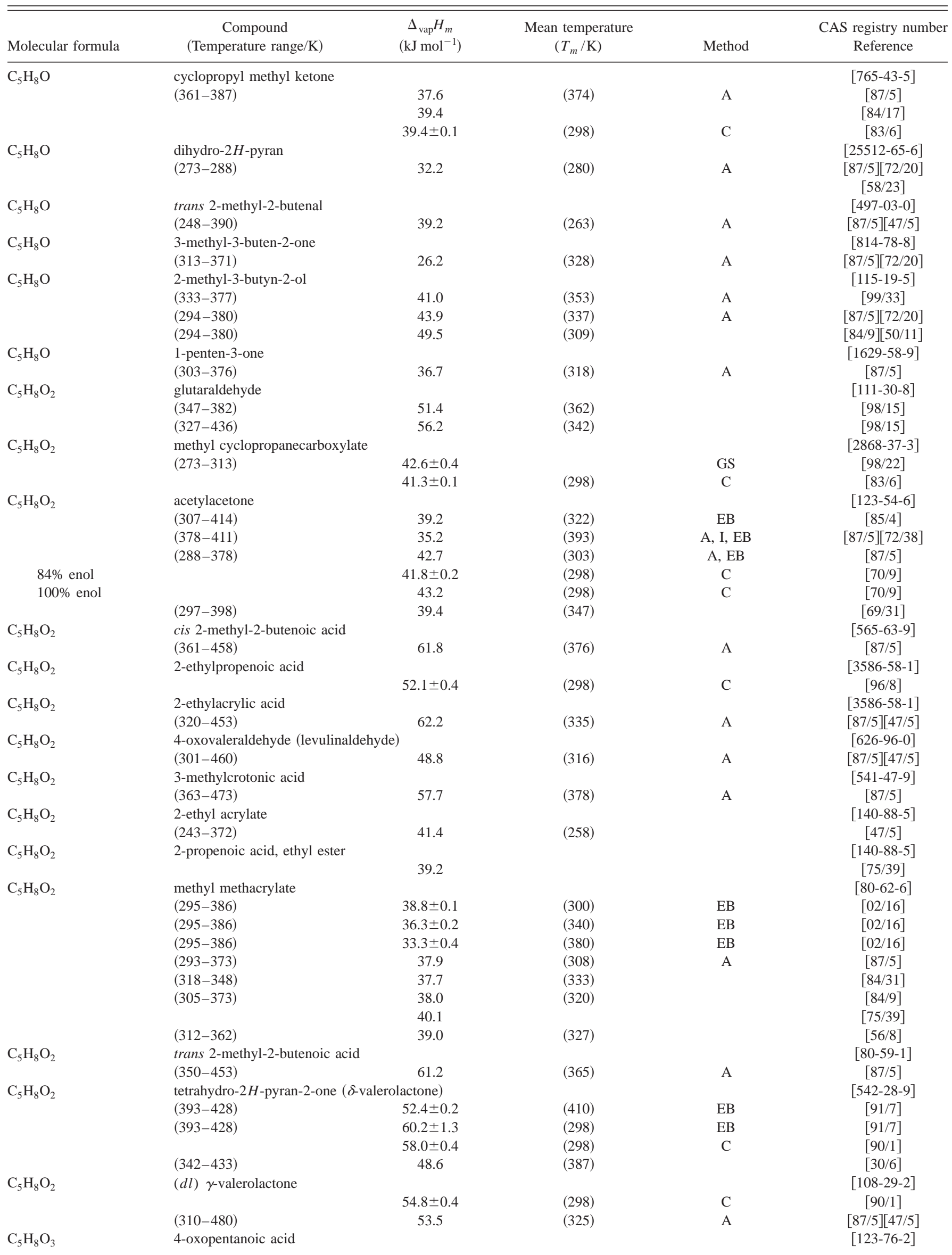


TABLE 6. Enthalpies of vaporization of organic compounds, 1880-2002-Continued

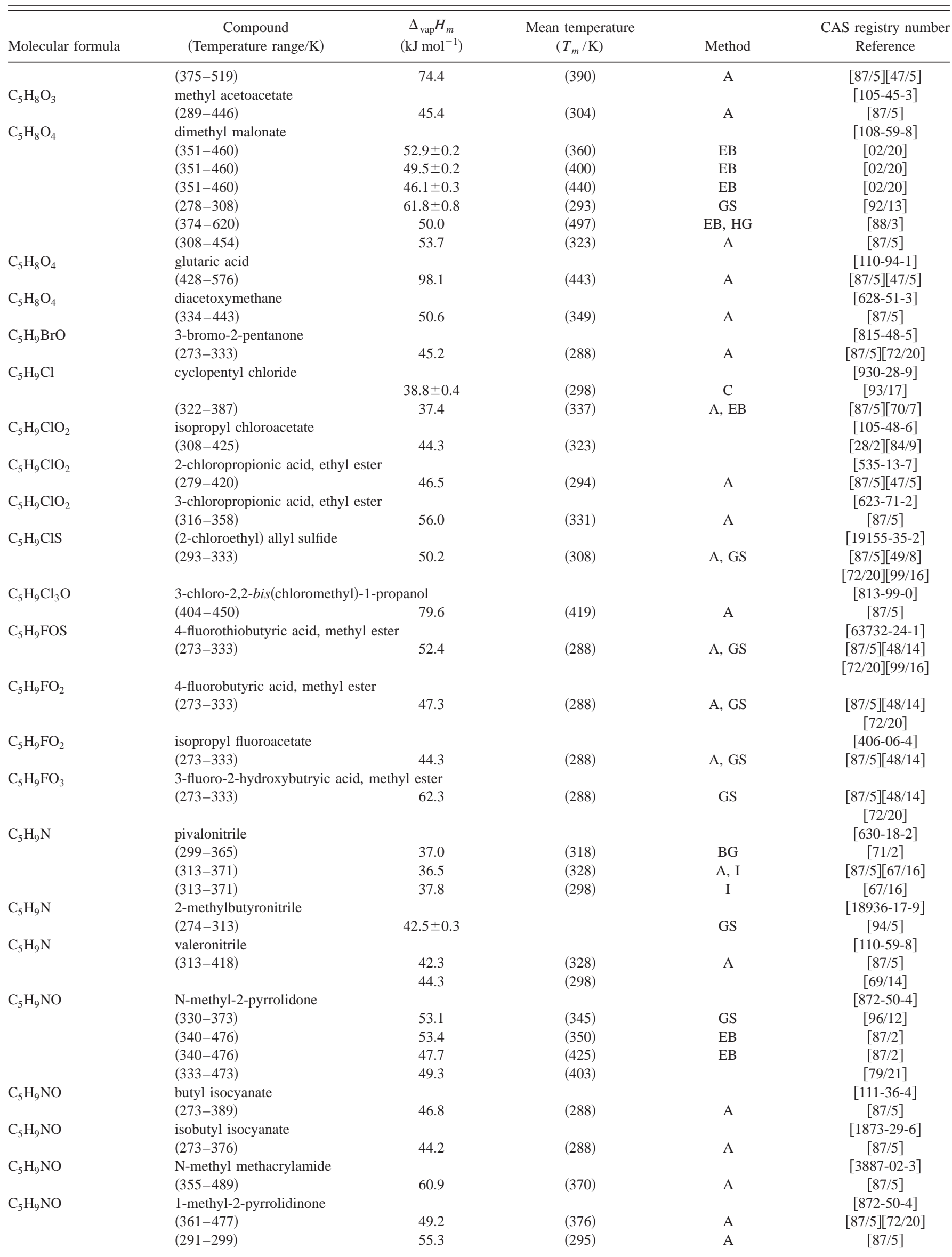


TABLE 6. Enthalpies of vaporization of organic compounds, 1880-2002-Continued

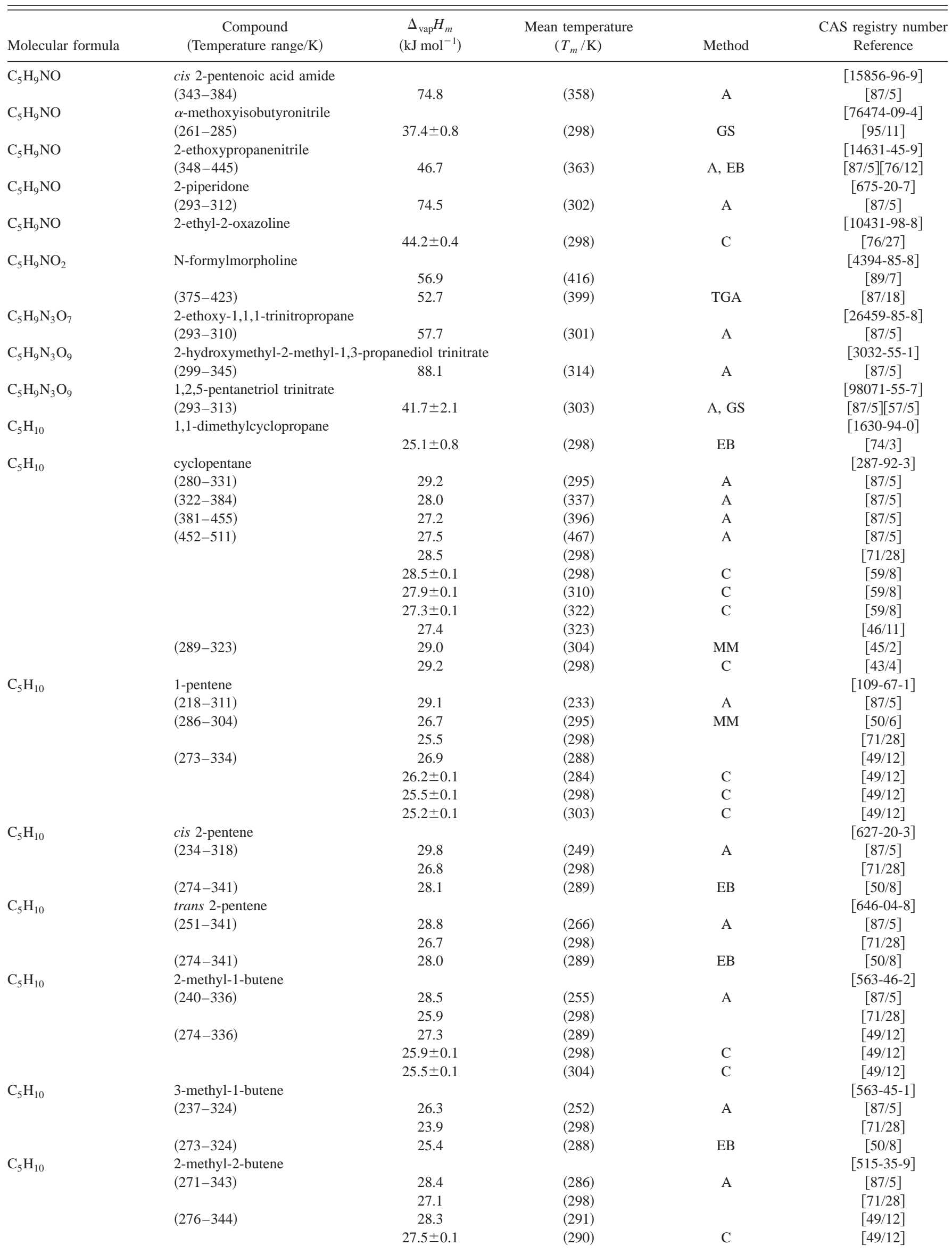


TABLE 6. Enthalpies of vaporization of organic compounds, 1880-2002-Continued

\begin{tabular}{|c|c|c|c|c|c|}
\hline Molecular formula & $\begin{array}{c}\text { Compound } \\
\text { (Temperature range/K) }\end{array}$ & $\begin{array}{c}\Delta_{\mathrm{vap}} H_{m} \\
\left(\mathrm{~kJ} \mathrm{~mol}^{-1}\right)\end{array}$ & $\begin{array}{l}\text { Mean temperature } \\
\qquad\left(T_{m} / \mathrm{K}\right)\end{array}$ & Method & $\begin{array}{c}\text { CAS registry number } \\
\text { Reference }\end{array}$ \\
\hline & & $27.1 \pm 0.1$ & $(298)$ & $\mathrm{C}$ & [49/12] \\
\hline & & $26.3 \pm 0.1$ & $(312)$ & $\mathrm{C}$ & {$[49 / 12]$} \\
\hline \multirow[t]{2}{*}{$\mathrm{C}_{5} \mathrm{H}_{10} \mathrm{Br}_{2}$} & 1,1-dibromopentane & & & & {$[13320-56-4]$} \\
\hline & $(360-501)$ & 48.8 & $(375)$ & A, EST & $\begin{array}{c}{[87 / 5][56 / 16]} \\
{[72 / 20]}\end{array}$ \\
\hline \multirow[t]{5}{*}{$\mathrm{C}_{5} \mathrm{H}_{10} \mathrm{Br}_{2}$} & 1,2-dibromopentane & & & & [3234-49-9] \\
\hline & $(348-465)$ & 46.5 & $(363)$ & A & {$[87 / 5]$} \\
\hline & $(350-450)$ & 49.0 & $(298)$ & & {$[75 / 15][91 / 2]$} \\
\hline & & $49.2 \pm 0.8$ & $(298)$ & EB & {$[75 / 15]$} \\
\hline & $(292-448)$ & 48.8 & $(307)$ & $\mathrm{A}$ & {$[87 / 5][47 / 5]$} \\
\hline \multirow[t]{2}{*}{$\mathrm{C}_{5} \mathrm{H}_{10} \mathrm{Br}_{2}$} & 1,4-dibromopentane & & & & [626-87-9] \\
\hline & $(377-524)$ & 51.8 & $(392)$ & A & {$[87 / 5][72 / 20]$} \\
\hline \multirow[t]{2}{*}{$\mathrm{C}_{5} \mathrm{H}_{10} \mathrm{Br}_{2}$} & 1,5-dibromopentane & & & & {$[111-24-0]$} \\
\hline & $(396-549)$ & 54.4 & $(411)$ & A & {$[87 / 5][72 / 20]$} \\
\hline \multirow[t]{3}{*}{$\mathrm{C}_{5} \mathrm{H}_{10} \mathrm{Cl}_{2}$} & 1,1-dichloropentane & & & & {$[820-55-3]$} \\
\hline & $(340-410)$ & 44.3 & $(298)$ & A & {$[87 / 12][91 / 2]$} \\
\hline & $(325-457)$ & 42.0 & $(340)$ & $\mathrm{A}, \mathrm{EST}$ & $\begin{array}{c}{[87 / 5][56 / 16]} \\
{[72 / 20]}\end{array}$ \\
\hline \multirow[t]{4}{*}{$\mathrm{C}_{5} \mathrm{H}_{10} \mathrm{Cl}_{2}$} & 1,2-dichloropentane & & & & {$[1674-33-5]$} \\
\hline & $(330-420)$ & 44.4 & $(298)$ & & {$[91 / 2]$} \\
\hline & $(332-418)$ & 41.9 & $(347)$ & A & {$[87 / 5]$} \\
\hline & & $43.8 \pm 0.7$ & $(298)$ & $\mathrm{EB}$ & {$[75 / 16]$} \\
\hline \multirow[t]{4}{*}{$\mathrm{C}_{5} \mathrm{H}_{10} \mathrm{Cl}_{2}$} & 1,4-dichloropentane & & & & {$[626-92-6]$} \\
\hline & $(350-440)$ & 48.9 & $(298)$ & & {$[91 / 2]$} \\
\hline & $(348-443)$ & 45.0 & $(363)$ & A & {$[87 / 5]$} \\
\hline & & $48.1 \pm 0.8$ & $(298)$ & $\mathrm{EB}$ & {$[75 / 16]$} \\
\hline \multirow[t]{4}{*}{$\mathrm{C}_{5} \mathrm{H}_{10} \mathrm{Cl}_{2}$} & 1,5-dichloropentane & & & & {$[628-76-2]$} \\
\hline & $(360-450)$ & 52.2 & $(298)$ & & {$[91 / 2]$} \\
\hline & $(362-453)$ & 47.2 & $(377)$ & A & {$[87 / 5]$} \\
\hline & & $51.3 \pm 0.8$ & $(298)$ & $\mathrm{EB}$ & {$[75 / 16]$} \\
\hline \multirow[t]{2}{*}{$\mathrm{C}_{5} \mathrm{H}_{10} \mathrm{Cl}_{2} \mathrm{O}$} & (2-chloroethyl) (2-chloroi & ther & & & {$[52250-75-6]$} \\
\hline & $(297-453)$ & 49.7 & $(312)$ & $\mathrm{A}$ & {$[87 / 5][47 / 5]$} \\
\hline \multirow[t]{2}{*}{$\mathrm{C}_{5} \mathrm{H}_{10} \mathrm{Cl}_{2} \mathrm{O}$} & (2-chloroethyl) (2-chlorop & & & & {$[42434-29-7]$} \\
\hline & $(302-467)$ & 49.3 & $(317)$ & A & {$[87 / 5][47 / 5]$} \\
\hline \multirow[t]{2}{*}{$\mathrm{C}_{5} \mathrm{H}_{10} \mathrm{Cl}_{2} \mathrm{O}_{2}$} & bis(2-chloroethoxy) meth & & & & {$[111-91-1]$} \\
\hline & $(326-488)$ & 54.2 & $(341)$ & A & {$[87 / 5][47 / 5]$} \\
\hline \multirow[t]{2}{*}{$\mathrm{C}_{5} \mathrm{H}_{10} \mathrm{~F}_{2}$} & 1,1-difluoropentane & & & & {$[62127-40-6]$} \\
\hline & $(268-378)$ & 34.4 & $(283)$ & $\mathrm{A}, \mathrm{EST}$ & $\begin{array}{c}{[87 / 5][56 / 16]} \\
{[72 / 20]}\end{array}$ \\
\hline \multirow[t]{2}{*}{$\mathrm{C}_{5} \mathrm{H}_{10} \mathrm{~F}_{2}$} & 2,2-difluoropentane & & & & [371-65-3] \\
\hline & $(262-367)$ & 33.7 & $(277)$ & $\mathrm{A}$ & {$[87 / 5][72 / 20]$} \\
\hline \multirow[t]{2}{*}{$\mathrm{C}_{5} \mathrm{H}_{10} \mathrm{~F}_{2}$} & 3,3-difluoropentane & & & & [358-03-2] \\
\hline & $(262-368)$ & 33.8 & $(277)$ & A & {$[87 / 5][72 / 20]$} \\
\hline \multirow[t]{2}{*}{$\mathrm{C}_{5} \mathrm{H}_{10} \mathrm{~F}_{2} \mathrm{O}_{2}$} & bis(2-fluoroethoxy) metha & & & & {$[373-40-0]$} \\
\hline & $(273-333)$ & 52.3 & $(288)$ & A, GS & $\begin{array}{c}{[87 / 5][48 / 14]} \\
{[72 / 20]}\end{array}$ \\
\hline \multirow[t]{6}{*}{$\mathrm{C}_{5} \mathrm{H}_{10} \mathrm{~N}_{2}$} & 3-(dimethylamino)propion & & & & {$[1738-25-6]$} \\
\hline & & $42.2 \pm 0.1$ & & & {$[92 / 20]$} \\
\hline & $(330-445)$ & 45.9 & $(345)$ & A & {$[87 / 5]$} \\
\hline & $(331-407)$ & 52.4 & $(346)$ & A & {$[87 / 5]$} \\
\hline & $(290-317)$ & $44.1 \pm 0.2$ & & & {$[84 / 28]$} \\
\hline & & 47.3 & & & {$[77 / 29]$} \\
\hline \multirow[t]{2}{*}{$\mathrm{C}_{5} \mathrm{H}_{10} \mathrm{~N}_{2} \mathrm{O}$} & 1-nitrosopiperidine & & & & {$[100-75-4]$} \\
\hline & $(333-383)$ & 47.7 & $(348)$ & A & {$[87 / 5]$} \\
\hline $\mathrm{C}_{5} \mathrm{H}_{10} \mathrm{~N}_{2} \mathrm{O}_{6}$ & 1,5-pentanediol dinitrate & & & & [3457-92-9] \\
\hline & $(293-313)$ & $78.9 \pm 5.9$ & $(303)$ & A, GS & $\begin{array}{c}{[87 / 5][57 / 5]} \\
{[72 / 20]}\end{array}$ \\
\hline $\mathrm{C}_{5} \mathrm{H}_{10} \mathrm{~N}_{2} \mathrm{O}_{6}$ & 2,4-pentanediol dinitrate & & & & {$[25385-63-1]$} \\
\hline & $(293-313)$ & $60.6 \pm 5.9$ & $(303)$ & A, GS & $\begin{array}{c}{[87 / 5][57 / 5]} \\
{[72 / 20]}\end{array}$ \\
\hline $\mathrm{C}_{5} \mathrm{H}_{10} \mathrm{~N}_{2} \mathrm{O}_{6}$ & 1-(methoxymethoxy)-2,2- & ane & & & {$[67727-92-8]$} \\
\hline & $(293-333)$ & 71.3 & $(308)$ & A & {$[87 / 5]$} \\
\hline $\mathrm{C}_{5} \mathrm{H}_{10} \mathrm{O}$ & allyl ethyl ether & & & & {$[557-31-3]$} \\
\hline & $(244-401)$ & 34.6 & $(259)$ & A & {$[87 / 5]$} \\
\hline
\end{tabular}


TABLE 6. Enthalpies of vaporization of organic compounds, 1880-2002-Continued

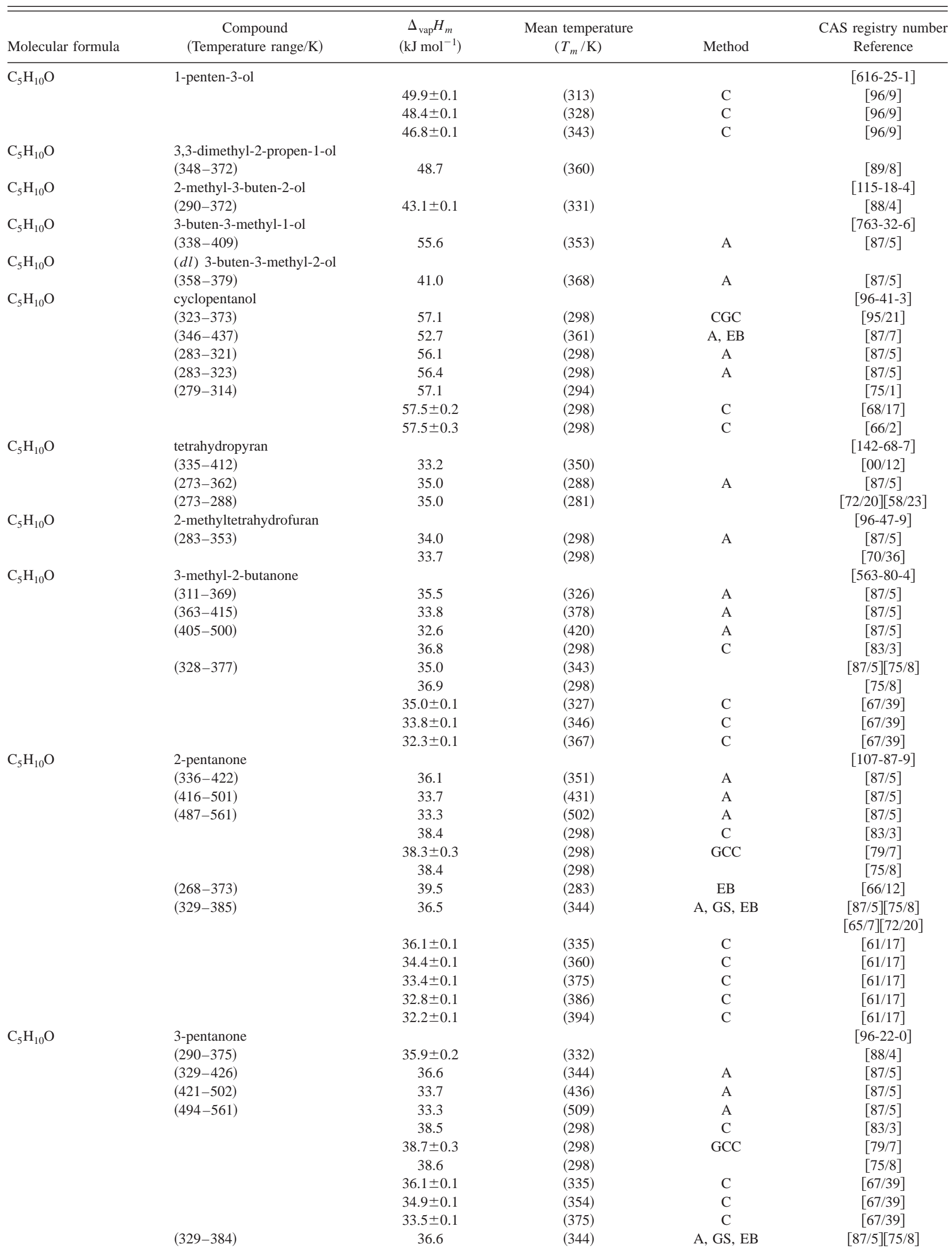


TABLE 6. Enthalpies of vaporization of organic compounds, 1880-2002-Continued

\begin{tabular}{|c|c|c|c|c|c|}
\hline Molecular formula & $\begin{array}{c}\text { Compound } \\
\text { (Temperature range/K) }\end{array}$ & $\begin{array}{c}\Delta_{\mathrm{vap}} H_{m} \\
\left(\mathrm{~kJ} \mathrm{~mol}^{-1}\right)\end{array}$ & $\begin{array}{l}\text { Mean temperature } \\
\qquad\left(T_{m} / \mathrm{K}\right)\end{array}$ & Method & $\begin{array}{c}\text { CAS registry number } \\
\text { Reference }\end{array}$ \\
\hline \multirow{7}{*}{$\mathrm{C}_{5} \mathrm{H}_{10} \mathrm{O}$} & & & & & {$[65 / 7][72 / 20]$} \\
\hline & $(283-323)$ & 36.9 & $(303)$ & & {$[37 / 9]$} \\
\hline & pentanal & & & & {$[110-62-3]$} \\
\hline & $(313-353)$ & 38.3 & $(298)$ & CGC & {$[95 / 21]$} \\
\hline & $(290-385)$ & U50.0 & (305) & A & {$[87 / 5]$} \\
\hline & & $38.1 \pm 0.1$ & (298) & & {$[81 / 18]$} \\
\hline & $(305-377)$ & 37.3 & (320) & & {$[79 / 15]$} \\
\hline \multirow[t]{2}{*}{$\mathrm{C}_{5} \mathrm{H}_{10} \mathrm{O}$} & pivaldehyde & & & & [630-19-3] \\
\hline & $(308-336)$ & 34.2 & $(322)$ & & {$[89 / 2]$} \\
\hline \multirow[t]{2}{*}{$\mathrm{C}_{5} \mathrm{H}_{10} \mathrm{O}$} & 3,3-dimethyloxetane & & & & {$[6921-35-3]$} \\
\hline & & $33.9 \pm 0.3$ & (298) & $\mathrm{C}$ & {$[71 / 25]$} \\
\hline \multirow[t]{2}{*}{$\mathrm{C}_{5} \mathrm{H}_{10} \mathrm{OS}$} & S-propyl thiolacetate & & & & {$[2307-10-0]$} \\
\hline & & $44.1 \pm 0.2$ & $(298)$ & $\mathrm{C}$ & {$[66 / 2]$} \\
\hline \multirow[t]{2}{*}{$\mathrm{C}_{5} \mathrm{H}_{10} \mathrm{OS}$} & S-isopropyl thiolacetate & & & & {$[926-73-8]$} \\
\hline & & $42.3 \pm 0.2$ & $(298)$ & $\mathrm{C}$ & {$[66 / 2]$} \\
\hline \multirow[t]{2}{*}{$\mathrm{C}_{5} \mathrm{H}_{10} \mathrm{OS}$} & 1-(methylthio)-2-(vinyloxy)ethane & & & & [6607-53-0] \\
\hline & $(316-347)$ & 47.5 & $(331)$ & A & {$[87 / 5][99 / 16]$} \\
\hline \multirow[t]{3}{*}{$\mathrm{C}_{5} \mathrm{H}_{10} \mathrm{O}_{2}$} & tetrahydrofurfuryl alcohol & & & & [97-99-4] \\
\hline & $(393-453)$ & 46.2 & (408) & A & {$[87 / 5]$} \\
\hline & $(333-443)$ & 46.5 & $(388)$ & & {$[79 / 21]$} \\
\hline \multirow{2}{*}{$\mathrm{C}_{5} \mathrm{H}_{10} \mathrm{O}_{2}$} & 2,2-dimethyl-1,3-dioxolane & & & & [2916-31-6] \\
\hline & $(278-318)$ & $41.1 \pm 0.2$ & & GS & {$[98 / 21][02 / 32]$} \\
\hline \multirow[t]{2}{*}{$\mathrm{C}_{5} \mathrm{H}_{10} \mathrm{O}_{2}$} & 4-methyl-1,3-dioxane & & & & [1120-97-4] \\
\hline & $(273-313)$ & $43.7 \pm 0.3$ & & GS & {$[98 / 21][02 / 32]$} \\
\hline \multirow[t]{2}{*}{$\mathrm{C}_{5} \mathrm{H}_{10} \mathrm{O}_{2}$} & 1-methoxy-2-butanone & & & & {$[50741-70-3]$} \\
\hline & $(297-408)$ & 44.9 & $(312)$ & A & $\begin{array}{c}{[87 / 5][34 / 3]} \\
{[72 / 20]}\end{array}$ \\
\hline \multirow[t]{2}{*}{$\mathrm{C}_{5} \mathrm{H}_{10} \mathrm{O}_{2}$} & 3-hydroxy-3-methyl-2-butanone & & & & {$[115-22-0]$} \\
\hline & $(317-419)$ & 41.1 & $(332)$ & A & $\begin{array}{c}{[87 / 5][72 / 20]} \\
{[50 / 11]}\end{array}$ \\
\hline \multirow[t]{3}{*}{$\mathrm{C}_{5} \mathrm{H}_{10} \mathrm{O}_{2}$} & 4-hydroxy-3-methyl-2-butanone & & & & [3393-64-4] \\
\hline & $(375-528)$ & 58.3 & (390) & A & {$[87 / 5][72 / 20]$} \\
\hline & $(317-458)$ & 59.0 & $(332)$ & & {$[47 / 5]$} \\
\hline \multirow[t]{8}{*}{$\mathrm{C}_{5} \mathrm{H}_{10} \mathrm{O}_{2}$} & butyl formate & & & & {$[592-84-7]$} \\
\hline & $(295-380)$ & 37.9 & $(310)$ & A & {$[87 / 5]$} \\
\hline & & $41.3 \pm 0.1$ & (298) & $\mathrm{C}$ & {$[80 / 13]$} \\
\hline & & $40.1 \pm 0.1$ & (313) & $\mathrm{C}$ & {$[80 / 13]$} \\
\hline & & $39.0 \pm 0.1$ & (328) & $\mathrm{C}$ & [80/13] \\
\hline & & $38.7 \pm 0.1$ & $(346)$ & $\mathrm{C}$ & {$[76 / 14]$} \\
\hline & & $38.1 \pm 0.1$ & $(355)$ & $\mathrm{C}$ & {$[76 / 14]$} \\
\hline & & $37.3 \pm 0.1$ & $(363)$ & $\mathrm{C}$ & {$[76 / 14]$} \\
\hline \multirow[t]{2}{*}{$\mathrm{C}_{5} \mathrm{H}_{10} \mathrm{O}_{2}$} & sec butyl formate & & & & [589-40-2] \\
\hline & $(238-367)$ & 37.7 & (253) & A & [87/5] \\
\hline \multirow{12}{*}{$\mathrm{C}_{5} \mathrm{H}_{10} \mathrm{O}_{2}$} & ethyl propionate & & & & [105-37-3] \\
\hline & $(315-420)$ & 36.7 & (330) & & [97/11] \\
\hline & $(372-538)$ & 34.4 & (387) & $\mathrm{A}$ & {$[87 / 5]$} \\
\hline & & $39.3 \pm 0.1$ & (298) & $\mathrm{C}$ & {$[80 / 13]$} \\
\hline & & $38.2 \pm 0.1$ & (313) & $\mathrm{C}$ & {$[80 / 13]$} \\
\hline & & $36.6 \pm 0.1$ & (336) & $\mathrm{C}$ & {$[77 / 12]$} \\
\hline & & $36.0 \pm 0.1$ & (344) & $\mathrm{C}$ & {$[77 / 12]$} \\
\hline & & $35.5 \pm 0.1$ & (351) & $\mathrm{C}$ & {$[77 / 12]$} \\
\hline & & $34.5 \pm 0.1$ & (363) & $\mathrm{C}$ & {$[77 / 12]$} \\
\hline & & $39.1 \pm 0.1$ & (298) & $\mathrm{C}$ & {$[72 / 3]$} \\
\hline & $(306-372)$ & 38.2 & (321) & A & {$[87 / 5][65 / 3]$} \\
\hline & & & & & {$[72 / 20]$} \\
\hline $\mathrm{C}_{5} \mathrm{H}_{10} \mathrm{O}_{2}$ & isobutyl formate & & & & {$[542-55-2]$} \\
\hline & $(371-507)$ & 36.6 & $(386)$ & A & {$[87 / 5]$} \\
\hline & $(240-372)$ & 38.6 & (255) & A & {$[87 / 5][47 / 5]$} \\
\hline $\mathrm{C}_{5} \mathrm{H}_{10} \mathrm{O}_{2}$ & isopropyl acetate & & & & {$[108-21-4]$} \\
\hline & $(313-353)$ & 37.0 & (298) & CGC & {$[95 / 21]$} \\
\hline & & $37.2 \pm 0.2$ & (298) & $\mathrm{C}$ & [66/2] \\
\hline & $(235-362)$ & 38.8 & (250) & $\mathrm{A}$ & {$[87 / 5][47 / 5]$} \\
\hline & $(273-363)$ & 36.3 & (288) & $\mathrm{A}$ & [29/5] \\
\hline $\mathrm{C}_{5} \mathrm{H}_{10} \mathrm{O}_{2}$ & methyl butyrate & & & & [623-42-7] \\
\hline
\end{tabular}


TABLE 6. Enthalpies of vaporization of organic compounds, 1880-2002-Continued

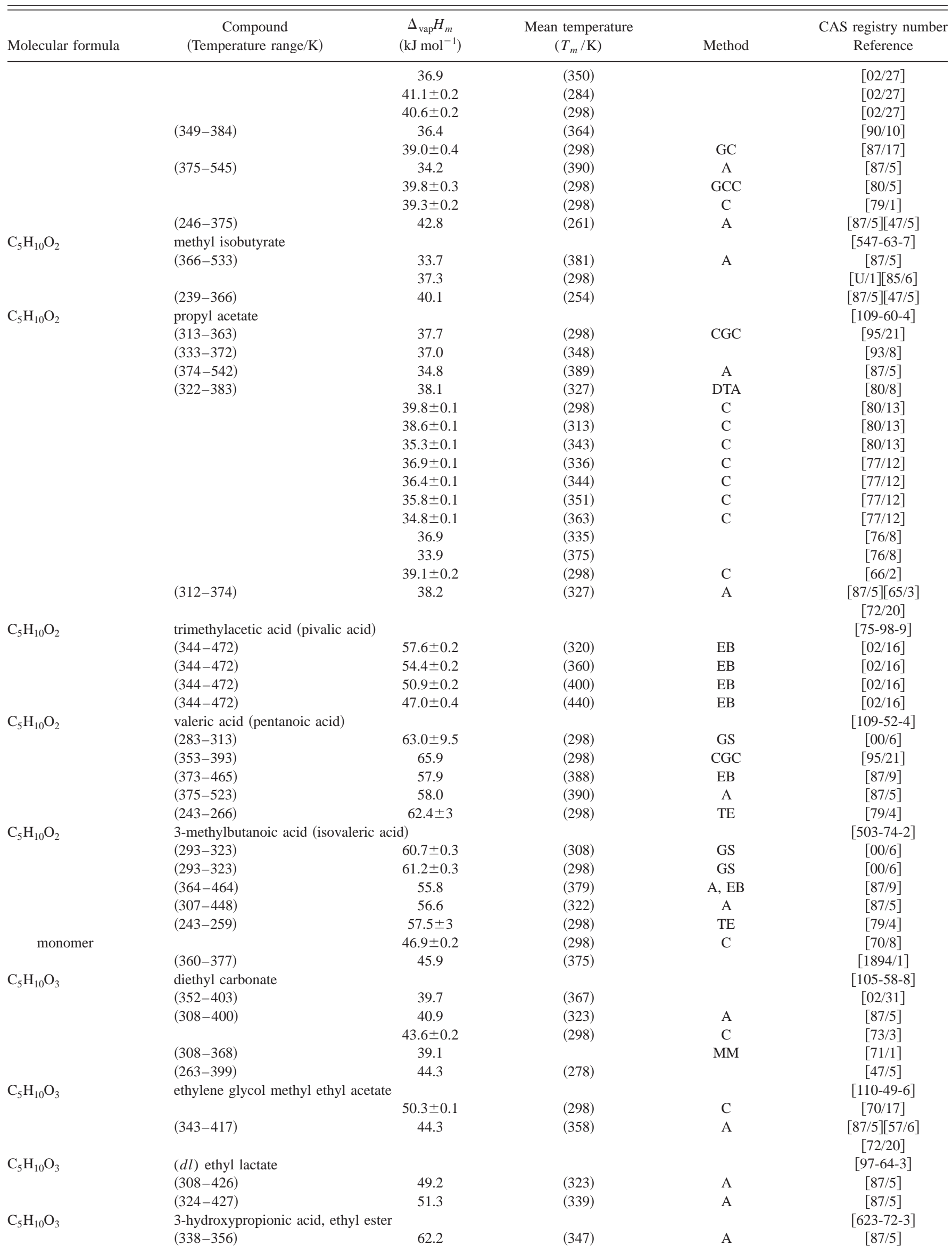


TABLE 6. Enthalpies of vaporization of organic compounds, 1880-2002-Continued

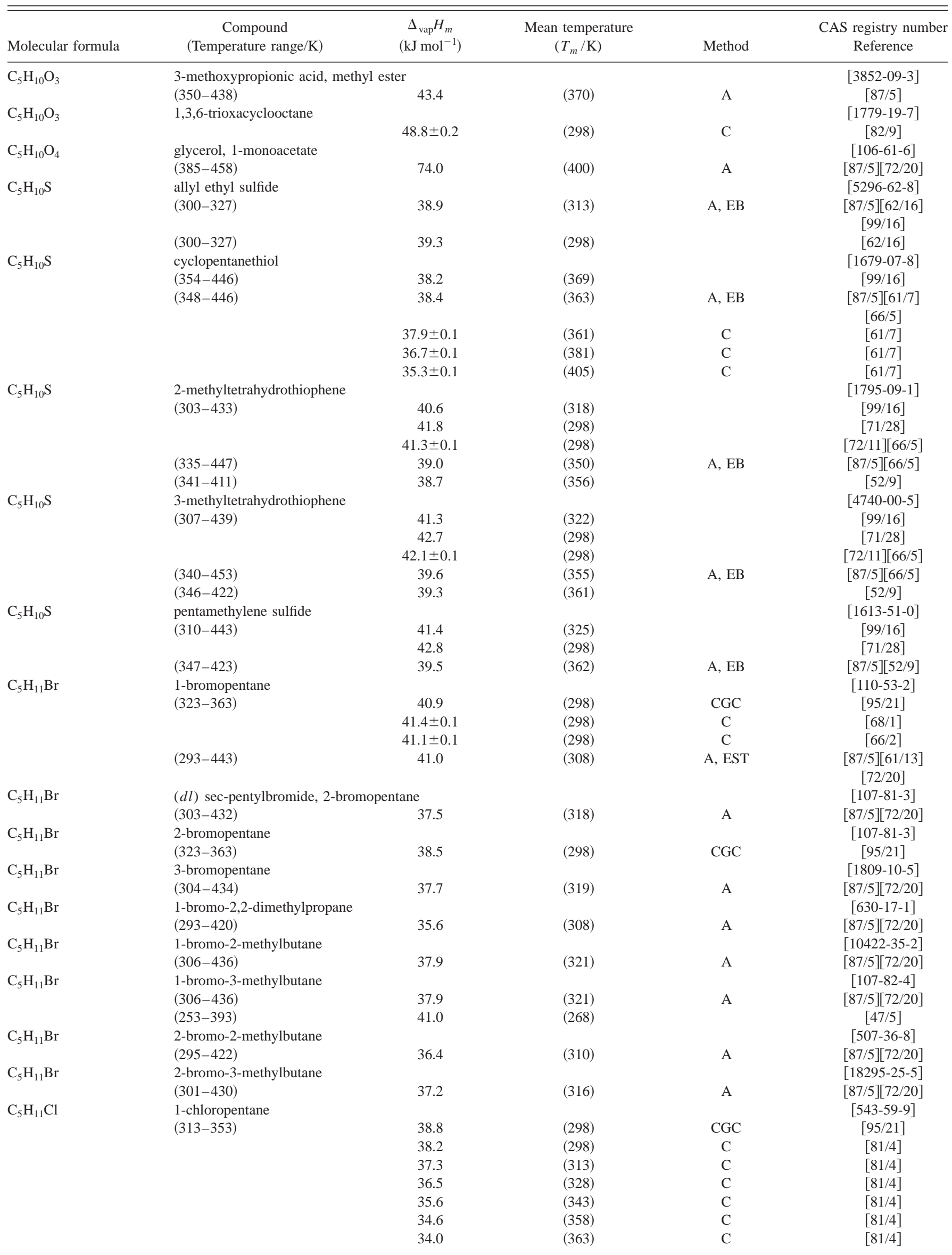


TABLE 6. Enthalpies of vaporization of organic compounds, 1880-2002-Continued

\begin{tabular}{|c|c|c|c|c|c|}
\hline Molecular formula & $\begin{array}{c}\text { Compound } \\
\text { (Temperature range/K) }\end{array}$ & $\begin{array}{c}\Delta_{\mathrm{vap}} H_{m} \\
\left(\mathrm{~kJ} \mathrm{~mol}^{-1}\right)\end{array}$ & $\begin{array}{l}\text { Mean temperature } \\
\qquad\left(T_{m} / \mathrm{K}\right)\end{array}$ & Method & $\begin{array}{c}\text { CAS registry number } \\
\text { Reference }\end{array}$ \\
\hline \multirow{11}{*}{$\mathrm{C}_{5} \mathrm{H}_{11} \mathrm{Cl}$} & & $38.2 \pm 0.1$ & $(298)$ & $\mathrm{C}$ & {$[68 / 1]$} \\
\hline & $(277-421)$ & 38.7 & $(292)$ & A, EST & {$[87 / 5][61 / 13]$} \\
\hline & & & & & {$[72 / 20]$} \\
\hline & $(283-323)$ & 36.2 & $(303)$ & & {$[37 / 9]$} \\
\hline & 2-chloropentane & & & & {$[625-29-6]$} \\
\hline & & 36.0 & (298) & $\mathrm{C}$ & {$[81 / 4]$} \\
\hline & & 35.2 & (313) & $\mathrm{C}$ & {$[81 / 4]$} \\
\hline & & 34.4 & (328) & $\mathrm{C}$ & {$[81 / 4]$} \\
\hline & & 33.5 & $(358)$ & $\mathrm{C}$ & {$[81 / 4]$} \\
\hline & & 31.9 & (368) & $\mathrm{C}$ & {$[81 / 4]$} \\
\hline & $(289-409)$ & 36.2 & $(304)$ & $\mathrm{A}$ & {$[87 / 5][72 / 20]$} \\
\hline \multirow{2}{*}{$\mathrm{C}_{5} \mathrm{H}_{11} \mathrm{Cl}$} & 3-chloropentane & & & & [616-20-6] \\
\hline & $(289-410)$ & 36.5 & $(304)$ & A & {$[87 / 5][72 / 20]$} \\
\hline \multirow[t]{2}{*}{$\mathrm{C}_{5} \mathrm{H}_{11} \mathrm{Cl}$} & 1-chloro-2,2-dimethylpropane & & & & [753-89-9] \\
\hline & $(279-395)$ & 34.9 & (294) & A & {$[87 / 5][72 / 20]$} \\
\hline \multirow[t]{2}{*}{$\mathrm{C}_{5} \mathrm{H}_{11} \mathrm{Cl}$} & ( $d l$ ) 1-chloro-2-methylbutane & & & & [616-13-7] \\
\hline & $(300-374)$ & 35.4 & $(315)$ & A & {$[87 / 5][72 / 20]$} \\
\hline \multirow[t]{2}{*}{$\mathrm{C}_{5} \mathrm{H}_{11} \mathrm{Cl}$} & 2-chloro-2-methylbutane & & & & [594-36-5] \\
\hline & $(280-396)$ & 35.0 & $(295)$ & A & {$[87 / 5][72 / 20]$} \\
\hline \multirow{2}{*}{$\mathrm{C}_{5} \mathrm{H}_{11} \mathrm{Cl}$} & 2-chloro-3-methylbutane & & & & {$[631-65-2]$} \\
\hline & $(285-405)$ & 35.9 & $(300)$ & A & {$[87 / 5][72 / 20]$} \\
\hline \multirow[t]{8}{*}{$\mathrm{C}_{5} \mathrm{H}_{11} \mathrm{Cl}$} & 1-chloro-3-methylbutane & & & & [107-84-6] \\
\hline & $(313-353)$ & 38.1 & $(298)$ & $\mathrm{CGC}$ & {$[95 / 21]$} \\
\hline & & 36.2 & (298) & $\mathrm{C}$ & {$[81 / 4]$} \\
\hline & & 35.4 & (313) & $\mathrm{C}$ & {$[81 / 4]$} \\
\hline & & 34.6 & $(328)$ & $\mathrm{C}$ & {$[81 / 4]$} \\
\hline & & 33.7 & $(343)$ & $\mathrm{C}$ & {$[81 / 4]$} \\
\hline & & 32.8 & $(358)$ & $\mathrm{C}$ & {$[81 / 4]$} \\
\hline & & 32.3 & $(368)$ & $\mathrm{C}$ & {$[81 / 4]$} \\
\hline \multirow{4}{*}{$\mathrm{C}_{5} \mathrm{H}_{11} \mathrm{ClO}_{2} \mathrm{~S}$} & 1-pentanesulfonyl chloride & & & & [6303-18-0] \\
\hline & $(293-387)$ & 58.5 & $(308)$ & & {$[99 / 16]$} \\
\hline & $(387-492)$ & 55.1 & $(402)$ & & {$[99 / 16]$} \\
\hline & $(263-293)$ & 60.5 & $(278)$ & A & {$[87 / 5][99 / 16]$} \\
\hline \multirow[t]{2}{*}{$\mathrm{C}_{5} \mathrm{H}_{11} \mathrm{Cl}_{2} \mathrm{~N}$} & $\mathrm{~N}$-methyl-bis(2-chloroethyl)amine & & & & {$[51-75-2]$} \\
\hline & $(273-333)$ & 54.6 & $(288)$ & A & {$[87 / 5]$} \\
\hline \multirow[t]{2}{*}{$\mathrm{C}_{5} \mathrm{H}_{11} \mathrm{~F}$} & 1-fluoropentane & & & & {$[592-50-7]$} \\
\hline & $(245-373)$ & 33.7 & $(260)$ & EST & $\begin{array}{c}{[87 / 5][61 / 13]} \\
{[72 / 20]}\end{array}$ \\
\hline \multirow[t]{2}{*}{$\mathrm{C}_{5} \mathrm{H}_{11} \mathrm{~F}$} & 1-fluoro-2-methylbutane & & & & [10086-64-3] \\
\hline & $(287-329)$ & 30.7 & $(302)$ & A & {$[87 / 5][72 / 20]$} \\
\hline \multirow[t]{2}{*}{$\mathrm{C}_{5} \mathrm{H}_{11} \mathrm{~F}$} & 2-fluoro-2-methylbutane & & & & {$[661-53-0]$} \\
\hline & $(249-341)$ & 31.8 & $(264)$ & A & {$[87 / 5][72 / 20]$} \\
\hline \multirow[t]{5}{*}{$\mathrm{C}_{5} \mathrm{H}_{11} \mathrm{I}$} & 1-iodopentane & & & & {$[628-17-1]$} \\
\hline & $(313-353)$ & 44.4 & $(298)$ & CGC & {$[95 / 21]$} \\
\hline & & $45.3 \pm 0.1$ & $(298)$ & $\mathrm{C}$ & {$[68 / 1]$} \\
\hline & $(312-473)$ & 43.1 & $(327)$ & A, EST & {$[87 / 5][61 / 13]$} \\
\hline & & & & & {$[72 / 20]$} \\
\hline \multirow[t]{2}{*}{$\mathrm{C}_{5} \mathrm{H}_{11} \mathrm{I}$} & 1-iodo-2-methylbutane & & & & [616-14-8] \\
\hline & $(339-406)$ & 39.8 & $(354)$ & A & {$[87 / 5][72 / 20]$} \\
\hline $\mathrm{C}_{5} \mathrm{H}_{11} \mathrm{I}$ & 1-iodo-3-methylbutane & & & & {$[541-28-6]$} \\
\hline & $(313-353)$ & 42.2 & $(298)$ & CGC & {$[95 / 21]$} \\
\hline & $(270-422)$ & 43.5 & $(285)$ & A & {$[87 / 5][47 / 5]$} \\
\hline $\mathrm{C}_{5} \mathrm{H}_{11} \mathrm{I}$ & 2-iodo-2-methylbutane & & & & [594-38-7] \\
\hline & $(308-398)$ & 40.4 & (323) & $\mathrm{A}$ & {$[87 / 5][72 / 20]$} \\
\hline $\mathrm{C}_{5} \mathrm{H}_{11} \mathrm{~N}$ & cyclopentylamine & & & & [1003-03-8] \\
\hline & $(317-419)$ & 38.3 & (332) & EB & {$[87 / 5][75 / 5]$} \\
\hline & $(317-419)$ & $40.2 \pm 0.4$ & (298) & $\mathrm{EB}$ & {$[75 / 7]$} \\
\hline $\mathrm{C}_{5} \mathrm{H}_{11} \mathrm{~N}$ & 1-methylpyrrolidine & & & & [120-94-5] \\
\hline & $(270-298)$ & $35.0 \pm 0.7$ & (284) & GS & {$[98 / 12]$} \\
\hline & $(270-298)$ & $34.2 \pm 0.7$ & (298) & GS & {$[98 / 12]$} \\
\hline & $(273-315)$ & 33.7 & (288) & $\mathrm{A}$ & {$[87 / 5]$} \\
\hline $\mathrm{C}_{5} \mathrm{H}_{11} \mathrm{~N}$ & piperidine & & & & [110-89-4] \\
\hline & & 36.6 & (338) & & {$[88 / 7]$} \\
\hline & & 35.3 & $(357)$ & & {$[88 / 7]$} \\
\hline
\end{tabular}


TABLE 6. Enthalpies of vaporization of organic compounds, 1880-2002-Continued

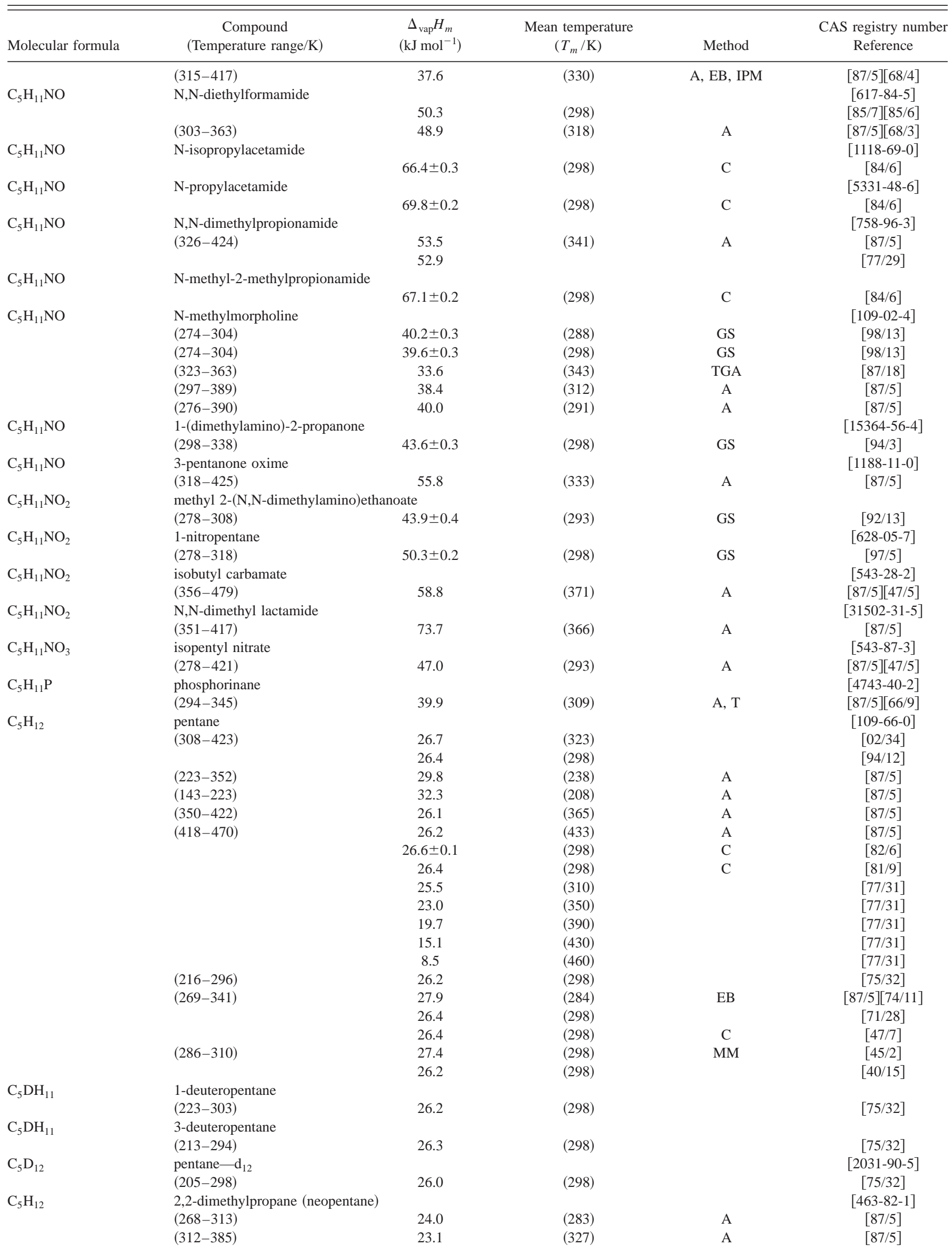


TABLE 6. Enthalpies of vaporization of organic compounds, 1880-2002-Continued

\begin{tabular}{|c|c|c|c|c|c|}
\hline Molecular formula & $\begin{array}{c}\text { Compound } \\
\text { (Temperature range/K) }\end{array}$ & $\begin{array}{c}\Delta_{\mathrm{vap}} H_{m} \\
\left(\mathrm{~kJ} \mathrm{~mol}^{-1}\right)\end{array}$ & $\begin{array}{l}\text { Mean temperature } \\
\qquad\left(T_{m} / \mathrm{K}\right)\end{array}$ & Method & $\begin{array}{c}\text { CAS registry number } \\
\text { Reference }\end{array}$ \\
\hline & $(382-433)$ & 23.1 & $(397)$ & A & {$[87 / 5]$} \\
\hline & & 21.8 & $(298)$ & $\mathrm{C}$ & {$[81 / 9]$} \\
\hline & & 22.2 & $(290)$ & & {$[77 / 32]$} \\
\hline & & 19.5 & $(330)$ & & {$[77 / 32]$} \\
\hline & & 16.2 & $(370)$ & & {$[77 / 32]$} \\
\hline & & 11.1 & $(410)$ & & {$[77 / 32]$} \\
\hline & $(257-293)$ & 24.3 & $(272)$ & & {$[75 / 26][84 / 9]$} \\
\hline & $(343-433)$ & 22.8 & $(358)$ & & {$[73 / 28][84 / 9]$} \\
\hline & & 21.85 & $(298)$ & & {$[71 / 28]$} \\
\hline & & $22.8 \pm 0.1$ & (283) & & {$[36 / 8]$} \\
\hline \multirow[t]{15}{*}{$\mathrm{C}_{5} \mathrm{H}_{12}$} & 2-methylbutane (isopentane) & & & & {$[78-78-4]$} \\
\hline & $(255-323)$ & 26.9 & $(270)$ & & {$[91 / 15]$} \\
\hline & $(216-323)$ & 28.5 & $(231)$ & A & {$[87 / 5]$} \\
\hline & $(300-460)$ & 25.2 & $(315)$ & A & {$[87 / 5]$} \\
\hline & $(320-391)$ & 25.2 & $(335)$ & A & {$[87 / 5]$} \\
\hline & $(385-416)$ & 24.8 & $(400)$ & A & {$[87 / 5]$} \\
\hline & $(412-460)$ & 25.3 & $(427)$ & A & {$[87 / 5]$} \\
\hline & & 24.4 & $(310)$ & & {$[77 / 33]$} \\
\hline & & 21.5 & $(350)$ & & {$[77 / 33]$} \\
\hline & & 18.0 & $(390)$ & & {$[77 / 33]$} \\
\hline & & 12.9 & $(430)$ & & {$[77 / 33]$} \\
\hline & & 24.8 & $(298)$ & & {$[71 / 28]$} \\
\hline & $(190-300)$ & 30.2 & $(205)$ & & {$[47 / 5]$} \\
\hline & $(289-301)$ & 26.2 & $(295)$ & $\mathrm{MM}$ & {$[45 / 2]$} \\
\hline & & 25.0 & $(298)$ & $\mathrm{C}$ & {$[42 / 5]$} \\
\hline \multirow[t]{2}{*}{$\mathrm{C}_{5} \mathrm{H}_{12} \mathrm{ClF}_{3} \mathrm{~N}_{2} \mathrm{~S}$} & chlorobis (N-methylmethanam & rifluorometh & & & {$[63265-71-4]$} \\
\hline & & 38.1 & $(368)$ & $\mathrm{I}$ & {$[77 / 15]$} \\
\hline \multirow[t]{2}{*}{$\mathrm{C}_{5} \mathrm{H}_{12} \mathrm{ClF}_{3} \mathrm{~N}_{2} \mathrm{OS}$} & chlorobis (N-methylmethanam & xo(trifluoron & ulfur & & {$[63265-73-6]$} \\
\hline & & 40.2 & $(477)$ & $\mathrm{I}$ & {$[77 / 15]$} \\
\hline \multirow[t]{2}{*}{$\mathrm{C}_{5} \mathrm{H}_{12} \mathrm{NO}_{3} \mathrm{PS}_{2}$} & phosphorodithioic acid, O,O- & $1-\mathrm{S}-[2-($ meth & )-2-oxoethyl] ester & & {$[60-51-5]$} \\
\hline & $(283-390)$ & 95.0 & $(298)$ & A & {$[87 / 5]$} \\
\hline \multirow[t]{2}{*}{$\mathrm{C}_{5} \mathrm{H}_{12} \mathrm{~N}_{2}$} & methyl butyldiazene & & & & {$[4426-46-4]$} \\
\hline & & $36.4 \pm 0.2$ & $(298)$ & $\mathrm{C}$ & {$[78 / 3]$} \\
\hline \multirow[t]{2}{*}{$\mathrm{C}_{5} \mathrm{H}_{12} \mathrm{~N}_{2}$} & N-methylpiperazine & & & & {$[109-01-3]$} \\
\hline & $(274-319)$ & 46.7 & $(289)$ & A & {$[87 / 5]$} \\
\hline \multirow[t]{3}{*}{$\mathrm{C}_{5} \mathrm{H}_{12} \mathrm{~N}_{2} \mathrm{O}$} & 1,1,3,3-tetramethylurea & & & & {$[632-22-4]$} \\
\hline & $(320-450)$ & 41.7 & $(450)$ & $\mathrm{A}, \mathrm{EB}$ & {$[87 / 2]$} \\
\hline & $(320-450)$ & 52.2 & $(325)$ & $\mathrm{A}, \mathrm{EB}$ & {$[87 / 2]$} \\
\hline \multirow[t]{2}{*}{$\mathrm{C}_{5} \mathrm{H}_{12} \mathrm{~N}_{2} \mathrm{~S}$} & 1,3-diethylthiourea & & & & {$[105-55-5]$} \\
\hline & $(351-384)$ & $101 \pm 3.0$ & $(368)$ & ME, TE & {$[94 / 21]$} \\
\hline \multirow[t]{7}{*}{$\mathrm{C}_{5} \mathrm{H}_{12} \mathrm{O}$} & 1-methoxybutane & & & & {$[628-28-4]$} \\
\hline & $(293-367)$ & 32.5 & $(308)$ & A & {$[87 / 5]$} \\
\hline & & 32.4 & (398) & $\mathrm{C}$ & {$[80 / 3]$} \\
\hline & $(265-367)$ & 32.4 & $(298)$ & & {$[76 / 2]$} \\
\hline & $(265-367)$ & 29.6 & $(343)$ & & {$[76 / 2]$} \\
\hline & & $32.5 \pm 0.1$ & $(298)$ & $\mathrm{C}$ & {$[75 / 3]$} \\
\hline & $(296-342)$ & 32.4 & $(311)$ & $\mathrm{EB}$ & {$[69 / 15]$} \\
\hline \multirow[t]{7}{*}{$\mathrm{C}_{5} \mathrm{H}_{12} \mathrm{O}$} & 1-ethoxypropane & & & & {$[628-32-0]$} \\
\hline & $(264-359)$ & 33.0 & $(279)$ & A & {$[87 / 5][76 / 2]$} \\
\hline & & 31.4 & $(298)$ & $\mathrm{C}$ & {$[80 / 3]$} \\
\hline & $(264-359)$ & 31.4 & $(298)$ & & {$[76 / 2]$} \\
\hline & $(264-359)$ & 29.0 & $(336)$ & & {$[76 / 2]$} \\
\hline & & $31.4 \pm 0.1$ & $(298)$ & $\mathrm{C}$ & {$[75 / 3]$} \\
\hline & $(293-335)$ & 31.6 & $(308)$ & & {$[69 / 15]$} \\
\hline \multirow[t]{2}{*}{$\mathrm{C}_{5} \mathrm{H}_{12} \mathrm{O}$} & ethyl isopropyl ether & & & & {$[625-54-7]$} \\
\hline & & 30.0 & $(298)$ & $\mathrm{C}$ & {$[80 / 3]$} \\
\hline \multirow[t]{8}{*}{$\mathrm{C}_{5} \mathrm{H}_{12} \mathrm{O}$} & methyl tert-butyl ether & & & & [1634-04-4] \\
\hline & $(300-328)$ & 29.9 & (314) & & {$[02 / 26]$} \\
\hline & $(315-365)$ & 29.6 & $(330)$ & & [98/9] \\
\hline & $(300-411)$ & 31.2 & $(315)$ & $\mathrm{EB}$ & {$[94 / 10]$} \\
\hline & $(287-326)$ & 30.4 & $(302)$ & & {$[91 / 3]$} \\
\hline & & 29.8 & $(298)$ & $\mathrm{C}$ & {$[80 / 3]$} \\
\hline & $(287-351)$ & 30.2 & $(302)$ & A & {$[87 / 5][76 / 2]$} \\
\hline & & 29.6 & $(298)$ & & {$[76 / 2]$} \\
\hline
\end{tabular}


TABLE 6. Enthalpies of vaporization of organic compounds, 1880-2002-Continued

\begin{tabular}{|c|c|c|c|c|c|}
\hline Molecular formula & $\begin{array}{c}\text { Compound } \\
\text { (Temperature range/K) }\end{array}$ & $\begin{array}{c}\Delta_{\mathrm{vap}} H_{m} \\
\left(\mathrm{~kJ} \mathrm{~mol}^{-1}\right)\end{array}$ & $\begin{array}{l}\text { Mean temperature } \\
\qquad\left(T_{m} / \mathrm{K}\right)\end{array}$ & Method & $\begin{array}{c}\text { CAS registry number } \\
\text { Reference }\end{array}$ \\
\hline & & 27.9 & $(328)$ & & {$[76 / 2]$} \\
\hline & & $30.4 \pm 0.1$ & $(298)$ & $\mathrm{C}$ & {$[75 / 3]$} \\
\hline \multirow[t]{29}{*}{$\mathrm{C}_{5} \mathrm{H}_{12} \mathrm{O}$} & 1-pentanol & & & & {$[71-41-0]$} \\
\hline & & 44.4 & $(411)$ & & {$[00 / 26]$} \\
\hline & & 40.1 & $(448)$ & & {$[00 / 26]$} \\
\hline & & 36.1 & (473) & & {$[00 / 26]$} \\
\hline & & 31.7 & $(498)$ & & {$[00 / 26]$} \\
\hline & & 26.4 & $(523)$ & & {$[00 / 26]$} \\
\hline & & 22.0 & $(548)$ & & {$[00 / 26]$} \\
\hline & & 14.1 & $(573)$ & & {$[00 / 26]$} \\
\hline & & 7.1 & $(586)$ & & {$[00 / 26]$} \\
\hline & & 43.5 & & & [99/32] \\
\hline & $(323-373)$ & 57.8 & (298) & CGC & {$[95 / 21]$} \\
\hline & $(323-373)$ & 57.4 & (298) & CGC & {$[95 / 21]$} \\
\hline & $(335-410)$ & 51.5 & $(350)$ & & [94/7] \\
\hline & $(388-420)$ & 47.2 & (403) & A & {$[87 / 5]$} \\
\hline & $(326-411)$ & 54.3 & $(341)$ & A & {$[87 / 5]$} \\
\hline & $(408-441)$ & 45.4 & $(423)$ & A & {$[87 / 5]$} \\
\hline & & $55.7 \pm 0.2$ & (313) & $\mathrm{C}$ & {$[85 / 1]$} \\
\hline & & $54.4 \pm 0.2$ & $(328)$ & $\mathrm{C}$ & {$[85 / 1]$} \\
\hline & & $53.0 \pm 0.2$ & (343) & $\mathrm{C}$ & {$[85 / 1]$} \\
\hline & & $51.2 \pm 0.2$ & $(358)$ & $\mathrm{C}$ & {$[85 / 1]$} \\
\hline & $(343-303)$ & 55.4 & (298) & & [83/14] \\
\hline & $(310-411)$ & 55.0 & $(325)$ & & {$[73 / 26]$} \\
\hline & & $50.5 \pm 0.1$ & $(362)$ & $\mathrm{C}$ & {$[70 / 20]$} \\
\hline & & $49.2 \pm 0.1$ & (374) & $\mathrm{C}$ & {$[70 / 20]$} \\
\hline & & $47.0 \pm 0.1$ & (392) & $\mathrm{C}$ & {$[70 / 20]$} \\
\hline & & $44.4 \pm 0.1$ & $(411)$ & $\mathrm{C}$ & {$[70 / 20]$} \\
\hline & $(347-429)$ & 51.6 & $(362)$ & $\mathrm{EB}$ & {$[87 / 5][70 / 2]$} \\
\hline & $(307-411)$ & 56.2 & $(322)$ & DTA & [69/5] \\
\hline & & $56.9 \pm 0.2$ & (298) & $\mathrm{C}$ & {$[66 / 2]$} \\
\hline \multirow[t]{13}{*}{$\mathrm{C}_{5} \mathrm{H}_{12} \mathrm{O}$} & 2-pentanol & & & & [6032-29-7] \\
\hline & $(323-373)$ & 53.6 & (298) & CGC & {$[95 / 21]$} \\
\hline & $(274-393)$ & 58.9 & (289) & A & {$[87 / 5]$} \\
\hline & & $54.2 \pm 0.2$ & (298) & $\mathrm{C}$ & {$[85 / 1]$} \\
\hline & & $52.7 \pm 0.2$ & (313) & $\mathrm{C}$ & {$[85 / 1]$} \\
\hline & & $50.9 \pm 0.2$ & $(328)$ & $\mathrm{C}$ & {$[85 / 1]$} \\
\hline & & $49.0 \pm 0.2$ & $(343)$ & $\mathrm{C}$ & {$[85 / 1]$} \\
\hline & & $46.9 \pm 0.1$ & $(358)$ & $\mathrm{C}$ & {$[85 / 1]$} \\
\hline & & $45.4 \pm 0.1$ & $(368)$ & $\mathrm{C}$ & {$[85 / 1]$} \\
\hline & $(322-393)$ & 50.3 & $(337)$ & & {$[84 / 10]$} \\
\hline & $(298-393)$ & 54.0 & (313) & & {$[73 / 26]$} \\
\hline & & 53.0 & $(298)$ & $\mathrm{C}$ & {$[63 / 2]$} \\
\hline & $(298-383)$ & 53.7 & $(313)$ & & {$[35 / 6][84 / 9]$} \\
\hline \multirow[t]{6}{*}{$\mathrm{C}_{5} \mathrm{H}_{12} \mathrm{O}$} & 3-pentanol & & & & {$[584-02-1]$} \\
\hline & $(245-390)$ & 59.9 & $(260)$ & A & {$[87 / 5]$} \\
\hline & $(317-389)$ & 49.6 & $(332)$ & & {$[84 / 10]$} \\
\hline & $(279-318)$ & 53.6 & (294) & & {$[75 / 1]$} \\
\hline & $(294-389)$ & 50.2 & (319) & & {$[73 / 26]$} \\
\hline & & 52.9 & $(298)$ & $\mathrm{C}$ & {$[63 / 2]$} \\
\hline \multirow[t]{9}{*}{$\mathrm{C}_{5} \mathrm{H}_{12} \mathrm{O}$} & 2-methyl-1-butanol & & & & [137-32-6] \\
\hline & $(330-405)$ & 51.2 & $(345)$ & & {$[94 / 7]$} \\
\hline & $(338-402)$ & 49.8 & $(353)$ & A & {$[87 / 5]$} \\
\hline & $(317-403)$ & 53.9 & $(332)$ & A & {$[87 / 5]$} \\
\hline & $(249-319)$ & 58.5 & $(264)$ & A & {$[87 / 5][79 / 16]$} \\
\hline & $(307-403)$ & 56.1 & $(322)$ & & {$[73 / 26]$} \\
\hline & & 54.1 & $(298)$ & $\mathrm{C}$ & {$[63 / 2]$} \\
\hline & $(302-410)$ & 43.4 & $(317)$ & & {$[57 / 13][84 / 9]$} \\
\hline & $(298-393)$ & 56.7 & $(313)$ & & {$[84 / 9][35 / 6]$} \\
\hline \multirow[t]{5}{*}{$\mathrm{C}_{5} \mathrm{H}_{12} \mathrm{O}$} & 2-methyl-2-butanol & & & & [75-85-4] \\
\hline & $(274-306)$ & $51.5 \pm 0.3$ & $(298)$ & GS & {$[01 / 7]$} \\
\hline & $(323-373)$ & 50.5 & $(298)$ & CGC & {$[95 / 21]$} \\
\hline & $(308-375)$ & 47.3 & $(323)$ & & {$[94 / 7]$} \\
\hline & $(280-375)$ & 49.0 & $(295)$ & A & {$[87 / 5]$} \\
\hline
\end{tabular}


TABLE 6. Enthalpies of vaporization of organic compounds, 1880-2002-Continued

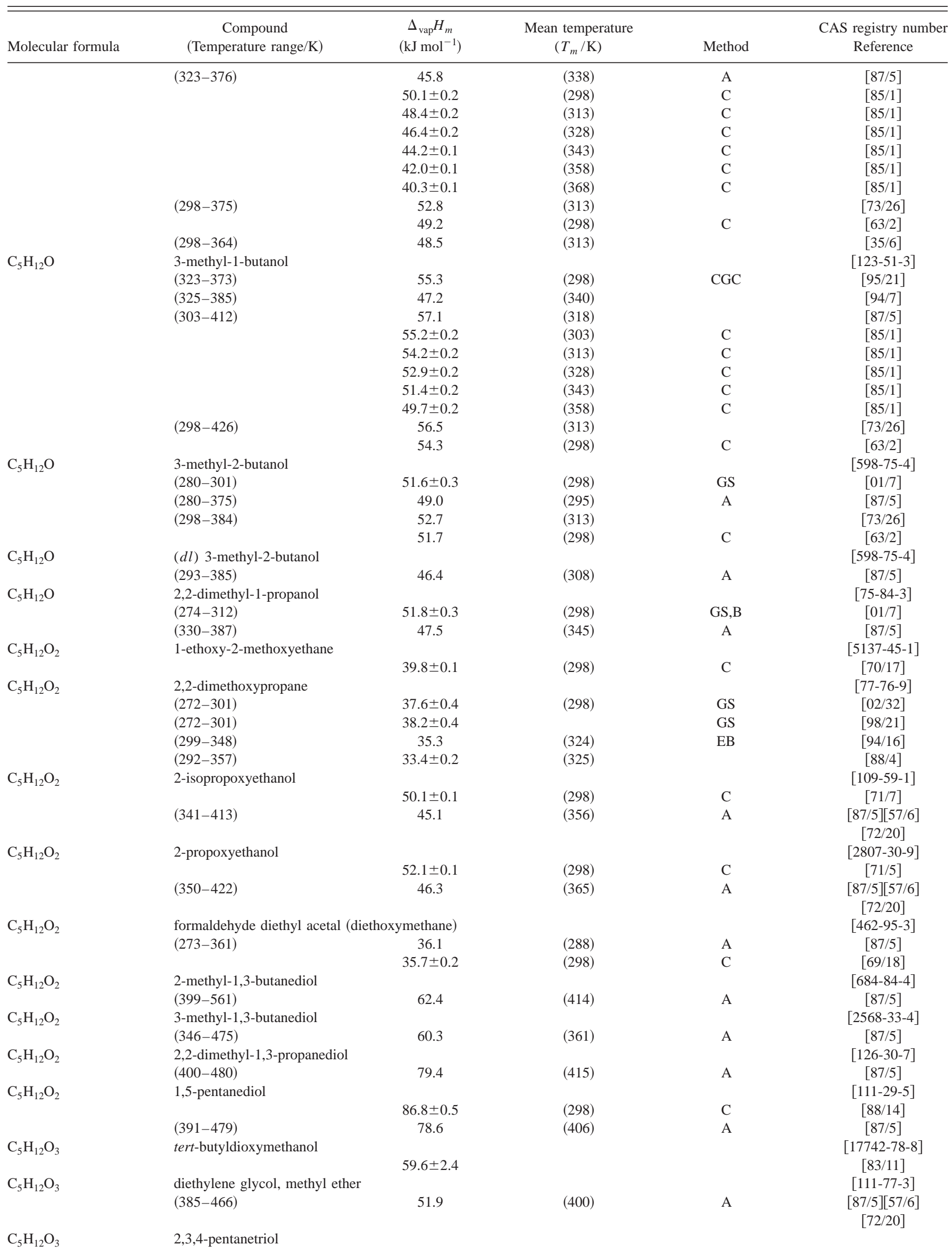


TABLE 6. Enthalpies of vaporization of organic compounds, 1880-2002-Continued

\begin{tabular}{|c|c|c|c|c|c|}
\hline Molecular formula & $\begin{array}{c}\text { Compound } \\
\text { (Temperature range/K) }\end{array}$ & $\begin{array}{c}\Delta_{\mathrm{vap}} H_{m} \\
\left(\mathrm{~kJ} \mathrm{~mol}^{-1}\right)\end{array}$ & $\begin{array}{c}\text { Mean temperature } \\
\qquad\left(T_{m} / \mathrm{K}\right)\end{array}$ & Method & $\begin{array}{c}\text { CAS registry number } \\
\text { Reference }\end{array}$ \\
\hline \multirow{3}{*}{$\mathrm{C}_{5} \mathrm{H}_{12} \mathrm{O}_{4}$} & $(428-600)$ & 78.9 & $(443)$ & & {$[47 / 5]$} \\
\hline & tetramethoxymethane & & & & [1850-14-2] \\
\hline & $(304-387)$ & 41.2 & $(319)$ & & {$[80 / 15]$} \\
\hline \multirow[t]{2}{*}{$\mathrm{C}_{5} \mathrm{H}_{12} \mathrm{O}_{5}$} & adonitol & & & & {$[488-81-3]$} \\
\hline & $(418-465)$ & $111.1 \pm 1.5$ & $(443)$ & TE & {$[90 / 16]$} \\
\hline \multirow[t]{2}{*}{$\mathrm{C}_{5} \mathrm{H}_{12} \mathrm{O}_{5}$} & D-arabinitol & & & & \\
\hline & $(414-461)$ & $110.1 \pm 1.5$ & $(440)$ & $\mathrm{TE}$ & {$[90 / 16]$} \\
\hline \multirow[t]{2}{*}{$\mathrm{C}_{5} \mathrm{H}_{12} \mathrm{O}_{5}$} & xylitol & & & & [87-99-0] \\
\hline & $(406-460)$ & $111.1 \pm 0.8$ & (433) & $\mathrm{TE}$ & {$[90 / 16]$} \\
\hline \multirow[t]{2}{*}{$\mathrm{C}_{5} \mathrm{H}_{12} \mathrm{~S}$} & 3-methyl-2-thiapentane & & & & [10359-64-5] \\
\hline & $(288-418)$ & 38.5 & $(303)$ & & {$[99 / 16]$} \\
\hline \multirow[t]{2}{*}{$\mathrm{C}_{5} \mathrm{H}_{12} \mathrm{~S}$} & 4-methyl-2-thiapentane & & & & [5008-69-5] \\
\hline & $(288-411)$ & 36.9 & $(303)$ & & {$[99 / 16]$} \\
\hline \multirow[t]{8}{*}{$\mathrm{C}_{5} \mathrm{H}_{12} \mathrm{~S}$} & butyl methyl sulfide & & & & [628-29-5] \\
\hline & $(297-423)$ & 40.4 & $(312)$ & & {$[99 / 16]$} \\
\hline & $(301-330)$ & 35.3 & $(315)$ & A & {$[87 / 5]$} \\
\hline & & 40.5 & $(298)$ & & {$[81 / 12]$} \\
\hline & & 41.0 & (298) & & {$[71 / 28]$} \\
\hline & & $40.9 \pm 0.8$ & (298) & GC & {$[64 / 7]$} \\
\hline & $(296-325)$ & 38.1 & $(313)$ & EB & {$[62 / 17]$} \\
\hline & $(343-436)$ & 38.0 & $(358)$ & A, EB & $\begin{array}{c}{[87 / 5][61 / 8]} \\
{[66 / 5]}\end{array}$ \\
\hline \multirow[t]{4}{*}{$\mathrm{C}_{5} \mathrm{H}_{12} \mathrm{~S}$} & methyl tert-butyl sulfide & & & & [6163-64-0] \\
\hline & $(276-397)$ & 36.5 & $(291)$ & & {$[99 / 16]$} \\
\hline & & 35.9 & (298) & & {$[71 / 28]$} \\
\hline & $(305-411)$ & 35.1 & $(320)$ & $\mathrm{A}, \mathrm{EB}$ & $\begin{array}{c}{[87 / 5][62 / 9]} \\
{[66 / 5]}\end{array}$ \\
\hline \multirow[t]{7}{*}{$\mathrm{C}_{5} \mathrm{H}_{12} \mathrm{~S}$} & ethyl isopropyl sulfide & & & & [5145-99-3] \\
\hline & $(284-406)$ & 38.1 & (299) & & {$[99 / 16]$} \\
\hline & & 37.8 & (298) & & [81/12] \\
\hline & & 38.5 & (298) & & {$[71 / 28]$} \\
\hline & & $37.9 \pm 0.8$ & (298) & GC & {$[64 / 17]$} \\
\hline & $(296-325)$ & 38.1 & (313) & EB & {$[62 / 17]$} \\
\hline & $(319-391)$ & 36.3 & (334) & $\mathrm{A}, \mathrm{EB}$ & {$[87 / 5][52 / 9]$} \\
\hline \multirow[t]{5}{*}{$\mathrm{C}_{5} \mathrm{H}_{12} \mathrm{~S}$} & ethyl propyl sulfide & & & & {$[4110-50-3]$} \\
\hline & $(293-418)$ & 39.8 & $(308)$ & & {$[99 / 16]$} \\
\hline & & 40.0 & (298) & & {$[81 / 12]$} \\
\hline & & 39.5 & (298) & $\mathrm{C}$ & {$[81 / 8]$} \\
\hline & $(331-398)$ & 37.8 & $(346)$ & $\mathrm{A}, \mathrm{EB}$ & {$[87 / 5][52 / 9]$} \\
\hline \multirow{7}{*}{$\mathrm{C}_{5} \mathrm{H}_{12} \mathrm{~S}$} & 1-pentanethiol & & & & {$[110-66-7]$} \\
\hline & $(300-426)$ & 40.6 & (315) & & [99/16] \\
\hline & & 41.1 & (298) & & {$[71 / 28]$} \\
\hline & & $37.1 \pm 0.1$ & $(356)$ & $\mathrm{C}$ & {$[65 / 14]$} \\
\hline & & $36.4 \pm 0.1$ & (376) & $\mathrm{C}$ & {$[65 / 14]$} \\
\hline & & $34.9 \pm 0.1$ & $(400)$ & $\mathrm{C}$ & {$[65 / 14]$} \\
\hline & $(347-440)$ & 38.1 & $(362)$ & $\mathrm{A}, \mathrm{EB}$ & $\begin{array}{c}{[87 / 5][52 / 11]} \\
{[66 / 5]}\end{array}$ \\
\hline \multirow[t]{3}{*}{$\mathrm{C}_{5} \mathrm{H}_{12} \mathrm{~S}$} & 2-pentanethiol & & & & [2084-19-7] \\
\hline & $(287-412)$ & 38.4 & $(302)$ & & [99/16] \\
\hline & $(347-435)$ & 37.8 & $(361)$ & A & {$[87 / 5]$} \\
\hline \multirow[t]{2}{*}{$\mathrm{C}_{5} \mathrm{H}_{12} \mathrm{~S}$} & 3-pentanethiol & & & & [616-31-9] \\
\hline & $(288-413)$ & 38.3 & (303) & & [99/16] \\
\hline \multirow[t]{5}{*}{$\mathrm{C}_{5} \mathrm{H}_{12} \mathrm{~S}$} & 2-methyl-1-butanethiol & & & & [1878-18-8] \\
\hline & $(293-418)$ & 39.2 & $(308)$ & & [99/16] \\
\hline & & $39.9 \pm 0.1$ & $(298)$ & & {$[72 / 11][66 / 5]$} \\
\hline & & 39.7 & (298) & & {$[71 / 28]$} \\
\hline & $(324-432)$ & 37.6 & (339) & $\mathrm{A}, \mathrm{EB}$ & {$[87 / 5][66 / 5]$} \\
\hline \multirow[t]{5}{*}{$\mathrm{C}_{5} \mathrm{H}_{12} \mathrm{~S}$} & 3-methyl-1-butanethiol & & & & {$[541-31-1]$} \\
\hline & $(292-418)$ & 39.3 & $(307)$ & & [99/16] \\
\hline & & 39.7 & (298) & & {$[71 / 28]$} \\
\hline & & $39.9 \pm 0.1$ & (298) & & {$[72 / 11][66 / 5]$} \\
\hline & $(323-431)$ & 37.7 & $(338)$ & $\mathrm{A}, \mathrm{EB}$ & {$[87 / 5][66 / 5]$} \\
\hline $\mathrm{C}_{5} \mathrm{H}_{12} \mathrm{~S}$ & 2-methyl-2-butanethiol & & & & [1679-09-0] \\
\hline & $(276-398)$ & 36.3 & (291) & & [99/16] \\
\hline
\end{tabular}


TABLE 6. Enthalpies of vaporization of organic compounds, 1880-2002-Continued

\begin{tabular}{|c|c|c|c|c|c|}
\hline Molecular formula & $\begin{array}{c}\text { Compound } \\
\text { (Temperature range/K) }\end{array}$ & $\begin{array}{c}\Delta_{\mathrm{vap}} H_{m} \\
\left(\mathrm{~kJ} \mathrm{~mol}^{-1}\right)\end{array}$ & $\begin{array}{l}\text { Mean temperature } \\
\qquad\left(T_{m} / \mathrm{K}\right)\end{array}$ & Method & $\begin{array}{c}\text { CAS registry number } \\
\text { Reference }\end{array}$ \\
\hline & & 35.6 & $(298)$ & & {$[71 / 28]$} \\
\hline & $(320-411)$ & 34.3 & $(335)$ & $\mathrm{A}, \mathrm{EB}$ & {$[87 / 5][62 / 10]$} \\
\hline & & $33.8 \pm 0.1$ & $(330)$ & $\mathrm{C}$ & {$[62 / 10]$} \\
\hline & & $32.7 \pm 0.1$ & $(350)$ & $\mathrm{C}$ & {$[62 / 10]$} \\
\hline & & $31.4 \pm 0.1$ & $(372)$ & $\mathrm{C}$ & {$[62 / 10]$} \\
\hline \multirow[t]{5}{*}{$\mathrm{C}_{5} \mathrm{H}_{12} \mathrm{~S}$} & 3-methyl-2-butanethiol & & & & [2084-18-6] \\
\hline & $(285-409)$ & 37.7 & $(300)$ & & {$[99 / 16]$} \\
\hline & & $37.5 \pm 0.1$ & (298) & & {$[72 / 11][66 / 5]$} \\
\hline & & 37.7 & $(298)$ & & {$[71 / 28]$} \\
\hline & $(315-422)$ & 36.2 & $(330)$ & A, EB & {$[87 / 5][66 / 5]$} \\
\hline \multirow{6}{*}{$\mathrm{C}_{5} \mathrm{H}_{12} \mathrm{~S}$} & 2,2-dimethyl-1-propanethiol & & & & [1679-08-9] \\
\hline & $(280-403)$ & 36.9 & $(295)$ & & {$[99 / 16]$} \\
\hline & & $36.4 \pm 0.1$ & (298) & & {$[72 / 11][66 / 5]$} \\
\hline & & 36.8 & (298) & & {$[71 / 28]$} \\
\hline & $(292-416)$ & 36.2 & $(307)$ & A, EB & {$[87 / 5][66 / 5]$} \\
\hline & $(213-415)$ & 42.1 & $(230)$ & $\mathrm{EB}, \mathrm{IPM}$ & {$[66 / 5]$} \\
\hline \multirow{3}{*}{$\mathrm{C}_{5} \mathrm{H}_{12} \mathrm{~S}_{2}$} & 1,5-pentanedithiol & & & & [928-98-3] \\
\hline & $(363-491)$ & 51.6 & (378) & A & {$[87 / 5][99 / 16]$} \\
\hline & & 59.3 & $(298)$ & & {$[62 / 11]$} \\
\hline \multirow[t]{3}{*}{$\mathrm{C}_{5} \mathrm{H}_{12} \mathrm{~S}_{2}$} & ethyl isopropyl disulfide & & & & [53966-36-2] \\
\hline & $(369-426)$ & 42.5 & $(384)$ & & {$[99 / 16]$} \\
\hline & $(363-427)$ & 42.9 & (378) & A, EB & {$[87 / 5][52 / 9]$} \\
\hline \multirow[t]{2}{*}{$\mathrm{C}_{5} \mathrm{H}_{12} \mathrm{~S}_{2}$} & ethyl propyl disulfide & & & & [30453-31-7] \\
\hline & $(373-414)$ & 44.0 & (388) & A, EB & $\begin{array}{c}{[87 / 5][52 / 9]} \\
{[99 / 16]}\end{array}$ \\
\hline \multirow[t]{6}{*}{$\mathrm{C}_{5} \mathrm{H}_{13} \mathrm{~N}$} & N-ethylisopropylamine & & & & [19961-27-4] \\
\hline & & $33.1 \pm 0.1$ & (298) & $\mathrm{C}$ & {$[79 / 8]$} \\
\hline & & $32.1 \pm 0.1$ & (313) & $\mathrm{C}$ & {$[79 / 8]$} \\
\hline & & $31.0 \pm 0.1$ & (328) & $\mathrm{C}$ & {$[79 / 8]$} \\
\hline & & $28.8 \pm 0.1$ & (358) & $\mathrm{C}$ & {$[79 / 8]$} \\
\hline & $(303-342)$ & 33.4 & (318) & $\mathrm{EB}$ & {$[79 / 8]$} \\
\hline \multirow[t]{2}{*}{$\mathrm{C}_{5} \mathrm{H}_{13} \mathrm{~N}$} & $\mathrm{~N}, \mathrm{~N}$-diethylmethylamine & & & & [616-39-7] \\
\hline & $(283-339)$ & 31.8 & (298) & A & {$[87 / 5]$} \\
\hline \multirow[t]{2}{*}{$\mathrm{C}_{5} \mathrm{H}_{13} \mathrm{~N}$} & N-methylbutylamine & & & & [110-68-9] \\
\hline & $(283-313)$ & 38.1 & (298) & $\mathrm{A}$ & {$[87 / 5]$} \\
\hline \multirow[t]{2}{*}{$\mathrm{C}_{5} \mathrm{H}_{13} \mathrm{~N}$} & tert-butylmethylamine & & & & [14610-37-8] \\
\hline & $(270-288)$ & $32.3 \pm 1.4$ & (297) & & [97/21] \\
\hline \multirow[t]{3}{*}{$\mathrm{C}_{5} \mathrm{H}_{13} \mathrm{~N}$} & pentylamine & & & & [110-58-7] \\
\hline & $(298-417)$ & 39.0 & (313) & $\mathrm{A}$ & {$[87 / 5][72 / 20]$} \\
\hline & & $40.1 \pm 0.1$ & (298) & $\mathrm{C}$ & {$[69 / 2]$} \\
\hline \multirow{2}{*}{$\mathrm{C}_{5} \mathrm{H}_{13} \mathrm{NO}_{2}$} & methyl diethanolamine & & & & [105-59-9] \\
\hline & $(390-520)$ & 73.0 & $(405)$ & $\mathrm{A}$ & {$[87 / 5]$} \\
\hline \multirow[t]{2}{*}{$\mathrm{C}_{5} \mathrm{H}_{13} \mathrm{NO}_{2} \mathrm{~S}$} & $\mathrm{~N}, \mathrm{~N}$-diethyl methanesulfonamide & & & & [2374-61-0] \\
\hline & $(384-528)$ & 52.1 & (399) & A & {$[87 / 5]$} \\
\hline \multirow[t]{2}{*}{$\mathrm{C}_{5} \mathrm{H}_{13} \mathrm{NS}$} & N-methyl-tert-butylsulfenamide & & & & \\
\hline & $(329-397)$ & 41.9 & $(364)$ & & {$[99 / 16]$} \\
\hline \multirow[t]{2}{*}{$\mathrm{C}_{5} \mathrm{H}_{13} \mathrm{~N}_{3}$} & 1,1,3,3-tetramethylguanidine & & & & {$[80-70-6]$} \\
\hline & & 46.9 & & & {$[67 / 29]$} \\
\hline $\mathrm{C}_{5} \mathrm{H}_{13} \mathrm{O}_{3} \mathrm{P}$ & diethyl methylphosphonate & & & & [683-08-9] \\
\hline & $(343-402)$ & 51.8 & $(358)$ & A & {$[87 / 5][72 / 20]$} \\
\hline & & $56.5 \pm 4.2$ & & & {$[56 / 23][82 / 15]$} \\
\hline $\mathrm{C}_{5} \mathrm{H}_{14} \mathrm{NP}$ & trimethylphosphine-N-ethylimine & & & & \\
\hline & & $61.5 \pm 4.2$ & & & {$[60 / 23][82 / 15]$} \\
\hline $\mathrm{C}_{5} \mathrm{H}_{14} \mathrm{~N}_{2}$ & $\mathrm{~N}, \mathrm{~N}$-dimethyl-1,3-propanediamine & & & & {$[111-33-1]$} \\
\hline & $(303-366)$ & 45.7 & $(318)$ & A & {$[87 / 5]$} \\
\hline & $(303-408)$ & 42.0 & (318) & $\mathrm{A}$ & {$[87 / 5]$} \\
\hline & & 52.7 & & & {$[77 / 29]$} \\
\hline $\mathrm{C}_{5} \mathrm{H}_{14} \mathrm{~N}_{2}$ & bis(dimethylamino)methane & & & & {$[51-80-9]$} \\
\hline & $(273-348)$ & 32.3 & $(310)$ & & {$[65 / 29]$} \\
\hline $\mathrm{C}_{6} \mathrm{BrF}_{5}$ & bromopentafluorobenzene & & & & [344-04-7] \\
\hline & $(400-522)$ & 38.2 & $(415)$ & A & {$[87 / 5][72 / 20]$} \\
\hline & $(414-522)$ & 38.0 & $(429)$ & $\mathrm{EB}$ & {$[69 / 11]$} \\
\hline $\mathrm{C}_{6} \mathrm{BrF}_{15} \mathrm{~N}_{2} \mathrm{~S}$ & bis $[1,2,2,2$-tetrafluoro-1-(trifluorome & thyl)ethyl]di & furyl bromide fluoride & & [62977-74-6] \\
\hline & & 41.0 & $(476)$ & I & {$[77 / 15]$} \\
\hline
\end{tabular}


TABLE 6. Enthalpies of vaporization of organic compounds, 1880-2002-Continued

\begin{tabular}{|c|c|c|c|c|c|}
\hline Molecular formula & $\begin{array}{c}\text { Compound } \\
\text { (Temperature range/K) }\end{array}$ & $\begin{array}{c}\Delta_{\mathrm{vap}} H_{m} \\
\left(\mathrm{~kJ} \mathrm{~mol}^{-1}\right)\end{array}$ & $\begin{array}{l}\text { Mean temperature } \\
\qquad\left(T_{m} / \mathrm{K}\right)\end{array}$ & Method & $\begin{array}{c}\text { CAS registry number } \\
\text { Reference }\end{array}$ \\
\hline \multirow[t]{2}{*}{$\mathrm{C}_{6} \mathrm{ClF}_{15} \mathrm{~N}_{2} \mathrm{~S}$} & \multicolumn{4}{|c|}{ bis $[1,2,2,2$-tetrafluoro-1-(trifluoromethyl)ethyl]diimidosulfuryl chloride fluoride } & {$[62977-72-4]$} \\
\hline & & 37.2 & $(458)$ & $\mathrm{I}$ & {$[77 / 15]$} \\
\hline \multirow[t]{8}{*}{$\mathrm{C}_{6} \mathrm{ClF}_{5}$} & \multicolumn{4}{|l|}{ chloropentafluorobenzene } & [344-07-0] \\
\hline & $(290-550)$ & 41.3 & $(298)$ & & {$[91 / 2]$} \\
\hline & $(348-402)$ & 37.7 & $(363)$ & A & {$[87 / 5]$} \\
\hline & \multirow[t]{4}{*}{$(307-417)$} & 40.0 & $(322)$ & & {$[87 / 5][68 / 10]$} \\
\hline & & $37.7 \pm 0.1$ & $(349)$ & & {$[68 / 26]$} \\
\hline & & $36.4 \pm 0.1$ & $(369)$ & & {$[68 / 26]$} \\
\hline & & $34.8 \pm 0.1$ & $(391)$ & & {$[68 / 26]$} \\
\hline & $(403-547)$ & 35.2 & $(418)$ & EB & {$[66 / 11]$} \\
\hline \multirow[t]{2}{*}{$\mathrm{C}_{6} \mathrm{ClF}_{13} \mathrm{~N}_{2}$} & \multicolumn{4}{|c|}{ 1-chloro-1',2,2,2,2',2',2-heptafluoro-1,1'-bis(trifluoromethyl)-azoethane } & [33757-14-1] \\
\hline & $(297-355)$ & 33.3 & $(312)$ & A & {$[87 / 5][71 / 17]$} \\
\hline \multirow[t]{2}{*}{$\mathrm{C}_{6} \mathrm{ClF}_{14} \mathrm{P}$} & \multicolumn{5}{|c|}{ bis(heptafluoropropyl) chlorophosphine } \\
\hline & $(283-373)$ & 37.5 & $(328)$ & & {$[59 / 21]$} \\
\hline \multirow{2}{*}{$\mathrm{C}_{6} \mathrm{Cl}_{2} \mathrm{~F}_{12} \mathrm{~N}_{2} \mathrm{~S}$} & \multicolumn{5}{|c|}{ bis(2-chlorohexafluoroisopropylimino) sulfur } \\
\hline & & 43.5 & $(404)$ & I & {$[72 / 22]$} \\
\hline \multirow[t]{3}{*}{$\mathrm{C}_{6} \mathrm{Cl}_{3} \mathrm{~F}_{3}$} & \multicolumn{4}{|c|}{ 1,3,5-trichloro-2,4,6-trifluorobenzene } & {$[319-88-0]$} \\
\hline & $(364-496)$ & 49.2 & $(379)$ & A & {$[87 / 5]$} \\
\hline & $(364-550)$ & 53.8 & $(298)$ & & {$[84 / 9][91 / 2]$} \\
\hline \multirow[t]{2}{*}{$\mathrm{C}_{6} \mathrm{Cl}_{3} \mathrm{~F}_{14} \mathrm{P}$} & trichloro bis(heptafluorop & phorane & & & \\
\hline & $(323-393)$ & 40.1 & $(358)$ & & {$[59 / 21]$} \\
\hline $\mathrm{C}_{6} \mathrm{Cl}_{3} \mathrm{~N}_{3} \mathrm{O}_{6}$ & 1,3,5-trichloro-2,4,6-trinit & & & & [2631-68-7] \\
\hline & $(503-543)$ & 68.9 & $(518)$ & A & {$[87 / 5][68 / 14]$} \\
\hline & $(503-543)$ & 43.2 & $(518)$ & & {$[72 / 20]$} \\
\hline $\mathrm{C}_{6} \mathrm{Cl}_{4} \mathrm{O}_{2}$ & 2,3,5,6-tetrachloro-1,4-ber & (chloranil) & & & {$[118-75-2]$} \\
\hline & $(343-435)$ & 88.5 & $(358)$ & & {$[47 / 5]$} \\
\hline $\mathrm{C}_{6} \mathrm{Cl}_{6}$ & hexachlorobenzene & & & & {$[118-74-1]$} \\
\hline & & $74.4 \pm 0.7$ & $(298)$ & GS & {$[01 / 1]$} \\
\hline & $(413-453)$ & 76.8 & $(298)$ & GC & {$[94 / 22]$} \\
\hline & $(258-313)$ & 81.3 & & GC & {$[94 / 23]$} \\
\hline & $(343-453)$ & 68.6 & (398) & GC & {$[90 / 2]$} \\
\hline & $(502-589)$ & 68.7 & $(517)$ & $\mathrm{A}$ & {$[87 / 5]$} \\
\hline & $(387-582)$ & 60.5 & $(402)$ & & {$[47 / 5]$} \\
\hline $\mathrm{C}_{6} \mathrm{~F}_{6}$ & hexafluorobenzene & & & & {$[392-56-3]$} \\
\hline & $(318-376)$ & 34.4 & (333) & $\mathrm{EB}$ & [90/7] \\
\hline & $(403-516)$ & 31.8 & $(425)$ & & {$[88 / 20]$} \\
\hline & $(278-354)$ & 36.5 & $(293)$ & A & {$[87 / 5]$} \\
\hline & $(348-389)$ & 33.2 & $(363)$ & A & {$[87 / 5]$} \\
\hline & $(384-462)$ & 32.2 & (399) & A & {$[87 / 5]$} \\
\hline & $(458-517)$ & 31.8 & $(473)$ & A & {$[87 / 5]$} \\
\hline & $(290-510)$ & 35.6 & $(298)$ & & {$[82 / 11][91 / 2]$} \\
\hline & & 35.7 & (298) & $\mathrm{C}$ & {$[81 / 8]$} \\
\hline & $(293-323)$ & 35.7 & $(308)$ & & {$[80 / 23]$} \\
\hline & $(278-321)$ & 36.2 & $(292)$ & MM & {$[69 / 9]$} \\
\hline & $(363-516)$ & 32.2 & $(378)$ & EB & {$[66 / 11]$} \\
\hline & $(275-387)$ & 36.5 & (293) & & {$[65 / 8]$} \\
\hline & $(293-356)$ & 35.1 & $(308)$ & & {$[64 / 16]$} \\
\hline & $(293-358)$ & 35.5 & $(308)$ & & {$[46 / 5][84 / 9]$} \\
\hline $\mathrm{C}_{6} \mathrm{~F}_{7} \mathrm{NOS}$ & $\mathrm{N}$-(pentafluorophenyl)imi & fluoride & & & [20094-84-2] \\
\hline & $(309-355)$ & 45.3 & $(332)$ & & {$[68 / 21]$} \\
\hline $\mathrm{C}_{6} \mathrm{~F}_{7} \mathrm{OP}$ & pentafluorophenoxydifluos & & & & \\
\hline & $(310-363)$ & 42.4 & $(325)$ & & {$[76 / 28]$} \\
\hline $\mathrm{C}_{6} \mathrm{~F}_{7} \mathrm{O}_{2} \mathrm{P}$ & pentafluorophenoxyphosp & oride & & & \\
\hline & $(323-367)$ & 46.4 & $(338)$ & & {$[76 / 28]$} \\
\hline $\mathrm{C}_{6} \mathrm{~F}_{8}$ & perfluoro(2-methyl-3-metl & obutene) & & & \\
\hline & $(243-306)$ & 31.0 & $(258)$ & A & {$[87 / 5]$} \\
\hline $\mathrm{C}_{6} \mathrm{~F}_{11} \mathrm{NO}$ & $2,2,3,3,3$-pentafluoro- $\mathrm{N}-[2$ & ro-1-(trifluor & )-ethylidene]propana & & {$[52225-58-8]$} \\
\hline & & 32.7 & $(338)$ & & {$[74 / 24]$} \\
\hline $\mathrm{C}_{6} \mathrm{~F}_{11} \mathrm{NO}_{2} \mathrm{~S}$ & $2,2,3,3,4,4,5,5$-octafluoro- & -hexahydro-1 & oroacetyl)-imino]thio & xide & {$[77589-41-4]$} \\
\hline & & 33.9 & $(383)$ & & {$[81 / 16]$} \\
\hline $\mathrm{C}_{6} \mathrm{~F}_{12}$ & perfluoromethylcyclopent & & & & \\
\hline & & 30.68 & $(298)$ & $\mathrm{EB}$ & [98/18] \\
\hline $\mathrm{C}_{6} \mathrm{~F}_{12}$ & perfluorocyclohexane & & & & {$[355-68-0]$} \\
\hline & $(373-457)$ & 28.0 & $(388)$ & & {$[88 / 20]$} \\
\hline
\end{tabular}


TABLE 6. Enthalpies of vaporization of organic compounds, 1880-2002-Continued

\begin{tabular}{|c|c|c|c|c|c|}
\hline Molecular formula & $\begin{array}{l}\text { Compound } \\
\text { (Temperature range/K) }\end{array}$ & $\begin{array}{c}\Delta_{\mathrm{vap}} H_{m} \\
\left(\mathrm{~kJ} \mathrm{~mol}^{-1}\right)\end{array}$ & $\begin{array}{l}\text { Mean temperature } \\
\qquad\left(T_{m} / \mathrm{K}\right)\end{array}$ & Method & $\begin{array}{l}\text { CAS registry number } \\
\text { Reference }\end{array}$ \\
\hline & $(350-451)$ & 28.1 & $(365)$ & A & {$[87 / 5]$} \\
\hline & $(336-394)$ & 29.6 & $(351)$ & & {$[57 / 11][84 / 9]$} \\
\hline \multirow[t]{2}{*}{$\mathrm{C}_{6} \mathrm{~F}_{12}$} & perfluoro(1,2-dimethylcyc & & & & [2994-71-0] \\
\hline & $(242-318)$ & 32.1 & $(257)$ & $\mathrm{A}$ & {$[87 / 5]$} \\
\hline \multirow[t]{2}{*}{$\mathrm{C}_{6} \mathrm{~F}_{12} \mathrm{~N}_{2}$} & $\mathrm{~N}, \mathrm{~N}, \mathrm{~N}, \mathrm{~N}$-tetrakis (trifluoro & 2-ethynylene & & & [19451-96-8] \\
\hline & $(305-328)$ & 32.1 & $(316)$ & A & {$[87 / 5]$} \\
\hline \multirow[t]{2}{*}{$\mathrm{C}_{6} \mathrm{~F}_{12} \mathrm{~N}_{2} \mathrm{OS}$} & \multicolumn{4}{|c|}{ 1,1,1,3,3,3-hexafluoro-2-isocyanato-N-[2,2,2-trifluoro-1-(trifluoro-methyl)ethylidene]-2-propanamine } & [34619-84-6] \\
\hline & & 39.3 & $(375)$ & I & {$[72 / 24]$} \\
\hline \multirow[t]{2}{*}{$\mathrm{C}_{6} \mathrm{~F}_{12} \mathrm{~N}_{2} \mathrm{O}_{2} \mathrm{~S}$} & \multicolumn{4}{|c|}{ 1,1,1-trifluoro-N'-(trifluoroacetyl)-N-[2,2,2-trifluoro-1-(trifluoromethyl)- } & {$[62609-66-9]$} \\
\hline & & 32.6 & $(404)$ & I & {$[77 / 19]$} \\
\hline \multirow[t]{2}{*}{$\mathrm{C}_{6} \mathrm{~F}_{12} \mathrm{~N}_{2} \mathrm{~S}$} & \multicolumn{4}{|c|}{ bis $[2,2,2$-trifluoro-1-(trifluoromethyl)ethylidene]sulfoxylic diamide } & [31340-33-7] \\
\hline & & 40.6 & $(391)$ & I & {$[72 / 24]$} \\
\hline \multirow[t]{2}{*}{$\mathrm{C}_{6} \mathrm{~F}_{12} \mathrm{~N}_{2} \mathrm{~S}_{2}$} & \multicolumn{5}{|c|}{ bis(hexafluoroisopropylidenimino) disulfide } \\
\hline & & 46.0 & $(417)$ & I & {$[72 / 22]$} \\
\hline \multirow[t]{2}{*}{$\mathrm{C}_{6} \mathrm{~F}_{12} \mathrm{O}$} & \multicolumn{4}{|c|}{ perfluoro(methoxycyclopentane) } & {$[788-40-9]$} \\
\hline & $(246-330)$ & 38.6 & (261) & A & {$[87 / 5][72 / 20]$} \\
\hline \multirow[t]{3}{*}{$\mathrm{C}_{6} \mathrm{~F}_{12} \mathrm{O}_{2}$} & \multicolumn{4}{|c|}{ trifluoroacetic acid, 2,2,2-trifluoro-1,1-bis(trifluoromethyl)ethyl ester } & [24165-10-4] \\
\hline & $(264-298)$ & 34.3 & $(279)$ & A & {$[87 / 5][75 / 22]$} \\
\hline & & 33.1 & (329) & HG & {$[73 / 20]$} \\
\hline \multirow[t]{2}{*}{$\mathrm{C}_{6} \mathrm{~F}_{12} \mathrm{O}_{4}$} & \multicolumn{4}{|c|}{$\begin{array}{l}\text { carbonoperoxoic acid, O-[2,2,2-trifluoro-1,1-bis(trifluoromethyl)ethyl]-O,O- } \\
\text { (trifluoromethyl) ester ester }\end{array}$} & [55100-93-1] \\
\hline & $(273-315)$ & 33.5 & $(288)$ & A & {$[87 / 5][75 / 22]$} \\
\hline $\mathrm{C}_{6} \mathrm{~F}_{13} \mathrm{NS}$ & $\begin{array}{l}\text { 2,2,2-trifluoro-N-[1,2,2,2- } \\
\text { trifluoromethyl ester }\end{array}$ & - (trifluorom & hyl]-ethanimidothioic & & [53120-07-9] \\
\hline & & 35.3 & $(360)$ & & {$[75 / 20]$} \\
\hline $\mathrm{C}_{6} \mathrm{~F}_{14}$ & perfluorohexane & & & & {$[355-42-0]$} \\
\hline & $(261-334)$ & 34.4 & $(276)$ & A & {$[87 / 5]$} \\
\hline & $(285-340)$ & 31.4 & (298) & & {$[84 / 9][91 / 2]$} \\
\hline & $(433-449)$ & 33.4 & (441) & A & {$[87 / 5][78 / 8]$} \\
\hline & $(303-330)$ & 31.5 & (316) & & {$[58 / 12][84 / 9]$} \\
\hline & $(284-342)$ & 32.4 & $(298)$ & & {$[52 / 1]$} \\
\hline $\mathrm{C}_{6} \mathrm{~F}_{14}$ & perfluoro-2-methylpentane & & & & {$[335-04-4]$} \\
\hline & $(280-340)$ & 31.4 & (298) & & {$[84 / 9][91 / 2]$} \\
\hline & $(253-329)$ & 34.5 & (268) & $\mathrm{A}$ & $\begin{array}{c}{[87 / 5][67 / 15]} \\
{[84 / 9]}\end{array}$ \\
\hline & $(277-341)$ & 32.5 & $(292)$ & & {$[52 / 1][84 / 9]$} \\
\hline $\mathrm{C}_{6} \mathrm{~F}_{14}$ & perfluoro-3-methylpentane & & & & {$[865-71-4]$} \\
\hline & $(282-333)$ & 30.8 & $(297)$ & A & {$[87 / 5]$} \\
\hline $\mathrm{C}_{6} \mathrm{~F}_{14}$ & perfluoro-2,3-dimethylbut & & & & {$[354-96-1]$} \\
\hline & $(260-340)$ & 31.6 & $(298)$ & & {$[84 / 9][91 / 2]$} \\
\hline & $(262-333)$ & 33.0 & $(277)$ & A & {$[87 / 5][67 / 15]$} \\
\hline $\mathrm{C}_{6} \mathrm{~F}_{14} \mathrm{IP}$ & bis(heptafluoropropyl) iod & & & & {$[84 / 9]$} \\
\hline & $(273-353)$ & 41.6 & (313) & & {$[59 / 21]$} \\
\hline $\mathrm{C}_{6} \mathrm{~F}_{14} \mathrm{~N}_{2} \mathrm{~S}$ & bis $[1,2,2,2$-tetrafluoro-1-(t & thyl)ethyl] st & mide & & [34451-12-2] \\
\hline & $(325-378)$ & 38.5 & $(340)$ & A & {$[87 / 5][72 / 21]$} \\
\hline $\mathrm{C}_{6} \mathrm{~F}_{14} \mathrm{O}$ & perfluorodipropyl ether & & & & {$[356-62-7]$} \\
\hline & $(306-327)$ & $31.2 \pm 0.4$ & $(298)$ & EB & {$[89 / 13]$} \\
\hline $\mathrm{C}_{6} \mathrm{~F}_{15} \mathrm{~N}$ & perfluorotriethylamine & & & & {$[359-70-6]$} \\
\hline & $(297-343)$ & $34.0 \pm 0.4$ & (298) & EB & {$[95 / 20]$} \\
\hline & & $34.2 \pm 0.1$ & (298) & $\mathrm{C}$ & {$[95 / 20]$} \\
\hline & $(320-334)$ & 32.8 & (327) & A & {$[87 / 5]$} \\
\hline & $(317-349)$ & 32.9 & $(332)$ & $\mathrm{A}$ & {$[87 / 5]$} \\
\hline $\mathrm{C}_{6} \mathrm{~F}_{15} \mathrm{NO}$ & $1,1,1,2,3,3,3$,-heptafluoro- & uoroethyl)-N & omethyl)-2-propanan & & [54566-82-4] \\
\hline & & 27.1 & (338) & & {$[75 / 19]$} \\
\hline $\mathrm{C}_{6} \mathrm{~F}_{15} \mathrm{O}_{4} \mathrm{~S}_{2}$ & 2,2,4,4-tetrafluoro-1,1,3,3 & -1,1,3,3-tetrc & loromethoxy)-1,3-dit & & [63441-15-6] \\
\hline & & 37.2 & (404) & I & {$[77 / 16]$} \\
\hline $\mathrm{C}_{6} \mathrm{~F}_{16} \mathrm{~N}_{2} \mathrm{~S}$ & bis $[1,2,2,2$-tetrafluoro-1-(t & $\begin{array}{l}\text { thyl)ethyl]di } \\
35.8\end{array}$ & furyl fluoride & & $\begin{array}{l}{[59617-31-1]} \\
{[76 / 29]}\end{array}$ \\
\hline $\mathrm{C}_{6} \mathrm{~F}_{16} \mathrm{~S}$ & difluorobis $[1,2,2,2$-tetraflu & luoromethyl) & lfur & & [1423-18-3] \\
\hline & $(273-383)$ & 36.6 & $(328)$ & $\mathrm{A}$ & {$[87 / 5][99 / 16]$} \\
\hline $\mathrm{C}_{6} \mathrm{~F}_{20} \mathrm{~N}_{3} \mathrm{O}_{3} \mathrm{P}$ & phosphorous tris [bis(triflu & nitroxide] d & & & \\
\hline & & 39.3 & $(421)$ & & {$[73 / 24]$} \\
\hline $\mathrm{C}_{6} \mathrm{~N}_{2}$ & dicyanobutadiyne & & & & [16419-78-6] \\
\hline
\end{tabular}


TABLE 6. Enthalpies of vaporization of organic compounds, 1880-2002-Continued

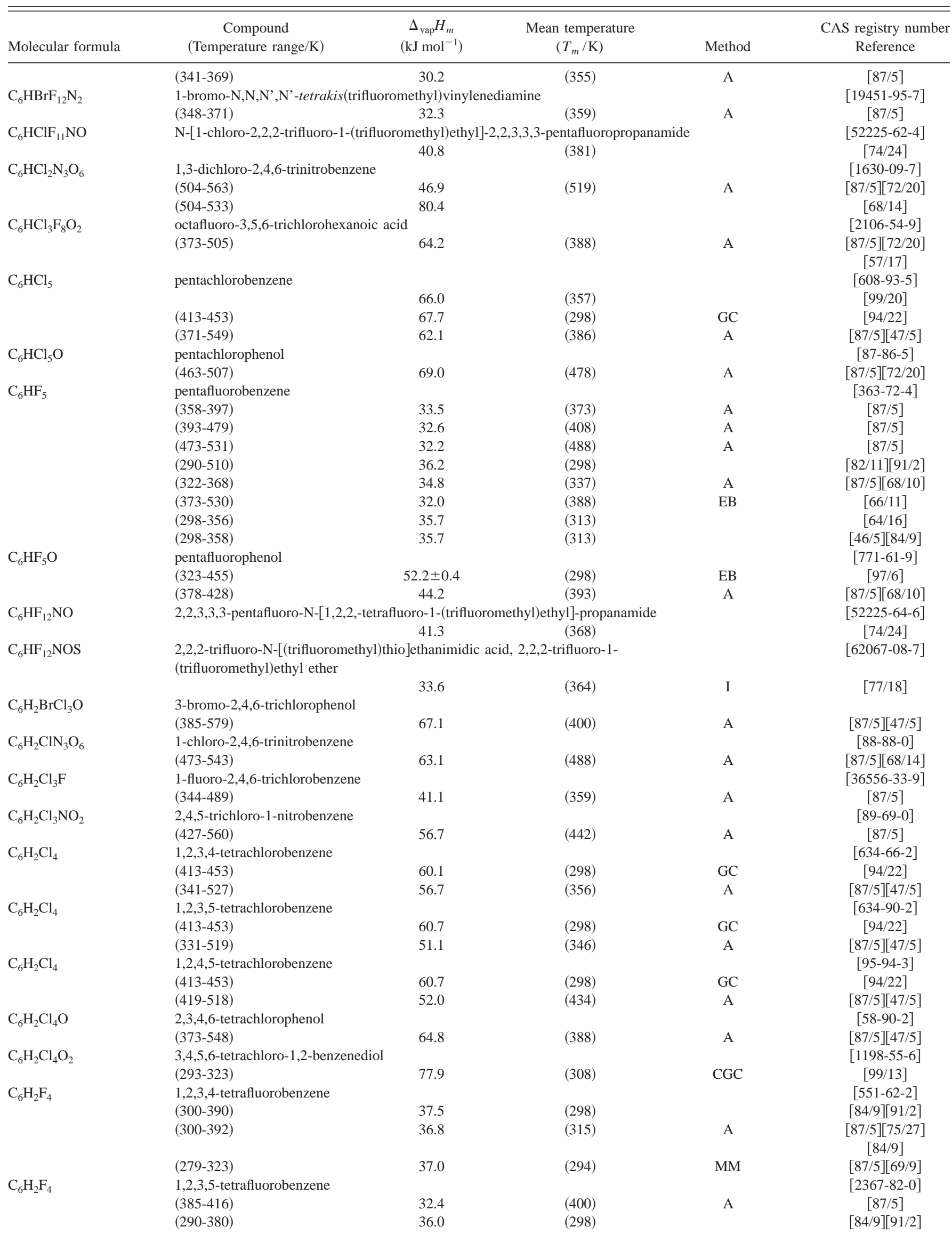


TABLE 6. Enthalpies of vaporization of organic compounds, 1880-2002-Continued

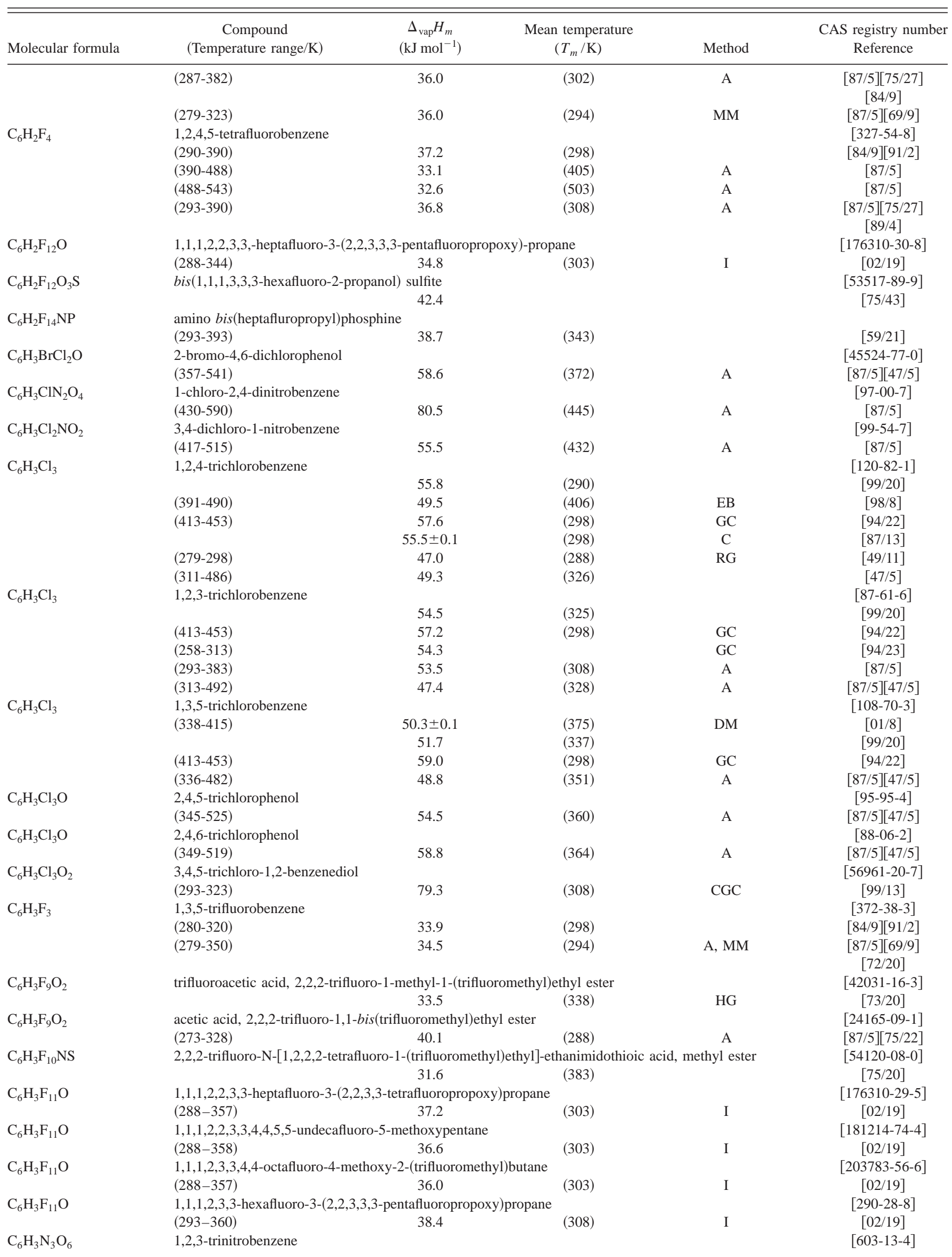


TABLE 6. Enthalpies of vaporization of organic compounds, 1880-2002-Continued

\begin{tabular}{|c|c|c|c|c|c|}
\hline Molecular formula & $\begin{array}{c}\text { Compound } \\
\text { (Temperature range/K) }\end{array}$ & $\begin{array}{c}\Delta_{\mathrm{vap}} H_{m} \\
\left(\mathrm{~kJ} \mathrm{~mol}^{-1}\right)\end{array}$ & $\begin{array}{l}\text { Mean temperature } \\
\qquad\left(T_{m} / \mathrm{K}\right)\end{array}$ & Method & $\begin{array}{c}\text { CAS registry number } \\
\text { Reference }\end{array}$ \\
\hline & $(523-573)$ & 60.3 & $(538)$ & A & $\begin{array}{c}{[87 / 5][68 / 14]} \\
{[72 / 20]}\end{array}$ \\
\hline \multirow[t]{2}{*}{$\mathrm{C}_{6} \mathrm{H}_{3} \mathrm{~N}_{3} \mathrm{O}_{6}$} & 1,2,4-trinitrobenzene & & & & [610-31-1] \\
\hline & $(523-573)$ & 82.6 & $(538)$ & A & $\begin{array}{c}{[87 / 5][68 / 14]} \\
{[72 / 20]}\end{array}$ \\
\hline \multirow[t]{2}{*}{$\mathrm{C}_{6} \mathrm{H}_{3} \mathrm{~N}_{3} \mathrm{O}_{6}$} & 1,3,5-trinitrobenzene & & & & [99-35-4] \\
\hline & $(475-585)$ & 70.3 & $(490)$ & A & $\begin{array}{c}{[87 / 5][68 / 14]} \\
{[72 / 20]}\end{array}$ \\
\hline \multirow[t]{2}{*}{$\mathrm{C}_{6} \mathrm{H}_{3} \mathrm{~N}_{3} \mathrm{O}_{7}$} & 2,4,6-trinitrophenol (picric acid) & & & & [88-89-1] \\
\hline & $(468-598)$ & 106.4 & $(483)$ & A & {$[87 / 5]$} \\
\hline \multirow[t]{2}{*}{$\mathrm{C}_{6} \mathrm{H}_{4} \mathrm{BrCl}$} & 1-bromo-3-chlorobenzene & & & & [108-37-2] \\
\hline & $(252-469)$ & 52.2 & $(267)$ & A & {$[87 / 5][72 / 20]$} \\
\hline \multirow[t]{3}{*}{$\mathrm{C}_{6} \mathrm{H}_{4} \mathrm{BrCl}$} & 1-bromo-4-chlorobenzene & & & & [106-39-8] \\
\hline & $(333-470)$ & 49.1 & $(348)$ & A & {$[87 / 5]$} \\
\hline & $(305-470)$ & 49.7 & $(320)$ & & [47/5] \\
\hline \multirow[t]{2}{*}{$\mathrm{C}_{6} \mathrm{H}_{4} \mathrm{Br}_{2}$} & 1,2-dibromobenzene & & & & [583-53-9] \\
\hline & $(388-568)$ & 50.1 & $(403)$ & A & {$[87 / 5][72 / 20]$} \\
\hline \multirow[t]{2}{*}{$\mathrm{C}_{6} \mathrm{H}_{4} \mathrm{Br}_{2}$} & 1,3-dibromobenzene & & & & [108-36-1] \\
\hline & $(417-500)$ & 48.3 & $(432)$ & A & {$[87 / 5]$} \\
\hline \multirow[t]{2}{*}{$\mathrm{C}_{6} \mathrm{H}_{4} \mathrm{Br}_{2}$} & 1,4-dibromobenzene & & & & [106-37-6] \\
\hline & $(373-493)$ & 49.9 & $(388)$ & $\mathrm{A}$ & {$[87 / 5][72 / 20]$} \\
\hline \multirow[t]{2}{*}{$\mathrm{C}_{6} \mathrm{H}_{4} \mathrm{ClF}$} & 1-chloro-3-fluorobenzene & & & & [625-98-9] \\
\hline & $(273-403)$ & 37.4 & $(288)$ & $\mathrm{A}$ & {$[87 / 5]$} \\
\hline \multirow[t]{2}{*}{$\mathrm{C}_{6} \mathrm{H}_{4} \mathrm{ClI}$} & 1-chloro-4-iodobenzene & & & & {$[352-33-0]$} \\
\hline & $(333-500)$ & 56.5 & $(348)$ & $\mathrm{A}$ & {$[87 / 5]$} \\
\hline \multirow[t]{2}{*}{$\mathrm{C}_{6} \mathrm{H}_{4} \mathrm{ClNO}_{2}$} & 1-chloro-2-nitrobenzene & & & & {$[88-73-3]$} \\
\hline & $(420-516)$ & 52.1 & $(435)$ & EB & {$[84 / 21]$} \\
\hline \multirow[t]{2}{*}{$\mathrm{C}_{6} \mathrm{H}_{4} \mathrm{ClNO}_{2}$} & 1-chloro-3-nitrobenzene & & & & {$[121-73-3]$} \\
\hline & $(414-506)$ & 51.5 & (429) & EB & {$[84 / 21]$} \\
\hline \multirow{2}{*}{$\mathrm{C}_{6} \mathrm{H}_{4} \mathrm{ClNO}_{2}$} & 1-chloro-4-nitrobenzene & & & & {$[100-00-5]$} \\
\hline & $(385-515)$ & 51.3 & $(400)$ & A & {$[87 / 5]$} \\
\hline \multirow[t]{9}{*}{$\mathrm{C}_{6} \mathrm{H}_{4} \mathrm{Cl}_{2}$} & 1,2-dichlorobenzene & & & & {$[95-50-1]$} \\
\hline & & 51.2 & $(256)$ & & {$[99 / 20]$} \\
\hline & $(363-454)$ & 44.5 & $(376)$ & EB & {$[98 / 8]$} \\
\hline & $(256-287)$ & 50.8 & (271) & & [96/19] \\
\hline & $(413-453)$ & 50.9 & $(298)$ & $\mathrm{GC}$ & {$[94 / 22]$} \\
\hline & $(258-313)$ & 51.2 & & $\mathrm{GC}$ & {$[94 / 23]$} \\
\hline & $(373-453)$ & 44.0 & $(388)$ & A & {$[87 / 5]$} \\
\hline & $(360-450)$ & 49.9 & (298) & & {$[84 / 9][91 / 2]$} \\
\hline & $(301-343)$ & 50.0 & $(322)$ & GS & {$[82 / 1]$} \\
\hline \multirow{7}{*}{$\mathrm{C}_{6} \mathrm{H}_{4} \mathrm{Cl}_{2}$} & 1,3-dichlorobenzene & & & & {$[541-73-1]$} \\
\hline & & 50.4 & (248) & & {$[99 / 20]$} \\
\hline & $(357-448)$ & 44.1 & (372) & EB & {$[98 / 8]$} \\
\hline & $(250-274)$ & 50.0 & (262) & & [96/19] \\
\hline & $(413-453)$ & 53.9 & (298) & GC & [94/22] \\
\hline & $(360-450)$ & 47.0 & (298) & & {$[84 / 9][91 / 2]$} \\
\hline & $(348-513)$ & 44.7 & $(363)$ & & {$[87 / 5][72 / 20]$} \\
\hline $\mathrm{C}_{6} \mathrm{H}_{4} \mathrm{Cl}_{2}$ & 1,4-dichlorobenzene & & & & [106-46-7] \\
\hline & & 46.4 & (326) & & [99/20] \\
\hline & $(358-448)$ & 44.2 & (373) & $\mathrm{EB}$ & {$[98 / 8]$} \\
\hline & $(413-453)$ & 54.8 & (298) & GC & {$[94 / 22]$} \\
\hline & $(258-313)$ & U35.0 & & GC & {$[94 / 23]$} \\
\hline & $(341-448)$ & 45.0 & (356) & A & {$[87 / 5]$} \\
\hline & $(370-450)$ & 47.8 & $(298)$ & & {$[84 / 9][91 / 2]$} \\
\hline $\mathrm{C}_{6} \mathrm{H}_{4} \mathrm{Cl}_{2} \mathrm{O}$ & 2,4-dichlorophenol & & & & {$[128-83-2]$} \\
\hline & $(326-483)$ & 60.8 & $(341)$ & A & {$[87 / 5][47 / 5]$} \\
\hline & & & & & {$[75 / 17]$} \\
\hline $\mathrm{C}_{6} \mathrm{H}_{4} \mathrm{Cl}_{2} \mathrm{O}$ & 2,6-dichlorophenol & & & & {$[87-65-0]$} \\
\hline & $(333-493)$ & 57.9 & $(348)$ & A & {$[87 / 5][47 / 5]$} \\
\hline $\mathrm{C}_{6} \mathrm{H}_{4} \mathrm{Cl}_{2} \mathrm{O}_{2}$ & 4,5-dichloro-1,2-benzenediol & & & & [3428-24-8] \\
\hline & $(293-323)$ & 70.5 & $(308)$ & CGC & [99/13] \\
\hline $\mathrm{C}_{6} \mathrm{H}_{4} \mathrm{Cl}_{2} \mathrm{O}_{3}$ & vinyl mucochlorate & & & & \\
\hline & $(273-333)$ & 63.9 & $(288)$ & A & {$[87 / 5]$} \\
\hline $\mathrm{C}_{6} \mathrm{H}_{4} \mathrm{Cl}_{3} \mathrm{~N}$ & 2,4,6-trichloroaniline & & & & {$[634-93-5]$} \\
\hline
\end{tabular}


TABLE 6. Enthalpies of vaporization of organic compounds, 1880-2002-Continued

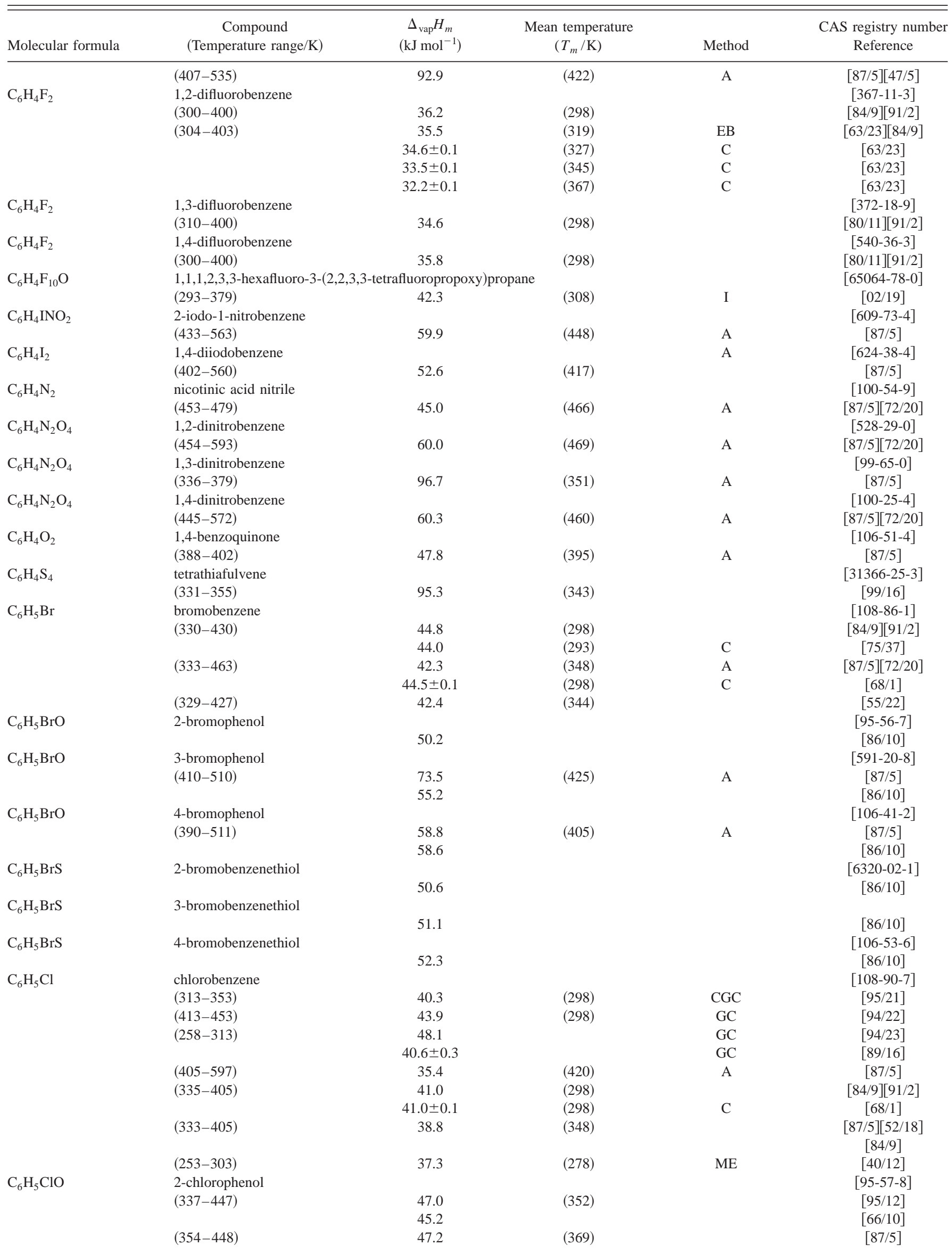


TABLE 6. Enthalpies of vaporization of organic compounds, 1880-2002-Continued

\begin{tabular}{|c|c|c|c|c|c|}
\hline Molecular formula & $\begin{array}{c}\text { Compound } \\
\text { (Temperature range/K) }\end{array}$ & $\begin{array}{c}\Delta_{\mathrm{vap}} H_{m} \\
\left(\mathrm{~kJ} \mathrm{~mol}^{-1}\right)\end{array}$ & $\begin{array}{l}\text { Mean temperature } \\
\qquad\left(T_{m} / \mathrm{K}\right)\end{array}$ & Method & $\begin{array}{c}\text { CAS registry number } \\
\text { Reference }\end{array}$ \\
\hline & $(333-449)$ & 50.1 & $(348)$ & A & {$[87 / 5][74 / 15]$} \\
\hline & $(285-447)$ & 45.2 & $(300)$ & & {$[47 / 5]$} \\
\hline \multirow[t]{3}{*}{$\mathrm{C}_{6} \mathrm{H}_{5} \mathrm{ClO}$} & 3-chlorophenol & & & & {$[108-43-0]$} \\
\hline & & 52.3 & & & {$[86 / 10]$} \\
\hline & $(317-487)$ & 53.1 & $(332)$ & A & {$[87 / 5][47 / 5]$} \\
\hline \multirow[t]{4}{*}{$\mathrm{C}_{6} \mathrm{H}_{5} \mathrm{ClO}$} & 4-chlorophenol & & & & [106-48-9] \\
\hline & $(373-493)$ & 60.6 & $(388)$ & A & {$[87 / 5]$} \\
\hline & & 54.0 & & & {$[86 / 10]$} \\
\hline & $(323-493)$ & 52.8 & $(338)$ & & {$[47 / 5]$} \\
\hline \multirow[t]{2}{*}{$\mathrm{C}_{6} \mathrm{H}_{5} \mathrm{ClS}$} & 2-chlorobenzenethiol & & & & {$[6320-03-2]$} \\
\hline & & 47.7 & & & {$[86 / 10]$} \\
\hline \multirow{2}{*}{$\mathrm{C}_{6} \mathrm{H}_{5} \mathrm{ClS}$} & 3-chlorobenzenethiol & & & & {$[2037-31-2]$} \\
\hline & & 48.5 & & & {$[86 / 10]$} \\
\hline \multirow[t]{2}{*}{$\mathrm{C}_{6} \mathrm{H}_{5} \mathrm{ClS}$} & 4-chlorobenzenethiol & & & & [106-54-7] \\
\hline & & 48.5 & & & {$[86 / 10]$} \\
\hline \multirow{2}{*}{$\mathrm{C}_{6} \mathrm{H}_{5} \mathrm{ClO}_{2}$} & 4-chloro-1,2-benzenediol & & & & [2138-22-9] \\
\hline & $(293-323)$ & 70.2 & $(308)$ & CGC & [99/13] \\
\hline \multirow[t]{3}{*}{$\mathrm{C}_{6} \mathrm{H}_{5} \mathrm{ClO}_{2} \mathrm{~S}$} & benzenesulfonyl chloride & & & & [98-09-9] \\
\hline & $(339-524)$ & 54.4 & $(354)$ & & {$[99 / 16]$} \\
\hline & $(338-525)$ & 57.2 & $(353)$ & A & {$[87 / 5][47 / 5]$} \\
\hline \multirow[t]{2}{*}{$\mathrm{C}_{6} \mathrm{H}_{5} \mathrm{Cl}_{2} \mathrm{~N}$} & 3,4-dichloroaniline & & & & {$[95-76-1]$} \\
\hline & $(420-545)$ & 58.6 & $(435)$ & A & {$[87 / 5]$} \\
\hline \multirow[t]{2}{*}{$\mathrm{C}_{6} \mathrm{H}_{5} \mathrm{Cl}_{2} \mathrm{O}_{2} \mathrm{P}$} & phenyl dichlorophosphate & & & & {$[770-12-7]$} \\
\hline & $(339-513)$ & 63.6 & $(354)$ & A & {$[87 / 5][47 / 5]$} \\
\hline \multirow[t]{11}{*}{$\mathrm{C}_{6} \mathrm{H}_{5} \mathrm{~F}$} & fluorobenzene & & & & {$[462-06-6]$} \\
\hline & $(358-530)$ & 31.9 & $(373)$ & A & {$[87 / 5]$} \\
\hline & $(373-419)$ & 31.8 & $(388)$ & A & {$[87 / 5]$} \\
\hline & $(414-501)$ & 31.0 & $(429)$ & A & {$[87 / 5]$} \\
\hline & $(497-561)$ & 30.9 & $(512)$ & A & {$[87 / 5]$} \\
\hline & $(255-360)$ & 34.5 & $(298)$ & & {$[84 / 9][91 / 2]$} \\
\hline & $(312-394)$ & 33.6 & $(327)$ & EB & {$[87 / 5][56 / 19]$} \\
\hline & & $33.5 \pm 0.1$ & $(318)$ & $\mathrm{C}$ & {$[56 / 19]$} \\
\hline & & $32.4 \pm 0.1$ & $(337)$ & $\mathrm{C}$ & {$[56 / 19]$} \\
\hline & & $31.2 \pm 0.1$ & $(358)$ & $\mathrm{C}$ & {$[56 / 19]$} \\
\hline & & $29.7 \pm 0.1$ & $(382)$ & $\mathrm{C}$ & {$[56 / 19]$} \\
\hline \multirow[t]{2}{*}{$\mathrm{C}_{6} \mathrm{H}_{5} \mathrm{FO}$} & 3-fluorophenol & & & & {$[372-20-3]$} \\
\hline & $(373-451)$ & 50.3 & $(388)$ & A & {$[87 / 5]$} \\
\hline \multirow[t]{2}{*}{$\mathrm{C}_{6} \mathrm{H}_{5} \mathrm{FO}$} & 4-fluorophenol & & & & [371-41-5] \\
\hline & $(360-460)$ & 48.8 & $(375)$ & A & {$[87 / 5]$} \\
\hline \multirow[t]{2}{*}{$\mathrm{C}_{6} \mathrm{H}_{5} \mathrm{~F}_{8} \mathrm{NOS}$} & 1-(ethylimino)-2,2,3,3,4,4,5 & loro- $1,1,2,3,4$ & nydro-thiophene-1-ox & & [77984-30-6] \\
\hline & & 31.4 & $(333)$ & & {$[81 / 15]$} \\
\hline \multirow[t]{2}{*}{$\mathrm{C}_{6} \mathrm{H}_{5} \mathrm{~F}_{9} \mathrm{O}$} & 1-ethoxy-1,1,2,2,3,3,4,4,4-1 & butane & & & [163702-05-4] \\
\hline & $(293-350)$ & 34.2 & $(308)$ & $\mathrm{I}$ & {$[02 / 19]$} \\
\hline $\mathrm{C}_{6} \mathrm{H}_{5} \mathrm{I}$ & iodobenzene & & & & {$[591-50-4]$} \\
\hline & $(313-353)$ & 47.4 & $(298)$ & CGC & {$[95 / 21]$} \\
\hline & $(462-679)$ & 41.1 & $(477)$ & A & {$[87 / 5]$} \\
\hline & $(320-460)$ & 48.9 & $(298)$ & & {$[84 / 9][91 / 2]$} \\
\hline & $(273-358)$ & 51.4 & $(288)$ & A & {$[87 / 5][72 / 20]$} \\
\hline & $(358-543)$ & 46.0 & (373) & A & {$[87 / 5][72 / 20]$} \\
\hline $\mathrm{C}_{6} \mathrm{H}_{5} \mathrm{NO}_{2}$ & nitrobenzene & & & & [98-95-3] \\
\hline & $(313-353)$ & 54.5 & $(298)$ & CGC & {$[95 / 21]$} \\
\hline & $(291-305)$ & $56.1 \pm 1.7$ & $(298)$ & $\mathrm{ME}$ & {$[71 / 10]$} \\
\hline & & 55.0 & $(298)$ & & {$[71 / 9]$} \\
\hline & $(279-296)$ & 54.7 & $(287)$ & $\mathrm{A}$ & {$[87 / 5][72 / 20]$} \\
\hline & & & & & {$[60 / 18]$} \\
\hline & $(407-483)$ & 48.5 & $(422)$ & & {$[52 / 19][84 / 9]$} \\
\hline & $(369-481)$ & 48.9 & $(425)$ & & [33/15] \\
\hline $\mathrm{C}_{6} \mathrm{H}_{5} \mathrm{NO}_{3}$ & 2-nitrophenol & & & & [88-75-5] \\
\hline & $(366-490)$ & 55.9 & $(381)$ & A & {$[87 / 5]$} \\
\hline & $(322-357)$ & 54.4 & $(337)$ & A & {$[47 / 5]$} \\
\hline $\mathrm{C}_{6} \mathrm{H}_{5} \mathrm{~N}_{3}$ & phenyl azide & & & & [622-37-3] \\
\hline & $(348-368)$ & 45.2 & $(358)$ & A & {$[87 / 5][72 / 20]$} \\
\hline $\mathrm{C}_{6} \mathrm{H}_{6}$ & benzene & & & & [71-43-2] \\
\hline & $(305-345)$ & 33.2 & $(320)$ & & [02/45] \\
\hline
\end{tabular}


TABLE 6. Enthalpies of vaporization of organic compounds, 1880-2002-Continued

\begin{tabular}{|c|c|c|c|c|c|}
\hline \multirow[t]{38}{*}{ Molecular formula } & $\begin{array}{c}\text { Compound } \\
\text { (Temperature range/K) }\end{array}$ & $\begin{array}{c}\Delta_{\mathrm{vap}} H_{m} \\
\left(\mathrm{~kJ} \mathrm{~mol}^{-1}\right)\end{array}$ & $\begin{array}{l}\text { Mean temperature } \\
\qquad\left(T_{m} / \mathrm{K}\right)\end{array}$ & Method & $\begin{array}{c}\text { CAS registry number } \\
\text { Reference }\end{array}$ \\
\hline & $(258-313)$ & 35.6 & & GC & [94/23] \\
\hline & $(296-377)$ & 33.5 & $(311)$ & $\mathrm{EB}$ & {$[90 / 7]$} \\
\hline & & $33.9 \pm 0.2$ & & GC & {$[89 / 16]$} \\
\hline & & 33.4 & $(307)$ & $\mathrm{C}$ & [88/19] \\
\hline & & 33.1 & (314) & $\mathrm{C}$ & [88/19] \\
\hline & & 32.4 & $(324)$ & $\mathrm{C}$ & [88/19] \\
\hline & & 31.9 & (332) & $\mathrm{C}$ & [88/19] \\
\hline & & 31.4 & $(344)$ & $\mathrm{C}$ & [88/19] \\
\hline & & 30.6 & (353) & $\mathrm{C}$ & [88/19] \\
\hline & $(279-377)$ & 34.4 & (294) & A & {$[87 / 5]$} \\
\hline & $(353-422)$ & 31.5 & $(368)$ & A & {$[87 / 5]$} \\
\hline & $(420-502)$ & 30.2 & $(435)$ & $\mathrm{A}$ & {$[87 / 5]$} \\
\hline & $(501-562)$ & 30.3 & $(516)$ & A & {$[87 / 5]$} \\
\hline & & 30.8 & $(352)$ & & [83/12] \\
\hline & & 30.5 & $(361)$ & & {$[83 / 12]$} \\
\hline & & 30.2 & $(366)$ & & {$[83 / 12]$} \\
\hline & $(313-373)$ & 35.3 & (343) & & {$[83 / 8]$} \\
\hline & & 31.0 & $(350)$ & & {$[77 / 30]$} \\
\hline & & $33.8 \pm 0.1$ & (298) & $\mathrm{C}$ & {$[73 / 13]$} \\
\hline & & $33.0 \pm 0.1$ & $(313)$ & $\mathrm{C}$ & {$[73 / 13]$} \\
\hline & & $32.2 \pm 0.1$ & $(328)$ & $\mathrm{C}$ & {$[73 / 13]$} \\
\hline & & $31.8 \pm 0.1$ & (333) & $\mathrm{C}$ & [73/13] \\
\hline & & $31.4 \pm 0.1$ & (343) & $\mathrm{C}$ & {$[73 / 13]$} \\
\hline & & $30.9 \pm 0.1$ & $(353)$ & $\mathrm{C}$ & {$[73 / 13]$} \\
\hline & & $32.6 \pm 0.4$ & (313) & DSC & {$[71 / 23]$} \\
\hline & & $32.5 \pm 0.5$ & $(328)$ & DSC & {$[71 / 23]$} \\
\hline & & 33.9 & (298) & & {$[71 / 28]$} \\
\hline & & $31.6 \pm 0.4$ & $(345)$ & DSC & {$[71 / 23]$} \\
\hline & & 34.1 & (293) & & {$[49 / 20]$} \\
\hline & $(284-354)$ & 34.1 & (299) & & [49/6] \\
\hline & & 33.8 & (298) & $\mathrm{C}$ & {$[47 / 7]$} \\
\hline & $(282-354)$ & 34.1 & $(297)$ & & {$[46 / 4]$} \\
\hline & & 31.2 & $(294)$ & & {$[46 / 8]$} \\
\hline & $(288-354)$ & 34.1 & (303) & MM & {$[45 / 2]$} \\
\hline & $(298-373)$ & 33.4 & (313) & $\mathrm{EB}$ & [41/9] \\
\hline & $(273-348)$ & 34.5 & $(288)$ & & {$[40 / 5]$} \\
\hline & & 34.0 & $(298)$ & & {$[27 / 3]$} \\
\hline \multirow[t]{2}{*}{$\mathrm{C}_{6} \mathrm{D}_{6}$} & benzene $-\mathrm{d}_{6}$ & & & & {$[1076-43-3]$} \\
\hline & $(283-352)$ & 34.2 & $(298)$ & & {$[53 / 10]$} \\
\hline \multirow[t]{2}{*}{$\mathrm{C}_{6} \mathrm{H}_{6}$} & 1,5-hexadien-3-yne & & & & [821-08-9] \\
\hline & $(223-357)$ & 40.4 & $(238)$ & A & {$[87 / 5]$} \\
\hline \multirow[t]{2}{*}{$\mathrm{C}_{6} \mathrm{H}_{6}$} & 1,3-hexadien-5-yne & & & & {$[10420-90-3]$} \\
\hline & $(223-303)$ & 44.0 & $(238)$ & A & {$[87 / 5]$} \\
\hline \multirow[t]{2}{*}{$\mathrm{C}_{6} \mathrm{H}_{6}$} & 2,4-hexadiyne & & & & [2809-69-0] \\
\hline & $(364-408)$ & 42.5 & $(298)$ & EB & {$[86 / 1]$} \\
\hline \multirow[t]{4}{*}{$\mathrm{C}_{6} \mathrm{H}_{6} \mathrm{ClN}$} & 2-chloroaniline & & & & {$[95-51-2]$} \\
\hline & $(397-482)$ & 50.7 & $(412)$ & A & {$[87 / 5]$} \\
\hline & $(287-336)$ & $58.2 \pm 1.4$ & $(311)$ & TE, ME & {$[85 / 11]$} \\
\hline & $(294-330)$ & $57.1 \pm 1.0$ & $(312)$ & $\mathrm{TE}, \mathrm{ME}$ & {$[85 / 11]$} \\
\hline \multirow[t]{4}{*}{$\mathrm{C}_{6} \mathrm{H}_{6} \mathrm{ClN}$} & 3-chloroaniline & & & & [108-42-9] \\
\hline & $(398-573)$ & 53.6 & $(413)$ & A & {$[87 / 5][72 / 20]$} \\
\hline & $(292-346)$ & $60.3 \pm 0.6$ & (319) & TE, ME & {$[85 / 11]$} \\
\hline & $(304-342)$ & $61.0 \pm 0.8$ & $(323)$ & $\mathrm{TE}, \mathrm{ME}$ & {$[85 / 11]$} \\
\hline \multirow[t]{2}{*}{$\mathrm{C}_{6} \mathrm{H}_{6} \mathrm{ClN}$} & 4-chloroaniline & & & & {$[106-47-8]$} \\
\hline & $(363-505)$ & 52.2 & $(378)$ & A & {$[87 / 5]$} \\
\hline \multirow[t]{2}{*}{$\mathrm{C}_{6} \mathrm{H}_{6} \mathrm{Cl}_{4}$} & $\alpha-3,4,5,6$-tetrachlorocyclo & & & & [41992-55-6] \\
\hline & $(353-399)$ & 58.0 & $(368)$ & A & {$[87 / 5]$} \\
\hline \multirow[t]{2}{*}{$\mathrm{C}_{6} \mathrm{H}_{6} \mathrm{Cl}_{6}$} & $\alpha$-hexachlorocyclohexane & & & & [319-84-6] \\
\hline & $(343-453)$ & 68.5 & $(398)$ & GC & {$[90 / 2]$} \\
\hline \multirow[t]{2}{*}{$\mathrm{C}_{6} \mathrm{H}_{6} \mathrm{Cl}_{6}$} & $\beta$-hexachlorocyclohexane & & & & {$[58-69-9]$} \\
\hline & $(343-453)$ & 70.5 & (398) & $\mathrm{GC}$ & {$[90 / 2]$} \\
\hline \multirow[t]{2}{*}{$\mathrm{C}_{6} \mathrm{H}_{6} \mathrm{~F}_{8} \mathrm{O}$} & \multicolumn{4}{|c|}{ 1,1,2,2,3,3,4,4-octafluoro-5-methoxypentane } & {$[77527-96-9]$} \\
\hline & $(293-396)$ & 44.8 & $(308)$ & I & {$[02 / 19]$} \\
\hline $\mathrm{C}_{6} \mathrm{H}_{6} \mathrm{~F}_{9} \mathrm{~N}_{3} \mathrm{~S}$ & \multicolumn{4}{|c|}{$\mathrm{N}$-[N,N'-dimethyl-S-(trifluoromethyl)sulfonodiimidoyl]-1,1,1,3,3,3-hexafluoro-2-propanimine } & {$[63265-76-9]$} \\
\hline
\end{tabular}


TABLE 6. Enthalpies of vaporization of organic compounds, 1880-2002-Continued

\begin{tabular}{|c|c|c|c|c|c|}
\hline Molecular formula & $\begin{array}{c}\text { Compound } \\
\text { (Temperature range/K) }\end{array}$ & $\begin{array}{c}\Delta_{\mathrm{vap}} H_{m} \\
\left(\mathrm{~kJ} \mathrm{~mol}^{-1}\right)\end{array}$ & $\begin{array}{l}\text { Mean temperature } \\
\qquad\left(T_{m} / \mathrm{K}\right)\end{array}$ & Method & $\begin{array}{c}\text { CAS registry number } \\
\text { Reference }\end{array}$ \\
\hline & & 32.6 & $(426)$ & $\mathrm{I}$ & [77/15] \\
\hline \multirow[t]{2}{*}{$\mathrm{C}_{6} \mathrm{H}_{6} \mathrm{~N}_{2}$} & 3-hexenedinitrile & & & & {$[1119-85-3]$} \\
\hline & $(353-448)$ & 49.4 & $(368)$ & A & {$[87 / 5]$} \\
\hline \multirow[t]{3}{*}{$\mathrm{C}_{6} \mathrm{H}_{6} \mathrm{~N}_{2} \mathrm{O}_{2}$} & 2-nitroaniline & & & & {$[88-74-4]$} \\
\hline & $(423-553)$ & 59.3 & $(438)$ & A & {$[87 / 5]$} \\
\hline & $(377-558)$ & 64.8 & (392) & & {$[47 / 5]$} \\
\hline \multirow{2}{*}{$\mathrm{C}_{6} \mathrm{H}_{6} \mathrm{~N}_{2} \mathrm{O}_{2}$} & 3-nitroaniline & & & & [99-09-2] \\
\hline & $(443-578)$ & 64.9 & $(458)$ & A & {$[87 / 5]$} \\
\hline \multirow[t]{3}{*}{$\mathrm{C}_{6} \mathrm{H}_{6} \mathrm{~N}_{2} \mathrm{O}_{2}$} & 4-nitroaniline & & & & {$[100-01-6]$} \\
\hline & $(473-538)$ & 77.9 & $(488)$ & $\mathrm{A}$ & {$[87 / 5]$} \\
\hline & $(415-609)$ & 70.0 & $(430)$ & & {$[47 / 5]$} \\
\hline \multirow{12}{*}{$\mathrm{C}_{6} \mathrm{H}_{6} \mathrm{O}$} & phenol & & & & {$[108-95-2]$} \\
\hline & $(363-391)$ & 53.2 & $(378)$ & EB & {$[01 / 17]$} \\
\hline & $(393-433)$ & 58.8 & $(298)$ & CGC & {$[95 / 21]$} \\
\hline & $(455-655)$ & 49.5 & $(470)$ & A & {$[87 / 5]$} \\
\hline & $(314-395)$ & 57.4 & (329) & A & {$[87 / 5]$} \\
\hline & $(387-456)$ & 50.9 & $(402)$ & A & {$[87 / 5]$} \\
\hline & $(449-526)$ & 46.8 & $(464)$ & A & {$[87 / 5]$} \\
\hline & $(520-625)$ & 43.8 & $(535)$ & A & {$[87 / 5]$} \\
\hline & & 51.1 & & & [86/10] \\
\hline & $(383-473)$ & 51.3 & $(398)$ & $\mathrm{EB}, \mathrm{GS}$ & $\begin{array}{c}{[87 / 5][60 / 4]} \\
{[72 / 20]}\end{array}$ \\
\hline & $(380-455)$ & 51.4 & $(395)$ & & {$[49 / 1][84 / 9]$} \\
\hline & $(414-454)$ & 48.1 & $(434)$ & & [39/4] \\
\hline \multirow[t]{3}{*}{$\mathrm{C}_{6} \mathrm{H}_{6} \mathrm{O}_{2}$} & 1,2-dihydroxybenzene (ca & & & & {$[120-80-9]$} \\
\hline & $(395-519)$ & 63.1 & $(410)$ & $\mathrm{A}$ & {$[87 / 5]$} \\
\hline & $(378-439)$ & 61.2 & (393) & $\mathrm{GC}$ & {$[75 / 24]$} \\
\hline \multirow[t]{3}{*}{$\mathrm{C}_{6} \mathrm{H}_{6} \mathrm{O}_{2}$} & 1,3-dihydroxybenzene (re & & & & {$[108-46-3]$} \\
\hline & $(419-550)$ & 74.3 & $(434)$ & A & {$[87 / 5]$} \\
\hline & $(392-463)$ & 74.3 & $(407)$ & GC & {$[87 / 5][75 / 24]$} \\
\hline \multirow[t]{2}{*}{$\mathrm{C}_{6} \mathrm{H}_{6} \mathrm{O}_{2}$} & 1,4-dihydroxybenzene (hy & & & & [123-31-9] \\
\hline & $(448-559)$ & 70.5 & $(463)$ & A & {$[87 / 5]$} \\
\hline \multirow[t]{2}{*}{$\mathrm{C}_{6} \mathrm{H}_{6} \mathrm{O}_{3}$} & 1,2,3-trihydroxybenzene & & & & [87-66-1] \\
\hline & $(425-582)$ & 69.5 & $(440)$ & A & {$[87 / 5][55 / 9]$} \\
\hline \multirow[t]{2}{*}{$\mathrm{C}_{6} \mathrm{H}_{6} \mathrm{O}_{4}$} & butynedioic acid, dimethy & & & & \\
\hline & $(273-460)$ & 56.3 & $(288)$ & A & {$[87 / 5][72 / 20]$} \\
\hline \multirow[t]{10}{*}{$\mathrm{C}_{6} \mathrm{H}_{6} \mathrm{~S}$} & benzenethiol (thiophenol) & & & & [108-98-5] \\
\hline & $(333-471)$ & 45.9 & $(348)$ & & [99/16] \\
\hline & & 43.5 & & & {$[86 / 10]$} \\
\hline & $(385-486)$ & 43.1 & $(400)$ & $\mathrm{A}, \mathrm{EB}$ & {$[87 / 5][66 / 5]$} \\
\hline & & & & & {$[56 / 13]$} \\
\hline & & $43.8 \pm 0.1$ & $(375)$ & $\mathrm{C}$ & {$[56 / 13]$} \\
\hline & & $42.6 \pm 0.1$ & $(395)$ & $\mathrm{C}$ & {$[56 / 13]$} \\
\hline & & $41.8 \pm 0.1$ & $(407)$ & $\mathrm{C}$ & {$[56 / 13]$} \\
\hline & & $41.3 \pm 0.1$ & $(417)$ & $\mathrm{C}$ & {$[56 / 13]$} \\
\hline & $(324-440)$ & 44.3 & (339) & & {$[55 / 9][84 / 9]$} \\
\hline \multirow[t]{2}{*}{$\mathrm{C}_{6} \mathrm{H}_{7} \mathrm{Cl}_{3} \mathrm{OS}$} & 2,3,3-trichloro-2-propenet & O-propyl ester & & & {$[76619-93-7]$} \\
\hline & $(383-433)$ & 69.4 & & $\mathrm{GC}$ & {$[80 / 24]$} \\
\hline \multirow[t]{2}{*}{$\mathrm{C}_{6} \mathrm{H}_{7} \mathrm{~N}$} & 3-methylenecyclobutaneca & & & & {$[15760-35-7]$} \\
\hline & $(348-435)$ & 45.9 & $(366)$ & BG & {$[71 / 2]$} \\
\hline \multirow[t]{2}{*}{$\mathrm{C}_{6} \mathrm{H}_{7} \mathrm{~N}$} & bicyclo[2.1.0]pentane-1-ca & & & & {$[31357-71-8]$} \\
\hline & $(332-390)$ & 41.8 & $(343)$ & BG & {$[71 / 2]$} \\
\hline $\mathrm{C}_{6} \mathrm{H}_{7} \mathrm{~N}$ & aniline & & & & [62-53-3] \\
\hline & $(350-499)$ & $51.0 \pm 0.2$ & $(360)$ & $\mathrm{EB}$ & {$[02 / 14]$} \\
\hline & $(350-499)$ & $48.0 \pm 0.2$ & $(400)$ & $\mathrm{EB}$ & {$[02 / 14]$} \\
\hline & $(350-499)$ & $45.2 \pm 0.2$ & $(440)$ & $\mathrm{EB}$ & {$[02 / 14]$} \\
\hline & $(350-499)$ & $42.2 \pm 0.4$ & $(480)$ & $\mathrm{EB}$ & {$[02 / 14]$} \\
\hline & $(421-591)$ & 45.8 & $(444)$ & & {$[92 / 1]$} \\
\hline & $(273-338)$ & 52.2 & $(288)$ & A & {$[87 / 5]$} \\
\hline & $(304-485)$ & 53.6 & (319) & $\mathrm{A}$ & {$[87 / 5]$} \\
\hline & $(373-458)$ & 48.6 & (388) & A & {$[87 / 5]$} \\
\hline & $(455-523)$ & 46.3 & $(470)$ & $\mathrm{A}$ & {$[87 / 5]$} \\
\hline & $(313-386)$ & 51.4 & $(350)$ & & {$[79 / 23]$} \\
\hline & $(304-457)$ & 54.0 & (319) & & {$[62 / 7]$} \\
\hline
\end{tabular}


TABLE 6. Enthalpies of vaporization of organic compounds, 1880-2002-Continued

\begin{tabular}{|c|c|c|c|c|c|}
\hline Molecular formula & $\begin{array}{c}\text { Compound } \\
\text { (Temperature range/K) }\end{array}$ & $\begin{array}{c}\Delta_{\mathrm{vap}} H_{m} \\
\left(\mathrm{~kJ} \mathrm{~mol}^{-1}\right)\end{array}$ & $\begin{array}{l}\text { Mean temperature } \\
\qquad\left(T_{m} / \mathrm{K}\right)\end{array}$ & Method & $\begin{array}{c}\text { CAS registry number } \\
\text { Reference }\end{array}$ \\
\hline & & 53.0 & (333) & $\mathrm{C}$ & {$[62 / 7]$} \\
\hline \multirow[t]{21}{*}{$\mathrm{C}_{6} \mathrm{H}_{7} \mathrm{~N}$} & 2-methylpyridine & & & & [109-06-8] \\
\hline & $(308-441)$ & $41.2 \pm 0.1$ & $(320)$ & $\mathrm{EB}$ & {$[99 / 24]$} \\
\hline & $(308-441)$ & $38.8 \pm 0.1$ & $(360)$ & EB & {$[99 / 24]$} \\
\hline & $(308-441)$ & $36.4 \pm 0.1$ & $(400)$ & EB & {$[99 / 24]$} \\
\hline & $(308-441)$ & $33.7 \pm 0.3$ & $(440)$ & EB & {$[99 / 24]$} \\
\hline & $(323-373)$ & 43.6 & $(298)$ & CGC & {$[95 / 21]$} \\
\hline & $(292-403)$ & 42.0 & $(307)$ & EB & {$[90 / 6]$} \\
\hline & $(209-245)$ & 46.9 & $(230)$ & $\mathrm{A}$ & {$[87 / 5]$} \\
\hline & $(429-537)$ & 36.5 & $(444)$ & A & {$[87 / 5]$} \\
\hline & $(521-621)$ & 35.4 & $(536)$ & A & {$[87 / 5]$} \\
\hline & & $42.5 \pm 0.1$ & (298) & $\mathrm{C}$ & {$[84 / 4]$} \\
\hline & & $41.6 \pm 0.1$ & (313) & $\mathrm{C}$ & {$[84 / 4]$} \\
\hline & & $40.7 \pm 0.1$ & $(328)$ & $\mathrm{C}$ & {$[84 / 4]$} \\
\hline & & $39.8 \pm 0.1$ & (343) & $\mathrm{C}$ & {$[84 / 4]$} \\
\hline & & $38.3 \pm 0.1$ & $(368)$ & $\mathrm{C}$ & {$[84 / 4]$} \\
\hline & $(352-445)$ & 39.1 & $(367)$ & $\mathrm{EB}, \mathrm{IPM}$ & {$[87 / 5][68 / 4]$} \\
\hline & $(352-442)$ & 39.1 & $(367)$ & EB & {$[87 / 5][63 / 11]$} \\
\hline & & $38.8 \pm 0.1$ & (359) & $\mathrm{C}$ & {$[63 / 11]$} \\
\hline & & $37.7 \pm 0.1$ & (379) & $\mathrm{C}$ & {$[63 / 11]$} \\
\hline & & $36.2 \pm 0.1$ & (402) & $\mathrm{C}$ & {$[63 / 11]$} \\
\hline & $(337-403)$ & 39.8 & $(352)$ & MG & {$[53 / 4]$} \\
\hline \multirow[t]{19}{*}{$\mathrm{C}_{6} \mathrm{H}_{7} \mathrm{~N}$} & 3-methylpyridine & & & & [108-99-6] \\
\hline & $(314-457)$ & $43.2 \pm 0.1$ & $(320)$ & EB & {$[99 / 24]$} \\
\hline & $(314-457)$ & $40.9 \pm 0.1$ & $(360)$ & $\mathrm{EB}$ & {$[99 / 24]$} \\
\hline & $(314-457)$ & $38.6 \pm 0.1$ & $(400)$ & EB & {$[99 / 24]$} \\
\hline & $(314-457)$ & $36.1 \pm 0.2$ & $(440)$ & EB & {$[99 / 24]$} \\
\hline & $(374-458)$ & 40.1 & $(389)$ & $\mathrm{A}$ & {$[87 / 5]$} \\
\hline & $(450-570)$ & 37.7 & $(465)$ & A & {$[87 / 5]$} \\
\hline & $(561-645)$ & 36.8 & $(576)$ & A & {$[87 / 5]$} \\
\hline & & $44.6 \pm 0.1$ & (298) & $\mathrm{C}$ & {$[84 / 4]$} \\
\hline & & $43.6 \pm 0.1$ & (313) & $\mathrm{C}$ & {$[84 / 4]$} \\
\hline & & $42.7 \pm 0.1$ & $(328)$ & $\mathrm{C}$ & {$[84 / 4]$} \\
\hline & & $42.0 \pm 0.1$ & (343) & $\mathrm{C}$ & {$[84 / 4]$} \\
\hline & & $40.4 \pm 0.1$ & $(368)$ & $\mathrm{C}$ & {$[84 / 4]$} \\
\hline & $(347-458)$ & 41.3 & $(362)$ & $\mathrm{EB}, \mathrm{IPM}$ & {$[87 / 5][68 / 4]$} \\
\hline & $(347-458)$ & 41.3 & $(362)$ & EB & {$[87 / 5][63 / 12]$} \\
\hline & & $40.2 \pm 0.1$ & $(372)$ & $\mathrm{C}$ & {$[63 / 12]$} \\
\hline & & $38.9 \pm 0.1$ & (393) & $\mathrm{C}$ & {$[63 / 12]$} \\
\hline & & $37.4 \pm 0.1$ & $(417)$ & $\mathrm{C}$ & {$[63 / 12]$} \\
\hline & $(354-418)$ & 41.0 & $(369)$ & MG & [53/4] \\
\hline \multirow[t]{18}{*}{$\mathrm{C}_{6} \mathrm{H}_{7} \mathrm{~N}$} & 4-methylpyridine & & & & [108-89-4] \\
\hline & $(328-459)$ & $43.4 \pm 0.1$ & $(320)$ & EB & {$[99 / 24]$} \\
\hline & $(328-459)$ & $41.1 \pm 0.1$ & $(360)$ & $\mathrm{EB}$ & {$[99 / 24]$} \\
\hline & $(328-459)$ & $38.8 \pm 0.1$ & $(400)$ & EB & {$[99 / 24]$} \\
\hline & $(328-459)$ & $36.2 \pm 0.2$ & $(440)$ & $\mathrm{EB}$ & {$[99 / 24]$} \\
\hline & $(323-373)$ & 44.7 & $(298)$ & CGC & {$[95 / 21]$} \\
\hline & $(348-460)$ & 41.4 & $(363)$ & A & {$[87 / 5]$} \\
\hline & $(348-347)$ & 42.1 & $(347)$ & A & {$[87 / 5]$} \\
\hline & $(381-460)$ & 40.0 & $(396)$ & A & {$[87 / 5]$} \\
\hline & $(452-573)$ & 37.9 & $(467)$ & A & {$[87 / 5]$} \\
\hline & $(564-646)$ & 37.2 & $(579)$ & A & {$[87 / 5]$} \\
\hline & & $44.9 \pm 0.1$ & $(298)$ & $\mathrm{C}$ & {$[84 / 4]$} \\
\hline & & $43.9 \pm 0.1$ & $(313)$ & $\mathrm{C}$ & {$[84 / 4]$} \\
\hline & & $42.9 \pm 0.1$ & $(328)$ & $\mathrm{C}$ & {$[84 / 4]$} \\
\hline & & $42.1 \pm 0.1$ & $(343)$ & $\mathrm{C}$ & {$[84 / 4]$} \\
\hline & & $44.8 \pm 0.1$ & $(298)$ & $\mathrm{C}$ & {$[81 / 8]$} \\
\hline & $(348-459)$ & 41.4 & $(363)$ & $\mathrm{EB}, \mathrm{IPM}$ & {$[87 / 5][68 / 4]$} \\
\hline & $(350-418)$ & 41.3 & $(365)$ & MG & {$[53 / 4]$} \\
\hline \multirow[t]{2}{*}{$\mathrm{C}_{6} \mathrm{H}_{7} \mathrm{~N}$} & 2-cyclopentene-1-carbonitrile & & & & {$[26555-56-5]$} \\
\hline & & $44.9 \pm 0.1$ & $(298)$ & $\mathrm{C}$ & {$[70 / 21]$} \\
\hline \multirow[t]{2}{*}{$\mathrm{C}_{6} \mathrm{H}_{7} \mathrm{NO}$} & 2-methoxypyridine & & & & {$[1628-89-3]$} \\
\hline & $(304-338)$ & 40.5 & $(319)$ & A & {$[87 / 5]$} \\
\hline $\mathrm{C}_{6} \mathrm{H}_{7} \mathrm{NO}$ & 1-methyl-2(1H)-pyridone & & & & [694-85-9] \\
\hline
\end{tabular}


TABLE 6. Enthalpies of vaporization of organic compounds, 1880-2002-Continued

\begin{tabular}{|c|c|c|c|c|c|}
\hline Molecular formula & $\begin{array}{c}\text { Compound } \\
\text { (Temperature range/K) }\end{array}$ & $\begin{array}{c}\Delta_{\mathrm{vap}} H_{m} \\
\left(\mathrm{~kJ} \mathrm{~mol}^{-1}\right)\end{array}$ & $\begin{array}{l}\text { Mean temperature } \\
\qquad\left(T_{m} / \mathrm{K}\right)\end{array}$ & Method & $\begin{array}{c}\text { CAS registry number } \\
\text { Reference }\end{array}$ \\
\hline \multirow{3}{*}{$\mathrm{C}_{6} \mathrm{H}_{7} \mathrm{NS}$} & $(353-399)$ & 60.2 & $(368)$ & A & {$[87 / 5]$} \\
\hline & 4-(methylthio)pyridine & & & & [22581-72-2] \\
\hline & $(346-383)$ & 55.8 & $(361)$ & A & {$[87 / 5]$} \\
\hline \multirow[t]{3}{*}{$\mathrm{C}_{6} \mathrm{H}_{8}$} & cis, anti, cis-tricyclo[3.1.0 & & & & \\
\hline & $(273-329)$ & 30.6 & $(293)$ & & {$[79 / 3]$} \\
\hline & $(273-329)$ & 29.7 & (313) & & {$[79 / 3]$} \\
\hline \multirow[t]{3}{*}{$\mathrm{C}_{6} \mathrm{H}_{8}$} & 1,3-cyclohexadiene & & & & [592-57-4] \\
\hline & $(307-364)$ & 32.6 & $(322)$ & $\mathrm{A}, \mathrm{EB}$ & {$[87 / 5][73 / 12]$} \\
\hline & $(304-322)$ & 32.4 & (313) & MM & {$[74 / 4]$} \\
\hline \multirow[t]{3}{*}{$\mathrm{C}_{6} \mathrm{H}_{8}$} & 1,4-cyclohexadiene & & & & [628-41-1] \\
\hline & $(304-360)$ & 34.0 & $(319)$ & A & {$[87 / 5]$} \\
\hline & $(304-322)$ & 33.9 & (313) & MM & {$[74 / 4]$} \\
\hline \multirow[t]{2}{*}{$\mathrm{C}_{6} \mathrm{H}_{8}$} & cis 1,3,5-hexatriene & & & & [2612-46-6] \\
\hline & $(306-323)$ & 33.3 & $(314)$ & A, MM & {$[87 / 5][74 / 4]$} \\
\hline \multirow[t]{2}{*}{$\mathrm{C}_{6} \mathrm{H}_{8} \mathrm{ClN}$} & 3-methylpyridine hydroch & & & & [14401-92-4] \\
\hline & $(420-471)$ & 68.7 & $(435)$ & A & {$[87 / 5]$} \\
\hline \multirow[t]{2}{*}{$\mathrm{C}_{6} \mathrm{H}_{8} \mathrm{ClN}$} & 4-methylpyridine hydroch & & & & [14401-93-5] \\
\hline & $(437-473)$ & 64.7 & $(452)$ & A & {$[87 / 5]$} \\
\hline \multirow[t]{2}{*}{$\mathrm{C}_{6} \mathrm{H}_{8} \mathrm{Cl}_{2} \mathrm{O}_{4}$} & ethylene glycol, bis chlor & & & & [6941-69-1] \\
\hline & $(385-557)$ & 73.9 & (400) & A & {$[87 / 5][47 / 5]$} \\
\hline \multirow{2}{*}{$\mathrm{C}_{6} \mathrm{H}_{8} \mathrm{~N}_{2}$} & 2,5-dimethylpyrazine & & & & {$[123-32-0]$} \\
\hline & $(303-411)$ & 44.5 & $(357)$ & & {$[95 / 4]$} \\
\hline \multirow[t]{2}{*}{$\mathrm{C}_{6} \mathrm{H}_{8} \mathrm{~N}_{2}$} & adiponitrile & & & & [111-69-3] \\
\hline & $(348-523)$ & 58.7 & $(363)$ & $\mathrm{A}$ & {$[87 / 5]$} \\
\hline \multirow[t]{2}{*}{$\mathrm{C}_{6} \mathrm{H}_{8} \mathrm{~N}_{2}$} & 2-methylaminopyridine & & & & [4597-87-9] \\
\hline & $(308-323)$ & 49.0 & $(316)$ & A & {$[87 / 5]$} \\
\hline \multirow[t]{2}{*}{$\mathrm{C}_{6} \mathrm{H}_{8} \mathrm{~N}_{2}$} & 3-methylaminopyridine & & & & [18364-47-1] \\
\hline & $(313-343)$ & 57.2 & (326) & A & {$[87 / 5]$} \\
\hline \multirow[t]{2}{*}{$\mathrm{C}_{6} \mathrm{H}_{8} \mathrm{~N}_{2}$} & 4-methylaminopyridine & & & & [1121-58-0] \\
\hline & $(313-343)$ & 54.1 & $(328)$ & A & {$[87 / 5]$} \\
\hline \multirow{2}{*}{$\mathrm{C}_{6} \mathrm{H}_{8} \mathrm{~N}_{2}$} & 1,3-diaminobenzene & & & & {$[108-45-2]$} \\
\hline & $(372-559)$ & 63.7 & $(387)$ & $\mathrm{A}$ & {$[87 / 5][47 / 5]$} \\
\hline \multirow[t]{4}{*}{$\mathrm{C}_{6} \mathrm{H}_{8} \mathrm{~N}_{2}$} & phenyl hydrazine & & & & [100-63-0] \\
\hline & $(413-518)$ & 57.3 & $(428)$ & $\mathrm{A}$ & {$[87 / 5][72 / 20]$} \\
\hline & $(345-517)$ & 59.2 & $(360)$ & & {$[47 / 5]$} \\
\hline & $(378-465)$ & 57.2 & (393) & $\mathrm{T}$ & {$[42 / 4]$} \\
\hline $\mathrm{C}_{6} \mathrm{H}_{8} \mathrm{O}$ & 2-cyclohexen-1-one & & & & [930-68-7] \\
\hline & $(335-481)$ & $49.5 \pm 0.4$ & $(298)$ & EB & {$[97 / 8]$} \\
\hline $\mathrm{C}_{6} \mathrm{H}_{8} \mathrm{O}$ & 2,5-dimethylfuran & & & & [625-36-3] \\
\hline & $(271-308)$ & $32.3 \pm 0.3$ & $(290)$ & GS & {$[98 / 2]$} \\
\hline & $(271-308)$ & $31.8 \pm 0.3$ & $(298)$ & GS & {$[98 / 2]$} \\
\hline $\mathrm{C}_{6} \mathrm{H}_{8} \mathrm{O}_{2}$ & methyl bicyclo[1.1.0]buta & xylate & & & {$[4935-01-7]$} \\
\hline & $(299-377)$ & 37.3 & $(318)$ & BG & {$[71 / 2]$} \\
\hline $\mathrm{C}_{6} \mathrm{H}_{8} \mathrm{O}_{3}$ & 2,2-dimethylsuccinic acid & & & & [17347-61-4] \\
\hline & $(334-493)$ & 57.3 & (349) & $\mathrm{A}$ & {$[87 / 5][47 / 5]$} \\
\hline $\mathrm{C}_{6} \mathrm{H}_{8} \mathrm{O}_{3}$ & 2-methylglutaric acid anh & & & & [31468-33-4] \\
\hline & $(366-556)$ & 60.7 & $(381)$ & A & {$[87 / 5][47 / 5]$} \\
\hline $\mathrm{C}_{6} \mathrm{H}_{8} \mathrm{O}_{4}$ & dimethyl fumarate & & & & {$[624-49-7]$} \\
\hline & $(361-466)$ & 53.8 & $(376)$ & $\mathrm{A}$ & {$[87 / 5]$} \\
\hline $\mathrm{C}_{6} \mathrm{H}_{8} \mathrm{O}_{4}$ & dimethyl maleate & & & & [624-48-6] \\
\hline & $(385-421)$ & 52.0 & $(400)$ & A & {$[87 / 5]$} \\
\hline & $(318-478)$ & 53.9 & (334) & & {$[47 / 5]$} \\
\hline $\mathrm{C}_{6} \mathrm{H}_{8} \mathrm{~S}$ & 2,3-dimethylthiophene & & & & [632-16-6] \\
\hline & $(353-473)$ & 39.4 & $(368)$ & $\mathrm{A}$ & {$[87 / 5][72 / 20]$} \\
\hline $\mathrm{C}_{6} \mathrm{H}_{8} \mathrm{~S}$ & 2,4-dimethylthiophene & & & & [638-00-6] \\
\hline & $(323-493)$ & 41.4 & (338) & $\mathrm{A}$ & $\begin{array}{c}{[87 / 5][72 / 20]} \\
{[99 / 16]}\end{array}$ \\
\hline $\mathrm{C}_{6} \mathrm{H}_{8} \mathrm{~S}$ & 2,5-dimethylthiophene & & & & {$[638-02-8]$} \\
\hline & $(333-374)$ & 39.7 & (348) & $\mathrm{I}, \mathrm{A}$ & $\begin{array}{c}{[87 / 5][71 / 3]} \\
{[99 / 16]}\end{array}$ \\
\hline $\mathrm{C}_{6} \mathrm{H}_{8} \mathrm{~S}$ & 3,4-dimethylthiophene & & & & [632-15-5] \\
\hline & $(328-478)$ & 41.1 & (343) & A & $\begin{array}{c}{[87 / 5][72 / 20]} \\
{[99 / 16]}\end{array}$ \\
\hline $\mathrm{C}_{6} \mathrm{H}_{8} \mathrm{~S}$ & 2-ethylthiophene & & & & {$[872-55-9]$} \\
\hline
\end{tabular}


TABLE 6. Enthalpies of vaporization of organic compounds, 1880-2002-Continued

\begin{tabular}{|c|c|c|c|c|c|}
\hline Molecular formula & $\begin{array}{c}\text { Compound } \\
\text { (Temperature range/K) }\end{array}$ & $\begin{array}{c}\Delta_{\mathrm{vap}} H_{m} \\
\left(\mathrm{~kJ} \mathrm{~mol}^{-1}\right)\end{array}$ & $\begin{array}{l}\text { Mean temperature } \\
\qquad\left(T_{m} / \mathrm{K}\right)\end{array}$ & Method & $\begin{array}{c}\text { CAS registry number } \\
\text { Reference }\end{array}$ \\
\hline & $(333-374)$ & 39.7 & $(348)$ & $\mathrm{I}, \mathrm{A}$ & $\begin{array}{c}{[87 / 5][71 / 3]} \\
{[99 / 16]}\end{array}$ \\
\hline \multirow[t]{2}{*}{$\mathrm{C}_{6} \mathrm{H}_{8} \mathrm{~S}$} & 3-ethylthiophene & & & & [1795-01-3] \\
\hline & $(318-473)$ & 40.7 & (333) & $\mathrm{A}$ & {$[87 / 5][72 / 20]$} \\
\hline \multirow[t]{2}{*}{$\mathrm{C}_{6} \mathrm{H}_{9} \mathrm{~F}_{3} \mathrm{O}_{2}$} & butyl trifluoroacetate & & & & {$[367-64-6]$} \\
\hline & $(343-377)$ & 37.8 & $(358)$ & $\mathrm{A}, \mathrm{EB}$ & {$[87 / 5][69 / 13]$} \\
\hline \multirow[t]{5}{*}{$\mathrm{C}_{6} \mathrm{H}_{9} \mathrm{~N}$} & cyclopentanecarbonitrile & & & & {$[4254-02-8]$} \\
\hline & & $48.1 \pm 0.1$ & $(298)$ & $\mathrm{C}$ & {$[83 / 6]$} \\
\hline & & $43.4 \pm 0.1$ & $(298)$ & & {$[73 / 6]$} \\
\hline & $(340-418)$ & 40.9 & (359) & BG & {$[71 / 2]$} \\
\hline & & $43.5 \pm 0.1$ & $(298)$ & $\mathrm{C}$ & {$[70 / 21]$} \\
\hline \multirow[t]{2}{*}{$\mathrm{C}_{6} \mathrm{H}_{9} \mathrm{~N}$} & 2,5-dimethylpyrrole & & & & {$[625-84-3]$} \\
\hline & $(373-443)$ & 49.5 & $(388)$ & A, IPM, EB & {$[87 / 5][68 / 4]$} \\
\hline \multirow[t]{2}{*}{$\mathrm{C}_{6} \mathrm{H}_{9} \mathrm{NO}_{2}$} & ethyl 2-cyanopropionate & & & & [1572-99-2] \\
\hline & $(283-323)$ & $58.6 \pm 0.3$ & (298) & GS & [95/11] \\
\hline \multirow[t]{2}{*}{$\mathrm{C}_{6} \mathrm{H}_{9} \mathrm{P}$} & trivinylphosphine & & & & [3746-01-8] \\
\hline & $(289-334)$ & 33.7 & (304) & & {$[57 / 15][84 / 9]$} \\
\hline \multirow[t]{3}{*}{$\mathrm{C}_{6} \mathrm{H}_{10}$} & cis bicyclo[3.1.0]hexane & & & & {$[285-58-5]$} \\
\hline & $(273-300)$ & 33.7 & (286) & A & {$[87 / 5]$} \\
\hline & & $33.5 \pm 0.4$ & (298) & & {$[70 / 30]$} \\
\hline \multirow[t]{12}{*}{$\mathrm{C}_{6} \mathrm{H}_{10}$} & cyclohexene & & & & [110-83-8] \\
\hline & $(312-356)$ & 32.6 & (327) & & {$[01 / 12]$} \\
\hline & $(285-357)$ & $33.5 \pm 0.5$ & (298) & EB & {$[96 / 4]$} \\
\hline & $(309-365)$ & 32.7 & (324) & $\mathrm{A}, \mathrm{EB}$ & {$[87 / 5][73 / 12]$} \\
\hline & $(305-322)$ & 33.1 & $(308)$ & MM & {$[74 / 4]$} \\
\hline & & $32.7 \pm 0.1$ & (313) & $\mathrm{C}$ & {$[73 / 13]$} \\
\hline & & $32.2 \pm 0.1$ & $(323)$ & $\mathrm{C}$ & {$[73 / 13]$} \\
\hline & & $31.7 \pm 0.1$ & (333) & $\mathrm{C}$ & {$[73 / 13]$} \\
\hline & & $31.2 \pm 0.1$ & $(343)$ & $\mathrm{C}$ & {$[73 / 13]$} \\
\hline & & $30.7 \pm 0.1$ & $(353)$ & $\mathrm{C}$ & {$[73 / 13]$} \\
\hline & $(285-357)$ & 33.7 & $(300)$ & MM & {$[50 / 6]$} \\
\hline & $(229-292)$ & 32.6 & $(300)$ & & {$[41 / 6]$} \\
\hline \multirow[t]{3}{*}{$\mathrm{C}_{6} \mathrm{H}_{10}$} & 1-methylcyclopentene & & & & [693-89-0] \\
\hline & & $32.6 \pm 0.2$ & (298) & GCC & {$[79 / 17]$} \\
\hline & $(268-403)$ & 33.4 & $(283)$ & A & {$[87 / 5][72 / 20]$} \\
\hline \multirow[t]{3}{*}{$\mathrm{C}_{6} \mathrm{H}_{10}$} & 3-methylcyclopentene & & & & {$[1120-62-3]$} \\
\hline & & $31.0 \pm 0.2$ & (298) & GCC & {$[79 / 17]$} \\
\hline & $(263-392)$ & 32.1 & $(278)$ & A & {$[87 / 5][72 / 20]$} \\
\hline \multirow[t]{2}{*}{$\mathrm{C}_{6} \mathrm{H}_{10}$} & 4-methylcyclopentene & & & & [1759-81-5] \\
\hline & $(271-403)$ & 33.2 & $(286)$ & A & {$[87 / 5][72 / 20]$} \\
\hline \multirow[t]{2}{*}{$\mathrm{C}_{6} \mathrm{H}_{10}$} & 1,3-dimethylcyclobutene & & & & [1489-61-8] \\
\hline & $(269-296)$ & 31.3 & (282) & A & {$[87 / 5]$} \\
\hline \multirow[t]{2}{*}{$\mathrm{C}_{6} \mathrm{H}_{10}$} & 2,3-dimethyl-1,3-butadiene & & & & {$[513-81-5]$} \\
\hline & $(273-342)$ & 32.2 & (288) & A & {$[87 / 5][55 / 3]$} \\
\hline \multirow[t]{2}{*}{$\mathrm{C}_{6} \mathrm{H}_{10}$} & trans 1,3-hexadiene & & & & {$[592-48-3]$} \\
\hline & $(299-319)$ & 32.1 & (309) & $\mathrm{A}, \mathrm{MM}$ & {$[87 / 5][74 / 4]$} \\
\hline \multirow[t]{2}{*}{$\mathrm{C}_{6} \mathrm{H}_{10}$} & trans 1,4 -hexadiene & & & & [7319-00-8] \\
\hline & $(304-323)$ & 30.2 & (313) & $\mathrm{A}, \mathrm{MM}$ & {$[87 / 5][74 / 4]$} \\
\hline $\mathrm{C}_{6} \mathrm{H}_{10}$ & 1,5-hexadiene & & & & {$[592-42-7]$} \\
\hline & $(299-333)$ & 29.4 & (314) & A & {$[87 / 5]$} \\
\hline & $(300-319)$ & 28.6 & (308) & & {$[74 / 4]$} \\
\hline & $(273-333)$ & 30.5 & (288) & $\mathrm{A}$ & $\begin{array}{c}{[87 / 5][55 / 3]} \\
{[72 / 20]}\end{array}$ \\
\hline $\mathrm{C}_{6} \mathrm{H}_{10}$ & trans trans 2,4 -hexadiene & & & & [5194-51-4] \\
\hline & $(304-354)$ & 33.2 & (319) & A & {$[87 / 5]$} \\
\hline & $(305-323)$ & 33.2 & (308) & MM & {$[74 / 4]$} \\
\hline $\mathrm{C}_{6} \mathrm{H}_{10}$ & 1-hexyne & & & & {$[693-02-7]$} \\
\hline & $(250-290)$ & 33.5 & $(270)$ & MM & {$[81 / 19]$} \\
\hline & $(237-287)$ & 34.2 & $(262)$ & HSA & [81/19] \\
\hline & $(265-391)$ & 33.4 & $(280)$ & A & {$[87 / 5][72 / 20]$} \\
\hline $\mathrm{C}_{6} \mathrm{H}_{10}$ & 3-hexyne & & & & {$[764-35-2]$} \\
\hline & $(253-354)$ & 30.5 & (268) & A & {$[87 / 5]$} \\
\hline & $(253-298)$ & 31.6 & $(275)$ & $\mathrm{T}$ & {$[65 / 13]$} \\
\hline $\mathrm{C}_{6} \mathrm{H}_{10} \mathrm{Br}_{2}$ & trans 1,2-dibromocyclohexane & & & & [7429-37-0] \\
\hline
\end{tabular}


TABLE 6. Enthalpies of vaporization of organic compounds, 1880-2002-Continued

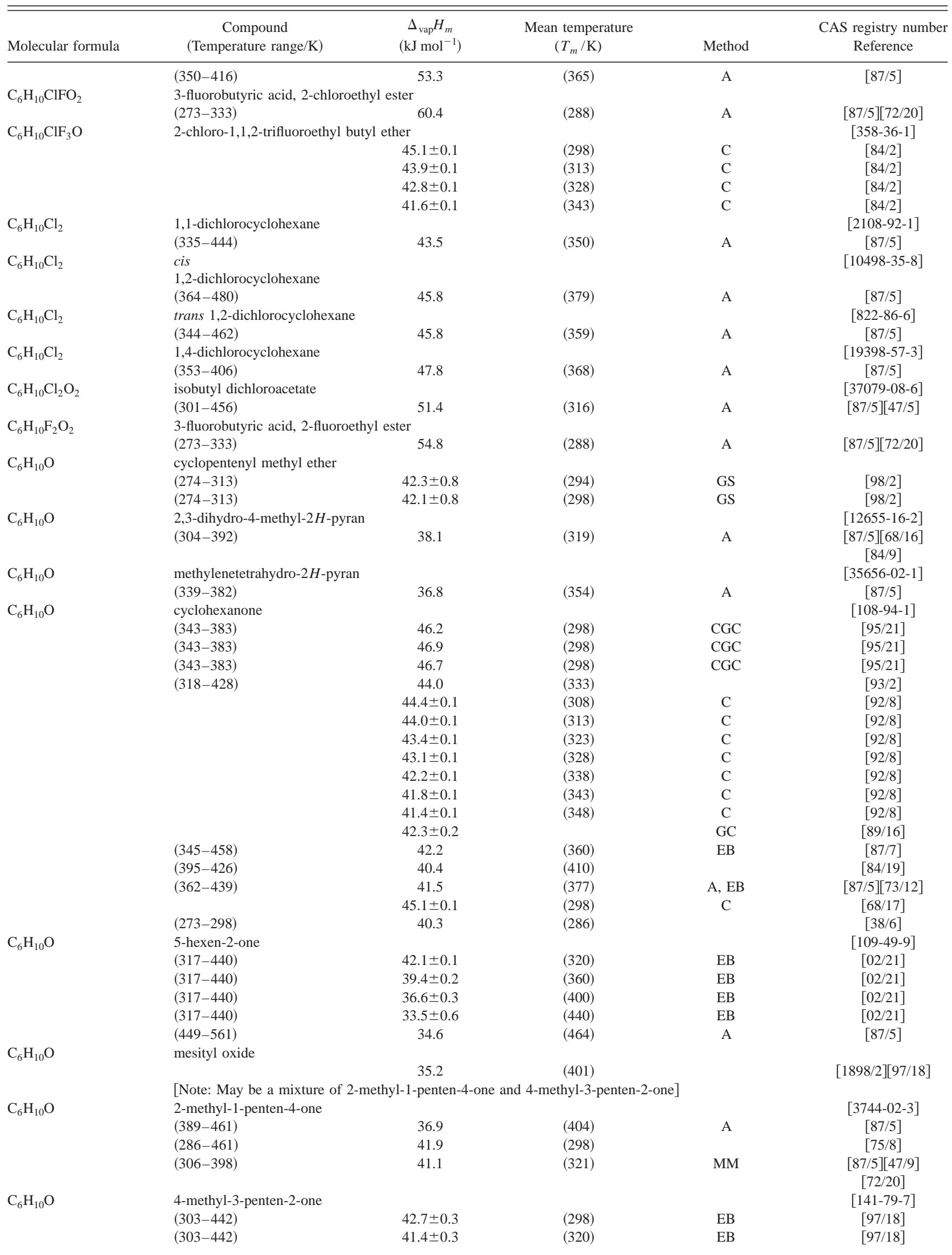


TABLE 6. Enthalpies of vaporization of organic compounds, 1880-2002-Continued

\begin{tabular}{|c|c|c|c|c|c|}
\hline Molecular formula & $\begin{array}{c}\text { Compound } \\
\text { (Temperature range/K) }\end{array}$ & $\begin{array}{c}\Delta_{\mathrm{vap}} H_{m} \\
\left(\mathrm{~kJ} \mathrm{~mol}^{-1}\right)\end{array}$ & $\begin{array}{l}\text { Mean temperature } \\
\qquad\left(T_{m} / \mathrm{K}\right)\end{array}$ & Method & $\begin{array}{c}\text { CAS registry number } \\
\text { Reference }\end{array}$ \\
\hline & $(303-442)$ & $39.1 \pm 0.3$ & $(360)$ & EB & {$[97 / 18]$} \\
\hline & $(303-442)$ & $36.5 \pm 0.3$ & $(400)$ & EB & {$[97 / 18]$} \\
\hline & $(303-442)$ & $33.5 \pm 0.6$ & $(440)$ & $\mathrm{EB}$ & {$[97 / 18]$} \\
\hline & $(343-383)$ & 44.8 & (298) & CGC & {$[95 / 21]$} \\
\hline & $(399-471)$ & 37.8 & (414) & A & {$[87 / 5]$} \\
\hline & $(292-471)$ & 43.3 & (298) & & {$[75 / 4]$} \\
\hline & $(313-405)$ & 41.5 & $(328)$ & MM & $\begin{array}{c}{[87 / 5][47 / 9]} \\
{[72 / 20]}\end{array}$ \\
\hline $\mathrm{C}_{6} \mathrm{H}_{10} \mathrm{O}_{2}$ & \multirow[t]{3}{*}{ methyl cyclobutanecarboxylate } & & & & {$[765-85-5]$} \\
\hline & & $44.2 \pm 0.2$ & & GS & {$[98 / 22]$} \\
\hline & & $44.7 \pm 0.1$ & (298) & $\mathrm{C}$ & {$[83 / 6]$} \\
\hline & $(319-378)$ & 41.4 & $(340)$ & BG & {$[71 / 2]$} \\
\hline \multirow[t]{2}{*}{$\mathrm{C}_{6} \mathrm{H}_{10} \mathrm{O}_{2}$} & \multicolumn{2}{|c|}{ cyclopropanecarboxylic acid ethyl ester } & & & \\
\hline & $(278-308)$ & $44.0 \pm 0.5$ & & GS & {$[98 / 22]$} \\
\hline \multirow[t]{2}{*}{$\mathrm{C}_{6} \mathrm{H}_{10} \mathrm{O}_{2}$} & allyl glycidyl ether & & & & {$[106-92-3]$} \\
\hline & $(323-420)$ & 47.0 & $(338)$ & A & {$[87 / 5]$} \\
\hline \multirow[t]{2}{*}{$\mathrm{C}_{6} \mathrm{H}_{10} \mathrm{O}_{2}$} & butyric acid, vinyl ester & & & & {$[123-20-6]$} \\
\hline & $(365-387)$ & 39.3 & (376) & A & {$[87 / 5]$} \\
\hline \multirow{2}{*}{$\mathrm{C}_{6} \mathrm{H}_{10} \mathrm{O}_{2}$} & 4-vinyl-1,3-dioxane & & & & {$[1072-96-4]$} \\
\hline & $(306-416)$ & 54.5 & $(321)$ & A & {$[87 / 5]$} \\
\hline \multirow[t]{3}{*}{$\mathrm{C}_{6} \mathrm{H}_{10} \mathrm{O}_{2}$} & caprolactone & & & & {$[502-44-3]$} \\
\hline & $(395-436)$ & $54.0 \pm 0.2$ & $(415)$ & EB & {$[91 / 7]$} \\
\hline & $(395-436)$ & $62.0 \pm 1.3$ & $(298)$ & EB & {$[91 / 7]$} \\
\hline \multirow[t]{2}{*}{$\mathrm{C}_{6} \mathrm{H}_{10} \mathrm{O}_{2}$} & ethyl crotonate & & & & [10544-63-5] \\
\hline & $(329-420)$ & 47.1 & $(344)$ & $\mathrm{A}$ & {$[87 / 5]$} \\
\hline \multirow[t]{2}{*}{$\mathrm{C}_{6} \mathrm{H}_{10} \mathrm{O}_{2}$} & ethyl methacrylate & & & & [97-63-2] \\
\hline & $(285-390)$ & 38.3 & $(300)$ & A & {$[87 / 5]$} \\
\hline \multirow[t]{2}{*}{$\mathrm{C}_{6} \mathrm{H}_{10} \mathrm{O}_{2}$} & 5,5-dimethyldihydro-2 $(3 \mathrm{H})$-furanone & & & & {$[3123-97-5]$} \\
\hline & $(311-480)$ & 52.7 & $(326)$ & A & {$[87 / 5]$} \\
\hline \multirow[t]{2}{*}{$\mathrm{C}_{6} \mathrm{H}_{10} \mathrm{O}_{2}$} & propyl acrylate & & & & {$[925-60-0]$} \\
\hline & $(287-395)$ & 37.9 & $(302)$ & A & {$[87 / 5]$} \\
\hline \multirow{2}{*}{$\mathrm{C}_{6} \mathrm{H}_{10} \mathrm{O}_{2}$} & 2,5-hexanedione & & & & [110-13-4] \\
\hline & $(386-474)$ & 50.1 & $(401)$ & A & {$[87 / 5]$} \\
\hline \multirow[t]{2}{*}{$\mathrm{C}_{6} \mathrm{H}_{10} \mathrm{O}_{3}$} & cis/trans 2,5-dimethoxy-2,5-dihydrofur & Iran & & & \\
\hline & & $44.2 \pm 0.3$ & $(298)$ & CGC & {$[00 / 9]$} \\
\hline $\mathrm{C}_{6} \mathrm{H}_{10} \mathrm{O}_{3}$ & cyclohexene ozonide & & & & [284-22-0] \\
\hline & $(276-311)$ & 74.2 & $(291)$ & A & {$[87 / 5]$} \\
\hline & $(353-403)$ & 58.6 & $(378)$ & & {$[77 / 9]$} \\
\hline $\mathrm{C}_{6} \mathrm{H}_{10} \mathrm{O}_{3}$ & ethyl acetoacetate & & & & {$[141-97-9]$} \\
\hline & $(301-454)$ & 52.5 & $(316)$ & $\mathrm{A}$ & {$[87 / 5]$} \\
\hline $\mathrm{C}_{6} \mathrm{H}_{10} \mathrm{O}_{3}$ & methyl levulinate & & & & {$[624-45-3]$} \\
\hline & $(312-471)$ & 50.4 & $(327)$ & A & {$[87 / 5][47 / 5]$} \\
\hline & & 51.1 & (410) & & {$[31 / 1]$} \\
\hline $\mathrm{C}_{6} \mathrm{H}_{10} \mathrm{O}_{3}$ & propionic anhydride & & & & {$[123-62-6]$} \\
\hline & $(293-440)$ & 48.2 & $(308)$ & A & {$[87 / 5]$} \\
\hline & $(341-440)$ & 52.2 & $(356)$ & & {$[1883 / 1]$} \\
\hline $\mathrm{C}_{6} \mathrm{H}_{10} \mathrm{O}_{3}$ & ethyl 3-oxobutanoate & & & & [141-97-9] \\
\hline & & $54.2 \pm 1.0$ & $(298)$ & $\mathrm{C}$ & {$[95 / 6]$} \\
\hline & & 55.0 & & & {$[75 / 39]$} \\
\hline $\mathrm{C}_{6} \mathrm{H}_{10} \mathrm{O}_{4}$ & 1,1-diacetoxyethane & & & & {$[542-10-9]$} \\
\hline & $(343-438)$ & 49.7 & $(358)$ & $\mathrm{A}$ & {$[87 / 5]$} \\
\hline $\mathrm{C}_{6} \mathrm{H}_{10} \mathrm{O}_{4}$ & 2-acetoxypropionic acid, methyl ester & & & & {$[6284-75-9]$} \\
\hline & $(337-445)$ & 52.9 & $(352)$ & A & {$[87 / 5]$} \\
\hline $\mathrm{C}_{6} \mathrm{H}_{10} \mathrm{O}_{4}$ & 3-acetoxypropionic acid, methyl ester & & & & [38003-42-8] \\
\hline & $(343-358)$ & 68.0 & $(350)$ & A & {$[87 / 5]$} \\
\hline $\mathrm{C}_{6} \mathrm{H}_{10} \mathrm{O}_{4}$ & diethyl oxalate & & & & {$[95-92-1]$} \\
\hline & $(343-457)$ & 53.9 & $(358)$ & A & {$[87 / 5]$} \\
\hline & $(320-459)$ & 62.3 & $(335)$ & A & {$[87 / 5][47 / 5]$} \\
\hline $\mathrm{C}_{6} \mathrm{H}_{10} \mathrm{O}_{4}$ & dimethyl succinate & & & & {$[106-65-0]$} \\
\hline & $(340-470)$ & 49.3 & $(364)$ & A & {$[87 / 5]$} \\
\hline $\mathrm{C}_{6} \mathrm{H}_{10} \mathrm{O}_{4}$ & ethylene glycol diacetate & & & & {$[111-55-7]$} \\
\hline & $(311-464)$ & 55.2 & $(326)$ & A & {$[87 / 5]$} \\
\hline & & $61.0 \pm 0.1$ & (298) & $\mathrm{C}$ & [70/17] \\
\hline & $(373-463)$ & 57.6 & (388) & & {$[26 / 5][84 / 9]$} \\
\hline
\end{tabular}


TABLE 6. Enthalpies of vaporization of organic compounds, 1880-2002-Continued

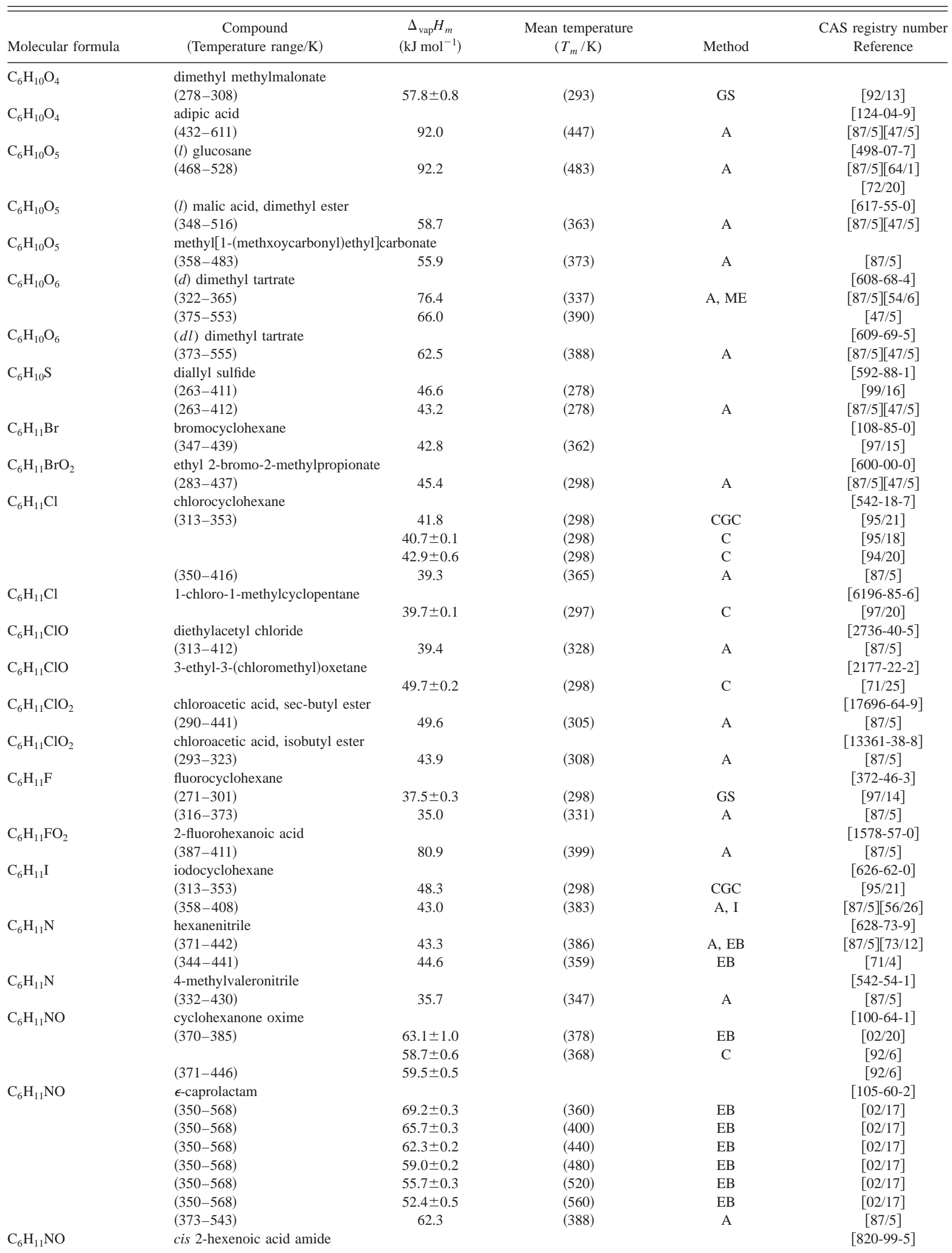


TABLE 6. Enthalpies of vaporization of organic compounds, 1880-2002-Continued

\begin{tabular}{|c|c|c|c|c|c|}
\hline Molecular formula & $\begin{array}{c}\text { Compound } \\
\text { (Temperature range/K) }\end{array}$ & $\begin{array}{c}\Delta_{\mathrm{vap}} H_{m} \\
\left(\mathrm{~kJ} \mathrm{~mol}^{-1}\right)\end{array}$ & $\begin{array}{l}\text { Mean temperature } \\
\qquad\left(T_{m} / \mathrm{K}\right)\end{array}$ & Method & $\begin{array}{l}\text { CAS registry number } \\
\text { Reference }\end{array}$ \\
\hline & $(343-383)$ & 61.7 & $(358)$ & A & {$[87 / 5]$} \\
\hline \multirow[t]{2}{*}{$\mathrm{C}_{6} \mathrm{H}_{11} \mathrm{NO}$} & 1-methyl-2-piperidone & & & & {$[931-20-4]$} \\
\hline & $(341-385)$ & 55.4 & $(356)$ & A & {$[87 / 5]$} \\
\hline \multirow[t]{2}{*}{$\mathrm{C}_{6} \mathrm{H}_{11} \mathrm{NO}$} & 2,3,4,5-tetrahydro-6-methoxypyridine & & & & {$[5693-62-9]$} \\
\hline & $(292-338)$ & 42.8 & $(307)$ & A & {$[87 / 5]$} \\
\hline \multirow[t]{2}{*}{$\mathrm{C}_{6} \mathrm{H}_{11} \mathrm{NO}_{2}$} & nitrocyclohexane & & & & {$[1122-60-7]$} \\
\hline & $(298-318)$ & $54.7 \pm 0.6$ & $(298)$ & GS & {$[97 / 5]$} \\
\hline \multirow[t]{2}{*}{$\mathrm{C}_{6} \mathrm{H}_{11} \mathrm{NO}_{2}$} & 1-aminocyclopentanecarboxylic acid & & & & {$[52-52-8]$} \\
\hline & $(443-468)$ & 123.3 & $(455)$ & A & {$[87 / 5]$} \\
\hline $\mathrm{C}_{6} \mathrm{H}_{11} \mathrm{NO}_{2}$ & $\begin{array}{l}\text { lactic acid N-allyl amide } \\
(359-419)\end{array}$ & 78.2 & $(374)$ & A & {$[87 / 5]$} \\
\hline \multirow[t]{2}{*}{$\mathrm{C}_{6} \mathrm{H}_{11} \mathrm{NO}_{3}$} & ethyl acetamidoacetate & & & & {$[1906-82-7]$} \\
\hline & $(383-466)$ & 69.4 & $(398)$ & A & {$[87 / 5][72 / 20]$} \\
\hline \multirow[t]{2}{*}{$\mathrm{C}_{6} \mathrm{H}_{11} \mathrm{NS}$} & 2-piperidinethione & & & & {$[13070-07-0]$} \\
\hline & $(363-370)$ & 63.3 & $(366)$ & A & {$[87 / 5]$} \\
\hline \multirow{2}{*}{$\mathrm{C}_{6} \mathrm{H}_{11} \mathrm{NS}$} & 2,3,4,5-tetrahydro-(methylthio)pyridine & & & & {$[19766-29-1]$} \\
\hline & $(313-351)$ & 52.6 & $(328)$ & A & {$[87 / 5]$} \\
\hline \multirow[t]{3}{*}{$\mathrm{C}_{6} \mathrm{H}_{12}$} & ethylcyclobutane & & & & {$[4806-61-5]$} \\
\hline & & $31.2 \pm 0.2$ & $(298)$ & $\mathrm{C}$ & {$[83 / 6]$} \\
\hline & & $32.6 \pm 0.8$ & $(298)$ & $\mathrm{EB}$ & {$[74 / 3]$} \\
\hline \multirow[t]{38}{*}{$\mathrm{C}_{6} \mathrm{H}_{12}$} & cyclohexane & & & & {$[110-82-7]$} \\
\hline & $(300-345)$ & 32.7 & $(315)$ & & {$[02 / 45]$} \\
\hline & $(360-470)$ & 32.2 & $(375)$ & & {$[93 / 3]$} \\
\hline & $(313-336)$ & 31.9 & (324) & $\mathrm{EB}$ & [95/9] \\
\hline & $(313-336)$ & 33.1 & (298) & $\mathrm{EB}$ & {$[95 / 9]$} \\
\hline & & 32.3 & (314) & $\mathrm{C}$ & [88/19] \\
\hline & & 31.1 & (332) & $\mathrm{C}$ & [88/19] \\
\hline & & 30.3 & $(345)$ & $\mathrm{C}$ & [88/19] \\
\hline & & 30.0 & $(355)$ & $\mathrm{C}$ & [88/19] \\
\hline & $(353-414)$ & 30.9 & $(368)$ & A & {$[87 / 5]$} \\
\hline & $(412-491)$ & 29.6 & $(427)$ & A & {$[87 / 5]$} \\
\hline & $(489-553)$ & 29.6 & $(504)$ & A & {$[87 / 5]$} \\
\hline & & $33.0 \pm 0.1$ & (298) & $\mathrm{C}$ & [82/18] \\
\hline & & 33.0 & (298) & & [81/12] \\
\hline & & $33.0 \pm 0.1$ & (298) & $\mathrm{C}$ & {$[79 / 13]$} \\
\hline & & $32.3 \pm 0.1$ & (313) & $\mathrm{C}$ & [79/13] \\
\hline & & $31.2 \pm 0.1$ & (333) & $\mathrm{C}$ & [79/13] \\
\hline & & $31.0 \pm 0.1$ & (338) & $\mathrm{C}$ & {$[79 / 13]$} \\
\hline & & $30.4 \pm 0.1$ & $(348)$ & $\mathrm{C}$ & [79/13] \\
\hline & & $30.1 \pm 0.1$ & (353) & $\mathrm{C}$ & [79/13] \\
\hline & & $32.2 \pm 0.1$ & (313) & $\mathrm{C}$ & {$[73 / 13]$} \\
\hline & & $31.9 \pm 0.1$ & (323) & $\mathrm{C}$ & {$[73 / 13]$} \\
\hline & & $31.1 \pm 0.1$ & (333) & $\mathrm{C}$ & [73/13] \\
\hline & & $30.6 \pm 0.1$ & (343) & $\mathrm{C}$ & {$[73 / 13]$} \\
\hline & & $30.1 \pm 0.1$ & (354) & $\mathrm{C}$ & [73/13] \\
\hline & & $32.9 \pm 0.3$ & (298) & ME & {$[72 / 33]$} \\
\hline & & 32.9 & (298) & & {$[71 / 7]$} \\
\hline & & 33.0 & (298) & & {$[71 / 28]$} \\
\hline & $(303-343)$ & 32.5 & (318) & & {$[68 / 6]$} \\
\hline & $(298-348)$ & 32.9 & (313) & & {$[67 / 21]$} \\
\hline & $(316-354)$ & 32.8 & (331) & & {$[65 / 9]$} \\
\hline & & $31.4 \pm 0.1$ & (324) & $\mathrm{C}$ & {$[51 / 2]$} \\
\hline & & $30.4 \pm 0.1$ & $(346)$ & $\mathrm{C}$ & {$[51 / 2]$} \\
\hline & & 33.0 & (298) & $\mathrm{C}$ & {$[47 / 7]$} \\
\hline & & 30.1 & $(354)$ & & {$[46 / 11]$} \\
\hline & $(293-355)$ & 32.9 & $(308)$ & $\mathrm{A}, \mathrm{MM}$ & {$[87 / 5][45 / 2]$} \\
\hline & & $33.3 \pm 0.1$ & (298) & $\mathrm{C}$ & {$[43 / 5]$} \\
\hline & & 33.5 & (298) & & {$[27 / 3]$} \\
\hline \multirow[t]{2}{*}{$\mathrm{C}_{6} \mathrm{D}_{12}$} & cyclohexane- $\mathrm{d}_{12}$ & & & & [1735-17-7] \\
\hline & $(283-353)$ & 33.1 & $(298)$ & & {$[53 / 10]$} \\
\hline \multirow[t]{4}{*}{$\mathrm{C}_{6} \mathrm{H}_{12}$} & methylcyclopentane & & & & [96-37-7] \\
\hline & & 31.6 & $(298)$ & & {$[71 / 28]$} \\
\hline & & $31.3 \pm 0.1$ & $(304)$ & $\mathrm{C}$ & {$[59 / 8]$} \\
\hline & & $30.2 \pm 0.1$ & $(326)$ & $\mathrm{C}$ & {$[59 / 8]$} \\
\hline
\end{tabular}


TABLE 6. Enthalpies of vaporization of organic compounds, 1880-2002-Continued

\begin{tabular}{|c|c|c|c|c|c|}
\hline Molecular formula & $\begin{array}{c}\text { Compound } \\
\text { (Temperature range/K) }\end{array}$ & $\begin{array}{c}\Delta_{\mathrm{vap}} H_{m} \\
\left(\mathrm{~kJ} \mathrm{~mol}^{-1}\right)\end{array}$ & $\begin{array}{l}\text { Mean temperature } \\
\qquad\left(T_{m} / \mathrm{K}\right)\end{array}$ & Method & $\begin{array}{c}\text { CAS registry number } \\
\text { Reference }\end{array}$ \\
\hline & & $29.1 \pm 0.1$ & $(345)$ & $\mathrm{C}$ & {$[59 / 8]$} \\
\hline & & $31.6 \pm 0.1$ & $(298)$ & $\mathrm{C}$ & {$[47 / 7]$} \\
\hline & $(288-346)$ & 31.9 & $(303)$ & A, MM & {$[87 / 5][45 / 2]$} \\
\hline \multirow[t]{6}{*}{$\mathrm{C}_{6} \mathrm{H}_{12}$} & 1-hexene & & & & {$[592-41-6]$} \\
\hline & $(300-337)$ & 30.6 & $(315)$ & & {$[01 / 12]$} \\
\hline & $(273-343)$ & 31.6 & (288) & A & {$[87 / 5]$} \\
\hline & & 30.6 & $(298)$ & & {$[71 / 28]$} \\
\hline & $(289-337)$ & 30.6 & $(298)$ & & {$[56 / 9]$} \\
\hline & $(289-337)$ & 31.0 & $(304)$ & $\mathrm{MM}$ & {$[50 / 6]$} \\
\hline \multirow[t]{4}{*}{$\mathrm{C}_{6} \mathrm{H}_{12}$} & cis 2-hexene & & & & {$[7688-21-3]$} \\
\hline & $(278-343)$ & 32.2 & (293) & A & {$[87 / 5]$} \\
\hline & & 31.5 & (298) & & {$[71 / 28]$} \\
\hline & $(298-342)$ & 31.5 & $(298)$ & & {$[56 / 9]$} \\
\hline \multirow[t]{4}{*}{$\mathrm{C}_{6} \mathrm{H}_{12}$} & trans 2-hexene & & & & {$[4050-45-7]$} \\
\hline & $(283-342)$ & 32.2 & (298) & A & {$[87 / 5]$} \\
\hline & & 31.6 & (298) & & {$[71 / 28]$} \\
\hline & $(292-341)$ & 31.5 & $(298)$ & & {$[56 / 9]$} \\
\hline \multirow[t]{4}{*}{$\mathrm{C}_{6} \mathrm{H}_{12}$} & cis 3-hexene & & & & [7642-09-3] \\
\hline & $(276-348)$ & 32.1 & $(291)$ & A & {$[87 / 5]$} \\
\hline & & 31.3 & $(298)$ & & {$[71 / 28]$} \\
\hline & $(185-340)$ & 31.3 & $(298)$ & & {$[56 / 7]$} \\
\hline \multirow[t]{4}{*}{$\mathrm{C}_{6} \mathrm{H}_{12}$} & trans 3-hexene & & & & [13269-52-8] \\
\hline & $(278-341)$ & 32.3 & (293) & A & {$[87 / 5]$} \\
\hline & & 31.6 & $(298)$ & & {$[71 / 28]$} \\
\hline & $(291-341)$ & 31.5 & $(298)$ & & {$[56 / 7]$} \\
\hline \multirow[t]{4}{*}{$\mathrm{C}_{6} \mathrm{H}_{12}$} & 2-methyl-1-pentene & & & & {$[763-29-1]$} \\
\hline & $(272-341)$ & 31.6 & $(287)$ & A & {$[87 / 5]$} \\
\hline & & 30.5 & $(298)$ & & {$[71 / 28]$} \\
\hline & $(300-335)$ & 30.5 & $(298)$ & & {$[56 / 9]$} \\
\hline \multirow[t]{4}{*}{$\mathrm{C}_{6} \mathrm{H}_{12}$} & 3-methyl-1-pentene & & & & {$[760-20-3]$} \\
\hline & $(265-333)$ & 30.0 & $(280)$ & A & {$[87 / 5]$} \\
\hline & & 28.6 & $(298)$ & & {$[71 / 28]$} \\
\hline & $(287-328)$ & 28.6 & $(298)$ & & {$[56 / 9]$} \\
\hline \multirow[t]{8}{*}{$\mathrm{C}_{6} \mathrm{H}_{12}$} & 4-methyl-1-pentene & & & & [691-37-2] \\
\hline & $(310-360)$ & $28.6 \pm 0.2$ & $(298)$ & $\mathrm{EB}$ & [97/18] \\
\hline & $(310-360)$ & $27.4 \pm 0.3$ & $(320)$ & $\mathrm{EB}$ & {$[97 / 18]$} \\
\hline & $(310-360)$ & $26.2 \pm 0.4$ & $(340)$ & EB & [97/18] \\
\hline & $(310-360)$ & $24.9 \pm 0.5$ & $(360)$ & $\mathrm{EB}$ & {$[97 / 18]$} \\
\hline & $(265-333)$ & 30.1 & $(280)$ & A & {$[87 / 5]$} \\
\hline & & 28.7 & $(298)$ & & {$[71 / 28]$} \\
\hline & $(287-328)$ & 28.7 & $(298)$ & & {$[56 / 7]$} \\
\hline \multirow[t]{4}{*}{$\mathrm{C}_{6} \mathrm{H}_{12}$} & 2-methyl-2-pentene & & & & {$[625-27-4]$} \\
\hline & $(277-346)$ & 32.4 & $(292)$ & A & {$[87 / 5]$} \\
\hline & & 31.6 & $(298)$ & & {$[71 / 28]$} \\
\hline & $(292-341)$ & 31.6 & $(298)$ & & {$[56 / 7]$} \\
\hline \multirow[t]{4}{*}{$\mathrm{C}_{6} \mathrm{H}_{12}$} & cis 3-methyl-2-pentene & & & & [922-62-3] \\
\hline & $(277-347)$ & 32.2 & $(292)$ & A & {$[87 / 5]$} \\
\hline & & 31.3 & (298) & & {$[71 / 28]$} \\
\hline & $(300-344)$ & 32.1 & $(298)$ & & {$[56 / 9]$} \\
\hline \multirow[t]{4}{*}{$\mathrm{C}_{6} \mathrm{H}_{12}$} & trans 3-methyl-2-pentene & & & & [616-12-6] \\
\hline & $(280-349)$ & 32.8 & $(295)$ & A & {$[87 / 5]$} \\
\hline & & 32.1 & $(298)$ & & {$[71 / 28]$} \\
\hline & $(292-341)$ & 31.3 & $(298)$ & & {$[56 / 9]$} \\
\hline \multirow[t]{4}{*}{$\mathrm{C}_{6} \mathrm{H}_{12}$} & cis 4-methyl-2-pentene & & & & {$[691-38-3]$} \\
\hline & $(267-330)$ & 30.8 & $(282)$ & A & {$[87 / 5]$} \\
\hline & & 29.5 & $(298)$ & & {$[71 / 28]$} \\
\hline & $(300-330)$ & 29.5 & $(298)$ & & {$[56 / 9]$} \\
\hline \multirow[t]{4}{*}{$\mathrm{C}_{6} \mathrm{H}_{12}$} & trans 4-methyl-2-pentene & & & & {$[674-76-0]$} \\
\hline & $(269-337)$ & 31.2 & $(284)$ & A & {$[87 / 5]$} \\
\hline & & 30.0 & $(298)$ & & {$[71 / 28]$} \\
\hline & $(291-332)$ & 30.0 & $(298)$ & & {$[56 / 9]$} \\
\hline \multirow[t]{3}{*}{$\mathrm{C}_{6} \mathrm{H}_{12}$} & 2,3-dimethyl-1-butene & & & & {$[563-78-0]$} \\
\hline & $(267-335)$ & 30.5 & $(282)$ & A & {$[87 / 5]$} \\
\hline & & 29.2 & $(298)$ & & {$[71 / 28]$} \\
\hline
\end{tabular}


TABLE 6. Enthalpies of vaporization of organic compounds, 1880-2002-Continued

\begin{tabular}{|c|c|c|c|c|c|}
\hline Molecular formula & $\begin{array}{c}\text { Compound } \\
\text { (Temperature range/K) }\end{array}$ & $\begin{array}{c}\Delta_{\mathrm{vap}} H_{m} \\
\left(\mathrm{~kJ} \mathrm{~mol}^{-1}\right)\end{array}$ & $\begin{array}{l}\text { Mean temperature } \\
\qquad\left(T_{m} / \mathrm{K}\right)\end{array}$ & Method & $\begin{array}{c}\text { CAS registry number } \\
\text { Reference }\end{array}$ \\
\hline & $(289-329)$ & 29.2 & $(298)$ & & {$[56 / 9]$} \\
\hline \multirow{4}{*}{$\mathrm{C}_{6} \mathrm{H}_{12}$} & 3,3-dimethyl-1-butene & & & & {$[558-37-2]$} \\
\hline & $(254-316)$ & 28.6 & $(269)$ & A & {$[87 / 5]$} \\
\hline & & 26.6 & (298) & & {$[71 / 28]$} \\
\hline & $(281-315)$ & 26.6 & $(298)$ & & {$[56 / 9]$} \\
\hline \multirow[t]{8}{*}{$\mathrm{C}_{6} \mathrm{H}_{12}$} & 2,3-dimethyl-2-butene & & & & {$[563-79-1]$} \\
\hline & $(289-347)$ & 32.6 & (298) & & {$[56 / 9]$} \\
\hline & & 32.5 & (298) & & {$[71 / 28]$} \\
\hline & $(282-348)$ & 33.1 & (297) & A & {$[87 / 5][55 / 3]$} \\
\hline & & $32.9 \pm 0.1$ & (292) & $\mathrm{C}$ & {$[55 / 13]$} \\
\hline & & $32.0 \pm 0.1$ & (308) & $\mathrm{C}$ & {$[55 / 13]$} \\
\hline & & $30.9 \pm 0.1$ & (326) & $\mathrm{C}$ & {$[55 / 13]$} \\
\hline & & $29.7 \pm 0.1$ & (346) & $\mathrm{C}$ & {$[55 / 13]$} \\
\hline \multirow[t]{2}{*}{$\mathrm{C}_{6} \mathrm{H}_{12}$} & 2-ethyl-1-butene & & & & {$[760-21-4]$} \\
\hline & $(289-338)$ & 31.0 & (298) & & {$[56 / 9]$} \\
\hline \multirow[t]{2}{*}{$\mathrm{C}_{6} \mathrm{H}_{12} \mathrm{Br}_{2}$} & 1,1-dibromohexane & & & & [58133-26-9] \\
\hline & $(378-526)$ & 51.6 & $(393)$ & A, EST & $\begin{array}{c}{[87 / 5][56 / 16]} \\
{[72 / 20]}\end{array}$ \\
\hline \multirow[t]{2}{*}{$\mathrm{C}_{6} \mathrm{H}_{12} \mathrm{ClNO}$} & 4-(2-chloroethyl)morpholine & & & & [3240-94-6] \\
\hline & $(273-333)$ & 53.8 & $(288)$ & A & {$[87 / 5][72 / 20]$} \\
\hline \multirow[t]{3}{*}{$\mathrm{C}_{6} \mathrm{H}_{12} \mathrm{Cl}_{2}$} & 1,1-dichlorohexane & & & & [62017-16-7] \\
\hline & $(330-440)$ & 48.7 & $(298)$ & & {$[87 / 12][91 / 2]$} \\
\hline & $(345-484)$ & 45.1 & $(360)$ & A, EST & $\begin{array}{c}{[87 / 5][56 / 16]} \\
{[72 / 20]}\end{array}$ \\
\hline \multirow[t]{4}{*}{$\mathrm{C}_{6} \mathrm{H}_{12} \mathrm{Cl}_{2}$} & $(d l)$ 1,2-dichlorohexane & & & & [2162-92-7] \\
\hline & $(350-440)$ & 48.8 & (298) & & [91/2] \\
\hline & $(352-442)$ & 44.9 & $(367)$ & A & {$[87 / 5]$} \\
\hline & & $47.9 \pm 0.7$ & $(298)$ & $\mathrm{EB}$ & {$[75 / 16]$} \\
\hline \multirow[t]{2}{*}{$\mathrm{C}_{6} \mathrm{H}_{12} \mathrm{Cl}_{2}$} & 1,6-dichlorohexane & & & & [2163-00-0] \\
\hline & $(380-480)$ & 56.3 & (298) & & {$[88 / 11][91 / 2]$} \\
\hline \multirow[t]{2}{*}{$\mathrm{C}_{6} \mathrm{H}_{12} \mathrm{Cl}_{2} \mathrm{O}$} & bis(2-chloro-1-methylethyl) ether & & & & [108-60-1] \\
\hline & $(302-456)$ & 53.6 & $(317)$ & A & {$[87 / 5][47 / 5]$} \\
\hline \multirow[t]{2}{*}{$\mathrm{C}_{6} \mathrm{H}_{12} \mathrm{Cl}_{2} \mathrm{O}_{2}$} & bis(2-chloroethyl)acetaldehyde acetal & & & & {$[14689-97-5]$} \\
\hline & $(329-486)$ & 59.4 & $(344)$ & A & {$[87 / 5][47 / 5]$} \\
\hline \multirow[t]{2}{*}{$\mathrm{C}_{6} \mathrm{H}_{12} \mathrm{Cl}_{3} \mathrm{~N}$} & $\operatorname{tris}(2$-chloroethyl)amine & & & & {$[555-77-1]$} \\
\hline & $(273-333)$ & 65.0 & $(288)$ & A, GS & $\begin{array}{c}{[87 / 5][48 / 13]} \\
{[72 / 20]}\end{array}$ \\
\hline \multirow[t]{2}{*}{$\mathrm{C}_{6} \mathrm{H}_{12} \mathrm{Cl}_{3} \mathrm{O}_{4} \mathrm{P}$} & tris(2-chloroethyl)phosphate & & & & \\
\hline & $(293-445)$ & 36.7 & $(308)$ & A & {$[87 / 5][72 / 20]$} \\
\hline \multirow[t]{2}{*}{$\mathrm{C}_{6} \mathrm{H}_{12} \mathrm{~F}_{2}$} & 1,1-difluorohexane & & & & [62127-41-7] \\
\hline & $(290-407)$ & 37.7 & $(305)$ & A, EST & $\begin{array}{c}{[87 / 5][56 / 16]} \\
{[72 / 20]}\end{array}$ \\
\hline \multirow{2}{*}{$\mathrm{C}_{6} \mathrm{H}_{12} \mathrm{~F}_{3} \mathrm{OP}$} & methyl (trifluoromethyl)phosphinous & acid, tert-bu & & & \\
\hline & $(273-329)$ & 39.7 & $(296)$ & & {$[70 / 26]$} \\
\hline \multirow[t]{2}{*}{$\mathrm{C}_{6} \mathrm{H}_{12} \mathrm{~F}_{3} \mathrm{PS}$} & methyl (trifluoromethyl)phosphinothio & ious acid, te & ester & & {$[26348-87-8]$} \\
\hline & $(296-337)$ & 43.2 & $(312)$ & & {$[70 / 26]$} \\
\hline \multirow[t]{2}{*}{$\mathrm{C}_{6} \mathrm{H}_{12} \mathrm{~F}_{4} \mathrm{~N}_{2}$} & $\mathrm{~N}, \mathrm{~N}, \mathrm{~N}^{\prime}, \mathrm{N}^{\prime}$-tetrafluoro-2-methyl-1,2-pe & pentanediam & & & [16096-76-7] \\
\hline & $(253-293)$ & 42.8 & $(278)$ & A, IPM & $\begin{array}{c}{[87 / 5][63 / 9]} \\
{[62 / 13]}\end{array}$ \\
\hline $\mathrm{C}_{6} \mathrm{H}_{12} \mathrm{~N}_{2}$ & (diethylamino)acetonitrile & & & & {$[3010-02-4]$} \\
\hline & $(283-318)$ & $49.9 \pm 0.3$ & & GS & {$[97 / 10]$} \\
\hline $\mathrm{C}_{6} \mathrm{H}_{12} \mathrm{~N}_{2} \mathrm{O}$ & 1,3-dimethyl-3,4,5,6-tetrahydro-2( $1 \mathrm{H}$ & H)pyrimidin & & & {$[7226-23-5]$} \\
\hline & $(370-520)$ & 58.0 & (400) & EB & {$[87 / 2]$} \\
\hline $\mathrm{C}_{6} \mathrm{H}_{12} \mathrm{~N}_{2} \mathrm{OS}$ & tetramethyl monothiooxamide & & & & \\
\hline & & 59 & (508) & TGA, DSC & {$[02 / 36]$} \\
\hline $\mathrm{C}_{6} \mathrm{H}_{12} \mathrm{~N}_{2} \mathrm{O}_{2}$ & tetramethyloxamide & & & & \\
\hline & & 52.5 & $(460)$ & TGA, DSC & {$[02 / 36]$} \\
\hline $\mathrm{C}_{6} \mathrm{H}_{12} \mathrm{~N}_{2} \mathrm{O}_{6}$ & 2,5-dinitroxyhexane & & & & \\
\hline & $(293-313)$ & 54.4 & (303) & B,GS & {$[57 / 5][72 / 20]$} \\
\hline $\mathrm{C}_{6} \mathrm{H}_{12} \mathrm{~N}_{2} \mathrm{O}_{8}$ & triethylene glycol dinitrate & & & & [111-22-8] \\
\hline & $(303-348)$ & 88.3 & $(318)$ & $\mathrm{A}$ & {$[87 / 5][72 / 20]$} \\
\hline $\mathrm{C}_{6} \mathrm{H}_{12} \mathrm{~N}_{2} \mathrm{~S}_{2}$ & tetramethyl dithiooxamide & & & & [35840-78-9] \\
\hline & & 60.5 & (533) & TGA, DSC & {$[02 / 36]$} \\
\hline $\mathrm{C}_{6} \mathrm{H}_{12} \mathrm{O}$ & $(d l)$ 2,5-dimethyltetrahydrofuran & & & & {$[100-38-9]$} \\
\hline
\end{tabular}


TABLE 6. Enthalpies of vaporization of organic compounds, 1880-2002-Continued

\begin{tabular}{|c|c|c|c|c|c|}
\hline Molecular formula & $\begin{array}{c}\text { Compound } \\
\text { (Temperature range/K) }\end{array}$ & $\begin{array}{c}\Delta_{\mathrm{vap}} H_{m} \\
\left(\mathrm{~kJ} \mathrm{~mol}^{-1}\right)\end{array}$ & $\begin{array}{l}\text { Mean temperature } \\
\qquad\left(T_{m} / \mathrm{K}\right)\end{array}$ & Method & $\begin{array}{c}\text { CAS registry number } \\
\text { Reference }\end{array}$ \\
\hline & $(278-370)$ & 35.4 & $(293)$ & A & {$[87 / 5]$} \\
\hline \multirow[t]{2}{*}{$\mathrm{C}_{6} \mathrm{H}_{12} \mathrm{O}$} & 1,2-epoxyhexane & & & & [1436-34-6] \\
\hline & $(300-390)$ & 43.1 & $(315)$ & A & $\begin{array}{c}{[87 / 5][69 / 21]} \\
{[84 / 9]}\end{array}$ \\
\hline \multirow[t]{2}{*}{$\mathrm{C}_{6} \mathrm{H}_{12} \mathrm{O}$} & 2-methyl-2,3-epoxypentane & & & & [1192-22-9] \\
\hline & $(306-369)$ & 40.6 & $(321)$ & A & {$[87 / 5]$} \\
\hline \multirow[t]{3}{*}{$\mathrm{C}_{6} \mathrm{H}_{12} \mathrm{O}$} & allyl isopropyl ether & & & & {$[6140-80-3]$} \\
\hline & $(253-415)$ & 36.1 & $(268)$ & $\mathrm{A}$ & {$[87 / 5]$} \\
\hline & $(229-353)$ & 36.8 & $(244)$ & A & {$[87 / 5][47 / 5]$} \\
\hline \multirow{3}{*}{$\mathrm{C}_{6} \mathrm{H}_{12} \mathrm{O}$} & allyl propyl ether & & & & [1471-03-0] \\
\hline & $(261-428)$ & 37.5 & $(276)$ & $\mathrm{A}$ & {$[87 / 5]$} \\
\hline & $(234-364)$ & 36.4 & (249) & A & {$[87 / 5][47 / 5]$} \\
\hline \multirow[t]{4}{*}{$\mathrm{C}_{6} \mathrm{H}_{12} \mathrm{O}$} & butyl vinyl ether & & & & {$[111-34-2]$} \\
\hline & $(311-403)$ & $36.7 \pm 0.2$ & $(298)$ & $\mathrm{EB}$ & {$[96 / 5]$} \\
\hline & $(353-393)$ & 36.5 & (298) & CGC & {$[95 / 21]$} \\
\hline & $(269-368)$ & 36.1 & $(284)$ & A & {$[87 / 5]$} \\
\hline \multirow[t]{2}{*}{$\mathrm{C}_{6} \mathrm{H}_{12} \mathrm{O}$} & isobutyl vinyl ether & & & & [109-53-5] \\
\hline & $(266-357)$ & 37.4 & $(281)$ & A & {$[87 / 5]$} \\
\hline \multirow[t]{15}{*}{$\mathrm{C}_{6} \mathrm{H}_{12} \mathrm{O}$} & cyclohexanol & & & & [108-93-0] \\
\hline & $(390-430)$ & 49.8 & $(405)$ & & {$[02 / 9]$} \\
\hline & & $62.0 \pm 0.3$ & $(298)$ & $\mathrm{C}$ & {$[99 / 6]$} \\
\hline & $(341-471)$ & $63.5 \pm 0.7$ & (298) & $\mathrm{EB}$ & {$[97 / 8]$} \\
\hline & $(323-373)$ & 61.3 & (298) & CGC & {$[95 / 21]$} \\
\hline & $(350-456)$ & 55.0 & (365) & EB & {$[87 / 7]$} \\
\hline & $(318-434)$ & 59.9 & (333) & $\mathrm{A}$ & {$[87 / 5]$} \\
\hline & $(300-434)$ & 62.7 & $(315)$ & A & {$[87 / 5]$} \\
\hline & $(404-432)$ & 49.3 & $(418)$ & & [84/19] \\
\hline & $(303-373)$ & 58.4 & $(318)$ & & {$[84 / 27]$} \\
\hline & $(299-319)$ & 60.4 & (309) & & {$[75 / 1]$} \\
\hline & & $62.0 \pm 0.9$ & (298) & & {$[75 / 1]$} \\
\hline & & $62.0 \pm 0.2$ & (298) & $\mathrm{C}$ & {$[68 / 17]$} \\
\hline & & $62.0 \pm 0.3$ & (298) & & {$[66 / 2]$} \\
\hline & $(307-422)$ & 54.8 & $(322)$ & & {$[46 / 4]$} \\
\hline \multirow[t]{2}{*}{$\mathrm{C}_{6} \mathrm{H}_{12} \mathrm{O}$} & 1-methylcyclopentanol & & & & [1462-03-9] \\
\hline & $(354-407)$ & 45.7 & $(369)$ & $\mathrm{A}$ & {$[87 / 5]$} \\
\hline \multirow[t]{4}{*}{$\mathrm{C}_{6} \mathrm{H}_{12} \mathrm{O}$} & 5-hexen-1-ol & & & & [821-41-0] \\
\hline & & $60.2 \pm 0.1$ & $(298)$ & $\mathrm{C}$ & {$[96 / 9]$} \\
\hline & & $58.0 \pm 0.1$ & (343) & $\mathrm{C}$ & {$[96 / 9]$} \\
\hline & & $55.7 \pm 0.1$ & $(358)$ & $\mathrm{C}$ & [96/9] \\
\hline \multirow[t]{5}{*}{$\mathrm{C}_{6} \mathrm{H}_{12} \mathrm{O}$} & (dl) 3-methyl-2-pentanone & & & & {$[565-61-7]$} \\
\hline & $(286-400)$ & 39.8 & $(301)$ & A & {$[87 / 5]$} \\
\hline & $(283-395)$ & 41.5 & (298) & A & {$[87 / 5]$} \\
\hline & $(385-455)$ & 36.5 & (400) & A & [87/5] \\
\hline & $(283-457)$ & 41.2 & (298) & & {$[75 / 8]$} \\
\hline \multirow[t]{17}{*}{$\mathrm{C}_{6} \mathrm{H}_{12} \mathrm{O}$} & 2-hexanone & & & & [591-78-6] \\
\hline & & $43.1 \pm 0.1$ & (298) & $\mathrm{C}$ & {$[92 / 8]$} \\
\hline & & $42.5 \pm 0.1$ & (308) & $\mathrm{C}$ & {$[92 / 8]$} \\
\hline & & $41.6 \pm 0.1$ & $(323)$ & $\mathrm{C}$ & {$[92 / 8]$} \\
\hline & & $40.7 \pm 0.1$ & (338) & $\mathrm{C}$ & {$[92 / 8]$} \\
\hline & & $40.1 \pm 0.1$ & (348) & $\mathrm{C}$ & {$[92 / 8]$} \\
\hline & & $39.5 \pm 0.1$ & (358) & $\mathrm{C}$ & {$[92 / 8]$} \\
\hline & $(293-411)$ & 40.8 & (308) & A & {$[87 / 5]$} \\
\hline & $(279-423)$ & 43.8 & (294) & A & {$[87 / 5]$} \\
\hline & $(310-427)$ & 41.5 & (325) & A & {$[87 / 5]$} \\
\hline & $(421-523)$ & 36.7 & (436) & A & [87/5] \\
\hline & $(513-587)$ & 36.1 & (528) & A & {$[87 / 5]$} \\
\hline & & $43.1 \pm 0.1$ & (298) & $\mathrm{C}$ & {$[83 / 3]$} \\
\hline & & $43.0 \pm 0.3$ & (298) & GCC & {$[79 / 7]$} \\
\hline & $(307-482)$ & 42.9 & (298) & & {$[75 / 8]$} \\
\hline & & $42.2 \pm 0.1$ & (298) & $\mathrm{C}$ & {$[70 / 19]$} \\
\hline & $(280-400)$ & 53.8 & (295) & & {$[47 / 5]$} \\
\hline \multirow[t]{3}{*}{$\mathrm{C}_{6} \mathrm{H}_{12} \mathrm{O}$} & 3-hexanone & & & & [589-38-8] \\
\hline & $(408-517)$ & 36.5 & (423) & A & {$[87 / 5]$} \\
\hline & $(511-583)$ & 35.4 & $(526)$ & A & {$[87 / 5]$} \\
\hline
\end{tabular}


TABLE 6. Enthalpies of vaporization of organic compounds, 1880-2002-Continued

\begin{tabular}{|c|c|c|c|c|c|}
\hline Molecular formula & $\begin{array}{c}\text { Compound } \\
\text { (Temperature range/K) }\end{array}$ & $\begin{array}{c}\Delta_{\mathrm{vap}} H_{m} \\
\left(\mathrm{~kJ} \mathrm{~mol}^{-1}\right)\end{array}$ & $\begin{array}{l}\text { Mean temperature } \\
\qquad\left(T_{m} / \mathrm{K}\right)\end{array}$ & Method & $\begin{array}{c}\text { CAS registry number } \\
\text { Reference }\end{array}$ \\
\hline & & $40.6 \pm 0.1$ & (298) & $\mathrm{C}$ & {$[83 / 3]$} \\
\hline & & $42.3 \pm 0.3$ & (298) & GCC & {$[79 / 7]$} \\
\hline & $(348-413)$ & 38.9 & $(363)$ & A & {$[87 / 5][75 / 8]$} \\
\hline & & 42.3 & (298) & & {$[75 / 8]$} \\
\hline & $(292-406)$ & 42.2 & (307) & A & {$[87 / 5][72 / 20]$} \\
\hline & & $41.9 \pm 0.2$ & (298) & $\mathrm{C}$ & [70/19] \\
\hline & & $38.4 \pm 0.1$ & $(354)$ & $\mathrm{C}$ & [67/39] \\
\hline & & $37.0 \pm 0.1$ & $(374)$ & $\mathrm{C}$ & {$[67 / 39]$} \\
\hline & & $35.4 \pm 0.1$ & (396) & $\mathrm{C}$ & [67/39] \\
\hline & $(349-406)$ & 38.8 & $(364)$ & GS, EB & {$[65 / 7]$} \\
\hline \multirow[t]{15}{*}{$\mathrm{C}_{6} \mathrm{H}_{12} \mathrm{O}$} & 3,3-dimethyl-2-butanone & & & & {$[75-97-8]$} \\
\hline & & $37.8 \pm 0.1$ & $(308)$ & $\mathrm{C}$ & {$[92 / 8]$} \\
\hline & & $37.5 \pm 0.1$ & (313) & $\mathrm{C}$ & {$[92 / 8]$} \\
\hline & & $36.9 \pm 0.1$ & (323) & $\mathrm{C}$ & {$[92 / 8]$} \\
\hline & & $36.7 \pm 0.1$ & (328) & $\mathrm{C}$ & {$[92 / 8]$} \\
\hline & & $35.8 \pm 0.1$ & (338) & $\mathrm{C}$ & {$[92 / 8]$} \\
\hline & & $35.4 \pm 0.1$ & $(343)$ & $\mathrm{C}$ & {$[92 / 8]$} \\
\hline & & $35.0 \pm 0.1$ & $(348)$ & $\mathrm{C}$ & [92/8] \\
\hline & $(311-381)$ & 36.9 & (326) & A & {$[87 / 5]$} \\
\hline & $(363-400)$ & 34.9 & $(378)$ & A & {$[87 / 5]$} \\
\hline & $(396-509)$ & 33.8 & $(411)$ & A & {$[87 / 5]$} \\
\hline & $(491-567)$ & 33.1 & $(506)$ & A & {$[87 / 5]$} \\
\hline & $(289-402)$ & 38.3 & $(304)$ & $\mathrm{A}$ & {$[87 / 5][75 / 8]$} \\
\hline & & 38.3 & $(298)$ & & {$[75 / 8]$} \\
\hline & & $37.9 \pm 0.1$ & (298) & $\mathrm{C}$ & [70/19] \\
\hline \multirow[t]{11}{*}{$\mathrm{C}_{6} \mathrm{H}_{12} \mathrm{O}$} & 4-methyl-2-pentanone & & & & {$[108-10-1]$} \\
\hline & & $40.1 \pm 0.1$ & $(308)$ & $\mathrm{C}$ & {$[92 / 8]$} \\
\hline & & $39.0 \pm 0.1$ & $(323)$ & $\mathrm{C}$ & {$[92 / 8]$} \\
\hline & & $38.0 \pm 0.1$ & (338) & $\mathrm{C}$ & {$[92 / 8]$} \\
\hline & & $37.4 \pm 0.1$ & $(348)$ & $\mathrm{C}$ & [92/8] \\
\hline & $(309-416)$ & 39.2 & (324) & & {$[88 / 6]$} \\
\hline & $(281-400)$ & 42.5 & $(296)$ & A & {$[87 / 5]$} \\
\hline & $(349-389)$ & 37.0 & $(365)$ & $\mathrm{EB}$ & {$[85 / 12]$} \\
\hline & & $42.5 \pm 0.1$ & $(298)$ & $\mathrm{C}$ & {$[83 / 3]$} \\
\hline & $(282-456)$ & 41.0 & $(298)$ & & {$[75 / 8]$} \\
\hline & $(294-390)$ & 41.2 & $(309)$ & A & {$[87 / 5][52 / 14]$} \\
\hline \multirow[t]{6}{*}{$\mathrm{C}_{6} \mathrm{H}_{12} \mathrm{O}$} & 2-methyl-3-pentanone & & & & {$[565-69-5]$} \\
\hline & $(300-387)$ & 43.4 & $(315)$ & A & {$[87 / 5]$} \\
\hline & $(280-387)$ & 41.0 & $(295)$ & A & {$[87 / 5]$} \\
\hline & $(377-450)$ & 36.2 & $(392)$ & A & {$[87 / 5]$} \\
\hline & $(280-452)$ & 40.5 & $(298)$ & & {$[75 / 8]$} \\
\hline & & $39.8 \pm 0.2$ & $(298)$ & $\mathrm{C}$ & {$[70 / 18]$} \\
\hline \multirow[t]{3}{*}{$\mathrm{C}_{6} \mathrm{H}_{12} \mathrm{O}$} & hexanal & & & & {$[66-25-1]$} \\
\hline & & $42.3 \pm 0.1$ & $(298)$ & & {$[81 / 18]$} \\
\hline & $(315-402)$ & 41.0 & $(330)$ & & {$[79 / 15]$} \\
\hline \multirow[t]{2}{*}{$\mathrm{C}_{6} \mathrm{H}_{12} \mathrm{OS}$} & S-butyl thiolacetate & & & & {$[926-47-2]$} \\
\hline & & $48.1 \pm 0.2$ & $(298)$ & $\mathrm{C}$ & {$[66 / 2]$} \\
\hline \multirow[t]{2}{*}{$\mathrm{C}_{6} \mathrm{H}_{12} \mathrm{OS}$} & S-tert-butyl thiolacetate & & & & [999-90-6] \\
\hline & & $42.9 \pm 0.2$ & $(298)$ & $\mathrm{C}$ & {$[66 / 2]$} \\
\hline \multirow[t]{3}{*}{$\mathrm{C}_{6} \mathrm{H}_{12} \mathrm{O}_{2}$} & 2-ethyl-2-methyl-1,3-diox & & & & [126-39-6] \\
\hline & $(274-313)$ & $44.8 \pm 0.3$ & $(298)$ & GS & [02/32] \\
\hline & $(274-313)$ & $43.1 \pm 0.3$ & & GS & {$[98 / 21]$} \\
\hline \multirow[t]{2}{*}{$\mathrm{C}_{6} \mathrm{H}_{12} \mathrm{O}_{2}$} & 2-propyl-1,3-dioxolane & & & & [3390-13-4] \\
\hline & $(278-313)$ & $45.3 \pm 0.3$ & $(298)$ & GS & {$[98 / 21][02 / 29]$} \\
\hline \multirow[t]{2}{*}{$\mathrm{C}_{6} \mathrm{H}_{12} \mathrm{O}_{2}$} & 4-ethyl-1,3-dioxane & & & & {$[1121-61-5]$} \\
\hline & $(362-412)$ & 39.3 & $(377)$ & A & {$[87 / 5]$} \\
\hline \multirow[t]{2}{*}{$\mathrm{C}_{6} \mathrm{H}_{12} \mathrm{O}_{2}$} & 2,4-dimethyl-1,3-dioxane & & & & {$[766-20-1]$} \\
\hline & $(274-313)$ & $44.9 \pm 0.6$ & $(298)$ & GS & {$[02 / 32]$} \\
\hline \multirow[t]{3}{*}{$\mathrm{C}_{6} \mathrm{H}_{12} \mathrm{O}_{2}$} & 4,4-dimethyl-1,3-dioxane & & & & [766-15-4] \\
\hline & $(333-407)$ & 37.1 & $(348)$ & A & {$[87 / 5][68 / 16]$} \\
\hline & $(363-406)$ & 38.8 & $(378)$ & & {$[69 / 8]$} \\
\hline \multirow[t]{2}{*}{$\mathrm{C}_{6} \mathrm{H}_{12} \mathrm{O}_{2}$} & cis 4,5-dimethyl-1,3-dioxa & & & & {$[2391-24-4]$} \\
\hline & $(353-410)$ & 48.5 & $(368)$ & A & {$[87 / 5]$} \\
\hline $\mathrm{C}_{6} \mathrm{H}_{12} \mathrm{O}_{2}$ & trans 4,5-dimethyl-1,3-dic & & & & {$[1121-20-6]$} \\
\hline
\end{tabular}


TABLE 6. Enthalpies of vaporization of organic compounds, 1880-2002-Continued

\begin{tabular}{|c|c|c|c|c|c|}
\hline Molecular formula & $\begin{array}{c}\text { Compound } \\
\text { (Temperature range/K) }\end{array}$ & $\begin{array}{c}\Delta_{\mathrm{vap}} H_{m} \\
\left(\mathrm{~kJ} \mathrm{~mol}^{-1}\right)\end{array}$ & $\begin{array}{l}\text { Mean temperature } \\
\qquad\left(T_{m} / \mathrm{K}\right)\end{array}$ & Method & $\begin{array}{c}\text { CAS registry number } \\
\text { Reference }\end{array}$ \\
\hline & $(353-408)$ & 39.1 & $(368)$ & A & {$[87 / 5]$} \\
\hline \multirow[t]{2}{*}{$\mathrm{C}_{6} \mathrm{H}_{12} \mathrm{O}_{2}$} & 2-hydroxymethyltetrahydropyran & & & & {$[100-72-1]$} \\
\hline & $(344-460)$ & 49.0 & $(359)$ & A & {$[87 / 5]$} \\
\hline \multirow[t]{4}{*}{$\mathrm{C}_{6} \mathrm{H}_{12} \mathrm{O}_{2}$} & 1,1-dimethoxycyclobutane & & & & {$[4415-90-1]$} \\
\hline & $(274-313)$ & $42.0 \pm 0.3$ & $(298)$ & GS & {$[02 / 32]$} \\
\hline & $(273-313)$ & $42.3 \pm 0.3$ & & GS & {$[98 / 21]$} \\
\hline & $(301-348)$ & 42.6 & (325) & $\mathrm{EB}$ & {$[94 / 16]$} \\
\hline \multirow{2}{*}{$\mathrm{C}_{6} \mathrm{H}_{12} \mathrm{O}_{2}$} & {$[(1$-methylethoxy)methyl]oxirane } & & & & [4016-14-2] \\
\hline & & $43.5 \pm 2.1$ & & & {$[87 / 14]$} \\
\hline \multirow[t]{2}{*}{$\mathrm{C}_{6} \mathrm{H}_{12} \mathrm{O}_{2}$} & (propoxymethyl)oxirane & & & & [3126-95-2] \\
\hline & & $48.5 \pm 0.4$ & & & {$[87 / 14]$} \\
\hline $\mathrm{C}_{6} \mathrm{H}_{12} \mathrm{O}_{2}$ & $\begin{array}{l}\text { 1,1-dimethoxy-3-butene } \\
(305-334)\end{array}$ & 42.0 & (320) & EB & [94/16] \\
\hline \multirow[t]{16}{*}{$\mathrm{C}_{6} \mathrm{H}_{12} \mathrm{O}_{2}$} & butyl acetate & & & & [123-86-4] \\
\hline & & 42.4 & $(298)$ & $\mathrm{GC}$ & {$[97 / 13]$} \\
\hline & $(313-363)$ & 42.7 & (298) & CGC & {$[95 / 21]$} \\
\hline & $(313-353)$ & 43.1 & (298) & CGC & {$[95 / 21]$} \\
\hline & & $41.0 \pm 0.5$ & (298) & GC & [87/17] \\
\hline & & $43.0 \pm 0.1$ & (313) & $\mathrm{C}$ & {$[80 / 13]$} \\
\hline & & $41.7 \pm 0.1$ & $(328)$ & $\mathrm{C}$ & {$[80 / 13]$} \\
\hline & & $40.6 \pm 0.1$ & $(343)$ & $\mathrm{C}$ & {$[80 / 13]$} \\
\hline & & $39.4 \pm 0.1$ & $(358)$ & $\mathrm{C}$ & {$[80 / 13]$} \\
\hline & $(326-404)$ & 41.3 & $(341)$ & DTA & {$[80 / 8]$} \\
\hline & & $43.6 \pm 0.5$ & (298) & GCC & {$[80 / 5]$} \\
\hline & & $43.7 \pm 0.2$ & (298) & GCC & {$[80 / 5]$} \\
\hline & $(341-399)$ & 40.5 & $(356)$ & $\mathrm{A}, \mathrm{EB}$ & {$[87 / 5][69 / 13]$} \\
\hline & & $43.6 \pm 0.2$ & $(298)$ & C & {$[66 / 2]$} \\
\hline & $(332-399)$ & 40.8 & $(347)$ & A & $\begin{array}{c}{[87 / 5][64 / 24]} \\
{[84 / 9]}\end{array}$ \\
\hline & & 37.5 & $(398)$ & & {$[61 / 23]$} \\
\hline \multirow[t]{2}{*}{$\mathrm{C}_{6} \mathrm{H}_{12} \mathrm{O}_{2}$} & diacetone alcohol & & & & [123-42-2] \\
\hline & $(301-388)$ & 47.5 & $(316)$ & $\mathrm{A}, \mathrm{I}$ & {$[87 / 5][52 / 14]$} \\
\hline \multirow[t]{3}{*}{$\mathrm{C}_{6} \mathrm{H}_{12} \mathrm{O}_{2}$} & 4-hydroxy-4-methyl-2-pentanone & & & & {$[123-42-2]$} \\
\hline & & 52.1 & & & {$[75 / 39]$} \\
\hline & $(295-441)$ & 51.0 & $(310)$ & & {$[47 / 5]$} \\
\hline \multirow[t]{8}{*}{$\mathrm{C}_{6} \mathrm{H}_{12} \mathrm{O}_{2}$} & ethyl butyrate & & & & {$[105-54-4]$} \\
\hline & $(330-435)$ & 39.4 & $(345)$ & & [97/11] \\
\hline & $(332-393)$ & 40.2 & $(347)$ & & {$[93 / 8]$} \\
\hline & $(310-336)$ & $42.1 \pm 0.1$ & $(323)$ & EB & [91/7] \\
\hline & $(310-336)$ & $43.7 \pm 1.3$ & $(298)$ & $\mathrm{EB}$ & {$[91 / 7]$} \\
\hline & $(263-404)$ & 48.3 & (278) & A & {$[87 / 5]$} \\
\hline & & $42.0 \pm 0.1$ & (298) & $\mathrm{C}$ & {$[66 / 2]$} \\
\hline & $(254-394)$ & 41.8 & $(270)$ & & {$[47 / 5]$} \\
\hline \multirow{4}{*}{$\mathrm{C}_{6} \mathrm{H}_{12} \mathrm{O}_{2}$} & ethyl isobutyrate & & & & {$[97-62-1]$} \\
\hline & $(383-483)$ & 36.0 & $(398)$ & A & {$[87 / 5]$} \\
\hline & & $39.8 \pm 0.1$ & (298) & $\mathrm{C}$ & {$[66 / 2]$} \\
\hline & $(249-393)$ & 44.1 & $(264)$ & A & {$[87 / 5][47 / 5]$} \\
\hline \multirow[t]{2}{*}{$\mathrm{C}_{6} \mathrm{H}_{12} \mathrm{O}_{2}$} & isobutyl acetate & & & & {$[110-19-0]$} \\
\hline & $(252-391)$ & 39.8 & $(267)$ & $\mathrm{A}$ & {$[87 / 5][47 / 5]$} \\
\hline \multirow[t]{2}{*}{$\mathrm{C}_{6} \mathrm{H}_{12} \mathrm{O}_{2}$} & tert-butyl acetate & & & & {$[540-88-5]$} \\
\hline & & $38.0 \pm 0.2$ & $(298)$ & $\mathrm{C}$ & {$[66 / 2][96 / 11]$} \\
\hline \multirow[t]{6}{*}{$\mathrm{C}_{6} \mathrm{H}_{12} \mathrm{O}_{2}$} & methyl 2,2-dimethylpropanoate & & & & [598-98-1] \\
\hline & $(313-363)$ & 37.7 & $(298)$ & $\mathrm{CGC}$ & {$[95 / 21]$} \\
\hline & & $39.0 \pm 0.5$ & (298) & $\mathrm{GC}$ & {$[87 / 17]$} \\
\hline & & 38.8 & $(298)$ & & {$[\mathrm{U} / 1][85 / 6]$} \\
\hline & & $39.7 \pm 0.3$ & (298) & GCC & {$[80 / 5]$} \\
\hline & $(299-356)$ & 35.2 & (319) & BG & {$[71 / 2]$} \\
\hline \multirow[t]{2}{*}{$\mathrm{C}_{6} \mathrm{H}_{12} \mathrm{O}_{2}$} & isopentyl formate & & & & [110-45-2] \\
\hline & $(255-397)$ & 38.9 & $(270)$ & A & {$[87 / 5]$} \\
\hline \multirow[t]{2}{*}{$\mathrm{C}_{6} \mathrm{H}_{12} \mathrm{O}_{2}$} & methyl isovalerate & & & & {$[556-24-1]$} \\
\hline & $(254-390)$ & 41.2 & (269) & $\mathrm{A}$ & {$[87 / 5][47 / 5]$} \\
\hline $\mathrm{C}_{6} \mathrm{H}_{12} \mathrm{O}_{2}$ & methyl valerate & & & & [624-24-8] \\
\hline & & 41.3 & $(350)$ & & {$[02 / 27]$} \\
\hline & & $43.7 \pm 0.2$ & (298) & & {$[02 / 27]$} \\
\hline
\end{tabular}


TABLE 6. Enthalpies of vaporization of organic compounds, 1880-2002-Continued

\begin{tabular}{|c|c|c|c|c|c|}
\hline Molecular formula & $\begin{array}{c}\text { Compound } \\
\text { (Temperature range/K) }\end{array}$ & $\begin{array}{c}\Delta_{\mathrm{vap}} H_{m} \\
\left(\mathrm{~kJ} \mathrm{~mol}^{-1}\right)\end{array}$ & $\begin{array}{l}\text { Mean temperature } \\
\qquad\left(T_{m} / \mathrm{K}\right)\end{array}$ & Method & $\begin{array}{c}\text { CAS registry number } \\
\text { Reference }\end{array}$ \\
\hline & $(297-411)$ & 42.5 & $(312)$ & A & {$[87 / 5]$} \\
\hline & & $43.3 \pm 0.5$ & (298) & GC & {$[87 / 17]$} \\
\hline & & $46.1 \pm 0.3$ & (298) & GCC & {$[80 / 5]$} \\
\hline & & $44.1 \pm 0.1$ & (298) & GCC & {$[80 / 5]$} \\
\hline & & $43.1 \pm 0.1$ & (298) & $\mathrm{C}$ & {$[77 / 1]$} \\
\hline \multirow[t]{8}{*}{$\mathrm{C}_{6} \mathrm{H}_{12} \mathrm{O}_{2}$} & propyl propionate & & & & [106-36-5] \\
\hline & $(378-406)$ & 37.6 & (392) & & [94/15] \\
\hline & $(336-394)$ & 39.9 & (351) & & {$[93 / 8]$} \\
\hline & $(259-396)$ & 43.1 & (274) & A & {$[87 / 5][47 / 5]$} \\
\hline & & $42.1 \pm 0.1$ & (313) & $\mathrm{C}$ & {$[80 / 13]$} \\
\hline & & $41.1 \pm 0.1$ & (328) & $\mathrm{C}$ & {$[80 / 13]$} \\
\hline & & $40.0 \pm 0.1$ & (343) & $\mathrm{C}$ & {$[80 / 13]$} \\
\hline & & $38.8 \pm 0.1$ & (358) & $\mathrm{C}$ & {$[80 / 13]$} \\
\hline \multirow[t]{9}{*}{$\mathrm{C}_{6} \mathrm{H}_{12} \mathrm{O}_{2}$} & hexanoic acid & & & & {$[142-62-1]$} \\
\hline & $(297-328)$ & $68.4 \pm 0.9$ & (313) & GS & {$[00 / 6]$} \\
\hline & $(297-328)$ & $69.2 \pm 0.9$ & (298) & GS & {$[00 / 6]$} \\
\hline & $(353-393)$ & 71.3 & (298) & CGC & {$[95 / 21]$} \\
\hline & & 70.9 & $(271)$ & & {$[82 / 4]$} \\
\hline & $(270-280)$ & $73.2 \pm 2.0$ & (298) & TE & {$[79 / 4]$} \\
\hline & $(335-487)$ & 65.9 & $(350)$ & A & {$[87 / 5][72 / 20]$} \\
\hline & $(371-452)$ & 66.6 & (386) & & {$[57 / 16][84 / 9]$} \\
\hline & & 64.6 & $(367)$ & I & {$[43 / 7]$} \\
\hline \multirow[t]{2}{*}{$\mathrm{C}_{6} \mathrm{H}_{12} \mathrm{O}_{2}$} & 2-ethyl butyric acid & & & & [88-09-5] \\
\hline & $(373-466)$ & 58.2 & $(388)$ & A & {$[87 / 5]$} \\
\hline \multirow[t]{2}{*}{$\mathrm{C}_{6} \mathrm{H}_{12} \mathrm{O}_{2}$} & 4-methylvaleric acid & & & & [646-07-1] \\
\hline & $(339-481)$ & 91.7 & $(354)$ & A & {$[87 / 5]$} \\
\hline \multirow[t]{5}{*}{$\mathrm{C}_{6} \mathrm{H}_{12} \mathrm{O}_{2}$} & 2,2-dimethylbutanoic acid & & & & {$[595-37-9]$} \\
\hline & $(364-498)$ & $59.4 \pm 0.3$ & $(370)$ & EB & {$[02 / 14]$} \\
\hline & $(364-498)$ & $54.6 \pm 0.3$ & $(410)$ & $\mathrm{EB}$ & {$[02 / 14]$} \\
\hline & $(364-498)$ & $50.0 \pm 0.4$ & $(450)$ & $\mathrm{EB}$ & {$[02 / 14]$} \\
\hline & $(364-498)$ & $46.0 \pm 0.7$ & $(490)$ & EB & {$[02 / 14]$} \\
\hline \multirow[t]{3}{*}{$\mathrm{C}_{6} \mathrm{H}_{12} \mathrm{O}_{2}$} & 3,3-dimethylbutanoic acid & & & & {$[1070-83-3]$} \\
\hline & $(283-325)$ & $63.6 \pm 0.9$ & $(304)$ & GS & {$[00 / 6]$} \\
\hline & $(283-325)$ & $64.0 \pm 0.9$ & (298) & GS & {$[00 / 6]$} \\
\hline \multirow[t]{2}{*}{$\mathrm{C}_{6} \mathrm{H}_{12} \mathrm{O}_{3}$} & 1-hexene ozonide & & & & [767-09-9] \\
\hline & $(353-373)$ & 43.9 & $(363)$ & MM & {$[77 / 9]$} \\
\hline \multirow[t]{2}{*}{$\mathrm{C}_{6} \mathrm{H}_{12} \mathrm{O}_{3}$} & sec-butyl glycolate & & & & \\
\hline & $(301-451)$ & 52.3 & $(316)$ & $\mathrm{A}$ & {$[87 / 5][47 / 5]$} \\
\hline \multirow[t]{3}{*}{$\mathrm{C}_{6} \mathrm{H}_{12} \mathrm{O}_{3}$} & 2,4,6-trimethyl-1,3,5-trioxane & & & & {$[123-63-7]$} \\
\hline & $(323-396)$ & 41.5 & $(338)$ & $\mathrm{A}$ & {$[87 / 5]$} \\
\hline & & $41.4 \pm 0.4$ & & & {$[59 / 23]$} \\
\hline \multirow[t]{2}{*}{$\mathrm{C}_{6} \mathrm{H}_{12} \mathrm{O}_{3}$} & glycerol 1-monoallyl ether & & & & \\
\hline & $(323-383)$ & 74.7 & $(338)$ & A & {$[87 / 5][72 / 20]$} \\
\hline \multirow[t]{2}{*}{$\mathrm{C}_{6} \mathrm{H}_{12} \mathrm{O}_{3}$} & 2-ethoxyacetic acid, ethyl ester & & & & [817-95-8] \\
\hline & $(330-430)$ & 46.1 & $(345)$ & A & {$[87 / 5]$} \\
\hline \multirow[t]{2}{*}{$\mathrm{C}_{6} \mathrm{H}_{12} \mathrm{O}_{3}$} & 3-ethoxypropionic acid, methyl ester & & & & [14144-33-3] \\
\hline & $(320-432)$ & 44.3 & $(335)$ & A & {$[87 / 5][72 / 20]$} \\
\hline \multirow[t]{4}{*}{$\mathrm{C}_{6} \mathrm{H}_{12} \mathrm{O}_{3}$} & 2-ethoxyethanol acetate & & & & [111-15-9] \\
\hline & $(322-430)$ & 50.9 & (337) & A & {$[87 / 5]$} \\
\hline & & $52.7 \pm 0.1$ & (298) & $\mathrm{C}$ & {$[70 / 17]$} \\
\hline & $(330-468)$ & $52.6 \pm 0.4$ & (298) & EB & {$[66 / 3]$} \\
\hline $\mathrm{C}_{6} \mathrm{H}_{12} \mathrm{O}_{3}$ & 3-hydroxypropionic acid, propyl ester & 609 & $(362)$ & A & {$[87 / 5]$} \\
\hline $\mathrm{C}_{6} \mathrm{H}_{12} \mathrm{O}_{3}$ & 3-methoxypropionic acid, ethyl ester & 60.9 & (362) & A & $\begin{array}{c}{[87 / 5]} \\
{[10606-42-5]}\end{array}$ \\
\hline & $(313-432)$ & 44.6 & $(328)$ & A & {$[87 / 5]$} \\
\hline $\mathrm{C}_{6} \mathrm{H}_{12} \mathrm{O}_{3}$ & propyl lactate & & & & {$[616-09-1]$} \\
\hline & $(334-442)$ & 52.1 & $(349)$ & $\mathrm{A}$ & {$[87 / 5]$} \\
\hline $\mathrm{C}_{6} \mathrm{H}_{12} \mathrm{O}_{3}$ & ethoxymethyl propionate & & & & [54078-53-4] \\
\hline & & $49.9 \pm 0.1$ & $(298)$ & $\mathrm{C}$ & {$[74 / 37]$} \\
\hline $\mathrm{C}_{6} \mathrm{H}_{12} \mathrm{O}_{4}$ & ( $d l$ ) glycerol 1-propionate & & & & {$[624-47-5]$} \\
\hline & $(388-456)$ & 75.8 & $(403)$ & $\mathrm{A}$ & {$[87 / 5][72 / 20]$} \\
\hline $\mathrm{C}_{6} \mathrm{H}_{12} \mathrm{O}_{6}$ & myo-inositol & & & & \\
\hline & $(497-524)$ & $119.0 \pm 1.4$ & (519) & $\mathrm{TE}$ & {$[90 / 16]$} \\
\hline $\mathrm{C}_{6} \mathrm{H}_{12} \mathrm{~S}$ & cyclopentyl methyl sulfide & & & & {$[7133-36-0]$} \\
\hline
\end{tabular}


TABLE 6. Enthalpies of vaporization of organic compounds, 1880-2002-Continued

\begin{tabular}{|c|c|c|c|c|c|}
\hline Molecular formula & $\begin{array}{c}\text { Compound } \\
\text { (Temperature range/K) }\end{array}$ & $\begin{array}{c}\Delta_{\mathrm{vap}} H_{m} \\
\left(\mathrm{~kJ} \mathrm{~mol}^{-1}\right)\end{array}$ & $\begin{array}{l}\text { Mean temperature } \\
\qquad\left(T_{m} / \mathrm{K}\right)\end{array}$ & Method & $\begin{array}{c}\text { CAS registry number } \\
\text { Reference }\end{array}$ \\
\hline \multirow{5}{*}{$\mathrm{C}_{6} \mathrm{H}_{12} \mathrm{~S}$} & & $45.1 \pm 0.1$ & $(298)$ & & {$[72 / 11][66 / 5]$} \\
\hline & $(354-473)$ & 41.7 & $(369)$ & $\mathrm{A}, \mathrm{EB}$ & {$[87 / 5][66 / 5]$} \\
\hline & cis 2,5-dimethyltetrahydrothiophene & & & & {$[5161-13-7]$} \\
\hline & $(311-444)$ & 41.7 & (326) & & {$[99 / 16]$} \\
\hline & $(349-427)$ & 39.7 & (364) & $\mathrm{A}, \mathrm{EB}$ & {$[87 / 5][52 / 9]$} \\
\hline \multirow[t]{2}{*}{$\mathrm{C}_{6} \mathrm{H}_{12} \mathrm{~S}$} & trans 2,5-dimethyltetrahydrothiophene & & & & {$[5161-14-8]$} \\
\hline & $(348-396)$ & 39.3 & (363) & EB & $\begin{array}{c}{[87 / 5][52 / 9]} \\
{[99 / 16]}\end{array}$ \\
\hline \multirow[t]{2}{*}{$\mathrm{C}_{6} \mathrm{H}_{12} \mathrm{~S}$} & 2-ethyltetrahydrothiophene & & & & {$[1551-32-2]$} \\
\hline & $(333-488)$ & 42.6 & $(348)$ & A & $\begin{array}{c}{[87 / 5][72 / 20]} \\
{[99 / 16]}\end{array}$ \\
\hline \multirow[t]{2}{*}{$\mathrm{C}_{6} \mathrm{H}_{12} \mathrm{~S}$} & 3-ethyltetrahydrothiophene & & & & [62184-67-2] \\
\hline & $(343-503)$ & 43.1 & $(358)$ & A & $\begin{array}{c}{[87 / 5][72 / 20]} \\
{[99 / 16]}\end{array}$ \\
\hline \multirow[t]{3}{*}{$\mathrm{C}_{6} \mathrm{H}_{12} \mathrm{~S}$} & 2-methyltetrahydro- $2 H$-thiopyrane & & & & [5161-16-0] \\
\hline & $(317-455)$ & 42.1 & $(332)$ & & {$[99 / 16]$} \\
\hline & $(356-438)$ & 40.2 & $(371)$ & $\mathrm{A}, \mathrm{EB}$ & {$[87 / 5][52 / 9]$} \\
\hline \multirow[t]{3}{*}{$\mathrm{C}_{6} \mathrm{H}_{12} \mathrm{~S}$} & 3-methyltetrahydro- $2 \mathrm{H}$-thiopyrane & & & & {$[5258-50-4]$} \\
\hline & $(321-460)$ & 42.5 & $(336)$ & & [99/16] \\
\hline & $(361-435)$ & 40.7 & $(376)$ & $\mathrm{A}, \mathrm{EB}$ & {$[87 / 5][52 / 9]$} \\
\hline \multirow{3}{*}{$\mathrm{C}_{6} \mathrm{H}_{12} \mathrm{~S}$} & 4-methyltetrahydro- $2 \mathrm{H}$-thiopyrane & & & & {$[5161-17-1]$} \\
\hline & $(321-461)$ & 42.8 & $(336)$ & & {$[99 / 16]$} \\
\hline & $(361-441)$ & 40.8 & $(376)$ & $\mathrm{A}, \mathrm{EB}$ & {$[87 / 5][52 / 9]$} \\
\hline \multirow[t]{2}{*}{$\mathrm{C}_{6} \mathrm{H}_{13} \mathrm{Br}$} & 2-bromo-3,3-dimethylbutane & & & & [26356-06-9] \\
\hline & $(315-449)$ & 39.5 & $(330)$ & A & {$[87 / 5][72 / 20]$} \\
\hline \multirow[t]{4}{*}{$\mathrm{C}_{6} \mathrm{H}_{12} \mathrm{~S}$} & cyclohexanethiol & & & & {$[1569-69-3]$} \\
\hline & & 44.9 & $(298)$ & $\mathrm{C}$ & {$[81 / 8]$} \\
\hline & & $44.6 \pm 0.1$ & $(298)$ & & {$[72 / 11][66 / 5]$} \\
\hline & $(355-476)$ & 41.2 & $(370)$ & $\mathrm{A}, \mathrm{EB}$ & $\begin{array}{c}{[87 / 5][66 / 5]} \\
{[99 / 16]}\end{array}$ \\
\hline \multirow{5}{*}{$\mathrm{C}_{6} \mathrm{H}_{13} \mathrm{Br}$} & 1-bromohexane & & & & {$[111-25-1]$} \\
\hline & $(323-363)$ & 45.5 & (298) & $\mathrm{CGC}$ & {$[95 / 21]$} \\
\hline & & $46.1 \pm 0.1$ & (298) & $\mathrm{C}$ & {$[68 / 1]$} \\
\hline & & $45.6 \pm 0.1$ & $(298)$ & $\mathrm{C}$ & {$[66 / 2]$} \\
\hline & $(333-456)$ & 43.2 & $(348)$ & A, EST & $\begin{array}{c}{[87 / 5][61 / 13]} \\
{[72 / 20]}\end{array}$ \\
\hline \multirow[t]{2}{*}{$\mathrm{C}_{6} \mathrm{H}_{13} \mathrm{Br}$} & $(d l)$ 2-bromohexane & & & & [3377-86-4] \\
\hline & $(303-416)$ & 43.8 & $(318)$ & A & {$[87 / 5]$} \\
\hline \multirow[t]{2}{*}{$\mathrm{C}_{6} \mathrm{H}_{13} \mathrm{Br}$} & 2-bromo-4-methylpentane & & & & [30310-22-6] \\
\hline & $(315-448)$ & 29.3 & $(330)$ & A & {$[87 / 5][72 / 20]$} \\
\hline \multirow[t]{10}{*}{$\mathrm{C}_{6} \mathrm{H}_{13} \mathrm{Cl}$} & 1-chlorohexane & & & & {$[544-10-5]$} \\
\hline & $(290-410)$ & 42.0 & (298) & & {$[84 / 9][91 / 2]$} \\
\hline & & $42.8 \pm 0.1$ & (298) & $\mathrm{C}$ & {$[81 / 4]$} \\
\hline & & $40.5 \pm 0.1$ & (328) & $\mathrm{C}$ & {$[81 / 4]$} \\
\hline & & $40.0 \pm 0.1$ & (343) & $\mathrm{C}$ & {$[81 / 4]$} \\
\hline & & $39.0 \pm 0.1$ & (358) & $\mathrm{C}$ & {$[81 / 4]$} \\
\hline & & $38.4 \pm 0.1$ & (368) & $\mathrm{C}$ & {$[81 / 4]$} \\
\hline & $(288-409)$ & 43.5 & $(303)$ & A, DTA & {$[87 / 5][69 / 5]$} \\
\hline & & & & & {$[72 / 20]$} \\
\hline & & $42.8 \pm 0.1$ & $(298)$ & $\mathrm{C}$ & {$[68 / 1]$} \\
\hline \multirow[t]{2}{*}{$\mathrm{C}_{6} \mathrm{H}_{13} \mathrm{Cl}$} & $(d l)$ 2-chlorohexane & & & & [638-28-8] \\
\hline & $(300-399)$ & 40.9 & $(315)$ & A & {$[87 / 5]$} \\
\hline \multirow[t]{2}{*}{$\mathrm{C}_{6} \mathrm{H}_{13} \mathrm{Cl}$} & 2-chloro-2,3-dimethylbutane & & & & {$[594-57-0]$} \\
\hline & $(301-426)$ & 38.0 & $(316)$ & $\mathrm{A}$ & {$[87 / 5][72 / 20]$} \\
\hline \multirow[t]{2}{*}{$\mathrm{C}_{6} \mathrm{H}_{13} \mathrm{Cl}$} & ( $d l$ ) 2-chloro-3,3-dimethylbutane & & & & {$[5750-00-5]$} \\
\hline & $(300-425)$ & 38.0 & $(315)$ & A & {$[87 / 5][72 / 20]$} \\
\hline \multirow[t]{4}{*}{$\mathrm{C}_{6} \mathrm{H}_{13} \mathrm{ClO}_{2} \mathrm{~S}$} & 1-hexanesulfonyl chloride & & & & [14532-24-2] \\
\hline & $(273-304)$ & 60.7 & $(288)$ & & {$[99 / 16]$} \\
\hline & $(303-400)$ & 61.7 & (318) & & [99/16] \\
\hline & $(400-507)$ & 57.2 & $(415)$ & & [99/16] \\
\hline $\mathrm{C}_{6} \mathrm{H}_{13} \mathrm{Cl}_{2} \mathrm{~N}$ & N-ethyl-bis(2-chloroethyl)amine & & & & [13426-57-8] \\
\hline & $(273-333)$ & 54.9 & $(288)$ & A, GS & [87/5][48/13] \\
\hline $\mathrm{C}_{6} \mathrm{H}_{13} \mathrm{~F}$ & 1-fluorohexane & & & & $\begin{array}{c}{[72 / 20]} \\
{[373-14-8]}\end{array}$ \\
\hline
\end{tabular}


TABLE 6. Enthalpies of vaporization of organic compounds, 1880-2002-Continued

\begin{tabular}{|c|c|c|c|c|c|}
\hline Molecular formula & $\begin{array}{c}\text { Compound } \\
\text { (Temperature range/K) }\end{array}$ & $\begin{array}{c}\Delta_{\mathrm{vap}} H_{m} \\
\left(\mathrm{~kJ} \mathrm{~mol}^{-1}\right)\end{array}$ & $\begin{array}{l}\text { Mean temperature } \\
\qquad\left(T_{m} / \mathrm{K}\right)\end{array}$ & Method & $\begin{array}{c}\text { CAS registry number } \\
\text { Reference }\end{array}$ \\
\hline & $(273-388)$ & 36.9 & $(288)$ & A, EST & $\begin{array}{c}{[87 / 5][61 / 13]} \\
{[72 / 20]}\end{array}$ \\
\hline \multirow[t]{2}{*}{$\mathrm{C}_{6} \mathrm{H}_{13} \mathrm{~F}$} & 3-fluorohexane & & & & {$[52688-75-2]$} \\
\hline & $(281-393)$ & 36.8 & $(296)$ & $\mathrm{A}$ & {$[87 / 5][72 / 20]$} \\
\hline \multirow[t]{3}{*}{$\mathrm{C}_{6} \mathrm{H}_{13} \mathrm{I}$} & 1-iodohexane & & & & [638-45-9] \\
\hline & & $49.8 \pm 0.1$ & (298) & $\mathrm{C}$ & {$[68 / 1]$} \\
\hline & $(331-485)$ & 46.2 & $(346)$ & A, EST & $\begin{array}{c}{[87 / 5][61 / 13]} \\
{[72 / 20]}\end{array}$ \\
\hline \multirow[t]{8}{*}{$\mathrm{C}_{6} \mathrm{H}_{13} \mathrm{~N}$} & cyclohexylamine & & & & [108-91-8] \\
\hline & $(363-407)$ & 40.6 & $(378)$ & & {$[87 / 5]$} \\
\hline & & $42.7 \pm 0.1$ & (313) & $\mathrm{C}$ & {$[79 / 9]$} \\
\hline & & $40.7 \pm 0.1$ & (343) & $\mathrm{C}$ & [79/9] \\
\hline & & $39.6 \pm 0.1$ & (358) & $\mathrm{C}$ & {$[79 / 9]$} \\
\hline & & $42.8 \pm 0.1$ & (298) & $\mathrm{C}$ & {$[75 / 9]$} \\
\hline & $(333-408)$ & 40.8 & $(348)$ & A & {$[87 / 5][72 / 20]$} \\
\hline & $(334-401)$ & 40.8 & (349) & & {$[60 / 17][84 / 9]$} \\
\hline \multirow[t]{3}{*}{$\mathrm{C}_{6} \mathrm{H}_{13} \mathrm{~N}$} & hexahydro- $1 H$-azepine & & & & [111-49-9] \\
\hline & $(348-423)$ & 37.7 & (363) & A & {$[87 / 5]$} \\
\hline & $(312-411)$ & 40.4 & $(327)$ & $\mathrm{A}$ & {$[87 / 5][72 / 20]$} \\
\hline \multirow[t]{2}{*}{$\mathrm{C}_{6} \mathrm{H}_{13} \mathrm{~N}$} & ( $d l$ ) 2-methylpiperidine & & & & [109-05-7] \\
\hline & $(323-431)$ & 38.2 & $(338)$ & EB, IPM & {$[87 / 5][68 / 4]$} \\
\hline \multirow[t]{3}{*}{$\mathrm{C}_{6} \mathrm{H}_{13} \mathrm{NO}$} & N-ethylmorpholine & & & & {$[100-74-3]$} \\
\hline & $(274-313)$ & $42.3 \pm 0.3$ & $(294)$ & GS & {$[98 / 13]$} \\
\hline & $(274-313)$ & $42.1 \pm 0.3$ & (298) & GS & {$[98 / 13]$} \\
\hline \multirow[t]{3}{*}{$\mathrm{C}_{6} \mathrm{H}_{13} \mathrm{~N}$} & $\mathrm{~N}$-methylpiperidine & & & & {$[626-67-5]$} \\
\hline & $(273-380)$ & 37.3 & $(288)$ & A & {$[87 / 5]$} \\
\hline & & $36.7 \pm 0.1$ & (298) & & {$[79 / 14][98 / 18]$} \\
\hline \multirow[t]{2}{*}{$\mathrm{C}_{6} \mathrm{H}_{13} \mathrm{NO}$} & $\mathrm{N}, \mathrm{N}$-diethylacetamide & & & & {$[127-19-5]$} \\
\hline & & 54.1 & $(298)$ & $\mathrm{A}$ & {$[85 / 7][85 / 6]$} \\
\hline \multirow[t]{2}{*}{$\mathrm{C}_{6} \mathrm{H}_{13} \mathrm{NO}$} & N-butylacetamide & & & & {$[1119-49-9]$} \\
\hline & & $75.0 \pm 0.3$ & $(298)$ & $\mathrm{C}$ & {$[84 / 6]$} \\
\hline \multirow[t]{3}{*}{$\mathrm{C}_{6} \mathrm{H}_{13} \mathrm{NO}$} & $\mathrm{N}, \mathrm{N}$-dimethyl butyramide & & & & {$[760-79-2]$} \\
\hline & $(251-432)$ & 50.8 & $(366)$ & A & {$[87 / 5]$} \\
\hline & & 55.2 & & & {$[77 / 29]$} \\
\hline \multirow[t]{2}{*}{$\mathrm{C}_{6} \mathrm{H}_{13} \mathrm{NO}_{2}$} & methyl 2-(N,N-dimethylam & noate & & & \\
\hline & $(278-306)$ & $46.1 \pm 1.1$ & $(290)$ & GS & {$[92 / 13]$} \\
\hline \multirow[t]{2}{*}{$\mathrm{C}_{6} \mathrm{H}_{13} \mathrm{NO}_{2}$} & ethyl 2-(N,N-dimethylamin & & & & \\
\hline & $(278-308)$ & $47.6 \pm 0.8$ & (293) & GS & {$[92 / 13]$} \\
\hline $\mathrm{C}_{6} \mathrm{H}_{13} \mathrm{NO}_{2}$ & $\begin{array}{l}\text { N-isopropyl lactamide } \\
(369-407)\end{array}$ & 69.9 & $(384)$ & $\mathrm{A}$ & {$[87 / 5]$} \\
\hline $\mathrm{C}_{6} \mathrm{H}_{13} \mathrm{NO}_{2}$ & $\begin{array}{l}\text { N-propyl lactamide } \\
(373-423)\end{array}$ & 74.0 & $(388)$ & A & {$[87 / 5]$} \\
\hline \multirow{22}{*}{$\mathrm{C}_{6} \mathrm{H}_{14}$} & hexane & & & & [110-54-3] \\
\hline & & $31.5 \pm 0.1$ & (298) & $\mathrm{C}$ & {$[96 / 18]$} \\
\hline & & 31.5 & (298) & & {$[94 / 12]$} \\
\hline & $(283-323)$ & 32.1 & $(298)$ & & {$[92 / 9]$} \\
\hline & & $31.3 \pm 0.3$ & & GC & {$[89 / 16]$} \\
\hline & $(238-298)$ & 34.9 & $(253)$ & A & {$[87 / 5]$} \\
\hline & $(189-259)$ & 35.7 & (244) & $\mathrm{A}$ & {$[87 / 5]$} \\
\hline & $(298-343)$ & 31.5 & (313) & A & {$[87 / 5]$} \\
\hline & $(341-377)$ & 30.1 & (356) & A & {$[87 / 5]$} \\
\hline & $(374-451)$ & 29.3 & (389) & A & [87/5] \\
\hline & $(445-508)$ & 29.4 & (460) & A & {$[87 / 5]$} \\
\hline & & 26.6 & (373) & $\mathrm{C}$ & [85/17] \\
\hline & & 22.5 & (423) & $\mathrm{C}$ & [85/17] \\
\hline & & 15.7 & (473) & $\mathrm{C}$ & [85/17] \\
\hline & & 8.9 & (498) & $\mathrm{C}$ & [85/17] \\
\hline & & 31.6 & (298) & & {$[\mathrm{U} / 1][85 / 6]$} \\
\hline & $(298-338)$ & 30.9 & (313) & & {$[84 / 15]$} \\
\hline & & $31.6 \pm 0.1$ & (298) & $\mathrm{C}$ & [79/13] \\
\hline & & $30.7 \pm 0.1$ & (313) & $\mathrm{C}$ & [79/13] \\
\hline & & $29.5 \pm 0.1$ & (333) & $\mathrm{C}$ & [79/13] \\
\hline & & $28.2 \pm 0.1$ & (353) & $\mathrm{C}$ & [79/13] \\
\hline & $(300-321)$ & 31.6 & $(310)$ & & {$[74 / 4][84 / 9]$} \\
\hline
\end{tabular}


TABLE 6. Enthalpies of vaporization of organic compounds, 1880-2002-Continued

\begin{tabular}{|c|c|c|c|c|c|}
\hline Molecular formula & $\begin{array}{c}\text { Compound } \\
\text { (Temperature range/K) }\end{array}$ & $\begin{array}{c}\Delta_{\mathrm{vap}} H_{m} \\
\left(\mathrm{~kJ} \mathrm{~mol}^{-1}\right)\end{array}$ & $\begin{array}{c}\text { Mean temperature } \\
\qquad\left(T_{m} / \mathrm{K}\right)\end{array}$ & Method & $\begin{array}{c}\text { CAS registry number } \\
\text { Reference }\end{array}$ \\
\hline & $(178-265)$ & 32.5 & $(250)$ & & {$[73 / 11]$} \\
\hline & & 31.55 & $(298)$ & & {$[71 / 28]$} \\
\hline & & $30.9 \pm 0.1$ & (309) & $\mathrm{C}$ & [47/12] \\
\hline & & $29.8 \pm 0.1$ & $(328)$ & $\mathrm{C}$ & {$[47 / 12]$} \\
\hline & & $31.5 \pm 0.1$ & (298) & $\mathrm{C}$ & {$[47 / 7]$} \\
\hline & $(286-343)$ & 32.0 & $(301)$ & A, MM & {$[87 / 5][45 / 2]$} \\
\hline & & $31.0 \pm 0.2$ & (298) & C & {$[43 / 3]$} \\
\hline & & $30.5 \pm 0.2$ & (313) & $\mathrm{C}$ & {$[43 / 3]$} \\
\hline & & $29.0 \pm 0.2$ & (333) & $\mathrm{C}$ & {$[43 / 3]$} \\
\hline & & $28.2 \pm 0.2$ & $(353)$ & $\mathrm{C}$ & {$[43 / 3]$} \\
\hline \multirow[t]{14}{*}{$\mathrm{C}_{6} \mathrm{H}_{14}$} & 2-methylpentane & & & & {$[107-83-5]$} \\
\hline & $(301-333)$ & 30.0 & $(316)$ & & {$[02 / 5]$} \\
\hline & $(310-359)$ & 29.7 & $(325)$ & & [98/9] \\
\hline & $(293-335)$ & 30.5 & $(308)$ & A & {$[87 / 5]$} \\
\hline & & 29.9 & (298) & & {$[71 / 28]$} \\
\hline & & $29.9 \pm 0.1$ & (298) & $\mathrm{C}$ & {$[49 / 8]$} \\
\hline & & $28.7 \pm 0.1$ & $(318)$ & $\mathrm{C}$ & {$[49 / 8]$} \\
\hline & & $27.8 \pm 0.1$ & $(333)$ & $\mathrm{C}$ & {$[49 / 8]$} \\
\hline & & $29.9 \pm 0.1$ & $(298)$ & $\mathrm{C}$ & {$[47 / 7]$} \\
\hline & $(286-334)$ & 30.4 & $(301)$ & $\mathrm{MM}$ & {$[45 / 2]$} \\
\hline & & $29.8 \pm 0.2$ & $(293)$ & $\mathrm{C}$ & {$[43 / 3]$} \\
\hline & & $29.0 \pm 0.2$ & (313) & $\mathrm{C}$ & {$[43 / 3]$} \\
\hline & & $27.6 \pm 0.2$ & $(333)$ & $\mathrm{C}$ & {$[43 / 3]$} \\
\hline & & $26.9 \pm 0.2$ & (353) & $\mathrm{C}$ & {$[43 / 3]$} \\
\hline \multirow[t]{13}{*}{$\mathrm{C}_{6} \mathrm{H}_{14}$} & 3-methylpentane & & & & {$[96-14-0]$} \\
\hline & $(316-361)$ & 29.9 & $(331)$ & & [99/28] \\
\hline & $(293-338)$ & 30.5 & $(308)$ & A & {$[87 / 5]$} \\
\hline & & $30.3 \pm 0.1$ & $(298)$ & $\mathrm{C}$ & {$[79 / 13]$} \\
\hline & & $29.5 \pm 0.1$ & (313) & $\mathrm{C}$ & {$[79 / 13]$} \\
\hline & & $28.3 \pm 0.1$ & $(333)$ & $\mathrm{C}$ & {$[79 / 13]$} \\
\hline & & $27.0 \pm 0.1$ & $(353)$ & $\mathrm{C}$ & {$[79 / 13]$} \\
\hline & & 30.3 & $(298)$ & & {$[71 / 28]$} \\
\hline & & $30.0 \pm 0.1$ & $(303)$ & $\mathrm{C}$ & {$[49 / 8]$} \\
\hline & & $28.8 \pm 0.1$ & $(324)$ & $\mathrm{C}$ & {$[49 / 8]$} \\
\hline & & $28.1 \pm 0.1$ & $(336)$ & $\mathrm{C}$ & {$[49 / 8]$} \\
\hline & & $30.3 \pm 0.1$ & $(298)$ & $\mathrm{C}$ & {$[47 / 7]$} \\
\hline & $(288-337)$ & 30.2 & $(303)$ & MM & {$[45 / 2]$} \\
\hline \multirow[t]{11}{*}{$\mathrm{C}_{6} \mathrm{H}_{14}$} & 2,3-dimethylbutane & & & & {$[79-29-8]$} \\
\hline & & 29.1 & $(298)$ & & {$[71 / 28]$} \\
\hline & & $29.2 \pm 0.1$ & $(296)$ & $\mathrm{C}$ & {$[49 / 8]$} \\
\hline & & $28.9 \pm 0.1$ & $(303)$ & $\mathrm{C}$ & {$[49 / 8]$} \\
\hline & & $28.3 \pm 0.1$ & $(313)$ & $\mathrm{C}$ & {$[49 / 8]$} \\
\hline & & $27.3 \pm 0.1$ & $(331)$ & $\mathrm{C}$ & {$[49 / 8]$} \\
\hline & $(287-332)$ & 29.6 & $(302)$ & $\mathrm{MM}$ & {$[45 / 2]$} \\
\hline & & $29.2 \pm 0.1$ & $(293)$ & $\mathrm{C}$ & {$[43 / 3]$} \\
\hline & & $28.2 \pm 0.1$ & (313) & $\mathrm{C}$ & {$[43 / 3]$} \\
\hline & & $27.0 \pm 0.1$ & $(333)$ & $\mathrm{C}$ & {$[43 / 3]$} \\
\hline & & $26.1 \pm 0.1$ & $(353)$ & $\mathrm{C}$ & {$[43 / 3]$} \\
\hline \multirow[t]{7}{*}{$\mathrm{C}_{6} \mathrm{H}_{14}$} & 2,2-dimethylbutane & & & & {$[75-83-2]$} \\
\hline & & 27.7 & $(298)$ & & {$[71 / 28]$} \\
\hline & $(273-318)$ & 28.7 & $(288)$ & & {$[49 / 2][84 / 9]$} \\
\hline & & $27.8 \pm 0.1$ & $(296)$ & $\mathrm{C}$ & {$[47 / 12]$} \\
\hline & & $26.3 \pm 0.1$ & $(323)$ & $\mathrm{C}$ & {$[47 / 12]$} \\
\hline & $(211-289)$ & 29.2 & $(274)$ & & {$[46 / 2]$} \\
\hline & $(288-323)$ & 28.3 & $(303)$ & MM & {$[45 / 2]$} \\
\hline \multirow[t]{2}{*}{$\mathrm{C}_{6} \mathrm{H}_{14} \mathrm{FO}_{3} \mathrm{P}$} & fluorophosphoric acid, dii & ster & & & {$[55-91-4]$} \\
\hline & $(273-348)$ & 29.4 & $(288)$ & A & {$[87 / 5]$} \\
\hline \multirow[t]{3}{*}{$\mathrm{C}_{6} \mathrm{H}_{14} \mathrm{NO}$} & N-(hydroxyethyl)piperazi & & & & \\
\hline & $(308-343)$ & $77.3 \pm 0.7$ & $(326)$ & GS & {$[02 / 28]$} \\
\hline & $(308-343)$ & $78.8 \pm 0.7$ & $(298)$ & GS & {$[02 / 28]$} \\
\hline \multirow[t]{4}{*}{$\mathrm{C}_{6} \mathrm{H}_{14} \mathrm{~N}_{2}$} & dipropyldiazene & & & & {$[821-67-0]$} \\
\hline & & $39.9 \pm 0.4$ & $(298)$ & $\mathrm{C}$ & {$[76 / 3]$} \\
\hline & $(295-305)$ & 39.5 & $(300)$ & UV & {$[74 / 32]$} \\
\hline & & 41.1 & & & {$[68 / 20][74 / 32]$} \\
\hline
\end{tabular}


TABLE 6. Enthalpies of vaporization of organic compounds, 1880-2002-Continued

\begin{tabular}{|c|c|c|c|c|c|}
\hline Molecular formula & $\begin{array}{c}\text { Compound } \\
\text { (Temperature range/K) }\end{array}$ & $\begin{array}{c}\Delta_{\mathrm{vap}} H_{m} \\
\left(\mathrm{~kJ} \mathrm{~mol}^{-1}\right)\end{array}$ & $\begin{array}{c}\text { Mean temperature } \\
\left(T_{m} / \mathrm{K}\right)\end{array}$ & Method & $\begin{array}{l}\text { CAS registry number } \\
\text { Reference }\end{array}$ \\
\hline \multirow[t]{5}{*}{$\mathrm{C}_{6} \mathrm{H}_{14} \mathrm{~N}_{2}$} & diisopropyldiazene & & & & {$[3880-49-7]$} \\
\hline & & $35.9 \pm 0.4$ & $(298)$ & $\mathrm{C}$ & {$[76 / 3]$} \\
\hline & $(296-308)$ & 36.1 & $(302)$ & UV & {$[74 / 32]$} \\
\hline & & 37.7 & & I & {$[74 / 32]$} \\
\hline & & 34.9 & & & {$[68 / 28][74 / 32]$} \\
\hline \multirow[t]{2}{*}{$\mathrm{C}_{6} \mathrm{H}_{14} \mathrm{~N}_{2}$} & 1,4-diaminocyclohexane & & & & [3114-70-3] \\
\hline & $(383-473)$ & 48.2 & (398) & A & {$[87 / 5]$} \\
\hline \multirow{4}{*}{$\mathrm{C}_{6} \mathrm{H}_{14} \mathrm{~N}_{2}$} & 1,4-dimethylpiperazine & & & & [106-58-1] \\
\hline & $(270-309)$ & $44.3 \pm 0.3$ & $(289)$ & GS & {$[98 / 13]$} \\
\hline & $(270-309)$ & $43.8 \pm 0.3$ & (298) & GS & [98/13] \\
\hline & $(276-405)$ & 41.6 & $(291)$ & $\mathrm{A}$ & {$[87 / 5]$} \\
\hline \multirow[t]{2}{*}{$\mathrm{C}_{6} \mathrm{H}_{14} \mathrm{~N}_{2}$} & 2,5-dimethylpiperazine & & & & [106-55-8] \\
\hline & $(437-609)$ & 48.4 & $(452)$ & A & {$[87 / 5]$} \\
\hline \multirow[t]{2}{*}{$\mathrm{C}_{6} \mathrm{H}_{14} \mathrm{~N}_{2}$} & propylhydrazone acetone & & & & \\
\hline & $(288-318)$ & 44.0 & $(300)$ & & {$[80 / 20]$} \\
\hline \multirow[t]{2}{*}{$\mathrm{C}_{6} \mathrm{H}_{14} \mathrm{~N}_{2}$} & isopropylhydrazone acetone & & & & \\
\hline & $(288-323)$ & 44.6 & $(303)$ & & {$[80 / 20]$} \\
\hline \multirow[t]{2}{*}{$\mathrm{C}_{6} \mathrm{H}_{14} \mathrm{~N}_{2} \mathrm{O}$} & dipropyldiazene $\mathrm{N}$-oxide & & & & [17697-55-1] \\
\hline & & $51.7 \pm 0.1$ & (298) & $\mathrm{C}$ & {$[81 / 7]$} \\
\hline \multirow[t]{6}{*}{$\mathrm{C}_{6} \mathrm{H}_{14} \mathrm{O}$} & butyl ethyl ether & & & & [62881-9] \\
\hline & & $36.3 \pm 0.1$ & (298) & $\mathrm{C}$ & {$[80 / 3]$} \\
\hline & $(311-365)$ & 36.5 & (298) & & {$[76 / 2]$} \\
\hline & $(311-365)$ & 32.1 & (365) & & {$[76 / 2]$} \\
\hline & $(311-365)$ & 35.2 & $(326)$ & A, EB & {$[87 / 5][76 / 2]$} \\
\hline & & & & & {$[69 / 15][72 / 20]$} \\
\hline \multirow[t]{11}{*}{$\mathrm{C}_{6} \mathrm{H}_{14} \mathrm{O}$} & tert-amyl methyl ether & & & & [994-05-8] \\
\hline & & $35.3 \pm 0.4$ & (298) & & {$[\mathrm{U} / 2][02 / 32]$} \\
\hline & $(309-396)$ & $36.6 \pm 0.1$ & $(320)$ & EB & {$[02 / 15]$} \\
\hline & $(309-396)$ & $34.5 \pm 0.2$ & $(360)$ & EB & {$[02 / 15]$} \\
\hline & $(309-396)$ & $32.1 \pm 0.5$ & $(400)$ & EB & {$[02 / 15]$} \\
\hline & $(314-362)$ & 33.4 & (329) & & {$[98 / 9]$} \\
\hline & $(283-308)$ & $35.7 \pm 1.0$ & (295) & GS & {$[98 / 2]$} \\
\hline & $(283-308)$ & $35.5 \pm 1.0$ & (298) & GS & {$[98 / 2]$} \\
\hline & $(306-359)$ & 33.8 & $(321)$ & EB & {$[94 / 10]$} \\
\hline & & 35.8 & (298) & $\mathrm{C}$ & [91/11] \\
\hline & $(294-359)$ & 34.3 & (309) & $\mathrm{EB}$ & {$[84 / 26]$} \\
\hline \multirow[t]{6}{*}{$\mathrm{C}_{6} \mathrm{H}_{14} \mathrm{O}$} & tert-butyl ethyl ether & & & & [637-92-3] \\
\hline & $(307-346)$ & 32.1 & $(322)$ & & {$[00 / 13]$} \\
\hline & $(306-345)$ & 32.2 & $(321)$ & EB & {$[94 / 10]$} \\
\hline & $(284-346)$ & 33.5 & (299) & $\mathrm{A}$ & {$[87 / 5]$} \\
\hline & $(248-350)$ & 35.3 & (263) & $\mathrm{A}$ & {$[87 / 5]$} \\
\hline & $(340-407)$ & 31.2 & $(355)$ & A & {$[87 / 5]$} \\
\hline \multirow{9}{*}{$\mathrm{C}_{6} \mathrm{H}_{14} \mathrm{O}$} & dipropyl ether & & & & [111-43-3] \\
\hline & $(385-467)$ & 32.2 & $(400)$ & A & {$[87 / 5]$} \\
\hline & $(465-530)$ & 32.4 & $(480)$ & $\mathrm{A}$ & {$[87 / 5]$} \\
\hline & & $35.7 \pm 0.1$ & (298) & $\mathrm{C}$ & {$[80 / 3]$} \\
\hline & $(292-389)$ & 35.6 & (307) & A & {$[87 / 5][76 / 2]$} \\
\hline & & 31.4 & (363) & & {$[76 / 2]$} \\
\hline & $(312-371)$ & 34.6 & (327) & $\mathrm{A}, \mathrm{EB}$ & {$[87 / 5][73 / 12]$} \\
\hline & $(300-362)$ & 35.1 & $(315)$ & EB & {$[69 / 15]$} \\
\hline & $(340-379)$ & 34.5 & $(360)$ & & [68/13] \\
\hline \multirow[t]{11}{*}{$\mathrm{C}_{6} \mathrm{H}_{14} \mathrm{O}$} & diisopropyl ether & & & & [108-20-3] \\
\hline & $(278-323)$ & 33.0 & (293) & & [99/14] \\
\hline & $(307-349)$ & 31.1 & (322) & & [99/10] \\
\hline & $(360-440)$ & 29.9 & (375) & A & {$[87 / 5]$} \\
\hline & $(436-500)$ & 29.5 & (451) & $\mathrm{A}$ & {$[87 / 5]$} \\
\hline & & $32.1 \pm 0.1$ & (298) & $\mathrm{C}$ & {$[80 / 3]$} \\
\hline & $(284-365)$ & 32.6 & (299) & A & {$[87 / 5][76 / 2]$} \\
\hline & $(284-365)$ & 29.2 & (341) & & {$[76 / 2]$} \\
\hline & $(296-342)$ & 32.1 & (311) & A, EB & {$[87 / 5][69 / 15]$} \\
\hline & $(321-350)$ & 30.1 & (336) & & {$[65 / 22][72 / 20]$} \\
\hline & $(273-333)$ & 33.2 & $(288)$ & & {$[49 / 2][84 / 9]$} \\
\hline \multirow[t]{2}{*}{$\mathrm{C}_{6} \mathrm{H}_{14} \mathrm{O}$} & 1-hexanol & & & & {$[111-27-3]$} \\
\hline & $(265-328)$ & 62.0 & (288) & GS & {$[01 / 3]$} \\
\hline
\end{tabular}


TABLE 6. Enthalpies of vaporization of organic compounds, 1880-2002-Continued

\begin{tabular}{|c|c|c|c|c|c|}
\hline Molecular formula & $\begin{array}{c}\text { Compound } \\
\text { (Temperature range/K) }\end{array}$ & $\begin{array}{c}\Delta_{\mathrm{vap}} H_{m} \\
\left(\mathrm{~kJ} \mathrm{~mol}^{-1}\right)\end{array}$ & $\begin{array}{c}\text { Mean temperature } \\
\qquad\left(T_{m} / \mathrm{K}\right)\end{array}$ & Method & $\begin{array}{l}\text { CAS registry number } \\
\text { Reference }\end{array}$ \\
\hline & $(265-328)$ & 61.1 & (298) & GS & {$[01 / 3]$} \\
\hline & $(373-423)$ & 61.5 & (298) & CGC & {$[95 / 21]$} \\
\hline & $(323-373)$ & 61.6 & (298) & CGC & {$[95 / 21]$} \\
\hline & $(253-338)$ & 61.2 & (296) & & {$[92 / 14]$} \\
\hline & $(298-343)$ & 57.7 & (313) & A & {$[87 / 5]$} \\
\hline & $(380-417)$ & 47.9 & (395) & EB & {$[85 / 12]$} \\
\hline & & $58.5 \pm 0.2$ & $(328)$ & $\mathrm{C}$ & {$[85 / 3]$} \\
\hline & & $57.6 \pm 0.2$ & $(343)$ & $\mathrm{C}$ & {$[85 / 3]$} \\
\hline & & $55.2 \pm 0.2$ & (358) & $\mathrm{C}$ & {$[85 / 3]$} \\
\hline & & $53.8 \pm 0.2$ & $(368)$ & $\mathrm{C}$ & {$[85 / 3]$} \\
\hline & $(243-303)$ & 59.1 & $(298)$ & & {$[83 / 14]$} \\
\hline & & $60.8 \pm 0.2$ & (298) & $\mathrm{C}$ & {$[77 / 1]$} \\
\hline & $(308-430)$ & 57.9 & (323) & & {$[73 / 26]$} \\
\hline & $(325-431)$ & 58.5 & $(340)$ & DTA & $\begin{array}{c}{[87 / 5][69 / 5]} \\
{[72 / 20]}\end{array}$ \\
\hline & & $61.6 \pm 0.2$ & (298) & $\mathrm{C}$ & {$[66 / 2]$} \\
\hline & $(334-381)$ & 56.0 & (349) & & {$[61 / 6]$} \\
\hline & $(308-428)$ & U55.8 & $(323)$ & I & {$[38 / 10]$} \\
\hline & $(333-425)$ & 57.9 & $(348)$ & & {$[35 / 6][84 / 9]$} \\
\hline \multirow[t]{13}{*}{$\mathrm{C}_{6} \mathrm{H}_{14} \mathrm{O}$} & $(d l)$ 2-hexanol & & & & {$[626-93-7]$} \\
\hline & $(274-309)$ & $58.3 \pm 0.3$ & (298) & GS & {$[01 / 7]$} \\
\hline & $(224-323)$ & 61.8 & (239) & & [99/11] \\
\hline & $(360-415)$ & 48.7 & $(375)$ & A & {$[87 / 5]$} \\
\hline & & $56.8 \pm 0.2$ & (313) & $\mathrm{C}$ & {$[85 / 1]$} \\
\hline & & $55.0 \pm 0.2$ & $(328)$ & $\mathrm{C}$ & {$[85 / 1]$} \\
\hline & & $53.0 \pm 0.2$ & $(343)$ & $\mathrm{C}$ & {$[85 / 1]$} \\
\hline & & $50.7 \pm 0.2$ & (358) & $\mathrm{C}$ & {$[85 / 1]$} \\
\hline & & $49.2 \pm 0.2$ & $(368)$ & $\mathrm{C}$ & {$[85 / 1]$} \\
\hline & $(337-413)$ & 52.4 & $(352)$ & & {$[84 / 10]$} \\
\hline & $(351-412)$ & 47.8 & $(366)$ & A & {$[87 / 5][75 / 23]$} \\
\hline & $(301-415)$ & 53.1 & (316) & & {$[73 / 26]$} \\
\hline & $(298-413)$ & 49.7 & $(356)$ & I & {$[38 / 10]$} \\
\hline \multirow[t]{8}{*}{$\mathrm{C}_{6} \mathrm{H}_{14} \mathrm{O}$} & $(d l)$ 3-hexanol & & & & {$[623-37-0]$} \\
\hline & $(278-311)$ & $58.6 \pm 0.4$ & $(298)$ & GS & {$[01 / 7]$} \\
\hline & $(244-318)$ & U50.7 & (259) & & {$[99 / 11]$} \\
\hline & $(354-410)$ & 46.1 & (369) & A & {$[87 / 5]$} \\
\hline & $(280-320)$ & 57.5 & $(295)$ & A & {$[87 / 5]$} \\
\hline & $(333-409)$ & 51.5 & $(348)$ & & {$[84 / 10]$} \\
\hline & $(280-316)$ & 57.4 & $(295)$ & & {$[75 / 1]$} \\
\hline & $(298-408)$ & 46.4 & $(353)$ & I & {$[38 / 10]$} \\
\hline \multirow[t]{10}{*}{$\mathrm{C}_{6} \mathrm{H}_{14} \mathrm{O}$} & $(d l)$ 2-methyl-1-pentanol & & & & {$[105-30-6]$} \\
\hline & $(275-313)$ & $59.4 \pm 0.3$ & $(298)$ & GS & {$[01 / 7]$} \\
\hline & $(367-423)$ & 49.3 & $(382)$ & A & {$[87 / 5]$} \\
\hline & $(261-294)$ & 64.9 & $(279)$ & $\mathrm{A}$ & {$[87 / 5][79 / 16]$} \\
\hline & & $57.4 \pm 0.2$ & $(328)$ & $\mathrm{C}$ & {$[85 / 1]$} \\
\hline & & $55.7 \pm 0.2$ & $(343)$ & $\mathrm{C}$ & {$[85 / 1]$} \\
\hline & & $53.9 \pm 0.2$ & $(358)$ & $\mathrm{C}$ & {$[85 / 1]$} \\
\hline & & $52.7 \pm 0.2$ & $(368)$ & $\mathrm{C}$ & {$[85 / 1]$} \\
\hline & $(298-423)$ & 54.2 & $(313)$ & & {$[73 / 26]$} \\
\hline & $(298-413)$ & 50.2 & $(356)$ & $\mathrm{I}$ & {$[38 / 10]$} \\
\hline \multirow[t]{5}{*}{$\mathrm{C}_{6} \mathrm{H}_{14} \mathrm{O}$} & $(d l)$ 3-methyl-1-pentanol & & & & {$[589-35-5]$} \\
\hline & $(280-316)$ & $61.7 \pm 0.3$ & $(298)$ & GS & {$[01 / 7]$} \\
\hline & $(328-427)$ & 54.8 & $(343)$ & $\mathrm{A}$ & {$[87 / 5]$} \\
\hline & $(298-427)$ & 59.7 & $(313)$ & & {$[73 / 26]$} \\
\hline & $(298-423)$ & 47.2 & $(360)$ & $\mathrm{I}$ & [40/9] \\
\hline \multirow[t]{5}{*}{$\mathrm{C}_{6} \mathrm{H}_{14} \mathrm{O}$} & 4-methyl-1-pentanol & & & & {$[626-89-1]$} \\
\hline & $(357-427)$ & 53.0 & $(372)$ & A & {$[87 / 5]$} \\
\hline & $(371-427)$ & 51.1 & $(386)$ & A & {$[87 / 5]$} \\
\hline & $(298-427)$ & 63.9 & $(313)$ & & {$[73 / 26]$} \\
\hline & $(298-423)$ & 46.5 & $(360)$ & $\mathrm{I}$ & [40/9] \\
\hline \multirow[t]{4}{*}{$\mathrm{C}_{6} \mathrm{H}_{14} \mathrm{O}$} & 2-methyl-2-pentanol & & & & {$[590-36-3]$} \\
\hline & $(341-396)$ & 44.2 & $(356)$ & A & {$[87 / 5]$} \\
\hline & $(330-397)$ & 48.9 & $(345)$ & A & {$[87 / 5]$} \\
\hline & & $54.7 \pm 0.2$ & $(298)$ & $\mathrm{C}$ & {$[85 / 1]$} \\
\hline
\end{tabular}


TABLE 6. Enthalpies of vaporization of organic compounds, 1880-2002-Continued

\begin{tabular}{|c|c|c|c|c|c|}
\hline Molecular formula & $\begin{array}{c}\text { Compound } \\
\text { (Temperature range/K) }\end{array}$ & $\begin{array}{c}\Delta_{\mathrm{vap}} H_{m} \\
\left(\mathrm{~kJ} \mathrm{~mol}^{-1}\right)\end{array}$ & $\begin{array}{l}\text { Mean temperature } \\
\qquad\left(T_{m} / \mathrm{K}\right)\end{array}$ & Method & $\begin{array}{c}\text { CAS registry number } \\
\text { Reference }\end{array}$ \\
\hline & & $52.8 \pm 0.2$ & $(313)$ & $\mathrm{C}$ & {$[85 / 1]$} \\
\hline & & $50.7 \pm 0.2$ & $(328)$ & $\mathrm{C}$ & {$[85 / 1]$} \\
\hline & & $48.5 \pm 0.2$ & $(343)$ & $\mathrm{C}$ & {$[85 / 1]$} \\
\hline & & $46.1 \pm 0.2$ & (358) & $\mathrm{C}$ & {$[85 / 1]$} \\
\hline & & $44.4 \pm 0.2$ & $(368)$ & $\mathrm{C}$ & {$[85 / 1]$} \\
\hline & $(288-396)$ & 58.3 & (303) & & {$[73 / 26]$} \\
\hline & $(268-394)$ & 49.1 & (283) & & {$[47 / 5]$} \\
\hline & $(288-396)$ & 51.3 & (303) & I & [33/9] \\
\hline \multirow{5}{*}{$\mathrm{C}_{6} \mathrm{H}_{14} \mathrm{O}$} & ( $d l$ ) 3-methyl-2-pentanol & & & & {$[565-60-5]$} \\
\hline & $(275-310)$ & $58.2 \pm 0.3$ & $(298)$ & GS & {$[01 / 7]$} \\
\hline & $(314-409)$ & 54.4 & (329) & A & {$[87 / 5]$} \\
\hline & $(255-295)$ & 60.4 & (280) & $\mathrm{A}$ & {$[87 / 5][79 / 16]$} \\
\hline & $(296-408)$ & 54.8 & $(311)$ & & {$[73 / 26]$} \\
\hline \multirow[t]{6}{*}{$\mathrm{C}_{6} \mathrm{H}_{14} \mathrm{O}$} & ( $d l$ ) 4-methyl-2-pentanol & & & & [108-11-2] \\
\hline & $(274-301)$ & $57.3 \pm 0.3$ & $(298)$ & GS & {$[01 / 7]$} \\
\hline & $(240-295)$ & 59.6 & $(280)$ & A & {$[87 / 5]$} \\
\hline & $(293-406)$ & 49.6 & $(308)$ & & {$[73 / 26]$} \\
\hline & $(353-404)$ & 47.3 & $(368)$ & $\mathrm{A}, \mathrm{EB}$ & {$[87 / 5][70 / 7]$} \\
\hline & $(298-403)$ & 45.6 & $(350)$ & I & {$[38 / 10]$} \\
\hline \multirow[t]{6}{*}{$\mathrm{C}_{6} \mathrm{H}_{14} \mathrm{O}$} & (dl) 2-methyl-3-pentanol & & & & {$[565-67-3]$} \\
\hline & $(275-307)$ & $56.0 \pm 0.5$ & $(298)$ & GS & {$[01 / 7]$} \\
\hline & $(307-401)$ & 52.2 & $(322)$ & A & {$[87 / 5]$} \\
\hline & $(342-400)$ & 45.4 & $(357)$ & $\mathrm{A}$ & {$[87 / 5][75 / 23]$} \\
\hline & $(298-401)$ & 52.0 & (313) & & {$[73 / 26]$} \\
\hline & $(298-399)$ & 44.4 & $(349)$ & I & {$[40 / 8]$} \\
\hline \multirow[t]{6}{*}{$\mathrm{C}_{6} \mathrm{H}_{14} \mathrm{O}$} & 3-methyl-3-pentanol & & & & {$[77-74-7]$} \\
\hline & $(275-301)$ & $55.7 \pm 0.3$ & $(298)$ & GS & {$[01 / 7]$} \\
\hline & $(322-397)$ & 40.1 & $(337)$ & A & {$[87 / 5]$} \\
\hline & $(338-396)$ & 46.4 & (353) & & {$[73 / 26]$} \\
\hline & & $56.7 \pm 0.8$ & (298) & & {$[91 / 8]$} \\
\hline & $(298-393)$ & 42.1 & $(346)$ & I & {$[40 / 8]$} \\
\hline \multirow[t]{4}{*}{$\mathrm{C}_{6} \mathrm{H}_{14} \mathrm{O}$} & 2,2-dimethyl-1-butanol & & & & [1185-33-7] \\
\hline & $(356-415)$ & 47.2 & $(371)$ & $\mathrm{A}$ & {$[87 / 5]$} \\
\hline & $(298-415)$ & 53.7 & (313) & & {$[73 / 26]$} \\
\hline & $(298-408)$ & 52.1 & $(313)$ & I & {$[40 / 7]$} \\
\hline \multirow[t]{3}{*}{$\mathrm{C}_{6} \mathrm{H}_{14} \mathrm{O}$} & ( $d l$ ) 2,3-dimethyl-1-butanol & & & & [19550-30-2] \\
\hline & $(324-431)$ & 51.4 & (339) & A & {$[87 / 5]$} \\
\hline & $(373-422)$ & 49.6 & $(388)$ & & {$[73 / 26]$} \\
\hline \multirow[t]{7}{*}{$\mathrm{C}_{6} \mathrm{H}_{14} \mathrm{O}$} & 3,3-dimethyl-1-butanol & & & & [624-95-3] \\
\hline & $(276-312)$ & $58.0 \pm 0.2$ & $(298)$ & GS & {$[01 / 7]$} \\
\hline & & $58.6 \pm 0.1$ & (328) & $\mathrm{C}$ & {$[96 / 9]$} \\
\hline & & $55.4 \pm 0.1$ & (343) & $\mathrm{C}$ & {$[96 / 9]$} \\
\hline & & $52.4 \pm 0.1$ & (358) & $\mathrm{C}$ & {$[96 / 9]$} \\
\hline & $(319-424)$ & 50.8 & $(334)$ & $\mathrm{A}$ & {$[87 / 5]$} \\
\hline & $(353-417)$ & 49.4 & $(368)$ & & {$[73 / 26]$} \\
\hline \multirow[t]{4}{*}{$\mathrm{C}_{6} \mathrm{H}_{14} \mathrm{O}$} & 2,3-dimethyl-2-butanol & & & & {$[594-60-5]$} \\
\hline & $(303-340)$ & $54.0 \pm 0.8$ & $(298)$ & & {$[91 / 8]$} \\
\hline & $(299-400)$ & 48.8 & (314) & $\mathrm{A}$ & {$[87 / 5]$} \\
\hline & $(298-393)$ & 49.1 & (313) & & {$[73 / 26]$} \\
\hline \multirow[t]{4}{*}{$\mathrm{C}_{6} \mathrm{H}_{14} \mathrm{O}$} & (dl) 3,3-dimethyl-2-butanol & & & & {$[464-07-3]$} \\
\hline & $(280-315)$ & $53.8 \pm 0.3$ & $(298)$ & GS & {$[01 / 7]$} \\
\hline & $(302-401)$ & 48.3 & (317) & A & {$[87 / 5]$} \\
\hline & $(338-393)$ & 46.8 & $(353)$ & & {$[73 / 26]$} \\
\hline \multirow[t]{6}{*}{$\mathrm{C}_{6} \mathrm{H}_{14} \mathrm{O}$} & 2-ethyl-1-butanol & & & & {$[97-95-0]$} \\
\hline & $(275-313)$ & $60.3 \pm 0.3$ & $(298)$ & GS & {$[01 / 7]$} \\
\hline & $(321-426)$ & 53.1 & (336) & $\mathrm{A}$ & {$[87 / 5]$} \\
\hline & $(262-295)$ & 65.4 & $(280)$ & $\mathrm{A}$ & {$[87 / 5][79 / 16]$} \\
\hline & $(298-426)$ & 59.6 & (313) & & {$[73 / 26]$} \\
\hline & $(298-418)$ & $\mathrm{U} 45.5$ & (313) & I & {$[40 / 7]$} \\
\hline \multirow[t]{2}{*}{$\mathrm{C}_{6} \mathrm{H}_{14} \mathrm{OS}$} & 2-methyl-2-propanesulfonic & hyl ester & & & \\
\hline & $(337-343)$ & U14.0 & $(340)$ & $\mathrm{A}$ & {$[87 / 5]$} \\
\hline \multirow[t]{3}{*}{$\mathrm{C}_{6} \mathrm{H}_{14} \mathrm{O}_{2}$} & 2-butoxyethanol & & & & {$[111-76-2]$} \\
\hline & $(363-382)$ & 51.2 & $(373)$ & MM & {$[99 / 15]$} \\
\hline & $(336-443)$ & 49.5 & $(351)$ & A & {$[87 / 5][72 / 20]$} \\
\hline
\end{tabular}


TABLE 6. Enthalpies of vaporization of organic compounds, 1880-2002-Continued

\begin{tabular}{|c|c|c|c|c|c|}
\hline Molecular formula & $\begin{array}{c}\text { Compound } \\
\text { (Temperature range/K) }\end{array}$ & $\begin{array}{c}\Delta_{\text {vap }} H_{m} \\
\left(\mathrm{~kJ} \mathrm{~mol}^{-1}\right)\end{array}$ & $\begin{array}{l}\text { Mean temperature } \\
\qquad\left(T_{m} / \mathrm{K}\right)\end{array}$ & Method & $\begin{array}{c}\text { CAS registry number } \\
\text { Reference }\end{array}$ \\
\hline & $(336-443)$ & 52.6 & $(351)$ & & {$[57 / 6][84 / 9]$} \\
\hline \multirow{2}{*}{$\mathrm{C}_{6} \mathrm{H}_{14} \mathrm{O}_{2}$} & 1,1-dimethoxybutane & & & & [4461-87-4] \\
\hline & $(304-329)$ & 41.2 & $(317)$ & EB & {$[94 / 16]$} \\
\hline \multirow[t]{5}{*}{$\mathrm{C}_{6} \mathrm{H}_{14} \mathrm{O}_{2}$} & 1,1-diethoxyethane & & & & [105-57-7] \\
\hline & $(275-308)$ & $39.6 \pm 0.3$ & (298) & GS & {$[98 / 21][02 / 32]$} \\
\hline & $(281-384)$ & 41.6 & (296) & A & {$[87 / 5][72 / 20]$} \\
\hline & $(273-343)$ & 39.8 & $(288)$ & & {$[49 / 2][84 / 9]$} \\
\hline & $(239-392)$ & 36.2 & $(255)$ & & {$[47 / 5]$} \\
\hline \multirow{4}{*}{$\mathrm{C}_{6} \mathrm{H}_{14} \mathrm{O}_{2}$} & 1,2-diethoxyethane & & & & [629-14-1] \\
\hline & $(339-382)$ & 39.3 & (361) & & {$[87 / 16]$} \\
\hline & $(239-393)$ & 37.9 & $(254)$ & A & {$[87 / 5]$} \\
\hline & & $43.2 \pm 0.1$ & $(298)$ & $\mathrm{C}$ & {$[70 / 17]$} \\
\hline \multirow[t]{2}{*}{$\mathrm{C}_{6} \mathrm{H}_{14} \mathrm{O}_{2}$} & 1-methoxy-2-propoxyethane & & & & [77078-18-3] \\
\hline & & $43.7 \pm 0.1$ & $(298)$ & $\mathrm{C}$ & {$[70 / 17]$} \\
\hline \multirow[t]{2}{*}{$\mathrm{C}_{6} \mathrm{H}_{14} \mathrm{O}_{2}$} & 2-isobutoxyethanol & & & & {$[4439-24-1]$} \\
\hline & $(344-432)$ & 48.1 & $(359)$ & A & $\begin{array}{c}{[87 / 5][72 / 20]} \\
{[57 / 6][84 / 9]}\end{array}$ \\
\hline \multirow{2}{*}{$\mathrm{C}_{6} \mathrm{H}_{14} \mathrm{O}_{2}$} & 1,6-hexanediol & & & & [629-11-8] \\
\hline & $(355-559)$ & $98.5 \pm 1.8$ & $(298)$ & $\mathrm{EB}, \mathrm{IPM}$ & {$[96 / 5]$} \\
\hline \multirow[t]{2}{*}{$\mathrm{C}_{6} \mathrm{H}_{14} \mathrm{O}_{2}$} & 3-methyl-1,5-pentanediol & & & & {$[4457-71-0]$} \\
\hline & $(402-485)$ & 76.9 & (417) & A & {$[87 / 5]$} \\
\hline \multirow[t]{2}{*}{$\mathrm{C}_{6} \mathrm{H}_{14} \mathrm{O}_{2}$} & ( $d l$ ) 2-methyl-2,4-pentanediol & & & & {$[107-41-5]$} \\
\hline & $(373-473)$ & 58.1 & $(388)$ & A & {$[87 / 5]$} \\
\hline \multirow[t]{2}{*}{$\mathrm{C}_{6} \mathrm{H}_{14} \mathrm{O}_{2}$} & 2,3-dimethyl-2,3-butanediol & & & & [76-09-5] \\
\hline & $(346-448)$ & 59.1 & $(361)$ & A & {$[87 / 5]$} \\
\hline \multirow[t]{3}{*}{$\mathrm{C}_{6} \mathrm{H}_{14} \mathrm{O}_{3}$} & diethylene glycol, dimethyl ether & & & & {$[111-96-6]$} \\
\hline & & $48.0 \pm 0.6$ & $(298)$ & CGC & {$[00 / 9]$} \\
\hline & $(286-433)$ & 47.4 & $(301)$ & A & {$[87 / 5][47 / 5]$} \\
\hline \multirow{2}{*}{$\mathrm{C}_{6} \mathrm{H}_{14} \mathrm{O}_{3}$} & 3,5,7-trioxanonane & & & & {$[5648-29-3]$} \\
\hline & & $44.7 \pm 0.2$ & (298) & $\mathrm{C}$ & {$[69 / 18]$} \\
\hline \multirow[t]{2}{*}{$\mathrm{C}_{6} \mathrm{H}_{14} \mathrm{O}_{3}$} & tert-butyl 2-hydroxyethyl peroxide & & & & {$[15476-85-4]$} \\
\hline & & $66.4 \pm 1.9$ & & & {$[83 / 11]$} \\
\hline \multirow[t]{2}{*}{$\mathrm{C}_{6} \mathrm{H}_{14} \mathrm{O}_{3}$} & diethylene glycol, monoethyl ether & & & & {$[111-90-0]$} \\
\hline & $(318-475)$ & 52.1 & $(333)$ & A & {$[87 / 5][47 / 5]$} \\
\hline \multirow[t]{2}{*}{$\mathrm{C}_{6} \mathrm{H}_{14} \mathrm{O}_{3}$} & dipropylene glycol & & & & {$[25265-71-8]$} \\
\hline & $(423-505)$ & 61.2 & $(438)$ & A & {$[87 / 5]$} \\
\hline $\mathrm{C}_{6} \mathrm{H}_{14} \mathrm{O}_{3}$ & 2-ethyl-2-hydroxymethyl-1,3-propan & ediol & & & {$[77-99-6]$} \\
\hline & $(433-570)$ & 81.4 & $(448)$ & A & {$[87 / 5]$} \\
\hline $\mathrm{C}_{6} \mathrm{H}_{14} \mathrm{O}_{3}$ & 1,2,6-trihydroxyhexane & & & & [106-69-4] \\
\hline & $(393-433)$ & 97.2 & $(408)$ & A & {$[87 / 5]$} \\
\hline $\mathrm{C}_{6} \mathrm{H}_{14} \mathrm{O}_{4}$ & 1,1,2,2-tetramethoxyethane & & & & {$[2517-44-4]$} \\
\hline & $(351-432)$ & 42.9 & $(366)$ & A & {$[87 / 5]$} \\
\hline $\mathrm{C}_{6} \mathrm{H}_{14} \mathrm{O}_{4}$ & triethylene glycol & & & & {$[112-27-6]$} \\
\hline & $(442-562)$ & $72.2 \pm 0.3$ & $(440)$ & EB & {$[02 / 17]$} \\
\hline & $(442-562)$ & $68.5 \pm 0.3$ & $(480)$ & $\mathrm{EB}$ & {$[02 / 17]$} \\
\hline & $(442-562)$ & $64.6 \pm 0.3$ & $(520)$ & $\mathrm{EB}$ & {$[02 / 17]$} \\
\hline & $(442-562)$ & $60.8 \pm 0.5$ & $(560)$ & $\mathrm{EB}$ & {$[02 / 17]$} \\
\hline & $(288-303)$ & 67.7 & $(295)$ & A & {$[87 / 5]$} \\
\hline & $(387-552)$ & 71.5 & $(402)$ & $\mathrm{A}$ & {$[87 / 5][47 / 5]$} \\
\hline $\mathrm{C}_{6} \mathrm{H}_{14} \mathrm{O}_{6}$ & dulcitol & & & & \\
\hline & $(464-496)$ & $133.8 \pm 1.4$ & $(482)$ & $\mathrm{TE}$ & {$[90 / 16]$} \\
\hline $\mathrm{C}_{6} \mathrm{H}_{14} \mathrm{O}_{6}$ & D-mannitiol & & & & \\
\hline & $(458-501)$ & $135.6 \pm 1.1$ & $(479)$ & $\mathrm{TE}$ & {$[90 / 16]$} \\
\hline $\mathrm{C}_{6} \mathrm{H}_{14} \mathrm{O}_{6}$ & D-sorbitol & & & & \\
\hline & $(461-497)$ & $132.4 \pm 2.0$ & $(477)$ & $\mathrm{TE}$ & {$[90 / 16]$} \\
\hline $\mathrm{C}_{6} \mathrm{H}_{14} \mathrm{~S}$ & methyl pentyl sulfide & & & & [1741-83-9] \\
\hline & $(321-349)$ & 44.2 & (336) & & [99/16] \\
\hline & & 45.2 & (298) & & [81/12] \\
\hline & & $44.6 \pm 0.8$ & (298) & GC & {$[64 / 17]$} \\
\hline & $(321-350)$ & 42.6 & (308) & EB & {$[61 / 17]$} \\
\hline $\mathrm{C}_{6} \mathrm{H}_{14} \mathrm{~S}$ & butyl ethyl sulfide & & & & {$[638-46-0]$} \\
\hline & $(314-445)$ & 43.7 & (319) & & [99/16] \\
\hline & & 44.5 & (298) & & {$[81 / 12]$} \\
\hline & & 44.9 & (298) & & {$[71 / 28]$} \\
\hline
\end{tabular}


TABLE 6. Enthalpies of vaporization of organic compounds, 1880-2002-Continued

\begin{tabular}{|c|c|c|c|c|c|}
\hline Molecular formula & $\begin{array}{c}\text { Compound } \\
\text { (Temperature range/K) }\end{array}$ & $\begin{array}{c}\Delta_{\mathrm{vap}} H_{m} \\
\left(\mathrm{~kJ} \mathrm{~mol}^{-1}\right)\end{array}$ & $\begin{array}{l}\text { Mean temperature } \\
\qquad\left(T_{m} / \mathrm{K}\right)\end{array}$ & Method & $\begin{array}{c}\text { CAS registry number } \\
\text { Reference }\end{array}$ \\
\hline \multirow{6}{*}{$\mathrm{C}_{6} \mathrm{H}_{14} \mathrm{~S}$} & & $44.6 \pm 0.8$ & $(298)$ & GC & {$[64 / 17]$} \\
\hline & $(316-348)$ & 43.5 & (333) & EB & {$[62 / 17]$} \\
\hline & $(354-424)$ & 40.7 & $(369)$ & $\mathrm{A}, \mathrm{EB}$ & {$[87 / 5][52 / 9]$} \\
\hline & sec-butyl ethyl sulfide & & & & {$[5008-72-0]$} \\
\hline & $(304-434)$ & 41.2 & (319) & & [99/16] \\
\hline & $(345-409)$ & 39.0 & $(360)$ & $\mathrm{A}, \mathrm{EB}$ & {$[87 / 5][52 / 9]$} \\
\hline \multirow[t]{4}{*}{$\mathrm{C}_{6} \mathrm{H}_{14} \mathrm{~S}$} & tert-butyl ethyl sulfide & & & & [14290-92-7] \\
\hline & $(293-420)$ & 39.2 & $(308)$ & & [99/16] \\
\hline & & 39.3 & (298) & & {$[71 / 28]$} \\
\hline & $(332-400)$ & 37.1 & $(347)$ & $\mathrm{A}, \mathrm{EB}$ & {$[87 / 5][52 / 9]$} \\
\hline \multirow[t]{7}{*}{$\mathrm{C}_{6} \mathrm{H}_{14} \mathrm{~S}$} & diisopropyl sulfide & & & & {$[625-80-9]$} \\
\hline & $(293-420)$ & 39.4 & (308) & & {$[99 / 16]$} \\
\hline & & $39.6 \pm 0.1$ & (298) & & {$[72 / 11][66 / 5]$} \\
\hline & $(324-433)$ & 37.7 & (339) & $\mathrm{A}, \mathrm{EB}$ & {$[87 / 5][66 / 5]$} \\
\hline & & $39.6 \pm 0.8$ & (298) & GC & {$[64 / 17]$} \\
\hline & $(303-328)$ & 38.5 & (318) & $\mathrm{EB}$ & {$[62 / 17]$} \\
\hline & $(330-400)$ & 37.4 & $(345)$ & EB & {$[52 / 9]$} \\
\hline \multirow[t]{7}{*}{$\mathrm{C}_{6} \mathrm{H}_{14} \mathrm{~S}$} & dipropyl sulfide & & & & [111-47-7] \\
\hline & $(313-411)$ & 42.9 & (328) & & {$[99 / 16]$} \\
\hline & & 44.2 & (298) & & {$[81 / 12]$} \\
\hline & & 44.5 & (298) & & {$[71 / 28]$} \\
\hline & & 39.5 & (298) & & {$[71 / 28]$} \\
\hline & & $44.7 \pm 0.8$ & (298) & GC & [64/17] \\
\hline & $(353-427)$ & 40.6 & (368) & $\mathrm{A}, \mathrm{EB}$ & {$[87 / 5][52 / 9]$} \\
\hline \multirow[t]{4}{*}{$\mathrm{C}_{6} \mathrm{H}_{14} \mathrm{~S}$} & isopropyl propyl sulfide & & & & {$[5008-73-1]$} \\
\hline & $(303-432)$ & 41.1 & $(318)$ & & {$[99 / 16]$} \\
\hline & & 41.8 & (298) & & [81/12] \\
\hline & $(343-416)$ & 39.0 & $(358)$ & A, EB & {$[87 / 5][52 / 9]$} \\
\hline \multirow[t]{3}{*}{$\mathrm{C}_{6} \mathrm{H}_{14} \mathrm{~S}$} & ethyl isobutyl sulfide & & & & {$[1613-45-2]$} \\
\hline & $(305-401)$ & 41.3 & $(320)$ & & [99/16] \\
\hline & $(345-414)$ & 39.2 & $(360)$ & $\mathrm{A}, \mathrm{EB}$ & {$[87 / 5][52 / 9]$} \\
\hline \multirow{4}{*}{$\mathrm{C}_{6} \mathrm{H}_{14} \mathrm{~S}$} & 1-hexanethiol & & & & {$[111-31-9]$} \\
\hline & $(320-454)$ & 43.9 & $(335)$ & & [99/16] \\
\hline & & $44.8 \pm 0.2$ & (298) & & {$[66 / 10][66 / 5]$} \\
\hline & $(352-468)$ & 42.4 & $(367)$ & $\mathrm{A}, \mathrm{EB}$ & {$[87 / 5][66 / 5]$} \\
\hline \multirow[t]{3}{*}{$\mathrm{C}_{6} \mathrm{H}_{14} \mathrm{~S}$} & 2-hexanethiol & & & & [1679-06-7] \\
\hline & $(310-440)$ & 42.7 & $(325)$ & & {$[99 / 16]$} \\
\hline & $(328-423)$ & 41.4 & (343) & $\mathrm{A}$ & {$[87 / 5]$} \\
\hline \multirow[t]{5}{*}{$\mathrm{C}_{6} \mathrm{H}_{14} \mathrm{~S}$} & 2,3-dimethyl-2-butanethiol & & & & [1639-01-6] \\
\hline & $(285-318)$ & 39.3 & $(300)$ & & [99/16] \\
\hline & $(318-441)$ & 37.8 & (333) & & [99/16] \\
\hline & & $39.3 \pm 0.1$ & (298) & & {$[72 / 11][66 / 5]$} \\
\hline & $(328-441)$ & 37.4 & (343) & $\mathrm{A}, \mathrm{EB}$ & {$[87 / 5][66 / 5]$} \\
\hline \multirow{3}{*}{$\mathrm{C}_{6} \mathrm{H}_{14} \mathrm{~S}$} & 2-methyl-2-pentanethiol & & & & [1633-97-2] \\
\hline & & $40.0 \pm 0.1$ & $(298)$ & & {$[72 / 11][66 / 5]$} \\
\hline & $(327-439)$ & 38.0 & $(342)$ & $\mathrm{A}, \mathrm{EB}$ & $\begin{array}{c}{[87 / 5][66 / 5]} \\
{[99 / 16]}\end{array}$ \\
\hline \multirow[t]{5}{*}{$\mathrm{C}_{6} \mathrm{H}_{14} \mathrm{~S}_{2}$} & diisopropyl disulfide & & & & {$[4253-89-8]$} \\
\hline & $(383-423)$ & 49.3 & $(298)$ & $\mathrm{CGC}$ & {$[95 / 21]$} \\
\hline & & 39.6 & $(298)$ & & {$[81 / 12]$} \\
\hline & $(377-447)$ & 43.8 & $(392)$ & $\mathrm{A}, \mathrm{EB}$ & {$[87 / 5][52 / 9]$} \\
\hline & & & & & {$[99 / 16]$} \\
\hline \multirow{7}{*}{$\mathrm{C}_{6} \mathrm{H}_{14} \mathrm{~S}_{2}$} & dipropyl disulfide & & & & {$[629-19-6]$} \\
\hline & $(354-499)$ & 47.8 & (369) & & {$[99 / 16]$} \\
\hline & & $53.8 \pm 0.1$ & (298) & $\mathrm{C}$ & {$[85 / 2]$} \\
\hline & & 53.8 & $(298)$ & & {$[81 / 12]$} \\
\hline & $(389-447)$ & 47.0 & (404) & $\mathrm{A}, \mathrm{EB}$ & {$[87 / 5][58 / 8]$} \\
\hline & & & & & {$[66 / 5]$} \\
\hline & $(395-456)$ & 46.6 & $(410)$ & EB & {$[52 / 9]$} \\
\hline \multirow[t]{3}{*}{$\mathrm{C}_{6} \mathrm{H}_{14} \mathrm{~S}_{2}$} & ethyl (1,1-dimethylethyl) disulfide & & & & [4151-69-3] \\
\hline & $(373-461)$ & 43.4 & $(388)$ & $\mathrm{A}, \mathrm{EB}$ & {$[87 / 5][52 / 9]$} \\
\hline & & & & & {$[99 / 16]$} \\
\hline \multirow[t]{2}{*}{$\mathrm{C}_{6} \mathrm{H}_{14} \mathrm{~S}_{2}$} & isopropyl propyl disulfide & & & & {$[33672-51-4]$} \\
\hline & $(383-433)$ & 45.4 & $(398)$ & A & {$[87 / 5][99 / 16]$} \\
\hline
\end{tabular}


TABLE 6. Enthalpies of vaporization of organic compounds, 1880-2002-Continued

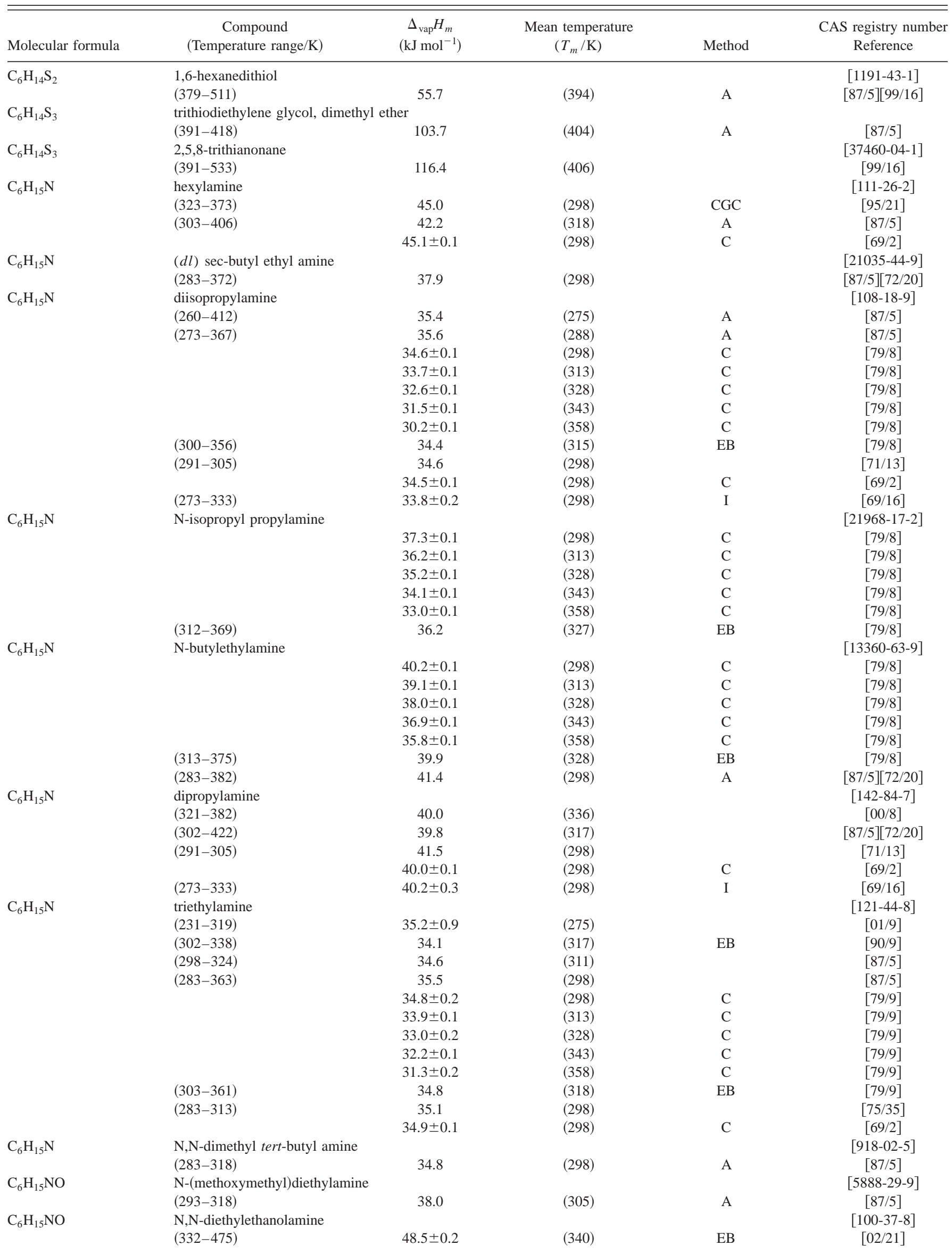


TABLE 6. Enthalpies of vaporization of organic compounds, 1880-2002-Continued

\begin{tabular}{|c|c|c|c|c|c|}
\hline Molecular formula & $\begin{array}{c}\text { Compound } \\
\text { (Temperature range/K) }\end{array}$ & $\begin{array}{c}\Delta_{\mathrm{vap}} H_{m} \\
\left(\mathrm{~kJ} \mathrm{~mol}^{-1}\right)\end{array}$ & $\begin{array}{l}\text { Mean temperature } \\
\qquad\left(T_{m} / \mathrm{K}\right)\end{array}$ & Method & $\begin{array}{c}\text { CAS registry number } \\
\text { Reference }\end{array}$ \\
\hline & $(332-475)$ & $45.0 \pm 0.2$ & $(380)$ & $\mathrm{EB}$ & {$[02 / 21]$} \\
\hline & $(332-475)$ & $41.6 \pm 0.4$ & $(420)$ & $\mathrm{EB}$ & {$[02 / 21]$} \\
\hline & $(332-475)$ & $37.8 \pm 0.7$ & $(460)$ & $\mathrm{EB}$ & {$[02 / 21]$} \\
\hline & $(328-433)$ & 48.5 & $(343)$ & A & {$[87 / 5]$} \\
\hline \multirow[t]{2}{*}{$\mathrm{C}_{6} \mathrm{H}_{15} \mathrm{NO}_{2}$} & diisopropanolamine & & & & [110-97-4] \\
\hline & $(390-521)$ & 68.0 & $(405)$ & A & {$[87 / 5][72 / 20]$} \\
\hline \multirow[t]{2}{*}{$\mathrm{C}_{6} \mathrm{H}_{15} \mathrm{NO}_{2}$} & $2-[2-($ dimethylamino $)$ etho & & & & {$[1704-62-7]$} \\
\hline & $(412-452)$ & 54.4 & $(427)$ & A & {$[87 / 5]$} \\
\hline \multirow[t]{2}{*}{$\mathrm{C}_{6} \mathrm{H}_{15} \mathrm{NO}_{2} \mathrm{~S}$} & $\mathrm{~N}, \mathrm{~N}$-diethyl ethanesulfona & & & & {$[33718-39-7]$} \\
\hline & $(392-526)$ & 55.4 & $(407)$ & A & {$[87 / 5]$} \\
\hline \multirow[t]{2}{*}{$\mathrm{C}_{6} \mathrm{H}_{15} \mathrm{NO}_{3}$} & triethanolamine & & & & {$[102-71-6]$} \\
\hline & $(523-579)$ & 79.3 & $(538)$ & A & $\begin{array}{c}{[87 / 5][59 / 1]} \\
{[84 / 9]}\end{array}$ \\
\hline \multirow[t]{2}{*}{$\mathrm{C}_{6} \mathrm{H}_{15} \mathrm{NS}$} & $\mathrm{N}, \mathrm{N}$-dimethyl-S-tert-butyl & xylamine & & & \\
\hline & $(328-334)$ & 28.3 & $(331)$ & A & {$[87 / 5][99 / 16]$} \\
\hline \multirow[t]{3}{*}{$\mathrm{C}_{6} \mathrm{H}_{15} \mathrm{~N}_{3}$} & 1,3,5-trimethylhexahydro- & & & & \\
\hline & $(284-328)$ & $50.8 \pm 0.8$ & $(306)$ & GS & {$[02 / 28]$} \\
\hline & $(284-328)$ & $51.2 \pm 0.8$ & $(298)$ & GS & {$[02 / 28]$} \\
\hline \multirow[t]{2}{*}{$\mathrm{C}_{6} \mathrm{H}_{15} \mathrm{O}_{2} \mathrm{PS}_{3}$} & O,O-dimethyl-S-[2-(ethylt & dithiophosph & & & [640-15-3] \\
\hline & $(283-394)$ & 76.8 & $(298)$ & A & {$[87 / 5][99 / 16]$} \\
\hline \multirow[t]{2}{*}{$\mathrm{C}_{6} \mathrm{H}_{15} \mathrm{O}_{3} \mathrm{P}$} & phosphonic acid, dipropyl & & & & {$[1809-21-8]$} \\
\hline & $(318-467)$ & 38.1 & $(333)$ & A & {$[87 / 5][72 / 20]$} \\
\hline \multirow[t]{2}{*}{$\mathrm{C}_{6} \mathrm{H}_{15} \mathrm{O}_{3} \mathrm{PS}$} & O,O,O-triethythiophospha & & & & {$[126-68-1]$} \\
\hline & $(305-335)$ & 87.5 & $(320)$ & A & {$[87 / 5][99 / 16]$} \\
\hline \multirow[t]{2}{*}{$\mathrm{C}_{6} \mathrm{H}_{15} \mathrm{O}_{3} \mathrm{PS}$} & $\mathrm{O}, \mathrm{O}, \mathrm{S}$-triethylthiophospha & & & & [1186-09-0] \\
\hline & $(312-352)$ & 76.3 & $(327)$ & A & {$[87 / 5][99 / 16]$} \\
\hline \multirow[t]{2}{*}{$\mathrm{C}_{6} \mathrm{H}_{15} \mathrm{O}_{3} \mathrm{PS}_{2}$} & phosphorothioic acid, O- & o)ethyl]-O,O & 1 ester & & [867-27-6] \\
\hline & $(283-379)$ & 71.0 & (298) & A & {$[87 / 5][99 / 16]$} \\
\hline \multirow[t]{2}{*}{$\mathrm{C}_{6} \mathrm{H}_{15} \mathrm{O}_{3} \mathrm{PS}_{2}$} & phosphorothioic acid, $\mathrm{S}-[2$ & o)ethyl]-O,O & 1 ester & & [919-86-8] \\
\hline & $(283-407)$ & 78.8 & $(298)$ & A & {$[87 / 5][99 / 16]$} \\
\hline \multirow[t]{2}{*}{$\mathrm{C}_{6} \mathrm{H}_{15} \mathrm{O}_{4} \mathrm{P}$} & triethylphosphate & & & & [78-40-0] \\
\hline & $(312-484)$ & 46.3 & $(327)$ & A & {$[87 / 5][47 / 5]$} \\
\hline \multirow[t]{2}{*}{$\mathrm{C}_{6} \mathrm{H}_{15} \mathrm{P}$} & triethylphosphine & & & & {$[554-70-1]$} \\
\hline & $(291-402)$ & 38.3 & $(306)$ & A & {$[87 / 5][72 / 20]$} \\
\hline $\mathrm{C}_{6} \mathrm{H}_{16} \mathrm{FN}_{2} \mathrm{OP}$ & $\mathrm{N}, \mathrm{N}^{\prime}$-diisopropyl phospho & c fluoride & & & [371-86-8] \\
\hline & $(278-398)$ & 58.1 & $(293)$ & A & {$[87 / 5]$} \\
\hline $\mathrm{C}_{6} \mathrm{H}_{16} \mathrm{~N}_{2}$ & 1,6-hexanediamine & & & & {$[124-09-4]$} \\
\hline & $(348-474)$ & 49.3 & $(363)$ & A & {$[87 / 5]$} \\
\hline & $(338-473)$ & 51.3 & $(353)$ & A & {$[87 / 5]$} \\
\hline $\mathrm{C}_{6} \mathrm{H}_{16} \mathrm{~N}_{2} \mathrm{O}_{2}$ & N,N-bis(2-hydroxyethyl)e & mine & & & {$[4439-20-7]$} \\
\hline & $(399-500)$ & $106.4 \pm 6.4$ & $(298)$ & $\mathrm{EB}, \mathrm{IPM}$ & {$[97 / 6][97 / 7]$} \\
\hline $\mathrm{C}_{6} \mathrm{H}_{18} \mathrm{~N}_{3} \mathrm{P}$ & tris(dimethylamino)phospl & & & & {$[1608-26-0]$} \\
\hline & & $41.5 \pm 0.6$ & $(298)$ & STG & {$[95 / 2]$} \\
\hline & $(298-333)$ & 63.2 & $(313)$ & & {$[84 / 15]$} \\
\hline $\mathrm{C}_{6} \mathrm{H}_{18} \mathrm{~N}_{4}$ & triethylenetetramine & & & & [112-24-3] \\
\hline & $(431-550)$ & 59.8 & $(446)$ & A & {$[87 / 5][72 / 20]$} \\
\hline $\mathrm{C}_{7} \mathrm{ClF}_{17} \mathrm{~N}_{2} \mathrm{~S}$ & chloro(trifluoromethyl) bis & roisopropylim & & & \\
\hline & & 38.9 & $(467)$ & I & {$[77 / 15]$} \\
\hline $\mathrm{C}_{7} \mathrm{~F}_{6} \mathrm{O}_{2}$ & carbonofluoridic acid pent & enyl ester & & & [59483-82-8] \\
\hline & & 42.3 & & & {$[76 / 30]$} \\
\hline $\mathrm{C}_{7} \mathrm{~F}_{8}$ & perfluorotoluene & & & & [434-64-0] \\
\hline & $(291-378)$ & 40.0 & $(306)$ & & {$[99 / 16]$} \\
\hline & $(285-376)$ & 40.9 & $(300)$ & $\mathrm{A}$ & {$[87 / 5]$} \\
\hline & $(290-400)$ & 40.4 & (298) & & {$[84 / 9][91 / 2]$} \\
\hline $\mathrm{C}_{7} \mathrm{~F}_{10}$ & 3,3-difluoro-1,2-bis(trifluo & -4-(difluoroet & yclobutene & & [14451-74-2] \\
\hline & $(272-316)$ & 31.5 & $(287)$ & A & {$[87 / 5][99 / 16]$} \\
\hline $\mathrm{C}_{7} \mathrm{~F}_{12} \mathrm{O}_{2} \mathrm{~S}_{4}$ & pentanebis(dithioperoxoic) & afluoro-bis $(\mathrm{t}$ & hethyl) ester & & {$[58936-62-2]$} \\
\hline & & 33.6 & $(370)$ & $\mathrm{I}$ & {$[76 / 18]$} \\
\hline $\mathrm{C}_{7} \mathrm{~F}_{12} \mathrm{O}_{6}$ & hexafluoroperoxyglutaric & ifluoromethy & & & {$[32751-20-5]$} \\
\hline & $(200-390)$ & 47.3 & $(215)$ & A & {$[87 / 5][99 / 16]$} \\
\hline $\mathrm{C}_{7} \mathrm{~F}_{14}$ & perfluoromethylcyclohexa & & & & {$[355-02-2]$} \\
\hline & $(305-414)$ & 33.1 & $(320)$ & & [99/16] \\
\hline & $(413-488)$ & 30.2 & $(428)$ & & {$[99 / 16]$} \\
\hline & $(306-384)$ & 34.0 & $(298)$ & & {$[84 / 9][91 / 2]$} \\
\hline
\end{tabular}


TABLE 6. Enthalpies of vaporization of organic compounds, 1880-2002-Continued

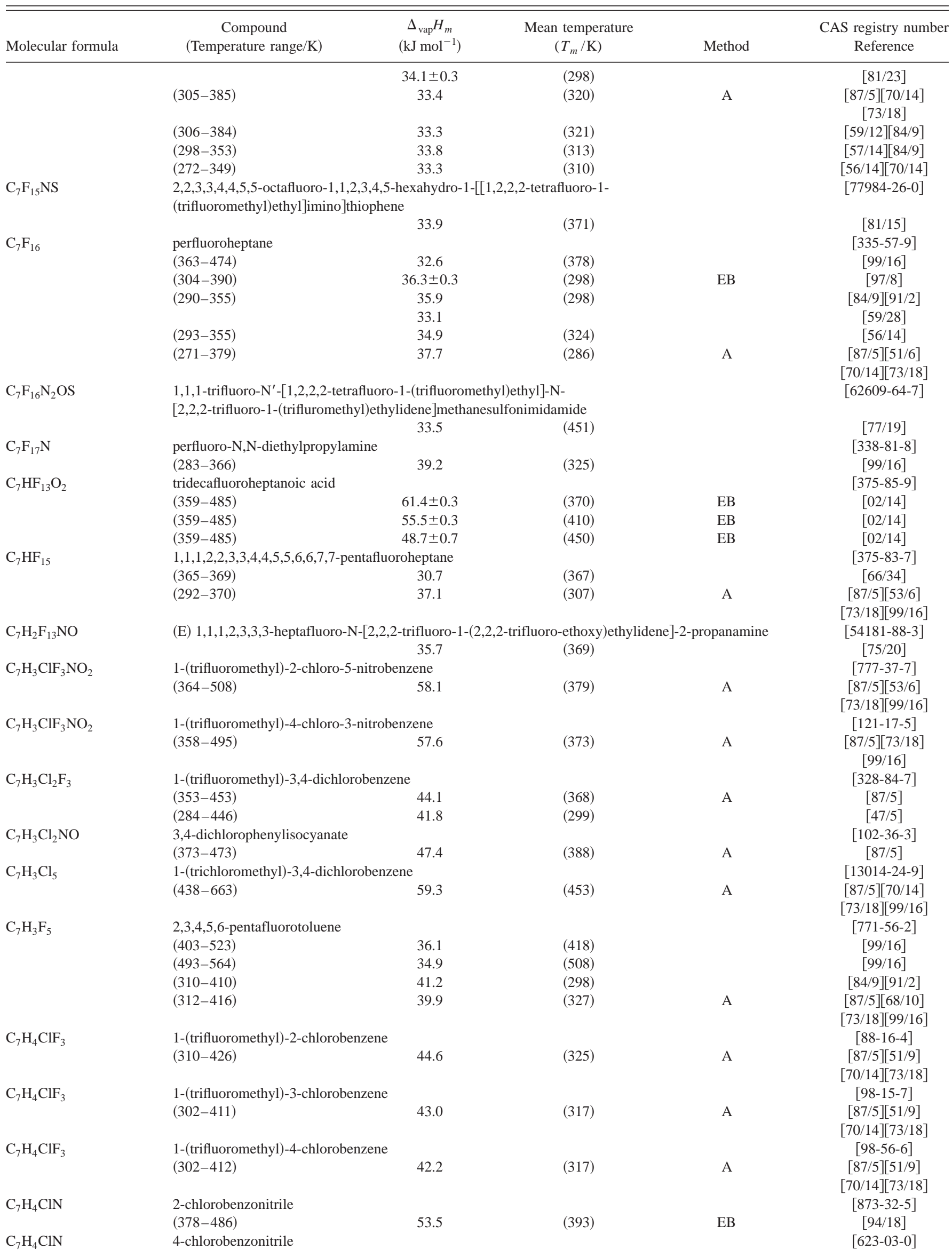


TABLE 6. Enthalpies of vaporization of organic compounds, 1880-2002-Continued

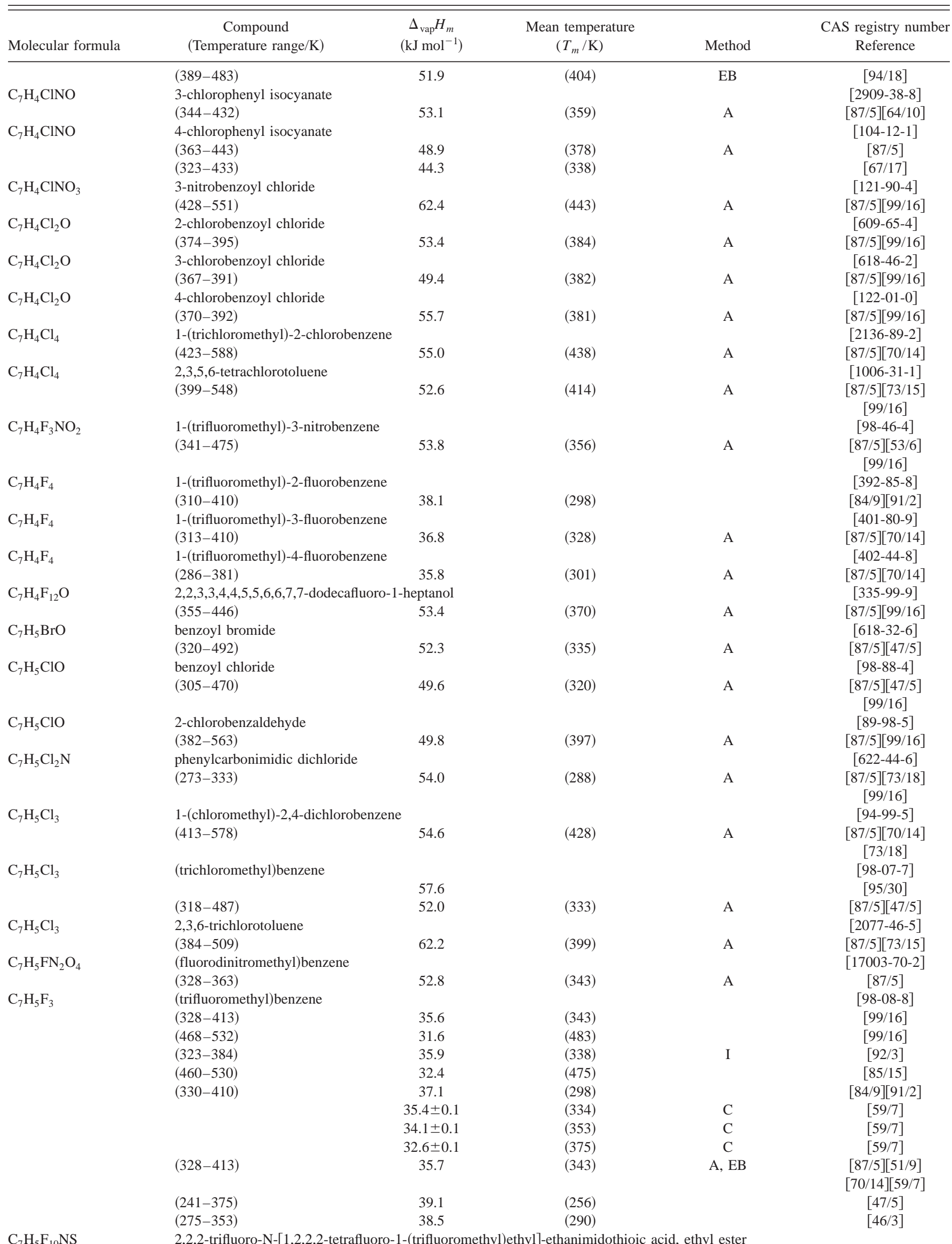


TABLE 6. Enthalpies of vaporization of organic compounds, 1880-2002-Continued

\begin{tabular}{|c|c|c|c|c|c|}
\hline Molecular formula & $\begin{array}{c}\text { Compound } \\
\text { (Temperature range/K) }\end{array}$ & $\begin{array}{c}\Delta_{\mathrm{vap}} H_{m} \\
\left(\mathrm{~kJ} \mathrm{~mol}^{-1}\right)\end{array}$ & $\begin{array}{l}\text { Mean temperature } \\
\qquad\left(T_{m} / \mathrm{K}\right)\end{array}$ & Method & $\begin{array}{c}\text { CAS registry number } \\
\text { Reference }\end{array}$ \\
\hline & & 30.1 & (394) & & {$[75 / 20]$} \\
\hline \multirow[t]{2}{*}{$\mathrm{C}_{7} \mathrm{H}_{5} \mathrm{~F}_{11} \mathrm{O}$} & 1-ethoxy-1,1,2,2,3,3,4,4,5 & afluoropentane & & & [181214-75-5] \\
\hline & $(288-373)$ & 39.0 & $(303)$ & I & {$[02 / 19]$} \\
\hline \multirow[t]{2}{*}{$\mathrm{C}_{7} \mathrm{H}_{5} \mathrm{~F}_{11} \mathrm{O}$} & 1-ethoxy-1,1,2,2,3,4,4,4-o & -(trifluorometh & ane & & [203783-57-7] \\
\hline & $(288-373)$ & 38.3 & $(303)$ & $\mathrm{I}$ & {$[02 / 19]$} \\
\hline \multirow[t]{2}{*}{$\mathrm{C}_{7} \mathrm{H}_{5} \mathrm{~N}$} & benzonitrile & & & & {$[100-47-0]$} \\
\hline & $(301-464)$ & 49.1 & $(316)$ & A & {$[87 / 5][47 / 5]$} \\
\hline \multirow[t]{2}{*}{$\mathrm{C}_{7} \mathrm{H}_{5} \mathrm{~N}$} & phenyl isocyanide & & & & [931-54-4] \\
\hline & $(285-438)$ & 46.2 & $(300)$ & A & {$[87 / 5][47 / 5]$} \\
\hline \multirow[t]{6}{*}{$\mathrm{C}_{7} \mathrm{H}_{5} \mathrm{NO}$} & benzoxazole & & & & {$[273-53-0]$} \\
\hline & & 51.2 & $(320)$ & $\mathrm{EB}$ & {$[92 / 10]$} \\
\hline & & 48.6 & $(360)$ & EB & {$[92 / 10]$} \\
\hline & & 46.1 & $(400)$ & EB & {$[92 / 10]$} \\
\hline & & 43.5 & $(440)$ & $\mathrm{EB}$ & {$[92 / 10]$} \\
\hline & & 40.7 & $(480)$ & $\mathrm{EB}$ & {$[92 / 10]$} \\
\hline \multirow[t]{3}{*}{$\mathrm{C}_{7} \mathrm{H}_{5} \mathrm{NO}$} & phenyl isocyanate & & & & {$[103-71-9]$} \\
\hline & $(329-445)$ & $46.5 \pm 0.3$ & $(298)$ & $\mathrm{EB}$ & [96/4] \\
\hline & $(283-439)$ & 45.0 & $(298)$ & A & {$[87 / 5][47 / 5]$} \\
\hline \multirow[t]{3}{*}{$\mathrm{C}_{7} \mathrm{H}_{5} \mathrm{NO}_{3}$} & 2-nitrobenzaldehyde & & & & {$[552-89-6]$} \\
\hline & $(390-547)$ & 58.7 & $(405)$ & A & {$[87 / 5]$} \\
\hline & $(359-547)$ & 59.5 & $(373)$ & & {$[47 / 5]$} \\
\hline \multirow[t]{2}{*}{$\mathrm{C}_{7} \mathrm{H}_{5} \mathrm{NO}_{3}$} & 3-nitrobenzaldehyde & & & & [99-61-6] \\
\hline & $(401-552)$ & 62.0 & $(416)$ & A & {$[87 / 5]$} \\
\hline \multirow[t]{7}{*}{$\mathrm{C}_{7} \mathrm{H}_{5} \mathrm{NS}$} & benzothiazole & & & & {$[95-16-9]$} \\
\hline & & 58.7 & $(320)$ & EB & {$[92 / 10]$} \\
\hline & & 56.0 & $(360)$ & $\mathrm{EB}$ & {$[92 / 10]$} \\
\hline & & 53.5 & $(400)$ & $\mathrm{EB}$ & {$[92 / 10]$} \\
\hline & & 50.9 & $(440)$ & $\mathrm{EB}$ & {$[92 / 10]$} \\
\hline & & 48.4 & $(480)$ & $\mathrm{EB}$ & {$[92 / 10]$} \\
\hline & & 45.7 & $(520)$ & $\mathrm{EB}$ & {$[92 / 10]$} \\
\hline \multirow[t]{2}{*}{$\mathrm{C}_{7} \mathrm{H}_{5} \mathrm{NS}$} & phenyl isothiocyanate & & & & {$[103-72-0]$} \\
\hline & $(320-492)$ & 52.6 & $(335)$ & A & {$[87 / 5][47 / 5]$} \\
\hline \multirow[t]{3}{*}{$\mathrm{C}_{7} \mathrm{H}_{5} \mathrm{~N}_{3} \mathrm{O}_{6}$} & 2,4,6-trinitrotoluene & & & & {$[118-96-7]$} \\
\hline & $(353-523)$ & 93.7 & $(368)$ & A & {$[87 / 5]$} \\
\hline & & $87.0 \pm 1.9$ & $(298)$ & $\mathrm{ME}$ & {$[78 / 2]$} \\
\hline \multirow[t]{2}{*}{$\mathrm{C}_{7} \mathrm{H}_{5} \mathrm{~N}_{3} \mathrm{O}_{7}$} & 2,4,6-trinitroanisole & & & & [606-35-9] \\
\hline & $(342-363)$ & 91.9 & $(352)$ & A & {$[87 / 5]$} \\
\hline \multirow[t]{2}{*}{$\mathrm{C}_{7} \mathrm{H}_{6} \mathrm{Cl}_{2}$} & (dichloromethyl)benzene & & & & [98-87-3] \\
\hline & $(308-487)$ & 49.5 & $(323)$ & A & {$[87 / 5][47 / 5]$} \\
\hline \multirow[t]{2}{*}{$\mathrm{C}_{7} \mathrm{H}_{6} \mathrm{Cl}_{2}$} & 2,4-dichlorotoluene & & & & [95-73-8] \\
\hline & $(346-475)$ & 50.6 & $(361)$ & A & $\begin{array}{c}{[87 / 5][73 / 15]} \\
{[99 / 16]}\end{array}$ \\
\hline \multirow[t]{2}{*}{$\mathrm{C}_{7} \mathrm{H}_{6} \mathrm{Cl}_{2}$} & 3,4-dichlorotoluene & & & & {$[95-75-0]$} \\
\hline & $(378-543)$ & 49.4 & $(393)$ & A & $\begin{array}{c}{[87 / 5][0 / 14]} \\
{[99 / 16]}\end{array}$ \\
\hline \multirow[t]{2}{*}{$\mathrm{C}_{7} \mathrm{H}_{6} \mathrm{Cl}_{3} \mathrm{NO}_{2}$} & 2,2,4-trichloro-5-(dimethy & cyclopentene-1 & & & {$[77765-42-5]$} \\
\hline & $(453-483)$ & 70.9 & $(468)$ & GC & {$[80 / 25]$} \\
\hline $\mathrm{C}_{7} \mathrm{H}_{6} \mathrm{~F}_{3} \mathrm{~N}$ & 1-(trifluoromethyl)-3-amin & & & & [98-16-8] \\
\hline & $(334-464)$ & 53.1 & $(349)$ & A & $\begin{array}{c}{[87 / 5][53 / 6]} \\
{[99 / 16]}\end{array}$ \\
\hline $\mathrm{C}_{7} \mathrm{H}_{6} \mathrm{~F}_{3} \mathrm{NS}$ & $\begin{array}{l}\mathrm{N} \text {-(trifluoromethyl)thioani } \\
(333-413)\end{array}$ & 47.0 & $(348)$ & A & {$[87 / 5]$} \\
\hline $\mathrm{C}_{7} \mathrm{H}_{6} \mathrm{~F}_{6} \mathrm{O}_{4}$ & dimethyl perfluoroglutarat & & & & \\
\hline & & 52.3 & $(298)$ & EB & {$[76 / 7]$} \\
\hline $\mathrm{C}_{7} \mathrm{H}_{6} \mathrm{~N}_{2} \mathrm{O}_{4}$ & 2,4-dinitrotoluene & & & & [121-14-2] \\
\hline & $(344-572)$ & 76.9 & (359) & A & {$[87 / 5]$} \\
\hline & $(473-572)$ & 58.2 & $(488)$ & & $\begin{array}{c}{[87 / 5][68 / 14]} \\
{[73 / 18]}\end{array}$ \\
\hline & $(354-439)$ & 70.2 & & & {$[77 / 6][58 / 1]$} \\
\hline $\mathrm{C}_{7} \mathrm{H}_{6} \mathrm{~N}_{2} \mathrm{O}_{4}$ & 2,6-dinitrotoluene & & & & [606-20-2] \\
\hline & $(330-533)$ & 77.8 & $(345)$ & A & [87/5] \\
\hline & $(423-553)$ & 56.9 & $(438)$ & A & {$[87 / 5][68 / 14]$} \\
\hline & $(344-427)$ & 68.7 & & & {$[77 / 6][58 / 1]$} \\
\hline $\mathrm{C}_{7} \mathrm{H}_{6} \mathrm{~N}_{2} \mathrm{O}_{4}$ & 3,5-dinitrotoluene & & & & [618-85-9] \\
\hline
\end{tabular}


TABLE 6. Enthalpies of vaporization of organic compounds, 1880-2002-Continued

\begin{tabular}{|c|c|c|c|c|c|}
\hline Molecular formula & $\begin{array}{c}\text { Compound } \\
\text { (Temperature range/K) }\end{array}$ & $\begin{array}{c}\Delta_{\mathrm{vap}} H_{m} \\
\left(\mathrm{~kJ} \mathrm{~mol}^{-1}\right)\end{array}$ & $\begin{array}{c}\text { Mean temperature } \\
\qquad\left(T_{m} / \mathrm{K}\right)\end{array}$ & Method & $\begin{array}{l}\text { CAS registry number } \\
\text { Reference }\end{array}$ \\
\hline & $(493-543)$ & 62.6 & $(508)$ & A & {$[87 / 5][68 / 14]$} \\
\hline \multirow[t]{12}{*}{$\mathrm{C}_{7} \mathrm{H}_{6} \mathrm{O}$} & benzaldehyde & & & & {$[100-52-7]$} \\
\hline & $(313-353)$ & 49.1 & $(298)$ & CGC & {$[95 / 21]$} \\
\hline & $(348-452)$ & 49.5 & (363) & A & {$[87 / 5]$} \\
\hline & $(409-481)$ & 43.8 & (424) & A & {$[87 / 5]$} \\
\hline & $(311-376)$ & 48.6 & (326) & $\mathrm{A}$ & {$[87 / 5]$} \\
\hline & $(370-475)$ & 45.5 & (385) & A & {$[87 / 5]$} \\
\hline & $(465-541)$ & 41.9 & $(480)$ & A & {$[87 / 5]$} \\
\hline & $(529-599)$ & 40.6 & (544) & $\mathrm{A}$ & {$[87 / 5]$} \\
\hline & $(311-404)$ & 50.3 & (298) & EB & {$[75 / 5]$} \\
\hline & $(311-404)$ & 42.5 & $(452)$ & $\mathrm{EB}$ & {$[75 / 5]$} \\
\hline & $(273-373)$ & 47.0 & $(288)$ & $\mathrm{A}, \mathrm{BG}$ & {$[87 / 5][73 / 4]$} \\
\hline & $(299-452)$ & 54.4 & $(314)$ & & {$[47 / 5]$} \\
\hline \multirow[t]{2}{*}{$\mathrm{C}_{7} \mathrm{H}_{6} \mathrm{O}$} & $2,4,6$-cycloheptatrienone (tropone) & & & & {$[539-80-0]$} \\
\hline & $(273-323)$ & 54.2 & $(288)$ & $\mathrm{A}$ & {$[87 / 5]$} \\
\hline \multirow[t]{5}{*}{$\mathrm{C}_{7} \mathrm{H}_{6} \mathrm{O}_{2}$} & benzoic acid & & & & {$[65-85-0]$} \\
\hline & $(353-393)$ & 78.9 & (298) & CGC & {$[95 / 21]$} \\
\hline & $(405-523)$ & 66.3 & $(420)$ & A & {$[87 / 5]$} \\
\hline & & 65.4 & $(428)$ & I & {$[43 / 7]$} \\
\hline & $(401-520)$ & 67.7 & $(416)$ & MM, A & {$[27 / 5]$} \\
\hline \multirow{2}{*}{$\mathrm{C}_{7} \mathrm{H}_{6} \mathrm{O}_{2}$} & 4-hydroxybenzaldehyde & & & & {$[123-08-0]$} \\
\hline & $(394-583)$ & 72.3 & (409) & A & {$[87 / 5][47 / 5]$} \\
\hline \multirow[t]{4}{*}{$\mathrm{C}_{7} \mathrm{H}_{6} \mathrm{O}_{2}$} & 2-hydroxybenzaldehyde & & & & {$[100-83-4]$} \\
\hline & $(383-470)$ & 30.6 & $(398)$ & A & {$[87 / 5]$} \\
\hline & & 47.7 & & & {$[86 / 10]$} \\
\hline & $(306-470)$ & 49.6 & $(321)$ & & {$[47 / 5]$} \\
\hline \multirow[t]{2}{*}{$\mathrm{C}_{7} \mathrm{H}_{6} \mathrm{O}_{2}$} & phenyl formate & & & & [1864-94-4] \\
\hline & $(287-305)$ & $52.9 \pm 0.6$ & $(298)$ & BG & {$[76 / 9][75 / 36]$} \\
\hline \multirow{2}{*}{$\mathrm{C}_{7} \mathrm{H}_{6} \mathrm{O}_{2}$} & 1,3-benzodioxole & & & & [274-09-9] \\
\hline & & 41.4 & & & {$[58 / 25]$} \\
\hline \multirow{2}{*}{$\mathrm{C}_{7} \mathrm{H}_{6} \mathrm{O}_{3}$} & 2-hydroxybenzoic acid & & & & {$[69-72-7]$} \\
\hline & $(445-504)$ & 79.4 & $(460)$ & A & {$[87 / 5]$} \\
\hline \multirow[t]{6}{*}{$\mathrm{C}_{7} \mathrm{H}_{7} \mathrm{Br}$} & benzylbromide & & & & [100-39-0] \\
\hline & $(284-306)$ & $53.3 \pm 0.7$ & (298) & GS & {$[02 / 29]$} \\
\hline & & 53.7 & (298) & CGC & [02/29] \\
\hline & $(340-409)$ & 48.1 & $(355)$ & $\mathrm{I}, \mathrm{A}$ & {$[76 / 11][87 / 5]$} \\
\hline & & $50.5 \pm 0.5$ & (298) & & {$[76 / 11]$} \\
\hline & $(305-472)$ & 46.9 & $(320)$ & A & $\begin{array}{c}{[87 / 5][47 / 5]} \\
{[99 / 16]}\end{array}$ \\
\hline \multirow[t]{5}{*}{$\mathrm{C}_{7} \mathrm{H}_{7} \mathrm{Br}$} & 2-bromotoluene & & & & {$[95-46-5]$} \\
\hline & $(322-455)$ & 47.2 & (337) & & [99/16] \\
\hline & $(353-518)$ & 45.3 & $(368)$ & A & $\begin{array}{c}{[87 / 5][70 / 14]} \\
{[73 / 18]}\end{array}$ \\
\hline & $(297-455)$ & 52.6 & $(312)$ & & {$[47 / 5]$} \\
\hline & $(273-348)$ & 48.8 & $(288)$ & & {$[40 / 5]$} \\
\hline \multirow[t]{4}{*}{$\mathrm{C}_{7} \mathrm{H}_{7} \mathrm{Br}$} & 3-bromotoluene & & & & [591-17-3] \\
\hline & $(351-457)$ & 47.7 & $(366)$ & & [99/16] \\
\hline & $(287-457)$ & 48.3 & (302) & A & {$[87 / 5][47 / 5]$} \\
\hline & $(273-348)$ & 49.4 & (288) & & {$[40 / 5]$} \\
\hline \multirow[t]{4}{*}{$\mathrm{C}_{7} \mathrm{H}_{7} \mathrm{Br}$} & 4-bromotoluene & & & & [108-38-7] \\
\hline & $(320-458)$ & 47.1 & (335) & & [99/16] \\
\hline & $(273-472)$ & 55.3 & (288) & & [99/16] \\
\hline & $(358-523)$ & 45.8 & (373) & A & $\begin{array}{c}{[87 / 5][70 / 14]} \\
{[73 / 18]}\end{array}$ \\
\hline \multirow[t]{2}{*}{$\mathrm{C}_{7} \mathrm{H}_{7} \mathrm{BrO}$} & 2-bromoanisole & & & & {$[578-57-4]$} \\
\hline & & 52.3 & & & {$[86 / 10]$} \\
\hline \multirow[t]{2}{*}{$\mathrm{C}_{7} \mathrm{H}_{7} \mathrm{BrO}$} & 3-bromoanisole & & & & [2398-37-0] \\
\hline & & 50.2 & & & {$[86 / 10]$} \\
\hline \multirow[t]{3}{*}{$\mathrm{C}_{7} \mathrm{H}_{7} \mathrm{BrO}$} & 4-bromoanisole & & & & [104-92-7] \\
\hline & & 50.6 & & & {$[86 / 10]$} \\
\hline & $(318-496)$ & 48.9 & (333) & & {$[47 / 5]$} \\
\hline $\mathrm{C}_{7} \mathrm{H}_{7} \mathrm{BrS}$ & 2-bromothioanisole & & & & [19614-16-5] \\
\hline & & 56.5 & & & {$[86 / 10]$} \\
\hline $\mathrm{C}_{7} \mathrm{H}_{7} \mathrm{BrS}$ & 3-bromothioanisole & & & & \\
\hline
\end{tabular}


TABLE 6. Enthalpies of vaporization of organic compounds, 1880-2002-Continued

\begin{tabular}{|c|c|c|c|c|c|}
\hline Molecular formula & $\begin{array}{c}\text { Compound } \\
\text { (Temperature range/K) }\end{array}$ & $\begin{array}{c}\Delta_{\mathrm{vap}} H_{m} \\
\left(\mathrm{~kJ} \mathrm{~mol}^{-1}\right)\end{array}$ & $\begin{array}{l}\text { Mean temperature } \\
\qquad\left(T_{m} / \mathrm{K}\right)\end{array}$ & Method & $\begin{array}{c}\text { CAS registry number } \\
\text { Reference }\end{array}$ \\
\hline & & 54.4 & & & [86/10] \\
\hline \multirow[t]{2}{*}{$\mathrm{C}_{7} \mathrm{H}_{7} \mathrm{BrS}$} & 4-bromothioanisole & & & & [104-95-0] \\
\hline & & 55.7 & & & {$[86 / 10]$} \\
\hline \multirow[t]{6}{*}{$\mathrm{C}_{7} \mathrm{H}_{7} \mathrm{Cl}$} & benzyl chloride & & & & [100-44-7] \\
\hline & $(276-309)$ & $50.1 \pm 0.3$ & $(298)$ & GS & [02/29] \\
\hline & & 49.9 & (298) & CGC & {$[02 / 29]$} \\
\hline & $(320-390)$ & 48.6 & (335) & $\mathrm{A}, \mathrm{I}$ & {$[87 / 5][76 / 11]$} \\
\hline & & $50.1 \pm 0.5$ & (298) & & {$[76 / 11][99 / 16]$} \\
\hline & $(295-453)$ & 48.6 & $(310)$ & & {$[87 / 5][47 / 5]$} \\
\hline \multirow[t]{7}{*}{$\mathrm{C}_{7} \mathrm{H}_{7} \mathrm{Cl}$} & 2-chlorotoluene & & & & [95-49-8] \\
\hline & $(370-432)$ & 41.6 & $(385)$ & & [99/16] \\
\hline & $(345-430)$ & 45.3 & (298) & & {$[84 / 9][91 / 2]$} \\
\hline & $(345-430)$ & 42.5 & (361) & & {$[84 / 9]$} \\
\hline & $(338-493)$ & 42.8 & (353) & A & $\begin{array}{c}{[87 / 5][73 / 18]} \\
{[70 / 14]}\end{array}$ \\
\hline & $(278-432)$ & 44.8 & $(293)$ & & {$[47 / 5]$} \\
\hline & $(273-348)$ & 45.8 & (288) & & {$[40 / 5]$} \\
\hline \multirow[t]{4}{*}{$\mathrm{C}_{7} \mathrm{H}_{7} \mathrm{Cl}$} & 3-chlorotoluene & & & & [108-41-8] \\
\hline & $(373-435)$ & 41.9 & $(388)$ & & [99/16] \\
\hline & $(277-436)$ & 43.7 & $(292)$ & $\mathrm{A}$ & {$[87 / 5][47 / 5]$} \\
\hline & $(273-348)$ & 46.2 & $(288)$ & & {$[40 / 5]$} \\
\hline \multirow[t]{6}{*}{$\mathrm{C}_{7} \mathrm{H}_{7} \mathrm{Cl}$} & 4-chlorotoluene & & & & [106-43-4] \\
\hline & $(362-435)$ & 41.8 & $(375)$ & & [99/16] \\
\hline & $(304-436)$ & 41.7 & (319) & A & {$[87 / 5]$} \\
\hline & $(340-430)$ & 46.0 & (298) & & {$[84 / 9][91 / 2]$} \\
\hline & $(338-433)$ & 43.5 & (353) & & {$[84 / 9]$} \\
\hline & $(279-435)$ & 44.1 & (293) & & {$[47 / 5]$} \\
\hline \multirow[t]{4}{*}{$\mathrm{C}_{7} \mathrm{H}_{7} \mathrm{ClO}$} & 2-chloroanisole & & & & [766-51-8] \\
\hline & & 49.4 & & & {$[86 / 10]$} \\
\hline & $(388-460)$ & 48.3 & $(403)$ & A & {$[87 / 5][73 / 18]$} \\
\hline & & & & & [99/16] \\
\hline \multirow{2}{*}{$\mathrm{C}_{7} \mathrm{H}_{7} \mathrm{ClO}$} & 3-chloroanisole & & & & [2845-89-8] \\
\hline & & 48.1 & & & {$[86 / 10]$} \\
\hline \multirow[t]{2}{*}{$\mathrm{C}_{7} \mathrm{H}_{7} \mathrm{ClO}$} & 4-chloroanisole & & & & {$[623-12-1]$} \\
\hline & & 47.7 & & & {$[86 / 10]$} \\
\hline \multirow[t]{2}{*}{$\mathrm{C}_{7} \mathrm{H}_{7} \mathrm{ClS}$} & 2-chlorothioanisole & & & & [17733-22-1] \\
\hline & & 53.6 & & & {$[86 / 10]$} \\
\hline \multirow[t]{2}{*}{$\mathrm{C}_{7} \mathrm{H}_{7} \mathrm{ClS}$} & 3-chlorothioanisole & & & & [4867-37-2] \\
\hline & & 51.9 & & & {$[86 / 10]$} \\
\hline \multirow[t]{2}{*}{$\mathrm{C}_{7} \mathrm{H}_{7} \mathrm{ClS}$} & 4-chlorothioanisole & & & & {$[123-09-1]$} \\
\hline & & 53.1 & & & {$[86 / 10]$} \\
\hline \multirow[t]{7}{*}{$\mathrm{C}_{7} \mathrm{H}_{7} \mathrm{~F}$} & benzyl fluoride & & & & {$[350-50-5]$} \\
\hline & $(278-318)$ & $46.2 \pm 0.3$ & (298) & GS & {$[02 / 29]$} \\
\hline & & 46.5 & $(298)$ & CGC & {$[02 / 29]$} \\
\hline & $(278-318)$ & $46.3 \pm 0.3$ & (298) & GS & [97/14] \\
\hline & $(297-410)$ & 43.7 & (312) & A & {$[87 / 5]$} \\
\hline & $(298-356)$ & 44.3 & (312) & I & {$[76 / 11]$} \\
\hline & & $44.5 \pm 0.4$ & (298) & & {$[76 / 11]$} \\
\hline \multirow[t]{7}{*}{$\mathrm{C}_{7} \mathrm{H}_{7} \mathrm{~F}$} & 2-fluorotoluene & & & & {$[95-52-3]$} \\
\hline & $(248-388)$ & 42.0 & $(263)$ & & [99/16] \\
\hline & $(452-531)$ & 31.5 & $(465)$ & & [99/16] \\
\hline & $(453-530)$ & 32.3 & $(468)$ & & [84/16] \\
\hline & $(308-348)$ & 38.0 & (323) & & {$[74 / 26][84 / 9]$} \\
\hline & $(295-388)$ & 38.7 & $(310)$ & A & {$[87 / 5][51 / 9]$} \\
\hline & $(248-387)$ & 40.5 & (264) & & {$[47 / 5]$} \\
\hline \multirow{4}{*}{$\mathrm{C}_{7} \mathrm{H}_{7} \mathrm{~F}$} & 3 -fluorotoluene & & & & {$[352-70-5]$} \\
\hline & $(250-390)$ & 41.6 & $(265)$ & & [99/16] \\
\hline & $(293-390)$ & 39.2 & (308) & $\mathrm{A}$ & {$[87 / 5][51 / 9]$} \\
\hline & $(250-389)$ & 40.7 & $(266)$ & & {$[47 / 5]$} \\
\hline \multirow[t]{3}{*}{$\mathrm{C}_{7} \mathrm{H}_{7} \mathrm{~F}$} & 4-fluorotoluene & & & & {$[352-32-9]$} \\
\hline & $(340-430)$ & 39.5 & (298) & & {$[84 / 9][91 / 2]$} \\
\hline & $(340-429)$ & 37.0 & $(355)$ & A & $\begin{array}{c}{[87 / 5][51 / 9]} \\
{[99 / 16]}\end{array}$ \\
\hline $\mathrm{C}_{7} \mathrm{H}_{7} \mathrm{~F}_{2} \mathrm{~N}$ & $\mathrm{~N}, \mathrm{~N}$-difluorobenzylamine & & & & [23162-99-4] \\
\hline
\end{tabular}


TABLE 6. Enthalpies of vaporization of organic compounds, 1880-2002-Continued

\begin{tabular}{|c|c|c|c|c|c|}
\hline Molecular formula & $\begin{array}{c}\text { Compound } \\
\text { (Temperature range/K) }\end{array}$ & $\begin{array}{c}\Delta_{\mathrm{vap}} H_{m} \\
\left(\mathrm{~kJ} \mathrm{~mol}^{-1}\right)\end{array}$ & $\begin{array}{c}\text { Mean temperature } \\
\left(T_{m} / \mathrm{K}\right)\end{array}$ & Method & $\begin{array}{c}\text { CAS registry number } \\
\text { Reference }\end{array}$ \\
\hline & $(313-333)$ & 77.8 & (323) & A & {$[87 / 5]$} \\
\hline \multirow[t]{2}{*}{$\mathrm{C}_{7} \mathrm{H}_{7} \mathrm{~F}_{9} \mathrm{O}$} & \multicolumn{2}{|c|}{$1,1,1,2,2,3,3,4,4$-nonafluoro-4-propoxybutane } & & & [72372-80-6] \\
\hline & $(288-369)$ & 37.9 & $(303)$ & I & {$[02 / 19]$} \\
\hline \multirow[t]{5}{*}{$\mathrm{C}_{7} \mathrm{H}_{7} \mathrm{I}$} & benzyl iodide & & & & {$[620-05-3]$} \\
\hline & $(301-337)$ & $57.4 \pm 0.3$ & $(298)$ & GS & {$[02 / 29]$} \\
\hline & & 57.7 & (298) & CGC & [02/29] \\
\hline & $(360-400)$ & 46.8 & (375) & $\mathrm{I}, \mathrm{A}$ & {$[87 / 5][76 / 11]$} \\
\hline & & $50.6 \pm 1.4$ & (298) & & {$[76 / 11]$} \\
\hline \multirow[t]{2}{*}{$\mathrm{C}_{7} \mathrm{H}_{7} \mathrm{I}$} & 2-iodotoluene & & & & {$[615-37-2]$} \\
\hline & $(310-484)$ & 49.7 & $(325)$ & A & {$[87 / 5][47 / 5]$} \\
\hline \multirow[t]{3}{*}{$\mathrm{C}_{7} \mathrm{H}_{7} \mathrm{IO}$} & 4-iodoanisole & & & & [696-62-8] \\
\hline & $(401-520)$ & 54.4 & (416) & A & {$[87 / 5][99 / 16]$} \\
\hline & $(401-479)$ & $53.1 \pm 0.4$ & $(440)$ & $\mathrm{I}$ & [56/26] \\
\hline \multirow[t]{2}{*}{$\mathrm{C}_{7} \mathrm{H}_{7} \mathrm{NO}_{2}$} & (nitromethyl)benzene & & & & {$[622-42-4]$} \\
\hline & $(363-413)$ & 53.8 & (378) & A & {$[87 / 5]$} \\
\hline \multirow[t]{6}{*}{$\mathrm{C}_{7} \mathrm{H}_{7} \mathrm{NO}_{2}$} & 2-nitrotoluene & & & & {$[88-72-2]$} \\
\hline & $(274-323)$ & $59.0 \pm 0.3$ & (299) & GS & {$[00 / 15]$} \\
\hline & & $59.1 \pm 0.3$ & (298) & & {$[00 / 15]$} \\
\hline & $(388-448)$ & 52.0 & (403) & EB & {$[94 / 8]$} \\
\hline & $(402-496)$ & 51.0 & (417) & $\mathrm{A}$ & {$[87 / 5]$} \\
\hline & $(387-493)$ & 52.2 & $(402)$ & & {$[38 / 9][94 / 8]$} \\
\hline \multirow[t]{3}{*}{$\mathrm{C}_{7} \mathrm{H}_{7} \mathrm{NO}_{2}$} & 3-nitrotoluene & & & & {$[99-08-1]$} \\
\hline & $(397-452)$ & 52.8 & (413) & EB & [94/8] \\
\hline & $(353-505)$ & 49.8 & (368) & A & {$[87 / 5]$} \\
\hline \multirow[t]{4}{*}{$\mathrm{C}_{7} \mathrm{H}_{7} \mathrm{NO}_{2}$} & 4-nitrotoluene & & & & [99-99-0] \\
\hline & $(407-457)$ & 52.8 & $(422)$ & EB & {$[94 / 8]$} \\
\hline & $(423-512)$ & 49.8 & (438) & A & {$[87 / 5]$} \\
\hline & $(387-493)$ & 54.2 & $(402)$ & & {$[38 / 9][94 / 8]$} \\
\hline \multirow[t]{2}{*}{$\mathrm{C}_{7} \mathrm{H}_{7} \mathrm{NO}_{3}$} & 2-nitroanisole & & & & {$[91-23-6]$} \\
\hline & $(424-545)$ & 58.6 & $(439)$ & A & {$[87 / 5]$} \\
\hline \multirow{2}{*}{$\mathrm{C}_{7} \mathrm{H}_{7} \mathrm{~N}_{3}$} & (azidomethyl)benzene & & & & {$[622-79-7]$} \\
\hline & $(333-363)$ & 48.0 & $(348)$ & $\mathrm{A}$ & {$[87 / 5]$} \\
\hline \multirow[t]{6}{*}{$\mathrm{C}_{7} \mathrm{H}_{8}$} & bicyclo[2.2.1]hepta-2,5-die & & & & [121-46-0] \\
\hline & & $34.8 \pm 0.1$ & (298) & $\mathrm{C}$ & {$[93 / 1]$} \\
\hline & $(300-364)$ & 33.6 & (315) & $\mathrm{A}$ & {$[87 / 5]$} \\
\hline & & $34.7 \pm 0.1$ & (298) & $\mathrm{C}$ & {$[85 / 2]$} \\
\hline & & $33.8 \pm 0.9$ & (298) & & {$[78 / 1]$} \\
\hline & $(300-353)$ & $32.9 \pm 0.8$ & $(298)$ & BG & {$[73 / 1]$} \\
\hline \multirow[t]{4}{*}{$\mathrm{C}_{7} \mathrm{H}_{8}$} & 1,3,5-cycloheptatriene & & & & [544-25-2] \\
\hline & $(273-338)$ & 40.8 & (288) & A & {$[87 / 5][73 / 18]$} \\
\hline & $(273-416)$ & 39.4 & (288) & $\mathrm{A}, \mathrm{EB}$ & {$[87 / 5][56 / 20]$} \\
\hline & & $38.7 \pm 0.2$ & (298) & & {$[56 / 20]$} \\
\hline \multirow[t]{5}{*}{$\mathrm{C}_{7} \mathrm{H}_{8}$} & tetracyclo[3.2.0.0 $\left.0^{2,7} \cdot 0^{4,6}\right]$ he & adricyclane) & & & {$[278-06-8]$} \\
\hline & & $37.9 \pm 0.1$ & (298) & $\mathrm{C}$ & [93/1] \\
\hline & & $37.9 \pm 0.1$ & (298) & $\mathrm{C}$ & {$[85 / 2]$} \\
\hline & & $37.0 \pm 0.8$ & (298) & & [78/1] \\
\hline & $(302-372)$ & $37.3 \pm 0.8$ & $(317)$ & BG & {$[87 / 5][73 / 1]$} \\
\hline $\mathrm{C}_{7} \mathrm{H}_{8}$ & toluene & & & & {$[108-88-3]$} \\
\hline & $(331-496)$ & 35.7 & (346) & & {$[93 / 3]$} \\
\hline & $(210-279)$ & 40.6 & (264) & A & {$[87 / 5]$} \\
\hline & $(383-445)$ & 34.4 & (398) & A & {$[87 / 5]$} \\
\hline & $(440-531)$ & 33.2 & (455) & A & {$[87 / 5]$} \\
\hline & $(530-592)$ & 33.3 & (545) & A & {$[87 / 5]$} \\
\hline & $(273-295)$ & 38.9 & (284) & A & {$[87 / 5]$} \\
\hline & & $33.5 \pm 0.1$ & $(380)$ & $\mathrm{C}$ & {$[85 / 10]$} \\
\hline & & $32.1 \pm 0.1$ & $(403)$ & $\mathrm{C}$ & {$[85 / 10]$} \\
\hline & & $29.4 \pm 0.1$ & $(441)$ & $\mathrm{C}$ & {$[85 / 10]$} \\
\hline & & $27.1 \pm 0.1$ & $(470)$ & $\mathrm{C}$ & {$[85 / 10]$} \\
\hline & & $24.0 \pm 0.1$ & $(505)$ & $\mathrm{C}$ & {$[85 / 10]$} \\
\hline & & 35.4 & (333) & & [84/29] \\
\hline & & 33.4 & $(373)$ & & [84/29] \\
\hline & & 31.4 & $(413)$ & & [84/29] \\
\hline & & 28.4 & (453) & & [84/29] \\
\hline & & 24.0 & (493) & & [84/29] \\
\hline
\end{tabular}


TABLE 6. Enthalpies of vaporization of organic compounds, 1880-2002-Continued

\begin{tabular}{|c|c|c|c|c|c|}
\hline Molecular formula & $\begin{array}{c}\text { Compound } \\
\text { (Temperature range/K) }\end{array}$ & $\begin{array}{c}\Delta_{\mathrm{vap}} H_{m} \\
\left(\mathrm{~kJ} \mathrm{~mol}^{-1}\right)\end{array}$ & $\begin{array}{l}\text { Mean temperature } \\
\qquad\left(T_{m} / \mathrm{K}\right)\end{array}$ & Method & $\begin{array}{c}\text { CAS registry number } \\
\text { Reference }\end{array}$ \\
\hline & $(343-383)$ & 35.4 & $(360)$ & & {$[75 / 33]$} \\
\hline & & 38.0 & (298) & & {$[71 / 28]$} \\
\hline & $(303-343)$ & 37.3 & (318) & & {$[68 / 23]$} \\
\hline & $(288-348)$ & 36.9 & (303) & & {$[67 / 10]$} \\
\hline & $(210-293)$ & 37.8 & (278) & & {$[56 / 5]$} \\
\hline & $(308-386)$ & 37.0 & (323) & & {$[87 / 5][49 / 6]$} \\
\hline & & 38.0 & (298) & $\mathrm{C}$ & {$[47 / 7]$} \\
\hline & $(286-362)$ & 37.8 & (301) & & {$[46 / 4]$} \\
\hline & $(308-384)$ & 37.0 & (323) & MM & {$[45 / 2]$} \\
\hline & $(273-323)$ & 38.8 & $(288)$ & & {$[43 / 1]$} \\
\hline \multirow[t]{13}{*}{$\mathrm{C}_{7} \mathrm{H}_{8} \mathrm{O}$} & anisole & & & & [100-66-3] \\
\hline & $(353-393)$ & 45.3 & $(298)$ & CGC & {$[95 / 21]$} \\
\hline & $(382-429)$ & 41.8 & (397) & & {$[93 / 4]$} \\
\hline & & 41.0 & & & {$[86 / 10]$} \\
\hline & $(382-437)$ & 41.9 & (397) & A & {$[87 / 5][76 / 2]$} \\
\hline & $(382-437)$ & 46.9 & (298) & & {$[76 / 2]$} \\
\hline & $(282-437)$ & 39.0 & $(426)$ & & {$[76 / 2]$} \\
\hline & & $46.8 \pm 0.2$ & (298) & C & {$[75 / 3]$} \\
\hline & & $42.9 \pm 0.1$ & (367) & $\mathrm{C}$ & {$[67 / 39]$} \\
\hline & & $42.0 \pm 0.1$ & $(382)$ & $\mathrm{C}$ & {$[67 / 39]$} \\
\hline & & $40.5 \pm 0.1$ & $(402)$ & $\mathrm{C}$ & {$[67 / 39]$} \\
\hline & & $38.9 \pm 0.1$ & $(427)$ & $\mathrm{C}$ & {$[67 / 39]$} \\
\hline & $(382-437)$ & 41.9 & (397) & & {$[55 / 9][65 / 7]$} \\
\hline \multirow[t]{8}{*}{$\mathrm{C}_{7} \mathrm{H}_{8} \mathrm{O}$} & benzyl alcohol & & & & {$[100-51-6]$} \\
\hline & $(277-381)$ & $64.8 \pm 0.6$ & $(298)$ & GS & {$[99 / 3]$} \\
\hline & $(323-373)$ & 69.5 & (298) & CGC & {$[95 / 21]$} \\
\hline & & 60.5 & & & {$[95 / 30]$} \\
\hline & $(303-333)$ & 66.2 & $(318)$ & GS & {$[82 / 1]$} \\
\hline & $(385-573)$ & 54.6 & $(400)$ & A & {$[87 / 5][73 / 18]$} \\
\hline & $(293-313)$ & 61.5 & (303) & $\mathrm{A}, \mathrm{ME}$ & {$[87 / 5][57 / 9]$} \\
\hline & & & & & {$[73 / 18]$} \\
\hline \multirow[t]{9}{*}{$\mathrm{C}_{7} \mathrm{H}_{8} \mathrm{O}$} & 2-hydroxytoluene & & & & [95-48-7] \\
\hline & $(304-409)$ & 58.5 & (319) & A & {$[87 / 5]$} \\
\hline & $(399-470)$ & 50.1 & (414) & $\mathrm{A}$ & {$[87 / 5]$} \\
\hline & $(463-526)$ & 46.2 & (478) & A & {$[87 / 5]$} \\
\hline & $(517-630)$ & 44.0 & $(532)$ & $\mathrm{A}$ & {$[87 / 5]$} \\
\hline & & 50.2 & & & [86/10] \\
\hline & $(383-473)$ & 51.3 & (398) & GS, EB & {$[87 / 5][60 / 4]$} \\
\hline & & & & & {$[73 / 18]$} \\
\hline & $(415-462)$ & 48.2 & $(438)$ & & [39/4] \\
\hline \multirow[t]{12}{*}{$\mathrm{C}_{7} \mathrm{H}_{8} \mathrm{O}$} & 3-hydroxytoluene & & & & [108-39-4] \\
\hline & $(393-433)$ & 62.5 & (298) & CGC & {$[95 / 21]$} \\
\hline & $(284-313)$ & 61.7 & (298) & A & {$[87 / 5]$} \\
\hline & $(285-416)$ & 63.1 & (300) & A & {$[87 / 5]$} \\
\hline & $(410-477)$ & 52.7 & (425) & $\mathrm{A}$ & {$[87 / 5]$} \\
\hline & $(471-531)$ & 47.6 & (486) & A & {$[87 / 5]$} \\
\hline & $(523-633)$ & 43.8 & (538) & A & [87/5] \\
\hline & $(383-473)$ & 55.0 & (398) & GS, EB & {$[87 / 5][60 / 4]$} \\
\hline & & & & & {$[73 / 18]$} \\
\hline & $(388-429)$ & 60.6 & (409) & GS & [80/17] \\
\hline & $(359-473)$ & 58.8 & (374) & & {$[55 / 9][84 / 9]$} \\
\hline & $(422-474)$ & 50.7 & (448) & & [39/4] \\
\hline \multirow[t]{9}{*}{$\mathrm{C}_{7} \mathrm{H}_{8} \mathrm{O}$} & 4-hydroxytoluene & & & & [106-44-5] \\
\hline & $(308-393)$ & 62.0 & (323) & A & [87/5] \\
\hline & $(385-477)$ & 55.4 & (400) & $\mathrm{A}$ & {$[87 / 5]$} \\
\hline & $(463-533)$ & 49.2 & (478) & $\mathrm{A}$ & {$[87 / 5]$} \\
\hline & $(523-635)$ & 46.0 & $(538)$ & $\mathrm{A}$ & {$[87 / 5]$} \\
\hline & & 54.0 & & & {$[86 / 10]$} \\
\hline & $(383-473)$ & 55.6 & (398) & $\mathrm{A}, \mathrm{GS}, \mathrm{EB}$ & {$[87 / 5][60 / 4]$} \\
\hline & & & & & {$[73 / 18]$} \\
\hline & $(419-474)$ & 51.3 & $(446)$ & & {$[39 / 4]$} \\
\hline \multirow[t]{2}{*}{$\mathrm{C}_{7} \mathrm{H}_{8} \mathrm{OS}$} & 4-methoxybenzenethiol & & & & [106-53-6] \\
\hline & & 52.3 & & & {$[86 / 10]$} \\
\hline $\mathrm{C}_{7} \mathrm{H}_{8} \mathrm{O}_{2}$ & 2,4-dihydroxytoluene & & & & {$[496-73-1]$} \\
\hline
\end{tabular}


TABLE 6. Enthalpies of vaporization of organic compounds, 1880-2002-Continued

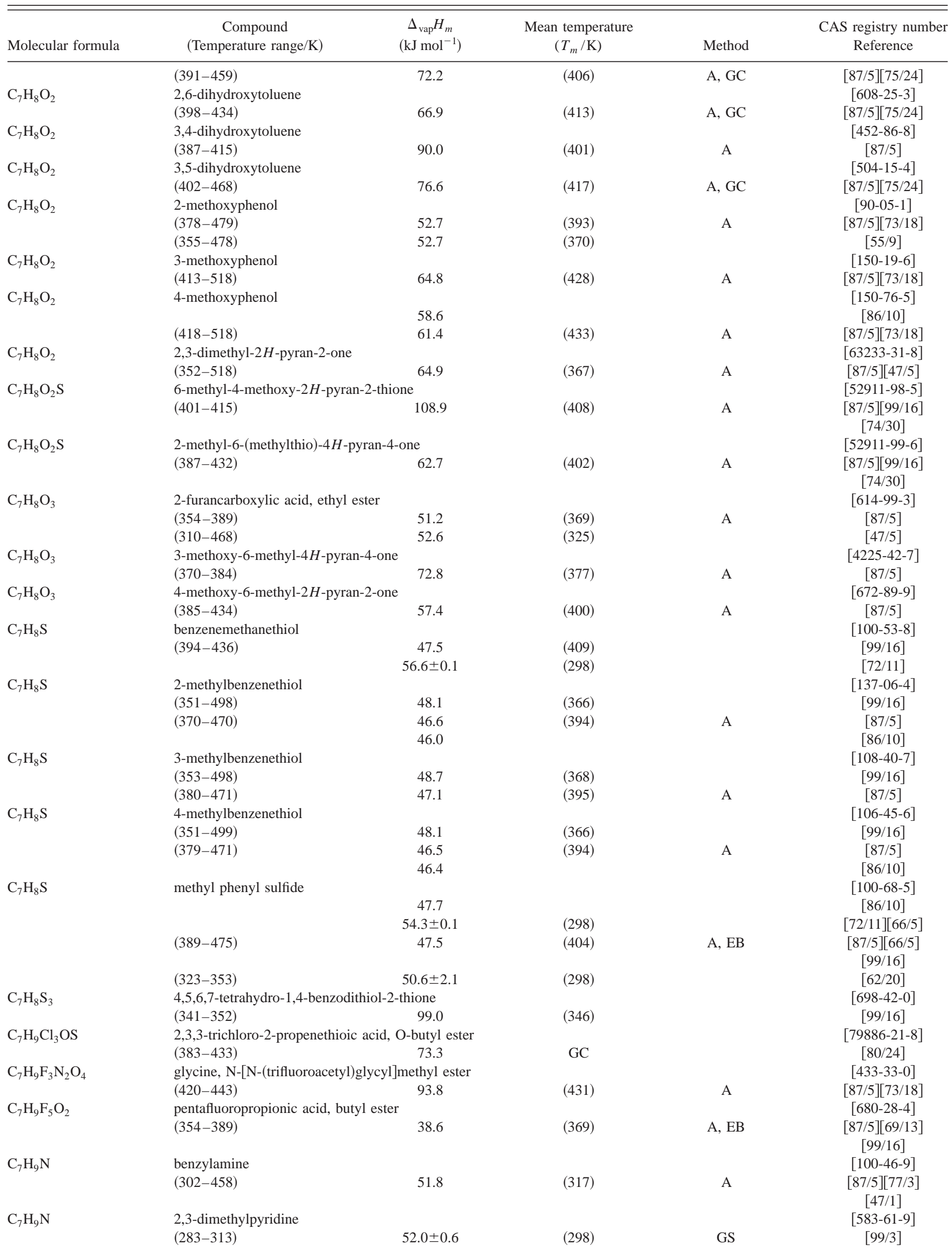


TABLE 6. Enthalpies of vaporization of organic compounds, 1880-2002-Continued

\begin{tabular}{|c|c|c|c|c|c|}
\hline Molecular formula & $\begin{array}{c}\text { Compound } \\
\text { (Temperature range/K) }\end{array}$ & $\begin{array}{c}\Delta_{\mathrm{vap}} H_{m} \\
\left(\mathrm{~kJ} \mathrm{~mol}^{-1}\right)\end{array}$ & $\begin{array}{l}\text { Mean temperature } \\
\qquad\left(T_{m} / \mathrm{K}\right)\end{array}$ & Method & $\begin{array}{c}\text { CAS registry number } \\
\text { Reference }\end{array}$ \\
\hline & $(328-476)$ & 45.2 & $(340)$ & $\mathrm{EB}$ & {$[95 / 22]$} \\
\hline & $(328-476)$ & 42.7 & $(380)$ & EB & {$[95 / 22]$} \\
\hline & $(328-476)$ & 40.2 & $(420)$ & EB & {$[95 / 22]$} \\
\hline & $(328-476)$ & 37.4 & $(460)$ & EB & {$[95 / 22]$} \\
\hline & $(323-373)$ & 47.6 & $(298)$ & CGC & {$[95 / 21]$} \\
\hline & & 46.9 & $(313)$ & $\mathrm{C}$ & {$[85 / 1]$} \\
\hline & & 45.0 & $(343)$ & $\mathrm{C}$ & {$[85 / 1]$} \\
\hline & & 43.5 & $(368)$ & $\mathrm{C}$ & {$[85 / 1]$} \\
\hline & $(372-436)$ & 43.0 & $(387)$ & A & {$[87 / 5][73 / 18]$} \\
\hline \multirow[t]{13}{*}{$\mathrm{C}_{7} \mathrm{H}_{9} \mathrm{~N}$} & 2,4-dimethylpyridine & & & & [108-47-4] \\
\hline & $(323-373)$ & 47.5 & $(298)$ & $\mathrm{CGC}$ & {$[95 / 21]$} \\
\hline & $(288-373)$ & 45.5 & $(330)$ & & {$[95 / 4]$} \\
\hline & $(331-473)$ & 44.8 & $(340)$ & $\mathrm{EB}$ & {$[95 / 22]$} \\
\hline & $(331-473)$ & 42.3 & $(380)$ & EB & {$[95 / 22]$} \\
\hline & $(331-473)$ & 39.8 & $(420)$ & EB & {$[95 / 22]$} \\
\hline & $(331-473)$ & 37.0 & $(460)$ & EB & {$[95 / 22]$} \\
\hline & $(298-431)$ & 47.1 & $(313)$ & EB & {$[90 / 6]$} \\
\hline & $(267-358)$ & 47.5 & $(282)$ & $\mathrm{MM}$ & {$[86 / 2]$} \\
\hline & & 46.5 & $(313)$ & $\mathrm{C}$ & {$[85 / 1]$} \\
\hline & & 44.6 & $(343)$ & $\mathrm{C}$ & {$[85 / 1]$} \\
\hline & & 43.9 & $(368)$ & $\mathrm{C}$ & {$[85 / 1]$} \\
\hline & $(349-433)$ & 43.5 & $(364)$ & A & {$[87 / 5][73 / 18]$} \\
\hline \multirow[t]{6}{*}{$\mathrm{C}_{7} \mathrm{H}_{9} \mathrm{~N}$} & 2,5-dimethylpyridine & & & & [589-93-5] \\
\hline & $(330-471)$ & 44.4 & $(340)$ & EB & {$[95 / 22]$} \\
\hline & $(330-471)$ & 41.9 & $(380)$ & EB & {$[95 / 22]$} \\
\hline & $(330-471)$ & 39.4 & $(420)$ & EB & {$[95 / 22]$} \\
\hline & $(330-471)$ & 36.5 & $(460)$ & EB & {$[95 / 22]$} \\
\hline & $(358-431)$ & 42.8 & $(373)$ & $\mathrm{A}, \mathrm{MG}$ & $\begin{array}{c}{[87 / 5][53 / 4]} \\
{[73 / 18]}\end{array}$ \\
\hline \multirow[t]{13}{*}{$\mathrm{C}_{7} \mathrm{H}_{9} \mathrm{~N}$} & 2,6-dimethylpyridine & & & & {$[108-48-5]$} \\
\hline & $(323-373)$ & 46.4 & $(298)$ & $\mathrm{CGC}$ & {$[95 / 21]$} \\
\hline & $(288-373)$ & 43.7 & $(330)$ & & {$[95 / 4]$} \\
\hline & $(315-457)$ & 43.9 & $(320)$ & EB & {$[95 / 22]$} \\
\hline & $(315-457)$ & 41.4 & $(360)$ & EB & {$[95 / 22]$} \\
\hline & $(315-457)$ & 38.8 & $(400)$ & EB & {$[95 / 22]$} \\
\hline & $(315-457)$ & 36.0 & $(440)$ & EB & {$[95 / 22]$} \\
\hline & $(295-417)$ & 45.0 & $(310)$ & EB & {$[90 / 6]$} \\
\hline & $(267-358)$ & 46.1 & $(282)$ & MM & {$[86 / 2]$} \\
\hline & & 44.4 & $(313)$ & $\mathrm{C}$ & {$[85 / 1]$} \\
\hline & & 42.5 & $(343)$ & $\mathrm{C}$ & {$[85 / 1]$} \\
\hline & & 40.8 & $(368)$ & C & {$[85 / 1]$} \\
\hline & $(352-418)$ & 41.6 & $(367)$ & $\mathrm{A}, \mathrm{MG}$ & {$[87 / 5][53 / 4]$} \\
\hline \multirow[t]{10}{*}{$\mathrm{C}_{7} \mathrm{H}_{9} \mathrm{~N}$} & 3,4-dimethylpyridine & & & & {$[583-58-4]$} \\
\hline & $(341-495)$ & 46.6 & $(360)$ & $\mathrm{EB}$ & {$[95 / 22]$} \\
\hline & $(341-495)$ & 44.2 & $(400)$ & $\mathrm{EB}$ & {$[95 / 22]$} \\
\hline & $(341-495)$ & 41.7 & $(440)$ & EB & {$[95 / 22]$} \\
\hline & $(341-495)$ & 39.0 & $(480)$ & $\mathrm{EB}$ & {$[95 / 22]$} \\
\hline & $(288-422)$ & 47.6 & $(355)$ & & {$[95 / 4]$} \\
\hline & & 48.8 & $(328)$ & $\mathrm{C}$ & {$[85 / 1]$} \\
\hline & & 47.6 & $(343)$ & $\mathrm{C}$ & {$[85 / 1]$} \\
\hline & & 45.9 & $(368)$ & $\mathrm{C}$ & {$[85 / 1]$} \\
\hline & $(385-454)$ & 44.8 & $(400)$ & A & {$[87 / 5][73 / 18]$} \\
\hline \multirow[t]{12}{*}{$\mathrm{C}_{7} \mathrm{H}_{9} \mathrm{~N}$} & 3,5-dimethylpyridine & & & & [591-22-0] \\
\hline & $(323-373)$ & 48.7 & $(298)$ & CGC & {$[95 / 21]$} \\
\hline & $(288-392)$ & 47.0 & $(340)$ & & {$[95 / 4]$} \\
\hline & $(335-487)$ & 46.7 & $(340)$ & $\mathrm{EB}$ & {$[95 / 22]$} \\
\hline & $(335-487)$ & 44.3 & $(380)$ & EB & {$[95 / 22]$} \\
\hline & $(335-487)$ & 41.8 & $(420)$ & $\mathrm{EB}$ & {$[95 / 22]$} \\
\hline & $(335-487)$ & 39.2 & $(460)$ & $\mathrm{EB}$ & {$[95 / 22]$} \\
\hline & $(273-358)$ & 49.1 & $(288)$ & MM & {$[86 / 2]$} \\
\hline & & 49.6 & $(313)$ & $\mathrm{C}$ & {$[85 / 1]$} \\
\hline & & 46.5 & $(343)$ & $\mathrm{C}$ & {$[85 / 1]$} \\
\hline & & 44.8 & $(368)$ & $\mathrm{C}$ & {$[85 / 1]$} \\
\hline & $(373-446)$ & 44.3 & (388) & $\mathrm{A}$ & {$[87 / 5][73 / 18]$} \\
\hline
\end{tabular}


TABLE 6. Enthalpies of vaporization of organic compounds, 1880-2002-Continued

\begin{tabular}{|c|c|c|c|c|c|}
\hline Molecular formula & $\begin{array}{c}\text { Compound } \\
\text { (Temperature range/K) }\end{array}$ & $\begin{array}{c}\Delta_{\mathrm{vap}} H_{m} \\
\left(\mathrm{~kJ} \mathrm{~mol}^{-1}\right)\end{array}$ & $\begin{array}{l}\text { Mean temperature } \\
\qquad\left(T_{m} / \mathrm{K}\right)\end{array}$ & Method & $\begin{array}{l}\text { CAS registry number } \\
\text { Reference }\end{array}$ \\
\hline $\mathrm{C}_{7} \mathrm{H}_{9} \mathrm{~N}$ & $\begin{array}{l}\text { 3-ethylpyridine } \\
\text { (334-373) }\end{array}$ & 44.6 & $(349)$ & A & $\begin{array}{c}{[536-78-7]} \\
{[87 / 5][73 / 18]}\end{array}$ \\
\hline $\mathrm{C}_{7} \mathrm{H}_{9} \mathrm{~N}$ & $\begin{array}{l}\text { 2-ethylpyridine } \\
\text { (323-373) }\end{array}$ & 43.7 & (338) & A & $\begin{array}{c}{[100-71-0]} \\
{[87 / 5][73 / 18]}\end{array}$ \\
\hline $\mathrm{C}_{7} \mathrm{H}_{9} \mathrm{~N}$ & $\begin{array}{l}\text { 4-ethylpyridine } \\
(333-372)\end{array}$ & 45.3 & $(348)$ & $\mathrm{A}$ & $\begin{array}{c}{[536-75-4]} \\
{[87 / 5][73 / 18]}\end{array}$ \\
\hline $\mathrm{C}_{7} \mathrm{H}_{9} \mathrm{~N}$ & $\begin{array}{l}\text { N-methylaniline } \\
(309-469)\end{array}$ & 53.6 & $(324)$ & $\mathrm{A}$ & $\begin{array}{c}{[100-61-8]} \\
{[87 / 5]}\end{array}$ \\
\hline $\mathrm{C}_{7} \mathrm{H}_{9} \mathrm{~N}$ & $\begin{array}{l}o \text {-toluidine } \\
(290-517) \\
(290-517) \\
(290-517) \\
(290-517) \\
(290-517) \\
(290-517) \\
(391-474)\end{array}$ & $\begin{array}{l}57.8 \\
54.5 \\
51.5 \\
48.6 \\
45.7 \\
42.7 \\
50.0\end{array}$ & $\begin{array}{l}(300) \\
(340) \\
(380) \\
(420) \\
(460) \\
(500) \\
(406)\end{array}$ & $\begin{array}{c}\text { EB, IPM } \\
\text { EB, IPM } \\
\text { EB, IPM } \\
\text { EB, IPM } \\
\text { EB, IPM } \\
\text { EB, IPM } \\
\text { A }\end{array}$ & $\begin{array}{c}{[95-53-4]} \\
{[94 / 17]} \\
{[94 / 17]} \\
{[94 / 17]} \\
{[94 / 17]} \\
{[94 / 17]} \\
{[94 / 17]} \\
{[87 / 5][49 / 1]} \\
{[84 / 9]}\end{array}$ \\
\hline $\mathrm{C}_{7} \mathrm{H}_{9} \mathrm{~N}$ & $\begin{array}{l}m \text {-toluidine } \\
(394-477)\end{array}$ & 51.1 & $(409)$ & A & $\begin{array}{c}{[108-44-1]} \\
{[87 / 5][49 / 1]} \\
{[84 / 9]}\end{array}$ \\
\hline $\mathrm{C}_{7} \mathrm{H}_{9} \mathrm{~N}$ & $\begin{array}{l}p \text {-toluidine } \\
(393-474) \\
(315-473)\end{array}$ & $\begin{array}{l}51.1 \\
54.9\end{array}$ & $\begin{array}{l}(408) \\
(330)\end{array}$ & A & $\begin{array}{l}{[106-49-0]} \\
{[87 / 5]} \\
{[47 / 5]}\end{array}$ \\
\hline $\mathrm{C}_{7} \mathrm{H}_{9} \mathrm{~N}$ & 1-cyclohexene-1-carbonitrile & $53.6 \pm 0.1$ & $(298)$ & $\mathrm{C}$ & $\begin{array}{c}{[1855-63-6]} \\
{[70 / 21]}\end{array}$ \\
\hline $\mathrm{C}_{7} \mathrm{H}_{9} \mathrm{~N}$ & $\begin{array}{l}\text { bicyclo[3.1.0]hexane-1-carbonitrile } \\
(366-444)\end{array}$ & $\mathrm{U} 43.2$ & $(382)$ & BG & $\begin{array}{c}{[31357-72-9]} \\
{[71 / 2]}\end{array}$ \\
\hline $\mathrm{C}_{7} \mathrm{H}_{9} \mathrm{NO}$ & $\begin{array}{l}\text { 2-methoxyaniline } \\
(334-492)\end{array}$ & 57.5 & $(349)$ & $\mathrm{A}$ & $\begin{array}{c}{[90-04-0]} \\
{[87 / 5][47 / 5]}\end{array}$ \\
\hline $\mathrm{C}_{7} \mathrm{H}_{10}$ & $\begin{array}{l}\text { bicyclo[2.2.1]hept-2-ene (norbornene) } \\
(338-406) \\
(301-350)\end{array}$ & $35.1 \pm 0.2$ & $\begin{array}{l}(298) \\
(316)\end{array}$ & $\begin{array}{c}\mathrm{EB} \\
\mathrm{A}\end{array}$ & $\begin{array}{c}{[498-66-8]} \\
{[96 / 5]} \\
{[87 / 5]}\end{array}$ \\
\hline $\mathrm{C}_{7} \mathrm{H}_{10}$ & $\begin{array}{l}\text { bicyclo[4.1.0]hept-3-ene } \\
(333-384)\end{array}$ & $\begin{array}{c}36.7 \\
38.4 \pm 0.6\end{array}$ & $\begin{array}{l}(348) \\
(298)\end{array}$ & $\begin{array}{c}\mathrm{A} \\
\mathrm{EB}\end{array}$ & $\begin{array}{c}{[16554-83-9]} \\
{[87 / 5]} \\
{[74 / 20]}\end{array}$ \\
\hline $\mathrm{C}_{7} \mathrm{H}_{10}$ & $\begin{array}{l}\text { tricyclo }\left[2 \cdot 2 \cdot 1 \cdot 0^{2,6}\right] \text { heptane } \\
(302-337)\end{array}$ & 38.3 & $(317)$ & A & $\begin{array}{c}{[279-19-6]} \\
{[87 / 5]}\end{array}$ \\
\hline $\mathrm{C}_{7} \mathrm{H}_{10}$ & tricyclo $\left[4 \cdot 1 \cdot 0.0^{2,4}\right]$ heptane & $36.5 \pm 0.5$ & $(298)$ & EB & [74/20] \\
\hline $\mathrm{C}_{7} \mathrm{H}_{10}$ & $\begin{array}{l}\text { tricyclo }\left[4 \cdot 1 \cdot 0.0^{2,6}\right] \text { heptane } \\
(322-373)\end{array}$ & 35.3 & $(337)$ & A & $\begin{array}{c}{[187-26-8]} \\
{[87 / 5]}\end{array}$ \\
\hline $\mathrm{C}_{7} \mathrm{H}_{10} \mathrm{~N}_{2}$ & 2,3,5-trimethylpyrazine & $53.9 \pm 1.6$ & $(298)$ & $\mathrm{C}$ & $\begin{array}{c}{[14667-55-1]} \\
{[96 / 2]}\end{array}$ \\
\hline $\mathrm{C}_{7} \mathrm{H}_{10} \mathrm{~N}_{2}$ & $\begin{array}{l}\text { diallylcyanamide } \\
(369-495)\end{array}$ & 52.3 & $(384)$ & A & $\begin{array}{c}{[538-08-9]} \\
{[87 / 5]}\end{array}$ \\
\hline $\mathrm{C}_{7} \mathrm{H}_{10} \mathrm{~N}_{2}$ & $\begin{array}{l}\text { 1,5-dicyanopentane } \\
(306-331)\end{array}$ & 74.5 & $(318)$ & A & $\begin{array}{c}{[646-20-8]} \\
{[87 / 5]}\end{array}$ \\
\hline $\mathrm{C}_{7} \mathrm{H}_{10} \mathrm{~N}_{2}$ & $\begin{array}{l}\text { 2,4-diaminotoluene } \\
(379-553)\end{array}$ & 67.7 & (394) & A & $\begin{array}{c}{[95-80-7]} \\
{[87 / 5][47 / 5]}\end{array}$ \\
\hline $\mathrm{C}_{7} \mathrm{H}_{10} \mathrm{~N}_{2}$ & $\begin{array}{l}\text { 4-tolyhydrazine } \\
(355-515)\end{array}$ & 65.4 & $(370)$ & A & $\begin{array}{l}{[539-44-6]} \\
{[87 / 5][47 / 5]}\end{array}$ \\
\hline $\mathrm{C}_{7} \mathrm{H}_{10} \mathrm{O}$ & $\begin{array}{l}\text { 1-ethynyl-1-cyclopentanol } \\
(323-373)\end{array}$ & 62.1 & $(298)$ & CGC & $\begin{array}{c}{[17356-19-3]} \\
{[95 / 21]}\end{array}$ \\
\hline $\mathrm{C}_{7} \mathrm{H}_{10} \mathrm{O}$ & $\begin{array}{l}\text { 7-norbornanone } \\
(322-348)\end{array}$ & 47.9 & $(335)$ & $\mathrm{EB}$ & $\begin{array}{c}{[10218-02-7]} \\
{[94 / 16]}\end{array}$ \\
\hline $\mathrm{C}_{7} \mathrm{H}_{10} \mathrm{O}$ & $\begin{array}{l}\text { 2-norbornanone } \\
(343-383) \\
(343-383)\end{array}$ & $\begin{array}{l}50.0 \\
51.5 \\
49.6\end{array}$ & $\begin{array}{l}(298) \\
(298) \\
(298)\end{array}$ & $\begin{array}{l}\text { GC } \\
\text { CGC } \\
\text { CGC }\end{array}$ & $\begin{array}{c}{[497-38-1]} \\
02 / 37] \\
{[95 / 21]} \\
{[95 / 21]}\end{array}$ \\
\hline $\mathrm{C}_{7} \mathrm{H}_{10} \mathrm{O}_{2}$ & $\begin{array}{l}\text { 5-methyl-5-hexene-2,4-dione } \\
(323-363)\end{array}$ & 26.4 & (338) & A & $\begin{array}{l}{[20583-46-4]} \\
{[87 / 5][73 / 18]}\end{array}$ \\
\hline $\begin{array}{l}\mathrm{C}_{7} \mathrm{H}_{10} \mathrm{O}_{3} \\
\mathrm{C}_{7} \mathrm{H}_{10} \mathrm{O}_{3}\end{array}$ & $\begin{array}{l}\text { 3-acetyl-2,4-pentanedione } \\
(369-477) \\
\text { glycidyl methacrylate }\end{array}$ & 54.9 & $(384)$ & A & $\begin{array}{c}{[815-68-9]} \\
{[87 / 5]} \\
{[106-91-2]}\end{array}$ \\
\hline
\end{tabular}


TABLE 6. Enthalpies of vaporization of organic compounds, 1880-2002-Continued

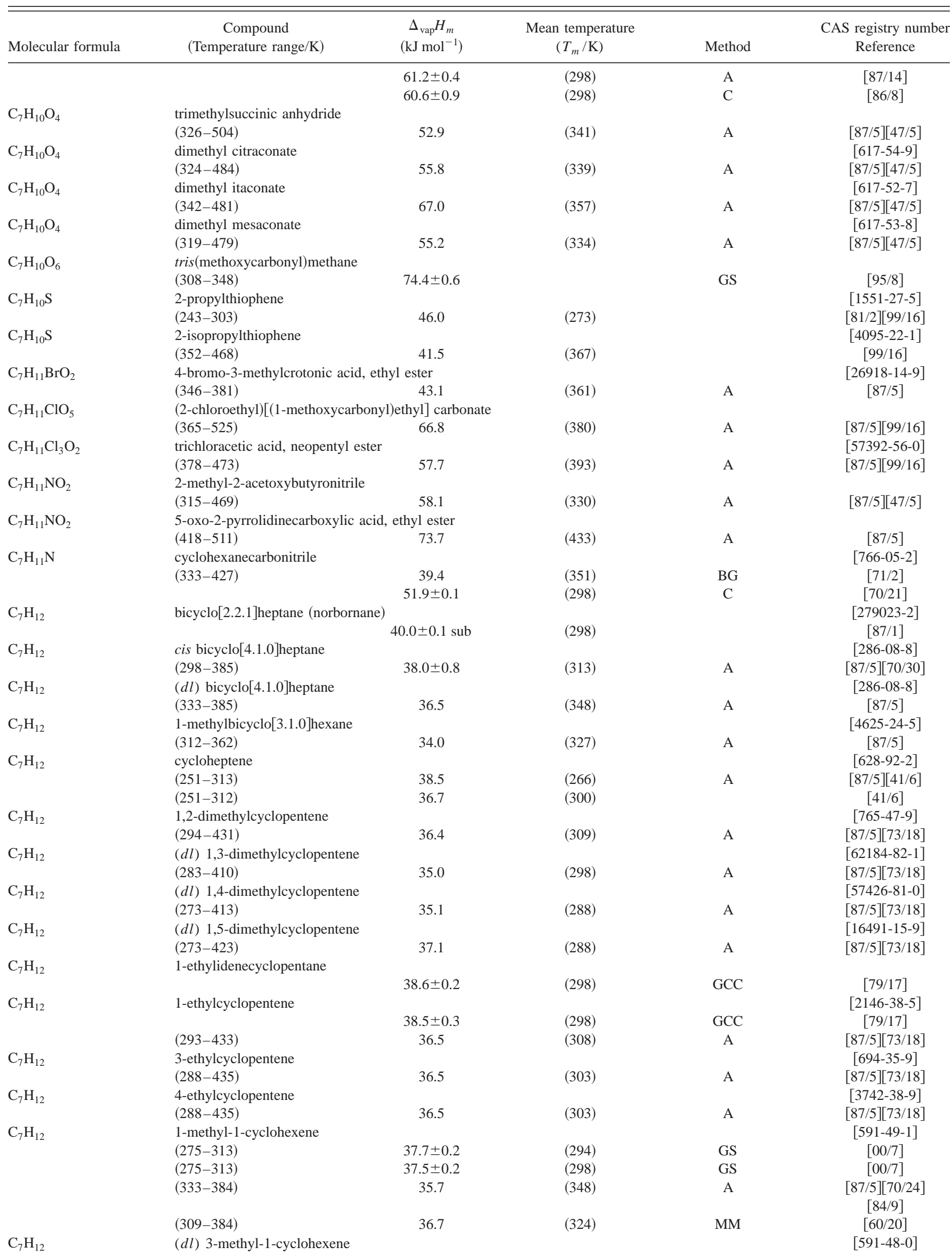


TABLE 6. Enthalpies of vaporization of organic compounds, 1880-2002-Continued

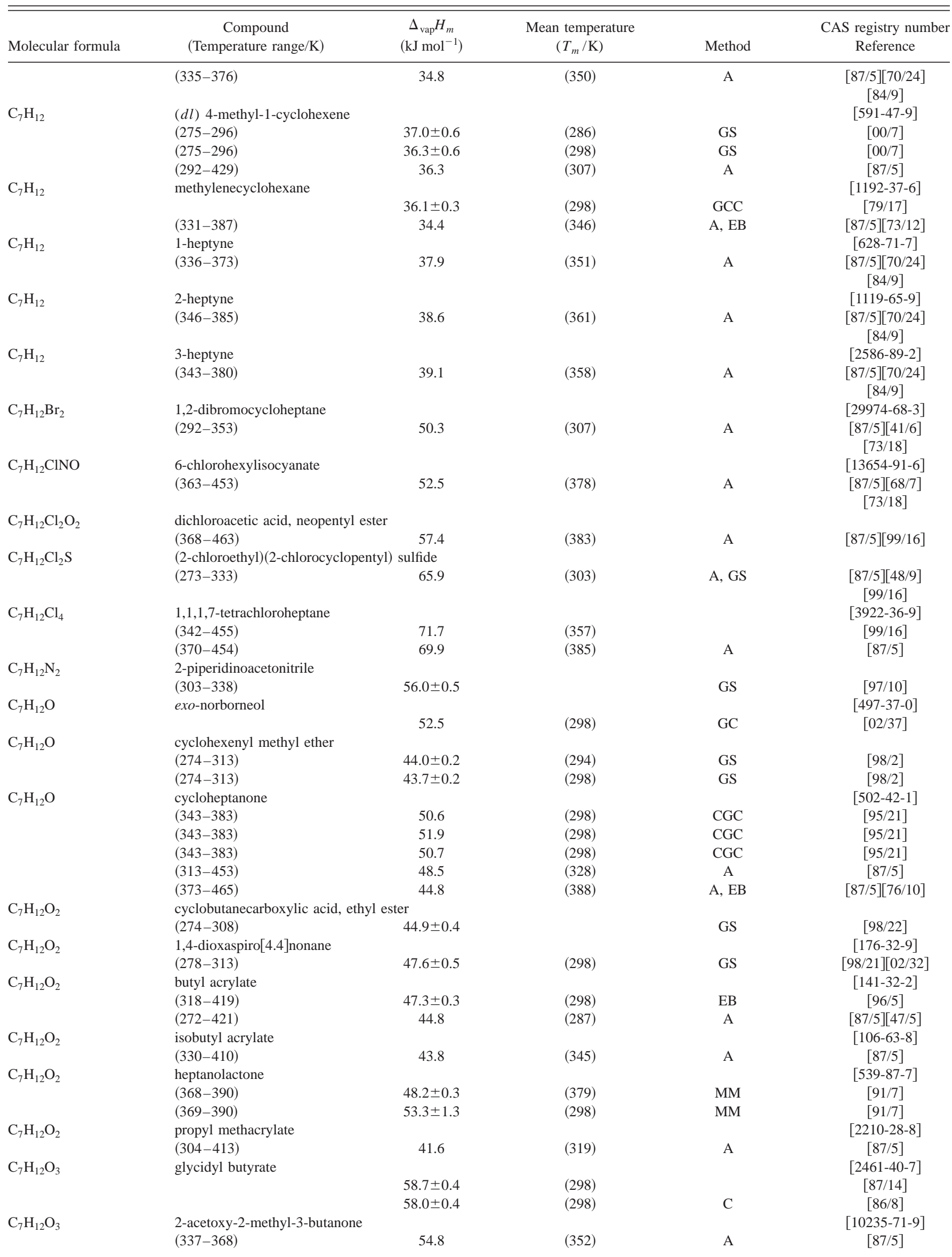


TABLE 6. Enthalpies of vaporization of organic compounds, 1880-2002-Continued

\begin{tabular}{|c|c|c|c|c|c|}
\hline Molecular formula & $\begin{array}{c}\text { Compound } \\
\text { (Temperature range/K) }\end{array}$ & $\begin{array}{c}\Delta_{\mathrm{vap}} H_{m} \\
\left(\mathrm{~kJ} \mathrm{~mol}^{-1}\right)\end{array}$ & $\begin{array}{l}\text { Mean temperature } \\
\qquad\left(T_{m} / \mathrm{K}\right)\end{array}$ & Method & $\begin{array}{c}\text { CAS registry number } \\
\text { Reference }\end{array}$ \\
\hline \multirow[t]{3}{*}{$\mathrm{C}_{7} \mathrm{H}_{12} \mathrm{O}_{3}$} & ethyl levulinate & & & & [539-88-8] \\
\hline & $(320-480)$ & 58.3 & (335) & A & {$[87 / 5][47 / 5]$} \\
\hline & & 51.6 & $(420)$ & & {$[31 / 1]$} \\
\hline \multirow[t]{2}{*}{$\mathrm{C}_{7} \mathrm{H}_{12} \mathrm{O}_{4}$} & 2-acetoxypropionic acid, ethyl ester & & & & {$[2985-28-6]$} \\
\hline & $(313-454)$ & 57.9 & $(328)$ & A & {$[87 / 5]$} \\
\hline \multirow[t]{2}{*}{$\mathrm{C}_{7} \mathrm{H}_{12} \mathrm{O}_{4}$} & 3-acetoxypropionic acid, ethyl ester & & & & [40326-37-2] \\
\hline & $(350-367)$ & 72.1 & (358) & A & {$[87 / 5][73 / 18]$} \\
\hline \multirow{2}{*}{$\mathrm{C}_{7} \mathrm{H}_{12} \mathrm{O}_{4}$} & 1,2-propylene glycol diacetate & & & & [623-84-7] \\
\hline & $(318-367)$ & 54.9 & $(323)$ & & {$[01 / 19]$} \\
\hline \multirow{5}{*}{$\mathrm{C}_{7} \mathrm{H}_{12} \mathrm{O}_{4}$} & diethyl malonate & & & & {$[105-53-3]$} \\
\hline & $(288-318)$ & $64.7 \pm 0.2$ & (293) & GS & {$[92 / 13]$} \\
\hline & $(293-318)$ & 63.3 & (305) & A & {$[87 / 5]$} \\
\hline & $(384-468)$ & 59.9 & (399) & A & {$[87 / 5]$} \\
\hline & $(313-472)$ & 51.2 & $(328)$ & $\mathrm{A}$ & {$[87 / 5][47 / 5]$} \\
\hline \multirow[t]{2}{*}{$\mathrm{C}_{7} \mathrm{H}_{12} \mathrm{O}_{4}$} & dimethyl dimethylmalonate & & & & \\
\hline & $(278-307)$ & $55.6 \pm 0.8$ & $(293)$ & GS & [92/13] \\
\hline \multirow[t]{2}{*}{$\mathrm{C}_{7} \mathrm{H}_{12} \mathrm{O}_{4}$} & glutaric acid, dimethyl ester & & & & [1119-40-0] \\
\hline & $(366-483)$ & 54.7 & (381) & A & {$[87 / 5]$} \\
\hline \multirow[t]{2}{*}{$\mathrm{C}_{7} \mathrm{H}_{12} \mathrm{O}_{4}$} & methyl adipate & & & & {$[627-91-8]$} \\
\hline & $(453-503)$ & 82.9 & $(468)$ & A & {$[87 / 5]$} \\
\hline \multirow[t]{2}{*}{$\mathrm{C}_{7} \mathrm{H}_{12} \mathrm{O}_{4}$} & heptanedioic acid (pimelic acid) & & & & [111-16-0] \\
\hline & $(436-615)$ & 88.6 & $(451)$ & A & {$[87 / 5][47 / 5]$} \\
\hline \multirow[t]{2}{*}{$\mathrm{C}_{7} \mathrm{H}_{12} \mathrm{O}_{4}$} & $2,4,8,10$-tetraoxaspiro[5.5] undecane & & & & {$[126-54-5]$} \\
\hline & & 56.0 & & & {$[59 / 23]$} \\
\hline \multirow[t]{2}{*}{$\mathrm{C}_{7} \mathrm{H}_{12} \mathrm{O}_{5}$} & ethyl[(1-methoxycarbonyl)ethyl $]$ carbo & onate & & & \\
\hline & $(343-473)$ & 60.0 & $(358)$ & A & {$[87 / 5]$} \\
\hline \multirow[t]{2}{*}{$\mathrm{C}_{7} \mathrm{H}_{12} \mathrm{O}_{5}$} & 2-(lactyloxy)propionic acid, methyl es & ester & & & \\
\hline & $(317-384)$ & 72.0 & $(332)$ & A & {$[87 / 5]$} \\
\hline \multirow[t]{2}{*}{$\mathrm{C}_{7} \mathrm{H}_{13} \mathrm{ClO}$} & heptanoyl chloride & & & & {$[2528-61-2]$} \\
\hline & $(307-418)$ & 63.7 & $(322)$ & A & {$[87 / 5][47 / 5]$} \\
\hline \multirow[t]{2}{*}{$\mathrm{C}_{7} \mathrm{H}_{13} \mathrm{ClO}_{2}$} & chloroacetic acid, neopentyl ester & & & & \\
\hline & $(378-448)$ & 55.6 & (393) & A & {$[87 / 5][99 / 16]$} \\
\hline $\mathrm{C}_{7} \mathrm{H}_{13} \mathrm{~F}_{3} \mathrm{O}_{3}$ & tris(2-fluoroethyl)orthoformate & & & & {$[2339-51-7]$} \\
\hline & $(273-333)$ & 59.7 & $(288)$ & A & {$[87 / 5][99 / 16]$} \\
\hline $\mathrm{C}_{7} \mathrm{H}_{13} \mathrm{~N}$ & 2,2-dimethylpentanenitrile & & & & \\
\hline & $(274-303)$ & $46.9 \pm 0.4$ & & GS & {$[94 / 5]$} \\
\hline $\mathrm{C}_{7} \mathrm{H}_{13} \mathrm{~N}$ & heptanonitrile & & & & {$[629-08-3]$} \\
\hline & $(313-473)$ & 46.0 & $(328)$ & $\mathrm{A}$ & {$[87 / 5]$} \\
\hline & $(294-457)$ & 46.4 & $(309)$ & & {$[47 / 5]$} \\
\hline $\mathrm{C}_{7} \mathrm{H}_{13} \mathrm{NO}$ & N-methylcaprolactam & & & & {$[2556-73-2]$} \\
\hline & $(340-400)$ & 49.4 & $(370)$ & & {$[84 / 18]$} \\
\hline $\mathrm{C}_{7} \mathrm{H}_{13} \mathrm{NO}$ & 2-butoxypropionitrile & & & & \\
\hline & $(373-423)$ & 46.7 & $(388)$ & A & {$[87 / 5][73 / 18]$} \\
\hline $\mathrm{C}_{7} \mathrm{H}_{13} \mathrm{NO}$ & 2-methoxy-3,3-dimethylbutanenitrile & & & & [162047-91-8] \\
\hline & $(295-324)$ & $58.8 \pm 1.1$ & $(298)$ & GS & {$[95 / 11]$} \\
\hline $\mathrm{C}_{7} \mathrm{H}_{13} \mathrm{NO}$ & 2-methoxy-2-methylpentanenitrile & & & & {$[162047-90-7]$} \\
\hline & $(278-308)$ & $48.5 \pm 0.6$ & $(298)$ & GS & {$[95 / 11]$} \\
\hline $\mathrm{C}_{7} \mathrm{H}_{13} \mathrm{NO}_{2}$ & lactic acid, $\mathrm{N}$-(methallyl) amide & & & & \\
\hline & $(360-428)$ & 81.8 & $(375)$ & A & {$[87 / 5]$} \\
\hline $\mathrm{C}_{7} \mathrm{H}_{13} \mathrm{NO}_{2}$ & N-lactylmorpholine & & & & \\
\hline & $(371-423)$ & 62.7 & $(386)$ & $\mathrm{A}$ & {$[87 / 5]$} \\
\hline $\mathrm{C}_{7} \mathrm{H}_{13} \mathrm{NO}_{3}$ & (dl) N-acetylalanine ethyl ester & & & & {$[5143-72-6]$} \\
\hline & $(372-460)$ & 65.2 & $(387)$ & A & {$[87 / 5][73 / 18]$} \\
\hline $\mathrm{C}_{7} \mathrm{H}_{13} \mathrm{O}_{6} \mathrm{P}$ & Mevinphos & & & & {$[7786-34-7]$} \\
\hline & $(293-383)$ & 68.1 & (308) & A & {$[87 / 5]$} \\
\hline $\mathrm{C}_{7} \mathrm{H}_{14}$ & cycloheptane & & & & {$[291-64-5]$} \\
\hline & $(282-333)$ & 38.6 & $(297)$ & A & {$[87 / 5]$} \\
\hline & $(476-604)$ & 31.7 & (491) & A & {$[87 / 5]$} \\
\hline & $(333-398)$ & 36.4 & (348) & $\mathrm{A}, \mathrm{EB}$ & {$[87 / 5][76 / 10]$} \\
\hline & $(341-433)$ & 36.1 & (356) & $\mathrm{A}, \mathrm{EB}$ & {$[87 / 5][56 / 20]$} \\
\hline & & $38.5 \pm 0.2$ & (298) & & {$[56 / 20]$} \\
\hline $\mathrm{C}_{7} \mathrm{H}_{14}$ & methylcyclohexane & & & & {$[108-87-2]$} \\
\hline & $(295-333)$ & 36.2 & $(310)$ & & {$[91 / 3]$} \\
\hline & & $35.1 \pm 0.4$ & (298) & $\mathrm{GC}$ & {$[87 / 17]$} \\
\hline
\end{tabular}


TABLE 6. Enthalpies of vaporization of organic compounds, 1880-2002-Continued

\begin{tabular}{|c|c|c|c|c|c|}
\hline Molecular formula & $\begin{array}{c}\text { Compound } \\
\text { (Temperature range/K) }\end{array}$ & $\begin{array}{c}\Delta_{\mathrm{vap}} H_{m} \\
\left(\mathrm{~kJ} \mathrm{~mol}^{-1}\right)\end{array}$ & $\begin{array}{l}\text { Mean temperature } \\
\qquad\left(T_{m} / \mathrm{K}\right)\end{array}$ & Method & $\begin{array}{c}\text { CAS registry number } \\
\text { Reference }\end{array}$ \\
\hline & $(373-511)$ & 32.3 & $(388)$ & A & {$[87 / 5]$} \\
\hline & $(501-573)$ & 31.2 & $(516)$ & A & {$[87 / 5]$} \\
\hline & & 32.2 & $(353)$ & & {$[84 / 29]$} \\
\hline & & 29.9 & (393) & & {$[84 / 29]$} \\
\hline & & 26.9 & $(433)$ & & {$[84 / 29]$} \\
\hline & & 23.4 & $(473)$ & & {$[84 / 29]$} \\
\hline & & $35.4 \pm 0.1$ & $(298)$ & $\mathrm{C}$ & {$[79 / 13]$} \\
\hline & & $34.6 \pm 0.1$ & $(313)$ & $\mathrm{C}$ & {$[79 / 13]$} \\
\hline & & $33.5 \pm 0.1$ & $(333)$ & $\mathrm{C}$ & {$[79 / 13]$} \\
\hline & & $32.5 \pm 0.1$ & $(353)$ & $\mathrm{C}$ & {$[79 / 13]$} \\
\hline & & 35.4 & $(298)$ & GCC & {$[78 / 16]$} \\
\hline & & 35.3 & $(298)$ & & {$[75 / 12]$} \\
\hline & $(308-368)$ & 34.6 & $(323)$ & A & $\begin{array}{c}{[87 / 5][70 / 25]} \\
{[84 / 9]}\end{array}$ \\
\hline & & 31.8 & $(374)$ & & {$[46 / 11]$} \\
\hline & $(299-375)$ & 34.9 & $(314)$ & MM & {$[45 / 2]$} \\
\hline & $(273-348)$ & 36.1 & $(288)$ & & {$[40 / 5]$} \\
\hline \multirow[t]{4}{*}{$\mathrm{C}_{7} \mathrm{H}_{14}$} & 1,1-dimethylcyclopentane & & & & {$[1638-26-2]$} \\
\hline & $(284-363)$ & 34.0 & $(299)$ & A & {$[87 / 5]$} \\
\hline & & 33.8 & $(298)$ & & {$[71 / 28]$} \\
\hline & $(289-362)$ & 33.8 & $(304)$ & & {$[49 / 6]$} \\
\hline \multirow[t]{4}{*}{$\mathrm{C}_{7} \mathrm{H}_{14}$} & cis 1,2-dimethylcyclopentane & & & & [1192-18-3] \\
\hline & $(293-375)$ & 35.5 & $(308)$ & A & {$[87 / 5]$} \\
\hline & & 35.8 & $(298)$ & & {$[71 / 28]$} \\
\hline & $(298-373)$ & 35.2 & $(313)$ & & {$[49 / 6]$} \\
\hline \multirow[t]{4}{*}{$\mathrm{C}_{7} \mathrm{H}_{14}$} & (dl) trans 1,2-dimethylcyclopentane & & & & {$[822-50-4]$} \\
\hline & $(295-367)$ & 34.2 & $(310)$ & A & {$[87 / 5]$} \\
\hline & & 34.6 & $(298)$ & & {$[71 / 28]$} \\
\hline & $(299-366)$ & 34.0 & $(314)$ & & {$[49 / 6]$} \\
\hline \multirow[t]{7}{*}{$\mathrm{C}_{7} \mathrm{H}_{14}$} & cis 1,3-dimethylcyclopentane & & & & {$[2532-58-3]$} \\
\hline & $(295-366)$ & 34.2 & $(310)$ & A & {$[87 / 5]$} \\
\hline & & 34.3 & $(298)$ & & {$[71 / 28]$} \\
\hline & & $32.8 \pm 0.1$ & $(323)$ & $\mathrm{C}$ & {$[59 / 8]$} \\
\hline & & $31.7 \pm 0.1$ & $(342)$ & $\mathrm{C}$ & {$[59 / 8]$} \\
\hline & & $30.4 \pm 0.1$ & $(364)$ & $\mathrm{C}$ & {$[59 / 8]$} \\
\hline & $(299-366)$ & 34.0 & $(314)$ & & {$[49 / 6]$} \\
\hline \multirow[t]{4}{*}{$\mathrm{C}_{7} \mathrm{H}_{14}$} & (dl) trans 1,3-dimethylcyclopentane & & & & {$[1759-58-6]$} \\
\hline & $(295-367)$ & 34.0 & $(310)$ & A & {$[87 / 5]$} \\
\hline & & 34.5 & $(298)$ & & {$[71 / 28]$} \\
\hline & $(291-365)$ & 34.2 & $(306)$ & & {$[49 / 6]$} \\
\hline \multirow[t]{11}{*}{$\mathrm{C}_{7} \mathrm{H}_{14}$} & ethylcyclopentane & & & & {$[1640-89-7]$} \\
\hline & $(308-387)$ & 35.5 & $(323)$ & A & {$[87 / 5]$} \\
\hline & $(386-507)$ & 32.9 & $(401)$ & A & {$[87 / 5]$} \\
\hline & $(499-569)$ & 31.9 & $(514)$ & A & {$[87 / 5]$} \\
\hline & & $35.6 \pm 0.1$ & (313) & $\mathrm{C}$ & {$[81 / 14]$} \\
\hline & & $34.8 \pm 0.1$ & $(328)$ & $\mathrm{C}$ & {$[81 / 14]$} \\
\hline & & $33.9 \pm 0.1$ & (343) & $\mathrm{C}$ & {$[81 / 14]$} \\
\hline & & $33.0 \pm 0.1$ & $(358)$ & $\mathrm{C}$ & [81/14] \\
\hline & & $32.5 \pm 0.1$ & $(368)$ & $\mathrm{C}$ & {$[81 / 14]$} \\
\hline & & 36.5 & (298) & & {$[71 / 28]$} \\
\hline & $(302-377)$ & 35.7 & $(317)$ & & [49/6] \\
\hline \multirow[t]{7}{*}{$\mathrm{C}_{7} \mathrm{H}_{14}$} & 1-heptene & & & & [592-76-7] \\
\hline & $(311-368)$ & 34.6 & $(326)$ & A & {$[87 / 5]$} \\
\hline & $(327-367)$ & 33.9 & $(342)$ & & {$[70 / 24][84 / 9]$} \\
\hline & & 35.7 & $(298)$ & & {$[71 / 28]$} \\
\hline & $(295-318)$ & 35.3 & $(310)$ & MM & {$[50 / 6]$} \\
\hline & $(255-312)$ & 35.9 & $(300)$ & & {$[41 / 6]$} \\
\hline & $(273-362)$ & 34.5 & $(288)$ & & {$[36 / 3]$} \\
\hline \multirow[t]{6}{*}{$\mathrm{C}_{7} \mathrm{H}_{14}$} & cis 2-heptene & & & & [6443-92-1] \\
\hline & $(276-304)$ & $39.0 \pm 0.3$ & $(290)$ & GS & {$[00 / 7]$} \\
\hline & $(276-304)$ & 38.6 & $(298)$ & GS & {$[00 / 7]$} \\
\hline & $(315-372)$ & 35.3 & $(330)$ & A & {$[87 / 5]$} \\
\hline & $(332-371)$ & 34.6 & $(347)$ & & {$[70 / 24][84 / 9]$} \\
\hline & & 36.0 & $(298)$ & & {$[71 / 28]$} \\
\hline
\end{tabular}


TABLE 6. Enthalpies of vaporization of organic compounds, 1880-2002-Continued

\begin{tabular}{|c|c|c|c|c|c|}
\hline Molecular formula & $\begin{array}{c}\text { Compound } \\
\text { (Temperature range/K) }\end{array}$ & $\begin{array}{c}\Delta_{\mathrm{vap}} H_{m} \\
\left(\mathrm{~kJ} \mathrm{~mol}^{-1}\right)\end{array}$ & $\begin{array}{l}\text { Mean temperature } \\
\qquad\left(T_{m} / \mathrm{K}\right)\end{array}$ & Method & $\begin{array}{c}\text { CAS registry number } \\
\text { Reference }\end{array}$ \\
\hline \multirow[t]{4}{*}{$\mathrm{C}_{7} \mathrm{H}_{14}$} & trans 2-heptene & & & & [14686-13-6] \\
\hline & $(314-373)$ & 35.3 & $(329)$ & A & {$[87 / 5]$} \\
\hline & & 36.0 & (298) & & {$[71 / 28]$} \\
\hline & $(331-370)$ & 34.6 & $(346)$ & & {$[70 / 24]$} \\
\hline \multirow[t]{3}{*}{$\mathrm{C}_{7} \mathrm{H}_{14}$} & cis 3-heptene & & & & [7642-10-6] \\
\hline & $(312-369)$ & 35.0 & $(327)$ & A & {$[87 / 5]$} \\
\hline & & 35.6 & (298) & & {$[71 / 28]$} \\
\hline \multirow[t]{3}{*}{$\mathrm{C}_{7} \mathrm{H}_{14}$} & trans 3-heptene & & & & [14686-14-7] \\
\hline & $(312-368)$ & 34.6 & $(327)$ & A & {$[87 / 5]$} \\
\hline & & 35.6 & (298) & & {$[71 / 28]$} \\
\hline \multirow[t]{3}{*}{$\mathrm{C}_{7} \mathrm{H}_{14}$} & 2-methyl-1-hexene & & & & [6094-02-6] \\
\hline & $(318-390)$ & 33.9 & (333) & A & {$[87 / 5][73 / 18]$} \\
\hline & & 35.1 & $(298)$ & & {$[71 / 28]$} \\
\hline \multirow[t]{3}{*}{$\mathrm{C}_{7} \mathrm{H}_{14}$} & $(d l)$ 3-methyl-1-hexene & & & & [3404-61-3] \\
\hline & $(311-381)$ & 33.4 & $(326)$ & $\mathrm{A}$ & {$[87 / 5][73 / 18]$} \\
\hline & & 34.3 & $(298)$ & & {$[71 / 28]$} \\
\hline \multirow[t]{3}{*}{$\mathrm{C}_{7} \mathrm{H}_{14}$} & $(d l)$ 4-methyl-1-hexene & & & & [3769-23-1] \\
\hline & $(313-384)$ & 33.6 & $(328)$ & A & {$[87 / 5][73 / 18]$} \\
\hline & & 34.7 & (298) & & {$[71 / 28]$} \\
\hline \multirow[t]{3}{*}{$\mathrm{C}_{7} \mathrm{H}_{14}$} & 5-methyl-1-hexene & & & & [3524-73-0] \\
\hline & $(313-393)$ & 33.5 & (328) & A & {$[87 / 5][73 / 18]$} \\
\hline & & 34.3 & $(298)$ & & {$[71 / 28]$} \\
\hline \multirow[t]{3}{*}{$\mathrm{C}_{7} \mathrm{H}_{14}$} & 2-methyl-2-hexene & & & & [2738-19-4] \\
\hline & $(322-394)$ & 34.0 & $(337)$ & A & {$[87 / 5][73 / 18]$} \\
\hline & & 35.6 & (298) & & {$[71 / 28]$} \\
\hline \multirow[t]{3}{*}{$\mathrm{C}_{7} \mathrm{H}_{14}$} & cis 3-methyl-2-hexene & & & & [10574-36-4] \\
\hline & $(322-396)$ & 34.2 & $(337)$ & A & {$[87 / 5][73 / 18]$} \\
\hline & & 35.6 & (298) & & {$[71 / 28]$} \\
\hline \multirow[t]{3}{*}{$\mathrm{C}_{7} \mathrm{H}_{14}$} & trans 3-methyl-2-hexene & & & & [20710-38-7] \\
\hline & $(321-394)$ & 34.1 & $(336)$ & A & {$[87 / 5][73 / 18]$} \\
\hline & & 35.6 & (298) & & {$[71 / 28]$} \\
\hline \multirow[t]{3}{*}{$\mathrm{C}_{7} \mathrm{H}_{14}$} & cis 4-methyl-2-hexene & & & & [3683-19-0] \\
\hline & $(313-384)$ & 33.5 & $(328)$ & $\mathrm{A}$ & {$[87 / 5][73 / 18]$} \\
\hline & & 34.7 & (298) & & {$[71 / 28]$} \\
\hline \multirow[t]{3}{*}{$\mathrm{C}_{7} \mathrm{H}_{14}$} & trans 4-methyl-2-hexene & & & & {$[3683-22-5]$} \\
\hline & $(314-385)$ & 33.6 & (329) & A & {$[87 / 5][73 / 18]$} \\
\hline & & 34.7 & (298) & & {$[71 / 28]$} \\
\hline \multirow[t]{3}{*}{$\mathrm{C}_{7} \mathrm{H}_{14}$} & cis 5-methyl-2-hexene & & & & [13151-17-2] \\
\hline & $(354-372)$ & 32.6 & $(363)$ & A & {$[87 / 5][73 / 18]$} \\
\hline & & 34.7 & (298) & & {$[71 / 28]$} \\
\hline \multirow[t]{3}{*}{$\mathrm{C}_{7} \mathrm{H}_{14}$} & trans 5-methyl-2-hexene & & & & {$[7385-82-2]$} \\
\hline & $(315-386)$ & 33.6 & $(330)$ & A & {$[87 / 5][73 / 18]$} \\
\hline & & 34.7 & (298) & & {$[71 / 28]$} \\
\hline $\mathrm{C}_{7} \mathrm{H}_{14}$ & cis 2-methyl-3-hexene & & & & [15840-60-5] \\
\hline & $(262-383)$ & 36.1 & $(277)$ & $\mathrm{A}$ & {$[87 / 5][73 / 18]$} \\
\hline & & 34.3 & $(298)$ & & {$[71 / 28]$} \\
\hline $\mathrm{C}_{7} \mathrm{H}_{14}$ & trans 2-methyl-3-hexene & & & & [692-24-0] \\
\hline & $(313-383)$ & 33.5 & $(328)$ & A & {$[87 / 5][73 / 18]$} \\
\hline & & 34.3 & $(298)$ & & {$[71 / 28]$} \\
\hline $\mathrm{C}_{7} \mathrm{H}_{14}$ & cis 3-methyl-3-hexene & & & & [4914-89-0] \\
\hline & $(307-375)$ & 35.4 & (322) & A & {$[87 / 5]$} \\
\hline & & 36.4 & (298) & & {$[71 / 28]$} \\
\hline & $(302-368)$ & 35.7 & $(317)$ & MM & {$[60 / 20]$} \\
\hline $\mathrm{C}_{7} \mathrm{H}_{14}$ & trans 3-methyl-3-hexene & & & & [3899-36-3] \\
\hline & $(310-368)$ & 34.8 & (325) & A & {$[87 / 5]$} \\
\hline & & 35.8 & (298) & & {$[71 / 28]$} \\
\hline & $(300-367)$ & 35.3 & $(315)$ & MM & {$[60 / 20]$} \\
\hline $\mathrm{C}_{7} \mathrm{H}_{14}$ & ( $d l$ ) 2,3-dimethylpent-1-ene & & & & [3404-72-6] \\
\hline & $(311-382)$ & 33.4 & $(326)$ & A & {$[87 / 5][73 / 18]$} \\
\hline $\mathrm{C}_{7} \mathrm{H}_{14}$ & 2,3-dimethylpent-2-ene & & & & \\
\hline & & 34.3 & $(298)$ & & {$[71 / 28]$} \\
\hline $\mathrm{C}_{7} \mathrm{H}_{14}$ & 2,4-dimethylpent-1-ene & & & & [2213-32-3] \\
\hline & $(311-361)$ & 32.3 & (326) & A & {$[87 / 5]$} \\
\hline & & 33.1 & $(298)$ & & {$[71 / 28]$} \\
\hline
\end{tabular}


TABLE 6. Enthalpies of vaporization of organic compounds, 1880-2002-Continued

\begin{tabular}{|c|c|c|c|c|c|}
\hline Molecular formula & $\begin{array}{c}\text { Compound } \\
\text { (Temperature range/K) }\end{array}$ & $\begin{array}{c}\Delta_{\mathrm{vap}} H_{m} \\
\left(\mathrm{~kJ} \mathrm{~mol}^{-1}\right)\end{array}$ & $\begin{array}{l}\text { Mean temperature } \\
\qquad\left(T_{m} / \mathrm{K}\right)\end{array}$ & Method & $\begin{array}{c}\text { CAS registry number } \\
\text { Reference }\end{array}$ \\
\hline & $(289-355)$ & 33.2 & $(304)$ & MM & {$[60 / 20]$} \\
\hline \multirow[t]{3}{*}{$\mathrm{C}_{7} \mathrm{H}_{14}$} & 3,3-dimethylpent-1-ene & & & & [3404-73-7] \\
\hline & $(306-374)$ & 33.0 & $(321)$ & A & {$[87 / 5][73 / 18]$} \\
\hline & & 33.5 & (298) & & {$[71 / 28]$} \\
\hline \multirow[t]{3}{*}{$\mathrm{C}_{7} \mathrm{H}_{14}$} & ( $d l$ ) 3,4-dimethylpent-1-ene & & & & [7385-78-6] \\
\hline & $(309-378)$ & 33.2 & $(324)$ & A & {$[87 / 5][73 / 18]$} \\
\hline & & 33.9 & $(298)$ & & {$[71 / 28]$} \\
\hline \multirow[t]{4}{*}{$\mathrm{C}_{7} \mathrm{H}_{14}$} & 4,4-dimethylpent-1-ene & & & & {$[762-62-9]$} \\
\hline & $(299-347)$ & 31.0 & (314) & A & {$[87 / 5]$} \\
\hline & & 31.2 & (298) & & {$[71 / 28]$} \\
\hline & $(290-346)$ & 31.0 & $(315)$ & MM & {$[60 / 20]$} \\
\hline \multirow[t]{3}{*}{$\mathrm{C}_{7} \mathrm{H}_{14}$} & 2,3-dimethylpent-2-ene & & & & [10574-37-5] \\
\hline & $(322-396)$ & 34.2 & $(337)$ & $\mathrm{A}$ & {$[87 / 5][73 / 18]$} \\
\hline & & 35.6 & (298) & & {$[71 / 28]$} \\
\hline \multirow[t]{6}{*}{$\mathrm{C}_{7} \mathrm{H}_{14}$} & 2,4-dimethylpent-2-ene & & & & {$[625-65-0]$} \\
\hline & $(276-297)$ & $35.2 \pm 1.5$ & (286) & GS & {$[00 / 7]$} \\
\hline & $(276-297)$ & $34.5 \pm 1.5$ & (298) & GS & {$[00 / 7]$} \\
\hline & $(286-363)$ & 34.5 & $(301)$ & A & {$[87 / 5]$} \\
\hline & & 34.3 & (298) & & {$[71 / 28]$} \\
\hline & $(292-357)$ & 34.2 & $(307)$ & MM & {$[60 / 20]$} \\
\hline \multirow[t]{3}{*}{$\mathrm{C}_{7} \mathrm{H}_{14}$} & cis 3,4-dimethylpent-2-ene & & & & [4914-91-4] \\
\hline & $(316-387)$ & 33.7 & $(331)$ & $\mathrm{A}$ & {$[87 / 5][73 / 18]$} \\
\hline & & 34.7 & $(298)$ & & {$[71 / 28]$} \\
\hline \multirow[t]{3}{*}{$\mathrm{C}_{7} \mathrm{H}_{14}$} & trans 3,4-dimethylpent-2-ene & & & & [4914-92-5] \\
\hline & $(317-390)$ & 33.9 & $(332)$ & $\mathrm{A}$ & {$[87 / 5][73 / 18]$} \\
\hline & & 35.1 & (298) & & {$[71 / 28]$} \\
\hline \multirow[t]{4}{*}{$\mathrm{C}_{7} \mathrm{H}_{14}$} & cis 4,4-dimethylpent-2-ene & & & & {$[762-63-0]$} \\
\hline & $(303-355)$ & 32.2 & $(318)$ & A & {$[87 / 5]$} \\
\hline & & 32.6 & $(298)$ & & {$[71 / 28]$} \\
\hline & $(291-354)$ & 32.6 & (306) & MM & {$[60 / 20]$} \\
\hline \multirow{4}{*}{$\mathrm{C}_{7} \mathrm{H}_{14}$} & trans 4,4-dimethylpent-2-ene & & & & {$[690-08-4]$} \\
\hline & $(295-352)$ & 32.8 & $(310)$ & A & {$[87 / 5]$} \\
\hline & & 32.8 & (298) & & {$[71 / 28]$} \\
\hline & $(289-350)$ & 33.0 & (304) & MM & {$[60 / 20]$} \\
\hline \multirow[t]{4}{*}{$\mathrm{C}_{7} \mathrm{H}_{14}$} & 2-ethyl-3-methyl-1-butene & & & & {$[7357-93-9]$} \\
\hline & $(303-381)$ & 33.8 & $(318)$ & A & {$[87 / 5]$} \\
\hline & & 34.3 & (298) & & {$[71 / 28]$} \\
\hline & $(290-360)$ & 34.4 & (305) & MM & {$[60 / 20]$} \\
\hline \multirow[t]{3}{*}{$\mathrm{C}_{7} \mathrm{H}_{14}$} & 2-ethyl-1-pentene & & & & [3404-71-5] \\
\hline & $(267-392)$ & 36.6 & (282) & A & {$[87 / 5][73 / 18]$} \\
\hline & & 35.1 & (298) & & {$[71 / 28]$} \\
\hline \multirow[t]{3}{*}{$\mathrm{C}_{7} \mathrm{H}_{14}$} & 3-ethyl-1-pentene & & & & [4038-04-4] \\
\hline & $(311-382)$ & 33.4 & $(326)$ & A & {$[87 / 5][73 / 18]$} \\
\hline & & 34.3 & (298) & & {$[71 / 28]$} \\
\hline \multirow[t]{3}{*}{$\mathrm{C}_{7} \mathrm{H}_{14}$} & 3-ethyl-2-pentene & & & & [816-79-5] \\
\hline & $(321-395)$ & 34.1 & $(336)$ & A & {$[87 / 5][73 / 18]$} \\
\hline & & 35.6 & (298) & & {$[71 / 28]$} \\
\hline \multirow[t]{4}{*}{$\mathrm{C}_{7} \mathrm{H}_{14}$} & 2,3,3-trimethyl-1-butene & & & & [594-56-9] \\
\hline & $(288-353)$ & 32.4 & (303) & A & {$[87 / 5]$} \\
\hline & & 34.3 & (298) & & {$[71 / 28]$} \\
\hline & $(288-351)$ & 32.1 & (303) & MM & {$[60 / 20]$} \\
\hline \multirow[t]{2}{*}{$\mathrm{C}_{7} \mathrm{H}_{14} \mathrm{Br}_{2}$} & 1,1-dibromoheptane & & & & [59104-79-9] \\
\hline & $(395-548)$ & 54.4 & (410) & A, EST & $\begin{array}{c}{[87 / 5][56 / 16]} \\
{[70 / 14]}\end{array}$ \\
\hline $\mathrm{C}_{7} \mathrm{H}_{14} \mathrm{Br}_{2}$ & ( $d l$ ) 1,2-dibromoheptane & & & & [42474-21-5] \\
\hline & $(295-553)$ & 52.9 & $(310)$ & A & $\begin{array}{c}{[87 / 5][73 / 18]} \\
{[99 / 16]}\end{array}$ \\
\hline & $(295-355)$ & 54.4 & $(300)$ & & {$[41 / 6]$} \\
\hline $\mathrm{C}_{7} \mathrm{H}_{14} \mathrm{Cl}_{2}$ & 1,1-dichloroheptane & & & & [821-25-0] \\
\hline & $(375-460)$ & 53.5 & (298) & & {$[87 / 12][91 / 2]$} \\
\hline & $(364-510)$ & 48.4 & $(379)$ & A, EST & $\begin{array}{c}{[87 / 5][56 / 16]} \\
{[70 / 14]}\end{array}$ \\
\hline $\mathrm{C}_{7} \mathrm{H}_{14} \mathrm{Cl}_{2}$ & 1,2-dichloroheptane & & & & [10575-87-8] \\
\hline & $(353-466)$ & 49.0 & (368) & & [99/16] \\
\hline
\end{tabular}


TABLE 6. Enthalpies of vaporization of organic compounds, 1880-2002-Continued

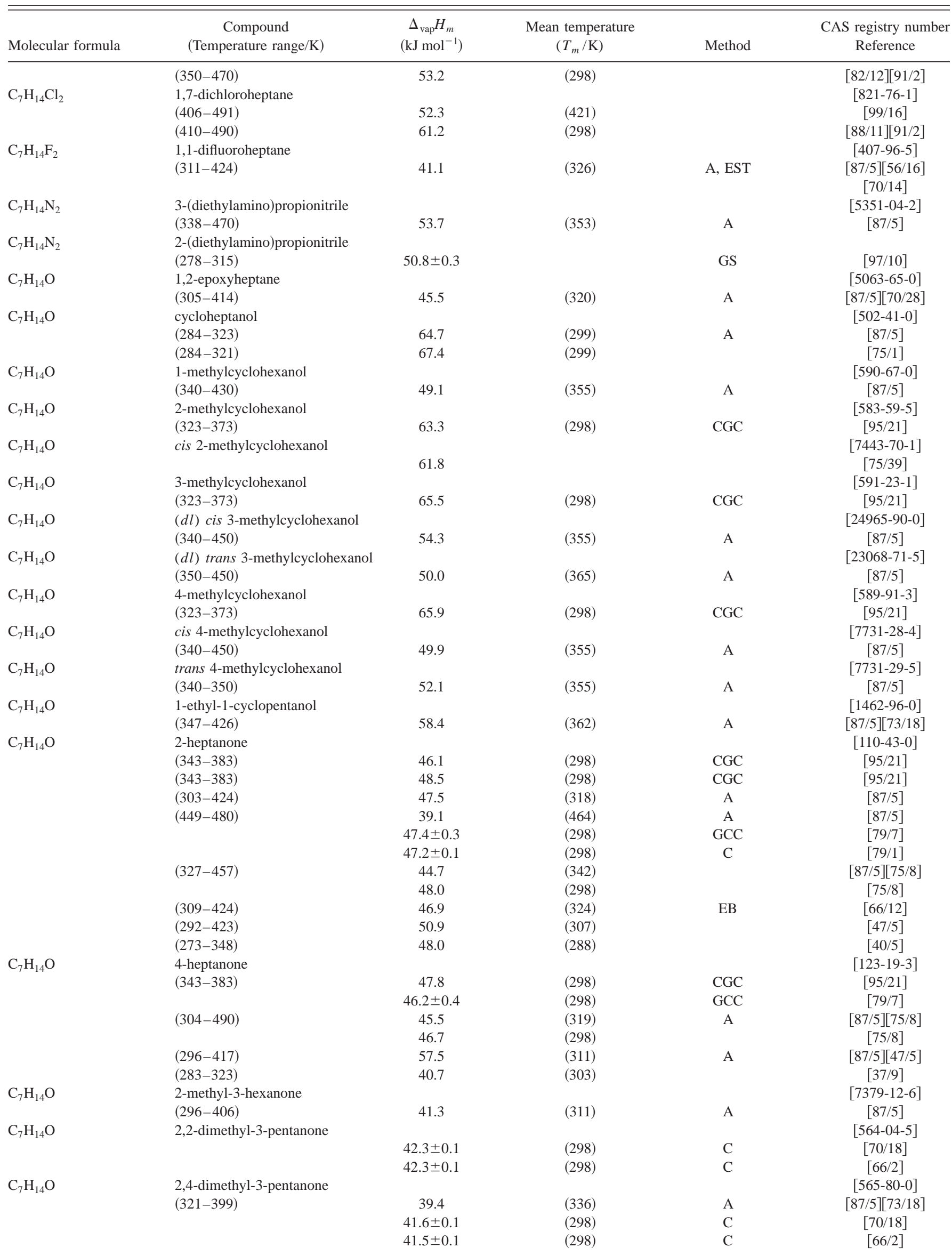


TABLE 6. Enthalpies of vaporization of organic compounds, 1880-2002-Continued

\begin{tabular}{|c|c|c|c|c|c|}
\hline Molecular formula & $\begin{array}{c}\text { Compound } \\
\text { (Temperature range/K) }\end{array}$ & $\begin{array}{c}\Delta_{\mathrm{vap}} H_{m} \\
\left(\mathrm{~kJ} \mathrm{~mol}^{-1}\right)\end{array}$ & $\begin{array}{l}\text { Mean temperature } \\
\qquad\left(T_{m} / \mathrm{K}\right)\end{array}$ & Method & $\begin{array}{c}\text { CAS registry number } \\
\text { Reference }\end{array}$ \\
\hline \multirow[t]{4}{*}{$\mathrm{C}_{7} \mathrm{H}_{14} \mathrm{O}$} & heptanal & & & & {$[111-71-7]$} \\
\hline & $(313-353)$ & 48.7 & $(298)$ & CGC & {$[95 / 21]$} \\
\hline & & $47.3 \pm 0.1$ & $(298)$ & & {$[81 / 18]$} \\
\hline & $(285-428)$ & 62.0 & $(300)$ & & {$[47 / 5]$} \\
\hline \multirow[t]{2}{*}{$\mathrm{C}_{7} \mathrm{H}_{14} \mathrm{O}$} & 3-methylhexanal & & & & [19269-28-4] \\
\hline & $(314-417)$ & 42.8 & $(329)$ & EB & {$[87 / 10]$} \\
\hline \multirow[t]{2}{*}{$\mathrm{C}_{7} \mathrm{H}_{14} \mathrm{O}$} & 3,4-dimethylpentanal & & & & [19353-21-0] \\
\hline & $(319-417)$ & 42.4 & $(334)$ & EB & {$[87 / 10]$} \\
\hline \multirow{3}{*}{$\mathrm{C}_{7} \mathrm{H}_{14} \mathrm{O}_{2}$} & 1,1-dimethoxycyclopentane & & & & [931-94-2] \\
\hline & $(278-318)$ & $44.5 \pm 0.3$ & (298) & GS & {$[98 / 21][02 / 32]$} \\
\hline & $(307-343)$ & 46.1 & $(325)$ & EB & [94/16] \\
\hline \multirow[t]{10}{*}{$\mathrm{C}_{7} \mathrm{H}_{14} \mathrm{O}_{2}$} & methyl hexanoate & & & & {$[106-70-7]$} \\
\hline & & 45.2 & $(350)$ & & {$[02 / 27]$} \\
\hline & & $46.4 \pm 0.1$ & (325) & & {$[02 / 27]$} \\
\hline & & $47.7 \pm 0.1$ & (298) & & {$[02 / 27]$} \\
\hline & $(313-363)$ & 47.9 & (298) & CGC & {$[95 / 21]$} \\
\hline & $(313-353)$ & 48.2 & (298) & CGC & {$[95 / 21]$} \\
\hline & & $47.8 \pm 0.5$ & (298) & GC & [87/17] \\
\hline & & $48.7 \pm 0.3$ & $(298)$ & GCC & {$[80 / 5]$} \\
\hline & & $48.0 \pm 0.1$ & (298) & $\mathrm{C}$ & {$[77 / 1]$} \\
\hline & $(315-383)$ & 45.3 & (330) & A & {$[87 / 5][63 / 16]$} \\
\hline \multirow[t]{3}{*}{$\mathrm{C}_{7} \mathrm{H}_{14} \mathrm{O}_{2}$} & butyl propionate & & & & [590-01-2] \\
\hline & $(305-417)$ & 49.1 & (320) & A & {$[87 / 5]$} \\
\hline & $(305-365)$ & 47.4 & $(320)$ & & {$[59 / 13][84 / 9]$} \\
\hline \multirow[t]{5}{*}{$\mathrm{C}_{7} \mathrm{H}_{14} \mathrm{O}_{2}$} & ethyl pivalate & & & & [3938-95-2] \\
\hline & $(308-429)$ & $39.8 \pm 0.1$ & $(320)$ & EB & {$[02 / 16]$} \\
\hline & $(308-429)$ & $36.9 \pm 0.2$ & $(360)$ & EB & {$[02 / 16]$} \\
\hline & $(308-429)$ & $33.8 \pm 0.6$ & $(400)$ & EB & {$[02 / 16]$} \\
\hline & & $41.3 \pm 0.1$ & (298) & $\mathrm{C}$ & {$[66 / 2]$} \\
\hline \multirow[t]{3}{*}{$\mathrm{C}_{7} \mathrm{H}_{14} \mathrm{O}_{2}$} & ethyl isovalerate & & & & {$[108-64-5]$} \\
\hline & $(301-418)$ & 42.8 & $(316)$ & $\mathrm{A}$ & {$[87 / 5]$} \\
\hline & $(267-407)$ & 44.5 & $(282)$ & & {$[47 / 5]$} \\
\hline \multirow[t]{2}{*}{$\mathrm{C}_{7} \mathrm{H}_{14} \mathrm{O}_{2}$} & isobutyl propionate & & & & {$[540-42-1]$} \\
\hline & $(271-410)$ & 44.9 & $(286)$ & A & {$[87 / 5][47 / 5]$} \\
\hline \multirow[t]{6}{*}{$\mathrm{C}_{7} \mathrm{H}_{14} \mathrm{O}_{2}$} & isopentyl acetate & & & & {$[123-92-2]$} \\
\hline & $(230-435)$ & 46.4 & $(300)$ & & {$[99 / 27]$} \\
\hline & $(278-305)$ & $46.8 \pm 0.2$ & $(292)$ & GS & {$[99 / 4]$} \\
\hline & $(278-305)$ & $46.4 \pm 0.2$ & $(298)$ & GS & {$[99 / 4]$} \\
\hline & $(308-424)$ & 44.3 & $(323)$ & A & {$[87 / 5]$} \\
\hline & $(313-368)$ & 45.1 & $(328)$ & & {$[59 / 15][84 / 9]$} \\
\hline \multirow[t]{2}{*}{$\mathrm{C}_{7} \mathrm{H}_{14} \mathrm{O}_{2}$} & isopropyl isobutyrate & & & & [617-50-5] \\
\hline & $(257-394)$ & 43.3 & $(272)$ & A & {$[87 / 5][47 / 5]$} \\
\hline \multirow[t]{2}{*}{$\mathrm{C}_{7} \mathrm{H}_{14} \mathrm{O}_{2}$} & 4-methoxy-4-methyl-2-pentanone & & & & {$[107-70-0]$} \\
\hline & $(343-423)$ & 45.0 & $(358)$ & A & {$[87 / 5]$} \\
\hline \multirow[t]{2}{*}{$\mathrm{C}_{7} \mathrm{H}_{14} \mathrm{O}_{2}$} & methyl tert-butylacetate & & & & {$[10250-48-3]$} \\
\hline & $(274-313)$ & $44.4 \pm 0.2$ & $(298)$ & GS & {$[96 / 11]$} \\
\hline \multirow[t]{2}{*}{$\mathrm{C}_{7} \mathrm{H}_{14} \mathrm{O}_{2}$} & neopentyl acetate & & & & {$[926-41-0]$} \\
\hline & $(301-400)$ & 49.1 & $(316)$ & A & {$[87 / 5]$} \\
\hline $\mathrm{C}_{7} \mathrm{H}_{14} \mathrm{O}_{2}$ & tert-pentyl acetate & & & & [625-16-1] \\
\hline & $(274-308)$ & $42.8 \pm 0.3$ & $(298)$ & GS & {$[96 / 11]$} \\
\hline $\mathrm{C}_{7} \mathrm{H}_{14} \mathrm{O}_{2}$ & pentyl acetate & & & & [628-63-7] \\
\hline & $(321-462)$ & $48.6 \pm 0.4$ & $(298)$ & EB & {$[96 / 3]$} \\
\hline & $(329-423)$ & 43.2 & $(344)$ & A & {$[87 / 5]$} \\
\hline $\mathrm{C}_{7} \mathrm{H}_{14} \mathrm{O}_{2}$ & propyl butyrate & & & & [105-66-8] \\
\hline & $(390-430)$ & 39.6 & $(405)$ & & {$[95 / 17]$} \\
\hline & $(355-416)$ & 42.0 & $(370)$ & & {$[93 / 8]$} \\
\hline & $(271-416)$ & 44.3 & (286) & A & {$[87 / 5][47 / 5]$} \\
\hline $\mathrm{C}_{7} \mathrm{H}_{14} \mathrm{O}_{2}$ & propyl isobutyrate & & & & {$[644-49-5]$} \\
\hline & $(267-407)$ & 50.5 & $(282)$ & A & {$[87 / 5][47 / 5]$} \\
\hline $\mathrm{C}_{7} \mathrm{H}_{14} \mathrm{O}_{2}$ & ethyl 2-methylbutanoate & & & & \\
\hline & $(288-308)$ & 64.7 & $(298)$ & GS & [92/13] \\
\hline $\mathrm{C}_{7} \mathrm{H}_{14} \mathrm{O}_{2}$ & butyl glycidyl ether & & & & [2426-08-6] \\
\hline & & $53.3 \pm 0.4$ & & & {$[87 / 14]$} \\
\hline $\mathrm{C}_{7} \mathrm{H}_{14} \mathrm{O}_{2}$ & tert-butyl glycidyl ether & & & & {$[7665-72-7]$} \\
\hline
\end{tabular}


TABLE 6. Enthalpies of vaporization of organic compounds, 1880-2002-Continued

\begin{tabular}{|c|c|c|c|c|c|}
\hline Molecular formula & $\begin{array}{c}\text { Compound } \\
\text { (Temperature range/K) }\end{array}$ & $\begin{array}{c}\Delta_{\mathrm{vap}} H_{m} \\
\left(\mathrm{~kJ} \mathrm{~mol}^{-1}\right)\end{array}$ & $\begin{array}{l}\text { Mean temperature } \\
\qquad\left(T_{m} / \mathrm{K}\right)\end{array}$ & Method & $\begin{array}{c}\text { CAS registry number } \\
\text { Reference }\end{array}$ \\
\hline & & $50.2 \pm 0.4$ & & & {$[87 / 14]$} \\
\hline \multirow[t]{2}{*}{$\mathrm{C}_{7} \mathrm{H}_{14} \mathrm{O}_{2}$} & 2,2,4-trimethyl-1,3-dioxane & & & & \\
\hline & & $41.9 \pm 1.2$ & $(298)$ & & {$[67 / 37]$} \\
\hline \multirow[t]{2}{*}{$\mathrm{C}_{7} \mathrm{H}_{14} \mathrm{O}_{2}$} & 2-methyl-2-propyl-1,3-dioxolane & & & & [4352-98-1] \\
\hline & $(278-313)$ & $46.3 \pm 0.3$ & $(298)$ & GS & {$[98 / 21][02 / 32]$} \\
\hline \multirow[t]{3}{*}{$\mathrm{C}_{7} \mathrm{H}_{14} \mathrm{O}_{2}$} & 2-methyl-2-isopropyl-1,3-dioxolane & & & & [4405-16-7] \\
\hline & $(274-303)$ & $43.9 \pm 0.2$ & $(298)$ & GS & {$[02 / 32]$} \\
\hline & $(273-303)$ & $44.6 \pm 0.2$ & & GS & {$[98 / 21]$} \\
\hline \multirow{2}{*}{$\mathrm{C}_{7} \mathrm{H}_{14} \mathrm{O}_{2}$} & [(1-methylpropoxy)methyl]oxirane & & & & [3814-55-9] \\
\hline & & $45.2 \pm 1.8$ & & & {$[87 / 14]$} \\
\hline \multirow{7}{*}{$\mathrm{C}_{7} \mathrm{H}_{14} \mathrm{O}_{2}$} & heptanoic acid & & & & {$[111-14-8]$} \\
\hline & $(283-328)$ & $72.5 \pm 0.8$ & $(306)$ & GS & {$[00 / 6]$} \\
\hline & $(283-328)$ & $72.9 \pm 0.8$ & (298) & GS & {$[00 / 6]$} \\
\hline & $(353-393)$ & 75.7 & (298) & $\mathrm{CGC}$ & {$[95 / 21]$} \\
\hline & & 76.0 & $(266)$ & & {$[82 / 4]$} \\
\hline & $(271-291)$ & $72.0 \pm 1.5$ & (298) & $\mathrm{TE}$ & {$[79 / 4]$} \\
\hline & $(351-495)$ & 68.3 & $(366)$ & A & {$[87 / 5][47 / 5]$} \\
\hline \multirow{2}{*}{$\mathrm{C}_{7} \mathrm{H}_{14} \mathrm{O}_{3}$} & tert-butylperoxymethyloxirane & & & & {$[33415-52-0]$} \\
\hline & & $53.9 \pm 0.4$ & & & {$[87 / 14]$} \\
\hline \multirow[t]{2}{*}{$\mathrm{C}_{7} \mathrm{H}_{14} \mathrm{O}_{3}$} & 2-propoxyethylacetate & & & & [20706-25-6] \\
\hline & & $55.6 \pm 0.1$ & $(298)$ & $\mathrm{C}$ & {$[70 / 17]$} \\
\hline \multirow[t]{2}{*}{$\mathrm{C}_{7} \mathrm{H}_{14} \mathrm{O}_{3}$} & $(d l)$ butyl lactate & & & & {$[138-22-7]$} \\
\hline & $(339-456)$ & 58.7 & $(354)$ & A & {$[87 / 5]$} \\
\hline \multirow[t]{2}{*}{$\mathrm{C}_{7} \mathrm{H}_{14} \mathrm{O}_{3}$} & 3-ethoxypropionic acid, ethyl ester & & & & {$[763-69-9]$} \\
\hline & $(312-446)$ & 45.5 & $(327)$ & A & {$[87 / 5]$} \\
\hline \multirow[t]{2}{*}{$\mathrm{C}_{7} \mathrm{H}_{14} \mathrm{O}_{3}$} & 1-heptene ozonide & & & & {$[768-63-8]$} \\
\hline & $(353-373)$ & 44.4 & $(363)$ & A & {$[87 / 5][77 / 9]$} \\
\hline \multirow[t]{2}{*}{$\mathrm{C}_{7} \mathrm{H}_{14} \mathrm{O}_{3}$} & 4-(2-hydroxyethyl)-4-methyl-1,3-diox & xane & & & \\
\hline & $(329-455)$ & 51.7 & $(344)$ & $\mathrm{A}$ & {$[87 / 5]$} \\
\hline \multirow[t]{2}{*}{$\mathrm{C}_{7} \mathrm{H}_{14} \mathrm{O}_{3}$} & 3-hydroxypropionic acid, butyl ester & & & & \\
\hline & $(361-382)$ & 60.3 & $(371)$ & A & {$[87 / 5][73 / 18]$} \\
\hline \multirow[t]{2}{*}{$\mathrm{C}_{7} \mathrm{H}_{14} \mathrm{O}_{3}$} & 3-methoxypropionic acid, propyl ester & & & & {$[5349-56-4]$} \\
\hline & $(323-433)$ & 47.0 & $(338)$ & A & {$[87 / 5]$} \\
\hline \multirow[t]{2}{*}{$\mathrm{C}_{7} \mathrm{H}_{14} \mathrm{O}_{3}$} & 3-propoxypropionic acid, methyl ester & & & & [14144-39-9] \\
\hline & $(323-453)$ & 46.6 & $(338)$ & A & {$[87 / 5]$} \\
\hline $\mathrm{C}_{7} \mathrm{H}_{14} \mathrm{O}_{3}$ & $(\mathrm{dl})$ butyric acid, 2,3-dihydroxypropy & yl ester & & & {$[557-25-5]$} \\
\hline & $(392-449)$ & 80.4 & $(407)$ & A & {$[87 / 5]$} \\
\hline $\mathrm{C}_{7} \mathrm{H}_{14} \mathrm{O}_{3}$ & 2-butoxypropionic acid & & & & {$[14620-87-2]$} \\
\hline & $(373-473)$ & 52.8 & $(388)$ & A & {$[87 / 5][73 / 18]$} \\
\hline $\mathrm{C}_{7} \mathrm{H}_{14} \mathrm{~S}$ & allyl tert-butyl sulfide & & & & [37850-75-2] \\
\hline & $(319-339)$ & 41.9 & $(332)$ & & {$[99 / 16]$} \\
\hline & $(319-339)$ & 43.1 & (329) & $\mathrm{A}, \mathrm{EB}$ & {$[87 / 5][62 / 16]$} \\
\hline & $(319-339)$ & 44.8 & $(298)$ & EB & {$[62 / 16]$} \\
\hline $\mathrm{C}_{7} \mathrm{H}_{15} \mathrm{Br}$ & 1-bromoheptane & & & & {$[629-04-9]$} \\
\hline & $(341-481)$ & 47.0 & $(356)$ & & {$[99 / 16]$} \\
\hline & $(323-363)$ & 50.2 & (298) & CGC & {$[95 / 21]$} \\
\hline & & $50.8 \pm 0.1$ & (298) & $\mathrm{C}$ & {$[68 / 1]$} \\
\hline & & $50.4 \pm 0.2$ & (298) & $\mathrm{C}$ & {$[66 / 2]$} \\
\hline & $(333-483)$ & 47.5 & $(348)$ & A, EST & {$[87 / 5][61 / 13]$} \\
\hline $\mathrm{C}_{7} \mathrm{H}_{15} \mathrm{Br}$ & (dl) 2-bromoheptane & & & & [1974-04-5] \\
\hline & $(333-440)$ & 45.0 & $(348)$ & A & {$[87 / 5][99 / 16]$} \\
\hline $\mathrm{C}_{7} \mathrm{H}_{15} \mathrm{Cl}$ & 1-chloroheptane & & & & [629-06-1] \\
\hline & $(326-462)$ & 45.1 & $(341)$ & & {$[99 / 16]$} \\
\hline & $(313-353)$ & 47.9 & $(298)$ & CGC & {$[95 / 21]$} \\
\hline & $(300-430)$ & 47.0 & $(298)$ & & {$[84 / 9][91 / 2]$} \\
\hline & $(307-434)$ & 46.9 & $(322)$ & A, DTA & {$[87 / 5][69 / 5]$} \\
\hline & & $47.7 \pm 0.1$ & $(298)$ & C & {$[68 / 1]$} \\
\hline $\mathrm{C}_{7} \mathrm{H}_{15} \mathrm{Cl}$ & ( $d l$ ) 2-chloroheptane & & & & {$[1001-89-4]$} \\
\hline & $(313-424)$ & 44.8 & $(328)$ & $\mathrm{A}$ & {$[87 / 5][99 / 16]$} \\
\hline $\mathrm{C}_{7} \mathrm{H}_{15} \mathrm{Cl}_{2} \mathrm{~N}$ & $\mathrm{~N}$-methyl-bis(2-chloropropyl)amine & & & & [52802-03-6] \\
\hline & $(273-333)$ & 54.6 & $(288)$ & A, GS & $\begin{array}{c}{[87 / 5][48 / 13]} \\
{[73 / 18]}\end{array}$ \\
\hline $\mathrm{C}_{7} \mathrm{H}_{15} \mathrm{Cl}_{2} \mathrm{~N}$ & N-propyl-bis(2-chloroethyl)amine & & & & {$[621-68-1]$} \\
\hline & $(273-369)$ & 56.8 & $(288)$ & A, GS & {$[87 / 5][48 / 13]$} \\
\hline
\end{tabular}


TABLE 6. Enthalpies of vaporization of organic compounds, 1880-2002-Continued

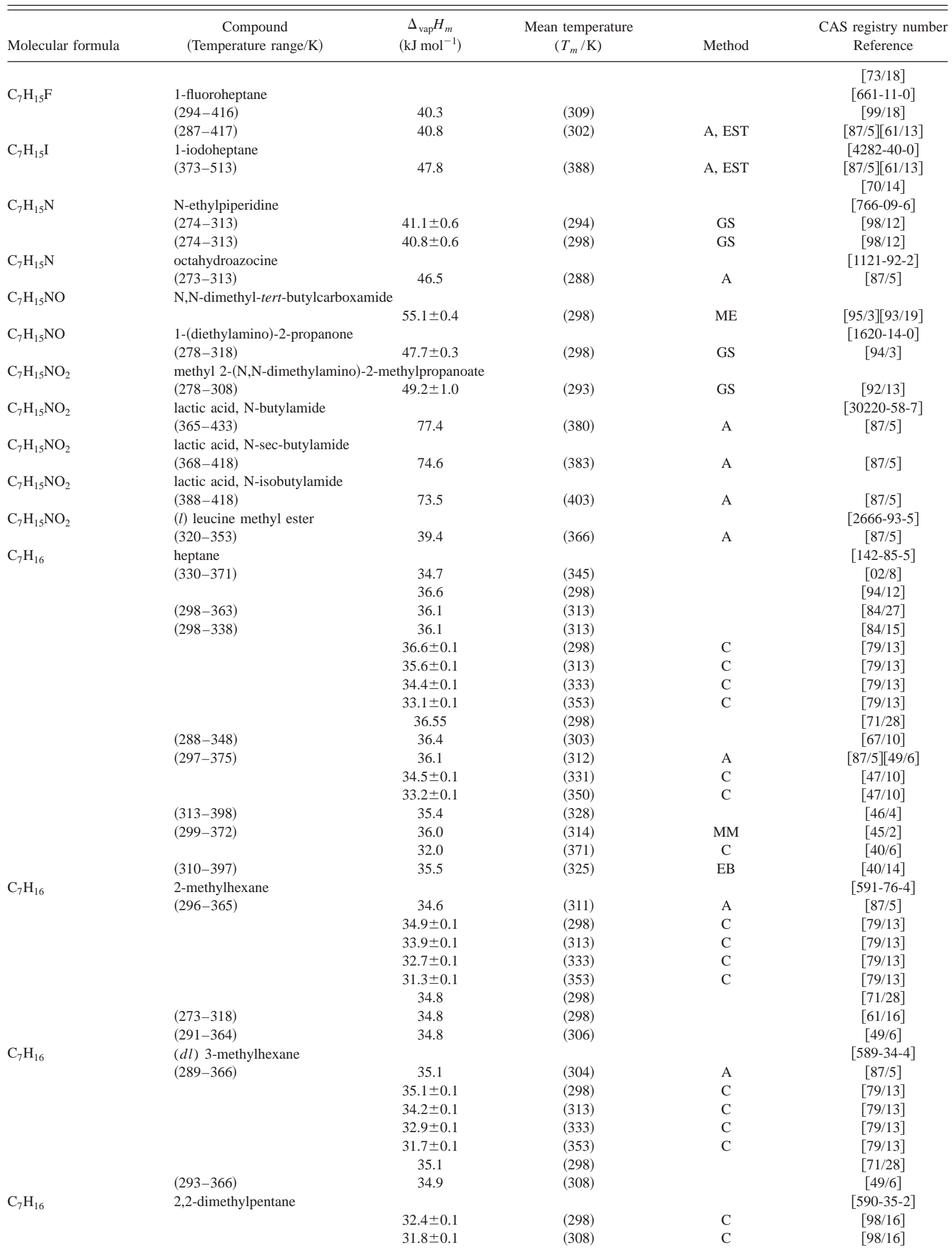


TABLE 6. Enthalpies of vaporization of organic compounds, 1880-2002-Continued

\begin{tabular}{|c|c|c|c|c|c|}
\hline Molecular formula & $\begin{array}{c}\text { Compound } \\
\text { (Temperature range/K) }\end{array}$ & $\begin{array}{c}\Delta_{\mathrm{vap}} H_{m} \\
\left(\mathrm{~kJ} \mathrm{~mol}^{-1}\right)\end{array}$ & $\begin{array}{c}\text { Mean temperature } \\
\qquad\left(T_{m} / \mathrm{K}\right)\end{array}$ & Method & $\begin{array}{c}\text { CAS registry number } \\
\text { Reference }\end{array}$ \\
\hline & & $31.4 \pm 0.1$ & $(315)$ & $\mathrm{C}$ & [98/16] \\
\hline & & $31.0 \pm 0.1$ & $(323)$ & $\mathrm{C}$ & {$[98 / 16]$} \\
\hline & & $30.5 \pm 0.1$ & $(330)$ & $\mathrm{C}$ & {$[98 / 16]$} \\
\hline & & $30.1 \pm 0.1$ & $(338)$ & $\mathrm{C}$ & {$[98 / 16]$} \\
\hline & & $29.4 \pm 0.1$ & $(348)$ & $\mathrm{C}$ & {$[98 / 16]$} \\
\hline & & $28.8 \pm 0.1$ & $(358)$ & $\mathrm{C}$ & {$[98 / 16]$} \\
\hline & & $28.1 \pm 0.1$ & $(368)$ & $\mathrm{C}$ & {$[98 / 16]$} \\
\hline & $(277-354)$ & 33.2 & $(292)$ & A & {$[87 / 5]$} \\
\hline & $(353-483)$ & 30.1 & $(368)$ & A & {$[87 / 5]$} \\
\hline & $(285-353)$ & 32.8 & $(300)$ & & {$[49 / 6]$} \\
\hline & & $32.4 \pm 0.1$ & (298) & $\mathrm{C}$ & {$[47 / 7]$} \\
\hline & & $32.2 \pm 0.1$ & $(298)$ & $\mathrm{C}$ & {$[47 / 7]$} \\
\hline & $(288-353)$ & 32.6 & $(303)$ & $\mathrm{MM}$ & {$[45 / 2]$} \\
\hline \multirow[t]{10}{*}{$\mathrm{C}_{7} \mathrm{H}_{16}$} & (dl) 2,3-dimethylpentane & & & & {$[565-59-3]$} \\
\hline & $(309-371)$ & 33.0 & $(324)$ & & {$[99 / 10]$} \\
\hline & $(208-286)$ & 35.9 & $(271)$ & A & {$[87 / 5]$} \\
\hline & & $34.3 \pm 0.1$ & $(298)$ & $\mathrm{C}$ & {$[79 / 13]$} \\
\hline & & $33.4 \pm 0.1$ & $(313)$ & $\mathrm{C}$ & {$[79 / 13]$} \\
\hline & & $32.2 \pm 0.1$ & $(333)$ & $\mathrm{C}$ & {$[79 / 13]$} \\
\hline & & $31.1 \pm 0.1$ & $(353)$ & $\mathrm{C}$ & {$[79 / 13]$} \\
\hline & $(286-365)$ & 34.5 & $(301)$ & & {$[87 / 5][73 / 18]$} \\
\hline & $(291-364)$ & 34.4 & $(306)$ & & {$[49 / 6]$} \\
\hline & & $34.2 \pm 0.1$ & $(298)$ & $\mathrm{C}$ & {$[47 / 7]$} \\
\hline \multirow[t]{11}{*}{$\mathrm{C}_{7} \mathrm{H}_{16}$} & 2,4-dimethylpentane & & & & [108-08-7] \\
\hline & & $32.7 \pm 0.1$ & $(298)$ & $\mathrm{C}$ & [98/16] \\
\hline & & $32.3 \pm 0.1$ & $(308)$ & $\mathrm{C}$ & {$[98 / 16]$} \\
\hline & & $31.9 \pm 0.1$ & $(315)$ & $\mathrm{C}$ & {$[98 / 16]$} \\
\hline & & $31.5 \pm 0.1$ & $(323)$ & $\mathrm{C}$ & {$[98 / 16]$} \\
\hline & & $31.0 \pm 0.1$ & $(330)$ & $\mathrm{C}$ & {$[98 / 16]$} \\
\hline & & $30.6 \pm 0.1$ & $(338)$ & $\mathrm{C}$ & {$[98 / 16]$} \\
\hline & & $30.0 \pm 0.1$ & $(348)$ & $\mathrm{C}$ & [98/16] \\
\hline & $(284-355)$ & 33.3 & (299) & A & {$[87 / 5][73 / 18]$} \\
\hline & $(287-354)$ & 33.2 & $(302)$ & & {$[49 / 6]$} \\
\hline & & $32.9 \pm 0.1$ & (298) & $\mathrm{C}$ & {$[47 / 7]$} \\
\hline \multirow[t]{8}{*}{$\mathrm{C}_{7} \mathrm{H}_{16}$} & 3,3-dimethylpentane & & & & {$[562-49-2]$} \\
\hline & $(213-281)$ & 34.8 & $(266)$ & & {$[87 / 5]$} \\
\hline & $(280-360)$ & 33.6 & $(295)$ & & {$[87 / 5]$} \\
\hline & & $33.0 \pm 0.1$ & $(298)$ & $\mathrm{C}$ & {$[81 / 9]$} \\
\hline & & 33.0 & $(298)$ & & {$[71 / 28]$} \\
\hline & $(287-360)$ & 33.2 & $(302)$ & & {$[49 / 6]$} \\
\hline & & $33.0 \pm 0.1$ & $(298)$ & $\mathrm{C}$ & {$[47 / 7]$} \\
\hline & $(285-360)$ & 33.3 & $(300)$ & $\mathrm{MM}$ & {$[87 / 5][45 / 2]$} \\
\hline \multirow[t]{13}{*}{$\mathrm{C}_{7} \mathrm{H}_{16}$} & 3-ethylpentane & & & & {$[617-78-7]$} \\
\hline & & $35.1 \pm 0.1$ & $(298)$ & $\mathrm{C}$ & {$[98 / 16]$} \\
\hline & & $34.5 \pm 0.1$ & $(308)$ & $\mathrm{C}$ & {$[98 / 16]$} \\
\hline & & $34.1 \pm 0.1$ & $(315)$ & $\mathrm{C}$ & {$[98 / 16]$} \\
\hline & & $33.7 \pm 0.1$ & $(232)$ & $\mathrm{C}$ & {$[98 / 16]$} \\
\hline & & $33.3 \pm 0.1$ & $(330)$ & $\mathrm{C}$ & {$[98 / 16]$} \\
\hline & & $32.7 \pm 0.1$ & $(338)$ & $\mathrm{C}$ & {$[98 / 16]$} \\
\hline & & $32.2 \pm 0.1$ & $(348)$ & $\mathrm{C}$ & {$[98 / 16]$} \\
\hline & $(291-368)$ & 35.2 & $(306)$ & & {$[87 / 5]$} \\
\hline & & 35.2 & $(298)$ & & {$[71 / 28]$} \\
\hline & & $35.2 \pm 0.1$ & $(298)$ & $\mathrm{C}$ & {$[47 / 7]$} \\
\hline & $(294-367)$ & 35.0 & (309) & & {$[45 / 2]$} \\
\hline & $(308-391)$ & 34.4 & (323) & EB & {$[41 / 9][84 / 9]$} \\
\hline \multirow[t]{7}{*}{$\mathrm{C}_{7} \mathrm{H}_{16}$} & 2,2,3-trimethylbutane & & & & [464-06-2] \\
\hline & $(284-355)$ & 32.4 & (299) & A & {$[87 / 5]$} \\
\hline & $(353-483)$ & 29.9 & $(368)$ & A & {$[87 / 5]$} \\
\hline & & 32.0 & (298) & & {$[71 / 28]$} \\
\hline & $(286-355)$ & 32.3 & $(301)$ & & {$[49 / 6]$} \\
\hline & & $31.2 \pm 0.1$ & $(314)$ & $\mathrm{C}$ & {$[47 / 10]$} \\
\hline & $(296-378)$ & 31.9 & $(311)$ & EB & {$[41 / 9]$} \\
\hline \multirow[t]{2}{*}{$\mathrm{C}_{7} \mathrm{H}_{16} \mathrm{~N}_{2} \mathrm{~S}$} & 1,3-dipropylthiourea & & & & [26536-60-7] \\
\hline & $(346-394)$ & $107 \pm 3.0$ & $(370)$ & $\mathrm{ME}, \mathrm{TE}$ & [94/21] \\
\hline
\end{tabular}


TABLE 6. Enthalpies of vaporization of organic compounds, 1880-2002-Continued

\begin{tabular}{|c|c|c|c|c|c|}
\hline Molecular formula & $\begin{array}{c}\text { Compound } \\
\text { (Temperature range/K) }\end{array}$ & $\begin{array}{c}\Delta_{\mathrm{vap}} H_{m} \\
\left(\mathrm{~kJ} \mathrm{~mol}^{-1}\right)\end{array}$ & $\begin{array}{l}\text { Mean temperature } \\
\qquad\left(T_{m} / \mathrm{K}\right)\end{array}$ & Method & $\begin{array}{c}\text { CAS registry number } \\
\text { Reference }\end{array}$ \\
\hline \multirow[t]{5}{*}{$\mathrm{C}_{7} \mathrm{H}_{16} \mathrm{O}$} & tert-amyl ethyl ether & & & & [919-94-8] \\
\hline & & $39.2 \pm 0.4$ & $(298)$ & & {$[\mathrm{U} / 2][02 / 32]$} \\
\hline & & $38.2 \pm 0.2$ & $(298)$ & $\mathrm{C}$ & {$[02 / 22]$} \\
\hline & $(318-374)$ & 35.7 & (333) & $\mathrm{EB}$ & {$[02 / 22]$} \\
\hline & $(320-374)$ & 35.6 & $(335)$ & $\mathrm{EB}$ & {$[94 / 10]$} \\
\hline \multirow[t]{4}{*}{$\mathrm{C}_{7} \mathrm{H}_{16} \mathrm{O}$} & propyl tert-butyl ether & & & & [29072-93-3] \\
\hline & & 38.3 & $(298)$ & & {$[\mathrm{U} / 2][02 / 32]$} \\
\hline & $(315-370)$ & $37.2 \pm 0.6$ & $(298)$ & $\mathrm{EB}$ & {$[02 / 10]$} \\
\hline & & $36.6 \pm 0.2$ & $(298)$ & $\mathrm{C}$ & {$[02 / 10]$} \\
\hline \multirow[t]{5}{*}{$\mathrm{C}_{7} \mathrm{H}_{16} \mathrm{O}$} & isopropyl tert-butyl ether & & & & {$[17348-59-3]$} \\
\hline & & 36.2 & $(298)$ & & {$[\mathrm{U} / 2][02 / 32]$} \\
\hline & $(305-360)$ & $34.4 \pm 0.6$ & $(298)$ & $\mathrm{EB}$ & {$[02 / 10]$} \\
\hline & & $34.5 \pm 0.2$ & $(298)$ & $\mathrm{C}$ & {$[02 / 10]$} \\
\hline & $(307-360)$ & 34.0 & $(322)$ & EB & {$[94 / 10]$} \\
\hline \multirow{9}{*}{$\mathrm{C}_{7} \mathrm{H}_{16} \mathrm{O}$} & 1-heptanol & & & & {$[111-70-6]$} \\
\hline & $(323-373)$ & 66.5 & $(298)$ & CGC & {$[95 / 21]$} \\
\hline & $(373-423)$ & 66.4 & $(298)$ & CGC & {$[95 / 21]$} \\
\hline & $(258-363)$ & 65.2 & $(310)$ & & {$[92 / 14]$} \\
\hline & $(335-450)$ & 62.5 & $(350)$ & A & {$[87 / 5]$} \\
\hline & & $66.8 \pm 0.2$ & $(298)$ & $\mathrm{C}$ & {$[77 / 1]$} \\
\hline & $(333-449)$ & 65.2 & $(348)$ & & {$[73 / 26]$} \\
\hline & $(336-450)$ & 62.6 & $(351)$ & DTA & {$[87 / 5][69 / 5]$} \\
\hline & $(333-425)$ & 62.9 & $(348)$ & & {$[35 / 6][84 / 9]$} \\
\hline \multirow[t]{5}{*}{$\mathrm{C}_{7} \mathrm{H}_{16} \mathrm{O}$} & (dl) 2-heptanol & & & & {$[543-49-7]$} \\
\hline & $(244-338)$ & 66.1 & $(259)$ & & {$[99 / 11]$} \\
\hline & $(351-433)$ & 54.4 & $(366)$ & & {$[84 / 10]$} \\
\hline & $(357-431)$ & 51.6 & $(372)$ & A & {$[87 / 5][75 / 23]$} \\
\hline & $(323-433)$ & 59.8 & $(338)$ & & {$[73 / 26]$} \\
\hline \multirow[t]{6}{*}{$\mathrm{C}_{7} \mathrm{H}_{16} \mathrm{O}$} & (dl) 3-heptanol & & & & [589-82-2] \\
\hline & $(244-333)$ & 67.0 & $(259)$ & & [99/11] \\
\hline & $(325-430)$ & 60.3 & $(340)$ & A & {$[87 / 5]$} \\
\hline & $(263-295)$ & 64.7 & $(280)$ & A & {$[87 / 5][79 / 16]$} \\
\hline & $(349-430)$ & 53.1 & $(364)$ & & {$[84 / 10]$} \\
\hline & $(328-429)$ & 59.2 & (343) & & {$[73 / 26]$} \\
\hline \multirow[t]{5}{*}{$\mathrm{C}_{7} \mathrm{H}_{16} \mathrm{O}$} & 4-heptanol & & & & [589-55-9] \\
\hline & $(320-428)$ & 58.2 & $(335)$ & A & {$[87 / 5]$} \\
\hline & $(349-428)$ & 53.1 & $(364)$ & & {$[84 / 10]$} \\
\hline & $(282-320)$ & 63.1 & $(297)$ & A & {$[87 / 5][75 / 1]$} \\
\hline & $(320-428)$ & 56.9 & $(335)$ & & {$[73 / 26]$} \\
\hline \multirow[t]{2}{*}{$\mathrm{C}_{7} \mathrm{H}_{16} \mathrm{O}$} & 2-methyl-1-hexanol & & & & {$[624-22-6]$} \\
\hline & $(343-438)$ & 53.5 & $(390)$ & & {$[73 / 26]$} \\
\hline \multirow{2}{*}{$\mathrm{C}_{7} \mathrm{H}_{16} \mathrm{O}$} & 3-methyl-1-hexanol & & & & [13231-81-7] \\
\hline & $(353-445)$ & 57.4 & $(399)$ & & {$[73 / 26]$} \\
\hline \multirow[t]{2}{*}{$\mathrm{C}_{7} \mathrm{H}_{16} \mathrm{O}$} & 4-methyl-1-hexanol & & & & {$[818-49-5]$} \\
\hline & $(348-448)$ & 62.6 & $(363)$ & & {$[73 / 26]$} \\
\hline \multirow[t]{2}{*}{$\mathrm{C}_{7} \mathrm{H}_{16} \mathrm{O}$} & 2-methyl-2-hexanol & & & & [625-23-0] \\
\hline & $(311-415)$ & 54.5 & $(326)$ & A & {$[87 / 5][73 / 26]$} \\
\hline \multirow[t]{2}{*}{$\mathrm{C}_{7} \mathrm{H}_{16} \mathrm{O}$} & 5-methyl-2-hexanol & & & & [627-59-8] \\
\hline & $(348-428)$ & 49.4 & $(388)$ & & {$[73 / 26]$} \\
\hline \multirow[t]{2}{*}{$\mathrm{C}_{7} \mathrm{H}_{16} \mathrm{O}$} & 2-methyl-3-hexanol & & & & {$[617-29-8]$} \\
\hline & $(323-420)$ & 55.7 & $(338)$ & & {$[73 / 26]$} \\
\hline \multirow[t]{2}{*}{$\mathrm{C}_{7} \mathrm{H}_{16} \mathrm{O}$} & 3-methyl-3-hexanol & & & & {$[597-96-6]$} \\
\hline & $(323-416)$ & 53.6 & $(338)$ & & {$[73 / 26]$} \\
\hline $\mathrm{C}_{7} \mathrm{H}_{16} \mathrm{O}$ & 3,4-dimethyl-1-pentanol & & & & {$[6570-87-2]$} \\
\hline & $(393-438)$ & 50.3 & $(388)$ & & {$[73 / 26]$} \\
\hline $\mathrm{C}_{7} \mathrm{H}_{16} \mathrm{O}$ & 2,4-dimethyl-2-pentanol & & & & {$[625-06-9]$} \\
\hline & $(328-408)$ & 49.7 & $(343)$ & & {$[73 / 26]$} \\
\hline $\mathrm{C}_{7} \mathrm{H}_{16} \mathrm{O}$ & 2,2-dimethyl-3-pentanol & & & & {$[3970-62-5]$} \\
\hline & $(318-411)$ & 51.4 & $(333)$ & & {$[73 / 26]$} \\
\hline $\mathrm{C}_{7} \mathrm{H}_{16} \mathrm{O}$ & 2,3-dimethyl-3-pentanol & & & & {$[595-41-5]$} \\
\hline & $(318-413)$ & 53.2 & $(333)$ & & {$[73 / 26]$} \\
\hline $\mathrm{C}_{7} \mathrm{H}_{16} \mathrm{O}$ & 2,4-dimethyl-3-pentanol & & & & {$[600-36-2]$} \\
\hline & & 51.8 & $(328)$ & $\mathrm{C}$ & {$[96 / 6]$} \\
\hline & & 48.8 & $(343)$ & $\mathrm{C}$ & {$[96 / 6]$} \\
\hline
\end{tabular}


TABLE 6. Enthalpies of vaporization of organic compounds, 1880-2002-Continued

\begin{tabular}{|c|c|c|c|c|c|}
\hline Molecular formula & $\begin{array}{c}\text { Compound } \\
\text { (Temperature range/K) }\end{array}$ & $\begin{array}{c}\Delta_{\mathrm{vap}} H_{m} \\
\left(\mathrm{~kJ} \mathrm{~mol}^{-1}\right)\end{array}$ & $\begin{array}{l}\text { Mean temperature } \\
\left(T_{m} / \mathrm{K}\right)\end{array}$ & Method & $\begin{array}{c}\text { CAS registry number } \\
\text { Reference }\end{array}$ \\
\hline & & 45.7 & $(358)$ & $\mathrm{C}$ & {$[96 / 6]$} \\
\hline & $(307-412)$ & 53.6 & $(322)$ & A & {$[87 / 5][73 / 26]$} \\
\hline \multirow[t]{3}{*}{$\mathrm{C}_{7} \mathrm{H}_{16} \mathrm{O}$} & 3-ethyl-3-pentanol & & & & {$[597-49-9]$} \\
\hline & $(317-408)$ & 51.3 & $(332)$ & A & {$[87 / 5][73 / 18]$} \\
\hline & $(308-416)$ & 55.2 & $(323)$ & & {$[73 / 26]$} \\
\hline \multirow[t]{2}{*}{$\mathrm{C}_{7} \mathrm{H}_{16} \mathrm{O}$} & 2-methyl-2-ethyl-1-butanol & & & & [18371-13-6] \\
\hline & $(358-428)$ & 55.7 & $(373)$ & & {$[73 / 26]$} \\
\hline \multirow[t]{2}{*}{$\mathrm{C}_{7} \mathrm{H}_{16} \mathrm{O}$} & 2,3,3-trimethyl-2-butanol & & & & {$[594-83-2]$} \\
\hline & $(298-363)$ & 48.7 & $(313)$ & MM & {$[85 / 5]$} \\
\hline \multirow[t]{2}{*}{$\mathrm{C}_{7} \mathrm{H}_{16} \mathrm{O}_{2}$} & 1-butoxy-2-methoxyethane & & & & {$[13343-98-1]$} \\
\hline & & $47.8 \pm 0.1$ & $(298)$ & $\mathrm{C}$ & {$[70 / 17]$} \\
\hline \multirow[t]{2}{*}{$\mathrm{C}_{7} \mathrm{H}_{16} \mathrm{O}_{2}$} & 1-propoxy-2-ethoxyethane & & & & {$[18854-31-4]$} \\
\hline & & $46.8 \pm 0.1$ & $(298)$ & $\mathrm{C}$ & {$[70 / 17]$} \\
\hline \multirow[t]{2}{*}{$\mathrm{C}_{7} \mathrm{H}_{16} \mathrm{O}_{2}$} & 1,3-diethoxypropane & & & & {$[3459-83-4]$} \\
\hline & & $45.9 \pm 0.2$ & $(298)$ & $\mathrm{C}$ & {$[72 / 42]$} \\
\hline \multirow[t]{4}{*}{$\mathrm{C}_{7} \mathrm{H}_{16} \mathrm{O}_{2}$} & 2,2-diethoxypropane & & & & {$[126-84-1]$} \\
\hline & $(273-308)$ & $43.2 \pm 0.4$ & $(298)$ & GS & {$[02 / 32]$} \\
\hline & $(273-308)$ & 43.9 & & GS & {$[98 / 21]$} \\
\hline & $(286-304)$ & $\mathrm{U} 28.2$ & $(295)$ & A, I & {$[87 / 5][62 / 14]$} \\
\hline \multirow{2}{*}{$\mathrm{C}_{7} \mathrm{H}_{16} \mathrm{O}_{2}$} & 1-tert-butoxy-2-propanol & & & & {$[57018-52-7]$} \\
\hline & $(346-420)$ & 45.4 & $(361)$ & EB & {$[01 / 6]$} \\
\hline \multirow[t]{2}{*}{$\mathrm{C}_{7} \mathrm{H}_{16} \mathrm{O}_{2}$} & 4-methyl-4-methoxy-2-pentanol & & & & {$[141-73-1]$} \\
\hline & $(343-423)$ & 46.6 & $(358)$ & $\mathrm{A}$ & {$[87 / 5][73 / 18]$} \\
\hline \multirow[t]{2}{*}{$\mathrm{C}_{7} \mathrm{H}_{16} \mathrm{O}_{3}$} & diethylene glycol monopropyl ether & & & & [6881-94-3] \\
\hline & $(369-404)$ & 65.3 & $(384)$ & $\mathrm{A}$ & {$[87 / 5]$} \\
\hline \multirow[t]{2}{*}{$\mathrm{C}_{7} \mathrm{H}_{16} \mathrm{O}_{3}$} & 2-(2-propoxyethoxy)ethanol & & & & [6881-94-3] \\
\hline & $(378-495)$ & $65.7 \pm 0.8$ & $(298)$ & EB & {$[96 / 3]$} \\
\hline \multirow[t]{4}{*}{$\mathrm{C}_{7} \mathrm{H}_{16} \mathrm{O}_{3}$} & triethoxymethane & & & & {$[122-51-0]$} \\
\hline & $(293-323)$ & 49.0 & $(308)$ & A & {$[87 / 5]$} \\
\hline & & $46.0 \pm 0.8$ & $(298)$ & & {$[71 / 26]$} \\
\hline & $(278-419)$ & 47.2 & $(293)$ & & {$[87 / 5][47 / 5]$} \\
\hline \multirow[t]{2}{*}{$\mathrm{C}_{7} \mathrm{H}_{16} \mathrm{O}_{3}$} & 3,5,7,9-tetraoxaundecane & & & & {$[4431-82-7]$} \\
\hline & & $53.6 \pm 0.7$ & $(298)$ & $\mathrm{C}$ & {$[69 / 18]$} \\
\hline \multirow[t]{2}{*}{$\mathrm{C}_{7} \mathrm{H}_{16} \mathrm{O}_{3}$} & tert-pentylperoxyethanol & & & & {$[51452-08-5]$} \\
\hline & & $70.1 \pm 2.5$ & & & {$[83 / 11]$} \\
\hline \multirow[t]{2}{*}{$\mathrm{C}_{7} \mathrm{H}_{16} \mathrm{O}_{4}$} & 3-tert-butyldioxy-1,2-propanediol & & & & {$[38578-50-6]$} \\
\hline & & $88.0 \pm 2.6$ & & & {$[83 / 11]$} \\
\hline $\mathrm{C}_{7} \mathrm{H}_{16} \mathrm{~S}$ & 1-heptanethiol & & & & {$[1639-09-4]$} \\
\hline & $(273-345)$ & 49.5 & $(288)$ & & {$[99 / 16]$} \\
\hline & & $50.6 \pm 0.2$ & $(298)$ & & {$[66 / 10][66 / 5]$} \\
\hline & $(373-472)$ & 45.0 & $(388)$ & $\mathrm{A}, \mathrm{EB}$ & $\begin{array}{c}{[87 / 5][65 / 8]} \\
{[66 / 5]}\end{array}$ \\
\hline $\mathrm{C}_{7} \mathrm{H}_{16} \mathrm{~S}$ & 2-heptanethiol & & & & {$[628-00-2]$} \\
\hline & $(343-437)$ & 44.1 & $(358)$ & & {$[99 / 16]$} \\
\hline & $(343-471)$ & 47.2 & $(360)$ & & [99/16] \\
\hline & $(341-443)$ & 44.2 & $(356)$ & A & {$[87 / 5][73 / 18]$} \\
\hline $\mathrm{C}_{7} \mathrm{H}_{16} \mathrm{~S}_{2}$ & 1,7-heptanedithiol & & & & {$[62224-02-6]$} \\
\hline & $(392-526)$ & 59.0 & $(407)$ & A & $\begin{array}{c}{[87 / 5][73 / 18]} \\
{[99 / 16]}\end{array}$ \\
\hline $\mathrm{C}_{7} \mathrm{H}_{17} \mathrm{~N}$ & tert-butylisopropylamine & & & & \\
\hline & $(275-299)$ & $35.7 \pm 1.0$ & $(287)$ & & {$[97 / 21]$} \\
\hline $\mathrm{C}_{7} \mathrm{H}_{17} \mathrm{~N}$ & N-butyl isopropylamine & & & & [39099-23-5] \\
\hline & & $42.1 \pm 0.1$ & $(298)$ & $\mathrm{C}$ & {$[79 / 8]$} \\
\hline & & $40.9 \pm 0.1$ & $(313)$ & $\mathrm{C}$ & {$[79 / 8]$} \\
\hline & & $39.9 \pm 0.1$ & $(328)$ & $\mathrm{C}$ & {$[79 / 8]$} \\
\hline & & $38.7 \pm 0.1$ & $(343)$ & $\mathrm{C}$ & {$[79 / 8]$} \\
\hline & & $37.6 \pm 0.1$ & $(358)$ & $\mathrm{C}$ & {$[79 / 8]$} \\
\hline & $(325-395)$ & 40.0 & $(340)$ & $\mathrm{C}$ & {$[79 / 8]$} \\
\hline $\mathrm{C}_{7} \mathrm{H}_{17} \mathrm{~N}$ & heptylamine & & & & {$[111-68-2]$} \\
\hline & $(323-373)$ & 49.9 & $(298)$ & CGC & {$[95 / 21]$} \\
\hline & $(326-430)$ & 46.5 & $(341)$ & A & {$[87 / 5]$} \\
\hline & & $50.0 \pm 0.1$ & $(298)$ & $\mathrm{C}$ & {$[69 / 2]$} \\
\hline $\mathrm{C}_{7} \mathrm{H}_{17} \mathrm{NO}$ & $\mathrm{N}$-(ethoxymethyl)diethylamine & & & & {$[7352-03-6]$} \\
\hline & $(285-400)$ & 39.6 & $(300)$ & A & {$[87 / 5]$} \\
\hline
\end{tabular}


TABLE 6. Enthalpies of vaporization of organic compounds, 1880-2002-Continued

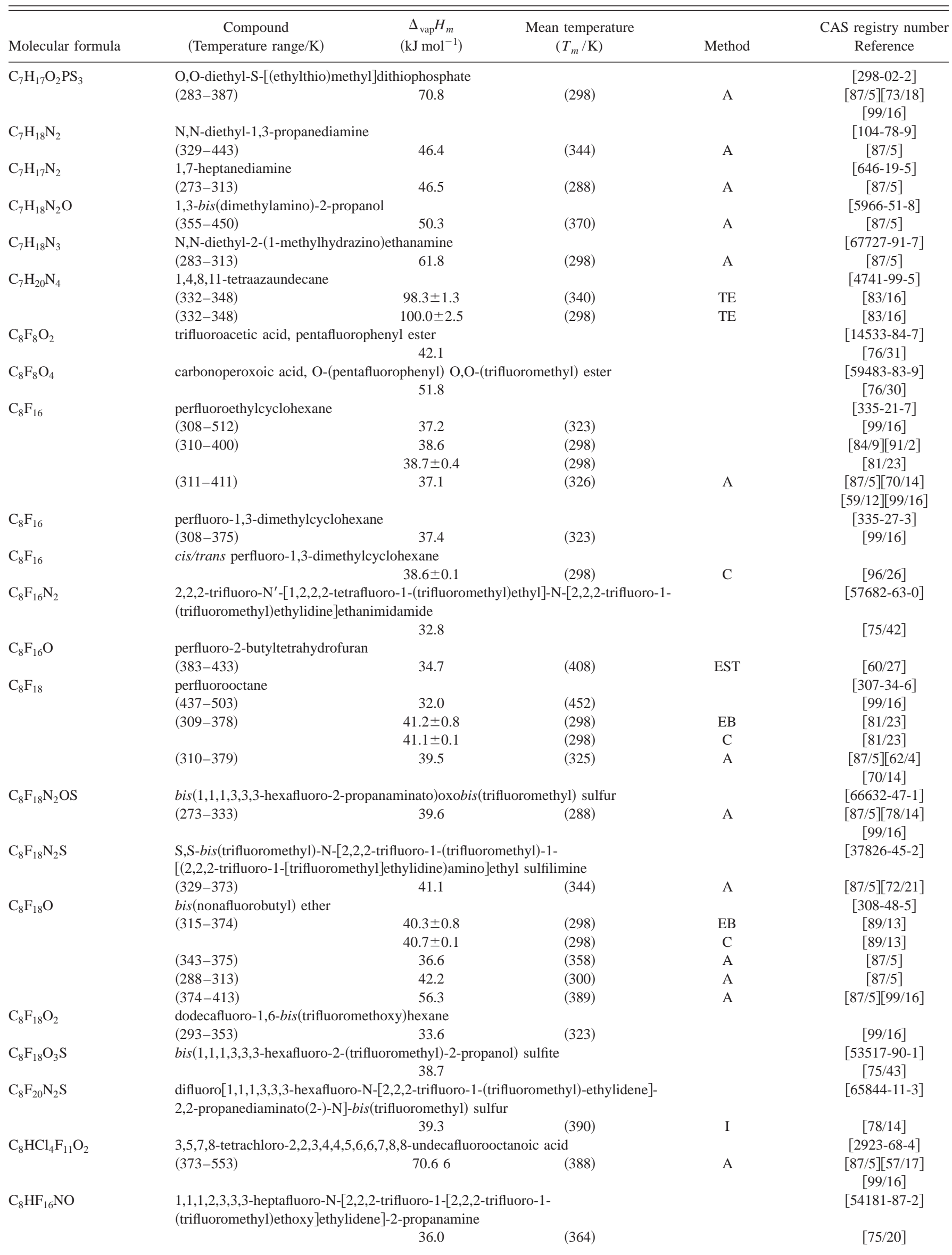


TABLE 6. Enthalpies of vaporization of organic compounds, 1880-2002-Continued

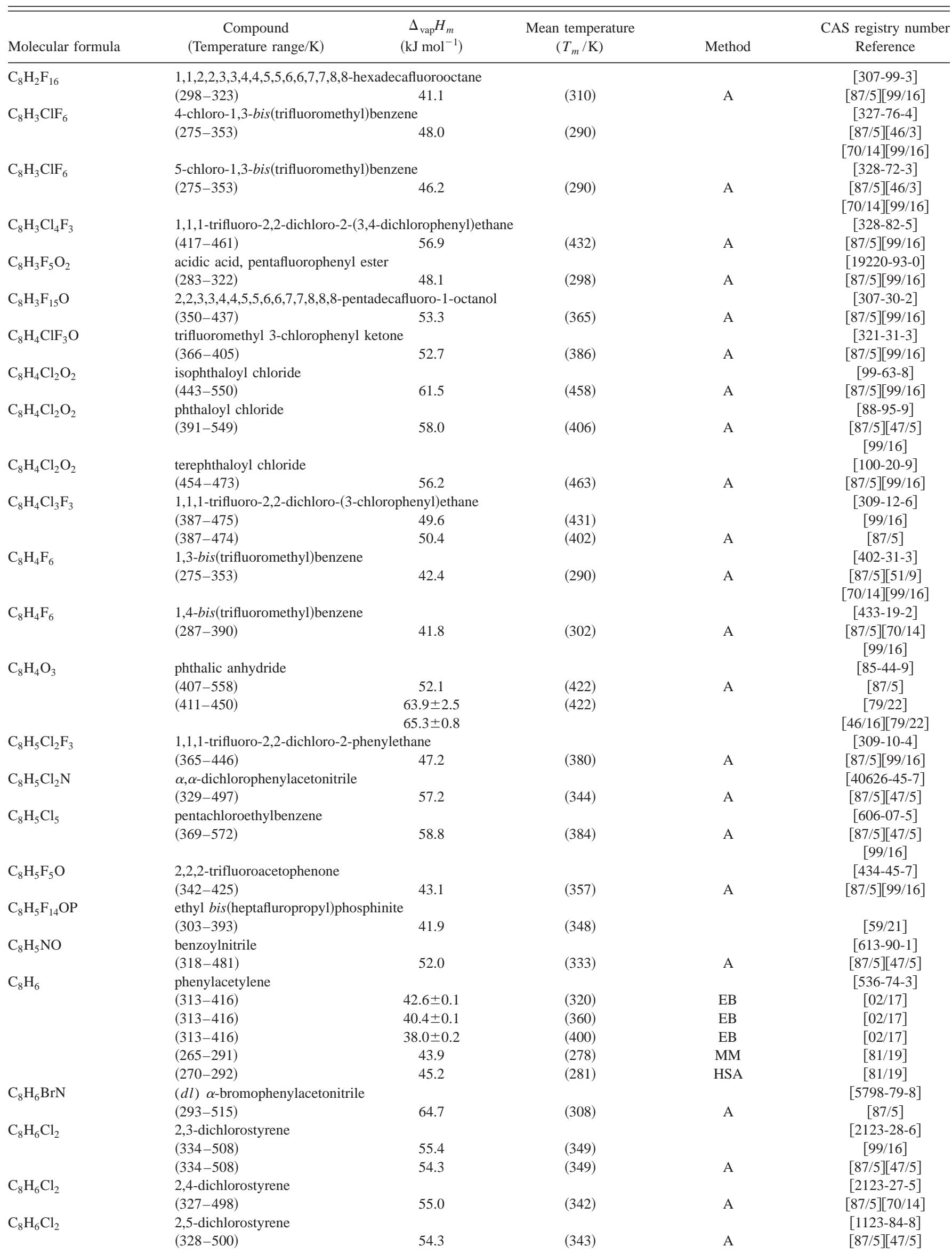


TABLE 6. Enthalpies of vaporization of organic compounds, 1880-2002-Continued

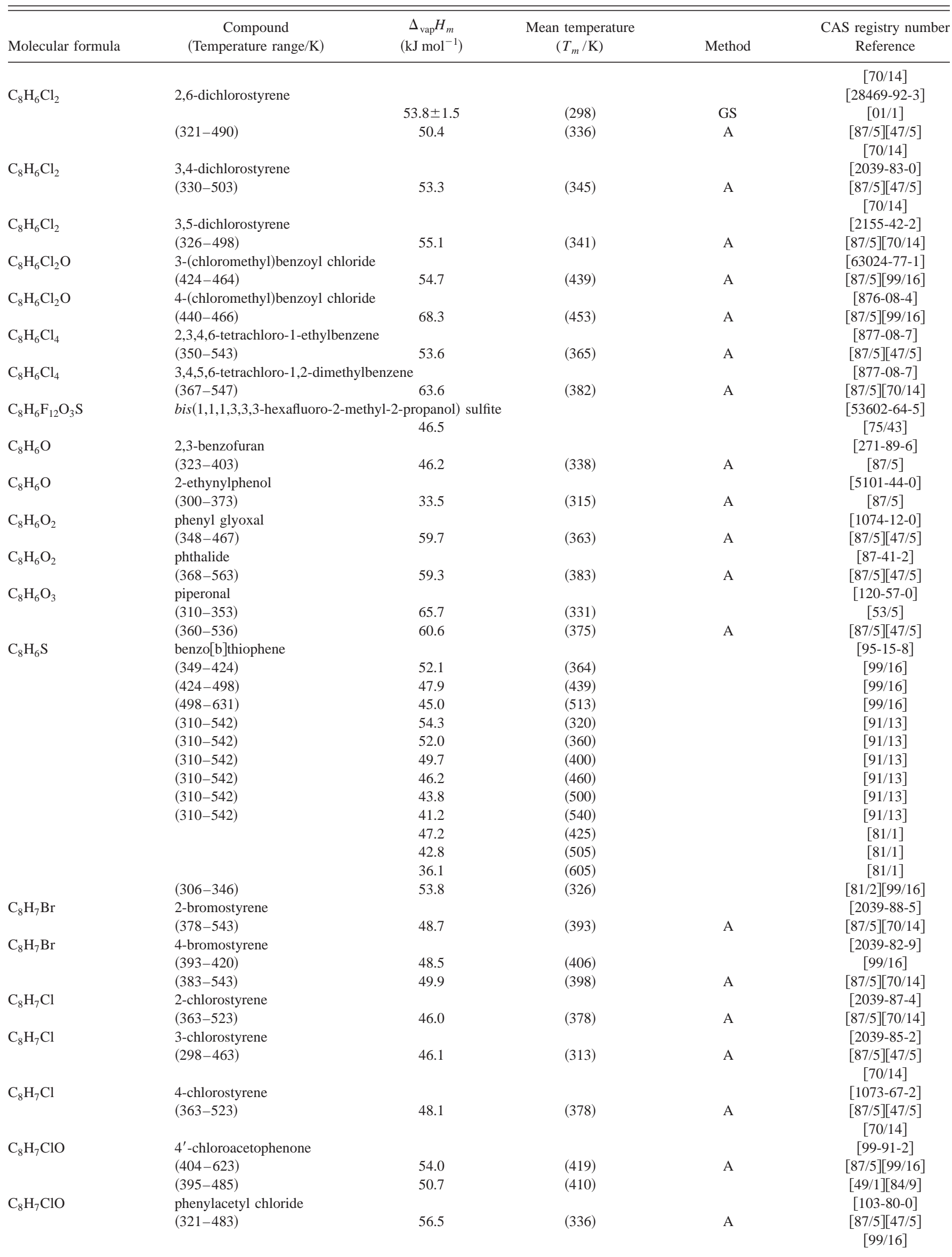


TABLE 6. Enthalpies of vaporization of organic compounds, 1880-2002-Continued

\begin{tabular}{|c|c|c|c|c|c|}
\hline Molecular formula & $\begin{array}{c}\text { Compound } \\
\text { (Temperature range/K) }\end{array}$ & $\begin{array}{c}\Delta_{\mathrm{vap}} H_{m} \\
\left(\mathrm{~kJ} \mathrm{~mol}^{-1}\right)\end{array}$ & $\begin{array}{l}\text { Mean temperature } \\
\qquad\left(T_{m} / \mathrm{K}\right)\end{array}$ & Method & $\begin{array}{l}\text { CAS registry number } \\
\text { Reference }\end{array}$ \\
\hline $\mathrm{C}_{8} \mathrm{H}_{7} \mathrm{ClO}_{2}$ & $\begin{array}{l}\text { benzyl chloroformate } \\
(293-303)\end{array}$ & $38.5 \pm 0.1$ & (298) & & $\begin{array}{c}{[501-53-1]} \\
{[90 / 12]}\end{array}$ \\
\hline $\mathrm{C}_{8} \mathrm{H}_{7} \mathrm{Cl}_{3} \mathrm{O}_{3}$ & $\begin{array}{l}\text { 3,4,5-trichloro-2,6-dimethoxyphenol } \\
(293-323)\end{array}$ & 77.4 & $(308)$ & CGC & $\begin{array}{c}{[2539-26-6]} \\
{[99 / 13]}\end{array}$ \\
\hline $\mathrm{C}_{8} \mathrm{H}_{7} \mathrm{FO}$ & $\begin{array}{l}\text { 2-fluoroacetophenone } \\
(273-333)\end{array}$ & 62.0 & (288) & A, GS & $\begin{array}{c}{[450-95-3]} \\
{[87 / 5][48 / 14]} \\
{[99 / 16]}\end{array}$ \\
\hline $\mathrm{C}_{8} \mathrm{H}_{7} \mathrm{~F}_{3}$ & $\begin{array}{l}\text { 1,1,1-trifluoro-2-phenylethane } \\
(273-313)\end{array}$ & $46.1 \pm 0.3$ & (298) & GS & $\begin{array}{c}{[21249-93-4]} \\
{[97 / 14]}\end{array}$ \\
\hline $\mathrm{C}_{8} \mathrm{H}_{7} \mathrm{~N}$ & $\begin{array}{l}\text { benzylcyanide } \\
(283-328) \\
(283-328) \\
(333-507)\end{array}$ & $\begin{array}{c}60.1 \pm 0.7 \\
60.5 \pm 0.7 \\
54.8\end{array}$ & $\begin{array}{l}(306) \\
(298) \\
(348)\end{array}$ & $\begin{array}{c}\text { GS } \\
\text { GS } \\
\text { A }\end{array}$ & $\begin{array}{c}{[140-29-4]} \\
{[00 / 2]} \\
{[00 / 2]} \\
{[87 / 5][47 / 5]}\end{array}$ \\
\hline $\mathrm{C}_{8} \mathrm{H}_{7} \mathrm{~N}$ & $\begin{array}{l}\text { 2-tolunitrile } \\
(309-479)\end{array}$ & 50.8 & $(324)$ & A & $\begin{array}{l}{[620-22-4]} \\
{[87 / 5][47 / 5]}\end{array}$ \\
\hline $\mathrm{C}_{8} \mathrm{H}_{7} \mathrm{~N}$ & $\begin{array}{l}\text { 4-tolunitrile } \\
(315-491)\end{array}$ & 48.0 & $(330)$ & A & $\begin{array}{l}{[104-85-8]} \\
{[87 / 5][47 / 5]}\end{array}$ \\
\hline $\mathrm{C}_{8} \mathrm{H}_{7} \mathrm{~N}$ & $\begin{array}{l}\text { 2-tolylisocyanide } \\
(298-457)\end{array}$ & 48.5 & $(313)$ & A & $\begin{array}{c}{[10468-64-1]} \\
{[87 / 5][47 / 5]}\end{array}$ \\
\hline $\mathrm{C}_{8} \mathrm{H}_{7} \mathrm{NO}$ & $\begin{array}{l}\text { benzyl isocyanate } \\
(333-393)\end{array}$ & 42.3 & $(348)$ & A & $\begin{array}{c}{[3173-56-6]} \\
{[87 / 5]}\end{array}$ \\
\hline $\mathrm{C}_{8} \mathrm{H}_{7} \mathrm{NO}_{3}$ & $\begin{array}{l}2^{\prime} \text {-nitroacetophenone } \\
(293-333)\end{array}$ & 103.6 & $(308)$ & A & $\begin{array}{l}{[577-59-3]} \\
{[87 / 5]}\end{array}$ \\
\hline $\mathrm{C}_{8} \mathrm{H}_{7} \mathrm{NO}_{4}$ & $\begin{array}{l}\text { 2-nitrobenzoic acid, methyl ester } \\
(423-453)\end{array}$ & 56.1 & $(438)$ & A & $\begin{array}{c}{[606-27-9]} \\
{[87 / 5]}\end{array}$ \\
\hline $\mathrm{C}_{8} \mathrm{H}_{7} \mathrm{NO}_{4}$ & $\begin{array}{l}\text { (2-nitrophenyl) acetate } \\
(373-526)\end{array}$ & 71.1 & $(388)$ & A & $\begin{array}{l}{[610-69-5]} \\
{[87 / 5][47 / 5]}\end{array}$ \\
\hline $\mathrm{C}_{8} \mathrm{H}_{7} \mathrm{NS}$ & $\begin{array}{l}\text { benzyl isothiocyanate } \\
(352-516)\end{array}$ & 62.2 & $(367)$ & A & $\begin{array}{l}{[622-78-6]} \\
{[87 / 5][47 / 5]}\end{array}$ \\
\hline $\mathrm{C}_{8} \mathrm{H}_{7} \mathrm{NS}$ & $\begin{array}{l}\text { 2-methylbenzothiazole } \\
(343-499)\end{array}$ & 61.3 & $(358)$ & A & $\begin{array}{c}{[120-75-2]} \\
{[87 / 5][47 / 5]} \\
{[99 / 16]}\end{array}$ \\
\hline $\mathrm{C}_{8} \mathrm{H}_{7} \mathrm{~N}_{3} \mathrm{O}_{7}$ & $\begin{array}{l}\text { 2,4,6-trinitrophenetole } \\
(342-351)\end{array}$ & 120.5 & $(346)$ & A & $\begin{array}{c}{[4732-14-3]} \\
{[87 / 5]}\end{array}$ \\
\hline $\mathrm{C}_{8} \mathrm{H}_{8}$ & $\begin{array}{l}\text { cyclooctatetraene } \\
(273-348)\end{array}$ & $\begin{array}{l}43.9 \\
43.1\end{array}$ & $\begin{array}{l}(288) \\
(298)\end{array}$ & A & $\begin{array}{c}{[629-20-9]} \\
{[87 / 5]} \\
49 / 23]\end{array}$ \\
\hline $\mathrm{C}_{8} \mathrm{H}_{8}$ & bicyclo[2.2.2]octa-2,5,7-triene & $42.9 \pm 0.1$ & (298) & $\mathrm{C}$ & $\begin{array}{c}{[500-24-3]} \\
{[85 / 2]}\end{array}$ \\
\hline $\mathrm{C}_{8} \mathrm{H}_{8}$ & $\begin{array}{l}\text { 1,5,7-octatriene-3-yene } \\
(313-429)\end{array}$ & 35.1 & $(328)$ & A & $\begin{array}{c}{[16607-77-5]} \\
{[87 / 5]}\end{array}$ \\
\hline $\mathrm{C}_{8} \mathrm{H}_{8}$ & $\begin{array}{l}\text { styrene } \\
(245-334) \\
(334-419) \\
(306-333) \\
(303-417) \\
(285-333) \\
(306-389) \\
(245-357)\end{array}$ & $\begin{array}{c}42.5 \\
41.5 \\
42.5 \\
43.1 \\
43.9 \\
43.5 \pm 0.4 \\
40.2 \\
43.2\end{array}$ & $\begin{array}{l}(260) \\
(349) \\
(319) \\
(318) \\
(298) \\
(298) \\
(348) \\
(298)\end{array}$ & $\begin{array}{l}\text { A } \\
\text { A }\end{array}$ & $\begin{array}{c}{[100-42-5]} \\
{[87 / 5]} \\
{[87 / 5]} \\
{[59 / 27]} \\
{[55 / 22]} \\
{[46 / 10]} \\
{[46 / 10]} \\
{[42 / 3]} \\
{[39 / 6]}\end{array}$ \\
\hline $\mathrm{C}_{8} \mathrm{H}_{8} \mathrm{Br}_{2}$ & $\begin{array}{l}\text { (1,2-dibromoethyl)benzene } \\
(359-527)\end{array}$ & 64.9 & (374) & A & $\begin{array}{c}{[93-52-7]} \\
{[87 / 5][47 / 5]} \\
{[70 / 14]}\end{array}$ \\
\hline $\mathrm{C}_{8} \mathrm{H}_{8} \mathrm{Cl}_{2}$ & $\begin{array}{l}\text { 2,5-dichloro-1,4-dimethylbenzene } \\
\text { (393-573) }\end{array}$ & 52.7 & (408) & $\mathrm{A}$ & $\begin{array}{c}{[1124-05-6]} \\
{[87 / 5][70 / 14]} \\
{[99 / 16]}\end{array}$ \\
\hline $\mathrm{C}_{8} \mathrm{H}_{8} \mathrm{Cl}_{2}$ & $\begin{array}{l}\text { 2,3-dichloro-1-ethylbenzene } \\
\text { (319-495) }\end{array}$ & 48.9 & (334) & A & $\begin{array}{c}{[54484-61-6]} \\
{[87 / 5][47 / 5]} \\
{[70 / 14]}\end{array}$ \\
\hline $\mathrm{C}_{8} \mathrm{H}_{8} \mathrm{Cl}_{2}$ & $\begin{array}{l}\text { 2,5-dichloro-1-ethylbenzene } \\
(311-490)\end{array}$ & 46.0 & (326) & A & $\begin{array}{c}{[54484-63-8]} \\
{[87 / 5][47 / 5]} \\
{[70 / 14]}\end{array}$ \\
\hline $\mathrm{C}_{8} \mathrm{H}_{8} \mathrm{Cl}_{2}$ & $\begin{array}{l}\text { 3,4-dichloro-1-ethylbenzene } \\
(320-500)\end{array}$ & 49.3 & (335) & A & $\begin{array}{l}{[6623-59-2]} \\
{[87 / 5][47 / 5]}\end{array}$ \\
\hline
\end{tabular}


TABLE 6. Enthalpies of vaporization of organic compounds, 1880-2002-Continued

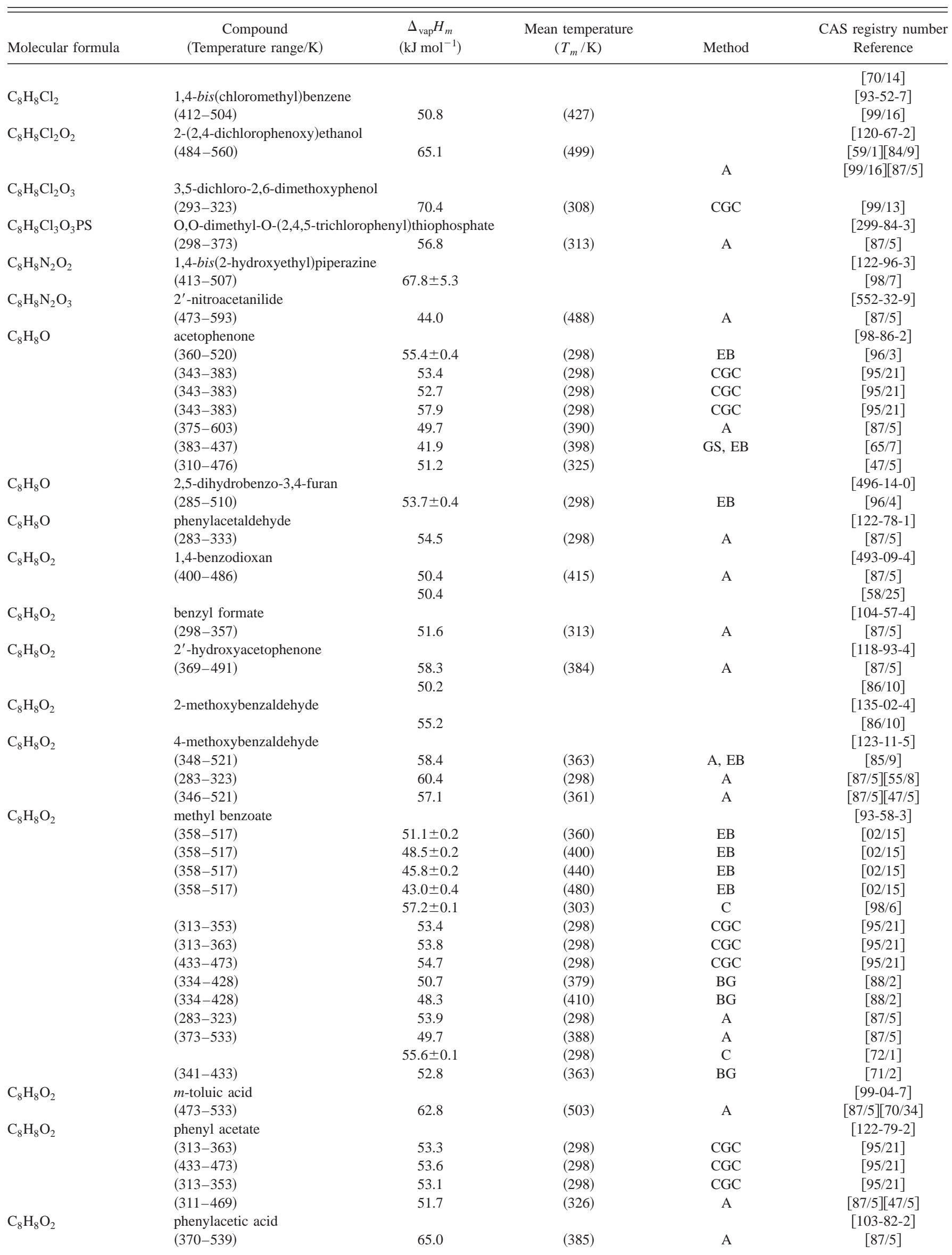


TABLE 6. Enthalpies of vaporization of organic compounds, 1880-2002-Continued

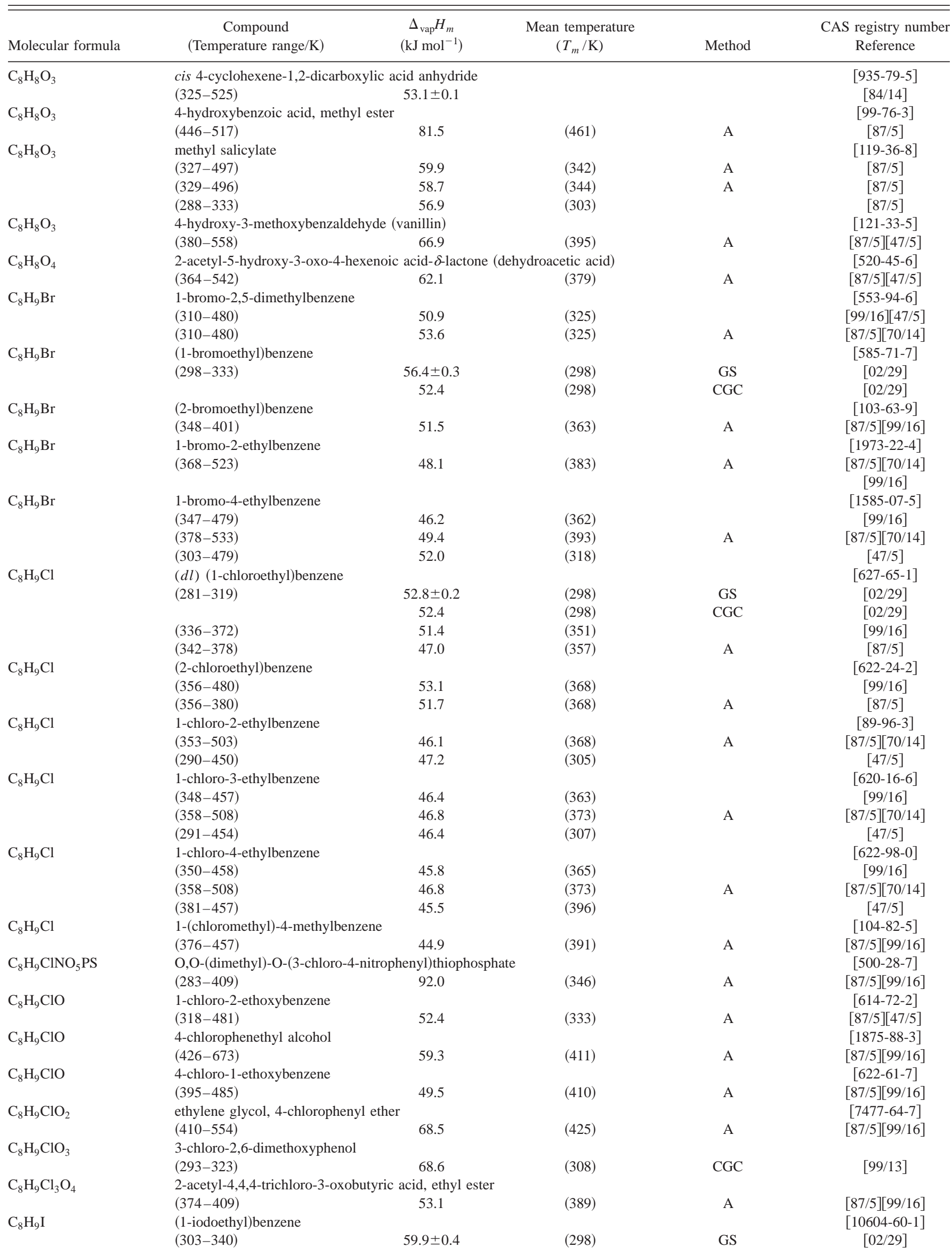


TABLE 6. Enthalpies of vaporization of organic compounds, 1880-2002-Continued

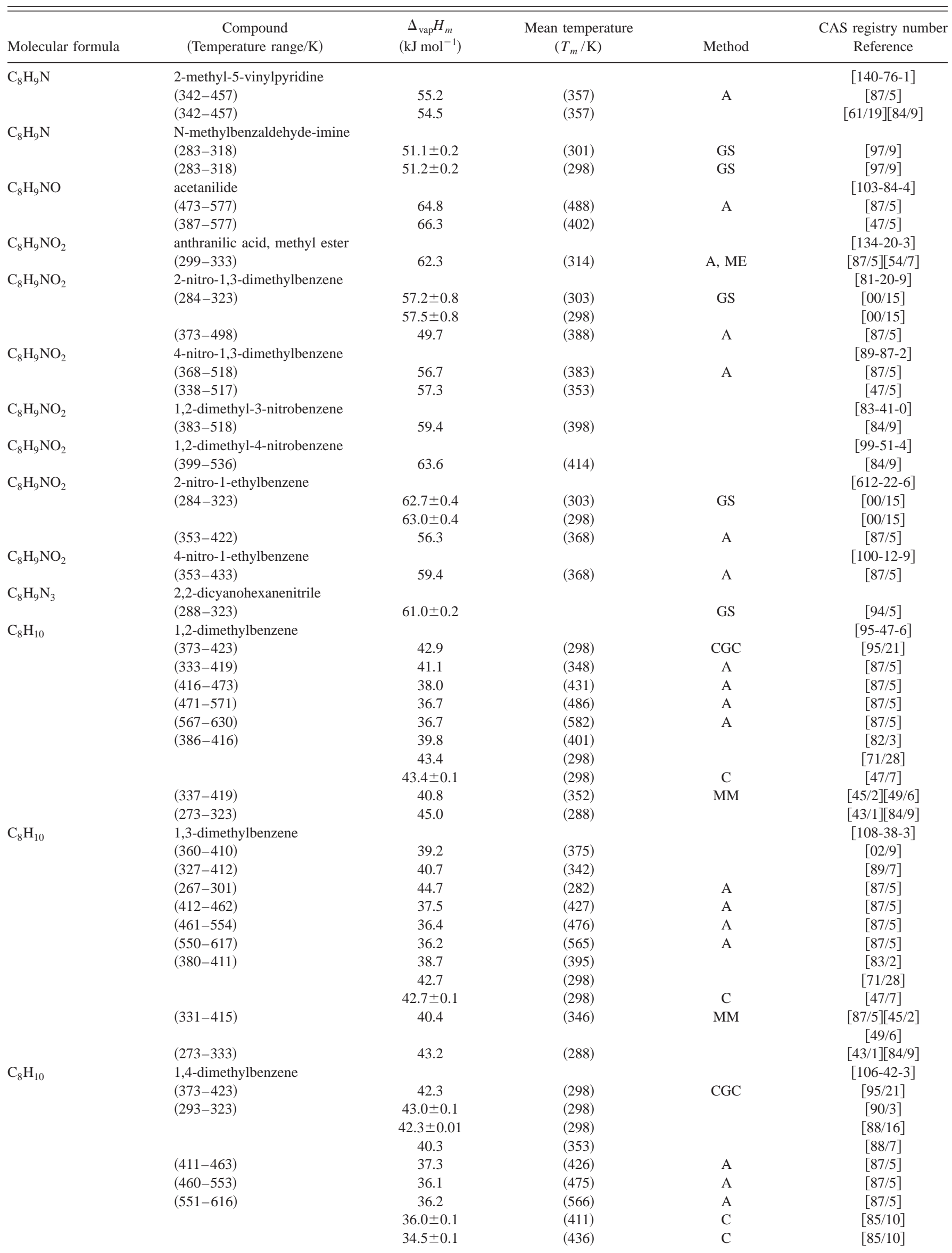


TABLE 6. Enthalpies of vaporization of organic compounds, 1880-2002-Continued

\begin{tabular}{|c|c|c|c|c|c|}
\hline Molecular formula & $\begin{array}{c}\text { Compound } \\
\text { (Temperature range/K) }\end{array}$ & $\begin{array}{c}\Delta_{\mathrm{vap}} H_{m} \\
\left(\mathrm{~kJ} \mathrm{~mol}^{-1}\right)\end{array}$ & $\begin{array}{l}\text { Mean temperature } \\
\qquad\left(T_{m} / \mathrm{K}\right)\end{array}$ & Method & $\begin{array}{l}\text { CAS registry number } \\
\text { Reference }\end{array}$ \\
\hline & & $30.5 \pm 0.1$ & $(484)$ & $\mathrm{C}$ & {$[85 / 10]$} \\
\hline & & $24.7 \pm 0.1$ & $(540)$ & $\mathrm{C}$ & {$[85 / 10]$} \\
\hline & $(380-410)$ & 37.3 & $(395)$ & & {$[82 / 3]$} \\
\hline & & $42.3 \pm 0.1$ & (298) & $\mathrm{C}$ & {$[81 / 10]$} \\
\hline & & 42.6 & (298) & & [74/13] \\
\hline & $(286-453)$ & 42.4 & $(301)$ & IPM, EB & {$[87 / 5][74 / 11]$} \\
\hline & & 42.4 & (298) & & {$[71 / 28]$} \\
\hline & $(303-343)$ & 41.6 & (318) & & {$[68 / 23]$} \\
\hline & & $42.4 \pm 0.1$ & (298) & $\mathrm{C}$ & [47/7] \\
\hline & $(332-413)$ & 40.1 & $(347)$ & MM & {$[45 / 2][49 / 6]$} \\
\hline \multirow[t]{13}{*}{$\mathrm{C}_{8} \mathrm{H}_{10}$} & ethylbenzene & & & & {$[100-41-4]$} \\
\hline & $(298-420)$ & 41.8 & (313) & A & {$[87 / 5]$} \\
\hline & $(409-459)$ & 37.0 & $(424)$ & A & [87/5] \\
\hline & $(457-554)$ & 35.8 & $(472)$ & A & {$[87 / 5]$} \\
\hline & $(320-400)$ & 40.6 & $(335)$ & & {$[86 / 13]$} \\
\hline & $(549-617)$ & 35.5 & $(564)$ & A & {$[87 / 5]$} \\
\hline & & $40.5 \pm 0.1$ & $(328)$ & $\mathrm{C}$ & {$[82 / 10]$} \\
\hline & & $39.5 \pm 0.1$ & (343) & $\mathrm{C}$ & {$[82 / 10]$} \\
\hline & & $38.6 \pm 0.1$ & $(358)$ & $\mathrm{C}$ & {$[82 / 10]$} \\
\hline & & $42.4 \pm 0.1$ & (298) & $\mathrm{C}$ & [81/10] \\
\hline & & 42.3 & (298) & & {$[71 / 28]$} \\
\hline & & $42.2 \pm 0.1$ & (298) & $\mathrm{C}$ & [47/7] \\
\hline & $(330-410)$ & 40.0 & $(345)$ & $\mathrm{MM}$ & {$[45 / 2][49 / 6]$} \\
\hline \multirow[t]{2}{*}{$\mathrm{C}_{8} \mathrm{H}_{10} \mathrm{~F}_{3} \mathrm{NO}_{3}$} & $\mathrm{~N}$-trifluoroacetyl-l-proline & ster & & & {$[715-58-2]$} \\
\hline & $(303-523)$ & 57.9 & (318) & A & {$[87 / 5][99 / 16]$} \\
\hline \multirow[t]{2}{*}{$\mathrm{C}_{8} \mathrm{H}_{10} \mathrm{~F}_{3} \mathrm{NO}_{5}$} & $\mathrm{~N}$-trifluoroacetyl- $l$-aspartic & lethyl ester & & & {$[81084-01-7]$} \\
\hline & $(303-423)$ & 58.2 & $(318)$ & A & {$[87 / 5][99 / 16]$} \\
\hline \multirow[t]{3}{*}{$\mathrm{C}_{8} \mathrm{H}_{10} \mathrm{NO}_{5} \mathrm{PS}$} & O,O-dimethyl-O-(4-nitrop & phosphate & & & [298-00-0] \\
\hline & $(293-427)$ & 88.9 & $(308)$ & A & {$[87 / 5]$} \\
\hline & & 87.0 & & GS & [79/19] \\
\hline \multirow[t]{3}{*}{$\mathrm{C}_{8} \mathrm{H}_{10} \mathrm{~N}_{2} \mathrm{O}_{2}$} & 3-nitro-N,N-dimethylanilir & & & & [619-31-8] \\
\hline & $(427-558)$ & 52.3 & $(442)$ & A, GS, EB & {$[87 / 5][60 / 4]$} \\
\hline & $(357-492)$ & 48.2 & $(372)$ & & {$[55 / 9]$} \\
\hline \multirow[t]{2}{*}{$\mathrm{C}_{8} \mathrm{H}_{10} \mathrm{O}$} & 2,3-dimethylphenol & & & & {$[526-75-0]$} \\
\hline & $(433-492)$ & 52.1 & $(448)$ & A, GS, EB & {$[87 / 5][60 / 4]$} \\
\hline \multirow[t]{4}{*}{$\mathrm{C}_{8} \mathrm{H}_{10} \mathrm{O}$} & 2,4-dimethylphenol & & & & {$[105-67-9]$} \\
\hline & $(393-433)$ & 64.6 & $(298)$ & $\mathrm{CGC}$ & {$[95 / 21]$} \\
\hline & $(282-318)$ & 65.9 & $(297)$ & A & {$[87 / 5]$} \\
\hline & $(429-486)$ & 51.8 & $(444)$ & A, GS, EB & {$[87 / 5][60 / 4]$} \\
\hline \multirow[t]{2}{*}{$\mathrm{C}_{8} \mathrm{H}_{10} \mathrm{O}$} & 2,5-dimethylphenol & & & & [95-87-4] \\
\hline & $(427-485)$ & 51.7 & $(442)$ & A, GS, EB & {$[87 / 5][60 / 4]$} \\
\hline \multirow[t]{4}{*}{$\mathrm{C}_{8} \mathrm{H}_{10} \mathrm{O}$} & 2,6-dimethylphenol & & & & [576-26-1] \\
\hline & & 75.6 & $(298)$ & & {$[71 / 7]$} \\
\hline & & 75.1 & $(298)$ & & {$[68 / 9]$} \\
\hline & $(417-476)$ & 48.5 & $(432)$ & A, GS, EB & {$[87 / 5][60 / 4]$} \\
\hline \multirow[t]{4}{*}{$\mathrm{C}_{8} \mathrm{H}_{10} \mathrm{O}$} & 3,4-dimethylphenol & & & & [96-65-8] \\
\hline & & 85.1 & $(298)$ & & {$[71 / 7]$} \\
\hline & & 85.0 & $(298)$ & & {$[68 / 9]$} \\
\hline & $(444-502)$ & 54.9 & $(459)$ & A, GS, EB & {$[87 / 5][60 / 4]$} \\
\hline \multirow[t]{3}{*}{$\mathrm{C}_{8} \mathrm{H}_{10} \mathrm{O}$} & 3,5-dimethylphenol & & & & [108-68-9] \\
\hline & & 82.0 & $(298)$ & & {$[71 / 7]$} \\
\hline & $(427-497)$ & 55.3 & $(442)$ & A, GS, EB & {$[87 / 5][60 / 4]$} \\
\hline \multirow[t]{2}{*}{$\mathrm{C}_{8} \mathrm{H}_{10} \mathrm{O}$} & benzyl methyl ether & & & & {$[538-86-3]$} \\
\hline & $(274-314)$ & $51.4 \pm 0.3$ & $(298)$ & GS & {$[02 / 29]$} \\
\hline \multirow[t]{6}{*}{$\mathrm{C}_{8} \mathrm{H}_{10} \mathrm{O}$} & ethoxybenzene & & & & {$[103-73-1]$} \\
\hline & $(390-454)$ & 44.5 & $(405)$ & A & {$[87 / 5][76 / 2]$} \\
\hline & $(390-454)$ & 50.7 & $(298)$ & & {$[76 / 2]$} \\
\hline & $(390-454)$ & 40.7 & $(443)$ & & {$[76 / 2]$} \\
\hline & & $51.0 \pm 0.1$ & (298) & $\mathrm{C}$ & {$[75 / 3]$} \\
\hline & $(400-454)$ & 44.0 & $(415)$ & & {$[65 / 7][84 / 9]$} \\
\hline \multirow[t]{4}{*}{$\mathrm{C}_{8} \mathrm{H}_{10} \mathrm{O}$} & 2-ethylphenol & & & & [90-00-6] \\
\hline & $(393-433)$ & 64.5 & $(298)$ & CGC & {$[95 / 21]$} \\
\hline & $(423-491)$ & 50.5 & $(438)$ & $\mathrm{A}, \mathrm{GS}, \mathrm{EB}$ & {$[87 / 5][63 / 10]$} \\
\hline & $(277-318)$ & 63.5 & $(292)$ & $\mathrm{A}, \mathrm{GS}, \mathrm{EB}$ & {$[87 / 5][63 / 10]$} \\
\hline
\end{tabular}


TABLE 6. Enthalpies of vaporization of organic compounds, 1880-2002-Continued

\begin{tabular}{|c|c|c|c|c|c|}
\hline Molecular formula & $\begin{array}{c}\text { Compound } \\
\text { (Temperature range/K) }\end{array}$ & $\begin{array}{c}\Delta_{\mathrm{vap}} H_{m} \\
\left(\mathrm{~kJ} \mathrm{~mol}^{-1}\right)\end{array}$ & $\begin{array}{l}\text { Mean temperature } \\
\qquad\left(T_{m} / \mathrm{K}\right)\end{array}$ & Method & $\begin{array}{c}\text { CAS registry number } \\
\text { Reference }\end{array}$ \\
\hline & $(359-480)$ & 51.6 & (374) & & {$[55 / 9]$} \\
\hline & $(321-492)$ & 51.4 & $(348)$ & & {$[53 / 9]$} \\
\hline & $(321-492)$ & 49.5 & $(373)$ & & {$[53 / 9]$} \\
\hline & $(321-492)$ & 48.6 & (398) & & {$[53 / 9]$} \\
\hline & $(321-492)$ & 47.0 & $(423)$ & & {$[53 / 9]$} \\
\hline & $(321-492)$ & 43.1 & $(473)$ & & {$[53 / 9]$} \\
\hline \multirow[t]{8}{*}{$\mathrm{C}_{8} \mathrm{H}_{10} \mathrm{O}$} & 3-ethylphenol & & & & [620-17-7] \\
\hline & $(445-503)$ & 53.1 & $(460)$ & A, GS, EB & {$[87 / 5][63 / 10]$} \\
\hline & $(277-323)$ & 68.1 & $(292)$ & $\mathrm{A}, \mathrm{GS}, \mathrm{EB}$ & {$[87 / 5][63 / 10]$} \\
\hline & $(334-501)$ & 58.3 & (348) & & {$[53 / 9]$} \\
\hline & $(334-501)$ & 56.5 & (373) & & {$[53 / 9]$} \\
\hline & $(334-501)$ & 55.2 & (398) & & {$[53 / 9]$} \\
\hline & $(334-501)$ & 53.7 & $(423)$ & & {$[53 / 9]$} \\
\hline & $(334-501)$ & 48.8 & (473) & & [53/9] \\
\hline \multirow[t]{7}{*}{$\mathrm{C}_{8} \mathrm{H}_{10} \mathrm{O}$} & 4-ethylphenol & & & & [123-07-9] \\
\hline & $(444-503)$ & 53.2 & $(459)$ & A, GS, EB & {$[87 / 5][63 / 10]$} \\
\hline & $(337-503)$ & 56.5 & $(348)$ & & {$[53 / 9]$} \\
\hline & $(337-503)$ & 54.7 & (373) & & {$[53 / 9]$} \\
\hline & $(337-503)$ & 53.8 & (398) & & {$[53 / 9]$} \\
\hline & $(337-503)$ & 51.3 & (423) & & {$[53 / 9]$} \\
\hline & $(337-503)$ & 47.6 & $(473)$ & & {$[53 / 9]$} \\
\hline \multirow[t]{2}{*}{$\mathrm{C}_{8} \mathrm{H}_{10} \mathrm{O}$} & 4-methylbenzyl alcohol & & & & [589-18-4] \\
\hline & $(338-376)$ & 64.2 & $(353)$ & $\mathrm{A}$ & {$[87 / 5]$} \\
\hline \multirow[t]{2}{*}{$\mathrm{C}_{8} \mathrm{H}_{10} \mathrm{O}$} & (dl) 1-phenylethanol & & & & {$[13323-81-4]$} \\
\hline & $(353-480)$ & 53.5 & $(368)$ & A & {$[87 / 5]$} \\
\hline \multirow[t]{3}{*}{$\mathrm{C}_{8} \mathrm{H}_{10} \mathrm{O}$} & 2-phenylethanol & & & & {$[60-12-8]$} \\
\hline & $(394-613)$ & 55.1 & (409) & A & {$[87 / 5]$} \\
\hline & $(283-318)$ & 68.4 & (298) & $\mathrm{A}, \mathrm{ME}$ & {$[87 / 5][54 / 10]$} \\
\hline \multirow[t]{2}{*}{$\mathrm{C}_{8} \mathrm{H}_{10} \mathrm{O}$} & 2-methylanisole & & & & {$[578-58-5]$} \\
\hline & & 45.2 & & & {$[86 / 10]$} \\
\hline \multirow[t]{2}{*}{$\mathrm{C}_{8} \mathrm{H}_{10} \mathrm{O}$} & 4-methylanisole & & & & [104-93-8] \\
\hline & & 46.0 & & & {$[86 / 10]$} \\
\hline \multirow[t]{2}{*}{$\mathrm{C}_{8} \mathrm{H}_{10} \mathrm{OS}$} & 4-methoxythioanisole & & & & [1879-16-9] \\
\hline & & 53.6 & & & {$[86 / 10]$} \\
\hline \multirow[t]{2}{*}{$\mathrm{C}_{8} \mathrm{H}_{10} \mathrm{O}_{2}$} & ethyl trans $\beta$-(2-furyl)acrylate & & & & \\
\hline & $(428-500)$ & 56.8 & (464) & & {$[56 / 11]$} \\
\hline \multirow[t]{3}{*}{$\mathrm{C}_{8} \mathrm{H}_{10} \mathrm{O}_{2}$} & 4-methoxybenzyl alcohol & & & & {$[105-13-5]$} \\
\hline & $(394-424)$ & 95.6 & (409) & A & {$[87 / 5]$} \\
\hline & $(354-453)$ & 71.7 & (369) & $\mathrm{EB}$ & [85/9] \\
\hline \multirow{2}{*}{$\mathrm{C}_{8} \mathrm{H}_{10} \mathrm{O}_{2}$} & 1,3-dihydroxy-2,5-dimethylbenzene & & & & [488-87-9] \\
\hline & $(393-459)$ & 74.7 & (408) & $\mathrm{A}, \mathrm{GC}$ & {$[87 / 5][75 / 24]$} \\
\hline \multirow[t]{2}{*}{$\mathrm{C}_{8} \mathrm{H}_{10} \mathrm{O}_{2}$} & 1,3-dihydroxy-4,5-dimethylbenzene & & & & {$[527-55-9]$} \\
\hline & $(424-453)$ & 67.5 & $(438)$ & $\mathrm{A}, \mathrm{GC}$ & {$[87 / 5][75 / 24]$} \\
\hline \multirow[t]{2}{*}{$\mathrm{C}_{8} \mathrm{H}_{10} \mathrm{O}_{2}$} & 1,3-dihydroxy-4,6-dimethylbenzene & & & & {$[615-89-4]$} \\
\hline & $(388-466)$ & 74.7 & $(403)$ & $\mathrm{A}, \mathrm{GC}$ & {$[75 / 24]$} \\
\hline \multirow[t]{2}{*}{$\mathrm{C}_{8} \mathrm{H}_{10} \mathrm{O}_{2}$} & 1,4-dihydroxy-2,5-dimethylbenzene & & & & {$[615-90-7]$} \\
\hline & $(331-361)$ & 101.1 & $(346)$ & $\mathrm{A}$ & {$[87 / 5]$} \\
\hline $\mathrm{C}_{8} \mathrm{H}_{10} \mathrm{O}_{2}$ & 1,3-dihydroxy-5-ethylbenzene & & & & [4299-72-3] \\
\hline & $(408-479)$ & 81.3 & $(423)$ & A, GC & {$[87 / 5][75 / 24]$} \\
\hline $\mathrm{C}_{8} \mathrm{H}_{10} \mathrm{O}_{2}$ & 1,2-dimethoxybenzene & & & & [91-16-7] \\
\hline & & 66.9 & & & {$[58 / 25]$} \\
\hline $\mathrm{C}_{8} \mathrm{H}_{10} \mathrm{O}_{2}$ & 1,3-dimethoxybenzene & & & & {$[151-10-0]$} \\
\hline & $(358-423)$ & 60.8 & (373) & $\mathrm{A}, \mathrm{GC}$ & {$[87 / 5][75 / 24]$} \\
\hline $\mathrm{C}_{8} \mathrm{H}_{10} \mathrm{O}_{2}$ & 1,4-dimethoxybenzene & & & & {$[150-78-7]$} \\
\hline & $(298-357)$ & 62.1 & (313) & A & {$[87 / 5]$} \\
\hline & & 51.5 & & & {$[86 / 10]$} \\
\hline $\mathrm{C}_{8} \mathrm{H}_{10} \mathrm{O}_{2}$ & 2-phenoxyethanol & & & & {$[122-99-6]$} \\
\hline & $(351-519)$ & 66.0 & $(366)$ & A & {$[87 / 5][47 / 5]$} \\
\hline $\mathrm{C}_{8} \mathrm{H}_{10} \mathrm{O}_{2}$ & 3-methoxy-4-hydroxytoluene & & & & {$[2896-67-5]$} \\
\hline & $(356-495)$ & 53.2 & $(371)$ & A & {$[87 / 5]$} \\
\hline $\mathrm{C}_{8} \mathrm{H}_{10} \mathrm{O}_{2} \mathrm{~S}$ & benzyl methyl sulfone & & & & {$[3112-90-1]$} \\
\hline & $(455-529)$ & 64.9 & $(470)$ & A & {$[87 / 5][99 / 16]$} \\
\hline $\mathrm{C}_{8} \mathrm{H}_{10} \mathrm{O}_{3}$ & cis cyclohexane-1,2-dicarboxylic aci & d anhydride & & & [13149-00-3] \\
\hline & $(325-525)$ & $48.8 \pm 0.1$ & & & {$[84 / 14]$} \\
\hline
\end{tabular}


TABLE 6. Enthalpies of vaporization of organic compounds, 1880-2002-Continued

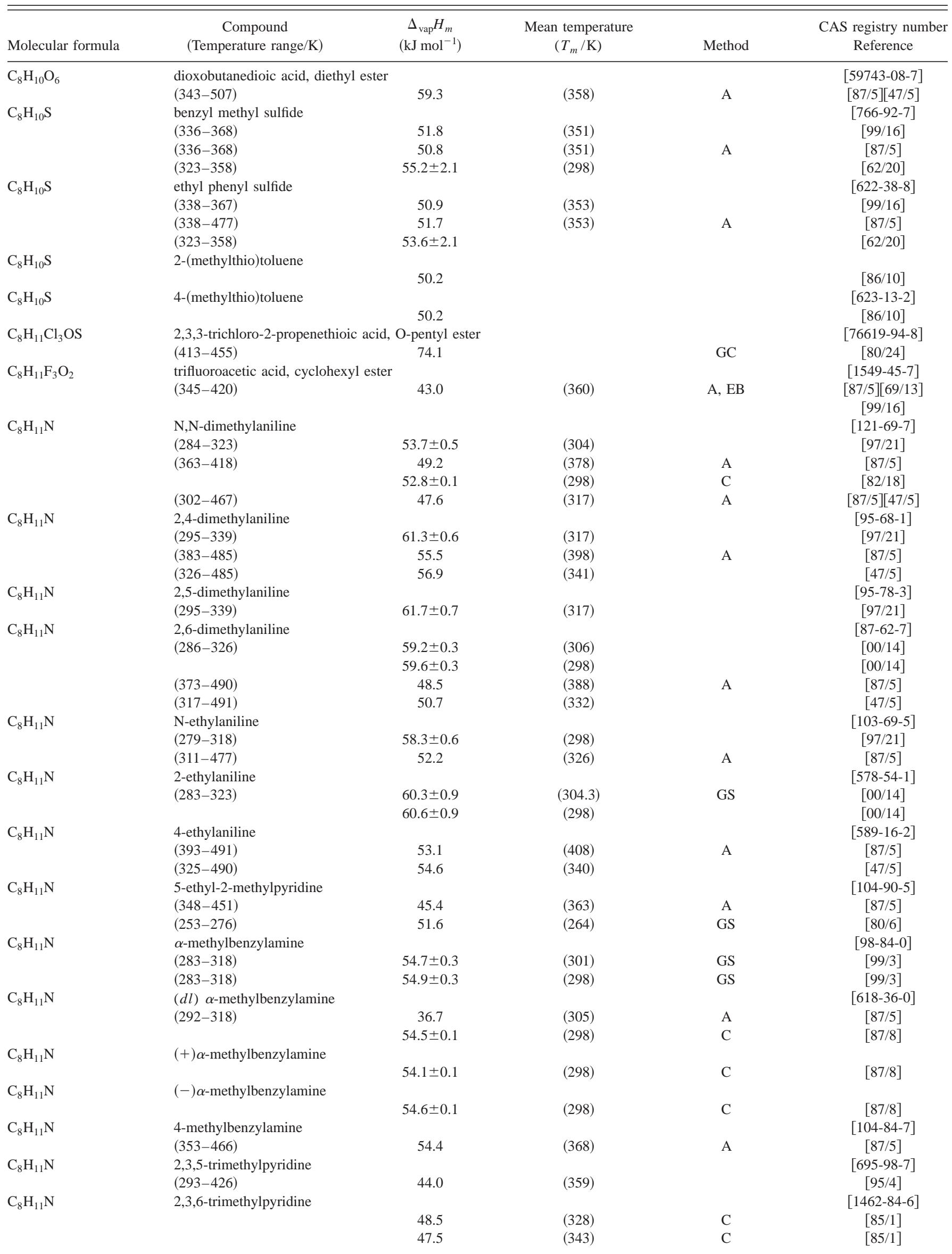


TABLE 6. Enthalpies of vaporization of organic compounds, 1880-2002-Continued

\begin{tabular}{|c|c|c|c|c|c|}
\hline Molecular formula & $\begin{array}{c}\text { Compound } \\
\text { (Temperature range/K) }\end{array}$ & $\begin{array}{c}\Delta_{\text {vap }} H_{m} \\
\left(\mathrm{~kJ} \mathrm{~mol}^{-1}\right)\end{array}$ & $\begin{array}{l}\text { Mean temperature } \\
\qquad\left(T_{m} / \mathrm{K}\right)\end{array}$ & Method & $\begin{array}{c}\text { CAS registry number } \\
\text { Reference }\end{array}$ \\
\hline & & 45.7 & $(368)$ & $\mathrm{C}$ & {$[85 / 1]$} \\
\hline \multirow[t]{8}{*}{$\mathrm{C}_{8} \mathrm{H}_{11} \mathrm{~N}$} & 2,4,6-trimethylpyridine & & & & {$[108-75-8]$} \\
\hline & $(323-373)$ & 50.2 & (298) & CGC & {$[95 / 21]$} \\
\hline & $(303-424)$ & 46.5 & $(363)$ & & {$[95 / 4]$} \\
\hline & $(298-444)$ & 51.2 & (313) & EB & {$[90 / 6]$} \\
\hline & & $50.3 \pm 0.2$ & (298) & & {$[85 / 1]$} \\
\hline & & 48.3 & (328) & $\mathrm{C}$ & {$[85 / 1]$} \\
\hline & & 47.2 & $(343)$ & $\mathrm{C}$ & {$[85 / 1]$} \\
\hline & & 45.5 & $(368)$ & $\mathrm{C}$ & {$[85 / 1]$} \\
\hline \multirow{2}{*}{$\mathrm{C}_{8} \mathrm{H}_{11} \mathrm{~N}$} & 2-propylpyridine & & & & [622-39-9] \\
\hline & $(338-445)$ & 46.6 & (353) & A & {$[87 / 5]$} \\
\hline \multirow{2}{*}{$\mathrm{C}_{8} \mathrm{H}_{11} \mathrm{~N}$} & 3-propylpyridine & & & & {$[4673-31-8]$} \\
\hline & $(350-450)$ & 49.9 & $(365)$ & $\mathrm{A}$ & {$[87 / 5]$} \\
\hline \multirow[t]{2}{*}{$\mathrm{C}_{8} \mathrm{H}_{11} \mathrm{~N}$} & 4-propypyridine & & & & {$[1122-81-2]$} \\
\hline & $(354-465)$ & 47.8 & $(369)$ & A & {$[87 / 5]$} \\
\hline \multirow[t]{2}{*}{$\mathrm{C}_{8} \mathrm{H}_{11} \mathrm{NO}$} & 2-anilinoethanol & & & & {$[122-98-5]$} \\
\hline & $(377-553)$ & 69.9 & $(392)$ & A & {$[87 / 5][47 / 5]$} \\
\hline \multirow[t]{2}{*}{$\mathrm{C}_{8} \mathrm{H}_{11} \mathrm{NO}$} & 2-ethoxyaniline & & & & {$[94-70-2]$} \\
\hline & $(373-458)$ & 57.3 & (388) & A & {$[87 / 5]$} \\
\hline \multirow[t]{2}{*}{$\mathrm{C}_{8} \mathrm{H}_{11} \mathrm{NO}$} & 4-ethoxyaniline & & & & {$[156-43-4]$} \\
\hline & $(421-523)$ & 61.2 & (436) & A & {$[87 / 5]$} \\
\hline \multirow[t]{4}{*}{$\mathrm{C}_{8} \mathrm{H}_{12}$} & tricyclo[ $\left[3 \cdot 3 \cdot 0 \cdot 0^{2,6}\right]$ octane & & & & {$[250-21-5]$} \\
\hline & $(273-343)$ & 40.0 & $(273)$ & & {$[88 / 9]$} \\
\hline & $(273-343)$ & 39.1 & (298) & & {$[88 / 9]$} \\
\hline & $(273-343)$ & 38.3 & (323) & & {$[88 / 9]$} \\
\hline \multirow[t]{2}{*}{$\mathrm{C}_{8} \mathrm{H}_{12}$} & cyclooctadiene (mixed isomers) & & & & \\
\hline & $(290-474)$ & 34.6 & $(305)$ & A & {$[87 / 5]$} \\
\hline \multirow[t]{2}{*}{$\mathrm{C}_{8} \mathrm{H}_{12}$} & cis cis 1,5 -cyclooctadiene & & & & {$[1552-12-1]$} \\
\hline & & $43.4 \pm 0.1$ & (298) & $\mathrm{C}$ & {$[96 / 18]$} \\
\hline \multirow[t]{2}{*}{$\mathrm{C}_{8} \mathrm{H}_{12}$} & 1,5-cyclooctadiene & & & & [10092-71-4] \\
\hline & $(348-386)$ & 40.9 & $(363)$ & A & {$[87 / 5]$} \\
\hline \multirow[t]{5}{*}{$\mathrm{C}_{8} \mathrm{H}_{12}$} & (dl) trans 1,2-divinylcyclobutane & & & & {$[6553-48-6]$} \\
\hline & $(319-371)$ & $38.9 \pm 0.5$ & $(298)$ & EB & {$[96 / 18]$} \\
\hline & $(350-385)$ & 39.1 & $(365)$ & $\mathrm{A}$ & {$[87 / 5]$} \\
\hline & & 42.3 & (298) & & {$[73 / 8]$} \\
\hline & & $39.0 \pm 0.5$ & $(367)$ & & {$[73 / 8]$} \\
\hline \multirow[t]{2}{*}{$\mathrm{C}_{8} \mathrm{H}_{12}$} & ( $d l$ ) 4-vinyl-1-cyclohexene & & & & {$[100-40-3]$} \\
\hline & $(292-405)$ & 40.1 & $(307)$ & A & {$[87 / 5]$} \\
\hline \multirow[t]{2}{*}{$\mathrm{C}_{8} \mathrm{H}_{12} \mathrm{Cl}_{2} \mathrm{O}_{5}$} & diethylene glycol bis(chloroacetate) & & & & \\
\hline & $(421-586)$ & 87.3 & (436) & & $\begin{array}{c}{[87 / 5][47 / 5]} \\
{[99 / 16]}\end{array}$ \\
\hline $\mathrm{C}_{8} \mathrm{H}_{12} \mathrm{~N}_{2}$ & suberic acid dinitrile & & & & {$[629-40-3]$} \\
\hline & $(303-339)$ & 77.3 & $(318)$ & A & {$[87 / 5]$} \\
\hline $\mathrm{C}_{8} \mathrm{H}_{12} \mathrm{O}$ & 1-methylnorcamphor & & & & [59348-18-4] \\
\hline & & 47.6 & & & {$[84 / 17]$} \\
\hline $\mathrm{C}_{8} \mathrm{H}_{12} \mathrm{O}$ & 2-tert-butylfuran & & & & [7040-43-9] \\
\hline & $(270-308)$ & $38.7 \pm 0.4$ & (289) & GS & {$[98 / 2]$} \\
\hline & $(270-308)$ & $38.1 \pm 0.4$ & (298) & GS & {$[98 / 2]$} \\
\hline $\mathrm{C}_{8} \mathrm{H}_{12} \mathrm{O}_{4}$ & diethyl fumerate & & & & {$[623-91-6]$} \\
\hline & $(326-492)$ & 53.2 & $(341)$ & A & {$[87 / 5][47 / 5]$} \\
\hline $\mathrm{C}_{8} \mathrm{H}_{12} \mathrm{O}_{4}$ & diethyl maleate & & & & {$[141-05-9]$} \\
\hline & $(330-498)$ & 55.2 & $(345)$ & $\mathrm{A}$ & {$[87 / 5][47 / 5]$} \\
\hline $\mathrm{C}_{8} \mathrm{H}_{12} \mathrm{O}_{4} \mathrm{~S}$ & thiodiacetic acid, diethyl ether & & & & {$[925-47-3]$} \\
\hline & $(385-448)$ & 77.7 & $(400)$ & & {$[99 / 16]$} \\
\hline $\mathrm{C}_{8} \mathrm{H}_{14}$ & cis bicyclo[3.3.0] octane & & & & {$[1755-05-1]$} \\
\hline & $(298-318)$ & 54.9 & $(308)$ & A & {$[87 / 5]$} \\
\hline & & $41.5 \pm 0.4$ & $(318)$ & & {$[70 / 30]$} \\
\hline & & $43.1 \pm 0.8$ & (298) & & {$[70 / 30]$} \\
\hline $\mathrm{C}_{8} \mathrm{H}_{14}$ & trans bicyclo[3.3.0]octane & & & & [5597-89-7] \\
\hline & $(298-320)$ & 49.6 & (309) & A & {$[87 / 5]$} \\
\hline & & $41.3 \pm 0.4$ & (320) & & {$[70 / 30]$} \\
\hline & & $42.7 \pm 0.8$ & (298) & & {$[70 / 30]$} \\
\hline $\mathrm{C}_{8} \mathrm{H}_{14}$ & cis bicyclo[4.2.0]octane & & & & [28282-35-1] \\
\hline & $(298-347)$ & 51.1 & (313) & $\mathrm{A}$ & {$[87 / 5]$} \\
\hline
\end{tabular}


TABLE 6. Enthalpies of vaporization of organic compounds, 1880-2002-Continued

\begin{tabular}{|c|c|c|c|c|c|}
\hline Molecular formula & $\begin{array}{c}\text { Compound } \\
\text { (Temperature range/K) }\end{array}$ & $\begin{array}{c}\Delta_{\mathrm{vap}} H_{m} \\
\left(\mathrm{~kJ} \mathrm{~mol}^{-1}\right)\end{array}$ & $\begin{array}{c}\text { Mean temperature } \\
\left(T_{m} / \mathrm{K}\right)\end{array}$ & Method & $\begin{array}{c}\text { CAS registry number } \\
\text { Reference }\end{array}$ \\
\hline & & $39.5 \pm 0.4$ & $(347)$ & & {$[70 / 30]$} \\
\hline & & $42.7 \pm 1.2$ & $(298)$ & & {$[70 / 30]$} \\
\hline \multirow[t]{2}{*}{$\mathrm{C}_{8} \mathrm{H}_{14}$} & cis bicyclo[5.1.0]octane & & & & [16526-90-2] \\
\hline & $(297-322)$ & $43.6 \pm 0.8$ & $(309)$ & $\mathrm{A}$ & {$[87 / 5][70 / 30]$} \\
\hline \multirow[t]{3}{*}{$\mathrm{C}_{8} \mathrm{H}_{14}$} & cyclooctene & & & & {$[931-88-4]$} \\
\hline & $(273-411)$ & 42.0 & (288) & A & {$[87 / 5]$} \\
\hline & $(273-333)$ & 41.6 & $(300)$ & & {$[41 / 6]$} \\
\hline \multirow[t]{2}{*}{$\mathrm{C}_{8} \mathrm{H}_{14}$} & vinylcyclohexane & & & & {$[695-12-5]$} \\
\hline & & $39.7 \pm 0.2$ & $(298)$ & GCC & {$[79 / 17]$} \\
\hline \multirow[t]{2}{*}{$\mathrm{C}_{8} \mathrm{H}_{14}$} & allylcyclopentane & & & & {$[3524-75-2]$} \\
\hline & & $40.4 \pm 0.2$ & $(298)$ & GCC & {$[79 / 17]$} \\
\hline $\mathrm{C}_{8} \mathrm{H}_{14}$ & $\begin{array}{l}\text { 2,5-dimethyl-1,5-hexadiene } \\
\text { (330-388) }\end{array}$ & 38.8 & $(345)$ & A & $\begin{array}{c}{[627-58-7]} \\
{[87 / 5]}\end{array}$ \\
\hline $\mathrm{C}_{8} \mathrm{H}_{14}$ & $\begin{array}{l}\text { 3,3-dimethyl-1,5-hexadiene } \\
(293-371)\end{array}$ & 35.2 & (308) & $\mathrm{A}$ & $\begin{array}{c}{[24253-25-6]} \\
{[87 / 5]}\end{array}$ \\
\hline \multirow[t]{3}{*}{$\mathrm{C}_{8} \mathrm{H}_{14}$} & 1-ethylcyclohexene & & & & {$[1453-24-3]$} \\
\hline & $(353-412)$ & 39.1 & $(368)$ & A & {$[87 / 5]$} \\
\hline & $(332-411)$ & 40.1 & $(347)$ & MM & {$[60 / 20]$} \\
\hline \multirow[t]{2}{*}{$\mathrm{C}_{8} \mathrm{H}_{14}$} & 1-methylbicyclo[4.1.0]heptane & & & & [2439-79-4] \\
\hline & $(340-394)$ & 37.2 & $(355)$ & $\mathrm{A}$ & {$[87 / 5]$} \\
\hline \multirow[t]{3}{*}{$\mathrm{C}_{8} \mathrm{H}_{14}$} & 1-octyne & & & & {$[629-05-0]$} \\
\hline & & $42.3 \pm 0.1$ & $(298)$ & $\mathrm{C}$ & {$[83 / 7]$} \\
\hline & $(357-400)$ & 38.5 & $(372)$ & A & $\begin{array}{c}{[87 / 5][70 / 24]} \\
{[84 / 9]}\end{array}$ \\
\hline \multirow[t]{3}{*}{$\mathrm{C}_{8} \mathrm{H}_{14}$} & 2-octyne & & & & [2809-67-8] \\
\hline & & $44.5 \pm 0.1$ & $(298)$ & $\mathrm{C}$ & {$[83 / 7]$} \\
\hline & $(368-412)$ & 39.9 & $(383)$ & A & $\begin{array}{c}{[87 / 5][70 / 24]} \\
{[84 / 9]}\end{array}$ \\
\hline \multirow[t]{3}{*}{$\mathrm{C}_{8} \mathrm{H}_{14}$} & 3-octyne & & & & {$[15232-76-5]$} \\
\hline & & 43.9 & (298) & & {$[\mathrm{U} / 1][85 / 6]$} \\
\hline & $(363-406)$ & 39.7 & $(378)$ & A & $\begin{array}{c}{[87 / 5][70 / 24]} \\
{[84 / 9]}\end{array}$ \\
\hline \multirow{3}{*}{$\mathrm{C}_{8} \mathrm{H}_{14}$} & 4-octyne & & & & {$[1942-45-6]$} \\
\hline & & $42.7 \pm 0.1$ & $(298)$ & $\mathrm{C}$ & {$[83 / 7]$} \\
\hline & $(362-405)$ & 39.6 & $(377)$ & A & $\begin{array}{c}{[87 / 5][70 / 24]} \\
{[84 / 9]}\end{array}$ \\
\hline \multirow[t]{2}{*}{$\mathrm{C}_{8} \mathrm{H}_{14} \mathrm{Br}_{2}$} & 1,2-dibromocyclooctane & & & & [29974-69-4] \\
\hline & $(292-354)$ & 50.3 & $(307)$ & $\mathrm{A}$ & {$[87 / 5][41 / 6]$} \\
\hline \multirow[t]{2}{*}{$\mathrm{C}_{8} \mathrm{H}_{14} \mathrm{Cl}_{2} \mathrm{~S}$} & (2-chlorocyclohexyl)(2-chloroe & sulfide & & & {$[16660-53-0]$} \\
\hline & $(293-333)$ & 62.5 & (308) & A, GS & $\begin{array}{c}{[87 / 5][48 / 9]} \\
{[99 / 16]}\end{array}$ \\
\hline $\mathrm{C}_{8} \mathrm{H}_{14} \mathrm{~N}_{2}$ & $\begin{array}{l}\text { 2-piperidinopropionitrile } \\
(283-318)\end{array}$ & $57.6 \pm 0.3$ & & GS & [97/10] \\
\hline \multirow[t]{6}{*}{$\mathrm{C}_{8} \mathrm{H}_{14} \mathrm{O}$} & cyclooctanone & & & & {$[502-49-8]$} \\
\hline & $(343-383)$ & 54.4 & $(298)$ & CGC & {$[95 / 21]$} \\
\hline & $(343-383)$ & 53.6 & (298) & CGC & {$[95 / 21]$} \\
\hline & $(343-383)$ & 54.2 & (298) & CGC & {$[95 / 21]$} \\
\hline & $(323-403)$ & 47.3 & (338) & A & {$[87 / 5]$} \\
\hline & $(394-484)$ & 46.8 & $(409)$ & $\mathrm{A}, \mathrm{EB}$ & {$[87 / 5][76 / 10]$} \\
\hline \multirow[t]{2}{*}{$\mathrm{C}_{8} \mathrm{H}_{14} \mathrm{O}$} & 2-ethyl-2-hexenal & & & & [66266-68-2] \\
\hline & $(326-448)$ & 48.4 & $(341)$ & A & {$[87 / 5][61 / 12]$} \\
\hline \multirow[t]{2}{*}{$\mathrm{C}_{8} \mathrm{H}_{14} \mathrm{O}$} & 2-ethyl-4-methyl-2-pentenal & & & & [28419-86-5] \\
\hline & $(311-436)$ & 46.7 & $(326)$ & A & {$[87 / 5][61 / 12]$} \\
\hline $\mathrm{C}_{8} \mathrm{H}_{14} \mathrm{O}$ & 6-methyl-5-hepten-2-one & & & & {$[110-93-0]$} \\
\hline & $(364-393)$ & 45.9 & (379) & & {$[89 / 8]$} \\
\hline & $(328-451)$ & $44.7 \pm 0.2$ & $(390)$ & & {$[88 / 4]$} \\
\hline $\mathrm{C}_{8} \mathrm{H}_{14} \mathrm{O}$ & ( $d l$ ) 2-propylcyclopentanone & & & & {$[1193-70-0]$} \\
\hline & $(332-457)$ & 46.0 & $(347)$ & A & {$[87 / 5]$} \\
\hline $\mathrm{C}_{8} \mathrm{H}_{14} \mathrm{O}_{2}$ & octanolactone & & & & {$[5698-29-3]$} \\
\hline & $(345-380)$ & $48.9 \pm 0.2$ & $(362)$ & MM & {$[91 / 7]$} \\
\hline & $(345-380)$ & $52.8 \pm 1.3$ & (298) & MM & {$[91 / 7]$} \\
\hline $\mathrm{C}_{8} \mathrm{H}_{14} \mathrm{O}_{2}$ & acrylic acid, neopentyl ester & & & & \\
\hline & $(301-325)$ & 45.7 & $(313)$ & A & {$[87 / 5]$} \\
\hline $\mathrm{C}_{8} \mathrm{H}_{14} \mathrm{O}_{2}$ & 1,4-butanediol divinyl ether & & & & [3891-33-6] \\
\hline
\end{tabular}


TABLE 6. Enthalpies of vaporization of organic compounds, 1880-2002-Continued

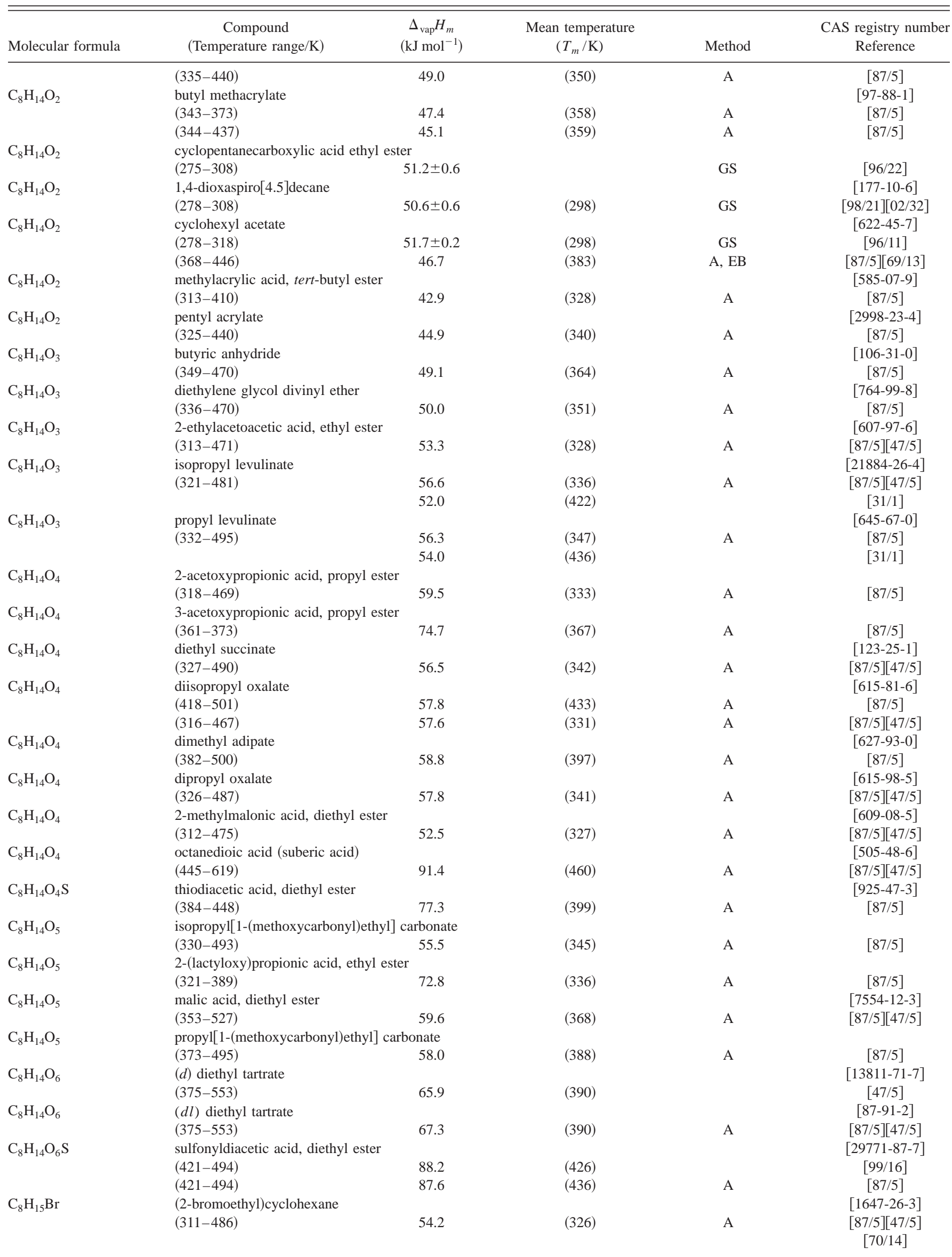


TABLE 6. Enthalpies of vaporization of organic compounds, 1880-2002-Continued

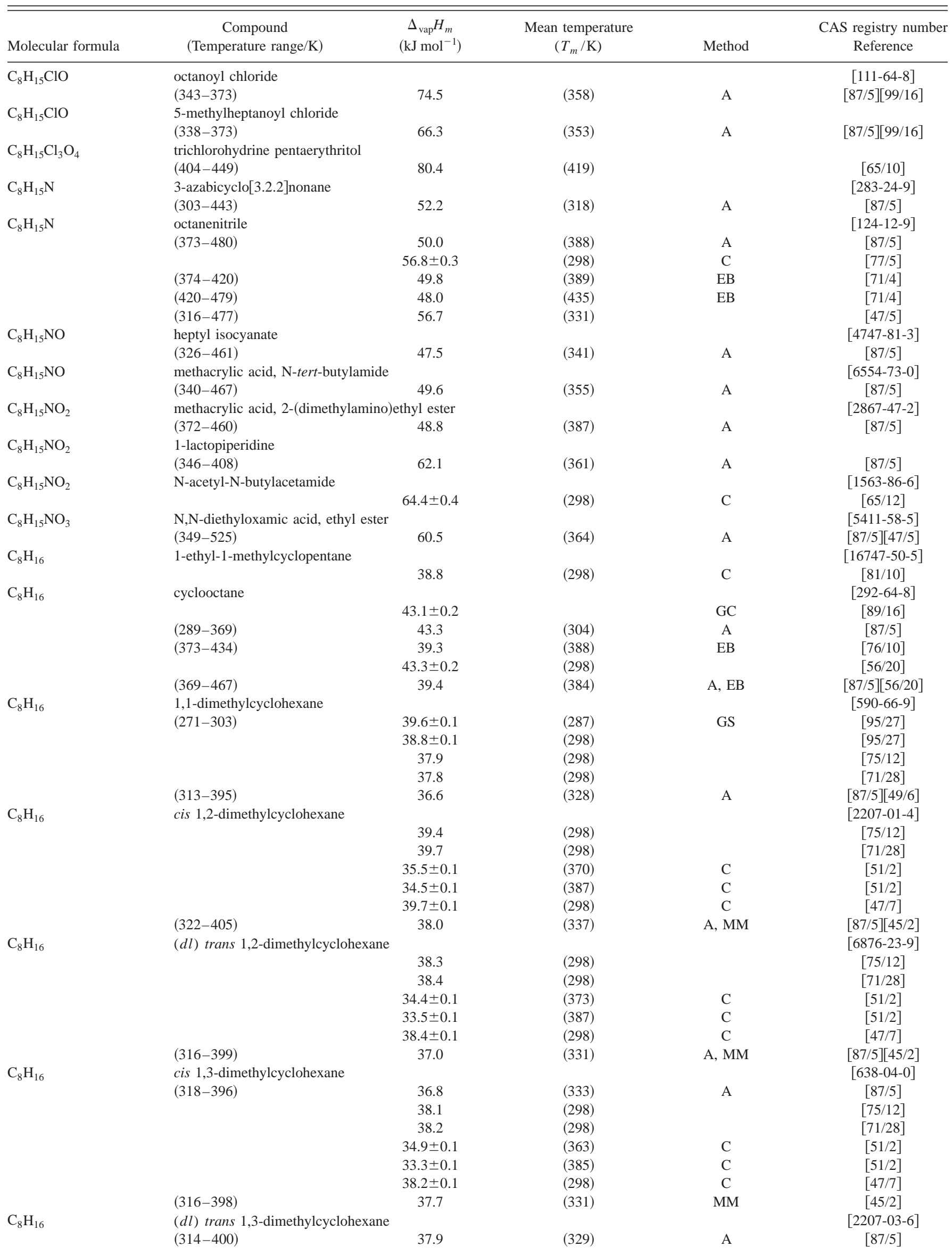


TABLE 6. Enthalpies of vaporization of organic compounds, 1880-2002-Continued

\begin{tabular}{|c|c|c|c|c|c|}
\hline Molecular formula & $\begin{array}{c}\text { Compound } \\
\text { (Temperature range/K) }\end{array}$ & $\begin{array}{c}\Delta_{\mathrm{vap}} H_{m} \\
\left(\mathrm{~kJ} \mathrm{~mol}^{-1}\right)\end{array}$ & $\begin{array}{c}\text { Mean temperature } \\
\left(T_{m} / \mathrm{K}\right)\end{array}$ & Method & $\begin{array}{c}\text { CAS registry number } \\
\text { Reference }\end{array}$ \\
\hline & & 39.1 & (298) & & {$[75 / 12]$} \\
\hline & & 39.2 & (298) & & {$[71 / 28]$} \\
\hline & & $39.2 \pm 0.1$ & (298) & $\mathrm{C}$ & {$[47 / 7]$} \\
\hline & $(314-394)$ & 37.4 & $(329)$ & MM & {$[45 / 2]$} \\
\hline \multirow[t]{5}{*}{$\mathrm{C}_{8} \mathrm{H}_{16}$} & cis 1,4-dimethylcyclohexane & & & & [624-29-3] \\
\hline & & 39.0 & (298) & & {$[75 / 12]$} \\
\hline & & 39.0 & (298) & & {$[71 / 28]$} \\
\hline & & $39.0 \pm 0.1$ & (298) & $\mathrm{C}$ & {$[47 / 7]$} \\
\hline & $(317-400)$ & 37.6 & (332) & $\mathrm{A}, \mathrm{MM}$ & {$[87 / 5][45 / 2]$} \\
\hline \multirow[t]{8}{*}{$\mathrm{C}_{8} \mathrm{H}_{16}$} & (dl) trans 1,4-dimethylcyclohexane & & & & [2207-04-7] \\
\hline & & 37.6 & $(298)$ & & {$[75 / 12]$} \\
\hline & & 37.9 & (298) & & {$[71 / 28]$} \\
\hline & & $35.6 \pm 0.1$ & $(341)$ & $\mathrm{C}$ & {$[51 / 2]$} \\
\hline & & $34.6 \pm 0.1$ & $(357)$ & $\mathrm{C}$ & {$[51 / 2]$} \\
\hline & & $33.5 \pm 0.1$ & (377) & $\mathrm{C}$ & {$[51 / 2]$} \\
\hline & & $39.9 \pm 0.1$ & $(298)$ & $\mathrm{C}$ & {$[47 / 7]$} \\
\hline & $(313-395)$ & 36.7 & $(328)$ & $\mathrm{A}, \mathrm{MM}$ & {$[87 / 5][45 / 2]$} \\
\hline \multirow[t]{12}{*}{$\mathrm{C}_{8} \mathrm{H}_{16}$} & ethylcyclohexane & & & & [1678-91-7] \\
\hline & & $39.2 \pm 0.4$ & (298) & GC & {$[87 / 17]$} \\
\hline & & $39.8 \pm 0.1$ & (313) & $\mathrm{C}$ & {$[81 / 14]$} \\
\hline & & $38.9 \pm 0.1$ & (328) & $\mathrm{C}$ & {$[81 / 14]$} \\
\hline & & $37.9 \pm 0.1$ & (343) & $\mathrm{C}$ & {$[81 / 14]$} \\
\hline & & $37.0 \pm 0.1$ & (358) & $\mathrm{C}$ & {$[81 / 14]$} \\
\hline & & $36.3 \pm 0.1$ & (368) & $\mathrm{C}$ & {$[81 / 14]$} \\
\hline & & $40.0 \pm 0.4$ & (298) & GCC & {$[78 / 16]$} \\
\hline & & 40.4 & (298) & & {$[75 / 12]$} \\
\hline & & 40.5 & (298) & & {$[41 / 28]$} \\
\hline & & $40.5 \pm 0.1$ & (298) & $\mathrm{C}$ & {$[47 / 7]$} \\
\hline & $(323-407)$ & 38.6 & $(338)$ & $\mathrm{A}, \mathrm{MM}$ & {$[87 / 5][45 / 2]$} \\
\hline \multirow[t]{4}{*}{$\mathrm{C}_{8} \mathrm{H}_{16}$} & propylcyclopentane & & & & [2040-96-2] \\
\hline & & 41.1 & (298) & & {$[71 / 28]$} \\
\hline & & $41.1 \pm 0.1$ & (298) & $\mathrm{C}$ & {$[47 / 7]$} \\
\hline & $(323-406)$ & 39.2 & (338) & $\mathrm{A}, \mathrm{MM}$ & {$[87 / 5][45 / 2]$} \\
\hline \multirow[t]{4}{*}{$\mathrm{C}_{8} \mathrm{H}_{16}$} & isopropylcyclopentane & & & & [3875-51-2] \\
\hline & & 37.9 & $(298)$ & & {$[71 / 28]$} \\
\hline & & $39.4 \pm 0.1$ & (298) & $\mathrm{C}$ & {$[47 / 7]$} \\
\hline & $(320-403)$ & 37.9 & $(335)$ & $\mathrm{A}, \mathrm{MM}$ & {$[87 / 5][45 / 2]$} \\
\hline \multirow[t]{2}{*}{$\mathrm{C}_{8} \mathrm{H}_{16}$} & 1,1,2-trimethylcyclopentane & & & & {$[4259-00-1]$} \\
\hline & $(309-389)$ & 36.3 & $(324)$ & $\mathrm{A}, \mathrm{MM}$ & {$[87 / 5][49 / 6]$} \\
\hline \multirow[t]{2}{*}{$\mathrm{C}_{8} \mathrm{H}_{16}$} & $1,1,3$-trimethylcyclopentane & & & & {$[4516-69-2]$} \\
\hline & $(301-379)$ & 35.4 & $(316)$ & $\mathrm{A}, \mathrm{MM}$ & {$[87 / 5][49 / 6]$} \\
\hline \multirow[t]{5}{*}{$\mathrm{C}_{8} \mathrm{H}_{16}$} & 1-ethyl-1-methylcyclopentane & & & & {$[16747-50-5]$} \\
\hline & $(331-397)$ & 36.7 & (346) & A & {$[87 / 5]$} \\
\hline & $(238-288)$ & 40.2 & $(273)$ & IPM & {$[87 / 5][74 / 11]$} \\
\hline & & 38.9 & (298) & & {$[71 / 28]$} \\
\hline & $(316-396)$ & 37.3 & $(331)$ & & {$[49 / 6]$} \\
\hline \multirow[t]{6}{*}{$\mathrm{C}_{8} \mathrm{H}_{16}$} & (dl) cis 1-ethyl-2-methylcyclopentane & & & & {$[930-89-2]$} \\
\hline & $(238-304)$ & 42.5 & (253) & $\mathrm{A}$ & {$[87 / 5]$} \\
\hline & $(303-403)$ & 39.3 & (318) & A & {$[87 / 5]$} \\
\hline & $(238-288)$ & 41.6 & (273) & IPM & {$[74 / 11]$} \\
\hline & & 40.2 & (298) & & {$[71 / 28]$} \\
\hline & $(322-402)$ & 38.3 & $(337)$ & & {$[49 / 6]$} \\
\hline \multirow[t]{2}{*}{$\mathrm{C}_{8} \mathrm{H}_{16}$} & trans 1-ethyl-2-methylcyclopentane & & & & \\
\hline & & 39.3 & $(298)$ & & {$[71 / 28]$} \\
\hline \multirow[t]{2}{*}{$\mathrm{C}_{8} \mathrm{H}_{16}$} & cis 1-ethyl-3-methylcyclopentane & & & & \\
\hline & & 39.3 & (298) & & {$[41 / 7]$} \\
\hline \multirow[t]{2}{*}{$\mathrm{C}_{8} \mathrm{H}_{16}$} & trans 1-ethyl-3-methylcyclopentane & & & & \\
\hline & & 38.9 & (298) & & {$[41 / 7]$} \\
\hline \multirow[t]{2}{*}{$\mathrm{C}_{8} \mathrm{H}_{16}$} & 1,1,2-trimethylcyclopentane & & & & {$[4259-00-1]$} \\
\hline & & 37.2 & $(298)$ & & {$[71 / 28]$} \\
\hline \multirow[t]{2}{*}{$\mathrm{C}_{8} \mathrm{H}_{16}$} & 1,1,3-trimethylcyclopentane & & & & {$[4516-69-2]$} \\
\hline & & 36.0 & $(298)$ & & {$[71 / 28]$} \\
\hline $\mathrm{C}_{8} \mathrm{H}_{16}$ & cis, cis 1,2,3-trimethylcyclopentane & & & & \\
\hline & & 38.9 & $(298)$ & & {$[71 / 28]$} \\
\hline
\end{tabular}


TABLE 6. Enthalpies of vaporization of organic compounds, 1880-2002-Continued

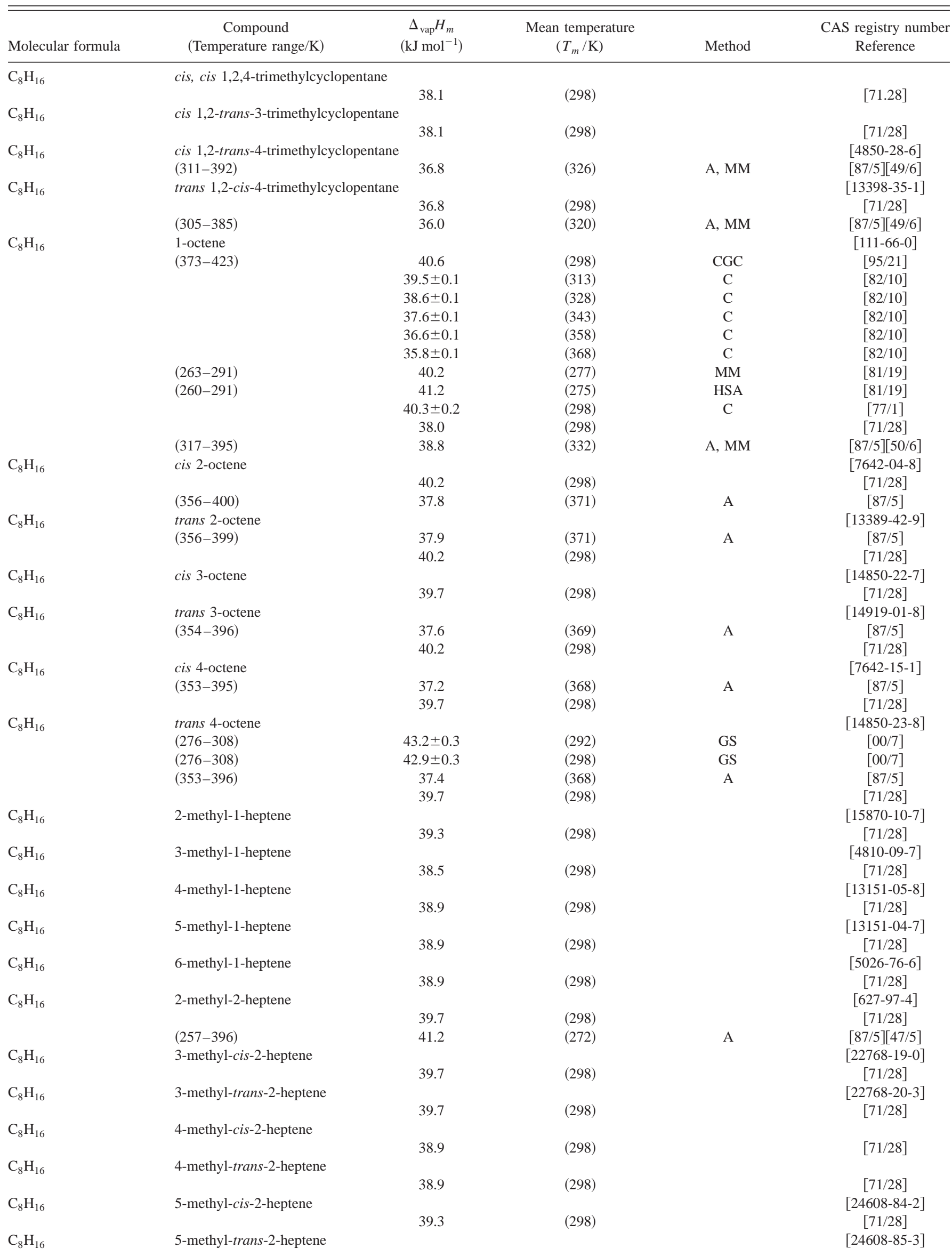


TABLE 6. Enthalpies of vaporization of organic compounds, 1880-2002-Continued

\begin{tabular}{|c|c|c|c|c|c|}
\hline Molecular formula & $\begin{array}{c}\text { Compound } \\
\text { (Temperature range/K) }\end{array}$ & $\begin{array}{c}\Delta_{\mathrm{vap}} H_{m} \\
\left(\mathrm{~kJ} \mathrm{~mol}^{-1}\right)\end{array}$ & $\begin{array}{l}\text { Mean temperature } \\
\qquad\left(T_{m} / \mathrm{K}\right)\end{array}$ & Method & $\begin{array}{c}\text { CAS registry number } \\
\text { Reference }\end{array}$ \\
\hline & & 39.3 & $(298)$ & & {$[71 / 28]$} \\
\hline \multirow[t]{2}{*}{$\mathrm{C}_{8} \mathrm{H}_{16}$} & 6-methyl-cis-2-heptene & & & & \\
\hline & & 39.3 & $(298)$ & & {$[71 / 28]$} \\
\hline \multirow[t]{2}{*}{$\mathrm{C}_{8} \mathrm{H}_{16}$} & 6-methyl-trans-2-heptene & & & & [51065-65-7] \\
\hline & & 39.3 & $(298)$ & & {$[71 / 28]$} \\
\hline \multirow[t]{2}{*}{$\mathrm{C}_{8} \mathrm{H}_{16}$} & 2-methyl-cis-3-heptene & & & & [20488-34-0] \\
\hline & & 38.9 & $(298)$ & & {$[71 / 28]$} \\
\hline \multirow[t]{2}{*}{$\mathrm{C}_{8} \mathrm{H}_{16}$} & 2-methyl-trans-3-heptene & & & & {$[692-96-6]$} \\
\hline & & 38.9 & $(298)$ & & {$[71 / 28]$} \\
\hline \multirow[t]{2}{*}{$\mathrm{C}_{8} \mathrm{H}_{16}$} & 3-methyl-cis-3-heptene & & & & [22768-17-8] \\
\hline & & 39.7 & $(298)$ & & {$[71 / 28]$} \\
\hline \multirow[t]{2}{*}{$\mathrm{C}_{8} \mathrm{H}_{16}$} & 3-methyl-trans-3-heptene & & & & [22768-18-9] \\
\hline & & 39.7 & $(298)$ & & {$[71 / 28]$} \\
\hline \multirow[t]{2}{*}{$\mathrm{C}_{8} \mathrm{H}_{16}$} & 4-methyl-cis-3-heptene & & & & {$[14255-24-4]$} \\
\hline & & 39.7 & $(298)$ & & {$[71 / 28]$} \\
\hline \multirow[t]{2}{*}{$\mathrm{C}_{8} \mathrm{H}_{16}$} & 4-methyl-trans-3-heptene & & & & [13714-85-7] \\
\hline & & 39.7 & $(298)$ & & {$[71 / 28]$} \\
\hline \multirow[t]{2}{*}{$\mathrm{C}_{8} \mathrm{H}_{16}$} & 5-methyl-cis-3-heptene & & & & [50422-80-5] \\
\hline & & 38.9 & $(298)$ & & {$[71 / 28]$} \\
\hline \multirow[t]{2}{*}{$\mathrm{C}_{8} \mathrm{H}_{16}$} & 5-methyl-trans-3-heptene & & & & [53510-18-2] \\
\hline & & 38.9 & $(298)$ & & {$[71 / 28]$} \\
\hline \multirow[t]{2}{*}{$\mathrm{C}_{8} \mathrm{H}_{16}$} & 6-methyl-cis-3-heptene & & & & [66225-19-2] \\
\hline & & 38.9 & (298) & & {$[71 / 28]$} \\
\hline \multirow[t]{2}{*}{$\mathrm{C}_{8} \mathrm{H}_{16}$} & 6-methyl-trans-3-heptene & & & & [66225-20-5] \\
\hline & & 38.9 & $(298)$ & & {$[71 / 28]$} \\
\hline \multirow[t]{2}{*}{$\mathrm{C}_{8} \mathrm{H}_{16}$} & 2-ethyl-1-hexene & & & & [1632-16-2] \\
\hline & & 39.7 & $(298)$ & & {$[71 / 28]$} \\
\hline \multirow[t]{2}{*}{$\mathrm{C}_{8} \mathrm{H}_{16}$} & 3-ethyl-1-hexene & & & & [3404-58-8] \\
\hline & & 38.5 & (298) & & {$[71 / 28]$} \\
\hline $\mathrm{C}_{8} \mathrm{H}_{16}$ & 4-ethyl-1-hexene & & & & [16746-85-3] \\
\hline & & 38.9 & (298) & & {$[71 / 28]$} \\
\hline $\mathrm{C}_{8} \mathrm{H}_{16}$ & 2,3-dimethyl-1-hexene & & & & [16746-86-4] \\
\hline & & 38.5 & $(298)$ & & {$[71 / 28]$} \\
\hline $\mathrm{C}_{8} \mathrm{H}_{16}$ & 2,4-dimethyl-1-hexene & & & & [16746-87-5] \\
\hline & & 38.5 & $(298)$ & & {$[71 / 28]$} \\
\hline $\mathrm{C}_{8} \mathrm{H}_{16}$ & 2,5-dimethyl-1-hexene & & & & [6795-92-4] \\
\hline & & 38.9 & $(298)$ & & {$[71 / 28]$} \\
\hline $\mathrm{C}_{8} \mathrm{H}_{16}$ & 3,3-dimethyl-1-hexene & & & & [3404-77-1] \\
\hline & & 36.0 & $(298)$ & & {$[71 / 28]$} \\
\hline $\mathrm{C}_{8} \mathrm{H}_{16}$ & 3,4-dimethyl-1-hexene & & & & [16745-94-1] \\
\hline & & 38.9 & $(298)$ & & {$[71 / 28]$} \\
\hline $\mathrm{C}_{8} \mathrm{H}_{16}$ & 3,5-dimethyl-1-hexene & & & & [7423-69-0] \\
\hline & & 38.1 & (298) & & {$[71 / 28]$} \\
\hline $\mathrm{C}_{8} \mathrm{H}_{16}$ & 4,4-dimethyl-1-hexene & & & & [1647-08-1] \\
\hline & & 31.0 & $(298)$ & & {$[71 / 28]$} \\
\hline $\mathrm{C}_{8} \mathrm{H}_{16}$ & 4,5-dimethyl-1-hexene & & & & [16106-59-5] \\
\hline & & 38.5 & $(298)$ & & {$[71 / 28]$} \\
\hline $\mathrm{C}_{8} \mathrm{H}_{16}$ & 5,5-dimethyl-1-hexene & & & & [7116-86-1] \\
\hline & & 37.7 & $(298)$ & & {$[71 / 28]$} \\
\hline $\mathrm{C}_{8} \mathrm{H}_{16}$ & 3-ethyl-cis-2-hexene & & & & [36880-72-5] \\
\hline & & 39.7 & $(298)$ & & {$[71 / 28]$} \\
\hline $\mathrm{C}_{8} \mathrm{H}_{16}$ & 3-ethyl-trans-2-hexene & & & & [19781-63-6] \\
\hline & & 39.7 & $(298)$ & & {$[71 / 28]$} \\
\hline $\mathrm{C}_{8} \mathrm{H}_{16}$ & 4-ethyl-cis-2-hexene & & & & [54616-49-8] \\
\hline & & 38.9 & $(298)$ & & {$[71 / 28]$} \\
\hline $\mathrm{C}_{8} \mathrm{H}_{16}$ & 4-ethyl-trans-2-hexene & & & & [19781-63-6] \\
\hline & & 38.9 & $(298)$ & & {$[71 / 28]$} \\
\hline $\mathrm{C}_{8} \mathrm{H}_{16}$ & 2,3-dimethyl-2-hexene & & & & [7145-20-2] \\
\hline & & 39.7 & $(298)$ & & {$[71 / 28]$} \\
\hline $\mathrm{C}_{8} \mathrm{H}_{16}$ & 2,4-dimethyl-2-hexene & & & & [14255-23-3] \\
\hline & & 38.5 & (298) & & {$[71 / 28]$} \\
\hline $\mathrm{C}_{8} \mathrm{H}_{16}$ & 2,5-dimethyl-2-hexene & & & & [3404-78-2] \\
\hline & & 38.9 & (298) & & {$[71 / 28]$} \\
\hline $\mathrm{C}_{8} \mathrm{H}_{16}$ & 3,4-dimethyl-cis-2-hexene & & & & [19550-81-3] \\
\hline
\end{tabular}


TABLE 6. Enthalpies of vaporization of organic compounds, 1880-2002-Continued

\begin{tabular}{|c|c|c|c|c|c|}
\hline Molecular formula & $\begin{array}{c}\text { Compound } \\
\text { (Temperature range/K) }\end{array}$ & $\begin{array}{c}\Delta_{\mathrm{vap}} H_{m} \\
\left(\mathrm{~kJ} \mathrm{~mol}^{-1}\right)\end{array}$ & $\begin{array}{l}\text { Mean temperature } \\
\qquad\left(T_{m} / \mathrm{K}\right)\end{array}$ & Method & $\begin{array}{c}\text { CAS registry number } \\
\text { Reference }\end{array}$ \\
\hline & \multirow{3}{*}{ 3,4-dimethyl-trans-2-hexene } & 39.3 & $(298)$ & & [71/28] \\
\hline \multirow[t]{2}{*}{$\mathrm{C}_{8} \mathrm{H}_{16}$} & & & & & [19550-82-4] \\
\hline & & 39.3 & (298) & & {$[71 / 28]$} \\
\hline \multirow[t]{2}{*}{$\mathrm{C}_{8} \mathrm{H}_{16}$} & \multirow[t]{2}{*}{ 3,5-dimethyl-cis-2-hexene } & & & & {$[66225-31-8]$} \\
\hline & & 38.9 & $(298)$ & & {$[71 / 28]$} \\
\hline \multirow[t]{2}{*}{$\mathrm{C}_{8} \mathrm{H}_{16}$} & \multirow[t]{2}{*}{ 3,5-dimethyl-trans-2-hexene } & & & & {$[66225-12-5]$} \\
\hline & & 38.9 & $(298)$ & & {$[71 / 28]$} \\
\hline \multirow[t]{2}{*}{$\mathrm{C}_{8} \mathrm{H}_{16}$} & \multirow[t]{2}{*}{ 4,4-dimethyl-cis-2-hexene } & & & & [66225-13-6] \\
\hline & & 38.1 & $(298)$ & & {$[71 / 28]$} \\
\hline \multirow[t]{2}{*}{$\mathrm{C}_{8} \mathrm{H}_{16}$} & \multirow[t]{2}{*}{ 4,4-dimethyl-trans-2-hexene } & & & & {$[19550-83-5]$} \\
\hline & & 38.1 & $(298)$ & & {$[71 / 28]$} \\
\hline \multirow[t]{2}{*}{$\mathrm{C}_{8} \mathrm{H}_{16}$} & \multirow[t]{2}{*}{ 4,5-dimethyl-cis-2-hexene } & & & & \\
\hline & & 38.5 & $(298)$ & & {$[71 / 28]$} \\
\hline \multirow[t]{2}{*}{$\mathrm{C}_{8} \mathrm{H}_{16}$} & \multirow[t]{2}{*}{ 4,5-dimethyl-trans-2-hexene } & & & & {$[66225-14-7]$} \\
\hline & & 38.5 & $(298)$ & & {$[71 / 28]$} \\
\hline $\mathrm{C}_{8} \mathrm{H}_{16}$ & 5,5-dimethyl-cis-2-hexene & & & & [39761-61-0] \\
\hline & & 38.1 & $(298)$ & & {$[71 / 28]$} \\
\hline $\mathrm{C}_{8} \mathrm{H}_{16}$ & 5,5-dimethyl-trans-2-hexene & & & & [39782-43-9] \\
\hline & & 38.1 & $(298)$ & & {$[71 / 28]$} \\
\hline $\mathrm{C}_{8} \mathrm{H}_{16}$ & 3-ethyl-3-hexene & & & & [16789-51-8] \\
\hline & & 39.3 & $(298)$ & & {$[71 / 28]$} \\
\hline $\mathrm{C}_{8} \mathrm{H}_{16}$ & cis 2,2-dimethyl-3-hexene & & & & {$[690-92-6]$} \\
\hline & $(319-380)$ & 35.3 & (334) & $\mathrm{A}$ & {$[87 / 5]$} \\
\hline & & 37.2 & (298) & & {$[71 / 28]$} \\
\hline & $(305-379)$ & 36.1 & $(320)$ & MM & {$[60 / 20]$} \\
\hline $\mathrm{C}_{8} \mathrm{H}_{16}$ & trans 2,2-dimethyl-3-hexene & & & & {$[690-93-7]$} \\
\hline & $(306-379)$ & 36.1 & $(321)$ & A & {$[87 / 5]$} \\
\hline & & 37.2 & (298) & & {$[71 / 28]$} \\
\hline & $(303-374)$ & 36.3 & $(318)$ & MM & {$[60 / 20]$} \\
\hline $\mathrm{C}_{8} \mathrm{H}_{16}$ & cis 2,3-dimethyl-3-hexene & & & & [59643-75-3] \\
\hline & & 38.9 & $(298)$ & & {$[71 / 28]$} \\
\hline $\mathrm{C}_{8} \mathrm{H}_{16}$ & trans 2,3-dimethyl-3-hexene & & & & [66225-30-7] \\
\hline & & 38.9 & $(298)$ & & {$[71 / 28]$} \\
\hline $\mathrm{C}_{8} \mathrm{H}_{16}$ & cis 2,4-dimethyl-3-hexene & & & & [37549-89-6] \\
\hline & & 38.5 & $(298)$ & & {$[71 / 28]$} \\
\hline $\mathrm{C}_{8} \mathrm{H}_{16}$ & trans 2,4-dimethyl-3-hexene & & & & [61847-78-7] \\
\hline & & 38.5 & $(298)$ & & {$[71 / 28]$} \\
\hline $\mathrm{C}_{8} \mathrm{H}_{16}$ & cis 2,5-dimethyl-3-hexene & & & & {$[10557-44-5]$} \\
\hline & & 37.2 & $(298)$ & & {$[71 / 28]$} \\
\hline $\mathrm{C}_{8} \mathrm{H}_{16}$ & trans 2,5-dimethyl-3-hexene & & & & {$[692-70-6]$} \\
\hline & & 37.5 & $(298)$ & & {$[71 / 28]$} \\
\hline $\mathrm{C}_{8} \mathrm{H}_{16}$ & cis 3,4-dimethyl-3-hexene & & & & [19550-87-9] \\
\hline & & 39.7 & $(298)$ & & {$[71 / 28]$} \\
\hline $\mathrm{C}_{8} \mathrm{H}_{16}$ & trans 3,4-dimethyl-3-hexene & & & & [19550-88-0] \\
\hline & & 39.7 & $(298)$ & & {$[71 / 28]$} \\
\hline $\mathrm{C}_{8} \mathrm{H}_{16}$ & 2- $n$-propyl-1-pentene & & & & \\
\hline & & 39.3 & $(298)$ & & {$[71 / 28]$} \\
\hline $\mathrm{C}_{8} \mathrm{H}_{16}$ & 2-isopropyl-1-pentene & & & & [61847-79-8] \\
\hline & & 38.8 & $(298)$ & & {$[71 / 28]$} \\
\hline $\mathrm{C}_{8} \mathrm{H}_{16}$ & 2-ethyl-3-methyl-1-pentene & & & & [3404-67-9] \\
\hline & $(307-389)$ & 36.4 & $(322)$ & A & {$[87 / 5]$} \\
\hline & & 38.9 & (298) & & {$[71 / 28]$} \\
\hline & $(308-383)$ & 36.4 & (323) & MM & {$[60 / 20]$} \\
\hline $\mathrm{C}_{8} \mathrm{H}_{16}$ & 2-ethyl-4-methyl-1-pentene & & & & [3404-80-6] \\
\hline & & 38.5 & $(298)$ & & {$[71 / 28]$} \\
\hline $\mathrm{C}_{8} \mathrm{H}_{16}$ & 3-ethyl-2-methyl-1-pentene & & & & [19780-66-6] \\
\hline & & 37.7 & $(298)$ & & {$[71 / 28]$} \\
\hline $\mathrm{C}_{8} \mathrm{H}_{16}$ & 3-ethyl-3-methyl-1-pentene & & & & [6196-60-7] \\
\hline & & 38.9 & $(298)$ & & {$[71 / 28]$} \\
\hline $\mathrm{C}_{8} \mathrm{H}_{16}$ & 3-ethyl-4-methyl-1-pentene & & & & [61847-80-1] \\
\hline & & 38.5 & $(298)$ & & {$[71 / 28]$} \\
\hline $\mathrm{C}_{8} \mathrm{H}_{16}$ & 2,3,3-trimethyl-1-pentene & & & & {$[560-23-6]$} \\
\hline & & 38.5 & $(298)$ & & {$[71 / 28]$} \\
\hline $\mathrm{C}_{8} \mathrm{H}_{16}$ & 2,3,4-trimethyl-1-pentene & & & & {$[565-76-4]$} \\
\hline
\end{tabular}


TABLE 6. Enthalpies of vaporization of organic compounds, 1880-2002-Continued

\begin{tabular}{|c|c|c|c|c|c|}
\hline Molecular formula & $\begin{array}{c}\text { Compound } \\
\text { (Temperature range/K) }\end{array}$ & $\begin{array}{c}\Delta_{\mathrm{vap}} H_{m} \\
\left(\mathrm{~kJ} \mathrm{~mol}^{-1}\right)\end{array}$ & $\begin{array}{l}\text { Mean temperature } \\
\qquad\left(T_{m} / \mathrm{K}\right)\end{array}$ & Method & $\begin{array}{c}\text { CAS registry number } \\
\text { Reference }\end{array}$ \\
\hline & & 38.5 & (298) & & {$[71 / 28]$} \\
\hline \multirow[t]{4}{*}{$\mathrm{C}_{8} \mathrm{H}_{16}$} & 2,4,4-trimethyl-1-pentene & & & & {$[107-39-1]$} \\
\hline & $(343-381)$ & 33.5 & $(358)$ & $\mathrm{A}$ & {$[87 / 5]$} \\
\hline & & 35.7 & (298) & & {$[71 / 28]$} \\
\hline & $(301-375)$ & 35.1 & (316) & MM & {$[60 / 20]$} \\
\hline \multirow[t]{2}{*}{$\mathrm{C}_{8} \mathrm{H}_{16}$} & 3,3,4-trimethyl-1-pentene & & & & {$[564-03-4]$} \\
\hline & & 38.1 & $(298)$ & & {$[71 / 28]$} \\
\hline \multirow[t]{2}{*}{$\mathrm{C}_{8} \mathrm{H}_{16}$} & 2-methyl-3-ethyl-2-pentene & & & & [19780-67-7] \\
\hline & & 39.3 & $(298)$ & & {$[71 / 28]$} \\
\hline \multirow[t]{2}{*}{$\mathrm{C}_{8} \mathrm{H}_{16}$} & 4-methyl-3-ethyl-cis-2-pentene & & & & [42067-48-1] \\
\hline & 4-methyl-3-ethyl-trans-2-pentene & 39.3 & $(298)$ & & $\begin{array}{c}{[71 / 28]} \\
{[42067-49-2]}\end{array}$ \\
\hline $\mathrm{C}_{8} \mathrm{H}_{16}$ & & 38.9 & $(298)$ & & {$[71 / 28]$} \\
\hline \multirow[t]{2}{*}{$\mathrm{C}_{8} \mathrm{H}_{16}$} & 2,3,4-trimethyl-2-pentene & & & & {$[565-77-5]$} \\
\hline & & 39.3 & $(298)$ & & {$[71 / 28]$} \\
\hline \multirow[t]{4}{*}{$\mathrm{C}_{8} \mathrm{H}_{16}$} & 2,4,4-trimethyl-2-pentene & & & & {$[107-40-4]$} \\
\hline & $(319-380)$ & 35.7 & $(334)$ & A & {$[87 / 5]$} \\
\hline & & 39.3 & $(298)$ & & {$[71 / 28]$} \\
\hline & $(305-378)$ & 37.2 & $(320)$ & MM & {$[60 / 20]$} \\
\hline \multirow[t]{2}{*}{$\mathrm{C}_{8} \mathrm{H}_{16}$} & 3,4,4-trimethyl-cis-2-pentene & & & & [39761-64-3] \\
\hline & & 38.9 & (298) & & {$[71 / 28]$} \\
\hline \multirow[t]{2}{*}{$\mathrm{C}_{8} \mathrm{H}_{16}$} & 3,4,4-trimethyl-trans-2-pentene & & & & [39761-64-3] \\
\hline & & 38.9 & (298) & & {$[71 / 28]$} \\
\hline \multirow[t]{2}{*}{$\mathrm{C}_{8} \mathrm{H}_{16}$} & 3-methyl-2-isopropyl-1-butene & & & & \\
\hline & & 38.1 & $(298)$ & & {$[71 / 28]$} \\
\hline \multirow[t]{2}{*}{$\mathrm{C}_{8} \mathrm{H}_{16}$} & 3,3-dimethyl-2-ethyl-1-butene & & & & \\
\hline & & 38.5 & $(298)$ & & {$[71 / 28]$} \\
\hline \multirow[t]{2}{*}{$\mathrm{C}_{8} \mathrm{H}_{16} \mathrm{Br}_{2}$} & 1,1-dibromooctane & & & & [62168-26-7] \\
\hline & $(412-571)$ & 57.1 & $(427)$ & A, EST & $\begin{array}{c}{[87 / 5][56 / 16]} \\
{[70 / 14][99 / 16]}\end{array}$ \\
\hline \multirow[t]{4}{*}{$\mathrm{C}_{8} \mathrm{H}_{16} \mathrm{Cl}_{2}$} & 1,1-dichlorooctane & & & & {$[20395-24-8]$} \\
\hline & $(380-480)$ & 57.7 & (298) & A & {$[87 / 12][91 / 2]$} \\
\hline & $(382-533)$ & 51.4 & (397) & A, EST & {$[87 / 5][56 / 16]$} \\
\hline & & & & & {$[70 / 14][99 / 16]$} \\
\hline \multirow[t]{3}{*}{$\mathrm{C}_{8} \mathrm{H}_{16} \mathrm{Cl}_{2}$} & 1,2-dichlorooctane & & & & [21948-46-9] \\
\hline & $(370-490)$ & 52.0 & $(385)$ & & {$[82 / 12][99 / 16]$} \\
\hline & $(370-490)$ & 57.6 & (298) & & {$[82 / 12][92 / 1]$} \\
\hline \multirow[t]{3}{*}{$\mathrm{C}_{8} \mathrm{H}_{16} \mathrm{Cl}_{2}$} & 1,8-dichlorooctane & & & & [2162-99-4] \\
\hline & $(410-510)$ & 55.9 & $(426)$ & & {$[99 / 16]$} \\
\hline & $(410-510)$ & 65.6 & $(298)$ & & {$[88 / 11][91 / 2]$} \\
\hline $\mathrm{C}_{8} \mathrm{H}_{16} \mathrm{Cl}_{2}$ & erythro-4,5-dichlorooctane & & & & [2162-99-4] \\
\hline & $(351-480)$ & 47.3 & $(415)$ & & {$[99 / 16]$} \\
\hline $\mathrm{C}_{8} \mathrm{H}_{16} \mathrm{~F}_{2}$ & 1,1-difluorooctane & & & & [61350-03-6] \\
\hline & $(329-459)$ & 44.2 & $(344)$ & A, EST & {$[87 / 5][56 / 16]$} \\
\hline & & & & & {$[70 / 14][99 / 16]$} \\
\hline $\mathrm{C}_{8} \mathrm{H}_{16} \mathrm{NO}_{2}$ & ethyl 2-(N,N-dimethylamino)-2-m & nylpropanoate & & & \\
\hline & $(278-313)$ & $51.6 \pm 0.5$ & $(298)$ & GS & {$[96 / 20]$} \\
\hline $\mathrm{C}_{8} \mathrm{H}_{16} \mathrm{~N}_{2}$ & methyl ethyl ketazine & & & & [5921-54-0] \\
\hline & $(439-524)$ & 40.0 & & & {$[93 / 21]$} \\
\hline $\mathrm{C}_{8} \mathrm{H}_{16} \mathrm{~N}_{2}$ & 1,1,4,4-tetramethyltetramethylenec & zine & & & \\
\hline & & $50.1 \pm 0.4$ & (298) & $\mathrm{C}$ & [76/3] \\
\hline $\mathrm{C}_{8} \mathrm{H}_{16} \mathrm{~N}_{2}$ & 2-diethylamino-2-methylpropionit & & & & \\
\hline & & $56.3 \pm 1.1$ & & GS & [97/10] \\
\hline $\mathrm{C}_{8} \mathrm{H}_{16} \mathrm{O}$ & octanal & & & & {$[124-13-0]$} \\
\hline & $(313-353)$ & 53.8 & (298) & CGC & {$[95 / 21]$} \\
\hline & $(293-438)$ & 43.4 & (308) & A & {$[87 / 5]$} \\
\hline & & $51.3 \pm 0.2$ & (298) & & {$[81 / 18]$} \\
\hline $\mathrm{C}_{8} \mathrm{H}_{16} \mathrm{O}$ & 2,4-dimethyl-3-hexanone & & & & [18641-70-8] \\
\hline & $(350-418)$ & 42.5 & $(365)$ & $\mathrm{A}$ & {$[87 / 5]$} \\
\hline $\mathrm{C}_{8} \mathrm{H}_{16} \mathrm{O}$ & 1-ethylcyclohexanol & & & & [1940-18-7] \\
\hline & $(324-440)$ & 46.9 & $(339)$ & $\mathrm{A}$ & {$[87 / 5]$} \\
\hline $\mathrm{C}_{8} \mathrm{H}_{16} \mathrm{O}$ & 2-methyl-3-heptanone & & & & [13019-20-0] \\
\hline & $(350-428)$ & 43.5 & $(365)$ & A & {$[87 / 5]$} \\
\hline $\mathrm{C}_{8} \mathrm{H}_{16} \mathrm{O}$ & 6-methyl-3-hepten-2-ol & & & & [51500-48-2] \\
\hline
\end{tabular}


TABLE 6. Enthalpies of vaporization of organic compounds, 1880-2002-Continued

\begin{tabular}{|c|c|c|c|c|c|}
\hline Molecular formula & $\begin{array}{c}\text { Compound } \\
\text { (Temperature range/K) }\end{array}$ & $\begin{array}{c}\Delta_{\mathrm{vap}} H_{m} \\
\left(\mathrm{~kJ} \mathrm{~mol}^{-1}\right)\end{array}$ & $\begin{array}{l}\text { Mean temperature } \\
\qquad\left(T_{m} / \mathrm{K}\right)\end{array}$ & Method & $\begin{array}{c}\text { CAS registry number } \\
\text { Reference }\end{array}$ \\
\hline & $(314-449)$ & 59.7 & (329) & A & {$[87 / 5][47 / 5]$} \\
\hline \multirow[t]{2}{*}{$\mathrm{C}_{8} \mathrm{H}_{16} \mathrm{O}$} & $(d l)$ 6-methyl-5-hepten-2-ol & & & & {$[4630-06-2]$} \\
\hline & $(314-448)$ & 57.0 & $(329)$ & A & {$[87 / 5][47 / 5]$} \\
\hline \multirow[t]{7}{*}{$\mathrm{C}_{8} \mathrm{H}_{16} \mathrm{O}$} & 2-octanone & & & & {$[111-13-7]$} \\
\hline & $(343-383)$ & 52.6 & $(298)$ & CGC & {$[95 / 21]$} \\
\hline & $(317-446)$ & 49.8 & $(332)$ & A & {$[87 / 5]$} \\
\hline & & $52.0 \pm 0.3$ & $(298)$ & GCC & {$[79 / 7]$} \\
\hline & $(324-520)$ & 49.1 & $(339)$ & A & {$[87 / 5][75 / 8]$} \\
\hline & $(324-520)$ & 51.8 & $(298)$ & & {$[75 / 8]$} \\
\hline & $(296-446)$ & 50.6 & $(311)$ & & {$[47 / 5]$} \\
\hline \multirow[t]{2}{*}{$\mathrm{C}_{8} \mathrm{H}_{16} \mathrm{O}$} & 3-octanone & & & & [106-68-3] \\
\hline & $(293-348)$ & 43.8 & $(308)$ & $\mathrm{A}$ & {$[87 / 5]$} \\
\hline \multirow[t]{2}{*}{$\mathrm{C}_{8} \mathrm{H}_{16} \mathrm{O}$} & 4-octanone & & & & {$[589-63-9]$} \\
\hline & $(288-433)$ & 36.4 & $(303)$ & A & {$[87 / 5]$} \\
\hline \multirow[t]{2}{*}{$\mathrm{C}_{8} \mathrm{H}_{16} \mathrm{O}$} & 1-propylcyclopentanol & & & & [1604-02-0] \\
\hline & $(344-447)$ & 64.2 & $(359)$ & A & {$[87 / 5]$} \\
\hline \multirow[t]{4}{*}{$\mathrm{C}_{8} \mathrm{H}_{16} \mathrm{O}$} & 2,2,4-trimethyl-3-pentanone & & & & {$[5857-36-2]$} \\
\hline & $(287-408)$ & 55.7 & $(302)$ & A & {$[87 / 5][47 / 5]$} \\
\hline & & $43.3 \pm 0.2$ & $(298)$ & $\mathrm{C}$ & {$[70 / 18]$} \\
\hline & & $43.3 \pm 0.1$ & $(298)$ & $\mathrm{C}$ & {$[66 / 2]$} \\
\hline \multirow[t]{2}{*}{$\mathrm{C}_{8} \mathrm{H}_{16} \mathrm{O}_{2}$} & 3-butoxy-2-butanone & & & & \\
\hline & $(323-398)$ & 36.7 & $(338)$ & A & {$[87 / 5]$} \\
\hline \multirow[t]{2}{*}{$\mathrm{C}_{8} \mathrm{H}_{16} \mathrm{O}_{2}$} & trans 2,2,4,6-tetramethyl-1,3-dioxane & & & & \\
\hline & & $41.9 \pm 1.2$ & $(298)$ & & {$[67 / 37]$} \\
\hline \multirow[t]{2}{*}{$\mathrm{C}_{8} \mathrm{H}_{16} \mathrm{O}_{2}$} & cis 2,2,4,6-tetramethyl-1,3-dioxane & & & & \\
\hline & & $42.3 \pm 1.2$ & $(298)$ & & {$[67 / 37]$} \\
\hline \multirow[t]{4}{*}{$\mathrm{C}_{8} \mathrm{H}_{16} \mathrm{O}_{2}$} & 1,1-dimethoxycyclohexane & & & & {$[933-40-4]$} \\
\hline & $(278-308)$ & $48.6 \pm 0.2$ & $(298)$ & GS & {$[02 / 32]$} \\
\hline & $(278-308)$ & $49.0 \pm 0.2$ & & GS & {$[98 / 21]$} \\
\hline & $(315-347)$ & 52.4 & $(331)$ & EB & {$[94 / 16]$} \\
\hline \multirow[t]{10}{*}{$\mathrm{C}_{8} \mathrm{H}_{16} \mathrm{O}_{2}$} & octanoic acid (caprylic acid) & & & & {$[124-07-2]$} \\
\hline & $(297-343)$ & $79.8 \pm 0.6$ & $(320)$ & GS & {$[00 / 6]$} \\
\hline & $(297-434)$ & $81.0 \pm 0.6$ & $(298)$ & GS & {$[00 / 6]$} \\
\hline & $(353-393)$ & 81.2 & $(298)$ & CGC & {$[95 / 21]$} \\
\hline & $(417-514)$ & 66.6 & $(432)$ & $\mathrm{A}, \mathrm{EB}$ & {$[87 / 9]$} \\
\hline & $(296-331)$ & 85.3 & $(311)$ & A & {$[87 / 5]$} \\
\hline & $(360-512)$ & 74.4 & $(375)$ & A & {$[87 / 5]$} \\
\hline & & 80.0 & $(290)$ & & {$[82 / 4]$} \\
\hline & $(291-303)$ & $82.9 \pm 1.0$ & $(298)$ & $\mathrm{TE}$ & {$[79 / 4]$} \\
\hline & & 70.0 & $(407)$ & I & {$[43 / 7]$} \\
\hline \multirow[t]{5}{*}{$\mathrm{C}_{8} \mathrm{H}_{16} \mathrm{O}_{2}$} & ethyl hexanoate & & & & {$[123-66-0]$} \\
\hline & $(345-374)$ & $47.4 \pm 0.3$ & $(359)$ & EB & [91/7] \\
\hline & $(345-379)$ & $51.5 \pm 1.3$ & $(298)$ & $\mathrm{EB}$ & {$[91 / 7]$} \\
\hline & $(396-449)$ & 51.8 & $(311)$ & A & {$[87 / 5]$} \\
\hline & $(300-376)$ & 48.6 & $(315)$ & A & {$[87 / 5]$} \\
\hline \multirow[t]{4}{*}{$\mathrm{C}_{8} \mathrm{H}_{16} \mathrm{O}_{2}$} & $(d l)$ 2-ethylhexanoic acid & & & & {$[149-57-5]$} \\
\hline & $(397-514)$ & $76.3 \pm 0.9$ & $(298)$ & EB & [97/8] \\
\hline & $(403-500)$ & 61.8 & $(418)$ & A & {$[87 / 5]$} \\
\hline & & $75.6 \pm 0.5$ & $(298)$ & $\mathrm{C}$ & {$[76 / 1]$} \\
\hline \multirow[t]{5}{*}{$\mathrm{C}_{8} \mathrm{H}_{16} \mathrm{O}_{2}$} & hexyl acetate & & & & [142-92-7] \\
\hline & & 52.1 & $(298)$ & $\mathrm{GC}$ & [97/13] \\
\hline & $(303-444)$ & 50.9 & $(318)$ & & {$[95 / 16]$} \\
\hline & $(304-381)$ & 48.9 & (319) & A & {$[87 / 5]$} \\
\hline & $(378-459)$ & 46.2 & $(387)$ & DTA & {$[80 / 8]$} \\
\hline $\mathrm{C}_{8} \mathrm{H}_{16} \mathrm{O}_{2}$ & isobutyl butyrate & & & & {$[539-90-2]$} \\
\hline & $(277-430)$ & 41.7 & $(292)$ & A & {$[87 / 5][47 / 5]$} \\
\hline $\mathrm{C}_{8} \mathrm{H}_{16} \mathrm{O}_{2}$ & isobutyl isobutyrate & & & & [97-85-8] \\
\hline & $(278-313)$ & $44.5 \pm 0.1$ & $(298)$ & GS & {$[96 / 11]$} \\
\hline & $(277-421)$ & 46.9 & $(292)$ & A & {$[87 / 5][47 / 5]$} \\
\hline $\mathrm{C}_{8} \mathrm{H}_{16} \mathrm{O}_{2}$ & isopentyl propionate & & & & {$[624-54-4]$} \\
\hline & $(281-434)$ & 44.1 & $(296)$ & A & {$[87 / 5]$} \\
\hline $\mathrm{C}_{8} \mathrm{H}_{16} \mathrm{O}_{2}$ & methyl heptanoate & & & & [106-73-0] \\
\hline & & 49.1 & $(350)$ & & {$[02 / 27]$} \\
\hline & & $50.2 \pm 0.1$ & (326) & & {$[02 / 27]$} \\
\hline
\end{tabular}


TABLE 6. Enthalpies of vaporization of organic compounds, 1880-2002-Continued

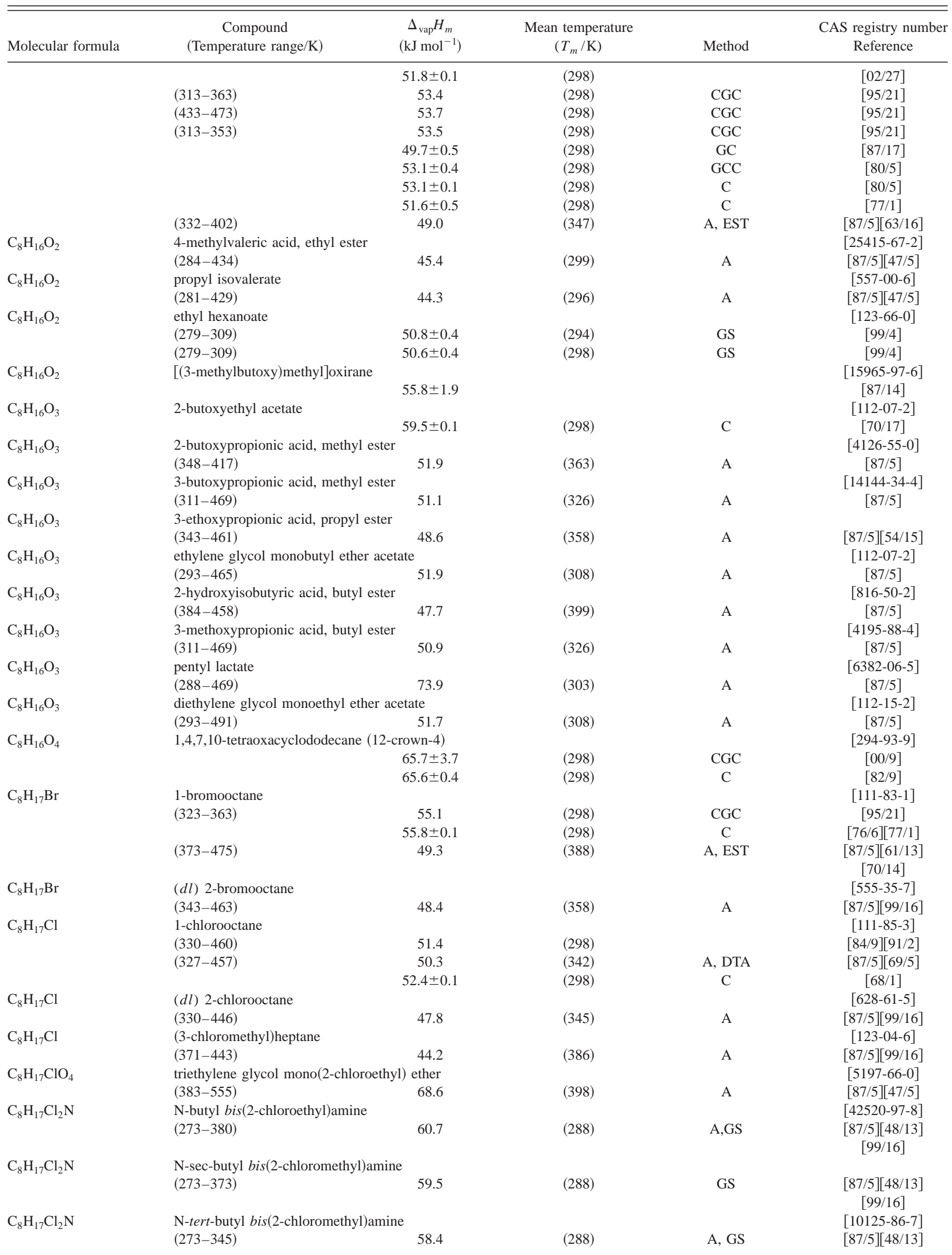


TABLE 6. Enthalpies of vaporization of organic compounds, 1880-2002-Continued

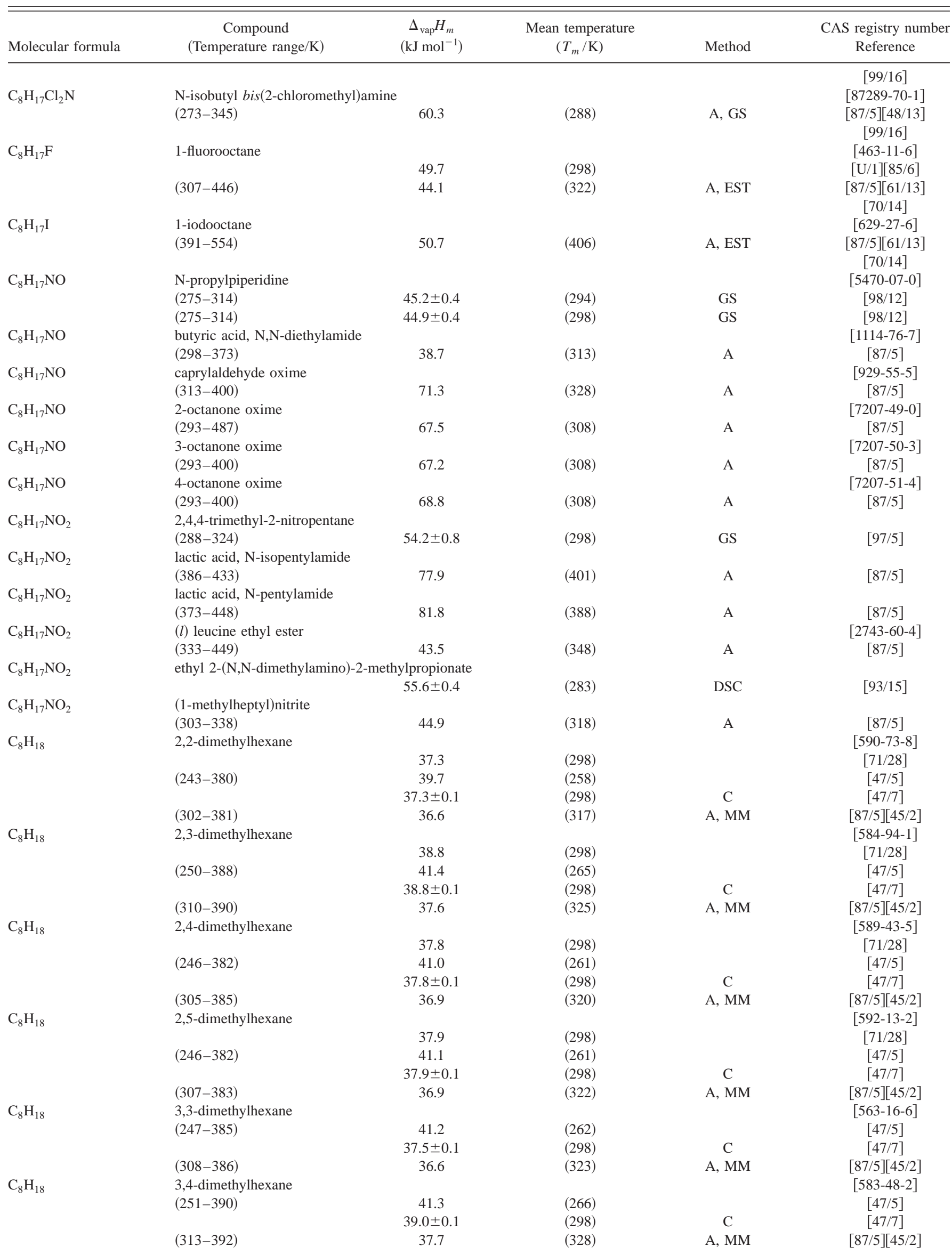


TABLE 6. Enthalpies of vaporization of organic compounds, 1880-2002-Continued

\begin{tabular}{|c|c|c|c|c|c|}
\hline Molecular formula & $\begin{array}{c}\text { Compound } \\
\text { (Temperature range/K) }\end{array}$ & $\begin{array}{c}\Delta_{\mathrm{vap}} H_{m} \\
\left(\mathrm{~kJ} \mathrm{~mol}^{-1}\right)\end{array}$ & $\begin{array}{l}\text { Mean temperature } \\
\qquad\left(T_{m} / \mathrm{K}\right)\end{array}$ & Method & $\begin{array}{c}\text { CAS registry number } \\
\text { Reference }\end{array}$ \\
\hline \multirow[t]{5}{*}{$\mathrm{C}_{8} \mathrm{H}_{18}$} & 3-ethylhexane & & & & [619-99-8] \\
\hline & & 39.7 & $(298)$ & & {$[71 / 28]$} \\
\hline & $(251-391)$ & 42.4 & (268) & & {$[47 / 5]$} \\
\hline & & $39.6 \pm 0.1$ & (298) & $\mathrm{C}$ & {$[47 / 7]$} \\
\hline & $(314-393)$ & 38.2 & (329) & $\mathrm{A}, \mathrm{MM}$ & {$[87 / 5][45 / 2]$} \\
\hline \multirow[t]{4}{*}{$\mathrm{C}_{8} \mathrm{H}_{18}$} & 3-ethyl-2-methylpentane & & & & {$[609-26-7]$} \\
\hline & & 38.5 & (298) & & {$[71 / 28]$} \\
\hline & & $38.5 \pm 0.1$ & (298) & $\mathrm{C}$ & {$[47 / 7]$} \\
\hline & $(311-390)$ & 37.4 & $(326)$ & $\mathrm{A}, \mathrm{MM}$ & {$[87 / 5][45 / 2]$} \\
\hline \multirow[t]{5}{*}{$\mathrm{C}_{8} \mathrm{H}_{18}$} & 3-ethyl-3-methylpentane & & & & [1067-08-9] \\
\hline & & 38.0 & $(298)$ & & {$[71 / 28]$} \\
\hline & $(249-391)$ & 40.2 & (264) & & {$[47 / 5]$} \\
\hline & & $38.0 \pm 0.1$ & (298) & $\mathrm{C}$ & {$[47 / 7]$} \\
\hline & $(312-393)$ & 36.9 & $(327)$ & $\mathrm{A}, \mathrm{MM}$ & {$[87 / 5][45 / 2]$} \\
\hline \multirow[t]{10}{*}{$\mathrm{C}_{8} \mathrm{H}_{18}$} & 2-methylheptane & & & & {$[592-27-8]$} \\
\hline & $(285-392)$ & 39.8 & $(300)$ & $\mathrm{A}$ & {$[87 / 5]$} \\
\hline & & $39.7 \pm 0.1$ & $(298)$ & $\mathrm{C}$ & [79/13] \\
\hline & & $38.7 \pm 0.1$ & (313) & $\mathrm{C}$ & {$[79 / 13]$} \\
\hline & & $37.3 \pm 0.1$ & (333) & $\mathrm{C}$ & {$[79 / 13]$} \\
\hline & & $36.0 \pm 0.1$ & (353) & $\mathrm{C}$ & {$[79 / 13]$} \\
\hline & $(233-283)$ & 41.6 & $(268)$ & IPM & {$[87 / 5][74 / 11]$} \\
\hline & & 39.7 & (298) & & {$[71 / 28]$} \\
\hline & & $39.8 \pm 0.1$ & (298) & $\mathrm{C}$ & {$[47 / 7]$} \\
\hline & $(315-391)$ & 38.1 & (330) & MM & {$[45 / 2]$} \\
\hline \multirow[t]{7}{*}{$\mathrm{C}_{8} \mathrm{H}_{18}$} & 3-methylheptane & & & & [589-81-1] \\
\hline & & $39.8 \pm 0.2$ & (298) & $\mathrm{C}$ & [87/19] \\
\hline & $(286-393)$ & 40.1 & (301) & A & {$[87 / 5]$} \\
\hline & $(238-286)$ & 41.6 & (271) & IPM & {$[87 / 5][74 / 11]$} \\
\hline & & 39.8 & (298) & & {$[71 / 28]$} \\
\hline & & $39.8 \pm 0.1$ & (298) & $\mathrm{C}$ & {$[47 / 7]$} \\
\hline & $(316-393)$ & 38.3 & (331) & MM & [45/2] \\
\hline \multirow{9}{*}{$\mathrm{C}_{8} \mathrm{H}_{18}$} & 4-methylheptane & & & & [589-53-7] \\
\hline & & $39.7 \pm 0.1$ & (298) & $\mathrm{C}$ & {$[79 / 13]$} \\
\hline & & $38.7 \pm 0.1$ & (313) & $\mathrm{C}$ & {$[79 / 13]$} \\
\hline & & $37.4 \pm 0.1$ & (333) & $\mathrm{C}$ & {$[79 / 13]$} \\
\hline & & $36.1 \pm 0.1$ & (353) & $\mathrm{C}$ & {$[79 / 13]$} \\
\hline & & 39.7 & (298) & & {$[71 / 28]$} \\
\hline & $(253-391)$ & 42.3 & $(268)$ & & {$[47 / 5]$} \\
\hline & & $39.7 \pm 0.1$ & (298) & $\mathrm{C}$ & [47/7] \\
\hline & $(312-392)$ & 38.2 & (327) & $\mathrm{A}, \mathrm{MM}$ & {$[87 / 5][45 / 2]$} \\
\hline \multirow[t]{21}{*}{$\mathrm{C}_{8} \mathrm{H}_{18}$} & octane & & & & [111-65-9] \\
\hline & & 41.6 & (298) & & [94/12] \\
\hline & $(297-400)$ & 41.0 & (312) & A & {$[87 / 5]$} \\
\hline & $(216-278)$ & 44.4 & (263) & A & {$[87 / 5]$} \\
\hline & $(396-432)$ & 36.3 & (411) & $\mathrm{A}$ & {$[87 / 5]$} \\
\hline & $(428-510)$ & 35.5 & (443) & $\mathrm{A}$ & {$[87 / 5]$} \\
\hline & $(506-569)$ & 34.9 & (521) & A & {$[87 / 5]$} \\
\hline & $(295-402)$ & 41.2 & (310) & & {$[86 / 13]$} \\
\hline & $(298-333)$ & 41.9 & (313) & & {$[84 / 15]$} \\
\hline & & $41.5 \pm 0.1$ & (298) & $\mathrm{C}$ & {$[81 / 9]$} \\
\hline & & $41.5 \pm 0.1$ & (298) & $\mathrm{C}$ & {$[79 / 13]$} \\
\hline & & $40.5 \pm 0.1$ & (313) & $\mathrm{C}$ & {$[79 / 13]$} \\
\hline & & $39.1 \pm 0.1$ & (333) & $\mathrm{C}$ & {$[79 / 13]$} \\
\hline & & $37.8 \pm 0.1$ & (353) & $\mathrm{C}$ & {$[79 / 13]$} \\
\hline & $(217-297)$ & 43.0 & $(282)$ & & {$[73 / 11]$} \\
\hline & & 41.5 & (298) & & {$[71 / 28]$} \\
\hline & & $38.0 \pm 0.1$ & (311) & $\mathrm{C}$ & {$[60 / 21]$} \\
\hline & & $36.7 \pm 0.1$ & (328) & $\mathrm{C}$ & {$[60 / 21]$} \\
\hline & & $35.4 \pm 0.1$ & (344) & $\mathrm{C}$ & {$[60 / 21]$} \\
\hline & & $41.5 \pm 0.1$ & $(298)$ & $\mathrm{C}$ & {$[47 / 7]$} \\
\hline & $(326-400)$ & 39.2 & $(341)$ & MM & {$[45 / 2]$} \\
\hline \multirow[t]{2}{*}{$\mathrm{C}_{8} \mathrm{H}_{18}$} & 2,2,3,3-tetramethylbutane & & & & [594-82-1] \\
\hline & $(377-390)$ & 333.0 & $(383)$ & A & {$[87 / 5]$} \\
\hline $\mathrm{C}_{8} \mathrm{H}_{18}$ & (dl) 2,2,3-trimethylpentane & & & & {$[560-21-4]$} \\
\hline
\end{tabular}


TABLE 6. Enthalpies of vaporization of organic compounds, 1880-2002-Continued

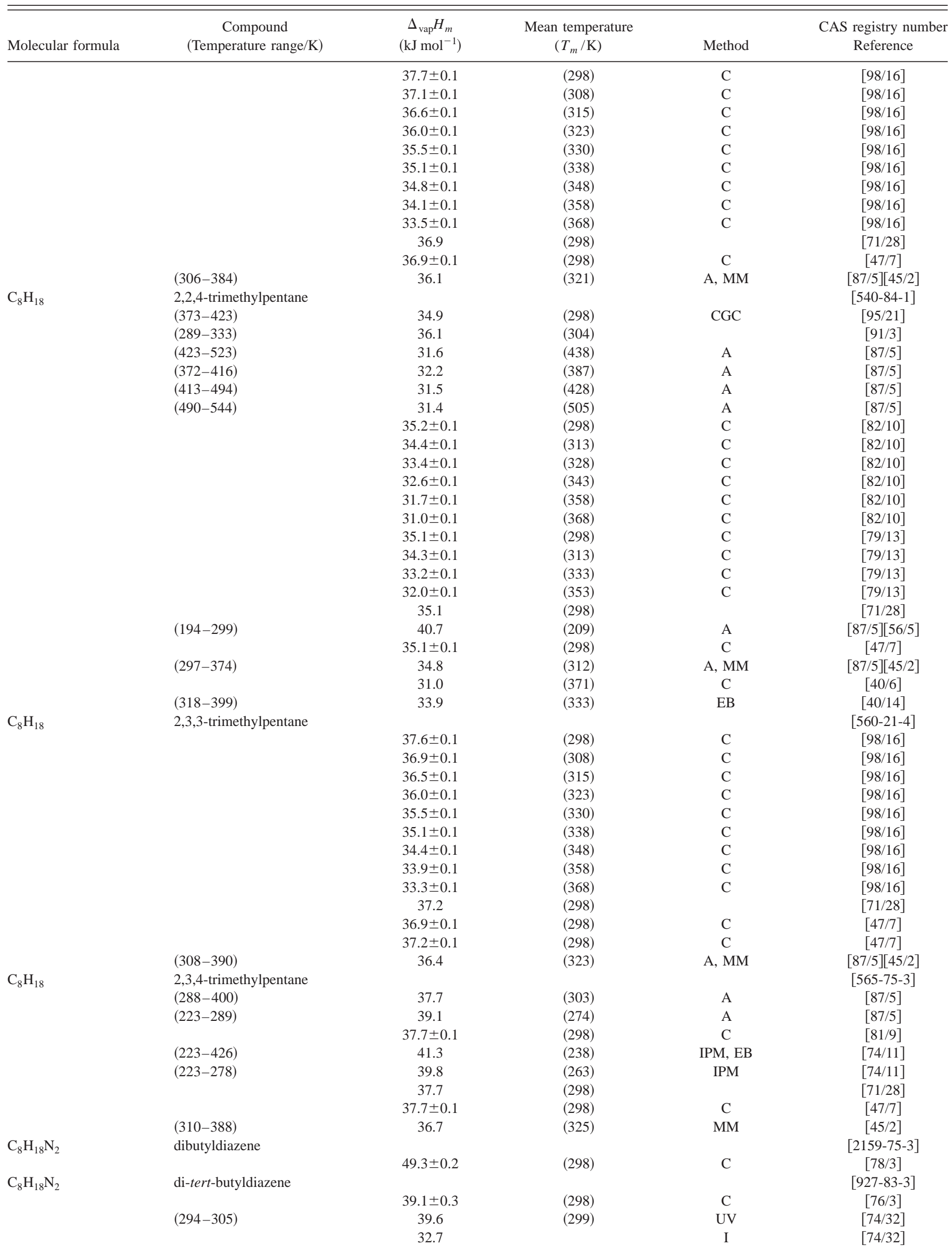


TABLE 6. Enthalpies of vaporization of organic compounds, 1880-2002-Continued

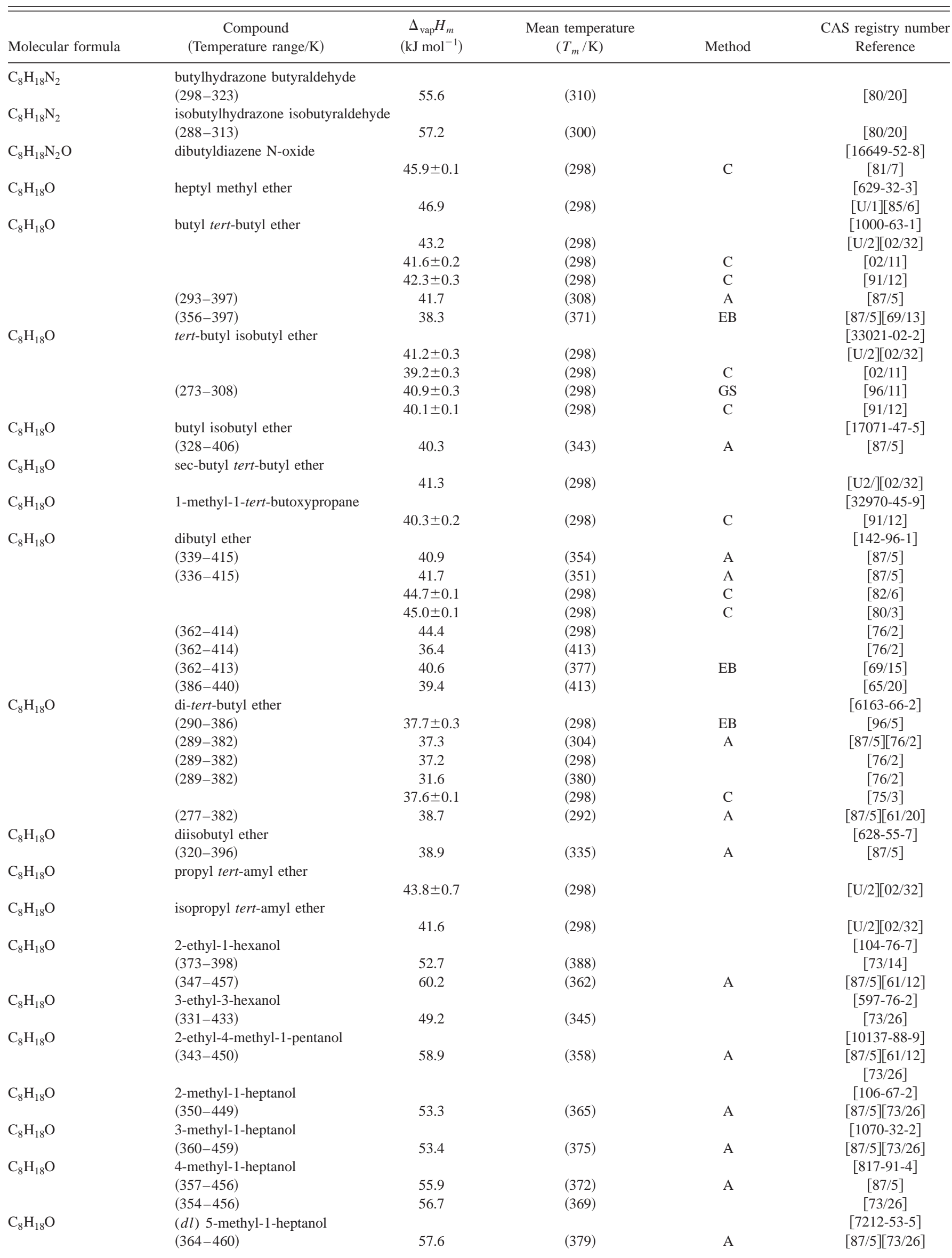


TABLE 6. Enthalpies of vaporization of organic compounds, 1880-2002-Continued

\begin{tabular}{|c|c|c|c|c|c|}
\hline Molecular formula & $\begin{array}{c}\text { Compound } \\
\text { (Temperature range/K) }\end{array}$ & $\begin{array}{c}\Delta_{\mathrm{vap}} H_{m} \\
\left(\mathrm{~kJ} \mathrm{~mol}^{-1}\right)\end{array}$ & $\begin{array}{l}\text { Mean temperature } \\
\qquad\left(T_{m} / \mathrm{K}\right)\end{array}$ & Method & $\begin{array}{c}\text { CAS registry number } \\
\text { Reference }\end{array}$ \\
\hline \multirow[t]{2}{*}{$\mathrm{C}_{8} \mathrm{H}_{18} \mathrm{O}$} & 6-methyl-1-heptanol & & & & [1653-40-3] \\
\hline & $(368-610)$ & 61.0 & (383) & A & {$[87 / 5][73 / 26]$} \\
\hline \multirow[t]{3}{*}{$\mathrm{C}_{8} \mathrm{H}_{18} \mathrm{O}$} & 2-methyl-2-heptanol & & & & {$[625-25-2]$} \\
\hline & $(343-430)$ & 53.1 & (358) & A & {$[87 / 5]$} \\
\hline & $(339-429)$ & 55.0 & $(354)$ & & {$[73 / 26]$} \\
\hline \multirow[t]{2}{*}{$\mathrm{C}_{8} \mathrm{H}_{18} \mathrm{O}$} & 3-methyl-2-heptanol & & & & [31367-46-1] \\
\hline & $(341-440)$ & 48.0 & (356) & A & {$[87 / 5][73 / 26]$} \\
\hline \multirow[t]{2}{*}{$\mathrm{C}_{8} \mathrm{H}_{18} \mathrm{O}$} & 4-methyl-2-heptanol & & & & [56298-90-9] \\
\hline & $(351-445)$ & 54.2 & $(366)$ & A & {$[87 / 5][73 / 26]$} \\
\hline \multirow[t]{3}{*}{$\mathrm{C}_{8} \mathrm{H}_{18} \mathrm{O}$} & 5-methyl-2-heptanol & & & & {$[54630-50-1]$} \\
\hline & $(348-445)$ & 51.9 & $(363)$ & A & {$[87 / 5]$} \\
\hline & $(348-445)$ & 47.2 & $(363)$ & & {$[73 / 26]$} \\
\hline \multirow[t]{2}{*}{$\mathrm{C}_{8} \mathrm{H}_{18} \mathrm{O}$} & $(d l)$ 6-methyl-2-heptanol & & & & {$[4730-22-7]$} \\
\hline & $(354-445)$ & 55.2 & (369) & A & {$[87 / 5][73 / 26]$} \\
\hline \multirow[t]{2}{*}{$\mathrm{C}_{8} \mathrm{H}_{18} \mathrm{O}$} & ( $d l$ ) 2-methyl-3-heptanol & & & & [18720-62-2] \\
\hline & $(349-441)$ & 54.8 & $(364)$ & $\mathrm{A}$ & {$[87 / 5][73 / 26]$} \\
\hline \multirow[t]{3}{*}{$\mathrm{C}_{8} \mathrm{H}_{18} \mathrm{O}$} & 3-methyl-3-heptanol & & & & {$[5582-82-1]$} \\
\hline & $(344-433)$ & 54.1 & $(359)$ & A & {$[87 / 5]$} \\
\hline & $(338-433)$ & 54.7 & (353) & & {$[73 / 26]$} \\
\hline \multirow[t]{2}{*}{$\mathrm{C}_{8} \mathrm{H}_{18} \mathrm{O}$} & 4-methyl-3-heptanol & & & & [14979-39-6] \\
\hline & $(330-429)$ & 43.9 & $(345)$ & $\mathrm{A}$ & {$[87 / 5][73 / 26]$} \\
\hline \multirow[t]{2}{*}{$\mathrm{C}_{8} \mathrm{H}_{18} \mathrm{O}$} & 5-methyl-3-heptanol & & & & {$[18720-65-5]$} \\
\hline & $(330-427)$ & 46.5 & $(345)$ & A & {$[87 / 5][73 / 26]$} \\
\hline \multirow[t]{2}{*}{$\mathrm{C}_{8} \mathrm{H}_{18} \mathrm{O}$} & $(d l)$ 6-methyl-3-heptanol & & & & [18720-66-6] \\
\hline & $(333-432)$ & 47.6 & $(348)$ & A & {$[87 / 5]$} \\
\hline \multirow[t]{3}{*}{$\mathrm{C}_{8} \mathrm{H}_{18} \mathrm{O}$} & 2-methyl-4-heptanol & & & & {$[21570-35-4]$} \\
\hline & $(348-440)$ & 54.8 & $(363)$ & A & {$[87 / 5]$} \\
\hline & $(345-437)$ & 56.3 & $(360)$ & & {$[73 / 26]$} \\
\hline \multirow[t]{2}{*}{$\mathrm{C}_{8} \mathrm{H}_{18} \mathrm{O}$} & ( $d l$ ) 3-methyl-4-heptanol & & & & [1838-73-9] \\
\hline & $(340-438)$ & 48.0 & $(355)$ & $\mathrm{A}$ & {$[87 / 5][73 / 26]$} \\
\hline \multirow[t]{3}{*}{$\mathrm{C}_{8} \mathrm{H}_{18} \mathrm{O}$} & 4-methyl-4-heptanol & & & & [598-01-6] \\
\hline & $(344-434)$ & 54.4 & $(359)$ & A & {$[87 / 5]$} \\
\hline & $(331-434)$ & 54.8 & $(345)$ & & {$[73 / 26]$} \\
\hline $\mathrm{C}_{8} \mathrm{H}_{18} \mathrm{O}$ & 2,5-dimethyl-3-hexanol & & & & [19550-07-3] \\
\hline & $(337-431)$ & 55.0 & $(352)$ & & {$[73 / 26]$} \\
\hline $\mathrm{C}_{8} \mathrm{H}_{18} \mathrm{O}$ & 1-octanol & & & & {$[111-87-5]$} \\
\hline & $(282-321)$ & 69.6 & $(303)$ & GS & {$[01 / 3]$} \\
\hline & $(282-321)$ & 70.1 & (298) & GS & {$[01 / 3]$} \\
\hline & $(373-423)$ & 71.6 & (298) & CGC & {$[95 / 21]$} \\
\hline & $(273-363)$ & 68.7 & $(318)$ & & {$[92 / 14]$} \\
\hline & $(328-400)$ & 67.3 & $(343)$ & A & {$[87 / 5]$} \\
\hline & $(430-474)$ & 52.5 & $(445)$ & A & {$[87 / 5]$} \\
\hline & $(397-479)$ & 56.6 & $(412)$ & A & {$[87 / 5]$} \\
\hline & $(475-555)$ & 47.8 & $(490)$ & $\mathrm{A}$ & {$[87 / 5]$} \\
\hline & & $71.0 \pm 0.4$ & (298) & $\mathrm{C}$ & {$[77 / 1]$} \\
\hline & $(343-468)$ & 67.5 & $(358)$ & & [73/26] \\
\hline & $(386-480)$ & 58.3 & $(401)$ & $\mathrm{EB}$ & {$[87 / 5][70 / 2]$} \\
\hline & $(352-468)$ & 65.0 & (367) & DTA & {$[69 / 5]$} \\
\hline & $(293-353)$ & 70.4 & (308) & & {$[66 / 7]$} \\
\hline & $(267-282)$ & 64.0 & (274) & $\mathrm{A}, \mathrm{ME}$ & {$[87 / 5][65 / 15]$} \\
\hline & $(365-427)$ & 61.6 & $(380)$ & & {$[58 / 2]$} \\
\hline $\mathrm{C}_{8} \mathrm{H}_{18} \mathrm{O}$ & $(d l)$ 2-octanol & & & & [123-96-6] \\
\hline & $(253-353)$ & 70.7 & $(268)$ & & [99/11] \\
\hline & $(333-453)$ & 60.7 & (348) & A & {$[87 / 5]$} \\
\hline & $(367-453)$ & 56.1 & (382) & & {$[84 / 10]$} \\
\hline & $(345-453)$ & 60.0 & $(360)$ & & {$[73 / 26]$} \\
\hline $\mathrm{C}_{8} \mathrm{H}_{18} \mathrm{O}$ & $(d l)$ 3-octanol & & & & [20296-29-1] \\
\hline & $(253-348)$ & 71.6 & $(268)$ & & [99/11] \\
\hline & $(313-450)$ & 64.1 & $(328)$ & A & {$[87 / 5]$} \\
\hline & $(366-450)$ & 54.5 & $(381)$ & & {$[84 / 10]$} \\
\hline & $(349-449)$ & 58.8 & (364) & & {$[73 / 26]$} \\
\hline $\mathrm{C}_{8} \mathrm{H}_{18} \mathrm{O}$ & $(d l)$ 4-octanol & & & & [589-62-8] \\
\hline & $(343-450)$ & 57.3 & $(358)$ & A & [87/5] \\
\hline
\end{tabular}


TABLE 6. Enthalpies of vaporization of organic compounds, 1880-2002-Continued

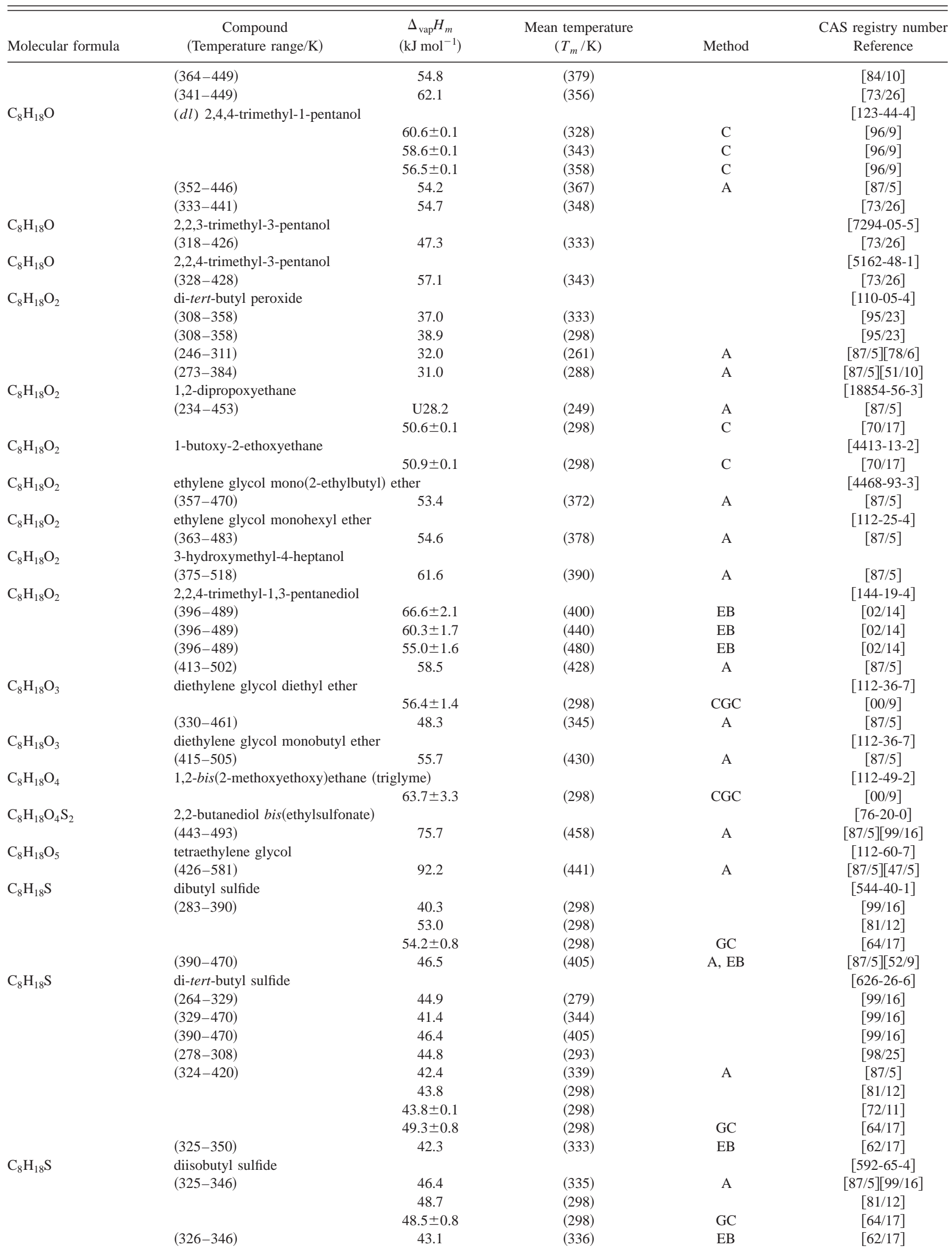


TABLE 6. Enthalpies of vaporization of organic compounds, 1880-2002-Continued

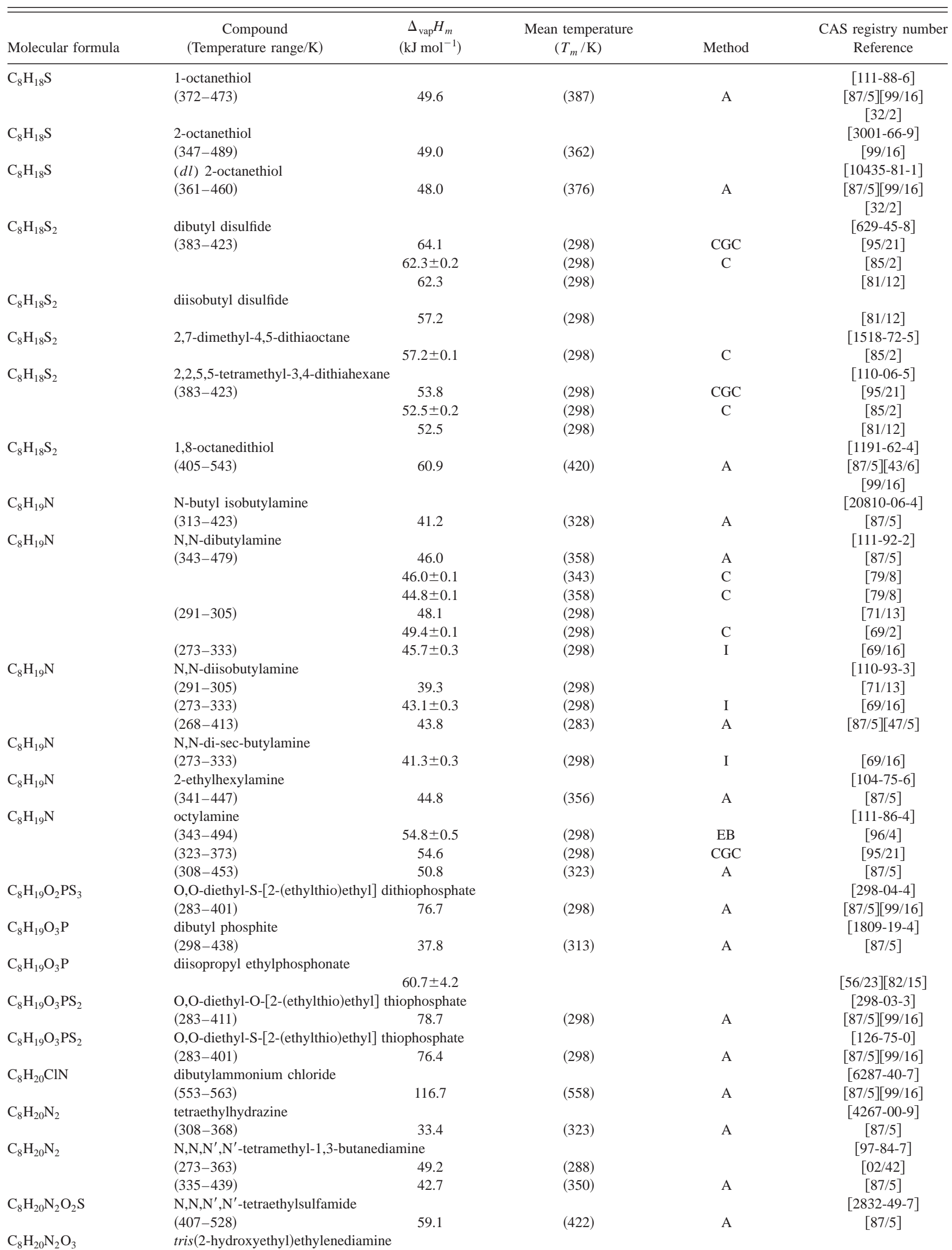


TABLE 6. Enthalpies of vaporization of organic compounds, 1880-2002-Continued

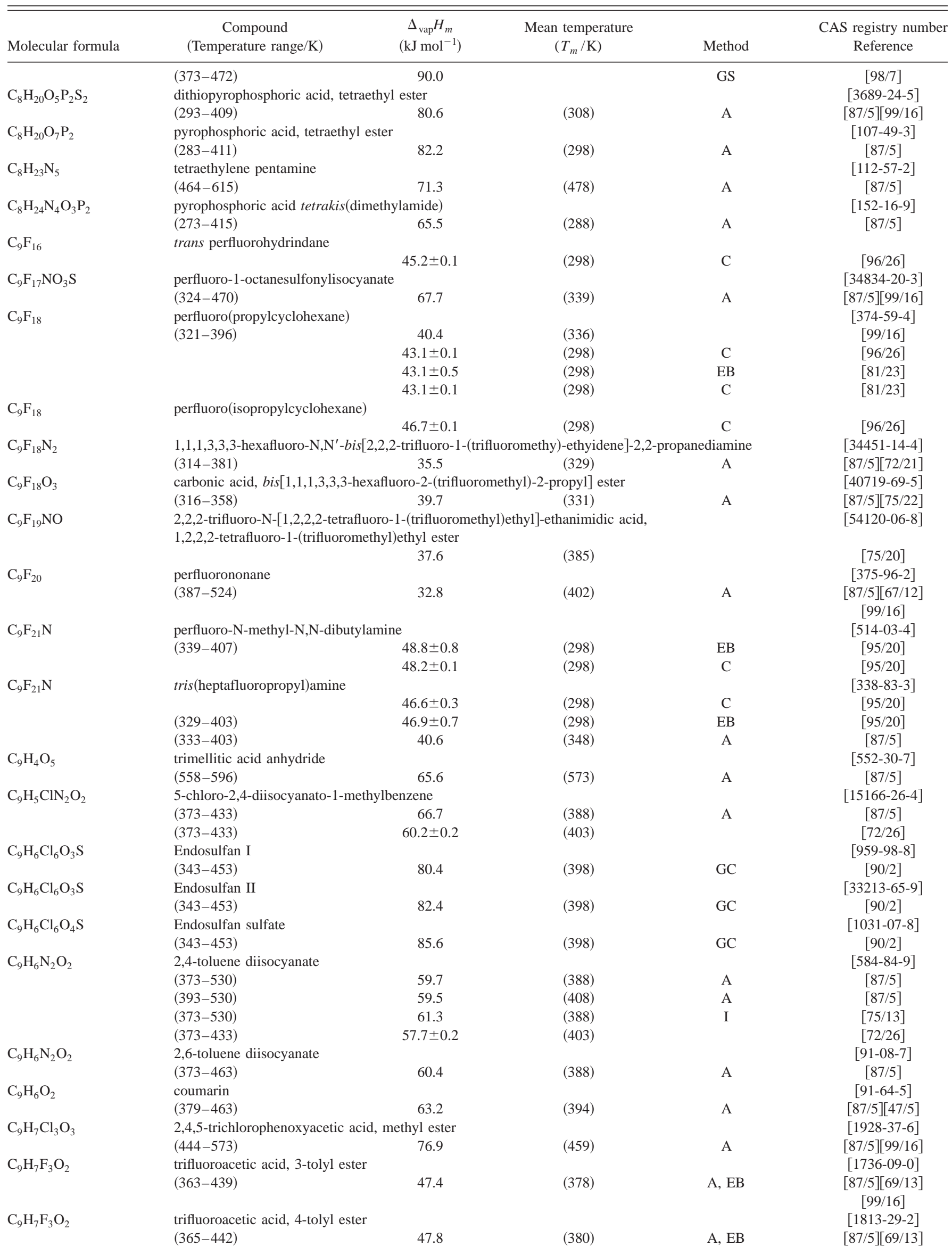


TABLE 6. Enthalpies of vaporization of organic compounds, 1880-2002-Continued

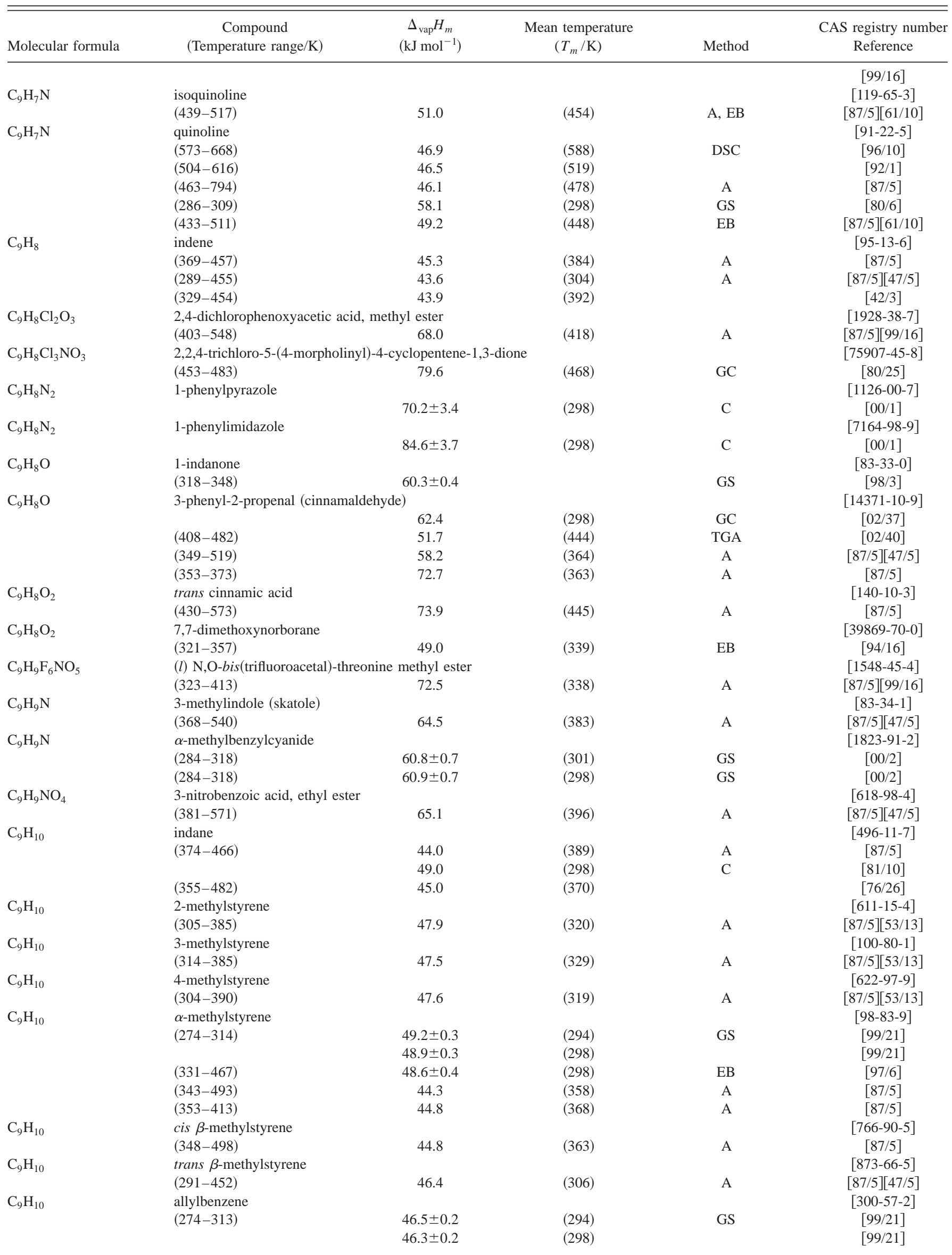


TABLE 6. Enthalpies of vaporization of organic compounds, 1880-2002-Continued

\begin{tabular}{|c|c|c|c|c|c|}
\hline Molecular formula & $\begin{array}{c}\text { Compound } \\
\text { (Temperature range/K) }\end{array}$ & $\begin{array}{c}\Delta_{\mathrm{vap}} H_{m} \\
\left(\mathrm{~kJ} \mathrm{~mol}^{-1}\right)\end{array}$ & $\begin{array}{l}\text { Mean temperature } \\
\qquad\left(T_{m} / \mathrm{K}\right)\end{array}$ & Method & $\begin{array}{l}\text { CAS registry number } \\
\text { Reference }\end{array}$ \\
\hline $\mathrm{C}_{9} \mathrm{H}_{10} \mathrm{Cl}_{2} \mathrm{O}_{4}$ & $\begin{array}{l}\text { 2,6-dichlorosyringaldehyde } \\
(293-323)\end{array}$ & 82.2 & $(308)$ & CGC & [99/13] \\
\hline $\mathrm{C}_{9} \mathrm{H}_{10} \mathrm{~F}_{2}$ & $\begin{array}{l}\text { 1,1-difluoro-3-phenylpropane } \\
(278-318)\end{array}$ & $53.3 \pm 0.4$ & (298) & GS & [97/14] \\
\hline $\mathrm{C}_{9} \mathrm{H}_{10} \mathrm{O}$ & $\begin{array}{l}\text { allyl phenyl ether } \\
(349-456)\end{array}$ & 49.4 & $(364)$ & A & $\begin{array}{c}{[1746-13-0]} \\
{[87 / 5]}\end{array}$ \\
\hline $\mathrm{C}_{9} \mathrm{H}_{10} \mathrm{O}$ & $\begin{array}{l}\text { cinnamyl alcohol } \\
(295-325) \\
(310-328) \\
(373-523)\end{array}$ & $\begin{array}{c}68.1 \pm 0.1 \\
79.8 \\
56.2\end{array}$ & $\begin{array}{l}(310) \\
(319) \\
(388)\end{array}$ & $\begin{array}{c}\text { TG,DTA } \\
\text { A } \\
\text { A }\end{array}$ & $\begin{array}{c}{[104-54-1]} \\
{[02 / 3]} \\
{[87 / 5]} \\
{[87 / 5]}\end{array}$ \\
\hline $\mathrm{C}_{9} \mathrm{H}_{10} \mathrm{O}$ & $\begin{array}{l}\text { 2,4-dimethylbenzaldehyde } \\
(358-489)\end{array}$ & 57.4 & (373) & A & $\begin{array}{c}{[15764-16-6]} \\
{[87 / 5]}\end{array}$ \\
\hline $\mathrm{C}_{9} \mathrm{H}_{10} \mathrm{O}$ & $\begin{array}{l}\text { 5-hydroxyindane } \\
(393-524)\end{array}$ & 55.4 & $(408)$ & $\mathrm{A}$ & $\begin{array}{c}{[1470-94-6]} \\
{[87 / 5]}\end{array}$ \\
\hline $\mathrm{C}_{9} \mathrm{H}_{10} \mathrm{O}$ & $\begin{array}{l}4^{\prime} \text {-methylacetophenone } \\
(288-333)\end{array}$ & 59.6 & $(303)$ & A & $\begin{array}{c}{[122-00-9]} \\
{[87 / 5]}\end{array}$ \\
\hline $\mathrm{C}_{9} \mathrm{H}_{10} \mathrm{O}$ & $\begin{array}{l}\text { 2-phenylpropionaldehyde } \\
(364-517) \\
(364-517) \\
(364-517) \\
(364-517)\end{array}$ & $\begin{array}{l}52.3 \pm 0.2 \\
49.4 \pm 0.2 \\
46.6 \pm 0.3 \\
43.4 \pm 0.5\end{array}$ & $\begin{array}{l}(360) \\
(400) \\
(440) \\
(480)\end{array}$ & $\begin{array}{l}\mathrm{EB} \\
\mathrm{EB} \\
\mathrm{EB} \\
\mathrm{EB}\end{array}$ & $\begin{array}{l}{[93-53-8]} \\
{[02 / 21]} \\
{[02 / 21]} \\
{[02 / 21]} \\
{[02 / 21]}\end{array}$ \\
\hline $\mathrm{C}_{9} \mathrm{H}_{10} \mathrm{O}$ & $\begin{array}{l}\text { 3-phenylpropionaldehyde } \\
(330-363)\end{array}$ & 67.5 & $(345)$ & A & $\begin{array}{c}{[104-53-0]} \\
{[87 / 5]}\end{array}$ \\
\hline $\mathrm{C}_{9} \mathrm{H}_{10} \mathrm{O}$ & $\begin{array}{l}\text { benzyl methyl ketone } \\
(343-383) \\
(343-383) \\
(273-328)\end{array}$ & $\begin{array}{c}56.1 \\
55.0 \\
53.5 \pm 0.3\end{array}$ & $\begin{array}{l}(298) \\
(298) \\
(298)\end{array}$ & $\begin{array}{l}\text { CGC } \\
\text { CGC }\end{array}$ & $\begin{array}{c}{[103-79-7]} \\
{[95 / 21]} \\
{[95 / 21]} \\
{[54 / 8]}\end{array}$ \\
\hline $\mathrm{C}_{9} \mathrm{H}_{10} \mathrm{O}$ & $\begin{array}{l}\text { ethyl phenyl ketone (propiophenone) } \\
(388-623) \\
(391-454)\end{array}$ & $\begin{array}{l}52.1 \\
44.4\end{array}$ & $\begin{array}{l}(403) \\
(406)\end{array}$ & $\begin{array}{c}\mathrm{A} \\
\mathrm{EB}, \mathrm{GS}\end{array}$ & $\begin{array}{c}{[93-55-0]} \\
{[87 / 5]} \\
{[65 / 7]}\end{array}$ \\
\hline $\mathrm{C}_{9} \mathrm{H}_{10} \mathrm{O}$ & $\begin{array}{l}\text { 2-vinylanisole } \\
(314-467)\end{array}$ & 56.7 & $(329)$ & $\mathrm{A}$ & $\begin{array}{c}{[612-15-7]} \\
{[87 / 5][47 / 5]}\end{array}$ \\
\hline $\mathrm{C}_{9} \mathrm{H}_{10} \mathrm{O}$ & $\begin{array}{l}\text { 3-vinylanisole } \\
(316-471)\end{array}$ & 55.9 & $(331)$ & A & $\begin{array}{c}{[626-20-0]} \\
{[87 / 5][47 / 5]}\end{array}$ \\
\hline $\mathrm{C}_{9} \mathrm{H}_{10} \mathrm{O}$ & $\begin{array}{l}\text { 4-vinylanisole } \\
(318-478)\end{array}$ & 54.9 & (333) & A & $\begin{array}{l}{[637-69-4]} \\
{[87 / 5][47 / 5]}\end{array}$ \\
\hline $\mathrm{C}_{9} \mathrm{H}_{10} \mathrm{O}_{2}$ & methyl $o$-toluate & $57.3 \pm 0.2$ & $(293)$ & $\mathrm{C}$ & $\begin{array}{c}{[89-71-4]} \\
{[98 / 6]}\end{array}$ \\
\hline $\mathrm{C}_{9} \mathrm{H}_{10} \mathrm{O}_{2}$ & methyl $m$-toluate & $\begin{array}{l}60.3 \pm 0.2 \\
53.5\end{array}$ & $\begin{array}{l}(296) \\
(388)\end{array}$ & $\mathrm{C}$ & $\begin{array}{c}{[99-36-5]} \\
{[98 / 6]} \\
{[74 / 1]}\end{array}$ \\
\hline $\mathrm{C}_{9} \mathrm{H}_{10} \mathrm{O}_{2}$ & $\begin{array}{l}\text { acetic acid, 3-tolyl ester } \\
(385-480)\end{array}$ & 55.7 & $(400)$ & $\mathrm{A}, \mathrm{EB}$ & $\begin{array}{c}{[122-46-3]} \\
{[87 / 5][69 / 13]}\end{array}$ \\
\hline $\mathrm{C}_{9} \mathrm{H}_{10} \mathrm{O}_{2}$ & $\begin{array}{l}\text { acetic acid, 4-tolyl ester } \\
(385-480)\end{array}$ & 55.9 & $(400)$ & $\mathrm{A}, \mathrm{EB}$ & $\begin{array}{c}{[140-39-6]} \\
{[87 / 5][69 / 13]}\end{array}$ \\
\hline $\mathrm{C}_{9} \mathrm{H}_{10} \mathrm{O}_{2}$ & 2-acetylanisole & 56.5 & & & $\begin{array}{c}{[579-74-8]} \\
{[86 / 10]}\end{array}$ \\
\hline $\mathrm{C}_{9} \mathrm{H}_{10} \mathrm{O}_{2}$ & $\begin{array}{l}\text { 4-acetylanisole } \\
(311-334)\end{array}$ & 66.5 & $(322)$ & $\mathrm{A}, \mathrm{ME}$ & $\begin{array}{l}{[100-06-1]} \\
{[87 / 5][54 / 9]}\end{array}$ \\
\hline $\mathrm{C}_{9} \mathrm{H}_{10} \mathrm{O}_{2}$ & 3,4-dihydro-2H-1,5-benzodioxepin & 55.6 & & & $\begin{array}{c}{[7216-18-4]} \\
{[58 / 25]}\end{array}$ \\
\hline $\mathrm{C}_{9} \mathrm{H}_{10} \mathrm{O}_{2}$ & $\begin{array}{l}\text { benzyl acetate } \\
(283-490) \\
(283-328)\end{array}$ & $\begin{array}{l}55.5 \\
60.4\end{array}$ & $\begin{array}{l}(298) \\
(305)\end{array}$ & $\begin{array}{c}\text { A } \\
\mathrm{ME}\end{array}$ & $\begin{array}{c}{[140-11-4]} \\
{[87 / 5]} \\
{[54 / 10]}\end{array}$ \\
\hline $\mathrm{C}_{9} \mathrm{H}_{10} \mathrm{O}_{2}$ & $\begin{array}{l}\text { ethylbenzoate } \\
(369-531) \\
(369-531) \\
(369-531) \\
(369-531) \\
(344-440) \\
(344-440) \\
(288-333) \\
(358-487) \\
(317-486)\end{array}$ & $\begin{array}{c}52.5 \pm 0.2 \\
49.6 \pm 0.2 \\
46.7 \pm 0.3 \\
43.6 \pm 0.5 \\
57.0 \\
50.5 \\
55.9 \\
50.4 \\
51.9\end{array}$ & $\begin{array}{l}(380) \\
(420) \\
(460) \\
(500) \\
(356) \\
(419) \\
(303) \\
(373) \\
(332)\end{array}$ & $\begin{array}{c}\text { EB } \\
\text { EB } \\
\text { EB } \\
\text { EB } \\
\text { BG } \\
\text { BG } \\
\text { A } \\
\text { A }\end{array}$ & $\begin{array}{c}{[93-89-0]} \\
{[02 / 15]} \\
{[02 / 15]} \\
{[02 / 15]} \\
{[02 / 15]} \\
{[88 / 2]} \\
{[88 / 2]} \\
{[87 / 5]} \\
{[87 / 5]} \\
{[47 / 5]}\end{array}$ \\
\hline
\end{tabular}


TABLE 6. Enthalpies of vaporization of organic compounds, 1880-2002-Continued

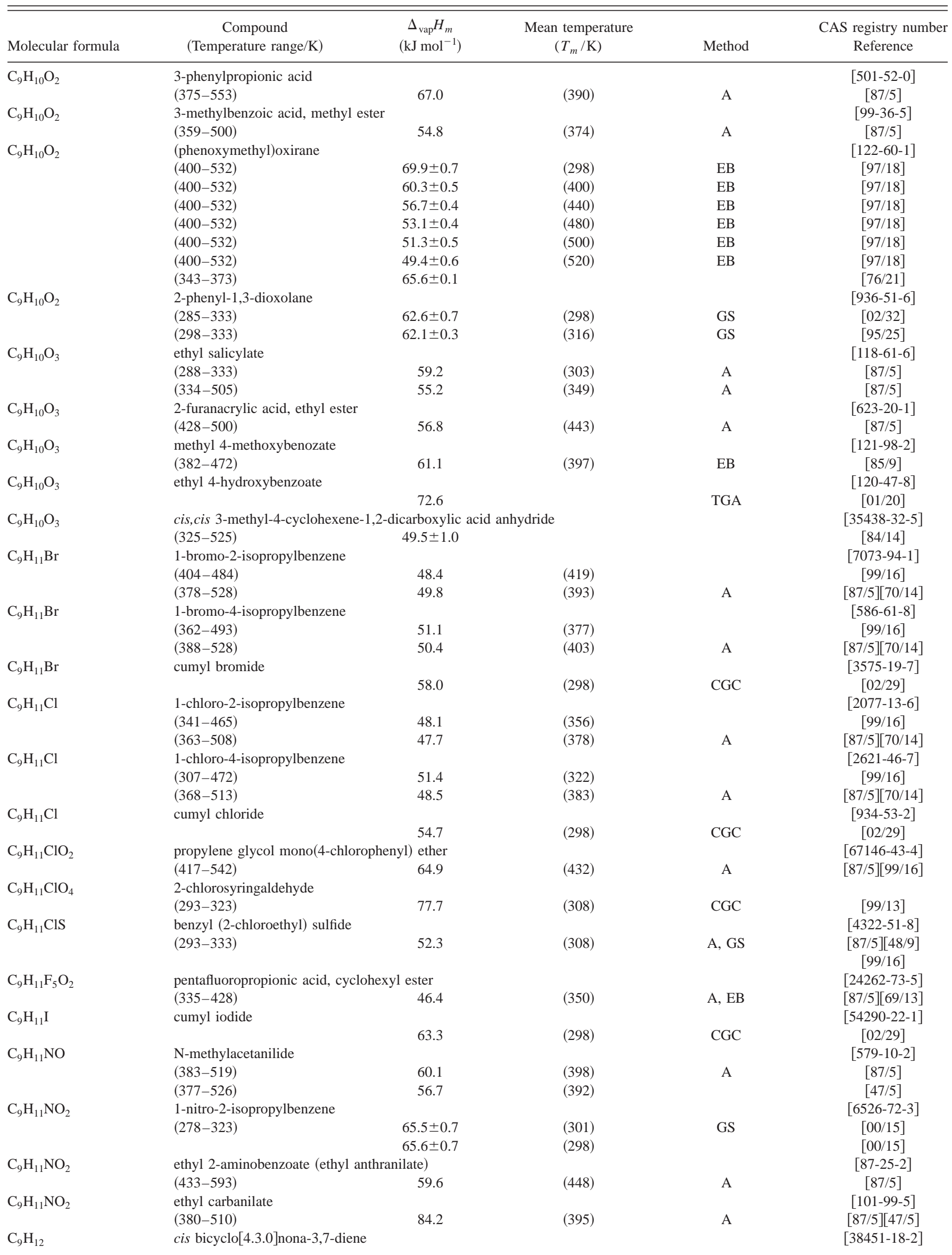


TABLE 6. Enthalpies of vaporization of organic compounds, 1880-2002-Continued

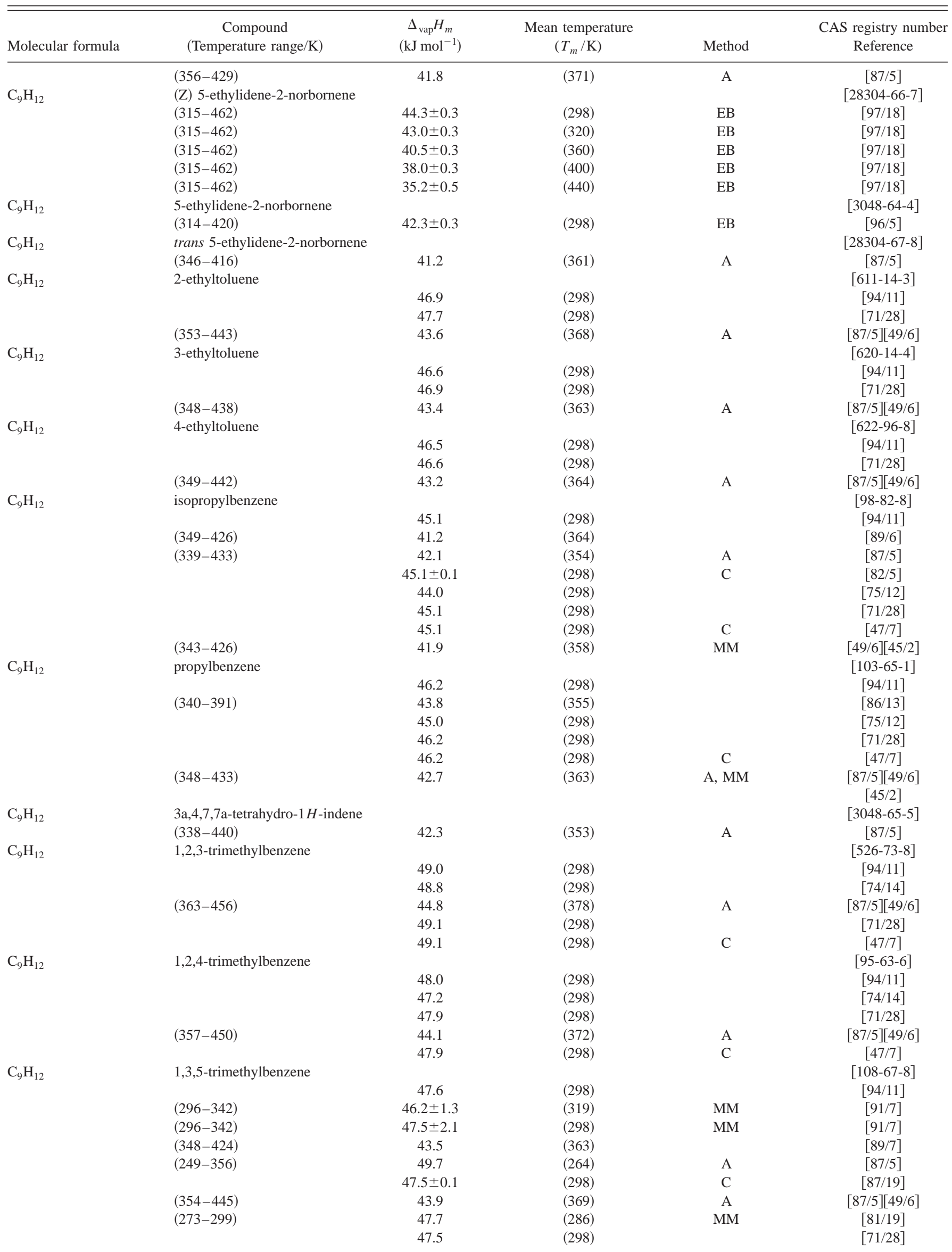


TABLE 6. Enthalpies of vaporization of organic compounds, 1880-2002-Continued

\begin{tabular}{|c|c|c|c|c|c|}
\hline Molecular formula & $\begin{array}{c}\text { Compound } \\
\text { (Temperature range/K) }\end{array}$ & $\begin{array}{c}\Delta_{\mathrm{vap}} H_{m} \\
\left(\mathrm{~kJ} \mathrm{~mol}^{-1}\right)\end{array}$ & $\begin{array}{c}\text { Mean temperature } \\
\left(T_{m} / \mathrm{K}\right)\end{array}$ & Method & $\begin{array}{l}\text { CAS registry number } \\
\text { Reference }\end{array}$ \\
\hline \multirow{4}{*}{$\mathrm{C}_{9} \mathrm{H}_{12}$} & & 47.5 & (298) & $\mathrm{C}$ & {$[47 / 7]$} \\
\hline & 5-vinyl-2-norbornene & & & & [3048-64-4] \\
\hline & $(301-410)$ & 42.0 & (316) & A & {$[87 / 5]$} \\
\hline & $(354-409)$ & 48.9 & $(369)$ & $\mathrm{A}$ & {$[87 / 5]$} \\
\hline \multirow[t]{2}{*}{$\mathrm{C}_{9} \mathrm{H}_{12} \mathrm{NO}_{5} \mathrm{PS}$} & O,O-dimethyl-O-(3-methyl & enyl)thiopho & & & [122-14-5] \\
\hline & $(293-382)$ & 78.0 & (308) & A & {$[87 / 5]$} \\
\hline \multirow[t]{2}{*}{$\mathrm{C}_{9} \mathrm{H}_{12} \mathrm{~N}_{2}$} & n-pentylmalodinitrile & & & & \\
\hline & $(298-328)$ & $66.9 \pm 0.4$ & & GS & {$[90 / 4]$} \\
\hline \multirow{2}{*}{$\mathrm{C}_{9} \mathrm{H}_{12} \mathrm{~N}_{2}$} & phenylhydrazone acetone & & & & [103-02-6] \\
\hline & $(413-436)$ & 74.6 & (424) & A & {$[87 / 5]$} \\
\hline \multirow[t]{3}{*}{$\mathrm{C}_{9} \mathrm{H}_{12} \mathrm{O}$} & (1-methoxyethyl)benzene & & & & \\
\hline & $(298-313)$ & $49.2 \pm 0.4$ & $(296)$ & GS & {$[01 / 16]$} \\
\hline & $(298-313)$ & $49.1 \pm 0.4$ & $(298)$ & GS & {$[01 / 16]$} \\
\hline \multirow[t]{3}{*}{$\mathrm{C}_{9} \mathrm{H}_{12} \mathrm{O}$} & benzyl ethyl ether & & & & {$[539-30-0]$} \\
\hline & $(278-314)$ & $53.5 \pm 0.4$ & $(298)$ & GS & {$[02 / 29]$} \\
\hline & $(299-460)$ & 48.0 & (314) & A & {$[87 / 5][47 / 5]$} \\
\hline \multirow[t]{2}{*}{$\mathrm{C}_{9} \mathrm{H}_{12} \mathrm{O}$} & 2-ethylanisole & & & & [14804-32-1] \\
\hline & $(302-460)$ & 49.8 & (317) & A & {$[87 / 5][47 / 5]$} \\
\hline \multirow[t]{2}{*}{$\mathrm{C}_{9} \mathrm{H}_{12} \mathrm{O}$} & 3-ethylanisole & & & & [10568-38-4] \\
\hline & $(306-470)$ & 49.3 & $(321)$ & A & {$[87 / 5][47 / 5]$} \\
\hline \multirow{2}{*}{$\mathrm{C}_{9} \mathrm{H}_{12} \mathrm{O}$} & 4-ethylanisole & & & & {$[1515-95-3]$} \\
\hline & $(306-470)$ & 51.9 & $(321)$ & $\mathrm{A}$ & {$[87 / 5][47 / 5]$} \\
\hline \multirow[t]{3}{*}{$\mathrm{C}_{9} \mathrm{H}_{12} \mathrm{O}$} & 5-ethyl-3-methylphenol & & & & \\
\hline & $(468-521)$ & 55.0 & $(483)$ & $\mathrm{A}, \mathrm{GS}, \mathrm{EB}$ & {$[87 / 5][64 / 14]$} \\
\hline & $(385-506)$ & 58.5 & & & {$[55 / 9]$} \\
\hline \multirow[t]{5}{*}{$\mathrm{C}_{9} \mathrm{H}_{12} \mathrm{O}$} & 2-isopropylphenol & & & & [88-69-7] \\
\hline & $(375-493)$ & 63.5 & $(390)$ & EB & {$[90 / 5]$} \\
\hline & $(370-489)$ & 55.1 & $(385)$ & A & {$[87 / 5]$} \\
\hline & $(375-493)$ & 56.1 & $(390)$ & & {$[86 / 7]$} \\
\hline & $(335-501)$ & 57.3 & $(350)$ & & {$[47 / 5]$} \\
\hline \multirow[t]{2}{*}{$\mathrm{C}_{9} \mathrm{H}_{12} \mathrm{O}$} & 3-isopropylphenol & & & & {$[618-45-1]$} \\
\hline & $(377-497)$ & 64.3 & $(392)$ & A & {$[87 / 5]$} \\
\hline \multirow[t]{3}{*}{$\mathrm{C}_{9} \mathrm{H}_{12} \mathrm{O}$} & 4-isopropylphenol & & & & [99-89-8] \\
\hline & $(391-507)$ & 63.7 & (406) & EB & {$[90 / 5]$} \\
\hline & $(380-496)$ & 63.1 & (395) & $\mathrm{A}$ & {$[87 / 5]$} \\
\hline \multirow[t]{2}{*}{$\mathrm{C}_{9} \mathrm{H}_{12} \mathrm{O}$} & isopropyl phenyl ether & & & & [2741-16-4] \\
\hline & $(345-448)$ & 49.5 & $(360)$ & A & $\begin{array}{c}{[87 / 5][65 / 25]} \\
{[84 / 9]}\end{array}$ \\
\hline $\mathrm{C}_{9} \mathrm{H}_{12} \mathrm{O}$ & 3-phenyl-1-propanol & & & & [122-97-4] \\
\hline & $(284-328)$ & 62.8 & (299) & A & {$[87 / 5]$} \\
\hline & $(347-508)$ & 62.6 & $(362)$ & & {$[47 / 5]$} \\
\hline $\mathrm{C}_{9} \mathrm{H}_{12} \mathrm{O}$ & 2-phenyl-2-propanol & & & & [617-94-7] \\
\hline & $(391-423)$ & 52.9 & $(406)$ & A & {$[87 / 5]$} \\
\hline $\mathrm{C}_{9} \mathrm{H}_{12} \mathrm{O}$ & phenyl propyl ether & & & & {$[622-85-5]$} \\
\hline & $(374-463)$ & 46.5 & $(389)$ & A & {$[87 / 5]$} \\
\hline $\mathrm{C}_{9} \mathrm{H}_{12} \mathrm{O}$ & 2-propylphenol & & & & [644-35-9] \\
\hline & $(377-495)$ & 56.9 & (392) & A & {$[87 / 5]$} \\
\hline & $(381-504)$ & 59.9 & (398) & & {$[53 / 9]$} \\
\hline & $(381-504)$ & 57.2 & (423) & & {$[53 / 9]$} \\
\hline & $(381-504)$ & 53.0 & (473) & & {$[53 / 9]$} \\
\hline $\mathrm{C}_{9} \mathrm{H}_{12} \mathrm{O}$ & 3-propylphenol & & & & [621-27-2] \\
\hline & $(408-538)$ & 60.2 & $(423)$ & A & {$[87 / 5]$} \\
\hline & $(386-512)$ & 59.9 & (398) & & {$[53 / 9]$} \\
\hline & $(386-512)$ & 57.2 & (423) & & {$[53 / 9]$} \\
\hline & $(386-512)$ & 53.0 & $(473)$ & & {$[53 / 9]$} \\
\hline $\mathrm{C}_{9} \mathrm{H}_{12} \mathrm{O}$ & 4-propylphenol & & & & [645-56-7] \\
\hline & $(383-508)$ & 56.7 & (398) & A & {$[87 / 5]$} \\
\hline & $(347-517)$ & 61.3 & (348) & & [53/9] \\
\hline & $(347-517)$ & 59.5 & (373) & & [53/9] \\
\hline & $(347-517)$ & 58.4 & (398) & & {$[53 / 9]$} \\
\hline & $(347-517)$ & 56.2 & (423) & & {$[53 / 9]$} \\
\hline & $(347-517)$ & 51.5 & $(473)$ & & [53/9] \\
\hline $\mathrm{C}_{9} \mathrm{H}_{12} \mathrm{O}$ & 2,3,5-trimethylphenol & & & & [697-82-5] \\
\hline & $(459-521)$ & 53.9 & (474) & A, GS, EB & {$[87 / 5][64 / 14]$} \\
\hline
\end{tabular}


TABLE 6. Enthalpies of vaporization of organic compounds, 1880-2002-Continued

\begin{tabular}{|c|c|c|c|c|c|}
\hline Molecular formula & $\begin{array}{c}\text { Compound } \\
\text { (Temperature range/K) }\end{array}$ & $\begin{array}{c}\Delta_{\mathrm{vap}} H_{m} \\
\left(\mathrm{~kJ} \mathrm{~mol}^{-1}\right)\end{array}$ & $\begin{array}{l}\text { Mean temperature } \\
\qquad\left(T_{m} / \mathrm{K}\right)\end{array}$ & Method & $\begin{array}{c}\text { CAS registry number } \\
\text { Reference }\end{array}$ \\
\hline & $(379-506)$ & 55.1 & $(394)$ & & {$[55 / 9]$} \\
\hline \multirow[t]{2}{*}{$\mathrm{C}_{9} \mathrm{H}_{12} \mathrm{O}$} & 2,4,5-trimethylphenol & & & & {$[496-78-6]$} \\
\hline & $(379-505)$ & 56.5 & (394) & $\mathrm{A}$ & {$[87 / 5][55 / 9]$} \\
\hline \multirow[t]{2}{*}{$\mathrm{C}_{9} \mathrm{H}_{12} \mathrm{O}$} & 2,4,6-trimethylphenol & & & & {$[527-60-6]$} \\
\hline & $(367-494)$ & 53.2 & (382) & A & {$[87 / 5][55 / 9]$} \\
\hline \multirow[t]{2}{*}{$\mathrm{C}_{9} \mathrm{H}_{12} \mathrm{O}$} & 3,4,5-trimethylphenol & & & & {$[527-54-8]$} \\
\hline & $(396-521)$ & 61.1 & $(411)$ & A & {$[87 / 5]$} \\
\hline \multirow[t]{2}{*}{$\mathrm{C}_{9} \mathrm{H}_{12} \mathrm{O}$} & 2,5,6-trimethylphenol & & & & [2416-94-6] \\
\hline & $(359-503)$ & $51.1 \pm 0.2$ & $(431)$ & & {$[88 / 4]$} \\
\hline \multirow[t]{3}{*}{$\mathrm{C}_{9} \mathrm{H}_{12} \mathrm{O}$} & $\alpha, \alpha$-dimethylbenzyl alcohol & & & & [617-94-7] \\
\hline & $(311-338)$ & $63.4 \pm 0.5$ & (325) & GS & [99/3] \\
\hline & $(311-338)$ & $65.0 \pm 0.5$ & $(298)$ & GS & {$[99 / 3]$} \\
\hline \multirow[t]{2}{*}{$\mathrm{C}_{9} \mathrm{H}_{12} \mathrm{O}_{2}$} & trimethylhydroquinone & & & & [700-13-0] \\
\hline & $(450-501)$ & $45.5 \pm 0.3$ & $(475)$ & & {$[88 / 4]$} \\
\hline \multirow[t]{3}{*}{$\mathrm{C}_{9} \mathrm{H}_{12} \mathrm{O}_{2}$} & cumene hydroperoxide & & & & [80-15-9] \\
\hline & $(283-333)$ & 69.9 & $(298)$ & A & {$[87 / 5]$} \\
\hline & $(347-390)$ & 74.0 & $(362)$ & A & {$[87 / 5]$} \\
\hline $\mathrm{C}_{9} \mathrm{H}_{12} \mathrm{O}_{2}$ & $\begin{array}{l}\text { 1,3-dihydroxy-5-methyl-2-etl } \\
(388-453)\end{array}$ & ene 77.1 & (403) & $\mathrm{A}, \mathrm{GC}$ & {$[87 / 5][75 / 24]$} \\
\hline \multirow[t]{2}{*}{$\mathrm{C}_{9} \mathrm{H}_{12} \mathrm{O}_{2}$} & 3,5-dimethoxytoluene & & & & [4179-19-5] \\
\hline & $(374-520)$ & 59.5 & $(389)$ & A & {$[87 / 5]$} \\
\hline \multirow[t]{2}{*}{$\mathrm{C}_{9} \mathrm{H}_{12} \mathrm{O}_{2}$} & ethylene glycol monobenzyl & & & & [622-08-2] \\
\hline & $(453-530)$ & 58.6 & $(468)$ & A & {$[87 / 5]$} \\
\hline \multirow[t]{2}{*}{$\mathrm{C}_{9} \mathrm{H}_{12} \mathrm{O}_{2}$} & propylene glycol 1-phenyl et & & & & {$[770-35-4]$} \\
\hline & $(389-509)$ & 59.5 & $(404)$ & A & {$[87 / 5]$} \\
\hline \multirow[t]{2}{*}{$\mathrm{C}_{9} \mathrm{H}_{12} \mathrm{O}_{2}$} & isopropyl catechol (isomer $n$ & fied) & & & \\
\hline & $(393-453)$ & 65.3 & $(423)$ & & [65/21] \\
\hline \multirow[t]{3}{*}{$\mathrm{C}_{9} \mathrm{H}_{12} \mathrm{O}_{2}$} & benzaldehyde dimethyl aceta & & & & [1125-88-8] \\
\hline & $(278-318)$ & $60.9 \pm 0.5$ & $(298)$ & GS & {$[02 / 32]$} \\
\hline & $(283-318)$ & $56.5 \pm 0.7$ & $(300)$ & GS & {$[95 / 25]$} \\
\hline \multirow[t]{2}{*}{$\mathrm{C}_{9} \mathrm{H}_{12} \mathrm{O}_{3}$} & 1,3,5-trimethoxybenzene & & & & [621-23-8] \\
\hline & & $68.2 \pm 2.0$ & $(298)$ & CGC & {$[00 / 9]$} \\
\hline $\mathrm{C}_{9} \mathrm{H}_{12} \mathrm{~S}$ & benzyl ethyl sulfide & & & & {$[6263-62-3]$} \\
\hline & $(346-370)$ & 56.0 & $(358)$ & & [99/16] \\
\hline & $(345-500)$ & 54.8 & $(360)$ & A & {$[87 / 5]$} \\
\hline & & $56.9 \pm 2.1$ & $(298)$ & & {$[62 / 20]$} \\
\hline $\mathrm{C}_{9} \mathrm{H}_{12} \mathrm{~S}$ & 2-ethylthioanisole & & & & [20760-06-9] \\
\hline & $(481-511)$ & 44.3 & $(496)$ & & {$[99 / 16]$} \\
\hline $\mathrm{C}_{9} \mathrm{H}_{12} \mathrm{~S}$ & ethyl $m$-tolyl sulfide & & & & [34786-24-8] \\
\hline & $(472-502)$ & 43.5 & (487) & & [99/16] \\
\hline $\mathrm{C}_{9} \mathrm{H}_{12} \mathrm{~S}$ & ethyl $p$-tolyl sulfide & & & & {$[622.63-9]$} \\
\hline & $(473-503)$ & 43.6 & (488) & & [99/16] \\
\hline $\mathrm{C}_{9} \mathrm{H}_{12} \mathrm{~S}$ & (isopropylthio)benzene & & & & [3019-20-3] \\
\hline & $(461-491)$ & U23.6 & $(476)$ & & [99/16] \\
\hline $\mathrm{C}_{9} \mathrm{H}_{12} \mathrm{~S}$ & (propylthio)benzene & & & & {$[874-79-3]$} \\
\hline & $(473-503)$ & 44.3 & $(488)$ & & [99/16] \\
\hline $\mathrm{C}_{9} \mathrm{H}_{13} \mathrm{Cl}_{3} \mathrm{OS}$ & 2,3,3-trichloro-2-propenethio & O-hexyl ester & & & [76619-95-9] \\
\hline & $(433-483)$ & 69.5 & & GC & {$[80 / 24]$} \\
\hline $\mathrm{C}_{9} \mathrm{H}_{13} \mathrm{~N}$ & $\alpha, \alpha$-dimethylbenzylamine & & & & \\
\hline & $(283-323)$ & $56.4 \pm 0.7$ & (303) & GS & {$[99 / 3]$} \\
\hline & $(283-323)$ & $56.7 \pm 0.7$ & $(298)$ & GS & {$[99 / 3]$} \\
\hline $\mathrm{C}_{9} \mathrm{H}_{13} \mathrm{~N}$ & $\mathrm{~N}, \mathrm{~N}$-dimethylbenzylamine & & & & [103-83-3] \\
\hline & $(288-328)$ & $48.9 \pm 0.4$ & (308) & GS & {$[99 / 3]$} \\
\hline & $(288-328)$ & $49.5 \pm 0.4$ & (298) & GS & {$[99 / 3]$} \\
\hline & & $50.1 \pm 0.9$ & $(298)$ & $\mathrm{C}$ & {$[96 / 21]$} \\
\hline $\mathrm{C}_{9} \mathrm{H}_{13} \mathrm{~N}$ & $\mathrm{~N}, \mathrm{~N}$-dimethyl-2-toluidine & & & & {$[609-72-3]$} \\
\hline & $(301-458)$ & 52.4 & $(316)$ & $\mathrm{A}$ & {$[87 / 5][47 / 5]$} \\
\hline $\mathrm{C}_{9} \mathrm{H}_{13} \mathrm{~N}$ & $\mathrm{~N}, \mathrm{~N}$-dimethyl-3-toluidine & & & & {$[121-72-2]$} \\
\hline & & $58.2 \pm 6.9$ & $(298)$ & CGC & {$[96 / 1]$} \\
\hline $\mathrm{C}_{9} \mathrm{H}_{13} \mathrm{~N}$ & $\mathrm{~N}, \mathrm{~N}$-dimethyl-4-toluidine & & & & {$[99-97-8]$} \\
\hline & $(323-483)$ & 60.7 & $(338)$ & $\mathrm{A}$ & {$[87 / 5][47 / 5]$} \\
\hline $\mathrm{C}_{9} \mathrm{H}_{13} \mathrm{~N}$ & N-ethyl-3-toluidine & & & & {$[102-27-2]$} \\
\hline & & $60.0 \pm 3.0$ & $(298)$ & CGC & {$[96 / 1]$} \\
\hline $\mathrm{C}_{9} \mathrm{H}_{13} \mathrm{~N}$ & 2-isopropylaniline & & & & [643-28-7] \\
\hline
\end{tabular}


TABLE 6. Enthalpies of vaporization of organic compounds, 1880-2002-Continued

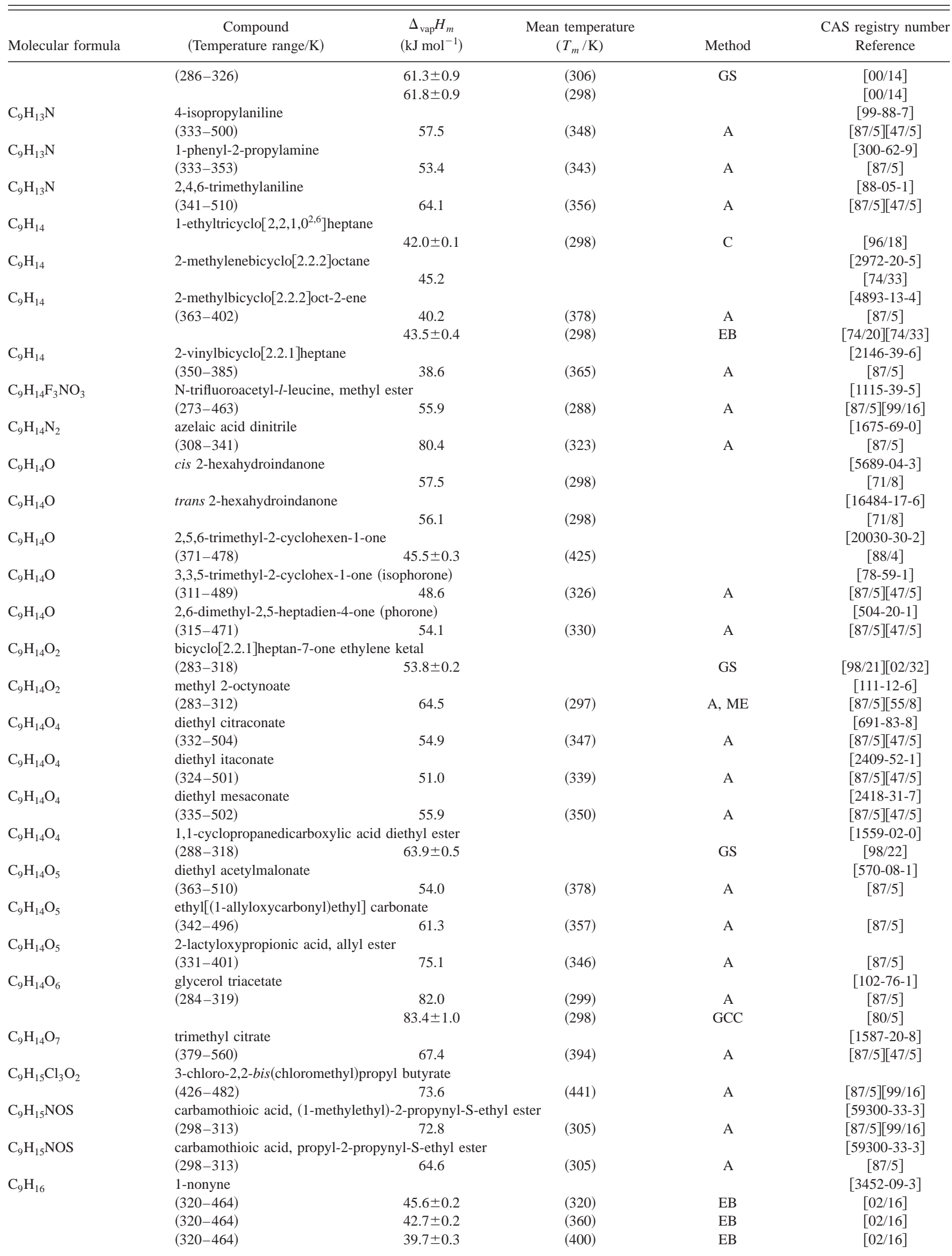


TABLE 6. Enthalpies of vaporization of organic compounds, 1880-2002-Continued

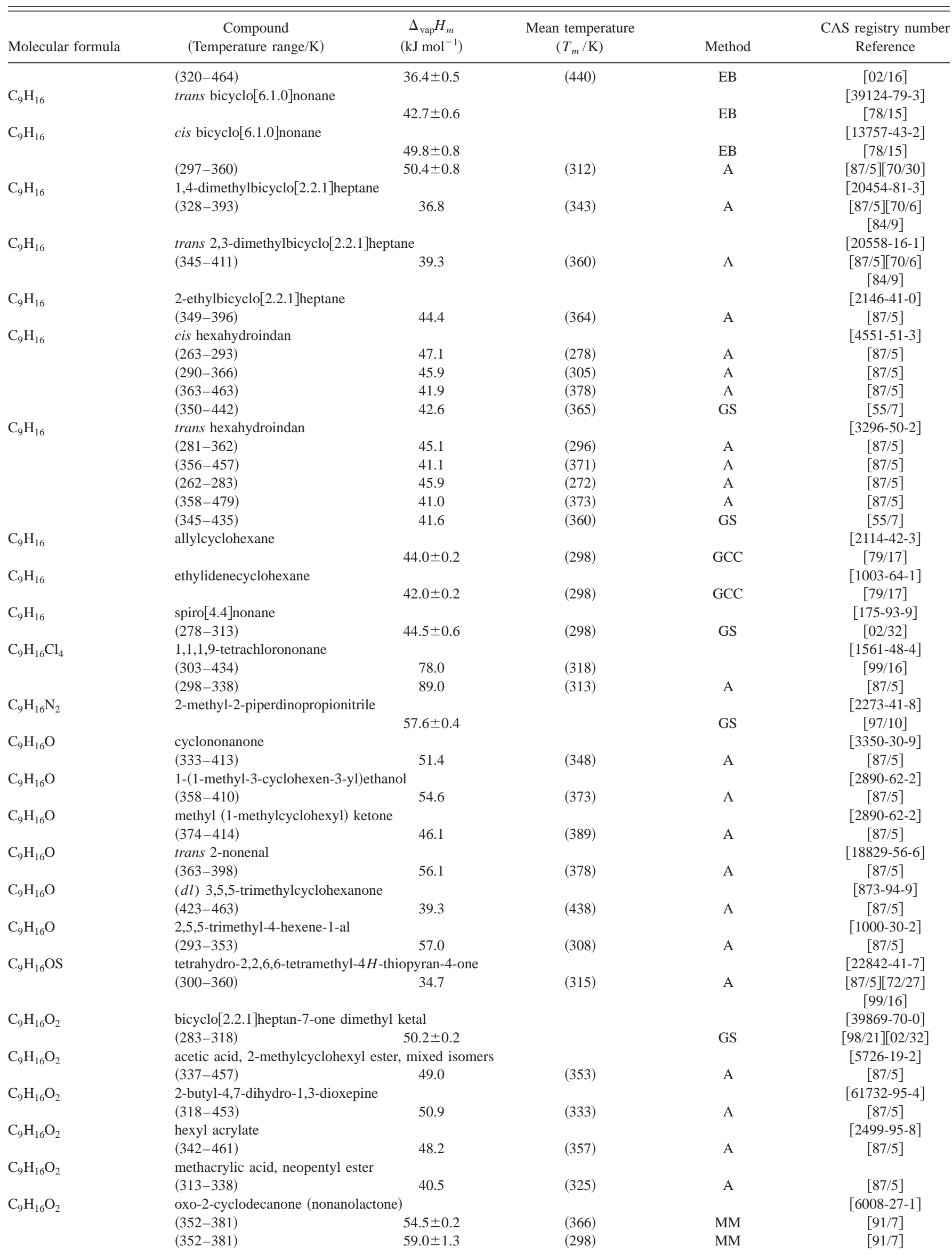


TABLE 6. Enthalpies of vaporization of organic compounds, 1880-2002-Continued

\begin{tabular}{|c|c|c|c|c|c|}
\hline Molecular formula & $\begin{array}{c}\text { Compound } \\
\text { (Temperature range/K) }\end{array}$ & $\begin{array}{c}\Delta_{\mathrm{vap}} H_{m} \\
\left(\mathrm{~kJ} \mathrm{~mol}^{-1}\right)\end{array}$ & $\begin{array}{l}\text { Mean temperature } \\
\qquad\left(T_{m} / \mathrm{K}\right)\end{array}$ & Method & $\begin{array}{l}\text { CAS registry number } \\
\text { Reference }\end{array}$ \\
\hline & $(333-383)$ & 60.9 & $(348)$ & A & {$[87 / 5]$} \\
\hline \multirow[t]{2}{*}{$\mathrm{C}_{9} \mathrm{H}_{16} \mathrm{O}_{2}$} & 2,6-dimethyl-3,5-heptanedione & & & & [18362-64-6] \\
\hline & & 56.1 & $(298)$ & & {$[78 / 18]$} \\
\hline \multirow[t]{2}{*}{$\mathrm{C}_{9} \mathrm{H}_{16} \mathrm{O}_{2}$} & pentyl methacrylate & & & & [2849-98-1] \\
\hline & $(339-456)$ & 47.6 & (354) & A & [87/5] \\
\hline \multirow[t]{3}{*}{$\mathrm{C}_{9} \mathrm{H}_{16} \mathrm{O}_{3}$} & butyl levulinate & & & & [2052-15-5] \\
\hline & $(338-511)$ & 55.5 & $(373)$ & A & {$[87 / 5]$} \\
\hline & & 56.0 & $(452)$ & & {$[31 / 1]$} \\
\hline \multirow[t]{2}{*}{$\mathrm{C}_{9} \mathrm{H}_{16} \mathrm{O}_{3}$} & sec-butyl levulinate & & & & {$[2052-15-5]$} \\
\hline & $(393-499)$ & 51.0 & (408) & A & {$[87 / 5]$} \\
\hline \multirow[t]{3}{*}{$\mathrm{C}_{9} \mathrm{H}_{16} \mathrm{O}_{3}$} & isobutyl levulinate & & & & {$[3757-32-2]$} \\
\hline & $(338-503)$ & 61.5 & (353) & A & {$[87 / 5][47 / 5]$} \\
\hline & & 54.7 & $(444)$ & & {$[31 / 1]$} \\
\hline $\mathrm{C}_{9} \mathrm{H}_{16} \mathrm{O}_{4}$ & $\begin{array}{l}\text { 2-acetoxypropionic acid, butyl ester } \\
(325-485)\end{array}$ & 63.2 & (340) & A & {$[87 / 5]$} \\
\hline \multirow[t]{2}{*}{$\mathrm{C}_{9} \mathrm{H}_{16} \mathrm{O}_{4}$} & 3-acetoxypropionic acid, butyl ester & & & & [40326-38-3] \\
\hline & $(373-391)$ & 75.4 & $(382)$ & $\mathrm{A}$ & {$[87 / 5]$} \\
\hline \multirow[t]{2}{*}{$\mathrm{C}_{9} \mathrm{H}_{16} \mathrm{O}_{4}$} & diethyl glutarate & & & & {$[818-38-2]$} \\
\hline & $(338-510)$ & 55.7 & (353) & A & {$[87 / 5][47 / 5]$} \\
\hline \multirow[t]{2}{*}{$\mathrm{C}_{9} \mathrm{H}_{16} \mathrm{O}_{4}$} & ethylmalonic acid, diethyl ester & & & & {$[133-13-1]$} \\
\hline & $(323-485)$ & 55.3 & (338) & A & {$[87 / 5][47 / 5]$} \\
\hline \multirow[t]{2}{*}{$\mathrm{C}_{9} \mathrm{H}_{16} \mathrm{O}_{4}$} & nonanedioic acid (azelaic acid) & & & & {$[123-99-9]$} \\
\hline & $(451-630)$ & 89.3 & (466) & A & {$[87 / 5][47 / 5]$} \\
\hline \multirow[t]{2}{*}{$\mathrm{C}_{9} \mathrm{H}_{16} \mathrm{O}_{5}$} & butyl[1-(methoxycarbonyl)ethyl] carl & bonate & & & \\
\hline & $(349-510)$ & 61.7 & $(364)$ & A & {$[87 / 5]$} \\
\hline \multirow[t]{2}{*}{$\mathrm{C}_{9} \mathrm{H}_{16} \mathrm{O}_{5}$} & isobutyl[1-(methoxycarbonyl)ethyl $]$ & carbonate & & & \\
\hline & $(340-501)$ & 59.1 & $(355)$ & A & {$[87 / 5]$} \\
\hline \multirow[t]{2}{*}{$\mathrm{C}_{9} \mathrm{H}_{16} \mathrm{O}_{5}$} & 2-lactoylpropionic acid, propyl ester & & & & \\
\hline & $(327-397)$ & 73.5 & $(342)$ & $\mathrm{A}$ & {$[87 / 5]$} \\
\hline \multirow[t]{2}{*}{$\mathrm{C}_{9} \mathrm{H}_{16} \mathrm{O}_{5}$} & methyl[1-(butoxycarbonyl)ethyl] carl & bonate & & & \\
\hline & $(311-503)$ & 60.2 & $(326)$ & A & {$[87 / 5]$} \\
\hline $\mathrm{C}_{9} \mathrm{H}_{17} \mathrm{~N}$ & trans $(\mathrm{R}, \mathrm{S})$-decahydroquinoline & & & & {$[767-92-0]$} \\
\hline & $(325-525)$ & 50.4 & (340) & EB, IPM & {$[94 / 17]$} \\
\hline & $(325-525)$ & 47.6 & $(380)$ & EB, IPM & {$[94 / 17]$} \\
\hline & $(325-525)$ & 45.0 & $(420)$ & $\mathrm{EB}, \mathrm{IPM}$ & {$[94 / 17]$} \\
\hline & $(325-525)$ & 42.3 & $(460)$ & $\mathrm{EB}, \mathrm{IPM}$ & {$[94 / 17]$} \\
\hline & $(325-525)$ & 39.5 & $(500)$ & EB,IPM & [94/17] \\
\hline $\mathrm{C}_{9} \mathrm{H}_{17} \mathrm{~N}$ & octyl cyanide & & & & {$[2243-27-8]$} \\
\hline & $(328-503)$ & 56.8 & $(343)$ & A & {$[87 / 5]$} \\
\hline $\mathrm{C}_{9} \mathrm{H}_{17} \mathrm{NO}_{3}$ & $(d l)$ N-acetylvaline ethyl ester & & & & {$[56430-36-5]$} \\
\hline & $(382-466)$ & 67.7 & (397) & A & {$[87 / 5]$} \\
\hline $\mathrm{C}_{9} \mathrm{H}_{17} \mathrm{NO}_{3} \mathrm{~S}$ & $(d l) \mathrm{N}$-acetylmethionine ethyl ester & & & & [33280-93-2] \\
\hline & $(432-519)$ & 81.6 & (447) & A & {$[87 / 5][99 / 16]$} \\
\hline $\mathrm{C}_{9} \mathrm{H}_{18}$ & butylcyclopentane & & & & [2040-95-1] \\
\hline & $(413-432)$ & 39.4 & $(422)$ & A & {$[87 / 5]$} \\
\hline & & $43.8 \pm 0.1$ & (328) & $\mathrm{C}$ & {$[81 / 14]$} \\
\hline & & $42.7 \pm 0.1$ & (343) & $\mathrm{C}$ & {$[81 / 14]$} \\
\hline & & $41.6 \pm 0.1$ & (358) & $\mathrm{C}$ & {$[81 / 14]$} \\
\hline & & $40.9 \pm 0.1$ & $(368)$ & $\mathrm{C}$ & {$[81 / 14]$} \\
\hline & & 46.0 & (298) & & {$[71 / 28]$} \\
\hline $\mathrm{C}_{9} \mathrm{H}_{18}$ & cis 1-ethyl-3-methylcyclohexane & & & & [19489-10-2] \\
\hline & $(373-465)$ & 39.0 & (388) & A & {$[87 / 5]$} \\
\hline $\mathrm{C}_{9} \mathrm{H}_{18}$ & isopropylcyclohexane & & & & {$[696-29-7]$} \\
\hline & $(295-431)$ & 44.1 & $(310)$ & A & {$[87 / 5]$} \\
\hline & $(344-429)$ & 41.1 & $(359)$ & & {$[49 / 6]$} \\
\hline $\mathrm{C}_{9} \mathrm{H}_{18}$ & propylcyclohexane & & & & {$[1678-92-8]$} \\
\hline & & $42.8 \pm 0.5$ & (298) & $\mathrm{GC}$ & {$[87 / 17]$} \\
\hline & & $44.7 \pm 0.4$ & (298) & GCC & {$[78 / 16]$} \\
\hline & & 45.2 & (298) & & {$[71 / 28]$} \\
\hline & & 45.2 & (298) & $\mathrm{C}$ & {$[47 / 7]$} \\
\hline & $(346-431)$ & 41.7 & $(361)$ & $\mathrm{A}, \mathrm{MM}$ & {$[87 / 5][47 / 5]$} \\
\hline & & & & & {$[49 / 6]$} \\
\hline $\mathrm{C}_{9} \mathrm{H}_{18}$ & 1,1,3-trimethylcyclohexane & & & & [3073-66-3] \\
\hline & $(348-411)$ & 37.7 & (363) & A & {$[87 / 5]$} \\
\hline
\end{tabular}


TABLE 6. Enthalpies of vaporization of organic compounds, 1880-2002-Continued

\begin{tabular}{|c|c|c|c|c|c|}
\hline Molecular formula & $\begin{array}{c}\text { Compound } \\
\text { (Temperature range/K) }\end{array}$ & $\begin{array}{c}\Delta_{\mathrm{vap}} H_{m} \\
\left(\mathrm{~kJ} \mathrm{~mol}^{-1}\right)\end{array}$ & $\begin{array}{l}\text { Mean temperature } \\
\qquad\left(T_{m} / \mathrm{K}\right)\end{array}$ & Method & $\begin{array}{c}\text { CAS registry number } \\
\text { Reference }\end{array}$ \\
\hline & $(327-410)$ & 38.6 & $(342)$ & & {$[62 / 24][84 / 9]$} \\
\hline & $(328-411)$ & 38.4 & $(343)$ & & {$[49 / 6]$} \\
\hline \multirow[t]{2}{*}{$\mathrm{C}_{9} \mathrm{H}_{18}$} & 1,1,4-trimethylcyclohexane & & & & {$[7094-27-1]$} \\
\hline & & 45.6 & & & {$[95 / 31]$} \\
\hline \multirow[t]{2}{*}{$\mathrm{C}_{9} \mathrm{H}_{18}$} & cis 1,3,5-trimethylcyclohexane & & & & {$[1795-27-3]$} \\
\hline & $(318-410)$ & 38.3 & $(333)$ & A & {$[87 / 5]$} \\
\hline \multirow[t]{3}{*}{$\mathrm{C}_{9} \mathrm{H}_{18}$} & 2,6-dimethyl-1-heptene & & & & {$[3074-78-0]$} \\
\hline & $(273-306)$ & $46.3 \pm 0.5$ & $(290)$ & GS & {$[00 / 7]$} \\
\hline & $(273-306)$ & $45.9 \pm 0.5$ & $(298)$ & GS & {$[00 / 7]$} \\
\hline \multirow[t]{4}{*}{$\mathrm{C}_{9} \mathrm{H}_{18}$} & 1-nonene & & & & [124-11-8] \\
\hline & $(278-318)$ & $44.7 \pm 0.2$ & $(298)$ & GS & {$[00 / 7]$} \\
\hline & & 45.5 & $(298)$ & & {$[71 / 28]$} \\
\hline & $(339-423)$ & 42.0 & $(354)$ & $\mathrm{A}, \mathrm{MM}$ & {$[87 / 5][50 / 6]$} \\
\hline \multirow[t]{2}{*}{$\mathrm{C}_{9} \mathrm{H}_{18}$} & cis 2-nonene & & & & {$[6434-77-1]$} \\
\hline & $(379-424)$ & 40.7 & $(394)$ & A & {$[87 / 5]$} \\
\hline \multirow{2}{*}{$\mathrm{C}_{9} \mathrm{H}_{18}$} & trans 2-nonene & & & & [6434-78-2] \\
\hline & $(379-422)$ & 40.8 & $(394)$ & A & {$[87 / 5]$} \\
\hline \multirow[t]{2}{*}{$\mathrm{C}_{9} \mathrm{H}_{18}$} & cis 3-nonene & & & & {$[20237-46-1]$} \\
\hline & $(376-422)$ & 40.3 & $(391)$ & A & {$[87 / 5]$} \\
\hline \multirow[t]{2}{*}{$\mathrm{C}_{9} \mathrm{H}_{18}$} & trans 3-nonene & & & & {$[20063-92-7]$} \\
\hline & $(377-421)$ & 40.6 & $(392)$ & A & {$[87 / 5]$} \\
\hline \multirow[t]{2}{*}{$\mathrm{C}_{9} \mathrm{H}_{18}$} & cis 4-nonene & & & & [10405-84-2] \\
\hline & $(376-421)$ & 40.1 & $(391)$ & A & {$[87 / 5]$} \\
\hline \multirow[t]{2}{*}{$\mathrm{C}_{9} \mathrm{H}_{18}$} & trans 4-nonene & & & & {$[10405-85-3]$} \\
\hline & $(376-420)$ & 40.4 & $(391)$ & A & {$[87 / 5]$} \\
\hline \multirow[t]{2}{*}{$\mathrm{C}_{9} \mathrm{H}_{18} \mathrm{Br}_{2}$} & 1,1-dibromononane & & & & [62168-27-8] \\
\hline & $(427-591)$ & 59.5 & $(442)$ & $\mathrm{A}, \mathrm{EST}$ & $\begin{array}{c}{[87 / 5][56 / 16]} \\
{[70 / 14][99 / 16]}\end{array}$ \\
\hline \multirow[t]{4}{*}{$\mathrm{C}_{9} \mathrm{H}_{18} \mathrm{Cl}_{2}$} & 1,1-dichlorononane & & & & [821-88-5] \\
\hline & $(420-490)$ & 62.3 & (298) & & {$[87 / 12][91 / 2]$} \\
\hline & $(398-556)$ & 54.0 & $(413)$ & A, EST & {$[87 / 5][56 / 16]$} \\
\hline & & & & & [70/14][99/16] \\
\hline \multirow[t]{3}{*}{$\mathrm{C}_{9} \mathrm{H}_{18} \mathrm{Cl}_{2}$} & 1,2-dichlorononane & & & & {$[56375-96-3]$} \\
\hline & $(430-510)$ & 52.1 & $(443)$ & & [99/16] \\
\hline & $(430-510)$ & 62.1 & $(298)$ & & {$[86 / 5][91 / 2]$} \\
\hline \multirow[t]{3}{*}{$\mathrm{C}_{9} \mathrm{H}_{18} \mathrm{~F}_{2}$} & 1,1-difluorononane & & & & {$[62127-42-8]$} \\
\hline & $(347-482)$ & 47.2 & $(362)$ & A, EST & {$[87 / 5][56 / 16]$} \\
\hline & & & & & {$[70 / 14][99 / 16]$} \\
\hline $\mathrm{C}_{9} \mathrm{H}_{18} \mathrm{~F}_{2}$ & 2,2-difluorononane & & & & \\
\hline & $(279-313)$ & $46.7 \pm 0.2$ & $(298)$ & GS & [97/14] \\
\hline $\mathrm{C}_{9} \mathrm{H}_{18} \mathrm{~N}_{2}$ & 2-(diethylamino)pentanenitrile & & & & {$[19340-91-9]$} \\
\hline & $(283-318)$ & $57.4 \pm 0.4$ & & GS & {$[97 / 10]$} \\
\hline & $(283-326)$ & 58.8 & $(298)$ & A & {$[87 / 5]$} \\
\hline $\mathrm{C}_{9} \mathrm{H}_{18} \mathrm{O}$ & 1-butylcyclopentanol & & & & {$[1462-97-1]$} \\
\hline & $(359-466)$ & 63.5 & $(374)$ & A & {$[87 / 5]$} \\
\hline $\mathrm{C}_{9} \mathrm{H}_{18} \mathrm{O}$ & 2,2,4,4-tetramethyl-3-pentanone & & & & {$[815-24-7]$} \\
\hline & & $45.5 \pm 0.4$ & $(298)$ & $\mathrm{C}$ & {$[77 / 7]$} \\
\hline & & $45.4 \pm 0.1$ & (298) & $\mathrm{C}$ & {$[70 / 18]$} \\
\hline & & $45.4 \pm 0.1$ & $(298)$ & $\mathrm{C}$ & {$[66 / 2]$} \\
\hline $\mathrm{C}_{9} \mathrm{H}_{18} \mathrm{O}$ & 2,6-dimethyl-4-heptanone & & & & {$[108-83-8]$} \\
\hline & & $49.8 \pm 0.1$ & $(308)$ & $\mathrm{C}$ & [92/8] \\
\hline & & $49.3 \pm 0.1$ & $(313)$ & $\mathrm{C}$ & {$[92 / 8]$} \\
\hline & & $48.4 \pm 0.1$ & $(323)$ & $\mathrm{C}$ & [92/8] \\
\hline & & $47.9 \pm 0.1$ & $(328)$ & $\mathrm{C}$ & {$[92 / 8]$} \\
\hline & & $47.1 \pm 0.1$ & $(338)$ & $\mathrm{C}$ & [92/8] \\
\hline & & $46.6 \pm 0.1$ & $(343)$ & $\mathrm{C}$ & {$[92 / 8]$} \\
\hline & & $46.1 \pm 0.1$ & $(348)$ & $\mathrm{C}$ & {$[92 / 8]$} \\
\hline & & $45.2 \pm 0.1$ & $(358)$ & $\mathrm{C}$ & {$[92 / 8]$} \\
\hline & $(322-471)$ & 51.0 & (298) & & {$[75 / 8]$} \\
\hline & & $50.9 \pm 0.1$ & (298) & C & [70/18] \\
\hline & $(336-451)$ & 46.8 & $(351)$ & A, MM & {$[87 / 5][47 / 8]$} \\
\hline $\mathrm{C}_{9} \mathrm{H}_{18} \mathrm{O}$ & 1-(1-methylcyclohexyl)ethanol & & & & \\
\hline & $(358-408)$ & 55.5 & $(373)$ & A & {$[87 / 5]$} \\
\hline $\mathrm{C}_{9} \mathrm{H}_{18} \mathrm{O}$ & nonanal & & & & [124-19-6] \\
\hline
\end{tabular}


TABLE 6. Enthalpies of vaporization of organic compounds, 1880-2002-Continued

\begin{tabular}{|c|c|c|c|c|c|}
\hline Molecular formula & $\begin{array}{c}\text { Compound } \\
\text { (Temperature range/K) }\end{array}$ & $\begin{array}{c}\Delta_{\mathrm{vap}} H_{m} \\
\left(\mathrm{~kJ} \mathrm{~mol}^{-1}\right)\end{array}$ & $\begin{array}{c}\text { Mean temperature } \\
\qquad\left(T_{m} / \mathrm{K}\right)\end{array}$ & Method & $\begin{array}{c}\text { CAS registry number } \\
\text { Reference }\end{array}$ \\
\hline & $(323-343)$ & 55.2 & (298) & CGC & {$[96 / 7][00 / 10]$} \\
\hline & $(313-353)$ & 58.9 & (298) & CGC & {$[95 / 21]$} \\
\hline & $(306-458)$ & 51.1 & $(321)$ & A & {$[87 / 5]$} \\
\hline & & $56.3 \pm 0.2$ & (298) & & [81/18] \\
\hline \multirow[t]{7}{*}{$\mathrm{C}_{9} \mathrm{H}_{18} \mathrm{O}$} & 2-nonanone & & & & [925-78-0] \\
\hline & $(285-454)$ & 55.6 & $(300)$ & & [99/27] \\
\hline & $(335-468)$ & 52.6 & $(350)$ & A & {$[87 / 5]$} \\
\hline & & $56.6 \pm 0.6$ & (298) & GCC & {$[79 / 7]$} \\
\hline & & $56.4 \pm 0.1$ & (298) & $\mathrm{C}$ & {$[77 / 2]$} \\
\hline & $(342-545)$ & 56.4 & (298) & & {$[75 / 8]$} \\
\hline & $(335-437)$ & 52.7 & $(348)$ & & {$[66 / 12]$} \\
\hline \multirow{7}{*}{$\mathrm{C}_{9} \mathrm{H}_{18} \mathrm{O}$} & 5-nonanone & & & & {$[502-56-7]$} \\
\hline & $(443-486)$ & 44.7 & $(458)$ & A & [87/5] \\
\hline & & $54.9 \pm 0.4$ & $(298)$ & GCC & {$[79 / 7]$} \\
\hline & $(357-468)$ & 49.7 & $(372)$ & A & {$[87 / 5][75 / 8]$} \\
\hline & & 55.0 & (298) & & {$[75 / 8]$} \\
\hline & & $53.3 \pm 0.1$ & $(298)$ & $\mathrm{C}$ & [70/19] \\
\hline & $(283-323)$ & 40.2 & $(298)$ & A & {$[87 / 5][37 / 9]$} \\
\hline \multirow[t]{2}{*}{$\mathrm{C}_{9} \mathrm{H}_{18} \mathrm{O}$} & 3,3,5-trimethylcyclohexanol & & & & {$[116-02-9]$} \\
\hline & $(343-473)$ & 61.8 & $(358)$ & A & {$[87 / 5]$} \\
\hline \multirow[t]{2}{*}{$\mathrm{C}_{9} \mathrm{H}_{18} \mathrm{O}$} & 2,2,5-trimethyl-4-hexene-1-ol & & & & {$[53965-16-5]$} \\
\hline & $(323-373)$ & 61.5 & $(338)$ & A & {$[87 / 5]$} \\
\hline \multirow[t]{2}{*}{$\mathrm{C}_{9} \mathrm{H}_{18} \mathrm{O}_{2}$} & 2-butoxy-3-pentanone & & & & {$[22432-66-2]$} \\
\hline & $(333-398)$ & 39.8 & $(348)$ & A & {$[87 / 5]$} \\
\hline \multirow[t]{2}{*}{$\mathrm{C}_{9} \mathrm{H}_{18} \mathrm{O}_{2}$} & 2-butyl-1,3-dioxepane & & & & {$[22432-66-2]$} \\
\hline & $(325-358)$ & 57.4 & $(340)$ & A & {$[87 / 5]$} \\
\hline \multirow[t]{2}{*}{$\mathrm{C}_{9} \mathrm{H}_{18} \mathrm{O}_{2}$} & 2-ethylheptanoic acid & & & & {$[3274-29-1]$} \\
\hline & $(386-475)$ & 63.4 & $(401)$ & $\mathrm{A}, \mathrm{EB}$ & {$[87 / 5][60 / 22]$} \\
\hline \multirow[t]{2}{*}{$\mathrm{C}_{9} \mathrm{H}_{18} \mathrm{O}_{2}$} & 2-methyl-2-pentyl-1,3-dioxolane & & & & [4352-95-8] \\
\hline & $(278-318)$ & $54.0 \pm 0.3$ & $(298)$ & GS & {$[98 / 21][02 / 32]$} \\
\hline \multirow[t]{2}{*}{$\mathrm{C}_{9} \mathrm{H}_{18} \mathrm{O}_{2}$} & 2,2-diisopropyl-1,3-dioxolane & & & & [4421-10-7] \\
\hline & $(278-318)$ & $49.9 \pm 0.3$ & $(293)$ & GS & {$[98 / 21][02 / 32]$} \\
\hline \multirow[t]{2}{*}{$\mathrm{C}_{9} \mathrm{H}_{18} \mathrm{O}_{2}$} & 2-hexyl-1,3-dioxolane & & & & {$[1708-34-5]$} \\
\hline & $(325-353)$ & 55.0 & $(339)$ & A & {$[87 / 5]$} \\
\hline \multirow[t]{2}{*}{$\mathrm{C}_{9} \mathrm{H}_{18} \mathrm{O}_{2}$} & methyl 2,4,4-trimethylpentanoate & & & & {$[64198-22-7]$} \\
\hline & $(278-318)$ & $48.4 \pm 0.2$ & $(298)$ & GS & {$[96 / 11]$} \\
\hline \multirow[t]{2}{*}{$\mathrm{C}_{9} \mathrm{H}_{18} \mathrm{O}_{2}$} & butyl pivalate & & & & {$[5129-37-3]$} \\
\hline & $(274-313)$ & $50.4 \pm 0.3$ & $(298)$ & GS & {$[96 / 11]$} \\
\hline \multirow[t]{2}{*}{$\mathrm{C}_{9} \mathrm{H}_{18} \mathrm{O}_{2}$} & isobutyl isovalerate & & & & {$[589-59-3]$} \\
\hline & $(289-442)$ & 47.3 & $(304)$ & A & {$[87 / 5][47 / 5]$} \\
\hline \multirow[t]{2}{*}{$\mathrm{C}_{9} \mathrm{H}_{18} \mathrm{O}_{2}$} & isopentyl butyrate & & & & {$[106-27-4]$} \\
\hline & $(294-452)$ & 47.4 & $(309)$ & A & {$[87 / 5][47 / 5]$} \\
\hline $\mathrm{C}_{9} \mathrm{H}_{18} \mathrm{O}_{2}$ & isopentyl isobutyrate & & & & {$[2050-01-3]$} \\
\hline & $(287-442)$ & 47.4 & $(302)$ & A & {$[87 / 5][47 / 5]$} \\
\hline $\mathrm{C}_{9} \mathrm{H}_{18} \mathrm{O}_{2}$ & isopropyl caproate & & & & {$[2311-46-8]$} \\
\hline & $(307-383)$ & 51.6 & $(322)$ & A & {$[87 / 5]$} \\
\hline $\mathrm{C}_{9} \mathrm{H}_{18} \mathrm{O}_{2}$ & methyl octanoate (methyl caprylate) & & & & {$[111-11-5]$} \\
\hline & & 53.3 & $(350)$ & & {$[02 / 27]$} \\
\hline & & $52.6 \pm 0.1$ & $(363)$ & & {$[02 / 27]$} \\
\hline & & $56.9 \pm 0.1$ & $(298)$ & & {$[02 / 27]$} \\
\hline & & $54.7 \pm 0.6$ & $(298)$ & GC & {$[87 / 17]$} \\
\hline & & $57.3 \pm 0.4$ & (298) & $\mathrm{GCC}$ & {$[80 / 5]$} \\
\hline & & $57.9 \pm 0.4$ & $(298)$ & $\mathrm{C}$ & {$[77 / 7]$} \\
\hline & & $56.4 \pm 0.5$ & (298) & $\mathrm{C}$ & {$[77 / 1]$} \\
\hline & $(347-470)$ & 52.4 & $(362)$ & A, EST & {$[87 / 5][63 / 16]$} \\
\hline & $(373-419)$ & 50.8 & $(388)$ & & {$[61 / 6][84 / 9]$} \\
\hline & $(307-350)$ & 55.2 & $(322)$ & $\mathrm{MG}, \mathrm{OM}$ & {$[52 / 13]$} \\
\hline $\mathrm{C}_{9} \mathrm{H}_{18} \mathrm{O}_{2}$ & nonanoic acid & & & & {$[112-05-0]$} \\
\hline & $(381-528)$ & 76.9 & $(396)$ & A & {$[87 / 5]$} \\
\hline & $(292-313)$ & $85.3 \pm 2.0$ & $(304)$ & $\mathrm{ME}, \mathrm{TE}$ & {$[82 / 4]$} \\
\hline & $(293-303)$ & $82.4 \pm 0.4$ & (298) & & {$[68 / 20]$} \\
\hline & $(387-483)$ & 64.2 & & EB & {$[60 / 22]$} \\
\hline $\mathrm{C}_{9} \mathrm{H}_{18} \mathrm{O}_{2}$ & propyl caproate & & & & [626-77-7] \\
\hline & $(315-394)$ & 52.8 & $(330)$ & A & [87/5] \\
\hline
\end{tabular}


TABLE 6. Enthalpies of vaporization of organic compounds, 1880-2002-Continued

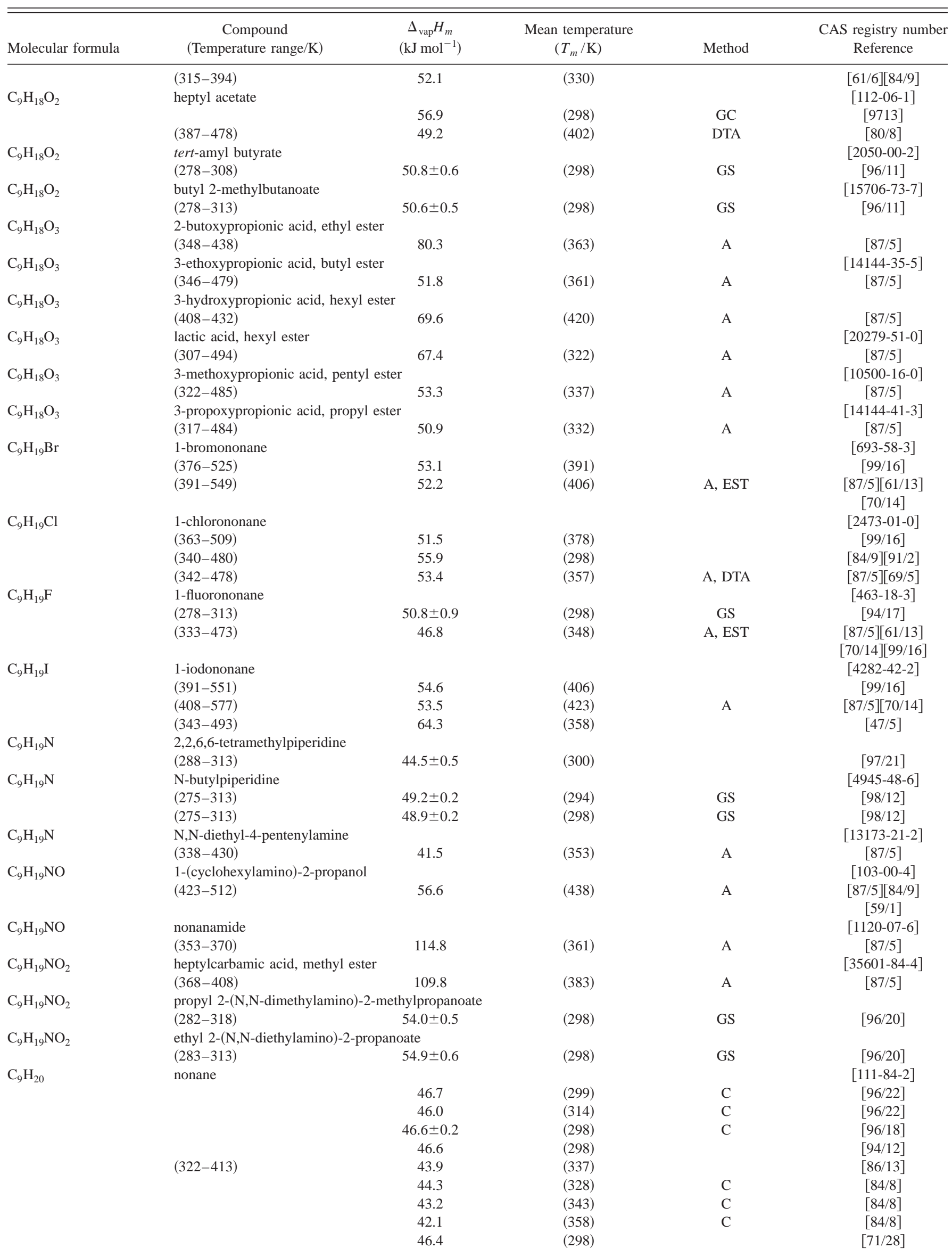


TABLE 6. Enthalpies of vaporization of organic compounds, 1880-2002-Continued

\begin{tabular}{|c|c|c|c|c|c|}
\hline Molecular formula & $\begin{array}{c}\text { Compound } \\
\text { (Temperature range/K) }\end{array}$ & $\begin{array}{c}\Delta_{\mathrm{vap}} H_{m} \\
\left(\mathrm{~kJ} \mathrm{~mol}^{-1}\right)\end{array}$ & $\begin{array}{l}\text { Mean temperature } \\
\qquad\left(T_{m} / \mathrm{K}\right)\end{array}$ & Method & $\begin{array}{c}\text { CAS registry number } \\
\text { Reference }\end{array}$ \\
\hline & $(219-308)$ & 48.3 & $(234)$ & A & {$[87 / 5][73 / 11]$} \\
\hline & & 46.4 & $(298)$ & $\mathrm{C}$ & {$[47 / 7]$} \\
\hline & $(344-426)$ & 42.7 & (359) & $\mathrm{A}, \mathrm{MM}$ & $\begin{array}{c}{[87 / 5][45 / 2]} \\
{[49 / 6]}\end{array}$ \\
\hline \multirow[t]{3}{*}{$\mathrm{C}_{9} \mathrm{H}_{20}$} & 2-methyloctane & & & & [3221-61-2] \\
\hline & $(305-417)$ & 43.2 & $(320)$ & A & {$[87 / 5]$} \\
\hline & & 44.9 & (298) & & {$[71 / 28][61 / 30]$} \\
\hline \multirow[t]{2}{*}{$\mathrm{C}_{9} \mathrm{H}_{20}$} & 3-methyloctane & & & & {$[2216-33-3]$} \\
\hline & & 44.9 & $(298)$ & & {$[71 / 28][61 / 30]$} \\
\hline \multirow[t]{2}{*}{$\mathrm{C}_{9} \mathrm{H}_{20}$} & 4-methyloctane & & & & [2216-34-4] \\
\hline & & 44.5 & (298) & & {$[71 / 28][61 / 30]$} \\
\hline \multirow[t]{2}{*}{$\mathrm{C}_{9} \mathrm{H}_{20}$} & 3-ethylheptane & & & & [15869-80-4] \\
\hline & & 44.5 & $(298)$ & & {$[71 / 28][61 / 30]$} \\
\hline \multirow[t]{2}{*}{$\mathrm{C}_{9} \mathrm{H}_{20}$} & 4-ethylheptane & & & & [2216-32-2] \\
\hline & & 44.1 & (298) & & {$[61 / 30]$} \\
\hline \multirow[t]{2}{*}{$\mathrm{C}_{9} \mathrm{H}_{20}$} & 2,2-dimethylheptane & & & & [1071-26-7] \\
\hline & & 42.3 & (298) & & {$[71 / 28][61 / 30]$} \\
\hline \multirow{2}{*}{$\mathrm{C}_{9} \mathrm{H}_{20}$} & 2,3-dimethylheptane & & & & [3074-71-3] \\
\hline & & 43.6 & (298) & & {$[71 / 28][61 / 30]$} \\
\hline \multirow[t]{2}{*}{$\mathrm{C}_{9} \mathrm{H}_{20}$} & 2,4-dimethylheptane & & & & [2213-23-2] \\
\hline & & 42.9 & (298) & & {$[71 / 28][61 / 30]$} \\
\hline \multirow[t]{2}{*}{$\mathrm{C}_{9} \mathrm{H}_{20}$} & 2,5-dimethylheptane & & & & [2216-30-0] \\
\hline & & 43.3 & $(298)$ & & {$[71 / 28][61 / 30]$} \\
\hline \multirow[t]{2}{*}{$\mathrm{C}_{9} \mathrm{H}_{20}$} & 2,6-dimethylheptane & & & & {$[1072-05-5]$} \\
\hline & & 43.3 & (298) & & {$[71 / 28][61 / 30]$} \\
\hline \multirow[t]{2}{*}{$\mathrm{C}_{9} \mathrm{H}_{20}$} & 3,3-dimethylheptane & & & & [4032-86-4] \\
\hline & & 42.6 & (298) & & {$[71 / 28][61 / 30]$} \\
\hline \multirow[t]{2}{*}{$\mathrm{C}_{9} \mathrm{H}_{20}$} & 3,4-dimethylheptane & & & & [922-28-1] \\
\hline & & 43.6 & (298) & & {$[71 / 28][61 / 30]$} \\
\hline \multirow{2}{*}{$\mathrm{C}_{9} \mathrm{H}_{20}$} & 3,5-dimethylheptane & & & & [926-82-9] \\
\hline & & 43.3 & $(298)$ & & {$[71 / 28]$} \\
\hline \multirow[t]{2}{*}{$\mathrm{C}_{9} \mathrm{H}_{20}$} & 4,4-dimethylheptane & & & & [1068-19-5] \\
\hline & & 42.2 & (298) & & {$[71 / 28][61 / 30]$} \\
\hline $\mathrm{C}_{9} \mathrm{H}_{20}$ & 3,3-diethylpentane & & & & {$[1067-20-5]$} \\
\hline & & $42.6 \pm 0.3$ & $(298)$ & $\mathrm{GCC}$ & {$[79 / 17]$} \\
\hline & & 43.6 & $(298)$ & & {$[71 / 28][61 / 30]$} \\
\hline & $(335-426)$ & 39.8 & $(350)$ & $\mathrm{A}$ & {$[87 / 5][49 / 6]$} \\
\hline $\mathrm{C}_{9} \mathrm{H}_{20}$ & 2-methyl-3-ethylhexane & & & & [16789-46-1] \\
\hline & & 43.2 & (298) & & {$[61 / 30]$} \\
\hline $\mathrm{C}_{9} \mathrm{H}_{20}$ & 2-methyl-4-ethylhexane & & & & {$[3074-75-7]$} \\
\hline & & 42.9 & (298) & & {$[61 / 30]$} \\
\hline $\mathrm{C}_{9} \mathrm{H}_{20}$ & 3-methyl-3-ethylhexane & & & & [3074-76-8] \\
\hline & & 42.9 & (298) & & {$[61 / 30]$} \\
\hline $\mathrm{C}_{9} \mathrm{H}_{20}$ & 3-methyl-4-ethylhexane & & & & \\
\hline & & 43.6 & (298) & & {$[61 / 30]$} \\
\hline $\mathrm{C}_{9} \mathrm{H}_{20}$ & 2,2,3-trimethylhexane & & & & {$[16747-25-4]$} \\
\hline & $(238-303)$ & 42.2 & $(288)$ & IPM & {$[74 / 11]$} \\
\hline & & 41.7 & (298) & & {$[71 / 28][61 / 30]$} \\
\hline $\mathrm{C}_{9} \mathrm{H}_{20}$ & 2,2,4-trimethylhexane & & & & {$[16747-26-5]$} \\
\hline & $(288-410)$ & 39.5 & (303) & A & {$[87 / 5]$} \\
\hline & $(238-393)$ & 41.0 & (278) & A & {$[87 / 5]$} \\
\hline & $(238-303)$ & 40.5 & $(288)$ & IPM & {$[74 / 11]$} \\
\hline & & 40.7 & (298) & & {$[71 / 28]$} \\
\hline $\mathrm{C}_{9} \mathrm{H}_{20}$ & 2,2,5-trimethylhexane & & & & [3522-94-9] \\
\hline & $(288-399)$ & 40.1 & (303) & A & {$[87 / 5]$} \\
\hline & $(238-303)$ & 41.1 & $(288)$ & A, IPM & {$[87 / 5][74 / 11]$} \\
\hline & & 40.2 & (298) & & {$[71 / 28]$} \\
\hline & $(319-398)$ & 38.5 & (334) & & {$[49 / 6]$} \\
\hline & & 40.2 & (298) & $\mathrm{C}$ & {$[47 / 7]$} \\
\hline $\mathrm{C}_{9} \mathrm{H}_{20}$ & 2,3,3-trimethylhexane & & & & [16747-28-7] \\
\hline & $(238-303)$ & 44.2 & (253) & A & {$[87 / 5]$} \\
\hline & $(288-422)$ & 39.4 & $(303)$ & A & {$[87 / 5]$} \\
\hline & & 42.1 & (298) & & {$[71 / 28][61 / 30]$} \\
\hline $\mathrm{C}_{9} \mathrm{H}_{20}$ & 2,3,4-trimethylhexane & & & & [921-47-1] \\
\hline
\end{tabular}


TABLE 6. Enthalpies of vaporization of organic compounds, 1880-2002-Continued

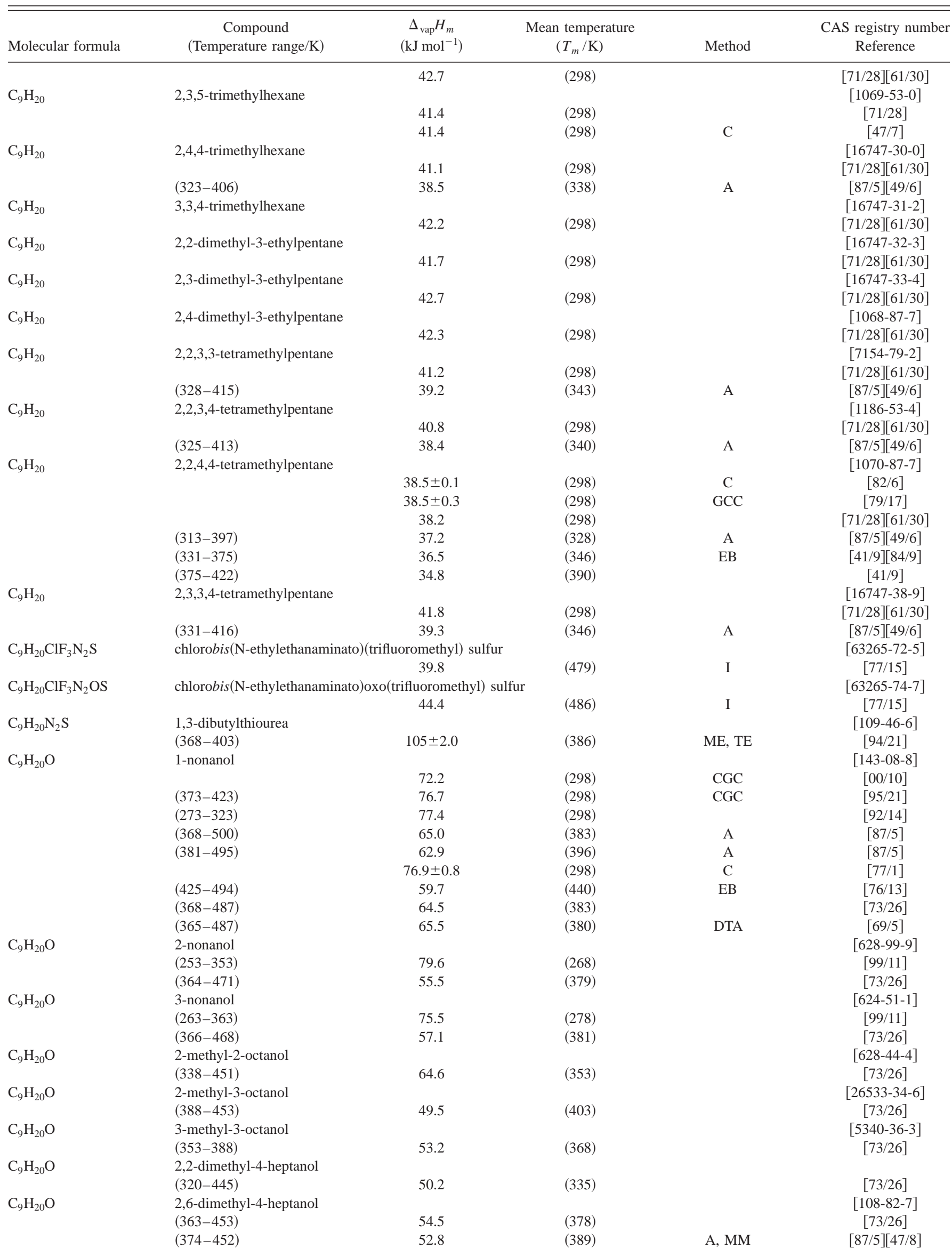


TABLE 6. Enthalpies of vaporization of organic compounds, 1880-2002-Continued

\begin{tabular}{|c|c|c|c|c|c|}
\hline Molecular formula & $\begin{array}{c}\text { Compound } \\
\text { (Temperature range/K) }\end{array}$ & $\begin{array}{c}\Delta_{\mathrm{vap}} H_{m} \\
\left(\mathrm{~kJ} \mathrm{~mol}^{-1}\right)\end{array}$ & $\begin{array}{l}\text { Mean temperature } \\
\qquad\left(T_{m} / \mathrm{K}\right)\end{array}$ & Method & $\begin{array}{c}\text { CAS registry number } \\
\text { Reference }\end{array}$ \\
\hline $\mathrm{C}_{9} \mathrm{H}_{20} \mathrm{O}$ & $\begin{array}{l}\text { 2,2,3-trimethyl-3-hexanol } \\
(343-441)\end{array}$ & 55.1 & $(358)$ & & $\begin{array}{c}{[5340-41-0]} \\
{[73 / 26]}\end{array}$ \\
\hline $\mathrm{C}_{9} \mathrm{H}_{20} \mathrm{O}$ & $\begin{array}{l}\text { 2,4-dimethyl-3-ethyl-3-pentanol } \\
(369-451)\end{array}$ & 50.0 & $(384)$ & & $\begin{array}{c}{[3970-59-0]} \\
{[73 / 26]}\end{array}$ \\
\hline $\mathrm{C}_{9} \mathrm{H}_{20} \mathrm{O}$ & $\begin{array}{l}\text { 2,2,3,4-tetramethyl-3-pentanol } \\
(329-448)\end{array}$ & 60.8 & $(344)$ & & $\begin{array}{c}{[29772-39-2]} \\
{[73 / 26]}\end{array}$ \\
\hline $\mathrm{C}_{9} \mathrm{H}_{20} \mathrm{O}$ & $\begin{array}{l}\text { butyl 1,1-dimethylpropyl ether } \\
\text { (278-308) }\end{array}$ & $46.1 \pm 0.3$ & (298) & GS & $\begin{array}{c}{[3249-47-6]} \\
{[96 / 11]}\end{array}$ \\
\hline $\mathrm{C}_{9} \mathrm{H}_{20} \mathrm{O}$ & $\begin{array}{l}\text { 2-methoxy-2,4,4-trimethylpentane } \\
(381-418)\end{array}$ & 38.5 & (396) & & {$[01 / 14]$} \\
\hline $\mathrm{C}_{9} \mathrm{H}_{20} \mathrm{O}$ & methyl tert-octyl ether & 45.3 & $(298)$ & & {$[\mathrm{U} / 2][02 / 32]$} \\
\hline $\mathrm{C}_{9} \mathrm{H}_{20} \mathrm{O}$ & $\begin{array}{l}\text { pentyl tert-butyl ether } \\
(319-365) \\
(319-365)\end{array}$ & $\begin{array}{c}48.3 \\
43.7 \\
46.9 \pm 1.0\end{array}$ & $\begin{array}{l}(298) \\
(334) \\
(298)\end{array}$ & $\begin{array}{l}\mathrm{EB} \\
\mathrm{EB}\end{array}$ & $\begin{array}{c}{[10100-95-5]} \\
{[\mathrm{U} / 2][02 / 32]} \\
{[90 / 15]} \\
{[90 / 15]}\end{array}$ \\
\hline $\mathrm{C}_{9} \mathrm{H}_{20} \mathrm{O}$ & isobutyl tert-amyl ether & 46.3 & (298) & & {$[\mathrm{U} / 2][02 / 32]$} \\
\hline $\mathrm{C}_{9} \mathrm{H}_{20} \mathrm{O}$ & sec-butyl tert-amyl ether & 46.8 & (298) & & {$[\mathrm{U} / 2][02 / 32]$} \\
\hline $\mathrm{C}_{9} \mathrm{H}_{20} \mathrm{O}$ & butyl tert-amyl ether & 48.3 & (298) & & {$[\mathrm{U} / 2][02 / 32]$} \\
\hline $\mathrm{C}_{9} \mathrm{H}_{20} \mathrm{O}_{2}$ & $\begin{array}{l}\text { 2,6,6-trimethyl-5-oxa-2-heptanol } \\
(329-454)\end{array}$ & 53.3 & $(344)$ & & {$[68 / 16][84 / 9]$} \\
\hline $\mathrm{C}_{9} \mathrm{H}_{20} \mathrm{O}_{2}$ & $\begin{array}{l}\text { dibutoxymethane } \\
(366-452)\end{array}$ & 47.9 & $(381)$ & EB & {$[00 / 17]$} \\
\hline $\mathrm{C}_{9} \mathrm{H}_{20} \mathrm{O}_{2}$ & 1-butoxy-2-propoxyethane & $54.7 \pm 0.1$ & $(298)$ & $\mathrm{C}$ & $\begin{array}{c}{[18854-58-5]} \\
{[70 / 17]}\end{array}$ \\
\hline $\mathrm{C}_{9} \mathrm{H}_{20} \mathrm{O}_{2}$ & $\begin{array}{l}\text { 2-butyl-2-ethyl-1,3-propanediol } \\
(424-523) \\
(424-523) \\
(424-523)\end{array}$ & $\begin{array}{l}74.3 \pm 0.3 \\
67.2 \pm 0.3 \\
61.4 \pm 0.6\end{array}$ & $\begin{array}{l}(420) \\
(460) \\
(500)\end{array}$ & $\begin{array}{l}\text { EB } \\
\text { EB } \\
\text { EB }\end{array}$ & $\begin{array}{l}{[115-84-4]} \\
{[02 / 14]} \\
{[02 / 14]} \\
{[02 / 14]}\end{array}$ \\
\hline $\mathrm{C}_{9} \mathrm{H}_{20} \mathrm{O}_{2}$ & $\begin{array}{l}\text { 4-tert-butoxy-2-methyl-2-butanol } \\
\text { (367-483) }\end{array}$ & 61.5 & $(382)$ & A & $\begin{array}{c}{[22419-28-9]} \\
{[87 / 5]}\end{array}$ \\
\hline $\mathrm{C}_{9} \mathrm{H}_{20} \mathrm{O}_{2}$ & $\begin{array}{l}\text { 2,2,4-trimethyl-1,6-hexanediol } \\
(419-541)\end{array}$ & 68.0 & $(434)$ & A & $\begin{array}{c}{[3089-24-5]} \\
{[87 / 5]}\end{array}$ \\
\hline $\mathrm{C}_{9} \mathrm{H}_{20} \mathrm{O}_{3}$ & $\begin{array}{l}\text { dipropylene glycol isopropyl ether } \\
(319-479)\end{array}$ & 55.0 & $(334)$ & $\mathrm{A}$ & {$[87 / 5][47 / 5]$} \\
\hline $\mathrm{C}_{9} \mathrm{H}_{20} \mathrm{O}_{4}$ & $\begin{array}{l}\text { tripropylene glycol } \\
(369-541)\end{array}$ & 63.3 & $(384)$ & A & {$[87 / 5]$} \\
\hline $\mathrm{C}_{9} \mathrm{H}_{20} \mathrm{~S}$ & $\begin{array}{l}\text { 1-nonanethiol } \\
(390-494)\end{array}$ & 52.6 & $(405)$ & A & $\begin{array}{c}{[1455-21-6]} \\
{[87 / 5][99 / 16]} \\
{[32 / 2]}\end{array}$ \\
\hline $\mathrm{C}_{9} \mathrm{H}_{20} \mathrm{~S}$ & $\begin{array}{l}\text { 2-nonanethiol } \\
(379-482)\end{array}$ & 50.3 & (394) & & $\begin{array}{l}{[13281-11-3]} \\
{[99 / 16][32 / 2]}\end{array}$ \\
\hline $\mathrm{C}_{9} \mathrm{H}_{20} \mathrm{~S}_{2}$ & $\begin{array}{l}\text { 1,9-nonanedithiol } \\
(418-557)\end{array}$ & 63.6 & (433) & A & $\begin{array}{c}{[3489-28-9]} \\
{[87 / 5][99 / 16]} \\
{[43 / 6]}\end{array}$ \\
\hline $\mathrm{C}_{9} \mathrm{H}_{21} \mathrm{~N}$ & $\begin{array}{l}\text { N-methyl octylamine } \\
(365-508)\end{array}$ & 49.2 & (380) & A & $\begin{array}{c}{[2439-54-5]} \\
{[87 / 5]}\end{array}$ \\
\hline $\mathrm{C}_{9} \mathrm{H}_{21} \mathrm{~N}$ & $\begin{array}{l}\text { nonylamine } \\
(377-478)\end{array}$ & 50.7 & (392) & A & $\begin{array}{c}{[112-20-9]} \\
{[87 / 5]}\end{array}$ \\
\hline $\mathrm{C}_{9} \mathrm{H}_{21} \mathrm{~N}$ & $\begin{array}{l}\text { tripropylamine } \\
(341-475)\end{array}$ & $\begin{array}{c}45.6 \\
46.2 \pm 0.1\end{array}$ & $\begin{array}{l}(356) \\
(298)\end{array}$ & $\begin{array}{l}\mathrm{A} \\
\mathrm{C}\end{array}$ & $\begin{array}{c}{[102-69-2]} \\
{[87 / 5]} \\
{[69 / 2]}\end{array}$ \\
\hline $\mathrm{C}_{9} \mathrm{H}_{21} \mathrm{NO}_{3}$ & $\begin{array}{l}\text { triisopropanolamine } \\
(428-573)\end{array}$ & 73.7 & (443) & A & $\begin{array}{l}{[122-20-3]} \\
{[87 / 5]}\end{array}$ \\
\hline $\mathrm{C}_{9} \mathrm{H}_{21} \mathrm{O}_{4} \mathrm{P}$ & $\begin{array}{l}\text { tripropylphosphate } \\
(394-525)\end{array}$ & 56.7 & (409) & A & $\begin{array}{c}{[513-08-6]} \\
{[87 / 5]}\end{array}$ \\
\hline $\mathrm{C}_{9} \mathrm{H}_{21} \mathrm{P}$ & $\begin{array}{l}\text { tripropylphosphine } \\
(324-368)\end{array}$ & $39.4 \pm 0.2$ & (346) & & $\begin{array}{c}{[2234-97-1]} \\
{[01 / 9]}\end{array}$ \\
\hline $\begin{array}{l}\mathrm{C}_{9} \mathrm{H}_{22} \mathrm{ClN}_{2} \mathrm{PS} \\
\mathrm{C}_{10} \mathrm{Cl}_{8}\end{array}$ & $\begin{array}{l}\mathrm{P} \text {-(chloromethyl)-N, } \mathrm{N}^{\prime} \text {-bis(1-methy } \\
\text { (333-368) } \\
\text { octachloronaphthalene }\end{array}$ & $\begin{array}{l}\text { propyl)phosp } \\
66.8\end{array}$ & $\begin{array}{l}\text { ic diamide } \\
\qquad(348)\end{array}$ & A & $\begin{array}{l}{[58023-20-4]} \\
{[87 / 5][99 / 16]} \\
{[2234-13-1]}\end{array}$ \\
\hline
\end{tabular}


TABLE 6. Enthalpies of vaporization of organic compounds, 1880-2002-Continued

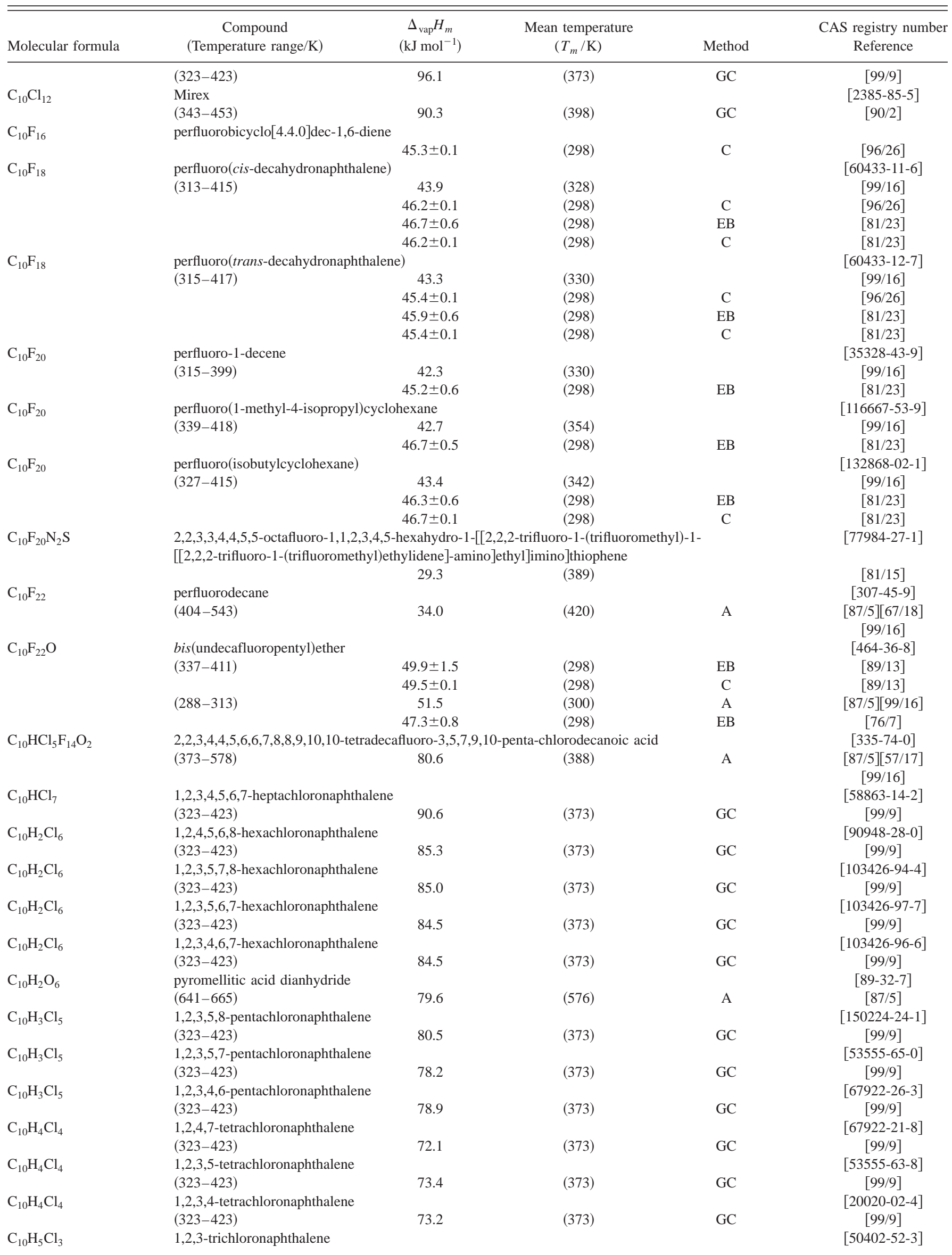


TABLE 6. Enthalpies of vaporization of organic compounds, 1880-2002-Continued

\begin{tabular}{|c|c|c|c|c|c|}
\hline Molecular formula & $\begin{array}{c}\text { Compound } \\
\text { (Temperature range/K) }\end{array}$ & $\begin{array}{c}\Delta_{\mathrm{vap}} H_{m} \\
\left(\mathrm{~kJ} \mathrm{~mol}^{-1}\right)\end{array}$ & $\begin{array}{l}\text { Mean temperature } \\
\qquad\left(T_{m} / \mathrm{K}\right)\end{array}$ & Method & $\begin{array}{c}\text { CAS registry number } \\
\text { Reference }\end{array}$ \\
\hline & $(323-423)$ & 68.0 & $(373)$ & GC & [99/9] \\
\hline \multirow{2}{*}{$\mathrm{C}_{10} \mathrm{H}_{5} \mathrm{Cl}_{7}$} & Heptachlor & & & & [76-44-8] \\
\hline & $(343-453)$ & 76.5 & $(398)$ & GC & {$[90 / 2]$} \\
\hline \multirow[t]{2}{*}{$\mathrm{C}_{10} \mathrm{H}_{5} \mathrm{Cl}_{9}$} & cis Nonachlor & & & & {$[5103-73-1]$} \\
\hline & $(343-453)$ & 83.8 & $(398)$ & GC & {$[90 / 2]$} \\
\hline \multirow[t]{2}{*}{$\mathrm{C}_{10} \mathrm{H}_{5} \mathrm{Cl}_{9}$} & trans Nonachlor & & & & {$[39765-80-5]$} \\
\hline & $(343-453)$ & 85.5 & $(398)$ & GC & {$[90 / 2]$} \\
\hline \multirow[t]{2}{*}{$\mathrm{C}_{10} \mathrm{H}_{6} \mathrm{Cl}_{2}$} & 1,2-dichloronaphthalene & & & & [2050-69-3] \\
\hline & $(323-423)$ & 60.7 & (373) & GC & [99/9] \\
\hline \multirow[t]{2}{*}{$\mathrm{C}_{10} \mathrm{H}_{6} \mathrm{Cl}_{2}$} & 1,4-dichloronaphthalene & & & & {$[1825-31-6]$} \\
\hline & $(323-423)$ & 58.7 & $(373)$ & GC & {$[99 / 9]$} \\
\hline \multirow[t]{3}{*}{$\mathrm{C}_{10} \mathrm{H}_{6} \mathrm{Cl}_{8}$} & cis Chlordane & & & & {$[5103-71-9]$} \\
\hline & $(323-409)$ & 83.0 & $(338)$ & & [99/16] \\
\hline & $(343-453)$ & 82.0 & (398) & GC & {$[90 / 2]$} \\
\hline \multirow[t]{3}{*}{$\mathrm{C}_{10} \mathrm{H}_{6} \mathrm{Cl}_{8}$} & trans Chlordane & & & & {$[5103-74-2]$} \\
\hline & $(373-409)$ & 81.7 & $(388)$ & & {$[99 / 16]$} \\
\hline & $(343-453)$ & 80.7 & $(398)$ & GC & {$[90 / 2]$} \\
\hline \multirow[t]{2}{*}{$\mathrm{C}_{10} \mathrm{H}_{6} \mathrm{~N}_{2} \mathrm{O}_{4}$} & 1,5-dinitronaphthalene & & & & {$[605-71-0]$} \\
\hline & $(506-642)$ & 74.7 & $(521)$ & A & {$[87 / 5]$} \\
\hline \multirow{2}{*}{$\mathrm{C}_{10} \mathrm{H}_{6} \mathrm{~N}_{2} \mathrm{O}_{4}$} & 1,8-dinitronaphthalene & & & & {$[602-38-0]$} \\
\hline & $(553-715)$ & 78.5 & $(568)$ & A & {$[87 / 5]$} \\
\hline \multirow[t]{4}{*}{$\mathrm{C}_{10} \mathrm{H}_{7} \mathrm{Br}$} & 1-bromonaphthalene & & & & {$[90-11-9]$} \\
\hline & $(357-555)$ & 58.5 & $(372)$ & & {$[87 / 5]$} \\
\hline & $(295-359)$ & $56 \pm 6$ & $(329)$ & $\mathrm{ME}$ & {$[80 / 7]$} \\
\hline & $(469-559)$ & 45.8 & $(484)$ & $\mathrm{A}, \mathrm{EB}$ & $\begin{array}{c}{[87 / 5][76 / 13]} \\
{[99 / 16]}\end{array}$ \\
\hline \multirow[t]{3}{*}{$\mathrm{C}_{10} \mathrm{H}_{7} \mathrm{Br}$} & 2-bromonaphthalene & & & & {$[580-13-2]$} \\
\hline & $(330-378)$ & 42.5 & $(354)$ & & [99/16] \\
\hline & $(322-359)$ & 40.4 & $(340)$ & $\mathrm{ME}, \mathrm{TE}$ & {$[81 / 22]$} \\
\hline \multirow[t]{5}{*}{$\mathrm{C}_{10} \mathrm{H}_{7} \mathrm{Cl}$} & 1-chloronaphthalene & & & & {$[90-13-1]$} \\
\hline & & $64.0 \pm 0.3$ & $(298)$ & GS & [01/1] \\
\hline & $(323-423)$ & 58.6 & $(373)$ & GC & [99/9] \\
\hline & $(353-553)$ & 59.6 & $(368)$ & A & {$[87 / 5][47 / 5]$} \\
\hline & $(400-435)$ & 57.8 & $(415)$ & A & {$[87 / 5]$} \\
\hline \multirow[t]{3}{*}{$\mathrm{C}_{10} \mathrm{H}_{7} \mathrm{Cl}$} & 2-chloronaphthalene & & & & {$[91-58-7]$} \\
\hline & $(400-435)$ & 57.9 & $(417)$ & & {$[99 / 16]$} \\
\hline & $(323-423)$ & 58.5 & $(373)$ & GC & {$[99 / 9]$} \\
\hline \multirow[t]{2}{*}{$\mathrm{C}_{10} \mathrm{H}_{7} \mathrm{Cl}_{7}$} & $1,4,5,6,7,8,8$-heptachloro- & trahydro- 4,7 & oindan & & [2589-15-3] \\
\hline & $(333-353)$ & 83.8 & $(343)$ & & [99/16] \\
\hline \multirow[t]{2}{*}{$\mathrm{C}_{10} \mathrm{H}_{7} \mathrm{~F}_{5} \mathrm{O}_{2}$} & pentafluoropropionic acid, & & & & {$[24277-51-0]$} \\
\hline & $(371-446)$ & 48.3 & $(386)$ & $\mathrm{A}, \mathrm{EB}$ & $\begin{array}{c}{[87 / 5][69 / 13]} \\
{[99 / 16]}\end{array}$ \\
\hline $\mathrm{C}_{10} \mathrm{H}_{7} \mathrm{~F}_{5} \mathrm{O}_{2}$ & pentafluoropropionic acid, & & & & {$[24271-52-1]$} \\
\hline & $(371-448)$ & 48.3 & $(386)$ & $\mathrm{A}, \mathrm{EB}$ & $\begin{array}{c}{[87 / 5][69 / 13]} \\
{[99 / 16]}\end{array}$ \\
\hline $\mathrm{C}_{10} \mathrm{H}_{7} \mathrm{I}$ & 1-iodonaphthalene & & & & [90-14-2] \\
\hline & $(321-428)$ & 78.9 & $(336)$ & & {$[99 / 16]$} \\
\hline $\mathrm{C}_{10} \mathrm{H}_{7} \mathrm{NO}_{2}$ & 1-nitronaphthalene & & & & {$[86-57-7]$} \\
\hline & $(332-580)$ & 66.4 & $(347)$ & A & {$[87 / 5]$} \\
\hline $\mathrm{C}_{10} \mathrm{H}_{8}$ & azulene & & & & {$[275-51-4]$} \\
\hline & & 52.8 & $(298)$ & CGC & {$[98 / 11]$} \\
\hline & $(369-515)$ & 53.0 & $(384)$ & A & {$[87 / 5]$} \\
\hline & $(442-534)$ & 51.2 & $(457)$ & $\mathrm{EB}$ & {$[77 / 10]$} \\
\hline & $(373-423)$ & 55.5 & $(373)$ & & {$[62 / 25]$} \\
\hline $\mathrm{C}_{10} \mathrm{H}_{8}$ & naphthalene & & & & [91-20-3] \\
\hline & $(323-473)$ & 56.1 & (398) & GC & [02/18] \\
\hline & & 53.4 & (298) & CGC & {$[98 / 11]$} \\
\hline & $(460-647)$ & 45.4 & $(475)$ & DSC & {$[96 / 10]$} \\
\hline & $(403-453)$ & 56.6 & (298) & CGC & {$[95 / 21]$} \\
\hline & & $48.7 \pm 0.3$ & $(400)$ & EB & [93/11] \\
\hline & & 46.4 & $(440)$ & $\mathrm{EB}$ & {$[93 / 11]$} \\
\hline & & 44.0 & $(480)$ & $\mathrm{EB}$ & {$[93 / 11]$} \\
\hline & & 41.5 & $(520)$ & $\mathrm{EB}$ & {$[93 / 11]$} \\
\hline & $(513-613)$ & 44.4 & $(528)$ & & [93/3] \\
\hline
\end{tabular}


TABLE 6. Enthalpies of vaporization of organic compounds, 1880-2002-Continued

\begin{tabular}{|c|c|c|c|c|c|}
\hline Molecular formula & $\begin{array}{c}\text { Compound } \\
\text { (Temperature range/K) }\end{array}$ & $\begin{array}{c}\Delta_{\mathrm{vap}} H_{m} \\
\left(\mathrm{~kJ} \mathrm{~mol}^{-1}\right)\end{array}$ & $\begin{array}{l}\text { Mean temperature } \\
\qquad\left(T_{m} / \mathrm{K}\right)\end{array}$ & Method & $\begin{array}{c}\text { CAS registry number } \\
\text { Reference }\end{array}$ \\
\hline & $(418-613)$ & 47.9 & $(423)$ & $\mathrm{EB}$ & {$[90 / 7]$} \\
\hline & $(352-500)$ & 50.6 & $(367)$ & $\mathrm{A}$ & {$[87 / 5]$} \\
\hline & $(491-565)$ & 44.8 & $(506)$ & A & {$[87 / 5]$} \\
\hline & $(563-663)$ & 43.2 & $(578)$ & A & {$[87 / 5]$} \\
\hline & $(661-750)$ & 43.3 & $(676)$ & A & {$[87 / 5]$} \\
\hline & $(441-727)$ & 44.7 & $(466)$ & & {$[81 / 21][86 / 15]$} \\
\hline & $(353-388)$ & $50.3 \pm 0.2$ & $(370)$ & & {$[81 / 6]$} \\
\hline & $(354-453)$ & 50.7 & $(369)$ & & {$[68 / 11]$} \\
\hline & $(399-491)$ & 49.0 & $(414)$ & & {$[55 / 7]$} \\
\hline & & 46.4 & $(441)$ & $\mathrm{C}$ & {$[51 / 7]$} \\
\hline & & 48.3 & $(379)$ & $\mathrm{I}$ & {$[43 / 7]$} \\
\hline & $(373-473)$ & 47.2 & $(423)$ & I & {$[23 / 1]$} \\
\hline & $(360-494)$ & 47.7 & $(427)$ & $\mathrm{I}$ & {$[22 / 1]$} \\
\hline \multirow[t]{2}{*}{$\mathrm{C}_{10} \mathrm{H}_{8} \mathrm{~N}_{2} \mathrm{O}_{2}$} & 1,3-bis(isocyanatomethyl) & & & & {$[3634-83-1]$} \\
\hline & $(403-473)$ & 46.7 & $(418)$ & A & {$[87 / 5]$} \\
\hline \multirow[t]{2}{*}{$\mathrm{C}_{10} \mathrm{H}_{8} \mathrm{~N}_{2} \mathrm{O}_{2}$} & $1,4-b i s$ (isocyanatomethyl) & & & & [1014-98-8] \\
\hline & $(403-473)$ & 56.9 & $(418)$ & A & {$[87 / 5]$} \\
\hline \multirow[t]{2}{*}{$\mathrm{C}_{10} \mathrm{H}_{8} \mathrm{~N}_{2} \mathrm{O}_{2}$} & benzene, ethyldiisocyanat & omers) & & & {$[64711-83-7]$} \\
\hline & $(363-473)$ & 60.7 & $(378)$ & A & {$[87 / 5][77 / 22]$} \\
\hline \multirow[t]{3}{*}{$\mathrm{C}_{10} \mathrm{H}_{8} \mathrm{O}$} & 1-naphthol & & & & [90-15-3] \\
\hline & $(399-556)$ & 58.5 & $(414)$ & A & {$[87 / 5]$} \\
\hline & $(423-563)$ & 60.8 & $(473)$ & & [27/4] \\
\hline \multirow[t]{5}{*}{$\mathrm{C}_{10} \mathrm{H}_{8} \mathrm{O}$} & 2-naphthol & & & & {$[135-19-3]$} \\
\hline & $(393-433)$ & 76.2 & $(298)$ & CGC & {$[95 / 21]$} \\
\hline & $(401-561)$ & 59.7 & $(416)$ & A & {$[87 / 5]$} \\
\hline & $(417-561)$ & 59.7 & $(432)$ & & {$[55 / 9]$} \\
\hline & $(423-563)$ & 61.8 & $(473)$ & & {$[27 / 4]$} \\
\hline \multirow[t]{2}{*}{$\mathrm{C}_{10} \mathrm{H}_{9} \mathrm{Cl}_{3} \mathrm{O}_{3}$} & (2,4,5-trichlorophenoxy)ac & ethyl ester & & & {$[1928-39-8]$} \\
\hline & $(444-573)$ & 76.4 & $(459)$ & A & {$[87 / 5][99 / 16]$} \\
\hline \multirow[t]{4}{*}{$\mathrm{C}_{10} \mathrm{H}_{9} \mathrm{~N}$} & 2-methylquinoline (quinal & & & & [91-63-4] \\
\hline & & $66.1 \pm 1.9$ & $(298)$ & $\mathrm{C}$ & {$[95 / 1]$} \\
\hline & $(281-313)$ & 61.2 & $(297)$ & GS & {$[80 / 6]$} \\
\hline & $(443-521)$ & 54.7 & $(548)$ & $\mathrm{A}, \mathrm{EB}$ & $\begin{array}{c}{[87 / 5][61 / 11]} \\
{[61 / 10]}\end{array}$ \\
\hline \multirow[t]{2}{*}{$\mathrm{C}_{10} \mathrm{H}_{9} \mathrm{~N}$} & 3-methylquinoline & & & & {$[612-58-8]$} \\
\hline & $(443-528)$ & 55.8 & $(458)$ & A & {$[87 / 5][61 / 11]$} \\
\hline \multirow[t]{3}{*}{$\mathrm{C}_{10} \mathrm{H}_{9} \mathrm{~N}$} & 4-methylquinoline (lepidir & & & & [491-35-0] \\
\hline & & $67.6 \pm 1.8$ & $(298)$ & $\mathrm{C}$ & {$[95 / 1]$} \\
\hline & $(463-539)$ & 58.2 & $(478)$ & $\mathrm{A}, \mathrm{EB}$ & $\begin{array}{c}{[87 / 5][61 / 11]} \\
{[61 / 10]}\end{array}$ \\
\hline \multirow[t]{3}{*}{$\mathrm{C}_{10} \mathrm{H}_{9} \mathrm{~N}$} & 6-methylquinoline & & & & [91-62-3] \\
\hline & & $67.7 \pm 1.8$ & $(298)$ & $\mathrm{C}$ & {$[95 / 1]$} \\
\hline & $(453-540)$ & 56.1 & $(468)$ & A & {$[87 / 5]$} \\
\hline \multirow[t]{2}{*}{$\mathrm{C}_{10} \mathrm{H}_{9} \mathrm{~N}$} & 7-methylquinoline & & & & [612-60-2] \\
\hline & $(493-532)$ & 56.7 & $(508)$ & $\mathrm{A}, \mathrm{EB}$ & {$[87 / 5][61 / 10]$} \\
\hline \multirow[t]{3}{*}{$\mathrm{C}_{10} \mathrm{H}_{9} \mathrm{~N}$} & 8-methylquinoline & & & & [611-32-5] \\
\hline & & $65.7 \pm 1.9$ & $(298)$ & C & {$[95 / 1]$} \\
\hline & $(493-523)$ & 52.2 & $(508)$ & $\mathrm{A}, \mathrm{EB}$ & {$[87 / 5][61 / 10]$} \\
\hline \multirow[t]{2}{*}{$\mathrm{C}_{10} \mathrm{H}_{9} \mathrm{~N}$} & 1-naphthylamine & & & & [134-32-7] \\
\hline & $(377-574)$ & 63.6 & $(392)$ & A & {$[87 / 5][47 / 5]$} \\
\hline \multirow[t]{2}{*}{$\mathrm{C}_{10} \mathrm{H}_{9} \mathrm{~N}$} & 2-naphthylamine & & & & {$[91-59-8]$} \\
\hline & $(388-579)$ & 63.5 & $(403)$ & A & {$[87 / 5][47 / 5]$} \\
\hline \multirow[t]{2}{*}{$\mathrm{C}_{10} \mathrm{H}_{9} \mathrm{NO}$} & 6-methoxyquinoline & & & & [5263-87-6] \\
\hline & & $78.1 \pm 2.3$ & $(298)$ & $\mathrm{C}$ & {$[02 / 35]$} \\
\hline $\mathrm{C}_{10} \mathrm{H}_{10}$ & 1,3-divinylbenzene & & & & {$[108-57-6]$} \\
\hline & $(305-453)$ & 48.3 & $(320)$ & A & {$[87 / 5][47 / 5]$} \\
\hline $\mathrm{C}_{10} \mathrm{H}_{10}$ & dicyclopentadiene & & & & \\
\hline & $(307-440)$ & 42.4 & $(322)$ & & {$[47 / 5]$} \\
\hline $\mathrm{C}_{10} \mathrm{H}_{10}$ & 1,2-dihydronaphthalene & & & & {$[447-53-0]$} \\
\hline & $(274-319)$ & $51.9 \pm 0.4$ & $(296)$ & GS & {$[99 / 21]$} \\
\hline & & $51.9 \pm 0.4$ & $(298)$ & & {$[99 / 21]$} \\
\hline $\mathrm{C}_{10} \mathrm{H}_{10}$ & 1,4-dihydronaphthalene & & & & [612-17-9] \\
\hline & $(300-333)$ & $53.2 \pm 0.4$ & $(296)$ & GS & [99/21] \\
\hline & & $54.2 \pm 0.4$ & $(298)$ & & {$[99 / 21]$} \\
\hline
\end{tabular}


TABLE 6. Enthalpies of vaporization of organic compounds, 1880-2002-Continued

\begin{tabular}{|c|c|c|c|c|c|}
\hline Molecular formula & $\begin{array}{c}\text { Compound } \\
\text { (Temperature range/K) }\end{array}$ & $\begin{array}{c}\Delta_{\mathrm{vap}} H_{m} \\
\left(\mathrm{~kJ} \mathrm{~mol}^{-1}\right)\end{array}$ & $\begin{array}{l}\text { Mean temperature } \\
\qquad\left(T_{m} / \mathrm{K}\right)\end{array}$ & Method & $\begin{array}{c}\text { CAS registry number } \\
\text { Reference }\end{array}$ \\
\hline \multirow[t]{2}{*}{$\mathrm{C}_{10} \mathrm{H}_{10}$} & \multirow{2}{*}{\multicolumn{4}{|c|}{ diisopropenyldiacetylene }} & {$[5187-81-5]$} \\
\hline & & & & & {$[77 / 25]$} \\
\hline \multirow[t]{2}{*}{$\mathrm{C}_{10} \mathrm{H}_{10} \mathrm{Cl}_{2} \mathrm{O}_{3}$} & \multicolumn{3}{|c|}{ (2,4-dichlorophenoxy)acetic acid, ethyl ester } & & {$[533-23-3]$} \\
\hline & $(444-573)$ & 72.6 & (459) & A & {$[87 / 5][99 / 16]$} \\
\hline \multirow{2}{*}{$\mathrm{C}_{10} \mathrm{H}_{10} \mathrm{~N}_{2}$} & \multicolumn{2}{|l|}{ 1-benzylpyrazole } & & & [10199-67-4] \\
\hline & & $73.8 \pm 2.0$ & $(298)$ & $\mathrm{C}$ & {$[99 / 5]$} \\
\hline \multirow[t]{7}{*}{$\mathrm{C}_{10} \mathrm{H}_{10} \mathrm{O}$} & \multicolumn{2}{|l|}{ 2-methyl-3-phenyl-2-propenal } & & & {$[101-39-3]$} \\
\hline & $(401-556)$ & $59.3 \pm 0.2$ & $(400)$ & EB & {$[02 / 16]$} \\
\hline & $(401-556)$ & $56.3 \pm 0.2$ & $(440)$ & $\mathrm{EB}$ & {$[02 / 16]$} \\
\hline & $(401-556)$ & $53.4 \pm 0.2$ & $(480)$ & $\mathrm{EB}$ & {$[02 / 16]$} \\
\hline & $(401-556)$ & $50.5 \pm 0.4$ & $(520)$ & EB & {$[02 / 16]$} \\
\hline & $(401-556)$ & $47.7 \pm 0.6$ & $(560)$ & $\mathrm{EB}$ & {$[02 / 16]$} \\
\hline & $(343-393)$ & 71.5 & $(358)$ & A & {$[87 / 5]$} \\
\hline \multirow[t]{2}{*}{$\mathrm{C}_{10} \mathrm{H}_{10} \mathrm{O}$} & \multicolumn{2}{|l|}{ 4-phenyl-3-buten-2-one } & & & {$[122-57-6]$} \\
\hline & $(354-534)$ & 58.5 & (369) & A & {$[87 / 5][47 / 5]$} \\
\hline \multirow[t]{3}{*}{$\mathrm{C}_{10} \mathrm{H}_{10} \mathrm{O}$} & \multicolumn{2}{|l|}{ 1-tetralone } & & & {$[529-34-0]$} \\
\hline & $(284-324)$ & $65.0 \pm 0.3$ & $(298)$ & GS & {$[98 / 4]$} \\
\hline & $(388-535)$ & 61.5 & $(403)$ & A & {$[87 / 5]$} \\
\hline \multirow{7}{*}{$\mathrm{C}_{10} \mathrm{H}_{10} \mathrm{O}_{2}$} & \multicolumn{2}{|l|}{ cinnamic acid, methyl ester } & & & [103-26-4] \\
\hline & $(409-557)$ & $59.9 \pm 0.2$ & $(420)$ & EB & {$[02 / 16]$} \\
\hline & $(409-557)$ & $56.9 \pm 0.2$ & $(460)$ & $\mathrm{EB}$ & {$[02 / 16]$} \\
\hline & $(409-557)$ & $53.8 \pm 0.3$ & $(500)$ & $\mathrm{EB}$ & {$[02 / 16]$} \\
\hline & $(409-557)$ & $50.5 \pm 0.5$ & $(540)$ & $\mathrm{EB}$ & {$[02 / 16]$} \\
\hline & $(288-333)$ & 62.4 & (303) & A & {$[87 / 5]$} \\
\hline & $(350-536)$ & 58.3 & $(365)$ & A & {$[87 / 5][47 / 5]$} \\
\hline $\mathrm{C}_{10} \mathrm{H}_{10} \mathrm{O}_{2}$ & 1,3-diacetylbenzene & & & & {$[6781-42-6]$} \\
\hline & $(323-418)$ & 43.2 & $(338)$ & A & {$[87 / 5]$} \\
\hline $\mathrm{C}_{10} \mathrm{H}_{10} \mathrm{O}_{2}$ & 1,4-diacetylbenzene & & & & [1009-61-6] \\
\hline & $(388-431)$ & 82.2 & (403) & A & {$[87 / 5]$} \\
\hline $\mathrm{C}_{10} \mathrm{H}_{10} \mathrm{O}_{2}$ & isosafrole & & & & {$[120-58-1]$} \\
\hline & $(393-531)$ & 59.4 & $(408)$ & A & {$[87 / 5]$} \\
\hline $\mathrm{C}_{10} \mathrm{H}_{10} \mathrm{O}_{2}$ & $\alpha$-methylcinnamic acid & & & & [1199-77-5] \\
\hline & $(398-561)$ & 78.5 & $(413)$ & A & {$[87 / 5][47 / 5]$} \\
\hline $\mathrm{C}_{10} \mathrm{H}_{10} \mathrm{O}_{2}$ & safrole & & & & {$[94-59-7]$} \\
\hline & $(336-506)$ & 54.6 & $(351)$ & $\mathrm{A}$ & {$[87 / 5][47 / 5]$} \\
\hline $\mathrm{C}_{10} \mathrm{H}_{10} \mathrm{O}_{2}$ & 4-carboxypentacyclo[4.3.0.0. & onane & & & \\
\hline & & 82.0 & & $\mathrm{C}$ & [84/12] \\
\hline $\mathrm{C}_{10} \mathrm{H}_{10} \mathrm{O}_{4}$ & 1,2-diacetoxybenzene & & & & {$[635-67-6]$} \\
\hline & $(371-551)$ & 62.9 & (386) & A & {$[87 / 5]$} \\
\hline $\mathrm{C}_{10} \mathrm{H}_{10} \mathrm{O}_{4}$ & dimethyl isophthalate & & & & [1459-93-4] \\
\hline & $(350-607)$ & $77.2 \pm 0.8$ & (298) & EB, IPM & {$[96 / 7]$} \\
\hline & $(393-550)$ & 60.5 & $(408)$ & A, GS & {$[87 / 5][63 / 13]$} \\
\hline $\mathrm{C}_{10} \mathrm{H}_{10} \mathrm{O}_{4}$ & dimethyl phthalate & & & & [131-11-3] \\
\hline & $(466-518)$ & 61.5 & $(481)$ & EB & {$[99 / 25]$} \\
\hline & & $69.4 \pm 0.1$ & (365) & $\mathrm{C}$ & {$[98 / 6]$} \\
\hline & & $72.5 \pm 0.6$ & (344) & $\mathrm{C}$ & {$[98 / 6]$} \\
\hline & & $74.5 \pm 0.3$ & (326) & $\mathrm{C}$ & {$[98 / 6]$} \\
\hline & $(304-371)$ & 78.7 & (319) & A & {$[87 / 5]$} \\
\hline & $(371-547)$ & 63.7 & (386) & $\mathrm{A}$ & {$[87 / 5]$} \\
\hline & $(377-440)$ & 68.6 & (409) & & [69/1] \\
\hline $\mathrm{C}_{10} \mathrm{H}_{10} \mathrm{O}_{4}$ & dimethyl terephthalate & & & & {$[120-61-6]$} \\
\hline & $(413-523)$ & 62.0 & $(428)$ & $\mathrm{A}$ & {$[87 / 5]$} \\
\hline $\mathrm{C}_{10} \mathrm{H}_{11} \mathrm{~N}$ & $\alpha, \alpha$-dimethylbenzylcyanide & & & & \\
\hline & $(284-323)$ & $60.3 \pm 0.6$ & $(303)$ & GS & {$[00 / 2]$} \\
\hline & $(284-323)$ & $60.6 \pm 0.6$ & $(298)$ & GS & {$[00 / 2]$} \\
\hline $\mathrm{C}_{10} \mathrm{H}_{11} \mathrm{~N}$ & $\alpha$-ethylbenzylcyanide & & & & \\
\hline & $(283-313)$ & $64.3 \pm 0.6$ & $(298)$ & GS & {$[00 / 2]$} \\
\hline $\mathrm{C}_{10} \mathrm{H}_{13} \mathrm{NO}_{2}$ & 2-nitro-1-tert-butylbenzene & & & & \\
\hline & $(278-323)$ & $64.8 \pm 0.6$ & $(301)$ & GS & {$[00 / 15]$} \\
\hline & & $65.0 \pm 0.6$ & $(298)$ & & {$[00 / 15]$} \\
\hline $\mathrm{C}_{10} \mathrm{H}_{12}$ & endo dicyclopentadiene & & & & {$[77-73-6]$} \\
\hline & $(350-446)$ & 43.6 & $(365)$ & A & {$[87 / 5]$} \\
\hline $\mathrm{C}_{10} \mathrm{H}_{12}$ & 2,4-dimethylstyrene & & & & {$[2234-20-0]$} \\
\hline & $(307-453)$ & 50.0 & $(322)$ & A & {$[87 / 5][47 / 5]$} \\
\hline
\end{tabular}


TABLE 6. Enthalpies of vaporization of organic compounds, 1880-2002-Continued

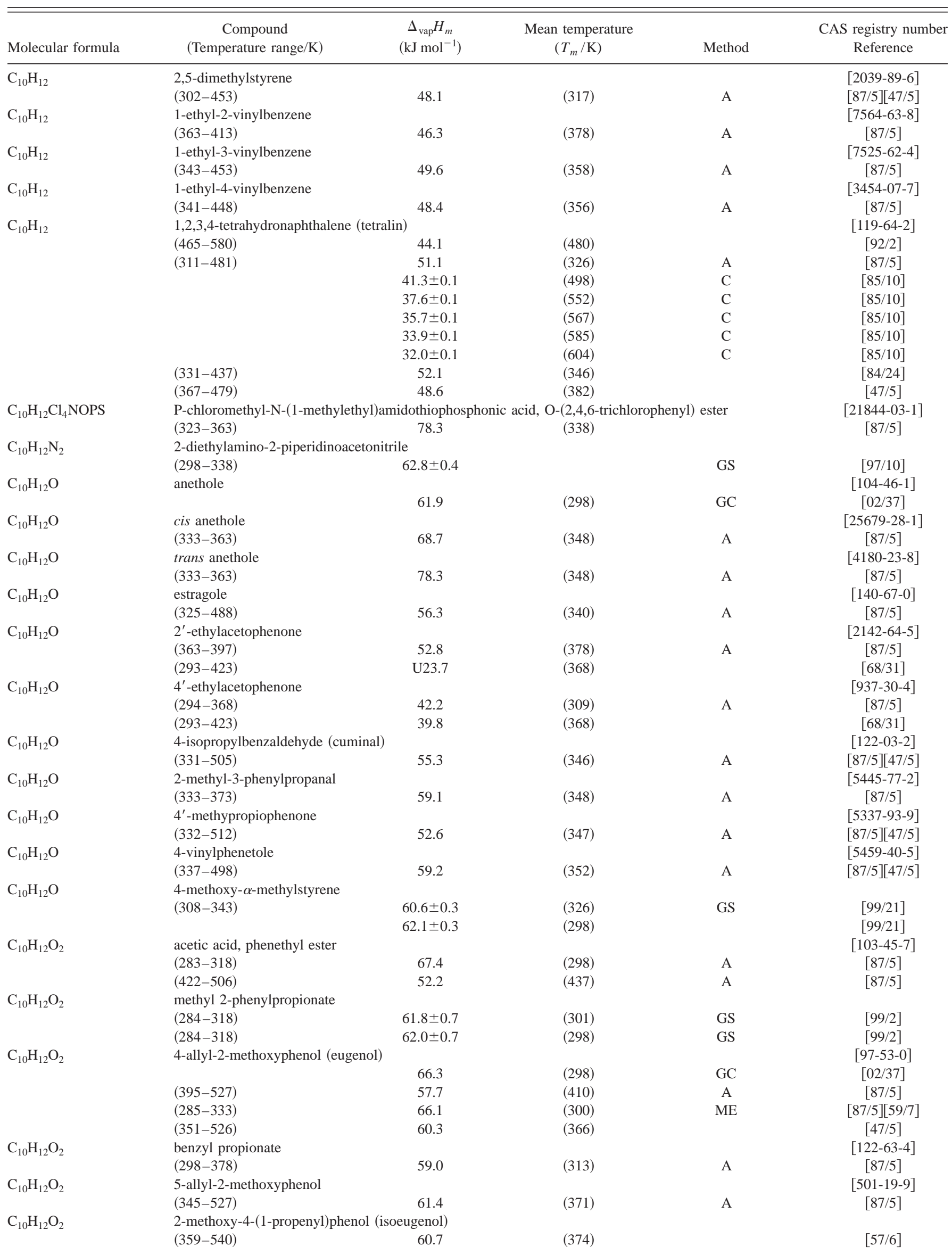


TABLE 6. Enthalpies of vaporization of organic compounds, 1880-2002-Continued

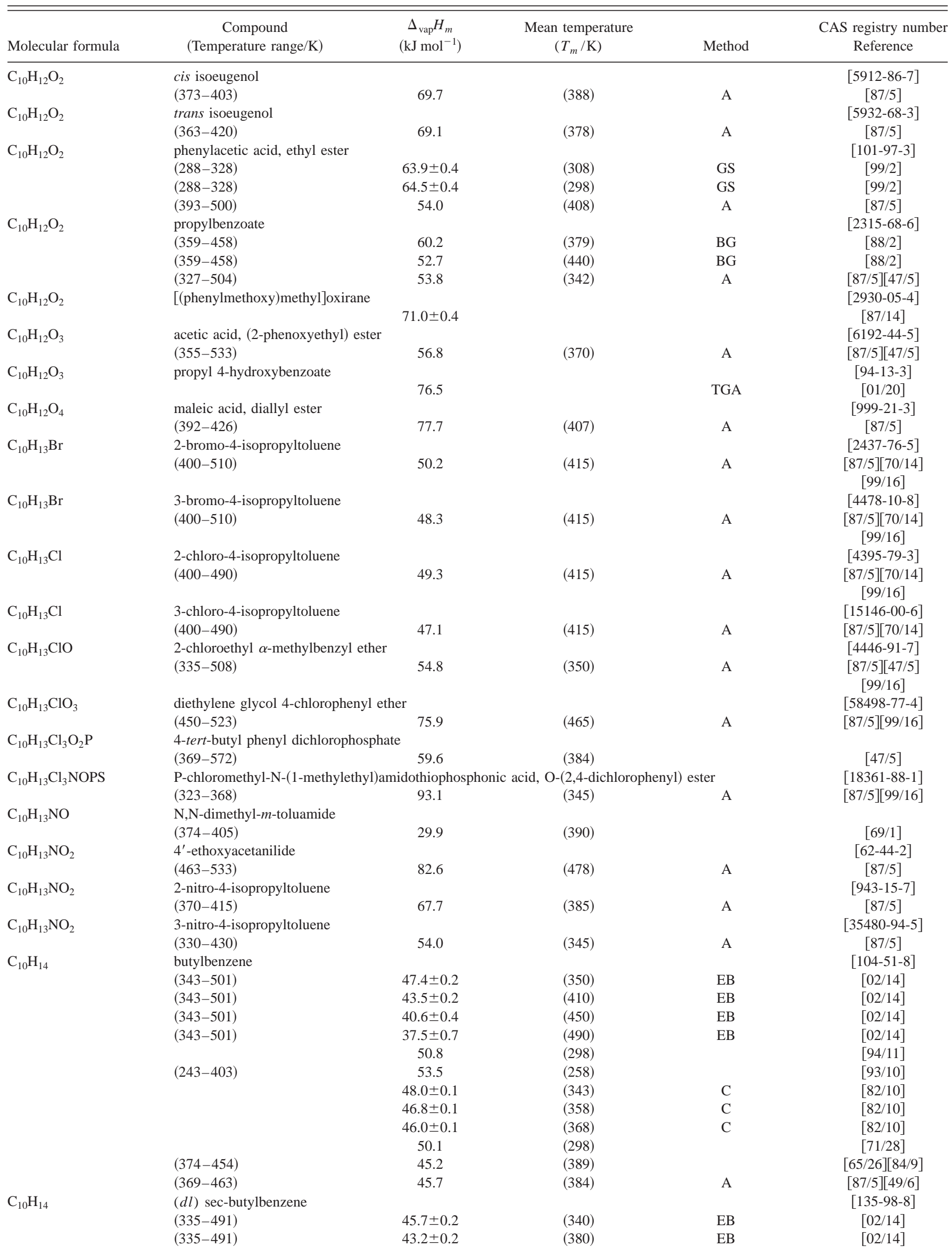


TABLE 6. Enthalpies of vaporization of organic compounds, 1880-2002-Continued

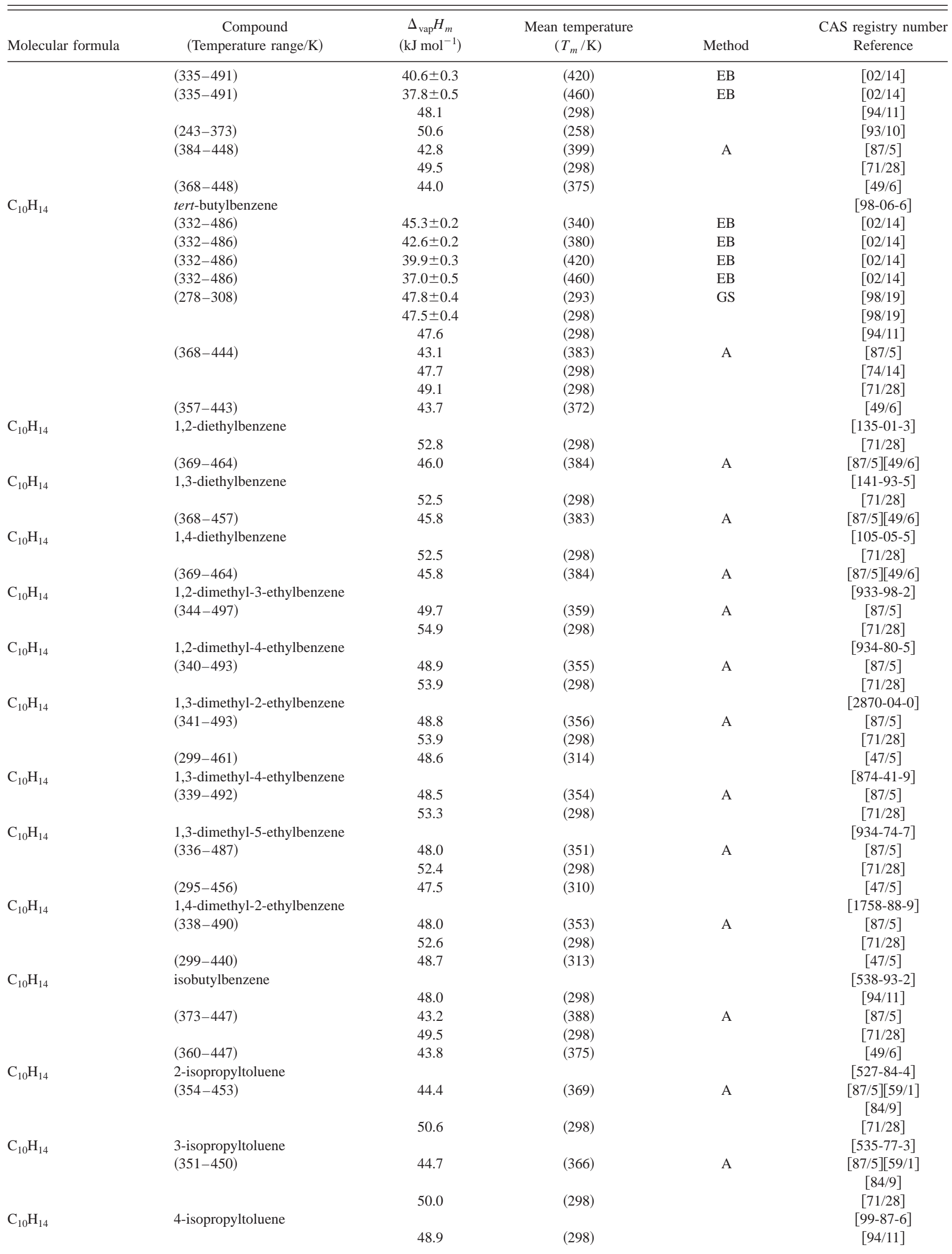


TABLE 6. Enthalpies of vaporization of organic compounds, 1880-2002-Continued

\begin{tabular}{|c|c|c|c|c|c|}
\hline Molecular formula & $\begin{array}{c}\text { Compound } \\
\text { (Temperature range/K) }\end{array}$ & $\begin{array}{c}\Delta_{\mathrm{vap}} H_{m} \\
\left(\mathrm{~kJ} \mathrm{~mol}^{-1}\right)\end{array}$ & $\begin{array}{l}\text { Mean temperature } \\
\qquad\left(T_{m} / \mathrm{K}\right)\end{array}$ & Method & $\begin{array}{c}\text { CAS registry number } \\
\text { Reference }\end{array}$ \\
\hline & $(380-452)$ & 44.0 & (395) & A & $\begin{array}{c}{[87 / 5][59 / 1]} \\
{[84 / 9]}\end{array}$ \\
\hline & & 50.3 & $(298)$ & & {$[71 / 28]$} \\
\hline \multirow[t]{3}{*}{$\mathrm{C}_{10} \mathrm{H}_{14}$} & 2-propyltoluene & & & & [1074-17-5] \\
\hline & $(337-488)$ & 48.0 & (352) & A & {$[87 / 5]$} \\
\hline & & 52.7 & $(298)$ & & {$[71 / 28]$} \\
\hline \multirow[t]{3}{*}{$\mathrm{C}_{10} \mathrm{H}_{14}$} & 3-propyltoluene & & & & [1074-43-7] \\
\hline & $(334-485)$ & 47.8 & (349) & $\mathrm{A}$ & {$[87 / 5]$} \\
\hline & & 52.1 & (298) & & {$[71 / 28]$} \\
\hline \multirow{3}{*}{$\mathrm{C}_{10} \mathrm{H}_{14}$} & 4-propyltoluene & & & & {$[1074-55-1]$} \\
\hline & $(335-487)$ & 47.6 & $(350)$ & A & {$[87 / 5]$} \\
\hline & & 51.9 & $(298)$ & & {$[71 / 28]$} \\
\hline \multirow[t]{6}{*}{$\mathrm{C}_{10} \mathrm{H}_{14}$} & 1,2,3,4-tetramethylbenzene & & & & {$[488-23-3]$} \\
\hline & & 54.0 & (298) & & [94/11] \\
\hline & & $52.6 \pm 0.2$ & (298) & $\mathrm{C}$ & {$[94 / 1]$} \\
\hline & $(352-509)$ & 50.7 & $(367)$ & A & {$[87 / 5]$} \\
\hline & & 57.2 & $(298)$ & & {$[71 / 28]$} \\
\hline & $(316-477)$ & 55.7 & $(331)$ & & {$[47 / 5]$} \\
\hline \multirow{6}{*}{$\mathrm{C}_{10} \mathrm{H}_{14}$} & $1,2,3,5$-tetramethylbenzene & & & & {$[527-53-7]$} \\
\hline & & 53.2 & (298) & & {$[94 / 11]$} \\
\hline & & $52.0 \pm 0.2$ & (298) & $\mathrm{C}$ & {$[94 / 1]$} \\
\hline & $(348-502)$ & 50.0 & $(363)$ & $\mathrm{A}$ & {$[87 / 5]$} \\
\hline & & 55.8 & (298) & & {$[71 / 28]$} \\
\hline & $(314-471)$ & 58.9 & $(329)$ & & {$[47 / 5]$} \\
\hline \multirow[t]{3}{*}{$\mathrm{C}_{10} \mathrm{H}_{14}$} & $1,2,4,5$-tetramethylbenzene & & & & {$[95-93-2]$} \\
\hline & $(363-381)$ & $47.7 \pm 0.3$ & $(375)$ & $\mathrm{DM}$ & {$[01 / 8]$} \\
\hline & $(353-500)$ & 49.4 & $(368)$ & A & {$[87 / 5]$} \\
\hline \multirow[t]{2}{*}{$\mathrm{C}_{10} \mathrm{H}_{14}$} & spirocyclopropane-1,6-tricy & $\left..0^{2,4}\right]$ octane & & & \\
\hline & & $47.8 \pm 0.1$ & $(298)$ & $\mathrm{C}$ & {$[98 / 17][96 / 18]$} \\
\hline \multirow{2}{*}{$\mathrm{C}_{10} \mathrm{H}_{14} \mathrm{NO}_{5} \mathrm{PS}$} & Parathion & & & & [58-38-2] \\
\hline & $(293-433)$ & 93.4 & (308) & A & {$[87 / 5][99 / 16]$} \\
\hline \multirow[t]{2}{*}{$\mathrm{C}_{10} \mathrm{H}_{14} \mathrm{NO}_{5} \mathrm{PS}$} & phosphorothioic acid, $\mathrm{O}, \mathrm{O}^{\prime}$ & S-(4-nitrophenyl) & & & [3270-86-8] \\
\hline & $(313-366)$ & 75.9 & (328) & A & {$[87 / 5][99 / 16]$} \\
\hline \multirow[t]{2}{*}{$\mathrm{C}_{10} \mathrm{H}_{14} \mathrm{NO}_{5} \mathrm{PS}$} & phosphorothioic acid, O,S-d & '-(4-nitrophenyl) & & & {$[597-88-6]$} \\
\hline & $(332-364)$ & 75.1 & $(347)$ & $\mathrm{A}$ & {$[87 / 5][99 / 16]$} \\
\hline \multirow[t]{2}{*}{$\mathrm{C}_{10} \mathrm{H}_{14} \mathrm{NO}_{6} \mathrm{P}$} & O,O-diethyl-O-(4-nitropheny & phate & & & [311-45-5] \\
\hline & $(273-422)$ & 87.9 & $(288)$ & A & {$[87 / 5]$} \\
\hline \multirow[t]{2}{*}{$\mathrm{C}_{10} \mathrm{H}_{14} \mathrm{~N}_{2}$} & $(d l)$ nicotine & & & & [22083-74-5] \\
\hline & $(406-520)$ & 53.3 & $(421)$ & A & {$[87 / 5]$} \\
\hline \multirow[t]{5}{*}{$\mathrm{C}_{10} \mathrm{H}_{14} \mathrm{O}$} & 2-butylphenol & & & & [3180-09-4] \\
\hline & $(403-533)$ & 55.1 & $(418)$ & $\mathrm{A}$ & {$[87 / 5][75 / 17]$} \\
\hline & $(382-520)$ & 52.9 & (398) & & {$[53 / 9]$} \\
\hline & $(382-520)$ & 51.0 & (423) & & {$[53 / 9]$} \\
\hline & $(382-520)$ & 47.0 & $(473)$ & & {$[53 / 9]$} \\
\hline \multirow[t]{2}{*}{$\mathrm{C}_{10} \mathrm{H}_{14} \mathrm{O}$} & 2-sec-butylphenol & & & & [89-72-5] \\
\hline & $(451-513)$ & 52.1 & $(466)$ & A, GS, EB & {$[87 / 5][64 / 14]$} \\
\hline $\mathrm{C}_{10} \mathrm{H}_{14} \mathrm{O}$ & 2-tert-butylphenol & & & & {$[88-18-6]$} \\
\hline & $(289-329)$ & $62.6 \pm 0.2$ & (309) & GS & [99/18] \\
\hline & & $63.2 \pm 0.2$ & (298) & & [99/18] \\
\hline & $(409-467)$ & 74.1 & $(424)$ & EB & {$[90 / 5]$} \\
\hline & $(409-465)$ & 52.9 & (424) & & {$[86 / 7]$} \\
\hline & $(353-498)$ & 54.9 & (368) & A & {$[87 / 5]$} \\
\hline & $(330-507)$ & 55.6 & $(348)$ & & [53/9] \\
\hline & $(330-507)$ & 53.9 & (373) & & [53/9] \\
\hline & $(330-507)$ & 51.0 & $(423)$ & & {$[53 / 9]$} \\
\hline & $(330-507)$ & 47.0 & (473) & & {$[53 / 9]$} \\
\hline $\mathrm{C}_{10} \mathrm{H}_{14} \mathrm{O}$ & 3-butylphenol & & & & [4074-43-5] \\
\hline & $(396-533)$ & 62.5 & $(411)$ & A & {$[87 / 5]$} \\
\hline & $(396-533)$ & 56.6 & (398) & & {$[53 / 9]$} \\
\hline & $(396-533)$ & 54.4 & (423) & & {$[53 / 9]$} \\
\hline & $(396-533)$ & 49.9 & (473) & & [53/9] \\
\hline $\mathrm{C}_{10} \mathrm{H}_{14} \mathrm{O}$ & 3-tert-butylphenol & & & & [585-34-2] \\
\hline & $(320-348)$ & $69.1 \pm 0.8$ & (334) & GS & {$[99 / 18]$} \\
\hline & & $71.3 \pm 0.8$ & (298) & & [99/18] \\
\hline
\end{tabular}


TABLE 6. Enthalpies of vaporization of organic compounds, 1880-2002-Continued

\begin{tabular}{|c|c|c|c|c|c|}
\hline Molecular formula & $\begin{array}{c}\text { Compound } \\
\text { (Temperature range/K) }\end{array}$ & $\begin{array}{c}\Delta_{\mathrm{vap}} H_{m} \\
\left(\mathrm{~kJ} \mathrm{~mol}^{-1}\right)\end{array}$ & $\begin{array}{c}\text { Mean temperature } \\
\left(T_{m} / \mathrm{K}\right)\end{array}$ & Method & $\begin{array}{c}\text { CAS registry number } \\
\text { Reference }\end{array}$ \\
\hline & $(391-524)$ & 62.4 & $(406)$ & $\mathrm{A}$ & {$[87 / 5]$} \\
\hline & $(391-524)$ & 56.6 & (398) & & {$[53 / 9]$} \\
\hline & $(391-524)$ & 54.4 & (423) & & {$[53 / 9]$} \\
\hline & $(391-524)$ & 49.9 & (473) & & [53/9] \\
\hline \multirow[t]{6}{*}{$\mathrm{C}_{10} \mathrm{H}_{14} \mathrm{O}$} & 4-butylphenol & & & & {$[1638-22-8]$} \\
\hline & $(395-653)$ & 61.7 & (410) & A & {$[87 / 5]$} \\
\hline & $(357-529)$ & 57.6 & (373) & & {$[53 / 9]$} \\
\hline & $(357-529)$ & 56.6 & (398) & & {$[53 / 9]$} \\
\hline & $(357-529)$ & 54.4 & (423) & & [53/9] \\
\hline & $(357-529)$ & 49.9 & (473) & & {$[53 / 9]$} \\
\hline \multirow{2}{*}{$\mathrm{C}_{10} \mathrm{H}_{14} \mathrm{O}$} & 4-sec-butylphenol & & & & {$[99-71-8]$} \\
\hline & $(344-516)$ & 59.0 & $(359)$ & $\mathrm{A}$ & {$[87 / 5][47 / 5]$} \\
\hline \multirow[t]{8}{*}{$\mathrm{C}_{10} \mathrm{H}_{14} \mathrm{O}$} & 4-tert-butylphenol & & & & {$[98-54-4]$} \\
\hline & & $67.9 \pm 1.0$ & (298) & $\mathrm{C}$ & {$[99 / 7]$} \\
\hline & $(471-525)$ & 54.3 & (486) & A, GS, EB & $\begin{array}{c}{[87 / 5][47 / 5]} \\
{[64 / 14]}\end{array}$ \\
\hline & $(346-523)$ & 59.6 & $(348)$ & & {$[53 / 9]$} \\
\hline & $(346-523)$ & 57.6 & (373) & & {$[53 / 9]$} \\
\hline & $(346-523)$ & 56.6 & (398) & & {$[53 / 9]$} \\
\hline & $(346-523)$ & 54.4 & (423) & & {$[53 / 9]$} \\
\hline & $(346-523)$ & 49.9 & $(473)$ & & {$[53 / 9]$} \\
\hline \multirow[t]{2}{*}{$\mathrm{C}_{10} \mathrm{H}_{14} \mathrm{O}$} & butyl phenyl ether & & & & [1126-79-0] \\
\hline & $(391-483)$ & 48.9 & $(406)$ & $\mathrm{A}$ & $\begin{array}{c}{[87 / 5][49 / 1]} \\
{[84 / 9]}\end{array}$ \\
\hline \multirow[t]{2}{*}{$\mathrm{C}_{10} \mathrm{H}_{14} \mathrm{O}$} & 3-isopropyl-2-methylphenol & & & & {$[4371-48-6]$} \\
\hline & $(365-516)$ & 60.2 & $(380)$ & EB & {$[69 / 23]$} \\
\hline \multirow[t]{2}{*}{$\mathrm{C}_{10} \mathrm{H}_{14} \mathrm{O}$} & 4-isopropyl-2-methylphenol & & & & {$[1740-97-2]$} \\
\hline & $(382-503)$ & 59.8 & $(397)$ & EB & {$[69 / 23]$} \\
\hline \multirow[t]{4}{*}{$\mathrm{C}_{10} \mathrm{H}_{14} \mathrm{O}$} & 5-isopropyl-2-methylphenol (carvacrol) & & & & {$[499-75-2]$} \\
\hline & & 68.2 & $(298)$ & GC & {$[02 / 37]$} \\
\hline & $(387-512)$ & 59.4 & (402) & $\mathrm{EB}$ & {$[69 / 23]$} \\
\hline & $(343-510)$ & 56.5 & (358) & $\mathrm{A}$ & {$[87 / 5][47 / 5]$} \\
\hline \multirow[t]{2}{*}{$\mathrm{C}_{10} \mathrm{H}_{14} \mathrm{O}$} & 6-isopropyl-2-methylphenol & & & & [3228-04-4] \\
\hline & $(371-499)$ & 54.5 & $(386)$ & EB & {$[69 / 23]$} \\
\hline \multirow[t]{2}{*}{$\mathrm{C}_{10} \mathrm{H}_{14} \mathrm{O}$} & 3,5-diethylphenol & & & & {$[1197-34-8]$} \\
\hline & $(387-521)$ & 54.3 & $(402)$ & A & {$[87 / 5][55 / 9]$} \\
\hline \multirow[t]{2}{*}{$\mathrm{C}_{10} \mathrm{H}_{14} \mathrm{O}$} & 4-isobutylphenol & & & & {$[4167-74-2]$} \\
\hline & $(345-510)$ & 58.1 & $(360)$ & A & {$[87 / 5][47 / 5]$} \\
\hline \multirow[t]{2}{*}{$\mathrm{C}_{10} \mathrm{H}_{14} \mathrm{O}$} & 2,3,5,6-tetramethylphenol & & & & {$[527-35-5]$} \\
\hline & $(381-522)$ & 51.2 & $(396)$ & A & {$[87 / 5][55 / 9]$} \\
\hline \multirow[t]{10}{*}{$\mathrm{C}_{10} \mathrm{H}_{14} \mathrm{O}$} & 2-isopropyl-5-methylphenol (thymol) & & & & {$[89-83-8]$} \\
\hline & & 68.7 & (298) & $\mathrm{GC}$ & {$[02 / 37]$} \\
\hline & $(393-433)$ & 70.5 & (298) & $\mathrm{CGC}$ & {$[95 / 21]$} \\
\hline & $(381-514)$ & 58.4 & (396) & A & {$[87 / 5]$} \\
\hline & $(339-514)$ & 63.2 & (373) & & {$[53 / 9]$} \\
\hline & $(339-514)$ & 58.4 & (398) & & {$[53 / 9]$} \\
\hline & $(339-514)$ & 55.2 & $(423)$ & & {$[53 / 9]$} \\
\hline & $(339-514)$ & 52.8 & $(448)$ & & {$[53 / 9]$} \\
\hline & $(339-514)$ & 51.5 & $(473)$ & & {$[53 / 9]$} \\
\hline & $(337-505)$ & 54.9 & $(352)$ & & {$[47 / 5]$} \\
\hline \multirow[t]{2}{*}{$\mathrm{C}_{10} \mathrm{H}_{14} \mathrm{O}$} & $(d l)$ carvone & & & & {$[22327-39-5]$} \\
\hline & $(330-501)$ & 55.0 & $(345)$ & A & {$[87 / 5][47 / 5]$} \\
\hline \multirow[t]{2}{*}{$\mathrm{C}_{10} \mathrm{H}_{14} \mathrm{O}$} & 4-ethylphenetole & & & & [1585-06-4] \\
\hline & $(321-481)$ & 54.3 & $(336)$ & A & {$[87 / 5][47 / 5]$} \\
\hline \multirow[t]{2}{*}{$\mathrm{C}_{10} \mathrm{H}_{14} \mathrm{O}$} & 2-(2-ethylphenyl)ethanol & & & & \\
\hline & $(420-653)$ & 59.5 & $(435)$ & $\mathrm{A}$ & {$[87 / 5]$} \\
\hline $\mathrm{C}_{10} \mathrm{H}_{14} \mathrm{O}$ & 2-(4-ethylphenyl)ethanol & & & & [22545-13-7] \\
\hline & $(420-653)$ & 59.1 & $(435)$ & A & {$[87 / 5]$} \\
\hline $\mathrm{C}_{10} \mathrm{H}_{14} \mathrm{O}$ & 4-isopropylbenzyl alcohol & & & & {$[536-60-7]$} \\
\hline & $(347-520)$ & 59.7 & $(362)$ & A & {$[87 / 5][47 / 5]$} \\
\hline $\mathrm{C}_{10} \mathrm{H}_{14} \mathrm{O}$ & 2-methyl-3-phenyl-1-propanol & & & & [7384-80-7] \\
\hline & $(343-393)$ & 71.9 & $(358)$ & A & {$[87 / 5]$} \\
\hline $\mathrm{C}_{10} \mathrm{H}_{14} \mathrm{O}$ & (1-ethoxyethyl)benzene & & & & \\
\hline & $(286-318)$ & $52.4 \pm 0.2$ & (302) & GS & {$[01 / 16]$} \\
\hline
\end{tabular}


TABLE 6. Enthalpies of vaporization of organic compounds, 1880-2002-Continued

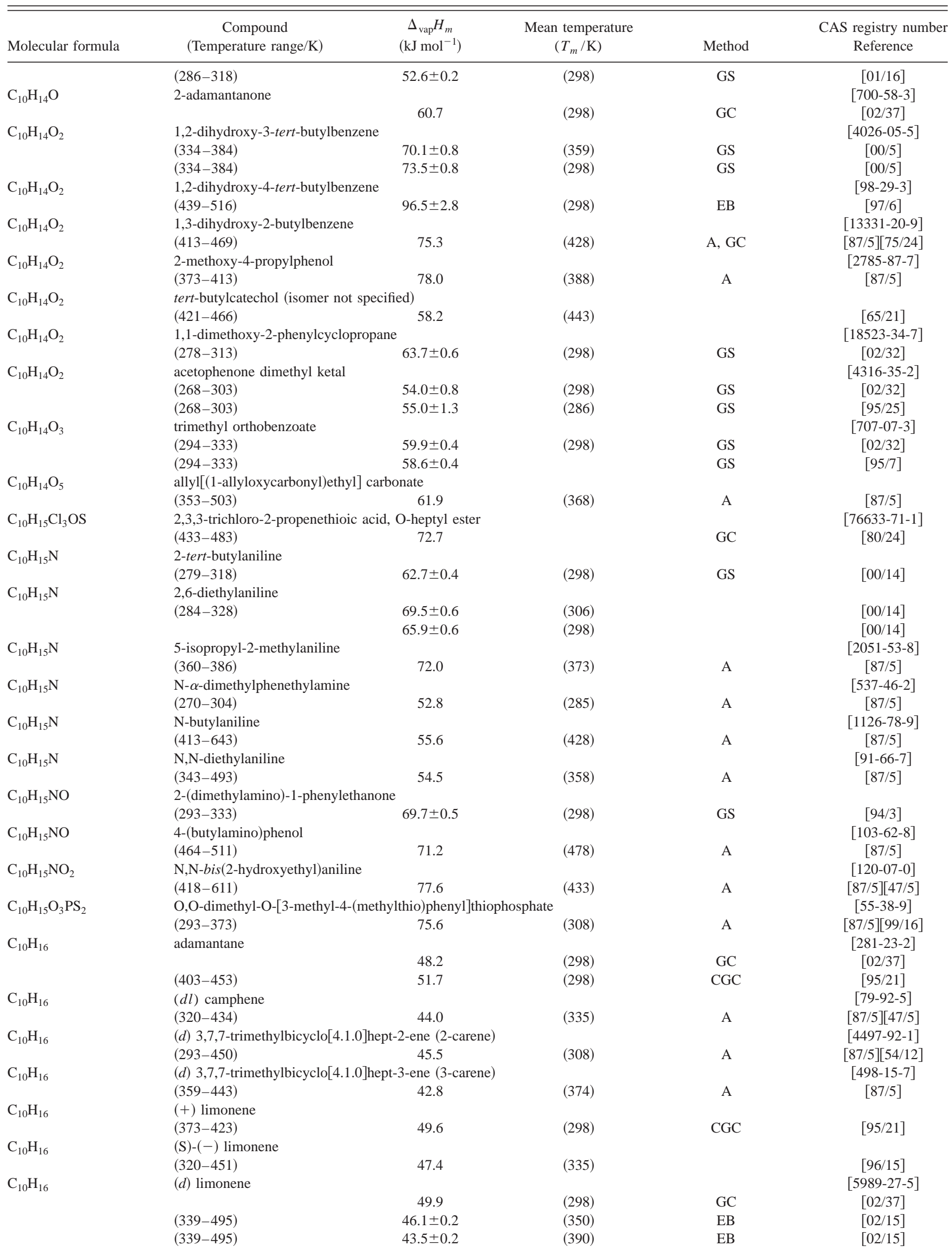


TABLE 6. Enthalpies of vaporization of organic compounds, 1880-2002-Continued

\begin{tabular}{|c|c|c|c|c|c|}
\hline Molecular formula & $\begin{array}{c}\text { Compound } \\
\text { (Temperature range/K) }\end{array}$ & $\begin{array}{c}\Delta_{\mathrm{vap}} H_{m} \\
\left(\mathrm{~kJ} \mathrm{~mol}^{-1}\right)\end{array}$ & $\begin{array}{l}\text { Mean temperature } \\
\qquad\left(T_{m} / \mathrm{K}\right)\end{array}$ & Method & $\begin{array}{c}\text { CAS registry number } \\
\text { Reference }\end{array}$ \\
\hline & $(339-495)$ & $40.9 \pm 0.3$ & $(430)$ & EB & {$[02 / 15]$} \\
\hline & $(339-495)$ & $37.9 \pm 0.6$ & $(470)$ & $\mathrm{EB}$ & {$[02 / 15]$} \\
\hline & $(250-434)$ & 49.2 & (300) & & {$[99 / 27]$} \\
\hline & & $48.9 \pm 0.1$ & (298) & $\mathrm{C}$ & {$[87 / 8]$} \\
\hline & $(287-448)$ & 44.5 & (302) & A & {$[87 / 5]$} \\
\hline & $(288-323)$ & 47.7 & (303) & A & {$[87 / 5]$} \\
\hline \multirow[t]{3}{*}{$\mathrm{C}_{10} \mathrm{H}_{16}$} & (l) limonene & & & & [5989-54-8] \\
\hline & & $49.0 \pm 0.1$ & (298) & $\mathrm{C}$ & {$[87 / 8]$} \\
\hline & $(303-363)$ & 45.5 & $(318)$ & A & {$[87 / 5][54 / 12]$} \\
\hline \multirow[t]{2}{*}{$\mathrm{C}_{10} \mathrm{H}_{16}$} & $(d l)$ limonene & & & & [138-86-3] \\
\hline & $(287-448)$ & 45.9 & (302) & A & {$[87 / 5]$} \\
\hline \multirow[t]{2}{*}{$\mathrm{C}_{10} \mathrm{H}_{16}$} & limonene & & & & \\
\hline & $(353-405)$ & 39.4 & $(379)$ & TGA & {$[02 / 40]$} \\
\hline \multirow[t]{2}{*}{$\mathrm{C}_{10} \mathrm{H}_{16}$} & $\beta$-myrcene & & & & \\
\hline & $(303-363)$ & 47.0 & $(318)$ & & [54/12] \\
\hline \multirow[t]{3}{*}{$\mathrm{C}_{10} \mathrm{H}_{16}$} & 7-methyl-3-methylene-1,6 & (myrcene) & & & {$[123-35-3]$} \\
\hline & & 50.6 & (298) & $\mathrm{GC}$ & {$[02 / 37]$} \\
\hline & $(287-445)$ & 45.7 & $(302)$ & A & {$[87 / 5][47 / 5]$} \\
\hline \multirow[t]{2}{*}{$\mathrm{C}_{10} \mathrm{H}_{16}$} & 5-isopropyl-2-methyl-1,3- & diene & & & [99-83-2] \\
\hline & $(293-448)$ & 47.7 & (308) & A & {$[87 / 5]$} \\
\hline \multirow[t]{2}{*}{$\mathrm{C}_{10} \mathrm{H}_{16}$} & 3-isopropyl-6-methylenec & & & & {$[555-10-2]$} \\
\hline & $(303-363)$ & 47.7 & (318) & $\mathrm{A}$ & {$[87 / 5][54 / 12]$} \\
\hline \multirow[t]{4}{*}{$\mathrm{C}_{10} \mathrm{H}_{16}$} & $\alpha$-pinene & & & & \\
\hline & $(320-429)$ & 42.5 & (335) & & {$[96 / 15]$} \\
\hline & $(365-430)$ & 40.2 & $(380)$ & & {$[93 / 4]$} \\
\hline & & $44.6 \pm 0.1$ & (298) & $\mathrm{C}$ & [87/19] \\
\hline \multirow[t]{3}{*}{$\mathrm{C}_{10} \mathrm{H}_{16}$} & (d) $\alpha$-pinene & & & & {$[80-56-8]$} \\
\hline & $(292-433)$ & 45.0 & $(307)$ & A & {$[87 / 5]$} \\
\hline & $(293-363)$ & 43.4 & (308) & & {$[54 / 12]$} \\
\hline \multirow[t]{4}{*}{$\mathrm{C}_{10} \mathrm{H}_{16}$} & $\beta$-pinene & & & & \\
\hline & $(290-439)$ & 46.0 & (305) & & {$[96 / 15]$} \\
\hline & $(364-439)$ & 41.6 & (379) & & {$[93 / 4]$} \\
\hline & & $45.8 \pm 0.1$ & (298) & $\mathrm{C}$ & [87/19] \\
\hline \multirow[t]{3}{*}{$\mathrm{C}_{10} \mathrm{H}_{16}$} & (l) $\beta$-pinene & & & & {$[127-91-3]$} \\
\hline & $(291-441)$ & 46.1 & $(306)$ & A & {$[87 / 5]$} \\
\hline & $(293-363)$ & 44.9 & $(308)$ & & {$[54 / 12]$} \\
\hline \multirow[t]{3}{*}{$\mathrm{C}_{10} \mathrm{H}_{16}$} & terpinolene & & & & [586-62-9] \\
\hline & $(313-363)$ & 50.8 & (328) & & {$[54 / 12]$} \\
\hline & $(305-458)$ & 50.5 & $(320)$ & $\mathrm{A}$ & {$[87 / 5][47 / 5]$} \\
\hline \multirow[t]{2}{*}{$\mathrm{C}_{10} \mathrm{H}_{16}$} & tetrahydrodicyclopentadie & & & & [6004-38-2] \\
\hline & $(358-465)$ & 43.5 & (373) & A & {$[87 / 5]$} \\
\hline \multirow{2}{*}{$\mathrm{C}_{10} \mathrm{H}_{16} \mathrm{ClO}_{6}$} & lactic acid, O-ethoxycarbo & chloroethoxy) & ter (solid) & & \\
\hline & $(406-523)$ & 83.8 & $(421)$ & A & {$[87 / 5]$} \\
\hline $\mathrm{C}_{10} \mathrm{H}_{16} \mathrm{Cl}_{3} \mathrm{NOS}$ & carbamothioic acid, bis(is & -(2,3,3-trichl & ester & & \\
\hline & $(293-318)$ & 84.3 & (305) & $\mathrm{A}$ & {$[87 / 5]$} \\
\hline $\mathrm{C}_{10} \mathrm{H}_{16} \mathrm{~N}_{2}$ & sebaconitrile & & & & [1871-96-1] \\
\hline & $(303-343)$ & 83.7 & $(318)$ & A & {$[87 / 5]$} \\
\hline $\mathrm{C}_{10} \mathrm{H}_{16} \mathrm{~N}_{2}$ & $\mathrm{~N}^{\prime}$-(2,4-dimethylphenyl)-I & rmamidine & & & \\
\hline & & 89.2 & $(303)$ & & [98/23] \\
\hline $\mathrm{C}_{10} \mathrm{H}_{16} \mathrm{O}$ & 3,7-dimethyl-6-octen-1-yn & ydrolinalool) & & & [29171-20-8] \\
\hline & $(406-471)$ & 52.1 & $(421)$ & EB & {$[01 / 13]$} \\
\hline & $(359-381)$ & Unreliable & & & [99/33] \\
\hline & $(369-445)$ & $50.4 \pm 0.1$ & (407) & & {$[88 / 4]$} \\
\hline $\mathrm{C}_{10} \mathrm{H}_{16} \mathrm{O}$ & camphor & & & & \\
\hline & $(343-383)$ & 54.4 & (298) & $\mathrm{CGC}$ & {$[95 / 21]$} \\
\hline & $(343-383)$ & 54.5 & (298) & CGC & {$[95 / 21]$} \\
\hline & $(343-383)$ & 55.2 & (298) & CGC & {$[95 / 21]$} \\
\hline $\mathrm{C}_{10} \mathrm{H}_{16} \mathrm{O}$ & $(+)$ camphor & & & & [464-49-3] \\
\hline & & 55.3 & $(298)$ & GC & {$[02 / 37]$} \\
\hline $\mathrm{C}_{10} \mathrm{H}_{16} \mathrm{O}$ & $\alpha$-pinene oxide & & & & [1686-14-2] \\
\hline & & 53.6 & $(298)$ & GC & {$[02 / 37]$} \\
\hline $\mathrm{C}_{10} \mathrm{H}_{16} \mathrm{O}$ & (d) 3-bornanone & & & & [13854-85-8] \\
\hline & $(452-488)$ & 44.6 & $(467)$ & A & {$[87 / 5]$} \\
\hline $\mathrm{C}_{10} \mathrm{H}_{16} \mathrm{O}$ & (d) 1,3,3-trimethylbicyclo & tan- 2 one (fen & & & [4695-62-9] \\
\hline
\end{tabular}


TABLE 6. Enthalpies of vaporization of organic compounds, 1880-2002-Continued

\begin{tabular}{|c|c|c|c|c|c|}
\hline Molecular formula & $\begin{array}{c}\text { Compound } \\
\text { (Temperature range/K) }\end{array}$ & $\begin{array}{c}\Delta_{\mathrm{vap}} H_{m} \\
\left(\mathrm{~kJ} \mathrm{~mol}^{-1}\right)\end{array}$ & $\begin{array}{l}\text { Mean temperature } \\
\qquad\left(T_{m} / \mathrm{K}\right)\end{array}$ & Method & $\begin{array}{c}\text { CAS registry number } \\
\text { Reference }\end{array}$ \\
\hline & $(365-384)$ & 47.0 & $(374)$ & & {$[02 / 13]$} \\
\hline & & $51.7 \pm 0.1$ & $(298)$ & $\mathrm{C}$ & {$[87 / 8]$} \\
\hline & & $51.4 \pm 0.1$ & (298) & $\mathrm{C}$ & {$[87 / 8]$} \\
\hline & & $51.1 \pm 0.1$ & $(298)$ & $\mathrm{C}$ & {$[85 / 2]$} \\
\hline & $(301-464)$ & 48.9 & $(316)$ & A & {$[87 / 5][47 / 5]$} \\
\hline \multirow[t]{4}{*}{$\mathrm{C}_{10} \mathrm{H}_{16} \mathrm{O}$} & (l) 1,3,3-trimethylbicyclo[ & n-2one (fenc & & & \\
\hline & & $51.1 \pm 0.1$ & $(298)$ & $\mathrm{C}$ & {$[87 / 8]$} \\
\hline & & $51.3 \pm 0.1$ & $(298)$ & $\mathrm{C}$ & {$[87 / 8]$} \\
\hline & & $51.4 \pm 0.1$ & $(298)$ & $\mathrm{C}$ & {$[85 / 2]$} \\
\hline \multirow[t]{3}{*}{$\mathrm{C}_{10} \mathrm{H}_{16} \mathrm{O}$} & pulegone & & & & {$[89-82-7]$} \\
\hline & & 62.0 & $(298)$ & GC & {$[02 / 37]$} \\
\hline & $(331-494)$ & U99.8 & $(346)$ & $\mathrm{A}$ & {$[87 / 5][47 / 5]$} \\
\hline \multirow[t]{2}{*}{$\mathrm{C}_{10} \mathrm{H}_{16} \mathrm{O}$} & (d) 1-isopropyl-4-methylb & 0]hexan-3-on & ne) & & {$[471-15-8]$} \\
\hline & $(311-474)$ & 51.8 & $(326)$ & $\mathrm{A}$ & {$[87 / 5][47 / 5]$} \\
\hline \multirow[t]{2}{*}{$\mathrm{C}_{10} \mathrm{H}_{16} \mathrm{O}$} & ( $d l$ ) 3-isopropyl-6-methyl & xene-1-one & & & {$[499-74-1]$} \\
\hline & $(364-507)$ & 56.9 & $(379)$ & A & {$[87 / 5]$} \\
\hline \multirow[t]{2}{*}{$\mathrm{C}_{10} \mathrm{H}_{16} \mathrm{O}$} & 5-isopropyl-2-methyl-2-cy & -one & & & {$[43205-82-9]$} \\
\hline & $(361-503)$ & 56.8 & $(376)$ & A & {$[87 / 5]$} \\
\hline \multirow[t]{2}{*}{$\mathrm{C}_{10} \mathrm{H}_{16} \mathrm{O}$} & 3,4-epoxycarane ( $\alpha$-isome & & & & \\
\hline & & 49.4 & & & {$[77 / 27]$} \\
\hline \multirow{2}{*}{$\mathrm{C}_{10} \mathrm{H}_{16} \mathrm{O}$} & 3,4-epoxycarane $(\beta$-isome & & & & \\
\hline & & 50.2 & & & {$[77 / 27]$} \\
\hline \multirow[t]{2}{*}{$\mathrm{C}_{10} \mathrm{H}_{16} \mathrm{O}$} & $(d l)$ dihydrocarvone & & & & [4584-09-2] \\
\hline & $(319-496)$ & 51.2 & $(334)$ & A & {$[87 / 5][47 / 5]$} \\
\hline \multirow[t]{3}{*}{$\mathrm{C}_{10} \mathrm{H}_{16} \mathrm{O}$} & geranial (citral) & & & & {$[141-27-5]$} \\
\hline & $(283-333)$ & 61.0 & $(298)$ & A & {$[87 / 5]$} \\
\hline & $(373-501)$ & 54.9 & $(388)$ & A & {$[87 / 5]$} \\
\hline \multirow[t]{2}{*}{$\mathrm{C}_{10} \mathrm{H}_{16} \mathrm{O}$} & 1-adamantanol & & & & {$[768-95-6]$} \\
\hline & & 60.8 & $(298)$ & GC & {$[02 / 37]$} \\
\hline \multirow[t]{2}{*}{$\mathrm{C}_{10} \mathrm{H}_{16} \mathrm{O}$} & trans octahydro-3a-methy & -2-one & & & {$[20379-99-1]$} \\
\hline & & $58.3 \pm 0.2$ & $(298)$ & $\mathrm{C}$ & {$[70 / 38]$} \\
\hline \multirow[t]{2}{*}{$\mathrm{C}_{10} \mathrm{H}_{16} \mathrm{O}_{2}$} & 3-acetyl-2,2-dimethylcycl & taldehyde (pi & hyde) & & \\
\hline & $(283-308)$ & $75.5 \pm 5.6$ & & ME & {$[97 / 24]$} \\
\hline \multirow[t]{2}{*}{$\mathrm{C}_{10} \mathrm{H}_{16} \mathrm{O}_{2}$} & 2,2-dimethyl-3-(2-oxoprop & opaneacetald & caronaldehyde) & & \\
\hline & $(283-308)$ & $77.4 \pm 6.9$ & & ME & {$[97 / 24]$} \\
\hline \multirow[t]{2}{*}{$\mathrm{C}_{10} \mathrm{H}_{16} \mathrm{O}_{2}$} & diosphenol & & & & {$[490-03-9]$} \\
\hline & $(339-505)$ & 56.2 & $(354)$ & A & {$[87 / 5][47 / 5]$} \\
\hline $\mathrm{C}_{10} \mathrm{H}_{16} \mathrm{O}_{2}$ & fencholic acid & & & & \\
\hline & $(374-537)$ & 77.5 & $(389)$ & & {$[47 / 5]$} \\
\hline $\mathrm{C}_{10} \mathrm{H}_{16} \mathrm{O}_{2}$ & (2,3,3-trimethyl-3-cyclope & Icetic acid & & & \\
\hline & $(370-529)$ & 71.3 & $(385)$ & A & {$[87 / 5][47 / 5]$} \\
\hline $\mathrm{C}_{10} \mathrm{H}_{16} \mathrm{O}_{4}$ & 1,1-cyclobutanedicarboxy & thyl ester & & & \\
\hline & $(288-318)$ & $65.8 \pm 0.4$ & & GS & {$[98 / 22]$} \\
\hline $\mathrm{C}_{10} \mathrm{H}_{16} \mathrm{O}_{6}$ & lactic acid, O-ethoxycarbc & arfuryl ester & & & \\
\hline & $(390-523)$ & 71.2 & $(405)$ & A & {$[87 / 5]$} \\
\hline $\mathrm{C}_{10} \mathrm{H}_{16} \mathrm{O}_{6}$ & tris-(carboethoxy)methane & & & & \\
\hline & $(297-338)$ & $79.1 \pm 0.7$ & $(298)$ & GS & {$[92 / 13]$} \\
\hline $\mathrm{C}_{10} \mathrm{H}_{16} \mathrm{~S}$ & $(1 \mathrm{R})(-)$-thiocamphor & & & & {$[53402-10-1]$} \\
\hline & & 55.5 & $(298)$ & $\mathrm{GC}$ & {$[02 / 37]$} \\
\hline $\mathrm{C}_{10} \mathrm{H}_{17} \mathrm{~N}$ & 1-cyclohexylimino-2-bute & & & & \\
\hline & & 58.3 & & & {$[93 / 20]$} \\
\hline $\mathrm{C}_{10} \mathrm{H}_{17} \mathrm{NOS}$ & carbamothioic acid, N,N-c & -(2-propynyl & solid) & & {$[59300-36-6]$} \\
\hline & $(298-313)$ & 92.4 & $(305)$ & A & {$[87 / 5]$} \\
\hline $\mathrm{C}_{10} \mathrm{H}_{17} \mathrm{NO}_{3}$ & 2-(2-cyanoethoxy)propion & tyl ester & & & \\
\hline & $(328-382)$ & 61.7 & $(343)$ & A & {$[87 / 5]$} \\
\hline $\mathrm{C}_{10} \mathrm{H}_{17} \mathrm{NO}_{5}$ & (l) N-acetylaspartic acid, & & & & {$[1069-39-2]$} \\
\hline & $(418-508)$ & 76.0 & $(433)$ & A & {$[87 / 5]$} \\
\hline $\mathrm{C}_{10} \mathrm{H}_{18}$ & spiro[4.5]decane & & & & {$[176-63-6]$} \\
\hline & & 54.8 & $(298)$ & $\mathrm{C}$ & {$[75 / 14]$} \\
\hline & $(348-389)$ & 44.0 & $(363)$ & & {$[65 / 11]$} \\
\hline $\mathrm{C}_{10} \mathrm{H}_{18}$ & cis bicyclo[5.3.0]decane & & & & {$[16189-46-1]$} \\
\hline & $(298-377)$ & 49.8 & $(313)$ & A & {$[87 / 5]$} \\
\hline & & $46.9 \pm 0.8$ & $(377)$ & & {$[70 / 30]$} \\
\hline & & $53.6 \pm 1.2$ & (298) & & {$[70 / 30]$} \\
\hline
\end{tabular}


TABLE 6. Enthalpies of vaporization of organic compounds, 1880-2002-Continued

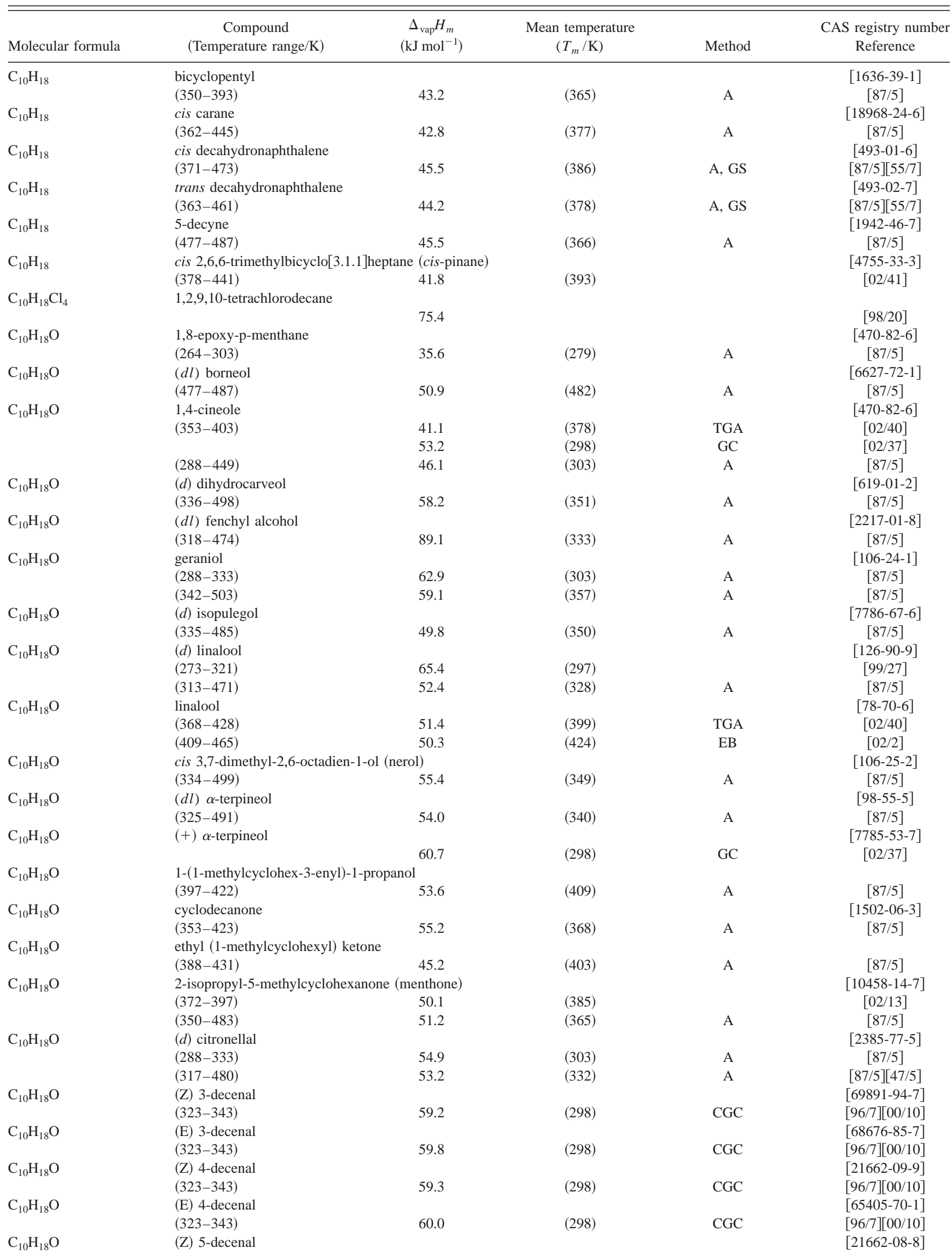


TABLE 6. Enthalpies of vaporization of organic compounds, 1880-2002-Continued

\begin{tabular}{|c|c|c|c|c|c|}
\hline Molecular formula & $\begin{array}{c}\text { Compound } \\
\text { (Temperature range/K) }\end{array}$ & $\begin{array}{c}\Delta_{\mathrm{vap}} H_{m} \\
\left(\mathrm{~kJ} \mathrm{~mol}^{-1}\right)\end{array}$ & $\begin{array}{l}\text { Mean temperature } \\
\qquad\left(T_{m} / \mathrm{K}\right)\end{array}$ & Method & $\begin{array}{c}\text { CAS registry number } \\
\text { Reference }\end{array}$ \\
\hline \multirow{3}{*}{$\mathrm{C}_{10} \mathrm{H}_{18} \mathrm{O}$} & $(323-343)$ & 58.5 & $(298)$ & CGC & {$[96 / 7][00 / 10]$} \\
\hline & (E) 5-decenal & & & & {$[21662-11-3]$} \\
\hline & $(323-343)$ & 59.2 & $(298)$ & CGC & {$[96 / 7][00 / 10]$} \\
\hline \multirow[t]{2}{*}{$\mathrm{C}_{10} \mathrm{H}_{18} \mathrm{O}$} & (Z) 6-decenal & & & & {$[147159-48-6]$} \\
\hline & $(323-343)$ & 59.3 & $(298)$ & CGC & {$[96 / 7][00 / 10]$} \\
\hline \multirow[t]{2}{*}{$\mathrm{C}_{10} \mathrm{H}_{18} \mathrm{O}$} & (E) 6-decenal & & & & {$[147159-48-6]$} \\
\hline & $(323-343)$ & 59.5 & $(298)$ & CGC & {$[96 / 7][00 / 10]$} \\
\hline \multirow[t]{2}{*}{$\mathrm{C}_{10} \mathrm{H}_{18} \mathrm{O}$} & (Z) 7-decenal & & & & [21661-97-2] \\
\hline & $(323-343)$ & 59.9 & $(298)$ & CGC & {$[96 / 7][00 / 10]$} \\
\hline \multirow[t]{2}{*}{$\mathrm{C}_{10} \mathrm{H}_{18} \mathrm{O}$} & (E) 7-decenal & & & & [21662-10-2] \\
\hline & $(323-343)$ & 59.8 & $(298)$ & CGC & {$[96 / 7][00 / 10]$} \\
\hline \multirow[t]{2}{*}{$\mathrm{C}_{10} \mathrm{H}_{18} \mathrm{O}$} & (Z) 8-decenal & & & & {$[174155-46-5]$} \\
\hline & $(323-343)$ & 60.5 & $(298)$ & CGC & {$[96 / 7][00 / 10]$} \\
\hline \multirow[t]{2}{*}{$\mathrm{C}_{10} \mathrm{H}_{18} \mathrm{O}$} & (E) 8-decenal & & & & {$[174155-47-6]$} \\
\hline & $(323-343)$ & 60.2 & (298) & CGC & {$[96 / 7][00 / 10]$} \\
\hline \multirow{2}{*}{$\mathrm{C}_{10} \mathrm{H}_{18} \mathrm{O}_{2}$} & 3,7-dimethyl-6-octenoic acid & & & & {$[502-47-6]$} \\
\hline & $(372-530)$ & 68.7 & $(387)$ & $\mathrm{A}$ & {$[87 / 5][47 / 5]$} \\
\hline \multirow[t]{2}{*}{$\mathrm{C}_{10} \mathrm{H}_{18} \mathrm{O}_{2}$} & 8,8-dimethyl-6,10-dioxaspiro[ & cane & & & \\
\hline & $(283-313)$ & $53.7 \pm 0.5$ & & GS & {$[98 / 21][02 / 32]$} \\
\hline \multirow[t]{2}{*}{$\mathrm{C}_{10} \mathrm{H}_{18} \mathrm{O}_{2}$} & 2,2,6-trimethyl-3,5-heptanedio & & & & {$[7333-23-5]$} \\
\hline & & 57.7 & $(298)$ & & {$[78 / 18]$} \\
\hline \multirow[t]{3}{*}{$\mathrm{C}_{10} \mathrm{H}_{18} \mathrm{O}_{2}$} & decanolactone & & & & [706-14-9] \\
\hline & $(365-387)$ & $57.7 \pm 0.8$ & $(376)$ & MM & {$[91 / 7]$} \\
\hline & $(365-387)$ & $63.0 \pm 1.5$ & $(298)$ & MM & {$[91 / 7]$} \\
\hline \multirow[t]{2}{*}{$\mathrm{C}_{10} \mathrm{H}_{18} \mathrm{O}_{2}$} & cyclohexyl butyrate & & & & [1551-44-6] \\
\hline & $(283-313)$ & $60.0 \pm 0.6$ & (298) & GS & {$[96 / 11]$} \\
\hline \multirow{2}{*}{$\mathrm{C}_{10} \mathrm{H}_{18} \mathrm{O}_{2}$} & heptyl acrylate & & & & [2499-58-3] \\
\hline & $(359-481)$ & 51.1 & $(374)$ & $\mathrm{A}$ & {$[87 / 5]$} \\
\hline \multirow[t]{2}{*}{$\mathrm{C}_{10} \mathrm{H}_{18} \mathrm{O}_{2}$} & hexyl methacrylate & & & & {$[142-09-6]$} \\
\hline & $(354-475)$ & 50.5 & (369) & A & {$[87 / 5]$} \\
\hline $\mathrm{C}_{10} \mathrm{H}_{18} \mathrm{O}_{2}$ & 1-methyl-3-isopropylcyclopen & arboxylic acid & & & {$[512-77-6]$} \\
\hline & $(374-538)$ & 91.6 & $(389)$ & $\mathrm{A}$ & {$[87 / 5]$} \\
\hline $\mathrm{C}_{10} \mathrm{H}_{18} \mathrm{O}_{3}$ & 3-hydroxy-2,3-dimethyl-4-hex & acid, ethyl este & & & \\
\hline & $(362-387)$ & 57.4 & $(374)$ & A & {$[87 / 5]$} \\
\hline $\mathrm{C}_{10} \mathrm{H}_{18} \mathrm{O}_{3}$ & isopentyl levulinate & & & & \\
\hline & $(403-521)$ & 59.4 & $(418)$ & A & {$[87 / 5]$} \\
\hline & & 56.3 & $(461)$ & & {$[31 / 1]$} \\
\hline $\mathrm{C}_{10} \mathrm{H}_{18} \mathrm{O}_{3}$ & 1-ethylpropyl levulinate & & & & \\
\hline & $(397-513)$ & 58.6 & $(412)$ & A & {$[87 / 5]$} \\
\hline $\mathrm{C}_{10} \mathrm{H}_{18} \mathrm{O}_{3}$ & 1-methylbutyl levulinate & & & & \\
\hline & $(397-513)$ & 57.2 & $(412)$ & A & {$[87 / 5]$} \\
\hline $\mathrm{C}_{10} \mathrm{H}_{18} \mathrm{O}_{3}$ & 2-methylbutyl levulinate & & & & \\
\hline & $(391-473)$ & 56.5 & $(406)$ & $\mathrm{A}$ & {$[87 / 5]$} \\
\hline $\mathrm{C}_{10} \mathrm{H}_{18} \mathrm{O}_{3}$ & pentyl levulinate & & & & [20279-49-6] \\
\hline & $(354-527)$ & 66.3 & (369) & A & {$[87 / 5][47 / 5]$} \\
\hline & & 56.2 & $(466)$ & & {$[31 / 1]$} \\
\hline $\mathrm{C}_{10} \mathrm{H}_{18} \mathrm{O}_{3}$ & trimethylacetic acid anhydride & & & & {$[1538-75-6]$} \\
\hline & $(355-513)$ & $50.7 \pm 0.2$ & $(360)$ & EB & {$[02 / 16]$} \\
\hline & $(355-513)$ & $47.4 \pm 0.2$ & $(400)$ & $\mathrm{EB}$ & {$[02 / 16]$} \\
\hline & $(355-513)$ & $44.0 \pm 0.4$ & $(440)$ & $\mathrm{EB}$ & {$[02 / 16]$} \\
\hline & $(355-513)$ & $40.3 \pm 0.7$ & $(480)$ & $\mathrm{EB}$ & {$[02 / 16]$} \\
\hline $\mathrm{C}_{10} \mathrm{H}_{18} \mathrm{O}_{4}$ & pentyl 2-acetoxypropionate & & & & \\
\hline & $(312-501)$ & 68.5 & $(327)$ & $\mathrm{A}$ & {$[87 / 5]$} \\
\hline $\mathrm{C}_{10} \mathrm{H}_{18} \mathrm{O}_{4}$ & diethyl adipate & & & & {$[141-28-6]$} \\
\hline & $(347-513)$ & 57.5 & $(362)$ & $\mathrm{A}$ & {$[87 / 5][47 / 5]$} \\
\hline $\mathrm{C}_{10} \mathrm{H}_{18} \mathrm{O}_{4}$ & diisobutyl oxalate & & & & [2050-61-5] \\
\hline & $(336-503)$ & 55.5 & $(351)$ & A & {$[87 / 5][47 / 5]$} \\
\hline $\mathrm{C}_{10} \mathrm{H}_{18} \mathrm{O}_{4}$ & dipropyl succinate & & & & {$[925-15-5]$} \\
\hline & $(350-524)$ & 59.4 & $(365)$ & A & {$[87 / 5][47 / 5]$} \\
\hline $\mathrm{C}_{10} \mathrm{H}_{18} \mathrm{O}_{4}$ & diethyl ethylmethylmalonate & & & & [2049-70-9] \\
\hline & $(317-481)$ & 53.2 & (332) & A & {$[87 / 5]$} \\
\hline $\mathrm{C}_{10} \mathrm{H}_{18} \mathrm{O}_{4}$ & sebacic acid & & & & [111-20-6] \\
\hline & $(456-625)$ & 85.9 & $(471)$ & & {$[47 / 5]$} \\
\hline $\mathrm{C}_{10} \mathrm{H}_{18} \mathrm{O}_{5}$ & ethyl[1-(butoxycarbonyl)ethyl & onate & & & \\
\hline
\end{tabular}


TABLE 6. Enthalpies of vaporization of organic compounds, 1880-2002-Continued

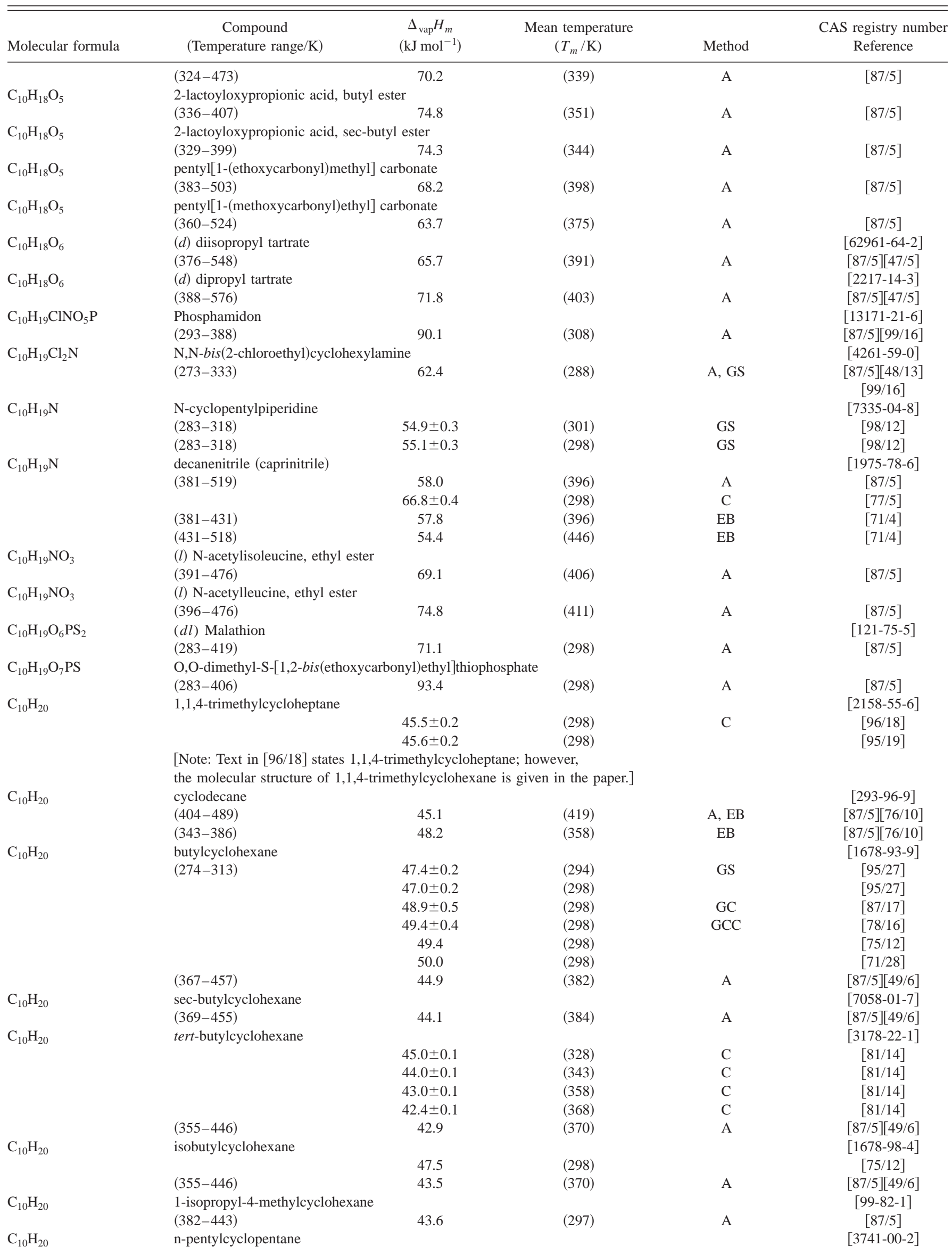


TABLE 6. Enthalpies of vaporization of organic compounds, 1880-2002-Continued

\begin{tabular}{|c|c|c|c|c|c|}
\hline Molecular formula & $\begin{array}{c}\text { Compound } \\
\text { (Temperature range/K) }\end{array}$ & $\begin{array}{c}\Delta_{\mathrm{vap}} H_{m} \\
\left(\mathrm{~kJ} \mathrm{~mol}^{-1}\right)\end{array}$ & $\begin{array}{l}\text { Mean temperature } \\
\qquad\left(T_{m} / \mathrm{K}\right)\end{array}$ & Method & $\begin{array}{c}\text { CAS registry number } \\
\text { Reference }\end{array}$ \\
\hline \multirow{6}{*}{$\mathrm{C}_{10} \mathrm{H}_{20}$} & & 51.0 & (298) & & [71/28] \\
\hline & 1-decene & & & & [872-05-9] \\
\hline & $(383-445)$ & 43.8 & (398) & $\mathrm{A}$ & {$[87 / 5]$} \\
\hline & & $50.4 \pm 0.2$ & (298) & $\mathrm{C}$ & {$[77 / 1]$} \\
\hline & & 50.5 & (298) & & {$[71 / 28]$} \\
\hline & $(360-445)$ & 45.1 & $(375)$ & & {$[50 / 6]$} \\
\hline \multirow[t]{2}{*}{$\mathrm{C}_{10} \mathrm{H}_{20}$} & cis 2-decene & & & & [20348-51-0] \\
\hline & $(401-447)$ & 43.6 & (416) & $\mathrm{A}$ & {$[87 / 5]$} \\
\hline \multirow[t]{2}{*}{$\mathrm{C}_{10} \mathrm{H}_{20}$} & trans 2-decene & & & & [20063-97-2] \\
\hline & $(401-447)$ & 43.7 & (416) & A & {$[87 / 5]$} \\
\hline \multirow[t]{2}{*}{$\mathrm{C}_{10} \mathrm{H}_{20}$} & cis 3-decene & & & & [19398-86-8] \\
\hline & $(398-444)$ & 43.1 & (413) & A & {$[87 / 5]$} \\
\hline \multirow[t]{2}{*}{$\mathrm{C}_{10} \mathrm{H}_{20}$} & trans 3-decene & & & & [19150-21-1] \\
\hline & $(398-445)$ & 43.4 & (413) & $\mathrm{A}$ & {$[87 / 5]$} \\
\hline \multirow[t]{2}{*}{$\mathrm{C}_{10} \mathrm{H}_{20}$} & cis 4-decene & & & & [19398-88-0] \\
\hline & $(397-444)$ & 43.0 & (412) & $\mathrm{A}$ & {$[87 / 5]$} \\
\hline \multirow[t]{2}{*}{$\mathrm{C}_{10} \mathrm{H}_{20}$} & trans 4-decene & & & & [19398-89-1] \\
\hline & $(398-444)$ & 43.2 & (413) & A & {$[87 / 5]$} \\
\hline \multirow[t]{2}{*}{$\mathrm{C}_{10} \mathrm{H}_{20}$} & cis 5-decene & & & & [7433-78-5] \\
\hline & $(397-443)$ & 42.9 & $(412)$ & A & {$[87 / 5]$} \\
\hline \multirow{2}{*}{$\mathrm{C}_{10} \mathrm{H}_{20}$} & trans 5-decene & & & & [7433-56-9] \\
\hline & $(398-444)$ & 42.3 & (413) & A & {$[87 / 5]$} \\
\hline \multirow[t]{2}{*}{$\mathrm{C}_{10} \mathrm{H}_{20}$} & 4-propyl-3-heptene & & & & [4485-13-6] \\
\hline & $(333-371)$ & 43.7 & $(348)$ & A, MG & {$[87 / 5][55 / 11]$} \\
\hline \multirow[t]{2}{*}{$\mathrm{C}_{10} \mathrm{H}_{20}$} & trans 2,2,4,4-tetramethyl-3-hexene & & & & \\
\hline & & $42.0 \pm 0.2$ & (298) & GCC & {$[79 / 17]$} \\
\hline \multirow[t]{2}{*}{$\mathrm{C}_{10} \mathrm{H}_{20} \mathrm{Br}_{2}$} & 1,1-dibromodecane & & & & [59104-80-2] \\
\hline & $(442-610)$ & 62.2 & $(457)$ & A, EST & $\begin{array}{c}{[87 / 5][56 / 16]} \\
{[70 / 14][99 / 16]}\end{array}$ \\
\hline \multirow{2}{*}{$\mathrm{C}_{10} \mathrm{H}_{20} \mathrm{Br}_{2}$} & 1,2-dibromodecane & & & & {$[28467-71-2]$} \\
\hline & $(368-524)$ & 67.0 & $(383)$ & A & $\begin{array}{c}{[87 / 5][47 / 5]} \\
{[70 / 14]}\end{array}$ \\
\hline \multirow[t]{2}{*}{$\mathrm{C}_{10} \mathrm{H}_{20} \mathrm{Cl}_{2}$} & 1,1-dichlorodecane & & & & {$[3162-62-7]$} \\
\hline & $(415-577)$ & 56.9 & $(430)$ & A, EST & $\begin{array}{c}{[87 / 5][56 / 16]} \\
{[70 / 14]}\end{array}$ \\
\hline \multirow[t]{4}{*}{$\mathrm{C}_{10} \mathrm{H}_{20} \mathrm{Cl}_{2}$} & 1,10-dichlorodecane & & & & [2162-98-3] \\
\hline & $(441-520)$ & 61.1 & $(456)$ & & {$[99 / 16]$} \\
\hline & & 67.3 & & & {$[98 / 20]$} \\
\hline & $(440-540)$ & 73.1 & $(298)$ & & {$[91 / 2]$} \\
\hline \multirow{2}{*}{$\mathrm{C}_{10} \mathrm{H}_{20} \mathrm{~F}_{2}$} & 1,1-difluorodecane & & & & [62127-43-9] \\
\hline & $(364-504)$ & 50.2 & $(379)$ & A, EST & $\begin{array}{c}{[87 / 5][56 / 16]} \\
{[70 / 14][99 / 16]}\end{array}$ \\
\hline $\mathrm{C}_{10} \mathrm{H}_{20} \mathrm{NO}_{2}$ & $\begin{array}{l}\text { ethyl 2-(N,N-diethylamino)butanoate } \\
(283-313)\end{array}$ & $57.3 \pm 0.2$ & (298) & GS & {$[96 / 20]$} \\
\hline $\mathrm{C}_{10} \mathrm{H}_{20} \mathrm{~N}_{2} \mathrm{O}_{2}$ & tetraethyloxamide & & & & [14288-05-2] \\
\hline & & 63 & $(464)$ & TGA, DSC & {$[02 / 36]$} \\
\hline $\mathrm{C}_{10} \mathrm{H}_{20} \mathrm{O}$ & bis(3-methyl-2-butenyl) ether & & & & \\
\hline & $(383-413)$ & 47.8 & (398) & & {$[89 / 8]$} \\
\hline $\mathrm{C}_{10} \mathrm{H}_{20} \mathrm{O}$ & (2-ethylhexyl) vinyl ether & & & & {$[103-44-6]$} \\
\hline & $(330-451)$ & 44.7 & $(345)$ & A & {$[87 / 5]$} \\
\hline $\mathrm{C}_{10} \mathrm{H}_{20} \mathrm{O}$ & 1-butylcyclohexanol & & & & [5445-30-7] \\
\hline & $(362-481)$ & 55.7 & $(377)$ & A & {$[87 / 5]$} \\
\hline $\mathrm{C}_{10} \mathrm{H}_{20} \mathrm{O}$ & 3,7-dimethyl-6-octene-1-ol & & & & [106-22-9] \\
\hline & $(293-333)$ & 72.6 & $(308)$ & A & {$[87 / 5]$} \\
\hline & $(373-500)$ & 65.9 & $(388)$ & A & {$[87 / 5]$} \\
\hline $\mathrm{C}_{10} \mathrm{H}_{20} \mathrm{O}$ & (l) menthol & & & & [2216-51-5] \\
\hline & $(372-488)$ & 59.1 & (387) & A & {$[87 / 5]$} \\
\hline & $(329-485)$ & 58.2 & (344) & & {$[47 / 5]$} \\
\hline $\mathrm{C}_{10} \mathrm{H}_{20} \mathrm{O}$ & 1-(1-methylcyclohexyl)-1-propanol & & & & \\
\hline & $(396-420)$ & 55.4 & (408) & A & {$[87 / 5]$} \\
\hline $\mathrm{C}_{10} \mathrm{H}_{20} \mathrm{O}$ & 2-(1-methylcyclohexyl)-2-propanol & & & & {$[27331-02-8]$} \\
\hline & $(393-418)$ & 53.0 & $(405)$ & A & {$[87 / 5]$} \\
\hline $\mathrm{C}_{10} \mathrm{H}_{20} \mathrm{O}$ & (Z) 3-decen-1-ol & & & & {$[10340-22-4]$} \\
\hline & $(323-363)$ & 78.7 & (298) & CGC & {$[00 / 10][94 / 13]$} \\
\hline
\end{tabular}


TABLE 6. Enthalpies of vaporization of organic compounds, 1880-2002-Continued

\begin{tabular}{|c|c|c|c|c|c|}
\hline Molecular formula & $\begin{array}{c}\text { Compound } \\
\text { (Temperature range/K) }\end{array}$ & $\begin{array}{c}\Delta_{\mathrm{vap}} H_{m} \\
\left(\mathrm{~kJ} \mathrm{~mol}^{-1}\right)\end{array}$ & $\begin{array}{l}\text { Mean temperature } \\
\qquad\left(T_{m} / \mathrm{K}\right)\end{array}$ & Method & $\begin{array}{c}\text { CAS registry number } \\
\text { Reference }\end{array}$ \\
\hline \multirow[t]{2}{*}{$\mathrm{C}_{10} \mathrm{H}_{20} \mathrm{O}$} & (E) 3-decen-1-ol & & & & [10339-60-3] \\
\hline & $(323-363)$ & 78.8 & $(298)$ & CGC & {$[00 / 10][94 / 13]$} \\
\hline \multirow[t]{2}{*}{$\mathrm{C}_{10} \mathrm{H}_{20} \mathrm{O}$} & (Z) 4-decen-1-ol & & & & {$[57074-37-0]$} \\
\hline & $(323-363)$ & 79.6 & $(298)$ & CGC & {$[00 / 10][94 / 13]$} \\
\hline \multirow[t]{2}{*}{$\mathrm{C}_{10} \mathrm{H}_{20} \mathrm{O}$} & (E) 4-decen-1-ol & & & & [10339-62-5] \\
\hline & $(323-363)$ & 80.3 & $(298)$ & CGC & {$[00 / 10][94 / 13]$} \\
\hline \multirow[t]{2}{*}{$\mathrm{C}_{10} \mathrm{H}_{20} \mathrm{O}$} & (Z) 5-decen-1-ol & & & & [51652-47-2] \\
\hline & $(323-363)$ & 80.3 & (298) & CGC & {$[00 / 10][94 / 13]$} \\
\hline \multirow{2}{*}{$\mathrm{C}_{10} \mathrm{H}_{20} \mathrm{O}$} & (E) 5-decen-1-ol & & & & {$[56578-18-8]$} \\
\hline & $(323-363)$ & 80.6 & $(298)$ & CGC & {$[00 / 10][94 / 13]$} \\
\hline \multirow[t]{2}{*}{$\mathrm{C}_{10} \mathrm{H}_{20} \mathrm{O}$} & (Z) 6-decen-1-ol & & & & {$[68760-59-8]$} \\
\hline & $(323-363)$ & 80.3 & $(298)$ & CGC & {$[00 / 10][94 / 13]$} \\
\hline \multirow[t]{2}{*}{$\mathrm{C}_{10} \mathrm{H}_{20} \mathrm{O}$} & (E) 6-decen-1-ol & & & & [38421-92-0] \\
\hline & $(323-363)$ & 80.6 & $(298)$ & CGC & {$[00 / 10][94 / 13]$} \\
\hline \multirow[t]{2}{*}{$\mathrm{C}_{10} \mathrm{H}_{20} \mathrm{O}$} & (Z) 7-decen-1-ol & & & & [16504-66-8] \\
\hline & $(323-363)$ & 80.8 & (298) & CGC & {$[00 / 10][94 / 13]$} \\
\hline \multirow[t]{2}{*}{$\mathrm{C}_{10} \mathrm{H}_{20} \mathrm{O}$} & (E) 7-decen-1-ol & & & & [52957-12-7] \\
\hline & $(323-363)$ & 81.1 & (298) & $\mathrm{CGC}$ & {$[00 / 10][94 / 13]$} \\
\hline \multirow[t]{2}{*}{$\mathrm{C}_{10} \mathrm{H}_{20} \mathrm{O}$} & (Z) 8-decen-1-ol & & & & [83799-67-1] \\
\hline & $(323-363)$ & 81.6 & $(298)$ & CGC & {$[00 / 10][94 / 13]$} \\
\hline \multirow{2}{*}{$\mathrm{C}_{10} \mathrm{H}_{20} \mathrm{O}$} & (E) 8-decen-1-ol & & & & [83799-68-2] \\
\hline & $(323-363)$ & 81.5 & $(298)$ & CGC & {$[00 / 10][94 / 13]$} \\
\hline \multirow[t]{5}{*}{$\mathrm{C}_{10} \mathrm{H}_{20} \mathrm{O}$} & 2-decanone & & & & [693-54-9] \\
\hline & $(317-484)$ & 51.7 & $(332)$ & A & {$[87 / 5][47 / 5]$} \\
\hline & $(357-560)$ & 55.1 & $(372)$ & & {$[87 / 5]$} \\
\hline & & $60.9 \pm 0.5$ & (298) & GCC & {$[79 / 7]$} \\
\hline & $(358-568)$ & 44.6 & $(487)$ & & {$[75 / 8]$} \\
\hline \multirow[t]{2}{*}{$\mathrm{C}_{10} \mathrm{H}_{20} \mathrm{O}$} & 2,2,5,5-tetramethyl-3-hexanone & & & & {$[868-91-7]$} \\
\hline & & $48.8 \pm 0.2$ & $(298)$ & $\mathrm{C}$ & {$[70 / 18]$} \\
\hline \multirow{6}{*}{$\mathrm{C}_{10} \mathrm{H}_{20} \mathrm{O}$} & decanal & & & & {$[112-31-2]$} \\
\hline & $(308-353)$ & 60.5 & $(298)$ & CGC & {$[96 / 7][00 / 10]$} \\
\hline & $(288-333)$ & 57.3 & $(303)$ & A & {$[87 / 5]$} \\
\hline & $(293-358)$ & 57.3 & $(308)$ & A & {$[87 / 5]$} \\
\hline & & $60.4 \pm 0.3$ & $(298)$ & & {$[81 / 18]$} \\
\hline & $(324-482)$ & 56.3 & $(339)$ & & {$[87 / 5][47 / 5]$} \\
\hline $\mathrm{C}_{10} \mathrm{H}_{20} \mathrm{O}_{2}$ & 2-heptyl-1,3-dioxolane & & & & {$[4359-57-3]$} \\
\hline & $(318-453)$ & 62.0 & $(333)$ & $\mathrm{A}$ & {$[87 / 5]$} \\
\hline $\mathrm{C}_{10} \mathrm{H}_{20} \mathrm{O}_{2}$ & 2-(1-ethylpentyl)-1,3-dioxolane & & & & {$[4359-47-1]$} \\
\hline & $(333-453)$ & 55.3 & $(348)$ & A & {$[87 / 5]$} \\
\hline $\mathrm{C}_{10} \mathrm{H}_{20} \mathrm{O}_{2}$ & 4-hexyl-1,3-dioxane & & & & [2244-85-1] \\
\hline & $(318-453)$ & 56.9 & $(333)$ & A & {$[87 / 5]$} \\
\hline $\mathrm{C}_{10} \mathrm{H}_{20} \mathrm{O}_{2}$ & 3-pentyl-4-hydroxytetrahydropyran & & & & [61827-60-9] \\
\hline & $(383-453)$ & 72.6 & $(398)$ & A & {$[87 / 5]$} \\
\hline $\mathrm{C}_{10} \mathrm{H}_{20} \mathrm{O}_{2}$ & 2-butoxy-3-hexanone & & & & \\
\hline & $(333-418)$ & 39.5 & $(348)$ & A & {$[87 / 5]$} \\
\hline $\mathrm{C}_{10} \mathrm{H}_{20} \mathrm{O}_{2}$ & hydroxycitronellal & & & & {$[107-75-5]$} \\
\hline & $(283-333)$ & 75.3 & $(298)$ & $\mathrm{A}, \mathrm{ME}$ & {$[87 / 5][55 / 8]$} \\
\hline $\mathrm{C}_{10} \mathrm{H}_{20} \mathrm{O}_{2}$ & octyl acetate & & & & {$[112-14-1]$} \\
\hline & & 61.7 & $(298)$ & GC & {$[97 / 22]$} \\
\hline & $(334-417)$ & 54.9 & (349) & A & {$[87 / 5]$} \\
\hline & $(345-472)$ & 47.8 & $(360)$ & A & {$[87 / 5]$} \\
\hline $\mathrm{C}_{10} \mathrm{H}_{20} \mathrm{O}_{2}$ & ethyl octanoate & & & & {$[106-32-1]$} \\
\hline & $(382-412)$ & $52.5 \pm 0.2$ & $(397)$ & EB & {$[91 / 7]$} \\
\hline & $(382-412)$ & $59.5 \pm 1.3$ & $(298)$ & $\mathrm{EB}$ & {$[91 / 7]$} \\
\hline & $(330-480)$ & 53.2 & $(345)$ & A & {$[87 / 5]$} \\
\hline $\mathrm{C}_{10} \mathrm{H}_{20} \mathrm{O}_{2}$ & 2-ethylhexylacetate & & & & [103-09-3] \\
\hline & $(333-472)$ & 50.1 & $(348)$ & $\mathrm{A}$ & {$[87 / 5]$} \\
\hline $\mathrm{C}_{10} \mathrm{H}_{20} \mathrm{O}_{2}$ & isopentyl isovalerate & & & & [659-70-1] \\
\hline & $(341-479)$ & 46.4 & $(356)$ & $\mathrm{A}$ & {$[87 / 5]$} \\
\hline & $(300-467)$ & 47.2 & $(315)$ & & {$[47 / 5]$} \\
\hline $\mathrm{C}_{10} \mathrm{H}_{20} \mathrm{O}_{2}$ & neopentyl pivalate & & & & [5340-26-1] \\
\hline & $(280-310)$ & $49.1 \pm 0.5$ & $(295)$ & GS & [99/4] \\
\hline & $(280-310)$ & $48.9 \pm 0.5$ & (298) & GS & [99/4] \\
\hline $\mathrm{C}_{10} \mathrm{H}_{20} \mathrm{O}_{2}$ & methyl nonanoate & & & & [1731-84-6] \\
\hline
\end{tabular}


TABLE 6. Enthalpies of vaporization of organic compounds, 1880-2002-Continued

\begin{tabular}{|c|c|c|c|c|c|}
\hline Molecular formula & $\begin{array}{c}\text { Compound } \\
\text { (Temperature range/K) }\end{array}$ & $\begin{array}{c}\Delta_{\mathrm{vap}} H_{m} \\
\left(\mathrm{~kJ} \mathrm{~mol}^{-1}\right)\end{array}$ & $\begin{array}{l}\text { Mean temperature } \\
\qquad\left(T_{m} / \mathrm{K}\right)\end{array}$ & Method & $\begin{array}{c}\text { CAS registry number } \\
\text { Reference }\end{array}$ \\
\hline \multirow{11}{*}{$\mathrm{C}_{10} \mathrm{H}_{20} \mathrm{O}_{2}$} & & 57.4 & $(350)$ & & {$[02 / 27]$} \\
\hline & & $56.7 \pm 0.3$ & $(364)$ & & {$[02 / 27]$} \\
\hline & & $61.6 \pm 0.4$ & $(298)$ & & {$[02 / 27]$} \\
\hline & & $57.7 \pm 0.7$ & $(298)$ & GC & {$[87 / 17]$} \\
\hline & & $62.0 \pm 0.5$ & $(298)$ & GCC & {$[80 / 5]$} \\
\hline & & $62.0 \pm 0.4$ & $(298)$ & $\mathrm{C}$ & {$[77 / 1]$} \\
\hline & $(364-439)$ & 55.6 & $(379)$ & A, EST & {$[87 / 5][63 / 16]$} \\
\hline & decanoic acid (capric acid) & & & & {$[334-48-5]$} \\
\hline & $(398-543)$ & 76.4 & $(413)$ & $\mathrm{A}$ & {$[87 / 5]$} \\
\hline & $(305-323)$ & 88.6 & $(314)$ & ME, TE & {$[82 / 4]$} \\
\hline & & 71.4 & $(418)$ & I & {$[43 / 7]$} \\
\hline \multirow{2}{*}{$\mathrm{C}_{10} \mathrm{H}_{20} \mathrm{O}_{3}$} & propyl 3-butoxypropionate & & & & {$[14144-47-9]$} \\
\hline & $(373-473)$ & 44.2 & $(388)$ & A & {$[87 / 5]$} \\
\hline \multirow[t]{2}{*}{$\mathrm{C}_{10} \mathrm{H}_{20} \mathrm{O}_{3}$} & pentyl 3-ethoxypropionate & & & & [14144-36-6] \\
\hline & $(374-498)$ & 54.1 & $(389)$ & A & {$[87 / 5]$} \\
\hline \multirow[t]{2}{*}{$\mathrm{C}_{10} \mathrm{H}_{20} \mathrm{O}_{3}$} & methyl 3-hexyloxypropionate & & & & {$[7419-97-8]$} \\
\hline & $(373-473)$ & 55.1 & $(388)$ & A & {$[87 / 5]$} \\
\hline \multirow[t]{2}{*}{$\mathrm{C}_{10} \mathrm{H}_{20} \mathrm{O}_{4}$} & diethylene glycol monobutyl & cetate & & & {$[124-17-4]$} \\
\hline & $(393-520)$ & 57.7 & $(408)$ & A & {$[87 / 5]$} \\
\hline \multirow{3}{*}{$\mathrm{C}_{10} \mathrm{H}_{20} \mathrm{O}_{5}$} & $1,4,7,10,13$-pentaoxacyclopen & e $(15$-crown-5) & & & {$[33100-27-5]$} \\
\hline & & $75.7 \pm 1.7$ & $(298)$ & CGC & {$[00 / 9]$} \\
\hline & & $79.6 \pm 0.3$ & $(298)$ & $\mathrm{C}$ & {$[82 / 9]$} \\
\hline \multirow[t]{3}{*}{$\mathrm{C}_{10} \mathrm{H}_{21} \mathrm{Br}$} & 1-bromodecane & & & & {$[112-29-8]$} \\
\hline & $(391-545)$ & 56.1 & $(406)$ & & {$[99 / 16]$} \\
\hline & $(383-570)$ & 56.6 & $(398)$ & $\mathrm{A}, \mathrm{EST}$ & {$[87 / 5][61 / 13]$} \\
\hline \multirow[t]{4}{*}{$\mathrm{C}_{10} \mathrm{H}_{21} \mathrm{Cl}$} & 1-chlorodecane & & & & {$[1002-69-3]$} \\
\hline & & $64.0 \pm 0.2$ & $(298)$ & GS & {$[01 / 1]$} \\
\hline & $(379-530)$ & 54.4 & $(394)$ & & [99/16] \\
\hline & $(359-499)$ & 56.2 & $(374)$ & A, DTA & {$[87 / 5][69 / 5]$} \\
\hline \multirow[t]{2}{*}{$\mathrm{C}_{10} \mathrm{H}_{21} \mathrm{~F}$} & 1-fluorodecane & & & & {$[334-56-5]$} \\
\hline & $(342-503)$ & 50.4 & $(357)$ & A & {$[87 / 5][61 / 13]$} \\
\hline \multirow[t]{3}{*}{$\mathrm{C}_{10} \mathrm{H}_{21} \mathrm{I}$} & 1-iododecane & & & & {$[2050-77-3]$} \\
\hline & $(407-571)$ & 57.4 & $(422)$ & & [99/16] \\
\hline & $(397-598)$ & 58.1 & $(412)$ & $\mathrm{A}, \mathrm{EST}$ & {$[87 / 5][61 / 13]$} \\
\hline \multirow[t]{2}{*}{$\mathrm{C}_{10} \mathrm{H}_{21} \mathrm{~N}$} & $\mathrm{~N}, \alpha$-dimethylcyclohexanethyl & & & & [101-40-6] \\
\hline & $(270-300)$ & 50.2 & $(285)$ & A & {$[87 / 5]$} \\
\hline \multirow[t]{2}{*}{$\mathrm{C}_{10} \mathrm{H}_{21} \mathrm{NO}$} & N,N-diethylhexanamide & & & & {$[6282-97-9]$} \\
\hline & $(373-443)$ & 47.7 & $(388)$ & A & {$[87 / 5]$} \\
\hline \multirow[t]{24}{*}{$\mathrm{C}_{10} \mathrm{H}_{22}$} & decane & & & & {$[124-18-5]$} \\
\hline & $(337-376)$ & 46.6 & $(352)$ & & {$[02 / 13]$} \\
\hline & & $51.1 \pm 3.9$ & $(298)$ & CGC & {$[00 / 9]$} \\
\hline & & 51.5 & (299) & $\mathrm{C}$ & {$[96 / 22]$} \\
\hline & & 50.5 & $(314)$ & $\mathrm{C}$ & {$[96 / 22]$} \\
\hline & & 50.1 & (324) & $\mathrm{C}$ & {$[96 / 22]$} \\
\hline & & 49.2 & $(334)$ & $\mathrm{C}$ & {$[96 / 22]$} \\
\hline & $(403-453)$ & 50.9 & (298) & CGC & {$[95 / 21]$} \\
\hline & $(423-473)$ & 51.5 & (298) & CGC & {$[95 / 21]$} \\
\hline & & 51.4 & (298) & & {$[94 / 12]$} \\
\hline & $(409-584)$ & 42.5 & $(424)$ & & [92/2] \\
\hline & $(268-490)$ & 48.1 & $(340)$ & $\mathrm{EB}, \mathrm{IPM}$ & {$[89 / 1]$} \\
\hline & $(268-490)$ & 51.4 & (298) & $\mathrm{EB}, \mathrm{IPM}$ & {$[89 / 1]$} \\
\hline & $(252-383)$ & 53.8 & $(267)$ & A & {$[87 / 5]$} \\
\hline & $(447-526)$ & 41.7 & $(462)$ & A & {$[87 / 5]$} \\
\hline & $(524-617)$ & 38.6 & $(539)$ & A & {$[87 / 5]$} \\
\hline & $(298-347)$ & 50.3 & (313) & GS & {$[86 / 6]$} \\
\hline & $(308-351)$ & $49.8 \pm 1.7$ & & & [84/12] \\
\hline & & $51.4 \pm 0.1$ & $(298)$ & $\mathrm{C}$ & {$[82 / 18]$} \\
\hline & $(243-310)$ & 55.9 & $(258)$ & & {$[73 / 11]$} \\
\hline & & 51.4 & $(298)$ & & [71/28] \\
\hline & $(373-443)$ & 45.3 & $(388)$ & & {$[87 / 5][70 / 6]$} \\
\hline & & 51.4 & $(298)$ & $\mathrm{C}$ & {$[47 / 7]$} \\
\hline & $(368-440)$ & 45.5 & $(383)$ & $\mathrm{MM}$ & {$[45 / 2]$} \\
\hline \multirow[t]{2}{*}{$\mathrm{C}_{10} \mathrm{H}_{22}$} & 2-methylnonane & & & & [871-83-0] \\
\hline & $(324-441)$ & $46.4 \pm 0.2$ & (339) & A & [87/5] \\
\hline
\end{tabular}


TABLE 6. Enthalpies of vaporization of organic compounds, 1880-2002-Continued

\begin{tabular}{|c|c|c|c|c|c|}
\hline Molecular formula & $\begin{array}{c}\text { Compound } \\
\text { (Temperature range/K) }\end{array}$ & $\begin{array}{c}\Delta_{\mathrm{vap}} H_{m} \\
\left(\mathrm{~kJ} \mathrm{~mol}^{-1}\right)\end{array}$ & $\begin{array}{l}\text { Mean temperature } \\
\qquad\left(T_{m} / \mathrm{K}\right)\end{array}$ & Method & $\begin{array}{c}\text { CAS registry number } \\
\text { Reference }\end{array}$ \\
\hline & & $47.3 \pm 0.2$ & $(328)$ & $\mathrm{C}$ & [84/8] \\
\hline & & $46.2 \pm 0.2$ & (343) & $\mathrm{C}$ & {$[84 / 8]$} \\
\hline & & $45.0 \pm 0.2$ & $(358)$ & $\mathrm{C}$ & {$[84 / 8]$} \\
\hline & & 51.0 & $(298)$ & & {$[71 / 28]$} \\
\hline \multirow[t]{5}{*}{$\mathrm{C}_{10} \mathrm{H}_{22}$} & 3-methylnonane & & & & [5911-04-6] \\
\hline & & $47.3 \pm 0.2$ & $(328)$ & $\mathrm{C}$ & {$[84 / 8]$} \\
\hline & & $46.2 \pm 0.2$ & $(343)$ & $\mathrm{C}$ & {$[84 / 8]$} \\
\hline & & $45.1 \pm 0.2$ & $(358)$ & $\mathrm{C}$ & {$[84 / 8]$} \\
\hline & & 50.2 & $(298)$ & & {$[71 / 28]$} \\
\hline \multirow[t]{2}{*}{$\mathrm{C}_{10} \mathrm{H}_{22}$} & 4-methylnonane & & & & [17301-94-9] \\
\hline & & 49.5 & $(298)$ & & {$[61 / 30]$} \\
\hline \multirow[t]{5}{*}{$\mathrm{C}_{10} \mathrm{H}_{22}$} & 5-methylnonane & & & & [15869-85-9] \\
\hline & & $47.0 \pm 0.2$ & $(328)$ & $\mathrm{C}$ & {$[84 / 8]$} \\
\hline & & $45.9 \pm 0.2$ & $(343)$ & $\mathrm{C}$ & {$[84 / 8]$} \\
\hline & & $44.6 \pm 0.2$ & $(358)$ & $\mathrm{C}$ & {$[84 / 8]$} \\
\hline & & 49.8 & $(298)$ & & {$[71 / 28]$} \\
\hline \multirow[t]{2}{*}{$\mathrm{C}_{10} \mathrm{H}_{22}$} & 3-ethyloctane & & & & [5881-17-4] \\
\hline & & 49.0 & $(298)$ & & [71/28] \\
\hline \multirow[t]{2}{*}{$\mathrm{C}_{10} \mathrm{H}_{22}$} & 4-ethyloctane & & & & [15869-86-0] \\
\hline & & 48.1 & $(298)$ & & [71/28] \\
\hline \multirow[t]{3}{*}{$\mathrm{C}_{10} \mathrm{H}_{22}$} & 4-propylheptane & & & & [3178-29-8] \\
\hline & & 48.5 & $(298)$ & & {$[71 / 28]$} \\
\hline & $(331-430)$ & 44.1 & $(346)$ & A, MG & {$[87 / 5][55 / 11]$} \\
\hline \multirow[t]{2}{*}{$\mathrm{C}_{10} \mathrm{H}_{22}$} & 4-isopropylheptane & & & & \\
\hline & & 47.3 & (298) & & {$[71 / 28]$} \\
\hline \multirow[t]{2}{*}{$\mathrm{C}_{10} \mathrm{H}_{22}$} & 2,2-dimethyloctane & & & & {$[15869-87-1]$} \\
\hline & & 49.0 & $(298)$ & & {$[71 / 28]$} \\
\hline \multirow[t]{2}{*}{$\mathrm{C}_{10} \mathrm{H}_{22}$} & 2,3-dimethyloctane & & & & [7146-60-3] \\
\hline & & 48.1 & $(298)$ & & {$[71 / 28]$} \\
\hline \multirow[t]{5}{*}{$\mathrm{C}_{10} \mathrm{H}_{22}$} & 2,4-dimethyloctane & & & & [4032-94-4] \\
\hline & & $44.9 \pm 0.2$ & $(328)$ & $\mathrm{C}$ & [84/4] \\
\hline & & $43.6 \pm 0.2$ & $(343)$ & $\mathrm{C}$ & {$[84 / 4]$} \\
\hline & & $42.4 \pm 0.2$ & $(358)$ & $\mathrm{C}$ & {$[84 / 4]$} \\
\hline & & 48.5 & $(298)$ & & {$[71 / 28]$} \\
\hline \multirow[t]{2}{*}{$\mathrm{C}_{10} \mathrm{H}_{22}$} & 2,5-dimethyloctane & & & & [15869-89-3] \\
\hline & & 49.0 & $(298)$ & & {$[71 / 28]$} \\
\hline \multirow{2}{*}{$\mathrm{C}_{10} \mathrm{H}_{22}$} & 2,6-dimethyloctane & & & & {$[2051-30-1]$} \\
\hline & & 49.3 & $(298)$ & & {$[71 / 28]$} \\
\hline \multirow[t]{3}{*}{$\mathrm{C}_{10} \mathrm{H}_{22}$} & 2,7-dimethyloctane & & & & [1072-16-8] \\
\hline & & 47.7 & $(298)$ & & [71/28] \\
\hline & $(279-433)$ & 45.2 & $(294)$ & A & {$[87 / 5][47 / 5]$} \\
\hline \multirow[t]{2}{*}{$\mathrm{C}_{10} \mathrm{H}_{22}$} & 3,3-dimethyloctane & & & & {$[4110-44-5]$} \\
\hline & & 48.5 & $(298)$ & & {$[71 / 28]$} \\
\hline $\mathrm{C}_{10} \mathrm{H}_{22}$ & 3,4-dimethyloctane & & & & [15869-92-8] \\
\hline & & 48.1 & $(298)$ & & {$[71 / 28]$} \\
\hline $\mathrm{C}_{10} \mathrm{H}_{22}$ & 3,5-dimethyloctane & & & & [15869-93-9] \\
\hline & & 48.5 & $(298)$ & & {$[71 / 28]$} \\
\hline $\mathrm{C}_{10} \mathrm{H}_{22}$ & 3,6-dimethyloctane & & & & [15869-94-0] \\
\hline & & 47.3 & $(298)$ & & {$[71 / 28]$} \\
\hline $\mathrm{C}_{10} \mathrm{H}_{22}$ & 4,4-dimethyloctane & & & & {$[15869-95-1]$} \\
\hline & & 48.1 & $(298)$ & & {$[71 / 28]$} \\
\hline $\mathrm{C}_{10} \mathrm{H}_{22}$ & 4,5-dimethyloctane & & & & [15869-96-2] \\
\hline & & 48.5 & $(298)$ & & {$[71 / 28]$} \\
\hline $\mathrm{C}_{10} \mathrm{H}_{22}$ & 2-methyl-3-ethylheptane & & & & {$[14676-29-0]$} \\
\hline & & 48.1 & $(298)$ & & {$[71 / 28]$} \\
\hline $\mathrm{C}_{10} \mathrm{H}_{22}$ & 2-methyl-4-ethylheptane & & & & {$[52896-88-5]$} \\
\hline & & 47.3 & $(298)$ & & {$[71 / 28]$} \\
\hline $\mathrm{C}_{10} \mathrm{H}_{22}$ & 2-methyl-5-ethylheptane & & & & {$[13475-78-0]$} \\
\hline & & 48.1 & $(298)$ & & {$[71 / 28]$} \\
\hline $\mathrm{C}_{10} \mathrm{H}_{22}$ & 3-methyl-3-ethylheptane & & & & {$[17302-01-1]$} \\
\hline & & 47.7 & $(298)$ & & {$[71 / 28]$} \\
\hline $\mathrm{C}_{10} \mathrm{H}_{22}$ & 3-methyl-4-ethylheptane & & & & [52896-89-6] \\
\hline & & 47.7 & $(298)$ & & {$[71 / 28]$} \\
\hline $\mathrm{C}_{10} \mathrm{H}_{22}$ & 3-methyl-5-ethylheptane & & & & \\
\hline
\end{tabular}


TABLE 6. Enthalpies of vaporization of organic compounds, 1880-2002-Continued

\begin{tabular}{|c|c|c|c|c|c|}
\hline Molecular formula & $\begin{array}{c}\text { Compound } \\
\text { (Temperature range/K) }\end{array}$ & $\begin{array}{c}\Delta_{\text {vap }} H_{m} \\
\left(\mathrm{~kJ} \mathrm{~mol}^{-1}\right)\end{array}$ & $\begin{array}{l}\text { Mean temperature } \\
\left(T_{m} / \mathrm{K}\right)\end{array}$ & Method & $\begin{array}{l}\text { CAS registry number } \\
\text { Reference }\end{array}$ \\
\hline & & 47.7 & (298) & & {$[71 / 28]$} \\
\hline $\mathrm{C}_{10} \mathrm{H}_{22}$ & 4-methyl-3-ethylheptane & 48.1 & (298) & & {$[61 / 30]$} \\
\hline \multirow{2}{*}{$\mathrm{C}_{10} \mathrm{H}_{22}$} & 4-methyl-4-ethylheptane & & & & \\
\hline & & 47.2 & (298) & & {$[61 / 30]$} \\
\hline \multirow[t]{2}{*}{$\mathrm{C}_{10} \mathrm{H}_{22}$} & 2,2,3-trimethylheptane & & & & [52896-92-1] \\
\hline & & 46.9 & (298) & & {$[71 / 28]$} \\
\hline $\mathrm{C}_{10} \mathrm{H}_{22}$ & 2,2,4-trimethylheptane & & & & [14720-74-2] \\
\hline \multirow{2}{*}{$\mathrm{C}_{10} \mathrm{H}_{22}$} & 2,2,5-trimethylheptane & 45.6 & (298) & & $\begin{array}{c}{[71 / 28]} \\
{[20291-95-6]}\end{array}$ \\
\hline & & 46.0 & (298) & & {$[71 / 28]$} \\
\hline \multirow{2}{*}{$\mathrm{C}_{10} \mathrm{H}_{22}$} & 2,2,6-trimethylheptane & & & & [1190-83-6] \\
\hline & & 46.4 & (298) & & {$[71 / 28]$} \\
\hline $\mathrm{C}_{10} \mathrm{H}_{22}$ & 2,3,3-trimethylheptane & 469 & (208) & & $\begin{array}{c}{[52896-93-2]} \\
{[71 / 28]}\end{array}$ \\
\hline \multirow{2}{*}{$\mathrm{C}_{10} \mathrm{H}_{22}$} & 2,3,4-trimethylheptane & & & & [52896-95-4] \\
\hline & & 47.3 & (298) & & {$[71 / 28]$} \\
\hline $\mathrm{C}_{10} \mathrm{H}_{22}$ & 2,3,5-trimethylheptane & 47.3 & (298) & & $\begin{array}{c}{[20278-85-7]} \\
{[71 / 28]}\end{array}$ \\
\hline \multirow{2}{*}{$\mathrm{C}_{10} \mathrm{H}_{22}$} & 2,3,6-trimethylheptane & & & & {$[4032-93-3]$} \\
\hline & & 47.3 & (298) & & {$[71 / 28]$} \\
\hline \multirow{2}{*}{$\mathrm{C}_{10} \mathrm{H}_{22}$} & 2,4,4-trimethylheptane & & & & [4032-92-2] \\
\hline & 24 -trimethylhentane & 45.2 & (298) & & [71/28] \\
\hline $\mathrm{C}_{10} \mathrm{H}_{22}$ & 2, & 46.9 & (298) & & $\begin{array}{c}{[20278-84-6]} \\
{[71 / 28]}\end{array}$ \\
\hline \multirow{2}{*}{$\mathrm{C}_{10} \mathrm{H}_{22}$} & 2,4,6-trimethylheptane & & & & [2613-61-8] \\
\hline & & 46.4 & (298) & & {$[71 / 28]$} \\
\hline \multirow{2}{*}{$\mathrm{C}_{10} \mathrm{H}_{22}$} & 2,5,5-trimethylheptane & & & & [1189-99-7] \\
\hline & & 46.0 & (298) & & [71/28] \\
\hline $\mathrm{C}_{10} \mathrm{H}_{22}$ & 3,3,4-trimethylheptane & 46.9 & (298) & & $\begin{array}{c}{[20278-88-0]} \\
{[71 / 28]}\end{array}$ \\
\hline \multirow[t]{2}{*}{$\mathrm{C}_{10} \mathrm{H}_{22}$} & 3,3,5-trimethylheptane & & & & [7154-80-5] \\
\hline & & 46.0 & (298) & & {$[71 / 28]$} \\
\hline \multirow[t]{2}{*}{$\mathrm{C}_{10} \mathrm{H}_{22}$} & 3,4,4-trimethylheptane & & & & [20278-88-0] \\
\hline & & 46.4 & (298) & & [71/28] \\
\hline \multirow{2}{*}{$\mathrm{C}_{10} \mathrm{H}_{22}$} & 3,4,5-trimethylheptane & & & & [20278-89-1] \\
\hline & 2-metbyl-3-isonronylhexane & 47.3 & (298) & & [71/28] \\
\hline $\mathrm{C}_{10} \mathrm{H}_{22}$ & 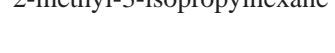 & 46.4 & (298) & & {$[71 / 28]$} \\
\hline $\mathrm{C}_{10} \mathrm{H}_{22}$ & 3,3-diethylhexane & & & & [17302-02-2] \\
\hline & & 47.3 & (298) & & [71/28] \\
\hline $\mathrm{C}_{10} \mathrm{H}_{22}$ & 3,4-diethylhexane & & & & [19398-77-7] \\
\hline $\mathrm{C}_{10} \mathrm{H}_{22}$ & 2,2-dimethyl-3-ethylhexane & 41.1 & (298) & & $\begin{array}{c}{[71 / 28]} \\
{[20291-91-2]}\end{array}$ \\
\hline & & 46.0 & (298) & & {$[71 / 28]$} \\
\hline $\mathrm{C}_{10} \mathrm{H}_{22}$ & 2,2-dimethyl-4-ethylhexane & & & & [52896-99-8] \\
\hline & & 45.2 & (298) & & {$[71 / 28]$} \\
\hline $\mathrm{C}_{10} \mathrm{H}_{22}$ & 2,3-dimethyl-3-ethylhexane & & & & [52897-00-4] \\
\hline & & 46.9 & (298) & & {$[71 / 28]$} \\
\hline $\mathrm{C}_{10} \mathrm{H}_{22}$ & 2,3-dimethyl-4-ethylhexane & & & & [52897-01-5] \\
\hline & & 46.9 & (298) & & [71/28] \\
\hline $\mathrm{C}_{10} \mathrm{H}_{22}$ & 2,4-dimethyl-3-ethylhexane & & & & {$[7220-26-0]$} \\
\hline $\mathrm{C}_{10} \mathrm{H}_{22}$ & 4-dimetbyl-4 etbylhexan & 46.9 & (298) & & $\begin{array}{c}{[71 / 28]} \\
5287\end{array}$ \\
\hline $\mathrm{C}_{10} \mathrm{H}_{22}$ & 2,4-dimethyl-4-ethylhexane & 46.4 & (298) & & $\begin{array}{c}{[52897-03-7]} \\
{[71 / 28]}\end{array}$ \\
\hline $\mathrm{C}_{10} \mathrm{H}_{22}$ & 2,5-dimethyl-3-ethylhexane & & & & {$[52897-04-8]$} \\
\hline & & 46.4 & (298) & & [71/28] \\
\hline $\mathrm{C}_{10} \mathrm{H}_{22}$ & 3,3-dimethyl-4-ethylhexane & & & & [52897-05-9] \\
\hline & & 46.4 & (298) & & [71/28] \\
\hline $\mathrm{C}_{10} \mathrm{H}_{22}$ & 3,4-dimethyl-3-ethylhexane & & & & [52897-06-0] \\
\hline & & 46.4 & (298) & & [71/28] \\
\hline $\mathrm{C}_{10} \mathrm{H}_{22}$ & 2,2,3,3-tetramethylhexane & & & & [13475-81-5] \\
\hline $\mathrm{C}_{10} \mathrm{H}_{22}$ & 2,2,3,4-tetramethylhexane & 45.2 & (298) & & $\begin{array}{c}{[71 / 28]} \\
{[52897-08-2]}\end{array}$ \\
\hline
\end{tabular}


TABLE 6. Enthalpies of vaporization of organic compounds, 1880-2002-Continued

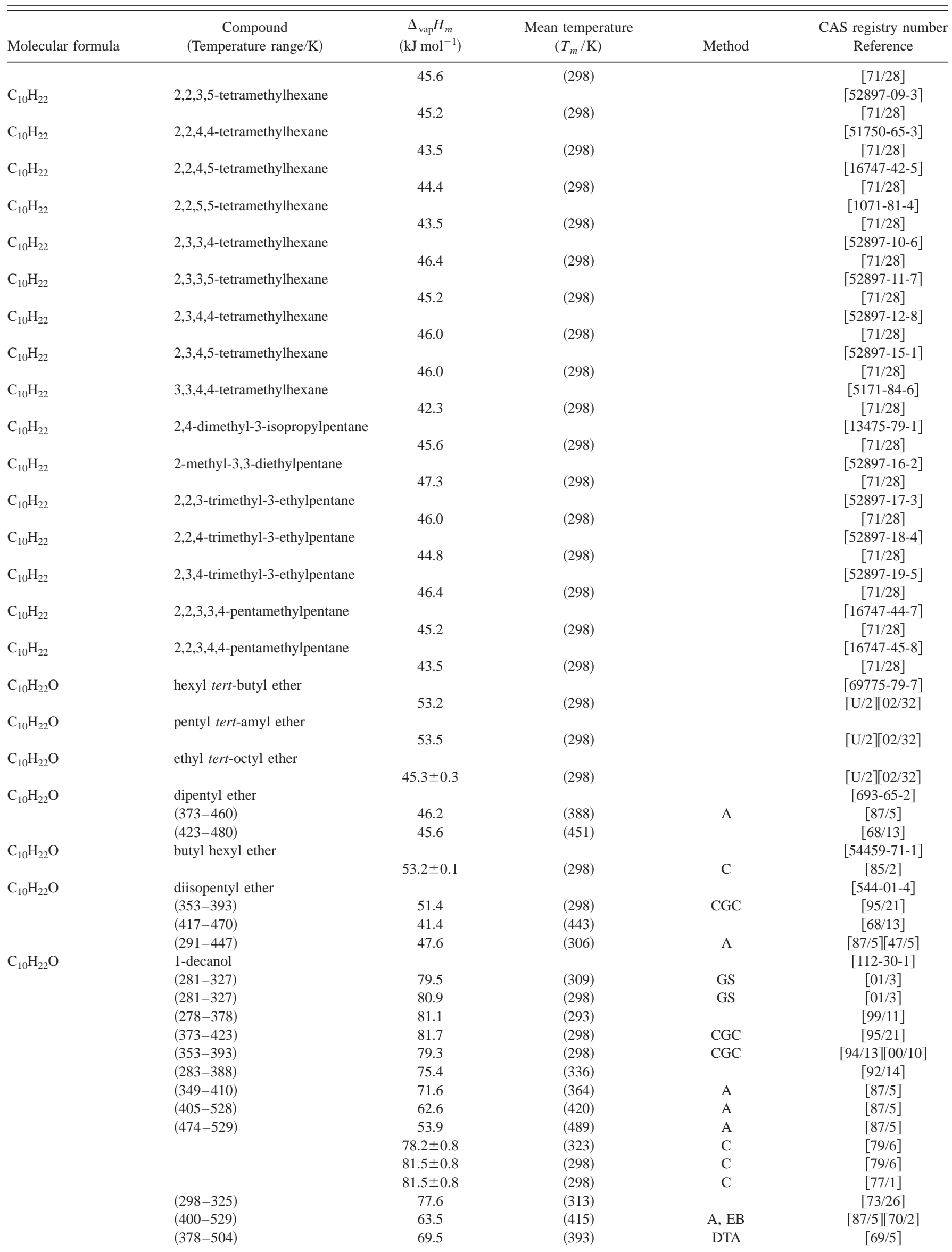


TABLE 6. Enthalpies of vaporization of organic compounds, 1880-2002-Continued

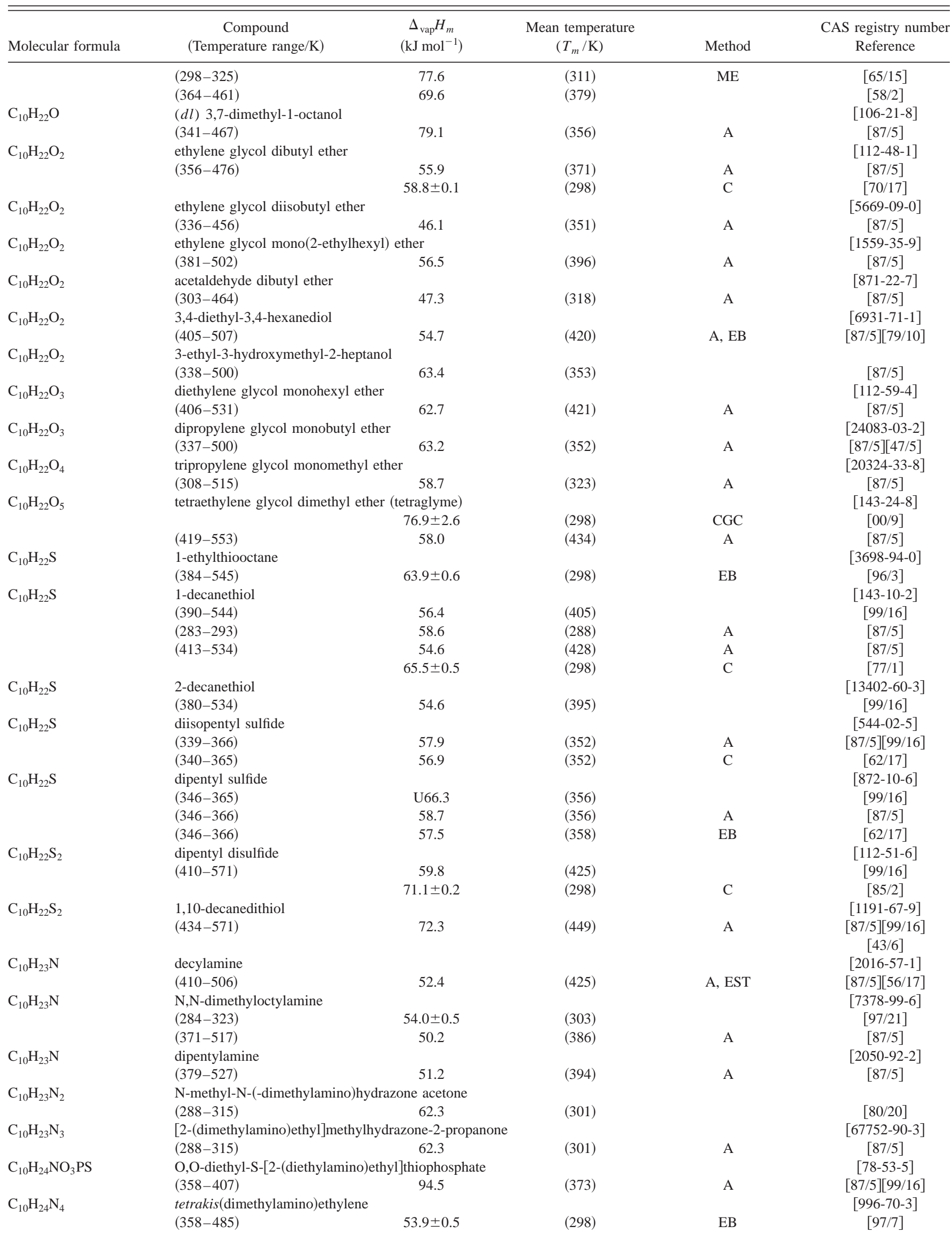


TABLE 6. Enthalpies of vaporization of organic compounds, 1880-2002-Continued

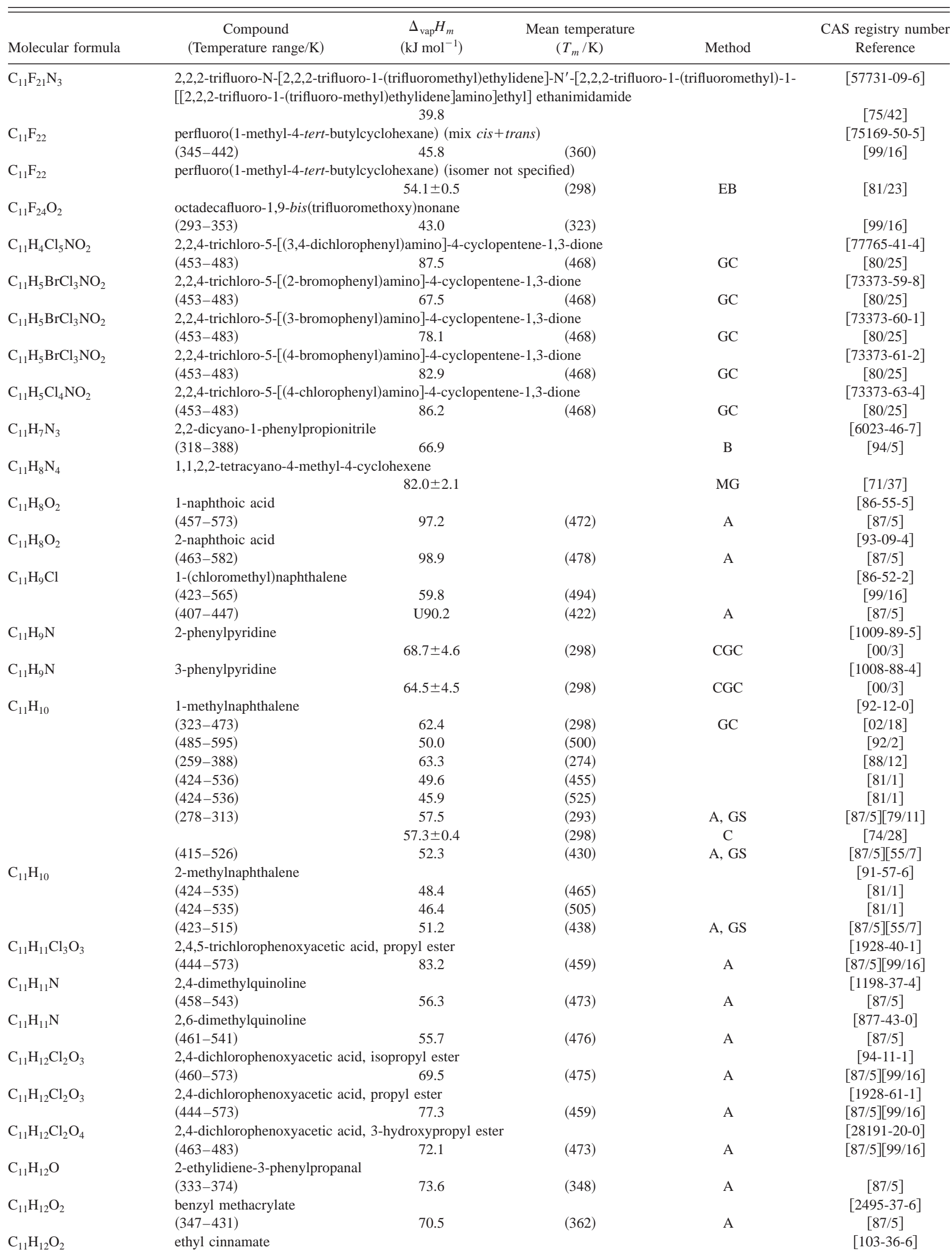


TABLE 6. Enthalpies of vaporization of organic compounds, 1880-2002-Continued

\begin{tabular}{|c|c|c|c|c|c|}
\hline Molecular formula & $\begin{array}{c}\text { Compound } \\
\text { (Temperature range/K) }\end{array}$ & $\begin{array}{c}\Delta_{\mathrm{vap}} H_{m} \\
\left(\mathrm{~kJ} \mathrm{~mol}^{-1}\right)\end{array}$ & $\begin{array}{l}\text { Mean temperature } \\
\qquad\left(T_{m} / \mathrm{K}\right)\end{array}$ & Method & $\begin{array}{c}\text { CAS registry number } \\
\text { Reference }\end{array}$ \\
\hline \multirow{3}{*}{$\mathrm{C}_{11} \mathrm{H}_{12} \mathrm{O}_{2}$} & $(453-544)$ & 57.8 & $(468)$ & A & {$[87 / 5]$} \\
\hline & 1-phenyl-1,3-pentanedione & & & & {$[5331-64-6]$} \\
\hline & $(371-550)$ & 64.6 & $(386)$ & A & {$[87 / 5]$} \\
\hline \multirow[t]{3}{*}{$\mathrm{C}_{11} \mathrm{H}_{12} \mathrm{O}_{2}$} & 1-phenyl-4,7-dioxaspiro[2.4]heptane & & & & {$[39522-76-4]$} \\
\hline & $(307-333)$ & $71.3 \pm 0.7$ & $(298)$ & GS & {$[02 / 32]$} \\
\hline & $(288-302)$ & $69.6 \pm 0.7$ & & GS & {$[98 / 21]$} \\
\hline \multirow{2}{*}{$\mathrm{C}_{11} \mathrm{H}_{12} \mathrm{O}_{2}$} & 4-carboxymethylpentacyclo[ $4 \cdot 3 \cdot 0.0 .^{2,5}$ & $\left.{ }^{5} 0^{4,7}\right]$ nonane & & & [40317-63-3] \\
\hline & $(303-343)$ & $80.0 \pm 1.7$ & $(333)$ & & {$[84 / 12]$} \\
\hline \multirow[t]{2}{*}{$\mathrm{C}_{11} \mathrm{H}_{12} \mathrm{O}_{3}$} & benzoylacetic acid, ethyl ester & & & & {$[94-02-0]$} \\
\hline & $(380-538)$ & 72.1 & (395) & A & {$[87 / 5]$} \\
\hline \multirow[t]{2}{*}{$\mathrm{C}_{11} \mathrm{H}_{12} \mathrm{O}_{3}$} & myristicin & & & & {$[607-91-0]$} \\
\hline & $(368-553)$ & 61.2 & $(383)$ & A & {$[87 / 5]$} \\
\hline $\mathrm{C}_{11} \mathrm{H}_{12} \mathrm{O}_{3}$ & 2-piperonylpropanal & & & & \\
\hline \multirow{2}{*}{$\mathrm{C}_{11} \mathrm{H}_{13} \mathrm{Cl}_{3}$} & $\begin{array}{l}\text { (373-423) } \\
\text { 4-tert-butyl-2,3,6-trichlorotoluene }\end{array}$ & 74.5 & $(388)$ & A & $\begin{array}{c}{[87 / 5]} \\
{[61468-36-8]}\end{array}$ \\
\hline & $(423-570)$ & 62.7 & $(438)$ & A & $\begin{array}{c}{[87 / 5][73 / 15]} \\
{[99 / 16]}\end{array}$ \\
\hline \multirow[t]{4}{*}{$\mathrm{C}_{11} \mathrm{H}_{14}$} & 1,1-dimethylindane & & & & [4912-92-9] \\
\hline & $(313-348)$ & 50.1 & $(328)$ & A & {$[87 / 5]$} \\
\hline & $(313-467)$ & 50.5 & $(328)$ & A & {$[87 / 5]$} \\
\hline & $(387-467)$ & 45.9 & $(402)$ & A & {$[87 / 5]$} \\
\hline \multirow[t]{4}{*}{$\mathrm{C}_{11} \mathrm{H}_{14}$} & 4,6-dimethylindane & & & & {$[1685-82-1]$} \\
\hline & $(313-467)$ & 56.9 & (328) & A & {$[87 / 5]$} \\
\hline & $(313-363)$ & 56.4 & (328) & A & {$[87 / 5]$} \\
\hline & $(415-467)$ & 50.3 & $(430)$ & $\mathrm{A}$ & {$[87 / 5]$} \\
\hline \multirow[t]{4}{*}{$\mathrm{C}_{11} \mathrm{H}_{14}$} & 4,7-dimethylindane & & & & [6682-71-9] \\
\hline & $(313-470)$ & 54.7 & $(328)$ & A & {$[87 / 5]$} \\
\hline & $(313-363)$ & 56.9 & $(328)$ & A & {$[87 / 5]$} \\
\hline & $(417-470)$ & 50.6 & $(432)$ & $\mathrm{A}$ & {$[87 / 5]$} \\
\hline \multirow{2}{*}{$\mathrm{C}_{11} \mathrm{H}_{14}$} & 4-isopropylstyrene & & & & {$[2055-40-5]$} \\
\hline & $(408-478)$ & 48.5 & $(423)$ & A & {$[87 / 5]$} \\
\hline \multirow[t]{2}{*}{$\mathrm{C}_{11} \mathrm{H}_{14}$} & 5-methyl-1,2,3,4-tetrahydronaphthalen & & & & [2809-64-5] \\
\hline & $(416-508)$ & 53.4 & $(431)$ & A & {$[87 / 5]$} \\
\hline \multirow[t]{2}{*}{$\mathrm{C}_{11} \mathrm{H}_{14}$} & 6-methyl-1,2,3,4-tetrahydronaphthalen & & & & [1680-51-9] \\
\hline & $(411-502)$ & 53.7 & $(426)$ & A & {$[87 / 5]$} \\
\hline \multirow[t]{2}{*}{$\mathrm{C}_{11} \mathrm{H}_{14}$} & 2,4,5-trimethylstyrene & & & & [3937-24-4] \\
\hline & $(352-490)$ & 56.4 & $(367)$ & A & {$[87 / 5][49 / 18]$} \\
\hline \multirow[t]{2}{*}{$\mathrm{C}_{11} \mathrm{H}_{14}$} & 2,4,6-trimethylstyrene & & & & {$[769-25-5]$} \\
\hline & $(362-483)$ & 50.9 & $(377)$ & A & {$[87 / 5][49 / 18]$} \\
\hline \multirow[t]{2}{*}{$\mathrm{C}_{11} \mathrm{H}_{14} \mathrm{Cl}_{2}$} & 4-tert-butyl-2,5-dichlorotoluene & & & & {$[61468-35-7]$} \\
\hline & $(395-538)$ & 57.0 & $(410)$ & A & $\begin{array}{c}{[87 / 5][73 / 15]} \\
{[99 / 16]}\end{array}$ \\
\hline $\mathrm{C}_{11} \mathrm{H}_{14} \mathrm{~N}_{2} \mathrm{O}_{4}$ & $\begin{array}{l}\text { 3-nitro-(4-nitrophenyl)pentane } \\
(321-358)\end{array}$ & $88.0 \pm 0.8$ & (298) & GS & [97/5] \\
\hline $\mathrm{C}_{11} \mathrm{H}_{14} \mathrm{O}$ & tert-butyl phenyl ketone & & & & {$[938-16-9]$} \\
\hline & $(330-493)$ & 55.5 & $(345)$ & A & {$[87 / 5][47 / 5]$} \\
\hline $\mathrm{C}_{11} \mathrm{H}_{14} \mathrm{O}$ & 2-ethyl-3-phenylpropanal & & & & \\
\hline & $(343-388)$ & 64.6 & $(358)$ & A & {$[87 / 5]$} \\
\hline $\mathrm{C}_{11} \mathrm{H}_{14} \mathrm{O}$ & isobutyl phenyl ketone & & & & {$[582-62-7]$} \\
\hline & $(331-501)$ & 55.7 & $(346)$ & A & {$[87 / 5][47 / 5]$} \\
\hline $\mathrm{C}_{11} \mathrm{H}_{14} \mathrm{O}$ & 2,3,5-trimethylacetophenone & & & & \\
\hline & $(352-557)$ & 57.9 & $(367)$ & A & {$[87 / 5][47 / 5]$} \\
\hline $\mathrm{C}_{11} \mathrm{H}_{14} \mathrm{O}_{2}$ & 1,1-dimethoxy-2-phenylcyclopropane & & & & \\
\hline & $(278-313)$ & $63.9 \pm 0.6$ & & GS & {$[98 / 21]$} \\
\hline $\mathrm{C}_{11} \mathrm{H}_{14} \mathrm{O}_{2}$ & 3-acetoxy-1-phenylpropane & & & & \\
\hline & $(293-333)$ & 74.3 & (306) & A & {$[87 / 5]$} \\
\hline $\mathrm{C}_{11} \mathrm{H}_{14} \mathrm{O}_{2}$ & butyl benzoate & & & & {$[120-50-3]$} \\
\hline & $(374-474)$ & 63.2 & (394) & BG & {$[88 / 2]$} \\
\hline & $(374-474)$ & 55.7 & $(452)$ & BG & {$[88 / 2]$} \\
\hline & $(343-405)$ & 59.1 & $(358)$ & A & {$[87 / 5]$} \\
\hline $\mathrm{C}_{11} \mathrm{H}_{14} \mathrm{O}_{2}$ & 1,2-dimethoxy-4-(1-propenyl)benzene & & & & {$[93-16-3]$} \\
\hline & $(358-521)$ & 61.9 & $(373)$ & A & {$[87 / 5][47 / 5]$} \\
\hline $\mathrm{C}_{11} \mathrm{H}_{14} \mathrm{O}_{2}$ & isobutyl benzoate & & & & {$[136-60-7]$} \\
\hline & $(370-467)$ & 60.4 & $(393)$ & BG & {$[88 / 2]$} \\
\hline
\end{tabular}


TABLE 6. Enthalpies of vaporization of organic compounds, 1880-2002-Continued

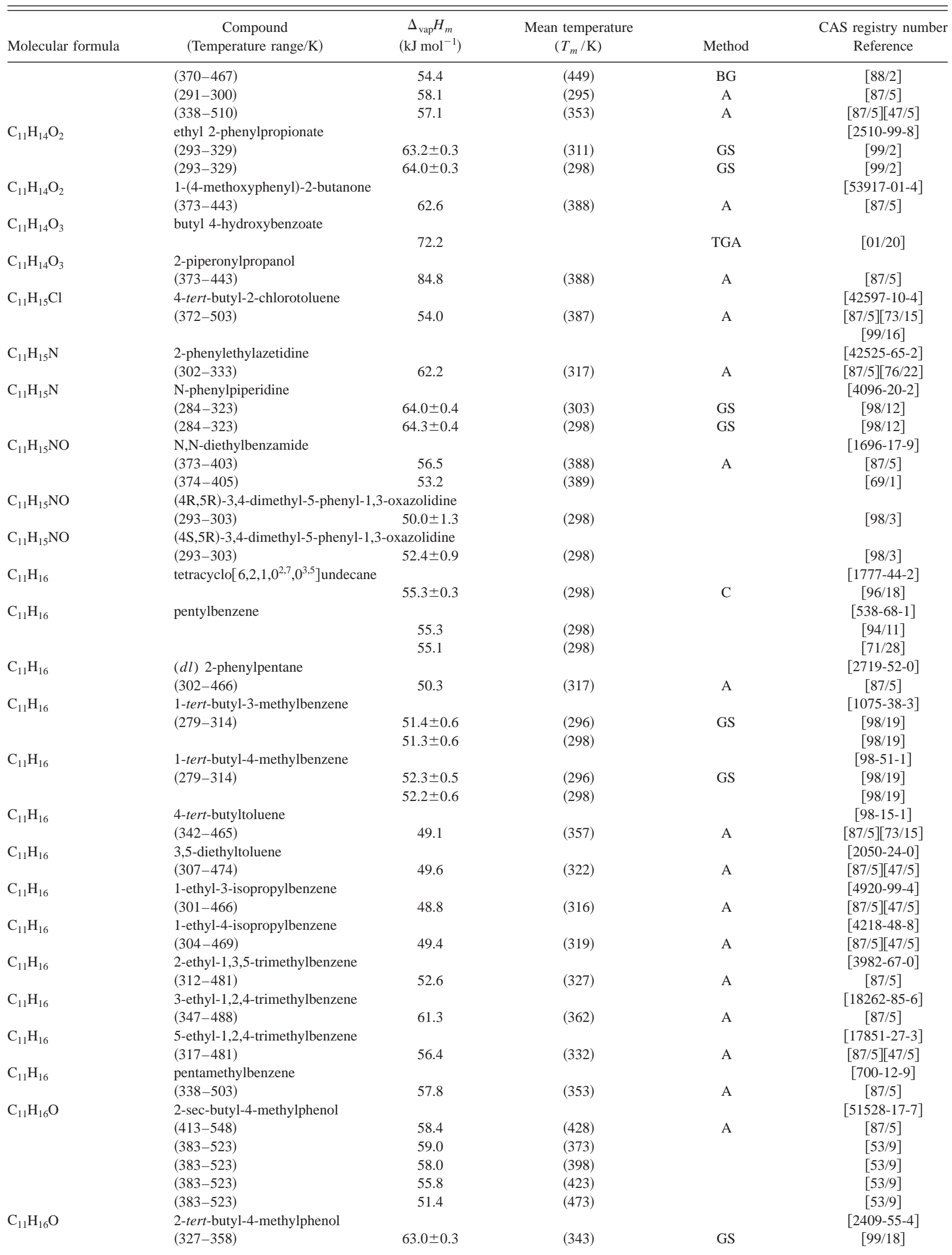


TABLE 6. Enthalpies of vaporization of organic compounds, 1880-2002-Continued

\begin{tabular}{|c|c|c|c|c|c|}
\hline Molecular formula & $\begin{array}{c}\text { Compound } \\
\text { (Temperature range/K) }\end{array}$ & $\begin{array}{c}\Delta_{\mathrm{vap}} H_{m} \\
\left(\mathrm{~kJ} \mathrm{~mol}^{-1}\right)\end{array}$ & $\begin{array}{l}\text { Mean temperature } \\
\qquad\left(T_{m} / \mathrm{K}\right)\end{array}$ & Method & $\begin{array}{c}\text { CAS registry number } \\
\text { Reference }\end{array}$ \\
\hline & & $65.7 \pm 0.3$ & $(298)$ & & {$[99 / 18]$} \\
\hline & $(385-517)$ & 58.9 & $(400)$ & A & {$[87 / 5]$} \\
\hline & $(343-507)$ & 57.7 & $(348)$ & & {$[53 / 9]$} \\
\hline & $(343-507)$ & 55.7 & $(373)$ & & {$[53 / 9]$} \\
\hline & $(343-507)$ & 52.6 & $(423)$ & & {$[53 / 9]$} \\
\hline & $(343-507)$ & 48.5 & $(473)$ & & {$[53 / 9]$} \\
\hline \multirow[t]{5}{*}{$\mathrm{C}_{11} \mathrm{H}_{16} \mathrm{O}$} & 2-tert-butyl-5-methylphenol & & & & {$[88-60-8]$} \\
\hline & $(296-343)$ & $65.9 \pm 0.3$ & $(320)$ & GS & {$[99 / 18]$} \\
\hline & & $67.2 \pm 0.3$ & $(298)$ & & {$[99 / 18]$} \\
\hline & $(378-490)$ & 59.8 & $(393)$ & A & {$[87 / 5]$} \\
\hline & $(383-518)$ & 53.0 & $(398)$ & A & {$[87 / 5]$} \\
\hline \multirow[t]{4}{*}{$\mathrm{C}_{11} \mathrm{H}_{16} \mathrm{O}$} & 2-tert-butyl-6-methylphenol & & & & {$[2219-82-1]$} \\
\hline & $(308-343)$ & $62.2 \pm 0.5$ & $(326)$ & GS & [99/19] \\
\hline & & $63.8 \pm 0.5$ & $(298)$ & & {$[99 / 19]$} \\
\hline & $(375-505)$ & 55.2 & $(390)$ & A & {$[87 / 5]$} \\
\hline \multirow[t]{10}{*}{$\mathrm{C}_{11} \mathrm{H}_{16} \mathrm{O}$} & 4-tert-butyl-2-methylphenol & & & & {$[98-27-1]$} \\
\hline & $(291-333)$ & $71.3 \pm 0.6$ & $(312)$ & GS & {$[99 / 18]$} \\
\hline & & $72.1 \pm 0.6$ & $(298)$ & & {$[99 / 18]$} \\
\hline & $(347-520)$ & 61.5 & $(362)$ & A & {$[87 / 5]$} \\
\hline & $(275-297)$ & 75.7 & $(286)$ & A & {$[87 / 5][60 / 1]$} \\
\hline & $(347-532)$ & 55.7 & $(348)$ & & {$[53 / 9]$} \\
\hline & $(347-532)$ & 53.9 & $(373)$ & & {$[53 / 9]$} \\
\hline & $(347-532)$ & 53.2 & $(398)$ & & {$[53 / 9]$} \\
\hline & $(347-532)$ & 50.9 & $(423)$ & & {$[53 / 9]$} \\
\hline & $(347-532)$ & 46.7 & $(473)$ & & {$[53 / 9]$} \\
\hline \multirow[t]{2}{*}{$\mathrm{C}_{11} \mathrm{H}_{16} \mathrm{O}$} & 2-ethyl-3-phenyl-1-propanol & & & & {$[3968-87-4]$} \\
\hline & $(348-393)$ & 70.9 & $(363)$ & A & {$[87 / 5]$} \\
\hline \multirow[t]{3}{*}{$\mathrm{C}_{11} \mathrm{H}_{16} \mathrm{O}$} & 2-(2-pentyl)phenol & & & & {$[87-26-3]$} \\
\hline & $(397-501)$ & 74.4 & $(413)$ & EB & {$[90 / 5]$} \\
\hline & $(397-501)$ & 59.6 & $(412)$ & & {$[93 / 10]$} \\
\hline \multirow[t]{2}{*}{$\mathrm{C}_{11} \mathrm{H}_{16} \mathrm{O}$} & 4-pentylphenol & & & & {$[14938-35-3]$} \\
\hline & $(423-563)$ & 60.9 & $(438)$ & $\mathrm{A}$ & {$[87 / 5]$} \\
\hline \multirow[t]{4}{*}{$\mathrm{C}_{11} \mathrm{H}_{16} \mathrm{O}$} & 4-tert-pentylphenol & & & & {$[80-46-6]$} \\
\hline & $(297-333)$ & $64.2 \pm 0.2$ & $(329)$ & GS & {$[99 / 18]$} \\
\hline & & $65.3 \pm 0.2$ & $(298)$ & & {$[99 / 18]$} \\
\hline & $(385-548)$ & 58.2 & $(400)$ & A & {$[87 / 5]$} \\
\hline \multirow[t]{2}{*}{$\mathrm{C}_{11} \mathrm{H}_{16} \mathrm{O}$} & 5-phenyl-1-pentanol & & & & {$[10521-91-2]$} \\
\hline & $(373-430)$ & 58.2 & $(388)$ & A & {$[87 / 5]$} \\
\hline \multirow[t]{3}{*}{$\mathrm{C}_{11} \mathrm{H}_{16} \mathrm{O}$} & (1-propoxyethyl)benzene & & & & \\
\hline & $(288-321)$ & $56.4 \pm 0.2$ & $(305)$ & GS & {$[01 / 16]$} \\
\hline & $(288-321)$ & $56.7 \pm 0.2$ & $(298)$ & GS & [01/16] \\
\hline \multirow[t]{2}{*}{$\mathrm{C}_{11} \mathrm{H}_{16} \mathrm{O}$} & (1-isopropoxyethyl)benzene & & & & {$[65757-61-1]$} \\
\hline & $(278-313)$ & $55.4 \pm 0.3$ & $(298)$ & GS & {$[02 / 29][02 / 38]$} \\
\hline \multirow[t]{3}{*}{$\mathrm{C}_{11} \mathrm{H}_{16} \mathrm{O}$} & cumyl ethyl ether & & & & [1712-74-9] \\
\hline & $(278-313)$ & $54.8 \pm 0.5$ & $(296)$ & GS & [01/18] \\
\hline & $(278-313)$ & $54.7 \pm 0.5$ & $(298)$ & GS & [01/18] \\
\hline \multirow[t]{2}{*}{$\mathrm{C}_{11} \mathrm{H}_{16} \mathrm{O}_{2}$} & 2-tert-butyl-4-methoxyphenol & & & & {$[121-00-6]$} \\
\hline & $(403-463)$ & 54.4 & $(418)$ & A & {$[87 / 5]$} \\
\hline \multirow[t]{2}{*}{$\mathrm{C}_{11} \mathrm{H}_{16} \mathrm{O}_{2}$} & 1,3-dihydroxy-4-pentylbenzene & & & & {$[533-24-4]$} \\
\hline & $(423-488)$ & 84.9 & $(438)$ & $\mathrm{A}, \mathrm{GC}$ & {$[87 / 5][75 / 24]$} \\
\hline \multirow[t]{2}{*}{$\mathrm{C}_{11} \mathrm{H}_{16} \mathrm{O}_{2}$} & phenyldiethoxymethane & & & & {$[774-48-1]$} \\
\hline & $(283-329)$ & $62.8 \pm 0.6$ & $(298)$ & GS & {$[02 / 32]$} \\
\hline $\mathrm{C}_{11} \mathrm{H}_{16} \mathrm{O}_{2}$ & 1,1-dimethoxy-1-phenylpropane & & & & {$[25310-92-3]$} \\
\hline & $(288-328)$ & $58.9 \pm 0.3$ & $(298)$ & GS & {$[02 / 32]$} \\
\hline & $(288-328)$ & $57.9 \pm 0.3$ & & GS & {$[98 / 21]$} \\
\hline $\mathrm{C}_{11} \mathrm{H}_{16} \mathrm{O}_{2}$ & tert-pentylcatechol (isomer not s & ified) & & & \\
\hline & $(398-473)$ & 58.2 & $(436)$ & & {$[65 / 21]$} \\
\hline $\mathrm{C}_{11} \mathrm{H}_{16} \mathrm{O}_{5}$ & ethylcamphoric acid anhydride & & & & \\
\hline & $(391-571)$ & 70.8 & $(406)$ & A & {$[87 / 5][47 / 5]$} \\
\hline $\mathrm{C}_{11} \mathrm{H}_{16} \mathrm{O}_{5}$ & (1-methylallyl)[1-(allyloxycarbo & ethyl]carbonate & & & \\
\hline & $(368-508)$ & 60.2 & $(383)$ & A & {$[87 / 5]$} \\
\hline $\mathrm{C}_{11} \mathrm{H}_{17} \mathrm{Cl}_{3} \mathrm{OS}$ & 2,3,3-trichloro-2-propenethioic a & O-octyl ester & & & {$[76619-96-0]$} \\
\hline & $(443-483)$ & 74.2 & & GC & {$[80 / 24]$} \\
\hline $\mathrm{C}_{11} \mathrm{H}_{17} \mathrm{NO}$ & 2-(dimethylamino)-1-phenyl-1-p & anone & & & \\
\hline
\end{tabular}


TABLE 6. Enthalpies of vaporization of organic compounds, 1880-2002-Continued

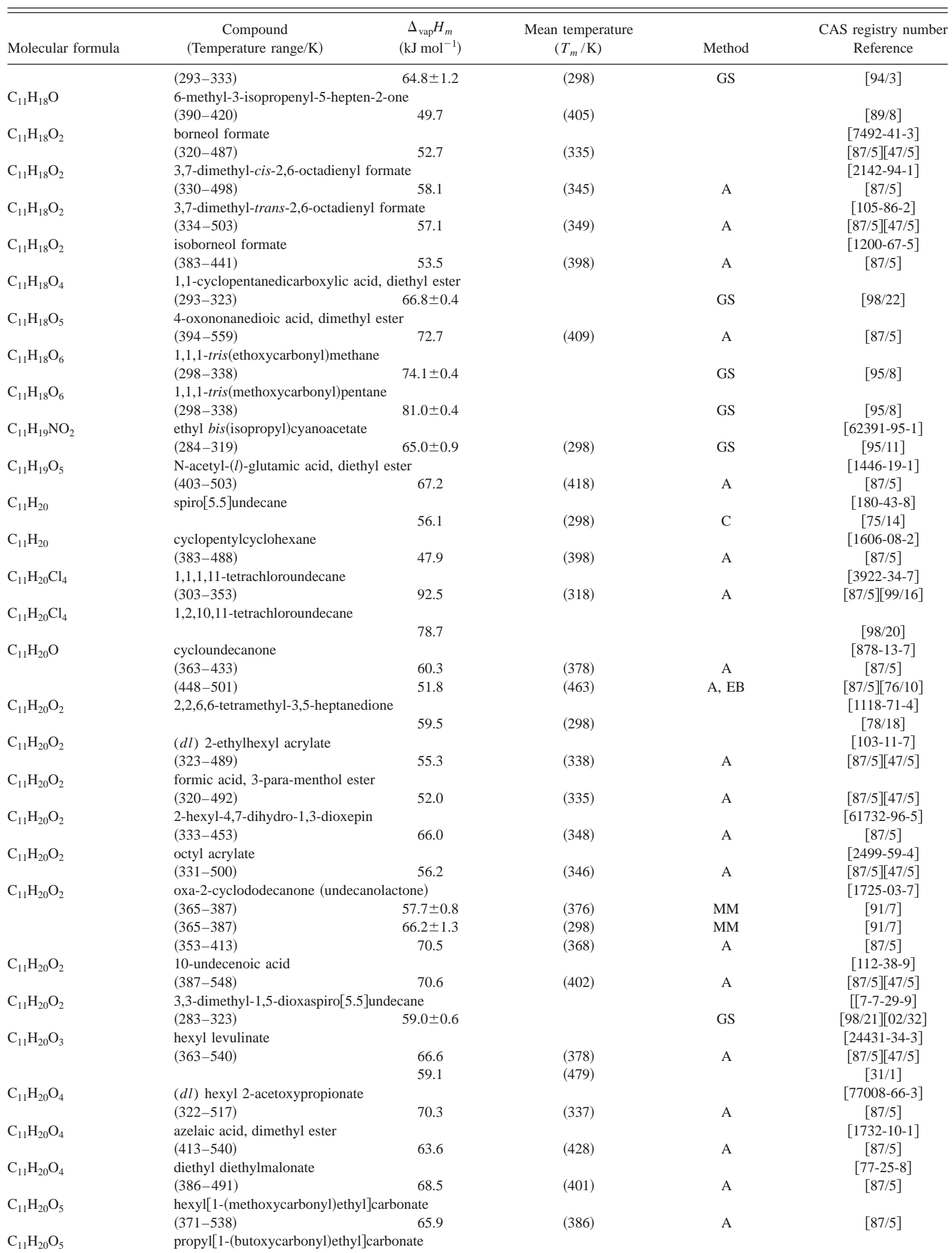


TABLE 6. Enthalpies of vaporization of organic compounds, 1880-2002-Continued

\begin{tabular}{|c|c|c|c|c|c|}
\hline Molecular formula & $\begin{array}{c}\text { Compound } \\
\text { (Temperature range/K) }\end{array}$ & $\begin{array}{c}\Delta_{\mathrm{vap}} H_{m} \\
\left(\mathrm{~kJ} \mathrm{~mol}^{-1}\right)\end{array}$ & $\begin{array}{l}\text { Mean temperature } \\
\qquad\left(T_{m} / \mathrm{K}\right)\end{array}$ & Method & $\begin{array}{c}\text { CAS registry number } \\
\text { Reference }\end{array}$ \\
\hline & $(330-463)$ & 66.4 & $(345)$ & A & {$[87 / 5]$} \\
\hline \multirow[t]{2}{*}{$\mathrm{C}_{11} \mathrm{H}_{21} \mathrm{~N}$} & 2-butyl-2-methylhexanenitrile & & & & {$[80606-32-2]$} \\
\hline & $(298-388)$ & $59.8 \pm 0.4$ & & GS & {$[94 / 5]$} \\
\hline \multirow[t]{3}{*}{$\mathrm{C}_{11} \mathrm{H}_{21} \mathrm{~N}$} & undecanonitrile & & & & {$[2244-07-1]$} \\
\hline & $(355-534)$ & 63.7 & $(370)$ & A & {$[87 / 5]$} \\
\hline & & $71.1 \pm 0.1$ & $(298)$ & $\mathrm{C}$ & {$[77 / 5]$} \\
\hline \multirow[t]{3}{*}{$\mathrm{C}_{11} \mathrm{H}_{21} \mathrm{~N}$} & N-cyclohexylpiperidine & & & & {$[3319-01-5]$} \\
\hline & $(288-328)$ & $59.9 \pm 0.6$ & $(308)$ & GS & {$[98 / 12]$} \\
\hline & $(288-328)$ & $60.5 \pm 0.6$ & (298) & GS & {$[98 / 12]$} \\
\hline \multirow[t]{2}{*}{$\mathrm{C}_{11} \mathrm{H}_{21} \mathrm{NO}$} & N-hexanoylpiperidone & & & & [15770-38-4] \\
\hline & $(383-433)$ & 66.3 & $(398)$ & A & {$[87 / 5]$} \\
\hline \multirow[t]{5}{*}{$\mathrm{C}_{11} \mathrm{H}_{22}$} & pentylcyclohexane & & & & {$[4292-92-6]$} \\
\hline & & $52.9 \pm 0.5$ & $(298)$ & & {$[87 / 17]$} \\
\hline & & $54.1 \pm 0.3$ & $(298)$ & GCC & {$[78 / 16]$} \\
\hline & & 53.9 & $(298)$ & & {$[75 / 12]$} \\
\hline & & 55.0 & $(298)$ & & {$[71 / 28]$} \\
\hline \multirow[t]{2}{*}{$\mathrm{C}_{11} \mathrm{H}_{22}$} & hexylcyclopentane & & & & {$[4457-00-5]$} \\
\hline & & 55.9 & (298) & & {$[71 / 28]$} \\
\hline \multirow[t]{4}{*}{$\mathrm{C}_{11} \mathrm{H}_{22}$} & 1-undecene & & & & {$[821-95-4]$} \\
\hline & $(283-312)$ & $54.3 \pm 0.3$ & $(298)$ & GS & {$[00 / 7]$} \\
\hline & & 55.4 & $(298)$ & & {$[71 / 28]$} \\
\hline & $(378-473)$ & 48.2 & (393) & A & {$[87 / 5][50 / 6]$} \\
\hline \multirow[t]{2}{*}{$\mathrm{C}_{11} \mathrm{H}_{22}$} & cis 2-undecene & & & & {$[821-96-5]$} \\
\hline & $(333-393)$ & 53.2 & $(348)$ & A & {$[87 / 5]$} \\
\hline \multirow[t]{2}{*}{$\mathrm{C}_{11} \mathrm{H}_{22}$} & trans 2-undecene & & & & {$[693-61-8]$} \\
\hline & $(333-393)$ & 53.0 & $(348)$ & A & {$[87 / 5]$} \\
\hline \multirow[t]{2}{*}{$\mathrm{C}_{11} \mathrm{H}_{22}$} & cis 3-undecene & & & & {$[821-97-6]$} \\
\hline & $(333-393)$ & 52.3 & $(348)$ & A & {$[87 / 5]$} \\
\hline \multirow[t]{2}{*}{$\mathrm{C}_{11} \mathrm{H}_{22}$} & trans 3-undecene & & & & {$[1002-68-2]$} \\
\hline & $(333-393)$ & 52.0 & $(348)$ & A & {$[87 / 5]$} \\
\hline \multirow[t]{2}{*}{$\mathrm{C}_{11} \mathrm{H}_{22}$} & cis 4-undecene & & & & {$[821-98-7]$} \\
\hline & $(333-393)$ & 51.6 & $(348)$ & A & {$[87 / 5]$} \\
\hline \multirow[t]{2}{*}{$\mathrm{C}_{11} \mathrm{H}_{22}$} & trans 4-undecene & & & & {$[693-62-9]$} \\
\hline & $(333-393)$ & 52.1 & $(348)$ & A & {$[87 / 5]$} \\
\hline \multirow[t]{2}{*}{$\mathrm{C}_{11} \mathrm{H}_{22}$} & cis 5-undecene & & & & {$[764-96-5]$} \\
\hline & $(333-393)$ & 51.4 & $(348)$ & A & {$[87 / 5]$} \\
\hline $\mathrm{C}_{11} \mathrm{H}_{22}$ & trans 5-undecene & & & & [764-97-6] \\
\hline & $(333-393)$ & 51.8 & $(348)$ & A & {$[87 / 5]$} \\
\hline $\mathrm{C}_{11} \mathrm{H}_{22}$ & 3-methyl-3-propyl-1-heptene & & & & \\
\hline & $(263-293)$ & $52.8 \pm 1.0$ & $(278)$ & HSA & {$[95 / 27]$} \\
\hline & & 50.9 & $(298)$ & & {$[95 / 27]$} \\
\hline & & 51.5 & $(298)$ & CGC & {$[95 / 27]$} \\
\hline $\mathrm{C}_{11} \mathrm{H}_{22} \mathrm{Cl}_{2}$ & 1,1-dichloroundecane & & & & {$[822-01-5]$} \\
\hline & $(430-500)$ & 59.5 & $(445)$ & & {$[99 / 16][87 / 12]$} \\
\hline & $(430-500)$ & 71.7 & $(298)$ & & {$[87 / 12][91 / 2]$} \\
\hline $\mathrm{C}_{11} \mathrm{H}_{22} \mathrm{~N}_{2}$ & bis(piperidino)methane & & & & {$[880-09-1]$} \\
\hline & $(283-322)$ & $61.9 \pm 0.9$ & $(303)$ & GS & {$[02 / 28]$} \\
\hline & $(283-322)$ & $62.2 \pm 0.9$ & $(298)$ & GS & {$[02 / 28]$} \\
\hline $\mathrm{C}_{11} \mathrm{H}_{22} \mathrm{O}$ & 1-hexylcyclopentanol & & & & {$[36633-49-5]$} \\
\hline & $(387-509)$ & 59.2 & $(402)$ & A & {$[87 / 5]$} \\
\hline $\mathrm{C}_{11} \mathrm{H}_{22} \mathrm{O}$ & cyclohexyl tert-amyl ether & & & & \\
\hline & & $54.3 \pm 0.2$ & $(298)$ & & {$[02 / 32]$} \\
\hline $\mathrm{C}_{11} \mathrm{H}_{22} \mathrm{O}$ & 2-undecanone & & & & [112-12-9] \\
\hline & $(461-538)$ & 51.5 & $(476)$ & A & [87/5] \\
\hline & & $69.7 \pm 0.5$ & $(298)$ & GCC & {$[79 / 7]$} \\
\hline & & $67.0 \pm 0.4$ & $(298)$ & $\mathrm{C}$ & {$[79 / 1]$} \\
\hline & $(393-523)$ & 56.2 & $(408)$ & A & {$[87 / 5][75 / 8]$} \\
\hline & & 46.4 & $(506)$ & & {$[75 / 8]$} \\
\hline & $(335-433)$ & 61.6 & $(350)$ & $\mathrm{A}, \mathrm{EB}$ & {$[87 / 5][66 / 12]$} \\
\hline & $(341-497)$ & 61.9 & $(356)$ & & {$[47 / 5]$} \\
\hline $\mathrm{C}_{11} \mathrm{H}_{22} \mathrm{O}$ & 6-undecanone & & & & {$[927-49-1]$} \\
\hline & $(343-383)$ & 59.0 & $(298)$ & CGC & [95/21] \\
\hline & $(343-383)$ & 61.8 & (298) & CGC & {$[95 / 21]$} \\
\hline & $(388-543)$ & 55.3 & $(403)$ & A & {$[87 / 5]$} \\
\hline
\end{tabular}


TABLE 6. Enthalpies of vaporization of organic compounds, 1880-2002-Continued

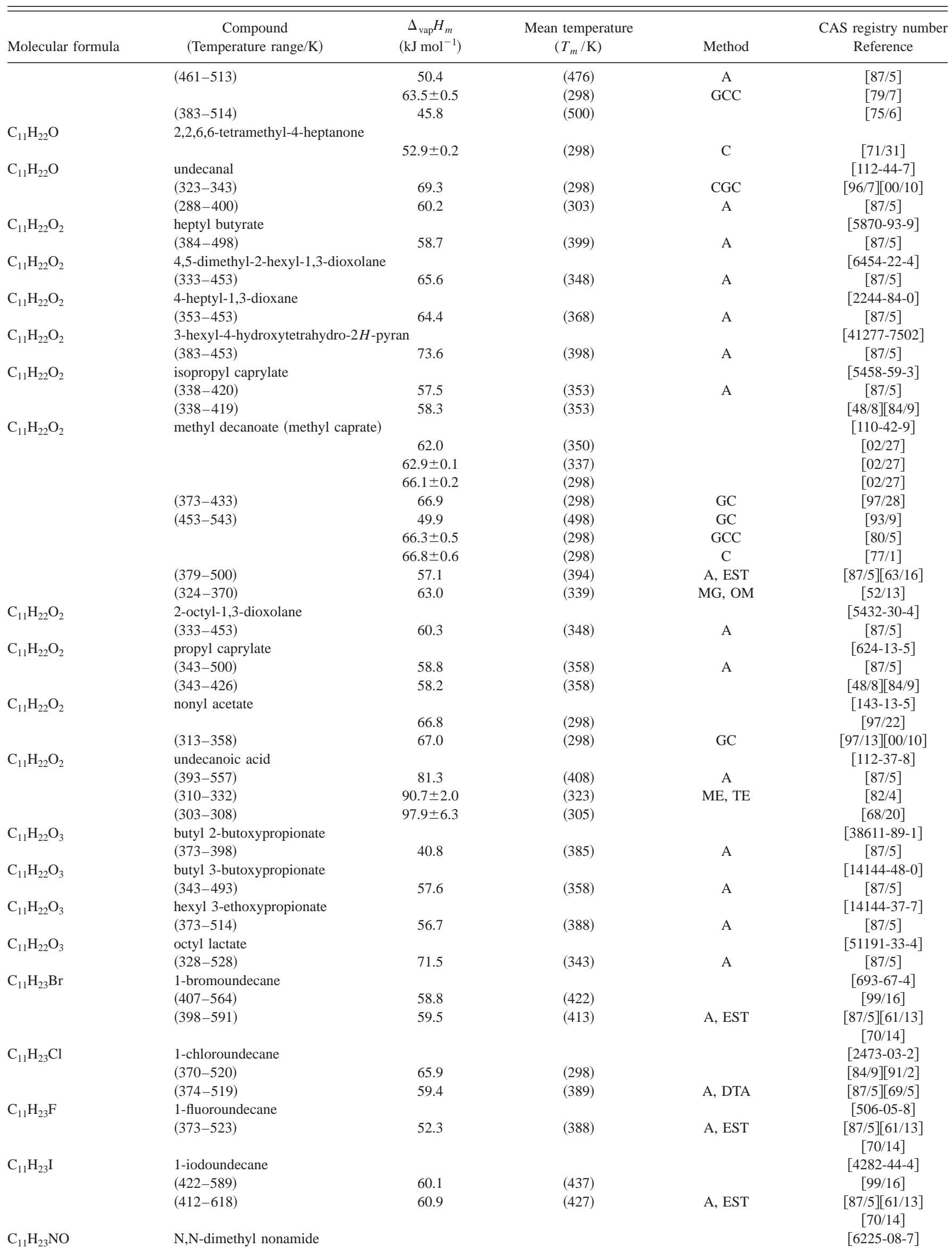


TABLE 6. Enthalpies of vaporization of organic compounds, 1880-2002-Continued

\begin{tabular}{|c|c|c|c|c|c|}
\hline Molecular formula & $\begin{array}{c}\text { Compound } \\
\text { (Temperature range/K) }\end{array}$ & $\begin{array}{c}\Delta_{\mathrm{vap}} H_{m} \\
\left(\mathrm{~kJ} \mathrm{~mol}^{-1}\right)\end{array}$ & $\begin{array}{l}\text { Mean temperature } \\
\qquad\left(T_{m} / \mathrm{K}\right)\end{array}$ & Method & $\begin{array}{c}\text { CAS registry number } \\
\text { Reference }\end{array}$ \\
\hline & $(411-509)$ & 69.3 & $(426)$ & A & [87/5] \\
\hline \multirow[t]{2}{*}{$\mathrm{C}_{11} \mathrm{H}_{23} \mathrm{NO}_{2}$} & $\mathrm{~N}, \mathrm{~N}$-dibutyl lactamide & & & & [6288-16-0] \\
\hline & $(393-418)$ & 88.3 & $(405)$ & A & {$[87 / 5]$} \\
\hline $\mathrm{C}_{11} \mathrm{H}_{23} \mathrm{NO}_{2}$ & $\begin{array}{l}\text { N-octyl lactamide } \\
(428-468)\end{array}$ & 96.3 & (443) & A & {$[87 / 5]$} \\
\hline \multirow[t]{10}{*}{$\mathrm{C}_{11} \mathrm{H}_{24}$} & undecane & & & & [1120-21-4] \\
\hline & & 56.2 & (299) & $\mathrm{C}$ & {$[96 / 22]$} \\
\hline & & 55.4 & (314) & $\mathrm{C}$ & {$[96 / 22]$} \\
\hline & & 54.5 & (324) & $\mathrm{C}$ & {$[96 / 22]$} \\
\hline & & 54.0 & (334) & $\mathrm{C}$ & {$[96 / 22]$} \\
\hline & & 53.1 & (344) & $\mathrm{C}$ & {$[96 / 22]$} \\
\hline & & 56.6 & (298) & & {$[94 / 12]$} \\
\hline & $(278-470)$ & 60.0 & (293) & A & {$[87 / 5]$} \\
\hline & & 56.3 & (298) & & {$[71 / 28]$} \\
\hline & $(378-470)$ & 49.1 & (393) & & {$[55 / 7]$} \\
\hline \multirow[t]{7}{*}{$\mathrm{C}_{11} \mathrm{H}_{24}$} & 2-methyldecane & & & & [6975-98-0] \\
\hline & $(273-353)$ & 55.5 & $(288)$ & A & {$[87 / 5]$} \\
\hline & $(379-463)$ & 47.4 & (394) & $\mathrm{A}$ & {$[87 / 5]$} \\
\hline & & 51.9 & (328) & $\mathrm{C}$ & [84/8] \\
\hline & & 50.6 & (343) & $\mathrm{C}$ & {$[84 / 8]$} \\
\hline & & 49.5 & (358) & $\mathrm{C}$ & {$[84 / 8]$} \\
\hline & $(273-293)$ & 55.4 & (283) & IPM & {$[74 / 11]$} \\
\hline \multirow[t]{2}{*}{$\mathrm{C}_{11} \mathrm{H}_{24}$} & 3-methyldecane & & & & [13151-34-3] \\
\hline & $(340-464)$ & 46.5 & (355) & A & {$[87 / 5]$} \\
\hline \multirow[t]{5}{*}{$\mathrm{C}_{11} \mathrm{H}_{24}$} & 4-methyldecane & & & & [2847-72-5] \\
\hline & $(339-460)$ & 46.6 & $(354)$ & A & {$[87 / 5]$} \\
\hline & & 50.4 & (343) & $\mathrm{C}$ & {$[84 / 8]$} \\
\hline & & 49.2 & $(358)$ & $\mathrm{C}$ & {$[84 / 8]$} \\
\hline & & 48.5 & $(368)$ & $\mathrm{C}$ & {$[84 / 8]$} \\
\hline \multirow[t]{2}{*}{$\mathrm{C}_{11} \mathrm{H}_{24}$} & 5-methyldecane & & & & [13151-35-4] \\
\hline & $(334-452)$ & 46.0 & (349) & $\mathrm{A}$ & {$[87 / 5]$} \\
\hline \multirow{2}{*}{$\mathrm{C}_{11} \mathrm{H}_{24}$} & 2,3-dimethylnonane & & & & [2884-06-2] \\
\hline & $(336-460)$ & 45.1 & $(351)$ & A & {$[87 / 5]$} \\
\hline \multirow[t]{2}{*}{$\mathrm{C}_{11} \mathrm{H}_{24}$} & 2,4-dimethylnonane & & & & [17302-24-8] \\
\hline & $(334-452)$ & 46.8 & (349) & A & {$[87 / 5]$} \\
\hline \multirow[t]{2}{*}{$\mathrm{C}_{11} \mathrm{H}_{24}$} & 2,4,6-trimethyloctane & & & & [62016-37-9] \\
\hline & $(325-442)$ & 44.9 & $(340)$ & $\mathrm{A}$ & {$[87 / 5]$} \\
\hline \multirow[t]{4}{*}{$\mathrm{C}_{11} \mathrm{H}_{24}$} & 2,4,7-trimethyloctane & & & & [62016-38-0] \\
\hline & & 47.6 & (328) & $\mathrm{C}$ & {$[84 / 8]$} \\
\hline & & 46.4 & (343) & $\mathrm{C}$ & {$[84 / 8]$} \\
\hline & & 45.3 & (358) & $\mathrm{C}$ & [84/8] \\
\hline \multirow[t]{6}{*}{$\mathrm{C}_{11} \mathrm{H}_{24} \mathrm{O}$} & decyl methyl ether & & & & [7289-52-3] \\
\hline & $(341-429)$ & 56.9 & $(356)$ & A & {$[87 / 5]$} \\
\hline & $(341-471)$ & 57.0 & $(356)$ & & {$[87 / 5][76 / 2]$} \\
\hline & $(341-471)$ & 62.6 & (298) & & {$[76 / 2]$} \\
\hline & $(341-471)$ & 45.5 & (489) & & {$[76 / 2]$} \\
\hline & & $62.3 \pm 0.3$ & (298) & $\mathrm{C}$ & {$[75 / 3]$} \\
\hline \multirow[t]{2}{*}{$\mathrm{C}_{11} \mathrm{H}_{24} \mathrm{O}$} & ethyl nonyl ether & & & & [16979-32-1] \\
\hline & & $60.3 \pm 0.1$ & $(298)$ & $\mathrm{C}$ & {$[85 / 2]$} \\
\hline \multirow[t]{2}{*}{$\mathrm{C}_{11} \mathrm{H}_{24} \mathrm{O}$} & propyl octyl ether & & & & [29379-41-7] \\
\hline & & $58.8 \pm 0.1$ & $(298)$ & $\mathrm{C}$ & {$[85 / 2]$} \\
\hline $\mathrm{C}_{11} \mathrm{H}_{24} \mathrm{O}$ & butyl heptyl ether & & & & [71112-90-8] \\
\hline & & $58.2 \pm 0.1$ & $(298)$ & $\mathrm{C}$ & {$[85 / 2]$} \\
\hline $\mathrm{C}_{11} \mathrm{H}_{24} \mathrm{O}$ & heptyl tert-butyl ether & & & & \\
\hline & & 56.6 & $(298)$ & & {$[\mathrm{U} / 2][02 / 32]$} \\
\hline $\mathrm{C}_{11} \mathrm{H}_{24} \mathrm{O}$ & hexyl tert-amyl ether & & & & \\
\hline & & 58.6 & $(298)$ & & {$[\mathrm{U} / 2][02 / 32]$} \\
\hline $\mathrm{C}_{11} \mathrm{H}_{24} \mathrm{O}$ & propyl tert-octyl ether & & & & \\
\hline & & $50.1 \pm 0.3$ & $(298)$ & & {$[\mathrm{U} / 2][02 / 32]$} \\
\hline $\mathrm{C}_{11} \mathrm{H}_{24} \mathrm{O}$ & 1-undecanol & & & & {$[112-42-5]$} \\
\hline & $(313-354)$ & 79.5 & $(336)$ & GS & {$[01 / 3]$} \\
\hline & $(313-354)$ & 84.7 & (298) & GS & {$[01 / 3]$} \\
\hline & $(373-423)$ & 86.8 & (298) & CGC & {$[95 / 21]$} \\
\hline & $(353-393)$ & 85.6 & (298) & CGC & {$[94 / 13][00 / 10]$} \\
\hline
\end{tabular}


TABLE 6. Enthalpies of vaporization of organic compounds, 1880-2002-Continued

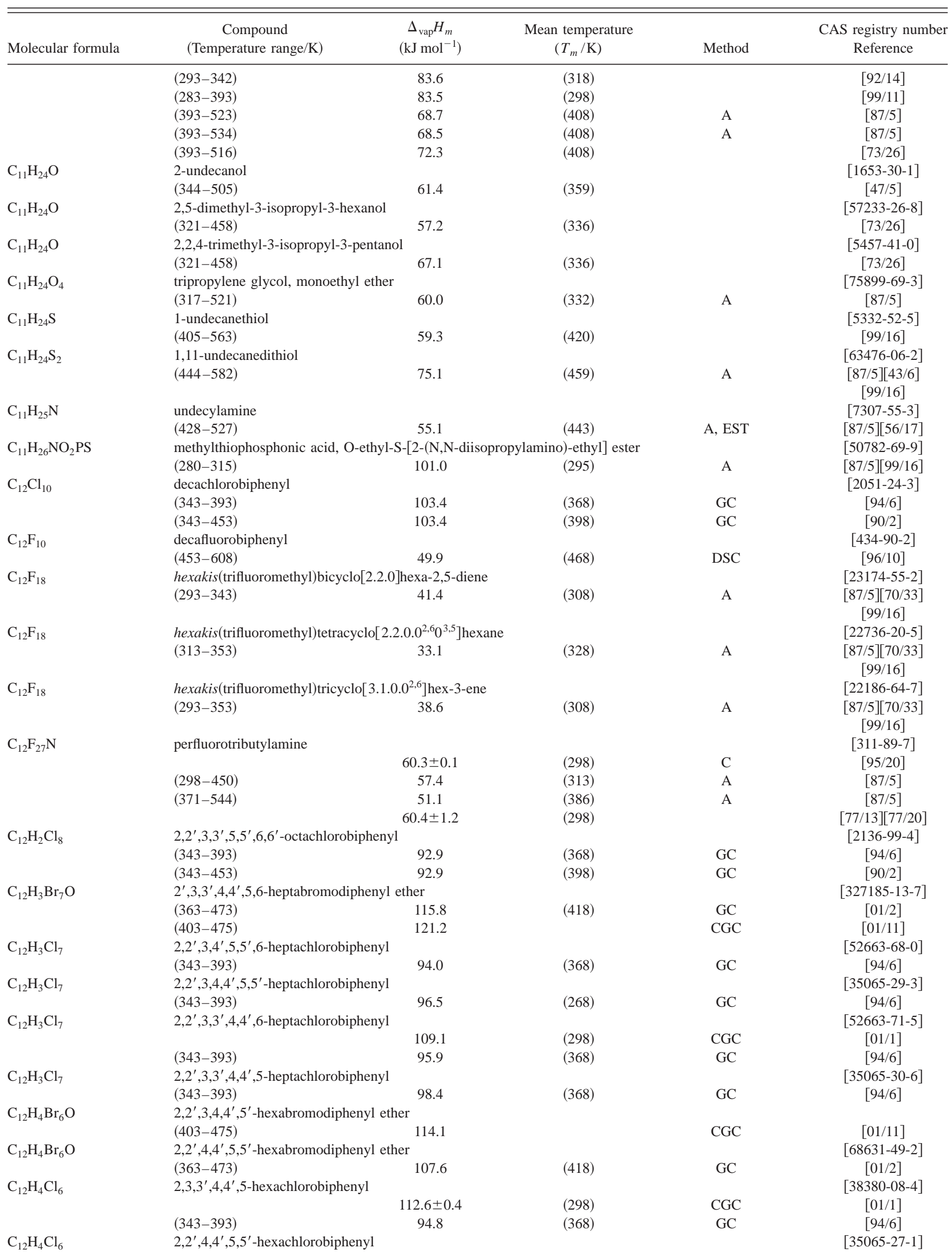


TABLE 6. Enthalpies of vaporization of organic compounds, 1880-2002-Continued

\begin{tabular}{|c|c|c|c|c|c|}
\hline Molecular formula & $\begin{array}{c}\text { Compound } \\
\text { (Temperature range/K) }\end{array}$ & $\begin{array}{c}\Delta_{\mathrm{vap}} H_{m} \\
\left(\mathrm{~kJ} \mathrm{~mol}^{-1}\right)\end{array}$ & $\begin{array}{l}\text { Mean temperature } \\
\qquad\left(T_{m} / \mathrm{K}\right)\end{array}$ & Method & $\begin{array}{l}\text { CAS registry number } \\
\text { Reference }\end{array}$ \\
\hline & & $103.5 \pm 0.1$ & $(298)$ & CGC & {$[01 / 1]$} \\
\hline & $(343-393)$ & 91.4 & $(368)$ & $\mathrm{GC}$ & {$[94 / 6]$} \\
\hline \multirow[t]{2}{*}{$\mathrm{C}_{12} \mathrm{H}_{4} \mathrm{Cl}_{6}$} & $2,2^{\prime}, 3,4^{\prime}, 5^{\prime}, 6$-hexachlorobiphenyl & & & & [38380-04-0] \\
\hline & $(343-393)$ & 89.8 & $(368)$ & GC & {$[94 / 6]$} \\
\hline \multirow[t]{2}{*}{$\mathrm{C}_{12} \mathrm{H}_{4} \mathrm{Cl}_{6}$} & $2,2^{\prime}, 3,4,4^{\prime}, 5^{\prime}$-hexachlorobiphenyl & & & & [35065-28-2] \\
\hline & $(343-393)$ & 91.9 & $(368)$ & GC & {$[94 / 6]$} \\
\hline $\mathrm{C}_{12} \mathrm{H}_{4} \mathrm{Cl}_{6}$ & $\begin{array}{l}2,2^{\prime}, 3,3^{\prime}, 4,4^{\prime} \text {-hexachlorobiphenyl } \\
(343-393)\end{array}$ & 93.5 & (368) & GC & $\begin{array}{c}{[38380-07-3]} \\
{[94 / 6]}\end{array}$ \\
\hline $\mathrm{C}_{12} \mathrm{H}_{5} \mathrm{Br}_{5} \mathrm{O}$ & $\begin{array}{l}2,2^{\prime}, 3,4,4^{\prime} \text {-pentabromodiphenyl ether } \\
(403-475)\end{array}$ & 111.0 & & CGC & [01/11] \\
\hline $\mathrm{C}_{12} \mathrm{H}_{5} \mathrm{Br}_{5} \mathrm{O}$ & $\begin{array}{l}2,2^{\prime}, 3,3^{\prime}, 4 \text {-pentabromodiphenyl ether } \\
(363-473)\end{array}$ & 99.1 & $(418)$ & GC & $\begin{array}{c}{[327185-11-5]} \\
{[01 / 2]}\end{array}$ \\
\hline \multirow[t]{3}{*}{$\mathrm{C}_{12} \mathrm{H}_{5} \mathrm{Br}_{5} \mathrm{O}$} & $2,2^{\prime}, 4,4^{\prime}, 5$-pentabromodiphenyl ether & & & & [60348-60-9] \\
\hline & $(363-473)$ & 100.3 & $(418)$ & $\mathrm{GC}$ & {$[01 / 2]$} \\
\hline & $(405-475)$ & 104.8 & & CGC & {$[01 / 11]$} \\
\hline \multirow[t]{2}{*}{$\mathrm{C}_{12} \mathrm{H}_{5} \mathrm{Br}_{5} \mathrm{O}$} & $2,2^{\prime}, 4,4^{\prime}, 6$-pentabromodiphenyl ether & & & & [189084-66-0] \\
\hline & $(363-473)$ & 101.8 & $(418)$ & GC & {$[01 / 2]$} \\
\hline $\mathrm{C}_{12} \mathrm{H}_{5} \mathrm{Cl}_{5}$ & $\begin{array}{l}2,3^{\prime}, 4,4^{\prime}, 5 \text {-pentachlorobiphenyl } \\
(343-393)\end{array}$ & 89.3 & (368) & $\mathrm{GC}$ & $\begin{array}{c}{[31508-00-6]} \\
{[94 / 6]}\end{array}$ \\
\hline $\mathrm{C}_{12} \mathrm{H}_{5} \mathrm{Cl}_{5}$ & $\begin{array}{l}2,3,3^{\prime}, 4,4^{\prime} \text {-pentachlorobiphenyl } \\
(343-393)\end{array}$ & 91.1 & (368) & $\mathrm{GC}$ & $\begin{array}{c}{[32598-14-4]} \\
{[94 / 6]}\end{array}$ \\
\hline \multirow[t]{3}{*}{$\mathrm{C}_{12} \mathrm{H}_{5} \mathrm{Cl}_{5}$} & $2,2^{\prime}, 4,5,5^{\prime}$-pentachlorobiphenyl & & & & [37680-73-2] \\
\hline & $(343-393)$ & 86.4 & $(368)$ & GC & {$[94 / 6]$} \\
\hline & $(343-453)$ & 83.7 & (398) & GC & {$[90 / 2]$} \\
\hline \multirow[t]{2}{*}{$\mathrm{C}_{12} \mathrm{H}_{5} \mathrm{Cl}_{5}$} & $2,2^{\prime}, 3,5^{\prime}, 6$-pentachlorobiphenyl & & & & [38379-99-6] \\
\hline & & $92.3 \pm 0.6$ & $(298)$ & CGC & {$[01 / 1]$} \\
\hline \multirow[t]{2}{*}{$\mathrm{C}_{12} \mathrm{H}_{5} \mathrm{Cl}_{5}$} & $2,2^{\prime}, 3,6,6^{\prime}$-pentachlorobiphenyl & & & & [73575-54-9] \\
\hline & & $89.6 \pm 0.2$ & $(298)$ & CGC & {$[01 / 1]$} \\
\hline \multirow[t]{2}{*}{$\mathrm{C}_{12} \mathrm{H}_{5} \mathrm{Cl}_{5}$} & $2,2^{\prime}, 4,5^{\prime}, 6$-pentachlorobiphenyl & & & & [60145-21-3] \\
\hline & & $91.6 \pm 0.5$ & $(298)$ & CGC & {$[01 / 1]$} \\
\hline \multirow{2}{*}{$\mathrm{C}_{12} \mathrm{H}_{5} \mathrm{Cl}_{5}$} & $2,2^{\prime}, 3,4,5^{\prime}$-pentachlorobiphenyl & & & & [38380-02-8] \\
\hline & $(343-393)$ & 87.3 & (368) & $\mathrm{GC}$ & {$[94 / 6]$} \\
\hline \multirow[t]{2}{*}{$\mathrm{C}_{12} \mathrm{H}_{5} \mathrm{Cl}_{5}$} & $2,2^{\prime}, 4,4^{\prime}, 5$-pentachlorobiphenyl & & & & {$[38380-01-7]$} \\
\hline & $(343-393)$ & 86.8 & $(368)$ & GC & {$[94 / 6]$} \\
\hline \multirow[t]{3}{*}{$\mathrm{C}_{12} \mathrm{H}_{6} \mathrm{Br}_{4} \mathrm{O}$} & $2,2^{\prime}, 4,4^{\prime}$-tetrabromodiphenyl ether & & & & {$[5436-43-1]$} \\
\hline & $(363-473)$ & 92.0 & $(418)$ & GC & {$[01 / 2]$} \\
\hline & $(403-475)$ & 103.1 & & CGC & {$[01 / 1]$} \\
\hline $\mathrm{C}_{12} \mathrm{H}_{6} \mathrm{Br}_{4} \mathrm{O}$ & $2,3^{\prime}, 4,4^{\prime}$-tetrabromodiphenyl ether & & & & [189084-61-5] \\
\hline & $(363-473)$ & 93.5 & $(418)$ & GC & {$[01 / 2]$} \\
\hline $\mathrm{C}_{12} \mathrm{H}_{6} \mathrm{Br}_{4} \mathrm{O}$ & $2,3^{\prime}, 4,6$-tetrabromodiphenyl ether & & & & [327185-09-1] \\
\hline & $(363-473)$ & 91.1 & $(418)$ & $\mathrm{GC}$ & {$[01 / 2]$} \\
\hline $\mathrm{C}_{12} \mathrm{H}_{6} \mathrm{Br}_{4} \mathrm{O}$ & $2,4,4^{\prime}, 6$-tetrabromodiphenyl ether & & & & [189084-63-7] \\
\hline & $(363-473)$ & 90.1 & (418) & $\mathrm{GC}$ & {$[01 / 2]$} \\
\hline $\mathrm{C}_{12} \mathrm{H}_{6} \mathrm{Br}_{4} \mathrm{O}$ & $3,3^{\prime}, 4,4^{\prime}$-tetrabromodiphenyl ether & & & & [93703-48-1] \\
\hline & $(363-473)$ & 95.3 & $(418)$ & $\mathrm{GC}$ & {$[01 / 2]$} \\
\hline $\mathrm{C}_{12} \mathrm{H}_{6} \mathrm{Cl}_{4}$ & $2,2^{\prime}, 3,3^{\prime}$-tetrachlorobiphenyl & & & & [38444-93-8] \\
\hline & $(343-398)$ & 81.8 & $(368)$ & GC & {$[94 / 6]$} \\
\hline $\mathrm{C}_{12} \mathrm{H}_{6} \mathrm{Cl}_{4}$ & $2,2^{\prime}, 5,5^{\prime}$-tetrachlorobiphenyl & & & & [35693-99-3] \\
\hline & $(343-398)$ & 80.8 & $(368)$ & GC & {$[94 / 6]$} \\
\hline & $(343-453)$ & 79.0 & $(398)$ & GC & {$[90 / 2]$} \\
\hline $\mathrm{C}_{12} \mathrm{H}_{6} \mathrm{Cl}_{4}$ & $2,3^{\prime}, 4,4^{\prime}$-tetrachlorobiphenyl & & & & {$[32598-10-0]$} \\
\hline & $(343-398)$ & 83.3 & (368) & $\mathrm{GC}$ & [94/6] \\
\hline $\mathrm{C}_{12} \mathrm{H}_{6} \mathrm{Cl}_{4}$ & $2,3^{\prime}, 4^{\prime}, 5$-tetrachlorobiphenyl & & & & [32598-11-1] \\
\hline & $(343-398)$ & 84.8 & $(368)$ & $\mathrm{GC}$ & {$[94 / 6]$} \\
\hline $\mathrm{C}_{12} \mathrm{H}_{6} \mathrm{Cl}_{4}$ & $2,2^{\prime}, 4,5^{\prime}$-tetrachlorobiphenyl & & & & [41464-40-8] \\
\hline & & $87.4 \pm 0.8$ & (298) & $\mathrm{CGC}$ & {$[01 / 1]$} \\
\hline $\mathrm{C}_{12} \mathrm{H}_{6} \mathrm{Cl}_{4}$ & $2,2^{\prime}, 5,6^{\prime}$-tetrachlorobiphenyl & & & & [41464-41-9] \\
\hline & & $84.9 \pm 0.6$ & $(298)$ & $\mathrm{CGC}$ & {$[01 / 1]$} \\
\hline & $(343-398)$ & 78.8 & $(368)$ & GC & {$[94 / 6]$} \\
\hline $\mathrm{C}_{12} \mathrm{H}_{6} \mathrm{Cl}_{4}$ & $3,3^{\prime}, 4,4^{\prime}$-tetrachlorobiphenyl & & & & [32598-13-3] \\
\hline & $(343-393)$ & 87.2 & $(368)$ & GC & {$[94 / 6]$} \\
\hline $\mathrm{C}_{12} \mathrm{H}_{7} \mathrm{Br}_{3} \mathrm{O}$ & $2,4,4^{\prime}$-tribromodiphenyl ether & & & & \\
\hline & $(403-475)$ & 94.1 & & $\mathrm{CGC}$ & {$[01 / 11]$} \\
\hline $\mathrm{C}_{12} \mathrm{H}_{7} \mathrm{Br}_{3} \mathrm{O}$ & $3,4,4^{\prime}$-tribromodiphenyl ether & & & & [147217-81-0] \\
\hline
\end{tabular}


TABLE 6. Enthalpies of vaporization of organic compounds, 1880-2002-Continued

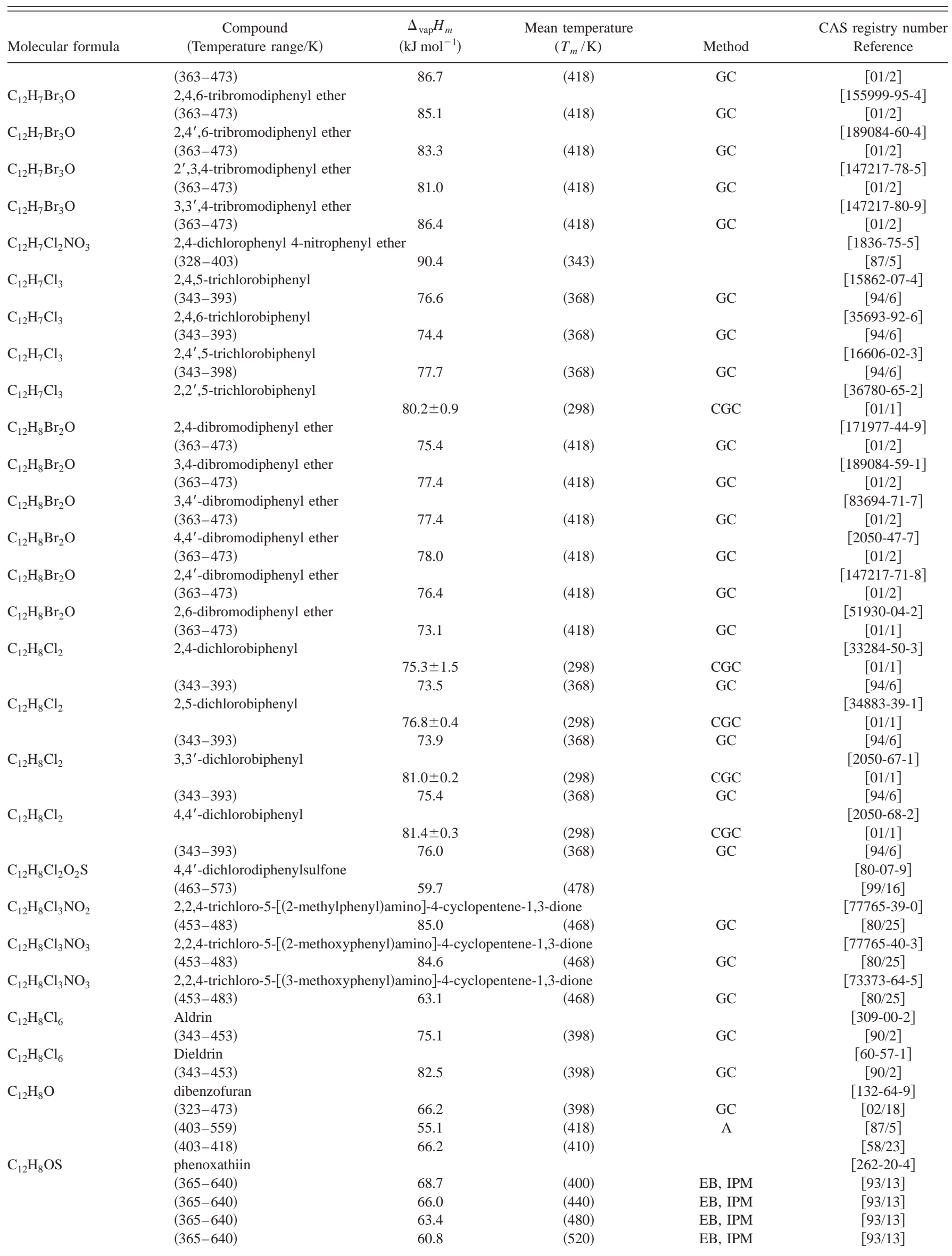


TABLE 6. Enthalpies of vaporization of organic compounds, 1880-2002-Continued

\begin{tabular}{|c|c|c|c|c|c|}
\hline Molecular formula & $\begin{array}{c}\text { Compound } \\
\text { (Temperature range/K) }\end{array}$ & $\begin{array}{c}\Delta_{\mathrm{vap}} H_{m} \\
\left(\mathrm{~kJ} \mathrm{~mol}^{-1}\right)\end{array}$ & $\begin{array}{l}\text { Mean temperature } \\
\qquad\left(T_{m} / \mathrm{K}\right)\end{array}$ & Method & $\begin{array}{c}\text { CAS registry number } \\
\text { Reference }\end{array}$ \\
\hline & $(365-640)$ & 58.0 & $(560)$ & EB, IPM & [93/13] \\
\hline & $(365-640)$ & 55.1 & $(600)$ & EB, IPM & {$[93 / 13]$} \\
\hline \multirow[t]{21}{*}{$\mathrm{C}_{12} \mathrm{H}_{8} \mathrm{~S}$} & dibenzothiophene & & & & [132-64-0] \\
\hline & $(373-424)$ & 65.6 & (388) & & [99/16] \\
\hline & $(424-608)$ & 63.4 & $(439)$ & & {$[99 / 16]$} \\
\hline & & 69.5 & $(380)$ & & {$[95 / 24]$} \\
\hline & & 66.8 & $(420)$ & & {$[95 / 24]$} \\
\hline & & 64.3 & $(460)$ & & {$[95 / 24]$} \\
\hline & & 61.8 & $(500)$ & & {$[95 / 24]$} \\
\hline & & 59.3 & $(540)$ & & {$[95 / 24]$} \\
\hline & & 56.8 & $(580)$ & & {$[95 / 24]$} \\
\hline & & 54.0 & $(620)$ & & {$[95 / 24]$} \\
\hline & & 68.0 & $(400)$ & & {$[91 / 10]$} \\
\hline & & 64.9 & $(450)$ & & {$[91 / 10]$} \\
\hline & & 61.8 & $(500)$ & & {$[91 / 10]$} \\
\hline & & 58.7 & $(550)$ & & {$[91 / 10]$} \\
\hline & & 55.4 & $(600)$ & & {$[91 / 10]$} \\
\hline & & 51.8 & $(650)$ & & [91/10] \\
\hline & $(385-574)$ & 60.1 & $(400)$ & A & {$[87 / 5]$} \\
\hline & & 56.9 & $(590)$ & $\mathrm{C}$ & {$[84 / 3]$} \\
\hline & & 55.3 & $(610)$ & $\mathrm{C}$ & {$[84 / 3]$} \\
\hline & & 53.6 & $(630)$ & $\mathrm{C}$ & {$[84 / 3]$} \\
\hline & $(373-403)$ & 69.4 & $(385)$ & & {$[81 / 2]$} \\
\hline \multirow[t]{11}{*}{$\mathrm{C}_{12} \mathrm{H}_{8} \mathrm{~S}_{2}$} & thianthrene & & & & {$[92-85-3]$} \\
\hline & $(429-460)$ & 71.2 & $(444)$ & & [99/16] \\
\hline & $(460-539)$ & 68.4 & $(475)$ & & [99/16] \\
\hline & $(395-639)$ & 72.7 & $(440)$ & EB, IPM & {$[93 / 13]$} \\
\hline & $(395-639)$ & 69.9 & $(480)$ & EB, IPM & {$[93 / 13]$} \\
\hline & $(395-639)$ & 67.2 & $(520)$ & EB, IPM & {$[93 / 13]$} \\
\hline & $(395-639)$ & 64.5 & $(560)$ & EB, IPM & {$[93 / 13]$} \\
\hline & $(395-639)$ & 61.7 & $(600)$ & EB, IPM & {$[93 / 13]$} \\
\hline & $(430-593)$ & 69.1 & $(465)$ & & {$[83 / 4]$} \\
\hline & $(430-593)$ & 68.7 & $(515)$ & & {$[83 / 4]$} \\
\hline & $(428-448)$ & 71.1 & $(438)$ & & {$[81 / 2]$} \\
\hline \multirow[t]{2}{*}{$\mathrm{C}_{12} \mathrm{H}_{9} \mathrm{Br}$} & 4-bromobiphenyl & & & & [92-66-0] \\
\hline & $(371-583)$ & 62.2 & $(386)$ & A & {$[87 / 5][47 / 5]$} \\
\hline \multirow{2}{*}{$\mathrm{C}_{12} \mathrm{H}_{9} \mathrm{BrO}$} & 2-bromodiphenyl ether & & & & {$[7025-06-1]$} \\
\hline & $(363-473)$ & 63.7 & $(418)$ & $\mathrm{GC}$ & {$[01 / 2]$} \\
\hline \multirow[t]{2}{*}{$\mathrm{C}_{12} \mathrm{H}_{9} \mathrm{BrO}$} & 3-bromodiphenyl ether & & & & {$[6876-00-2]$} \\
\hline & $(363-473)$ & 65.4 & $(418)$ & GC & {$[01 / 2]$} \\
\hline \multirow[t]{2}{*}{$\mathrm{C}_{12} \mathrm{H}_{9} \mathrm{BrO}$} & 4-bromodiphenyl ether & & & & {$[101-55-3]$} \\
\hline & $(463-673)$ & 64.6 & $(478)$ & A & {$[87 / 5]$} \\
\hline \multirow[t]{2}{*}{$\mathrm{C}_{12} \mathrm{H}_{9} \mathrm{BrO}$} & 2-bromo-4-phenylphenol & & & & [92-03-5] \\
\hline & $(373-584)$ & 57.8 & $(388)$ & A & {$[87 / 5][47 / 5]$} \\
\hline \multirow[t]{7}{*}{$\mathrm{C}_{12} \mathrm{H}_{9} \mathrm{Cl}$} & 2-chlorobiphenyl & & & & {$[2051-60-7]$} \\
\hline & & $72.1 \pm 2.0$ & $(298)$ & CGC & {$[01 / 1]$} \\
\hline & $(343-393)$ & 64.4 & $(368)$ & GC & {$[94 / 6]$} \\
\hline & $(409-540)$ & 57.8 & $(424)$ & A & {$[87 / 5]$} \\
\hline & $(306-350)$ & 74.5 & $(328)$ & $\mathrm{ME}$ & {$[83 / 9]$} \\
\hline & $(410-540)$ & 55.8 & $(424)$ & QM & {$[75 / 28]$} \\
\hline & $(362-541)$ & 61.1 & $(377)$ & A & {$[87 / 5][47 / 5]$} \\
\hline \multirow[t]{6}{*}{$\mathrm{C}_{12} \mathrm{H}_{9} \mathrm{Cl}$} & 3-chlorobiphenyl & & & & [2051-61-8] \\
\hline & & $74.3 \pm 1.1$ & $(298)$ & CGC & {$[01 / 1]$} \\
\hline & $(343-393)$ & 66.6 & $(368)$ & GC & {$[94 / 6]$} \\
\hline & $(310-359)$ & 66.2 & $(335)$ & ME & {$[83 / 9]$} \\
\hline & $(341-402)$ & 69.2 & $(372)$ & $\mathrm{TE}$ & {$[83 / 9]$} \\
\hline & $(452-536)$ & 66.0 & $(494)$ & QM & {$[75 / 28]$} \\
\hline \multirow[t]{6}{*}{$\mathrm{C}_{12} \mathrm{H}_{9} \mathrm{Cl}$} & 4-chlorobiphenyl & & & & [2051-62-9] \\
\hline & & $71.6 \pm 0.7$ & $(298)$ & CGC & {$[01 / 1]$} \\
\hline & $(343-393)$ & 66.8 & $(368)$ & GC & {$[94 / 6]$} \\
\hline & $(451-536)$ & 65.9 & $(466)$ & & {$[87 / 5]$} \\
\hline & $(348-409)$ & 67.8 & $(378)$ & $\mathrm{TE}$ & [83/9] \\
\hline & $(369-566)$ & 59.0 & $(384)$ & A & {$[87 / 5][47 / 5]$} \\
\hline $\mathrm{C}_{12} \mathrm{H}_{9} \mathrm{ClO}$ & 2-chloro-3-phenylphenol & & & & \\
\hline
\end{tabular}


TABLE 6. Enthalpies of vaporization of organic compounds, 1880-2002-Continued

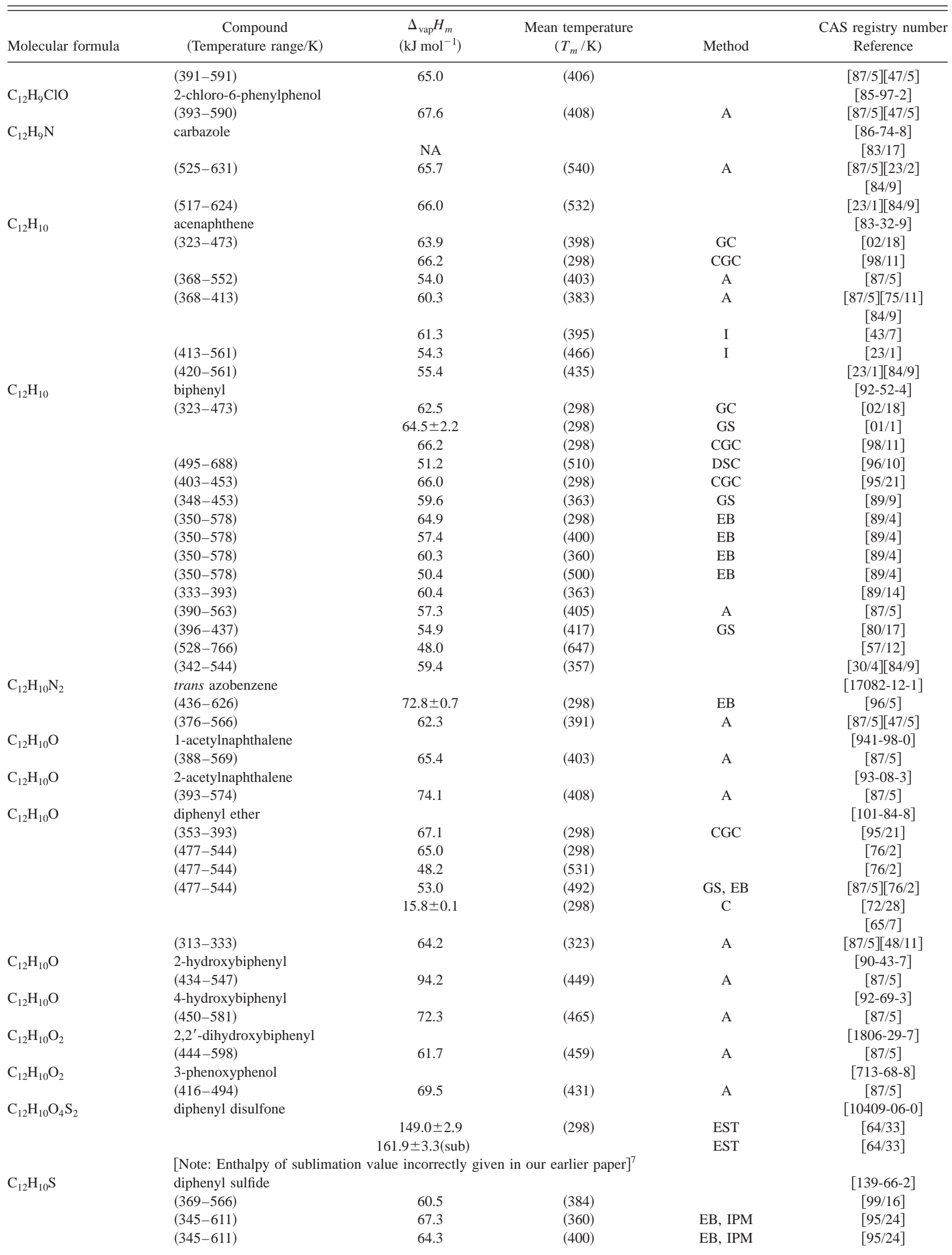


TABLE 6. Enthalpies of vaporization of organic compounds, 1880-2002-Continued

\begin{tabular}{|c|c|c|c|c|c|}
\hline Molecular formula & $\begin{array}{c}\text { Compound } \\
\text { (Temperature range/K) }\end{array}$ & $\begin{array}{c}\Delta_{\mathrm{vap}} H_{m} \\
\left(\mathrm{~kJ} \mathrm{~mol}^{-1}\right)\end{array}$ & $\begin{array}{l}\text { Mean temperature } \\
\qquad\left(T_{m} / \mathrm{K}\right)\end{array}$ & Method & $\begin{array}{c}\text { CAS registry number } \\
\text { Reference }\end{array}$ \\
\hline & $(345-611)$ & 61.3 & $(440)$ & EB, IPM & {$[95 / 24]$} \\
\hline & $(345-611)$ & 58.3 & $(480)$ & EB, IPM & {$[95 / 24]$} \\
\hline & $(345-611)$ & 55.3 & $(520)$ & EB, IPM & {$[95 / 24]$} \\
\hline & $(345-611)$ & 52.0 & $(560)$ & $\mathrm{EB}, \mathrm{IPM}$ & {$[95 / 24]$} \\
\hline & $(369-566)$ & 58.2 & $(384)$ & A & {$[87 / 5][49 / 5]$} \\
\hline \multirow[t]{4}{*}{$\mathrm{C}_{12} \mathrm{H}_{10} \mathrm{~S}_{2}$} & diphenyl disulfide & & & & {$[882-33-7]$} \\
\hline & $(405-583)$ & 72.4 & $(420)$ & & [99/16] \\
\hline & & $78.7 \pm 2.9$ & $(298)$ & & {$[62 / 16]$} \\
\hline & $(404-583)$ & 74.4 & $(419)$ & & {$[87 / 5][47 / 5]$} \\
\hline \multirow[t]{7}{*}{$\mathrm{C}_{12} \mathrm{H}_{11} \mathrm{~N}$} & 2-aminobiphenyl & & & & [90-41-5] \\
\hline & $(340-623)$ & 68.6 & $(400)$ & EB, IPM & [91/14] \\
\hline & $(340-623)$ & 65.1 & $(440)$ & EB, IPM & {$[91 / 14]$} \\
\hline & $(340-623)$ & 61.8 & $(480)$ & EB, IPM & [91/14] \\
\hline & $(340-623)$ & 58.5 & $(520)$ & EB, IPM & {$[91 / 14]$} \\
\hline & $(340-623)$ & 55.2 & $(560)$ & EB, IPM & {$[91 / 14]$} \\
\hline & $(340-623)$ & 51.7 & $(600)$ & EB, IPM & [91/14] \\
\hline \multirow[t]{3}{*}{$\mathrm{C}_{12} \mathrm{H}_{11} \mathrm{~N}$} & diphenylamine & & & & [122-39-4] \\
\hline & $(381-575)$ & 64.1 & (396) & A & {$[87 / 5][47 / 5]$} \\
\hline & $(573-673)$ & 54.2 & $(588)$ & A & {$[87 / 5]$} \\
\hline \multirow[t]{2}{*}{$\mathrm{C}_{12} \mathrm{H}_{12}$} & 1,5-dimethylnaphthalene & & & & {$[571-61-7]$} \\
\hline & $(323-473)$ & 64.1 & $(398)$ & GC & [02/18] \\
\hline \multirow[t]{2}{*}{$\mathrm{C}_{12} \mathrm{H}_{12}$} & 1,6-dimethylnaphthalene & & & & {$[575-43-9]$} \\
\hline & $(323-473)$ & 63.6 & $(398)$ & GC & {$[02 / 18]$} \\
\hline \multirow[t]{3}{*}{$\mathrm{C}_{12} \mathrm{H}_{12}$} & 1,8-dimethylnaphthalene & & & & {$[569-41-5]$} \\
\hline & $(338-413)$ & 62.8 & $(353)$ & A & $\begin{array}{c}{[87 / 5][75 / 11]} \\
{[84 / 9]}\end{array}$ \\
\hline & & 64.8 & $(336)$ & & {$[77 / 22]$} \\
\hline \multirow[t]{3}{*}{$\mathrm{C}_{12} \mathrm{H}_{12}$} & 2,3-dimethylnaphthalene & & & & {$[581-40-8]$} \\
\hline & & $60.9 \pm 0.7$ & $(380)$ & & {$[88 / 16]$} \\
\hline & $(378-408)$ & 60.0 & $(393)$ & A & {$[87 / 5]$} \\
\hline \multirow[t]{2}{*}{$\mathrm{C}_{12} \mathrm{H}_{12}$} & 2,6-dimethylnaphthalene & & & & {$[581-42-0]$} \\
\hline & $(384-418)$ & 57.3 & $(399)$ & A & $\begin{array}{c}{[87 / 5][75 / 11]} \\
{[84 / 9]}\end{array}$ \\
\hline \multirow[t]{7}{*}{$\mathrm{C}_{12} \mathrm{H}_{12}$} & 2,7-dimethylnaphthalene & & & & [582-16-1] \\
\hline & & 57.3 & $(400)$ & & [93/11] \\
\hline & & 54.8 & $(440)$ & & {$[93 / 11]$} \\
\hline & & 52.2 & $(480)$ & & {$[93 / 11]$} \\
\hline & & 49.5 & $(520)$ & & {$[93 / 11]$} \\
\hline & & 46.6 & $(560)$ & & [93/11] \\
\hline & $(369-400)$ & 58.5 & $(384)$ & A & $\begin{array}{c}{[87 / 5][75 / 11]} \\
{[84 / 9]}\end{array}$ \\
\hline \multirow[t]{2}{*}{$\mathrm{C}_{12} \mathrm{H}_{12}$} & 1-ethylnaphthalene & & & & {$[1127-76-0]$} \\
\hline & $(393-565)$ & 57.3 & $(408)$ & A, GS & {$[87 / 5][79 / 11]$} \\
\hline \multirow[t]{5}{*}{$\mathrm{C}_{12} \mathrm{H}_{12}$} & 2-ethylnaphthalene & & & & [939-27-5] \\
\hline & $(323-473)$ & 64.7 & $(398)$ & GC & [02/18] \\
\hline & $(269-398)$ & 69.3 & $(284)$ & & {$[88 / 12]$} \\
\hline & $(286-319)$ & 61.9 & $(301)$ & A & {$[87 / 5]$} \\
\hline & $(393-565)$ & 56.7 & $(408)$ & A & {$[87 / 5]$} \\
\hline \multirow[t]{2}{*}{$\mathrm{C}_{12} \mathrm{H}_{12} \mathrm{~N}_{2}$} & 1,1-diphenylhydrazine & & & & {$[530-50-7]$} \\
\hline & $(399-596)$ & 68.8 & $(414)$ & A & {$[87 / 5][47 / 5]$} \\
\hline \multirow[t]{2}{*}{$\mathrm{C}_{12} \mathrm{H}_{12} \mathrm{O}_{6}$} & 1,2,3-benzenetricarboxylic & ethyl ester & & & {$[2672-57-3]$} \\
\hline & $(453-513)$ & 72.5 & $(468)$ & A, GS & {$[87 / 5][63 / 13]$} \\
\hline \multirow[t]{3}{*}{$\mathrm{C}_{12} \mathrm{H}_{12} \mathrm{O}_{6}$} & 1,2,4-benzenetricarboxylic & ethyl ester & & & [28904-30-5] \\
\hline & & $78.5 \pm 0.4$ & $(399)$ & C & {$[98 / 6]$} \\
\hline & $(443-493)$ & 61.1 & $(458)$ & A, GS & {$[87 / 5][63 / 13]$} \\
\hline \multirow[t]{2}{*}{$\mathrm{C}_{12} \mathrm{H}_{12} \mathrm{O}_{6}$} & 1,3,5-benzenetricarboxylic & ethyl ester & & & {$[2672-58-4]$} \\
\hline & $(443-513)$ & 75.4 & $(458)$ & A & {$[87 / 5]$} \\
\hline $\mathrm{C}_{12} \mathrm{H}_{13} \mathrm{Cl}_{3} \mathrm{O}_{3}$ & 2,4,5-trichlorophenoxyace & tyl ester & & & {$[93-79-8]$} \\
\hline & $(460-573)$ & 87.3 & $(475)$ & A & {$[87 / 5]$} \\
\hline $\mathrm{C}_{12} \mathrm{H}_{14} \mathrm{Cl}_{2}$ & cyclohexyl-3,4-dichlorobe & & & & \\
\hline & $(383-488)$ & 64.7 & $(398)$ & & {$[81 / 20]$} \\
\hline $\mathrm{C}_{12} \mathrm{H}_{14} \mathrm{Cl}_{2} \mathrm{O}_{3}$ & 2,4-dichlorophenoxyacetic & 1 ester & & & [94-80-4] \\
\hline & $(444-573)$ & 76.3 & $(459)$ & A & {$[87 / 5][99 / 16]$} \\
\hline $\mathrm{C}_{12} \mathrm{H}_{14} \mathrm{Cl}_{2} \mathrm{O}_{3}$ & 2,4-dichlorophenoxyacetic & butyl ester & & & [94-79-1] \\
\hline
\end{tabular}


TABLE 6. Enthalpies of vaporization of organic compounds, 1880-2002-Continued

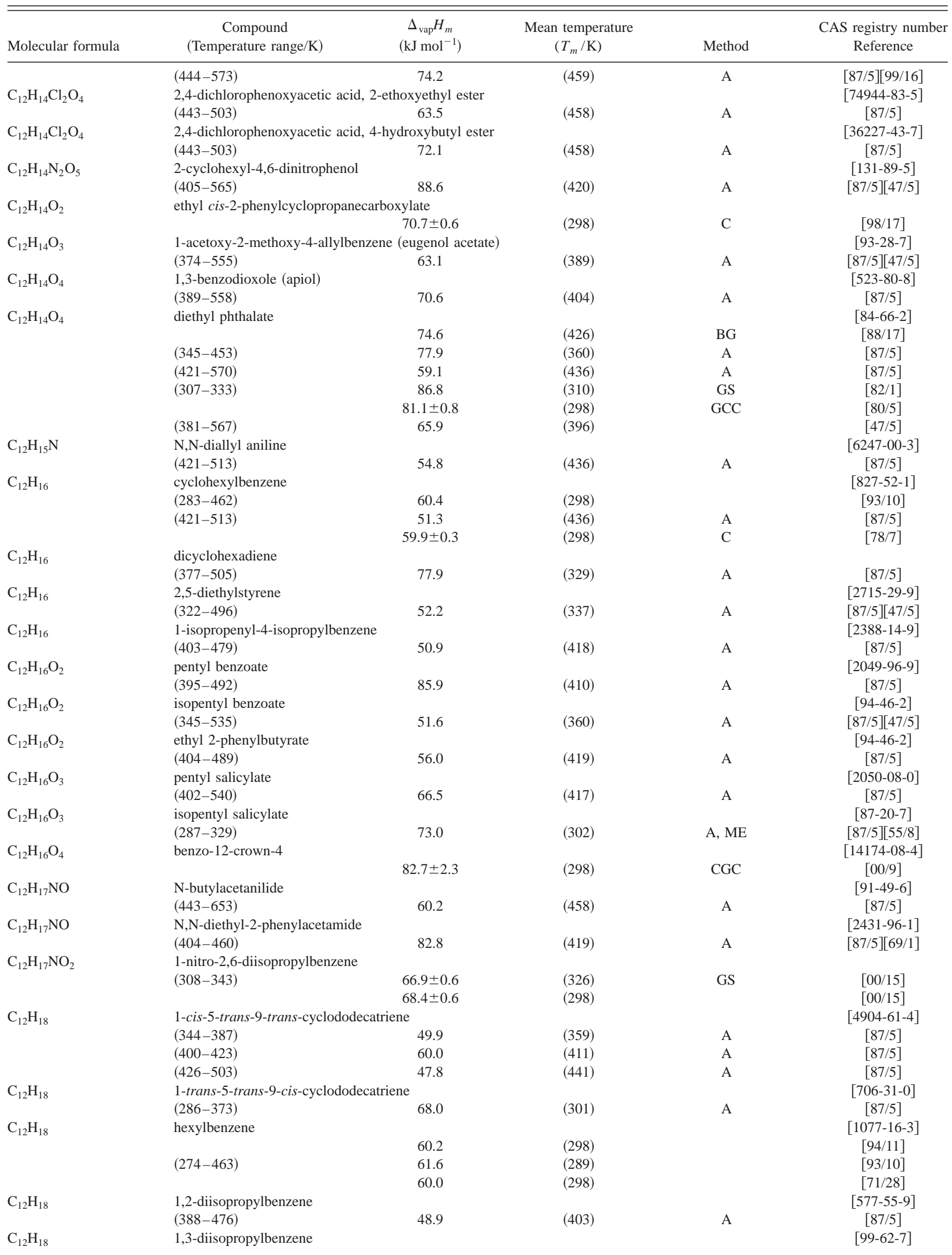


TABLE 6. Enthalpies of vaporization of organic compounds, 1880-2002-Continued

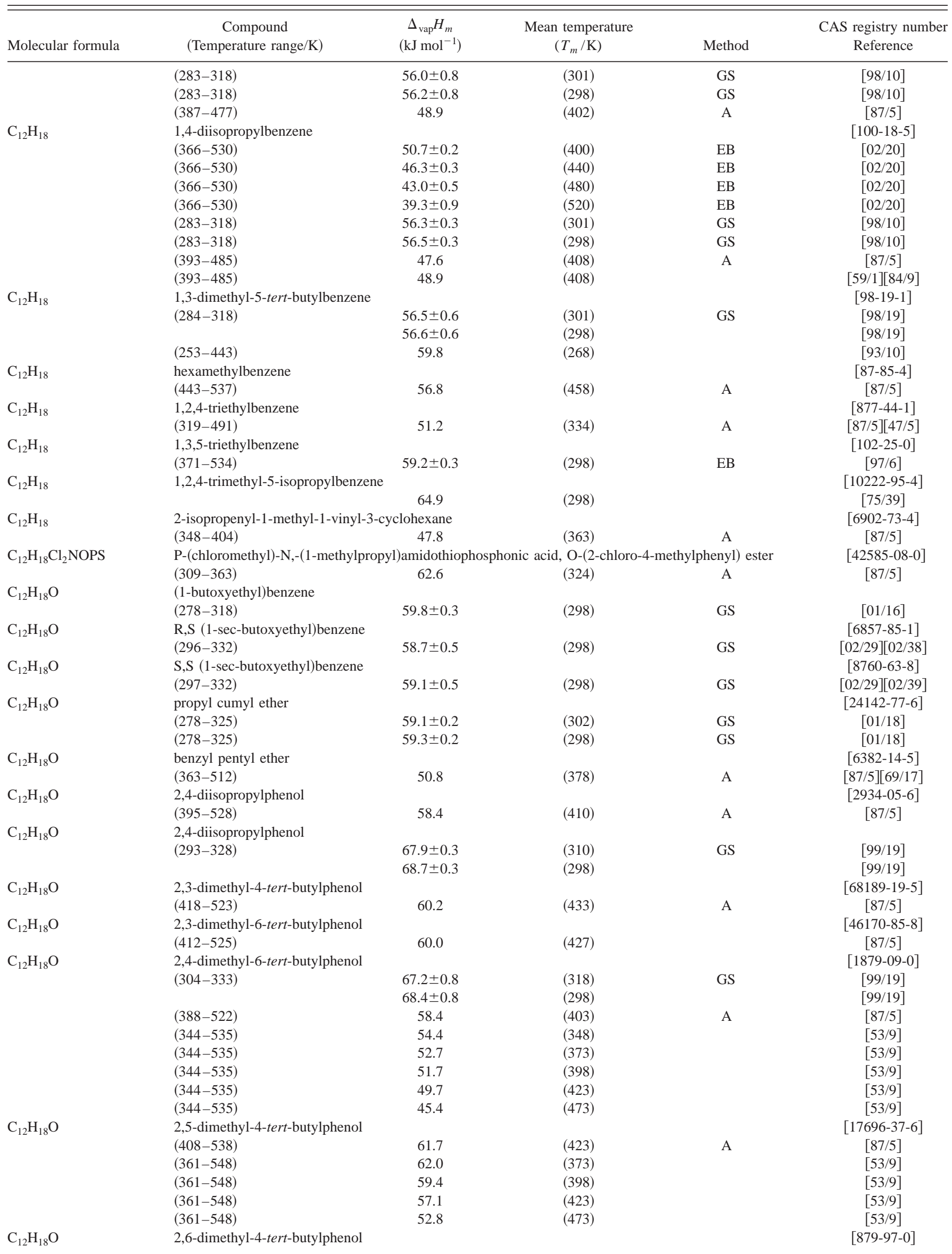


TABLE 6. Enthalpies of vaporization of organic compounds, 1880-2002-Continued

\begin{tabular}{|c|c|c|c|c|c|}
\hline Molecular formula & $\begin{array}{c}\text { Compound } \\
\text { (Temperature range/K) }\end{array}$ & $\begin{array}{c}\Delta_{\mathrm{vap}} H_{m} \\
\left(\mathrm{~kJ} \mathrm{~mol}^{-1}\right)\end{array}$ & $\begin{array}{l}\text { Mean temperature } \\
\qquad\left(T_{m} / \mathrm{K}\right)\end{array}$ & Method & $\begin{array}{c}\text { CAS registry number } \\
\text { Reference }\end{array}$ \\
\hline & $(392-522)$ & 59.7 & $(407)$ & A & {$[87 / 5]$} \\
\hline & $(347-530)$ & 58.4 & $(348)$ & & {$[53 / 9]$} \\
\hline & $(347-530)$ & 57.0 & $(373)$ & & {$[53 / 9]$} \\
\hline & $(347-530)$ & 55.4 & $(398)$ & & {$[53 / 9]$} \\
\hline & $(347-530)$ & 54.2 & $(423)$ & & {$[53 / 9]$} \\
\hline & $(347-530)$ & 49.3 & $(473)$ & & {$[53 / 9]$} \\
\hline \multirow[t]{2}{*}{$\mathrm{C}_{12} \mathrm{H}_{18} \mathrm{O}$} & 3,4-dimethyl-6-tert-butylphenol & & & & {$[1445-23-4]$} \\
\hline & $(413-532)$ & 62.7 & $(428)$ & A & {$[87 / 5]$} \\
\hline \multirow[t]{5}{*}{$\mathrm{C}_{12} \mathrm{H}_{18} \mathrm{O}$} & 2-ethyl-4-tert-butylphenol & & & & {$[63452-61-9]$} \\
\hline & $(428-623)$ & 61.6 & $(443)$ & A & {$[87 / 5]$} \\
\hline & $(397-543)$ & 55.4 & $(398)$ & & {$[53 / 9]$} \\
\hline & $(397-543)$ & 54.2 & $(423)$ & & {$[53 / 9]$} \\
\hline & $(397-543)$ & 49.3 & $(473)$ & & {$[53 / 9]$} \\
\hline \multirow[t]{2}{*}{$\mathrm{C}_{12} \mathrm{H}_{18} \mathrm{O}$} & 2-ethyl-6-tert-butylphenol & & & & {$[63551-41-7]$} \\
\hline & $(393-443)$ & 58.2 & $(408)$ & A & {$[87 / 5]$} \\
\hline \multirow{2}{*}{$\mathrm{C}_{12} \mathrm{H}_{18} \mathrm{O}$} & 3-ethyl-6-tert-butylphenol & & & & {$[4237-25-6]$} \\
\hline & $(415-530)$ & 59.5 & $(430)$ & A & {$[87 / 5]$} \\
\hline \multirow[t]{6}{*}{$\mathrm{C}_{12} \mathrm{H}_{18} \mathrm{O}$} & 4-ethyl-2-tert-butylphenol & & & & {$[96-70-8]$} \\
\hline & $(394-523)$ & 59.2 & $(409)$ & A & {$[87 / 5]$} \\
\hline & $(349-533)$ & 57.0 & $(373)$ & & {$[53 / 9]$} \\
\hline & $(349-533)$ & 55.4 & $(398)$ & & {$[53 / 9]$} \\
\hline & $(349-533)$ & 54.2 & $(423)$ & & {$[53 / 9]$} \\
\hline & $(349-533)$ & 49.3 & $(473)$ & & {$[53 / 9]$} \\
\hline \multirow[t]{4}{*}{$\mathrm{C}_{12} \mathrm{H}_{18} \mathrm{O}$} & 2-methyl-4-tert-pentylphenol & & & & {$[71745-63-6]$} \\
\hline & $(443-653)$ & 65.6 & $(458)$ & A & {$[87 / 5]$} \\
\hline & $(409-561)$ & 55.3 & $(423)$ & & {$[53 / 9]$} \\
\hline & $(409-561)$ & 50.7 & $(473)$ & & {$[53 / 9]$} \\
\hline \multirow[t]{4}{*}{$\mathrm{C}_{12} \mathrm{H}_{18} \mathrm{O}$} & 3-methyl-4-tert-pentylphenol & & & & \\
\hline & $(443-683)$ & 65.1 & $(458)$ & A & {$[87 / 5]$} \\
\hline & $(409-561)$ & 55.3 & $(423)$ & & {$[53 / 9]$} \\
\hline & $(409-561)$ & 50.7 & $(473)$ & & {$[53 / 9]$} \\
\hline \multirow[t]{5}{*}{$\mathrm{C}_{12} \mathrm{H}_{18} \mathrm{O}$} & 4-methyl-2-tert-pentylphenol & & & & {$[34072-71-4]$} \\
\hline & $(423-653)$ & 61.4 & $(438)$ & A & {$[87 / 5]$} \\
\hline & $(394-538)$ & 58.1 & $(398)$ & & {$[53 / 9]$} \\
\hline & $(394-538)$ & 55.3 & $(423)$ & & {$[53 / 9]$} \\
\hline & $(394-538)$ & 50.7 & $(473)$ & & {$[53 / 9]$} \\
\hline \multirow[t]{2}{*}{$\mathrm{C}_{12} \mathrm{H}_{18} \mathrm{O}_{2}$} & 1,3-dihydroxy-2-hexylbenzene & & & & {$[5673-09-6]$} \\
\hline & $(433-494)$ & 76.8 & $(448)$ & $\mathrm{A}, \mathrm{GC}$ & {$[87 / 5][75 / 24]$} \\
\hline \multirow[t]{2}{*}{$\mathrm{C}_{12} \mathrm{H}_{18} \mathrm{O}_{2}$} & 1,3-dihydroxy-4-hexylbenzene & & & & {$[136-77-6]$} \\
\hline & $(434-494)$ & 88.1 & $(449)$ & $\mathrm{A}, \mathrm{GC}$ & {$[87 / 5][75 / 24]$} \\
\hline \multirow[t]{2}{*}{$\mathrm{C}_{12} \mathrm{H}_{18} \mathrm{O}_{4}$} & 3,4-dihydro-2,2-dimethyl-4-oxo & pyran-6-carb & cid, butyl ester & & {$[532-34-3]$} \\
\hline & $(357-435)$ & 64.7 & $(372)$ & A & {$[87 / 5]$} \\
\hline \multirow[t]{2}{*}{$\mathrm{C}_{12} \mathrm{H}_{18} \mathrm{O}_{6}$} & triethyl aconitrate & & & & \\
\hline & $(423-540)$ & 79.6 & $(438)$ & A & {$[87 / 5]$} \\
\hline \multirow[t]{3}{*}{$\mathrm{C}_{12} \mathrm{H}_{19} \mathrm{~N}$} & 2,6-diisopropylaniline & & & & {$[24544-04-5]$} \\
\hline & $(284-323)$ & $69.2 \pm 0.3$ & $(303)$ & & {$[00 / 14]$} \\
\hline & & $69.5 \pm 0.3$ & $(298)$ & & {$[00 / 14]$} \\
\hline \multirow[t]{3}{*}{$\mathrm{C}_{12} \mathrm{H}_{19} \mathrm{~N}$} & N-methyl-3-methyl-3-phenyl-2- & leamine & & & \\
\hline & $(283-330)$ & $67.0 \pm 0.8$ & $(307)$ & GS & {$[98 / 1]$} \\
\hline & $(283-330)$ & $67.5 \pm 0.8$ & $(298)$ & GS & {$[98 / 1]$} \\
\hline $\mathrm{C}_{12} \mathrm{H}_{19} \mathrm{NO}$ & 2-(dimethylamino)-2-methyl-1-1 & lpropanone & & & \\
\hline & $(298-338)$ & $66.7 \pm 0.4$ & $(298)$ & GS & {$[94 / 3]$} \\
\hline $\mathrm{C}_{12} \mathrm{H}_{19} \mathrm{NO}$ & 2-(diethylamino)-1-phenylethan & & & & \\
\hline & $(293-338)$ & $71.6 \pm 0.9$ & $(298)$ & GS & {$[94 / 3]$} \\
\hline $\mathrm{C}_{12} \mathrm{H}_{19} \mathrm{~F}_{3} \mathrm{~N}_{2} \mathrm{O}_{4}$ & $\mathrm{~N}[\mathrm{~N}$-(trifluoroacetyl)valyl $]$ alani & nyl ester & & & \\
\hline & $(425-453)$ & 86.4 & $(439)$ & A & {$[87 / 5][99 / 16]$} \\
\hline $\mathrm{C}_{12} \mathrm{H}_{20}$ & 1-ethyladamantane & & & & {$[770-69-4]$} \\
\hline & & $55.3 \pm 1.1$ & $(298)$ & & {$[00 / 22]$} \\
\hline & $(383-492)$ & 49.1 & $(398)$ & A & {$[87 / 5]$} \\
\hline $\mathrm{C}_{12} \mathrm{H}_{20}$ & 1,3-dimethyladamantane & & & & {$[707-79-4]$} \\
\hline & & $49.2 \pm 0.2$ & $(308)$ & $\mathrm{C}$ & {$[01 / 4]$} \\
\hline & & $49.7 \pm 0.2$ & $(298)$ & $\mathrm{C}$ & {$[01 / 4]$} \\
\hline & $(352-526)$ & $49.4 \pm 0.3$ & $(298)$ & $\mathrm{EB}$ & {$[96 / 3]$} \\
\hline & & $67.8 \pm 1.3$ & $(298)$ & EB & {$[77 / 4]$} \\
\hline
\end{tabular}


TABLE 6. Enthalpies of vaporization of organic compounds, 1880-2002-Continued

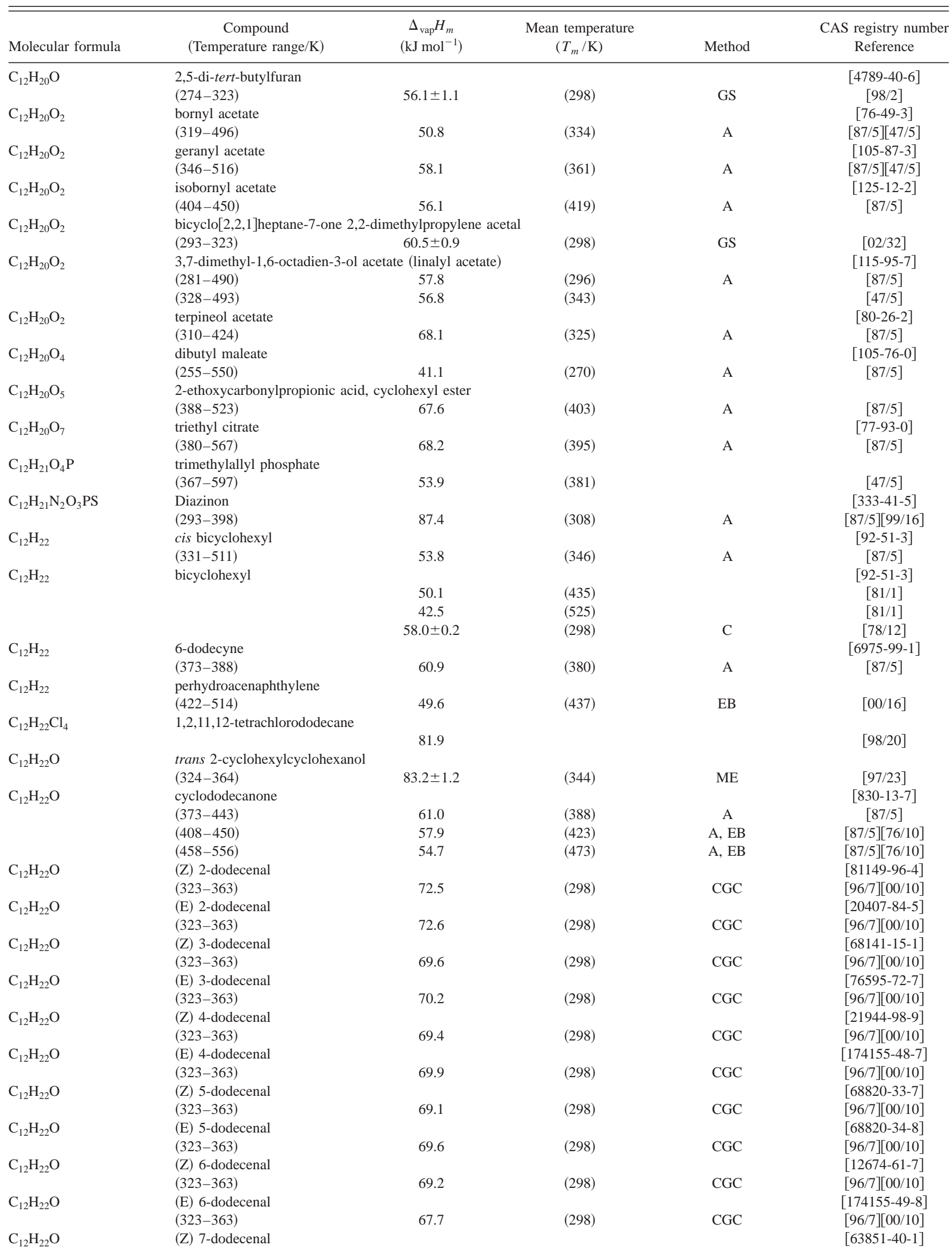


TABLE 6. Enthalpies of vaporization of organic compounds, 1880-2002-Continued

\begin{tabular}{|c|c|c|c|c|c|}
\hline Molecular formula & $\begin{array}{c}\text { Compound } \\
\text { (Temperature range/K) }\end{array}$ & $\begin{array}{c}\Delta_{\mathrm{vap}} H_{m} \\
\left(\mathrm{~kJ} \mathrm{~mol}^{-1}\right)\end{array}$ & $\begin{array}{l}\text { Mean temperature } \\
\qquad\left(T_{m} / \mathrm{K}\right)\end{array}$ & Method & $\begin{array}{c}\text { CAS registry number } \\
\text { Reference }\end{array}$ \\
\hline & $(323-363)$ & 69.4 & $(298)$ & CGC & {$[96 / 7][00 / 10]$} \\
\hline \multirow[t]{2}{*}{$\mathrm{C}_{12} \mathrm{H}_{22} \mathrm{O}$} & (E) 7-dodecenal & & & & [82944-76-1] \\
\hline & $(323-363)$ & 69.6 & $(298)$ & CGC & {$[96 / 7][00 / 10]$} \\
\hline \multirow[t]{2}{*}{$\mathrm{C}_{12} \mathrm{H}_{22} \mathrm{O}$} & (Z) 8-dodecenal & & & & [139909-65-2] \\
\hline & $(323-363)$ & 70.0 & $(298)$ & CGC & {$[96 / 7][00 / 10]$} \\
\hline \multirow[t]{2}{*}{$\mathrm{C}_{12} \mathrm{H}_{22} \mathrm{O}$} & (E) 8-dodecenal & & & & [144298-64-6] \\
\hline & $(323-363)$ & 69.8 & $(298)$ & CGC & {$[96 / 7][00 / 10]$} \\
\hline \multirow[t]{2}{*}{$\mathrm{C}_{12} \mathrm{H}_{22} \mathrm{O}$} & (Z) 9-dodecenal & & & & {$[56219-03-5]$} \\
\hline & $(323-363)$ & 70.1 & $(298)$ & CGC & {$[96 / 7][00 / 10]$} \\
\hline \multirow[t]{2}{*}{$\mathrm{C}_{12} \mathrm{H}_{22} \mathrm{O}$} & (E) 9-dodecenal & & & & {$[155235-07-7]$} \\
\hline & $(323-363)$ & 70.4 & $(298)$ & $\mathrm{CGC}$ & {$[96 / 7][00 / 10]$} \\
\hline \multirow[t]{2}{*}{$\mathrm{C}_{12} \mathrm{H}_{22} \mathrm{O}$} & (Z) 10-dodecenal & & & & {$[81892-61-7]$} \\
\hline & $(323-363)$ & 71.0 & $(298)$ & CGC & {$[96 / 7][00 / 10]$} \\
\hline \multirow[t]{2}{*}{$\mathrm{C}_{12} \mathrm{H}_{22} \mathrm{O}$} & (E) 10-dodecenal & & & & {$[81892-62-8]$} \\
\hline & $(323-363)$ & 70.9 & $(298)$ & CGC & {$[96 / 7][00 / 10]$} \\
\hline \multirow[t]{3}{*}{$\mathrm{C}_{12} \mathrm{H}_{22} \mathrm{O}_{2}$} & dodecanolactone & & & & [947-05-7] \\
\hline & $(377-403)$ & $64.2 \pm 1.1$ & $(390)$ & MM & {$[91 / 7]$} \\
\hline & $(377-403)$ & $70.5 \pm 1.7$ & $(298)$ & MM & {$[91 / 7]$} \\
\hline \multirow[t]{2}{*}{$\mathrm{C}_{12} \mathrm{H}_{22} \mathrm{O}_{2}$} & acetic acid, 4-tert-butylcy & ster & & & \\
\hline & $(285-318)$ & 63.8 & $(300)$ & $\mathrm{A}, \mathrm{ME}$ & $\begin{array}{c}{[87 / 5][58 / 9]} \\
{[57 / 9]}\end{array}$ \\
\hline \multirow[t]{2}{*}{$\mathrm{C}_{12} \mathrm{H}_{22} \mathrm{O}_{2}$} & (d) menthyl acetate & & & & [16409-45-3] \\
\hline & $(330-500)$ & 55.3 & $(345)$ & $\mathrm{A}$ & {$[87 / 5][47 / 5]$} \\
\hline \multirow[t]{2}{*}{$\mathrm{C}_{12} \mathrm{H}_{22} \mathrm{O}_{2}$} & citronellyl acetate & & & & {$[150-84-5]$} \\
\hline & $(347-490)$ & 68.7 & $(362)$ & A & {$[87 / 5][47 / 5]$} \\
\hline \multirow[t]{2}{*}{$\mathrm{C}_{12} \mathrm{H}_{22} \mathrm{O}_{2}$} & 2-(1-ethylpentyl)-4,7-dihy & xepin & & & {$[61732-97-6]$} \\
\hline & $(333-453)$ & 66.3 & $(348)$ & A & {$[87 / 5]$} \\
\hline \multirow[t]{2}{*}{$\mathrm{C}_{12} \mathrm{H}_{22} \mathrm{O}_{2}$} & octyl methacrylate & & & & [2157-01-9] \\
\hline & $(384-513)$ & 55.6 & $(399)$ & A & {$[87 / 5]$} \\
\hline \multirow[t]{2}{*}{$\mathrm{C}_{12} \mathrm{H}_{22} \mathrm{O}_{2}$} & methyl 10 -undecenoate & & & & {$[111-81-9]$} \\
\hline & $(397-524)$ & 59.2 & $(412)$ & A & {$[87 / 5]$} \\
\hline $\mathrm{C}_{12} \mathrm{H}_{22} \mathrm{O}_{2}$ & (Z) 3-decenyl acetate & & & & [81634-99-3] \\
\hline & $(313-358)$ & 69.5 & $(298)$ & GC & {$[97 / 13][00 / 10]$} \\
\hline & $(299-313)$ & 72 & $(306)$ & GC & {$[83 / 10]$} \\
\hline $\mathrm{C}_{12} \mathrm{H}_{22} \mathrm{O}_{2}$ & (E) 3-decenyl acetate & & & & [81634-98-2] \\
\hline & $(313-358)$ & 70.1 & $(298)$ & GC & {$[97 / 13][00 / 10]$} \\
\hline $\mathrm{C}_{12} \mathrm{H}_{22} \mathrm{O}_{2}$ & (Z) 4-decenyl acetate & & & & {$[67452-27-1]$} \\
\hline & $(313-358)$ & 69.0 & $(298)$ & GC & {$[97 / 13][00 / 10]$} \\
\hline $\mathrm{C}_{12} \mathrm{H}_{22} \mathrm{O}_{2}$ & (E) 4-decenyl acetate & & & & [69222-16-8] \\
\hline & $(313-358)$ & 70.1 & $(298)$ & GC & [97/13][00/10] \\
\hline $\mathrm{C}_{12} \mathrm{H}_{22} \mathrm{O}_{2}$ & (Z) 5-decenyl acetate & & & & {$[67446-07-5]$} \\
\hline & $(313-358)$ & 69.7 & $(298)$ & GC & {$[97 / 13][00 / 10]$} \\
\hline & $(299-313)$ & 72 & $(306)$ & GC & {$[83 / 10]$} \\
\hline $\mathrm{C}_{12} \mathrm{H}_{22} \mathrm{O}_{2}$ & (E) 5-decenyl acetate & & & & [38421-90-8] \\
\hline & $(313-358)$ & 70.6 & $(298)$ & GC & {$[97 / 13][00 / 10]$} \\
\hline $\mathrm{C}_{12} \mathrm{H}_{22} \mathrm{O}_{2}$ & (Z) 6-decenyl acetate & & & & {$[68760-70-3]$} \\
\hline & $(313-358)$ & 70.1 & $(298)$ & GC & {$[97 / 13][00 / 10]$} \\
\hline $\mathrm{C}_{12} \mathrm{H}_{22} \mathrm{O}_{2}$ & (E) 6-decenyl acetate & & & & \\
\hline & $(313-358)$ & 70.6 & $(298)$ & GC & {$[97 / 13][00 / 10]$} \\
\hline & $(299-313)$ & 72 & $(306)$ & GC & {$[83 / 10]$} \\
\hline $\mathrm{C}_{12} \mathrm{H}_{22} \mathrm{O}_{2}$ & (Z) 7-decenyl acetate & & & & [13857-03-9] \\
\hline & $(313-358)$ & 70.7 & $(298)$ & $\mathrm{GC}$ & {$[97 / 13][00 / 10]$} \\
\hline $\mathrm{C}_{12} \mathrm{H}_{22} \mathrm{O}_{2}$ & (E) 7-decenyl acetate & & & & [13857-04-0] \\
\hline & $(313-358)$ & 71.1 & $(298)$ & GC & {$[97 / 13][00 / 10]$} \\
\hline $\mathrm{C}_{12} \mathrm{H}_{22} \mathrm{O}_{2}$ & (Z) 8-decenyl acetate & & & & {$[83808-51-9]$} \\
\hline & $(313-358)$ & 71.5 & $(298)$ & GC & {$[97 / 13][00 / 10]$} \\
\hline $\mathrm{C}_{12} \mathrm{H}_{22} \mathrm{O}_{2}$ & (E) 8-decenyl acetate & & & & [83808-51-9] \\
\hline & $(313-358)$ & 71.5 & $(298)$ & GC & {$[97 / 13][00 / 10]$} \\
\hline $\mathrm{C}_{12} \mathrm{H}_{22} \mathrm{O}_{3}$ & heptyl levulinate & & & & \\
\hline & $(393-558)$ & 62.6 & $(408)$ & $\mathrm{A}$ & {$[87 / 5]$} \\
\hline & & 60.0 & $(496)$ & & {$[31 / 1]$} \\
\hline $\mathrm{C}_{12} \mathrm{H}_{22} \mathrm{O}_{3}$ & 3-pentyl-4-acetoxytetrahy & ran & & & [18871-14-2] \\
\hline & $(383-453)$ & 65.8 & $(398)$ & A & {$[87 / 5]$} \\
\hline $\mathrm{C}_{12} \mathrm{H}_{22} \mathrm{O}_{4}$ & dipropyl adipate & & & & [106-19-4] \\
\hline
\end{tabular}


TABLE 6. Enthalpies of vaporization of organic compounds, 1880-2002-Continued

\begin{tabular}{|c|c|c|c|c|c|}
\hline Molecular formula & $\begin{array}{c}\text { Compound } \\
\text { (Temperature range/K) }\end{array}$ & $\begin{array}{c}\Delta_{\mathrm{vap}} H_{m} \\
\left(\mathrm{~kJ} \mathrm{~mol}^{-1}\right)\end{array}$ & $\begin{array}{l}\text { Mean temperature } \\
\qquad\left(T_{m} / \mathrm{K}\right)\end{array}$ & Method & $\begin{array}{c}\text { CAS registry number } \\
\text { Reference }\end{array}$ \\
\hline \multirow{3}{*}{$\mathrm{C}_{12} \mathrm{H}_{22} \mathrm{O}_{4}$} & $(413-540)$ & 63.6 & $(428)$ & A & {$[87 / 5]$} \\
\hline & \multicolumn{2}{|l|}{ isopentylmalonic acid, diethyl ester } & & & {$[5398-08-3]$} \\
\hline & $(377-420)$ & 64.1 & $(392)$ & A & {$[87 / 5]$} \\
\hline \multirow[t]{2}{*}{$\mathrm{C}_{12} \mathrm{H}_{22} \mathrm{O}_{4}$} & \multicolumn{2}{|c|}{ (1-methylbutyl)malonic acid, diethyl ester } & & & {$[22328-91-2]$} \\
\hline & $(395-516)$ & 67.4 & $(410)$ & A & {$[87 / 5]$} \\
\hline \multirow[t]{2}{*}{$\mathrm{C}_{12} \mathrm{H}_{22} \mathrm{O}_{4}$} & \multicolumn{2}{|l|}{ diisopentyl oxalate } & & & {$[2051-00-5]$} \\
\hline & $(358-538)$ & 58.6 & $(373)$ & A & {$[87 / 5][47 / 5]$} \\
\hline \multirow[t]{2}{*}{$\mathrm{C}_{12} \mathrm{H}_{22} \mathrm{O}_{4} \mathrm{~S}$} & \multicolumn{2}{|l|}{ thiodiglycolic acid, diethyl ester } & & & {$[4121-12-4]$} \\
\hline & $(298-383)$ & 75.7 & $(313)$ & A & {$[87 / 5][99 / 16]$} \\
\hline \multirow[t]{2}{*}{$\mathrm{C}_{12} \mathrm{H}_{22} \mathrm{O}_{5}$} & \multicolumn{4}{|c|}{ butyl[1-(butoxycarbonyl)ethyl] carbonate } & \\
\hline & $(338-513)$ & 68.1 & $(353)$ & A & {$[87 / 5]$} \\
\hline \multirow[t]{2}{*}{$\mathrm{C}_{12} \mathrm{H}_{22} \mathrm{O}_{5}$} & \multicolumn{4}{|c|}{ pentyl[1-(ethoxycarbonyl)isopropyl] carbonate } & \\
\hline & $(368-513)$ & 63.8 & $(383)$ & A & {$[87 / 5]$} \\
\hline \multirow[t]{2}{*}{$\mathrm{C}_{12} \mathrm{H}_{22} \mathrm{O}_{6}$} & \multicolumn{4}{|c|}{ lactic acid, O-ethoxycarbonyl, 2-butoxyethyl ester } & \\
\hline & $(383-521)$ & 74.6 & (398) & A & {$[87 / 5]$} \\
\hline $\mathrm{C}_{12} \mathrm{H}_{22} \mathrm{O}_{6}$ & dibutyl tartrate & & & & {$[87-92-3]$} \\
\hline & $(428-511)$ & 79.8 & $(443)$ & A & {$[87 / 5]$} \\
\hline $\mathrm{C}_{12} \mathrm{H}_{22} \mathrm{O}_{6}$ & (d) diisobutyl tartrate & & & & [4054-82-4] \\
\hline & $(390-597)$ & 64.6 & $(405)$ & A & {$[87 / 5]$} \\
\hline $\mathrm{C}_{12} \mathrm{H}_{22} \mathrm{~S}$ & dicyclohexyl sulfide & & & & {$[7133-46-2]$} \\
\hline & $(421-523)$ & $69.0 \pm 0.7$ & $(298)$ & $\mathrm{EB}$ & {$[97 / 7]$} \\
\hline $\mathrm{C}_{12} \mathrm{H}_{23} \mathrm{~N}$ & dicyclohexylamine & & & & {$[101-83-7]$} \\
\hline & $(408-529)$ & 54.0 & $(423)$ & A & {$[87 / 5]$} \\
\hline $\mathrm{C}_{12} \mathrm{H}_{23} \mathrm{~N}$ & lauronitrile & & & & {$[2437-25-4]$} \\
\hline & & $76.1 \pm 0.1$ & $(298)$ & $\mathrm{C}$ & {$[77 / 5]$} \\
\hline & $(393-462)$ & 65.2 & $(408)$ & EB & {$[71 / 4]$} \\
\hline & $(440-556)$ & 60.7 & $(455)$ & $\mathrm{A}, \mathrm{EB}$ & $\begin{array}{c}{[87 / 5][71 / 4]} \\
{[73 / 12]}\end{array}$ \\
\hline $\mathrm{C}_{12} \mathrm{H}_{24}$ & cyclododecane & & & & [294-62-2] \\
\hline & & 63.0 & $(298)$ & CGC & [98/11] \\
\hline & $(403-453)$ & 62.8 & $(298)$ & CGC & {$[95 / 21]$} \\
\hline & $(386-441)$ & 52.6 & $(401)$ & $\mathrm{A}, \mathrm{EB}$ & {$[87 / 5][76 / 10]$} \\
\hline & $(440-529)$ & 49.8 & $(455)$ & $\mathrm{A}, \mathrm{EB}$ & {$[87 / 5][76 / 10]$} \\
\hline $\mathrm{C}_{12} \mathrm{H}_{24}$ & 1-dodecene & & & & [112-41-4] \\
\hline & & $60.8 \pm 0.3$ & $(298)$ & $\mathrm{C}$ & {$[76 / 5][77 / 1]$} \\
\hline & & 60.3 & $(298)$ & & {$[71 / 28]$} \\
\hline & $(396-493)$ & 51.1 & $(411)$ & A & {$[87 / 5][50 / 6]$} \\
\hline $\mathrm{C}_{12} \mathrm{H}_{24}$ & hexylcyclohexane & & & & {$[4292-75-5]$} \\
\hline & & $55.9 \pm 0.5$ & $(298)$ & GC & [87/17] \\
\hline & & $59.0 \pm 0.5$ & $(298)$ & GCC & {$[78 / 16]$} \\
\hline & & 59.9 & $(298)$ & & {$[71 / 28]$} \\
\hline $\mathrm{C}_{12} \mathrm{H}_{24}$ & heptylcyclopentane & & & & {$[5617-42-5]$} \\
\hline & & 60.8 & $(298)$ & & [71/28] \\
\hline $\mathrm{C}_{12} \mathrm{H}_{24}$ & $\operatorname{trans}$ 2,2,4,6,6-pentamethyl-3-hepten & & & & \\
\hline & $(291-318)$ & $65.6 \pm 0.5$ & $(305)$ & GS & {$[00 / 7]$} \\
\hline & $(291-318)$ & $65.9 \pm 0.3$ & $(298)$ & GS & {$[00 / 7]$} \\
\hline $\mathrm{C}_{12} \mathrm{H}_{24}$ & cis 2,2,4,6,6-pentamethyl-3-heptene & & & & \\
\hline & $(288-318)$ & $63.0 \pm 0.5$ & $(303)$ & GS & {$[00 / 7]$} \\
\hline & $(288-318)$ & $63.2 \pm 0.5$ & $(298)$ & GS & {$[00 / 7]$} \\
\hline $\mathrm{C}_{12} \mathrm{H}_{24} \mathrm{Cl}_{2}$ & 1,12-dichlorododecane & & & & \\
\hline & & 73.1 & & & {$[98 / 20]$} \\
\hline $\mathrm{C}_{12} \mathrm{H}_{24} \mathrm{O}$ & cyclododecanol & & & & {$[1724-39-6]$} \\
\hline & $(405-468)$ & 68.8 & $(420)$ & A & {$[87 / 5]$} \\
\hline & $(467-557)$ & 57.1 & $(482)$ & A & {$[87 / 5]$} \\
\hline $\mathrm{C}_{12} \mathrm{H}_{24} \mathrm{O}$ & dodecanal & & & & [112-54-9] \\
\hline & $(308-353)$ & 70.2 & $(298)$ & CGC & {$[96 / 7][00 / 10]$} \\
\hline & $(350-530)$ & 56.5 & $(365)$ & A & {$[87 / 5][47 / 5]$} \\
\hline $\mathrm{C}_{12} \mathrm{H}_{24} \mathrm{O}$ & 2-dodecanone & & & & {$[6175-49-1]$} \\
\hline & $(350-520)$ & 61.1 & $(365)$ & A & {$[87 / 5][47 / 5]$} \\
\hline & & $71.8 \pm 0.6$ & $(298)$ & $\mathrm{C}$ & {$[77 / 2]$} \\
\hline & $(386-609)$ & 60.8 & $(401)$ & A & {$[87 / 5][75 / 8]$} \\
\hline & $(386-609)$ & 48.1 & $(524)$ & & {$[75 / 8]$} \\
\hline $\mathrm{C}_{12} \mathrm{H}_{24} \mathrm{O}$ & ethyl p-menthyl ether & & & & {$[19321-39-2]$} \\
\hline & $(366-414)$ & 50.9 & $(381)$ & A & {$[87 / 5]$} \\
\hline
\end{tabular}


TABLE 6. Enthalpies of vaporization of organic compounds, 1880-2002-Continued

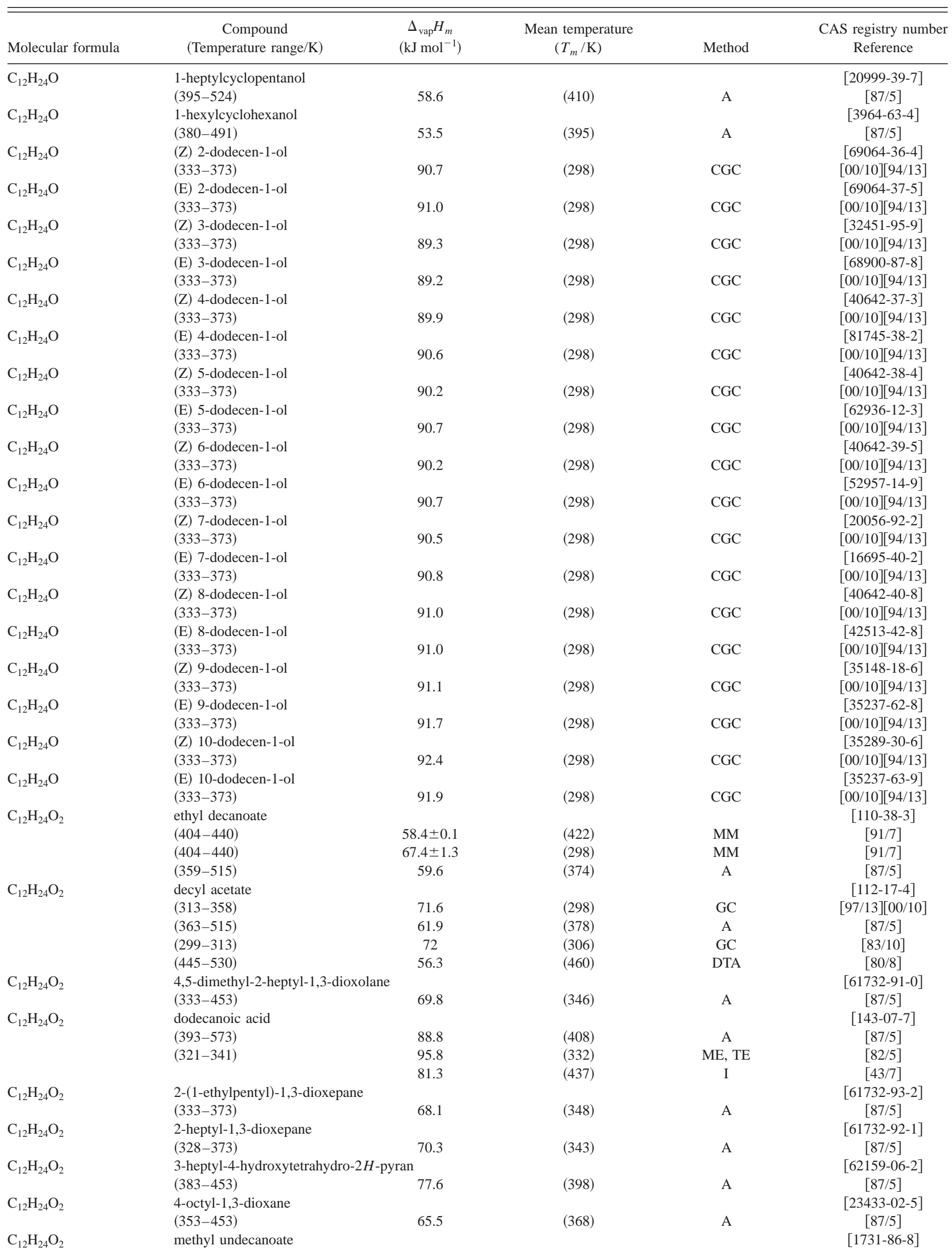


TABLE 6. Enthalpies of vaporization of organic compounds, 1880-2002-Continued

\begin{tabular}{|c|c|c|c|c|c|}
\hline Molecular formula & $\begin{array}{c}\text { Compound } \\
\text { (Temperature range/K) }\end{array}$ & $\begin{array}{c}\Delta_{\mathrm{vap}} H_{m} \\
\left(\mathrm{~kJ} \mathrm{~mol}^{-1}\right)\end{array}$ & $\begin{array}{l}\text { Mean temperature } \\
\qquad\left(T_{m} / \mathrm{K}\right)\end{array}$ & Method & $\begin{array}{c}\text { CAS registry number } \\
\text { Reference }\end{array}$ \\
\hline & & 66.1 & $(350)$ & & {$[02 / 27]$} \\
\hline & & $67.0 \pm 0.1$ & $(340)$ & & {$[02 / 27]$} \\
\hline & & $70.8 \pm 0.4$ & $(298)$ & & {$[02 / 27]$} \\
\hline & $(433-473)$ & 70.6 & $(298)$ & CGC & {$[95 / 21]$} \\
\hline & & $71.4 \pm 0.3$ & $(298)$ & C & {$[77 / 1]$} \\
\hline & $(393-473)$ & 60.9 & $(408)$ & A, EST & {$[87 / 5][63 / 16]$} \\
\hline \multirow[t]{2}{*}{$\mathrm{C}_{12} \mathrm{H}_{24} \mathrm{O}_{3}$} & pentyl 2-butoxypropionate & & & & \\
\hline & $(373-398)$ & 47.3 & $(385)$ & A & {$[87 / 5]$} \\
\hline \multirow[t]{2}{*}{$\mathrm{C}_{12} \mathrm{H}_{24} \mathrm{O}_{3}$} & methyl 3-octyloxypropionate & & & & [7419-98-9] \\
\hline & $(373-513)$ & 59.8 & $(388)$ & A & {$[87 / 5]$} \\
\hline \multirow[t]{2}{*}{$\mathrm{C}_{12} \mathrm{H}_{24} \mathrm{O}_{6}$} & 18-crown-6 & & & & {$[17455-13-9]$} \\
\hline & & $86.1 \pm 6.7$ & $(298)$ & CGC & {$[00 / 9]$} \\
\hline \multirow[t]{3}{*}{$\mathrm{C}_{12} \mathrm{H}_{25} \mathrm{Br}$} & 1-bromododecane & & & & {$[143-15-7]$} \\
\hline & & $74.8 \pm 0.4$ & $(298)$ & $\mathrm{C}$ & {$[76 / 6][77 / 1]$} \\
\hline & $(411-610)$ & 62.2 & $(426)$ & $\mathrm{A}, \mathrm{EST}$ & $\begin{array}{c}{[87 / 5][61 / 13]} \\
{[70 / 14]}\end{array}$ \\
\hline \multirow[t]{6}{*}{$\mathrm{C}_{12} \mathrm{H}_{25} \mathrm{Cl}$} & 1-chlorododecane & & & & {$[112-52-7]$} \\
\hline & & $73.9 \pm 1.4$ & $(298)$ & GS & {$[01 / 1]$} \\
\hline & $(390-520)$ & 70.5 & $(298)$ & & {$[84 / 9][91 / 2]$} \\
\hline & & $71.9 \pm 0.3$ & $(298)$ & $\mathrm{C}$ & {$[77 / 1]$} \\
\hline & & $70.3 \pm 0.5$ & $(298)$ & $\mathrm{C}$ & {$[75 / 6]$} \\
\hline & $(389-519)$ & 62.4 & $(404)$ & A, DTA & {$[87 / 5][69 / 5]$} \\
\hline \multirow[t]{2}{*}{$\mathrm{C}_{12} \mathrm{H}_{25} \mathrm{Cl}$} & (dl) 2-chlorododecane & & & & {$[2350-12-1]$} \\
\hline & $(283-328)$ & 65.3 & $(298)$ & A & $\begin{array}{c}{[87 / 5][70 / 14]} \\
{[62 / 30]}\end{array}$ \\
\hline \multirow[t]{2}{*}{$\mathrm{C}_{12} \mathrm{H}_{25} \mathrm{Cl}$} & (dl) 3-chlorododecane & & & & {$[2350-12-1]$} \\
\hline & $(283-328)$ & 65.9 & $(298)$ & A & $\begin{array}{c}{[87 / 5][70 / 14]} \\
{[62 / 30]}\end{array}$ \\
\hline \multirow[t]{2}{*}{$\mathrm{C}_{12} \mathrm{H}_{25} \mathrm{Cl}$} & (dl) 4-chlorododecane & & & & {$[2350-13-2]$} \\
\hline & $(283-328)$ & 64.1 & $(298)$ & A & $\begin{array}{c}{[87 / 5][70 / 14]} \\
{[62 / 30]}\end{array}$ \\
\hline \multirow[t]{2}{*}{$\mathrm{C}_{12} \mathrm{H}_{25} \mathrm{Cl}$} & ( $d l$ ) 5-chlorododecane & & & & {$[2350-14-3]$} \\
\hline & $(283-328)$ & 65.9 & $(298)$ & A & $\begin{array}{c}{[87 / 5][70 / 14]} \\
{[62 / 30]}\end{array}$ \\
\hline \multirow[t]{2}{*}{$\mathrm{C}_{12} \mathrm{H}_{25} \mathrm{Cl}$} & 6-chlorododecane & & & & [26535-66-0] \\
\hline & $(283-328)$ & 65.5 & $(298)$ & A & $\begin{array}{c}{[87 / 5][70 / 14]} \\
{[62 / 30]}\end{array}$ \\
\hline \multirow[t]{3}{*}{$\mathrm{C}_{12} \mathrm{H}_{25} \mathrm{~F}$} & 1-fluorododecane & & & & {$[334-68-9]$} \\
\hline & $(288-328)$ & $64.0 \pm 0.2$ & $(298)$ & GS & {$[97 / 14]$} \\
\hline & $(374-533)$ & 56.2 & $(389)$ & $\mathrm{A}, \mathrm{EST}$ & $\begin{array}{c}{[87 / 5][61 / 13]} \\
{[70 / 14]}\end{array}$ \\
\hline \multirow[t]{2}{*}{$\mathrm{C}_{12} \mathrm{H}_{25} \mathrm{I}$} & 1-iodododecane & & & & [4292-19-7] \\
\hline & $(426-636)$ & 63.5 & $(441)$ & $\mathrm{A}, \mathrm{EST}$ & $\begin{array}{c}{[87 / 5][61 / 13]} \\
{[70 / 14]}\end{array}$ \\
\hline \multirow[t]{2}{*}{$\mathrm{C}_{12} \mathrm{H}_{25} \mathrm{NO}$} & N,N-diethylcaprylamide & & & & {$[996-97-4]$} \\
\hline & $(373-510)$ & 71.2 & $(388)$ & A & {$[87 / 5]$} \\
\hline \multirow[t]{17}{*}{$\mathrm{C}_{12} \mathrm{H}_{26}$} & dodecane & & & & {$[112-40-3]$} \\
\hline & & $62.1 \pm 0.2$ & $(298)$ & GS & {$[01 / 1]$} \\
\hline & & $60.3 \pm 0.8$ & $(298)$ & CGC & {$[00 / 9]$} \\
\hline & & 61.4 & (299) & $\mathrm{C}$ & {$[96 / 22]$} \\
\hline & & 58.1 & $(334)$ & $\mathrm{C}$ & {$[96 / 22]$} \\
\hline & & 57.4 & $(344)$ & $\mathrm{C}$ & {$[96 / 22]$} \\
\hline & $(373-423)$ & 60.7 & $(298)$ & CGC & {$[95 / 21]$} \\
\hline & $(363-413)$ & 61.2 & (298) & $\mathrm{CGC}$ & {$[95 / 21]$} \\
\hline & $(423-473)$ & 61.2 & $(298)$ & CGC & {$[95 / 21]$} \\
\hline & & 61.5 & (298) & & {$[94 / 12]$} \\
\hline & $(263-371)$ & 65.7 & $(278)$ & & {$[88 / 12]$} \\
\hline & $(278-400)$ & 61.8 & (293) & $\mathrm{A}$ & {$[87 / 5]$} \\
\hline & $(298-389)$ & 61.1 & $(313)$ & GS & {$[86 / 6]$} \\
\hline & & $61.8 \pm 0.5$ & (298) & $\mathrm{C}$ & {$[76 / 4]$} \\
\hline & & $60.4 \pm 0.3$ & $(298)$ & $\mathrm{C}$ & {$[72 / 29]$} \\
\hline & & 61.3 & (298) & & {$[71 / 28]$} \\
\hline & $(400-492)$ & 51.6 & $(415)$ & A, MM & {$[87 / 5][45 / 2]$} \\
\hline $\mathrm{C}_{12} \mathrm{H}_{26}$ & 2-methylundecane & & & & {$[7045-71-8]$} \\
\hline
\end{tabular}


TABLE 6. Enthalpies of vaporization of organic compounds, 1880-2002-Continued

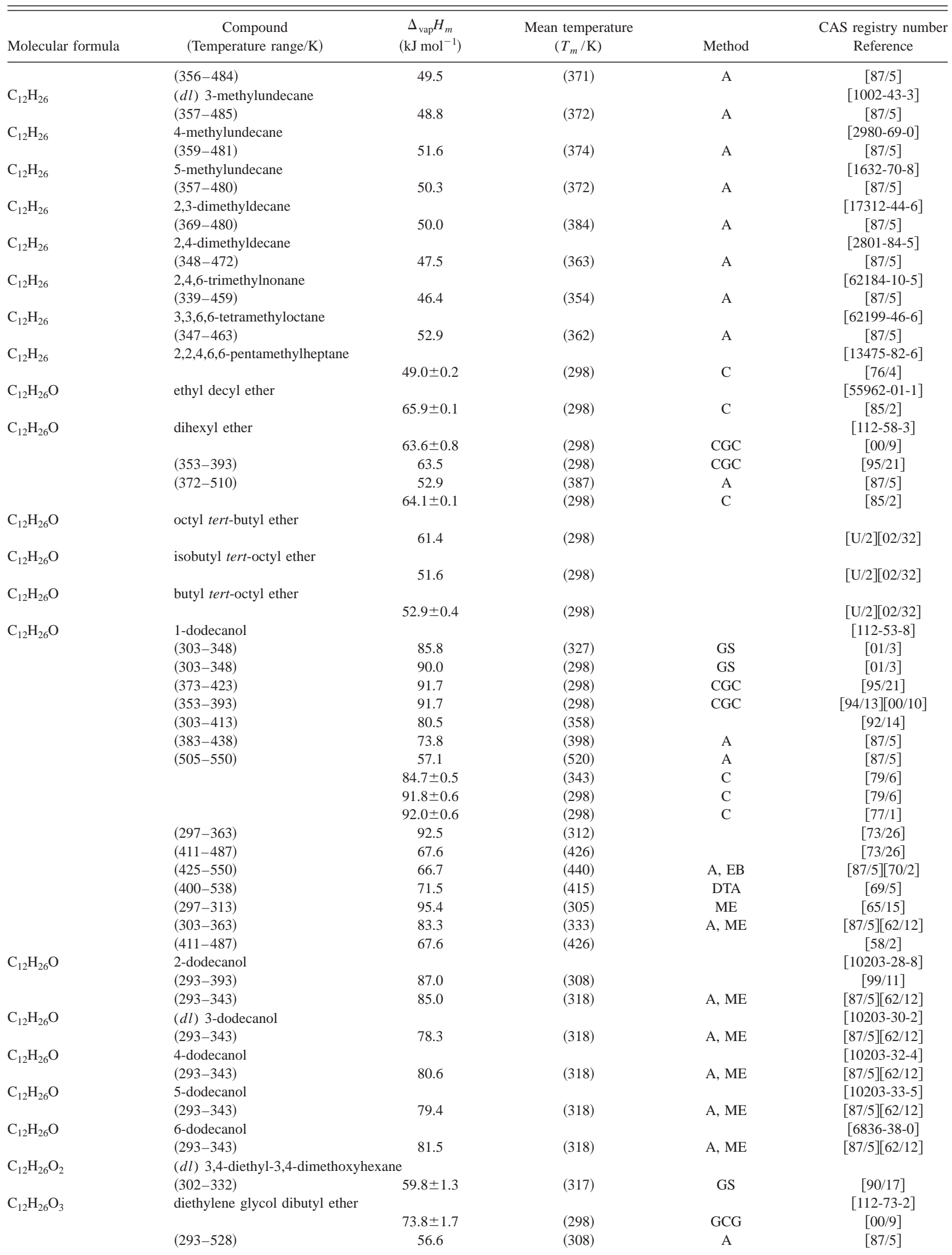


TABLE 6. Enthalpies of vaporization of organic compounds, 1880-2002-Continued

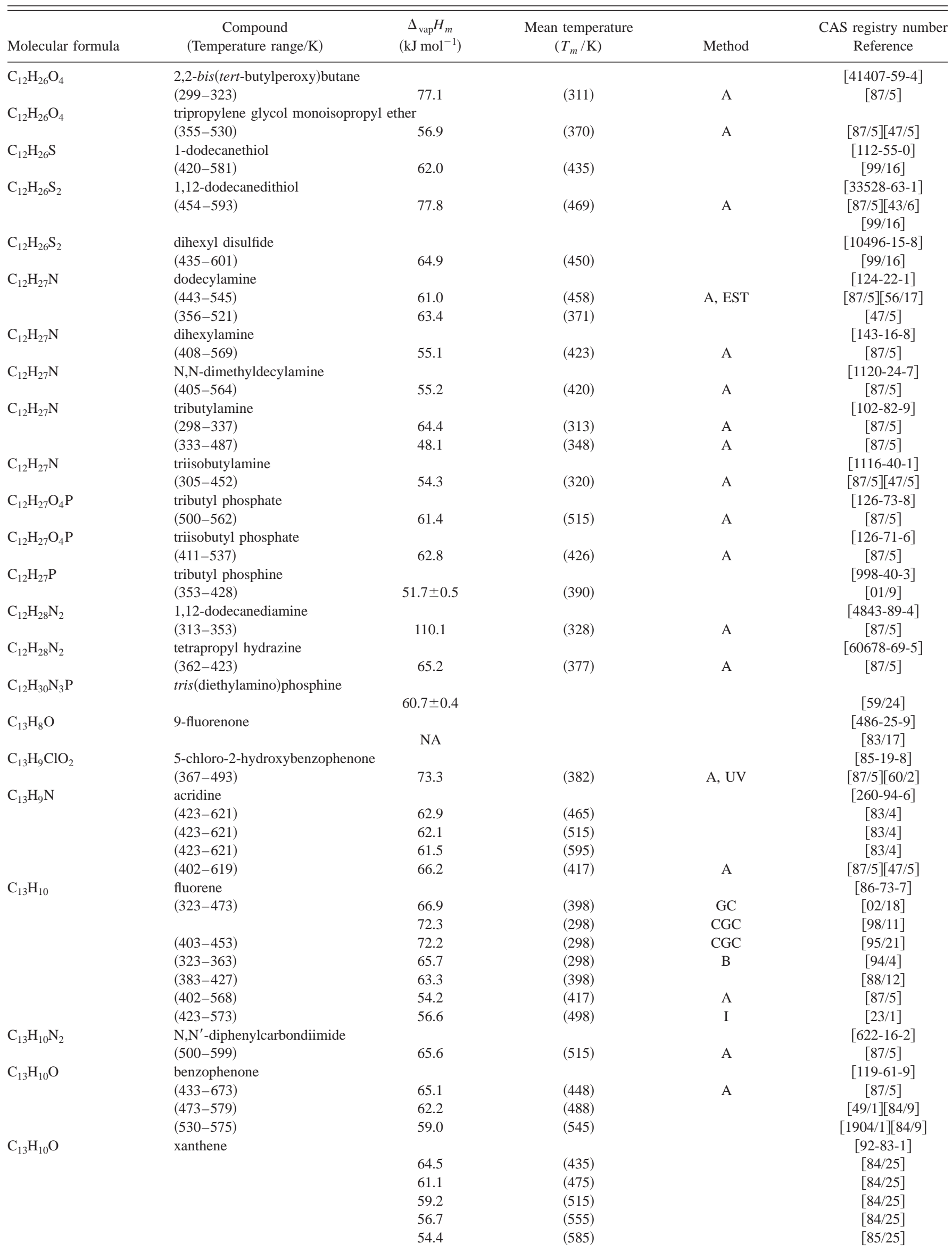


TABLE 6. Enthalpies of vaporization of organic compounds, 1880-2002-Continued

\begin{tabular}{|c|c|c|c|c|c|}
\hline Molecular formula & $\begin{array}{c}\text { Compound } \\
\text { (Temperature range/K) }\end{array}$ & $\begin{array}{c}\Delta_{\mathrm{vap}} H_{m} \\
\left(\mathrm{~kJ} \mathrm{~mol}^{-1}\right)\end{array}$ & $\begin{array}{l}\text { Mean temperature } \\
\qquad\left(T_{m} / \mathrm{K}\right)\end{array}$ & Method & $\begin{array}{c}\text { CAS registry number } \\
\text { Reference }\end{array}$ \\
\hline & $(413-433)$ & 88.7 & $(423)$ & A & {$[87 / 5][58 / 23]$} \\
\hline \multirow[t]{2}{*}{$\mathrm{C}_{13} \mathrm{H}_{10} \mathrm{O}$} & 9-hydroxyfluorene & & & & {$[1689-64-1]$} \\
\hline & & NA & & & [83/17] \\
\hline \multirow[t]{2}{*}{$\mathrm{C}_{13} \mathrm{H}_{10} \mathrm{O}_{2}$} & phenylbenzoate & & & & [93-99-2] \\
\hline & $(379-587)$ & 62.4 & $(394)$ & A & {$[87 / 5][47 / 5]$} \\
\hline \multirow[t]{2}{*}{$\mathrm{C}_{13} \mathrm{H}_{10} \mathrm{O}_{3}$} & 2,4-dihydroxybenzophenone & & & & {$[131-56-6]$} \\
\hline & $(418-485)$ & 87.1 & $(433)$ & A, UV & {$[87 / 5][60 / 2]$} \\
\hline \multirow[t]{2}{*}{$\mathrm{C}_{13} \mathrm{H}_{10} \mathrm{O}_{3}$} & phenyl salicyate & & & & {$[118-55-8]$} \\
\hline & $(423-587)$ & 69.9 & $(438)$ & A, UV & {$[87 / 5][60 / 2]$} \\
\hline \multirow[t]{2}{*}{$\mathrm{C}_{13} \mathrm{H}_{11} \mathrm{Cl}$} & chlorodiphenylmethane & & & & [90-99-3] \\
\hline & $(381-450)$ & 70.4 & $(396)$ & A & {$[87 / 5]$} \\
\hline \multirow{2}{*}{$\mathrm{C}_{13} \mathrm{H}_{11} \mathrm{~F}$} & fluorodiphenylmethane & & & & {$[579-55-5]$} \\
\hline & $(288-333)$ & $69.8 \pm 0.4$ & $(298)$ & GS & {$[97 / 14]$} \\
\hline \multirow[t]{4}{*}{$\mathrm{C}_{13} \mathrm{H}_{11} \mathrm{~N}$} & benzophenone imine & & & & {$[1013-88-3]$} \\
\hline & $(308-338)$ & $74.2 \pm 1.0$ & $(323)$ & GS & [97/9] \\
\hline & $(308-338)$ & $75.7 \pm 1.0$ & (298) & GS & {$[97 / 9]$} \\
\hline & $(373-422)$ & 62.3 & $(388)$ & A & {$[87 / 5]$} \\
\hline \multirow[t]{9}{*}{$\mathrm{C}_{13} \mathrm{H}_{11} \mathrm{~N}$} & 9-methylcarbazole & & & & {$[1484-12-4]$} \\
\hline & $(373-673)$ & 73.4 & $(400)$ & EB, IPM & {$[92 / 15]$} \\
\hline & $(373-673)$ & 70.5 & $(440)$ & $\mathrm{EB}, \mathrm{IPM}$ & {$[92 / 15]$} \\
\hline & $(373-673)$ & 67.7 & $(480)$ & EB, IPM & {$[92 / 15]$} \\
\hline & $(373-673)$ & 65.0 & $(520)$ & EB, IPM & {$[92 / 15]$} \\
\hline & $(373-673)$ & 62.1 & $(560)$ & EB, IPM & {$[92 / 15]$} \\
\hline & $(373-673)$ & 59.1 & $(600)$ & $\mathrm{EB}, \mathrm{IPM}$ & {$[92 / 15]$} \\
\hline & $(373-673)$ & 55.9 & $(640)$ & $\mathrm{EB}, \mathrm{IPM}$ & {$[92 / 15]$} \\
\hline & $(348-384)$ & 74.9 & $(366)$ & GS & {$[80 / 6]$} \\
\hline \multirow[t]{3}{*}{$\mathrm{C}_{13} \mathrm{H}_{11} \mathrm{~N}_{3} \mathrm{O}$} & 2-(2'-hydroxy-5'-methylphen & otriazole & & & {$[2440-22-4]$} \\
\hline & $(413-433)$ & 79.1 & $(423)$ & $\mathrm{ME}$ & {$[84 / 1]$} \\
\hline & $(404-435)$ & 70.6 & $(419)$ & A, UV & {$[87 / 5][60 / 2]$} \\
\hline \multirow[t]{2}{*}{$\mathrm{C}_{13} \mathrm{H}_{12}$} & 3-methylbiphenyl & & & & {$[643-93-6]$} \\
\hline & $(283-463)$ & 69.6 & $(298)$ & & {$[93 / 10]$} \\
\hline \multirow[t]{11}{*}{$\mathrm{C}_{13} \mathrm{H}_{12}$} & diphenylmethane & & & & {$[101-81-5]$} \\
\hline & & 65.7 & $(298)$ & $\mathrm{GC}$ & {$[02 / 37]$} \\
\hline & $(303-343)$ & $66.4 \pm 0.5$ & $(323)$ & GS & [99/8] \\
\hline & $(303-343)$ & $67.9 \pm 0.5$ & $(298)$ & GS & {$[99 / 8]$} \\
\hline & $(303-402)$ & 63.7 & $(363)$ & & {$[89 / 14]$} \\
\hline & $(295-383)$ & 72.2 & $(310)$ & A & {$[87 / 5]$} \\
\hline & $(423-583)$ & 56.7 & $(438)$ & A & {$[87 / 5]$} \\
\hline & & 55.8 & $(445)$ & & {$[81 / 1]$} \\
\hline & & 49.0 & $(535)$ & & {$[81 / 1]$} \\
\hline & & $66.6 \pm 0.1$ & $(298)$ & $\mathrm{C}$ & {$[72 / 28]$} \\
\hline & $(490-555)$ & 54.2 & $(505)$ & & {$[15 / 1][84 / 9]$} \\
\hline \multirow[t]{2}{*}{$\mathrm{C}_{13} \mathrm{H}_{12} \mathrm{O}$} & benzyl phenyl ether & & & & {$[946-80-5]$} \\
\hline & $(368-560)$ & 58.8 & $(383)$ & A & {$[87 / 5][47 / 5]$} \\
\hline \multirow[t]{2}{*}{$\mathrm{C}_{13} \mathrm{H}_{12} \mathrm{O}$} & diphenylmethanol & & & & {$[91-01-0]$} \\
\hline & $(438-574)$ & 65.4 & $(453)$ & A & {$[87 / 5]$} \\
\hline \multirow[t]{2}{*}{$\mathrm{C}_{13} \mathrm{H}_{12} \mathrm{O}$} & ethyl 1-naphthyl ketone & & & & {$[2876-63-3]$} \\
\hline & $(397-579)$ & 74.1 & $(412)$ & A & {$[87 / 5][47 / 5]$} \\
\hline $\mathrm{C}_{13} \mathrm{H}_{13} \mathrm{~N}$ & N-methyldiphenylamine & & & & {$[552-82-9]$} \\
\hline & $(376-555)$ & 65.2 & $(391)$ & A & {$[87 / 5][47 / 5]$} \\
\hline $\mathrm{C}_{13} \mathrm{H}_{13} \mathrm{~N}$ & N-benzylaniline & & & & {$[103-32-2]$} \\
\hline & $(316-343)$ & $79.6 \pm 1.1$ & $(330)$ & & {$[97 / 21]$} \\
\hline & & 79.5 & & & {$[80 / 21]$} \\
\hline & $\begin{array}{l}\text { [Note: The value reported in } \\
\text { is larger than the value given }\end{array}$ & $\begin{array}{l}\text { for the entl } \\
\text { enthalpy of }\end{array}$ & $\begin{array}{l}\text { vaporization } \\
\text { tion.] }\end{array}$ & & \\
\hline $\mathrm{C}_{13} \mathrm{H}_{14}$ & 1,6,7-trimethylnaphthalene & & & & {$[2245-38-7]$} \\
\hline & $(323-473)$ & 68.6 & $(398)$ & GC & {$[02 / 18]$} \\
\hline $\mathrm{C}_{13} \mathrm{H}_{14}$ & 1-isopropylnaphthalene & & & & {$[6158-45-8]$} \\
\hline & $(402-541)$ & 50.4 & $(417)$ & A & {$[87 / 5]$} \\
\hline $\mathrm{C}_{13} \mathrm{H}_{14}$ & 2-isopropylnaphthalene & & & & {$[2027-17-0]$} \\
\hline & $(402-541)$ & 60.3 & $(417)$ & A & {$[87 / 5]$} \\
\hline $\mathrm{C}_{13} \mathrm{H}_{14} \mathrm{~N}_{2}$ & $2,4^{\prime}$-diaminodiphenylmethane & & & & {$[1208-52-2]$} \\
\hline & $(353-403)$ & 111.5 & $(368)$ & A & {$[87 / 5]$} \\
\hline $\mathrm{C}_{13} \mathrm{H}_{14} \mathrm{~N}_{2}$ & $4,4^{\prime}$-diaminodiphenylmethane & & & & {$[101-77-9]$} \\
\hline
\end{tabular}


TABLE 6. Enthalpies of vaporization of organic compounds, 1880-2002-Continued

\begin{tabular}{|c|c|c|c|c|c|}
\hline Molecular formula & $\begin{array}{c}\text { Compound } \\
\text { (Temperature range/K) }\end{array}$ & $\begin{array}{c}\Delta_{\mathrm{vap}} H_{m} \\
\left(\mathrm{~kJ} \mathrm{~mol}^{-1}\right)\end{array}$ & $\begin{array}{l}\text { Mean temperature } \\
\qquad\left(T_{m} / \mathrm{K}\right)\end{array}$ & Method & $\begin{array}{c}\text { CAS registry number } \\
\text { Reference }\end{array}$ \\
\hline & $(343-393)$ & 109.3 & $(358)$ & A & {$[87 / 5]$} \\
\hline & $(486-545)$ & 98.0 & $(501)$ & A & {$[87 / 5]$} \\
\hline & $(471-545)$ & 100.6 & $(502)$ & A & {$[66 / 28]$} \\
\hline \multirow{2}{*}{$\mathrm{C}_{13} \mathrm{H}_{15} \mathrm{Cl}_{3} \mathrm{O}_{3}$} & \multicolumn{4}{|c|}{ 2,4,6-trichlorophenoxyacetic acid, pentyl ester } & {$[120-39-8]$} \\
\hline & $(460-573)$ & 78.8 & $(475)$ & A & {$[87 / 5]$} \\
\hline \multirow[t]{8}{*}{$\mathrm{C}_{13} \mathrm{H}_{15} \mathrm{~N}$} & \multicolumn{4}{|c|}{ 1,2,3,4-tetrahydro-9-methylcarbazole } & {$[17058-12-7]$} \\
\hline & $(370-655)$ & 72.5 & $(400)$ & EB, IPM & {$[92 / 15]$} \\
\hline & $(370-655)$ & 69.6 & $(440)$ & EB, IPM & {$[92 / 15]$} \\
\hline & $(370-655)$ & 66.7 & $(480)$ & $\mathrm{EB}, \mathrm{IPM}$ & {$[92 / 15]$} \\
\hline & $(370-655)$ & 63.8 & $(520)$ & $\mathrm{EB}, \mathrm{IPM}$ & {$[92 / 15]$} \\
\hline & $(370-655)$ & 60.7 & $(560)$ & $\mathrm{EB}, \mathrm{IPM}$ & {$[92 / 15]$} \\
\hline & $(370-655)$ & 57.4 & $(600)$ & $\mathrm{EB}, \mathrm{IPM}$ & {$[92 / 15]$} \\
\hline & $(370-655)$ & 53.8 & $(640)$ & $\mathrm{EB}, \mathrm{IPM}$ & {$[92 / 15]$} \\
\hline \multirow[t]{2}{*}{$\mathrm{C}_{13} \mathrm{H}_{15} \mathrm{NO}$} & \multicolumn{5}{|c|}{ 1-(1-isocyanato-1-methylethyl)-4-(1-methylethylbenzene) } \\
\hline & $(298-463)$ & 68.5 & $(308)$ & $\begin{array}{l}\text { DTA, T, } \\
\text { HSA }\end{array}$ & {$[86 / 3]$} \\
\hline \multirow[t]{2}{*}{$\mathrm{C}_{13} \mathrm{H}_{16} \mathrm{Cl}_{2} \mathrm{O}_{3}$} & \multicolumn{4}{|c|}{ 2,4-dichlorophenoxyacetic acid, isopentyl ester } & {$[67821-07-2]$} \\
\hline & $(460-573)$ & 75.8 & $(475)$ & A & {$[87 / 5]$} \\
\hline \multirow[t]{2}{*}{$\mathrm{C}_{13} \mathrm{H}_{16} \mathrm{Cl}_{2} \mathrm{O}_{3}$} & \multicolumn{4}{|c|}{ 2,4-dichlorophenoxyacetic acid, pentyl ester } & {$[1917-96-6]$} \\
\hline & $(444-573)$ & 73.6 & $(459)$ & A & {$[87 / 5]$} \\
\hline $\mathrm{C}_{13} \mathrm{H}_{16} \mathrm{~N}_{2}$ & $\begin{array}{l}\text { 2-phenyl-2-piperidinoacet } \\
(338-378)\end{array}$ & $73.2 \pm 0.4$ & & GS & {$[97 / 10]$} \\
\hline \multirow[t]{3}{*}{$\mathrm{C}_{13} \mathrm{H}_{17} \mathrm{NO}$} & \multicolumn{4}{|l|}{ 1-(phenacyl)piperidine } & {$[3626-62-8]$} \\
\hline & $(381-446)$ & 51.4 & $(396)$ & A & {$[87 / 5][69 / 1]$} \\
\hline & $(382-450)$ & 47.2 & $(416)$ & & {$[69 / 1]$} \\
\hline \multirow[t]{2}{*}{$\mathrm{C}_{13} \mathrm{H}_{17} \mathrm{NO}$} & 1-(m-toluoyl)piperidine & & & & {$[13290-48-7]$} \\
\hline & $(373-403)$ & 53.8 & $(388)$ & A & {$[87 / 5][69 / 1]$} \\
\hline $\mathrm{C}_{13} \mathrm{H}_{17} \mathrm{NO}_{3}$ & $(d l) \mathrm{N}$-acetylphenylalanin & & & & [4134-09-2] \\
\hline & $(438-528)$ & 82.4 & $(453)$ & A & {$[87 / 5]$} \\
\hline $\mathrm{C}_{13} \mathrm{H}_{18}$ & 1,1,4,6-tetramethylindane & & & & [941-60-6] \\
\hline & $(313-383)$ & 59.4 & $(328)$ & A & {$[87 / 5]$} \\
\hline & $(313-469)$ & 60.2 & $(328)$ & A & {$[87 / 5]$} \\
\hline & $(242-469)$ & 51.9 & $(439)$ & A & {$[87 / 5]$} \\
\hline $\mathrm{C}_{13} \mathrm{H}_{18}$ & 1,1,4,7-tetramethylindane & & & & [1078-04-2] \\
\hline & $(313-388)$ & 59.6 & $(328)$ & A & {$[87 / 5]$} \\
\hline & $(313-469)$ & 60.4 & $(328)$ & A & {$[87 / 5]$} \\
\hline & $(431-469)$ & 52.0 & $(446)$ & A & {$[87 / 5]$} \\
\hline $\mathrm{C}_{13} \mathrm{H}_{18} \mathrm{O}$ & 4,4-dimethyl-1-phenyl-3-p & & & & {$[5195-24-4]$} \\
\hline & $(405-520)$ & 63.5 & $(420)$ & A & {$[87 / 5]$} \\
\hline $\mathrm{C}_{13} \mathrm{H}_{18} \mathrm{O}$ & p-isopropyl- $\alpha$-methylhydr & dehyde & & & {$[103-95-7]$} \\
\hline & $(283-499)$ & 72.6 & $(298)$ & A & {$[87 / 5]$} \\
\hline $\mathrm{C}_{13} \mathrm{H}_{18} \mathrm{O}$ & 1-phenyl-1-heptanone & & & & {$[1671-75-6]$} \\
\hline & $(373-550)$ & 64.6 & $(388)$ & A & {$[87 / 5][47 / 5]$} \\
\hline $\mathrm{C}_{13} \mathrm{H}_{19} \mathrm{NO}$ & 3-phenylpropionic acid, $\mathrm{N}$ & amide & & & \\
\hline & $(353-439)$ & 46.5 & $(368)$ & A & {$[87 / 5]$} \\
\hline $\mathrm{C}_{13} \mathrm{H}_{19} \mathrm{NO}$ & $(4 \mathrm{R}, 5 \mathrm{R})-2,2,3,4$-tetrameth & 1-1,3-oxazoli & & & [141271-51-4] \\
\hline & $(293-301)$ & $61.6 \pm 1.8$ & $(298)$ & & {$[98 / 3]$} \\
\hline $\mathrm{C}_{13} \mathrm{H}_{20}$ & heptylbenzene & & & & {$[1078-71-3]$} \\
\hline & $(423-527)$ & 54.0 & $(438)$ & A & {$[87 / 5]$} \\
\hline & & 64.9 & $(298)$ & & {$[71 / 28]$} \\
\hline $\mathrm{C}_{13} \mathrm{H}_{20} \mathrm{O}$ & butyl cumyl ether & & & & \\
\hline & $(278-318)$ & $63.8 \pm 0.5$ & $(298)$ & GS & [01/18] \\
\hline $\mathrm{C}_{13} \mathrm{H}_{20} \mathrm{O}$ & $\alpha$-ionone & & & & {$[127-41-3]$} \\
\hline & $(352-523)$ & 62.0 & $(367)$ & A & {$[87 / 5][47 / 5]$} \\
\hline & $(286-333)$ & 67.5 & $(301)$ & $\mathrm{A}, \mathrm{ME}$ & {$[87 / 5][57 / 9]$} \\
\hline $\mathrm{C}_{13} \mathrm{H}_{20} \mathrm{O}$ & $\beta$-ionone & & & & [14901-07-6] \\
\hline & $(291-334)$ & 69.0 & $(306)$ & $\mathrm{A}, \mathrm{ME}$ & {$[87 / 5][57 / 9]$} \\
\hline $\mathrm{C}_{13} \mathrm{H}_{20} \mathrm{O}$ & 6,10-dimethyl-4,5,9-undec & & & & {$[16647-05-5]$} \\
\hline & $(349-421)$ & $63.6 \pm 1.4$ & $(385)$ & & {$[88 / 4]$} \\
\hline $\mathrm{C}_{13} \mathrm{H}_{20} \mathrm{O}$ & 6,10-dimethyl-3,5,9-undec & & & & {$[141-10-6]$} \\
\hline & $(382-457)$ & $67.6 \pm 1.1$ & $(420)$ & & {$[88 / 4]$} \\
\hline $\mathrm{C}_{13} \mathrm{H}_{20} \mathrm{O}$ & 4-(2,6,6-trimethyl-1-cyclo & -3-buten-2-c & & & {$[79-77-6]$} \\
\hline & $(373-442)$ & $49.6 \pm 1.1$ & $(408)$ & & {$[88 / 4]$} \\
\hline $\mathrm{C}_{13} \mathrm{H}_{20} \mathrm{O}_{2}$ & 1,3-dihydroxy-5-heptylber & & & & {$[500-67-4]$} \\
\hline
\end{tabular}


TABLE 6. Enthalpies of vaporization of organic compounds, 1880-2002-Continued

\begin{tabular}{|c|c|c|c|c|c|}
\hline Molecular formula & $\begin{array}{c}\text { Compound } \\
\text { (Temperature range/K) }\end{array}$ & $\begin{array}{c}\Delta_{\mathrm{vap}} H_{m} \\
\left(\mathrm{~kJ} \mathrm{~mol}^{-1}\right)\end{array}$ & $\begin{array}{l}\text { Mean temperature } \\
\qquad\left(T_{m} / \mathrm{K}\right)\end{array}$ & Method & $\begin{array}{c}\text { CAS registry number } \\
\text { Reference }\end{array}$ \\
\hline & $(443-504)$ & 91.6 & $(458)$ & $\mathrm{A}, \mathrm{GC}$ & {$[87 / 5][75 / 24]$} \\
\hline \multirow{2}{*}{$\mathrm{C}_{13} \mathrm{H}_{20} \mathrm{O}_{2}$} & \multicolumn{4}{|c|}{ 1,3-dihydroxy-5-methyl-2-hexylbenzene } & {$[41395-27-1]$} \\
\hline & $(433-493)$ & 82.8 & $(448)$ & $\mathrm{A}, \mathrm{GC}$ & {$[87 / 5][75 / 24]$} \\
\hline \multirow{2}{*}{$\mathrm{C}_{13} \mathrm{H}_{21} \mathrm{Cl}_{3} \mathrm{OS}$} & \multicolumn{4}{|c|}{ 2,3,3-trichloro-2-propenethioic acid, O-decyl ester } & [76619-97-1] \\
\hline & $(483-503)$ & 79.9 & & GC & {$[80 / 24]$} \\
\hline \multirow[t]{3}{*}{$\mathrm{C}_{13} \mathrm{H}_{21} \mathrm{~N}$} & \multicolumn{5}{|c|}{ N,N-dimethyl-3-methyl-3-phenyl-2-butaneamine } \\
\hline & $(283-330)$ & $59.8 \pm 0.7$ & $(307)$ & GS & {$[98 / 1]$} \\
\hline & $(283-330)$ & $60.3 \pm 0.7$ & $(298)$ & GS & {$[98 / 1]$} \\
\hline \multirow[t]{3}{*}{$\mathrm{C}_{13} \mathrm{H}_{21} \mathrm{~N}$} & \multicolumn{5}{|c|}{ N-methyl-2,3-dimethyl-3-phenyl-2-butaneamine } \\
\hline & $(285-332)$ & $71.9 \pm 1.1$ & $(309)$ & GS & {$[98 / 1]$} \\
\hline & $(285-332)$ & $72.5 \pm 1.1$ & $(298)$ & GS & {$[98 / 1]$} \\
\hline \multirow[t]{2}{*}{$\mathrm{C}_{13} \mathrm{H}_{21} \mathrm{NO}$} & \multicolumn{5}{|c|}{ 2-(diethylamino)-1-phenyl-1-propanone } \\
\hline & $(293-333)$ & $71.6 \pm 1.0$ & $(298)$ & GS & {$[94 / 3]$} \\
\hline $\mathrm{C}_{13} \mathrm{H}_{22}$ & \multicolumn{4}{|c|}{ 2-allyl-cis-decahydronaphthalene } & {$[87 / 5]$} \\
\hline $\mathrm{C}_{13} \mathrm{H}_{22}$ & \multicolumn{4}{|c|}{ 2-allyl-trans-decahydronaphthalene } & {$[87 / 5]$} \\
\hline $\mathrm{C}_{13} \mathrm{H}_{22}$ & \multicolumn{4}{|l|}{ dodecahydrofluorene } & $\begin{array}{c}{[5744-03-6]} \\
{[87 / 5]}\end{array}$ \\
\hline $\mathrm{C}_{13} \mathrm{H}_{22}$ & \multicolumn{2}{|l|}{ 1,3,5-trimethyladamantane } & $(298)$ & & $\begin{array}{c}{[707-35-7]} \\
{[00 / 21][00 / 22]}\end{array}$ \\
\hline $\mathrm{C}_{13} \mathrm{H}_{22} \mathrm{Cl}_{2} \mathrm{O}_{4}$ & $\begin{array}{l}\text { 2,2-bis(chloromethyl)-1,3 } \\
(454-572)\end{array}$ & $\begin{array}{l}\text { ol dibutyrate } \\
43.1\end{array}$ & $(469)$ & A & {$[87 / 5]$} \\
\hline $\mathrm{C}_{13} \mathrm{H}_{22} \mathrm{O}_{2}$ & $\begin{array}{l}\text { bornyl propionate } \\
(337-508)\end{array}$ & 55.9 & $(352)$ & A & $\begin{array}{l}{[78548-53-5]} \\
{[87 / 5][47 / 5]}\end{array}$ \\
\hline $\mathrm{C}_{13} \mathrm{H}_{24} \mathrm{Cl}_{4}$ & $\begin{array}{l}\text { 1,1,1,13-tetrachlorotrideca } \\
(320-370)\end{array}$ & 97.4 & $(335)$ & A & $\begin{array}{c}{[3922-33-6]} \\
{[87 / 5]}\end{array}$ \\
\hline $\mathrm{C}_{13} \mathrm{H}_{24} \mathrm{O}$ & $\begin{array}{l}\text { 5-methyl-2-ethyl-2-butyl- } \\
(323-393)\end{array}$ & 69.1 & $(338)$ & A & $\begin{array}{c}{[42023-59-6]} \\
{[87 / 5]}\end{array}$ \\
\hline $\mathrm{C}_{13} \mathrm{H}_{24} \mathrm{O}_{2}$ & decyl acrylate & & & & {$[2156-96-9]$} \\
\hline & $(404-536)$ & 59.6 & $(419)$ & A & {$[87 / 5]$} \\
\hline $\mathrm{C}_{13} \mathrm{H}_{24} \mathrm{O}_{2}$ & oxa-2-cyclotetradecanone & lactone) & & & {$[1725-04-8]$} \\
\hline & $(375-405)$ & $66.6 \pm 1.1$ & $(390)$ & MM & {$[91 / 7]$} \\
\hline & $(375-405)$ & $72.9 \pm 1.7$ & $(298)$ & MM & {$[91 / 7]$} \\
\hline & $(393-443)$ & 67.5 & $(408)$ & A & {$[87 / 5]$} \\
\hline $\mathrm{C}_{13} \mathrm{H}_{24} \mathrm{O}_{2}$ & ethyl 10 -undecenoate & & & & {$[692-86-4]$} \\
\hline & $(404-532)$ & 77.4 & $(419)$ & A & {$[87 / 5]$} \\
\hline $\mathrm{C}_{13} \mathrm{H}_{24} \mathrm{O}_{3}$ & 1,4-dioxa-5-cyclopentadec & & & & [1898-91-1] \\
\hline & $(403-443)$ & 69.6 & $(418)$ & $\mathrm{A}, \mathrm{GC}$ & {$[87 / 5][71 / 33]$} \\
\hline $\mathrm{C}_{13} \mathrm{H}_{24} \mathrm{O}_{3}$ & 1,6-dioxa-7-cyclopentadec & & & & {$[36575-54-9]$} \\
\hline & $(403-443)$ & 75.7 & $(418)$ & $\mathrm{A}, \mathrm{GC}$ & {$[87 / 5][71 / 33]$} \\
\hline $\mathrm{C}_{13} \mathrm{H}_{24} \mathrm{O}_{3}$ & 1,8-dioxa-9-cyclopentadec & & & & {$[36575-53-8]$} \\
\hline & $(403-443)$ & 66.5 & $(418)$ & $\mathrm{A}, \mathrm{GC}$ & {$[87 / 5][71 / 33]$} \\
\hline $\mathrm{C}_{13} \mathrm{H}_{24} \mathrm{O}_{3}$ & 3-hexyl-4-acetoxytetrahyd & & & & {$[18871-17-5]$} \\
\hline & $(383-453)$ & 72.1 & $(398)$ & A & {$[87 / 5]$} \\
\hline $\mathrm{C}_{13} \mathrm{H}_{24} \mathrm{O}_{3}$ & octyl levulinate & & & & {$[41780-57-8]$} \\
\hline & $(413-565)$ & 66.3 & $(428)$ & A & {$[87 / 5]$} \\
\hline & & 65.1 & $(507)$ & & {$[33 / 6]$} \\
\hline $\mathrm{C}_{13} \mathrm{H}_{24} \mathrm{O}_{4}$ & octyl 3-acetoxypropionate & & & & \\
\hline & $(420-440)$ & 88.4 & $(430)$ & A & {$[87 / 5]$} \\
\hline $\mathrm{C}_{13} \mathrm{H}_{24} \mathrm{O}_{4}$ & ethylisopentylmalonic aci & thyl ester & & & [72030-39-8] \\
\hline & $(392-501)$ & 73.1 & $(407)$ & A & {$[87 / 5]$} \\
\hline $\mathrm{C}_{13} \mathrm{H}_{24} \mathrm{O}_{5}$ & octyl[1-(methoxycarbonyl & bonate & & & \\
\hline & $(391-566)$ & 70.0 & $(406)$ & A & {$[87 / 5]$} \\
\hline $\mathrm{C}_{13} \mathrm{H}_{24} \mathrm{O}_{5}$ & pentyl[1-(butoxycarbonyl) & onate & & & \\
\hline & $(348-513)$ & 70.1 & $(363)$ & A & {$[87 / 5]$} \\
\hline $\mathrm{C}_{13} \mathrm{H}_{25} \mathrm{~N}$ & tridecanonitrile & & & & [629-60-7] \\
\hline & $(380-566)$ & 69.5 & $(395)$ & A & {$[87 / 5]$} \\
\hline $\mathrm{C}_{13} \mathrm{H}_{25} \mathrm{NO}$ & 1-octanoyl piperidine & & & & [20299-83-6] \\
\hline & $(373-443)$ & 50.0 & $(388)$ & A & {$[87 / 5]$} \\
\hline $\mathrm{C}_{13} \mathrm{H}_{26}$ & 5-butyl-4-nonene & & & & {$[7367-38-6]$} \\
\hline & $(310-361)$ & 55.8 & $(325)$ & $\mathrm{A}, \mathrm{MG}$ & {$[87 / 5][55 / 11]$} \\
\hline $\mathrm{C}_{13} \mathrm{H}_{26}$ & 1-tridecene & & & & {$[2437-56-1]$} \\
\hline & & 65.3 & $(298)$ & & {$[71 / 28]$} \\
\hline
\end{tabular}


TABLE 6. Enthalpies of vaporization of organic compounds, 1880-2002-Continued

\begin{tabular}{|c|c|c|c|c|c|}
\hline Molecular formula & $\begin{array}{c}\text { Compound } \\
\text { (Temperature range/K) }\end{array}$ & $\begin{array}{c}\Delta_{\mathrm{vap}} H_{m} \\
\left(\mathrm{~kJ} \mathrm{~mol}^{-1}\right)\end{array}$ & $\begin{array}{c}\text { Mean temperature } \\
\qquad\left(T_{m} / \mathrm{K}\right)\end{array}$ & Method & $\begin{array}{c}\text { CAS registry number } \\
\text { Reference }\end{array}$ \\
\hline & $(413-509)$ & 53.9 & $(428)$ & A & {$[87 / 5][55 / 7]$} \\
\hline \multirow[t]{2}{*}{$\mathrm{C}_{13} \mathrm{H}_{26}$} & n-octylcyclopentane & & & & {$[1795-20-6]$} \\
\hline & & 65.8 & (298) & & {$[71 / 28]$} \\
\hline \multirow[t]{2}{*}{$\mathrm{C}_{13} \mathrm{H}_{26}$} & n-heptylcyclohexane & & & & {$[5617-41-4]$} \\
\hline & & 64.9 & $(298)$ & & {$[71 / 28]$} \\
\hline \multirow[t]{2}{*}{$\mathrm{C}_{13} \mathrm{H}_{26} \mathrm{O}$} & 5-methyl-2-ethyl-2-butyl-4-hexene-1-ol & & & & [53144-53-9] \\
\hline & $(333-393)$ & 76.9 & $(348)$ & A & {$[87 / 5]$} \\
\hline \multirow[t]{2}{*}{$\mathrm{C}_{13} \mathrm{H}_{26} \mathrm{O}$} & 1-octylcyclopentanol & & & & [30089-09-9] \\
\hline & $(468-541)$ & 60.9 & $(483)$ & A & {$[87 / 5]$} \\
\hline \multirow[t]{6}{*}{$\mathrm{C}_{13} \mathrm{H}_{26} \mathrm{O}$} & 2-tridecanone & & & & {$[593-08-8]$} \\
\hline & $(335-534)$ & 69.6 & $(350)$ & A & {$[87 / 5]$} \\
\hline & $(424-510)$ & 61.0 & $(439)$ & $\mathrm{A}$ & {$[87 / 5]$} \\
\hline & $(400-628)$ & 49.6 & $(541)$ & & {$[75 / 8]$} \\
\hline & $(335-431)$ & 69.8 & $(348)$ & EB & {$[66 / 12]$} \\
\hline & $(360-535)$ & 62.1 & (375) & & {$[47 / 5]$} \\
\hline \multirow[t]{3}{*}{$\mathrm{C}_{13} \mathrm{H}_{26} \mathrm{O}$} & 7-tridecanone & & & & {$[462-18-0]$} \\
\hline & $(395-600)$ & 62.7 & $(410)$ & A & {$[87 / 5]$} \\
\hline & $(396-623)$ & 49.3 & $(536)$ & & {$[75 / 8]$} \\
\hline \multirow[t]{2}{*}{$\mathrm{C}_{13} \mathrm{H}_{26} \mathrm{O}$} & (Z) 7-tridecen-1-ol & & & & {$[64470-31-1]$} \\
\hline & $(343-383)$ & 95.1 & $(298)$ & CGC & {$[00 / 10][94 / 13]$} \\
\hline \multirow{2}{*}{$\mathrm{C}_{13} \mathrm{H}_{26} \mathrm{O}$} & (E) 7-tridecen-1-ol & & & & {$[64437-28-1]$} \\
\hline & $(343-383)$ & 95.6 & (298) & $\mathrm{CGC}$ & {$[00 / 10][94 / 13]$} \\
\hline \multirow[t]{2}{*}{$\mathrm{C}_{13} \mathrm{H}_{26} \mathrm{O}$} & (Z) 9-tridecen-1-ol & & & & {$[52957-10-5]$} \\
\hline & $(343-383)$ & 95.8 & (298) & $\mathrm{CGC}$ & {$[00 / 10][94 / 13]$} \\
\hline \multirow[t]{2}{*}{$\mathrm{C}_{13} \mathrm{H}_{26} \mathrm{O}$} & (E) 9-tridecen-1-ol & & & & [52957-15-0] \\
\hline & $(343-383)$ & 96.4 & (298) & CGC & {$[00 / 10][94 / 13]$} \\
\hline \multirow[t]{2}{*}{$\mathrm{C}_{13} \mathrm{H}_{26} \mathrm{O}$} & (Z) 11-tridecen-1-ol & & & & {$[34010-24-7]$} \\
\hline & $(343-383)$ & 97.1 & $(298)$ & CGC & {$[00 / 10][94 / 13]$} \\
\hline \multirow[t]{2}{*}{$\mathrm{C}_{13} \mathrm{H}_{26} \mathrm{O}$} & (E) 1,1-tridecen-1-ol & & & & [56195-34-7] \\
\hline & $(343-383)$ & 97.2 & $(298)$ & CGC & {$[00 / 10][94 / 13]$} \\
\hline \multirow{2}{*}{$\mathrm{C}_{13} \mathrm{H}_{26} \mathrm{O}$} & 6,10-dimethyl-2-undecanone & & & & [1604-34-8] \\
\hline & $(379-473)$ & $59.3 \pm 0.4$ & $(426)$ & & {$[88 / 4]$} \\
\hline \multirow[t]{2}{*}{$\mathrm{C}_{13} \mathrm{H}_{26} \mathrm{O}_{2}$} & 4,5-dimethyl-2-octyl-1,3-dioxolane & & & & [5452-11-9] \\
\hline & $(333-453)$ & 72.8 & (348) & A & {$[87 / 5]$} \\
\hline $\mathrm{C}_{13} \mathrm{H}_{26} \mathrm{O}_{2}$ & 2-octyl-1,3-dioxepane & & & & [61732-94-3] \\
\hline & $(323-373)$ & 61.2 & $(338)$ & A & {$[87 / 5]$} \\
\hline $\mathrm{C}_{13} \mathrm{H}_{26} \mathrm{O}_{2}$ & undecyl acetate & & & & [1731-81-3] \\
\hline & $(333-378)$ & 77.2 & $(298)$ & GC & {$[97 / 13][00 / 10]$} \\
\hline $\mathrm{C}_{13} \mathrm{H}_{26} \mathrm{O}_{2}$ & isopropyl decanoate & & & & [2311-59-3] \\
\hline & $(363-451)$ & 60.8 & (378) & A & {$[87 / 5]$} \\
\hline $\mathrm{C}_{13} \mathrm{H}_{26} \mathrm{O}_{2}$ & propyl decanoate & & & & [30673-60-0] \\
\hline & $(369-459)$ & 62.4 & (384) & A & {$[87 / 5]$} \\
\hline $\mathrm{C}_{13} \mathrm{H}_{26} \mathrm{O}_{2}$ & methyl laurate & & & & {$[111-82-0]$} \\
\hline & & 71.4 & $(350)$ & & {$[02 / 27]$} \\
\hline & & $70.7 \pm 0.2$ & $(356)$ & & {$[02 / 27]$} \\
\hline & & $76.6 \pm 0.4$ & (298) & & {$[02 / 27]$} \\
\hline & $(295-452)$ & 74.9 & $(310)$ & & {$[01 / 10]$} \\
\hline & $(393-463)$ & 76.8 & (298) & GC & {$[97 / 28]$} \\
\hline & $(453-543)$ & 53.3 & $(498)$ & GC & {$[93 / 9]$} \\
\hline & $(287-333)$ & 83.6 & $(302)$ & A & {$[87 / 5]$} \\
\hline & & $76.5 \pm 0.7$ & (298) & $\mathrm{C}, \mathrm{GC}$ & {$[80 / 5]$} \\
\hline & & $77.2 \pm 0.6$ & (298) & $\mathrm{C}$ & {$[77 / 1]$} \\
\hline & $(407-540)$ & 63.6 & $(422)$ & A & {$[87 / 5][63 / 16]$} \\
\hline & $(336-409)$ & 71.4 & $(351)$ & MG,OM & {$[52 / 13]$} \\
\hline & $(373-439)$ & 62.3 & $(388)$ & & {$[44 / 7]$} \\
\hline $\mathrm{C}_{13} \mathrm{H}_{26} \mathrm{O}_{2}$ & tridecanoic acid & & & & [638-53-9] \\
\hline & $(409-585)$ & 90.1 & $(424)$ & A & {$[87 / 5]$} \\
\hline & $(328-350)$ & $100.4 \pm 2.0$ & $(340)$ & ME, TE & {$[82 / 4]$} \\
\hline $\mathrm{C}_{13} \mathrm{H}_{26} \mathrm{O}_{3}$ & decyl lactate & & & & [42175-34-8] \\
\hline & $(349-556)$ & 76.6 & $(364)$ & A & {$[87 / 5]$} \\
\hline $\mathrm{C}_{13} \mathrm{H}_{26} \mathrm{O}_{3}$ & octyl 3-ethoxypropionate & & & & \\
\hline & $(398-543)$ & 56.9 & $(413)$ & A & {$[87 / 5]$} \\
\hline $\mathrm{C}_{13} \mathrm{H}_{26} \mathrm{O}_{3}$ & pentyl 3-pentyloxypropionate & & & & [14144-56-0] \\
\hline & $(378-498)$ & 62.3 & (393) & A & {$[87 / 5]$} \\
\hline
\end{tabular}


TABLE 6. Enthalpies of vaporization of organic compounds, 1880-2002-Continued

\begin{tabular}{|c|c|c|c|c|c|}
\hline Molecular formula & $\begin{array}{c}\text { Compound } \\
\text { (Temperature range/K) }\end{array}$ & $\begin{array}{c}\Delta_{\mathrm{vap}} H_{m} \\
\left(\mathrm{~kJ} \mathrm{~mol}^{-1}\right)\end{array}$ & $\begin{array}{l}\text { Mean temperature } \\
\qquad\left(T_{m} / \mathrm{K}\right)\end{array}$ & Method & $\begin{array}{l}\text { CAS registry number } \\
\text { Reference }\end{array}$ \\
\hline $\mathrm{C}_{13} \mathrm{H}_{27} \mathrm{Br}$ & $\begin{array}{l}\text { 1-bromotridecane } \\
(425-628)\end{array}$ & 64.6 & (440) & A, EST & $\begin{array}{c}{[765-09-3]} \\
{[87 / 5][61 / 13]} \\
{[70 / 14]}\end{array}$ \\
\hline $\mathrm{C}_{13} \mathrm{H}_{27} \mathrm{Cl}$ & $\begin{array}{l}\text { 1-chlorotridecane } \\
(414-611)\end{array}$ & 63.0 & (429) & A, EST & $\begin{array}{c}{[822-13-9]} \\
{[87 / 5][61 / 13]} \\
{[70 / 14]}\end{array}$ \\
\hline $\mathrm{C}_{13} \mathrm{H}_{27} \mathrm{~F}$ & $\begin{array}{l}\text { 1-fluorotridecane } \\
(387-558)\end{array}$ & 58.9 & $(402)$ & A, EST & $\begin{array}{c}{[1536-21-6]} \\
{[87 / 5][61 / 13]} \\
{[70 / 14]}\end{array}$ \\
\hline $\mathrm{C}_{13} \mathrm{H}_{27} \mathrm{I}$ & $\begin{array}{l}\text { 1-iodotridecane } \\
(440-655)\end{array}$ & 66.1 & $(455)$ & A, EST & $\begin{array}{c}{[35599-77-0]} \\
{[87 / 5][61 / 13]} \\
{[70 / 14]}\end{array}$ \\
\hline $\mathrm{C}_{13} \mathrm{H}_{27} \mathrm{NO}_{2}$ & $\begin{array}{l}\text { N-decyl lactamide } \\
(413-483)\end{array}$ & 97.9 & (428) & $\mathrm{A}$ & {$[87 / 5]$} \\
\hline $\mathrm{C}_{13} \mathrm{H}_{27} \mathrm{NO}_{2}$ & $\begin{array}{l}\text { O-decyl lactamide } \\
(413-483)\end{array}$ & 95.0 & $(428)$ & A & [87/5] \\
\hline $\mathrm{C}_{13} \mathrm{H}_{28}$ & $(417-511)$ & $\begin{array}{c}65.3 \\
64.9 \\
64.2 \\
63.3 \\
62.4 \\
62.3 \\
66.7 \\
65.6 \\
64.6 \\
61.7 \\
66.5 \pm 0.2 \\
66.4 \pm 0.3 \\
66.2 \\
54.5\end{array}$ & $\begin{array}{l}(309) \\
(314) \\
(324) \\
(334) \\
(344) \\
(349) \\
(298) \\
(308) \\
(318) \\
(348) \\
(298) \\
(298) \\
(298) \\
(432)\end{array}$ & $\begin{array}{l}\mathrm{C} \\
\mathrm{C} \\
\mathrm{C} \\
\mathrm{C} \\
\mathrm{C} \\
\mathrm{C} \\
\\
\mathrm{C} \\
\mathrm{C} \\
\mathrm{C} \\
\mathrm{C} \\
\mathrm{C}\end{array}$ & $\begin{array}{c}{[629-50-5]} \\
{[96 / 22]} \\
{[96 / 22]} \\
{[96 / 22]} \\
{[96 / 22]} \\
{[96 / 22]} \\
{[96 / 22]} \\
{[94 / 12]} \\
{[79 / 2]} \\
{[79 / 2]} \\
{[79 / 2]} \\
{[79 / 2]} \\
{[72 / 29]} \\
{[71 / 28]} \\
{[87 / 5][55 / 7]}\end{array}$ \\
\hline $\mathrm{C}_{13} \mathrm{H}_{28}$ & $\begin{array}{l}\text { 2-methyldodecane } \\
(373-503)\end{array}$ & 52.5 & $(388)$ & A & $\begin{array}{c}{[1560-97-0]} \\
{[87 / 5]}\end{array}$ \\
\hline $\mathrm{C}_{13} \mathrm{H}_{28}$ & $\begin{array}{l}\text { 3-methyldodecane } \\
(372-504)\end{array}$ & 51.4 & $(387)$ & $\mathrm{A}$ & $\begin{array}{c}{[17312-57-1]} \\
{[87 / 5]}\end{array}$ \\
\hline $\mathrm{C}_{13} \mathrm{H}_{28}$ & $\begin{array}{l}\text { 4-methyldodecane } \\
(372-501)\end{array}$ & 52.0 & $(387)$ & A & $\begin{array}{c}{[6117-97-1]} \\
{[87 / 5]}\end{array}$ \\
\hline $\mathrm{C}_{13} \mathrm{H}_{28}$ & $\begin{array}{l}\text { 5-methyldodecane } \\
(368-500)\end{array}$ & 50.6 & $(383)$ & $\mathrm{A}$ & $\begin{array}{c}{[17453-93-9]} \\
{[87 / 5]}\end{array}$ \\
\hline $\mathrm{C}_{13} \mathrm{H}_{28}$ & $\begin{array}{l}\text { 2,3-dimethylundecane } \\
(383-500)\end{array}$ & 53.2 & (398) & $\mathrm{A}$ & $\begin{array}{c}{[17312-77-5]} \\
{[87 / 5]}\end{array}$ \\
\hline $\mathrm{C}_{13} \mathrm{H}_{28}$ & $\begin{array}{l}\text { 2,4-dimethylundecane } \\
(365-490)\end{array}$ & 52.1 & $(380)$ & A & $\begin{array}{c}{[17312-80-0]} \\
{[87 / 5]}\end{array}$ \\
\hline $\mathrm{C}_{13} \mathrm{H}_{28}$ & $\begin{array}{l}\text { 2,4,6-trimethyldecane } \\
(352-478)\end{array}$ & 48.7 & $(367)$ & A & [87/5] \\
\hline $\mathrm{C}_{13} \mathrm{H}_{28}$ & $\begin{array}{l}\text { 5-ethyl-5-methyldecane } \\
(273-307)\end{array}$ & $\begin{array}{l}61.4 \pm 1.1 \\
60.5 \pm 1.1 \\
61.4 \pm 1.8\end{array}$ & $\begin{array}{l}(290) \\
(298) \\
(298)\end{array}$ & $\begin{array}{l}\text { HSA } \\
\text { CGC }\end{array}$ & $\begin{array}{l}{[95 / 27]} \\
{[95 / 27]} \\
{[95 / 27]}\end{array}$ \\
\hline $\mathrm{C}_{13} \mathrm{H}_{28}$ & $\begin{array}{l}\text { 5-butylnonane } \\
(298-365)\end{array}$ & 52.6 & $(313)$ & A, MG & $\begin{array}{l}{[17312-63-9]} \\
{[87 / 5][55 / 11]}\end{array}$ \\
\hline $\mathrm{C}_{13} \mathrm{H}_{28} \mathrm{O}$ & pentyl tert-octyl ether & $55.9 \pm 0.3$ & (298) & & {$[\mathrm{U} / 2][02 / 32]$} \\
\hline $\mathrm{C}_{13} \mathrm{H}_{28} \mathrm{O}$ & $\begin{array}{l}\text { 1-tridecanol } \\
(307-348) \\
(307-348) \\
(313-373) \\
(431-568)\end{array}$ & $\begin{array}{l}91.1 \\
95.8 \\
87.4 \\
69.2\end{array}$ & $\begin{array}{l}(327) \\
(298) \\
(343) \\
(446)\end{array}$ & $\begin{array}{r}\text { GS } \\
\text { GS } \\
\text { A }\end{array}$ & $\begin{array}{c}{[112-70-9]} \\
{[01 / 3]} \\
{[01 / 3]} \\
{[92 / 14]} \\
{[87 / 5]}\end{array}$ \\
\hline $\mathrm{C}_{13} \mathrm{H}_{28} \mathrm{O}$ & $\begin{array}{l}\text { 2,2-dimethyl-3-tert-butyl-3-heptanol } \\
\text { (379-513) }\end{array}$ & 58.3 & (394) & & $\begin{array}{c}{[42930-67-6]} \\
{[73 / 26]}\end{array}$ \\
\hline $\mathrm{C}_{13} \mathrm{H}_{28} \mathrm{O}$ & $\begin{array}{l}\text { 3,3,5,5-tetramethyl-4-ethyl-4-heptanol } \\
(393-526)\end{array}$ & 55.9 & $(408)$ & & {$[73 / 26]$} \\
\hline $\mathrm{C}_{13} \mathrm{H}_{28} \mathrm{O}$ & $\begin{array}{l}\text { 3,3,6-trimethyl-4-isopropyl-4-heptanol } \\
(381-512)\end{array}$ & 59.1 & (396) & & {$[73 / 26]$} \\
\hline
\end{tabular}


TABLE 6. Enthalpies of vaporization of organic compounds, 1880-2002-Continued

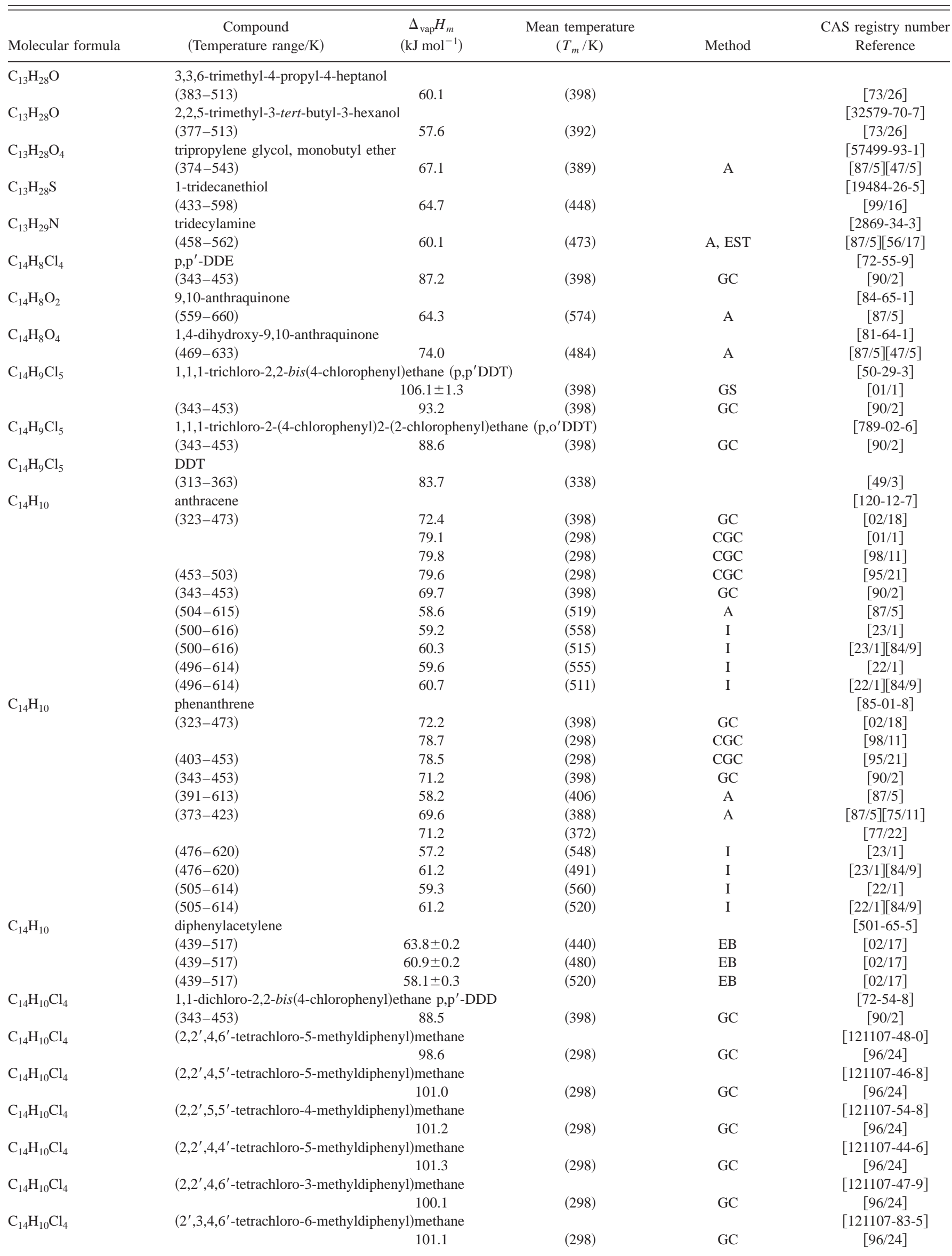


TABLE 6. Enthalpies of vaporization of organic compounds, 1880-2002-Continued

\begin{tabular}{|c|c|c|c|c|c|}
\hline Molecular formula & $\begin{array}{c}\text { Compound } \\
\text { (Temperature range/K) }\end{array}$ & $\begin{array}{c}\Delta_{\mathrm{vap}} H_{m} \\
\left(\mathrm{~kJ} \mathrm{~mol}^{-1}\right)\end{array}$ & $\begin{array}{l}\text { Mean temperature } \\
\qquad\left(T_{m} / \mathrm{K}\right)\end{array}$ & Method & $\begin{array}{c}\text { CAS registry number } \\
\text { Reference }\end{array}$ \\
\hline \multirow{2}{*}{$\mathrm{C}_{14} \mathrm{H}_{10} \mathrm{Cl}_{4}$} & \multicolumn{4}{|c|}{$\left(2,2^{\prime}, 4,4^{\prime}\right.$-tetrachloro-3-methyldiphenyl)methane } & {$[121107-43-5]$} \\
\hline & & 101.8 & $(298)$ & GC & {$[96 / 24]$} \\
\hline \multirow[t]{2}{*}{$\mathrm{C}_{14} \mathrm{H}_{10} \mathrm{Cl}_{4}$} & \multicolumn{4}{|c|}{$\left(2,3^{\prime}, 4,4^{\prime}\right.$-tetrachloro-5-methyldiphenyl)methane } & {$[121107-65-1]$} \\
\hline & & 103.8 & $(298)$ & $\mathrm{GC}$ & {$[96 / 24]$} \\
\hline \multirow[t]{2}{*}{$\mathrm{C}_{14} \mathrm{H}_{10} \mathrm{Cl}_{4}$} & \multicolumn{4}{|c|}{ (2'3,4,4'-tetrachloro-6-methyldiphenyl)methane } & {$[121107-77-5]$} \\
\hline & & 103.0 & $(298)$ & GC & {$[96 / 24]$} \\
\hline \multirow[t]{2}{*}{$\mathrm{C}_{14} \mathrm{H}_{10} \mathrm{O}_{2}$} & \multicolumn{4}{|l|}{ benzil } & {$[134-81-6]$} \\
\hline & $(401-620)$ & 69.2 & $(416)$ & A & {$[87 / 5][47 / 5]$} \\
\hline \multirow{2}{*}{$\mathrm{C}_{14} \mathrm{H}_{10} \mathrm{O}_{3}$} & \multicolumn{4}{|l|}{ benzoic acid anhydride } & [93-97-0] \\
\hline & $(416-633)$ & 69.1 & $(431)$ & A & {$[87 / 5][47 / 5]$} \\
\hline \multirow[t]{2}{*}{$\mathrm{C}_{14} \mathrm{H}_{11} \mathrm{~F}_{3}$} & \multicolumn{4}{|c|}{ 1,1,1-trifluoro-2,2-diphenylethane } & {$[384-94-1]$} \\
\hline & $(286-328)$ & $69.1 \pm 0.9$ & $(298)$ & GS & {$[97 / 14]$} \\
\hline \multirow{2}{*}{$\mathrm{C}_{14} \mathrm{H}_{12}$} & \multicolumn{4}{|l|}{ 1-methylfluorene } & {$[1730-37-6]$} \\
\hline & $(323-473)$ & 71.1 & $(398)$ & GC & {$[02 / 18]$} \\
\hline $\mathrm{C}_{14} \mathrm{H}_{12}$ & 9-methylfluorene & & & & {$[2523-37-7]$} \\
\hline & $(318-358)$ & 66.5 & $(298)$ & $\mathrm{B}$ & [94/4] \\
\hline $\mathrm{C}_{14} \mathrm{H}_{12}$ & 9,10-dihydrophenanthene & & & & {$[776-35-2]$} \\
\hline & $(417-453)$ & 64.0 & $(432)$ & A & {$[87 / 5]$} \\
\hline & $(353-418)$ & $72.3 \pm 0.6$ & $(340)$ & & {$[79 / 5]$} \\
\hline & $(353-418)$ & $76.6 \pm 0.1$ & $(298)$ & & {$[79 / 5]$} \\
\hline $\mathrm{C}_{14} \mathrm{H}_{12}$ & 1,1-diphenylethylene & & & & {$[530-48-3]$} \\
\hline & $(360-550)$ & 59.3 & $(375)$ & A & {$[87 / 5][47 / 5]$} \\
\hline $\mathrm{C}_{14} \mathrm{H}_{12}$ & cis 1,2-diphenylethylene ( & & & & {$[645-49-8]$} \\
\hline & $(373-428)$ & 66.5 & $(388)$ & A & {$[87 / 5]$} \\
\hline $\mathrm{C}_{14} \mathrm{H}_{12}$ & trans 1,2-diphenylethylen & lbene) & & & [103-30-0] \\
\hline & & 79.7 & $(298)$ & CGC & {$[98 / 11]$} \\
\hline & $(453-503)$ & 79.8 & $(298)$ & CGC & {$[95 / 21]$} \\
\hline & $(403-453)$ & 79.6 & (298) & CGC & {$[95 / 21]$} \\
\hline & $(419-580)$ & 65.5 & $(434)$ & A & {$[87 / 5]$} \\
\hline $\mathrm{C}_{14} \mathrm{H}_{12} \mathrm{O}$ & benzyl phenyl ketone & & & & {$[451-40-1]$} \\
\hline & $(396-594)$ & 68.1 & $(411)$ & A & {$[87 / 5][47 / 5]$} \\
\hline $\mathrm{C}_{14} \mathrm{H}_{12} \mathrm{O}$ & 2-methylbenzophenone & & & & {$[131-58-8]$} \\
\hline & $(435-580)$ & 65.1 & $(450)$ & A & {$[87 / 5]$} \\
\hline $\mathrm{C}_{14} \mathrm{H}_{12} \mathrm{O}$ & 3-methylbenzophenone & & & & [643-65-2] \\
\hline & $(445-585)$ & 68.4 & $(460)$ & A & {$[87 / 5]$} \\
\hline $\mathrm{C}_{14} \mathrm{H}_{12} \mathrm{O}$ & 4-methylbenzophenone & & & & [134-84-9] \\
\hline & $(450-492)$ & 72.0 & $(465)$ & A & {$[87 / 5]$} \\
\hline $\mathrm{C}_{14} \mathrm{H}_{12} \mathrm{O}_{2}$ & $(d l)$ benzoin & & & & [579-44-2] \\
\hline & $(408-616)$ & 69.0 & $(423)$ & A & {$[87 / 5][47 / 5]$} \\
\hline $\mathrm{C}_{14} \mathrm{H}_{12} \mathrm{O}_{2}$ & benzyl benzoate & & & & {$[120-51-4]$} \\
\hline & $(497-602)$ & 59.7 & $(512)$ & $\mathrm{A}, \mathrm{EB}$ & {$[87 / 5][76 / 13]$} \\
\hline & $(297-353)$ & 77.7 & $(312)$ & $\mathrm{A}, \mathrm{ME}$ & {$[87 / 5][57 / 9]$} \\
\hline $\mathrm{C}_{14} \mathrm{H}_{12} \mathrm{O}_{3}$ & benzyl salicylate & & & & {$[118-58-1]$} \\
\hline & $(295-334)$ & 78.7 & $(310)$ & $\mathrm{A}, \mathrm{ME}$ & {$[87 / 5][55 / 8]$} \\
\hline $\mathrm{C}_{14} \mathrm{H}_{12} \mathrm{O}_{3}$ & 2-hydroxy-4-methoxybenz & & & & {$[131-57-7]$} \\
\hline & $(337-413)$ & 74.7 & $(352)$ & A, UV & {$[87 / 5][60 / 2]$} \\
\hline $\mathrm{C}_{14} \mathrm{H}_{12} \mathrm{O}_{4}$ & $2,2^{\prime}$-dihydroxy-4-methoxy & hone & & & {$[131-53-3]$} \\
\hline & $(342-481)$ & 75.6 & $(357)$ & A, UV & {$[87 / 5][60 / 2]$} \\
\hline $\mathrm{C}_{14} \mathrm{H}_{13} \mathrm{~N}$ & N-benzylbenzaldehyde-im & & & & \\
\hline & $(309-340)$ & $83.4 \pm 1.2$ & $(324)$ & GS & {$[97 / 9]$} \\
\hline & $(309-340)$ & $85.0 \pm 1.2$ & $(298)$ & GS & {$[97 / 9]$} \\
\hline $\mathrm{C}_{14} \mathrm{H}_{14}$ & (4-methylphenyl)phenylm & & & & \\
\hline & $(293-333)$ & $68.6 \pm 0.3$ & $(313)$ & GS & {$[99 / 8]$} \\
\hline & $(293-333)$ & $69.5 \pm 0.3$ & $(298)$ & GS & [99/8] \\
\hline $\mathrm{C}_{14} \mathrm{H}_{14}$ & 3,3'-dimethylbiphenyl & & & & {$[612-75-9]$} \\
\hline & $(288-308)$ & 71.9 & $(298)$ & A & {$[87 / 5]$} \\
\hline $\mathrm{C}_{14} \mathrm{H}_{14}$ & 1,1-diphenylethane & & & & {$[612-00-0]$} \\
\hline & $(293-328)$ & $68.2 \pm 0.6$ & $(313)$ & GS & {$[99 / 8]$} \\
\hline & $(293-328)$ & $68.9 \pm 0.6$ & $(298)$ & GS & {$[99 / 8]$} \\
\hline & $(348-405)$ & 62.4 & $(363)$ & A & {$[87 / 5]$} \\
\hline $\mathrm{C}_{14} \mathrm{H}_{14}$ & 1,2-diphenylethane & & & & {$[103-29-7]$} \\
\hline & $(323-473)$ & 67.4 & $(398)$ & GC & {$[02 / 18]$} \\
\hline & & $66.2 \pm 0.2$ & $(340)$ & & {$[88 / 16]$} \\
\hline & $(333-413)$ & 64.1 & $(373)$ & & {$[89 / 14]$} \\
\hline
\end{tabular}


TABLE 6. Enthalpies of vaporization of organic compounds, 1880-2002-Continued

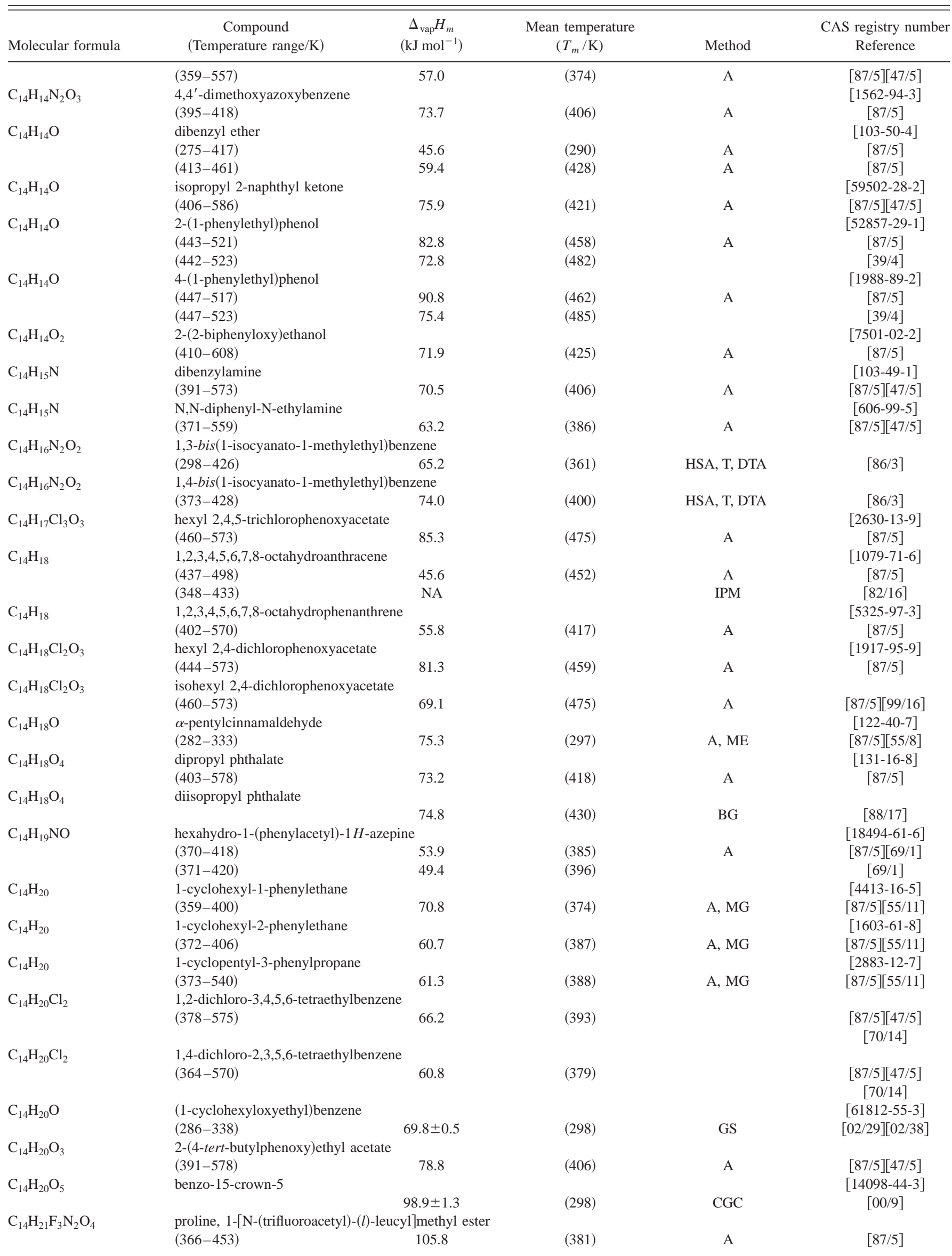


TABLE 6. Enthalpies of vaporization of organic compounds, 1880-2002-Continued

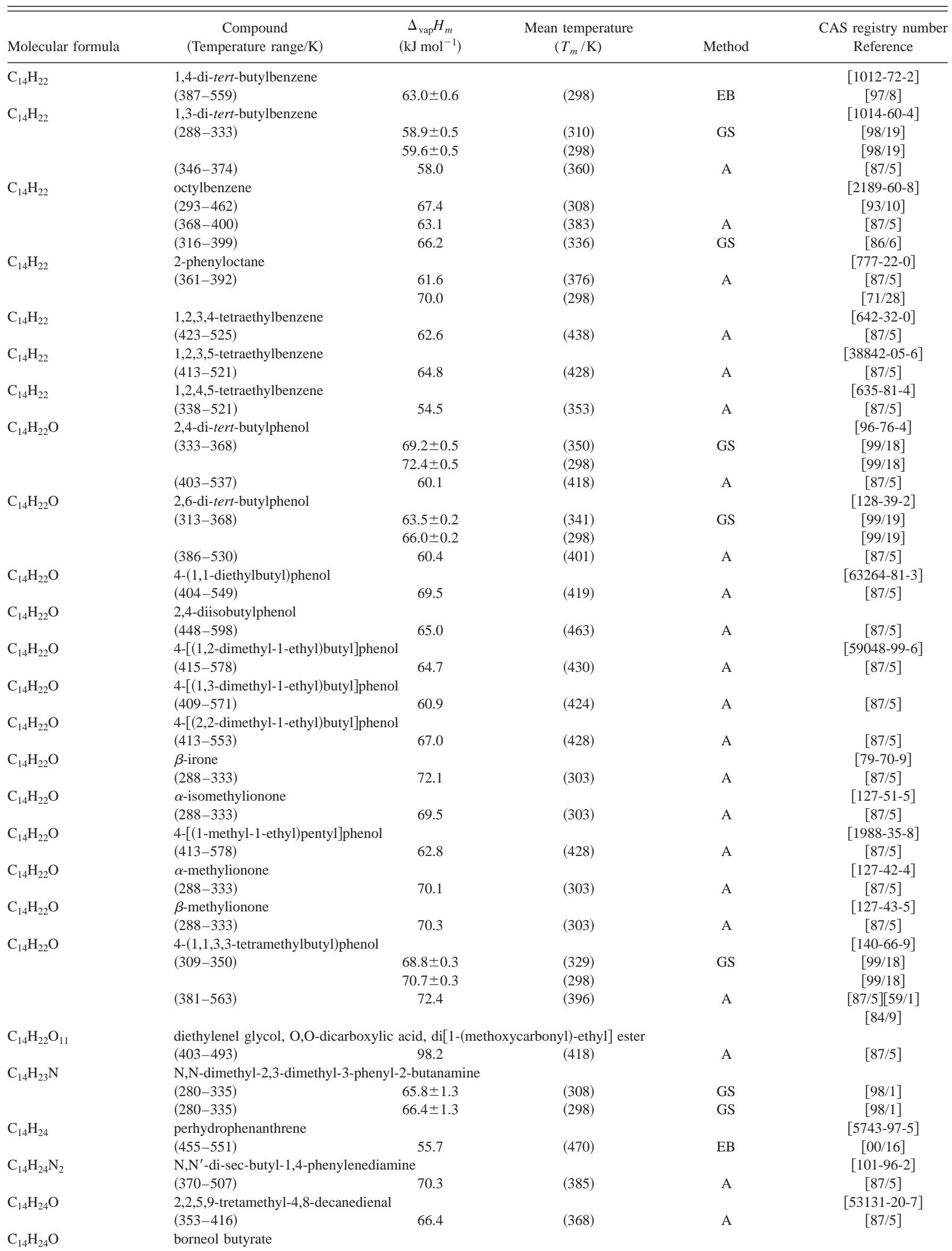


TABLE 6. Enthalpies of vaporization of organic compounds, 1880-2002-Continued

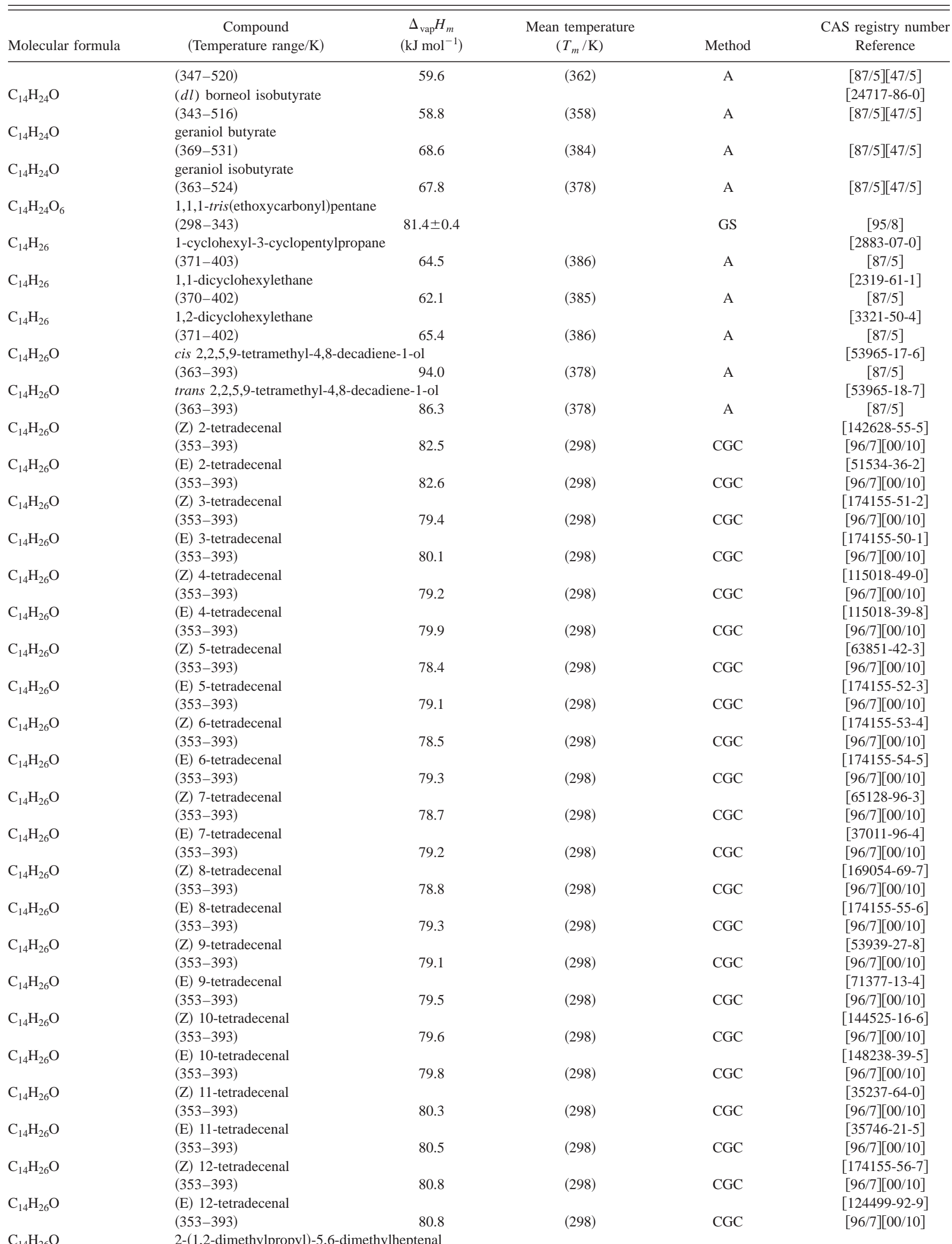


TABLE 6. Enthalpies of vaporization of organic compounds, 1880-2002-Continued

\begin{tabular}{|c|c|c|c|c|c|}
\hline Molecular formula & $\begin{array}{c}\text { Compound } \\
\text { (Temperature range/K) }\end{array}$ & $\begin{array}{c}\Delta_{\mathrm{vap}} H_{m} \\
\left(\mathrm{~kJ} \mathrm{~mol}^{-1}\right)\end{array}$ & $\begin{array}{l}\text { Mean temperature } \\
\qquad\left(T_{m} / \mathrm{K}\right)\end{array}$ & Method & $\begin{array}{l}\text { CAS registry number } \\
\text { Reference }\end{array}$ \\
\hline & $(385-535)$ & 60.0 & $(400)$ & EB & {$[87 / 3]$} \\
\hline \multirow[t]{2}{*}{$\mathrm{C}_{14} \mathrm{H}_{26} \mathrm{O}$} & 2-pentyl-2-nonenal & & & & [3021-89-4] \\
\hline & $(385-553)$ & 65.0 & (409) & $\mathrm{EB}$ & {$[87 / 3]$} \\
\hline \multirow[t]{2}{*}{$\mathrm{C}_{14} \mathrm{H}_{26} \mathrm{O}_{2}$} & decyl methacrylate & & & & {$[3179-47-3]$} \\
\hline & $(350-541)$ & 62.7 & $(365)$ & $\mathrm{A}$ & {$[87 / 5]$} \\
\hline \multirow[t]{2}{*}{$\mathrm{C}_{14} \mathrm{H}_{26} \mathrm{O}_{2}$} & (Z) 2-dodecenyl acetate & & & & [84801-15-0] \\
\hline & $(333-378)$ & 79.7 & (298) & $\mathrm{GC}$ & {$[97 / 13][00 / 10]$} \\
\hline \multirow[t]{2}{*}{$\mathrm{C}_{14} \mathrm{H}_{26} \mathrm{O}_{2}$} & (E) 2-dodecenyl acetate & & & & [84801-16-1] \\
\hline & $(333-378)$ & 81.0 & (298) & $\mathrm{GC}$ & {$[97 / 13][00 / 10]$} \\
\hline \multirow{2}{*}{$\mathrm{C}_{14} \mathrm{H}_{26} \mathrm{O}_{2}$} & (Z) 3-dodecenyl acetate & & & & {$[38363-24-5]$} \\
\hline & $(333-378)$ & 79.3 & $(298)$ & GC & {$[97 / 13][00 / 10]$} \\
\hline \multirow[t]{2}{*}{$\mathrm{C}_{14} \mathrm{H}_{26} \mathrm{O}_{2}$} & (E) 3-dodecenyl acetate & & & & {$[56218-63-4]$} \\
\hline & $(333-378)$ & 79.8 & (298) & GC & {$[97 / 13][00 / 10]$} \\
\hline \multirow[t]{2}{*}{$\mathrm{C}_{14} \mathrm{H}_{26} \mathrm{O}_{2}$} & (Z) 4-dodecenyl acetate & & & & {$[38363-25-6]$} \\
\hline & $(333-378)$ & 78.6 & (298) & GC & {$[97 / 13][00 / 10]$} \\
\hline \multirow[t]{2}{*}{$\mathrm{C}_{14} \mathrm{H}_{26} \mathrm{O}_{2}$} & (E) 4-dodecenyl acetate & & & & [38363-26-7] \\
\hline & $(333-378)$ & 79.8 & (298) & GC & {$[97 / 13][00 / 10]$} \\
\hline \multirow[t]{2}{*}{$\mathrm{C}_{14} \mathrm{H}_{26} \mathrm{O}_{2}$} & (Z) 5-dodecenyl acetate & & & & [16676-96-3] \\
\hline & $(333-378)$ & 79.2 & (298) & $\mathrm{GC}$ & {$[97 / 13][00 / 10]$} \\
\hline \multirow[t]{2}{*}{$\mathrm{C}_{14} \mathrm{H}_{26} \mathrm{O}_{2}$} & (E) 5-dodecenyl acetate & & & & {$[16676-97-4]$} \\
\hline & $(333-378)$ & 80.0 & (298) & GC & {$[97 / 13][00 / 10]$} \\
\hline \multirow[t]{2}{*}{$\mathrm{C}_{14} \mathrm{H}_{26} \mathrm{O}_{2}$} & (Z) 6-dodecenyl acetate & & & & [16974-12-2] \\
\hline & $(333-378)$ & 79.3 & $(298)$ & $\mathrm{GC}$ & {$[97 / 13][00 / 10]$} \\
\hline \multirow[t]{2}{*}{$\mathrm{C}_{14} \mathrm{H}_{26} \mathrm{O}_{2}$} & (E) 6-dodecenyl acetate & & & & [29868-16-4] \\
\hline & $(333-378)$ & 80.0 & $(298)$ & $\mathrm{GC}$ & {$[97 / 13][00 / 10]$} \\
\hline \multirow[t]{3}{*}{$\mathrm{C}_{14} \mathrm{H}_{26} \mathrm{O}_{2}$} & (Z) 7-dodecenyl acetate & & & & {$[14959-86-5]$} \\
\hline & $(333-378)$ & 79.8 & (298) & GC & {$[97 / 13][00 / 10]$} \\
\hline & $(303-317)$ & 77.5 & $(310)$ & GC & {$[83 / 10]$} \\
\hline \multirow[t]{2}{*}{$\mathrm{C}_{14} \mathrm{H}_{26} \mathrm{O}_{2}$} & (E) 7-dodecenyl acetate & & & & [16695-41-3] \\
\hline & $(333-378)$ & 80.2 & (298) & $\mathrm{GC}$ & {$[97 / 13][00 / 10]$} \\
\hline $\mathrm{C}_{14} \mathrm{H}_{26} \mathrm{O}_{2}$ & (Z) 8-dodecenyl acetate & & & & [28079-04-1] \\
\hline & $(333-378)$ & 80.2 & (298) & $\mathrm{GC}$ & {$[97 / 13][00 / 10]$} \\
\hline $\mathrm{C}_{14} \mathrm{H}_{26} \mathrm{O}_{2}$ & (E) 8-dodecenyl acetate & & & & [38363-29-0] \\
\hline & $(333-378)$ & 80.5 & $(298)$ & $\mathrm{GC}$ & {$[97 / 13][00 / 10]$} \\
\hline $\mathrm{C}_{14} \mathrm{H}_{26} \mathrm{O}_{2}$ & (Z) 9-dodecenyl acetate & & & & [16974-11-1] \\
\hline & $(333-378)$ & 80.7 & $(298)$ & $\mathrm{GC}$ & {$[97 / 13][00 / 10]$} \\
\hline $\mathrm{C}_{14} \mathrm{H}_{26} \mathrm{O}_{2}$ & (E) 9-dodecenyl acetate & & & & [35148-19-7] \\
\hline & $(333-378)$ & 81.0 & $(298)$ & GC & {$[97 / 13][00 / 10]$} \\
\hline $\mathrm{C}_{14} \mathrm{H}_{26} \mathrm{O}_{2}$ & (Z) 10-dodecenyl acetate & & & & {$[35148-20-0]$} \\
\hline & $(333-378)$ & 81.4 & (298) & $\mathrm{GC}$ & {$[97 / 13][00 / 10]$} \\
\hline $\mathrm{C}_{14} \mathrm{H}_{26} \mathrm{O}_{2}$ & (E) 10 -dodecenyl acetate & & & & [35153-09-4] \\
\hline & $(333-378)$ & 81.5 & (298) & $\mathrm{GC}$ & {$[97 / 13][00 / 10]$} \\
\hline $\mathrm{C}_{14} \mathrm{H}_{26} \mathrm{O}_{3}$ & 1,7-dioxa-8-cyclohexadeca & & & & {$[5963-13-3]$} \\
\hline & $(403-453)$ & 73.3 & $(418)$ & A & {$[87 / 5]$} \\
\hline $\mathrm{C}_{14} \mathrm{H}_{26} \mathrm{O}_{3}$ & 3-heptyl-4-acetoxytetrahyd & ran & & & [23144-23-2] \\
\hline & $(383-453)$ & 74.4 & (398) & A & {$[87 / 5]$} \\
\hline $\mathrm{C}_{14} \mathrm{H}_{26} \mathrm{O}_{3}$ & nonyl levulinate & & & & \\
\hline & $(423-571)$ & 69.4 & $(438)$ & A & {$[87 / 5]$} \\
\hline & & 68.4 & (516) & & {$[33 / 6]$} \\
\hline $\mathrm{C}_{14} \mathrm{H}_{26} \mathrm{O}_{4}$ & dibutyl adipate & & & & [105-99-7] \\
\hline & $(435-563)$ & 68.7 & $(450)$ & A & {$[87 / 5]$} \\
\hline $\mathrm{C}_{14} \mathrm{H}_{26} \mathrm{O}_{4}$ & diethyl isopentylmalonate & & & & \\
\hline & $(388-526)$ & 75.3 & $(403)$ & A & {$[87 / 5]$} \\
\hline $\mathrm{C}_{14} \mathrm{H}_{26} \mathrm{O}_{4}$ & 2-methylheptane-5,5-dicarl & id, diethyl e & & & \\
\hline $141_{26} U_{4}$ & $(394-427)$ & 70.1 & (409) & A & {$[87 / 5]$} \\
\hline $\mathrm{C}_{14} \mathrm{H}_{26} \mathrm{O}_{4}$ & diethyl decanedioate & & & & {$[110-40-7]$} \\
\hline & $(398-579)$ & 74.1 & $(413)$ & A & {$[87 / 5][47 / 5]$} \\
\hline $\mathrm{C}_{14} \mathrm{H}_{26} \mathrm{O}_{5}$ & ethyl[1-(1-octyloxycarbony & arbonate & & & \\
\hline & $(413-513)$ & 74.0 & (428) & A & {$[87 / 5]$} \\
\hline $\mathrm{C}_{14} \mathrm{H}_{26} \mathrm{O}_{5}$ & hexyl[1-(1-butoxycarbonyl & bonate & & & \\
\hline & $(357-501)$ & 72.1 & $(372)$ & A & {$[87 / 5]$} \\
\hline $\mathrm{C}_{14} \mathrm{H}_{27} \mathrm{~N}$ & myristonitrile & & & & [629-63-0] \\
\hline & $(391-580)$ & 71.4 & (406) & A & {$[87 / 5]$} \\
\hline & & $85.3 \pm 0.5$ & (298) & $\mathrm{C}$ & {$[77 / 5]$} \\
\hline
\end{tabular}


TABLE 6. Enthalpies of vaporization of organic compounds, 1880-2002-Continued

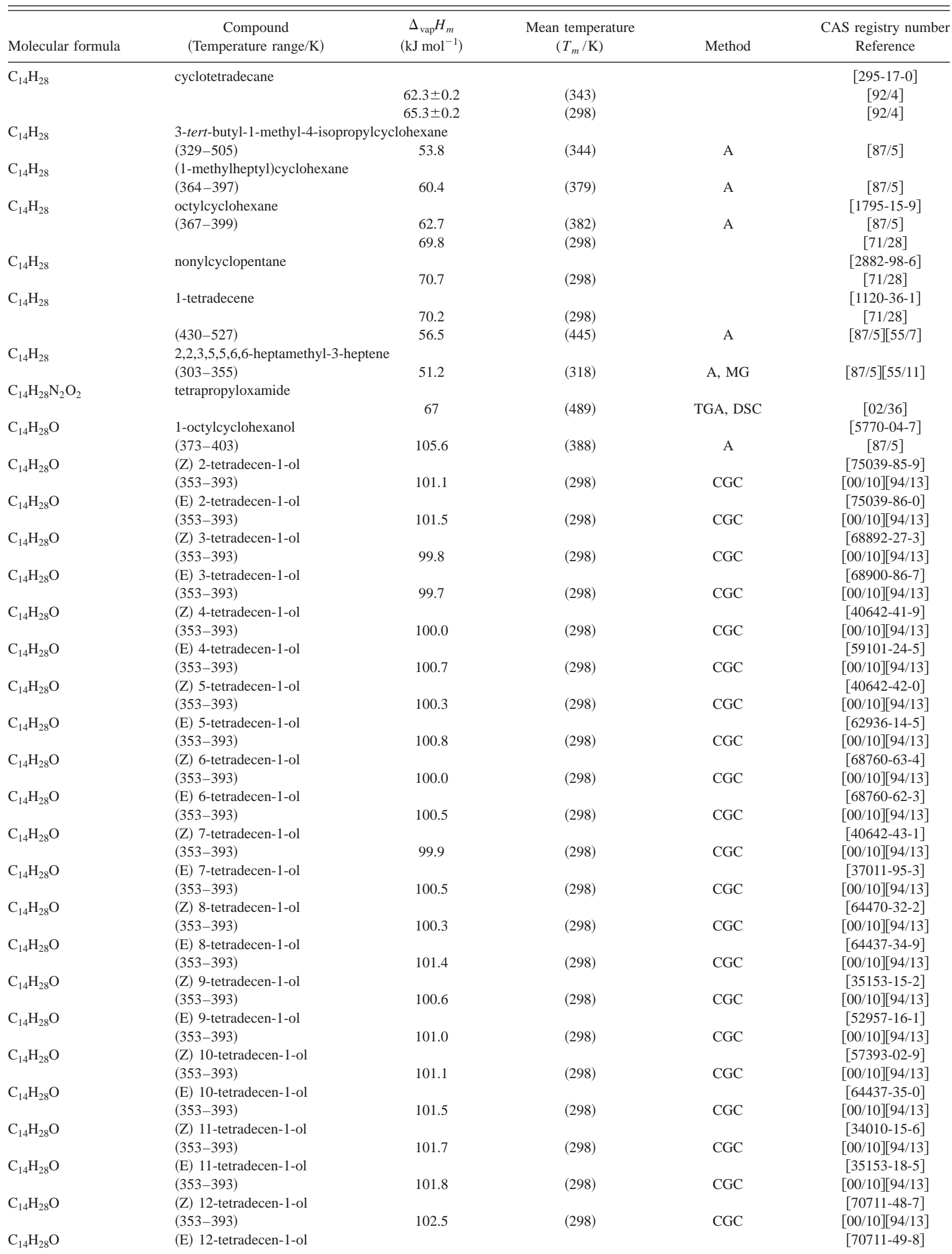


TABLE 6. Enthalpies of vaporization of organic compounds, 1880-2002-Continued

\begin{tabular}{|c|c|c|c|c|c|}
\hline Molecular formula & $\begin{array}{c}\text { Compound } \\
\text { (Temperature range/K) }\end{array}$ & $\begin{array}{c}\Delta_{\mathrm{vap}} H_{m} \\
\left(\mathrm{~kJ} \mathrm{~mol}^{-1}\right)\end{array}$ & $\begin{array}{l}\text { Mean temperature } \\
\qquad\left(T_{m} / \mathrm{K}\right)\end{array}$ & Method & $\begin{array}{c}\text { CAS registry number } \\
\text { Reference }\end{array}$ \\
\hline & $(353-393)$ & 102.5 & (298) & CGC & {$[00 / 10][94 / 13]$} \\
\hline \multirow{5}{*}{$\mathrm{C}_{14} \mathrm{H}_{28} \mathrm{O}$} & 2-tetradecanone & & & & [2345-27-9] \\
\hline & $(411-560)$ & 65.6 & $(426)$ & A & [87/5] \\
\hline & $(549-643)$ & 55.6 & $(564)$ & A & {$[87 / 5]$} \\
\hline & $(412-643)$ & 51.6 & $(556)$ & & [75/8] \\
\hline & $(372-551)$ & 64.4 & $(387)$ & A & {$[87 / 5][47 / 5]$} \\
\hline \multirow[t]{2}{*}{$\mathrm{C}_{14} \mathrm{H}_{28} \mathrm{O}$} & 7-tetradecanone & & & & {$[6137-34-4]$} \\
\hline & $(438-462)$ & 66.9 & $(450)$ & $\mathrm{A}, \mathrm{ME}$ & {$[87 / 5][38 / 8]$} \\
\hline \multirow[t]{3}{*}{$\mathrm{C}_{14} \mathrm{H}_{28} \mathrm{O}$} & tetradecanal & & & & {$[124-25-4]$} \\
\hline & $(343-383)$ & 80.2 & (298) & CGC & {$[96 / 7][00 / 10]$} \\
\hline & $(372-571)$ & 63.4 & $(387)$ & A & {$[87 / 5][47 / 5]$} \\
\hline \multirow{3}{*}{$\mathrm{C}_{14} \mathrm{H}_{28} \mathrm{O}_{2}$} & dodecyl acetate & & & & [112-66-3] \\
\hline & $(333-378)$ & 81.8 & (298) & GC & [97/13][00/10] \\
\hline & $(398-540)$ & 70.5 & $(413)$ & $\mathrm{A}$ & {$[87 / 5]$} \\
\hline \multirow[t]{3}{*}{$\mathrm{C}_{14} \mathrm{H}_{28} \mathrm{O}_{2}$} & ethyl dodecanoate & & & & {$[106-33-2]$} \\
\hline & $(423-483)$ & 80.0 & (298) & GC & {$[97 / 28]$} \\
\hline & $(386-435)$ & 67.2 & $(401)$ & $\mathrm{A}$ & {$[87 / 5]$} \\
\hline \multirow[t]{7}{*}{$\mathrm{C}_{14} \mathrm{H}_{28} \mathrm{O}_{2}$} & methyl tridecanoate & & & & {$[1731-88-0]$} \\
\hline & & 74.0 & $(350)$ & & {$[02 / 27]$} \\
\hline & & $72.3 \pm 0.1$ & $(368)$ & & {$[02 / 27]$} \\
\hline & & $80.0 \pm 0.5$ & (298) & & {$[02 / 27]$} \\
\hline & & $81.3 \pm 0.7$ & (298) & $\mathrm{GC}, \mathrm{C}$ & {$[80 / 5]$} \\
\hline & & $82.7 \pm 0.8$ & $(298)$ & C & {$[77 / 1]$} \\
\hline & $(377-504)$ & 72.6 & (392) & A, EST & {$[87 / 5][63 / 16]$} \\
\hline \multirow[t]{5}{*}{$\mathrm{C}_{14} \mathrm{H}_{28} \mathrm{O}_{2}$} & tetradecanoic acid & & & & {$[544-63-8]$} \\
\hline & $(383-459)$ & 100.4 & (398) & A & {$[87 / 5]$} \\
\hline & $(423-599)$ & 91.6 & $(438)$ & $\mathrm{A}$ & {$[87 / 5]$} \\
\hline & $(339-358)$ & $104.1 \pm 2.0$ & $(349)$ & $\mathrm{ME}, \mathrm{TE}$ & {$[82 / 4]$} \\
\hline & & 88.9 & $(455)$ & I & {$[43 / 7]$} \\
\hline \multirow[t]{2}{*}{$\mathrm{C}_{14} \mathrm{H}_{28} \mathrm{O}_{3}$} & decyl 3-methoxypropionate & & & & \\
\hline & $(403-513)$ & 68.9 & $(418)$ & A & {$[87 / 5]$} \\
\hline \multirow[t]{2}{*}{$\mathrm{C}_{14} \mathrm{H}_{29} \mathrm{Br}$} & 1-bromotetradecane & & & & {$[112-71-0]$} \\
\hline & $(437-645)$ & 67.1 & $(452)$ & $\mathrm{A}, \mathrm{EST}$ & $\begin{array}{c}{[87 / 5][61 / 13]} \\
{[70 / 14]}\end{array}$ \\
\hline \multirow[t]{6}{*}{$\mathrm{C}_{14} \mathrm{H}_{29} \mathrm{Cl}$} & 1-chlorotetradecane & & & & {$[2425-54-9]$} \\
\hline & $(313-373)$ & 80.2 & $(313)$ & GC & {$[80 / 14]$} \\
\hline & $(313-373)$ & 78.0 & $(333)$ & GC & {$[80 / 14]$} \\
\hline & $(313-373)$ & 74.4 & $(353)$ & GC & {$[80 / 14]$} \\
\hline & $(313-373)$ & 72.9 & $(373)$ & GC & [80/14] \\
\hline & $(414-570)$ & 68.7 & $(429)$ & A, DTA & {$[87 / 5][69 / 5]$} \\
\hline \multirow[t]{3}{*}{$\mathrm{C}_{14} \mathrm{H}_{29} \mathrm{~F}$} & 1-fluorotetradecane & & & & {$[593-33-9]$} \\
\hline & $(288-335)$ & $73.5 \pm 0.4$ & $(298)$ & GS & [97/14] \\
\hline & $(400-593)$ & 61.4 & $(415)$ & $\mathrm{A}, \mathrm{EST}$ & $\begin{array}{c}{[87 / 5][61 / 13]} \\
{[70 / 14]}\end{array}$ \\
\hline \multirow[t]{2}{*}{$\mathrm{C}_{14} \mathrm{H}_{29} \mathrm{I}$} & 1-iodotetradecane & & & & [19218-94-1] \\
\hline & $(452-672)$ & 68.6 & $(467)$ & A, EST & $\begin{array}{c}{[87 / 5][61 / 13]} \\
{[70 / 14]}\end{array}$ \\
\hline \multirow[t]{17}{*}{$\mathrm{C}_{14} \mathrm{H}_{30}$} & tetradecane & & & & [629-54-4] \\
\hline & & 72.1 & $(298)$ & GS & {$[01 / 1]$} \\
\hline & & $72.0 \pm 2.4$ & $(298)$ & CGC & {$[00 / 9]$} \\
\hline & & 69.0 & $(324)$ & $\mathrm{C}$ & {$[96 / 22]$} \\
\hline & & 68.6 & $(329)$ & $\mathrm{C}$ & {$[96 / 22]$} \\
\hline & & 67.9 & $(334)$ & $\mathrm{C}$ & {$[96 / 22]$} \\
\hline & & 66.8 & $(344)$ & $\mathrm{C}$ & {$[96 / 22]$} \\
\hline & & 65.7 & $(359)$ & $\mathrm{C}$ & {$[96 / 22]$} \\
\hline & $(423-473)$ & 71.2 & (298) & CGC & {$[95 / 21]$} \\
\hline & $(363-413)$ & 71.4 & (298) & $\mathrm{CGC}$ & {$[95 / 21]$} \\
\hline & & 71.7 & $(298)$ & & {$[94 / 12]$} \\
\hline & $(313-433)$ & 67.8 & $(328)$ & $\mathrm{A}$ & {$[87 / 5]$} \\
\hline & $(343-395)$ & 64.1 & $(361)$ & GS & {$[86 / 6]$} \\
\hline & & 70.1 & (313) & $\mathrm{C}$ & {$[79 / 2]$} \\
\hline & & 68.9 & $(328)$ & $\mathrm{C}$ & {$[79 / 2]$} \\
\hline & & $71.8 \pm 0.6$ & (298) & $\mathrm{C}$ & {$[79 / 2]$} \\
\hline & & $71.1 \pm 0.4$ & $(298)$ & $\mathrm{C}$ & {$[72 / 29]$} \\
\hline
\end{tabular}


TABLE 6. Enthalpies of vaporization of organic compounds, 1880-2002-Continued

\begin{tabular}{|c|c|c|c|c|c|}
\hline Molecular formula & $\begin{array}{c}\text { Compound } \\
\text { (Temperature range/K) }\end{array}$ & $\begin{array}{c}\Delta_{\mathrm{vap}} H_{m} \\
\left(\mathrm{~kJ} \mathrm{~mol}^{-1}\right)\end{array}$ & $\begin{array}{l}\text { Mean temperature } \\
\qquad\left(T_{m} / \mathrm{K}\right)\end{array}$ & Method & $\begin{array}{c}\text { CAS registry number } \\
\text { Reference }\end{array}$ \\
\hline & & 71.7 & $(298)$ & & {$[71 / 28]$} \\
\hline & $(432-529)$ & 57.1 & $(447)$ & A & {$[87 / 5][55 / 7]$} \\
\hline & $(429-468)$ & 57.8 & $(449)$ & ME & {$[38 / 8]$} \\
\hline \multirow{2}{*}{$\mathrm{C}_{14} \mathrm{H}_{30}$} & 2-methyltridecane & & & & {$[1560-96-9]$} \\
\hline & $(388-530)$ & 56.3 & $(403)$ & A & {$[87 / 5]$} \\
\hline \multirow[t]{2}{*}{$\mathrm{C}_{14} \mathrm{H}_{30}$} & 3-methyltridecane & & & & [6418-41-3] \\
\hline & $(389-521)$ & 55.1 & $(404)$ & $\mathrm{A}$ & {$[87 / 5]$} \\
\hline \multirow[t]{2}{*}{$\mathrm{C}_{14} \mathrm{H}_{30}$} & 4-methyltridecane & & & & {$[26730-12-1]$} \\
\hline & $(386-520)$ & 54.2 & $(401)$ & A & {$[87 / 5]$} \\
\hline \multirow[t]{2}{*}{$\mathrm{C}_{14} \mathrm{H}_{30}$} & 5-methyltridecane & & & & {$[25117-31-1]$} \\
\hline & $(385-518)$ & 53.8 & $(400)$ & A & {$[87 / 5]$} \\
\hline \multirow[t]{2}{*}{$\mathrm{C}_{14} \mathrm{H}_{30}$} & 7-methyltridecane & & & & [26730-14-3] \\
\hline & $(357-389)$ & 59.0 & $(372)$ & A & {$[87 / 5]$} \\
\hline \multirow[t]{2}{*}{$\mathrm{C}_{14} \mathrm{H}_{30}$} & 2,3-dimethyldodecane & & & & [6117-98-2] \\
\hline & $(385-519)$ & 53.4 & $(400)$ & A & {$[87 / 5]$} \\
\hline \multirow{2}{*}{$\mathrm{C}_{14} \mathrm{H}_{30}$} & 2,4-dimethyldodecane & & & & [6117-99-3] \\
\hline & $(379-509)$ & 54.0 & (394) & A & {$[87 / 5]$} \\
\hline \multirow[t]{2}{*}{$\mathrm{C}_{14} \mathrm{H}_{30}$} & 2,4,6-trimethylundecane & & & & \\
\hline & $(368-491)$ & 53.2 & $(383)$ & A & {$[87 / 5]$} \\
\hline \multirow[t]{2}{*}{$\mathrm{C}_{14} \mathrm{H}_{30}$} & 2,2,3,4,6,6-heptamethylhe & & & & {$[7225-67-4]$} \\
\hline & $(313-366)$ & 54.5 & $(366)$ & A, MG & {$[87 / 5][55 / 11]$} \\
\hline \multirow[t]{4}{*}{$\mathrm{C}_{14} \mathrm{H}_{30}$} & hexaethylethane $(3,3,4,4$-t & xane) & & & {$[5171-86-8]$} \\
\hline & $(298-307)$ & $63.9 \pm 1.2$ & $(298)$ & GS & {$[97 / 29]$} \\
\hline & $(283-302)$ & $65.7 \pm 1.2$ & $(292)$ & GS & {$[73 / 30][95 / 27]$} \\
\hline & & $65.0 \pm 1.2$ & $(298)$ & & {$[73 / 30]$} \\
\hline \multirow[t]{2}{*}{$\mathrm{C}_{14} \mathrm{H}_{30}$} & $2,2,3,3,4,4,5,5$-octamethyl & & & & [65149-84-0] \\
\hline & $(288-325)$ & $56.9 \pm 0.7$ & $(298)$ & GS & [97/29] \\
\hline \multirow[t]{2}{*}{$\mathrm{C}_{14} \mathrm{H}_{30} \mathrm{O}$} & diheptyl ether & & & & {$[629-64-1]$} \\
\hline & $(360-547)$ & 63.1 & $(375)$ & A & {$[87 / 5]$} \\
\hline \multirow[t]{2}{*}{$\mathrm{C}_{14} \mathrm{H}_{30} \mathrm{O}$} & 4-methylpentyl tert-octyl & & & & \\
\hline & & 57.5 & $(298)$ & & {$[\mathrm{U} / 2][02 / 32]$} \\
\hline \multirow[t]{2}{*}{$\mathrm{C}_{14} \mathrm{H}_{30} \mathrm{O}$} & 3-methylpentyl tert-octyl & & & & \\
\hline & & 58.0 & $(298)$ & & {$[\mathrm{U} / 2][02 / 32]$} \\
\hline $\mathrm{C}_{14} \mathrm{H}_{30} \mathrm{O}$ & 3,3-dimethylbutyl tert-octy & & & & \\
\hline & & 56.4 & $(298)$ & & {$[\mathrm{U} / 2][02 / 32]$} \\
\hline $\mathrm{C}_{14} \mathrm{H}_{30} \mathrm{O}$ & hexyl tert-octyl ether & & & & \\
\hline & & 59.2 & $(298)$ & & {$[\mathrm{U} / 2][02 / 32]$} \\
\hline $\mathrm{C}_{14} \mathrm{H}_{30} \mathrm{O}$ & 1-tetradecanol & & & & {$[112-72-1]$} \\
\hline & $(312-346)$ & 93.6 & $(328)$ & GS & {$[01 / 3]$} \\
\hline & $(312-346)$ & 98.7 & $(298)$ & GS & {$[01 / 3]$} \\
\hline & $(333-438)$ & 81.8 & $(386)$ & & {$[92 / 14]$} \\
\hline & $(317-358)$ & 109.0 & $(332)$ & A & {$[87 / 5]$} \\
\hline & & $102.2 \pm 2.3$ & $(298)$ & $\mathrm{C}$ & {$[77 / 1]$} \\
\hline & $(313-358)$ & 106.4 & $(328)$ & & {$[73 / 26]$} \\
\hline & $(424-569)$ & 76.6 & $(439)$ & $\mathrm{A}$ & {$[87 / 5][69 / 5]$} \\
\hline & $(313-326)$ & 104.2 & $(320)$ & ME & {$[65 / 15]$} \\
\hline $\mathrm{C}_{14} \mathrm{H}_{30} \mathrm{O}$ & 2-tetradecanol & & & & [4706-81-4] \\
\hline & $(313-428)$ & 95.7 & $(328)$ & & {$[99 / 11]$} \\
\hline $\mathrm{C}_{14} \mathrm{H}_{30} \mathrm{O}_{2}$ & 2-(dodecyloxy)ethanol & & & & {$[4536-30-5]$} \\
\hline & $(414-467)$ & 71.5 & $(429)$ & A & {$[87 / 5]$} \\
\hline $\mathrm{C}_{14} \mathrm{H}_{30} \mathrm{~S}$ & 1-tetradecanethiol & & & & [2079-95-0] \\
\hline & $(446-614)$ & 67.3 & $(461)$ & & {$[99 / 16]$} \\
\hline $\mathrm{C}_{14} \mathrm{H}_{30} \mathrm{~S}_{2}$ & diheptyl disulfide & & & & [10496-16-9] \\
\hline & $(458-630)$ & 69.8 & $(473)$ & & {$[99 / 16]$} \\
\hline $\mathrm{C}_{14} \mathrm{H}_{31} \mathrm{~N}$ & diheptylamine & & & & [2470-68-0] \\
\hline & $(435-605)$ & 60.0 & $(450)$ & A & {$[87 / 5]$} \\
\hline $\mathrm{C}_{14} \mathrm{H}_{31} \mathrm{~N}$ & $\mathrm{~N}, \mathrm{~N}$-dimethyldodecylamin & & & & {$[112-18-5]$} \\
\hline & $(380-604)$ & 64.4 & $(395)$ & A & {$[87 / 5]$} \\
\hline $\mathrm{C}_{14} \mathrm{H}_{31} \mathrm{~N}$ & tetradecylamine & & & & [2016-42-4] \\
\hline & $(471-577)$ & 62.4 & $(486)$ & A, EST & {$[87 / 5][56 / 17]$} \\
\hline $\mathrm{C}_{14} \mathrm{H}_{31} \mathrm{O}_{2} \mathrm{P}$ & diheptylphosphinic acid & & & & \\
\hline & $(482-664)$ & 64.1 & $(573)$ & & {$[71 / 32]$} \\
\hline $\mathrm{C}_{15} \mathrm{H}_{8} \mathrm{Cl}_{3} \mathrm{NO}_{2}$ & 2,2,4-trichloro-5-(2-naphth & ino)-4-cyclo & 1,3-dione & & {$[77765-38-9]$} \\
\hline & $(453-483)$ & 91.4 & $(468)$ & GC & {$[80 / 25]$} \\
\hline
\end{tabular}


TABLE 6. Enthalpies of vaporization of organic compounds, 1880-2002-Continued

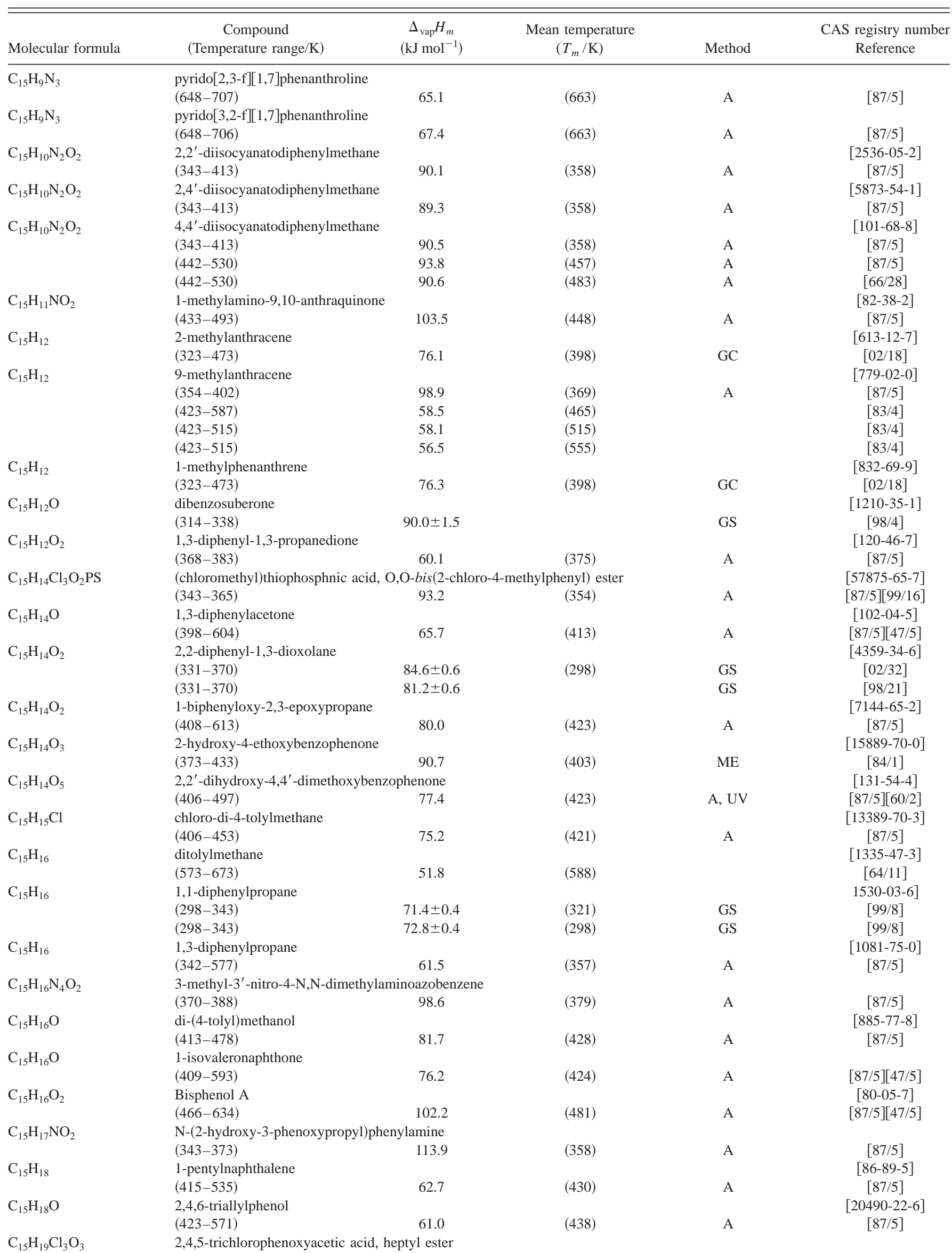


TABLE 6. Enthalpies of vaporization of organic compounds, 1880-2002-Continued

\begin{tabular}{|c|c|c|c|c|c|}
\hline Molecular formula & $\begin{array}{c}\text { Compound } \\
\text { (Temperature range/K) }\end{array}$ & $\begin{array}{c}\Delta_{\mathrm{vap}} H_{m} \\
\left(\mathrm{~kJ} \mathrm{~mol}^{-1}\right)\end{array}$ & $\begin{array}{l}\text { Mean temperature } \\
\qquad\left(T_{m} / \mathrm{K}\right)\end{array}$ & Method & $\begin{array}{c}\text { CAS registry number } \\
\text { Reference }\end{array}$ \\
\hline & $(460-573)$ & 92.3 & $(475)$ & A & {$[87 / 5]$} \\
\hline \multirow[t]{2}{*}{$\mathrm{C}_{15} \mathrm{H}_{20} \mathrm{Cl}_{2} \mathrm{O}_{3}$} & \multicolumn{4}{|c|}{ 2,4-dichlorophenoxyacetic acid, heptyl ester } & [1917-96-0] \\
\hline & $(460-573)$ & 88.3 & $(475)$ & A & {$[87 / 5]$} \\
\hline \multirow[t]{2}{*}{$\mathrm{C}_{15} \mathrm{H}_{20} \mathrm{Cl}_{2} \mathrm{O}_{3}$} & \multicolumn{5}{|c|}{ 2,4-dichlorophenoxyacetic acid, 1-propylbutyl ester } \\
\hline & $(460-573)$ & 77.3 & $(475)$ & A & {$[87 / 5]$} \\
\hline \multirow[t]{2}{*}{$\mathrm{C}_{15} \mathrm{H}_{20} \mathrm{Cl}_{2} \mathrm{O}_{4}$} & \multicolumn{4}{|c|}{ 2,4-dichlorophenoxyacetic acid, (1-methyl-2-butoxy)ethyl ester } & [3966-11-8] \\
\hline & $(443-573)$ & 82.5 & $(458)$ & A & {$[87 / 5]$} \\
\hline \multirow{2}{*}{$\mathrm{C}_{15} \mathrm{H}_{20} \mathrm{O}_{2}$} & helenine, alantolactone & & & & {$[1407-13-3]$} \\
\hline & $(430-548)$ & 112.7 & $(445)$ & A & {$[87 / 5]$} \\
\hline \multirow[t]{2}{*}{$\mathrm{C}_{15} \mathrm{H}_{22} \mathrm{~N}_{2} \mathrm{O}_{2}$} & dicyclohexylmethane- $4,4^{\prime}$ & & & & {$[5124-30-1]$} \\
\hline & $(326-404)$ & 80.4 & $(341)$ & A & {$[87 / 5]$} \\
\hline \multirow{3}{*}{$\mathrm{C}_{15} \mathrm{H}_{24}$} & nonylbenzene & & & & {$[1081-77-2]$} \\
\hline & $(316-415)$ & 69.7 & $(331)$ & GS & {$[86 / 6]$} \\
\hline & & 74.8 & $(298)$ & & {$[71 / 28]$} \\
\hline \multirow[t]{4}{*}{$\mathrm{C}_{15} \mathrm{H}_{24}$} & 1,3,5-triisopropylbenzene & & & & [717-74-8] \\
\hline & $(283-323)$ & $64.3 \pm 0.3$ & $(303)$ & GS & {$[98 / 10]$} \\
\hline & $(283-323)$ & $64.6 \pm 0.6$ & $(298)$ & GS & {$[98 / 10]$} \\
\hline & $(282-388)$ & 67.4 & $(297)$ & & {$[93 / 10]$} \\
\hline \multirow[t]{3}{*}{$\mathrm{C}_{15} \mathrm{H}_{24}$} & 1,3-di-tert-butyl-5-methyll & & & & \\
\hline & $(309-338)$ & $61.8 \pm 0.9$ & $(310)$ & GS & [98/19] \\
\hline & & $63.3 \pm 0.9$ & $(298)$ & & [98/19] \\
\hline \multirow[t]{2}{*}{$\mathrm{C}_{15} \mathrm{H}_{24} \mathrm{O}$} & 2,4-di-tert-butyl-5-methylp & & & & [497-39-2] \\
\hline & $(376-555)$ & 67.0 & $(391)$ & A & {$[87 / 5][47 / 5]$} \\
\hline \multirow[t]{2}{*}{$\mathrm{C}_{15} \mathrm{H}_{24} \mathrm{O}$} & 2,4-di-tert-butyl-6-methyl & & & & {$[616-55-7]$} \\
\hline & $(359-543)$ & 59.8 & $(374)$ & A & {$[87 / 5]$} \\
\hline \multirow[t]{3}{*}{$\mathrm{C}_{15} \mathrm{H}_{24} \mathrm{O}$} & 2,6-di-tert-butyl-4-methyl & & & & {$[128-37-0]$} \\
\hline & $(303-343)$ & 87.8 & $(318)$ & A & {$[87 / 5]$} \\
\hline & $(358-536)$ & 61.5 & (373) & A & {$[87 / 5][47 / 5]$} \\
\hline $\mathrm{C}_{15} \mathrm{H}_{24} \mathrm{O}$ & 2-methyl-4-(1,1,3,3-tetram & phenol & & & [2219-84-3] \\
\hline & $(447-683)$ & 67.1 & $(462)$ & A & {$[87 / 5]$} \\
\hline $\mathrm{C}_{15} \mathrm{H}_{24} \mathrm{O}$ & 3-methyl-4-(1,1,3,3-tetram & phenol & & & [2219-84-3] \\
\hline & $(436-549)$ & 65.5 & $(451)$ & A & {$[87 / 5]$} \\
\hline $\mathrm{C}_{15} \mathrm{H}_{24} \mathrm{O}$ & 4-methyl-2-(1,1,3,3-tetram & phenol & & & {$[4979-46-8]$} \\
\hline & $(415-545)$ & 65.0 & $(430)$ & A & {$[87 / 5]$} \\
\hline $\mathrm{C}_{15} \mathrm{H}_{24} \mathrm{O}$ & 4- $\left(3^{\prime}, 6^{\prime}\right.$-dimethyl-3'-hepty & & & & \\
\hline & & 89.4 & $(298)$ & $\mathrm{ME}$ & {$[01 / 21]$} \\
\hline $\mathrm{C}_{15} \mathrm{H}_{24} \mathrm{O}$ & 4-nonylphenol & & & & [104-40-5] \\
\hline & $(487-595)$ & 65.0 & $(502)$ & $\mathrm{A}, \mathrm{EB}$ & {$[87 / 5][76 / 13]$} \\
\hline $\mathrm{C}_{15} \mathrm{H}_{24} \mathrm{O}$ & $\alpha$-santalol & & & & [115-71-9] \\
\hline & $(293-450)$ & 58.3 & $(308)$ & A & {$[87 / 5]$} \\
\hline $\mathrm{C}_{15} \mathrm{H}_{24} \mathrm{O}_{2}$ & 2,5-di-tert-butyl-4-methox & & & & {$[1991-52-2]$} \\
\hline & $(423-453)$ & 64.4 & $(438)$ & A & {$[87 / 5]$} \\
\hline $\mathrm{C}_{15} \mathrm{H}_{24} \mathrm{O}_{2}$ & 1,3-dimethoxy-5-heptylber & & & & [6121-64-8] \\
\hline & $(419-488)$ & 75.5 & $(434)$ & A, GC & {$[87 / 5][75 / 24]$} \\
\hline $\mathrm{C}_{15} \mathrm{H}_{24} \mathrm{O}_{2}$ & 1,3-dimethoxy-5-methyl-2 & ene & & & {$[41442-51-7]$} \\
\hline & $(410-475)$ & 72.3 & $(425)$ & $\mathrm{A}, \mathrm{GC}$ & {$[87 / 5][75 / 24]$} \\
\hline $\mathrm{C}_{15} \mathrm{H}_{24} \mathrm{O}_{6}$ & aconitic acid, tripropyl est & & & & [64617-28-3] \\
\hline & $(359-500)$ & 72.3 & $(374)$ & A & {$[87 / 5]$} \\
\hline $\mathrm{C}_{15} \mathrm{H}_{26} \mathrm{O}$ & guaiol & & & & [489-86-1] \\
\hline & $(373-561)$ & 62.2 & $(388)$ & A & {$[87 / 5]$} \\
\hline $\mathrm{C}_{15} \mathrm{H}_{26} \mathrm{O}_{6}$ & camphorenic acid, triethyl & & & & \\
\hline & $(423-574)$ & 69.0 & $(438)$ & A & {$[87 / 5][47 / 5]$} \\
\hline $\mathrm{C}_{15} \mathrm{H}_{26} \mathrm{O}_{6}$ & tripropyl 1,2,3-propanetric & & & & {$[5333-54-0]$} \\
\hline & $(360-460)$ & 76.5 & $(375)$ & A & {$[87 / 5]$} \\
\hline $\mathrm{C}_{15} \mathrm{H}_{26} \mathrm{O}_{6}$ & glycerol tributyrate & & & & {$[60-01-1]$} \\
\hline & $(318-364)$ & 81.4 & $(333)$ & A & {$[87 / 5]$} \\
\hline $\mathrm{C}_{15} \mathrm{H}_{28} \mathrm{Cl}_{4}$ & 1,1,1,15-tetrachloropentad & & & & {$[3922-32-5]$} \\
\hline & $(340-392)$ & 103.5 & $(355)$ & A & {$[87 / 5]$} \\
\hline $\mathrm{C}_{15} \mathrm{H}_{28} \mathrm{O}$ & 3,7,11-trimethyl-1-dodecy & & & & [1604-35-9] \\
\hline & $(401-524)$ & $43.2 \pm 1.1$ & $(463)$ & & {$[88 / 4][86 / 16]$} \\
\hline $\mathrm{C}_{15} \mathrm{H}_{28} \mathrm{O}_{2}$ & dodecyl acrylate & & & & {$[2156-97-0]$} \\
\hline & $(432-573)$ & 64.6 & $(447)$ & A & {$[87 / 5]$} \\
\hline $\mathrm{C}_{15} \mathrm{H}_{28} \mathrm{O}_{2}$ & pentadecanolide & & & & {$[106-02-5]$} \\
\hline & $(363-443)$ & 78.2 & $(378)$ & A & {$[87 / 5]$} \\
\hline
\end{tabular}


TABLE 6. Enthalpies of vaporization of organic compounds, 1880-2002-Continued

\begin{tabular}{|c|c|c|c|c|c|}
\hline Molecular formula & $\begin{array}{c}\text { Compound } \\
\text { (Temperature range/K) }\end{array}$ & $\begin{array}{c}\Delta_{\mathrm{vap}} H_{m} \\
\left(\mathrm{~kJ} \mathrm{~mol}^{-1}\right)\end{array}$ & $\begin{array}{c}\text { Mean temperature } \\
\left(T_{m} / \mathrm{K}\right)\end{array}$ & Method & $\begin{array}{l}\text { CAS registry number } \\
\text { Reference }\end{array}$ \\
\hline & $(310-320)$ & 74.2 & $(315)$ & $\mathrm{A}, \mathrm{ME}$ & {$[87 / 5][54 / 9]$} \\
\hline \multirow[t]{2}{*}{$\mathrm{C}_{15} \mathrm{H}_{28} \mathrm{O}_{2}$} & (Z) 7-tridecenyl acetate & & & & [34270-22-9] \\
\hline & $(343-388)$ & 84.3 & $(298)$ & $\mathrm{GC}$ & {$[97 / 13][00 / 10]$} \\
\hline \multirow[t]{2}{*}{$\mathrm{C}_{15} \mathrm{H}_{28} \mathrm{O}_{2}$} & (E) 7-tridecenyl acetate & & & & {$[56577-30-1]$} \\
\hline & $(343-388)$ & 84.8 & $(298)$ & GC & {$[97 / 13][00 / 10]$} \\
\hline \multirow[t]{2}{*}{$\mathrm{C}_{15} \mathrm{H}_{28} \mathrm{O}_{2}$} & (Z) 9-tridecenyl acetate & & & & {$[35835-78-0]$} \\
\hline & $(343-388)$ & 85.1 & $(298)$ & $\mathrm{GC}$ & {$[97 / 13][00 / 10]$} \\
\hline \multirow[t]{2}{*}{$\mathrm{C}_{15} \mathrm{H}_{28} \mathrm{O}_{2}$} & (E) 9-tridecenyl acetate & & & & [52957-19-4] \\
\hline & $(343-388)$ & 85.5 & $(298)$ & GC & {$[97 / 13][00 / 10]$} \\
\hline \multirow[t]{2}{*}{$\mathrm{C}_{15} \mathrm{H}_{28} \mathrm{O}_{2}$} & (Z) 11-tridecenyl acetate & & & & {$[33951-95-0]$} \\
\hline & $(343-388)$ & 86.4 & $(298)$ & GC & {$[97 / 13][00 / 10]$} \\
\hline \multirow[t]{2}{*}{$\mathrm{C}_{15} \mathrm{H}_{28} \mathrm{O}_{2}$} & (E) 11-tridecenyl acetate & & & & [56195-36-9] \\
\hline & $(343-388)$ & 86.4 & $(298)$ & GC & {$[97 / 13][00 / 10]$} \\
\hline \multirow[t]{3}{*}{$\mathrm{C}_{15} \mathrm{H}_{28} \mathrm{O}_{3}$} & decyl levulinate & & & & {$[37826-51-0]$} \\
\hline & $(423-580)$ & 76.1 & $(438)$ & A & {$[87 / 5]$} \\
\hline & & 72.0 & $(524)$ & & [33/6] \\
\hline \multirow{2}{*}{$\mathrm{C}_{15} \mathrm{H}_{28} \mathrm{O}_{3}$} & 1,6-dioxa-7-cycloheptade & & & & [6707-60-4] \\
\hline & $(403-463)$ & 75.9 & $(418)$ & A & {$[87 / 5]$} \\
\hline \multirow{2}{*}{$\mathrm{C}_{15} \mathrm{H}_{28} \mathrm{O}_{5}$} & decyl[1-(methoxycarbony & bonate & & & \\
\hline & $(411-592)$ & 73.8 & $(426)$ & A & {$[87 / 5]$} \\
\hline \multirow{2}{*}{$\mathrm{C}_{15} \mathrm{H}_{29} \mathrm{~N}$} & pentadecanenitrile & & & & [2570-26-5] \\
\hline & $(403-596)$ & 75.5 & $(418)$ & A & {$[87 / 5]$} \\
\hline \multirow[t]{2}{*}{$\mathrm{C}_{15} \mathrm{H}_{29} \mathrm{NO}_{3}$} & 2-[2-ethyl(hexanoyloxy) $]$ & icid, butylam & & & \\
\hline & $(378-433)$ & 81.0 & $(393)$ & A & {$[87 / 5]$} \\
\hline \multirow[t]{4}{*}{$\mathrm{C}_{15} \mathrm{H}_{30}$} & decylcyclopentane & & & & [1795-21-7] \\
\hline & $(358-411)$ & 71.1 & $(373)$ & $\mathrm{A}$ & {$[87 / 5]$} \\
\hline & & 75.7 & $(298)$ & & {$[71 / 28]$} \\
\hline & $(453-553)$ & 59.7 & $(468)$ & A, MM & {$[87 / 5][54 / 7]$} \\
\hline \multirow{2}{*}{$\mathrm{C}_{15} \mathrm{H}_{30}$} & nonylcyclohexane & & & & {$[2883-02-5]$} \\
\hline & & 74.7 & (298) & & {$[71 / 28]$} \\
\hline \multirow[t]{5}{*}{$\mathrm{C}_{15} \mathrm{H}_{30}$} & 1-pentadecene & & & & [13360-61-7] \\
\hline & $(375-407)$ & 65.2 & $(390)$ & A & {$[87 / 5]$} \\
\hline & $(423-658)$ & 53.2 & $(570)$ & & {$[75 / 8]$} \\
\hline & & 75.1 & (298) & & {$[71 / 28]$} \\
\hline & $(443-543)$ & 59.3 & $(458)$ & A & {$[87 / 5][55 / 7]$} \\
\hline $\mathrm{C}_{15} \mathrm{H}_{30} \mathrm{O}$ & (Z) 9-pentadecen-1-ol & & & & [56218-94-1] \\
\hline & $(363-403)$ & 105.3 & $(298)$ & CGC & {$[00 / 10][94 / 13]$} \\
\hline $\mathrm{C}_{15} \mathrm{H}_{30} \mathrm{O}$ & (E) 9-pentadecen-1-ol & & & & {$[64437-40-7]$} \\
\hline & $(363-403)$ & 105.9 & $(298)$ & CGC & {$[00 / 10][94 / 13]$} \\
\hline $\mathrm{C}_{15} \mathrm{H}_{30} \mathrm{O}$ & (Z) 10-pentadecen-1-ol & & & & [64437-42-9] \\
\hline & $(363-403)$ & 105.9 & (298) & CGC & {$[00 / 10][94 / 13]$} \\
\hline $\mathrm{C}_{15} \mathrm{H}_{30} \mathrm{O}$ & (E) 10-pentadecen-1-ol & & & & [64437-44-1] \\
\hline & $(363-403)$ & 106.2 & $(298)$ & CGC & {$[00 / 10][94 / 13]$} \\
\hline $\mathrm{C}_{15} \mathrm{H}_{30} \mathrm{O}$ & (Z) 11-pentadecen-1-ol & & & & [69282-63-9] \\
\hline & $(363-403)$ & 106.3 & $(298)$ & CGC & {$[00 / 10][94 / 13]$} \\
\hline $\mathrm{C}_{15} \mathrm{H}_{30} \mathrm{O}$ & (E) 11-pentadecen-1-ol & & & & [69222-14-6] \\
\hline & $(363-403)$ & 106.5 & $(298)$ & CGC & {$[00 / 10][94 / 13]$} \\
\hline $\mathrm{C}_{15} \mathrm{H}_{30} \mathrm{O}$ & (Z) 12-pentadecen-1-ol & & & & [158906-50-4] \\
\hline & $(363-403)$ & 106.7 & $(298)$ & CGC & {$[00 / 10][94 / 13]$} \\
\hline $\mathrm{C}_{15} \mathrm{H}_{30} \mathrm{O}$ & (E) 12-pentadecen-1-ol & & & & [69222-15-7] \\
\hline & $(363-403)$ & 107.0 & (298) & CGC & {$[00 / 10][94 / 13]$} \\
\hline $\mathrm{C}_{15} \mathrm{H}_{30} \mathrm{O}$ & (Z) 13-pentadecen-1-ol & & & & [158906-51-5] \\
\hline & $(363-403)$ & 107.7 & (298) & CGC & {$[00 / 10][94 / 13]$} \\
\hline $\mathrm{C}_{15} \mathrm{H}_{30} \mathrm{O}$ & (E) 13-pentadecen-1-ol & & & & {$[158906-52-6]$} \\
\hline & $(363-403)$ & 107.7 & $(298)$ & CGC & {$[00 / 10][94 / 13]$} \\
\hline $\mathrm{C}_{15} \mathrm{H}_{30} \mathrm{O}$ & 2-pentadecanone & & & & {$[2345-28-0]$} \\
\hline & $(422-575)$ & 67.8 & $(437)$ & A & {$[87 / 5]$} \\
\hline & $(559-658)$ & 57.9 & $(574)$ & A & {$[87 / 5]$} \\
\hline $\mathrm{C}_{15} \mathrm{H}_{30} \mathrm{O}$ & 8-pentadecanone & & & & [818-23-5] \\
\hline & $(443-568)$ & 65.3 & $(458)$ & A & {$[87 / 5]$} \\
\hline & $(443-589)$ & 65.4 & $(458)$ & $\mathrm{A}$ & {$[87 / 5][75 / 8]$} \\
\hline & $(444-590)$ & 53.0 & $(567)$ & & {$[75 / 8]$} \\
\hline & $(438-462)$ & 61.9 & $(450)$ & $\mathrm{A}, \mathrm{ME}$ & {$[87 / 5][38 / 8]$} \\
\hline $\mathrm{C}_{15} \mathrm{H}_{30} \mathrm{O}_{2}$ & methyl tetradecanoate $(\mathrm{m}$ & state) & & & {$[124-10-7]$} \\
\hline
\end{tabular}


TABLE 6. Enthalpies of vaporization of organic compounds, 1880-2002-Continued

\begin{tabular}{|c|c|c|c|c|c|}
\hline Molecular formula & $\begin{array}{c}\text { Compound } \\
\text { (Temperature range/K) }\end{array}$ & $\begin{array}{c}\Delta_{\mathrm{vap}} H_{m} \\
\left(\mathrm{~kJ} \mathrm{~mol}^{-1}\right)\end{array}$ & $\begin{array}{l}\text { Mean temperature } \\
\qquad\left(T_{m} / \mathrm{K}\right)\end{array}$ & Method & $\begin{array}{c}\text { CAS registry number } \\
\text { Reference }\end{array}$ \\
\hline & & 79.8 & $(350)$ & & {$[02 / 27]$} \\
\hline & & $76.0 \pm 0.2$ & $(382)$ & & {$[02 / 27]$} \\
\hline & & $85.9 \pm 0.8$ & (298) & & {$[02 / 27]$} \\
\hline & $(393-473)$ & 86.6 & $(298)$ & GC & {$[97 / 28]$} \\
\hline & $(453-543)$ & 65.3 & $(498)$ & GC & [93/9] \\
\hline & & $86.2 \pm 1.0$ & (298) & $\mathrm{GC}, \mathrm{C}$ & {$[80 / 5]$} \\
\hline & & $87.0 \pm 0.9$ & $(298)$ & C & {$[77 / 1]$} \\
\hline & $(389-519)$ & 75.6 & (404) & A & {$[87 / 5][63 / 16]$} \\
\hline & $(364-417)$ & 77.4 & $(379)$ & $\mathrm{MG}, \mathrm{OM}$ & {$[52 / 13]$} \\
\hline \multirow[t]{3}{*}{$\mathrm{C}_{15} \mathrm{H}_{30} \mathrm{O}_{2}$} & isopropyl dodecanoate & & & & [10233-13-3] \\
\hline & $(305-452)$ & 81.5 & $(320)$ & & {$[01 / 10]$} \\
\hline & $(390-469)$ & 66.1 & $(405)$ & A & $\begin{array}{c}{[87 / 5][48 / 8]} \\
{[84 / 9]}\end{array}$ \\
\hline \multirow[t]{3}{*}{$\mathrm{C}_{15} \mathrm{H}_{30} \mathrm{O}_{2}$} & propyl dodecanoate & & & & {$[3681-78-5]$} \\
\hline & $(423-483)$ & 84.7 & $(298)$ & GC & [97/28] \\
\hline & $(396-479)$ & 66.9 & $(411)$ & A & $\begin{array}{c}{[87 / 5][48 / 8]} \\
{[84 / 9]}\end{array}$ \\
\hline \multirow[t]{2}{*}{$\mathrm{C}_{15} \mathrm{H}_{30} \mathrm{O}_{2}$} & tridecyl acetate & & & & [1072-33-9] \\
\hline & $(313-358)$ & 87.2 & $(298)$ & GC & {$[97 / 13][00 / 10]$} \\
\hline \multirow[t]{3}{*}{$\mathrm{C}_{15} \mathrm{H}_{30} \mathrm{O}_{2}$} & pentadecanoic acid & & & & [1002-84-2] \\
\hline & $(431-613)$ & 94.0 & $(446)$ & A & {$[87 / 5]$} \\
\hline & $(347-367)$ & $108.5 \pm 2.0$ & $(357)$ & $\mathrm{ME}, \mathrm{TE}$ & {$[82 / 4]$} \\
\hline \multirow[t]{2}{*}{$\mathrm{C}_{15} \mathrm{H}_{30} \mathrm{O}_{3}$} & dodecyl lactate & & & & {$[6283-92-7]$} \\
\hline & $(367-583)$ & 80.5 & $(382)$ & A & {$[87 / 5]$} \\
\hline \multirow[t]{2}{*}{$\mathrm{C}_{15} \mathrm{H}_{30} \mathrm{O}_{3}$} & decyl 2-ethoxypropionate & & & & [70160-09-7] \\
\hline & $(423-523)$ & 69.8 & $(438)$ & A & {$[87 / 5]$} \\
\hline \multirow[t]{2}{*}{$\mathrm{C}_{15} \mathrm{H}_{31} \mathrm{Br}$} & 1-bromopentadecane & & & & {$[629-72-1]$} \\
\hline & $(450-661)$ & 69.5 & $(465)$ & $\mathrm{A}, \mathrm{EST}$ & $\begin{array}{c}{[87 / 5][61 / 13]} \\
{[70 / 14]}\end{array}$ \\
\hline \multirow[t]{2}{*}{$\mathrm{C}_{15} \mathrm{H}_{31} \mathrm{Cl}$} & 1-chloropentadecane & & & & [4862-03-7] \\
\hline & $(439-645)$ & 55.4 & $(454)$ & $\mathrm{A}, \mathrm{EST}$ & $\begin{array}{c}{[87 / 5][61 / 13]} \\
{[70 / 14]}\end{array}$ \\
\hline \multirow[t]{2}{*}{$\mathrm{C}_{15} \mathrm{H}_{31} \mathrm{~F}$} & 1-fluoropentadecane & & & & {$[1555-17-5]$} \\
\hline & $(413-593)$ & 63.8 & $(428)$ & A, EST & $\begin{array}{c}{[87 / 5][61 / 13]} \\
{[70 / 14]}\end{array}$ \\
\hline \multirow[t]{2}{*}{$\mathrm{C}_{15} \mathrm{H}_{31} \mathrm{I}$} & 1-iodopentadecane & & & & [35599-78-1] \\
\hline & $(464-673)$ & 70.6 & $(479)$ & A, EST & $\begin{array}{c}{[87 / 5][61 / 13]} \\
{[70 / 14]}\end{array}$ \\
\hline $\mathrm{C}_{15} \mathrm{H}_{31} \mathrm{NO}_{2}$ & $\begin{array}{l}\text { N,N-dihexyl lactamide } \\
(418-453)\end{array}$ & 79.4 & $(433)$ & $\mathrm{A}$ & {$[87 / 5]$} \\
\hline $\mathrm{C}_{15} \mathrm{H}_{31} \mathrm{NO}_{2}$ & $\begin{array}{l}\text { N-dodecyl lactamide } \\
(408-476)\end{array}$ & 103.9 & $(423)$ & A & {$[87 / 5]$} \\
\hline \multirow[t]{17}{*}{$\mathrm{C}_{15} \mathrm{H}_{32}$} & pentadecane & & & & [629-62-9] \\
\hline & & 72.9 & $(334)$ & $\mathrm{C}$ & {$[96 / 22]$} \\
\hline & & 71.8 & $(344)$ & $\mathrm{C}$ & {$[96 / 22]$} \\
\hline & $(453-503)$ & 75.7 & (298) & CGC & {$[95 / 21]$} \\
\hline & $(423-473)$ & 76.2 & $(298)$ & CGC & {$[95 / 21]$} \\
\hline & $(363-413)$ & 76.4 & (298) & CGC & {$[95 / 21]$} \\
\hline & & 76.8 & $(298)$ & & [94/12] \\
\hline & $(366-409)$ & 67.5 & $(381)$ & A & {$[87 / 5]$} \\
\hline & $(333-409)$ & 66.4 & $(350)$ & GS & {$[86 / 6]$} \\
\hline & & $75.4 \pm 1.2$ & (298) & $\mathrm{C}$ & {$[79 / 2]$} \\
\hline & & 70.8 & $(353)$ & $\mathrm{C}$ & {$[79 / 2]$} \\
\hline & & 68.8 & $(373)$ & $\mathrm{C}$ & {$[79 / 2]$} \\
\hline & & $72.2 \pm 1.2$ & (333) & $\mathrm{C}$ & {$[79 / 2]$} \\
\hline & & $76.2 \pm 0.4$ & (298) & $\mathrm{C}$ & {$[72 / 29]$} \\
\hline & & 76.2 & $(298)$ & & {$[71 / 28]$} \\
\hline & $(447-546)$ & 59.6 & $(462)$ & A & {$[87 / 5][55 / 7]$} \\
\hline & $(430-464)$ & 61.9 & $(447)$ & ME & {$[38 / 8]$} \\
\hline \multirow[t]{2}{*}{$\mathrm{C}_{15} \mathrm{H}_{32}$} & 2-methyltetradecane & & & & [1560-95-8] \\
\hline & $(402-537)$ & 58.8 & $(417)$ & A & {$[87 / 5]$} \\
\hline \multirow[t]{2}{*}{$\mathrm{C}_{15} \mathrm{H}_{32}$} & 3-methyltetradecane & & & & {$[18435-22-8]$} \\
\hline & $(403-538)$ & 58.4 & $(418)$ & $\mathrm{A}$ & {$[87 / 5]$} \\
\hline $\mathrm{C}_{15} \mathrm{H}_{32}$ & 4-methyltetradecane & & & & [25117-24-2] \\
\hline
\end{tabular}


TABLE 6. Enthalpies of vaporization of organic compounds, 1880-2002-Continued

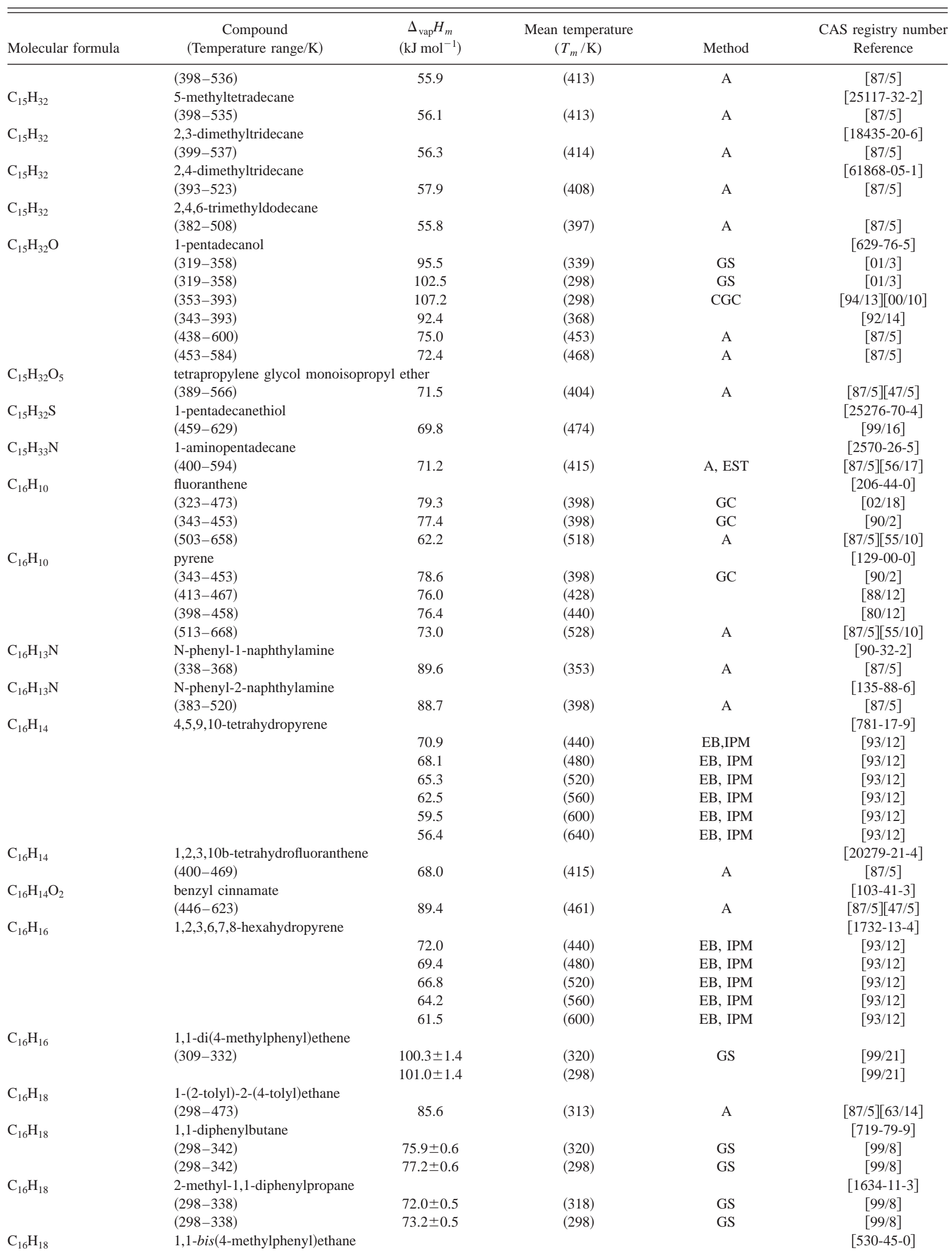


TABLE 6. Enthalpies of vaporization of organic compounds, 1880-2002-Continued

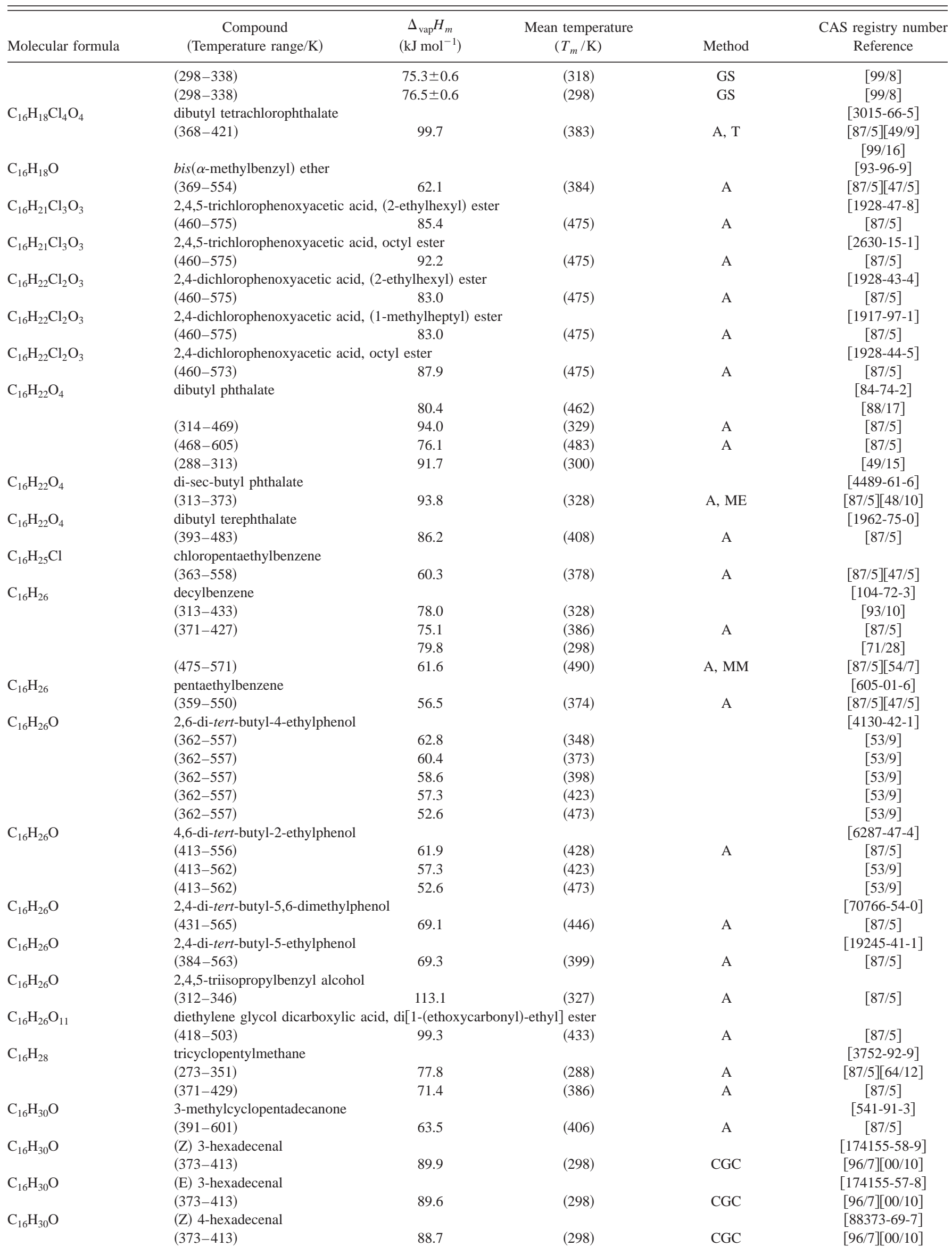


TABLE 6. Enthalpies of vaporization of organic compounds, 1880-2002-Continued

\begin{tabular}{|c|c|c|c|c|c|}
\hline Molecular formula & $\begin{array}{c}\text { Compound } \\
\text { (Temperature range/K) }\end{array}$ & $\begin{array}{c}\Delta_{\mathrm{vap}} H_{m} \\
\left(\mathrm{~kJ} \mathrm{~mol}^{-1}\right)\end{array}$ & $\begin{array}{l}\text { Mean temperature } \\
\qquad\left(T_{m} / \mathrm{K}\right)\end{array}$ & Method & $\begin{array}{c}\text { CAS registry number } \\
\text { Reference }\end{array}$ \\
\hline \multirow[t]{2}{*}{$\mathrm{C}_{16} \mathrm{H}_{30} \mathrm{O}$} & (E) 4-hexadecenal & & & & [174155-59-0] \\
\hline & $(373-413)$ & 88.9 & $(298)$ & CGC & {$[96 / 7][00 / 10]$} \\
\hline \multirow[t]{2}{*}{$\mathrm{C}_{16} \mathrm{H}_{30} \mathrm{O}$} & (Z) 5-hexadecenal & & & & [88373-68-6] \\
\hline & $(373-413)$ & 87.8 & $(298)$ & CGC & {$[96 / 7][00 / 10]$} \\
\hline \multirow[t]{2}{*}{$\mathrm{C}_{16} \mathrm{H}_{30} \mathrm{O}$} & (E) 5-hexadecenal & & & & [99142-11-7] \\
\hline & $(373-413)$ & 88.6 & (298) & CGC & {$[96 / 7][00 / 10]$} \\
\hline \multirow[t]{2}{*}{$\mathrm{C}_{16} \mathrm{H}_{30} \mathrm{O}$} & (Z) 6-hexadecenal & & & & [88373-67-5] \\
\hline & $(373-413)$ & 87.9 & (298) & $\mathrm{CGC}$ & {$[96 / 7][00 / 10]$} \\
\hline \multirow[t]{2}{*}{$\mathrm{C}_{16} \mathrm{H}_{30} \mathrm{O}$} & (E) 6-hexadecenal & & & & [103346-18-5] \\
\hline & $(373-413)$ & 88.5 & $(298)$ & CGC & {$[96 / 7][00 / 10]$} \\
\hline \multirow[t]{2}{*}{$\mathrm{C}_{16} \mathrm{H}_{30} \mathrm{O}$} & (Z) 7-hexadecenal & & & & [56797-40-1] \\
\hline & $(373-413)$ & 87.8 & (298) & CGC & {$[96 / 7][00 / 10]$} \\
\hline \multirow[t]{2}{*}{$\mathrm{C}_{16} \mathrm{H}_{30} \mathrm{O}$} & (E) 7-hexadecenal & & & & {$[72698-27-2]$} \\
\hline & $(373-413)$ & 88.6 & $(298)$ & CGC & {$[96 / 7][00 / 10]$} \\
\hline \multirow[t]{2}{*}{$\mathrm{C}_{16} \mathrm{H}_{30} \mathrm{O}$} & (Z) 8-hexadecenal & & & & [66644-98-2] \\
\hline & $(373-413)$ & 87.7 & $(298)$ & CGC & {$[96 / 7][00 / 10]$} \\
\hline \multirow[t]{2}{*}{$\mathrm{C}_{16} \mathrm{H}_{30} \mathrm{O}$} & (E) 8-hexadecenal & & & & [72698-28-3] \\
\hline & $(373-413)$ & 88.4 & (298) & CGC & {$[96 / 7][00 / 10]$} \\
\hline \multirow[t]{2}{*}{$\mathrm{C}_{16} \mathrm{H}_{30} \mathrm{O}$} & (Z) 9-hexadecenal & & & & [56219-04-6] \\
\hline & $(373-413)$ & 88.0 & (298) & $\mathrm{CGC}$ & {$[96 / 7][00 / 10]$} \\
\hline \multirow[t]{2}{*}{$\mathrm{C}_{16} \mathrm{H}_{30} \mathrm{O}$} & (E) 9-hexadecenal & & & & [72698-29-4] \\
\hline & $(373-413)$ & 88.6 & $(298)$ & CGC & {$[96 / 7][00 / 10]$} \\
\hline \multirow[t]{2}{*}{$\mathrm{C}_{16} \mathrm{H}_{30} \mathrm{O}$} & (Z) 10-hexadecenal & & & & [68279-24-3] \\
\hline & $(373-413)$ & 88.2 & $(298)$ & CGC & {$[96 / 7][00 / 10]$} \\
\hline \multirow[t]{2}{*}{$\mathrm{C}_{16} \mathrm{H}_{30} \mathrm{O}$} & (E) 10-hexadecenal & & & & [72698-30-7] \\
\hline & $(373-413)$ & 88.8 & $(298)$ & CGC & {$[96 / 7][00 / 10]$} \\
\hline \multirow[t]{2}{*}{$\mathrm{C}_{16} \mathrm{H}_{30} \mathrm{O}$} & (Z) 11-hexadecenal & & & & [53939-28-9] \\
\hline & $(373-413)$ & 88.5 & $(298)$ & CGC & {$[96 / 7][00 / 10]$} \\
\hline $\mathrm{C}_{16} \mathrm{H}_{30} \mathrm{O}$ & (E) 11-hexadecenal & & & & {$[57491-33-5]$} \\
\hline & $(373-413)$ & 89.2 & (298) & CGC & {$[96 / 7][00 / 10]$} \\
\hline $\mathrm{C}_{16} \mathrm{H}_{30} \mathrm{O}$ & (Z) 12-hexadecenal & & & & [72698-31-8] \\
\hline & $(373-413)$ & 89.3 & $(298)$ & $\mathrm{CGC}$ & {$[96 / 7][00 / 10]$} \\
\hline $\mathrm{C}_{16} \mathrm{H}_{30} \mathrm{O}$ & (E) 12-hexadecenal & & & & [72698-32-9] \\
\hline & $(373-413)$ & 89.3 & $(298)$ & CGC & {$[96 / 7][00 / 10]$} \\
\hline $\mathrm{C}_{16} \mathrm{H}_{30} \mathrm{O}$ & (Z) 13-hexadecenal & & & & [71545-96-5] \\
\hline & $(373-413)$ & 89.7 & $(298)$ & CGC & {$[96 / 7][00 / 10]$} \\
\hline $\mathrm{C}_{16} \mathrm{H}_{30} \mathrm{O}$ & (E) 13-hexadecenal & & & & [72698-33-0] \\
\hline & $(373-413)$ & 90.0 & (298) & CGC & {$[96 / 7][00 / 10]$} \\
\hline $\mathrm{C}_{16} \mathrm{H}_{30} \mathrm{O}_{2}$ & dodecyl methacrylate & & & & [142-90-5] \\
\hline & $(438-580)$ & 64.9 & $(453)$ & A & {$[87 / 5]$} \\
\hline $\mathrm{C}_{16} \mathrm{H}_{30} \mathrm{O}_{2}$ & oxa-2-cycloheptadecanone & & & & [109-29-5] \\
\hline & $(403-463)$ & 71.6 & $(418)$ & $\mathrm{A}$ & {$[87 / 5]$} \\
\hline $\mathrm{C}_{16} \mathrm{H}_{30} \mathrm{O}_{2}$ & (Z) 2-tetradecenyl acetate & & & & [51309-20-7] \\
\hline & $(353-398)$ & 89.1 & $(298)$ & GC & {$[97 / 13][00 / 10]$} \\
\hline $\mathrm{C}_{16} \mathrm{H}_{30} \mathrm{O}_{2}$ & (E) 2-tetradecenyl acetate & & & & {$[51309-21-8]$} \\
\hline & $(353-398)$ & 90.3 & $(298)$ & GC & {$[97 / 13][00 / 10]$} \\
\hline $\mathrm{C}_{16} \mathrm{H}_{30} \mathrm{O}_{2}$ & (Z) 3-tetradecenyl acetate & & & & {$[54897-65-3]$} \\
\hline & $(353-398)$ & 88.5 & $(298)$ & GC & {$[97 / 13][00 / 10]$} \\
\hline $\mathrm{C}_{16} \mathrm{H}_{30} \mathrm{O}_{2}$ & (E) 3-tetradecenyl acetate & & & & {$[56221-90-0]$} \\
\hline & $(353-398)$ & 89.2 & (298) & GC & {$[97 / 13][00 / 10]$} \\
\hline $\mathrm{C}_{16} \mathrm{H}_{30} \mathrm{O}_{2}$ & (Z) 4-tetradecenyl acetate & & & & [54897-66-4] \\
\hline & $(353-398)$ & 87.8 & $(298)$ & GC & {$[97 / 13][00 / 10]$} \\
\hline $\mathrm{C}_{16} \mathrm{H}_{30} \mathrm{O}_{2}$ & (E) 4-tetradecenyl acetate & & & & {$[56209-67-7]$} \\
\hline & $(353-398)$ & 89.0 & $(298)$ & GC & {$[97 / 13][00 / 10]$} \\
\hline $\mathrm{C}_{16} \mathrm{H}_{30} \mathrm{O}_{2}$ & (Z) 5-tetradecenyl acetate & & & & [35153-13-0] \\
\hline & $(353-398)$ & 88.3 & $(298)$ & GC & {$[97 / 13][00 / 10]$} \\
\hline $\mathrm{C}_{16} \mathrm{H}_{30} \mathrm{O}_{2}$ & (E) 5-tetradecenyl acetate & & & & [34010-13-4] \\
\hline & $(353-398)$ & 89.1 & $(298)$ & GC & {$[97 / 13][00 / 10]$} \\
\hline $\mathrm{C}_{16} \mathrm{H}_{30} \mathrm{O}_{2}$ & (Z) 6-tetradecenyl acetate & & & & {$[39650-11-8]$} \\
\hline & $(353-398)$ & 88.1 & $(298)$ & GC & {$[97 / 13][00 / 10]$} \\
\hline $\mathrm{C}_{16} \mathrm{H}_{30} \mathrm{O}_{2}$ & (E) 6-tetradecenyl acetate & & & & [39650-10-7] \\
\hline & $(353-398)$ & 88.9 & $(298)$ & GC & {$[97 / 13][00 / 10]$} \\
\hline $\mathrm{C}_{16} \mathrm{H}_{30} \mathrm{O}_{2}$ & (Z) 7-tetradecenyl acetate & & & & [16974-10-0] \\
\hline & $(353-398)$ & 88.4 & $(298)$ & GC & {$[97 / 13][00 / 10]$} \\
\hline
\end{tabular}


TABLE 6. Enthalpies of vaporization of organic compounds, 1880-2002-Continued

\begin{tabular}{|c|c|c|c|c|c|}
\hline Molecular formula & $\begin{array}{c}\text { Compound } \\
\text { (Temperature range/K) }\end{array}$ & $\begin{array}{c}\Delta_{\mathrm{vap}} H_{m} \\
\left(\mathrm{~kJ} \mathrm{~mol}^{-1}\right)\end{array}$ & $\begin{array}{l}\text { Mean temperature } \\
\qquad\left(T_{m} / \mathrm{K}\right)\end{array}$ & Method & $\begin{array}{c}\text { CAS registry number } \\
\text { Reference }\end{array}$ \\
\hline \multirow[t]{2}{*}{$\mathrm{C}_{16} \mathrm{H}_{30} \mathrm{O}_{2}$} & (E) 7-tetradecenyl acetate & & & & [28540-79-6] \\
\hline & $(353-398)$ & 89.0 & $(298)$ & GC & {$[97 / 13][00 / 10]$} \\
\hline \multirow[t]{2}{*}{$\mathrm{C}_{16} \mathrm{H}_{30} \mathrm{O}_{2}$} & (Z) 8-tetradecenyl acetate & & & & [35835-80-4] \\
\hline & $(353-398)$ & 88.7 & $(298)$ & GC & {$[97 / 13][00 / 10]$} \\
\hline \multirow[t]{2}{*}{$\mathrm{C}_{16} \mathrm{H}_{30} \mathrm{O}_{2}$} & (E) 8-tetradecenyl acetate & & & & [56218-64-5] \\
\hline & $(353-398)$ & 89.2 & $(298)$ & GC & {$[97 / 13][00 / 10]$} \\
\hline \multirow[t]{3}{*}{$\mathrm{C}_{16} \mathrm{H}_{30} \mathrm{O}_{2}$} & (Z) 9-tetradecenyl acetate & & & & {$[16725-53-4]$} \\
\hline & $(353-398)$ & 89.1 & (298) & $\mathrm{GC}$ & {$[97 / 13][00 / 10]$} \\
\hline & $(303-317)$ & 90 & $(310)$ & GC & {$[83 / 10]$} \\
\hline \multirow[t]{2}{*}{$\mathrm{C}_{16} \mathrm{H}_{30} \mathrm{O}_{2}$} & (E) 9-tetradecenyl acetate & & & & [23192-82-7] \\
\hline & $(353-398)$ & 89.6 & $(298)$ & GC & {$[97 / 13][00 / 10]$} \\
\hline \multirow[t]{2}{*}{$\mathrm{C}_{16} \mathrm{H}_{30} \mathrm{O}_{2}$} & (Z) 10-tetradecenyl acetate & & & & [35153-16-3] \\
\hline & $(353-398)$ & 89.6 & $(298)$ & GC & {$[97 / 13][00 / 10]$} \\
\hline \multirow[t]{2}{*}{$\mathrm{C}_{16} \mathrm{H}_{30} \mathrm{O}_{2}$} & (E) 10-tetradecenyl acetate & & & & {$[35153-17-4]$} \\
\hline & $(353-398)$ & 89.9 & (298) & GC & {$[97 / 13][00 / 10]$} \\
\hline \multirow[t]{2}{*}{$\mathrm{C}_{16} \mathrm{H}_{30} \mathrm{O}_{2}$} & (Z) 11-tetradecenyl acetate & & & & [20711-10-8] \\
\hline & $(353-398)$ & 90.0 & $(298)$ & GC & {$[97 / 13][00 / 10]$} \\
\hline \multirow[t]{2}{*}{$\mathrm{C}_{16} \mathrm{H}_{30} \mathrm{O}_{2}$} & (E) 11-tetradecenyl acetate & & & & [33189-72-9] \\
\hline & $(353-398)$ & 90.4 & $(298)$ & GC & {$[97 / 13][00 / 10]$} \\
\hline \multirow[t]{2}{*}{$\mathrm{C}_{16} \mathrm{H}_{30} \mathrm{O}_{2}$} & (Z) 12-tetradecenyl acetate & & & & [35153-20-9] \\
\hline & $(353-398)$ & 90.9 & $(298)$ & GC & {$[97 / 13][00 / 10]$} \\
\hline \multirow[t]{2}{*}{$\mathrm{C}_{16} \mathrm{H}_{30} \mathrm{O}_{2}$} & (E) 12-tetradecenyl acetate & & & & {$[35153-21-0]$} \\
\hline & $(353-398)$ & 90.8 & $(298)$ & $\mathrm{GC}$ & {$[97 / 13][00 / 10]$} \\
\hline \multirow[t]{2}{*}{$\mathrm{C}_{16} \mathrm{H}_{30} \mathrm{O}_{3}$} & 1,7-dioxa-8-cyclooctadecanone & & & & {$[6720-22-5]$} \\
\hline & $(403-463)$ & 73.3 & (418) & A & {$[87 / 5]$} \\
\hline \multirow[t]{2}{*}{$\mathrm{C}_{16} \mathrm{H}_{30} \mathrm{O}_{3}$} & 1,9-dioxa-2-cyclooctadecanone & & & & [36575-58-3] \\
\hline & $(403-463)$ & 74.5 & $(418)$ & A & {$[87 / 5]$} \\
\hline \multirow[t]{2}{*}{$\mathrm{C}_{16} \mathrm{H}_{30} \mathrm{O}_{4}$} & dipentyl adipate & & & & [14027-78-2] \\
\hline & $(449-575)$ & 74.7 & $(464)$ & $\mathrm{A}$ & {$[87 / 5]$} \\
\hline $\mathrm{C}_{16} \mathrm{H}_{30} \mathrm{O}_{5}$ & octyl[1-(butoxycarbonyl)ethyl]c & nate & & & \\
\hline & $(374-503)$ & 76.2 & $(389)$ & A & {$[87 / 5]$} \\
\hline $\mathrm{C}_{16} \mathrm{H}_{31} \mathrm{~N}$ & palmitonitrile & & & & {$[629-79-8]$} \\
\hline & $(503-608)$ & 70.1 & $(518)$ & A & {$[87 / 5]$} \\
\hline $\mathrm{C}_{16} \mathrm{H}_{32}$ & tetraisobutylene & & & & \\
\hline & $(381-440)$ & 54.5 & $(397)$ & & {$[43 / 2]$} \\
\hline $\mathrm{C}_{16} \mathrm{H}_{32}$ & decylcyclohexane & & & & {$[1795-16-0]$} \\
\hline & $(371-425)$ & 76.7 & $(386)$ & A & {$[87 / 5]$} \\
\hline & & 79.7 & (298) & & {$[71 / 28]$} \\
\hline & $(469-571)$ & 61.6 & $(484)$ & $\mathrm{A}, \mathrm{MM}$ & {$[87 / 5][54 / 7]$} \\
\hline $\mathrm{C}_{16} \mathrm{H}_{32}$ & undecylcyclopentane & & & & {$[6785-23-5]$} \\
\hline & & 80.6 & $(298)$ & & {$[71 / 28]$} \\
\hline $\mathrm{C}_{16} \mathrm{H}_{32}$ & 1-hexadecene & & & & [629-73-2] \\
\hline & & $80.3 \pm 0.4$ & $(298)$ & $\mathrm{C}$ & {$[77 / 1]$} \\
\hline & & $80.3 \pm 0.4$ & (298) & $\mathrm{C}$ & {$[76 / 5]$} \\
\hline & & 80.1 & (298) & & {$[71 / 28]$} \\
\hline & $(461-558)$ & 61.5 & $(476)$ & A & {$[87 / 5][54 / 7]$} \\
\hline $\mathrm{C}_{16} \mathrm{H}_{32} \mathrm{O}$ & (Z) 3-hexadecen-1-ol & & & & [141694-91-9] \\
\hline & $(373-413)$ & 110.7 & $(298)$ & CGC & {$[00 / 10][94 / 13]$} \\
\hline $\mathrm{C}_{16} \mathrm{H}_{32} \mathrm{O}$ & (E) 3-hexadecen-1-ol & & & & [128999-42-8] \\
\hline & $(373-413)$ & 110.8 & (298) & $\mathrm{CGC}$ & {$[00 / 10][94 / 13]$} \\
\hline $\mathrm{C}_{16} \mathrm{H}_{32} \mathrm{O}$ & (Z) 4-hexadecen-1-ol & & & & [145235-63-8] \\
\hline & $(373-413)$ & 110.6 & (298) & $\mathrm{CGC}$ & {$[00 / 10][94 / 13]$} \\
\hline $\mathrm{C}_{16} \mathrm{H}_{32} \mathrm{O}$ & (E) 4-hexadecen-1-ol & & & & [59101-23-4] \\
\hline & $(373-413)$ & 111.5 & $(298)$ & CGC & {$[00 / 10][94 / 13]$} \\
\hline $\mathrm{C}_{16} \mathrm{H}_{32} \mathrm{O}$ & (Z) 5-hexadecen-1-ol & & & & [106463-48-3] \\
\hline & $(373-413)$ & 110.9 & $(298)$ & $\mathrm{CGC}$ & {$[00 / 10][94 / 13]$} \\
\hline $\mathrm{C}_{16} \mathrm{H}_{32} \mathrm{O}$ & (E) 5-hexadecen-1-ol & & & & [85388-16-5] \\
\hline & $(373-413)$ & 111.4 & $(298)$ & CGC & {$[00 / 10][94 / 13]$} \\
\hline $\mathrm{C}_{16} \mathrm{H}_{32} \mathrm{O}$ & (Z) 6-hexadecen-1-ol & & & & [40642-45-3] \\
\hline & $(373-413)$ & 110.5 & $(298)$ & CGC & {$[00 / 10][94 / 13]$} \\
\hline $\mathrm{C}_{16} \mathrm{H}_{32} \mathrm{O}$ & (E) 6-hexadecen-1-ol & & & & [34500-33-9] \\
\hline & $(373-413)$ & 111.0 & (298) & $\mathrm{CGC}$ & {$[00 / 10][94 / 13]$} \\
\hline $\mathrm{C}_{16} \mathrm{H}_{32} \mathrm{O}$ & (Z) 7-hexadecen-1-ol & & & & [24880-48-6] \\
\hline & $(373-413)$ & 110.2 & (298) & $\mathrm{CGC}$ & {$[00 / 10][94 / 13]$} \\
\hline
\end{tabular}


TABLE 6. Enthalpies of vaporization of organic compounds, 1880-2002-Continued

\begin{tabular}{|c|c|c|c|c|c|}
\hline Molecular formula & $\begin{array}{c}\text { Compound } \\
\text { (Temperature range/K) }\end{array}$ & $\begin{array}{c}\Delta_{\mathrm{vap}} H_{m} \\
\left(\mathrm{~kJ} \mathrm{~mol}^{-1}\right)\end{array}$ & $\begin{array}{l}\text { Mean temperature } \\
\qquad\left(T_{m} / \mathrm{K}\right)\end{array}$ & Method & $\begin{array}{c}\text { CAS registry number } \\
\text { Reference }\end{array}$ \\
\hline \multirow[t]{2}{*}{$\mathrm{C}_{16} \mathrm{H}_{32} \mathrm{O}$} & (E) 7-hexadecen-1-ol & & & & [51824-10-3] \\
\hline & $(373-413)$ & 111.4 & $(298)$ & CGC & {$[00 / 10][94 / 13]$} \\
\hline \multirow[t]{2}{*}{$\mathrm{C}_{16} \mathrm{H}_{32} \mathrm{O}$} & (Z) 8-hexadecen-1-ol & & & & [64437-46-3] \\
\hline & $(373-413)$ & 110.4 & $(298)$ & CGC & {$[00 / 10][94 / 13]$} \\
\hline \multirow[t]{2}{*}{$\mathrm{C}_{16} \mathrm{H}_{32} \mathrm{O}$} & (E) 8-hexadecen-1-ol & & & & [64470-33-3] \\
\hline & $(373-413)$ & 111.1 & (298) & $\mathrm{CGC}$ & {$[00 / 10][94 / 13]$} \\
\hline \multirow[t]{2}{*}{$\mathrm{C}_{16} \mathrm{H}_{32} \mathrm{O}$} & (Z) 9-hexadecen-1-ol & & & & [10378-01-5] \\
\hline & $(373-413)$ & 110.6 & $(298)$ & CGC & {$[00 / 10][94 / 13]$} \\
\hline \multirow{2}{*}{$\mathrm{C}_{16} \mathrm{H}_{32} \mathrm{O}$} & (E) 9-hexadecen-1-ol & & & & {$[64437-47-4]$} \\
\hline & $(373-413)$ & 111.3 & $(298)$ & CGC & {$[00 / 10][94 / 13]$} \\
\hline \multirow[t]{2}{*}{$\mathrm{C}_{16} \mathrm{H}_{32} \mathrm{O}$} & (Z) 10-hexadecen-1-ol & & & & {$[64437-48-5]$} \\
\hline & $(373-413)$ & 111.0 & $(298)$ & CGC & {$[00 / 10][94 / 13]$} \\
\hline \multirow[t]{2}{*}{$\mathrm{C}_{16} \mathrm{H}_{32} \mathrm{O}$} & (E) 10-hexadecen-1-ol & & & & [54502-94-2] \\
\hline & $(373-413)$ & 111.5 & $(298)$ & CGC & {$[00 / 10][94 / 13]$} \\
\hline \multirow[t]{2}{*}{$\mathrm{C}_{16} \mathrm{H}_{32} \mathrm{O}$} & (Z) 11-hexadecen-1-ol & & & & [56683-54-6] \\
\hline & $(373-413)$ & 111.3 & (298) & CGC & {$[00 / 10][94 / 13]$} \\
\hline \multirow[t]{2}{*}{$\mathrm{C}_{16} \mathrm{H}_{32} \mathrm{O}$} & (E) 11-hexadecen-1-ol & & & & [61301-56-2] \\
\hline & $(373-413)$ & 111.8 & (298) & $\mathrm{CGC}$ & {$[00 / 10][94 / 13]$} \\
\hline \multirow[t]{2}{*}{$\mathrm{C}_{16} \mathrm{H}_{32} \mathrm{O}$} & (Z) 12-hexadecen-1-ol & & & & [72698-34-1] \\
\hline & $(373-413)$ & 111.8 & $(298)$ & CGC & {$[00 / 10][94 / 13]$} \\
\hline \multirow{2}{*}{$\mathrm{C}_{16} \mathrm{H}_{32} \mathrm{O}$} & (E) 12-hexadecen-1-ol & & & & {$[72698-35-2]$} \\
\hline & $(373-413)$ & 112.1 & (298) & $\mathrm{CGC}$ & {$[00 / 10][94 / 13]$} \\
\hline \multirow[t]{2}{*}{$\mathrm{C}_{16} \mathrm{H}_{32} \mathrm{O}$} & (Z) 13-hexadecen-1-ol & & & & {$[69282-65-1]$} \\
\hline & $(373-413)$ & 112.3 & $(298)$ & CGC & {$[00 / 10][94 / 13]$} \\
\hline \multirow[t]{2}{*}{$\mathrm{C}_{16} \mathrm{H}_{32} \mathrm{O}$} & (E) 13-hexadecen-1-ol & & & & [69282-66-2] \\
\hline & $(373-413)$ & 112.6 & $(298)$ & CGC & {$[00 / 10][94 / 13]$} \\
\hline \multirow[t]{2}{*}{$\mathrm{C}_{16} \mathrm{H}_{32} \mathrm{O}$} & 2-hexadecanone & & & & [18787-63-8] \\
\hline & $(382-580)$ & 72.3 & (397) & A & {$[87 / 5]$} \\
\hline $\mathrm{C}_{16} \mathrm{H}_{32} \mathrm{O}$ & hexadecanal & & & & [629-80-1] \\
\hline & $(343-383)$ & 89.7 & (298) & CGC & {$[96 / 7][00 / 10]$} \\
\hline & $(394-594)$ & 67.6 & (409) & $\mathrm{A}$ & {$[87 / 5][47 / 5]$} \\
\hline $\mathrm{C}_{16} \mathrm{H}_{32} \mathrm{O}_{2}$ & methyl pentadecanoate & & & & [7132-64-1] \\
\hline & & 82.1 & $(350)$ & & {$[02 / 26]$} \\
\hline & & $79.8 \pm 0.2$ & $(372)$ & & {$[02 / 26]$} \\
\hline & & $89.3 \pm 0.8$ & (298) & & {$[02 / 26]$} \\
\hline & $(433-473)$ & 88.8 & $(298)$ & $\mathrm{CGC}$ & {$[95 / 21]$} \\
\hline & & $91.6 \pm 0.9$ & (298) & GC,C & {$[80 / 5]$} \\
\hline & & $93.5 \pm 1.0$ & (298) & $\mathrm{C}$ & {$[77 / 1]$} \\
\hline & $(295-303)$ & $87.9 \pm 1.3$ & (299) & & {$[68 / 20]$} \\
\hline & $(400-527)$ & 78.3 & $(415)$ & A, EST & {$[87 / 5][63 / 16]$} \\
\hline $\mathrm{C}_{16} \mathrm{H}_{32} \mathrm{O}_{2}$ & ethyl tetradecanoate & & & & [124-06-1] \\
\hline & $(407-568)$ & 71.8 & $(422)$ & A & {$[87 / 5]$} \\
\hline $\mathrm{C}_{16} \mathrm{H}_{32} \mathrm{O}_{2}$ & butyl dodecanoate & & & & [106-18-3] \\
\hline & $(423-483)$ & 89.2 & $(298)$ & GC & {$[97 / 28]$} \\
\hline & $(343-383)$ & 75.8 & (358) & A & {$[87 / 5]$} \\
\hline $\mathrm{C}_{16} \mathrm{H}_{32} \mathrm{O}_{2}$ & isobutyl dodecanoate & & & & \\
\hline & $(345-452)$ & 80.0 & $(360)$ & & [01/10] \\
\hline $\mathrm{C}_{16} \mathrm{H}_{32} \mathrm{O}_{2}$ & tetradecyl acetate & & & & [638-59-5] \\
\hline & $(353-398)$ & 91.7 & (298) & GC & {$[97 / 13][00 / 10]$} \\
\hline & $(411-462)$ & 72.7 & $(426)$ & A & {$[87 / 5]$} \\
\hline $\mathrm{C}_{16} \mathrm{H}_{32} \mathrm{O}_{2}$ & hexadecanoic acid (palmitic acid) & & & & {$[57-10-3]$} \\
\hline & $(440-625)$ & 97.5 & $(455)$ & A & {$[87 / 5]$} \\
\hline & $(347-374)$ & $110.2 \pm 2.0$ & (364) & ME, TE & {$[82 / 4]$} \\
\hline & & 90.1 & $(475)$ & I & {$[43 / 7]$} \\
\hline $\mathrm{C}_{16} \mathrm{H}_{33} \mathrm{Br}$ & 1-bromohexadecane & & & & {$[112-82-3]$} \\
\hline & & $94.4 \pm 1.5$ & $(298)$ & $\mathrm{C}$ & {$[96 / 6]$} \\
\hline & $(461-673)$ & 71.9 & $(476)$ & A, EST & $\begin{array}{c}{[87 / 5][61 / 13]} \\
{[70 / 14]}\end{array}$ \\
\hline $\mathrm{C}_{16} \mathrm{H}_{33} \mathrm{Cl}$ & 1-chlorohexadecane & & & & {$[4860-03-1]$} \\
\hline & & $96.4 \pm 0.9$ & $(298)$ & GS & {$[01 / 1]$} \\
\hline & & $91.8 \pm 1.1$ & (298) & $\mathrm{C}$ & {$[77 / 1]$} \\
\hline & $(439-600)$ & 73.3 & $(454)$ & DTA & {$[69 / 5]$} \\
\hline $\mathrm{C}_{16} \mathrm{H}_{33} \mathrm{~F}$ & 1-fluorohexadecane & & & & {$[408-38-8]$} \\
\hline & $(425-608)$ & 66.1 & $(440)$ & A, EST & {$[87 / 5][61 / 13]$} \\
\hline
\end{tabular}


TABLE 6. Enthalpies of vaporization of organic compounds, 1880-2002-Continued

\begin{tabular}{|c|c|c|c|c|c|}
\hline Molecular formula & $\begin{array}{c}\text { Compound } \\
\text { (Temperature range/K) }\end{array}$ & $\begin{array}{c}\Delta_{\mathrm{vap}} H_{m} \\
\left(\mathrm{~kJ} \mathrm{~mol}^{-1}\right)\end{array}$ & $\begin{array}{l}\text { Mean temperature } \\
\qquad\left(T_{m} / \mathrm{K}\right)\end{array}$ & Method & $\begin{array}{c}\text { CAS registry number } \\
\text { Reference }\end{array}$ \\
\hline & & & & & [70/14] \\
\hline \multirow[t]{2}{*}{$\mathrm{C}_{16} \mathrm{H}_{33} \mathrm{I}$} & 1-iodohexadecane & & & & {$[544-77-4]$} \\
\hline & $(475-673)$ & 73.0 & $(490)$ & $\mathrm{A}, \mathrm{EST}$ & $\begin{array}{c}{[87 / 5][61 / 13]} \\
{[70 / 14]}\end{array}$ \\
\hline \multirow[t]{20}{*}{$\mathrm{C}_{16} \mathrm{H}_{34}$} & hexadecane & & & & [544-76-3] \\
\hline & & $81.8 \pm 1.3$ & $(298)$ & CGC & {$[00 / 9]$} \\
\hline & $(453-503)$ & 81.4 & (298) & $\mathrm{CGC}$ & {$[95 / 21]$} \\
\hline & $(423-473)$ & 81.4 & $(298)$ & CGC & {$[95 / 21]$} \\
\hline & $(363-413)$ & 81.2 & (298) & CGC & {$[95 / 21]$} \\
\hline & $(393-583)$ & 68.5 & $(408)$ & & {$[94 / 14]$} \\
\hline & & 81.4 & $(298)$ & & {$[94 / 12]$} \\
\hline & $(505-589)$ & 59.8 & $(520)$ & & {$[92 / 2]$} \\
\hline & $(323-423)$ & 74.9 & (338) & A & {$[87 / 5]$} \\
\hline & & 66.9 & $(343)$ & $\mathrm{GC}$ & {$[77 / 34]$} \\
\hline & & 66.2 & (353) & GC & {$[77 / 34]$} \\
\hline & & 65.6 & $(363)$ & $\mathrm{GC}$ & {$[77 / 34]$} \\
\hline & & 64.9 & (373) & GC & {$[77 / 34]$} \\
\hline & & 64.2 & (383) & GC & {$[77 / 34]$} \\
\hline & & $81.4 \pm 0.4$ & (298) & $\mathrm{C}$ & {$[72 / 29]$} \\
\hline & & 81.1 & (298) & & {$[71 / 28]$} \\
\hline & $(467-563)$ & 61.7 & $(482)$ & $\mathrm{A}, \mathrm{MM}$ & {$[87 / 5][54 / 7]$} \\
\hline & $(299-324)$ & 93.4 & $(311)$ & $\mathrm{ME}$ & [49/14] \\
\hline & $(293-308)$ & 80.2 & $(300)$ & $\mathrm{ME}$ & {$[49 / 17]$} \\
\hline & $(442-469)$ & 65.7 & $(455)$ & $\mathrm{ME}$ & [38/8] \\
\hline \multirow[t]{2}{*}{$\mathrm{C}_{16} \mathrm{H}_{34}$} & 2-methylpentadecane & & & & [1560-93-6] \\
\hline & $(417-554)$ & 62.0 & $(432)$ & A & {$[87 / 5]$} \\
\hline \multirow{2}{*}{$\mathrm{C}_{16} \mathrm{H}_{34}$} & 3-methylpentadecane & & & & [2882-96-4] \\
\hline & $(417-555)$ & 61.0 & $(432)$ & A & {$[87 / 5]$} \\
\hline \multirow[t]{2}{*}{$\mathrm{C}_{16} \mathrm{H}_{34}$} & 4-methylpentadecane & & & & {$[2801-87-8]$} \\
\hline & $(411-553)$ & 57.8 & $(426)$ & A & {$[87 / 5]$} \\
\hline \multirow[t]{2}{*}{$\mathrm{C}_{16} \mathrm{H}_{34}$} & 5-methylpentadecane & & & & {$[25117-33-3]$} \\
\hline & $(408-551)$ & 57.3 & $(423)$ & A & {$[87 / 5]$} \\
\hline \multirow[t]{2}{*}{$\mathrm{C}_{16} \mathrm{H}_{34}$} & 7-methylpentadecane & & & & {$[6165-40-8]$} \\
\hline & $(355-410)$ & 66.3 & $(370)$ & A & {$[87 / 5]$} \\
\hline \multirow[t]{2}{*}{$\mathrm{C}_{16} \mathrm{H}_{34}$} & 2,3-dimethyltetradecane & & & & [18435-23-9] \\
\hline & $(412-554)$ & 57.4 & $(427)$ & A & {$[87 / 5]$} \\
\hline \multirow[t]{2}{*}{$\mathrm{C}_{16} \mathrm{H}_{34}$} & 2,4-dimethyltetradecane & & & & {$[61868-06-2]$} \\
\hline & $(404-539)$ & 60.6 & $(419)$ & A & {$[87 / 5]$} \\
\hline \multirow[t]{2}{*}{$\mathrm{C}_{16} \mathrm{H}_{34}$} & 2,4,6-trimethyltridecane & & & & \\
\hline & $(395-521)$ & 59.1 & $(410)$ & A & {$[87 / 5]$} \\
\hline \multirow[t]{2}{*}{$\mathrm{C}_{16} \mathrm{H}_{34}$} & 2,2,4,4,6,8,8-heptamethylnonane & & & & {$[4390-04-9]$} \\
\hline & $(423-545)$ & 52.4 & $(438)$ & & {$[88 / 8]$} \\
\hline \multirow[t]{4}{*}{$\mathrm{C}_{16} \mathrm{H}_{34}$} & 3,3.6,6-tetraethyloctane & & & & \\
\hline & $(301-330)$ & $73.0 \pm 1.9$ & $(308)$ & HSA & {$[95 / 27]$} \\
\hline & & $74.3 \pm 1.9$ & $(298)$ & & {$[95 / 27]$} \\
\hline & & $72.3 \pm 1.8$ & $(298)$ & CGC & {$[95 / 27]$} \\
\hline \multirow[t]{2}{*}{$\mathrm{C}_{16} \mathrm{H}_{34} \mathrm{~N}_{2}$} & bis(1,1,3,3-tetramethylbutyl)diazene & & & & [39198-34-0] \\
\hline & & $66.5 \pm 0.6$ & $(298)$ & $\mathrm{C}$ & {$[76 / 3]$} \\
\hline \multirow[t]{12}{*}{$\mathrm{C}_{16} \mathrm{H}_{34} \mathrm{O}$} & 1-hexadecanol & & & & {$[36653-82-4]$} \\
\hline & $(328-362)$ & 100.4 & $(347)$ & GS & {$[01 / 3]$} \\
\hline & $(328-362)$ & 108.8 & $(298)$ & GS & {$[01 / 3]$} \\
\hline & & 112.5 & $(298)$ & CGC & {$[00 / 10]$} \\
\hline & $(343-463)$ & 88.2 & $(403)$ & & {$[92 / 14]$} \\
\hline & $(509-569)$ & 68.9 & $(524)$ & A & {$[87 / 5]$} \\
\hline & $(415-487)$ & 83.2 & $(430)$ & A & {$[87 / 5][74 / 13]$} \\
\hline & $(323-376)$ & 112.3 & $(338)$ & & {$[73 / 26]$} \\
\hline & $(418-463)$ & 78.8 & $(423)$ & & {$[73 / 26]$} \\
\hline & $(498-569)$ & 70.0 & $(513)$ & $\mathrm{A}, \mathrm{EB}$ & {$[87 / 5][70 / 2]$} \\
\hline & $(445-598)$ & 77.3 & $(460)$ & DTA & {$[69 / 5]$} \\
\hline & $(323-335)$ & 109.5 & $(329)$ & $\mathrm{ME}$ & {$[65 / 15]$} \\
\hline $\mathrm{C}_{16} \mathrm{H}_{34} \mathrm{O}$ & 2-hexadecanol & & & & {$[14852-31-4]$} \\
\hline & $(333-453)$ & 102.2 & $(348)$ & & [99/11] \\
\hline $\mathrm{C}_{16} \mathrm{H}_{34} \mathrm{O}_{3}$ & $2[2$-(dodecyloxy)ethoxy]ethanol & & & & {$[3055-93-4]$} \\
\hline & $(448-489)$ & 82.1 & $(463)$ & A & {$[87 / 5]$} \\
\hline
\end{tabular}


TABLE 6. Enthalpies of vaporization of organic compounds, 1880-2002-Continued

\begin{tabular}{|c|c|c|c|c|c|}
\hline Molecular formula & $\begin{array}{c}\text { Compound } \\
\text { (Temperature range/K) }\end{array}$ & $\begin{array}{c}\Delta_{\text {vap }} H_{m} \\
\left(\mathrm{~kJ} \mathrm{~mol}^{-1}\right)\end{array}$ & $\begin{array}{l}\text { Mean temperature } \\
\qquad\left(T_{m} / \mathrm{K}\right)\end{array}$ & Method & $\begin{array}{l}\text { CAS registry number } \\
\text { Reference }\end{array}$ \\
\hline $\mathrm{C}_{16} \mathrm{H}_{34} \mathrm{~S}$ & $\begin{array}{l}\text { 1-hexadecanethiol } \\
(470-643)\end{array}$ & 72.4 & (485) & & $\begin{array}{c}{[2917-26-2]} \\
{[99 / 16]}\end{array}$ \\
\hline $\mathrm{C}_{16} \mathrm{H}_{34} \mathrm{~S}$ & dioctyl sulfide & & & & [2690-08-6] \\
\hline & $(465-550)$ & $95.0 \pm 10.7$ & (298) & EB & [97/7] \\
\hline $\mathrm{C}_{16} \mathrm{H}_{34} \mathrm{~S}_{2}$ & $\begin{array}{l}\text { dioctyl disulfide } \\
(479-656)\end{array}$ & 73.9 & $(494)$ & & $\begin{array}{c}{[822-27-5]} \\
{[99 / 16]}\end{array}$ \\
\hline $\mathrm{C}_{16} \mathrm{H}_{35} \mathrm{~N}$ & $\begin{array}{l}\text { dioctylamine } \\
(448-597)\end{array}$ & $87.1 \pm 1.3$ & (298) & EB & $\begin{array}{c}{[1120-48-5]} \\
{[96 / 4]}\end{array}$ \\
\hline $\mathrm{C}_{16} \mathrm{H}_{35} \mathrm{~N}$ & $\begin{array}{l}\text { hexadecylamine } \\
(498-609)\end{array}$ & 66.9 & (513) & $\mathrm{A}$ & $\begin{array}{c}{[143-27-1]} \\
{[87 / 5]}\end{array}$ \\
\hline $\mathrm{C}_{16} \mathrm{H}_{35} \mathrm{~N}$ & $\begin{array}{l}\text { N,N-dimethyl-2-pentylnonylamine } \\
(401-552)\end{array}$ & 64.8 & $(425)$ & EB & {$[87 / 3]$} \\
\hline $\mathrm{C}_{16} \mathrm{H}_{36} \mathrm{~N}_{2}$ & $\begin{array}{l}\text { tetrabutyl hydrazine } \\
(392-453)\end{array}$ & 51.1 & $(407)$ & $\mathrm{A}$ & $\begin{array}{c}{[60678-70-8]} \\
{[87 / 5]}\end{array}$ \\
\hline $\mathrm{C}_{17} \mathrm{H}_{10} \mathrm{O}$ & $\begin{array}{l}\text { benzanthrone } \\
(498-673)\end{array}$ & 91.4 & (513) & A & $\begin{array}{c}{[82-05-3]} \\
{[87 / 5][47 / 5]}\end{array}$ \\
\hline $\mathrm{C}_{17} \mathrm{H}_{12}$ & $\begin{array}{l}\text { 1,2-benzofluorene } \\
(323-473)\end{array}$ & 83.7 & (398) & GC & $\begin{array}{c}{[238-84-6]} \\
{[02 / 18]}\end{array}$ \\
\hline $\mathrm{C}_{17} \mathrm{H}_{12}$ & $\begin{array}{l}\text { 2,3-benzofluorene } \\
(323-473)\end{array}$ & 84.7 & (398) & GC & $\begin{array}{c}{[243-17-4]} \\
{[02 / 18]}\end{array}$ \\
\hline $\mathrm{C}_{17} \mathrm{H}_{13} \mathrm{~N}$ & $\begin{array}{l}\text { 5-methyl-5 } H \text {-indeno[2,1-b]quinoline } \\
(375-388)\end{array}$ & 122.2 & $(381)$ & $\mathrm{A}$ & $\begin{array}{c}{[6626-64-8]} \\
{[87 / 5]}\end{array}$ \\
\hline $\mathrm{C}_{17} \mathrm{H}_{18} \mathrm{O}_{3}$ & $\begin{array}{l}\text { 2-hydroxy-4-butoxybenzophenone } \\
\text { (393-443) }\end{array}$ & 92.7 & (418) & ME & {$[84 / 1]$} \\
\hline $\mathrm{C}_{17} \mathrm{H}_{18} \mathrm{O}_{3}$ & $\begin{array}{l}\text { 4-(tert-butylphenyl) salicylate } \\
(336-438)\end{array}$ & 90.4 & $(351)$ & A, UV & $\begin{array}{c}{[87-18-3]} \\
{[87 / 5][60 / 2]}\end{array}$ \\
\hline $\mathrm{C}_{17} \mathrm{H}_{24} \mathrm{O}_{2}$ & $\begin{array}{l}\text { menthyl benzoate } \\
(396-574)\end{array}$ & 69.9 & (411) & A & $\begin{array}{l}{[6284-35-1]} \\
{[87 / 5][47 / 5]}\end{array}$ \\
\hline $\mathrm{C}_{17} \mathrm{H}_{28}$ & $\begin{array}{l}\text { undecylbenzene } \\
(450-622)\end{array}$ & $\begin{array}{l}66.7 \\
84.7\end{array}$ & $\begin{array}{l}(465) \\
(298)\end{array}$ & & $\begin{array}{c}{[6742-54-7]} \\
{[99 / 16]} \\
{[71 / 28]}\end{array}$ \\
\hline $\mathrm{C}_{17} \mathrm{H}_{28} \mathrm{O}$ & $\begin{array}{l}\text { 4-methyl-2,6-di-tert-pentylphenol } \\
(438-556)\end{array}$ & 65.9 & (453) & A & $\begin{array}{c}{[56103-67-4]} \\
{[87 / 5]}\end{array}$ \\
\hline $\mathrm{C}_{17} \mathrm{H}_{28} \mathrm{O}_{2}$ & $\begin{array}{l}\text { 1,3-dimethoxy-2-nonylbenzene } \\
(443-509)\end{array}$ & 79.2 & (458) & $\mathrm{A}, \mathrm{GC}$ & $\begin{array}{l}{[55095-35-7]} \\
{[87 / 5][75 / 24]}\end{array}$ \\
\hline $\mathrm{C}_{17} \mathrm{H}_{32}$ & $\begin{array}{l}\text { 1-heptadecyne } \\
(438-607)\end{array}$ & 62.7 & $(453)$ & & $\begin{array}{c}{[26186-00-5]} \\
{[99 / 16]}\end{array}$ \\
\hline $\mathrm{C}_{17} \mathrm{H}_{32}$ & $\begin{array}{l}\text { 2-heptadecyne } \\
(446-619)\end{array}$ & 63.7 & $(461)$ & & $\begin{array}{c}{[61847-96-9]} \\
{[99 / 16]}\end{array}$ \\
\hline $\mathrm{C}_{17} \mathrm{H}_{32}$ & $\begin{array}{l}\text { 3-heptadecyne } \\
(438-607)\end{array}$ & 62.5 & (453) & & $\begin{array}{c}{[61886-63-3]} \\
{[99 / 16]}\end{array}$ \\
\hline $\mathrm{C}_{17} \mathrm{H}_{32} \mathrm{Cl}_{4}$ & $\begin{array}{l}\text { 1,1,1,17-tetrachloroheptadecane } \\
(351-418)\end{array}$ & 108.0 & (366) & $\mathrm{A}$ & $\begin{array}{c}{[93479-16-4]} \\
{[87 / 5]}\end{array}$ \\
\hline $\mathrm{C}_{17} \mathrm{H}_{32} \mathrm{O}_{2}$ & $\begin{array}{l}\text { oxa-2-cyclooctadecanone } \\
(403-463)\end{array}$ & 73.5 & (418) & A & $\begin{array}{c}{[5637-97-8]} \\
{[87 / 5]}\end{array}$ \\
\hline $\mathrm{C}_{17} \mathrm{H}_{32} \mathrm{O}_{2}$ & $\begin{array}{l}\text { tetradecyl acrylate } \\
(458-601)\end{array}$ & 69.4 & $(473)$ & A & $\begin{array}{c}{[21643-42-5]} \\
{[87 / 5]}\end{array}$ \\
\hline $\mathrm{C}_{17} \mathrm{H}_{32} \mathrm{O}_{2}$ & $\begin{array}{l}\text { (Z) 9-pentadecenyl acetate } \\
(363-408)\end{array}$ & 93.6 & (298) & GC & $\begin{array}{c}{[35835-77-9]} \\
{[97 / 13][00 / 10]}\end{array}$ \\
\hline $\mathrm{C}_{17} \mathrm{H}_{32} \mathrm{O}_{2}$ & $\begin{array}{l}\text { (E) 9-pentadecenyl acetate } \\
(363-408)\end{array}$ & 94.3 & (298) & $\mathrm{GC}$ & $\begin{array}{c}{[64437-41-8]} \\
{[97 / 13][00 / 10]}\end{array}$ \\
\hline $\mathrm{C}_{17} \mathrm{H}_{32} \mathrm{O}_{2}$ & $\begin{array}{l}\text { (Z) 10-pentadecenyl acetate } \\
(363-408)\end{array}$ & 94.1 & $(298)$ & $\mathrm{GC}$ & $\begin{array}{l}{[64437-43-0]} \\
{[97 / 13][00 / 10]}\end{array}$ \\
\hline $\mathrm{C}_{17} \mathrm{H}_{32} \mathrm{O}_{2}$ & $\begin{array}{l}\text { (E) 10-pentadecenyl acetate } \\
(363-408)\end{array}$ & 94.6 & (298) & GC & $\begin{array}{c}{[64437-45-2]} \\
{[97 / 13][00 / 10]}\end{array}$ \\
\hline $\mathrm{C}_{17} \mathrm{H}_{32} \mathrm{O}_{2}$ & $\begin{array}{l}\text { (Z) 11-pentadecenyl acetate } \\
(363-408)\end{array}$ & 94.6 & (298) & GC & $\begin{array}{l}{[35153-25-4]} \\
{[97 / 13][00 / 10]}\end{array}$ \\
\hline $\mathrm{C}_{17} \mathrm{H}_{32} \mathrm{O}_{2}$ & $\begin{array}{l}\text { (E) 11-pentadecenyl acetate } \\
\text { (363-408) }\end{array}$ & 94.9 & (298) & GC & $\begin{array}{l}{[40535-40-8]} \\
{[97 / 13][00 / 10]}\end{array}$ \\
\hline $\mathrm{C}_{17} \mathrm{H}_{32} \mathrm{O}_{2}$ & $\begin{array}{l}\text { (Z) 12-pentadecenyl acetate } \\
\text { (363-408) }\end{array}$ & 95.1 & (298) & GC & $\begin{array}{c}{[70711-45-4]} \\
{[97 / 13][00 / 10]}\end{array}$ \\
\hline $\mathrm{C}_{17} \mathrm{H}_{32} \mathrm{O}_{2}$ & $\begin{array}{l}\text { (E) 12-pentadecenyl acetate } \\
\text { (363-408) } \\
\text { (Z) 13-pentadecenvl acetate }\end{array}$ & 94.5 & (298) & GC & $\begin{array}{l}{[73304-17-3]} \\
{[97 / 13][00 / 10]} \\
{[70711-46-5]}\end{array}$ \\
\hline
\end{tabular}


TABLE 6. Enthalpies of vaporization of organic compounds, 1880-2002-Continued

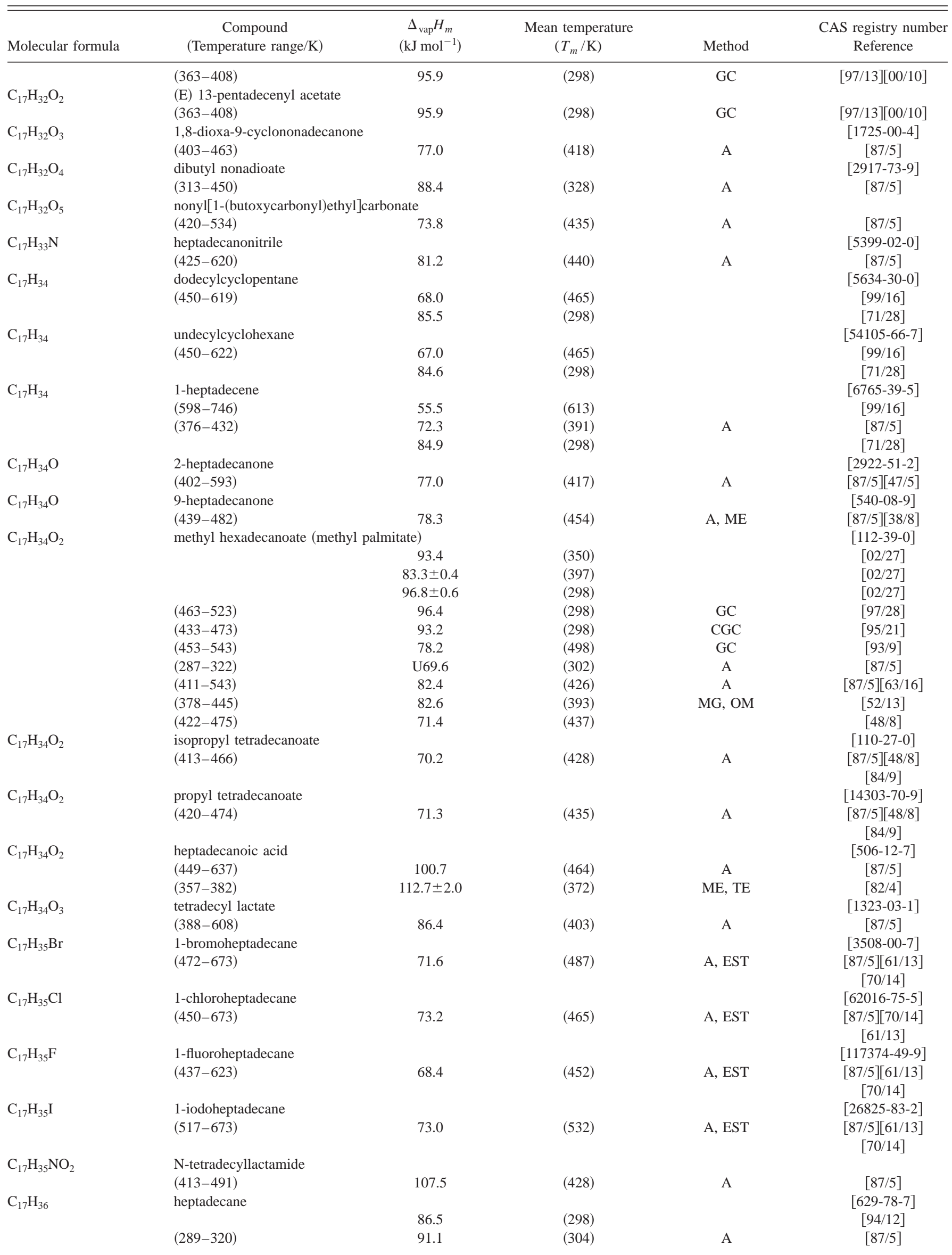


TABLE 6. Enthalpies of vaporization of organic compounds, 1880-2002-Continued

\begin{tabular}{|c|c|c|c|c|c|}
\hline Molecular formula & $\begin{array}{c}\text { Compound } \\
\text { (Temperature range/K) }\end{array}$ & $\begin{array}{c}\Delta_{\mathrm{vap}} H_{m} \\
\left(\mathrm{~kJ} \mathrm{~mol}^{-1}\right)\end{array}$ & $\begin{array}{l}\text { Mean temperature } \\
\qquad\left(T_{m} / \mathrm{K}\right)\end{array}$ & Method & $\begin{array}{l}\text { CAS registry number } \\
\text { Reference }\end{array}$ \\
\hline & $(488-577)$ & 62.9 & $(503)$ & A & {$[87 / 5]$} \\
\hline & & $86.0 \pm 0.8$ & (298) & $\mathrm{C}$ & {$[72 / 29]$} \\
\hline & & 86.2 & (298) & & {$[71 / 28]$} \\
\hline & $(445-470)$ & 71.6 & $(457)$ & ME & {$[38 / 8]$} \\
\hline \multirow[t]{2}{*}{$\mathrm{C}_{17} \mathrm{H}_{36}$} & 2-methylhexadecane & & & & {$[1560-92-5]$} \\
\hline & $(428-569)$ & 63.5 & $(443)$ & $\mathrm{A}$ & {$[87 / 5][59 / 15]$} \\
\hline \multirow[t]{2}{*}{$\mathrm{C}_{17} \mathrm{H}_{36}$} & 3-methylhexadecane & & & & [6418-43-5] \\
\hline & $(428-567)$ & 63.4 & $(443)$ & A & {$[87 / 5][59 / 15]$} \\
\hline \multirow[t]{2}{*}{$\mathrm{C}_{17} \mathrm{H}_{36}$} & 4-methylhexadecane & & & & [25117-26-4] \\
\hline & $(420-567)$ & 58.7 & $(435)$ & A & {$[87 / 5][59 / 15]$} \\
\hline \multirow[t]{2}{*}{$\mathrm{C}_{17} \mathrm{H}_{36}$} & 5-methylhexadecane & & & & [25117-34-4] \\
\hline & $(422-566)$ & 59.8 & $(437)$ & A & {$[87 / 5][59 / 15]$} \\
\hline \multirow[t]{2}{*}{$\mathrm{C}_{17} \mathrm{H}_{36}$} & 2,3-dimethylpentadecane & & & & {$[2882-97-5]$} \\
\hline & $(424-569)$ & 60.6 & $(439)$ & A & {$[87 / 5][59 / 15]$} \\
\hline \multirow[t]{2}{*}{$\mathrm{C}_{17} \mathrm{H}_{36}$} & 2,4-dimethylpentadecane & & & & [61868-07-3] \\
\hline & $(419-546)$ & 65.2 & $(434)$ & A & {$[87 / 5][59 / 15]$} \\
\hline \multirow[t]{2}{*}{$\mathrm{C}_{17} \mathrm{H}_{36}$} & 2,4,6-trimethyltetradecane & & & & [101791-53-1] \\
\hline & $(411-534)$ & 64.5 & $(426)$ & $\mathrm{A}$ & {$[87 / 5]$} \\
\hline \multirow[t]{2}{*}{$\mathrm{C}_{17} \mathrm{H}_{36}$} & 4,4-dipropylundecane & & & & \\
\hline & & $78.0 \pm 1.8$ & $(298)$ & $\mathrm{CGC}$ & {$[95 / 27]$} \\
\hline \multirow[t]{3}{*}{$\mathrm{C}_{17} \mathrm{H}_{36} \mathrm{O}$} & 1-heptadecanol & & & & [1454-85-9] \\
\hline & $(460-620)$ & 78.3 & $(475)$ & A & {$[87 / 5]$} \\
\hline & $(473-623)$ & 75.9 & $(488)$ & A & [87/5] \\
\hline \multirow[t]{2}{*}{$\mathrm{C}_{17} \mathrm{H}_{36} \mathrm{~S}$} & 1-heptadecanethiol & & & & [53193-22-9] \\
\hline & $(481-657)$ & 74.6 & $(496)$ & & [99/16] \\
\hline \multirow[t]{2}{*}{$\mathrm{C}_{17} \mathrm{H}_{37} \mathrm{~N}$} & heptadecylamine & & & & [4200-95-7] \\
\hline & $(522-636)$ & 68.2 & $(537)$ & A & {$[87 / 5][56 / 17]$} \\
\hline \multirow[t]{2}{*}{$\mathrm{C}_{18} \mathrm{H}_{12}$} & benz[a]anthracene & & & & {$[56-55-3]$} \\
\hline & $(343-453)$ & 91.0 & (398) & GC & {$[90 / 2]$} \\
\hline \multirow[t]{3}{*}{$\mathrm{C}_{18} \mathrm{H}_{12}$} & triphenylene & & & & [217-59-4] \\
\hline & $(323-473)$ & 88.5 & (398) & $\mathrm{GC}$ & {$[02 / 18]$} \\
\hline & $(535-768)$ & 67.7 & $(550)$ & & [99/16] \\
\hline \multirow[t]{2}{*}{$\mathrm{C}_{18} \mathrm{H}_{12}$} & chrysene & & & & [218-01-9] \\
\hline & $(323-473)$ & 89.6 & (398) & $\mathrm{GC}$ & {$[02 / 18]$} \\
\hline $\mathrm{C}_{18} \mathrm{H}_{14}$ & $o$-terphenyl & & & & [84-15-1] \\
\hline & $(335-368)$ & $81.0 \pm 0.4$ & $(352)$ & GS & {$[97 / 1]$} \\
\hline & $(335-368)$ & $84.2 \pm 0.4$ & (298) & GS & {$[97 / 1]$} \\
\hline & $(576-786)$ & 60.5 & (591) & DSC & {$[96 / 10]$} \\
\hline & $(343-462)$ & 77.6 & (403) & & [89/14] \\
\hline & $(462-650)$ & 68.5 & $(477)$ & A & {$[87 / 5]$} \\
\hline $\mathrm{C}_{18} \mathrm{H}_{14}$ & $m$-terphenyl & & & & [92-06-8] \\
\hline & & $97.2 \pm 0.3$ & (298) & $\mathrm{CGC}$ & {$[01 / 1]$} \\
\hline & $(462-691)$ & 76.1 & (477) & A & {$[87 / 5]$} \\
\hline $\mathrm{C}_{18} \mathrm{H}_{14}$ & $p$-terphenyl & & & & [92-94-4] \\
\hline & $(323-473)$ & 79.2 & (398) & GC & {$[02 / 18]$} \\
\hline & $(499-700)$ & 79.2 & $(514)$ & A & {$[87 / 5]$} \\
\hline $\mathrm{C}_{18} \mathrm{H}_{14} \mathrm{~N}_{4} \mathrm{O}_{2}$ & 1,4-bis[(4-hydroxyphenyl)azo]benzene & & & & [21811-64-3] \\
\hline & $(473-533)$ & 68.0 & $(488)$ & A & {$[87 / 5]$} \\
\hline $\mathrm{C}_{18} \mathrm{H}_{15} \mathrm{~N}$ & triphenylamine & & & & [603-34-9] \\
\hline & $(473-640)$ & 65.2 & $(488)$ & A & {$[87 / 5]$} \\
\hline $\mathrm{C}_{18} \mathrm{H}_{15} \mathrm{NO}_{2}$ & N-9-anthryldiacetamide & & & & {$[3808-37-5]$} \\
\hline & $(399-455)$ & 106.3 & $(414)$ & A & {$[87 / 5]$} \\
\hline $\mathrm{C}_{18} \mathrm{H}_{15} \mathrm{O}_{4} \mathrm{P}$ & triphenyl phosphate & & & & {$[115-86-6]$} \\
\hline & $(548-683)$ & 81.4 & $(563)$ & IA & {$[87 / 5][57 / 8]$} \\
\hline $\mathrm{C}_{18} \mathrm{H}_{15} \mathrm{P}$ & triphenylphosphine & & & & {$[603-35-0]$} \\
\hline & $(483-660)$ & 71.2 & $(498)$ & A & {$[87 / 5]$} \\
\hline & $(364-392)$ & $91.4 \pm 2$ & $(378)$ & TE, ME & {$[81 / 3]$} \\
\hline $\mathrm{C}_{18} \mathrm{H}_{16} \mathrm{O}_{2}$ & 2-tert-butyl-9,10-anthraquinone & & & & {$[84-47-9]$} \\
\hline & $(483-523)$ & 101.4 & $(498)$ & A & {$[87 / 5]$} \\
\hline $\mathrm{C}_{28} \mathrm{H}_{18}$ & 2-(tert-butyl)anthracene & & & & \\
\hline & $(323-473)$ & 84.5 & (398) & GC & {$[02 / 18]$} \\
\hline $\mathrm{C}_{18} \mathrm{H}_{18}$ & 9-butylanthracene & & & & [1498-69-7] \\
\hline & $(422-492)$ & 77.1 & (437) & A & {$[87 / 5]$} \\
\hline & $(328-373)$ & 83.9 & $(343)$ & A & {$[87 / 5][64 / 12]$} \\
\hline
\end{tabular}


TABLE 6. Enthalpies of vaporization of organic compounds, 1880-2002-Continued

\begin{tabular}{|c|c|c|c|c|c|}
\hline Molecular formula & $\begin{array}{c}\text { Compound } \\
\text { (Temperature range/K) }\end{array}$ & $\begin{array}{c}\Delta_{\mathrm{vap}} H_{m} \\
\left(\mathrm{~kJ} \mathrm{~mol}^{-1}\right)\end{array}$ & $\begin{array}{l}\text { Mean temperature } \\
\qquad\left(T_{m} / \mathrm{K}\right)\end{array}$ & Method & $\begin{array}{l}\text { CAS registry number } \\
\text { Reference }\end{array}$ \\
\hline $\mathrm{C}_{18} \mathrm{H}_{18}$ & $\begin{array}{l}\text { 1-methyl-7-isopropylphenanthrene } \\
\text { (539-678) }\end{array}$ & 54.0 & $(554)$ & A & $\begin{array}{l}{[483-65-8]} \\
{[87 / 5]}\end{array}$ \\
\hline $\mathrm{C}_{18} \mathrm{H}_{22}$ & $\begin{array}{l}\text { 1,6-diphenylhexane } \\
(293-373)\end{array}$ & 88.0 & (308) & A & $\begin{array}{l}{[1087-49-6]} \\
{[87 / 5][64 / 12]}\end{array}$ \\
\hline $\mathrm{C}_{18} \mathrm{H}_{22}$ & $\begin{array}{l}\text { 2,2-di(p-tolyl)butane } \\
(298-473)\end{array}$ & 85.4 & (313) & & [99/16] \\
\hline $\mathrm{C}_{18} \mathrm{H}_{22}$ & $\begin{array}{l}\text { 1-p-tolyl-(1-p-propylphenyl)ethane } \\
(298-473)\end{array}$ & 85.4 & (313) & & {$[99 / 16]$} \\
\hline $\mathrm{C}_{18} \mathrm{H}_{22}$ & $\begin{array}{l}\text { 1-o-tolyl-p-tolylbutane } \\
(298-473)\end{array}$ & 85.4 & (313) & & [99/16] \\
\hline $\mathrm{C}_{18} \mathrm{H}_{22} \mathrm{~N}_{4}$ & $\begin{array}{l}\text { trans, trans-1,6-diphenyl-3,3,4,4-tetr } \\
(348-388)\end{array}$ & $\begin{array}{l}\text { amethyl-1,2, } \\
92.8 \pm 1.5\end{array}$ & $\begin{array}{l}\text { azahexane } \\
\qquad(368)\end{array}$ & GS & [93/16] \\
\hline $\mathrm{C}_{18} \mathrm{H}_{24}$ & $\begin{array}{l}1,2,3,4,4 a, 7,8,9,10,12,12 a-d o d e c a h y d \\
(318-358)\end{array}$ & $\begin{array}{l}\text { Arochrysene } \\
84.2\end{array}$ & $(333)$ & $\mathrm{A}$ & $\begin{array}{l}{[1610-22-6]} \\
{[87 / 5][47 / 5]}\end{array}$ \\
\hline $\mathrm{C}_{18} \mathrm{H}_{24} \mathrm{O}_{4}$ & $\begin{array}{l}\text { butylcyclohexylphthalate } \\
(368-485)\end{array}$ & 94.3 & (383) & $\mathrm{A}$ & $\begin{array}{c}{[84-64-0]} \\
{[87 / 5]}\end{array}$ \\
\hline $\mathrm{C}_{18} \mathrm{H}_{26} \mathrm{O}_{4}$ & $\begin{array}{l}\text { diisopentylphthalate } \\
(390-610)\end{array}$ & 81.6 & $(405)$ & A & $\begin{array}{l}{[606-50-5]} \\
{[87 / 5]}\end{array}$ \\
\hline $\mathrm{C}_{18} \mathrm{H}_{26} \mathrm{O}_{4}$ & $\begin{array}{l}\text { dipentylphthalate } \\
(323-390) \\
(303-500)\end{array}$ & $\begin{array}{l}87.3 \\
99.4\end{array}$ & $\begin{array}{l}(338) \\
(318)\end{array}$ & $\begin{array}{c}\mathrm{T} \\
\mathrm{A}, \mathrm{ME}\end{array}$ & $\begin{array}{c}{[131-18-0]} \\
{[49 / 9]} \\
{[87 / 5][48 / 10]}\end{array}$ \\
\hline $\mathrm{C}_{18} \mathrm{H}_{28}$ & $\begin{array}{l}\text { 1,2,3,4-tetrahydro-6-octylnaphthalene } \\
(503-574)\end{array}$ & 103.3 & $(538)$ & & $\begin{array}{c}{[66553-12-6]} \\
{[99 / 16]}\end{array}$ \\
\hline $\mathrm{C}_{18} \mathrm{H}_{30}$ & $\begin{array}{l}\text { dodecylbenzene } \\
(333-453) \\
(496-609) \\
(336-456)\end{array}$ & $\begin{array}{l}92.0 \\
83.2 \\
67.4 \\
80.6 \\
89.6\end{array}$ & $\begin{array}{l}(275) \\
(348) \\
(511) \\
(356) \\
(298)\end{array}$ & $\begin{array}{c}\text { A } \\
\text { GS }\end{array}$ & $\begin{array}{c}{[123-01-3]} \\
{[00 / 4]} \\
{[93 / 10]} \\
{[87 / 5]} \\
{[86 / 6]} \\
{[71 / 28]}\end{array}$ \\
\hline $\mathrm{C}_{18} \mathrm{H}_{30}$ & $\begin{array}{l}\text { perhydrochrysene } \\
(273-353)\end{array}$ & 82.4 & $(288)$ & & $\begin{array}{c}{[2090-14-4]} \\
{[64 / 12]}\end{array}$ \\
\hline $\mathrm{C}_{18} \mathrm{H}_{30}$ & $\begin{array}{l}\text { hexaethylbenzene } \\
(407-572)\end{array}$ & 62.6 & $(422)$ & A & $\begin{array}{c}{[604-88-6]} \\
{[87 / 5][47 / 5]}\end{array}$ \\
\hline $\mathrm{C}_{18} \mathrm{H}_{30}$ & $\begin{array}{l}\text { 1,2,4,5-tetraisopropylbenzene } \\
(410-575) \\
(410-575) \\
(410-575) \\
(410-575)\end{array}$ & $\begin{array}{l}61.1 \pm 0.3 \\
56.8 \pm 0.3 \\
52.3 \pm 0.5 \\
47.5 \pm 0.9\end{array}$ & $\begin{array}{l}(420) \\
(460) \\
(500) \\
(540)\end{array}$ & $\begin{array}{l}\text { EB } \\
\text { EB } \\
\text { EB } \\
\text { EB }\end{array}$ & $\begin{array}{l}{[635-11-0]} \\
{[02 / 20]} \\
{[02 / 20]} \\
{[02 / 20]} \\
{[02 / 20]}\end{array}$ \\
\hline $\mathrm{C}_{18} \mathrm{H}_{30} \mathrm{O}_{2}$ & $\begin{array}{l}\text { 1,3-dimethoxy-4-decylbenzene } \\
(443-493)\end{array}$ & 76.6 & $(458)$ & $\mathrm{A}, \mathrm{GC}$ & $\begin{array}{l}{[59968-12-6]} \\
{[87 / 5][75 / 24]}\end{array}$ \\
\hline $\mathrm{C}_{18} \mathrm{H}_{30} \mathrm{O}_{2}$ & $\begin{array}{l}\text { 1,3-dimethoxy-5-decylbenzene } \\
(459-519)\end{array}$ & 78.4 & $(474)$ & $\mathrm{A}, \mathrm{GC}$ & $\begin{array}{l}{[41442-52-8]} \\
{[87 / 5][75 / 24]}\end{array}$ \\
\hline $\mathrm{C}_{18} \mathrm{H}_{30} \mathrm{O}_{4}$ & $\begin{array}{l}\text { 1,4-bis (1,1-diethoxyethyl)lbenzene } \\
(329-347)\end{array}$ & 88.5 & $(338)$ & A & $\begin{array}{c}{[47189-08-2]} \\
{[87 / 5]}\end{array}$ \\
\hline $\mathrm{C}_{18} \mathrm{H}_{30} \mathrm{O}_{6}$ & $\begin{array}{l}\text { trans aconitic acid, tributyl ester } \\
(385-483)\end{array}$ & 87.4 & $(400)$ & A & $\begin{array}{c}{[7568-58-3]} \\
{[87 / 5]}\end{array}$ \\
\hline $\mathrm{C}_{18} \mathrm{H}_{30} \mathrm{O}_{6}$ & $\begin{array}{l}\text { diethylene glycol dicarboxylic acid, } \\
(418-493)\end{array}$ & $\begin{array}{l}\text { di }[1 \text {-(isoprop } \\
\quad 97.6\end{array}$ & (433) & $\mathrm{A}$ & {$[87 / 5]$} \\
\hline $\mathrm{C}_{18} \mathrm{H}_{30} \mathrm{O}_{6}$ & $\begin{array}{l}\text { diethylene glycol dicarboxylic acid, } \\
(418-514)\end{array}$ & $\begin{array}{l}\text { di }[1-(\text { propox } \\
\quad 101.5\end{array}$ & $\begin{array}{c}\text { yl) ethyl] ester } \\
\text { (433) }\end{array}$ & A & {$[87 / 5]$} \\
\hline $\mathrm{C}_{18} \mathrm{H}_{32}$ & $\begin{array}{l}\text { 9-butyltetrahydroanthracene } \\
(420-456)\end{array}$ & 72.8 & $(435)$ & $\mathrm{A}$ & [87/5] \\
\hline $\mathrm{C}_{18} \mathrm{H}_{32}$ & $\begin{array}{l}\text { 1,2-dicyclohexylcyclohexane } \\
(375-563)\end{array}$ & 72.8 & $(390)$ & A & $\begin{array}{c}{[2456-43-1]} \\
{[87 / 5]}\end{array}$ \\
\hline $\mathrm{C}_{18} \mathrm{H}_{32}$ & $\begin{array}{l}\text { 1,6-dicyclohexylcyclohexane } \\
(288-373)\end{array}$ & 85.9 & (303) & $\mathrm{A}$ & $\begin{array}{c}{[1610-23-7]} \\
{[87 / 5][64 / 12]}\end{array}$ \\
\hline $\mathrm{C}_{18} \mathrm{H}_{32} \mathrm{O}$ & $\begin{array}{l}\text { 6,10,14-trimethyl-3,5-pentadecadien- } \\
(404-560)\end{array}$ & $\begin{array}{l}\text {-2-one } \\
43.4 \pm 0.5\end{array}$ & $(482)$ & & $\begin{array}{c}{[1604-32-6]} \\
{[88 / 4]}\end{array}$ \\
\hline $\mathrm{C}_{18} \mathrm{H}_{32} \mathrm{O}_{6}$ & $\begin{array}{l}\text { tributyl } 1,2,3 \text {-propanetricarboxylate } \\
(385-482) \\
(323-368)\end{array}$ & $\begin{array}{l}87.8 \\
81.9\end{array}$ & $\begin{array}{l}(400) \\
(328)\end{array}$ & $\begin{array}{l}\mathrm{A} \\
\mathrm{T}\end{array}$ & $\begin{array}{c}{[38094-11-0]} \\
{[87 / 5]} \\
{[49 / 16]}\end{array}$ \\
\hline $\mathrm{C}_{18} \mathrm{H}_{34}$ & $\begin{array}{l}\text { 1-octadecyne } \\
(450-623)\end{array}$ & 64.9 & $(465)$ & & $\begin{array}{c}{[629-89-0]} \\
{[99 / 16]}\end{array}$ \\
\hline $\mathrm{C}_{18} \mathrm{H}_{34}$ & 2-octadecyne & & & & [61847-97-0] \\
\hline
\end{tabular}


TABLE 6. Enthalpies of vaporization of organic compounds, 1880-2002-Continued

\begin{tabular}{|c|c|c|c|c|c|}
\hline Molecular formula & $\begin{array}{c}\text { Compound } \\
\text { (Temperature range/K) }\end{array}$ & $\begin{array}{c}\Delta_{\mathrm{vap}} H_{m} \\
\left(\mathrm{~kJ} \mathrm{~mol}^{-1}\right)\end{array}$ & $\begin{array}{l}\text { Mean temperature } \\
\qquad\left(T_{m} / \mathrm{K}\right)\end{array}$ & Method & $\begin{array}{c}\text { CAS registry number } \\
\text { Reference }\end{array}$ \\
\hline \multirow{3}{*}{$\mathrm{C}_{18} \mathrm{H}_{34}$} & $(458-633)$ & 65.7 & (473) & & {$[99 / 16]$} \\
\hline & 3-octadecyne & & & & [61886-64-4] \\
\hline & $(449-622)$ & 64.5 & $(464)$ & & [99/16] \\
\hline \multirow[t]{2}{*}{$\mathrm{C}_{18} \mathrm{H}_{34} \mathrm{O}_{2}$} & cis 9-octadecenoic acid & & & & {$[112-80-1]$} \\
\hline & $(441-633)$ & 83.8 & $(456)$ & A & {$[87 / 5]$} \\
\hline \multirow[t]{2}{*}{$\mathrm{C}_{18} \mathrm{H}_{34} \mathrm{O}_{2}$} & trans 9-octadecenoic acid & & & & {$[112-79-8]$} \\
\hline & $(444-635)$ & 82.3 & $(459)$ & A & {$[87 / 5][47 / 5]$} \\
\hline \multirow[t]{2}{*}{$\mathrm{C}_{18} \mathrm{H}_{34} \mathrm{O}_{2}$} & tetradecyl methacrylate & & & & [2549-53-3] \\
\hline & $(463-611)$ & 69.1 & $(478)$ & $\mathrm{A}$ & {$[87 / 5]$} \\
\hline \multirow[t]{2}{*}{$\mathrm{C}_{18} \mathrm{H}_{34} \mathrm{O}_{2}$} & (Z) 3-hexadecenyl acetate & & & & [141694-86-2] \\
\hline & $(373-418)$ & 98.5 & $(298)$ & GC & {$[97 / 13][00 / 10]$} \\
\hline \multirow[t]{2}{*}{$\mathrm{C}_{18} \mathrm{H}_{34} \mathrm{O}_{2}$} & (E) 3-hexadecenyl acetate & & & & [128984-60-1] \\
\hline & $(373-418)$ & 99.1 & $(298)$ & GC & {$[97 / 13][00 / 10]$} \\
\hline \multirow[t]{2}{*}{$\mathrm{C}_{18} \mathrm{H}_{34} \mathrm{O}_{2}$} & (Z) 4-hexadecenyl acetate & & & & {$[65954-24-7]$} \\
\hline & $(373-418)$ & 97.7 & $(298)$ & GC & {$[97 / 13][00 / 10]$} \\
\hline \multirow[t]{2}{*}{$\mathrm{C}_{18} \mathrm{H}_{34} \mathrm{O}_{2}$} & (E) 4-hexadecenyl acetate & & & & [155055-27-9] \\
\hline & $(373-418)$ & 98.9 & $(298)$ & GC & {$[97 / 13][00 / 10]$} \\
\hline \multirow[t]{2}{*}{$\mathrm{C}_{18} \mathrm{H}_{34} \mathrm{O}_{2}$} & (Z) 5-hexadecenyl acetate & & & & {$[34010-18-9]$} \\
\hline & $(373-418)$ & 98.0 & (298) & GC & {$[97 / 13][00 / 10]$} \\
\hline \multirow[t]{2}{*}{$\mathrm{C}_{18} \mathrm{H}_{34} \mathrm{O}_{2}$} & (E) 5-hexadecenyl acetate & & & & [56218-65-6] \\
\hline & $(373-418)$ & 98.8 & $(298)$ & GC & {$[97 / 13][00 / 10]$} \\
\hline \multirow[t]{2}{*}{$\mathrm{C}_{18} \mathrm{H}_{34} \mathrm{O}_{2}$} & (Z) 6-hexadecenyl acetate & & & & [34010-19-0] \\
\hline & $(373-418)$ & 97.8 & $(298)$ & $\mathrm{GC}$ & {$[97 / 13][00 / 10]$} \\
\hline \multirow[t]{2}{*}{$\mathrm{C}_{18} \mathrm{H}_{34} \mathrm{O}_{2}$} & (E) 6-hexadecenyl acetate & & & & [56218-66-7] \\
\hline & $(373-418)$ & 98.6 & $(298)$ & GC & {$[97 / 13][00 / 10]$} \\
\hline \multirow[t]{2}{*}{$\mathrm{C}_{18} \mathrm{H}_{34} \mathrm{O}_{2}$} & (Z) 7-hexadecenyl acetate & & & & [23192-42-9] \\
\hline & $(373-418)$ & 97.8 & (298) & $\mathrm{GC}$ & {$[97 / 13][00 / 10]$} \\
\hline \multirow[t]{2}{*}{$\mathrm{C}_{18} \mathrm{H}_{34} \mathrm{O}_{2}$} & (E) 7-hexadecenyl acetate & & & & [23192-83-8] \\
\hline & $(373-418)$ & 98.5 & $(298)$ & GC & {$[97 / 13][00 / 10]$} \\
\hline $\mathrm{C}_{18} \mathrm{H}_{34} \mathrm{O}_{2}$ & (Z) 8-hexadecenyl acetate & & & & {$[56218-67-8]$} \\
\hline & $(373-418)$ & 97.8 & $(298)$ & $\mathrm{GC}$ & {$[97 / 13][00 / 10]$} \\
\hline $\mathrm{C}_{18} \mathrm{H}_{34} \mathrm{O}_{2}$ & (E) 8-hexadecenyl acetate & & & & [56218-68-9] \\
\hline & $(373-418)$ & 98.6 & $(298)$ & GC & {$[97 / 13][00 / 10]$} \\
\hline $\mathrm{C}_{18} \mathrm{H}_{34} \mathrm{O}_{2}$ & (Z) 9-hexadecenyl acetate & & & & {$[34010-20-3]$} \\
\hline & $(373-418)$ & 98.2 & $(298)$ & GC & {$[97 / 13][00 / 10]$} \\
\hline $\mathrm{C}_{18} \mathrm{H}_{34} \mathrm{O}_{2}$ & (E) 9-hexadecenyl acetate & & & & [56218-69-0] \\
\hline & $(373-418)$ & 98.9 & $(298)$ & GC & {$[97 / 13][00 / 10]$} \\
\hline $\mathrm{C}_{18} \mathrm{H}_{34} \mathrm{O}_{2}$ & (Z) 10-hexadecenyl acetate & & & & {$[56218-70-3]$} \\
\hline & $(373-418)$ & 98.5 & (298) & GC & {$[97 / 13][00 / 10]$} \\
\hline $\mathrm{C}_{18} \mathrm{H}_{34} \mathrm{O}_{2}$ & (E) 10-hexadecenyl acetate & & & & [56218-71-4] \\
\hline & $(373-418)$ & 99.1 & $(298)$ & $\mathrm{GC}$ & {$[97 / 13][00 / 10]$} \\
\hline $\mathrm{C}_{18} \mathrm{H}_{34} \mathrm{O}_{2}$ & (Z) 11-hexadecenyl acetate & & & & {$[34010-21-4]$} \\
\hline & $(373-418)$ & 98.9 & $(298)$ & GC & {$[97 / 13][00 / 10]$} \\
\hline $\mathrm{C}_{18} \mathrm{H}_{34} \mathrm{O}_{2}$ & (E) 11-hexadecenyl acetate & & & & {$[56218-72-5]$} \\
\hline & $(373-418)$ & 99.5 & $(298)$ & $\mathrm{GC}$ & {$[97 / 13][00 / 10]$} \\
\hline $\mathrm{C}_{18} \mathrm{H}_{34} \mathrm{O}_{2}$ & (Z) 12-hexadecenyl acetate & & & & {$[56218-73-6]$} \\
\hline & $(373-418)$ & 99.5 & $(298)$ & GC & {$[97 / 13][00 / 10]$} \\
\hline $\mathrm{C}_{18} \mathrm{H}_{34} \mathrm{O}_{2}$ & (E) 12-hexadecenyl acetate & & & & [64789-90-8] \\
\hline & $(373-418)$ & 99.8 & $(298)$ & GC & {$[97 / 13][00 / 10]$} \\
\hline $\mathrm{C}_{18} \mathrm{H}_{34} \mathrm{O}_{2}$ & (Z) 13-hexadecenyl acetate & & & & {$[56218-74-7]$} \\
\hline & $(373-418)$ & 100.0 & (298) & $\mathrm{GC}$ & {$[97 / 13][00 / 10]$} \\
\hline $\mathrm{C}_{18} \mathrm{H}_{34} \mathrm{O}_{2}$ & (E) 13-hexadecenyl acetate & & & & [69282-67-3] \\
\hline & $(373-418)$ & 100.3 & $(298)$ & $\mathrm{GC}$ & {$[97 / 13][00 / 10]$} \\
\hline $\mathrm{C}_{18} \mathrm{H}_{34} \mathrm{O}_{4}$ & dihexyl adipate & & & & [110-33-8] \\
\hline & $(470-595)$ & 80.4 & $(485)$ & A & {$[87 / 5]$} \\
\hline $\mathrm{C}_{18} \mathrm{H}_{34} \mathrm{O}_{4}$ & dibutyl decanedioate & & & & [109-43-3] \\
\hline & $(401-520)$ & 94.3 & $(416)$ & $\mathrm{A}$ & {$[87 / 5]$} \\
\hline $\mathrm{C}_{18} \mathrm{H}_{34} \mathrm{O}_{5}$ & decyl[1-(butoxycarbonyl)eth & nate & & & \\
\hline & $(391-503)$ & 79.3 & $(406)$ & A & {$[87 / 5]$} \\
\hline $\mathrm{C}_{18} \mathrm{H}_{34} \mathrm{O}_{6}$ & triethylene glycol, bis(2-eth & & & & [95-08-9] \\
\hline & $(313-528)$ & 91.7 & $(328)$ & A & {$[87 / 5]$} \\
\hline $\mathrm{C}_{18} \mathrm{H}_{35} \mathrm{~N}$ & stearonitrile & & & & {$[638-65-3]$} \\
\hline & $(478-631)$ & 78.6 & (493) & A & {$[87 / 5]$} \\
\hline $\mathrm{C}_{18} \mathrm{H}_{36}$ & tridecylcyclopentane & & & & [6006-34-4] \\
\hline
\end{tabular}


TABLE 6. Enthalpies of vaporization of organic compounds, 1880-2002-Continued

\begin{tabular}{|c|c|c|c|c|c|}
\hline Molecular formula & $\begin{array}{c}\text { Compound } \\
\text { (Temperature range/K) }\end{array}$ & $\begin{array}{c}\Delta_{\mathrm{vap}} H_{m} \\
\left(\mathrm{~kJ} \mathrm{~mol}^{-1}\right)\end{array}$ & $\begin{array}{l}\text { Mean temperature } \\
\qquad\left(T_{m} / \mathrm{K}\right)\end{array}$ & Method & $\begin{array}{c}\text { CAS registry number } \\
\text { Reference }\end{array}$ \\
\hline & $(463-634)$ & 70.9 & $(478)$ & & [99/16] \\
\hline & & 90.5 & (298) & & {$[71 / 28]$} \\
\hline \multirow[t]{4}{*}{$\mathrm{C}_{18} \mathrm{H}_{36}$} & dodecylcyclohexane & & & & {$[1795-17-1]$} \\
\hline & & $88.9 \pm 0.8$ & $(298)$ & GCC & {$[78 / 16]$} \\
\hline & & 89.5 & (298) & & {$[71 / 28]$} \\
\hline & $(299-324)$ & 93.4 & $(311)$ & $\mathrm{A}, \mathrm{ME}$ & {$[87 / 5][49 / 14]$} \\
\hline \multirow{3}{*}{$\mathrm{C}_{18} \mathrm{H}_{36}$} & 1-octadecene & & & & {$[112-88-9]$} \\
\hline & $(399-589)$ & 76.4 & $(414)$ & A & {$[87 / 5]$} \\
\hline & & 90.0 & $(298)$ & & {$[71 / 28]$} \\
\hline \multirow[t]{2}{*}{$\mathrm{C}_{18} \mathrm{H}_{36} \mathrm{O}$} & (Z) 3-octadecen-1-ol & & & & [41207-35-6] \\
\hline & $(393-433)$ & 120.5 & $(298)$ & CGC & {$[00 / 10][94 / 13]$} \\
\hline \multirow{2}{*}{$\mathrm{C}_{18} \mathrm{H}_{36} \mathrm{O}$} & (E) 3-octadecen-1-ol & & & & {$[41207-36-7]$} \\
\hline & $(393-433)$ & 120.0 & $(298)$ & CGC & {$[00 / 10][94 / 13]$} \\
\hline \multirow[t]{2}{*}{$\mathrm{C}_{18} \mathrm{H}_{36} \mathrm{O}$} & (Z) 9-octadecen-1-ol & & & & [143-28-2] \\
\hline & $(393-433)$ & 119.3 & (298) & CGC & {$[00 / 10][94 / 13]$} \\
\hline \multirow{2}{*}{$\mathrm{C}_{18} \mathrm{H}_{36} \mathrm{O}$} & (E) 9-octadecen-1-ol & & & & {$[506-42-3]$} \\
\hline & $(393-433)$ & 120.1 & $(298)$ & CGC & {$[00 / 10][94 / 13]$} \\
\hline \multirow[t]{2}{*}{$\mathrm{C}_{18} \mathrm{H}_{36} \mathrm{O}$} & (Z) 11-octadecen-1-ol & & & & {$[57716-88-8]$} \\
\hline & $(393-433)$ & 119.6 & (298) & CGC & {$[00 / 10][94 / 13]$} \\
\hline \multirow{2}{*}{$\mathrm{C}_{18} \mathrm{H}_{36} \mathrm{O}$} & (E) 11-octadecen-1-ol & & & & [62972-93-4] \\
\hline & $(393-433)$ & 120.4 & $(298)$ & CGC & {$[00 / 10][94 / 13]$} \\
\hline \multirow[t]{2}{*}{$\mathrm{C}_{18} \mathrm{H}_{36} \mathrm{O}$} & (Z) 13-octadecen-1-ol & & & & {$[69820-27-5]$} \\
\hline & $(393-433)$ & 120.8 & $(298)$ & CGC & {$[00 / 10][94 / 13]$} \\
\hline \multirow{2}{*}{$\mathrm{C}_{18} \mathrm{H}_{36} \mathrm{O}$} & (E) 13-octadecen-1-ol & & & & [76836-10-7] \\
\hline & $(393-433)$ & 121.2 & $(298)$ & CGC & {$[00 / 10][94 / 13]$} \\
\hline \multirow[t]{2}{*}{$\mathrm{C}_{18} \mathrm{H}_{36} \mathrm{O}$} & 6,10,14-trimethyl-2-pentadecanone & & & & {$[502-69-2]$} \\
\hline & $(402-500)$ & $56.0 \pm 0.6$ & $(451)$ & & {$[88 / 4]$} \\
\hline \multirow[t]{2}{*}{$\mathrm{C}_{18} \mathrm{H}_{36} \mathrm{O}$} & octadecanal & & & & {$[638-66-4]$} \\
\hline & $(413-616)$ & 75.7 & $(428)$ & $\mathrm{A}$ & {$[87 / 5][47 / 5]$} \\
\hline \multirow[t]{3}{*}{$\mathrm{C}_{18} \mathrm{H}_{36} \mathrm{O}_{2}$} & hexadecyl acetate & & & & [629-70-9] \\
\hline & $(373-418)$ & 102.3 & $(298)$ & GC & {$[97 / 13][00 / 10]$} \\
\hline & $(431-469)$ & 70.3 & $(446)$ & A & {$[87 / 5]$} \\
\hline \multirow[t]{3}{*}{$\mathrm{C}_{18} \mathrm{H}_{36} \mathrm{O}_{2}$} & ethyl palmitate & & & & {$[628-97-7]$} \\
\hline & $(429-466)$ & 73.9 & $(444)$ & $\mathrm{A}$ & {$[87 / 5]$} \\
\hline & $(298-318)$ & 100.7 & $(308)$ & $\mathrm{ME}$ & {$[87 / 5][67 / 22]$} \\
\hline \multirow[t]{5}{*}{$\mathrm{C}_{18} \mathrm{H}_{36} \mathrm{O}_{2}$} & methyl heptadecanoate & & & & {$[1731-92-6]$} \\
\hline & & 89.3 & $(350)$ & & {$[02 / 27]$} \\
\hline & & $89.0 \pm 0.7$ & (353) & & {$[02 / 27]$} \\
\hline & & $97.0 \pm 1.2$ & $(298)$ & & {$[02 / 27]$} \\
\hline & $(421-525)$ & 84.4 & $(436)$ & A, EST & {$[87 / 5][63 / 16]$} \\
\hline $\mathrm{C}_{18} \mathrm{H}_{36} \mathrm{O}_{2}$ & octadecanoic acid (stearic acid) & & & & {$[55-11-4]$} \\
\hline & $(349-415)$ & 124.3 & $(364)$ & A & {$[87 / 5]$} \\
\hline & $(457-649)$ & 100.6 & $(472)$ & A & {$[87 / 5]$} \\
\hline & $(366-389)$ & $118.9 \pm 2.0$ & $(379)$ & $\mathrm{ME}, \mathrm{TE}$ & {$[82 / 4]$} \\
\hline & & 79.8 & $(515)$ & I & {$[43 / 7]$} \\
\hline $\mathrm{C}_{18} \mathrm{H}_{37} \mathrm{Br}$ & 1-bromooctadecane & & & & [112-89-0] \\
\hline & $(430-673)$ & 81.0 & $(445)$ & $\mathrm{A}, \mathrm{EST}$ & $\begin{array}{c}{[87 / 5][61 / 13]} \\
{[70 / 14]}\end{array}$ \\
\hline $\mathrm{C}_{18} \mathrm{H}_{37} \mathrm{Cl}$ & 1-chlorooctadecane & & & & [3386-33-2] \\
\hline & $(333-393)$ & 96.9 & $(333)$ & GC & {$[80 / 14]$} \\
\hline & $(333-393)$ & 93.4 & $(353)$ & GC & {$[80 / 14]$} \\
\hline & $(333-393)$ & 88.4 & $(373)$ & GC & {$[80 / 14]$} \\
\hline & $(333-393)$ & 86.7 & $(393)$ & GC & {$[80 / 14]$} \\
\hline & $(472-673)$ & 74.2 & $(487)$ & A & {$[87 / 5][70 / 14]$} \\
\hline $\mathrm{C}_{18} \mathrm{H}_{37} \mathrm{~F}$ & 1-fluorooctadecane & & & & {$[1649-73-6]$} \\
\hline & $(477-633)$ & 68.2 & $(492)$ & A, EST & $\begin{array}{c}{[87 / 5][61 / 13]} \\
{[70 / 14]}\end{array}$ \\
\hline $\mathrm{C}_{18} \mathrm{H}_{37} \mathrm{I}$ & 1-iodooctadecane & & & & [629-93-6] \\
\hline & $(496-673)$ & 77.2 & $(511)$ & $\mathrm{A}, \mathrm{EST}$ & $\begin{array}{c}{[87 / 5][61 / 13]} \\
{[70 / 14]}\end{array}$ \\
\hline $\mathrm{C}_{18} \mathrm{H}_{38}$ & octadecane & & & & [593-45-3] \\
\hline & & $90.6 \pm 1.0$ & $(298)$ & CGC & {$[02 / 44]$} \\
\hline & & $91.3 \pm 2.9$ & $(298)$ & GS & {$[01 / 1]$} \\
\hline & & $91.4 \pm 1.3$ & $(298)$ & CGC & {$[00 / 9]$} \\
\hline
\end{tabular}


TABLE 6. Enthalpies of vaporization of organic compounds, 1880-2002-Continued

\begin{tabular}{|c|c|c|c|c|c|}
\hline Molecular formula & $\begin{array}{c}\text { Compound } \\
\text { (Temperature range/K) }\end{array}$ & $\begin{array}{c}\Delta_{\mathrm{vap}} H_{m} \\
\left(\mathrm{~kJ} \mathrm{~mol}^{-1}\right)\end{array}$ & $\begin{array}{l}\text { Mean temperature } \\
\qquad\left(T_{m} / \mathrm{K}\right)\end{array}$ & Method & $\begin{array}{c}\text { CAS registry number } \\
\text { Reference }\end{array}$ \\
\hline & $(363-413)$ & 91.8 & $(298)$ & CGC & {$[95 / 21]$} \\
\hline & $(423-473)$ & 91.8 & (298) & $\mathrm{CGC}$ & {$[95 / 21]$} \\
\hline & $(453-503)$ & 92.8 & $(298)$ & CGC & {$[95 / 21]$} \\
\hline & $(413-588)$ & 74.4 & $(428)$ & & {$[94 / 14]$} \\
\hline & & 91.4 & $(298)$ & & {$[94 / 12]$} \\
\hline & $(501-548)$ & 64.8 & $(516)$ & & {$[87 / 5]$} \\
\hline & $(335-439)$ & 80.0 & $(348)$ & GS & {$[86 / 6]$} \\
\hline & $(318-361)$ & 84.3 & (333) & A, GS & {$[87 / 5][79 / 11]$} \\
\hline & & 72.5 & (343) & GC & {$[77 / 34]$} \\
\hline & & 71.8 & $(353)$ & GC & {$[77 / 34]$} \\
\hline & & 71.1 & $(363)$ & GC & {$[77 / 34]$} \\
\hline & & 70.5 & $(373)$ & GC & {$[77 / 34]$} \\
\hline & & 69.8 & $(383)$ & GC & {$[77 / 34]$} \\
\hline & & 90.8 & $(298)$ & & {$[71 / 28]$} \\
\hline & $(447-474)$ & 78.1 & $(460)$ & $\mathrm{ME}$ & {$[38 / 8]$} \\
\hline & $(447-590)$ & 69.4 & $(462)$ & & {$[1882 / 1][84 / 9]$} \\
\hline \multirow[t]{2}{*}{$\mathrm{C}_{18} \mathrm{H}_{38}$} & 2-methylheptadecane & & & & {$[1560-89-0]$} \\
\hline & $(442-581)$ & 67.8 & $(457)$ & A & {$[87 / 5][59 / 15]$} \\
\hline \multirow[t]{2}{*}{$\mathrm{C}_{18} \mathrm{H}_{38}$} & 3-methylheptadecane & & & & [6418-44-6] \\
\hline & $(441-583)$ & 65.6 & $(456)$ & A & {$[87 / 5][59 / 15]$} \\
\hline \multirow[t]{2}{*}{$\mathrm{C}_{18} \mathrm{H}_{38}$} & 4-methylheptadecane & & & & {$[26429-11-8]$} \\
\hline & $(429-580)$ & 58.9 & $(444)$ & A & {$[87 / 5][59 / 15]$} \\
\hline \multirow[t]{2}{*}{$\mathrm{C}_{18} \mathrm{H}_{38}$} & 5-methylheptadecane & & & & [26730-95-0] \\
\hline & $(432-581)$ & 61.1 & $(447)$ & A & {$[87 / 5][59 / 15]$} \\
\hline \multirow[t]{2}{*}{$\mathrm{C}_{18} \mathrm{H}_{38}$} & 2,3-dimethylhexadecane & & & & {$[61868-02-8]$} \\
\hline & $(466-583)$ & 64.9 & $(481)$ & A & {$[87 / 5][59 / 15]$} \\
\hline \multirow[t]{2}{*}{$\mathrm{C}_{18} \mathrm{H}_{38}$} & 2,4-dimethylhexadecane & & & & {$[61868-08-4]$} \\
\hline & $(434-562)$ & 69.0 & $(449)$ & A & {$[87 / 5][59 / 15]$} \\
\hline \multirow[t]{2}{*}{$\mathrm{C}_{18} \mathrm{H}_{38}$} & 2,4,6-trimethylpentadecane & & & & {$[101882-67-1]$} \\
\hline & $(420-550)$ & 64.3 & $(435)$ & A & {$[87 / 5][99 / 16]$} \\
\hline \multirow[t]{2}{*}{$\mathrm{C}_{18} \mathrm{H}_{38}$} & 4,9-diisopropyldodecane & & & & {$[59 / 15]$} \\
\hline & $(368-424)$ & 70.0 & $(383)$ & $\mathrm{A}$ & {$[87 / 5]$} \\
\hline \multirow[t]{5}{*}{$\mathrm{C}_{18} \mathrm{H}_{38} \mathrm{O}$} & 1-octadecanol & & & & {$[112-92-5]$} \\
\hline & $(435-504)$ & 86.4 & $(450)$ & A & {$[87 / 5]$} \\
\hline & $(500-573)$ & 76.3 & $(515)$ & A & {$[87 / 5]$} \\
\hline & $(494-575)$ & 76.9 & $(509)$ & $\mathrm{A}, \mathrm{EB}$ & {$[87 / 5][70 / 2]$} \\
\hline & $(334-356)$ & 113.5 & $(345)$ & $\mathrm{A}, \mathrm{ME}$ & {$[87 / 5][65 / 15]$} \\
\hline \multirow[t]{2}{*}{$\mathrm{C}_{18} \mathrm{H}_{38} \mathrm{~S}$} & 1-octadecanethiol & & & & {$[2885-00-9]$} \\
\hline & $(492-670)$ & 77.1 & $(507)$ & EST & {$[99 / 16]$} \\
\hline \multirow[t]{2}{*}{$\mathrm{C}_{18} \mathrm{H}_{38} \mathrm{~S}_{2}$} & dinonyl disulfide & & & & {$[4485-77-2]$} \\
\hline & $(490-650)$ & 78.3 & $(514)$ & & {$[99 / 16]$} \\
\hline \multirow[t]{2}{*}{$\mathrm{C}_{18} \mathrm{H}_{38} \mathrm{O}_{4}$} & $2-[2-(2-[$ dodecyloxy $]$ ethoxy & thanol & & & {$[3055-94-5]$} \\
\hline & $(475-523)$ & 102.7 & $(490)$ & A & {$[87 / 5]$} \\
\hline \multirow[t]{2}{*}{$\mathrm{C}_{18} \mathrm{H}_{39} \mathrm{~N}$} & $\mathrm{~N}, \mathrm{~N}$-dimethylhexadecylami & & & & {$[112-69-6]$} \\
\hline & $(483-671)$ & 67.3 & $(498)$ & A & {$[87 / 5]$} \\
\hline \multirow[t]{2}{*}{$\mathrm{C}_{18} \mathrm{H}_{39} \mathrm{~N}$} & dinonylamine & & & & [2044-21-5] \\
\hline & $(486-676)$ & 67.7 & $(501)$ & A & {$[87 / 5]$} \\
\hline $\mathrm{C}_{18} \mathrm{H}_{39} \mathrm{~N}$ & N-ethylhexadecylamine & & & & {$[5877-76-9]$} \\
\hline & $(406-613)$ & 66.4 & $(421)$ & A & {$[87 / 5][47 / 5]$} \\
\hline $\mathrm{C}_{18} \mathrm{H}_{39} \mathrm{~N}$ & octadecylamine & & & & {$[124-30-1]$} \\
\hline & $(450-635)$ & 76.2 & $(465)$ & A & {$[87 / 5]$} \\
\hline $\mathrm{C}_{19} \mathrm{H}_{13} \mathrm{NO}$ & 2-(1-naphthyl)-5-phenyloxa & & & & [846-63-9] \\
\hline & $(510-595)$ & 89.2 & $(525)$ & A & {$[87 / 5]$} \\
\hline $\mathrm{C}_{19} \mathrm{H}_{16}$ & triphenylmethane & & & & {$[519-73-3]$} \\
\hline & & 94.6 & $(298)$ & CGC & [98/11] \\
\hline & $(453-503)$ & 95.0 & (298) & $\mathrm{CGC}$ & {$[95 / 21]$} \\
\hline & $(343-462)$ & 82.0 & $(403)$ & & {$[89 / 14]$} \\
\hline & $(512-643)$ & 58.6 & $(527)$ & A & {$[87 / 5]$} \\
\hline $\mathrm{C}_{19} \mathrm{H}_{17} \mathrm{NO}_{2}$ & 1-piperidinoanthraquinone & & & & {$[4946-83-2]$} \\
\hline & $(395-404)$ & 82.0 & $(399)$ & A & {$[87 / 5]$} \\
\hline $\mathrm{C}_{19} \mathrm{H}_{20} \mathrm{O}_{2}$ & 3-(diphenylmethyl)-3-methy & tanedione & & & \\
\hline & $(353-386)$ & $83.1 \pm 0.5$ & $(370)$ & GS & [95/28] \\
\hline $\mathrm{C}_{19} \mathrm{H}_{20} \mathrm{O}_{4}$ & butyl benzyl phthalate & & & & {$[85-68-7]$} \\
\hline & $(416-516)$ & 89.0 & $(431)$ & A & {$[87 / 5]$} \\
\hline
\end{tabular}


TABLE 6. Enthalpies of vaporization of organic compounds, 1880-2002-Continued

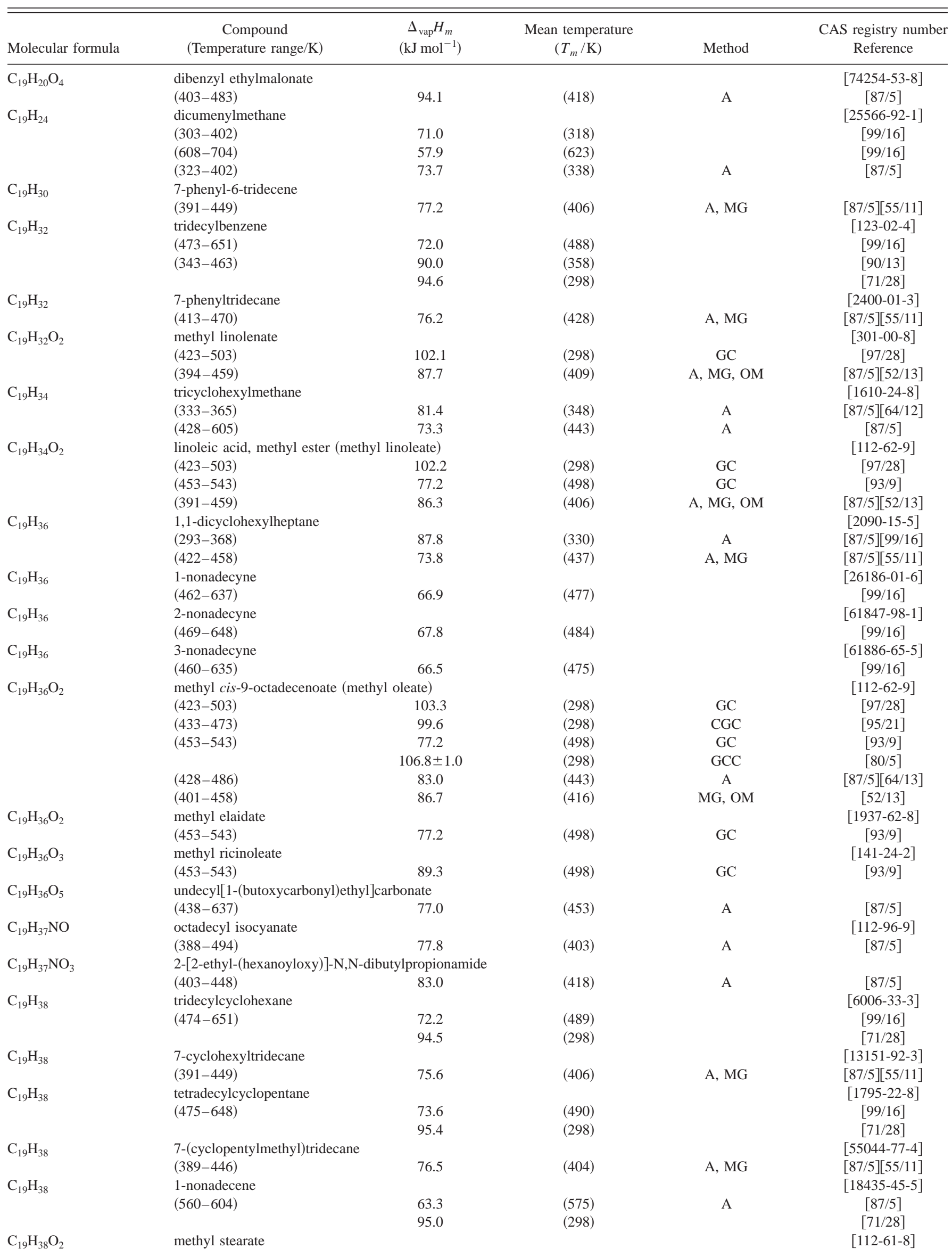


TABLE 6. Enthalpies of vaporization of organic compounds, 1880-2002-Continued

\begin{tabular}{|c|c|c|c|c|c|}
\hline Molecular formula & $\begin{array}{c}\text { Compound } \\
\text { (Temperature range/K) }\end{array}$ & $\begin{array}{c}\Delta_{\mathrm{vap}} H_{m} \\
\left(\mathrm{~kJ} \mathrm{~mol}^{-1}\right)\end{array}$ & $\begin{array}{l}\text { Mean temperature } \\
\qquad\left(T_{m} / \mathrm{K}\right)\end{array}$ & Method & $\begin{array}{c}\text { CAS registry number } \\
\text { Reference }\end{array}$ \\
\hline \multirow{8}{*}{$\mathrm{C}_{19} \mathrm{H}_{38} \mathrm{O}_{2}$} & & 98.0 & $(350)$ & & {$[02 / 27]$} \\
\hline & & $90.0 \pm 0.3$ & $(401)$ & & {$[02 / 27]$} \\
\hline & & $105.9 \pm 1.4$ & $(298)$ & & {$[02 / 27]$} \\
\hline & $(463-523)$ & 106.2 & $(298)$ & $\mathrm{GC}$ & {$[97 / 28]$} \\
\hline & $(453-543)$ & 75.4 & $(498)$ & $\mathrm{GC}$ & {$[93 / 9]$} \\
\hline & $(427-484)$ & 83.2 & $(442)$ & A & {$[87 / 5][64 / 13]$} \\
\hline & propyl palmitate & & & & {$[2239-78-3]$} \\
\hline & $(439-477)$ & 74.5 & $(454)$ & $\mathrm{A}$ & $\begin{array}{c}{[87 / 5][48 / 8]} \\
{[84 / 9]}\end{array}$ \\
\hline \multirow{2}{*}{$\mathrm{C}_{19} \mathrm{H}_{38} \mathrm{O}_{2}$} & isopropyl palmitate & & & & {$[142-91-6]$} \\
\hline & $(433-471)$ & 73.6 & $(448)$ & A & $\begin{array}{c}{[87 / 5][48 / 8]} \\
{[84 / 9]}\end{array}$ \\
\hline \multirow[t]{3}{*}{$\mathrm{C}_{19} \mathrm{H}_{38} \mathrm{O}_{2}$} & nonadecanoic acid & & & & {$[646-30-0]$} \\
\hline & $(511-659)$ & 94.4 & $(526)$ & A & {$[87 / 5]$} \\
\hline & $(371-394)$ & 121.8 & $(386)$ & ME, TE & {$[82 / 4]$} \\
\hline \multirow[t]{2}{*}{$\mathrm{C}_{19} \mathrm{H}_{38} \mathrm{O}_{3}$} & hexadecyl lactate & & & & [35274-05-6] \\
\hline & $(405-556)$ & 90.5 & $(420)$ & A & {$[87 / 5]$} \\
\hline $\mathrm{C}_{19} \mathrm{H}_{38} \mathrm{O}_{3}$ & $\begin{array}{l}\text { 3-octyloxypropionic acid, octyl ester } \\
(443-513)\end{array}$ & 73.6 & $(458)$ & A & [87/5] \\
\hline \multirow[t]{2}{*}{$\mathrm{C}_{19} \mathrm{H}_{39} \mathrm{Br}$} & 1-bromononadecane & & & & [4434-66-6] \\
\hline & $(493-673)$ & 77.9 & (508) & A, EST & $\begin{array}{c}{[87 / 5][61 / 13]} \\
{[70 / 14]}\end{array}$ \\
\hline \multirow[t]{2}{*}{$\mathrm{C}_{19} \mathrm{H}_{39} \mathrm{Cl}$} & 1-chlorononadecane & & & & [62016-76-6] \\
\hline & $(483-673)$ & 76.3 & (498) & A & {$[87 / 5][70 / 14]$} \\
\hline \multirow[t]{2}{*}{$\mathrm{C}_{19} \mathrm{H}_{39} \mathrm{~F}$} & 1-fluorononadecane & & & & [1480-63-3] \\
\hline & $(458-648)$ & 72.5 & (473) & A, EST & $\begin{array}{c}{[87 / 5][61 / 13]} \\
{[70 / 14]}\end{array}$ \\
\hline \multirow[t]{2}{*}{$\mathrm{C}_{19} \mathrm{H}_{39} \mathrm{I}$} & 1-iodononadecane & & & & [62127-51-9] \\
\hline & $(506-673)$ & 79.1 & $(521)$ & A, EST & $\begin{array}{c}{[87 / 5][61 / 13]} \\
{[70 / 14]}\end{array}$ \\
\hline $\mathrm{C}_{19} \mathrm{H}_{39} \mathrm{NO}_{2}$ & $\begin{array}{l}\text { N-hexadecyl lactamide } \\
(423-508)\end{array}$ & 111.0 & (438) & $\mathrm{A}$ & {$[87 / 5]$} \\
\hline $\mathrm{C}_{19} \mathrm{H}_{39} \mathrm{NO}_{2}$ & $\begin{array}{l}\text { N,N-dioctyl lactamide } \\
(453-488)\end{array}$ & 99.3 & $(468)$ & A & {$[87 / 5]$} \\
\hline \multirow[t]{5}{*}{$\mathrm{C}_{19} \mathrm{H}_{40}$} & nonadecane & & & & [629-92-5] \\
\hline & $(423-588)$ & 76.2 & $(438)$ & & {$[94 / 14]$} \\
\hline & & 96.4 & (298) & & {$[94 / 12]$} \\
\hline & $(456-606)$ & 73.0 & $(471)$ & A & {$[87 / 5]$} \\
\hline & & 95.8 & (298) & & {$[71 / 28]$} \\
\hline \multirow[t]{2}{*}{$\mathrm{C}_{19} \mathrm{H}_{40}$} & 2-methyloctadecane & & & & [1560-88-9] \\
\hline & $(451-595)$ & 67.5 & (466) & A & $\begin{array}{c}{[87 / 5][99 / 16]} \\
{[59 / 15]}\end{array}$ \\
\hline \multirow[t]{2}{*}{$\mathrm{C}_{19} \mathrm{H}_{40}$} & 3-methyloctadecane & & & & [6561-44-0] \\
\hline & $(455-597)$ & 69.2 & $(470)$ & A & $\begin{array}{c}{[87 / 5][99 / 16]} \\
{[59 / 15]}\end{array}$ \\
\hline \multirow[t]{2}{*}{$\mathrm{C}_{19} \mathrm{H}_{40}$} & 4-methyloctadecane & & & & [10544-95-3] \\
\hline & $(445-596)$ & 63.3 & $(460)$ & A & $\begin{array}{c}{[87 / 5][99 / 16]} \\
{[59 / 15]}\end{array}$ \\
\hline \multirow[t]{2}{*}{$\mathrm{C}_{19} \mathrm{H}_{40}$} & 5-methyloctadecane & & & & [25117-35-5] \\
\hline & $(445-595)$ & 63.8 & $(460)$ & A & $\begin{array}{c}{[87 / 5][99 / 16]} \\
{[59 / 15]}\end{array}$ \\
\hline \multirow[t]{3}{*}{$\mathrm{C}_{19} \mathrm{H}_{40}$} & 2,3-dimethylheptadecane & & & & [61868-03-9] \\
\hline & $(447-598)$ & 64.1 & $(462)$ & & {$[99 / 16][59 / 15]$} \\
\hline & $(493-598)$ & 67.2 & $(508)$ & A & {$[87 / 5]$} \\
\hline \multirow[t]{2}{*}{$\mathrm{C}_{19} \mathrm{H}_{40}$} & 2,4-dimethylheptadecane & & & & [61868-09-5] \\
\hline & $(444-574)$ & 70.6 & (459) & A & $\begin{array}{c}{[87 / 5][99 / 16]} \\
{[59 / 15]}\end{array}$ \\
\hline \multirow[t]{2}{*}{$\mathrm{C}_{19} \mathrm{H}_{40}$} & 2,4,6-trimethylhexadecane & & & & [102013-94-5] \\
\hline & $(435-568)$ & 67.3 & $(450)$ & A & $\begin{array}{c}{[87 / 5][99 / 16]} \\
{[59 / 15]}\end{array}$ \\
\hline \multirow[t]{2}{*}{$\mathrm{C}_{19} \mathrm{H}_{40}$} & 7-hexyltridecane & & & & [7225-66-3] \\
\hline & $(411-444)$ & 75.2 & $(426)$ & A & {$[87 / 5]$} \\
\hline \multirow[t]{2}{*}{$\mathrm{C}_{19} \mathrm{H}_{40} \mathrm{O}$} & 1-nonadecanol & & & & [1454-84-8] \\
\hline & $(479-640)$ & 81.7 & (494) & A & {$[87 / 5]$} \\
\hline
\end{tabular}


TABLE 6. Enthalpies of vaporization of organic compounds, 1880-2002-Continued

\begin{tabular}{|c|c|c|c|c|c|}
\hline Molecular formula & $\begin{array}{c}\text { Compound } \\
\text { (Temperature range/K) }\end{array}$ & $\begin{array}{c}\Delta_{\mathrm{vap}} H_{m} \\
\left(\mathrm{~kJ} \mathrm{~mol}^{-1}\right)\end{array}$ & $\begin{array}{l}\text { Mean temperature } \\
\qquad\left(T_{m} / \mathrm{K}\right)\end{array}$ & Method & $\begin{array}{l}\text { CAS registry number } \\
\text { Reference }\end{array}$ \\
\hline & $(494-635)$ & 80.0 & $(509)$ & A & {$[87 / 5]$} \\
\hline \multirow[t]{2}{*}{$\mathrm{C}_{19} \mathrm{H}_{40} \mathrm{~S}$} & 1-nonadecanethiol & & & & [53193-23-0] \\
\hline & $(502-682)$ & 79.2 & $(517)$ & EST & [99/16] \\
\hline \multirow[t]{2}{*}{$\mathrm{C}_{19} \mathrm{H}_{41} \mathrm{~N}$} & nonadecylamine & & & & [14130-05-3] \\
\hline & $(532-647)$ & 72.7 & $(547)$ & A, EST & {$[87 / 5][56 / 17]$} \\
\hline \multirow[t]{2}{*}{$\mathrm{C}_{20} \mathrm{H}_{10}$} & corannulene & & & & {$[5821-51-2]$} \\
\hline & & $115.5 \pm 2.5$ & $(298)$ & CGC & {$[02 / 44]$} \\
\hline \multirow[t]{3}{*}{$\mathrm{C}_{20} \mathrm{H}_{12}$} & perylene & & & & [198-55-0] \\
\hline & & $123.1 \pm 1.7$ & (298) & CGC & {$[02 / 44]$} \\
\hline & $(323-473)$ & 89.9 & (398) & $\mathrm{GC}$ & {$[02 / 18]$} \\
\hline \multirow[t]{3}{*}{$\mathrm{C}_{20} \mathrm{H}_{12}$} & benzo[a]pyrene & & & & {$[50-32-8]$} \\
\hline & $(323-473)$ & 91.0 & (398) & $\mathrm{GC}$ & {$[02 / 18]$} \\
\hline & $(343-453)$ & 95.5 & (398) & GC & {$[90 / 2]$} \\
\hline \multirow[t]{2}{*}{$\mathrm{C}_{20} \mathrm{H}_{12}$} & benzo[e]pyrene & & & & [192-97-2] \\
\hline & $(343-453)$ & 92.0 & (398) & $\mathrm{GC}$ & {$[90 / 2]$} \\
\hline \multirow[t]{2}{*}{$\mathrm{C}_{20} \mathrm{H}_{12}$} & benzo[k]fluoranthene & & & & [207-08-9] \\
\hline & $(323-473)$ & 88.5 & (398) & GC & {$[02 / 18]$} \\
\hline \multirow[t]{2}{*}{$\mathrm{C}_{20} \mathrm{H}_{12}$} & benzo[b]fluoranthene & & & & [205-99-2] \\
\hline & $(323-473)$ & 89.7 & (398) & GC & {$[02 / 18]$} \\
\hline \multirow[t]{4}{*}{$\mathrm{C}_{20} \mathrm{H}_{14}$} & 9-phenylanthracene & & & & {$[602-55-1]$} \\
\hline & $(323-473)$ & 91.6 & (398) & GC & {$[02 / 18]$} \\
\hline & $(430-510)$ & 84.4 & (445) & A & {$[87 / 5]$} \\
\hline & $(435-513)$ & 86.2 & $(450)$ & & {$[99 / 16][74 / 35]$} \\
\hline \multirow[t]{2}{*}{$\mathrm{C}_{20} \mathrm{H}_{14} \mathrm{O}_{4}$} & dibenzoyl resorcinol & & & & [94-01-9] \\
\hline & $(399-493)$ & 76.0 & $(414)$ & A, UV & {$[87 / 5][60 / 2]$} \\
\hline \multirow[t]{3}{*}{$\mathrm{C}_{20} \mathrm{H}_{16}$} & 7,12-dimethylbenz[a]anthracene & & & & {$[313-74-6]$} \\
\hline & $(323-473)$ & 88.9 & $(398)$ & GC & {$[02 / 18]$} \\
\hline & $(396-408)$ & 112.9 & $(402)$ & $\mathrm{A}, \mathrm{ME}$ & $\begin{array}{c}{[87 / 5][64 / 22]} \\
{[99 / 16]}\end{array}$ \\
\hline \multirow[t]{2}{*}{$\mathrm{C}_{20} \mathrm{H}_{16}$} & 5,6-dimethylchrysene & & & & {$[3697-27-6]$} \\
\hline & $(380-394)$ & 121.7 & $(387)$ & A & {$[87 / 5]$} \\
\hline $\mathrm{C}_{20} \mathrm{H}_{22} \mathrm{~N}_{2} \mathrm{O}_{2}$ & $\begin{array}{l}\text { 1,4-bis(propylamino)anthraquinone } \\
(409-463)\end{array}$ & 118.3 & $(424)$ & A & {$[87 / 5]$} \\
\hline \multirow[t]{2}{*}{$\mathrm{C}_{20} \mathrm{H}_{24} \mathrm{O}_{6}$} & dibenzo-18-crown-6 & & & & {$[14187-32-7]$} \\
\hline & & $137.0 \pm 7.4$ & $(298)$ & $\mathrm{CGC}$ & {$[00 / 9]$} \\
\hline \multirow[t]{2}{*}{$\mathrm{C}_{20} \mathrm{H}_{26} \mathrm{O}_{4}$} & dicyclohexyl phthalate & & & & {$[84-61-7]$} \\
\hline & $(391-475)$ & 97.0 & $(406)$ & A & {$[87 / 5]$} \\
\hline \multirow[t]{2}{*}{$\mathrm{C}_{20} \mathrm{H}_{28}$} & 2-butyl-3-hexylnaphthalene & & & & [55000-56-1] \\
\hline & $(422-485)$ & 80.8 & $(437)$ & & $\begin{array}{c}{[63 / 24][84 / 9]} \\
{[99 / 16]}\end{array}$ \\
\hline \multirow[t]{2}{*}{$\mathrm{C}_{20} \mathrm{H}_{16}$} & triphenylethylene & & & & {$[58-72-0]$} \\
\hline & $(353-443)$ & 89.7 & $(398)$ & & [89/14] \\
\hline \multirow[t]{2}{*}{$\mathrm{C}_{20} \mathrm{H}_{28}$} & 7-butyl-1-hexylnaphthalene & & & & {$[55000-55-0]$} \\
\hline & $(418-481)$ & 78.1 & $(433)$ & & $\begin{array}{c}{[63 / 24][84 / 9]} \\
{[99 / 16]}\end{array}$ \\
\hline $\mathrm{C}_{20} \mathrm{H}_{28}$ & 1,4-dimethyl-5-octylnaphthalene & & & & [55000-53-8] \\
\hline & $(432-496)$ & 81.6 & $(447)$ & & $\begin{array}{c}{[63 / 24][84 / 9]} \\
{[99 / 16]}\end{array}$ \\
\hline $\mathrm{C}_{20} \mathrm{H}_{28}$ & 2,6-dimethyl-3-octylnaphthalene & & & & [55000-54-9] \\
\hline & $(430-494)$ & 80.8 & $(445)$ & & $\begin{array}{c}{[63 / 24][84 / 9]} \\
{[99 / 16]}\end{array}$ \\
\hline $\mathrm{C}_{20} \mathrm{H}_{30}$ & hexacyclopropylethane & & & & {$[26902-55-6]$} \\
\hline & $(333-373)$ & $85.8 \pm 0.2$ & $(298)$ & GS & {$[95 / 27]$} \\
\hline $\mathrm{C}_{20} \mathrm{H}_{30} \mathrm{O}_{4}$ & dihexyl phthalate & & & & {$[84-75-3]$} \\
\hline & $(453-533)$ & 92.0 & (468) & A & {$[87 / 5]$} \\
\hline & $(343-387)$ & 103.0 & $(358)$ & $\mathrm{A}, \mathrm{ME}$ & {$[87 / 5][48 / 10]$} \\
\hline $\mathrm{C}_{20} \mathrm{H}_{32}$ & 1,2,3,4-tetrahydro-6-butyl-7-hexylna & phthalene & & & [66538-96-3] \\
\hline & $(413-475)$ & 78.1 & $(428)$ & & $\begin{array}{c}{[63 / 24][84 / 9]} \\
{[99 / 16]}\end{array}$ \\
\hline $\mathrm{C}_{20} \mathrm{H}_{32}$ & 1,2,3,4-tetrahydro-7-butyl-1-hexylna & phthalene & & & {$[66205-02-5]$} \\
\hline & $(409-471)$ & 76.7 & $(424)$ & & $\begin{array}{c}{[63 / 24][84 / 9]} \\
{[99 / 16]}\end{array}$ \\
\hline $\mathrm{C}_{20} \mathrm{H}_{32}$ & 1,2,3,4-tetrahydro-2,6-dimethyl-7-oc & tylnaphthalene & & & [55255-59-9] \\
\hline & $(418-480)$ & 79.4 & $(433)$ & & {$[63 / 24][84 / 9]$} \\
\hline
\end{tabular}


TABLE 6. Enthalpies of vaporization of organic compounds, 1880-2002-Continued

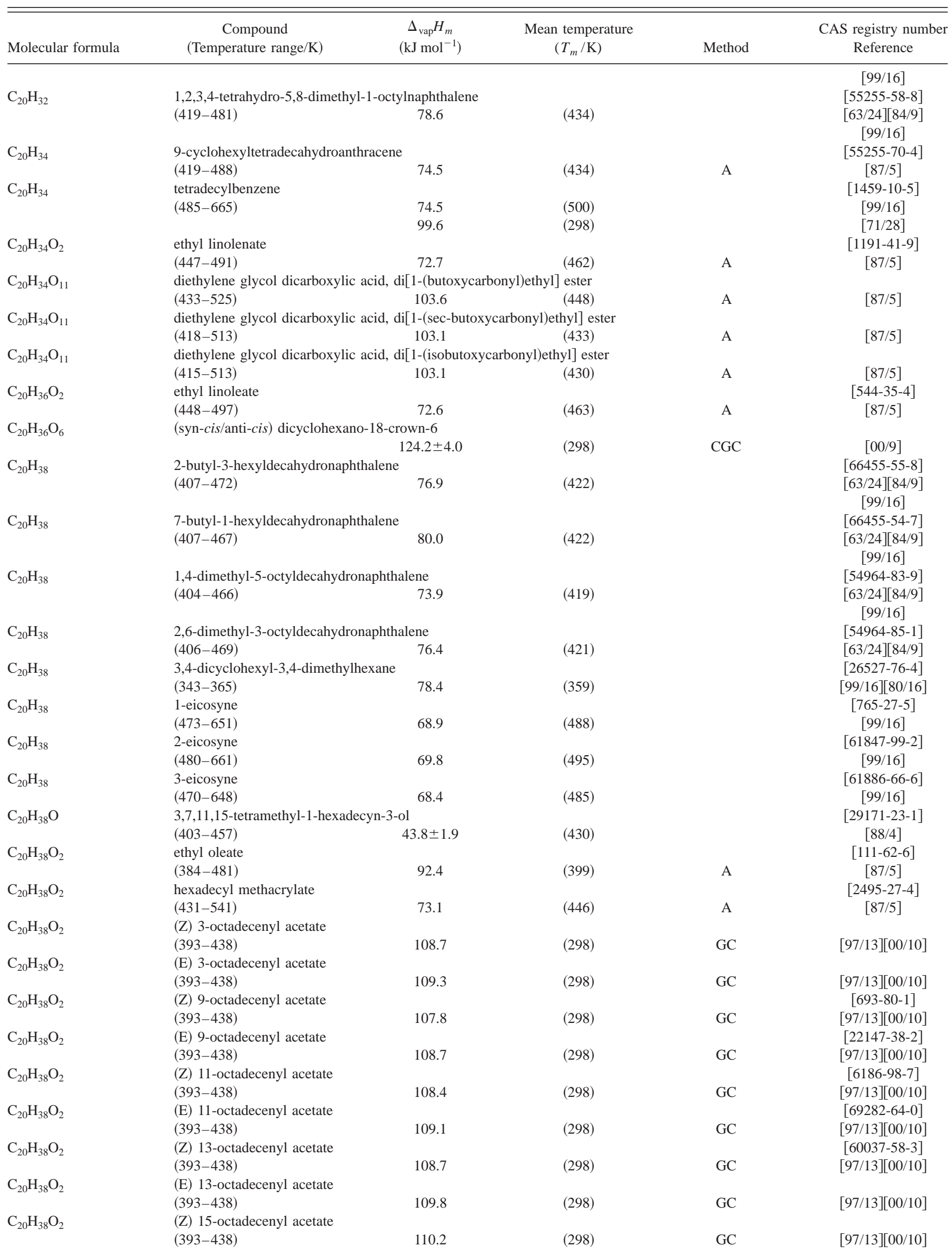


TABLE 6. Enthalpies of vaporization of organic compounds, 1880-2002-Continued

\begin{tabular}{|c|c|c|c|c|c|}
\hline Molecular formula & $\begin{array}{c}\text { Compound } \\
\text { (Temperature range/K) }\end{array}$ & $\begin{array}{c}\Delta_{\mathrm{vap}} H_{m} \\
\left(\mathrm{~kJ} \mathrm{~mol}^{-1}\right)\end{array}$ & $\begin{array}{l}\text { Mean temperature } \\
\qquad\left(T_{m} / \mathrm{K}\right)\end{array}$ & Method & $\begin{array}{c}\text { CAS registry number } \\
\text { Reference }\end{array}$ \\
\hline $\mathrm{C}_{20} \mathrm{H}_{38} \mathrm{O}_{2}$ & $\begin{array}{l}\text { (E) } 15 \text {-octadecenyl acetate } \\
(393-438)\end{array}$ & 110.5 & $(298)$ & GC & {$[97 / 13][00 / 10]$} \\
\hline $\mathrm{C}_{20} \mathrm{H}_{38} \mathrm{O}_{4}$ & $\begin{array}{l}\text { dioctyl succinate } \\
(503-523)\end{array}$ & 94.2 & $(513)$ & A & $\begin{array}{c}{[14491-66-8]} \\
{[87 / 5]}\end{array}$ \\
\hline $\mathrm{C}_{20} \mathrm{H}_{38} \mathrm{O}_{4}$ & $\begin{array}{l}\text { dipentyl sebacate } \\
(353-408)\end{array}$ & 99.2 & $(368)$ & A & $\begin{array}{c}{[6819-09-6]} \\
{[87 / 5]}\end{array}$ \\
\hline $\mathrm{C}_{20} \mathrm{H}_{38} \mathrm{O}_{5}$ & $\begin{array}{l}\text { dodecyl[1-(butoxycarbony } \\
(408-498)\end{array}$ & $\begin{array}{r}\text { rbonate } \\
82.8\end{array}$ & (423) & A & {$[87 / 5]$} \\
\hline $\mathrm{C}_{20} \mathrm{H}_{40}$ & $\begin{array}{l}1 \text {-eicosene } \\
(478-638) \\
(573-615)\end{array}$ & $\begin{array}{c}74.3 \\
65.0 \\
100.0\end{array}$ & $\begin{array}{l}(493) \\
(588) \\
(298)\end{array}$ & A & $\begin{array}{c}{[3452-07-1]} \\
{[99 / 16]} \\
{[87 / 5]} \\
{[71 / 28]}\end{array}$ \\
\hline $\mathrm{C}_{20} \mathrm{H}_{40}$ & $\begin{array}{l}\text { tetradecylcyclohexane } \\
(486-665)\end{array}$ & $\begin{array}{l}74.7 \\
99.4\end{array}$ & $\begin{array}{l}(501) \\
(298)\end{array}$ & & $\begin{array}{c}{[1795-18-2]} \\
{[99 / 16]} \\
{[71 / 28]}\end{array}$ \\
\hline $\mathrm{C}_{20} \mathrm{H}_{40}$ & $\begin{array}{l}\text { pentadecylcyclopentane } \\
(486-661)\end{array}$ & $\begin{array}{c}76.5 \\
100.3\end{array}$ & $\begin{array}{l}(501) \\
(298)\end{array}$ & & $\begin{array}{c}{[4669-01-6]} \\
{[99 / 16]} \\
{[71 / 28]}\end{array}$ \\
\hline $\mathrm{C}_{20} \mathrm{H}_{40} \mathrm{O}$ & $\begin{array}{l}\text { 3,7,11,15-tetramethyl-1-he } \\
(439-468)\end{array}$ & $\begin{array}{l}- \text { ol } \\
67.0 \pm 2.0\end{array}$ & $(453)$ & & $\begin{array}{c}{[60046-87-9]} \\
{[88 / 4]}\end{array}$ \\
\hline $\mathrm{C}_{20} \mathrm{H}_{40} \mathrm{O}_{2}$ & $\begin{array}{l}\text { octadecyl acetate } \\
(393-438) \\
(341-500)\end{array}$ & $\begin{array}{c}113.5 \\
94.3\end{array}$ & $\begin{array}{l}(298) \\
(356)\end{array}$ & $\begin{array}{c}\mathrm{GC} \\
\mathrm{A}\end{array}$ & $\begin{array}{c}{[822-23-1]} \\
{[97 / 13][00 / 10]} \\
{[87 / 5]}\end{array}$ \\
\hline $\mathrm{C}_{20} \mathrm{H}_{40} \mathrm{O}_{2}$ & $\begin{array}{l}\text { butyl palmitate } \\
(353-383)\end{array}$ & 93.8 & $(368)$ & A & $\begin{array}{c}{[111-06-8]} \\
{[87 / 5]}\end{array}$ \\
\hline $\mathrm{C}_{20} \mathrm{H}_{40} \mathrm{O}_{2}$ & $\begin{array}{l}\text { decyl decanoate } \\
(341-398)\end{array}$ & 97.8 & $(356)$ & $\mathrm{A}$ & $\begin{array}{c}{[1654-86-0]} \\
{[87 / 5]}\end{array}$ \\
\hline $\mathrm{C}_{20} \mathrm{H}_{40} \mathrm{O}_{2}$ & $\begin{array}{l}\text { eicosanoic acid } \\
(477-670) \\
(380-404)\end{array}$ & $\begin{array}{l}114.5 \\
125.5\end{array}$ & $\begin{array}{l}(492) \\
(392)\end{array}$ & $\begin{array}{c}\text { A } \\
\mathrm{ME}, \mathrm{TE}\end{array}$ & $\begin{array}{c}{[506-30-9]} \\
{[87 / 5]} \\
{[82 / 4]}\end{array}$ \\
\hline $\mathrm{C}_{20} \mathrm{H}_{40} \mathrm{O}_{2}$ & $\begin{array}{l}\text { ethyl stearate } \\
(454-469) \\
(310-328)\end{array}$ & $\begin{array}{l}111.9 \\
106.8\end{array}$ & $\begin{array}{l}(461) \\
(319)\end{array}$ & $\begin{array}{c}\mathrm{A} \\
\mathrm{A}, \mathrm{ME}\end{array}$ & $\begin{array}{c}{[111-61-5]} \\
{[87 / 5]} \\
{[87 / 5][67 / 22]}\end{array}$ \\
\hline $\mathrm{C}_{20} \mathrm{H}_{40} \mathrm{O}_{2}$ & methyl nonadecanoate & $\begin{array}{c}101.2 \\
105.0 \pm 2.4 \\
109.5 \pm 2.7 \\
90.1\end{array}$ & $\begin{array}{l}(350) \\
(326) \\
(298) \\
(456)\end{array}$ & A, EST & $\begin{array}{c}{[1731-94-8]} \\
{[02 / 27]} \\
{[02 / 27]} \\
{[02 / 27]} \\
{[87 / 5][63 / 16]}\end{array}$ \\
\hline $\mathrm{C}_{20} \mathrm{H}_{40} \mathrm{O}_{2}$ & $\begin{array}{l}\text { 2-ethylhexyl laurate } \\
(371-452) \\
(443-503)\end{array}$ & $\begin{array}{c}91.4 \\
104.5\end{array}$ & $\begin{array}{l}(386) \\
(298)\end{array}$ & GC & $\begin{array}{l}{[01 / 10]} \\
{[97 / 28]}\end{array}$ \\
\hline $\mathrm{C}_{20} \mathrm{H}_{41} \mathrm{Br}$ & $\begin{array}{l}\text { 1-bromoeicosane } \\
(502-673)\end{array}$ & 79.8 & $(517)$ & A, EST & $\begin{array}{c}{[4276-49-7]} \\
{[87 / 5][61 / 13]} \\
{[70 / 14]}\end{array}$ \\
\hline $\mathrm{C}_{20} \mathrm{H}_{41} \mathrm{Cl}$ & $\begin{array}{l}\text { 1-chloroeicosane } \\
(492-673)\end{array}$ & 78.3 & $(507)$ & A & $\begin{array}{l}{[42217-02-7]} \\
{[87 / 5][70 / 14]}\end{array}$ \\
\hline $\mathrm{C}_{20} \mathrm{H}_{41} \mathrm{~F}$ & $\begin{array}{l}\text { 1-fluoroeicosane } \\
(468-663)\end{array}$ & 74.3 & (483) & A, EST & $\begin{array}{c}{[676-44-8]} \\
{[87 / 5][61 / 13]} \\
{[70 / 14]}\end{array}$ \\
\hline $\mathrm{C}_{20} \mathrm{H}_{41} \mathrm{I}$ & $\begin{array}{l}\text { 1-iodoeicosane } \\
(516-673)\end{array}$ & 80.9 & $(531)$ & A, EST & $\begin{array}{c}{[34994-81-5]} \\
{[87 / 5][61 / 13]} \\
{[70 / 14]}\end{array}$ \\
\hline $\mathrm{C}_{20} \mathrm{H}_{42}$ & $\begin{array}{l}\text { 5-butylhexadecane } \\
(423-457)\end{array}$ & 77.3 & $(438)$ & $\mathrm{A}, \mathrm{MG}$ & $\begin{array}{l}{[6912-07-8]} \\
{[87 / 5][55 / 11]}\end{array}$ \\
\hline $\mathrm{C}_{20} \mathrm{H}_{42}$ & $\begin{array}{l}\text { 2,3-dimethyloctadecane } \\
(458-612)\end{array}$ & 65.7 & $(473)$ & A & $\begin{array}{l}{[61868-04-0]} \\
{[87 / 5][59 / 15]}\end{array}$ \\
\hline $\mathrm{C}_{20} \mathrm{H}_{42}$ & $\begin{array}{l}\text { 2,4-dimethyloctadecane } \\
(456-583)\end{array}$ & 75.8 & $(471)$ & A & $\begin{array}{l}{[61868-10-8]} \\
{[87 / 5][59 / 15]}\end{array}$ \\
\hline $\mathrm{C}_{20} \mathrm{H}_{42}$ & $\begin{array}{l}(453-503) \\
(433-583)\end{array}$ & $\begin{array}{c}102.6 \pm 1.0 \\
102.8 \pm 2.2 \\
101.1 \pm 2.0 \\
103.5 \\
78.0\end{array}$ & $\begin{array}{l}(298) \\
(298) \\
(298) \\
(298) \\
(448)\end{array}$ & $\begin{array}{c}\text { CGC } \\
\text { GS } \\
\text { CGC } \\
\text { CGC }\end{array}$ & $\begin{array}{c}{[112-95-8]} \\
{[02 / 44]} \\
{[01 / 1]} \\
{[00 / 9]} \\
{[95 / 21]} \\
{[94 / 14]}\end{array}$ \\
\hline
\end{tabular}


TABLE 6. Enthalpies of vaporization of organic compounds, 1880-2002-Continued

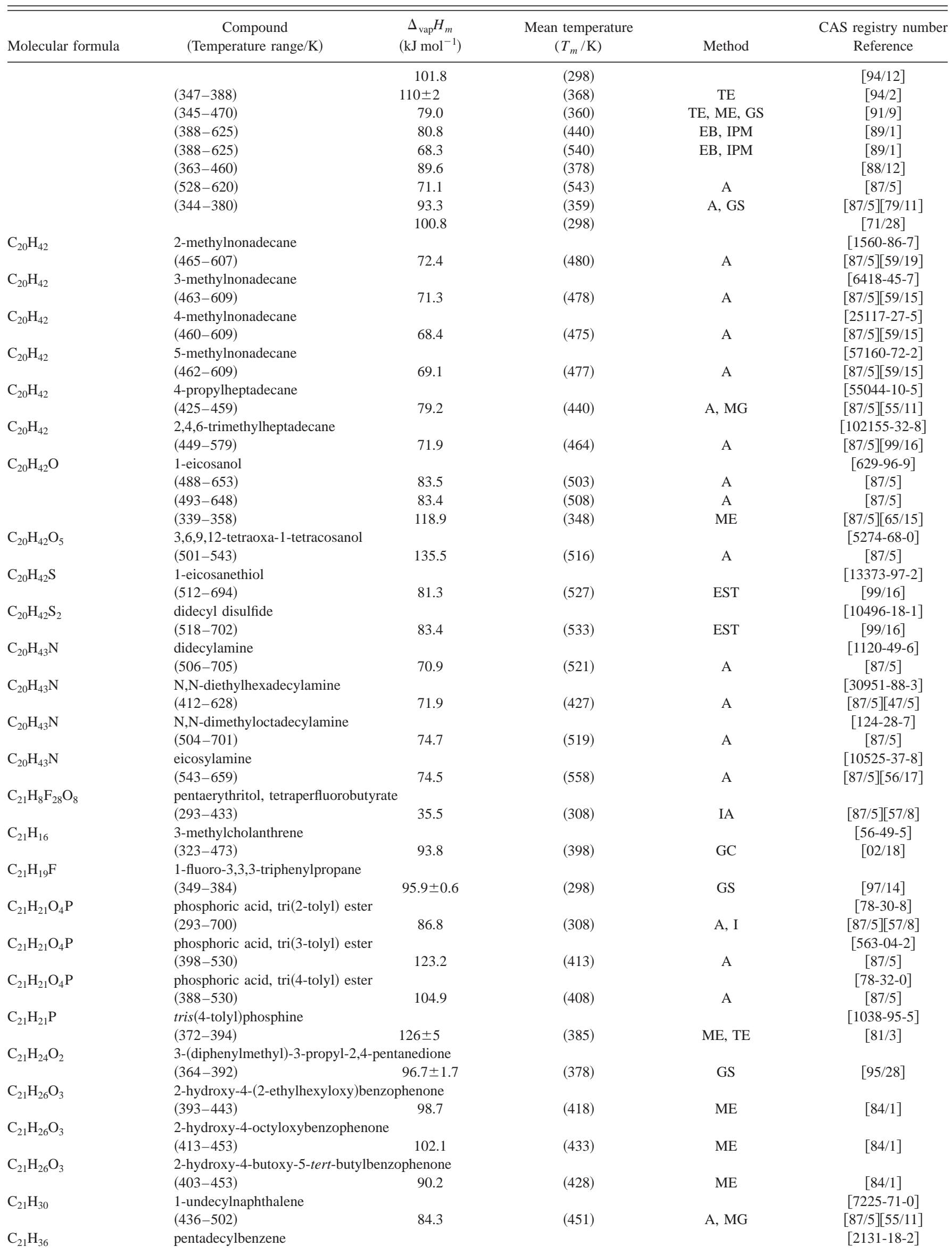


TABLE 6. Enthalpies of vaporization of organic compounds, 1880-2002-Continued

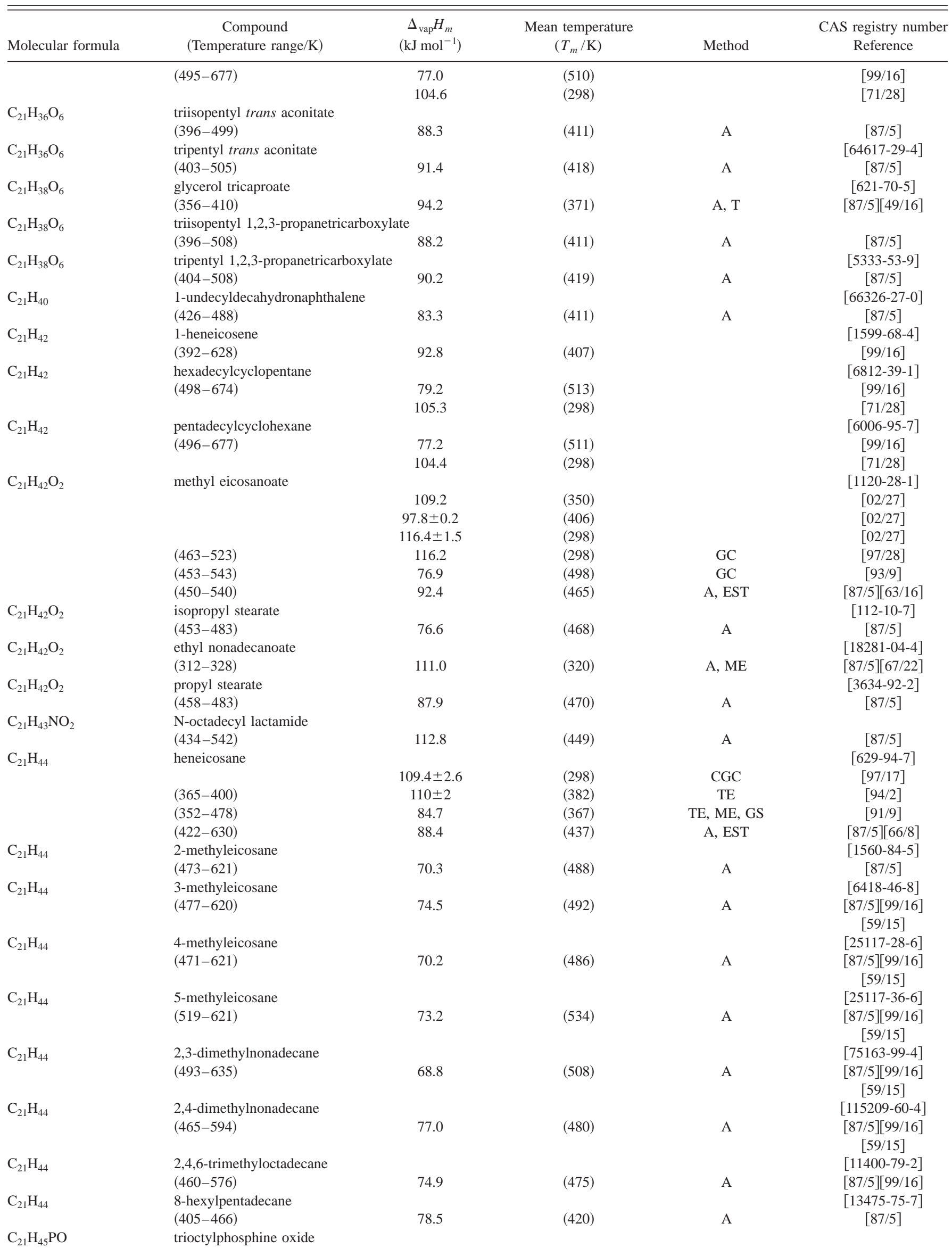


TABLE 6. Enthalpies of vaporization of organic compounds, 1880-2002-Continued

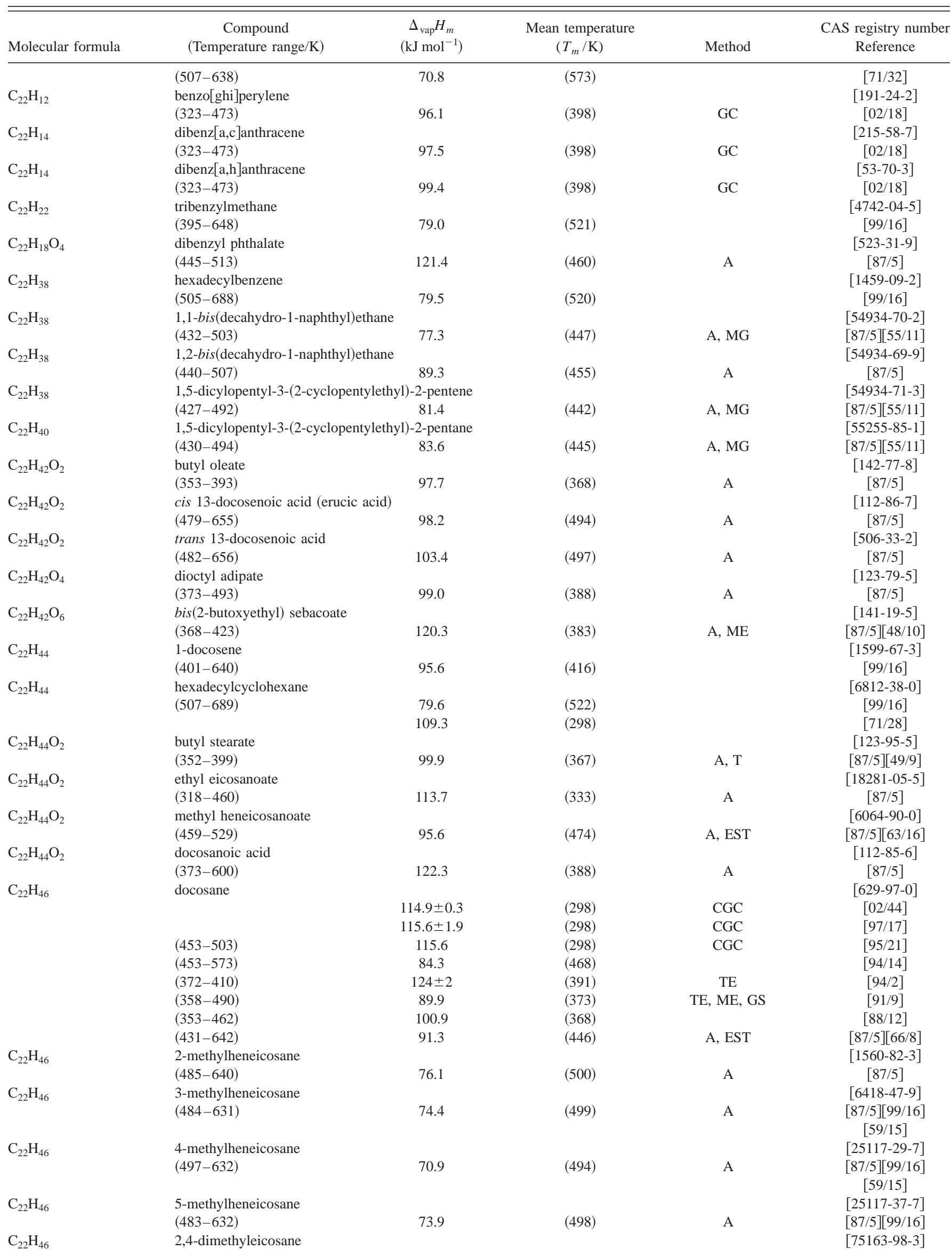


TABLE 6. Enthalpies of vaporization of organic compounds, 1880-2002-Continued

\begin{tabular}{|c|c|c|c|c|c|}
\hline Molecular formula & $\begin{array}{c}\text { Compound } \\
\text { (Temperature range/K) }\end{array}$ & $\begin{array}{c}\Delta_{\mathrm{vap}} H_{m} \\
\left(\mathrm{~kJ} \mathrm{~mol}^{-1}\right)\end{array}$ & $\begin{array}{l}\text { Mean temperature } \\
\qquad\left(T_{m} / \mathrm{K}\right)\end{array}$ & Method & $\begin{array}{c}\text { CAS registry number } \\
\text { Reference }\end{array}$ \\
\hline & $(471-603)$ & 77.5 & $(486)$ & A & $\begin{array}{c}{[87 / 5][99 / 16]} \\
{[59 / 15]}\end{array}$ \\
\hline \multirow[t]{2}{*}{$\mathrm{C}_{22} \mathrm{H}_{46}$} & 2,4,6-trimethylnonadecane & & & & [102886-19-1] \\
\hline & $(470-587)$ & 77.3 & $(485)$ & A & $\begin{array}{c}{[87 / 5][99 / 16]} \\
{[59 / 15]}\end{array}$ \\
\hline \multirow[t]{2}{*}{$\mathrm{C}_{22} \mathrm{H}_{46}$} & 8-heptylpentadecane & & & & {$[71005-15-7]$} \\
\hline & $(298-313)$ & 107.7 & $(305)$ & A & {$[87 / 5]$} \\
\hline \multirow[t]{2}{*}{$\mathrm{C}_{22} \mathrm{H}_{46} \mathrm{O}$} & 1-docosanol & & & & [661-19-8] \\
\hline & $(344-459)$ & 115.3 & $(351)$ & $\mathrm{A}, \mathrm{ME}$ & {$[87 / 5][65 / 15]$} \\
\hline \multirow[t]{2}{*}{$\mathrm{C}_{22} \mathrm{H}_{46} \mathrm{~S}$} & 1-docosanethiol & & & & {$[7773-83-3]$} \\
\hline & $(437-680)$ & 107.7 & $(452)$ & EST & {$[99 / 16]$} \\
\hline \multirow{2}{*}{$\mathrm{C}_{23} \mathrm{H}_{24} \mathrm{O}_{6}$} & tris(ethoxycarbonyl)-9-fluor & lane & & & \\
\hline & $(359-393)$ & $107.5 \pm 0.7$ & & GS & {$[95 / 8]$} \\
\hline \multirow[t]{2}{*}{$\mathrm{C}_{23} \mathrm{H}_{26} \mathrm{O}_{6}$} & 1,1,1-tris(ethoxycarbonyl)- & nylethane & & & \\
\hline & $(344-394)$ & $109.3 \pm 1.0$ & & GS & {$[95 / 8]$} \\
\hline \multirow{2}{*}{$\mathrm{C}_{23} \mathrm{H}_{40}$} & heptadecylbenzene & & & & {$[14752-75-1]$} \\
\hline & $(414-664)$ & 98.5 & $(429)$ & & {$[99 / 16]$} \\
\hline \multirow[t]{2}{*}{$\mathrm{C}_{23} \mathrm{H}_{42} \mathrm{O}_{3}$} & tetrahydrofurfuryl oleate & & & & {$[5420-17-7]$} \\
\hline & $(353-398)$ & 98.7 & $(368)$ & A & {$[87 / 5]$} \\
\hline \multirow[t]{3}{*}{$\mathrm{C}_{23} \mathrm{H}_{44} \mathrm{O}_{2}$} & methyl erucate & & & & [1120-34-9] \\
\hline & $(463-523)$ & 123.8 & $(298)$ & GC & {$[97 / 28]$} \\
\hline & $(453-543)$ & 93.5 & $(498)$ & GC & {$[93 / 9]$} \\
\hline \multirow[t]{2}{*}{$\mathrm{C}_{23} \mathrm{H}_{45} \mathrm{NO}_{3}$} & 2-lauryloxy-N,N-dibutylpro & & & & \\
\hline & $(443-458)$ & 90.6 & $(450)$ & $\mathrm{A}$ & {$[87 / 5]$} \\
\hline \multirow[t]{2}{*}{$\mathrm{C}_{23} \mathrm{H}_{46}$} & 9-cyclohexylheptadecane & & & & {$[55124-77-1]$} \\
\hline & $(456-492)$ & 83.9 & $(471)$ & A & {$[87 / 5]$} \\
\hline \multirow[t]{2}{*}{$\mathrm{C}_{23} \mathrm{H}_{46}$} & hexadecylcyclohexane & & & & {$[19781-73-8]$} \\
\hline & $(414-664)$ & 97.6 & $(429)$ & & {$[99 / 16]$} \\
\hline \multirow[t]{2}{*}{$\mathrm{C}_{23} \mathrm{H}_{46}$} & 1-tricosene & & & & {$[18835-32-0]$} \\
\hline & $(409-652)$ & 98.5 & $(424)$ & & {$[99 / 16]$} \\
\hline \multirow[t]{4}{*}{$\mathrm{C}_{23} \mathrm{H}_{46} \mathrm{O}_{2}$} & methyl docosanoate (methy & & & & [929-77-1] \\
\hline & $(463-523)$ & 126.1 & $(298)$ & GC & {$[97 / 28]$} \\
\hline & $(453-543)$ & 81.0 & $(498)$ & & [93/9] \\
\hline & $(467-539)$ & 98.2 & $(482)$ & A & {$[87 / 5][63 / 16]$} \\
\hline \multirow[t]{2}{*}{$\mathrm{C}_{23} \mathrm{H}_{46} \mathrm{O}_{3}$} & decyl 3-decyloxypropionate & & & & \\
\hline & $(453-523)$ & 90.2 & $(468)$ & A & {$[87 / 5]$} \\
\hline \multirow[t]{2}{*}{$\mathrm{C}_{23} \mathrm{H}_{48}$} & 9-hexylheptadecane & & & & {$[55124-79-3]$} \\
\hline & $(450-486)$ & 82.6 & $(465)$ & A & {$[87 / 5]$} \\
\hline $\mathrm{C}_{23} \mathrm{H}_{48}$ & 2-methyldocosane & & & & {$[1560-81-2]$} \\
\hline & $(495-652)$ & 79.7 & $(510)$ & A & {$[87 / 5]$} \\
\hline $\mathrm{C}_{23} \mathrm{H}_{48}$ & 4-methyldocosane & & & & {$[25117-30-0]$} \\
\hline & $(493-643)$ & 76.3 & $(508)$ & A & $\begin{array}{c}{[87 / 5][99 / 16]} \\
{[59 / 15]}\end{array}$ \\
\hline $\mathrm{C}_{23} \mathrm{H}_{48}$ & 5-methyldocosane & & & & {$[25163-52-4]$} \\
\hline & $(492-644)$ & 75.6 & $(507)$ & A & $\begin{array}{c}{[87 / 5][99 / 16]} \\
{[59 / 15]}\end{array}$ \\
\hline $\mathrm{C}_{23} \mathrm{H}_{48}$ & tricosane & & & & {$[638-67-5]$} \\
\hline & & $118.7 \pm 0.1$ & $(298)$ & GS & {$[01 / 1]$} \\
\hline & & $119.7 \pm 2.3$ & $(298)$ & CGC & {$[00 / 9]$} \\
\hline & & $120.5 \pm 2.0$ & $(298)$ & CGC & {$[91 / 17]$} \\
\hline & $(370-416)$ & $123 \pm 1$ & (393) & TE & {$[94 / 2]$} \\
\hline & $(370-490)$ & 92.0 & $(385)$ & TE, ME, GS & {$[91 / 9]$} \\
\hline & $(314-353)$ & 110.4 & $(329)$ & $\mathrm{A}$ & {$[87 / 5]$} \\
\hline & $(440-653)$ & 94.0 & $(455)$ & A, EST & {$[87 / 5][66 / 8]$} \\
\hline $\mathrm{C}_{23} \mathrm{H}_{48} \mathrm{~S}$ & 1-tricosanethiol & & & & {$[66375-01-7]$} \\
\hline & $(444-690)$ & 110.1 & $(459)$ & EST & {$[99 / 16]$} \\
\hline $\mathrm{C}_{24} \mathrm{H}_{12}$ & coronene & & & & [191-07-1] \\
\hline & & $148.0 \pm 0.5$ & $(298)$ & $\mathrm{CGC}$ & {$[02 / 44]$} \\
\hline & $(323-473)$ & 104.2 & $(398)$ & GC & {$[02 / 18]$} \\
\hline $\mathrm{C}_{24} \mathrm{H}_{18}$ & 1,3,5-triphenylbenzene & & & & {$[612-71-5]$} \\
\hline & & 140.0 & $(298)$ & CGC & {$[98 / 11]$} \\
\hline & $(500-735)$ & 77.5 & $(515)$ & A & {$[87 / 5]$} \\
\hline & $(454-500)$ & 118.0 & $(469)$ & A & {$[87 / 5][74 / 29]$} \\
\hline $\mathrm{C}_{24} \mathrm{H}_{20} \mathrm{O}_{6}$ & glycerol tribenzoate & & & & [614-33-5] \\
\hline
\end{tabular}


TABLE 6. Enthalpies of vaporization of organic compounds, 1880-2002-Continued

\begin{tabular}{|c|c|c|c|c|c|}
\hline Molecular formula & $\begin{array}{c}\text { Compound } \\
\text { (Temperature range/K) }\end{array}$ & $\begin{array}{c}\Delta_{\text {vap }} H_{m} \\
\left(\mathrm{~kJ} \mathrm{~mol}^{-1}\right)\end{array}$ & $\begin{array}{l}\text { Mean temperature } \\
\qquad\left(T_{m} / \mathrm{K}\right)\end{array}$ & Method & $\begin{array}{l}\text { CAS registry number } \\
\text { Reference }\end{array}$ \\
\hline & $(423-476)$ & 123.5 & $(438)$ & $\mathrm{A}, \mathrm{T}$ & {$[87 / 5][49 / 6]$} \\
\hline \multirow[t]{2}{*}{$\mathrm{C}_{24} \mathrm{H}_{27} \mathrm{NO}_{4}$} & \multicolumn{4}{|c|}{$b i s[\mathrm{~N}, \mathrm{~N}-(2$-hydroxy-3-phenoxy)propyl]phenylamine } & [3088-05-9] \\
\hline & $(388-423)$ & 131.0 & $(403)$ & A & {$[87 / 5]$} \\
\hline \multirow[t]{4}{*}{$\mathrm{C}_{24} \mathrm{H}_{30} \mathrm{O}_{4}$} & dibenzyl sebacate & & & & {$[140-24-9]$} \\
\hline & $(368-550)$ & 114.3 & (383) & A & {$[87 / 5]$} \\
\hline & $(405-463)$ & 112.2 & $(420)$ & $\mathrm{T}$ & {$[49 / 9]$} \\
\hline & $(373-432)$ & 121 & (388) & $\mathrm{T}$ & {$[39 / 3]$} \\
\hline \multirow{3}{*}{$\mathrm{C}_{24} \mathrm{H}_{38} \mathrm{O}_{4}$} & bis(2-ethylhexyl)phthalate & & & & {$[117-81-7]$} \\
\hline & $(373-660)$ & 102.5 & (388) & A & {$[87 / 5]$} \\
\hline & $(385-440)$ & 110.7 & $(390)$ & $\mathrm{T}$ & {$[49 / 9]$} \\
\hline \multirow[t]{2}{*}{$\mathrm{C}_{24} \mathrm{H}_{38} \mathrm{O}_{4}$} & bis(1-methylheptyl)phthalate & & & & {$[131-15-7]$} \\
\hline & $(393-435)$ & 93.1 & $(408)$ & A & {$[87 / 5]$} \\
\hline \multirow[t]{2}{*}{$\mathrm{C}_{24} \mathrm{H}_{38} \mathrm{O}_{4}$} & bis(6-methylheptyl)phthalate & & & & [131-20-4] \\
\hline & $(383-490)$ & 92.4 & $(398)$ & A & {$[87 / 5]$} \\
\hline \multirow[t]{3}{*}{$\mathrm{C}_{24} \mathrm{H}_{38} \mathrm{O}_{4}$} & dioctyl phthalate & & & & {$[117-84-0]$} \\
\hline & $(423-523)$ & 99.5 & $(438)$ & A & {$[87 / 5]$} \\
\hline & $(383-433)$ & 107.6 & $(398)$ & $\mathrm{T}$ & [49/9] \\
\hline \multirow[t]{2}{*}{$\mathrm{C}_{24} \mathrm{H}_{42}$} & hexapropylbenzene & & & & {$[2456-68-0]$} \\
\hline & $(458-606)$ & 68.4 & $(473)$ & A & {$[87 / 5][37 / 8]$} \\
\hline \multirow[t]{2}{*}{$\mathrm{C}_{24} \mathrm{H}_{42}$} & octadecylbenzene & & & & {$[4445-07-2]$} \\
\hline & $(423-675)$ & 101.0 & (438) & & {$[99 / 16]$} \\
\hline \multirow[t]{2}{*}{$\mathrm{C}_{24} \mathrm{H}_{42} \mathrm{O}_{6}$} & trans trihexyl aconitate & & & & [64617-30-7] \\
\hline & $(423-512)$ & 98.2 & $(438)$ & $\mathrm{A}$ & {$[87 / 5]$} \\
\hline $\mathrm{C}_{24} \mathrm{H}_{42} \mathrm{O}_{11}$ & \multicolumn{5}{|c|}{ di[1-(2-ethylbutyloxycarbonyl)ethyl] diethylene glycol dicarboxylate } \\
\hline \multirow{2}{*}{$\mathrm{C}_{24} \mathrm{H}_{42} \mathrm{O}_{11}$} & \multicolumn{5}{|c|}{ di[1-(2-hexyloxycarbonyl)ethyl] diethylene glycol dicarboxylate } \\
\hline & $(443-548)$ & 111.0 & (458) & A & {$[87 / 5]$} \\
\hline $\mathrm{C}_{24} \mathrm{H}_{44}$ & $\begin{array}{l}\text { 9-decyltetradecahydroanthracene } \\
(501-536)\end{array}$ & 103.2 & $(516)$ & A & {$[87 / 5]$} \\
\hline $\mathrm{C}_{24} \mathrm{H}_{44}$ & $\begin{array}{l}\text { 9-decyltetradecahydrophenanthrene } \\
(502-542)\end{array}$ & 92.0 & $(517)$ & A & {$[87 / 5]$} \\
\hline $\mathrm{C}_{24} \mathrm{H}_{44} \mathrm{O}_{6}$ & $\begin{array}{l}\text { O-acetylricinoleic acid, butyl ester } \\
(378-423)\end{array}$ & 105.2 & (393) & A & $\begin{array}{c}{[140-04-5]} \\
{[87 / 5]}\end{array}$ \\
\hline $\mathrm{C}_{24} \mathrm{H}_{44} \mathrm{O}_{6}$ & trihexyl 1,2,3-propanetricarboxylate & & & & [38094-13-2] \\
\hline & $(422-526)$ & 98.1 & $(437)$ & A & {$[87 / 5]$} \\
\hline $\mathrm{C}_{24} \mathrm{H}_{44} \mathrm{O}_{6}$ & $\begin{array}{l}\text { glycerol triheptanoate } \\
(401-452)\end{array}$ & 84.4 & (416) & & {$[01 / 10]$} \\
\hline $\mathrm{C}_{24} \mathrm{H}_{46} \mathrm{O}_{4}$ & bis(3,5,5-trimethylhexyl)adipate & & & & {$[20270-50-2]$} \\
\hline & $(353-413)$ & 107.6 & $(368)$ & $\mathrm{A}, \mathrm{ME}$ & {$[87 / 5][48 / 10]$} \\
\hline $\mathrm{C}_{24} \mathrm{H}_{48}$ & octadecylcyclohexane & & & & [4445-06-1] \\
\hline & $(422-675)$ & 100.3 & $(437)$ & & [99/16] \\
\hline $\mathrm{C}_{24} \mathrm{H}_{48}$ & 1-tetracosene & & & & [10192-32-2] \\
\hline & $(418-663)$ & 101.0 & (433) & & [99/16] \\
\hline $\mathrm{C}_{24} \mathrm{H}_{48} \mathrm{O}_{2}$ & ethyl docosanoate & & & & [5908-87-2] \\
\hline & $(327-344)$ & 127.5 & (335) & $\mathrm{A}, \mathrm{ME}$ & {$[87 / 5][67 / 12]$} \\
\hline $\mathrm{C}_{24} \mathrm{H}_{48} \mathrm{O}_{2}$ & methyl tricosanoate & & & & [2433-97-8] \\
\hline & $(473-528)$ & 99.8 & $(488)$ & A, EST & {$[87 / 5][63 / 16]$} \\
\hline $\mathrm{C}_{24} \mathrm{H}_{49} \mathrm{Cl}$ & 1-chlorotetracosane & & & & {$[6422-18-0]$} \\
\hline & $(543-774)$ & 72.4 & $(558)$ & $\mathrm{A}$ & {$[87 / 5][70 / 14]$} \\
\hline $\mathrm{C}_{24} \mathrm{H}_{50}$ & 2-methyltricosane & & & & [1928-30-9] \\
\hline & $(450-664)$ & 89.3 & $(465)$ & $\mathrm{A}$ & {$[87 / 5]$} \\
\hline $\mathrm{C}_{24} \mathrm{H}_{50}$ & 5-methyltricosane & & & & [22331-09-5] \\
\hline & $(503-653)$ & 79.6 & $(518)$ & $\mathrm{A}$ & $\begin{array}{c}{[87 / 5][99 / 16]} \\
{[59 / 15]}\end{array}$ \\
\hline $\mathrm{C}_{24} \mathrm{H}_{50}$ & tetracosane & & & & [646-31-1] \\
\hline & & $126.8 \pm 0.4$ & $(298)$ & CGC & {$[02 / 44]$} \\
\hline & & $125.7 \pm 1.6$ & (298) & CGC & {$[00 / 9]$} \\
\hline & & $126.2 \pm 2.3$ & (298) & $\mathrm{CGC}$ & {$[97 / 17]$} \\
\hline & $(453-588)$ & 92.6 & $(468)$ & & [94/14] \\
\hline & $(386-425)$ & $126 \pm 2$ & (405) & TE & {$[94 / 2]$} \\
\hline & $(382-523)$ & 95.2 & (397) & TE, ME, GS & {$[91 / 9]$} \\
\hline & $(451-497)$ & $86.2 \pm 4.6$ & (474) & GS & {$[90 / 14]$} \\
\hline & $(373-463)$ & 111.2 & (388) & & [88/12] \\
\hline & $(498-573)$ & 86.6 & (513) & $\mathrm{A}, \mathrm{EST}$ & {$[87 / 5][66 / 8]$} \\
\hline
\end{tabular}


TABLE 6. Enthalpies of vaporization of organic compounds, 1880-2002-Continued

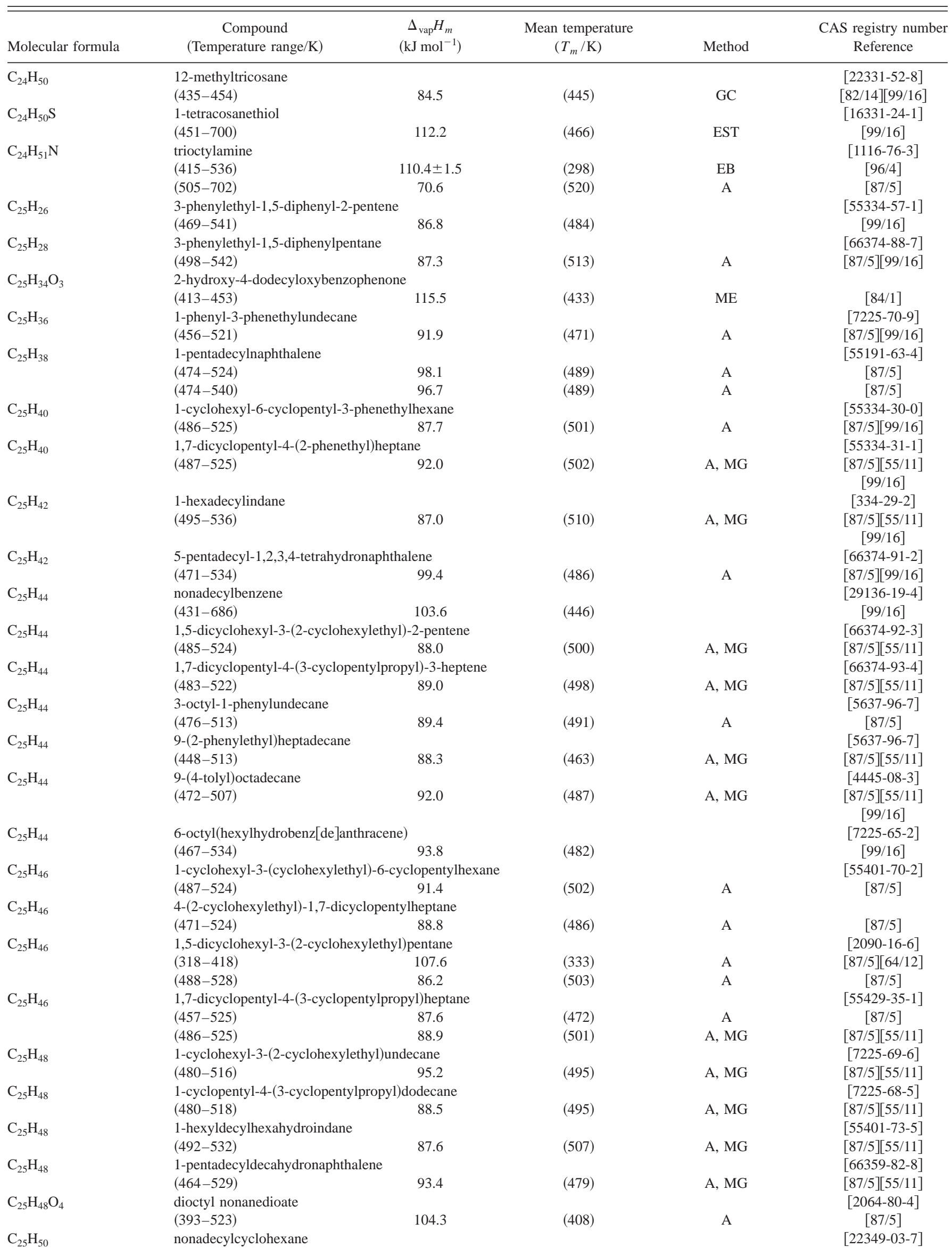


TABLE 6. Enthalpies of vaporization of organic compounds, 1880-2002-Continued

\begin{tabular}{|c|c|c|c|c|c|}
\hline Molecular formula & $\begin{array}{c}\text { Compound } \\
\text { (Temperature range/K) }\end{array}$ & $\begin{array}{c}\Delta_{\mathrm{vap}} H_{m} \\
\left(\mathrm{~kJ} \mathrm{~mol}^{-1}\right)\end{array}$ & $\begin{array}{c}\text { Mean temperature } \\
\qquad\left(T_{m} / \mathrm{K}\right)\end{array}$ & Method & $\begin{array}{c}\text { CAS registry number } \\
\text { Reference }\end{array}$ \\
\hline & $(430-686)$ & 102.8 & $(445)$ & & [99/16] \\
\hline \multirow[t]{2}{*}{$\mathrm{C}_{25} \mathrm{H}_{50}$} & 1-pentacosene & & & & {$[16980-85-1]$} \\
\hline & $(426-674)$ & 103.7 & $(441)$ & & {$[99 / 16]$} \\
\hline \multirow[t]{2}{*}{$\mathrm{C}_{25} \mathrm{H}_{50}$} & 9-(2-cyclohexylethyl)heptadecane & & & & [25446-35-9] \\
\hline & $(490-513)$ & 88.6 & $(495)$ & $\mathrm{A}, \mathrm{MG}$ & {$[87 / 5][55 / 11]$} \\
\hline \multirow[t]{2}{*}{$\mathrm{C}_{25} \mathrm{H}_{50}$} & 9-(3-cyclopentylpropyl)heptadecane & & & & {$[5638-09-5]$} \\
\hline & $(476-514)$ & 86.9 & $(491)$ & A & {$[87 / 5][99 / 16]$} \\
\hline \multirow[t]{2}{*}{$\mathrm{C}_{25} \mathrm{H}_{50}$} & 9-octyl-8-heptadecene & & & & [24306-18-1] \\
\hline & $(441-500)$ & 92.3 & $(456)$ & A & {$[87 / 5][99 / 16]$} \\
\hline \multirow[t]{3}{*}{$\mathrm{C}_{25} \mathrm{H}_{50} \mathrm{O}_{2}$} & methyl tetracosanoate & & & & {$[2442-49-1]$} \\
\hline & $(422-452)$ & 146.2 & $(437)$ & & {$[01 / 10]$} \\
\hline & $(483-536)$ & 100.8 & $(498)$ & A & {$[87 / 5]$} \\
\hline \multirow[t]{2}{*}{$\mathrm{C}_{25} \mathrm{H}_{50} \mathrm{O}_{2}$} & ethyl tricosanoate & & & & {$[18281-07-7]$} \\
\hline & $(336-359)$ & 121.8 & $(347)$ & $\mathrm{A}, \mathrm{ME}$ & {$[87 / 5][67 / 12]$} \\
\hline \multirow[t]{2}{*}{$\mathrm{C}_{25} \mathrm{H}_{52}$} & 9-octylheptadecane & & & & {$[7225-64-1]$} \\
\hline & $(470-505)$ & 93.4 & $(485)$ & $\mathrm{A}, \mathrm{MG}$ & {$[87 / 5][55 / 11]$} \\
\hline \multirow[t]{8}{*}{$\mathrm{C}_{25} \mathrm{H}_{52}$} & pentacosane & & & & [629-99-2] \\
\hline & & $128.6 \pm 2.2$ & (298) & GS & {$[01 / 1]$} \\
\hline & & $127.6 \pm 0.8$ & (298) & $\mathrm{CGC}$ & {$[00 / 9]$} \\
\hline & & $129.8 \pm 2.9$ & (298) & CGC & {$[97 / 17]$} \\
\hline & $(397-434)$ & $126 \pm 1$ & (415) & TE & {$[94 / 2]$} \\
\hline & $(390-531)$ & 97.6 & (405) & TE, ME, GS & {$[91 / 9]$} \\
\hline & $(461-498)$ & $90.9 \pm 5.7$ & $(479)$ & GS & {$[90 / 14]$} \\
\hline & $(457-675)$ & 99.2 & $(472)$ & $\mathrm{A}, \mathrm{EST}$ & {$[87 / 5][66 / 8]$} \\
\hline \multirow[t]{2}{*}{$\mathrm{C}_{25} \mathrm{H}_{52}$} & 12-ethyltricosane & & & & {$[79370-85-7]$} \\
\hline & $(435-454)$ & 84.6 & $(444)$ & $\mathrm{GC}$ & {$[82 / 14][99 / 16]$} \\
\hline \multirow[t]{2}{*}{$\mathrm{C}_{25} \mathrm{H}_{52}$} & 2-methyltetracosane & & & & {$[1560-78-7]$} \\
\hline & $(425-670)$ & 104.6 & $(440)$ & & {$[99 / 16]$} \\
\hline \multirow[t]{2}{*}{$\mathrm{C}_{25} \mathrm{H}_{52}$} & 5,5-bis (3,3'-dimethylbutyl)-2,2,8,8-tet & tramethylno & & & \\
\hline & & $91.9 \pm 1.8$ & $(298)$ & CGC & [95/27] \\
\hline \multirow[t]{2}{*}{$\mathrm{C}_{25} \mathrm{H}_{52}$} & 7,7-dihexyltridecane & & & & \\
\hline & & $115.3 \pm 1.8$ & $(298)$ & CGC & {$[95 / 27]$} \\
\hline \multirow[t]{2}{*}{$\mathrm{C}_{25} \mathrm{H}_{52} \mathrm{~S}$} & 1-pentacosanethiol & & & & {$[66359-74-8]$} \\
\hline & $(458-709)$ & 114.2 & $(473)$ & EST & {$[99 / 16]$} \\
\hline \multirow[t]{2}{*}{$\mathrm{C}_{26} \mathrm{H}_{18}$} & 9,10-diphenylanthracene & & & & [1499-10-1] \\
\hline & $(323-473)$ & 102.7 & (398) & GC & {$[02 / 18]$} \\
\hline \multirow[t]{2}{*}{$\mathrm{C}_{26} \mathrm{H}_{18}$} & 9,9'-bifluorenyl & & & & {$[1530-12-7]$} \\
\hline & $(383-408)$ & 95.7 & & B & [94/4] \\
\hline $\mathrm{C}_{26} \mathrm{H}_{18} \mathrm{~N}_{2} \mathrm{O}_{4}$ & disperse violet 31 & & & & \\
\hline & $(453-523)$ & 59.9 & $(468)$ & A & {$[87 / 5]$} \\
\hline $\mathrm{C}_{26} \mathrm{H}_{32}$ & 6-octyl-1,2,3,4-tetrahydronaphthacene & 1032 & $(518)$ & $A$ & $\lceil 8715]$ \\
\hline $\mathrm{C}_{26} \mathrm{H}_{34}$ & 9-dodecylanthracene & 103.2 & (518) & A & {$[2883-70-7]$} \\
\hline & $(495-566)$ & 99.4 & $(510)$ & A & {$[87 / 5]$} \\
\hline $\mathrm{C}_{26} \mathrm{H}_{34}$ & 9-dodecylphenathrene & & & & {$[3788-61-2]$} \\
\hline & $(495-568)$ & 95.7 & $(510)$ & A & {$[87 / 5]$} \\
\hline $\mathrm{C}_{26} \mathrm{H}_{38}$ & 1,1-diphenyltetradecane & & & & {$[55268-63-8]$} \\
\hline & $(467-530)$ & 98.2 & $(482)$ & A & {$[87 / 5]$} \\
\hline $\mathrm{C}_{26} \mathrm{H}_{38}$ & 1,1-di(4-tolyl)dodecane & & & & {$[55268-62-7]$} \\
\hline & $(466-529)$ & 98.3 & $(481)$ & A & {$[87 / 5]$} \\
\hline $\mathrm{C}_{26} \mathrm{H}_{40}$ & 5-octyl-1,2,3,4,4a,5,7,8,9,10,12,12a-do & lodecahydro & cene & & {$[95258-25-6]$} \\
\hline & $(479-549)$ & 91.9 & $(494)$ & A, MG & $\begin{array}{c}{[87 / 5][55 / 11]} \\
{[99 / 16]}\end{array}$ \\
\hline $\mathrm{C}_{26} \mathrm{H}_{42}$ & 1,1-bis(dodecahydroacenaphthylene-5 & 5-yl)ethane & & & \\
\hline & $(482-541)$ & 110.9 & (497) & $\mathrm{A}, \mathrm{MG}$ & {$[87 / 5][55 / 11]$} \\
\hline $\mathrm{C}_{26} \mathrm{H}_{42} \mathrm{O}_{4}$ & bis(3,5,5-trimethylhexyl)phthalate & & & & [14103-61-8] \\
\hline & $(333-393)$ & 113.6 & $(348)$ & $\mathrm{A}$ & {$[87 / 5]$} \\
\hline $\mathrm{C}_{26} \mathrm{H}_{42} \mathrm{O}_{4}$ & dinonyl phthalate & & & & {$[84-76-4]$} \\
\hline & $(333-393)$ & 108.9 & $(348)$ & A & {$[87 / 5]$} \\
\hline $\mathrm{C}_{26} \mathrm{H}_{46}$ & 1,4-didecylbenzene & & & & {$[2655-95-0]$} \\
\hline & $(468-536)$ & 95.2 & $(483)$ & A & {$[87 / 5]$} \\
\hline $\mathrm{C}_{26} \mathrm{H}_{46}$ & 1-phenyleicosane & & & & [2398-68-7] \\
\hline & $(499-538)$ & 94.7 & $(514)$ & $\mathrm{A}, \mathrm{MG}$ & {$[87 / 5][55 / 11]$} \\
\hline $\mathrm{C}_{26} \mathrm{H}_{46}$ & 2-phenyleicosane & & & & {$[2398-66-5]$} \\
\hline
\end{tabular}


TABLE 6. Enthalpies of vaporization of organic compounds, 1880-2002-Continued

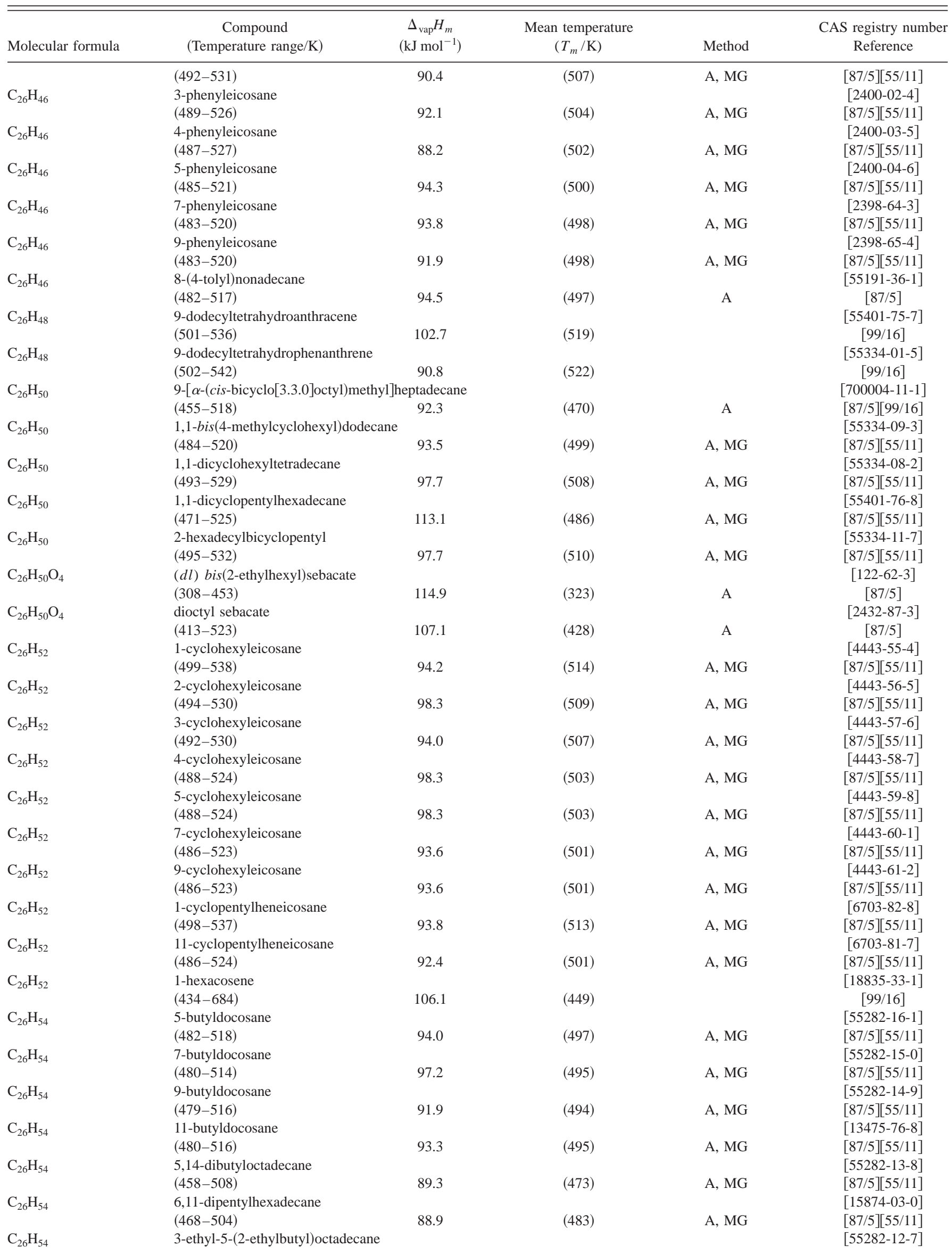


TABLE 6. Enthalpies of vaporization of organic compounds, 1880-2002-Continued

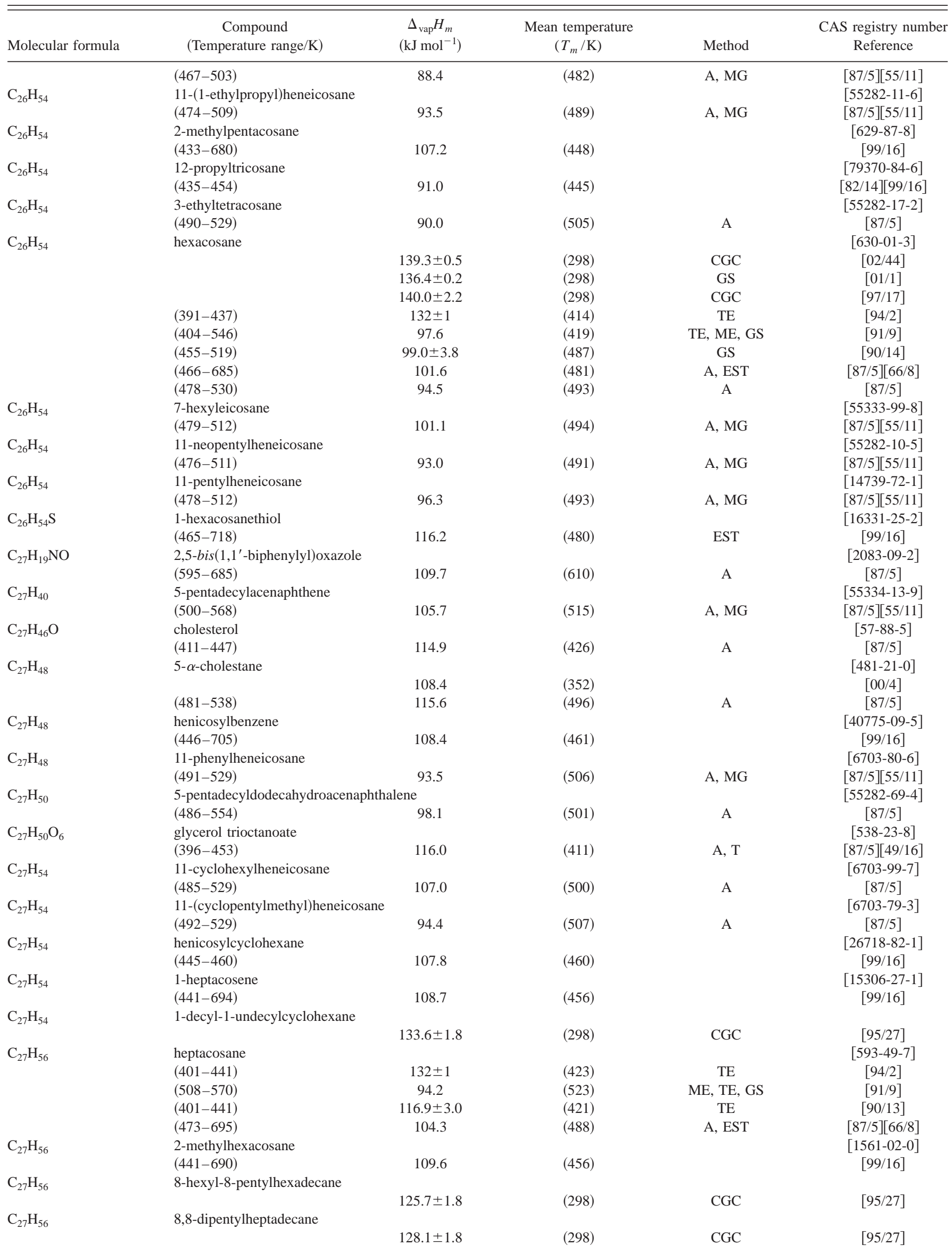


TABLE 6. Enthalpies of vaporization of organic compounds, 1880-2002-Continued

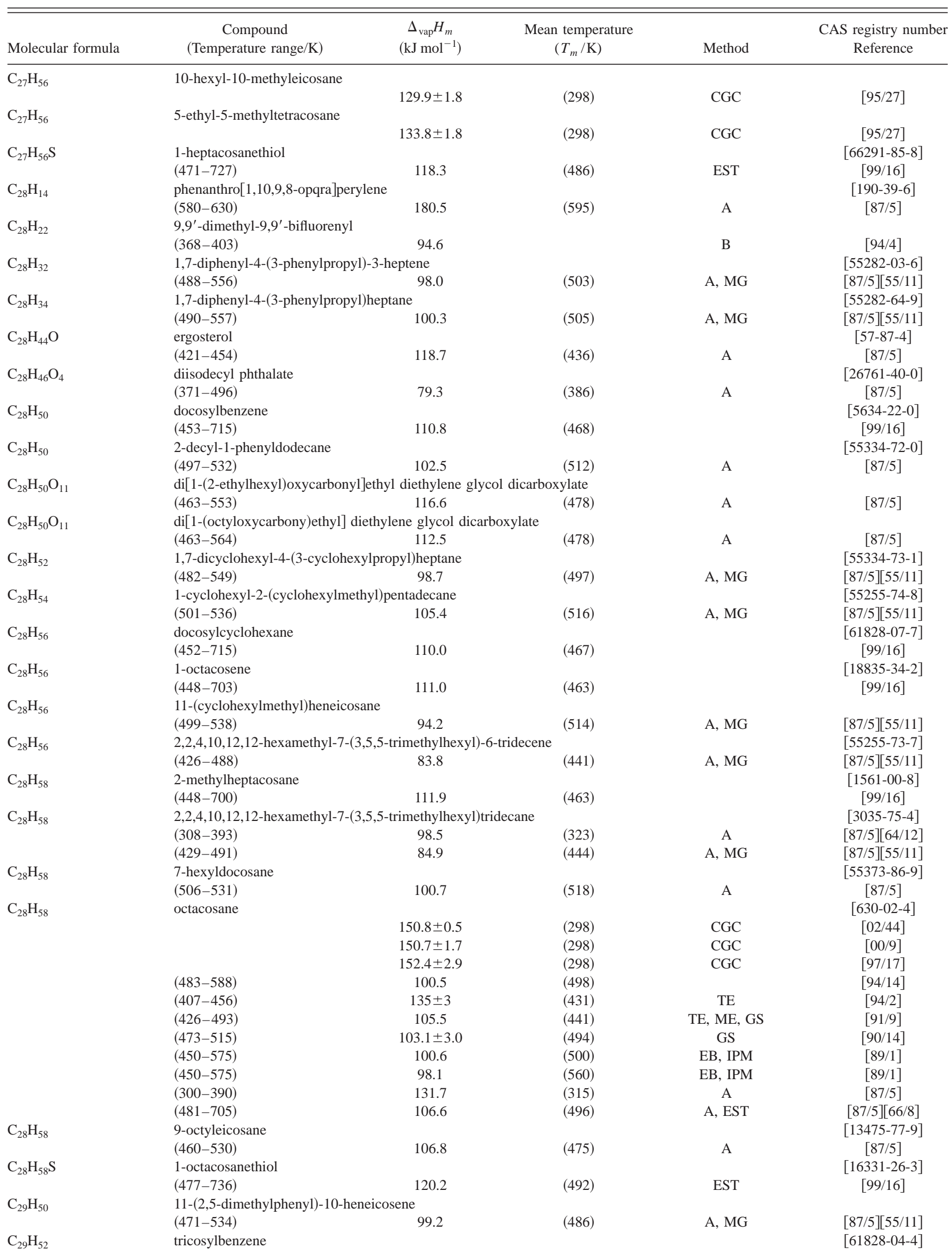


TABLE 6. Enthalpies of vaporization of organic compounds, 1880-2002-Continued

\begin{tabular}{|c|c|c|c|c|c|}
\hline Molecular formula & $\begin{array}{c}\text { Compound } \\
\text { (Temperature range/K) }\end{array}$ & $\begin{array}{c}\Delta_{\mathrm{vap}} H_{m} \\
\left(\mathrm{~kJ} \mathrm{~mol}^{-1}\right)\end{array}$ & $\begin{array}{l}\text { Mean temperature } \\
\qquad\left(T_{m} / \mathrm{K}\right)\end{array}$ & Method & $\begin{array}{c}\text { CAS registry number } \\
\text { Reference }\end{array}$ \\
\hline & $(459-724)$ & 113.2 & $(474)$ & & [99/16] \\
\hline \multirow[t]{2}{*}{$\mathrm{C}_{29} \mathrm{H}_{52}$} & 1-nonacosene & & & & {$[18835-35-3]$} \\
\hline & $(455-713)$ & 113.3 & $(470)$ & & {$[99 / 16]$} \\
\hline \multirow[t]{2}{*}{$\mathrm{C}_{29} \mathrm{H}_{52}$} & 11-(2,5-dimethylphenyl)heneicosane & & & & {$[55373-91-6]$} \\
\hline & $(472-535)$ & 100.8 & $(487)$ & $\mathrm{A}, \mathrm{MG}$ & {$[87 / 5][55 / 11]$} \\
\hline \multirow[t]{2}{*}{$\mathrm{C}_{29} \mathrm{H}_{58}$} & tricosylcyclohexane & & & & [61828-08-8] \\
\hline & $(459-724)$ & 112.3 & $(474)$ & & {$[99 / 16]$} \\
\hline $\mathrm{C}_{29} \mathrm{H}_{60}$ & $\begin{array}{l}\text { 2-methyloctacosane } \\
(455-709)\end{array}$ & 114.2 & (470) & & $\begin{array}{c}{[1560-98-1]} \\
{[99 / 16]}\end{array}$ \\
\hline \multirow{4}{*}{$\mathrm{C}_{29} \mathrm{H}_{60}$} & nonacosane & & & & {$[630-03-5]$} \\
\hline & $(423-457)$ & $137 \pm 3$ & $(440)$ & $\mathrm{TE}$ & {$[94 / 2]$} \\
\hline & $(423-456)$ & $137.1 \pm 3.0$ & (439) & $\mathrm{TE}$ & {$[90 / 13]$} \\
\hline & $(488-714)$ & 109.0 & (503) & A, EST & {$[87 / 5][66 / 8]$} \\
\hline \multirow[t]{2}{*}{$\mathrm{C}_{29} \mathrm{H}_{60} \mathrm{~S}$} & 1-nonacosanethiol & & & & [66213-92-1] \\
\hline & $(483-744)$ & 122.0 & (498) & EST & {$[99 / 16]$} \\
\hline \multirow[t]{2}{*}{$\mathrm{C}_{30} \mathrm{H}_{30}$} & 1,1,6,6-tetraphenylhexane & & & & [2819-41-2] \\
\hline & $(511-579)$ & 108.1 & $(526)$ & A, MG & {$[87 / 5][55 / 11]$} \\
\hline \multirow[t]{2}{*}{$\mathrm{C}_{30} \mathrm{H}_{34}$} & 1,10-di(1-naphthyl)decane & & & & [40339-27-3] \\
\hline & $(540-616)$ & 108.6 & $(555)$ & $\mathrm{A}, \mathrm{MG}$ & {$[87 / 5][55 / 11]$} \\
\hline \multirow{2}{*}{$\mathrm{C}_{30} \mathrm{H}_{54}$} & 1,10-bis(decahydro-1-naphthyl)decane & & & & [55268-64-9] \\
\hline & $(520-583)$ & 119.7 & $(535)$ & A, MG & {$[87 / 5][55 / 11]$} \\
\hline \multirow[t]{2}{*}{$\mathrm{C}_{30} \mathrm{H}_{54}$} & 1,1,6,6-tetracyclohexylhexane & & & & [55281-91-9] \\
\hline & $(501-569)$ & 103.0 & $(516)$ & A & {$[87 / 5]$} \\
\hline \multirow[t]{2}{*}{$\mathrm{C}_{30} \mathrm{H}_{54}$} & tetracosylbenzene & & & & {$[61828-05-5]$} \\
\hline & $(466-732)$ & 115.3 & $(481)$ & & {$[99 / 16]$} \\
\hline \multirow[t]{2}{*}{$\mathrm{C}_{30} \mathrm{H}_{54} \mathrm{O}_{6}$} & trans, tris(2-ethylhexyl) aconitate & & & & [52193-50-7] \\
\hline & $(437-551)$ & 97.1 & $(452)$ & A & {$[87 / 5]$} \\
\hline \multirow[t]{2}{*}{$\mathrm{C}_{30} \mathrm{H}_{54} \mathrm{O}_{6}$} & tris(2-ethylhexyl) 1,2,3-propanetricarb & boxylate & & & [5400-99-7] \\
\hline & $(438-551)$ & 97.9 & $(453)$ & $\mathrm{A}$ & {$[87 / 5]$} \\
\hline \multirow[t]{2}{*}{$\mathrm{C}_{30} \mathrm{H}_{60}$} & tetracosylcyclohexane & & & & [61828-09-9] \\
\hline & $(465-733)$ & 114.6 & (480) & & {$[99 / 16]$} \\
\hline $\mathrm{C}_{30} \mathrm{H}_{60}$ & 1-tricontene & & & & {$[18435-53-5]$} \\
\hline$-30^{11} 60$ & $(462-721)$ & 115.4 & $(477)$ & & {$[99 / 16]$} \\
\hline $\mathrm{C}_{30} \mathrm{H}_{62}$ & $2,6,10,15,19,23$-hexamethyltetracosan & ne (squalane) & & & [111-01-3] \\
\hline & $(363-513)$ & 116.2 & (378) & $\mathrm{A}$ & {$[87 / 5]$} \\
\hline $\mathrm{C}_{30} \mathrm{H}_{62}$ & 9-octyldocosane & & & & {$[55319-83-0]$} \\
\hline & $(518-588)$ & 109.3 & $(533)$ & A & {$[87 / 5]$} \\
\hline $\mathrm{C}_{30} \mathrm{H}_{62}$ & triacontane & & & & {$[638-68-6]$} \\
\hline & & $164.5 \pm 0.4$ & (298) & CGC & {$[00 / 9]$} \\
\hline & $(422-487)$ & $143 \pm 2$ & (454) & TE & [94/2] \\
\hline & $(495-723)$ & 111.3 & $(510)$ & A, EST & {$[87 / 5][66 / 8]$} \\
\hline $\mathrm{C}_{30} \mathrm{H}_{62}$ & 2-methylnonacosane & & & & {$[1560-75-4]$} \\
\hline & $(461-718)$ & 116.8 & $(476)$ & & {$[99 / 16]$} \\
\hline $\mathrm{C}_{30} \mathrm{H}_{62} \mathrm{~S}$ & 1-triacontanethiol & & & & [66213-99-8] \\
\hline & $(488-751)$ & 124.0 & (503) & EST & {$[99 / 16]$} \\
\hline $\mathrm{C}_{30} \mathrm{H}_{63} \mathrm{~N}$ & tridecylamine & & & & {$[2869-34-3]$} \\
\hline & $(545-759)$ & 76.8 & $(560)$ & A & {$[87 / 5]$} \\
\hline $\mathrm{C}_{31} \mathrm{H}_{34}$ & 1,1-di(1-naphthyl)-1-undecene & & & & {$[56247-76-8]$} \\
\hline & $(518-588)$ & 109.3 & (533) & A & {$[87 / 5]$} \\
\hline $\mathrm{C}_{31} \mathrm{H}_{48}$ & 1-(1-decylundec-1-enyl)naphthalene & & & & [55319-81-8] \\
\hline & $(499-567)$ & 105.1 & (514) & $\mathrm{A}, \mathrm{MG}$ & {$[87 / 5][55 / 11]$} \\
\hline $\mathrm{C}_{31} \mathrm{H}_{52} \mathrm{O}_{3}$ & $\alpha$-tocopherol acetate & & & & {$[58-95-7]$} \\
\hline & $(466-524)$ & $60.1 \pm 1.3$ & (496) & & {$[88 / 4]$} \\
\hline $\mathrm{C}_{31} \mathrm{H}_{56}$ & 1,1-bis(decahydro-1-naphthyl)undecar & & & & [55373-96-1] \\
\hline & $(525-561)$ & 110.5 & $(540)$ & A, MG & {$[87 / 5][55 / 11]$} \\
\hline $\mathrm{C}_{31} \mathrm{H}_{56}$ & pentacosylbenzene & & & & [61828-06-6] \\
\hline & $(472-741)$ & 117.5 & $(487)$ & & {$[99 / 16]$} \\
\hline $\mathrm{C}_{31} \mathrm{H}_{56}$ & 13-phenylpentacosane & & & & [6006-90-2] \\
\hline & $(495-560)$ & 106.7 & $(510)$ & $\mathrm{A}, \mathrm{MG}$ & {$[87 / 5][55 / 11]$} \\
\hline $\mathrm{C}_{31} \mathrm{H}_{60}$ & 1-(1-decylundecyl)decahydronaphthal & lene & & & [55320-00-8] \\
\hline & $(523-560)$ & 107.0 & $(538)$ & $\mathrm{A}, \mathrm{MG}$ & {$[87 / 5][55 / 11]$} \\
\hline $\mathrm{C}_{31} \mathrm{H}_{62}$ & 1-hentriacontene & & & & [18435-54-6] \\
\hline & $(468-730)$ & 117.7 & $(483)$ & & {$[99 / 16]$} \\
\hline $\mathrm{C}_{31} \mathrm{H}_{62}$ & pentacosylcyclohexane & & & & [61828-10-2] \\
\hline
\end{tabular}


TABLE 6. Enthalpies of vaporization of organic compounds, 1880-2002-Continued

\begin{tabular}{|c|c|c|c|c|c|}
\hline Molecular formula & $\begin{array}{c}\text { Compound } \\
\text { (Temperature range/K) }\end{array}$ & $\begin{array}{c}\Delta_{\mathrm{vap}} H_{m} \\
\left(\mathrm{~kJ} \mathrm{~mol}^{-1}\right)\end{array}$ & $\begin{array}{l}\text { Mean temperature } \\
\qquad\left(T_{m} / \mathrm{K}\right)\end{array}$ & Method & $\begin{array}{l}\text { CAS registry number } \\
\text { Reference }\end{array}$ \\
\hline & $(472-741)$ & 116.6 & $(487)$ & & [99/16] \\
\hline \multirow[t]{2}{*}{$\mathrm{C}_{31} \mathrm{H}_{62}$} & 13-cyclohexylpentaconsane & & & & [6697-15-0] \\
\hline & $(495-560)$ & 106.7 & $(510)$ & $\mathrm{A}, \mathrm{MG}$ & {$[87 / 5][55 / 11]$} \\
\hline $\mathrm{C}_{31} \mathrm{H}_{64}$ & $\begin{array}{l}\text { 2-methyltriacontane } \\
(468-726)\end{array}$ & 118.8 & $(483)$ & & $\begin{array}{c}{[1560-72-1]} \\
{[99 / 16]}\end{array}$ \\
\hline \multirow[t]{2}{*}{$\mathrm{C}_{31} \mathrm{H}_{64}$} & 11-decylheneicosane & & & & [55320-06-4] \\
\hline & $(298-313)$ & 110.9 & $(305)$ & $\mathrm{A}, \mathrm{MG}$ & {$[87 / 5][55 / 11]$} \\
\hline \multirow[t]{3}{*}{$\mathrm{C}_{31} \mathrm{H}_{64}$} & hentriacontane & & & & [630-04-6] \\
\hline & $(433-474)$ & $146 \pm 2$ & $(450)$ & TE & [94/2] \\
\hline & $(503-732)$ & 113.8 & $(518)$ & A, EST & {$[87 / 5][66 / 8]$} \\
\hline \multirow[t]{2}{*}{$\mathrm{C}_{31} \mathrm{H}_{64} \mathrm{~S}$} & 1-hentriacontanethiol & & & & {$[534-24-9]$} \\
\hline & (494-759) & 125.7 & $(509)$ & EST & {$[99 / 16]$} \\
\hline \multirow[t]{2}{*}{$\mathrm{C}_{32} \mathrm{H}_{58}$} & hexacosylbenzene & & & & [13024-80-1] \\
\hline & $(478-749)$ & 119.6 & $(493)$ & & {$[99 / 16]$} \\
\hline $\mathrm{C}_{32} \mathrm{H}_{64}$ & $\begin{array}{l}\text { 1-dotriacontene } \\
(474-738)\end{array}$ & 119.8 & $(489)$ & & $\begin{array}{c}{[18435-55-7]} \\
{[99 / 16]}\end{array}$ \\
\hline $\mathrm{C}_{32} \mathrm{H}_{64}$ & $\begin{array}{l}\text { hexacosylcyclohexane } \\
(478-749)\end{array}$ & 118.6 & (493) & & $\begin{array}{c}{[61828-11-3]} \\
{[99 / 16]}\end{array}$ \\
\hline \multirow[t]{2}{*}{$\mathrm{C}_{32} \mathrm{H}_{66}$} & 11-decyldocosane & & & & [55401-55-3] \\
\hline & $(523-559)$ & 108.7 & $(538)$ & $\mathrm{A}, \mathrm{MG}$ & {$[87 / 5][55 / 11]$} \\
\hline \multirow[t]{4}{*}{$\mathrm{C}_{32} \mathrm{H}_{66}$} & dotriacontane & & & & {$[544-85-4]$} \\
\hline & $(437-477)$ & $147 \pm 1$ & (456) & $\mathrm{TE}$ & [94/2] \\
\hline & $(361-395)$ & 130.5 & (376) & A & {$[87 / 5]$} \\
\hline & $(510-741)$ & 116.0 & $(535)$ & $\mathrm{A}, \mathrm{EST}$ & {$[87 / 5][66 / 8]$} \\
\hline \multirow[t]{2}{*}{$\mathrm{C}_{32} \mathrm{H}_{66}$} & 9-octyltetracosane & & & & [55401-54-2] \\
\hline & $(501-563)$ & 114.8 & $(516)$ & $\mathrm{A}, \mathrm{MG}$ & {$[87 / 5][55 / 11]$} \\
\hline \multirow[t]{2}{*}{$\mathrm{C}_{32} \mathrm{H}_{66}$} & 2-methylhentriacontane & & & & {$[1720-12-3]$} \\
\hline & $(474-735)$ & 120.9 & $(489)$ & & [99/16] \\
\hline $\mathrm{C}_{32} \mathrm{H}_{66} \mathrm{~S}$ & $\begin{array}{l}\text { 1-dotriacontanethiol } \\
(499-766)\end{array}$ & 127.5 & (514) & EST & $\begin{array}{c}{[66256-05-1]} \\
{[99 / 16]}\end{array}$ \\
\hline $\mathrm{C}_{33} \mathrm{H}_{54} \mathrm{O}_{6}$ & $\begin{array}{l}\text { tri(2-ethylhexyl)trimellitate } \\
(331-371)\end{array}$ & 81.1 & $(346)$ & ME & {$[00 / 11]$} \\
\hline $\mathrm{C}_{33} \mathrm{H}_{54} \mathrm{O}_{6}$ & $\begin{array}{l}\text { triisooctyltrimellitate } \\
(331-372)\end{array}$ & 79.0 & (346) & ME & {$[00 / 11]$} \\
\hline $\mathrm{C}_{33} \mathrm{H}_{60}$ & heptacosylbenzene & 1215 & (499) & & {$[61828-25-9]$} \\
\hline \multirow[t]{2}{*}{$\mathrm{C}_{33} \mathrm{H}_{62} \mathrm{O}_{6}$} & glycerol tricaprate & & & & [621-71-6] \\
\hline & $(437-485)$ & 124.6 & $(452)$ & $\mathrm{A}$ & {$[87 / 5]$} \\
\hline \multirow[t]{2}{*}{$\mathrm{C}_{33} \mathrm{H}_{66}$} & heptacosylcyclohexane & & & & [61828-12-4] \\
\hline & $(484-757)$ & 120.6 & (499) & & {$[99 / 16]$} \\
\hline $\mathrm{C}_{33} \mathrm{H}_{66}$ & 1-tritriacontene & & & & [61868-11-9] \\
\hline & $(480-746)$ & 121.8 & $(495)$ & & {$[99 / 16]$} \\
\hline $\mathrm{C}_{33} \mathrm{H}_{68}$ & tritriacontane & & & & {$[630-05-7]$} \\
\hline & $(438-480)$ & $148 \pm 1$ & $(458)$ & TE & {$[94 / 2]$} \\
\hline & $(517-749)$ & 118.0 & $(532)$ & $\mathrm{A}, \mathrm{EST}$ & {$[87 / 5][66 / 8]$} \\
\hline $\mathrm{C}_{33} \mathrm{H}_{68}$ & $\begin{array}{l}\text { 2-methyldotriacontane } \\
(480-743)\end{array}$ & 122.9 & (495) & & $\begin{array}{c}{[1720-11-2]} \\
{[99 / 16]}\end{array}$ \\
\hline $\mathrm{C}_{33} \mathrm{H}_{68} \mathrm{~S}$ & 1-tritriacontanethiol & & & & [66214-20-8] \\
\hline & $(504-773)$ & 129.1 & $(519)$ & EST & {$[99 / 16]$} \\
\hline $\mathrm{C}_{34} \mathrm{H}_{62}$ & octacosylbenzene & & & & [61828-26-0] \\
\hline & $(490-764)$ & 123.4 & (505) & & [99/16] \\
\hline $\mathrm{C}_{34} \mathrm{H}_{68}$ & octacosylcyclohexane & & & & [61828-13-5] \\
\hline & $(490-764)$ & 122.4 & $(505)$ & & {$[99 / 16]$} \\
\hline $\mathrm{C}_{34} \mathrm{H}_{68}$ & 1-tetratriacontene & & & & [61868-12-0] \\
\hline & $(486-754)$ & 123.7 & $(501)$ & & {$[99 / 16]$} \\
\hline $\mathrm{C}_{34} \mathrm{H}_{70}$ & 11-decyltetracosane & & & & [55429-84-0] \\
\hline & $(537-574)$ & 113.1 & $(552)$ & $\mathrm{A}, \mathrm{MG}$ & {$[87 / 5][55 / 11]$} \\
\hline $\mathrm{C}_{34} \mathrm{H}_{70}$ & 9-octylhexacosane & & & & [55429-83-9] \\
\hline & $(537-575)$ & 110.3 & $(552)$ & $\mathrm{A}, \mathrm{MG}$ & {$[87 / 5][55 / 11]$} \\
\hline $\mathrm{C}_{34} \mathrm{H}_{70}$ & tetratriacontane & & & & [14167-59-0] \\
\hline & $(446-497)$ & $152 \pm 2$ & $(471)$ & $\mathrm{TE}$ & {$[94 / 2]$} \\
\hline & $(372-402)$ & 149.7 & (387) & A & {$[87 / 5]$} \\
\hline & $(523-756)$ & 120.3 & $(538)$ & $\mathrm{A}, \mathrm{EST}$ & {$[87 / 5][66 / 8]$} \\
\hline $\mathrm{C}_{34} \mathrm{H}_{70}$ & 2-methyltritriacontane & & & & [66214-27-5] \\
\hline
\end{tabular}


TABLE 6. Enthalpies of vaporization of organic compounds, 1880-2002-Continued

\begin{tabular}{|c|c|c|c|c|c|}
\hline Molecular formula & $\begin{array}{c}\text { Compound } \\
\text { (Temperature range/K) }\end{array}$ & $\begin{array}{c}\Delta_{\mathrm{vap}} H_{m} \\
\left(\mathrm{~kJ} \mathrm{~mol}^{-1}\right)\end{array}$ & $\begin{array}{l}\text { Mean temperature } \\
\qquad\left(T_{m} / \mathrm{K}\right)\end{array}$ & Method & $\begin{array}{l}\text { CAS registry number } \\
\text { Reference }\end{array}$ \\
\hline & $(486-750)$ & 124.8 & $(501)$ & & [99/16] \\
\hline \multirow[t]{2}{*}{$\mathrm{C}_{34} \mathrm{H}_{70} \mathrm{~S}$} & 1-tetratriacontanethiol & & & & [66214-28-6] \\
\hline & $(509-780)$ & 130.7 & $(524)$ & EST & {$[99 / 16]$} \\
\hline \multirow[t]{2}{*}{$\mathrm{C}_{35} \mathrm{H}_{64}$} & 15-phenylnonacosane & & & & [56247-97-3] \\
\hline & $(523-550)$ & 126.5 & $(536)$ & $\mathrm{A}, \mathrm{MG}$ & {$[87 / 5][55 / 11]$} \\
\hline \multirow[t]{2}{*}{$\mathrm{C}_{35} \mathrm{H}_{64}$} & nonacosylbenzene & & & & [61828-27-1] \\
\hline & $(495-771)$ & 125.4 & $(510)$ & & {$[99 / 16]$} \\
\hline \multirow[t]{2}{*}{$\mathrm{C}_{35} \mathrm{H}_{70}$} & nonacosylcyclohexane & & & & [61828-14-6] \\
\hline & $(495-771)$ & 124.4 & $(510)$ & & [99/16] \\
\hline \multirow{2}{*}{$\mathrm{C}_{35} \mathrm{H}_{70}$} & 1-pentatriacontene & & & & [61868-13-1] \\
\hline & $(492-761)$ & 125.5 & $(507)$ & & {$[99 / 16]$} \\
\hline \multirow[t]{2}{*}{$\mathrm{C}_{35} \mathrm{H}_{70}$} & 15-cyclohexylnonacosane & & & & [55521-27-2] \\
\hline & $(548-581)$ & 129.0 & $(563)$ & $\mathrm{A}, \mathrm{MG}$ & {$[87 / 5][55 / 11]$} \\
\hline \multirow[t]{2}{*}{$\mathrm{C}_{35} \mathrm{H}_{72}$} & pentatriacontane & & & & [630-07-9] \\
\hline & $(529-764)$ & 122.4 & $(544)$ & $\mathrm{A}, \mathrm{EST}$ & {$[87 / 5][66 / 8]$} \\
\hline \multirow[t]{2}{*}{$\mathrm{C}_{35} \mathrm{H}_{72}$} & 2-methyltetratriacontane & & & & [14167-65-8] \\
\hline & $(491-758)$ & 126.9 & $(506)$ & & {$[99 / 16]$} \\
\hline \multirow{2}{*}{$\mathrm{C}_{35} \mathrm{H}_{72} \mathrm{~S}$} & 1-pentatriacontanethiol & & & & [66576-86-1] \\
\hline & $(514-787)$ & 132.2 & $(529)$ & EST & {$[99 / 16]$} \\
\hline \multirow[t]{2}{*}{$\mathrm{C}_{36} \mathrm{H}_{60} \mathrm{O}_{6}$} & triisononanyltrimellitate & & & & \\
\hline & $(334-372)$ & 102.2 & (349) & ME & [00/11] \\
\hline \multirow[t]{2}{*}{$\mathrm{C}_{36} \mathrm{H}_{62} \mathrm{O}_{4}$} & ditetradecyl phthalate & & & & [2915-60-8] \\
\hline & $(416-465)$ & 126.0 & $(431)$ & $\mathrm{T}$ & {$[87 / 5][49 / 9]$} \\
\hline \multirow[t]{2}{*}{$\mathrm{C}_{36} \mathrm{H}_{66}$} & triacontylbenzene & & & & {$[50715-02-1]$} \\
\hline & $(501-778)$ & 127.0 & $(516)$ & & {$[99 / 16]$} \\
\hline \multirow[t]{2}{*}{$\mathrm{C}_{36} \mathrm{H}_{72}$} & 1-hexatriacontene & & & & [61868-14-2] \\
\hline & $(497-768)$ & 127.4 & $(512)$ & & {$[99 / 16]$} \\
\hline \multirow[t]{2}{*}{$\mathrm{C}_{36} \mathrm{H}_{72}$} & triacontylcyclohexane & & & & [61828-15-7] \\
\hline & $(500-778)$ & 126.3 & (515) & & [99/16] \\
\hline $\mathrm{C}_{36} \mathrm{H}_{74}$ & hexatriacontane & & & & [630-06-8] \\
\hline & $(452-516)$ & $157 \pm 2$ & $(484)$ & $\mathrm{TE}$ & {$[94 / 2]$} \\
\hline & $(535-571)$ & 124.4 & $(550)$ & A, EST & {$[87 / 5][66 / 8]$} \\
\hline $\mathrm{C}_{36} \mathrm{H}_{74}$ & 13-undecylpentacosane & & & & [55517-89-0] \\
\hline & $(548-580)$ & 132.9 & $(563)$ & $\mathrm{A}, \mathrm{MG}$ & {$[87 / 5][55 / 11]$} \\
\hline $\mathrm{C}_{36} \mathrm{H}_{74}$ & 2-methylpentatriacontane & & & & [66576-73-6] \\
\hline & $(497-765)$ & 128.7 & $(512)$ & & {$[99 / 16]$} \\
\hline $\mathrm{C}_{36} \mathrm{H}_{74} \mathrm{~S}$ & 1-hexatriacontanethiol & & & & [66577-23-9] \\
\hline & $(518-793)$ & 134.0 & $(533)$ & EST & {$[99 / 16]$} \\
\hline $\mathrm{C}_{36} \mathrm{H}_{75} \mathrm{~N}$ & tridodecylamine & & & & {$[102-87-4]$} \\
\hline & $(579-807)$ & 82.1 & $(594)$ & A & {$[87 / 5]$} \\
\hline $\mathrm{C}_{37} \mathrm{H}_{68}$ & hexatriacontylbenzene & & & & [61828-28-2] \\
\hline & $(506-785)$ & 128.8 & $(521)$ & & {$[99 / 16]$} \\
\hline $\mathrm{C}_{37} \mathrm{H}_{70} \mathrm{O}_{6}$ & 1-caprylic-2-lauryl-3-myristic glycerol & & & & [30283-10-4] \\
\hline & $(464-526)$ & 131.7 & $(479)$ & $\mathrm{A}, \mathrm{T}$ & {$[87 / 5][49 / 16]$} \\
\hline $\mathrm{C}_{37} \mathrm{H}_{74}$ & hentriacontylcyclohexane & & & & [61828-16-8] \\
\hline & $(505-785)$ & 128.1 & $(520)$ & & {$[99 / 16]$} \\
\hline $\mathrm{C}_{37} \mathrm{H}_{74}$ & 1-heptatriacontene & & & & [61868-15-3] \\
\hline & $(502-775)$ & 129.2 & $(517)$ & & {$[99 / 16]$} \\
\hline $\mathrm{C}_{37} \mathrm{H}_{76}$ & heptatriacontane & & & & [7194-84-5] \\
\hline & $(471-511)$ & $155 \pm 2$ & $(491)$ & TE & {$[94 / 2]$} \\
\hline & $(541-778)$ & 126.4 & $(556)$ & $\mathrm{A}, \mathrm{EST}$ & {$[87 / 5][66 / 8]$} \\
\hline $\mathrm{C}_{37} \mathrm{H}_{76}$ & 2-methylhexatriacontane & & & & [66577-06-8] \\
\hline & $(502-772)$ & 130.5 & $(517)$ & & {$[99 / 16]$} \\
\hline $\mathrm{C}_{37} \mathrm{H}_{76} \mathrm{~S}$ & 1-heptatriacontanethiol & & & & [66577-07-9] \\
\hline & $(523-799)$ & 135.3 & $(538)$ & EST & {$[99 / 16]$} \\
\hline $\mathrm{C}_{38} \mathrm{H}_{70}$ & dotriacontylbenzene & & & & [61828-29-3] \\
\hline & $(511-791)$ & 130.4 & $(526)$ & & {$[99 / 16]$} \\
\hline $\mathrm{C}_{38} \mathrm{H}_{74} \mathrm{O}_{4}$ & ditetradecyl sebacate & & & & [26719-47-1] \\
\hline & $(431-483)$ & 135.5 & $(446)$ & $\mathrm{A}, \mathrm{T}$ & {$[87 / 5][49 / 9]$} \\
\hline $\mathrm{C}_{38} \mathrm{H}_{76}$ & dotriacontylcyclohexane & & & & [61828-17-9] \\
\hline & $(510-792)$ & 129.8 & $(525)$ & & {$[99 / 16]$} \\
\hline $\mathrm{C}_{38} \mathrm{H}_{76}$ & 1-octatriacontene & & & & [61868-16-4] \\
\hline & $(507-782)$ & 131.0 & (522) & & [99/16] \\
\hline $\mathrm{C}_{38} \mathrm{H}_{78}$ & octatriacontane & & & & [7194-85-6] \\
\hline
\end{tabular}


TABLE 6. Enthalpies of vaporization of organic compounds, 1880-2002-Continued

\begin{tabular}{|c|c|c|c|c|c|}
\hline Molecular formula & $\begin{array}{c}\text { Compound } \\
\text { (Temperature range/K) }\end{array}$ & $\begin{array}{c}\Delta_{\mathrm{vap}} H_{m} \\
\left(\mathrm{~kJ} \mathrm{~mol}^{-1}\right)\end{array}$ & $\begin{array}{l}\text { Mean temperature } \\
\qquad\left(T_{m} / \mathrm{K}\right)\end{array}$ & Method & $\begin{array}{c}\text { CAS registry number } \\
\text { Reference }\end{array}$ \\
\hline & $(471-511)$ & $160 \pm 2$ & $(491)$ & TE & [94/2] \\
\hline & $(546-785)$ & 128.5 & $(561)$ & A, EST & {$[87 / 5][66 / 8]$} \\
\hline \multirow[t]{2}{*}{$\mathrm{C}_{38} \mathrm{H}_{78}$} & 2-methylheptatriacontane & & & & [66576-92-9] \\
\hline & $(507-779)$ & 132.2 & $(522)$ & & [99/16] \\
\hline \multirow[t]{2}{*}{$\mathrm{C}_{38} \mathrm{H}_{78} \mathrm{~S}$} & 1-octatriacontanethiol & & & & [66576-93-0] \\
\hline & $(527-805)$ & 136.7 & $(542)$ & EST & [99/16] \\
\hline \multirow[t]{2}{*}{$\mathrm{C}_{39} \mathrm{H}_{72}$} & 17-phenyltritriacontane & & & & [55517-74-3] \\
\hline & $(544-571)$ & 147.1 & (557) & $\mathrm{A}, \mathrm{MG}$ & {$[87 / 5][55 / 11]$} \\
\hline \multirow[t]{2}{*}{$\mathrm{C}_{39} \mathrm{H}_{72}$} & tritriacontylbenzene & & & & [61828-30-6] \\
\hline & $(516-798)$ & 132.0 & $(531)$ & & {$[99 / 16]$} \\
\hline \multirow[t]{3}{*}{$\mathrm{C}_{39} \mathrm{H}_{74} \mathrm{O}_{6}$} & glycerol trilaurate & & & & [538-24-9] \\
\hline & & 221.1 & & TGA & {$[02 / 33]$} \\
\hline & $(458-520)$ & 137.6 & (473) & $\mathrm{A}, \mathrm{T}$ & {$[87 / 5][49 / 16]$} \\
\hline \multirow[t]{2}{*}{$\mathrm{C}_{39} \mathrm{H}_{78}$} & 1-nontriacontene & & & & [61868-17-5] \\
\hline & $(512-788)$ & 132.5 & (527) & & {$[99 / 16]$} \\
\hline \multirow[t]{2}{*}{$\mathrm{C}_{39} \mathrm{H}_{78}$} & triatriacontylcyclohexane & & & & [61828-18-0] \\
\hline & $(515-798)$ & 131.4 & $(530)$ & & {$[99 / 16]$} \\
\hline \multirow[t]{2}{*}{$\mathrm{C}_{39} \mathrm{H}_{78}$} & 17-cyclohexyltritricontane & & & & [55517-75-4] \\
\hline & $(570-602)$ & 131.9 & (585) & $\mathrm{A}, \mathrm{MG}$ & {$[87 / 5][55 / 11]$} \\
\hline \multirow[t]{2}{*}{$\mathrm{C}_{39} \mathrm{H}_{80}$} & nonatriacontane & & & & [7194-86-7] \\
\hline & $(552-791)$ & 130.3 & (567) & A, EST & {$[87 / 5][66 / 8]$} \\
\hline \multirow{2}{*}{$\mathrm{C}_{39} \mathrm{H}_{80}$} & 2-methyloctatriacontane & & & & [66576-59-8] \\
\hline & $(512-785)$ & 133.8 & (527) & & {$[99 / 16]$} \\
\hline \multirow[t]{2}{*}{$\mathrm{C}_{39} \mathrm{H}_{80} \mathrm{~S}$} & 1-nonatriacontanethiol & & & & [66576-60-1] \\
\hline & $(531-811)$ & 138.1 & (546) & EST & {$[99 / 16]$} \\
\hline \multirow[t]{2}{*}{$\mathrm{C}_{40} \mathrm{H}_{74}$} & tetratriacontylbenzene & & & & [61828-31-7] \\
\hline & $(520-804)$ & 133.8 & (535) & & {$[99 / 16]$} \\
\hline \multirow[t]{2}{*}{$\mathrm{C}_{40} \mathrm{H}_{80}$} & 1-tetracontene & & & & [61868-18-6] \\
\hline & $(517-794)$ & 134.0 & $(532)$ & & {$[99 / 16]$} \\
\hline \multirow[t]{2}{*}{$\mathrm{C}_{40} \mathrm{H}_{80}$} & tetratriacontylcyclohexane & & & & [61828-19-1] \\
\hline & $(520-804)$ & 132.9 & $(535)$ & & {$[99 / 16]$} \\
\hline $\mathrm{C}_{40} \mathrm{H}_{82}$ & tetracontane & & & & [4181-95-7] \\
\hline $40^{1+182}$ & $(557-798)$ & 132.2 & $(572)$ & A, EST & {$[87 / 5][66 / 8]$} \\
\hline $\mathrm{C}_{40} \mathrm{H}_{82}$ & 2-methylnonatricontane & & & & [66576-48-5] \\
\hline & $(517-791)$ & 135.3 & $(532)$ & & [99/16] \\
\hline $\mathrm{C}_{40} \mathrm{H}_{82} \mathrm{~S}$ & 1-tetracontanethiol & & & & [66576-49-6] \\
\hline & $(535-817)$ & 139.6 & $(550)$ & EST & [99/16] \\
\hline $\mathrm{C}_{41} \mathrm{H}_{76}$ & pentatriacontylbenzene & & & & [61828-32-8] \\
\hline & $(525-810)$ & 135.2 & (540) & & {$[99 / 16]$} \\
\hline $\mathrm{C}_{41} \mathrm{H}_{82}$ & 1-hentetracontene & & & & [66576-37-2] \\
\hline & $(521-800)$ & 135.8 & (536) & & {$[99 / 16]$} \\
\hline $\mathrm{C}_{41} \mathrm{H}_{82}$ & pentatriacontylcyclohexane & & & & [61828-20-4] \\
\hline & $(524-810)$ & 134.5 & (539) & & {$[99 / 16]$} \\
\hline $\mathrm{C}_{41} \mathrm{H}_{84}$ & hentetracontane & & & & [7194-87-8] \\
\hline & $(562-804)$ & 134.1 & $(577)$ & A, EST & {$[87 / 5][66 / 8]$} \\
\hline $\mathrm{C}_{41} \mathrm{H}_{84}$ & 2-methyltetracontane & & & & [66575-38-3] \\
\hline & $(521-797)$ & 137.1 & (536) & & [99/16] \\
\hline $\mathrm{C}_{41} \mathrm{H}_{84} \mathrm{~S}$ & 1-hentetracontanethiol & & & & [66576-39-4] \\
\hline & $(539-822)$ & 140.8 & (554) & EST & {$[99 / 16]$} \\
\hline $\mathrm{C}_{42} \mathrm{H}_{78}$ & hexatriacontylbenzene & & & & [61828-33-9] \\
\hline & $(529-815)$ & 129.0 & (544) & & {$[99 / 16]$} \\
\hline $\mathrm{C}_{42} \mathrm{H}_{84}$ & 1-dotetracontene & & & & [21807-60-3] \\
\hline & $(526-806)$ & 137.1 & $(541)$ & & [99/16] \\
\hline $\mathrm{C}_{42} \mathrm{H}_{84}$ & hexatriactonylcyclohexane & & & & [61828-21-5] \\
\hline & $(529-816)$ & 135.8 & (544) & & {$[99 / 16]$} \\
\hline $\mathrm{C}_{42} \mathrm{H}_{86}$ & dotetracontane & & & & [7098-20-6] \\
\hline & $(567-810)$ & 136.0 & (582) & $\mathrm{A}, \mathrm{EST}$ & {$[87 / 5][66 / 8]$} \\
\hline $\mathrm{C}_{42} \mathrm{H}_{86}$ & 2-methylhentetracontane & & & & [66576-40-7] \\
\hline & $(526-803)$ & 138.5 & $(541)$ & & {$[99 / 16]$} \\
\hline $\mathrm{C}_{42} \mathrm{H}_{86}$ & $2,2,4,15,17,17$-hexamethyl- & 3,5,5-trimeth & octadecane & & [55470-97-8] \\
\hline & $(512-575)$ & 118.3 & $(527)$ & A, MG & {$[87 / 5][55 / 11]$} \\
\hline $\mathrm{C}_{42} \mathrm{H}_{86} \mathrm{~S}$ & 1-dotetracontanethiol & & & & [66576-41-8] \\
\hline & $(543-828)$ & 142.1 & (558) & EST & [99/16] \\
\hline $\mathrm{C}_{42} \mathrm{H}_{87} \mathrm{~N}$ & tritetradecylamine & & & & [27911-72-4] \\
\hline
\end{tabular}


TABLE 6. Enthalpies of vaporization of organic compounds, 1880-2002-Continued

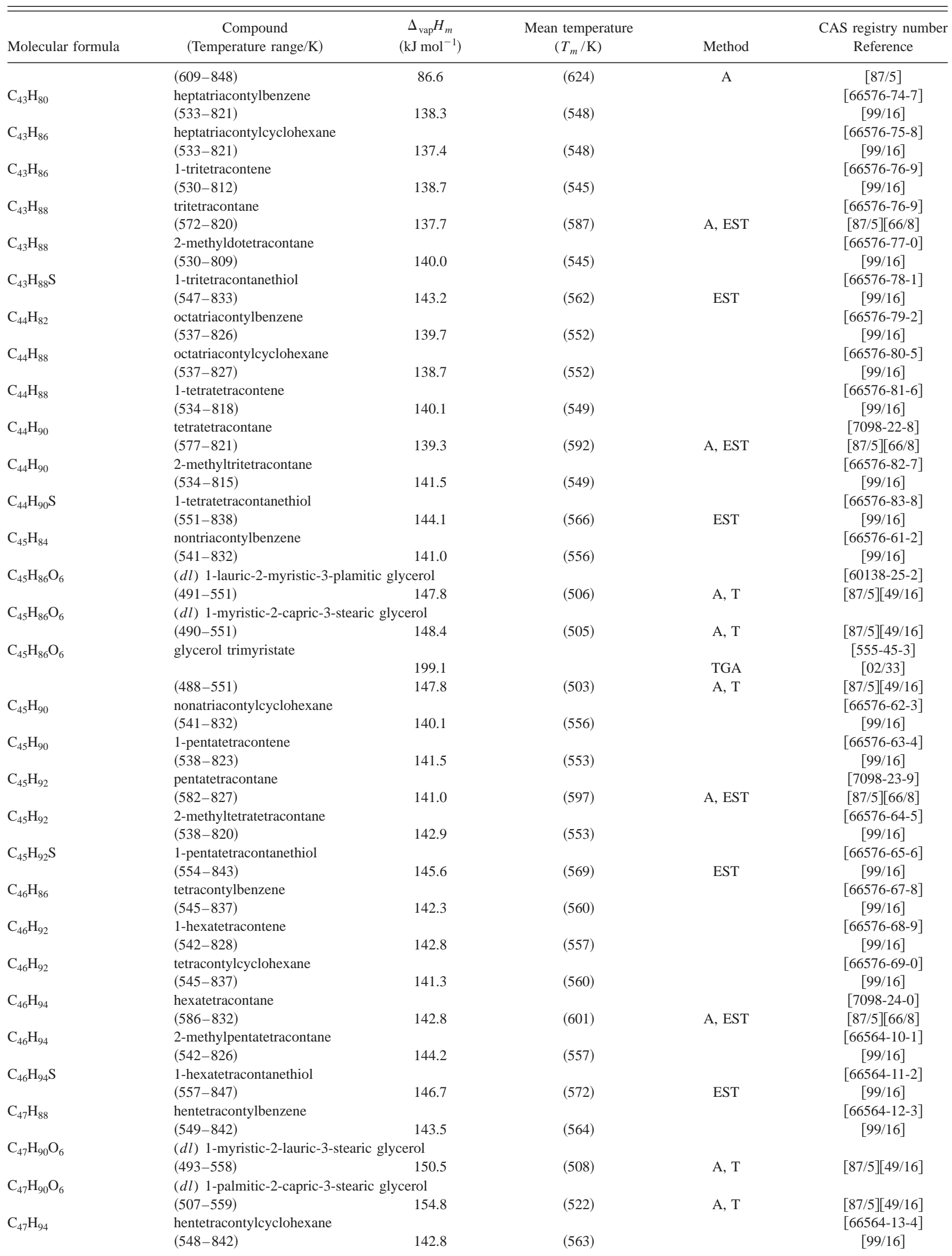


TABLE 6. Enthalpies of vaporization of organic compounds, 1880-2002-Continued

\begin{tabular}{|c|c|c|c|c|c|}
\hline Molecular formula & $\begin{array}{c}\text { Compound } \\
\text { (Temperature range/K) }\end{array}$ & $\begin{array}{c}\Delta_{\mathrm{vap}} H_{m} \\
\left(\mathrm{~kJ} \mathrm{~mol}^{-1}\right)\end{array}$ & $\begin{array}{l}\text { Mean temperature } \\
\qquad\left(T_{m} / \mathrm{K}\right)\end{array}$ & Method & $\begin{array}{c}\text { CAS registry number } \\
\text { Reference }\end{array}$ \\
\hline $\mathrm{C}_{47} \mathrm{H}_{94}$ & $\begin{array}{l}\text { 1-heptatetracontene } \\
(546-833)\end{array}$ & 143.9 & $(561)$ & & $\begin{array}{c}{[66576-01-0]} \\
{[99 / 16]}\end{array}$ \\
\hline $\mathrm{C}_{47} \mathrm{H}_{96}$ & heptatetracontane & & & & [7098-25-1] \\
\hline & $(591-837)$ & 144.2 & $(606)$ & A, EST & {$[87 / 5][66 / 8]$} \\
\hline $\mathrm{C}_{47} \mathrm{H}_{96}$ & $\begin{array}{l}\text { 2-methylhexatetracontane } \\
(546-831)\end{array}$ & 145.3 & $(561)$ & & $\begin{array}{c}{[66576-02-1]} \\
{[99 / 16]}\end{array}$ \\
\hline $\mathrm{C}_{47} \mathrm{H}_{96} \mathrm{~S}$ & $\begin{array}{l}\text { 1-heptatetracontanethiol } \\
\text { (561-852) }\end{array}$ & 147.6 & $(576)$ & EST & $\begin{array}{c}{[66576-03-2]} \\
{[99 / 16]}\end{array}$ \\
\hline $\mathrm{C}_{48} \mathrm{H}_{90}$ & $\begin{array}{l}\text { dotetracontylbenzene } \\
(552-846)\end{array}$ & 144.9 & $(567)$ & & $\begin{array}{c}{[66576-04-3]} \\
{[99 / 16]}\end{array}$ \\
\hline $\mathrm{C}_{48} \mathrm{H}_{96}$ & $\begin{array}{l}\text { dotetracontylcyclohexane } \\
(552-847)\end{array}$ & 143.9 & $(567)$ & & $\begin{array}{c}{[66576-05-4]} \\
{[99 / 16]}\end{array}$ \\
\hline $\mathrm{C}_{48} \mathrm{H}_{96}$ & $\begin{array}{l}\text { 1-octatetracontene } \\
(549-838)\end{array}$ & 145.4 & $(564)$ & & $\begin{array}{c}{[66576-06-5]} \\
{[99 / 16]}\end{array}$ \\
\hline $\mathrm{C}_{48} \mathrm{H}_{98}$ & $\begin{array}{l}\text { octatetracontane } \\
(595-843)\end{array}$ & 145.9 & $(610)$ & A, EST & $\begin{array}{l}{[7098-26-2]} \\
{[87 / 5][66 / 8]}\end{array}$ \\
\hline $\mathrm{C}_{48} \mathrm{H}_{98}$ & $\begin{array}{l}\text { 2-methylheptatetracontane } \\
(550-836)\end{array}$ & 146.5 & $(565)$ & & $\begin{array}{c}{[66576-07-6]} \\
{[99 / 16]}\end{array}$ \\
\hline $\mathrm{C}_{48} \mathrm{H}_{98} \mathrm{~S}$ & $\begin{array}{l}\text { 1-octatetracontanethiol } \\
(564-856)\end{array}$ & 148.7 & $(579)$ & EST & $\begin{array}{c}{[66576-08-7]} \\
{[99 / 16]}\end{array}$ \\
\hline $\mathrm{C}_{49} \mathrm{H}_{92}$ & $\begin{array}{l}\text { tritetracontylbenzene } \\
(556-851)\end{array}$ & 145.9 & $(571)$ & & $\begin{array}{c}{[66576-09-8]} \\
{[99 / 16]}\end{array}$ \\
\hline $\mathrm{C}_{49} \mathrm{H}_{94} \mathrm{O}_{6}$ & $\begin{array}{l}(d l) \text { 1-palmitic-2-lauryl-3-s } \\
(506-567)\end{array}$ & $\begin{array}{l}\text { ycerol } \\
160.0\end{array}$ & (521) & $\mathrm{A}, \mathrm{T}$ & {$[87 / 5][49 / 16]$} \\
\hline $\mathrm{C}_{49} \mathrm{H}_{98}$ & $\begin{array}{l}\text { 1-nonatetracontene } \\
(553-843)\end{array}$ & 146.4 & $(568)$ & & $\begin{array}{c}{[66576-19-1]} \\
{[99 / 16]}\end{array}$ \\
\hline $\mathrm{C}_{49} \mathrm{H}_{98}$ & $\begin{array}{l}\text { tritetracontylcyclohexane } \\
(556-852)\end{array}$ & 144.9 & $(571)$ & & $\begin{array}{c}{[66576-11-2]} \\
{[99 / 16]}\end{array}$ \\
\hline $\mathrm{C}_{49} \mathrm{H}_{100}$ & $\begin{array}{l}\text { nonatetracontane } \\
(599-847)\end{array}$ & 147.5 & $(614)$ & A, EST & $\begin{array}{l}{[7098-27-3]} \\
{[87 / 5][66 / 8]}\end{array}$ \\
\hline $\mathrm{C}_{49} \mathrm{H}_{100}$ & $\begin{array}{l}\text { 2-methyloctatetracontane } \\
(553-840)\end{array}$ & 147.9 & $(568)$ & & $\begin{array}{c}{[66576-12-3]} \\
{[99 / 16]}\end{array}$ \\
\hline $\mathrm{C}_{49} \mathrm{H}_{100} \mathrm{~S}$ & $\begin{array}{l}\text { 1-nonatetracontanethiol } \\
(567-861)\end{array}$ & 149.7 & $(582)$ & EST & $\begin{array}{c}{[66576-13-4]} \\
{[99 / 16]}\end{array}$ \\
\hline $\mathrm{C}_{50} \mathrm{H}_{94}$ & $\begin{array}{l}\text { tetratetracontylbenzene } \\
(559-856)\end{array}$ & 147.1 & $(574)$ & & $\begin{array}{c}{[66576-14-5]} \\
{[99 / 16]}\end{array}$ \\
\hline $\mathrm{C}_{50} \mathrm{H}_{100}$ & $\begin{array}{l}\text { 1-pentacontene } \\
(556-848)\end{array}$ & 147.8 & $(571)$ & & $\begin{array}{c}{[63911-02-4]} \\
{[99 / 16]}\end{array}$ \\
\hline $\mathrm{C}_{50} \mathrm{H}_{100}$ & $\begin{array}{l}\text { tetratetracontylcyclohexane } \\
(559-856)\end{array}$ & 146.2 & $(574)$ & & $\begin{array}{c}{[66576-15-6]} \\
{[99 / 16]}\end{array}$ \\
\hline $\mathrm{C}_{50} \mathrm{H}_{102}$ & $\begin{array}{l}\text { pentacontane } \\
(603-852)\end{array}$ & 149.0 & $(618)$ & A, EST & $\begin{array}{l}{[6596-40-3]} \\
{[87 / 5][66 / 8]}\end{array}$ \\
\hline $\mathrm{C}_{50} \mathrm{H}_{102}$ & $\begin{array}{l}\text { 2-methylnonatetracontane } \\
(557-845)\end{array}$ & 148.8 & $(572)$ & & $\begin{array}{c}{[66576-16-7]} \\
{[99 / 16]}\end{array}$ \\
\hline $\mathrm{C}_{50} \mathrm{H}_{102} \mathrm{~S}$ & $\begin{array}{l}\text { 1-pentacontanethiol } \\
(570-865)\end{array}$ & 150.7 & $(585)$ & EST & $\begin{array}{c}{[66576-17-8]} \\
{[99 / 16]}\end{array}$ \\
\hline $\mathrm{C}_{51} \mathrm{H}_{96}$ & $\begin{array}{l}\text { pentatetracontylbenzene } \\
(562-860)\end{array}$ & 148.3 & $(577)$ & & $\begin{array}{c}{[66576-18-9]} \\
{[99 / 16]}\end{array}$ \\
\hline $\mathrm{C}_{51} \mathrm{H}_{98} \mathrm{O}_{6}$ & $\begin{array}{l}\text { 1-myristic-2-palmitic-3-stea } \\
(508-572)\end{array}$ & rol 157.9 & $(523)$ & $\mathrm{A}, \mathrm{T}$ & $\begin{array}{l}{[60138-20-7]} \\
{[87 / 5][49 / 16]}\end{array}$ \\
\hline $\mathrm{C}_{51} \mathrm{H}_{98} \mathrm{O}_{6}$ & $\begin{array}{l}\text { glycerol tripalmitate } \\
(506-572)\end{array}$ & $\begin{array}{l}474.3 \\
160.8\end{array}$ & (521) & $\begin{array}{l}\text { TGA } \\
\text { A, T }\end{array}$ & $\begin{array}{c}{[555-44-2]} \\
{[02 / 33]} \\
{[87 / 5][49 / 16]}\end{array}$ \\
\hline $\mathrm{C}_{51} \mathrm{H}_{102}$ & $\begin{array}{l}\text { 1-henpentacontene } \\
(560-852)\end{array}$ & 148.6 & $(575)$ & $\mathrm{A}, 1$ & $\begin{array}{c}{[66576-19-0]} \\
{[99 / 16]}\end{array}$ \\
\hline $\mathrm{C}_{51} \mathrm{H}_{102}$ & $\begin{array}{l}\text { pentatetracontylcyclohexane } \\
(562-861)\end{array}$ & 147.4 & $(577)$ & & $\begin{array}{c}{[66576-20-3]} \\
{[99 / 16]}\end{array}$ \\
\hline $\mathrm{C}_{51} \mathrm{H}_{104}$ & $\begin{array}{l}\text { henpentacontane } \\
(607-857)\end{array}$ & 150.6 & $(622)$ & A, EST & $\begin{array}{l}{[7667-76-7]} \\
{[87 / 5][66 / 8]}\end{array}$ \\
\hline $\mathrm{C}_{51} \mathrm{H}_{104}$ & $\begin{array}{l}\text { 2-methylpentacontane } \\
(560-850)\end{array}$ & 150.0 & $(575)$ & & $\begin{array}{c}{[66575-81-3]} \\
{[99 / 16]}\end{array}$ \\
\hline $\mathrm{C}_{51} \mathrm{H}_{104} \mathrm{~S}$ & $\begin{array}{l}\text { 1-henpentacontanethiol } \\
(573-869)\end{array}$ & 151.6 & $(588)$ & EST & $\begin{array}{c}{[66575-82-4]} \\
{[99 / 16]}\end{array}$ \\
\hline $\mathrm{C}_{52} \mathrm{H}_{98}$ & hexatetracontylbenzene & & & & [66575-84-6] \\
\hline
\end{tabular}


TABLE 6. Enthalpies of vaporization of organic compounds, 1880-2002-Continued

\begin{tabular}{|c|c|c|c|c|c|}
\hline Molecular formula & $\begin{array}{c}\text { Compound } \\
\text { (Temperature range/K) }\end{array}$ & $\begin{array}{c}\Delta_{\mathrm{vap}} H_{m} \\
\left(\mathrm{~kJ} \mathrm{~mol}^{-1}\right)\end{array}$ & $\begin{array}{l}\text { Mean temperature } \\
\qquad\left(T_{m} / \mathrm{K}\right)\end{array}$ & Method & $\begin{array}{l}\text { CAS registry number } \\
\text { Reference }\end{array}$ \\
\hline & $(566-864)$ & 149.1 & $(581)$ & & [99/16] \\
\hline $\mathrm{C}_{52} \mathrm{H}_{104}$ & 1-dopentacontene & & & & {$[66575-85-7]$} \\
\hline & $(563-857)$ & 149.8 & (578) & & [99/16] \\
\hline $\mathrm{C}_{52} \mathrm{H}_{104}$ & hexatetracontylcyclohexane & & & & {$[66575-86-8]$} \\
\hline & $(565-865)$ & 148.5 & $(580)$ & & {$[99 / 16]$} \\
\hline $\mathrm{C}_{52} \mathrm{H}_{106}$ & dopentacontane & & & & [7719-79-1] \\
\hline & $(611-861)$ & 152.0 & $(626)$ & A, EST & {$[87 / 5][66 / 8]$} \\
\hline $\mathrm{C}_{52} \mathrm{H}_{106}$ & $\begin{array}{l}\text { 2-methylhenpentacontane } \\
(563-854)\end{array}$ & 151.2 & (578) & & $\begin{array}{c}{[66575-87-9]} \\
{[99 / 16]}\end{array}$ \\
\hline $\mathrm{C}_{52} \mathrm{H}_{106} \mathrm{~S}$ & $\begin{array}{l}\text { 1-dopentacontanethiol } \\
\text { (575-873) }\end{array}$ & 152.6 & $(590)$ & EST & $\begin{array}{c}{[66575-88-0]} \\
{[99 / 16]}\end{array}$ \\
\hline $\mathrm{C}_{53} \mathrm{H}_{100}$ & $\begin{array}{l}\text { heptatetracontylbenzene } \\
(569-868)\end{array}$ & 150.1 & $(584)$ & & $\begin{array}{c}{[66575-89-1]} \\
{[99 / 16]}\end{array}$ \\
\hline $\mathrm{C}_{53} \mathrm{H}_{106}$ & $\begin{array}{l}\text { heptatetracontylcyclohexane } \\
(568-869)\end{array}$ & 149.6 & (583) & & $\begin{array}{c}{[66563-49-3]} \\
{[99 / 16]}\end{array}$ \\
\hline $\mathrm{C}_{53} \mathrm{H}_{106}$ & $\begin{array}{l}\text { 1-tripentacontene } \\
(566-861)\end{array}$ & 150.9 & $(581)$ & & $\begin{array}{c}{[66577-50-2]} \\
{[99 / 16]}\end{array}$ \\
\hline $\mathrm{C}_{53} \mathrm{H}_{108}$ & $\begin{array}{l}\text { tripentacontane } \\
(615-866)\end{array}$ & 153.4 & $(630)$ & A, EST & $\begin{array}{l}{[7719-80-4]} \\
{[87 / 5][66 / 8]}\end{array}$ \\
\hline $\mathrm{C}_{53} \mathrm{H}_{108}$ & $\begin{array}{l}\text { 2-methyldopentacontane } \\
(566-858)\end{array}$ & 152.3 & $(581)$ & & $\begin{array}{c}{[66575-90-4]} \\
{[99 / 16]}\end{array}$ \\
\hline $\mathrm{C}_{53} \mathrm{H}_{108} \mathrm{~S}$ & $\begin{array}{l}\text { 1-tripentacontanethiol } \\
(578-877)\end{array}$ & 153.7 & $(593)$ & EST & $\begin{array}{c}{[66575-91-5]} \\
{[99 / 16]}\end{array}$ \\
\hline $\mathrm{C}_{54} \mathrm{H}_{102}$ & $\begin{array}{l}\text { octatetracontylbenzene } \\
(572-873)\end{array}$ & 151.1 & $(587)$ & & $\begin{array}{c}{[66575-92-6]} \\
{[99 / 16]}\end{array}$ \\
\hline $\mathrm{C}_{54} \mathrm{H}_{108}$ & $\begin{array}{l}\text { octatetracontylcyclohexane } \\
(571-873)\end{array}$ & 150.6 & $(586)$ & & $\begin{array}{c}{[66575-93-7]} \\
{[99 / 16]}\end{array}$ \\
\hline $\mathrm{C}_{54} \mathrm{H}_{110}$ & $\begin{array}{l}\text { 1-tetrapentacontene } \\
(569-865)\end{array}$ & 151.9 & $(584)$ & & $\begin{array}{c}{[66575-94-8]} \\
{[99 / 16]}\end{array}$ \\
\hline $\mathrm{C}_{54} \mathrm{H}_{110}$ & $\begin{array}{l}\text { tetrapentacontane } \\
(618-870)\end{array}$ & 155.0 & (633) & A, EST & $\begin{array}{l}{[5856-66-6]} \\
{[87 / 5][66 / 8]}\end{array}$ \\
\hline $\mathrm{C}_{54} \mathrm{H}_{110}$ & $\begin{array}{l}\text { 2-methyltripentacontane } \\
(569-863)\end{array}$ & 153.4 & (584) & & $\begin{array}{c}{[66575-95-9]} \\
{[99 / 16]}\end{array}$ \\
\hline $\mathrm{C}_{54} \mathrm{H}_{110} \mathrm{~S}$ & $\begin{array}{l}\text { 1-tetrapentacontanethiol } \\
(581-881)\end{array}$ & 154.4 & $(596)$ & EST & $\begin{array}{c}{[66575-96-0]} \\
{[99 / 16]}\end{array}$ \\
\hline $\mathrm{C}_{55} \mathrm{H}_{104}$ & $\begin{array}{l}\text { nontetracontylbenzene } \\
(575-877)\end{array}$ & 152.1 & $(590)$ & & $\begin{array}{c}{[66575-98-2]} \\
{[99 / 16]}\end{array}$ \\
\hline $\mathrm{C}_{55} \mathrm{H}_{110}$ & $\begin{array}{l}\text { nonatetracontylcyclohexane } \\
(574-877)\end{array}$ & 151.6 & $(589)$ & & $\begin{array}{c}{[66575-99-3]} \\
{[99 / 16]}\end{array}$ \\
\hline $\mathrm{C}_{55} \mathrm{H}_{110}$ & $\begin{array}{l}\text { 1-pentapentacontene } \\
(572-869)\end{array}$ & 152.9 & (587) & & $\begin{array}{c}{[66576-00-9]} \\
{[99 / 16]}\end{array}$ \\
\hline $\mathrm{C}_{55} \mathrm{H}_{112}$ & $\begin{array}{l}\text { pentapentacontane } \\
(622-874)\end{array}$ & 156.3 & (637) & A, EST & $\begin{array}{l}{[5846-40-2]} \\
{[87 / 5][66 / 8]}\end{array}$ \\
\hline $\mathrm{C}_{55} \mathrm{H}_{112}$ & $\begin{array}{l}\text { 2-methyltetrapentacontane } \\
(572-867)\end{array}$ & 154.3 & $(587)$ & & $\begin{array}{c}{[66575-60-8]} \\
{[99 / 16]}\end{array}$ \\
\hline $\mathrm{C}_{55} \mathrm{H}_{112} \mathrm{~S}$ & $\begin{array}{l}\text { 1-pentapentacontanethiol } \\
(584-885)\end{array}$ & 155.0 & (599) & EST & $\begin{array}{c}{[66575-61-9]} \\
{[99 / 16]}\end{array}$ \\
\hline $\mathrm{C}_{56} \mathrm{H}_{106}$ & $\begin{array}{l}\text { pentacontylbenzene } \\
(577-880)\end{array}$ & 153.2 & (592) & & $\begin{array}{c}{[66575-62-0]} \\
{[99 / 16]}\end{array}$ \\
\hline $\mathrm{C}_{56} \mathrm{H}_{108}$ & $\begin{array}{l}\text { 1-hexapentacontene } \\
(575-873)\end{array}$ & 154.5 & (588) & & $\begin{array}{c}{[66575-63-1]} \\
{[99 / 16]}\end{array}$ \\
\hline $\mathrm{C}_{56} \mathrm{H}_{108}$ & $\begin{array}{l}\text { pentacontylcyclohexane } \\
(577-881)\end{array}$ & 152.4 & $(592)$ & & $\begin{array}{c}{[66575-64-2]} \\
{[99 / 16]}\end{array}$ \\
\hline $\mathrm{C}_{56} \mathrm{H}_{114}$ & $\begin{array}{l}\text { hexapentacontane } \\
(625-878)\end{array}$ & 157.8 & (640) & A, EST & $\begin{array}{l}{[7719-82-6]} \\
{[87 / 5][66 / 8]}\end{array}$ \\
\hline $\mathrm{C}_{56} \mathrm{H}_{114}$ & $\begin{array}{l}\text { 2-methylpentapentacontane } \\
(575-871)\end{array}$ & 155.9 & (588) & & $\begin{array}{c}{[66575-65-3]} \\
{[99 / 16]}\end{array}$ \\
\hline $\mathrm{C}_{56} \mathrm{H}_{114} \mathrm{~S}$ & $\begin{array}{l}\text { 1-hexapentacontanethiol } \\
(586-888)\end{array}$ & 156.0 & $(601)$ & EST & $\begin{array}{c}{[66575-66-4]} \\
{[99 / 16]}\end{array}$ \\
\hline $\mathrm{C}_{57} \mathrm{H}_{108}$ & $\begin{array}{l}\text { henpentacontylbenzene } \\
(580-884)\end{array}$ & 154.1 & (595) & & $\begin{array}{c}{[66575-67-5]} \\
{[99 / 16]}\end{array}$ \\
\hline $\begin{array}{l}\mathrm{C}_{57} \mathrm{H}_{108} \mathrm{O}_{6} \\
\mathrm{C}_{57} \mathrm{H}_{108} \mathrm{O}_{6}\end{array}$ & $\begin{array}{l}\text { 1,3-distearic-2-oleic glycerol } \\
(523-593) \\
\text { glycerol tristearate }\end{array}$ & 165.8 & (538) & $\mathrm{A}, \mathrm{T}$ & $\begin{array}{c}{[2846-04-0]} \\
{[87 / 5][49 / 16]} \\
{[555-43-1]}\end{array}$ \\
\hline
\end{tabular}


TABLE 6. Enthalpies of vaporization of organic compounds, 1880-2002-Continued

\begin{tabular}{|c|c|c|c|c|c|}
\hline Molecular formula & $\begin{array}{c}\text { Compound } \\
\text { (Temperature range/K) }\end{array}$ & $\begin{array}{c}\Delta_{\mathrm{vap}} H_{m} \\
\left(\mathrm{~kJ} \mathrm{~mol}^{-1}\right)\end{array}$ & $\begin{array}{l}\text { Mean temperature } \\
\qquad\left(T_{m} / \mathrm{K}\right)\end{array}$ & Method & $\begin{array}{c}\text { CAS registry number } \\
\text { Reference }\end{array}$ \\
\hline & & 220.8 & & TGA & {$[02 / 33]$} \\
\hline & $(521-588)$ & 167.5 & $(536)$ & $\mathrm{A}, \mathrm{T}$ & {$[87 / 5][49 / 16]$} \\
\hline \multirow[t]{2}{*}{$\mathrm{C}_{57} \mathrm{H}_{114}$} & henpentacontylcyclohexane & & & & {$[66575-68-6]$} \\
\hline & $(580-885)$ & 153.3 & $(595)$ & & {$[99 / 16]$} \\
\hline \multirow[t]{2}{*}{$\mathrm{C}_{57} \mathrm{H}_{114}$} & 1-heptapentacontene & & & & {$[66575-69-7]$} \\
\hline & $(578-877)$ & 154.6 & $(593)$ & & [99/16] \\
\hline \multirow[t]{2}{*}{$\mathrm{C}_{57} \mathrm{H}_{116}$} & heptapentacontane & & & & {$[5856-67-7]$} \\
\hline & $(629-882)$ & 158.9 & $(644)$ & $\mathrm{A}, \mathrm{EST}$ & {$[87 / 5][66 / 8]$} \\
\hline \multirow[t]{2}{*}{$\mathrm{C}_{57} \mathrm{H}_{116}$} & 2-methylhexapentacontane & & & & {$[66575-70-0]$} \\
\hline & $(578-875)$ & 155.9 & $(593)$ & & [99/16] \\
\hline \multirow[t]{2}{*}{$\mathrm{C}_{57} \mathrm{H}_{116} \mathrm{~S}$} & 1-heptapentacontanethiol & & & & {$[66575-75-1]$} \\
\hline & $(589-892)$ & 156.7 & $(604)$ & EST & {$[99 / 16]$} \\
\hline \multirow[t]{2}{*}{$\mathrm{C}_{58} \mathrm{H}_{110}$} & dopentacontylbenzene & & & & {$[66575-73-3]$} \\
\hline & $(583-888)$ & 155.7 & $(598)$ & & [99/16] \\
\hline \multirow[t]{2}{*}{$\mathrm{C}_{58} \mathrm{H}_{116}$} & dopentacontylcyclohexane & & & & {$[66575-74-4]$} \\
\hline & $(582-888)$ & 154.3 & $(597)$ & & {$[99 / 16]$} \\
\hline \multirow[t]{2}{*}{$\mathrm{C}_{58} \mathrm{H}_{116}$} & 1-octapentacontene & & & & {$[66575-75-5]$} \\
\hline & $(580-881)$ & 155.8 & $(595)$ & & {$[99 / 16]$} \\
\hline \multirow{2}{*}{$\mathrm{C}_{58} \mathrm{H}_{118}$} & octapentacontane & & & & {$[7667-78-9]$} \\
\hline & $(632-886)$ & 160.3 & $(647)$ & A, EST & {$[87 / 5][66 / 8]$} \\
\hline \multirow[t]{2}{*}{$\mathrm{C}_{58} \mathrm{H}_{118}$} & 2-methylheptapentacontane & & & & {$[66575-76-6]$} \\
\hline & $(581-879)$ & 156.8 & $(596)$ & & {$[99 / 16]$} \\
\hline \multirow[t]{2}{*}{$\mathrm{C}_{58} \mathrm{H}_{118} \mathrm{~S}$} & 1-octapentacontanethiol & & & & {$[66575-77-7]$} \\
\hline & $(591-895)$ & 157.4 & $(606)$ & EST & {$[99 / 16]$} \\
\hline \multirow[t]{2}{*}{$\mathrm{C}_{59} \mathrm{H}_{112}$} & tripentacontylbenzene & & & & {$[66575-78-8]$} \\
\hline & $(585-891)$ & 155.9 & $(600)$ & & {$[99 / 16]$} \\
\hline \multirow[t]{2}{*}{$\mathrm{C}_{59} \mathrm{H}_{118}$} & tripentacontylcyclohexane & & & & {$[66575-80-2]$} \\
\hline & $(585-892)$ & 155.0 & $(600)$ & & {$[99 / 16]$} \\
\hline \multirow[t]{2}{*}{$\mathrm{C}_{59} \mathrm{H}_{118}$} & 1-nonapentacontene & & & & {$[66575-79-9]$} \\
\hline & $(583-885)$ & 156.4 & $(598)$ & & {$[99 / 16]$} \\
\hline $\mathrm{C}_{59} \mathrm{H}_{120}$ & nonapentacontane & & & & {$[7667-70-0]$} \\
\hline & $(635-890)$ & 161.8 & $(650)$ & A, EST & {$[87 / 5][66 / 8]$} \\
\hline $\mathrm{C}_{59} \mathrm{H}_{120}$ & 2-methyloctapentacontane & & & & {$[66575-49-3]$} \\
\hline & $(583-882)$ & 157.9 & $(598)$ & & {$[99 / 16]$} \\
\hline $\mathrm{C}_{59} \mathrm{H}_{120} \mathrm{~S}$ & 1-nonapentacontanethiol & & & & {$[66575-50-6]$} \\
\hline & $(593-899)$ & 158.3 & $(608)$ & EST & {$[99 / 16]$} \\
\hline $\mathrm{C}_{60} \mathrm{H}_{114}$ & tetrapentacontylbenzene & & & & {$[66575-51-7]$} \\
\hline & $(588-895)$ & 156.6 & $(603)$ & & {$[99 / 16]$} \\
\hline $\mathrm{C}_{60} \mathrm{H}_{120}$ & 1-hexacontene & & & & {$[66575-52-8]$} \\
\hline & $(586-888)$ & 157.1 & $(601)$ & & {$[99 / 16]$} \\
\hline $\mathrm{C}_{60} \mathrm{H}_{120}$ & tetrapentacontylcyclohexane & & & & {$[66575-53-9]$} \\
\hline & $(587-895)$ & 156.0 & $(602)$ & & {$[99 / 16]$} \\
\hline $\mathrm{C}_{60} \mathrm{H}_{122}$ & hexacontane & & & & {$[7667-80-3]$} \\
\hline & $(638-893)$ & 163.0 & $(653)$ & A, EST & {$[87 / 5][66 / 8]$} \\
\hline $\mathrm{C}_{60} \mathrm{H}_{122}$ & 2-methylnonapentacontane & & & & {$[66575-54-0]$} \\
\hline & $(586-886)$ & 158.5 & $(601)$ & & {$[99 / 16]$} \\
\hline $\mathrm{C}_{60} \mathrm{H}_{122} \mathrm{~S}$ & 1-hexacontanethiol & & & & {$[66575-55-1]$} \\
\hline & $(595-902)$ & 159.1 & $(610)$ & EST & [99/16] \\
\hline $\mathrm{C}_{61} \mathrm{H}_{116}$ & pentapentacontylbenzene & & & & {$[66563-50-6]$} \\
\hline & $(590-898)$ & 157.5 & $(605)$ & & {$[99 / 16]$} \\
\hline $\mathrm{C}_{61} \mathrm{H}_{122}$ & 1-henhexacontene & & & & {$[66563-51-7]$} \\
\hline & $(588-891)$ & 158.0 & $(603)$ & & [99/16] \\
\hline $\mathrm{C}_{61} \mathrm{H}_{122}$ & pentapentacontylcyclohexane & & & & {$[66563-52-8]$} \\
\hline & $(590-899)$ & 156.6 & $(605)$ & & {$[99 / 16]$} \\
\hline $\mathrm{C}_{61} \mathrm{H}_{124}$ & henhexacontane & & & & {$[7667-81-4]$} \\
\hline & $(642-897)$ & 163.9 & $(657)$ & $\mathrm{A}, \mathrm{EST}$ & {$[87 / 5][66 / 8]$} \\
\hline $\mathrm{C}_{61} \mathrm{H}_{124}$ & 2-methylhexacontane & & & & {$[66563-53-9]$} \\
\hline & $(588-889)$ & 159.4 & $(603)$ & & {$[99 / 16]$} \\
\hline $\mathrm{C}_{61} \mathrm{H}_{124} \mathrm{~S}$ & 1-henhexacontanethiol & & & & {$[66563-54-0]$} \\
\hline & $(597-905)$ & 159.6 & $(612)$ & EST & {$[99 / 16]$} \\
\hline $\mathrm{C}_{62} \mathrm{H}_{118}$ & hexapentacontylbenzene & & & & {$[66563-55-1]$} \\
\hline & $(592-902)$ & 158.4 & $(607)$ & & {$[99 / 16]$} \\
\hline $\mathrm{C}_{62} \mathrm{H}_{124}$ & 1-dohexacontene & & & & {$[66563-56-2]$} \\
\hline & $(590-895)$ & 158.6 & $(605)$ & & {$[99 / 16]$} \\
\hline
\end{tabular}


TABLE 6. Enthalpies of vaporization of organic compounds, 1880-2002-Continued

\begin{tabular}{|c|c|c|c|c|c|}
\hline Molecular formula & $\begin{array}{c}\text { Compound } \\
\text { (Temperature range/K) }\end{array}$ & $\begin{array}{c}\Delta_{\text {vap }} H_{m} \\
\left(\mathrm{~kJ} \mathrm{~mol}^{-1}\right)\end{array}$ & $\begin{array}{l}\text { Mean temperature } \\
\qquad\left(T_{m} / \mathrm{K}\right)\end{array}$ & Method & $\begin{array}{l}\text { CAS registry number } \\
\text { Reference }\end{array}$ \\
\hline $\mathrm{C}_{62} \mathrm{H}_{126}$ & $\begin{array}{l}\text { hexapentacontylcyclohexane } \\
(592-902)\end{array}$ & 157.5 & $(607)$ & & $\begin{array}{c}{[66563-57-3]} \\
{[99 / 16]}\end{array}$ \\
\hline $\mathrm{C}_{62} \mathrm{H}_{126}$ & dohexacontane & & & & [7719-83-7] \\
\hline & $(645-901)$ & 165.2 & $(660)$ & A, EST & {$[87 / 5][66 / 8]$} \\
\hline $\mathrm{C}_{62} \mathrm{H}_{126}$ & $\begin{array}{l}\text { 2-methylhenhexacontane } \\
(590-892)\end{array}$ & 160.1 & $(605)$ & & $\begin{array}{c}{[66563-58-4]} \\
{[99 / 16]}\end{array}$ \\
\hline $\mathrm{C}_{62} \mathrm{H}_{122} \mathrm{~S}$ & $\begin{array}{l}\text { 1-dohexacontanethiol } \\
\text { (599-908) }\end{array}$ & 160.1 & $(614)$ & EST & $\begin{array}{c}{[66563-59-5]} \\
{[99 / 16]}\end{array}$ \\
\hline $\mathrm{C}_{63} \mathrm{H}_{120}$ & $\begin{array}{l}\text { heptapentacontylbenzene } \\
(595-905)\end{array}$ & 158.9 & (610) & & $\begin{array}{c}{[66563-60-8]} \\
{[99 / 16]}\end{array}$ \\
\hline $\mathrm{C}_{63} \mathrm{H}_{126}$ & $\begin{array}{l}\text { heptapentacontylcyclohexane } \\
(594-905)\end{array}$ & 158.2 & $(609)$ & & $\begin{array}{c}{[66563-61-9]} \\
{[99 / 16]}\end{array}$ \\
\hline $\mathrm{C}_{63} \mathrm{H}_{126}$ & $\begin{array}{l}\text { 1-trihexacontene } \\
(593-899)\end{array}$ & 159.8 & $(608)$ & & $\begin{array}{c}{[66563-62-0]} \\
{[99 / 16]}\end{array}$ \\
\hline $\mathrm{C}_{63} \mathrm{H}_{128}$ & $\begin{array}{l}\text { trihexacontane } \\
(647-904)\end{array}$ & 116.7 & $(662)$ & A, EST & $\begin{array}{l}{[7719-84-8]} \\
{[87 / 5][66 / 8]}\end{array}$ \\
\hline $\mathrm{C}_{63} \mathrm{H}_{128}$ & $\begin{array}{l}\text { 2-methyldohexacontane } \\
(593-897)\end{array}$ & 161.3 & $(608)$ & & $\begin{array}{c}{[66563-63-1]} \\
{[99 / 16]}\end{array}$ \\
\hline $\mathrm{C}_{63} \mathrm{H}_{128} \mathrm{~S}$ & $\begin{array}{l}\text { 1-trihexacontanethiol } \\
(602-911)\end{array}$ & 161.1 & $(617)$ & EST & $\begin{array}{c}{[66563-64-2]} \\
{[99 / 16]}\end{array}$ \\
\hline $\mathrm{C}_{64} \mathrm{H}_{122}$ & $\begin{array}{l}\text { octapentacontylbenzene } \\
(597-908)\end{array}$ & 159.5 & (612) & & $\begin{array}{c}{[66563-65-3]} \\
{[99 / 16]}\end{array}$ \\
\hline $\mathrm{C}_{64} \mathrm{H}_{128}$ & $\begin{array}{l}\text { octapentacontylcyclohexane } \\
(596-908)\end{array}$ & 158.8 & (611) & & $\begin{array}{c}{[66563-66-4]} \\
{[99 / 16]}\end{array}$ \\
\hline $\mathrm{C}_{64} \mathrm{H}_{128}$ & $\begin{array}{l}\text { 1-tetrahexacontene } \\
(595-902)\end{array}$ & 160.5 & $(610)$ & & $\begin{array}{c}{[66563-36-8]} \\
{[99 / 16]}\end{array}$ \\
\hline $\mathrm{C}_{64} \mathrm{H}_{130}$ & $\begin{array}{l}\text { tetrahexacontane } \\
(650-907)\end{array}$ & 168.3 & $(665)$ & A, EST & $\begin{array}{l}{[7719-87-1]} \\
{[87 / 5][66 / 8]}\end{array}$ \\
\hline $\mathrm{C}_{64} \mathrm{H}_{130}$ & $\begin{array}{l}\text { 2-methyltrihexacontane } \\
(595-900)\end{array}$ & 161.9 & $(610)$ & & $\begin{array}{c}{[66563-37-9]} \\
{[99 / 16]}\end{array}$ \\
\hline $\mathrm{C}_{64} \mathrm{H}_{130} \mathrm{~S}$ & $\begin{array}{l}\text { 1-tetrahexacontanethiol } \\
\text { (604-914) }\end{array}$ & 161.6 & (619) & EST & $\begin{array}{c}{[66563-38-0]} \\
{[99 / 16]}\end{array}$ \\
\hline $\mathrm{C}_{65} \mathrm{H}_{124}$ & $\begin{array}{l}\text { nonapentacontylbenzene } \\
\text { (599-911) }\end{array}$ & 160.1 & $(614)$ & & $\begin{array}{c}{[66563-39-1]} \\
{[99 / 16]}\end{array}$ \\
\hline $\mathrm{C}_{65} \mathrm{H}_{130}$ & $\begin{array}{l}\text { nonapentacontylcyclohexane } \\
(599-912)\end{array}$ & 159.9 & $(614)$ & & $\begin{array}{c}{[66563-40-4]} \\
{[99 / 16]}\end{array}$ \\
\hline $\mathrm{C}_{65} \mathrm{H}_{130}$ & $\begin{array}{l}\text { 1-pentahexacontene } \\
(597-905)\end{array}$ & 161.1 & $(612)$ & & $\begin{array}{c}{[66563-41-5]} \\
{[99 / 16]}\end{array}$ \\
\hline $\mathrm{C}_{65} \mathrm{H}_{132}$ & $\begin{array}{l}\text { pentahexacontane } \\
(653-910)\end{array}$ & 169.0 & $(668)$ & A, EST & $\begin{array}{l}{[7719-88-2]} \\
{[87 / 5][66 / 8]}\end{array}$ \\
\hline $\mathrm{C}_{65} \mathrm{H}_{132}$ & $\begin{array}{l}\text { 2-methyltetrahexacontane } \\
\text { (597-903) }\end{array}$ & 162.5 & (612) & & $\begin{array}{c}{[66563-42-6]} \\
{[99 / 16]}\end{array}$ \\
\hline $\mathrm{C}_{65} \mathrm{H}_{132} \mathrm{~S}$ & $\begin{array}{l}\text { 1-pentahexacontanethiol } \\
(606-917)\end{array}$ & 162.1 & $(621)$ & EST & $\begin{array}{c}{[66563-43-7]} \\
{[99 / 16]}\end{array}$ \\
\hline $\mathrm{C}_{66} \mathrm{H}_{126}$ & $\begin{array}{l}\text { hexacontylbenzene } \\
(602-914)\end{array}$ & 161.2 & (617) & & $\begin{array}{c}{[66563-44-8]} \\
{[99 / 16]}\end{array}$ \\
\hline $\mathrm{C}_{66} \mathrm{H}_{132}$ & $\begin{array}{l}\text { hexacontylcyclohexane } \\
(601-915)\end{array}$ & 160.5 & $(616)$ & & $\begin{array}{c}{[66563-45-9]} \\
{[99 / 16]}\end{array}$ \\
\hline $\mathrm{C}_{66} \mathrm{H}_{132}$ & $\begin{array}{l}\text { 1-hexahexacontene } \\
(599-908)\end{array}$ & 161.7 & $(614)$ & & $\begin{array}{c}{[66563-46-0]} \\
{[99 / 16]}\end{array}$ \\
\hline $\mathrm{C}_{66} \mathrm{H}_{134}$ & $\begin{array}{l}\text { hexahexacontane } \\
(656-914)\end{array}$ & 170.0 & (671) & A, EST & $\begin{array}{l}{[7719-89-3]} \\
{[87 / 5][66 / 8]}\end{array}$ \\
\hline $\mathrm{C}_{66} \mathrm{H}_{134}$ & $\begin{array}{l}\text { 2-methylpentahexacontane } \\
\text { (599-906) }\end{array}$ & 163.1 & (614) & & $\begin{array}{c}{[66563-47-1]} \\
{[99 / 16]}\end{array}$ \\
\hline $\mathrm{C}_{66} \mathrm{H}_{134} \mathrm{~S}$ & $\begin{array}{l}\text { 1-hexahexacontanethiol } \\
(607-920)\end{array}$ & 162.8 & $(622)$ & EST & $\begin{array}{c}{[66563-48-2]} \\
{[99 / 16]}\end{array}$ \\
\hline $\mathrm{C}_{67} \mathrm{H}_{128}$ & $\begin{array}{l}\text { henhexacontylbenzene } \\
(603-917)\end{array}$ & 162.1 & (618) & & $\begin{array}{c}{[66563-72-2]} \\
{[99 / 16]}\end{array}$ \\
\hline $\mathrm{C}_{67} \mathrm{H}_{134}$ & $\begin{array}{l}\text { henhexacontylcyclohexane } \\
(603-917)\end{array}$ & 160.9 & (618) & & $\begin{array}{c}{[66563-73-3]} \\
{[99 / 16]}\end{array}$ \\
\hline $\mathrm{C}_{67} \mathrm{H}_{134}$ & $\begin{array}{l}\text { 1-heptahexacontene } \\
(601-911)\end{array}$ & 162.3 & (616) & & $\begin{array}{c}{[66563-74-4]} \\
{[99 / 16]}\end{array}$ \\
\hline $\mathrm{C}_{67} \mathrm{H}_{136}$ & $\begin{array}{l}\text { heptahexacontane } \\
(659-937)\end{array}$ & 170.9 & $(674)$ & A, EST & $\begin{array}{l}{[7719-90-6]} \\
{[87 / 5][66 / 8]}\end{array}$ \\
\hline
\end{tabular}


TABLE 6. Enthalpies of vaporization of organic compounds, 1880-2002-Continued

\begin{tabular}{|c|c|c|c|c|c|}
\hline Molecular formula & $\begin{array}{c}\text { Compound } \\
\text { (Temperature range/K) }\end{array}$ & $\begin{array}{c}\Delta_{\mathrm{vap}} H_{m} \\
\left(\mathrm{~kJ} \mathrm{~mol}^{-1}\right)\end{array}$ & $\begin{array}{l}\text { Mean temperature } \\
\qquad\left(T_{m} / \mathrm{K}\right)\end{array}$ & Method & $\begin{array}{c}\text { CAS registry number } \\
\text { Reference }\end{array}$ \\
\hline $\mathrm{C}_{67} \mathrm{H}_{136}$ & $\begin{array}{l}\text { 2-methylhexahexacontane } \\
(601-909)\end{array}$ & 163.7 & $(616)$ & & $\begin{array}{c}{[66563-75-5]} \\
{[99 / 16]}\end{array}$ \\
\hline $\mathrm{C}_{67} \mathrm{H}_{136} \mathrm{~S}$ & $\begin{array}{l}\text { 1-heptahexacontanethiol } \\
(609-922)\end{array}$ & 163.2 & $(624)$ & EST & $\begin{array}{c}{[66563-76-6]} \\
{[99 / 16]}\end{array}$ \\
\hline $\mathrm{C}_{68} \mathrm{H}_{130}$ & $\begin{array}{l}\text { dohexacontylbenzene } \\
(605-920)\end{array}$ & 162.6 & $(620)$ & & $\begin{array}{c}{[66563-75-5]} \\
{[99 / 16]}\end{array}$ \\
\hline $\mathrm{C}_{68} \mathrm{H}_{136}$ & $\begin{array}{l}\text { dohexacontylcyclohexane } \\
(605-920)\end{array}$ & 161.5 & $(620)$ & & $\begin{array}{c}{[66563-78-8]} \\
{[99 / 16]}\end{array}$ \\
\hline $\mathrm{C}_{68} \mathrm{H}_{136}$ & $\begin{array}{l}\text { 1-octahexacontene } \\
(603-913)\end{array}$ & 162.8 & $(618)$ & & $\begin{array}{c}{[66563-79-9]} \\
{[99 / 16]}\end{array}$ \\
\hline $\mathrm{C}_{68} \mathrm{H}_{138}$ & $\begin{array}{l}\text { octahexacontane } \\
(661-920)\end{array}$ & 172.3 & $(676)$ & A, EST & $\begin{array}{l}{[7719-91-7]} \\
{[87 / 5][66 / 8]}\end{array}$ \\
\hline $\mathrm{C}_{68} \mathrm{H}_{138}$ & $\begin{array}{l}\text { 2-methylheptahexacontane } \\
(603-912)\end{array}$ & 164.3 & $(618)$ & & $\begin{array}{c}{[66563-80-2]} \\
{[99 / 16]}\end{array}$ \\
\hline $\mathrm{C}_{68} \mathrm{H}_{138} \mathrm{~S}$ & $\begin{array}{l}\text { 1-octahexacontanethiol } \\
(611-925)\end{array}$ & 163.6 & $(626)$ & EST & $\begin{array}{c}{[66563-81-3]} \\
{[99 / 16]}\end{array}$ \\
\hline $\mathrm{C}_{69} \mathrm{H}_{132}$ & $\begin{array}{l}\text { trihexacontylbenzene } \\
(607-923)\end{array}$ & 163.1 & $(622)$ & & $\begin{array}{c}{[66563-82-4]} \\
{[99 / 16]}\end{array}$ \\
\hline $\mathrm{C}_{69} \mathrm{H}_{138}$ & $\begin{array}{l}\text { 1-nonhexacontene } \\
(605-916)\end{array}$ & 163.4 & $(620)$ & & $\begin{array}{c}{[66563-83-5]} \\
{[99 / 16]}\end{array}$ \\
\hline $\mathrm{C}_{69} \mathrm{H}_{138}$ & $\begin{array}{l}\text { trihexacontylcyclohexane } \\
(607-923)\end{array}$ & 162.0 & $(622)$ & & $\begin{array}{c}{[66563-93-7]} \\
{[99 / 16]}\end{array}$ \\
\hline $\mathrm{C}_{69} \mathrm{H}_{140}$ & $\begin{array}{l}\text { nonahexacontane } \\
(664-923)\end{array}$ & 173.2 & (679) & A, EST & $\begin{array}{l}{[7719-92-8]} \\
{[87 / 5][66 / 8]}\end{array}$ \\
\hline $\mathrm{C}_{69} \mathrm{H}_{140}$ & $\begin{array}{l}\text { 2-methyloctahexacontane } \\
(605-914)\end{array}$ & 164.9 & $(620)$ & & $\begin{array}{c}{[66563-94-8]} \\
{[99 / 16]}\end{array}$ \\
\hline $\mathrm{C}_{69} \mathrm{H}_{140} \mathrm{~S}$ & $\begin{array}{l}\text { 1-nonahexacontanethiol } \\
(612-928)\end{array}$ & 164.4 & $(627)$ & EST & $\begin{array}{c}{[66577-83-1]} \\
{[99 / 16]}\end{array}$ \\
\hline $\mathrm{C}_{70} \mathrm{H}_{134}$ & $\begin{array}{l}\text { tetrahexacontylbenzene } \\
(609-925)\end{array}$ & 163.6 & $(624)$ & & $\begin{array}{c}{[66577-84-2]} \\
{[99 / 16]}\end{array}$ \\
\hline $\mathrm{C}_{70} \mathrm{H}_{140}$ & $\begin{array}{l}\text { 1-heptacontene } \\
(607-919)\end{array}$ & 163.9 & $(622)$ & & $\begin{array}{c}{[66577-85-3]} \\
{[99 / 16]}\end{array}$ \\
\hline $\mathrm{C}_{70} \mathrm{H}_{140}$ & $\begin{array}{l}\text { tetrahexacontylcyclohexane } \\
(608-926)\end{array}$ & 162.8 & (623) & & $\begin{array}{c}{[66577-86-4]} \\
{[99 / 16]}\end{array}$ \\
\hline $\mathrm{C}_{70} \mathrm{H}_{142}$ & $\begin{array}{l}\text { heptacontane } \\
(666-926)\end{array}$ & 174.4 & $(681)$ & A, EST & $\begin{array}{l}{[7719-93-9]} \\
{[87 / 5][66 / 8]}\end{array}$ \\
\hline $\mathrm{C}_{70} \mathrm{H}_{142}$ & $\begin{array}{l}\text { 2-methylnonahexacontane } \\
(607-917)\end{array}$ & 165.4 & $(622)$ & & $\begin{array}{c}{[66577-87-5]} \\
{[99 / 16]}\end{array}$ \\
\hline $\mathrm{C}_{70} \mathrm{H}_{142} \mathrm{~S}$ & $\begin{array}{l}\text { 1-heptacontanethiol } \\
(614-930)\end{array}$ & 164.8 & $(621)$ & EST & $\begin{array}{c}{[66577-88-6]} \\
{[99 / 16]}\end{array}$ \\
\hline $\mathrm{C}_{71} \mathrm{H}_{136}$ & $\begin{array}{l}\text { pentahexacontylbenzene } \\
(611-928)\end{array}$ & 164.4 & $(626)$ & & $\begin{array}{c}{[66577-89-7]} \\
{[99 / 16]}\end{array}$ \\
\hline $\mathrm{C}_{71} \mathrm{H}_{142}$ & $\begin{array}{l}\text { 1-henheptacontene } \\
\text { (609-922) }\end{array}$ & 164.4 & (624) & & $\begin{array}{c}{[66577-90-0]} \\
{[99 / 16]}\end{array}$ \\
\hline $\mathrm{C}_{71} \mathrm{H}_{142}$ & $\begin{array}{l}\text { pentahexacontylcyclohexane } \\
(610-928)\end{array}$ & 163.3 & $(625)$ & & $\begin{array}{c}{[66577-91-1]} \\
{[99 / 16]}\end{array}$ \\
\hline $\mathrm{C}_{71} \mathrm{H}_{144}$ & $\begin{array}{l}\text { henheptacontane } \\
(669-928)\end{array}$ & 175.2 & $(684)$ & $\mathrm{A}, \mathrm{EST}$ & $\begin{array}{l}{[7667-82-5]} \\
{[87 / 5][66 / 8]}\end{array}$ \\
\hline $\mathrm{C}_{71} \mathrm{H}_{144}$ & $\begin{array}{l}\text { 2-methylheptacontane } \\
(609-920)\end{array}$ & 165.9 & (624) & & $\begin{array}{c}{[66577-92-2]} \\
{[99 / 16]}\end{array}$ \\
\hline $\mathrm{C}_{71} \mathrm{H}_{144} \mathrm{~S}$ & $\begin{array}{l}\text { 1-henheptacontanethiol } \\
(616-933)\end{array}$ & 165.1 & (631) & EST & $\begin{array}{c}{[66577-93-3]} \\
{[99 / 16]}\end{array}$ \\
\hline $\mathrm{C}_{72} \mathrm{H}_{138}$ & $\begin{array}{l}\text { hexahexacontylbenzene } \\
(613-931)\end{array}$ & 164.5 & (628) & & $\begin{array}{c}{[66577-94-4]} \\
{[99 / 16]}\end{array}$ \\
\hline $\mathrm{C}_{72} \mathrm{H}_{144}$ & $\begin{array}{l}\text { 1-doheptacontene } \\
(610-924)\end{array}$ & 165.3 & $(625)$ & & $\begin{array}{c}{[66577-95-5]} \\
{[99 / 16]}\end{array}$ \\
\hline $\mathrm{C}_{72} \mathrm{H}_{144}$ & $\begin{array}{l}\text { hexahexacontylcyclohexane } \\
(612-931)\end{array}$ & 163.8 & $(627)$ & & $\begin{array}{c}{[66577-96-6]} \\
{[99 / 16]}\end{array}$ \\
\hline $\mathrm{C}_{72} \mathrm{H}_{146}$ & $\begin{array}{l}\text { doheptacontane } \\
(671-931)\end{array}$ & 176.4 & $(686)$ & A, EST & $\begin{array}{l}{[7668-83-6]} \\
{[87 / 5][66 / 8]}\end{array}$ \\
\hline $\mathrm{C}_{72} \mathrm{H}_{146}$ & $\begin{array}{l}\text { 2-methylhenheptacontane } \\
\text { (611-923) }\end{array}$ & 166.4 & (626) & & $\begin{array}{c}{[66577-97-7]} \\
{[99 / 16]}\end{array}$ \\
\hline $\mathrm{C}_{72} \mathrm{H}_{146} \mathrm{~S}$ & $\begin{array}{l}\text { 1-doheptacontanethiol } \\
\text { (617-935) }\end{array}$ & 165.8 & $(632)$ & EST & $\begin{array}{c}{[66577-98-8]} \\
{[99 / 16]}\end{array}$ \\
\hline
\end{tabular}


TABLE 6. Enthalpies of vaporization of organic compounds, 1880-2002-Continued

\begin{tabular}{|c|c|c|c|c|c|}
\hline Molecular formula & $\begin{array}{c}\text { Compound } \\
\text { (Temperature range/K) }\end{array}$ & $\begin{array}{c}\Delta_{\mathrm{vap}} H_{m} \\
\left(\mathrm{~kJ} \mathrm{~mol}^{-1}\right)\end{array}$ & $\begin{array}{l}\text { Mean temperature } \\
\qquad\left(T_{m} / \mathrm{K}\right)\end{array}$ & Method & $\begin{array}{l}\text { CAS registry number } \\
\text { Reference }\end{array}$ \\
\hline $\mathrm{C}_{73} \mathrm{H}_{140}$ & $\begin{array}{l}\text { heptahexacontylbenzene } \\
(614-933)\end{array}$ & 165.4 & $(629)$ & & $\begin{array}{c}{[66577-99-9]} \\
{[99 / 16]}\end{array}$ \\
\hline $\mathrm{C}_{73} \mathrm{H}_{146}$ & $\begin{array}{l}\text { heptahexacontylcyclohexane } \\
(614-933)\end{array}$ & 164.2 & $(629)$ & & $\begin{array}{c}{[66578-00-5]} \\
{[99 / 16]}\end{array}$ \\
\hline $\mathrm{C}_{73} \mathrm{H}_{146}$ & $\begin{array}{l}\text { 1-triheptacontene } \\
(612-927)\end{array}$ & 165.7 & $(627)$ & & $\begin{array}{c}{[66578-01-6]} \\
{[99 / 16]}\end{array}$ \\
\hline $\mathrm{C}_{73} \mathrm{H}_{148}$ & $\begin{array}{l}\text { triheptacontane } \\
(674-934)\end{array}$ & 177.1 & $(689)$ & A, EST & $\begin{array}{l}{[7667-84-7]} \\
{[87 / 5][66 / 8]}\end{array}$ \\
\hline $\mathrm{C}_{73} \mathrm{H}_{148}$ & $\begin{array}{l}\text { 2-methyldoheptacontane } \\
\text { (613-926) }\end{array}$ & 166.9 & (628) & & $\begin{array}{c}{[66578-02-7]} \\
{[99 / 16]}\end{array}$ \\
\hline $\mathrm{C}_{73} \mathrm{H}_{148} \mathrm{~S}$ & $\begin{array}{l}\text { 1-triheptacontanethiol } \\
\text { (619-938) }\end{array}$ & 166.2 & $(634)$ & EST & $\begin{array}{c}{[66577-64-8]} \\
{[99 / 16]}\end{array}$ \\
\hline $\mathrm{C}_{74} \mathrm{H}_{142}$ & $\begin{array}{l}\text { octahexacontylbenzene } \\
(616-936)\end{array}$ & 165.8 & $(631)$ & & $\begin{array}{c}{[66577-65-9]} \\
{[99 / 16]}\end{array}$ \\
\hline $\mathrm{C}_{74} \mathrm{H}_{148}$ & $\begin{array}{l}\text { octahexacontylcyclohexane } \\
(615-936)\end{array}$ & 165.0 & $(630)$ & & $\begin{array}{c}{[66577-66-0]} \\
{[99 / 16]}\end{array}$ \\
\hline $\mathrm{C}_{74} \mathrm{H}_{148}$ & $\begin{array}{l}\text { 1-tetraheptacontene } \\
(614-930)\end{array}$ & 166.2 & $(629)$ & & $\begin{array}{c}{[66577-67-1]} \\
{[99 / 16]}\end{array}$ \\
\hline $\mathrm{C}_{74} \mathrm{H}_{150}$ & $\begin{array}{l}\text { tetraheptacontane } \\
(676-936)\end{array}$ & 178.2 & $(691)$ & A, EST & $\begin{array}{l}{[7667-85-8]} \\
{[87 / 5][66 / 8]}\end{array}$ \\
\hline $\mathrm{C}_{74} \mathrm{H}_{150}$ & $\begin{array}{l}\text { 2-methyltriheptacontane } \\
(615-928)\end{array}$ & 167.4 & (630) & & $\begin{array}{c}{[66577-68-2]} \\
{[99 / 16]}\end{array}$ \\
\hline $\mathrm{C}_{74} \mathrm{H}_{150} \mathrm{~S}$ & $\begin{array}{l}\text { 1-tetraheptacontanethiol } \\
(620-940)\end{array}$ & 166.9 & $(635)$ & EST & $\begin{array}{c}{[66577-69-3]} \\
{[99 / 16]}\end{array}$ \\
\hline $\mathrm{C}_{75} \mathrm{H}_{144}$ & $\begin{array}{l}\text { nonahexacontylbenzene } \\
(618-938)\end{array}$ & 166.3 & $(633)$ & & $\begin{array}{c}{[66577-70-6]} \\
{[99 / 16]}\end{array}$ \\
\hline $\mathrm{C}_{75} \mathrm{H}_{150}$ & $\begin{array}{l}\text { nonahexacontylcyclohexane } \\
(617-632)\end{array}$ & 165.4 & $(632)$ & & $\begin{array}{c}{[66577-71-7]} \\
{[99 / 16]}\end{array}$ \\
\hline $\mathrm{C}_{75} \mathrm{H}_{150}$ & $\begin{array}{l}\text { 1-pentaheptacontene } \\
(616-932)\end{array}$ & 166.7 & $(631)$ & & $\begin{array}{c}{[66577-72-8]} \\
{[99 / 16]}\end{array}$ \\
\hline $\mathrm{C}_{75} \mathrm{H}_{152}$ & $\begin{array}{l}\text { pentaheptacontane } \\
(678-939)\end{array}$ & 179.4 & (693) & A, EST & $\begin{array}{l}{[7667-86-9]} \\
{[87 / 5][66 / 8]}\end{array}$ \\
\hline $\mathrm{C}_{75} \mathrm{H}_{152}$ & $\begin{array}{l}\text { 2-methyltetraheptacontane } \\
(616-931)\end{array}$ & 168.2 & (631) & & $\begin{array}{c}{[66577-73-9]} \\
{[99 / 16]}\end{array}$ \\
\hline $\mathrm{C}_{75} \mathrm{H}_{152} \mathrm{~S}$ & $\begin{array}{l}\text { 1-pentaheptacontanethiol } \\
(622-942)\end{array}$ & 167.2 & $(637)$ & EST & $\begin{array}{c}{[66577-74-0]} \\
{[99 / 16]}\end{array}$ \\
\hline $\mathrm{C}_{76} \mathrm{H}_{146}$ & $\begin{array}{l}\text { heptacontylbenzene } \\
\text { (619-941) }\end{array}$ & 167.0 & $(634)$ & & $\begin{array}{c}{[66577-75-1]} \\
{[99 / 16]}\end{array}$ \\
\hline $\mathrm{C}_{76} \mathrm{H}_{152}$ & $\begin{array}{l}\text { heptacontylcyclohexane } \\
(619-941)\end{array}$ & 165.8 & $(634)$ & & $\begin{array}{c}{[66577-76-2]} \\
{[99 / 16]}\end{array}$ \\
\hline $\mathrm{C}_{76} \mathrm{H}_{152}$ & $\begin{array}{l}\text { 1-hexaheptacontene } \\
(617-935)\end{array}$ & 167.5 & (632) & & $\begin{array}{c}{[66577-77-3]} \\
{[99 / 16]}\end{array}$ \\
\hline $\mathrm{C}_{76} \mathrm{H}_{154}$ & $\begin{array}{l}\text { hexaheptacontane } \\
(680-941)\end{array}$ & 180.4 & (695) & A, EST & $\begin{array}{l}{[7667-87-0]} \\
{[87 / 5][66 / 8]}\end{array}$ \\
\hline $\mathrm{C}_{76} \mathrm{H}_{154}$ & $\begin{array}{l}\text { 2-methylpentaheptacontane } \\
(618-933)\end{array}$ & 168.7 & (633) & & $\begin{array}{c}{[66577-78-4]} \\
{[99 / 16]}\end{array}$ \\
\hline $\mathrm{C}_{76} \mathrm{H}_{154} \mathrm{~S}$ & $\begin{array}{l}\text { 1-hexaheptacontanethiol } \\
(623-945)\end{array}$ & 169.8 & $(638)$ & EST & $\begin{array}{c}{[66577-79-5]} \\
{[99 / 16]}\end{array}$ \\
\hline $\mathrm{C}_{77} \mathrm{H}_{148}$ & $\begin{array}{l}\text { henheptacontylbenzene } \\
(621-943)\end{array}$ & 167.4 & $(636)$ & & $\begin{array}{c}{[66577-80-8]} \\
{[99 / 16]}\end{array}$ \\
\hline $\mathrm{C}_{77} \mathrm{H}_{154}$ & $\begin{array}{l}\text { henheptacontylcyclohexane } \\
(620-943)\end{array}$ & 166.6 & $(635)$ & & $\begin{array}{c}{[66577-81-9]} \\
{[99 / 16]}\end{array}$ \\
\hline $\mathrm{C}_{77} \mathrm{H}_{154}$ & $\begin{array}{l}\text { 1-heptaheptacontene } \\
(619-937)\end{array}$ & 167.9 & (634) & & $\begin{array}{c}{[66577-82-0]} \\
{[99 / 16]}\end{array}$ \\
\hline $\mathrm{C}_{77} \mathrm{H}_{156}$ & $\begin{array}{l}\text { heptaheptacontane } \\
(682-944)\end{array}$ & 181.4 & $(697)$ & A, EST & $\begin{array}{l}{[7719-94-0]} \\
{[87 / 5][66 / 8]}\end{array}$ \\
\hline $\mathrm{C}_{77} \mathrm{H}_{156}$ & $\begin{array}{l}\text { 2-methylhexaheptacontane } \\
(620-936)\end{array}$ & 169.1 & $(635)$ & & $\begin{array}{c}{[66575-56-2]} \\
{[99 / 16]}\end{array}$ \\
\hline $\mathrm{C}_{77} \mathrm{H}_{156} \mathrm{~S}$ & $\begin{array}{l}\text { 1-heptaheptacontanethiol } \\
(625-947)\end{array}$ & 168.2 & (640) & EST & $\begin{array}{c}{[66575-57-3]} \\
{[99 / 16]}\end{array}$ \\
\hline $\mathrm{C}_{78} \mathrm{H}_{150}$ & $\begin{array}{l}\text { doheptacontylbenzene } \\
(622-945)\end{array}$ & 167.3 & (637) & & $\begin{array}{c}{[66327-30-8]} \\
{[99 / 16]}\end{array}$ \\
\hline $\mathrm{C}_{78} \mathrm{H}_{156}$ & $\begin{array}{l}\text { doheptacontylcyclohexane } \\
(622-945)\end{array}$ & 166.9 & (637) & & $\begin{array}{c}{[66327-31-9]} \\
{[99 / 16]}\end{array}$ \\
\hline
\end{tabular}


TABLE 6. Enthalpies of vaporization of organic compounds, 1880-2002-Continued

\begin{tabular}{|c|c|c|c|c|c|}
\hline Molecular formula & $\begin{array}{c}\text { Compound } \\
\text { (Temperature range/K) }\end{array}$ & $\begin{array}{c}\Delta_{\mathrm{vap}} H_{m} \\
\left(\mathrm{~kJ} \mathrm{~mol}^{-1}\right)\end{array}$ & $\begin{array}{l}\text { Mean temperature } \\
\qquad\left(T_{m} / \mathrm{K}\right)\end{array}$ & Method & $\begin{array}{c}\text { CAS registry number } \\
\text { Reference }\end{array}$ \\
\hline $\mathrm{C}_{78} \mathrm{H}_{156}$ & $\begin{array}{l}\text { 1-octaheptacontene } \\
(621-940)\end{array}$ & 168.3 & $(636)$ & & $\begin{array}{c}{[66327-32-0]} \\
{[99 / 16]}\end{array}$ \\
\hline $\mathrm{C}_{78} \mathrm{H}_{158}$ & $\begin{array}{l}\text { octaheptacontane } \\
(685-946)\end{array}$ & 181.8 & $(700)$ & A, EST & $\begin{array}{l}{[7719-85-9]} \\
{[87 / 5][66 / 8]}\end{array}$ \\
\hline $\mathrm{C}_{78} \mathrm{H}_{158}$ & $\begin{array}{l}\text { 2-methylheptaheptacontane } \\
(621-939)\end{array}$ & 169.9 & $(636)$ & & $\begin{array}{c}{[66327-33-1]} \\
{[99 / 16]}\end{array}$ \\
\hline $\mathrm{C}_{78} \mathrm{H}_{158} \mathrm{~S}$ & $\begin{array}{l}\text { 1-octaheptacontanethiol } \\
(626-949)\end{array}$ & 168.8 & $(641)$ & EST & $\begin{array}{c}{[66375-13-1]} \\
{[99 / 16]}\end{array}$ \\
\hline $\mathrm{C}_{79} \mathrm{H}_{152}$ & $\begin{array}{l}\text { triheptacontylbenzene } \\
(623-947)\end{array}$ & 168.0 & $(638)$ & & $\begin{array}{c}{[66327-34-2]} \\
{[99 / 16]}\end{array}$ \\
\hline $\mathrm{C}_{79} \mathrm{H}_{158}$ & $\begin{array}{l}\text { 1-nonaheptacontene } \\
(622-942)\end{array}$ & 169.1 & $(637)$ & & $\begin{array}{c}{[66327-35-3]} \\
{[99 / 16]}\end{array}$ \\
\hline $\mathrm{C}_{79} \mathrm{H}_{158}$ & $\begin{array}{l}\text { triheptacontylcyclohexane } \\
(623-948)\end{array}$ & 167.7 & $(638)$ & & $\begin{array}{c}{[66327-36-4]} \\
{[99 / 16]}\end{array}$ \\
\hline $\mathrm{C}_{79} \mathrm{H}_{160}$ & $\begin{array}{l}\text { nonaheptacontane } \\
(687-949)\end{array}$ & 182.7 & $(702)$ & A, EST & $\begin{array}{l}{[7719-86-0]} \\
{[87 / 5][66 / 8]}\end{array}$ \\
\hline $\mathrm{C}_{79} \mathrm{H}_{160}$ & $\begin{array}{l}\text { 2-methyloctaheptacontane } \\
(622-940)\end{array}$ & 167.8 & $(637)$ & & $\begin{array}{c}{[66327-37-5]} \\
{[99 / 16]}\end{array}$ \\
\hline $\mathrm{C}_{79} \mathrm{H}_{160} \mathrm{~S}$ & $\begin{array}{l}\text { 1-nonaheptacontanethiol } \\
(628-952)\end{array}$ & 169.1 & $(643)$ & EST & $\begin{array}{c}{[66327-38-6]} \\
{[99 / 16]}\end{array}$ \\
\hline $\mathrm{C}_{80} \mathrm{H}_{154}$ & $\begin{array}{l}\text { tetraheptacontylbenzene } \\
(625-949)\end{array}$ & 168.4 & $(640)$ & & $\begin{array}{c}{[66327-39-7]} \\
{[99 / 16]}\end{array}$ \\
\hline $\mathrm{C}_{80} \mathrm{H}_{160}$ & $\begin{array}{l}\text { 1-octacontene } \\
(624-945)\end{array}$ & 169.4 & $(639)$ & & $\begin{array}{c}{[66327-40-0]} \\
{[99 / 16]}\end{array}$ \\
\hline $\mathrm{C}_{80} \mathrm{H}_{160}$ & $\begin{array}{l}\text { tetraheptacontylcyclohexane } \\
(625-950)\end{array}$ & 168.0 & $(640)$ & & $\begin{array}{c}{[66327-41-1]} \\
{[99 / 16]}\end{array}$ \\
\hline $\mathrm{C}_{80} \mathrm{H}_{162}$ & $\begin{array}{l}\text { octacontane } \\
(689-951)\end{array}$ & 183.6 & $(704)$ & $\mathrm{A}, \mathrm{EST}$ & $\begin{array}{l}{[7667-88-1]} \\
{[87 / 5][66 / 8]}\end{array}$ \\
\hline $\mathrm{C}_{80} \mathrm{H}_{162}$ & $\begin{array}{l}\text { 2-methylnonaheptacontane } \\
(624-943)\end{array}$ & 170.2 & (639) & & $\begin{array}{c}{[66327-42-2]} \\
{[99 / 16]}\end{array}$ \\
\hline $\mathrm{C}_{80} \mathrm{H}_{162} \mathrm{~S}$ & $\begin{array}{l}\text { 1-octacontanethiol } \\
(629-954)\end{array}$ & 169.6 & $(644)$ & EST & $\begin{array}{c}{[66327-43-3]} \\
{[99 / 16]}\end{array}$ \\
\hline $\mathrm{C}_{81} \mathrm{H}_{156}$ & $\begin{array}{l}\text { pentaheptacontylbenzene } \\
(636-952)\end{array}$ & 169.1 & $(641)$ & & $\begin{array}{c}{[66327-44-4]} \\
{[99 / 16]}\end{array}$ \\
\hline $\mathrm{C}_{81} \mathrm{H}_{162}$ & $\begin{array}{l}\text { 1-henoctacontene } \\
(625-946)\end{array}$ & 169.3 & $(640)$ & & $\begin{array}{c}{[66327-45-5]} \\
{[99 / 16]}\end{array}$ \\
\hline $\mathrm{C}_{81} \mathrm{H}_{162}$ & $\begin{array}{l}\text { pentaheptacontylcyclohexane } \\
(627-952)\end{array}$ & 168.4 & $(642)$ & & $\begin{array}{c}{[66327-46-6]} \\
{[99 / 16]}\end{array}$ \\
\hline $\mathrm{C}_{81} \mathrm{H}_{164}$ & $\begin{array}{l}\text { henoctacontane } \\
(691-953)\end{array}$ & 184.5 & $(706)$ & A, EST & $\begin{array}{l}{[7667-89-2]} \\
{[87 / 5][66 / 8]}\end{array}$ \\
\hline $\mathrm{C}_{81} \mathrm{H}_{164}$ & $\begin{array}{l}\text { 2-methyloctancontane } \\
(625-945)\end{array}$ & 170.9 & $(640)$ & & $\begin{array}{c}{[66327-47-7]} \\
{[99 / 16]}\end{array}$ \\
\hline $\mathrm{C}_{81} \mathrm{H}_{164} \mathrm{~S}$ & $\begin{array}{l}\text { 1-henoctacontanethiol } \\
(630-955)\end{array}$ & 169.4 & $(645)$ & EST & $\begin{array}{c}{[66327-48-8]} \\
{[99 / 16]}\end{array}$ \\
\hline $\mathrm{C}_{82} \mathrm{H}_{158}$ & $\begin{array}{l}\text { hexaheptacontylbenzene } \\
(628-954)\end{array}$ & 169.4 & $(643)$ & & $\begin{array}{c}{[66327-49-9]} \\
{[99 / 16]}\end{array}$ \\
\hline $\mathrm{C}_{82} \mathrm{H}_{164}$ & $\begin{array}{l}\text { 1-dooctacontene } \\
(626-949)\end{array}$ & 157.6 & $(641)$ & & $\begin{array}{l}{[66327-50-2]} \\
{[99 / 16]}\end{array}$ \\
\hline $\mathrm{C}_{82} \mathrm{H}_{164}$ & $\begin{array}{l}\text { hexaheptacontylcyclohexane } \\
(627-954)\end{array}$ & 168.5 & $(642)$ & & $\begin{array}{c}{[66327-09-1]} \\
{[99 / 16]}\end{array}$ \\
\hline $\mathrm{C}_{82} \mathrm{H}_{166}$ & $\begin{array}{l}\text { dooctacontane } \\
(693-955)\end{array}$ & 185.3 & $(708)$ & A, EST & $\begin{array}{l}{[7719-95-1]} \\
{[87 / 5][66 / 8]}\end{array}$ \\
\hline $\mathrm{C}_{82} \mathrm{H}_{166}$ & $\begin{array}{l}\text { 2-methylhenoctacontane } \\
(627-947)\end{array}$ & 171.3 & $(642)$ & & $\begin{array}{c}{[66327-10-4]} \\
{[99 / 16]}\end{array}$ \\
\hline $\mathrm{C}_{82} \mathrm{H}_{166} \mathrm{~S}$ & $\begin{array}{l}\text { 1-dooctacontanethiol } \\
(631-957)\end{array}$ & 170.0 & $(646)$ & EST & $\begin{array}{l}{[66327-11-5]} \\
{[99 / 16]}\end{array}$ \\
\hline $\mathrm{C}_{83} \mathrm{H}_{160}$ & $\begin{array}{l}\text { heptaheptacontylbenzene } \\
(628-955)\end{array}$ & 169.6 & (643) & & $\begin{array}{c}{[66327-12-6]} \\
{[99 / 16]}\end{array}$ \\
\hline $\mathrm{C}_{83} \mathrm{H}_{166}$ & $\begin{array}{l}\text { heptaheptaconylcyclohexane } \\
(629-956)\end{array}$ & 168.9 & $(644)$ & & $\begin{array}{c}{[66327-13-7]} \\
{[99 / 16]}\end{array}$ \\
\hline $\mathrm{C}_{83} \mathrm{H}_{166}$ & $\begin{array}{l}\text { 1-trioctacontene } \\
(628-951)\end{array}$ & 170.4 & (643) & & $\begin{array}{c}{[66327-14-8]} \\
{[99 / 16]}\end{array}$ \\
\hline $\mathrm{C}_{83} \mathrm{H}_{168}$ & $\begin{array}{l}\text { trioctacontane } \\
(694-957)\end{array}$ & 186.5 & (709) & A, EST & $\begin{array}{l}{[7667-90-5]} \\
{[87 / 5][66 / 8]}\end{array}$ \\
\hline
\end{tabular}


TABLE 6. Enthalpies of vaporization of organic compounds, 1880-2002-Continued

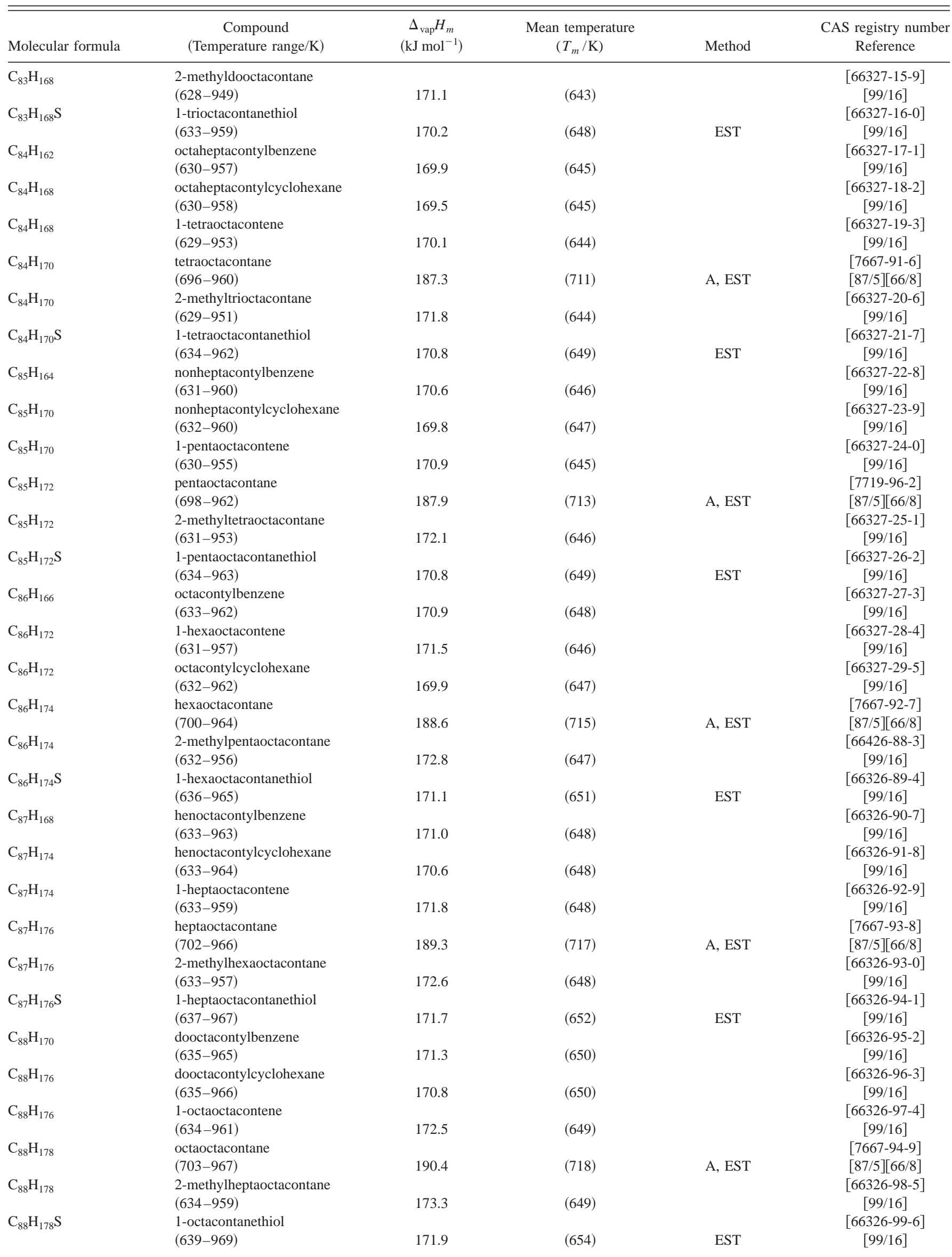


TABLE 6. Enthalpies of vaporization of organic compounds, 1880-2002-Continued

\begin{tabular}{|c|c|c|c|c|c|}
\hline Molecular formula & $\begin{array}{c}\text { Compound } \\
\text { (Temperature range/K) }\end{array}$ & $\begin{array}{c}\Delta_{\mathrm{vap}} H_{m} \\
\left(\mathrm{~kJ} \mathrm{~mol}^{-1}\right)\end{array}$ & $\begin{array}{l}\text { Mean temperature } \\
\qquad\left(T_{m} / \mathrm{K}\right)\end{array}$ & Method & $\begin{array}{c}\text { CAS registry number } \\
\text { Reference }\end{array}$ \\
\hline \multirow[t]{2}{*}{$\mathrm{C}_{89} \mathrm{H}_{172}$} & trioctacontylbenzene & & & & [66327-00-2] \\
\hline & $(636-967)$ & 172.0 & $(651)$ & & [99/16] \\
\hline \multirow[t]{2}{*}{$\mathrm{C}_{89} \mathrm{H}_{178}$} & 1-nonaoctacontene & & & & [66327-01-3] \\
\hline & $(635-962)$ & 172.3 & $(650)$ & & [99/16] \\
\hline \multirow[t]{2}{*}{$\mathrm{C}_{89} \mathrm{H}_{178}$} & trioctacontylcyclohexane & & & & [66327-02-4] \\
\hline & $(636-968)$ & 171.5 & $(651)$ & & {$[99 / 16]$} \\
\hline \multirow[t]{2}{*}{$\mathrm{C}_{89} \mathrm{H}_{180}$} & nonaoctacontane & & & & {$[7719-76-8]$} \\
\hline & $(705-969)$ & 190.9 & $(720)$ & A, EST & {$[87 / 5][66 / 8]$} \\
\hline \multirow[t]{2}{*}{$\mathrm{C}_{89} \mathrm{H}_{180}$} & 2-methyloctaoctacontane & & & & {$[66327-03-5]$} \\
\hline & $(636-962)$ & 173.6 & $(651)$ & & [99/16] \\
\hline \multirow[t]{2}{*}{$\mathrm{C}_{89} \mathrm{H}_{180} \mathrm{~S}$} & 1-nonaoctacontanethiol & & & & [66327-04-6] \\
\hline & $(639-970)$ & 171.9 & $(654)$ & EST & {$[99 / 16]$} \\
\hline \multirow[t]{2}{*}{$\mathrm{C}_{90} \mathrm{H}_{174}$} & tetraoctacontylbenzene & & & & [66327-05-7] \\
\hline & $(637-968)$ & 171.7 & $(652)$ & & {$[99 / 16]$} \\
\hline \multirow[t]{2}{*}{$\mathrm{C}_{90} \mathrm{H}_{182}$} & nonacontane & & & & {$[7667-51-8]$} \\
\hline & $(707-971)$ & 191.6 & $(722)$ & A, EST & {$[87 / 5][66 / 8]$} \\
\hline \multirow[t]{2}{*}{$\mathrm{C}_{91} \mathrm{H}_{184}$} & hennonacontane & & & & [7719-97-3] \\
\hline & $(708-973)$ & 192.5 & $(723)$ & A, EST & {$[87 / 5][66 / 8]$} \\
\hline \multirow[t]{2}{*}{$\mathrm{C}_{92} \mathrm{H}_{186}$} & dononacontane & & & & {$[7667-95-0]$} \\
\hline & $(710-975)$ & 193.0 & $(725)$ & A, EST & {$[87 / 5][66 / 8]$} \\
\hline \multirow[t]{2}{*}{$\mathrm{C}_{93} \mathrm{H}_{188}$} & trinonacontane & & & & {$[7667-96-1]$} \\
\hline & $(711-977)$ & 194.1 & $(726)$ & A, EST & {$[87 / 5][66 / 8]$} \\
\hline \multirow[t]{2}{*}{$\mathrm{C}_{94} \mathrm{H}_{190}$} & tetranonacontane & & & & {$[1574-32-9]$} \\
\hline & $(713-978)$ & 194.5 & $(728)$ & $\mathrm{A}, \mathrm{EST}$ & {$[87 / 5][66 / 8]$} \\
\hline \multirow[t]{2}{*}{$\mathrm{C}_{95} \mathrm{H}_{192}$} & pentanonacontane & & & & {$[7667-97-2]$} \\
\hline & $(714-980)$ & 195.4 & $(729)$ & A, EST & {$[87 / 5][66 / 8]$} \\
\hline \multirow[t]{2}{*}{$\mathrm{C}_{96} \mathrm{H}_{194}$} & hexanonacontane & & & & {$[7763-13-5]$} \\
\hline & $(716-982)$ & 195.8 & $(731)$ & A, EST & {$[87 / 5][66 / 8]$} \\
\hline \multirow[t]{2}{*}{$\mathrm{C}_{97} \mathrm{H}_{196}$} & heptanonacontane & & & & {$[7670-25-9]$} \\
\hline & $(717-983)$ & 196.6 & $(732)$ & $\mathrm{A}, \mathrm{EST}$ & {$[87 / 5][66 / 8]$} \\
\hline \multirow[t]{2}{*}{$\mathrm{C}_{98} \mathrm{H}_{198}$} & octanonacontane & & & & {$[7670-26-0]$} \\
\hline & $(719-985)$ & 196.9 & $(734)$ & A, EST & {$[87 / 5][66 / 8]$} \\
\hline \multirow[t]{2}{*}{$\mathrm{C}_{99} \mathrm{H}_{200}$} & nonanonacontane & & & & {$[7670-27-1]$} \\
\hline & $(720-986)$ & 197.8 & $(735)$ & A, EST & {$[87 / 5][66 / 8]$} \\
\hline \multirow[t]{2}{*}{$\mathrm{C}_{100} \mathrm{H}_{202}$} & hectane & & & & [6703-98-6] \\
\hline & $(721-988)$ & 198.5 & $(736)$ & A, EST & {$[87 / 5][66 / 8]$} \\
\hline
\end{tabular}


TABLE 7. Enthalpies of vaporization of select organo-metallic and inorganic compounds, 1880-2002

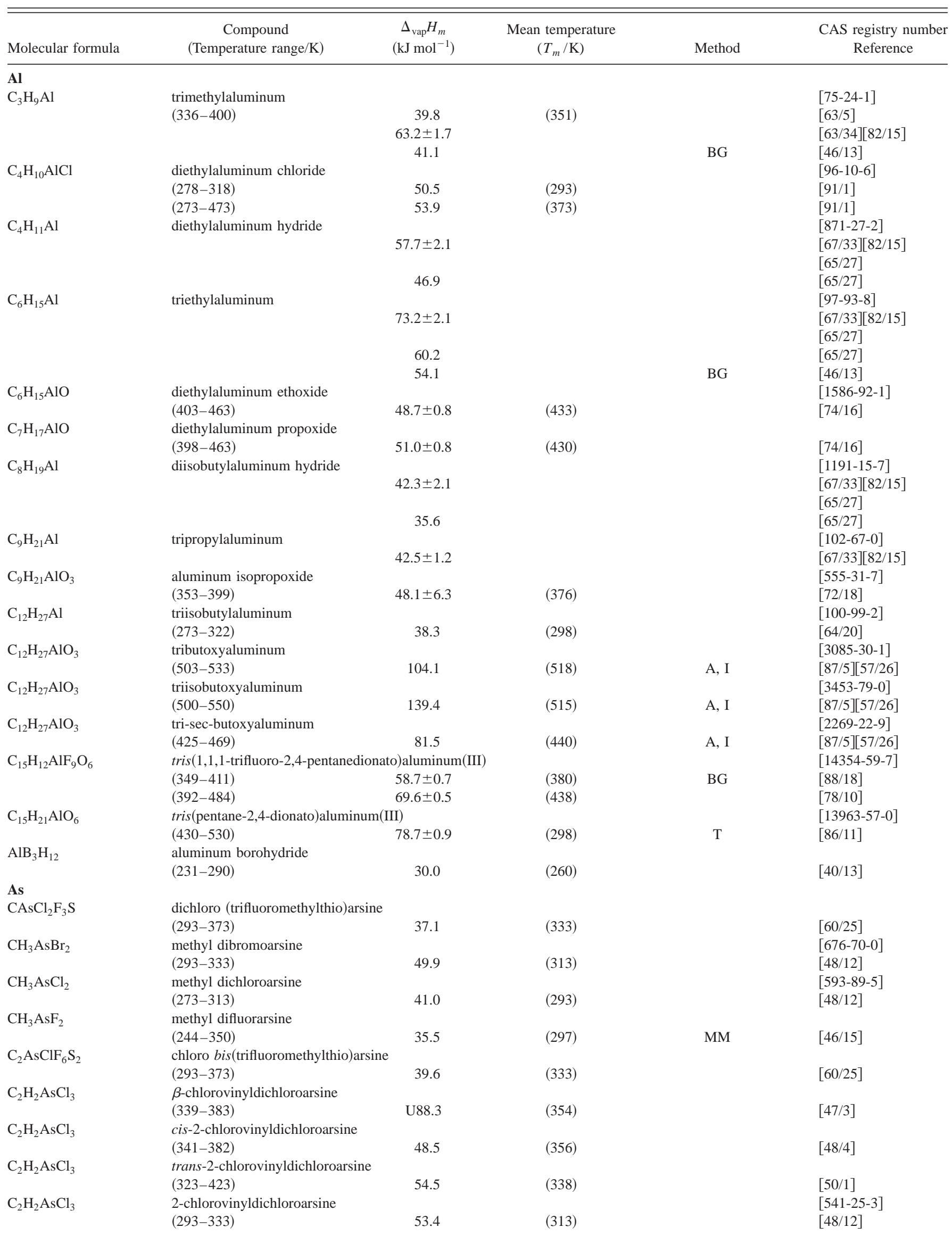


TABLE 7. Enthalpies of vaporization of select organo-metallic and inorganic compounds, 1880-2002-Continued

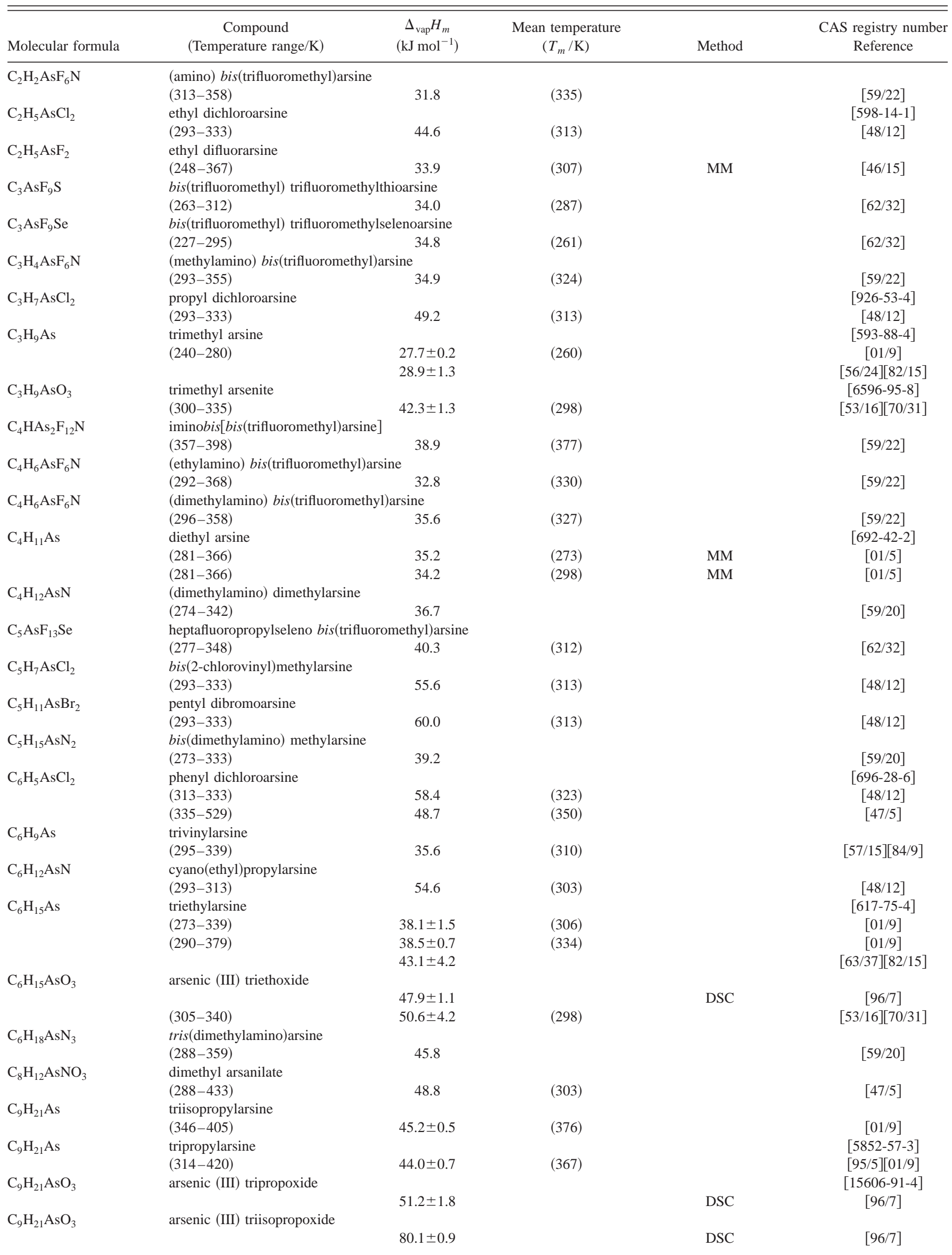


TABLE 7. Enthalpies of vaporization of select organo-metallic and inorganic compounds, 1880-2002-Continued

\begin{tabular}{|c|c|c|c|c|c|}
\hline Molecular formula & $\begin{array}{c}\text { Compound } \\
\text { (Temperature range/K) }\end{array}$ & $\begin{array}{c}\Delta_{\mathrm{vap}} H_{m} \\
\left(\mathrm{~kJ} \mathrm{~mol}^{-1}\right)\end{array}$ & $\begin{array}{l}\text { Mean temperature } \\
\qquad\left(T_{m} / \mathrm{K}\right)\end{array}$ & Method & $\begin{array}{l}\text { CAS registry number } \\
\text { Reference }\end{array}$ \\
\hline $\mathrm{C}_{10} \mathrm{H}_{16} \mathrm{AsNO}_{3}$ & $\begin{array}{l}\text { diethyl arsanilate } \\
(311-454)\end{array}$ & 54.2 & $(326)$ & A & {$[87 / 5][47 / 5]$} \\
\hline $\mathrm{C}_{12} \mathrm{H}_{27} \mathrm{AsO}_{3}$ & arsenic (III) tributoxide & $64.0 \pm 1.8$ & & DSC & $\begin{array}{c}{[3141-10-4]} \\
{[96 / 7]}\end{array}$ \\
\hline $\mathrm{C}_{12} \mathrm{H}_{27} \mathrm{AsO}_{3}$ & arsenic (III) triisobutoxide & $75.7 \pm 1.2$ & & DSC & {$[96 / 7]$} \\
\hline $\mathrm{C}_{13} \mathrm{H}_{10} \mathrm{AsN}$ & $\begin{array}{l}\text { diphenylarsine carbonitrile } \\
(296-326)\end{array}$ & 84.6 & $(311)$ & $\mathrm{A}$ & $\begin{array}{c}{[23525-22-6]} \\
{[87 / 5]}\end{array}$ \\
\hline $\mathrm{C}_{15} \mathrm{H}_{33} \mathrm{As}$ & $\begin{array}{l}\text { tripentylarsine } \\
(408-466)\end{array}$ & 62.3 & $(432)$ & & {$[32 / 4]$} \\
\hline $\mathrm{C}_{18} \mathrm{H}_{15} \mathrm{As}$ & $\begin{array}{l}\text { triphenylarsine } \\
(493-563)\end{array}$ & 75.7 & $(508)$ & A & $\begin{array}{c}{[603-32-7]} \\
{[87 / 5]}\end{array}$ \\
\hline $\mathrm{AsF}_{3}$ & arsenic trifluoride & 35.8 & (293) & & $\begin{array}{c}{[7784-35-2]} \\
{[41 / 11]}\end{array}$ \\
\hline $\mathrm{AsH}_{3}$ & arsine & 16.7 & $(210)$ & & $\begin{array}{c}{[7784-42-1]} \\
{[55 / 19]}\end{array}$ \\
\hline $\mathrm{As}_{2} \mathrm{~S}_{2}$ & $\begin{array}{l}\operatorname{arsenic}(\mathrm{II}) \text { sulfide } \\
(663-838)\end{array}$ & 69.6 & $(750)$ & & $\begin{array}{c}{[1303-32-8]} \\
{[68 / 25]}\end{array}$ \\
\hline B & & & & & \\
\hline $\mathrm{CH}_{3} \mathrm{BO}$ & $\begin{array}{l}\text { borine carbonyl } \\
(134-209)\end{array}$ & 19.7 & (194) & & {$[47 / 5]$} \\
\hline $\mathrm{CH}_{11} \mathrm{~B}_{5}$ & $\begin{array}{l}\text { 1-methylpentaborane (9) } \\
(241-349)\end{array}$ & 32.7 & $(295)$ & & {$[63 / 26]$} \\
\hline $\mathrm{C}_{2} \mathrm{BF}_{5}$ & $\begin{array}{l}\text { perfluorovinyldifluoroborine } \\
(177-238)\end{array}$ & 26.6 & $(207)$ & $\mathrm{T}$ & $\begin{array}{c}{[32038-87-2]} \\
{[60 / 12]}\end{array}$ \\
\hline $\mathrm{C}_{2} \mathrm{BCl}_{2} \mathrm{~F}_{3}$ & $\begin{array}{l}\text { perfluorovinyldichloroborine } \\
(238-301)\end{array}$ & 31.5 & $(269)$ & $\mathrm{T}$ & $\begin{array}{c}{[758-99-6]} \\
{[60 / 12]}\end{array}$ \\
\hline $\mathrm{C}_{2} \mathrm{H}_{3} \mathrm{BF}_{2}$ & $\begin{array}{l}\text { vinyldifluoroborane } \\
(178-228)\end{array}$ & 22.6 & $(203)$ & $\mathrm{T}$ & $\begin{array}{c}{[358-95-2]} \\
{[60 / 10]}\end{array}$ \\
\hline $\mathrm{C}_{2} \mathrm{H}_{3} \mathrm{BCl}_{2}$ & $\begin{array}{l}\text { vinyldichloroborane } \\
(237-282)\end{array}$ & 27.7 & $(260)$ & $\mathrm{T}$ & $\begin{array}{c}{[3677-80-3]} \\
{[60 / 10]}\end{array}$ \\
\hline $\mathrm{C}_{2} \mathrm{H}_{5} \mathrm{BCl}_{2} \mathrm{O}$ & ethoxydichloroborane & $35.1 \pm 0.8$ & $(298)$ & & {$[31 / 3][70 / 31]$} \\
\hline $\mathrm{C}_{2} \mathrm{H}_{6} \mathrm{BCl}_{2} \mathrm{~N}$ & dimethylaminodichloroborane & $37.2 \pm 1.3$ & $(298)$ & & {$[51 / 14][70 / 31]$} \\
\hline $\mathrm{C}_{2} \mathrm{H}_{6} \mathrm{~B}_{4}$ & $\begin{array}{l}\text { carborane-4 } \\
(241-287)\end{array}$ & 26.2 & $(272)$ & & {$[63 / 3]$} \\
\hline $\mathrm{C}_{2} \mathrm{H}_{6} \mathrm{O}-\mathrm{BF}_{3}$ & $\begin{array}{l}\text { dimethyl ether-boron trifluoride co } \\
(311-346)\end{array}$ & $\begin{array}{l}\text { mplex } \\
\quad 53.1\end{array}$ & $(328)$ & & {$[60 / 7]$} \\
\hline $\mathrm{C}_{2} \mathrm{H}_{6} \mathrm{ClBO}_{2}$ & dimethoxychloroborane & $34.3 \pm 1.2$ & (298) & & {$[31 / 3][70 / 31]$} \\
\hline $\mathrm{C}_{2} \mathrm{H}_{6} \mathrm{~S}-\mathrm{BH}_{3}$ & $\begin{array}{l}\text { dimethyl sulfide-borane complex } \\
(273-314)\end{array}$ & 44.9 & $(293)$ & & $\begin{array}{c}{[13292-87-0]} \\
{[99 / 16]}\end{array}$ \\
\hline $\mathrm{C}_{2} \mathrm{H}_{7} \mathrm{~B}_{5}$ & $\begin{array}{l}\text { 2,4-dicarba-closo-heptaborane } \\
(273-323)\end{array}$ & 31.6 & $(288)$ & I & $\begin{array}{c}{[20693-69-0]} \\
{[76 / 17]}\end{array}$ \\
\hline $\mathrm{C}_{2} \mathrm{H}_{8} \mathrm{BSb}$ & $\begin{array}{l}\text { dimethylstibinoborine } \\
(234-273)\end{array}$ & 32.1 & $(254)$ & & {$[59 / 6]$} \\
\hline $\mathrm{C}_{2} \mathrm{H}_{10} \mathrm{BP}$ & $\begin{array}{l}\text { dimethylphosphine borine } \\
(303-383)\end{array}$ & 45.1 & $(318)$ & & {$[53 / 4]$} \\
\hline $\mathrm{C}_{2} \mathrm{H}_{11} \mathrm{~B}_{2} \mathrm{~N}$ & $\begin{array}{l}\text { N-dimethylaminodiborane } \\
(220-267)\end{array}$ & 29.3 & $(252)$ & & {$[55 / 14]$} \\
\hline $\mathrm{C}_{2} \mathrm{H}_{13} \mathrm{~B}_{5}$ & $\begin{array}{l}\text { 1-ethylpentaborane (9) } \\
\text { (273-383) }\end{array}$ & 35.0 & $(328)$ & & {$[63 / 26]$} \\
\hline $\mathrm{C}_{3} \mathrm{BF}_{9} \mathrm{~S}_{3}$ & $\begin{array}{l}\text { tris(trifluoromethylthio)borane } \\
(242-298)\end{array}$ & 33.9 & $(270)$ & & $\begin{array}{c}{[36884-78-3]} \\
{[99 / 16]}\end{array}$ \\
\hline $\mathrm{C}_{3} \mathrm{H}_{5} \mathrm{BF}_{2}$ & $\begin{array}{l}\text { allyldifluoroborane } \\
(194-249)\end{array}$ & 28.0 & $(221)$ & $\mathrm{T}$ & {$[60 / 10]$} \\
\hline $\mathrm{C}_{3} \mathrm{H}_{7} \mathrm{BF}_{2}$ & $\begin{array}{l}\text { propyldifluoroborane } \\
(195-248)\end{array}$ & 29.4 & $(221)$ & $\mathrm{T}$ & {$[60 / 10]$} \\
\hline $\mathrm{C}_{3} \mathrm{H}_{9} \mathrm{BO}_{3}$ & $\begin{array}{l}\text { trimethylborate } \\
(304-340)\end{array}$ & 34.2 & (319) & & $\begin{array}{c}{[121-43-7]} \\
{[67 / 2]}\end{array}$ \\
\hline $\mathrm{C}_{3} \mathrm{H}_{9} \mathrm{~B}$ & trimethylborane & $\begin{array}{c}20.2 \pm 0.1 \\
23.9\end{array}$ & & BG & $\begin{array}{c}{[61 / 13][61 / 15]} \\
{[46 / 13]}\end{array}$ \\
\hline
\end{tabular}


TABLE 7. Enthalpies of vaporization of select organo-metallic and inorganic compounds, 1880-2002-Continued

\begin{tabular}{|c|c|c|c|c|c|}
\hline Molecular formula & $\begin{array}{c}\text { Compound } \\
\text { (Temperature range/K) }\end{array}$ & $\begin{array}{c}\Delta_{\mathrm{vap}} H_{m} \\
\left(\mathrm{~kJ} \mathrm{~mol}^{-1}\right)\end{array}$ & $\begin{array}{l}\text { Mean temperature } \\
\qquad\left(T_{m} / \mathrm{K}\right)\end{array}$ & Method & $\begin{array}{l}\text { CAS registry number } \\
\text { Reference }\end{array}$ \\
\hline $\mathrm{C}_{3} \mathrm{H}_{9} \mathrm{BS}$ & $\begin{array}{l}\text { dimethyl(methylthio)borane } \\
(227-304)\end{array}$ & 31.6 & (265) & & $\begin{array}{c}{[19163-05-4]} \\
{[99 / 16]}\end{array}$ \\
\hline $\mathrm{C}_{3} \mathrm{H}_{9} \mathrm{BS}_{2}$ & $\begin{array}{l}\text { methylbis(methylthio)borane } \\
(300-373)\end{array}$ & 44.7 & (315) & & $\begin{array}{c}{[19163-08-7]} \\
{[99 / 16]}\end{array}$ \\
\hline $\mathrm{C}_{3} \mathrm{H}_{9} \mathrm{BS}_{3}$ & $\begin{array}{l}\text { tris (methylthio)borane } \\
(325-462) \\
(303-493) \\
(303-493)\end{array}$ & $\begin{array}{c}44.9 \\
51.6 \\
54.0 \pm 0.8\end{array}$ & $\begin{array}{l}(394) \\
(398) \\
(298)\end{array}$ & & $\begin{array}{c}{[997-49-9]} \\
{[99 / 16]} \\
{[67 / 8]} \\
{[67 / 8]}\end{array}$ \\
\hline $\mathrm{C}_{3} \mathrm{H}_{9} \mathrm{~B}_{3} \mathrm{O}_{3}$ & $\begin{array}{l}\text { methylboric acid anhydride } \\
(273-327)\end{array}$ & 37.4 & (288) & & {$[40 / 4]$} \\
\hline $\mathrm{C}_{3} \mathrm{H}_{12} \mathrm{BN}$ & $\begin{array}{l}\text { borine trimethylamine } \\
(136-195)\end{array}$ & 19.9 & $(180)$ & & {$[37 / 6]$} \\
\hline $\mathrm{C}_{3} \mathrm{H}_{15} \mathrm{~B}_{5}$ & $\begin{array}{l}\text { 1-isopropylpentaborane (9) } \\
(273-398)\end{array}$ & 37.2 & (335) & & {$[63 / 26]$} \\
\hline $\mathrm{C}_{4} \mathrm{BClF}_{6}$ & $\begin{array}{l}\text { bis(perfluorovinyl)chloroborine } \\
(280-322)\end{array}$ & 35.6 & $(301)$ & $\mathrm{T}$ & {$[60 / 12]$} \\
\hline $\mathrm{C}_{4} \mathrm{H}_{6} \mathrm{BF}$ & $\begin{array}{l}\text { divinylfluoroborane } \\
(193-273)\end{array}$ & 25.8 & $(233)$ & $\mathrm{T}$ & {$[60 / 10]$} \\
\hline $\mathrm{C}_{4} \mathrm{H}_{6} \mathrm{BCl}$ & $\begin{array}{l}\text { divinylchloroborane } \\
(275-298)\end{array}$ & 33.0 & $(286)$ & $\mathrm{T}$ & {$[60 / 10]$} \\
\hline $\mathrm{C}_{5} \mathrm{H}_{10} \mathrm{O}-\mathrm{BF}_{3}$ & $\begin{array}{l}\text { tetrahydopyran-boron trifluoric } \\
(323-368)\end{array}$ & $\begin{array}{l}\text { mplex } \\
60.9\end{array}$ & (345) & & {$[60 / 8]$} \\
\hline $\mathrm{C}_{4} \mathrm{H}_{10} \mathrm{O}-\mathrm{BF}_{3}$ & $\begin{array}{l}\text { diethyl ether-boron trifluoride } \\
(283-353)\end{array}$ & $\begin{array}{l}\text { plex } \\
55.1\end{array}$ & $(318)$ & & {$[60 / 7]$} \\
\hline $\mathrm{C}_{4} \mathrm{H}_{10} \mathrm{BClO}_{2}$ & diethoxychloroborane & $38.9 \pm 0.8$ & (298) & & {$[31 / 3][70 / 31]$} \\
\hline $\mathrm{C}_{4} \mathrm{H}_{12} \mathrm{BClN}_{2}$ & bis(dimethylamino)chloroborane & $41.8 \pm 2.1$ & $(298)$ & & {$[51 / 14][70 / 31]$} \\
\hline $\mathrm{C}_{4} \mathrm{H}_{11} \mathrm{~N}-\mathrm{C}_{3} \mathrm{H}_{9} \mathrm{~B}$ & $\begin{array}{l}\text { N,N-dimethylethylamine- - trime } \\
(303-339)\end{array}$ & $\begin{array}{l}\text { borane complex } \\
58.2\end{array}$ & (321) & & {$[60 / 9]$} \\
\hline $\mathrm{C}_{4} \mathrm{H}_{12} \mathrm{~B}_{2} \mathrm{O}_{4}$ & $\begin{array}{l}\text { tetramethoxydiboron } \\
(273-348)\end{array}$ & $\begin{array}{l}44.0 \\
44.7\end{array}$ & $(310)$ & & $\begin{array}{l}{[60 / 13]} \\
{[72 / 32]}\end{array}$ \\
\hline $\mathrm{C}_{4} \mathrm{H}_{17} \mathrm{~B}_{5}$ & $\begin{array}{l}\text { 1-sec-butylpentaborane (9) } \\
(299-428)\end{array}$ & 41.4 & $(364)$ & & {$[63 / 26]$} \\
\hline $\mathrm{C}_{5} \mathrm{H}_{16} \mathrm{~B}_{10}$ & $\begin{array}{l}\text { isopropenyl-o-carborane } \\
(323-473)\end{array}$ & 36.7 & $(398)$ & & {$[63 / 46]$} \\
\hline $\mathrm{C}_{5} \mathrm{H}_{16} \mathrm{~B}_{10} \mathrm{O}_{2}$ & 1-acetoxymethyl-o-carborane & 56.5 & (569) & & $\begin{array}{c}{[19528-60-0]} \\
{[74 / 31]}\end{array}$ \\
\hline $\mathrm{C}_{5} \mathrm{H}_{19} \mathrm{~B}_{5}$ & $\begin{array}{l}\text { 1-methyl-2-sec-butylpentaboran } \\
(301-423)\end{array}$ & 41.0 & $(362)$ & & {$[63 / 26]$} \\
\hline $\mathrm{C}_{6} \mathrm{BF}_{9}$ & $\begin{array}{l}\text { tris(perfluorovinyl)borine } \\
(297-335)\end{array}$ & 41.1 & (316) & $\mathrm{T}$ & {$[60 / 12]$} \\
\hline $\mathrm{C}_{6} \mathrm{H}_{5} \mathrm{BBr}_{2}$ & $\begin{array}{l}\text { phenylboron dibromide } \\
(391-433)\end{array}$ & $43.9 \pm 2.1$ & $(412)$ & $\mathrm{T}$ & {$[67 / 1]$} \\
\hline $\mathrm{C}_{6} \mathrm{H}_{5} \mathrm{BCl}_{2}$ & $\begin{array}{l}\text { phenylboron dichloride } \\
(273-318)\end{array}$ & $33.7 \pm 0.8$ & $(296)$ & $\mathrm{T}$ & {$[67 / 1]$} \\
\hline $\mathrm{C}_{6} \mathrm{H}_{12} \mathrm{BCl}_{3} \mathrm{O}_{3}$ & $\begin{array}{l}\text { tris(2-chloroethyl) orthoborate } \\
(390-448)\end{array}$ & 57.7 & $(419)$ & & {$[46 / 9]$} \\
\hline $\mathrm{C}_{6} \mathrm{H}_{13} \mathrm{BO}_{2}$ & 1-butaneboronic acid, cyclic eth & $\begin{array}{l}\text { ester } \\
40.2\end{array}$ & (329) & & $\begin{array}{c}{[10173-39-4]} \\
{[70 / 29]}\end{array}$ \\
\hline $\mathrm{C}_{6} \mathrm{H}_{15} \mathrm{~B}$ & triethylborane & $\begin{array}{c}33.6 \\
36.8 \pm 0.4\end{array}$ & (293) & & $\begin{array}{c}{[97-94-9]} \\
{[83 / 1]} \\
{[63 / 32][82 / 15]}\end{array}$ \\
\hline $\mathrm{C}_{6} \mathrm{H}_{15} \mathrm{BO}_{3}$ & $\begin{array}{l}\text { triethylborate } \\
(302-382) \\
(302-382)\end{array}$ & $\begin{array}{l}41.0 \\
38.2\end{array}$ & $\begin{array}{l}(317) \\
(391)\end{array}$ & & $\begin{array}{c}{[150-46-9]} \\
{[67 / 2]} \\
{[67 / 2]}\end{array}$ \\
\hline $\mathrm{C}_{6} \mathrm{H}_{15} \mathrm{BS}_{3}$ & triethylthioborane & $61.5 \pm 2.1$ & & & {$[66 / 27][70 / 31]$} \\
\hline $\mathrm{C}_{6} \mathrm{H}_{15} \mathrm{~B}_{3} \mathrm{O}_{3}$ & $\begin{array}{l}\text { triethylboroxin } \\
(347-424)\end{array}$ & 46.0 & $(362)$ & EB & {$[90 / 19]$} \\
\hline $\mathrm{C}_{6} \mathrm{H}_{16} \mathrm{BN}$ & (N-ethylamino)diethylborane & $60.7 \pm 0.8$ & & & {$[67 / 32][82 / 15]$} \\
\hline $\mathrm{C}_{6} \mathrm{H}_{17} \mathrm{~B}_{5} \mathrm{Br}_{2} \mathrm{Si}_{2}$ & 2,4-bis(bromodimethylsilyl)-2,4 & rbo-closo-heptab & ne (7) & & {$[66798-29-6]$} \\
\hline
\end{tabular}


TABLE 7. Enthalpies of vaporization of select organo-metallic and inorganic compounds, 1880-2002-Continued

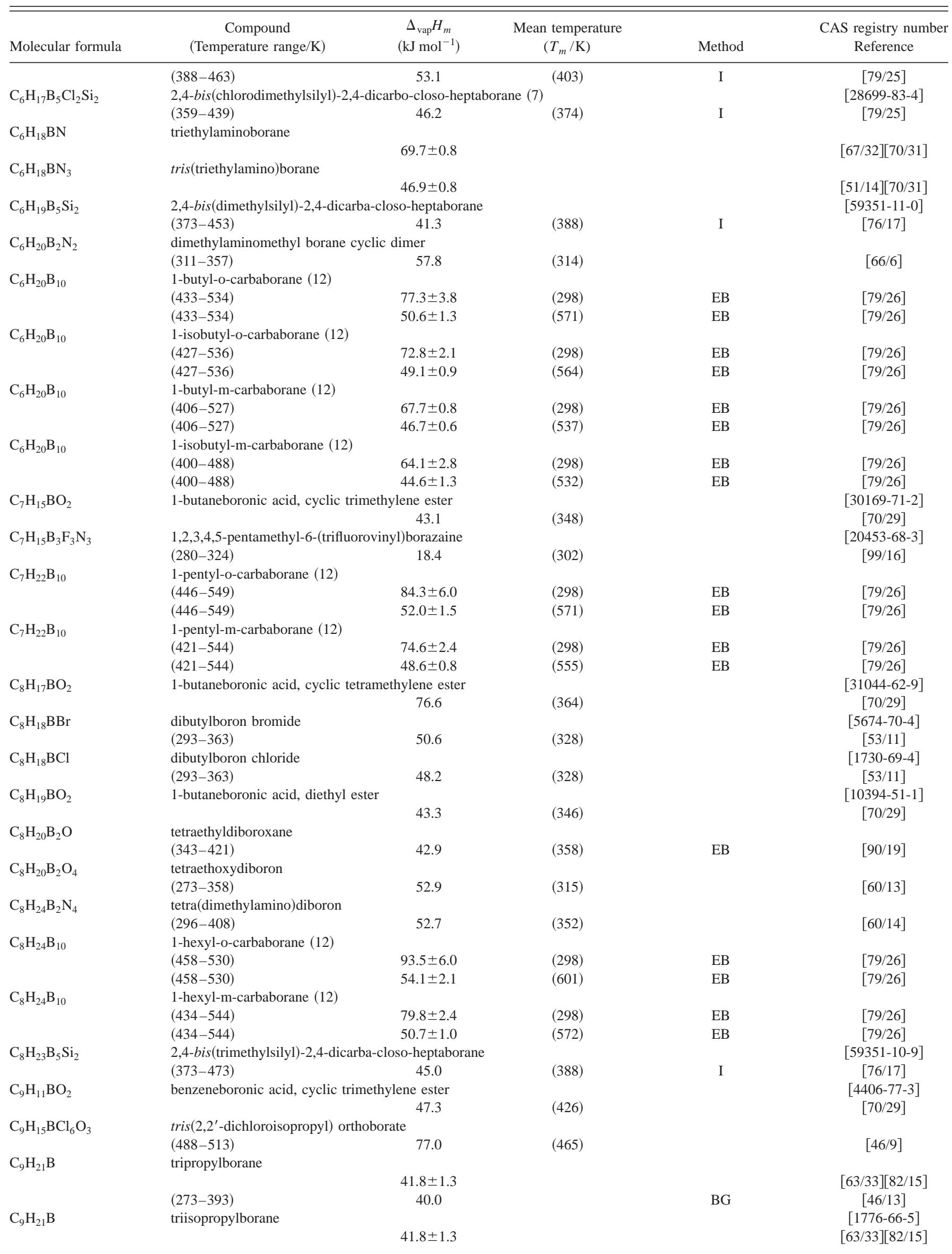


TABLE 7. Enthalpies of vaporization of select organo-metallic and inorganic compounds, 1880-2002-Continued

\begin{tabular}{|c|c|c|c|c|c|}
\hline Molecular formula & $\begin{array}{c}\text { Compound } \\
\text { (Temperature range/K) }\end{array}$ & $\begin{array}{c}\Delta_{\mathrm{vap}} H_{m} \\
\left(\mathrm{~kJ} \mathrm{~mol}^{-1}\right)\end{array}$ & $\begin{array}{l}\text { Mean temperature } \\
\qquad\left(T_{m} / \mathrm{K}\right)\end{array}$ & Method & $\begin{array}{c}\text { CAS registry number } \\
\text { Reference }\end{array}$ \\
\hline \multirow{4}{*}{$\mathrm{C}_{9} \mathrm{H}_{21} \mathrm{BO}_{3}$} & $(273-393)$ & 40.0 & & BG & [46/13] \\
\hline & tripropylborate & & & & {$[688-71-1]$} \\
\hline & $(340-453)$ & 52.3 & $(355)$ & & [80/15] \\
\hline & $(358-452)$ & 47.6 & $(452)$ & & {$[67 / 2]$} \\
\hline \multirow[t]{2}{*}{$\mathrm{C}_{9} \mathrm{H}_{21} \mathrm{BO}_{3}$} & triisopropylborate & & & & {$[5419-55-6]$} \\
\hline & $(338-412)$ & 42.4 & $(412)$ & & {$[67 / 2]$} \\
\hline \multirow[t]{3}{*}{$\mathrm{C}_{9} \mathrm{H}_{21} \mathrm{BS}_{3}$} & tri(propylthio)borane & & & & [998-38-9] \\
\hline & $(423-483)$ & 76.2 & $(453)$ & & {$[67 / 8]$} \\
\hline & $(423-483)$ & $87.0 \pm 2.1$ & $(298)$ & & {$[67 / 8]$} \\
\hline \multirow[t]{3}{*}{$\mathrm{C}_{9} \mathrm{H}_{22} \mathrm{BNO}$} & butyl(dimethylamino)methoxyborane & & & & \\
\hline & $(369-427)$ & 48.1 & $(384)$ & EB & {$[73 / 17]$} \\
\hline & & $58.2 \pm 2.5$ & (298) & & {$[73 / 17]$} \\
\hline \multirow[t]{2}{*}{$\mathrm{C}_{10} \mathrm{H}_{13} \mathrm{BO}_{2}$} & benzeneboronic acid, cyclic tetrameth & hylene ester & & & {$[4406-76-2]$} \\
\hline & & 57.3 & $(441)$ & & {$[70 / 29]$} \\
\hline \multirow[t]{2}{*}{$\mathrm{C}_{10} \mathrm{H}_{15} \mathrm{BO}_{2}$} & benzeneboronic acid, diethyl ester & & & & [31044-59-4] \\
\hline & & 67.4 & $(332)$ & & {$[70 / 29]$} \\
\hline \multirow{2}{*}{$\mathrm{C}_{11} \mathrm{H}_{24} \mathrm{~B}_{10} \mathrm{O}_{3}$} & 3-methyl-3-(7-isopropyl-m-carboranoy & oylperoxy)-1- & & & \\
\hline & $(353-368)$ & $140.6 \pm 4.4$ & $(360)$ & ME & [99/17] \\
\hline \multirow[t]{2}{*}{$\mathrm{C}_{12} \mathrm{H}_{10} \mathrm{BBr}$} & diphenylboron bromide & & & & {$[5123-17-1]$} \\
\hline & $(436-516)$ & $60.2 \pm 2.5$ & (476) & $\mathrm{T}$ & {$[67 / 1]$} \\
\hline \multirow{2}{*}{$\mathrm{C}_{12} \mathrm{H}_{10} \mathrm{BCl}$} & diphenylboron chloride & & & & {$[3677-81-4]$} \\
\hline & $(363-485)$ & $41.4 \pm 2.1$ & $(424)$ & $\mathrm{T}$ & {$[67 / 1]$} \\
\hline \multirow{2}{*}{$\mathrm{C}_{12} \mathrm{H}_{21} \mathrm{~B}$} & dodecahydro-9-boraphenalene & & & & [16664-33-8] \\
\hline & $(304-404)$ & 53.1 & $(319)$ & A & {$[87 / 5]$} \\
\hline \multirow[t]{2}{*}{$\mathrm{C}_{12} \mathrm{H}_{27} \mathrm{~B}$} & tributylboron & & & & {$[122-56-5]$} \\
\hline & $(293-363)$ & 54.7 & $(328)$ & & {$[53 / 11]$} \\
\hline \multirow[t]{3}{*}{$\mathrm{C}_{12} \mathrm{H}_{27} \mathrm{BO}_{3}$} & tributylborate & & & & {$[688-74-4]$} \\
\hline & $(380-504)$ & 58.1 & $(395)$ & & {$[80 / 15]$} \\
\hline & $(390-491)$ & 55.9 & $(405)$ & & {$[67 / 2]$} \\
\hline \multirow{2}{*}{$\mathrm{C}_{12} \mathrm{H}_{27} \mathrm{BO}_{3}$} & triisobutylborate & & & & [13195-76-1] \\
\hline & $(372-472)$ & 51.7 & $(483)$ & & {$[67 / 2]$} \\
\hline \multirow[t]{3}{*}{$\mathrm{C}_{12} \mathrm{H}_{27} \mathrm{BS}_{3}$} & tri(butylthio)borane & & & & {$[998-46-9]$} \\
\hline & $(440-503)$ & 83.9 & $(471)$ & & {$[67 / 8]$} \\
\hline & $(440-503)$ & $95.8 \pm 2.1$ & (298) & & {$[67 / 8]$} \\
\hline $\mathrm{C}_{15} \mathrm{H}_{32} \mathrm{~B}_{10} \mathrm{O}_{5}$ & 2,5-dimethyl-(2-tert-butylperoxy-5-m- & १-carboranoyl & )-3-hexyne & & \\
\hline & $(353-366)$ & $86.8 \pm 5.4$ & $(360)$ & ME & {$[99 / 17]$} \\
\hline $\mathrm{C}_{15} \mathrm{H}_{33} \mathrm{BO}_{3}$ & tripentylborate & & & & \\
\hline & $(410-505)$ & 67.7 & $(425)$ & & {$[80 / 15]$} \\
\hline $\mathrm{C}_{15} \mathrm{H}_{33} \mathrm{BS}_{3}$ & tri(penthylthio)borane & & & & \\
\hline & $(446-503)$ & 92.3 & $(474)$ & & {$[67 / 8]$} \\
\hline & $(446-503)$ & $104.6 \pm 2.1$ & $(298)$ & & {$[67 / 8]$} \\
\hline $\mathrm{C}_{18} \mathrm{H}_{12} \mathrm{BCl}_{3} \mathrm{O}_{3}$ & tris(4-chlorophenoxy)borane & & & & {$[7539-58-2]$} \\
\hline & $(428-476)$ & $30.6 \pm 0.9$ & $(452)$ & MM & {$[73 / 2]$} \\
\hline $\mathrm{C}_{18} \mathrm{H}_{12} \mathrm{BCl}_{3} \mathrm{O}_{3}$ & tris(3-chlorophenoxy)borane & & & & {$[42080-72-8]$} \\
\hline & $(476-524)$ & $49.6 \pm 1.6$ & $(500)$ & MM & {$[73 / 2]$} \\
\hline $\mathrm{C}_{18} \mathrm{H}_{15} \mathrm{~B}$ & triphenylborane & & & & {$[960-71-4]$} \\
\hline & $(423-568)$ & 64.3 & $(438)$ & A & {$[87 / 5]$} \\
\hline & $(423-548)$ & $64.4 \pm 2.1$ & $(486)$ & & {$[67 / 1]$} \\
\hline $\mathrm{C}_{21} \mathrm{H}_{12} \mathrm{BN}_{3} \mathrm{O}_{3}$ & tris(4-cyanophenoxy)borane & & & & \\
\hline & $(448-506)$ & $46.2 \pm 2.0$ & $(477)$ & MM & {$[73 / 2]$} \\
\hline $\mathrm{C}_{21} \mathrm{H}_{21} \mathrm{BO}_{3}$ & tris(4-methylphenoxy)borane & & & & [14643-62-0] \\
\hline & $(475-525)$ & $76.1 \pm 1.7$ & $(500)$ & MM & {$[73 / 2]$} \\
\hline $\mathrm{C}_{21} \mathrm{H}_{21} \mathrm{BO}_{3}$ & tris(3-methylphenoxy)borane & & & & \\
\hline & $(477-523)$ & $77.1 \pm 2.2$ & $(500)$ & MM & {$[73 / 2]$} \\
\hline $\mathrm{C}_{21} \mathrm{H}_{21} \mathrm{BO}_{6}$ & tris(3-methoxyphenoxy)borane & & & & [42080-76-2] \\
\hline & $(440-496)$ & $57.8 \pm 2.4$ & $(468)$ & MM & {$[73 / 2]$} \\
\hline $\mathrm{C}_{21} \mathrm{H}_{21} \mathrm{BO}_{6}$ & tris(4-methoxyphenoxy)borane & & & & [42080-75-1] \\
\hline & $(448-500)$ & $42.4 \pm 2.7$ & $(474)$ & MM & {$[73 / 2]$} \\
\hline $\mathrm{B}_{2} \mathrm{D}_{6}$ & perdeuterodiborane & & & & \\
\hline & $(118-179)$ & 15.3 & $(164)$ & & [61/29] \\
\hline $\mathrm{B}_{2} \mathrm{H}_{6}$ & diborane & & & & [19287-45-7] \\
\hline & $(118-179)$ & 15.3 & $(164)$ & & {$[61 / 29]$} \\
\hline & & 14.2 & $(180)$ & $\mathrm{C}$ & {$[59 / 19]$} \\
\hline & & 12.6 & $(210)$ & $\mathrm{C}$ & {$[59 / 19]$} \\
\hline
\end{tabular}


TABLE 7. Enthalpies of vaporization of select organo-metallic and inorganic compounds, 1880-2002-Continued

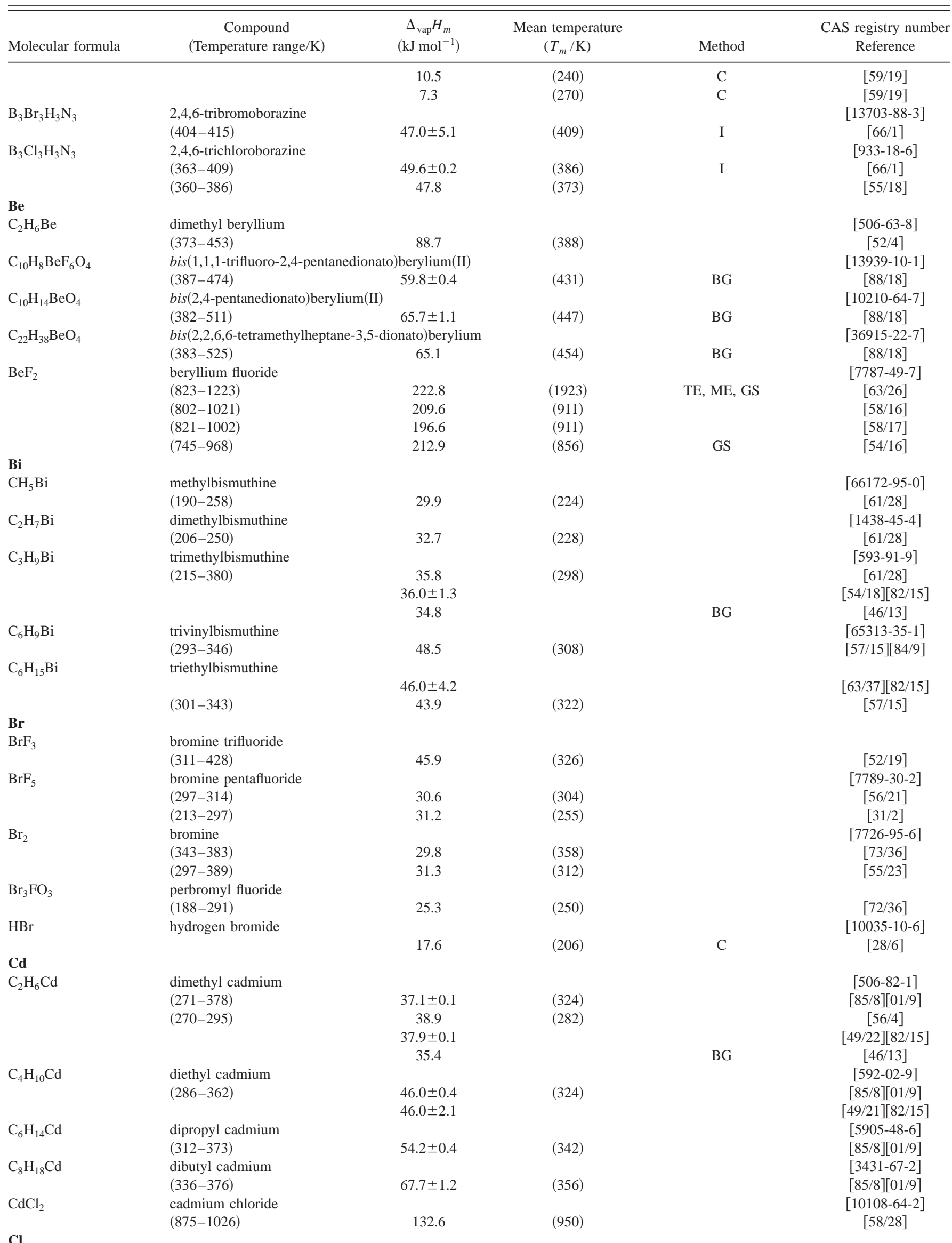


TABLE 7. Enthalpies of vaporization of select organo-metallic and inorganic compounds, 1880-2002-Continued

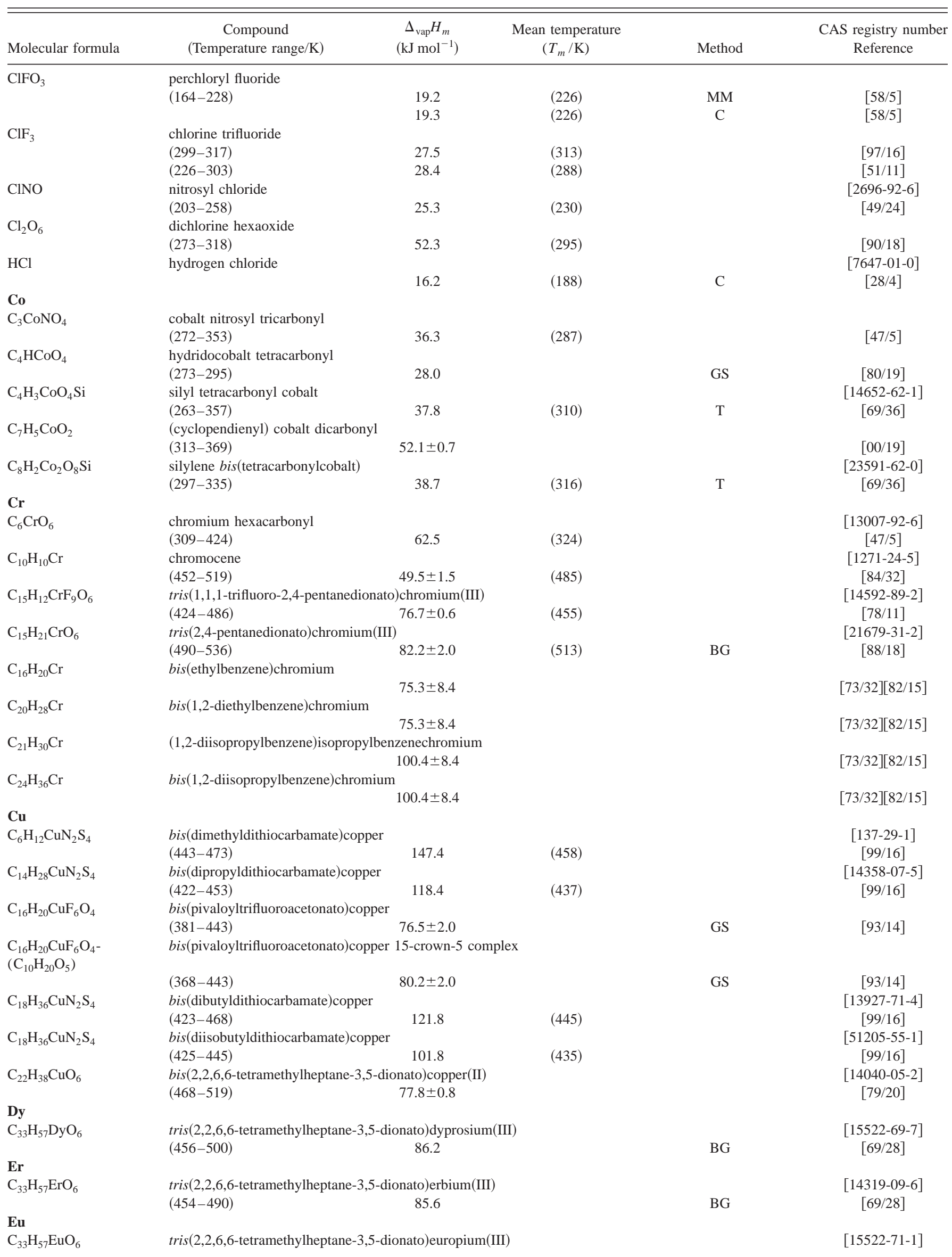


TABLE 7. Enthalpies of vaporization of select organo-metallic and inorganic compounds, 1880-2002-Continued

\begin{tabular}{|c|c|c|c|c|c|}
\hline Molecular formula & $\begin{array}{c}\text { Compound } \\
\text { (Temperature range/K) }\end{array}$ & $\begin{array}{c}\Delta_{\mathrm{vap}} H_{m} \\
\left(\mathrm{~kJ} \mathrm{~mol}^{-1}\right)\end{array}$ & $\begin{array}{l}\text { Mean temperature } \\
\qquad\left(T_{m} / \mathrm{K}\right)\end{array}$ & Method & $\begin{array}{c}\text { CAS registry number } \\
\text { Reference }\end{array}$ \\
\hline & $(466-490)$ & 87.4 & & BG & {$[69 / 28]$} \\
\hline \multicolumn{6}{|l|}{$\mathbf{F}$} \\
\hline \multirow{2}{*}{$\mathrm{F}_{3} \mathrm{NO}$} & trifluoroamine oxide & & & & [13847-65-9] \\
\hline & $(116-191)$ & 16.1 & & & {$[68 / 30]$} \\
\hline \multirow[t]{2}{*}{$\mathrm{F}_{5} \mathrm{I}$} & iodine pentafluoride & & & & [7783-66-6] \\
\hline & $(283-378)$ & 39.3 & (330) & & {$[71 / 35]$} \\
\hline \multirow[t]{3}{*}{$\mathrm{HF}$} & hydrogen fluoride & & & & {$[7664-39-3]$} \\
\hline & $(240-290)$ & 25.2 & (265) & & {$[34 / 8]$} \\
\hline & $(190-320)$ & 25.2 & $(255)$ & & {$[24 / 1]$} \\
\hline \multicolumn{6}{|l|}{$\mathbf{F e}$} \\
\hline \multirow[t]{2}{*}{$\mathrm{C}_{4} \mathrm{H}_{6} \mathrm{FeO}_{4} \mathrm{Si}_{2}$} & tetracarbonyl disilyl iron & & & & [26469-80-7] \\
\hline & $(329-377)$ & 43.8 & $(353)$ & $\mathrm{T}$ & {$[69 / 38]$} \\
\hline \multirow[t]{6}{*}{$\mathrm{C}_{5} \mathrm{FeO}_{5}$} & iron pentacarbonyl & & & & [13463-40-6] \\
\hline & $(254-304)$ & $40.1 \pm 0.5$ & $(279)$ & & {$[74 / 27]$} \\
\hline & $(266-353)$ & 39.0 & (309) & & {$[70 / 25]$} \\
\hline & & $38.1 \pm 0.4$ & (298) & & {$[59 / 14]$} \\
\hline & & $40.2 \pm 0.8$ & & & {$[59 / 18][82 / 15]$} \\
\hline & $(266-378)$ & 37.6 & (281) & & {$[47 / 5]$} \\
\hline \multirow[t]{2}{*}{$\mathrm{C}_{7} \mathrm{H}_{6} \mathrm{FeO}_{3}$} & 1,3-butadiene iron tricarbo & & & & \\
\hline & & $49.0 \pm 4.2$ & & & {$[76 / 23][82 / 15]$} \\
\hline \multirow[t]{5}{*}{$\mathrm{C}_{10} \mathrm{H}_{10} \mathrm{Fe}$} & ferrocene & & & & [102-54-5] \\
\hline & $(456-523)$ & 47.3 & $(471)$ & A & {$[87 / 5][99 / 16]$} \\
\hline & $(451-523)$ & 49.8 & (466) & $\mathrm{A}$ & {$[87 / 5]$} \\
\hline & $(519-604)$ & 44.7 & $(561)$ & & {$[72 / 34]$} \\
\hline & & 47.3 & $(456)$ & & {$[52 / 21]$} \\
\hline \multirow[t]{2}{*}{$\mathrm{C}_{15} \mathrm{H}_{12} \mathrm{~F}_{9} \mathrm{FeO}_{6}$} & $\operatorname{tris}(1,1,1$-trifluoro-2,4-pent & )iron(III) & & & [14526-22-8] \\
\hline & $(392-428)$ & $87.0 \pm 1.2$ & $(410)$ & & {$[78 / 11]$} \\
\hline \multicolumn{6}{|c|}{ (10) } \\
\hline \multirow[t]{3}{*}{$\mathrm{C}_{3} \mathrm{H}_{9} \mathrm{Ga}$} & trimethyl gallium & & & & [1445-79-0] \\
\hline & & $33.1 \pm 0.8$ & & & {$[58 / 18][82 / 15]$} \\
\hline & & 32.6 & & & {$[33 / 14][58 / 20]$} \\
\hline \multirow{2}{*}{$\mathrm{C}_{4} \mathrm{H}_{10} \mathrm{ClGa}$} & diethylgallium chloride & & & & [30914-08-0] \\
\hline & $(273-333)$ & 59.9 & (303) & & {$[91 / 1]$} \\
\hline \multirow[t]{3}{*}{$\mathrm{C}_{6} \mathrm{H}_{9} \mathrm{Ga}$} & trivinyl gallium & & & & [1188-13-2] \\
\hline & $(298-373)$ & U72.6 & $(335)$ & & {$[62 / 27]$} \\
\hline & [Note: Decomposition note & $33 \mathrm{~K}]$. & & & \\
\hline $\mathrm{C}_{6} \mathrm{H}_{15} \mathrm{Ga}$ & triethyl gallium & & & & [1115-99-7] \\
\hline & $(299-387)$ & $43.1 \pm 1.6$ & $(343)$ & & {$[01 / 9][01 / 24]$} \\
\hline & & $38.5 \pm 0.4$ & & & {$[73 / 31][82 / 15]$} \\
\hline $\mathrm{C}_{9} \mathrm{H}_{21} \mathrm{Ga}$ & triisopropyl gallium & & & & [54614-59-9] \\
\hline & $(298-373)$ & 49.0 & $(335)$ & & {$[62 / 27]$} \\
\hline $\mathrm{C}_{9} \mathrm{H}_{21} \mathrm{Ga}$ & tripropyl gallium & & & & [29868-77-7] \\
\hline & $(316-385)$ & $46.6 \pm 0.5$ & $(350)$ & & [01/9] \\
\hline & $(298-373)$ & 49.2 & $(335)$ & & {$[62 / 27]$} \\
\hline $\mathrm{C}_{11} \mathrm{H}_{24} \mathrm{GaNS}_{2}$ & di-tert-butyl gallium dimet & arbamate & & & \\
\hline & $(374-427)$ & $43 \pm 1$ & & TGA & {$[99 / 33]$} \\
\hline $\mathrm{C}_{11} \mathrm{H}_{24} \mathrm{GaNS}_{2}$ & dibutyl gallium dimethyldi & nate & & & \\
\hline & $(385-424)$ & $53 \pm 1$ & & TGA & {$[99 / 33]$} \\
\hline $\mathrm{C}_{11} \mathrm{H}_{24} \mathrm{GaNS}_{2}$ & di-sec-butyl gallium dimet & arbamate & & & \\
\hline & $(366-425)$ & $44 \pm 1$ & & TGA & [99/33] \\
\hline $\mathrm{C}_{12} \mathrm{H}_{27} \mathrm{Ga}$ & tributyl gallium & & & & [15677-44-8] \\
\hline & $(330-378)$ & $51.6 \pm 1.3$ & $(354)$ & & [01/9] \\
\hline & $(426-507)$ & 56.2 & $(441)$ & A & {$[87 / 5]$} \\
\hline $\mathrm{C}_{13} \mathrm{H}_{28} \mathrm{GaNS}_{2}$ & di-tert-butyl gallium diethy & bamate & & & \\
\hline & $(372-419)$ & $48 \pm 6$ & & TGA & [99/33] \\
\hline $\mathrm{C}_{15} \mathrm{H}_{12} \mathrm{~F}_{9} \mathrm{GaO}_{6}$ & tris(1,1,1-trifluoro-2,4-pent & )gallium(III) & & & [15453-83-5] \\
\hline & $(401-459)$ & $75.6 \pm 0.5$ & $(430)$ & & {$[78 / 10]$} \\
\hline $\mathrm{C}_{15} \mathrm{H}_{32} \mathrm{GaNS}_{2}$ & di-tert-butyl gallium diprol & arbamate & & & \\
\hline & $(365-424)$ & $46 \pm 1$ & & TGA & [99/33] \\
\hline Gd & & & & & \\
\hline $\mathrm{C}_{33} \mathrm{H}_{57} \mathrm{GdO}_{6}$ & $\operatorname{tris}(2,2,6,6$-tetramethylhep & onato)gadolir & & & {$[14768-15-1]$} \\
\hline & $(456-500)$ & 90.2 & & BG & {$[69 / 28]$} \\
\hline Ge & & & & & \\
\hline $\mathrm{CHCl}_{5} \mathrm{Ge}$ & trichloro(dichloromethyl)g & & & & \\
\hline
\end{tabular}


TABLE 7. Enthalpies of vaporization of select organo-metallic and inorganic compounds, 1880-2002-Continued

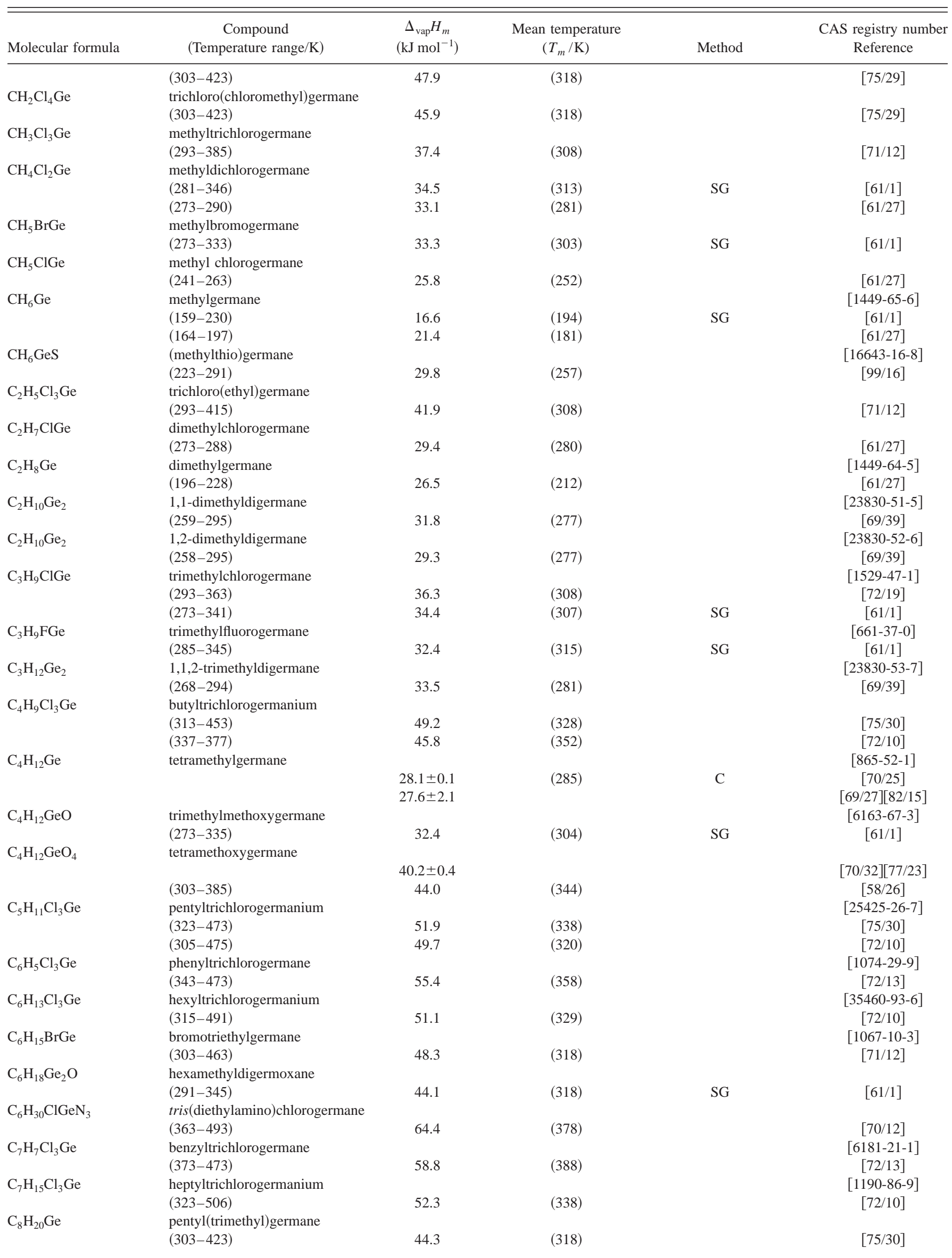


TABLE 7. Enthalpies of vaporization of select organo-metallic and inorganic compounds, 1880-2002-Continued

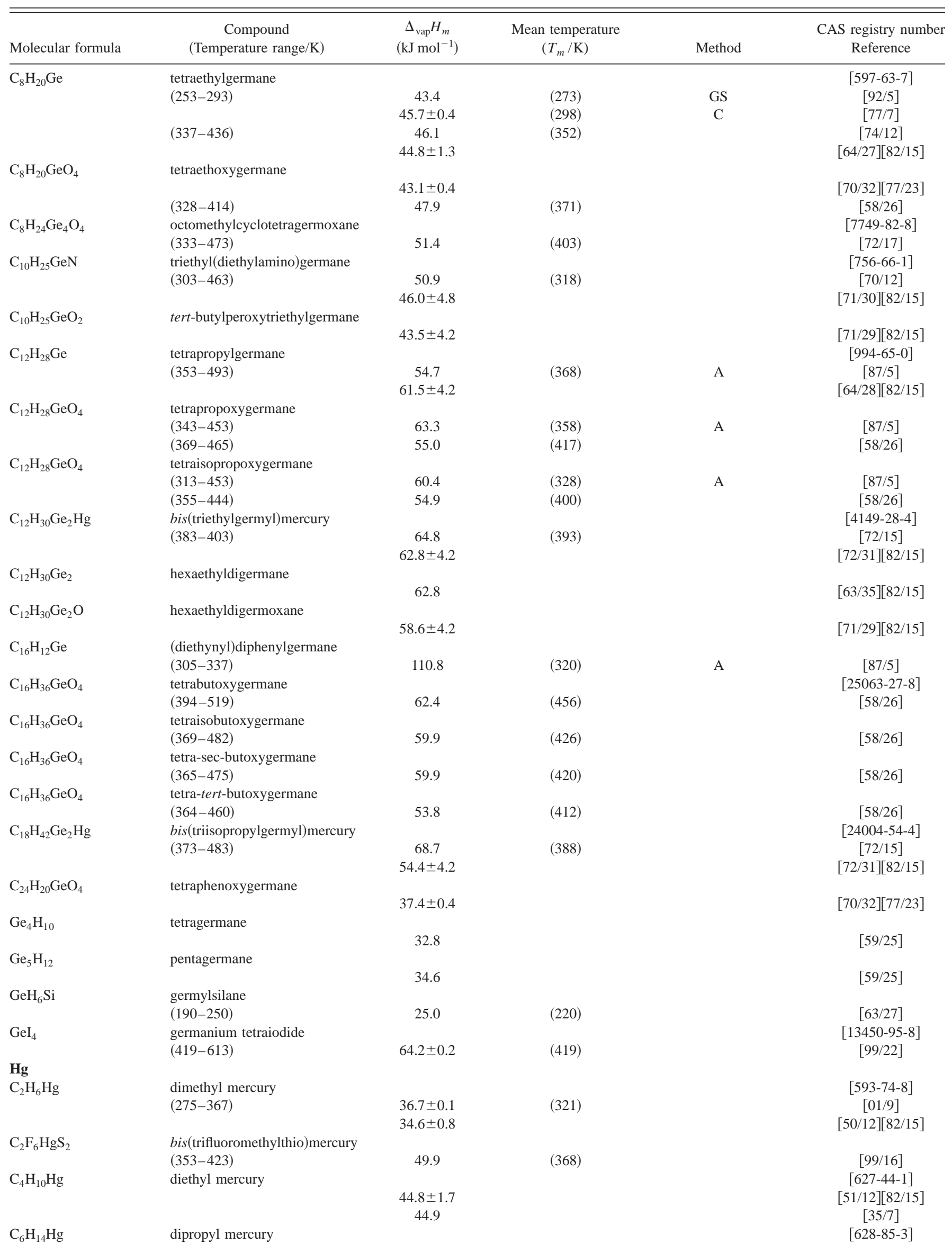


TABLE 7. Enthalpies of vaporization of select organo-metallic and inorganic compounds, 1880-2002-Continued

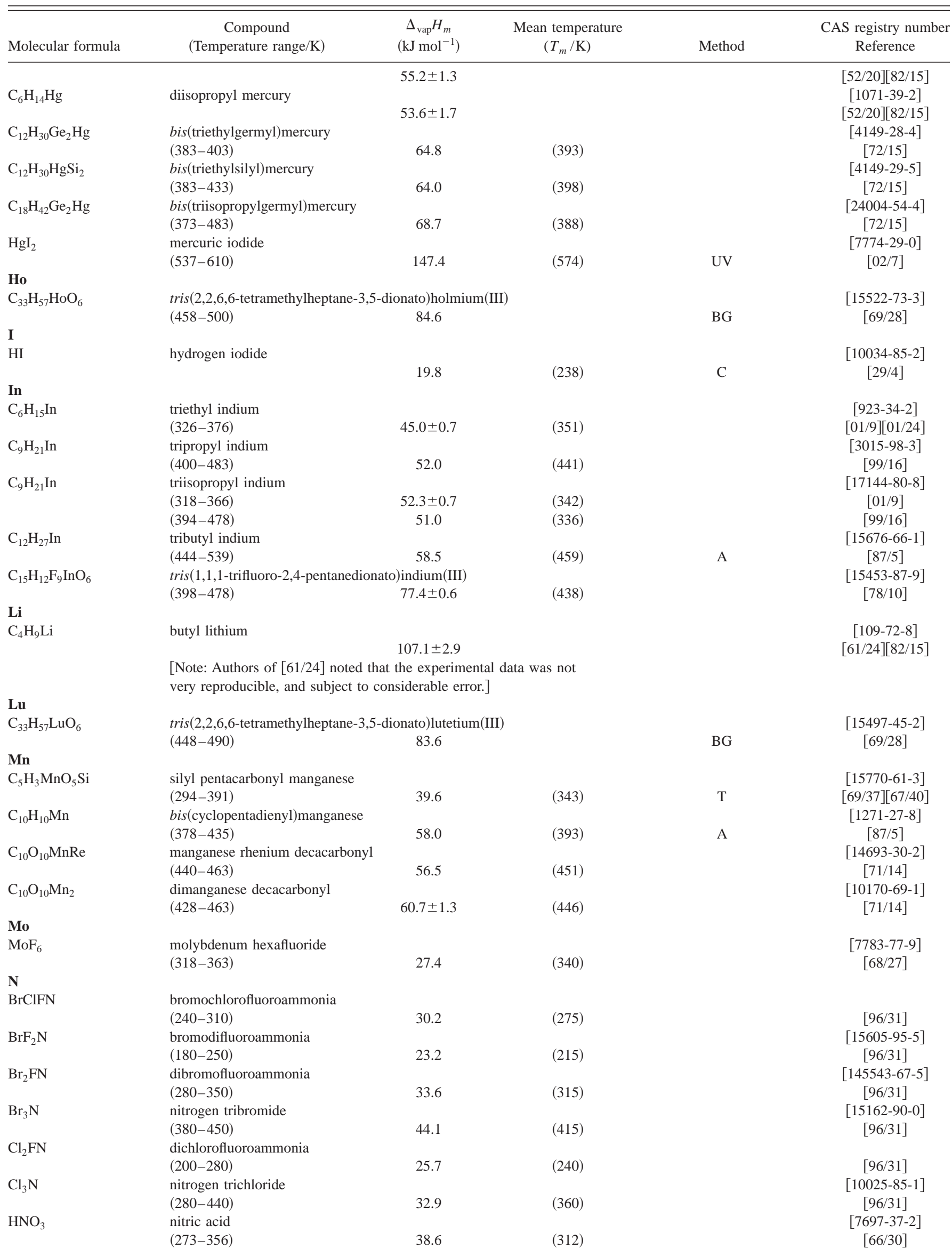


TABLE 7. Enthalpies of vaporization of select organo-metallic and inorganic compounds, 1880-2002-Continued

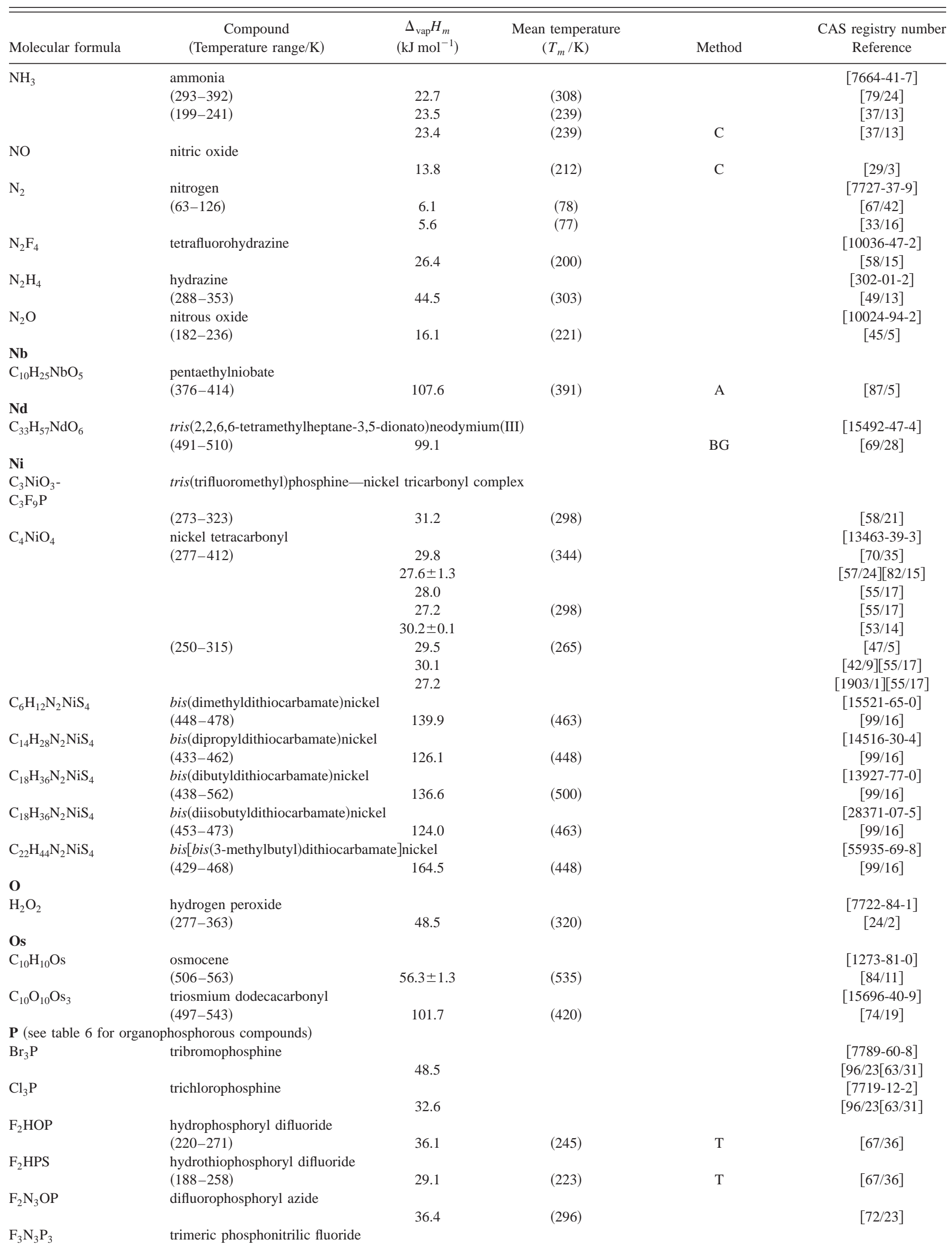


TABLE 7. Enthalpies of vaporization of select organo-metallic and inorganic compounds, 1880-2002-Continued

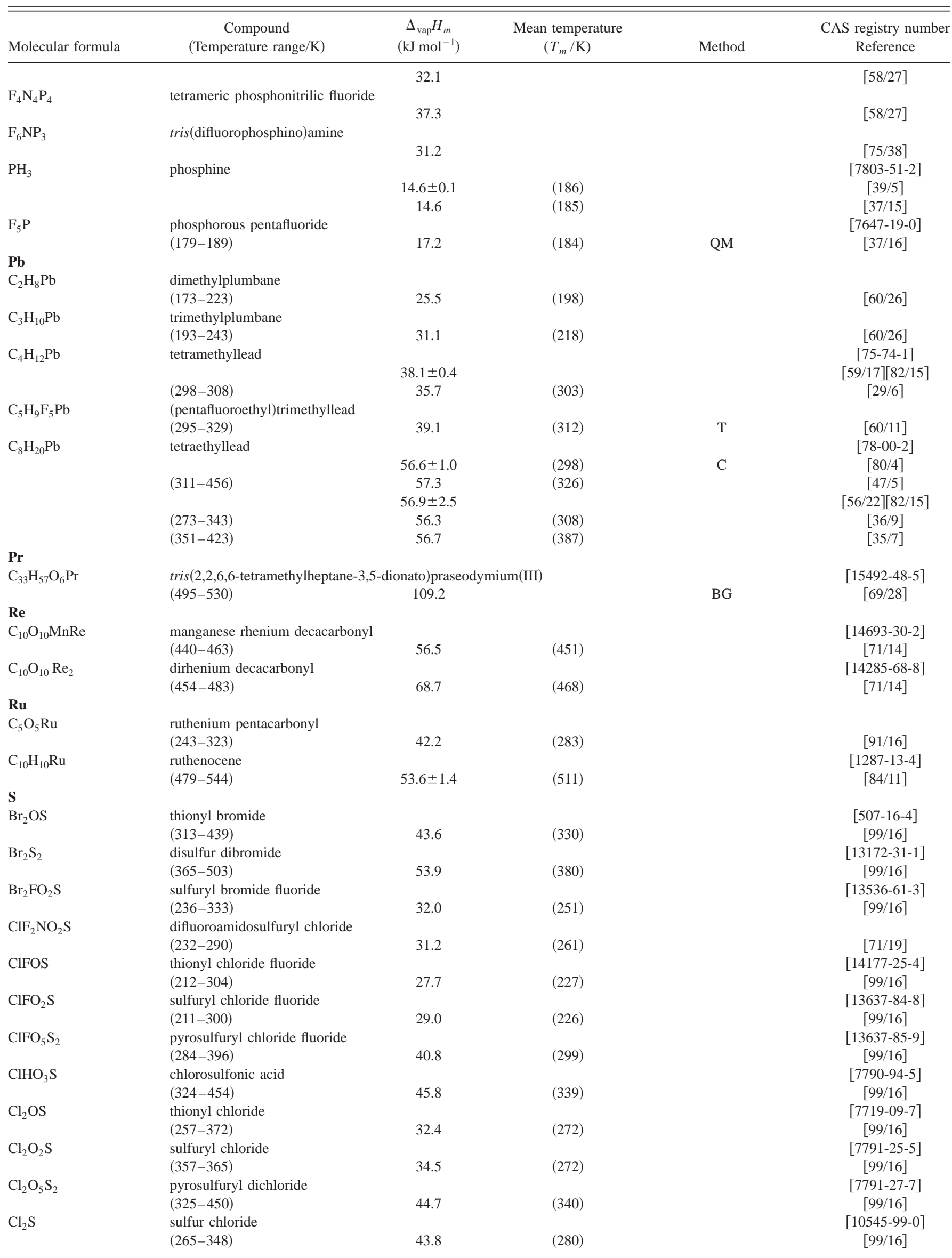


TABLE 7. Enthalpies of vaporization of select organo-metallic and inorganic compounds, 1880-2002-Continued

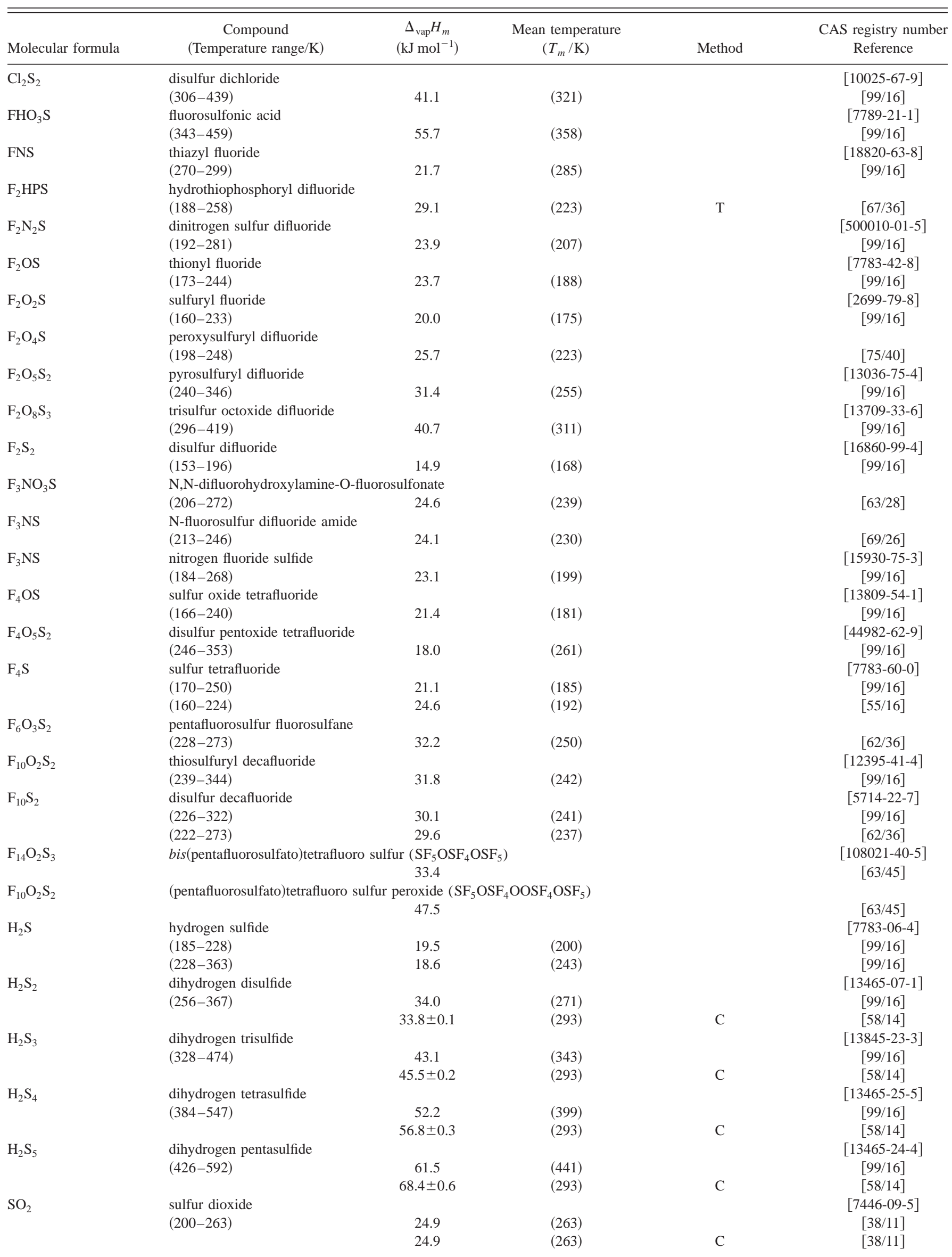


TABLE 7. Enthalpies of vaporization of select organo-metallic and inorganic compounds, 1880-2002-Continued

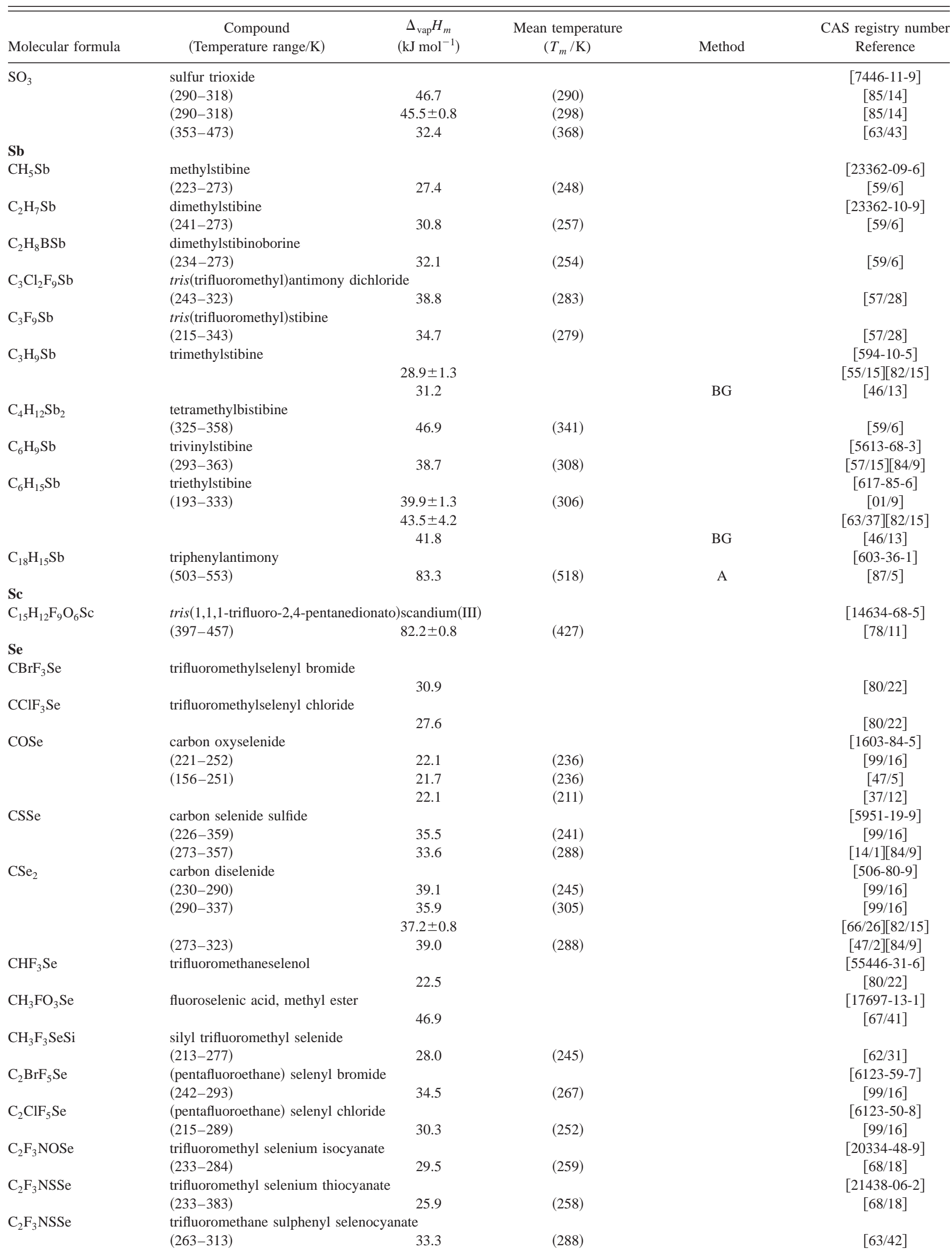


TABLE 7. Enthalpies of vaporization of select organo-metallic and inorganic compounds, 1880-2002-Continued

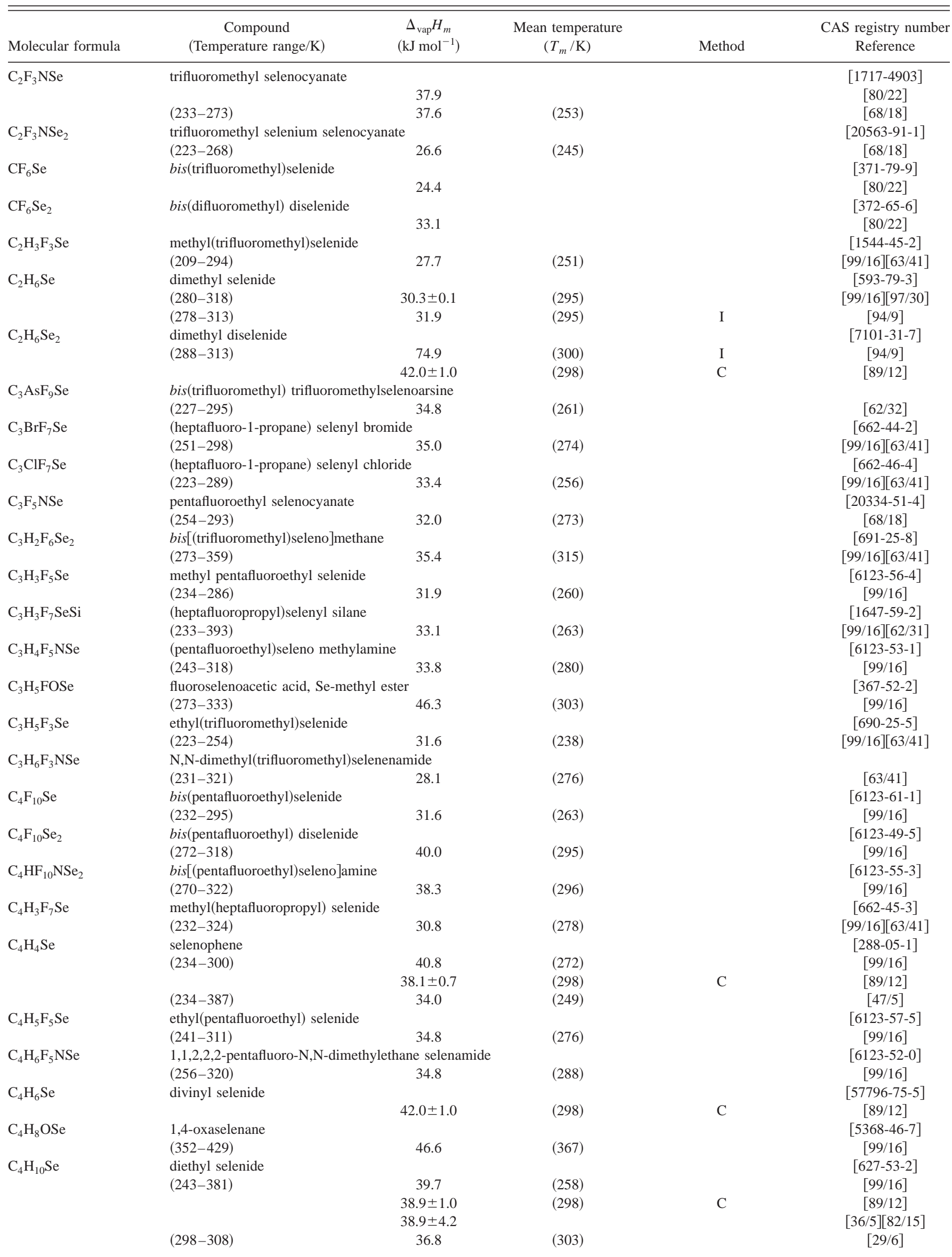


TABLE 7. Enthalpies of vaporization of select organo-metallic and inorganic compounds, 1880-2002-Continued

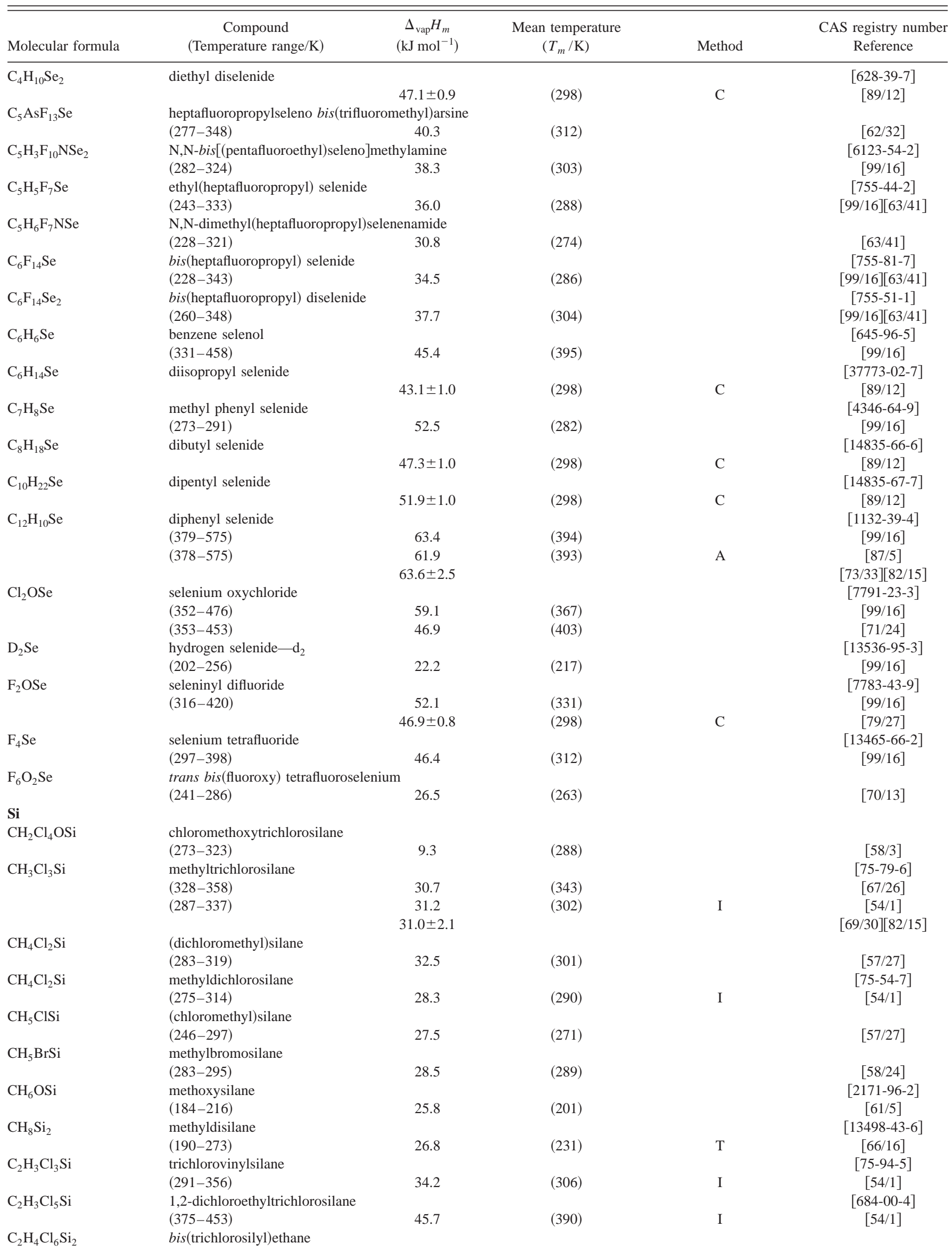


TABLE 7. Enthalpies of vaporization of select organo-metallic and inorganic compounds, 1880-2002-Continued

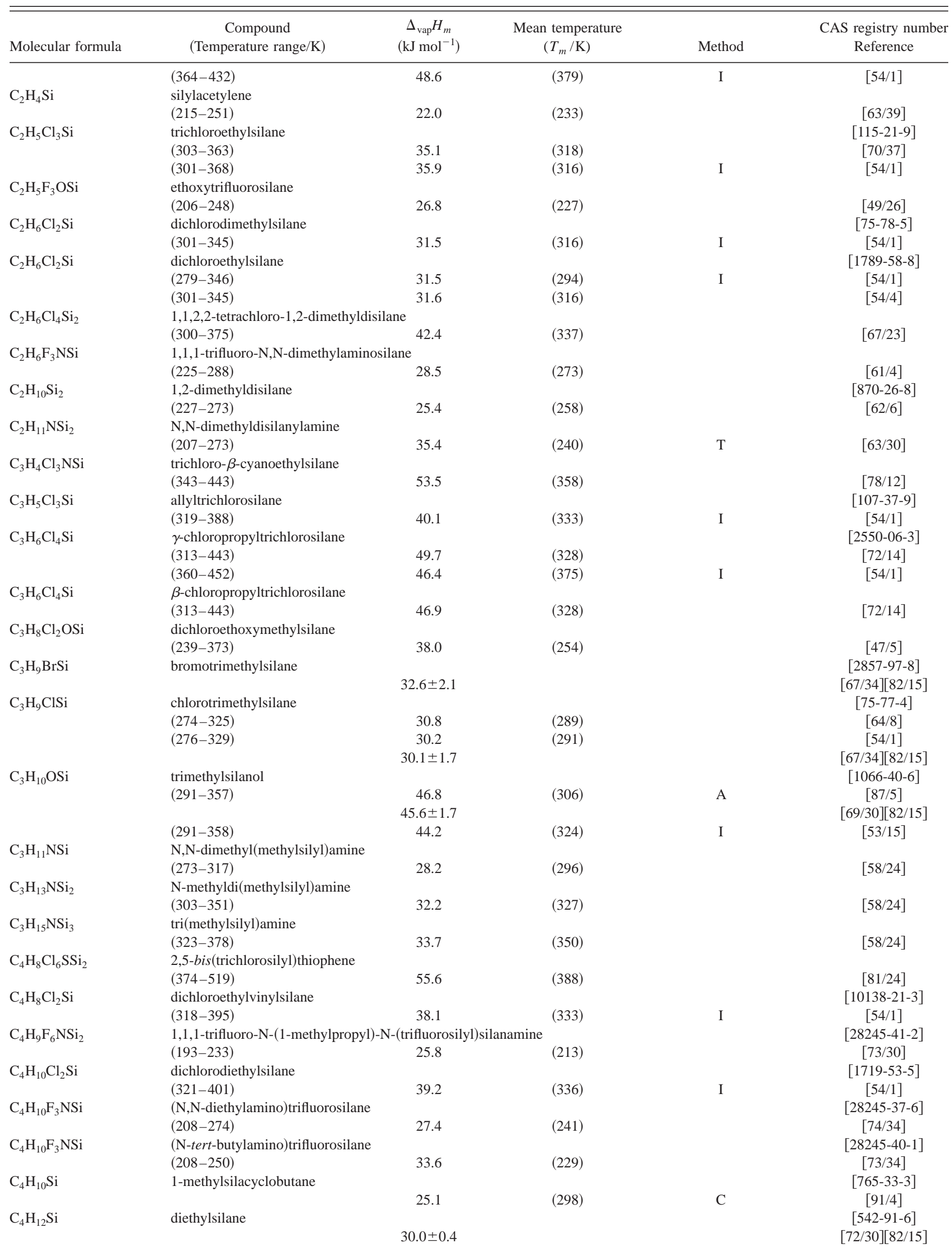


TABLE 7. Enthalpies of vaporization of select organo-metallic and inorganic compounds, 1880-2002-Continued

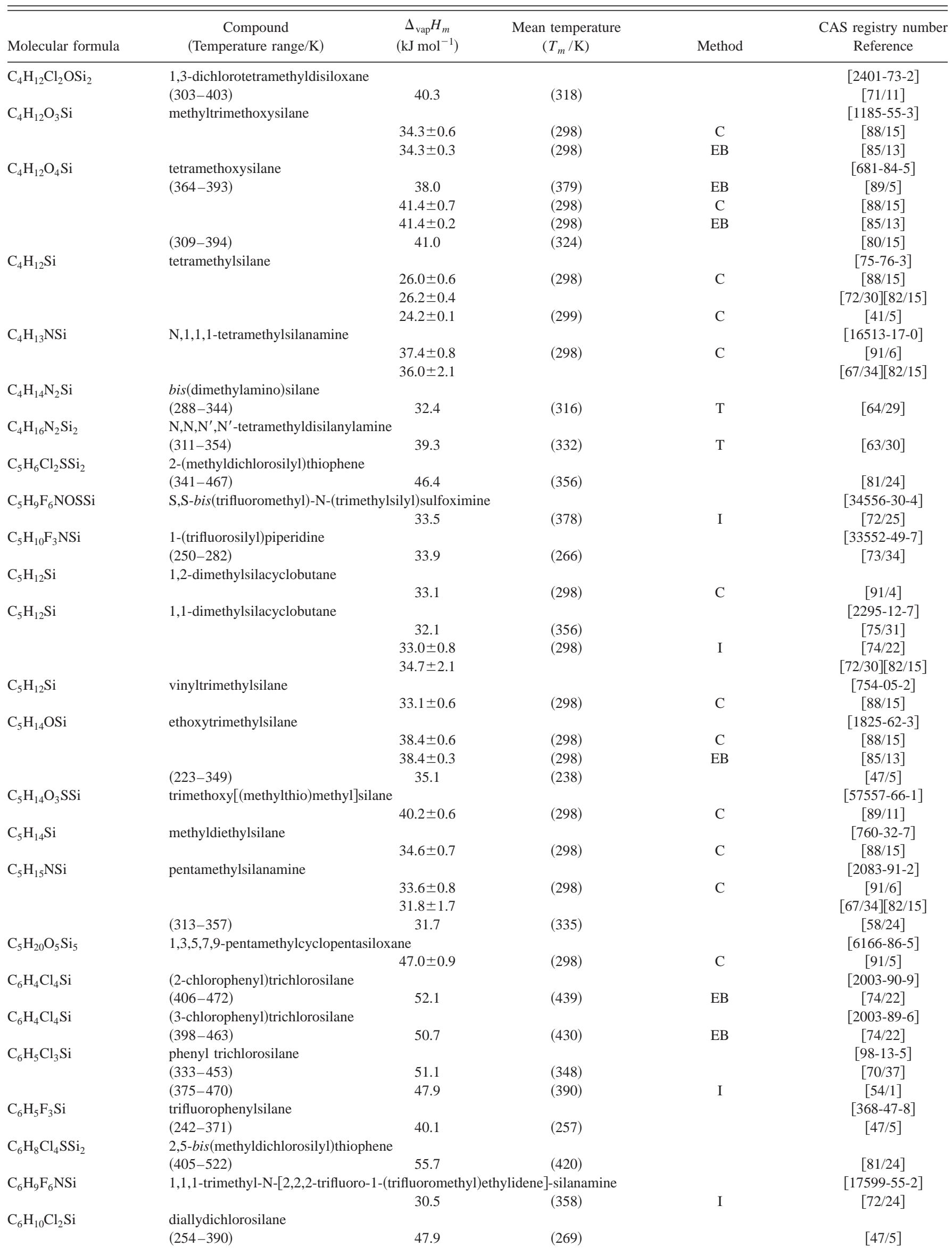


TABLE 7. Enthalpies of vaporization of select organo-metallic and inorganic compounds, 1880-2002-Continued

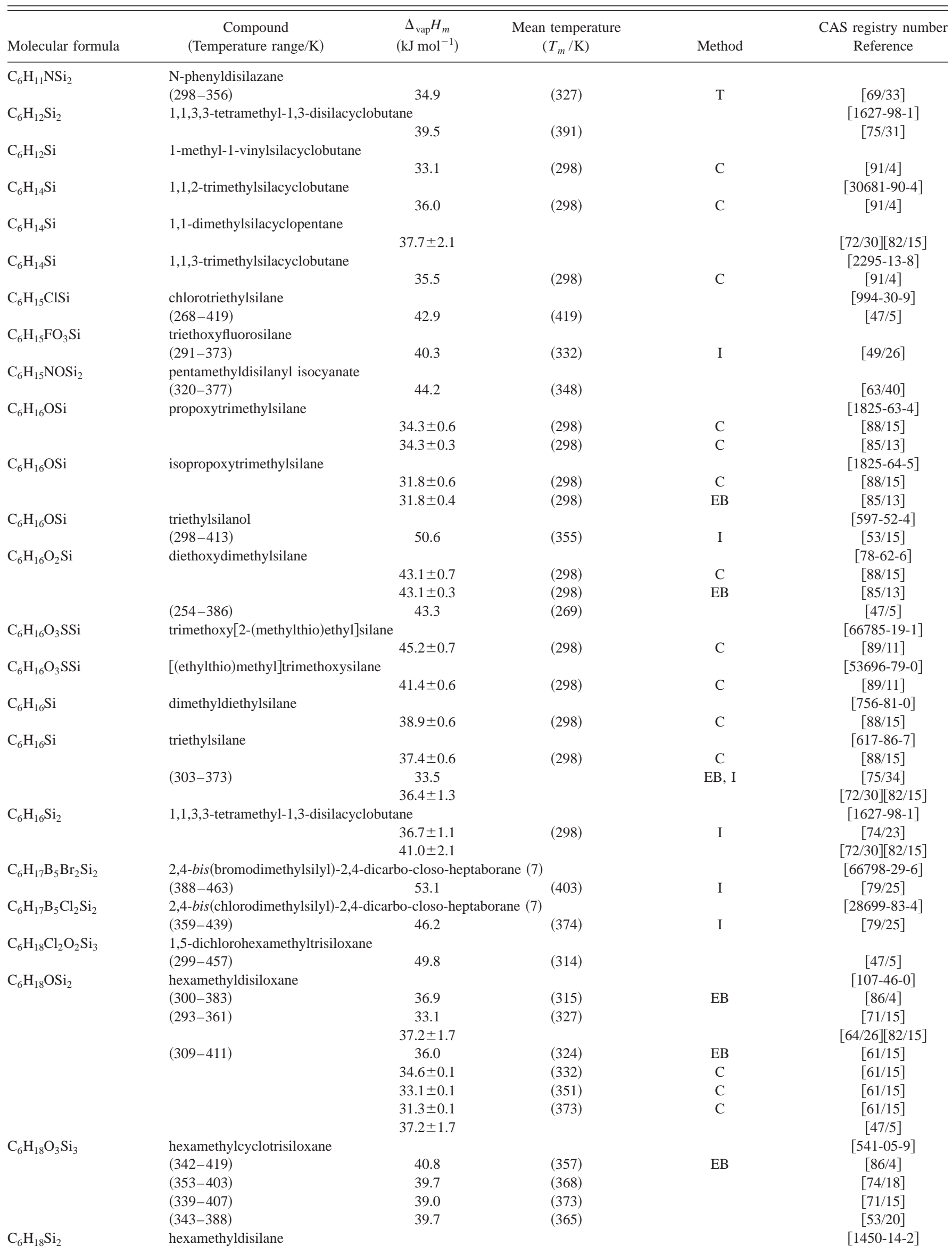


TABLE 7. Enthalpies of vaporization of select organo-metallic and inorganic compounds, 1880-2002-Continued

\begin{tabular}{|c|c|c|c|c|c|}
\hline Molecular formula & $\begin{array}{c}\text { Compound } \\
\text { (Temperature range/K) }\end{array}$ & $\begin{array}{c}\Delta_{\mathrm{vap}} H_{m} \\
\left(\mathrm{~kJ} \mathrm{~mol}^{-1}\right)\end{array}$ & $\begin{array}{l}\text { Mean temperature } \\
\qquad\left(T_{m} / \mathrm{K}\right)\end{array}$ & Method & $\begin{array}{c}\text { CAS registry number } \\
\text { Reference }\end{array}$ \\
\hline & $(305-387)$ & 36.3 & $(320)$ & $\mathrm{EB}$ & {$[86 / 12]$} \\
\hline & & $37.4 \pm 0.4$ & & & {$[72 / 30][82 / 15]$} \\
\hline & $(288-310)$ & 37.2 & & & {$[59 / 26][86 / 12]$} \\
\hline & $(294-334)$ & 36.8 & & & {$[41 / 12][86 / 12]$} \\
\hline \multirow[t]{2}{*}{$\mathrm{C}_{6} \mathrm{H}_{19} \mathrm{~B}_{5} \mathrm{Si}_{2}$} & \multicolumn{4}{|c|}{ 2,4-bis(dimethylsilyl)-2,4-dicarba-closo-heptaborane } & [59351-11-0] \\
\hline & $(373-453)$ & 41.3 & $(388)$ & $\mathrm{I}$ & {$[76 / 17]$} \\
\hline \multirow{4}{*}{$\mathrm{C}_{6} \mathrm{H}_{19} \mathrm{NSi}_{2}$} & \multirow[t]{2}{*}{ hexamethyldisilazane } & & & & {$[999-97-3]$} \\
\hline & & $42.2 \pm 0.9$ & $(298)$ & $\mathrm{C}$ & {$[91 / 6]$} \\
\hline & \multirow[t]{2}{*}{$(294-395)$} & 36.0 & $(344)$ & & {$[72 / 19]$} \\
\hline & & $41.4 \pm 2.1$ & & & {$[66 / 22][82 / 15]$} \\
\hline \multirow[t]{2}{*}{$\mathrm{C}_{6} \mathrm{H}_{19} \mathrm{~N}_{3} \mathrm{Si}$} & tris(dimethylamino)silane & & & & \\
\hline & $(309-387)$ & 41.1 & $(348)$ & $\mathrm{T}$ & {$[64 / 29]$} \\
\hline \multirow[t]{2}{*}{$\mathrm{C}_{6} \mathrm{H}_{21} \mathrm{~N}_{3} \mathrm{Si}_{3}$} & hexamethylcyclotrisilazane & & & & {$[1009-93-4]$} \\
\hline & $(342-456)$ & 45.6 & (399) & & {$[72 / 19]$} \\
\hline \multirow{2}{*}{$\mathrm{C}_{7} \mathrm{H}_{8} \mathrm{Cl}_{2} \mathrm{Si}$} & benzyl dichlorosilane & & & & [18173-99-4] \\
\hline & $(318-467)$ & 58.5 & $(333)$ & & {$[47 / 5][99 / 16]$} \\
\hline \multirow[t]{2}{*}{$\mathrm{C}_{7} \mathrm{H}_{8} \mathrm{Cl}_{2} \mathrm{Si}$} & phenyldichloromethylsilane & & & & {$[149-74-6]$} \\
\hline & $(309-479)$ & 51.2 & $(323)$ & & {$[47 / 5][99 / 16]$} \\
\hline \multirow{2}{*}{$\mathrm{C}_{7} \mathrm{H}_{8} \mathrm{Cl}_{2} \mathrm{Si}$} & dichloro-4-tolylsilane & & & & {$[13272-80-5]$} \\
\hline & $(319-469)$ & 58.0 & $(334)$ & & {$[47 / 5][99 / 16]$} \\
\hline \multirow[t]{2}{*}{$\mathrm{C}_{7} \mathrm{H}_{8} \mathrm{~F}_{2} \mathrm{Si}$} & difluoromethylphenylsilane & & & & {$[328-57-4]$} \\
\hline & $(303-413)$ & 44.6 & $(318)$ & & [99/16] \\
\hline $\mathrm{C}_{7} \mathrm{H}_{9} \mathrm{~F}_{8} \mathrm{NOSSi}$ & \multicolumn{4}{|c|}{$2,2,3,3,4,4,5,5$-octafluoro-1,1,2,3,4,5-hexahydro-1-[(trifluoromethyl)-silyl $]$ imino $]$ thiophene-1-oxide } & $\begin{array}{c}{[77589-40-3]} \\
{[81 / 16]}\end{array}$ \\
\hline \multirow[t]{2}{*}{$\mathrm{C}_{7} \mathrm{H}_{9} \mathrm{~F}_{9} \mathrm{~N}_{2} \mathrm{OSSi}$} & 1,1,1-trifluoro-N-[2,2,2-trifl & rifluoromethy & idene]-N'-(trimethyl & anesulfonimidamide & {$[62609-67-0]$} \\
\hline & & 39.3 & $(429)$ & I & {$[77 / 19][99 / 16]$} \\
\hline $\mathrm{C}_{7} \mathrm{H}_{16} \mathrm{O}_{3} \mathrm{SSi}$ & trimethoxy[(2-propenylthio) & ilane & & & {$[57877-58-4]$} \\
\hline & & $38.6 \pm 0.5$ & $(298)$ & $\mathrm{C}$ & {$[89 / 11]$} \\
\hline $\mathrm{C}_{7} \mathrm{H}_{17} \mathrm{ClSi}$ & (1-chloroethyl)diethylmethy & & & & {$[18817-17-9]$} \\
\hline & $(353-445)$ & 41.8 & $(400)$ & & {$[99 / 16]$} \\
\hline $\mathrm{C}_{7} \mathrm{H}_{18} \mathrm{OSi}$ & butyl trimethylsilyl ether & & & & {$[1825-65-6]$} \\
\hline & $(344-397)$ & 38.5 & $(359)$ & $\mathrm{EB}$ & {$[69 / 13][84 / 9]$} \\
\hline $\mathrm{C}_{7} \mathrm{H}_{18} \mathrm{O}_{3} \mathrm{SSi}$ & trimethoxy[3-(methylthio)p & & & & {$[94358-36-8]$} \\
\hline & & $43.5 \pm 0.6$ & $(298)$ & $\mathrm{C}$ & {$[89 / 11]$} \\
\hline $\mathrm{C}_{7} \mathrm{H}_{18} \mathrm{O}_{3} \mathrm{SSi}$ & [2-(ethylthio)ethyl] trimetho & & & & {$[40532-52-3]$} \\
\hline & & $41.4 \pm 0.7$ & $(298)$ & $\mathrm{C}$ & {$[89 / 11]$} \\
\hline $\mathrm{C}_{7} \mathrm{H}_{18} \mathrm{O}_{3} \mathrm{Si}$ & triethoxymethylsilane & & & & [2031-67-6] \\
\hline & & $45.1 \pm 0.7$ & $(298)$ & $\mathrm{C}$ & {$[88 / 15]$} \\
\hline & & $45.1 \pm 0.4$ & $(298)$ & $\mathrm{EB}$ & {$[85 / 13]$} \\
\hline & $(272-416)$ & 45.2 & $(287)$ & & {$[47 / 5]$} \\
\hline $\mathrm{C}_{7} \mathrm{H}_{18} \mathrm{SSi}$ & (n-butylthio)trimethylsilane & & & & \\
\hline & & $40.6 \pm 2.1$ & & & {$[67 / 34][82 / 15]$} \\
\hline $\mathrm{C}_{7} \mathrm{H}_{18} \mathrm{Si}$ & methyltriethylsilane & & & & [757-21-1] \\
\hline & & $40.5 \pm 0.6$ & $(298)$ & $\mathrm{C}$ & {$[88 / 15]$} \\
\hline $\mathrm{C}_{7} \mathrm{H}_{18} \mathrm{Si}$ & methyldipropylsilane & & & & [999-03-1] \\
\hline & & $35.9 \pm 0.7$ & $(298)$ & $\mathrm{C}$ & {$[88 / 15]$} \\
\hline $\mathrm{C}_{7} \mathrm{H}_{18} \mathrm{Si}$ & methyldiisopropylsilane & & & & {$[18442-00-7]$} \\
\hline & & $32.4 \pm 0.8$ & $(298)$ & $\mathrm{C}$ & {$[88 / 15]$} \\
\hline $\mathrm{C}_{7} \mathrm{H}_{19} \mathrm{NSi}$ & N,N-diethyl-1,1,1-trimethyl & & & & [996-50-9] \\
\hline & & $37.9 \pm 0.8$ & $(298)$ & $\mathrm{C}$ & {$[91 / 6]$} \\
\hline $\mathrm{C}_{7} \mathrm{H}_{20} \mathrm{Si}_{2}$ & methylene-bis(trimethylsilar & & & & {$[2117-28-4]$} \\
\hline & $(323-407)$ & $40.3 \pm 0.3$ & & QM & {$[76 / 25]$} \\
\hline & $(323-407)$ & $40.3 \pm 0.3$ & $(365)$ & & {$[75 / 25][75 / 31]$} \\
\hline $\mathrm{C}_{7} \mathrm{H}_{21} \mathrm{NSi}_{2}$ & $\mathrm{~N}, 1,1,1$-tetramethyl-N-(trim & )silanamine & & & [920-68-3] \\
\hline & & $38.1 \pm 0.8$ & $(298)$ & $\mathrm{C}$ & {$[91 / 6]$} \\
\hline & & $38.9 \pm 2.1$ & & & {$[67 / 34][82 / 15]$} \\
\hline $\mathrm{C}_{8} \mathrm{H}_{10} \mathrm{Cl}_{2} \mathrm{OSi}$ & dichloroethoxyphenylsilane & & & & {$[18236-80-1]$} \\
\hline & $(325-496)$ & 56.3 & $(340)$ & & {$[99 / 16]$} \\
\hline $\mathrm{C}_{8} \mathrm{H}_{10} \mathrm{Cl}_{2} \mathrm{Si}$ & dichloroethylphenylsilane & & & & {$[1125-27-5]$} \\
\hline & $(316-503)$ & 51.3 & $(331)$ & & {$[99 / 16]$} \\
\hline $\mathrm{C}_{8} \mathrm{H}_{11} \mathrm{ClSi}$ & chlorodimethylphenylsilane & & & & {$[768-33-2]$} \\
\hline & $(302-467)$ & 52.2 & $(317)$ & & {$[99 / 16]$} \\
\hline & $(303-466)$ & 49.7 & $(318)$ & & {$[47 / 5]$} \\
\hline $\mathrm{C}_{8} \mathrm{H}_{11} \mathrm{FSi}$ & fluorodimethylphenylsilane & & & & {$[454-57-9]$} \\
\hline
\end{tabular}


TABLE 7. Enthalpies of vaporization of select organo-metallic and inorganic compounds, 1880-2002-Continued

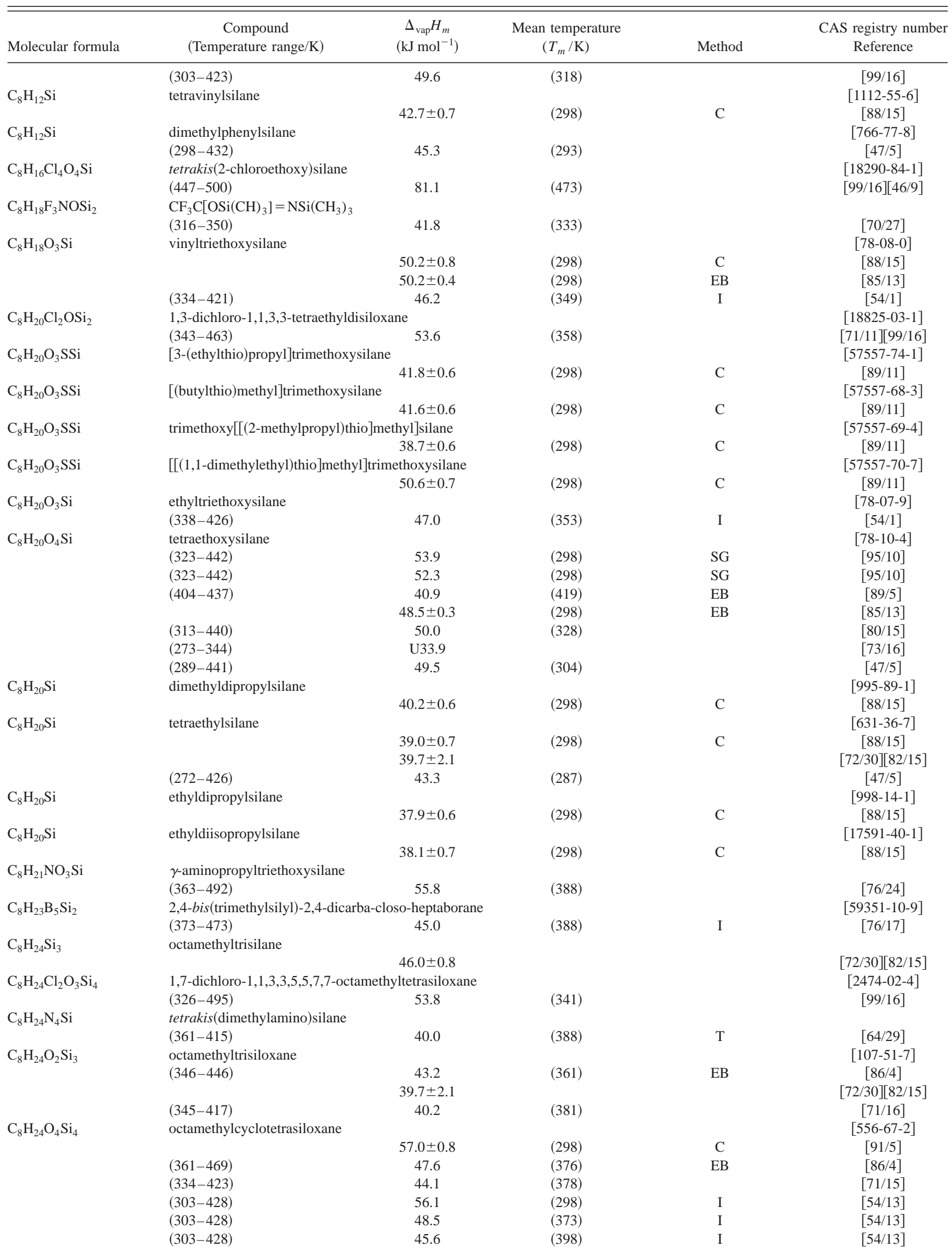


TABLE 7. Enthalpies of vaporization of select organo-metallic and inorganic compounds, 1880-2002-Continued

\begin{tabular}{|c|c|c|c|c|c|}
\hline Molecular formula & $\begin{array}{c}\text { Compound } \\
\text { (Temperature range/K) }\end{array}$ & $\begin{array}{c}\Delta_{\mathrm{vap}} H_{m} \\
\left(\mathrm{~kJ} \mathrm{~mol}^{-1}\right)\end{array}$ & $\begin{array}{l}\text { Mean temperature } \\
\qquad\left(T_{m} / \mathrm{K}\right)\end{array}$ & Method & $\begin{array}{l}\text { CAS registry number } \\
\text { Reference }\end{array}$ \\
\hline $\mathrm{C}_{8} \mathrm{H}_{28} \mathrm{~N}_{4} \mathrm{Si}_{4}$ & $\begin{array}{l}\text { octamethylcyclotetrasilazane } \\
(388-513)\end{array}$ & 52.3 & $(450)$ & & $\begin{array}{c}{[1020-84-4]} \\
{[72 / 19]}\end{array}$ \\
\hline $\mathrm{C}_{9} \mathrm{H}_{14} \mathrm{OSi}$ & phenoxytrimethylsilane & $56.9 \pm 0.8$ & (298) & $\mathrm{C}$ & $\begin{array}{c}{[1529-17-5]} \\
{[88 / 15]}\end{array}$ \\
\hline $\mathrm{C}_{9} \mathrm{H}_{20} \mathrm{OSi}$ & $\begin{array}{l}\text { cyclohexyl trimethylsilyl ether } \\
(364-441)\end{array}$ & 45.1 & $(379)$ & $\mathrm{EB}$ & $\begin{array}{c}{[13871-89-1]} \\
{[69 / 13]}\end{array}$ \\
\hline $\mathrm{C}_{9} \mathrm{H}_{22} \mathrm{O}_{3} \mathrm{SSi}$ & triethoxy[(ethylthio)methyl]silane & $42.3 \pm 0.6$ & $(298)$ & $\mathrm{C}$ & $\begin{array}{c}{[53696-83-6]} \\
{[89 / 11]}\end{array}$ \\
\hline $\mathrm{C}_{9} \mathrm{H}_{22} \mathrm{Si}$ & propyltriethylsilane & $40.0 \pm 0.7$ & $(298)$ & $\mathrm{C}$ & $\begin{array}{c}{[994-44-5]} \\
{[88 / 15]}\end{array}$ \\
\hline $\mathrm{C}_{9} \mathrm{H}_{22} \mathrm{Si}$ & tripropylsilane & $39.1 \pm 0.7$ & $(298)$ & $\mathrm{C}$ & $\begin{array}{c}{[998-29-8]} \\
{[88 / 15]}\end{array}$ \\
\hline $\mathrm{C}_{9} \mathrm{H}_{22} \mathrm{Si}$ & methyldibutylsilane & $36.2 \pm 0.7$ & $(298)$ & $\mathrm{C}$ & $\begin{array}{c}{[999-35-9]} \\
{[88 / 15]}\end{array}$ \\
\hline $\mathrm{C}_{9} \mathrm{H}_{23} \mathrm{NSi}$ & 1,1,1-triethyl-N-(1-methylethyl)silana & $\begin{array}{l}\text { amine } \\
38.6 \pm 0.8\end{array}$ & $(298)$ & $\mathrm{C}$ & $\begin{array}{c}{[5277-20-3]} \\
{[91 / 6]}\end{array}$ \\
\hline $\mathrm{C}_{9} \mathrm{H}_{23} \mathrm{NSi}$ & 1,1,1-triethyl-N-propylsilanamine & $41.5 \pm 0.8$ & $(298)$ & $\mathrm{C}$ & $\begin{array}{c}{[17887-11-5]} \\
{[91 / 6]}\end{array}$ \\
\hline $\mathrm{C}_{9} \mathrm{H}_{24} \mathrm{Si}_{2}$ & $\begin{array}{l}\text { 1,3-propanediyl-bis(trimethylsilane) } \\
(338-443) \\
(338-443)\end{array}$ & $\begin{array}{l}43.1 \pm 0.5 \\
43.1 \pm 0.5\end{array}$ & $(390)$ & QM & $\begin{array}{c}{[2295-05-8]} \\
{[76 / 25]} \\
{[75 / 25][75 / 31]}\end{array}$ \\
\hline $\mathrm{C}_{9} \mathrm{H}_{27} \mathrm{NSi}_{3}$ & tris(trimethylsilyl)amine & $54.4 \pm 8.4$ & & & {$[67 / 34][82 / 15]$} \\
\hline $\mathrm{C}_{10} \mathrm{H}_{11} \mathrm{NSi}_{4}$ & $\begin{array}{l}\text { 1,1,3,3-tetramethyl-1,3-bis (trimethylsi } \\
(378-435)\end{array}$ & $\begin{array}{l}\text { ilyl)disilazane } \\
58.0\end{array}$ & $(393)$ & A & {$[87 / 5]$} \\
\hline $\mathrm{C}_{10} \mathrm{H}_{16} \mathrm{OSi}$ & (2-methoxyphenyl)trimethylsilane & $59.4 \pm 0.8$ & $(298)$ & $\mathrm{C}$ & $\begin{array}{c}{[704-43-8]} \\
{[88 / 15]}\end{array}$ \\
\hline $\mathrm{C}_{10} \mathrm{H}_{16} \mathrm{OSi}$ & (3-methoxyphenyl)trimethylsilane & $56.1 \pm 0.8$ & $(298)$ & $\mathrm{C}$ & $\begin{array}{c}{[17876-90-3]} \\
{[88 / 15]}\end{array}$ \\
\hline $\mathrm{C}_{10} \mathrm{H}_{16} \mathrm{OSi}$ & (4-methoxyphenyl)trimethylsilane & $56.9 \pm 0.8$ & $(298)$ & $\mathrm{C}$ & $\begin{array}{c}{[877-68-9]} \\
{[88 / 15]}\end{array}$ \\
\hline $\mathrm{C}_{10} \mathrm{H}_{16} \mathrm{OSi}$ & $\begin{array}{l}m \text {-tolyl trimethylsilyl ether } \\
(371-398)\end{array}$ & 49.7 & $(384)$ & EB & $\begin{array}{c}{[17902-31-7]} \\
{[69 / 13]}\end{array}$ \\
\hline $\mathrm{C}_{10} \mathrm{H}_{16} \mathrm{OSi}$ & $\begin{array}{l}p \text {-tolyl trimethylsilyl ether } \\
(374-402)\end{array}$ & 49.8 & $(388)$ & EB & $\begin{array}{c}{[17902-32-8]} \\
{[69 / 13]}\end{array}$ \\
\hline & & $56.4 \pm 0.7$ & $(298)$ & $\mathrm{C}$ & {$[89 / 11]$} \\
\hline $\begin{array}{l}\mathrm{C}_{10} \mathrm{H}_{20} \mathrm{O}_{2} \mathrm{Si} \\
\mathrm{C}_{10} \mathrm{H}_{24} \mathrm{O}_{2} \mathrm{Si}\end{array}$ & $\begin{array}{l}\text { diallyl(diethoxy)silane } \\
\text { (342-459) } \\
\text { dipropyldiethoxysilane }\end{array}$ & 48.3 & $(357)$ & A & $\begin{array}{c}{[13081-67-9]} \\
{[87 / 5]} \\
{[2031-63-2]}\end{array}$ \\
\hline & & $\begin{array}{l}46.5 \pm 0.7 \\
46.4 \pm 0.3\end{array}$ & $\begin{array}{l}(298) \\
(298)\end{array}$ & $\begin{array}{c}\mathrm{C} \\
\mathrm{EB}\end{array}$ & $\begin{array}{l}{[88 / 15]} \\
{[85 / 13]}\end{array}$ \\
\hline $\mathrm{C}_{10} \mathrm{H}_{24} \mathrm{O}_{3} \mathrm{SSi}$ & triethoxy[2-(ethylthio)ethyl]silane & $46.9 \pm 0.7$ & $(298)$ & $\mathrm{C}$ & $\begin{array}{c}{[57557-72-9]} \\
{[89 / 11]}\end{array}$ \\
\hline $\mathrm{C}_{10} \mathrm{H}_{24} \mathrm{Si}$ & diethyldipropylsilane & $41.5 \pm 0.7$ & $(298)$ & $\mathrm{C}$ & $\begin{array}{c}{[994-59-2]} \\
{[88 / 15]}\end{array}$ \\
\hline $\mathrm{C}_{10} \mathrm{H}_{24} \mathrm{Si}$ & methyltripropylsilane & $42.6 \pm 0.6$ & $(298)$ & $\mathrm{C}$ & $\begin{array}{c}{[995-24-4]} \\
{[88 / 15]}\end{array}$ \\
\hline $\mathrm{C}_{10} \mathrm{H}_{24} \mathrm{Si}$ & ethyldibutylsilane & $39.9 \pm 0.7$ & $(298)$ & $\mathrm{C}$ & $\begin{array}{c}{[998-61-8]} \\
{[88 / 15]}\end{array}$ \\
\hline $\mathrm{C}_{10} \mathrm{H}_{24} \mathrm{Si}$ & ethyldiisobutylsilane & $39.8 \pm 0.7$ & $(298)$ & $\mathrm{C}$ & $\begin{array}{c}{[17591-42-3]} \\
{[88 / 15]}\end{array}$ \\
\hline $\mathrm{C}_{10} \mathrm{H}_{25} \mathrm{NO}_{2} \mathrm{Si}_{3}$ & $\begin{array}{l}\text { 1,1,1,3,5,5,5-heptamethyl-3-(2-cyanoe } \\
(367-511)\end{array}$ & $\begin{array}{l}\text { ethyl)trisiloxane } \\
59.5\end{array}$ & $(382)$ & A & {$[87 / 5]$} \\
\hline $\mathrm{C}_{10} \mathrm{H}_{25} \mathrm{NSi}$ & pentaethylsilanamine & $42.2 \pm 1.0$ & $(298)$ & $\mathrm{C}$ & $\begin{array}{c}{[6022-10-2]} \\
{[91 / 6]}\end{array}$ \\
\hline $\mathrm{C}_{10} \mathrm{H}_{25} \mathrm{NSi}$ & $\mathrm{N}$-(1,1-dimethylethyl)-1,1,1-triethylsil & $\begin{array}{l}\text { lanamine } \\
40.3 \pm 0.9\end{array}$ & $(298)$ & $\mathrm{C}$ & $\begin{array}{c}{[17940-20-4]} \\
{[91 / 6]}\end{array}$ \\
\hline $\mathrm{C}_{10} \mathrm{H}_{28} \mathrm{O}_{4} \mathrm{Si}_{3}$ & $\begin{array}{l}\text { 1,5-diethoxy-1,1,3,3,5,5-hexamethyltr } \\
(314-470)\end{array}$ & $\begin{array}{l}\text { risiloxane } \\
56.2\end{array}$ & $(329)$ & A & $\begin{array}{c}{[17928-13-1]} \\
{[87 / 5]}\end{array}$ \\
\hline $\mathrm{C}_{10} \mathrm{H}_{30} \mathrm{O}_{3} \mathrm{Si}_{4}$ & $\begin{array}{l}\text { methyl tris(trimethylsiloxy)silane } \\
(362-476)\end{array}$ & 49.5 & $(377)$ & $\mathrm{EB}$ & {$[86 / 4]$} \\
\hline $\mathrm{C}_{10} \mathrm{H}_{30} \mathrm{O}_{3} \mathrm{Si}_{4}$ & $\begin{array}{l}\text { decamethyl tetrasiloxane } \\
(366-479)\end{array}$ & 50.3 & $(381)$ & $\mathrm{EB}$ & {$[86 / 4]$} \\
\hline
\end{tabular}


TABLE 7. Enthalpies of vaporization of select organo-metallic and inorganic compounds, 1880-2002-Continued

\begin{tabular}{|c|c|c|c|c|c|}
\hline Molecular formula & $\begin{array}{c}\text { Compound } \\
\text { (Temperature range/K) }\end{array}$ & $\begin{array}{c}\Delta_{\mathrm{vap}} H_{m} \\
\left(\mathrm{~kJ} \mathrm{~mol}^{-1}\right)\end{array}$ & $\begin{array}{l}\text { Mean temperature } \\
\qquad\left(T_{m} / \mathrm{K}\right)\end{array}$ & Method & $\begin{array}{c}\text { CAS registry number } \\
\text { Reference }\end{array}$ \\
\hline \multirow{6}{*}{$\mathrm{C}_{10} \mathrm{H}_{30} \mathrm{O}_{5} \mathrm{Si}_{5}$} & $(343-454)$ & 48.2 & $(358)$ & A & {$[87 / 5][71 / 16]$} \\
\hline & decamethyl cyclopentasiloxane & & & & \\
\hline & & $59.0 \pm 1.0$ & (298) & $\mathrm{C}$ & [91/5] \\
\hline & $(383-496)$ & 52.1 & (398) & EB & {$[86 / 4]$} \\
\hline & $(364-472)$ & 49.0 & (379) & A & {$[87 / 5][71 / 15]$} \\
\hline & & $48.1 \pm 2.1$ & & & {$[72 / 30][82 / 15]$} \\
\hline \multirow[t]{2}{*}{$\mathrm{C}_{10} \mathrm{H}_{30} \mathrm{Si}_{4}$} & decamethyltetrasilane & & & & \\
\hline & & $52.3 \pm 1.7$ & & & {$[72 / 30][82 / 15]$} \\
\hline \multirow[t]{3}{*}{$\mathrm{C}_{10} \mathrm{H}_{31} \mathrm{NSi}_{4}$} & bis(pentamethyldisilanyl)amine & & & & \\
\hline & $(376-435)$ & 57.8 & $(405)$ & & {$[63 / 40]$} \\
\hline & [Note: Molecular formula give & oaper is not $\mathrm{cc}$ & nt with chemical nan & & \\
\hline \multirow[t]{2}{*}{$\mathrm{C}_{11} \mathrm{H}_{18} \mathrm{O}_{3} \mathrm{SSi}$} & trimethoxy $[[$ (phenylmethyl)thic & hyl]silane & & & {$[53696-80-3]$} \\
\hline & & $56.1 \pm 0.7$ & $(298)$ & $\mathrm{C}$ & [89/11] \\
\hline \multirow[t]{2}{*}{$\mathrm{C}_{11} \mathrm{H}_{20} \mathrm{OSi}$} & triallylethoxy silane & & & & [17962-20-8] \\
\hline & $(349-473)$ & 48.4 & (364) & A & {$[87 / 5]$} \\
\hline \multirow[t]{3}{*}{$\mathrm{C}_{11} \mathrm{H}_{20} \mathrm{OSi}_{2}$} & pentamethylphenyl disiloxane & & & & [14920-92-4] \\
\hline & $(347-474)$ & 53.3 & $(362)$ & A & {$[87 / 5]$} \\
\hline & $(347-474)$ & 44.4 & $(410)$ & & {$[74 / 32]$} \\
\hline \multirow[t]{2}{*}{$\mathrm{C}_{11} \mathrm{H}_{20} \mathrm{O}_{3} \mathrm{Si}_{3}$} & 1,1,3,3,5-pentamethyl-5-pheny & trisiloxane & & & \\
\hline & $(396-503)$ & 48.0 & $(450)$ & & {$[74 / 17]$} \\
\hline \multirow[t]{3}{*}{$\mathrm{C}_{11} \mathrm{H}_{24} \mathrm{O}_{3} \mathrm{Si}$} & vinyltripropoxysilane & & & & \\
\hline & & $52.3 \pm 0.9$ & $(298)$ & $\mathrm{C}$ & {$[88 / 15]$} \\
\hline & & $52.3 \pm 0.4$ & $(298)$ & EB & {$[85 / 13]$} \\
\hline \multirow[t]{2}{*}{$\mathrm{C}_{11} \mathrm{H}_{26} \mathrm{Si}$} & ethyltripropylsilane & & & & [994-63-8] \\
\hline & & $41.0 \pm 0.7$ & $(298)$ & $\mathrm{C}$ & {$[88 / 15]$} \\
\hline \multirow[t]{2}{*}{$\mathrm{C}_{11} \mathrm{H}_{26} \mathrm{Si}$} & methyldipentylsilane & & & & [1001-48-5] \\
\hline & & $40.3 \pm 0.7$ & $(298)$ & $\mathrm{C}$ & {$[88 / 15]$} \\
\hline \multirow[t]{2}{*}{$\mathrm{C}_{11} \mathrm{H}_{26} \mathrm{Si}$} & methyldi(2,2-dimethylpropyl)si & & & & \\
\hline & & $38.1 \pm 0.1$ & $(298)$ & $\mathrm{C}$ & {$[88 / 15]$} \\
\hline \multirow[t]{2}{*}{$\mathrm{C}_{11} \mathrm{H}_{27} \mathrm{NSi}$} & 1,1,1-triethyl-N-(1-methylbutyl & lamine & & & [133943-80-3] \\
\hline & & $46.9 \pm 1.0$ & $(298)$ & $\mathrm{C}$ & {$[91 / 6]$} \\
\hline \multirow[t]{3}{*}{$\mathrm{C}_{11} \mathrm{H}_{28} \mathrm{O}_{4} \mathrm{Si}_{4}$} & $8,8,10,10,12,12$-hexamethyl- 7, & 3 -tetrasiloxa- & 12-tetrasilaspiro $[5,7]$ & & [35331-58-9] \\
\hline & $(393-504)$ & 47.6 & $(408)$ & A & {$[87 / 5]$} \\
\hline & $(393-504)$ & 48.8 & $(449)$ & & {$[74 / 17]$} \\
\hline \multirow[t]{2}{*}{$\mathrm{C}_{11} \mathrm{H}_{28} \mathrm{O}_{4} \mathrm{Si}_{4}$} & hexamethyl(silacyclohexyl)cyc & asiloxane & & & \\
\hline & $(403-504)$ & 48.89 & $(453)$ & & {$[74 / 21]$} \\
\hline $\mathrm{C}_{12} \mathrm{H}_{9} \mathrm{Cl}_{3} \mathrm{Si}$ & 2-(trichlorosilyl)biphenyl & & & & {$[18030-62-1]$} \\
\hline & $(461-552)$ & 67.1 & (476) & A & {$[87 / 5]$} \\
\hline $\mathrm{C}_{12} \mathrm{H}_{9} \mathrm{Cl}_{3} \mathrm{Si}$ & 4-(trichlorosilyl)biphenyl & & & & [18030-61-0] \\
\hline & $(479-573)$ & 75.7 & $(494)$ & A & {$[87 / 5]$} \\
\hline $\mathrm{C}_{12} \mathrm{H}_{10} \mathrm{Cl}_{2} \mathrm{Si}$ & dichlorodiphenylsilane & & & & {$[80-10-4]$} \\
\hline & $(465-555)$ & 62.5 & $(480)$ & A, I & {$[87 / 5][54 / 1]$} \\
\hline & & $69.5 \pm 4.2$ & & & {$[66 / 23][82 / 15]$} \\
\hline $\mathrm{C}_{12} \mathrm{H}_{10} \mathrm{~F}_{2} \mathrm{Si}$ & difluorodiphenylsilane & & & & [312-40-3] \\
\hline & $(392-516)$ & 50.7 & (407) & A & [87/5] \\
\hline $\mathrm{C}_{12} \mathrm{H}_{13} \mathrm{NSi}$ & (N,N-diphenylamino)silane & & & & \\
\hline & $(425-495)$ & 50.4 & $(460)$ & $\mathrm{T}$ & {$[69 / 34]$} \\
\hline $\mathrm{C}_{12} \mathrm{H}_{20} \mathrm{Cl}_{8} \mathrm{O}_{4} \mathrm{Si}$ & $\operatorname{tris}\left(2,2^{\prime}\right.$-dichloroisopropyl) ort & cate & & & \\
\hline & $(517-532)$ & U172.7(524) & & & {$[46 / 9]$} \\
\hline $\mathrm{C}_{12} \mathrm{H}_{20} \mathrm{O}_{3} \mathrm{Si}$ & triethoxyphenylsilane & & & & {$[780-69-8]$} \\
\hline & & $58.3 \pm 0.9$ & $(298)$ & $\mathrm{C}$ & {$[88 / 15]$} \\
\hline & $(344-506)$ & 61.8 & $(359)$ & & {$[47 / 5]$} \\
\hline $\mathrm{C}_{12} \mathrm{H}_{28} \mathrm{O}_{4} \mathrm{Si}$ & tetrapropoxysilane & & & & {$[682-01-9]$} \\
\hline & & $49.8 \pm 0.8$ & (298) & $\mathrm{C}$ & {$[88 / 15]$} \\
\hline & $(307-563)$ & 66.9 & $(322)$ & A & {$[87 / 5]$} \\
\hline & & $49.8 \pm 0.4$ & (298) & EB & [85/13] \\
\hline $\mathrm{C}_{12} \mathrm{H}_{28} \mathrm{O}_{4} \mathrm{Si}$ & tetraisopropoxysilane & & & & [1992-48-9] \\
\hline & $(327-438)$ & 52.7 & $(342)$ & & {$[80 / 15]$} \\
\hline $\mathrm{C}_{12} \mathrm{H}_{28} \mathrm{Si}$ & tetrapropylsilane & & & & {$[994-66-1]$} \\
\hline & & $42.2 \pm 0.7$ & $(298)$ & $\mathrm{C}$ & {$[88 / 15]$} \\
\hline $\mathrm{C}_{12} \mathrm{H}_{28} \mathrm{Si}$ & tributylsilane & & & & [998-41-4] \\
\hline & & $42.9 \pm 0.7$ & $(298)$ & $\mathrm{C}$ & {$[88 / 15]$} \\
\hline $\mathrm{C}_{12} \mathrm{H}_{28} \mathrm{Si}$ & triisobutylsilane & & & & {$[6485-81-0]$} \\
\hline & & $40.0 \pm 0.7$ & (298) & $\mathrm{C}$ & {$[88 / 15]$} \\
\hline
\end{tabular}


TABLE 7. Enthalpies of vaporization of select organo-metallic and inorganic compounds, 1880-2002-Continued

\begin{tabular}{|c|c|c|c|c|c|}
\hline Molecular formula & $\begin{array}{c}\text { Compound } \\
\text { (Temperature range/K) }\end{array}$ & $\begin{array}{c}\Delta_{\mathrm{vap}} H_{m} \\
\left(\mathrm{~kJ} \mathrm{~mol}^{-1}\right)\end{array}$ & $\begin{array}{l}\text { Mean temperature } \\
\qquad\left(T_{m} / \mathrm{K}\right)\end{array}$ & Method & $\begin{array}{l}\text { CAS registry number } \\
\text { Reference }\end{array}$ \\
\hline $\mathrm{C}_{12} \mathrm{H}_{28} \mathrm{Si}$ & ethyldipentylsilane & $41.2 \pm 0.7$ & (298) & $\mathrm{C}$ & $\begin{array}{c}{[17591-43-4]} \\
{[88 / 15]}\end{array}$ \\
\hline $\mathrm{C}_{12} \mathrm{H}_{28} \mathrm{Si}$ & ethyldiisopentylsilane & $42.6 \pm 0.7$ & (298) & $\mathrm{C}$ & $\begin{array}{c}{[18159-61-0]} \\
{[88 / 15]}\end{array}$ \\
\hline $\mathrm{C}_{12} \mathrm{H}_{30} \mathrm{HgSi}_{2}$ & $\begin{array}{l}\text { bis(triethylsilyl)mercury } \\
(383-433)\end{array}$ & 64.0 & (398) & & {$[72 / 15]$} \\
\hline $\mathrm{C}_{12} \mathrm{H}_{30} \mathrm{O}_{3} \mathrm{Si}_{3}$ & $\begin{array}{l}\text { hexaethyl cyclotrisiloxane } \\
(385-524) \\
(434-516)\end{array}$ & $\begin{array}{l}57.9 \\
58.7\end{array}$ & $\begin{array}{l}(400) \\
(449)\end{array}$ & $\begin{array}{l}\mathrm{A} \\
\mathrm{A}\end{array}$ & $\begin{array}{c}{[2031-79-0]} \\
{[87 / 5]} \\
{[87 / 5][54 / 1]}\end{array}$ \\
\hline $\mathrm{C}_{12} \mathrm{H}_{31} \mathrm{~N}_{3} \mathrm{Si}$ & $\mathrm{N}, \mathrm{N}, \mathrm{N}^{\prime}, \mathrm{N}^{\prime}, \mathrm{N}^{\prime \prime}, \mathrm{N}^{\prime \prime}$-hexamethylsilanetri & $\begin{array}{l}\text { iamine } \\
58.4 \pm 1.0\end{array}$ & (298) & $\mathrm{C}$ & $\begin{array}{c}{[15730-66-2]} \\
{[91 / 6]}\end{array}$ \\
\hline $\mathrm{C}_{12} \mathrm{H}_{36} \mathrm{O}_{4} \mathrm{Si}_{5}$ & $\begin{array}{l}\text { dodecamethyl pentasiloxane } \\
(395-515) \\
(389-498)\end{array}$ & $\begin{array}{c}55.4 \\
50.3 \\
53.1 \pm 2.1\end{array}$ & $\begin{array}{l}(410) \\
(404)\end{array}$ & $\begin{array}{c}\mathrm{EB} \\
\mathrm{A}\end{array}$ & $\begin{array}{c}{[141-63-9]} \\
{[86 / 4]} \\
{[87 / 5][71 / 16]} \\
{[72 / 30][82 / 15]}\end{array}$ \\
\hline $\mathrm{C}_{12} \mathrm{H}_{36} \mathrm{O}_{6} \mathrm{Si}_{6}$ & $\begin{array}{l}\text { dodecamethyl cyclohexasiloxane } \\
(411-531) \\
(340-509)\end{array}$ & $\begin{array}{l}56.1 \\
62.6\end{array}$ & $\begin{array}{l}(426) \\
(355)\end{array}$ & $\begin{array}{c}\mathrm{EB} \\
\mathrm{A}\end{array}$ & $\begin{array}{c}{[540-97-6]} \\
{[86 / 4]} \\
{[87 / 5][71 / 15]}\end{array}$ \\
\hline $\mathrm{C}_{12} \mathrm{H}_{48} \mathrm{O}_{4} \mathrm{Si}_{5}$ & $\begin{array}{l}\text { tetrakis (trimethylsiloxy)silane } \\
(398-494)\end{array}$ & 52.3 & $(413)$ & EB & {$[86 / 4]$} \\
\hline $\mathrm{C}_{13} \mathrm{H}_{14} \mathrm{Si}$ & methyldiphenylsilane & $64.6 \pm 0.8$ & (298) & $\mathrm{C}$ & $\begin{array}{c}{[776-76-1]} \\
{[88 / 15]}\end{array}$ \\
\hline $\mathrm{C}_{13} \mathrm{H}_{26} \mathrm{O}_{2} \mathrm{Si}_{3}$ & $\begin{array}{l}\text { 1,1,1,3,5,5,5-heptamethyl-3-phenyl tr } \\
(357-492)\end{array}$ & $\begin{array}{l}\text { isiloxane } \\
\quad 61.5\end{array}$ & $(372)$ & A & $\begin{array}{l}{[546-44-1]} \\
{[87 / 5]}\end{array}$ \\
\hline $\mathrm{C}_{13} \mathrm{H}_{26} \mathrm{O}_{4} \mathrm{Si}_{4}$ & $\begin{array}{l}\text { 2,4,4,6,6,8,8-heptamethyl-2-phenylcy } \\
(397-514)\end{array}$ & $\begin{array}{l}\text { clotetrasiloxane } \\
\quad 65.6\end{array}$ & $(412)$ & A & $\begin{array}{c}{[10448-09-6]} \\
{[87 / 5]}\end{array}$ \\
\hline $\mathrm{C}_{13} \mathrm{H}_{30} \mathrm{O}_{3} \mathrm{SSi}$ & [3-(butylthio)propyl]triethoxysilane & $47.1 \pm 0.6$ & $(298)$ & $\mathrm{C}$ & $\begin{array}{c}{[57557-75-2]} \\
{[89 / 11]}\end{array}$ \\
\hline $\mathrm{C}_{13} \mathrm{H}_{30} \mathrm{Si}$ & $\begin{array}{l}\text { decyltrimethylsilane } \\
(340-513)\end{array}$ & 57.8 & $(355)$ & & {$[47 / 5]$} \\
\hline $\mathrm{C}_{13} \mathrm{H}_{30} \mathrm{Si}$ & methyldihexylsilane & $42.6 \pm 0.7$ & (298) & $\mathrm{C}$ & $\begin{array}{c}{[1001-46-3]} \\
{[88 / 15]}\end{array}$ \\
\hline $\mathrm{C}_{14} \mathrm{H}_{16} \mathrm{O}_{2} \mathrm{Si}$ & diphenoxydimethylsilane & $64.4 \pm 0.9$ & (298) & $\mathrm{C}$ & $\begin{array}{c}{[6843-66-9]} \\
{[88 / 15]}\end{array}$ \\
\hline $\mathrm{C}_{14} \mathrm{H}_{16} \mathrm{Si}$ & ethyldiphenylsilane & $66.1 \pm 0.8$ & $(298)$ & $\mathrm{C}$ & $\begin{array}{c}{[7535-07-1]} \\
{[88 / 15]}\end{array}$ \\
\hline $\mathrm{C}_{14} \mathrm{H}_{32} \mathrm{Si}$ & $\begin{array}{l}\text { triethyloctylsilane } \\
(347-535)\end{array}$ & 56.1 & $(361)$ & & $\begin{array}{c}{[10175-53-8]} \\
{[47 / 5]}\end{array}$ \\
\hline $\mathrm{C}_{14} \mathrm{H}_{32} \mathrm{Si}$ & ethyldihexylsilane & $44.8 \pm 0.7$ & $(298)$ & $\mathrm{C}$ & $\begin{array}{c}{[17591-45-6]} \\
{[88 / 15]}\end{array}$ \\
\hline $\mathrm{C}_{14} \mathrm{H}_{32} \mathrm{Si}$ & dipropyldibutylsilane & $44.0 \pm 0.8$ & (298) & $\mathrm{C}$ & {$[88 / 15]$} \\
\hline $\mathrm{C}_{14} \mathrm{H}_{33} \mathrm{NSi}$ & $\mathrm{N}, \mathrm{N}$-dibutyl-1,1,1-triethylsilanamine & $56.3 \pm 1.0$ & $(298)$ & $\mathrm{C}$ & $\begin{array}{c}{[17995-32-3]} \\
{[91 / 6]}\end{array}$ \\
\hline $\mathrm{C}_{14} \mathrm{H}_{33} \mathrm{NSi}$ & 1,1,1-triethyl-N,N-bis(1-methylpropy & $\begin{array}{l}\text { 1)silanamine } \\
51.4 \pm 0.9\end{array}$ & (298) & $\mathrm{C}$ & $\begin{array}{c}{[133943-79-0]} \\
{[91 / 6]}\end{array}$ \\
\hline $\mathrm{C}_{14} \mathrm{H}_{33} \mathrm{NSi}$ & 1,1,1-triethyl-N-octylsilanamine & $59.1 \pm 1.0$ & $(298)$ & $\mathrm{C}$ & $\begin{array}{c}{[133943-81-4]} \\
{[91 / 6]}\end{array}$ \\
\hline $\mathrm{C}_{14} \mathrm{H}_{42} \mathrm{O}_{5} \mathrm{Si}_{6}$ & $\begin{array}{l}\text { tetradecamethyl hexasiloxane } \\
(449-545)\end{array}$ & 56.9 & (464) & $\mathrm{EB}$ & $\begin{array}{c}{[107-52-8]} \\
{[86 / 4]}\end{array}$ \\
\hline & $(397-522)$ & 56.6 & (412) & A & {$[87 / 5][71 / 16]$} \\
\hline $\mathrm{C}_{14} \mathrm{H}_{42} \mathrm{O}_{7} \mathrm{Si}_{7}$ & $\begin{array}{l}\text { tetradecamethyl cycloheptasiloxane } \\
(359-537) \\
(431-548)\end{array}$ & $\begin{array}{l}58.6 \\
60.6\end{array}$ & $\begin{array}{l}(374) \\
(446)\end{array}$ & $\begin{array}{c}\mathrm{A} \\
\mathrm{EB}\end{array}$ & $\begin{array}{c}{[107-50-6]} \\
{[87 / 5]} \\
{[86 / 4]}\end{array}$ \\
\hline $\mathrm{C}_{15} \mathrm{H}_{34} \mathrm{Si}$ & propyltributylsilane & $45.0 \pm 0.8$ & (298) & $\mathrm{C}$ & $\begin{array}{c}{[994-78-5]} \\
{[88 / 15]}\end{array}$ \\
\hline $\mathrm{C}_{15} \mathrm{H}_{34} \mathrm{Si}$ & tripentylsilane & $48.1 \pm 0.8$ & (298) & $\mathrm{C}$ & $\begin{array}{c}{[6485-78-5]} \\
{[88 / 15]}\end{array}$ \\
\hline $\mathrm{C}_{15} \mathrm{H}_{34} \mathrm{Si}$ & triisopentylsilane & $43.8 \pm 0.7$ & (298) & $\mathrm{C}$ & $\begin{array}{c}{[17922-08-6]} \\
{[88 / 15]}\end{array}$ \\
\hline $\mathrm{C}_{15} \mathrm{H}_{34} \mathrm{Si}$ & $\begin{array}{l}\text { dodecyltrimethylsilane } \\
(364-546)\end{array}$ & 62.0 & (379) & & $\begin{array}{c}{[17908-09-7]} \\
{[47 / 5]}\end{array}$ \\
\hline $\mathrm{C}_{16} \mathrm{H}_{20} \mathrm{O}_{2} \mathrm{Si}$ & bis(2-methylphenoxy)dimethylsilane & $63.6 \pm 0.8$ & (298) & $\mathrm{C}$ & $\begin{array}{c}{[17964-48-6]} \\
{[88 / 15]}\end{array}$ \\
\hline
\end{tabular}


TABLE 7. Enthalpies of vaporization of select organo-metallic and inorganic compounds, 1880-2002-Continued

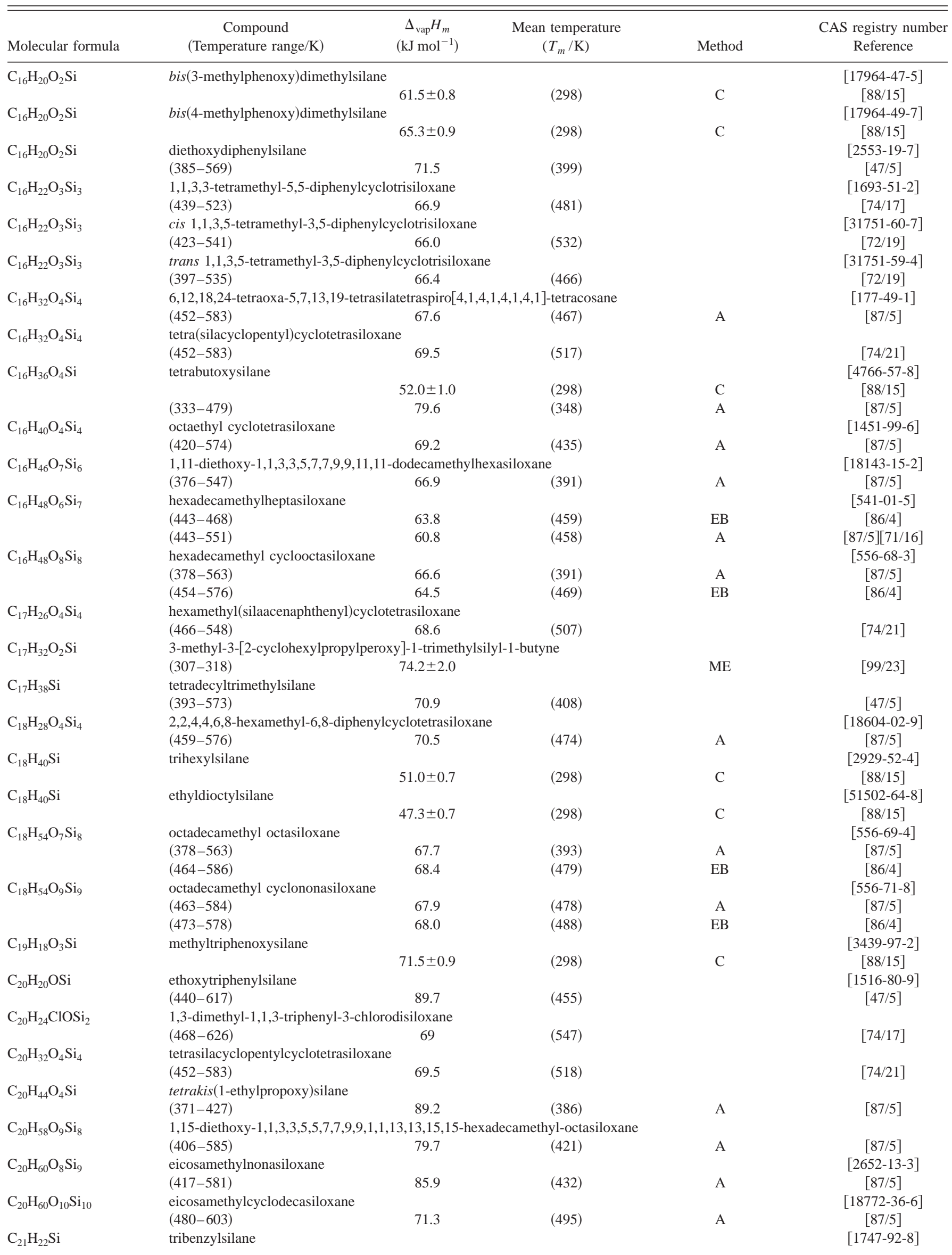


TABLE 7. Enthalpies of vaporization of select organo-metallic and inorganic compounds, 1880-2002-Continued

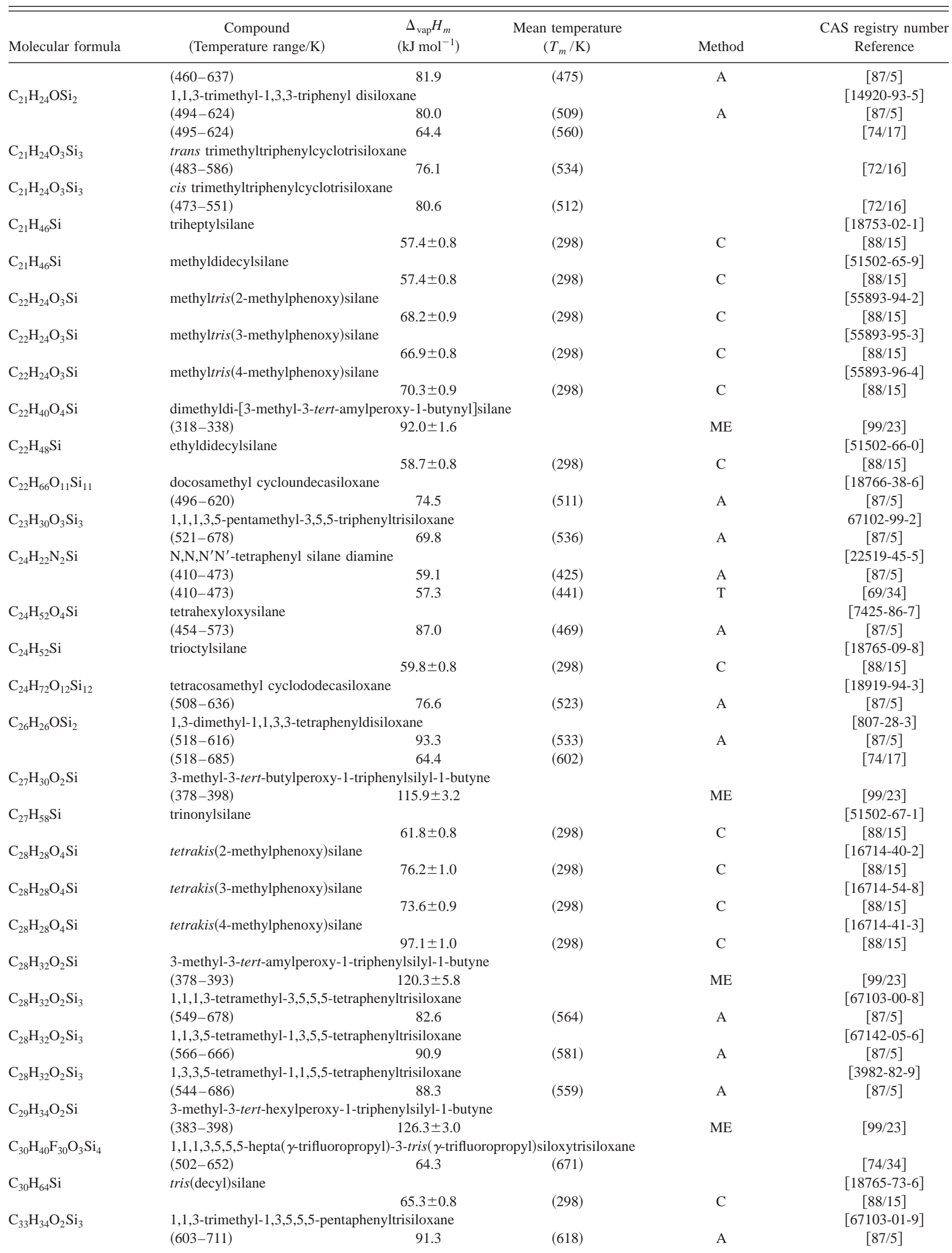


TABLE 7. Enthalpies of vaporization of select organo-metallic and inorganic compounds, 1880-2002-Continued

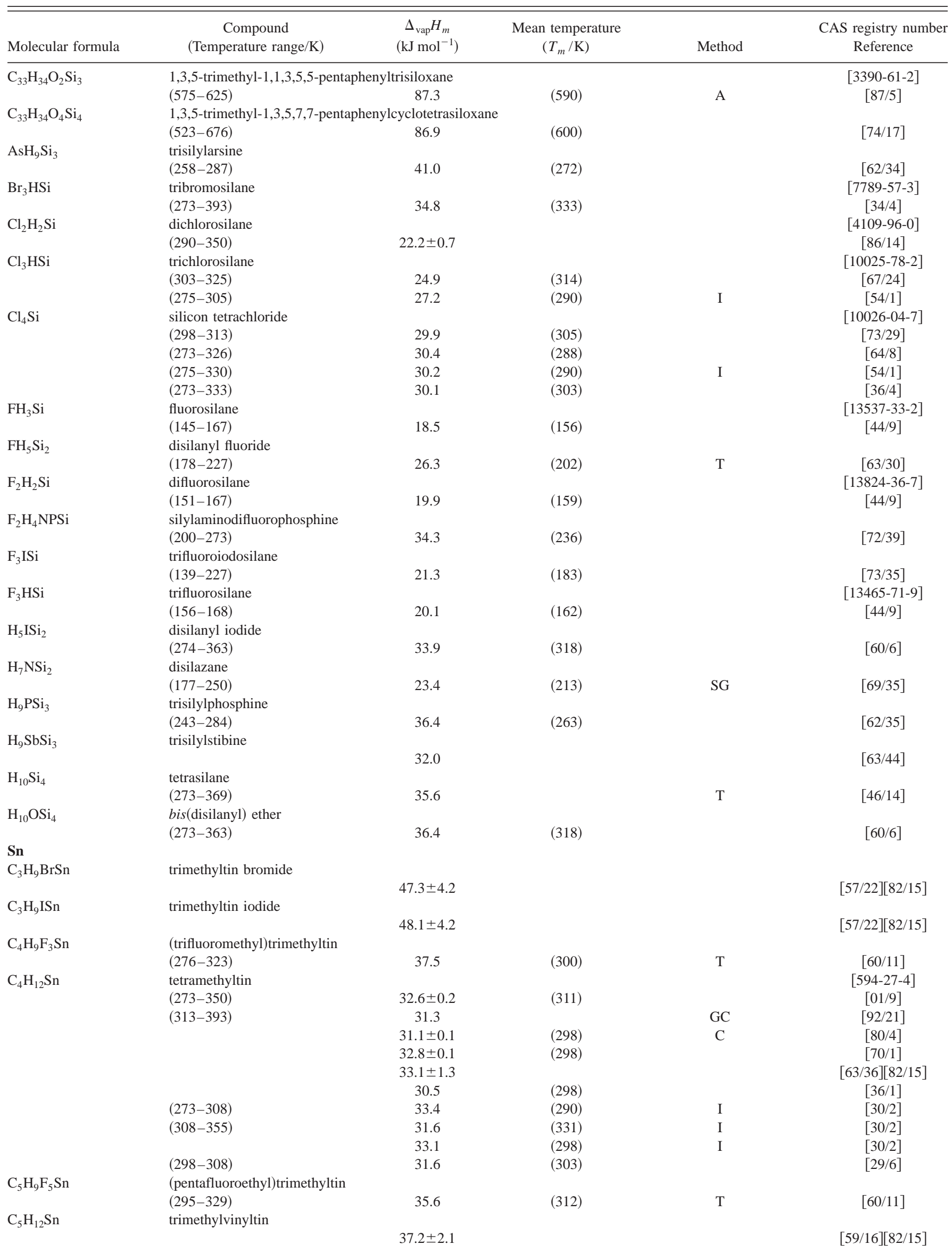


TABLE 7. Enthalpies of vaporization of select organo-metallic and inorganic compounds, 1880-2002-Continued

\begin{tabular}{|c|c|c|c|c|c|}
\hline Molecular formula & $\begin{array}{c}\text { Compound } \\
\text { (Temperature range/K) }\end{array}$ & $\begin{array}{c}\Delta_{\text {vap }} H_{m} \\
\left(\mathrm{~kJ} \mathrm{~mol}^{-1}\right)\end{array}$ & $\begin{array}{l}\text { Mean temperature } \\
\qquad\left(T_{m} / \mathrm{K}\right)\end{array}$ & Method & $\begin{array}{c}\text { CAS registry numbe } \\
\text { Reference }\end{array}$ \\
\hline \multirow[t]{5}{*}{$\mathrm{C}_{5} \mathrm{H}_{14} \mathrm{Sn}$} & ethyl trimethyltin & & & & [3531-44-0] \\
\hline & & $37.7 \pm 1.7$ & & & {$[63 / 36][82 / 15]$} \\
\hline & $(243-381)$ & 38.4 & $(258)$ & & {$[47 / 5]$} \\
\hline & $(273-336)$ & 37.0 & (304) & I & {$[30 / 2]$} \\
\hline & $(336-384)$ & 34.9 & (360) & I & {$[30 / 2]$} \\
\hline \multirow[t]{4}{*}{$\mathrm{C}_{6} \mathrm{H}_{16} \mathrm{Sn}$} & trimethylpropyltin & & & & {$[3531-45-1]$} \\
\hline & $(261-405)$ & 43.8 & (276) & & {$[47 / 5]$} \\
\hline & $(286-328)$ & 41.4 & (307) & I & {$[30 / 2]$} \\
\hline & $(328-405)$ & 38.0 & (366) & I & {$[30 / 2]$} \\
\hline \multirow[t]{2}{*}{$\mathrm{C}_{6} \mathrm{H}_{16} \mathrm{Sn}$} & trimethylisopropyltin & & & & \\
\hline & & $40.6 \pm 2.1$ & & & {$[66 / 24][82 / 15]$} \\
\hline \multirow{2}{*}{$\mathrm{C}_{6} \mathrm{H}_{18} \mathrm{Sn}_{2}$} & hexamethyldistannane & & & & \\
\hline & & $50.2 \pm 4.2$ & & & {$[57 / 22][82 / 15]$} \\
\hline \multirow[t]{3}{*}{$\mathrm{C}_{7} \mathrm{H}_{18} \mathrm{OSn}$} & triethylmethoxystannane & & & & {$[1067-21-6]$} \\
\hline & $(312-435)$ & 49.9 & (273) & MM & {$[01 / 5]$} \\
\hline & $(312-435)$ & 48.7 & (298) & MM & {$[01 / 5]$} \\
\hline \multirow[t]{2}{*}{$\mathrm{C}_{7} \mathrm{H}_{18} \mathrm{Sn}$} & tert-butyltrimethyltin & & & & \\
\hline & & $54.0 \pm 4.2$ & & & {$[66 / 24][82 / 15]$} \\
\hline \multirow[t]{2}{*}{$\mathrm{C}_{8} \mathrm{H}_{12} \mathrm{Sn}$} & tetravinyltin & & & & [1112-55-6] \\
\hline & $(313-393)$ & 40.5 & & $\mathrm{GC}$ & {$[92 / 21]$} \\
\hline \multirow[t]{2}{*}{$\mathrm{C}_{8} \mathrm{H}_{15} \mathrm{~F}_{5} \mathrm{Sn}$} & (pentafluoroethyl)triethyltin & & & & \\
\hline & $(303-343)$ & 39.2 & $(323)$ & $\mathrm{T}$ & {$[60 / 11]$} \\
\hline \multirow[t]{5}{*}{$\mathrm{C}_{8} \mathrm{H}_{20} \mathrm{Sn}$} & tetraethyltin & & & & [597-64-8] \\
\hline & $(293-455)$ & $46.6 \pm 0.6$ & (374) & & {$[01 / 9]$} \\
\hline & $(313-393)$ & 42.4 & & $\mathrm{GC}$ & {$[92 / 21]$} \\
\hline & & $50.6 \pm 0.2$ & (298) & $\mathrm{C}$ & {$[80 / 4]$} \\
\hline & & $51.0 \pm 2.1$ & & & {$[63 / 36][82 / 15]$} \\
\hline \multirow[t]{2}{*}{$\mathrm{C}_{9} \mathrm{H}_{14} \mathrm{Sn}$} & phenyltrimethyltin & & & & [934-56-5] \\
\hline & & $52.3 \pm 4.2$ & & & {$[59 / 16][82 / 15]$} \\
\hline \multirow[t]{2}{*}{$\mathrm{C}_{10} \mathrm{H}_{16} \mathrm{Sn}$} & benzyltrimethyltin & & & & [4314-94-7] \\
\hline & & $56.5 \pm 4.2$ & & & {$[59 / 16][82 / 15]$} \\
\hline \multirow[t]{2}{*}{$\mathrm{C}_{10} \mathrm{H}_{24} \mathrm{O}_{2} \mathrm{Sn}$} & triethyltin tert-butylperoxide & & & & \\
\hline & & $48.8 \pm 2.1$ & & & {$[71 / 29][82 / 15]$} \\
\hline \multirow[t]{2}{*}{$\mathrm{C}_{10} \mathrm{H}_{25} \mathrm{NSn}$} & (N,N-diethylamino)triethyltin & & & & \\
\hline & & $50.2 \pm 4.2$ & & & {$[71 / 30][82 / 15]$} \\
\hline \multirow[t]{2}{*}{$\mathrm{C}_{12} \mathrm{H}_{27} \mathrm{BrSn}$} & tributyltin bromide & & & & \\
\hline & & $83.7 \pm 12.6$ & & & {$[59 / 16][82 / 15]$} \\
\hline $\mathrm{C}_{12} \mathrm{H}_{20} \mathrm{Sn}$ & tetraallyltin & & & & [7393-43-3] \\
\hline & $(333-393)$ & 52.0 & & $\mathrm{GC}$ & {$[92 / 21]$} \\
\hline $\mathrm{C}_{12} \mathrm{H}_{28} \mathrm{Sn}$ & tetrapropyl tin & & & & {$[2176-98-9]$} \\
\hline & $(343-457)$ & $55.0 \pm 0.7$ & $(400)$ & & {$[01 / 9]$} \\
\hline & $(333-393)$ & 60.8 & GC & & {$[92 / 21]$} \\
\hline & $(361-470)$ & 52.5 & (376) & A & {$[87 / 5]$} \\
\hline & & $65.4 \pm 2.5$ & (298) & $\mathrm{C}$ & {$[80 / 4]$} \\
\hline & & $66.9 \pm 2.1$ & & & {$[63 / 36][82 / 15]$} \\
\hline & & 60.7 & & & {$[35 / 7]$} \\
\hline $\mathrm{C}_{12} \mathrm{H}_{28} \mathrm{Sn}$ & tetraisopropyl tin & & & & [2949-42-0] \\
\hline & $(342-441)$ & $48.0 \pm 0.7$ & $(392)$ & & {$[01 / 9]$} \\
\hline & $(333-393)$ & 56.4 & & GC & {$[92 / 21]$} \\
\hline & & $64.9 \pm 4.2$ & & & {$[66 / 24][82 / 15]$} \\
\hline $\mathrm{C}_{15} \mathrm{H}_{26} \mathrm{OSn}$ & bis(triethyltin)oxide & & & & \\
\hline & & $52.3 \pm 2.1$ & & & {$[71 / 29][82 / 15]$} \\
\hline $\mathrm{C}_{15} \mathrm{H}_{26} \mathrm{O}_{2} \mathrm{Sn}$ & triethyltin dimethylphenylperoxide & & & & \\
\hline & & $56.5 \pm 2.1$ & & & {$[71 / 29][82 / 15]$} \\
\hline $\mathrm{C}_{12} \mathrm{H}_{36} \mathrm{Sn}_{2}$ & hexaethyldistannane & & & & \\
\hline & & $62.8 \pm 4.2$ & & & {$[66 / 25][82 / 15]$} \\
\hline $\mathrm{C}_{16} \mathrm{H}_{36} \mathrm{Sn}$ & tetrabutyl tin & & & & {$[1461-25-2]$} \\
\hline & $(389-462)$ & $67.8 \pm 0.5$ & $(425)$ & & {$[01 / 9]$} \\
\hline & & $82.8 \pm 2.1$ & & & {$[63 / 36][82 / 15]$} \\
\hline $\mathrm{C}_{16} \mathrm{H}_{36} \mathrm{Sn}$ & tetraisobutyl tin & & & & [3531-43-9] \\
\hline & $(391-451)$ & $53.6 \pm 1.1$ & $(421)$ & & {$[01 / 9]$} \\
\hline $\mathrm{SnI}_{4}$ & stannic iodide & & & & {$[7790-47-8]$} \\
\hline & $(418-523)$ & 57.2 & (423) & & {$[36 / 7]$} \\
\hline
\end{tabular}


TABLE 7. Enthalpies of vaporization of select organo-metallic and inorganic compounds, 1880-2002-Continued

\begin{tabular}{|c|c|c|c|c|c|}
\hline Molecular formula & $\begin{array}{c}\text { Compound } \\
\text { (Temperature range/K) }\end{array}$ & $\begin{array}{c}\Delta_{\mathrm{vap}} H_{m} \\
\left(\mathrm{~kJ} \mathrm{~mol}^{-1}\right)\end{array}$ & $\begin{array}{c}\text { Mean temperature } \\
\left(T_{m} / \mathrm{K}\right)\end{array}$ & Method & $\begin{array}{c}\text { CAS registry number } \\
\text { Reference }\end{array}$ \\
\hline $\mathrm{C}_{33} \mathrm{H}_{57} \mathrm{O}_{6} \mathrm{Sm}$ & \multicolumn{4}{|c|}{ tris(2,2,6,6-tetramethylheptane-3,5-dionato)samarium(III) } & [15492-50-9] \\
\hline & $(468-500)$ & 93.0 & BG & & {$[69 / 28]$} \\
\hline \multicolumn{6}{|c|}{ (1) } \\
\hline $\mathrm{C}_{10} \mathrm{H}_{25} \mathrm{O}_{5} \mathrm{Ta}$ & $\begin{array}{l}\text { pentaethyltantalate } \\
(388-424)\end{array}$ & 72.6 & (403) & A & [87/5] \\
\hline \multicolumn{6}{|c|}{ 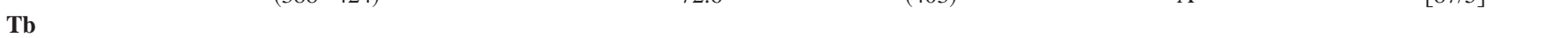 } \\
\hline $\mathrm{C}_{33} \mathrm{H}_{57} \mathrm{O}_{6} \mathrm{~Tb}$ & \multicolumn{4}{|c|}{$\operatorname{tris}(2,2,6,6$-tetramethylheptane-3,5-dionato)terbium(III) } & $\begin{array}{c}{[15492-51-0]} \\
{[69 / 28]}\end{array}$ \\
\hline \multicolumn{6}{|c|}{ (1) } \\
\hline \multirow{7}{*}{$\mathrm{C}_{2} \mathrm{H}_{6} \mathrm{Te}$} & dimethyl telluride & & & & {$[593-80-6]$} \\
\hline & $(298-367)$ & 34.4 & $(313)$ & & {$[99 / 16]$} \\
\hline & $(273-372)$ & $35.6 \pm 0.1$ & $(323)$ & & {$[97 / 30][96 / 29]$} \\
\hline & $(267-369)$ & 36.9 & $(282)$ & BG & {$[96 / 30]$} \\
\hline & $(267-369)$ & $36.1 \pm 1.0$ & $(298)$ & $\mathrm{BG}$ & {$[96 / 30]$} \\
\hline & & $37.4 \pm 0.7$ & $(298)$ & $\mathrm{C}$ & {$[89 / 12]$} \\
\hline & & $36.0 \pm 2.1$ & & & {$[88 / 1]$} \\
\hline \multirow[t]{3}{*}{$\mathrm{C}_{4} \mathrm{H}_{6} \mathrm{Te}$} & divinyl telluride & & & & {$[63000-06-6]$} \\
\hline & & $44.8 \pm 0.8$ & $(298)$ & $\mathrm{C}$ & [89/12] \\
\hline & & $38.1 \pm 2.1$ & & & [88/1] \\
\hline \multirow[t]{4}{*}{$\mathrm{C}_{4} \mathrm{H}_{10} \mathrm{Te}$} & diethyl telluride & & & & {$[627-54-3]$} \\
\hline & $(295-411)$ & 41.8 & (310) & & [99/16] \\
\hline & $(273-415)$ & $41.6 \pm 0.2$ & $(344)$ & & [96/29] \\
\hline & & $41.6 \pm 0.8$ & (298) & $\mathrm{C}$ & [89/12] \\
\hline \multirow[t]{3}{*}{$\mathrm{C}_{6} \mathrm{H}_{14} \mathrm{Te}$} & dipropyl telluride & & & & [64501-17-3] \\
\hline & $(298-434)$ & $45.5 \pm 0.3$ & $(366)$ & & {$[96 / 29]$} \\
\hline & & $46.5 \pm 0.7$ & (298) & $\mathrm{C}$ & {$[89 / 12]$} \\
\hline \multirow[t]{2}{*}{$\mathrm{C}_{6} \mathrm{H}_{14} \mathrm{Te}$} & diisopropyl telluride & & & & \\
\hline & $(298-399)$ & $40.4 \pm 0.1$ & (349) & & [96/29] \\
\hline \multirow[t]{2}{*}{$\mathrm{C}_{6} \mathrm{H}_{14} \mathrm{Te}_{2}$} & dipropyl ditelluride & & & & [79971-42-9] \\
\hline & & $52.7 \pm 1.0$ & (298) & $\mathrm{C}$ & [89/12] \\
\hline \multirow[t]{3}{*}{$\mathrm{C}_{8} \mathrm{H}_{18} \mathrm{Te}$} & dibutyl telluride & & & & [38788-38-4] \\
\hline & $(303-423)$ & $53.4 \pm 0.1$ & $(358)$ & & {$[96 / 29]$} \\
\hline & & $51.0 \pm 1.0$ & (298) & $\mathrm{C}$ & [89/12] \\
\hline \multirow[t]{2}{*}{$\mathrm{C}_{8} \mathrm{H}_{18} \mathrm{Te}$} & diisobutyl telluride & & & & \\
\hline & $(303-410)$ & $47.6 \pm 0.1$ & $(356)$ & & {$[96 / 29]$} \\
\hline $\mathrm{C}_{8} \mathrm{H}_{18} \mathrm{Te}$ & di-sec-butyl telluride & & & & \\
\hline & $(303-372)$ & $49.6 \pm 0.9$ & $(338)$ & & [96/29] \\
\hline $\mathrm{C}_{8} \mathrm{H}_{18} \mathrm{Te}_{2}$ & dibutyl ditelluride & & & & [77129-69-2] \\
\hline & & $57.3 \pm 1.0$ & $(298)$ & $\mathrm{C}$ & [89/12] \\
\hline $\mathrm{C}_{10} \mathrm{H}_{22} \mathrm{Te}$ & dipentyl telluride & & & & \\
\hline & $(343-403)$ & $59.5 \pm 0.8$ & (373) & & {$[96 / 29]$} \\
\hline $\mathrm{C}_{10} \mathrm{H}_{22} \mathrm{Te}$ & diisopentyl telluride & & & & \\
\hline & $(343-403)$ & $51.9 \pm 0.7$ & $(373)$ & & {$[96 / 29]$} \\
\hline $\mathrm{TeCl}_{4}$ & tellurium tetrachloride & & & & \\
\hline & $(506-660)$ & 77.0 & $(583)$ & GS & {$[30 / 7]$} \\
\hline $\mathbf{T i}$ & & & & & \\
\hline $\mathrm{C}_{8} \mathrm{H}_{24} \mathrm{~N}_{4} \mathrm{Ti}$ & titanium tetradimethylamide & & & & [3275-24-9] \\
\hline & $(353-418)$ & $53.8 \pm 3.0$ & $(383)$ & & {$[84 / 33][01 / 23]$} \\
\hline $\mathrm{C}_{12} \mathrm{H}_{28} \mathrm{O}_{4} \mathrm{Ti}$ & tetraisopropyl titanate & & & & \\
\hline & $(336-459)$ & 62.3 & $(351)$ & A & {$[87 / 5]$} \\
\hline $\mathrm{C}_{12} \mathrm{H}_{28} \mathrm{O}_{4} \mathrm{Ti}$ & tetrapropyl titanate & & & & \\
\hline & $(411-479)$ & 111.9 & $(426)$ & A & {$[87 / 5]$} \\
\hline $\mathrm{C}_{16} \mathrm{H}_{36} \mathrm{O}_{4} \mathrm{Ti}$ & tetrabutoxy titanium & & & & \\
\hline & $(462-564)$ & 89.7 & $(477)$ & A & {$[87 / 5]$} \\
\hline & $(443-493)$ & $85.0 \pm 3.1$ & $(458)$ & $\mathrm{A}$ & {$[87 / 5][78 / 13]$} \\
\hline $\mathrm{C}_{16} \mathrm{H}_{36} \mathrm{O}_{4} \mathrm{Ti}$ & tetraisobutoxy titanium & & & & \\
\hline & $(436-529)$ & 77.4 & $(451)$ & A & {$[87 / 5]$} \\
\hline $\mathrm{C}_{16} \mathrm{H}_{36} \mathrm{O}_{4} \mathrm{Ti}$ & tetra-sec-butoxy titanium & & & & \\
\hline & $(378-414)$ & 76.8 & $(393)$ & A & {$[87 / 5]$} \\
\hline & $(370-476)$ & 67.1 & $(385)$ & A & {$[87 / 5]$} \\
\hline $\mathrm{C}_{16} \mathrm{H}_{36} \mathrm{O}_{4} \mathrm{Ti}$ & tetra-tert-butoxy titanium & & & & \\
\hline & $(386-486)$ & 55.9 & $(401)$ & $\mathrm{A}$ & {$[87 / 5]$} \\
\hline & $(322-388)$ & 62.6 & (337) & SG & {$[58 / 13][84 / 9]$} \\
\hline & & $66.1 \pm 3.3$ & (298) & SG & {$[58 / 13][66 / 20]$} \\
\hline
\end{tabular}


TABLE 7. Enthalpies of vaporization of select organo-metallic and inorganic compounds, 1880-2002-Continued

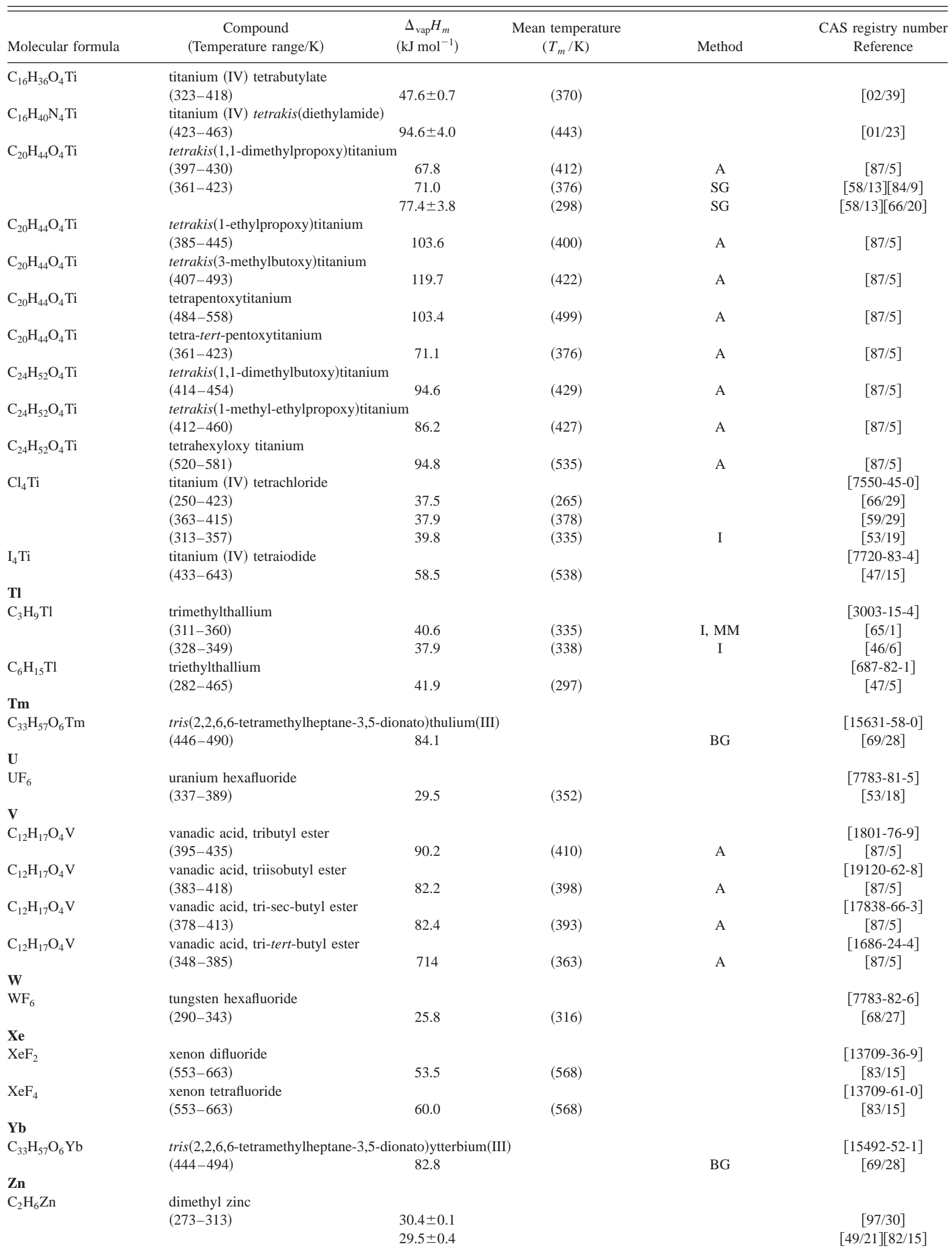


TABLE 7. Enthalpies of vaporization of select organo-metallic and inorganic compounds, 1880-2002-Continued

\begin{tabular}{|c|c|c|c|c|c|}
\hline Molecular formula & $\begin{array}{c}\text { Compound } \\
\text { (Temperature range/K) }\end{array}$ & $\begin{array}{c}\Delta_{\mathrm{vap}} H_{m} \\
\left(\mathrm{~kJ} \mathrm{~mol}^{-1}\right)\end{array}$ & $\begin{array}{c}\text { Mean temperature } \\
\left(T_{m} / \mathrm{K}\right)\end{array}$ & Method & $\begin{array}{c}\text { CAS registry number } \\
\text { Reference }\end{array}$ \\
\hline & $(248-318)$ & 29.9 & $(283)$ & BG & [46/13] \\
\hline \multirow[t]{5}{*}{$\mathrm{C}_{4} \mathrm{H}_{10} \mathrm{Zn}$} & diethyl zinc & & & & [557-20-0] \\
\hline & & 37.9 & (298) & & {$[83 / 1]$} \\
\hline & & $40.2 \pm 2.1$ & & & {$[49 / 21][82 / 15]$} \\
\hline & $(250-391)$ & 39.9 & (265) & & {$[47 / 5]$} \\
\hline & & 40.2 & & BG & [46/13] \\
\hline \multirow[t]{6}{*}{$\mathrm{C}_{6} \mathrm{H}_{14} \mathrm{Zn}$} & dipropyl zinc & & & & [628-91-1] \\
\hline & & $43 \pm 2$ & & & {$[02 / 43]$} \\
\hline & $(313-370)$ & $42.1 \pm 0.4$ & $(341)$ & & {$[84 / 34]$} \\
\hline & & $45.6 \pm 2.5$ & & & {$[49 / 21][82 / 15]$} \\
\hline & & 39.5 & & & [49/25] \\
\hline & & 40.3 & & BG & [46/13] \\
\hline \multirow[t]{3}{*}{$\mathrm{C}_{6} \mathrm{H}_{14} \mathrm{Zn}$} & diisopropyl zinc & & & & [625-81-0] \\
\hline & $(303-345)$ & $41.8 \pm 0.5$ & (324) & & {$[84 / 34]$} \\
\hline & $(310-338)$ & 47.4 & (324) & & {$[46 / 4]$} \\
\hline \multirow[t]{5}{*}{$\mathrm{C}_{8} \mathrm{H}_{18} \mathrm{Zn}$} & dibutyl zinc & & & & [1119-90-0] \\
\hline & $(305-379)$ & $50.7 \pm 0.3$ & $(342)$ & & [84/13] \\
\hline & & $54.4 \pm 3.3$ & & & {$[49 / 21][82 / 15]$} \\
\hline & & 45.3 & & & [49/25] \\
\hline & & 42.9 & & BG & {$[46 / 13]$} \\
\hline \multirow[t]{2}{*}{$\mathrm{C}_{8} \mathrm{H}_{18} \mathrm{Zn}$} & di-sec-butyl zinc & & & & [7446-94-8] \\
\hline & $(287-372)$ & $40.9 \pm 0.2$ & $(330)$ & & {$[84 / 13]$} \\
\hline \multirow[t]{2}{*}{$\mathrm{C}_{8} \mathrm{H}_{18} \mathrm{Zn}$} & diisobutyl zinc & & & & \\
\hline & $(288-372)$ & $44.6 \pm 0.2$ & $(330)$ & & [84/13] \\
\hline \multirow[t]{2}{*}{$\mathrm{C}_{8} \mathrm{H}_{18} \mathrm{Zn}$} & di-tert-butyl zinc & & & & [7446-94-8] \\
\hline & $(300-322)$ & $49.3 \pm 0.8$ & $(311)$ & & [84/13] \\
\hline \multirow[t]{2}{*}{$\mathrm{C}_{10} \mathrm{H}_{22} \mathrm{Zn}$} & dipentyl zinc & & & & [14402-93-8] \\
\hline & & 48.6 & & & [49/25] \\
\hline \multirow[t]{2}{*}{$\mathrm{C}_{12} \mathrm{H}_{26} \mathrm{Zn}$} & dihexyl zinc & & & & [13822-55-4] \\
\hline & & 56.2 & & & [49/25] \\
\hline \multirow[t]{2}{*}{$\mathrm{C}_{14} \mathrm{H}_{30} \mathrm{Zn}$} & diheptyl zinc & & & & {$[14402-95-0]$} \\
\hline & & 62.3 & & & {$[49 / 25]$} \\
\hline \multirow[t]{2}{*}{$\mathrm{Cl}_{2} \mathrm{Zn}$} & zinc chloride & & & & [7646-85-7] \\
\hline & $(695-826)$ & 134.5 & $(760)$ & & {$[58 / 28]$} \\
\hline \multicolumn{6}{|l|}{$\mathrm{Zr}$} \\
\hline \multirow[t]{2}{*}{$\mathrm{C}_{16} \mathrm{H}_{36} \mathrm{O}_{4} \mathrm{Zr}$} & tetra-tert-butoxy zirconium & & & & \\
\hline & $(374-587)$ & 56.6 & $(389)$ & A & {$[87 / 5]$} \\
\hline \multirow[t]{2}{*}{$\mathrm{C}_{20} \mathrm{H}_{44} \mathrm{O}_{4} \mathrm{Zr}$} & tetrakis 1 1,1-dimethylpropox & ium 680 & & & \\
\hline & $(392-426)$ & 68.0 & $(407)$ & A & {$[87 / 5]$} \\
\hline \multirow[t]{2}{*}{$\mathrm{C}_{20} \mathrm{H}_{44} \mathrm{O}_{4} \mathrm{Zr}$} & tetra-tert-pentoxyzirconium & & & & \\
\hline & $(361-435)$ & 74.1 & $(361)$ & A & {$[87 / 5]$} \\
\hline \multirow[t]{2}{*}{$\mathrm{C}_{24} \mathrm{H}_{52} \mathrm{O}_{4} \mathrm{Zr}$} & tetrakis $(1,1$-dimethylbutoxy & & & & \\
\hline & $(406-449)$ & 93.3 & $(421)$ & A & {$[87 / 5]$} \\
\hline \multirow[t]{2}{*}{$\mathrm{C}_{24} \mathrm{H}_{52} \mathrm{O}_{4} \mathrm{Zr}$} & tetrakis(1-methyl-1-ethylpro & conium & & & \\
\hline & $(423-460)$ & 91.4 & $(438)$ & A & {$[87 / 5]$} \\
\hline
\end{tabular}


TABLE 8. References to Tables 6 and 7

1882

82/1 F. Kraft, Ber. 15, 1687 (1882).

1883

83/1 G. W. A. Kahlbaum, Ber. 16, 2476-2484 (1883).

1886

86/1 A. Richardson, J. Chem. Soc. (London) 49, 761-776 (1886).

1894

94/1 G. W. A. Kahlbaum, Z. Phys. Chem. 13, 14-55 (1894).

1898

98/1 G. W. A. Kahlbaum, Z. Phys. Chem. 26, 577 (1898).

98/2 W. Louguinine, Ann. Chim. (Paris) 7, 334-377 (1898).

1901

01/1 M. De Forgrand, Comp. Rend. 132, 688 (1901).

1903

03/1 J. Dewar and H. O. Jones, Proc. Roy. Soc. London 71A, 427

(1903).

1904

04/1 A. Jaquerod and E. Wassmer, Ber. 3, 2531-2535 (1904).

1906

06/1 A. Rex, Z. Physik. Chem. 55, 355-370 (1906).

1910

10/1 E. C. Bingham, Am. Chem. J. 43, 287-309 (1910).

1912

12/1 W. Herz and W. Rathmann, Chem.-Ztg. 36, 1417-1418 (1912).

1914

14/1 A. Stock and E. Willfroth, Ber. 47, 144-154 (1914).

1915

15/1 J. M. Crafts, J. Chim. Phys. Phys.-Chim. Biol. 13, 105-161 (1915).

1919

19/1 E. Moles and T. Batuecas, J. Chim. Phys. Phys.-Chim. Biol. 17, 537-588 (1919).

\section{1}

21/1 A. Stock, F. Henning, and E. Kuss, Ber. 54, 1119-1129 (1931).

21/2 O. Maas and C. H. Wright, J. Am. Chem. Soc. 43, 1098-1111

(1921).

21/3 O. Maas and C. H. Wright, J. Am. Chem. Soc. 43, 1098-1111

(1921).

1922

22/1 O. A. Nelson and C. E. Senseman, Ind. Eng. Chem. 14, 58-62 (1922).

22/2 R. S. Taylor and L. B. Smith, J. Am. Chem. Soc. 44, 2450-2463

(1922).

1923

23/1 F. S. Mortimer and R. V. Murphy, Ind. Eng. Chem. 15, 1140-1142 (1923).

23/2 C. E. Senseman and O. A. Nelson, Ind. Eng. Chem. 15, 382-383

(1923).

1924

24/1 J. Simons, J. Am. Chem. Soc. 46, 2179-2183 (1924).

24/2 O. Maas and P. G. Hiebert, J. Am. Chem. Soc. 46, 2693-2700

(1924).

1925

25/1 J. H. Perry, J. Am. Chem. Soc. 47, 2629-2632 (1925).

1926

26/1 H. Sinozaki, R. Hara, and S. Mitsukuri, Bull Chem. Soc. Jpn. 1, 59-61 (1926).

26/2 A. G. Loomis and J. E. Walters, J. Am. Chem. Soc. 48, 2051-2055 (1926).

26/3 F. Straus, Ber. 59, 1664-1681 (1926).

26/4 W. V. Evans and M. B. Aylesworth, Ind. Eng. Chem. 18, 24-27 (1926).

26/5 C. A. Taylor and W. H. Rinkenbach, J. Chem. Soc. 48, 1305-1309 (1926).

26/6 G. W. C. Yates, Philos. Mag. 817-826 (1926).

26/7 W. A. Felsing and S. A. Durban, J. Am. Chem. Soc. 48, 2885-2893 (1926).

26/8 J. H. Perry and F. Porter, J. Am. Chem. Soc. 48, 299-302 (1926).
26/9 H. Sinozaki, R. Hara, and S. Mitsukuri, Bull. Chem. Soc. Jpn. 1, 59-61 (1926).

1927

27/1 W. H. Rinkenbach, Ind. Eng. Chem. 19, 474-476 (1927).

27/2 J. H. Perry, J. Phys. Chem. 31, 1737-1741 (1927).

27/3 N. N. Nagornov, Ann. Inst. Anal. Phys. Chem. (Leningrad) 3, 562-583 (1927); Chem. Zentr. II, 2668 (1927); Chem. Abstracts 22, 4298 (1928)

27/4 O. E. May, J. F. T. Berliner, and D. F. J. Lynch, J. Am. Chem. Soc. 49, 1012-1016 (1927).

$27 / 5$ S. Klosky, L. P. L. Woo, and R. J. Flanigan, J. Am. Chem. Soc. 49, 1280-1281 (1927).

1928

28/1 O. A. Nelson, Ind. Eng. Chem. 20, 1382-1384 (1928).

28/2 O. A. Nelson, Ind. Eng. Chem. 20, 1380-1382 (1928).

28/3 M. Roland, Bull. Soc. Chim. Belg. 37, 117-140 (1928).

28/4 W. F. Giauque and R. Wiebe, J. Am. Chem. Soc. 50, 101-122

(1928).

28/5 G. S. Parks and B. Barton, J. Am. Chem. Soc. 50, 24-27 (1928).

28/6 W. F. Giauque and R. Wiebe, J. Am. Chem. Soc. 50, 2193-2202

(1928).

1929

29/1 J. N. Pearce and P. E. Peters, J. Phys. Chem. 33, 873-878 (1929).

29/2 C. P. Smyth and E. W. Engel, J. Am. Chem. Soc. 51, 2646-2660

(1929).

29/3 H. L. Johnston and W. F. Giauque, J. Am. Chem. Soc. 51, 31943215 (1929).

29/4 W. F. Giauque and R. Wiebe, J. Am. Chem. Soc. 51, 1441-1449

(1929).

29/5 C. F. Haggerty and J. F. Weiler, J. Am. Chem. Soc. 51, 1623-1626

(1929)

29/6 Y. Tanaka and Y. Nagai, Proc. Imp. Acad. (Japan) 5, 78-79 (1929);

Chem. Abstracts 23, 2622 (1929).

1930

30/1 O. A. Nelson, Ind. Eng. Chem. 22, 971-972 (1930).

30/2 R. H. Bullard and A. C. Haussman, J. Phys. Chem. 34, 743-747

(1930).

30/3 M. A. Zmaczynski, J. Chim. Phys. 27, 503-517 (1930).

30/4 G. B. Cunningham, Power 72, 374-377 (1930).

30/5 R. Livingston and G. B. Heisig, J. Am. Chem. Soc. 52, 2409-2410

(1930)

30/6 H. A. Schuette and R. W. Thomas, J. Am. Chem. Soc. 52, 2028 -

2030 (1930)

30/7 J. H. Simons, J. Am. Chem. Soc. 52, 3488-3493 (1930).

30/8 A. S. Coolidge, J. Am. Chem. Soc. 52, 1874-1887 (1930).

1931

31/1 H. A. Scheulte and M. A. Cowley, J. Am. Chem. Soc. 53, 3485-

3489 (1931)

31/2 O. Ruff and W. Menzel, Z. Anorg. Allgem. Chem. 202, 49-61

(1931)

31/3 E. Wiberg and W. Sütterlin, Z. Anorg. Allgem. Chem. 202, 1-21

(1931)

1932

32/1 W. E. Vaughan, J. Am. Chem. Soc. 54, 3863-3876 (1932).

32/2 L. M. Ellis, Jr. and E. E. Reid, J. Am. Chem. Soc. 54, 1674-1687

(1932).

32/3 J. O. Clayton and W. F. Giauque, J. Am. Chem. Soc. 54, 2610-2626 (1932).

32/4 W. J. Jones, W. J. C. Dyke, G. Davies, D. C. Griffiths, and J. H. E. Webb, J. Chem. Soc. 2285-2293 (1932).

1933

33/1 W. Menzel and F. Mohry, Z. Anorg. Allgem. Chem. 210, 257-263 (1933).

33/2 G. B. Heisig and O. D. Murd, J. Am. Chem. Soc. 55, 3485-3487

(1933).

33/3 H. S. Booth, P. E. Burchfield, E. M. Bixly, and J. B. MacKelvey, J.

Am. Chem. Soc. 53, 2231-2235 (1933). 
33/4 F. Hovorka and F. E. Geiger, J. Am. Chem. Soc. 55, 4759-4761 (1933).

33/5 O. A. Nelson and H. D. Young, J. Am. Chem. Soc. 55, 2429-2431

(1933).

33/6 M. A. Cowley and H. A. Schuelte, J. Am. Chem. Soc. 55, 387-391 (1933).

33/7 A. Taylor and E. T. Layng, J. Chem. Phys. 1, 798-808 (1933).

33/8 H. S. Booth, P. E. Burchfield, E. M. Bixly, and J. B. MacKelvey, J. Am. Chem. Soc. 53, 2231-2235 (1933).

33/9 F. Hovorka, H. P. Lankelma, and C. K. Naujoks, J. Am. Chem. Soc. 53, 4820-4822 (1933).

33/10 H. Tannelberger, Ber. 66B, 484-486 (1933); Chem. Abstracts 27, 3374 (1933)

33/11 G. B. Heisig, J. Am. Chem. Soc. 55, 2304-2311 (1933).

33/12 E. G. V. Barrett and L. J. Burrage, J. Phys. Chem. 37, 1029-1035 (1933).

33/13 F. T. Miles and A. W. C. Menzies, J. Phys. Chem. 37, 435-430 (1933).

33/14 C. A. Kraus and F. E. Toonder, Proc. Natl. Acad. Sci. (USA) 19, 292-298 (1933); Chem. Abstracts 27, 2646 (1933).

33/15 M. T. Toral and E. Moles, Anales Soc. Espan. Fis. Quim. 31, 735-745 (1933); Chem. Abstracts 28, 954 (1934).

33/16 W. F. Giauque and J. O. Clayton, J. Am. Chem. Soc. 55, 48754889 (1933).

\section{4}

34/1 W. Mehl, Z. Physik. Chem. A164, 312-313 (1934).

34/2 W. Hieber and A. Woerner, Z. Elektrochem. 40, 252-256 (1934).

$34 / 3$ N. E. Rigler, W. A. Felsing, and H. R. Henze, J. Am. Chem. Soc. 56, 1499 (1934).

34/4 W. C. Schumb and F. A. Bickford, J. Am. Chem. Soc. 56, 852-858 (1934).

$34 / 5$ G. N. Lewis and P. W. Schultz, J. Am. Chem. Soc. 56, 1002 (1934). 34/6 J. W. Goodeve, Trans. Faraday Soc. 30, 501-504 (1934). [Vapor pressure data are presented graphically in paper as $\log P$ versus $1 / T$.] 34/7 S. Oguri, S. Anjo and Y. Kuwabara, Bull. Waseda Appl. Chem. Soc. 22, 1-4 (1934); Chem. Abstracts 28, 4298 (1934).

34/8 A. N. Campbell and A. J. R. Campbell, Trans. Faraday Soc. 30, 1109-1114 (1934).

1935

35/1 R. Spence and W. Wild, J. Chem. Soc. 138, 506-509 (1935).

$35 / 2$ H. W. Thompson and J. W. Linnett, Trans. Faraday Soc. 31, 1743-1747 (1935).

35/3 H. Bauer and K. Burschkies, Ber. 68, 1238-1243 (1935).

35/4 H. Schierholtz and M. L. Staples, J. Am. Chem. Soc. 57, 27092711 (1935).

$35 / 5$ V. A. Kireev and A. A. Popov, J. Gen. Chem. (U.S.S.R.) 5, 13991401 (1935); Chem. Abstracts 30, 2441 (1936).

35/6 J. A. V. Butler, C. N. Ramchandani, and D. W. Thompson, J. Chem. Soc. 280-285 (1935).

35/7 W. J. Jones, D. P. Evans, T. Gulwell, and D. C. Griffiths, J. Chem. Soc. 39-47 (1935).

1936

36/1 H. W. Thompson and J. W. Linnett, Trans. Faraday Soc. 32, 681-685 (1936)

36/2 M. Ewert, Bull. Soc. Chim. Belg. 45, 493-515 (1936).

36/3 H. E. Bent, G. R. Cuthbertson, M. Dorfman, and R. E. Leary, J.

Am. Chem. Soc. 58, 165-170 (1936).

36/4 K. Kearby, J. Am. Chem. Soc. 58, 374-375 (1936).

36/5 H. Merten and H. Schlüter, Chem. Ber. 69B, 1364-1366 (1936).

36/6 F. Hovorka, R. A. Schaefer, and D. Dreisback, J. Am. Chem. Soc.

58, 2264 (1936).

36/7 G. R. Negishi, J. Am. Chem. Soc. 58, $2293-2296$ (1936).

36/8 J. G. Aston and G. H. Messerly, J. Am. Chem. Soc. 58, 2354-2361 (1936).

36/9 E. J. Buckler and R. G. W. Norrish, J. Chem. Soc. 1567-1569

(1936).

1937

37/1 A. Klemenc and G. Wagner, Ber. 70B, 1880-1882 (1937); Chem. Abstracts 31, 8412 (1937).
37/2 J. D. Kemp and W. F. Giauque, J. Am. Chem. Soc. 59, 79-84 (1937).

37/3 H. W. Thompson and F. S. Dainton, Trans. Faraday Soc. 33, 1546-1555 (1937).

$37 / 4$ A. Guyer, W. Schutze, and M. Weidenmann, Helv. Chim. Acta 20, 936-949 (1937).

$37 / 5$ C. J. Egan and J. D. Kemp, J. Am. Chem. Soc. 59, 1264-1268 (1937).

37/6 A. B. Burg and W. I. Schlesinger, J. Am. Chem. Soc. 59, 780-787 (1937).

37/7 V. G. Moor, E. K. Kanep, and I. E. Dobkin, Trans. Exptl. Research Lab. Khemgas, Materials on Cracking and Chemical Treatment of Cracking Products U.S.S.R. 3, 320-328 (1937); Chem. Abstracts 31, 6072 (1937)

37/8 A. V. Grosse and V. N. Ipatieff, J. Org. Chem. 2, 447-458 (1937). 37/9 J. C. Rintelen, Jr., J. H. Saylor, and P. M. Gross, J. Am. Chem. Soc. 59, 1129-1130 (1937).

37/10 J. G. Aston, C. W. Siller, and G. H. Messerly, J. Am. Chem. Soc. 59, 1743-1751 (1937).

37/11 G. S. Gardner and J. E. Brewer, Ind. Eng. Chem. 29, 179-181 (1937).

37/12 R. H. Purcell and F. D. Zahoorbux, J. Chem. Soc. 1029-1035 (1937)

37/13 R. Overstreet and W. F. Giauque, J. Am. Chem. Soc. 59, 254-259

(1937).

37/14 R. K. Witt and J. D. Kemp, J. Am. Chem. Soc. 59, 273-276

(1937).

37/15 C. C. Stephenson and W. F. Giauque, J. Chem. Phys. 5, 149-158 (1937).

37/16 R. Linke and W. Rohrmann, Z. Physik. Chem. B35, 256-260

(1937).

1938

38/1 J. D. Brandner, Ind. Eng. Chem. 30, 681-684 (1938).

38/2 M. Linhard, Z. Anorg. Allgem. Chem. 236, 200-208 (1938).

38/3 C. G. Egan and J. D. Kemp, J. Am. Chem. Soc. 60, 2097-2101

(1938)

38/4 L. Reidel, Z. Ges. Kalte-Ind. 45, 221 (1938).

$38 / 5$ G. Scatchard and C. L. Raymond, J. Am. Chem. Soc. 60, 1278 1287 (1938) [Erratum 60, 3099 (1938)].

38/6 D. Radalescu and M. Alexa, Bull. Chem. Soc. Romania 20A, 89-113 (1938).

38/7 K. Kuchinskaya, Sbornik Trudov. Opytnogo Zavoda im. Akad. S. V. Lebedeva 27-30 (1938); Chem. Abstracts 34, 3147 (1940).

38/8 A. R. Ubbelohde, Trans. Faraday Soc. 34, 283-292 (1938).

38/9 E. Levin and I. Shtern, Zh. Prikl. Khim. (Moscow) 11, 426-441

(1938)

38/10 F. Hovorka, H. P. Lankelma, and S. C. Stanford, J. Am. Chem. Soc. 60, 820-827 (1938).

38/11 W. F. Giauque and C. C. Stephenson, J. Am. Chem. Soc. 60, 1389-1394 (1938).

38/12 E. Eftring, Dissertation, University of Lund, 1938; as quoted in $[56 / 25]$.

1939

39/1 J. G. Aston, M. L. Eidinoff, and W. S. Forster, J. Am. Chem. Soc. 61, 1539-1543 (1939).

39/2 T. M. Powell and W. F. Giauque, J. Am. Chem. Soc. 61, 2366-2370 (1939).

39/3 F. H. Verhoek and A. L. Marshall, J. Am. Chem. Soc. 61, 2736-

2742 (1939)

39/4 K. B. Goldblum, R. W. Martin, and R. B. Young, Ind. Eng. Chem. 39, 1474-1476 (1947).

39/5 A. Frank and K. Clusius, Z. Phys. Chem. 42B, 395-421 (1939).

39/6 W. Patnode and W. J. Scheiber, J. Am. Chem. Soc. 61, 3449-3451 (1939).

39/7 L. Reidel, Bull. Intern. Inst. Refrig. 20, 1-10 (1939).

\section{0}

40/1 A. F. Benning and R. C. McHarness, Ind. Eng. Chem. 32, 497-499 (1940). 
40/2 G. H. Messerly and J. C. Aston, J. Am. Chem. Soc. 62, 886-890 (1940).

40/3 A. B. Lamb and E. E. Roper, J. Am. Chem. Soc. 62, 806-814 (1940).

40/4 A. B. Burg, J. Am. Chem. Soc. 62, 2228-2234 (1940).

40/5 J. M. Stuckey and J. H. Saylor, J. Am. Chem. Soc. 62, 2922-2955 (1940).

40/6 K. S. Pitzer, J. Am. Chem. Soc. 62, 1224-1227 (1940).

40/7 F. Hovorka, H. P. Lankelma, and W. R. Smith, J. Am. Chem. Soc. 62, 2372-2374 (1940).

40/8 F. Hovorka, H. P. Lankelma, and A. E. Axelrod, J. Am. Chem. Soc. 62, 187-189 (1940).

40/9 F. Hovorka, H. P. Lankelma, and I. Schneider, J. Am. Chem. Soc. 62, 1096-1098 (1940).

40/10 J. G. Aston and G. H. Messerly, J. Am. Chem. Soc. 62, 19171923 (1940).

40/11 J. G. Aston, R. M. Kennedy, and S. C. Schumann, J. Am. Chem. Soc. 62, 2059 (1940); 62, 2063 (1940).

40/12 A. A. Zil'berman-Granovskaya and E. A. Shugam, J. Phys. Chem. (USSR) 14, 759-767 (1940).

40/13 H. I. Schlesinger, R. T. Sanderson, and A. B. Burg, J. Am. Chem. Soc. 62, 3421-3425 (1940).

40/14 E. R. Smith, J. Res. National Bureau Stand. 24, 229-234 (1940). 40/15 G. H. Messerly and R. M. Kennedy, J. Am. Chem. Soc. 62, 2988-2991 (1940).

\section{1}

41/1 A. F. Belyaev, Acta Physicochem. U.S.S.R. 14, 523 (1941).

41/2 D. M. Youst, C. S. Garner, D. W. Osborne, T. R. Rubin, and H. Russell, Jr., J. Am. Chem. Soc. 63, 2267-2272 (1941).

41/3 R. M. Kennedy, M. Sagenkahn, and J. G. Aston, J. Am. Chem. Soc. 63, 2267-2272 (1941).

$41 / 4$ D. W. Osborne, C. S. Garner, R. N. Doecher, and D. M. Yost, J.

Am. Chem. Soc. 63, 3496-3499 (1941).

41/5 J. G. Aston, R. M. Kennedy, and G. H. Messerly, J. Am. Chem.

Soc. 63, 2243-2348 (1941).

41/6 M. W. Lister, J. Am. Chem. Soc. 63, 143-149 (1941).

41/7 V. A. Kireev and I. P. Sitnikov, J. Appl. Chem. (USSR) 14,

483-485 (1941); Chem. Abstracts 36, 2189 (1942).

41/8 C. B. Heisig, J. Am. Chem. Soc. 63, 1698-1699 (1941).

41/9 E. R. Smith, J. Res. National Bureau Stand. 26, 129-134 (1941).

41/10 J. W. Stout and L. H. Fisher, J. Chem. Phys. 9, 163-168 (1941).

41/11 H. Russell, Jr., R. E. Rundle, and D. M. Yost, J. Am. Chem. Soc. 63, 2825-2828 (1941).

41/12 L. O. Brockway and N. R. Davidson, J. Am. Chem. Soc. 63, 3287 (1941).

41/13 L. Riedel, Z. Ges Kalte-Ind. 48, 9-13 (1941).

1942

42/1 H. Russell, Jr., D. W. Osborne, and D. M. Yost, J. Am. Chem. Soc. 64, 165-169 (1942).

42/2 D. W. Osborne, R. W. Doescher, and D. M. Yost, J. Am. Chem.

Soc. 64, 169-172 (1942).

42/3 P. E. Burchfield, J. Am. Chem. Soc. 64, 2501 (1942).

42/4 G. E. Williams and E. C. Gilbert, J. Am. Chem. Soc. 64, 27762777 (1942).

$42 / 5$ S. C. Schumann, J. G. Aston, and M. Sagenkahn, J. Am. Chem.

Soc. 64, 1039-1043 (1942) [Erratum 64, 30690 (1942)].

$42 / 6$ J. A. Beattie, H. G. Ingersoll, and W. H. Stockmayer, J. Am. Chem. Soc. 64, 546-548 (1942).

42/7 S. W. Benson and G. B. Kistiakowsky, J. Am. Chem. Soc. 64, 80-86 (1942).

42/8 S. W. Benson, Ind. Eng. Chem., Anal. Ed. 13, 189-191 (1942).

42/9 B. Suginuma and K. Satozaki, Rikagaku Kenkyusho Iho 41, 432 (1942).

1943

43/1 K. S. Pitzer and D. W. Scott, J. Am. Chem. Soc. 65, 803-811 (1943).

43/2 D. R. Stevens, Ind. Eng. Chem. 35, 655-660 (1943).

$43 / 3$ J. F. Lemons and W. A. Felsing, J. Am. Chem. Soc. 65, 46-48 (1943).
43/4 J. G. Aston, H. L. Finke, and S. C. Schumann, J. Am. Chem. Soc. 65, 341-346 (1943).

43/5 J. G. Aston, G. J. Szasz, and H. L. Finke, J. Am. Chem. Soc. 65, $1135-1139$ (1943).

43/6 W. P. Hall and E. E. Reid, J. Am. Chem. Soc. 65, 1466-1468

(1943).

43/7 J. S. N. Cramer, Rec. Trav. Chem. 62, 606-610 (1943); Chem.

Zentr. II, 2234 (1943); Chem. Abstracts 38, 6148 (1944).

43/8 G. Holst and L. Melander, Svensk. Kem. Tid. 55, 131-135 (1943);

Chem. Abstracts 40, 2706 (1946).

1944

44/1 H. J. McDonald, J. Phys. Chem. 48, 47-50 (1944).

44/2 T. R. Rubin, B. D. Levedahl, and D. M. Yost, J. Am. Chem. Soc. 66, 279-282 (1944).

44/3 H. Russell, Jr., D. R. V. Golding, and D. M. Yost, J. Am. Chem. Soc. 66, 16-20 (1944).

44/4 G. Milazzo, Gazz. Chim. Ital. 74, 49-57 (1944).

$44 / 5$ I. I. Ioffe and E. S. Yamp'olskaya, Zh. Prikl. Khim. 17, 527-528 (1944); Chem. Abstracts 39, 3986 (1945).

44/6 J. G. Aston, M. L. Sagenkahn, G. J. Szasz, G. W. Moessen, and H. F. Zuhr, J. Am. Chem. Soc. 66, 1171-1177 (1944).

44/7 P. M. Althouse and H. O. Triebold, Ind. Eng. Chem., Anal. Ed. 16, 605-606 (1944).

44/8 R. B. Scott, W. J. Ferbuson, and F. G. Brickwedde, J. Res. National Bureau Stand. 33, 1-20 (1944).

44/9 H. J. Emeleus and A. G. Maddock, J. Chem. Soc. 293-296 (1944). 1945

45/1 E. S. Swift, Jr. and H. P. Hochanadel, J. Am. Chem. Soc. 67, 880-881 (1945).

45/2 C. B. Willingham, W. J. Taylor, J. M. Pignocco, and F. D. Rossini, J. Res. National Bureau Stand. 35, 219-224 (1945).

45/3 L. Guttman and K. S. Pitzer, J. Am. Chem. Soc. 67, 324-327 (1945).

45/4 R. C. Wackher, C. B. Linn, and A. V. Grosse, Ind. Eng. Chem. 37, 464-468 (1945)

45/5 H. J. Hoge, J. Res. National Bureau Stand. 34, 281-293 (1945).

45/6 H. S. Davis and O. F. Wiedeman, Ind. Eng. Chem. 37, 482-485

(1945).

45/7 E. J. Prosen and F. D. Rossini, J. Res. National Bureau Stand. 34, 59-63 (1945).

45/8 F. S. Fawcett and H. E. Rasmussen, J. Am. Chem. Soc. 67,

1705-1709 (1945).

1946

46/1 R. A. Ruehrkein and T. M. Powell, J. Am. Chem. Soc. 68, 10631066 (1946).

46/2 J. E. Kilpatrick and K. S. Pitzer, J. Am. Chem. Soc. 68, 1066-1072 (1946).

46/3 F. H. Field and J. H. Saylor, J. Am. Chem. Soc. 68, 2649-2650

(1946).

46/4 G. W. Thompson, Chem. Rev. 38, 1-39 (1946).

46/5 C. R. Patrick and G. S. Prosser, Trans. Faraday Soc. 60, 700-704

(1946).

46/6 H. Gilman and R. G. Jones, J. Am. Chem. Soc. 68, 517-520

(1946).

46/7 J. G. Aston, H. L. Finke, A. B. Bestul, E. L. Pace, and G. J. Szasz, J. Am. Chem. Soc. 68, 52-57 (1946).

46/8 R. B. Scott and R. G. Brickwedde, J. Res. National Bureau Stand. 35, 501-512 (1946).

46/9 W. J. Jones, L. H. Thomas, E. H. Pritchard, and S. T. Bowden, J. Chem. Soc. 824-827 (1946).

46/10 K. S. Pitzer, L. Guttman, and E. F. Westrum, Jr., J. Am. Chem.

Soc. 68, 2209-2212 (1946).

46/11 R. Spitzer and K. S. Pitzer, J. Am. Chem. Soc. 68, 2537-2538 (1946).

46/12 J. W. Knowlton, N. C. Schieltz, and D. MacMillan, J. Am. Chem. Soc. 68, 208-210 (1946).

46/13 C. H. Bamford, D. L. Levi, and D. M. Newitt, J. Chem. Soc.

468-471 (1946). 
46/14 H. J. Emeleus and A. G. Maddock, J. Chem. Soc. 1131-1134 (1946).

46/15 L. H. Long, H. J. Emeleus, and H. V. A. Briscoe, J. Chem. Soc. 1123-1126 (1946)

46/16 D. A. Crooks and F. M. Feetham, J. Chem. Soc. 899-901 (1946).

46/17 L. S. Dzung, Brown Boveri Rev. 33, 158-163 (1946).

1947

47/1 J. Beersmans and J. C. Jungers, Bull. Soc. Chim. Belg. 56, 238-250 (1947).

47/2 D. J. G. Ives, R. W. Pittman, and W. Wardlaw, J. Chem. Soc.

1080-1083 (1947).

47/3 C. Gould, G. Holzman, and C. Niemann, Anal. Chem. 19, 204-206 (1947).

$47 / 4$ J. A. A. Ketalaar, P. F. Van Velden, and J. S. Zalm, Rec. Trav. Chim. 66, 721-732 (1947).

47/5 D. R. Stull, Ind. Eng. Chem. 39, 517-540 (1947).

47/6 E. W. Balson, K. G. Denbigh, and N. K. Adam, Trans. Faraday Soc. 43, 42-48 (1947).

$47 / 7$ N. S. Osborne and D. C. Ginnings, J. Res. National Bureau Stand. 39, 453-477 (1947).

47/8 F. H. Stross, C. M. Gable, and G. C. Rounds, J. Am. Chem. Soc. 69, 1629-1630 (1947).

47/9 F. H. Stross, J. M. Monger, and H. De V. Finch, J. Am. Chem. Soc. 69, 1627-1628 (1947).

47/10 G. Waddington, S. S. Todd, and H. M. Huffman, J. Am. Chem.

Soc. 69, 22-30 (1947).

47/11 W. M. Jones and W. F. Giauque, J. Am. Chem. Soc. 69, 983-987 (1947).

47/12 G. Waddington and D. R. Douslin, J. Am. Chem. Soc. 69, 2275-2279 (1947).

47/13 J. G. Aston and G. J. Szasz, J. Am. Chem. Soc. 69, 3108-3114 (1947).

47/14 R. D. Fowler, J. M. Hamilton, Jr., J. S. Kasper, C. E. Weber, W. B. Burford III, and H. C. Anderson, Ind. Eng. Chem. 39, 375-378 (1947). 47/15 J. M. Blocher, Jr. and I. E. Campbell, J. Am. Chem. Soc. 69, 2100-2101 (1947).

1948

48/1 K. B. Kellogg and G. H. Cady, J. Am. Chem. Soc. 70, 3986-3990 (1948).

48/2 W. F. Giauque and W. M. Jones, J. Am. Chem. Soc. 70, 120-124

(1948).

48/3 E. L. Pace and J. G. Aston, J. Chem. Soc. 566-570 (1948).

48/4 G. H. Whiting, J. Chem. Soc. 1209-1210 (1948).

$48 / 5$ J. F. Ganeff and J. C. Jungers, Bull. Soc. Chim. Belg. 57, 82-87 (1948).

48/6 J. Gordon and W. F. Giauque, J. Am. Chem. Soc. 70, 1506-1510 (1948).

48/7 A. A. Banks, H. J. Emeleus, R. N. Haszeldine, and V. Kerrigan, J. Chem. Soc. 2188-2190 (1948).

48/8 C. E. Bonhorst and P. M. Athouse, Ind. Eng. Chem. 40, 2379-2384 (1948).

48/9 C. E. Redemann, S. W. Chaikin, and R. B. Fearing, J. Am. Chem. Soc. 70, 631-634 (1948).

48/10 P. A. Small, K. W. Small, and P. Cowley, Trans. Faraday Soc. 44, 810-816 (1948).

48/11 H. E. Bent and R. J. Francel, J. Am. Chem. Soc. 70, 634-637 (1948).

48/12 C. E. Redemann, S. W. Chaiken, R. B. Fearing, and D. Benedict, J. Am. Chem. Soc. 70, 637-639 (1948)

48/13 C. E. Redemann, S. W. Chaikin, and R. B. Fearing, J. Am. Chem. Soc. 70, 1648-1650 (1948).

48/14 C. E. Redemann, S. W. Chaikin, R. B. Fearing, G. J. Rotariu, J. Savit, and D. Van Hoesen, J. Am. Chem. Soc. 70, 3604-3606 (1948).

48/15 A. Michels and T. Wassenaar, Physica (Amsterdam) 14, 104-110 (1948).

48/16 O. Glemser and V. Haüser, Z. Naturforsch. 3B, 159-163 (1948).

48/17 H. J. Emeleus and J. F. Wood, J. Chem. Soc. 2183-2188 (1948). 1949
49/1 R. R. Dreisbach and S. A. Shrader, Ind. Eng. Chem. 41, 2879-2880 (1949); R. R. Dreisbach and R. A. Martin, Ind. Eng. Chem. 41, 28752878 (1949)

49/2 E. Nicolini and P. Laffitte, Comptes Rend. 229, 757-759 (1949); 229, 935-936 (1949).

49/3 W. Kuhn and P. Massini, Helv. Chim. Acta 32, 1530-1542 (1949). 49/4 W. F. Giauque and J. Gordon, J. Am. Chem. Soc. 71, 2176-2181 (1949).

49/5 C. B. Kretschmer and R. Wiebe, J. Am. Chem. Soc. 71, 1793-1797 (1949)

49/6 A. F. Forziati, W. R. Norris, and F. D. Rossini, J. Res. National Bureau Stand. 43, 555-563 (1949).

49/7 G. Waddinton, J. W. Knowlton, D. W. Scott, S. D. Oliver, S. S. Todd, W. N. Hubbard, J. C. Smith, and H. M. Huffman, J. Am. Chem. Soc. 71, 797-808 (1949).

49/8 G. Waddington, J. C. Smith, D. W. Scott, and H. M. Huffman, J. Am. Chem. Soc. 71, 3902-3906 (1949).

49/9 E. S. Perry and W. H. Weber, J. Am. Chem. Soc. 71, 3726-3730 (1949).

49/10 T. Higuchi, N. Endow, and J. E. Willard, J. Am. Chem. Soc. 71, 365-366 (1949).

49/11 G. W. Sears and E. R. Hopke, J. Am. Chem. Soc. 71, 2575-2576

(1949)

49/12 D. W. Scott, G. Waddington, J. C. Smith, and H. M. Huffman, J. Am. Chem. Soc. 71, 2767-2773 (1949).

49/13 D. W. Scott, G. D. Oliver, M. E. Gross, W. N. Hubbard, and H. M Huffman, J. Am. Chem. Soc. 71, 2293-2297 (1949).

49/14 G. S. Parks and G. E. Moore, J. Chem. Phys. 17, 1151-1153 (1949)

49/15 J. Birks and R. S. Bradley, Proc. Roy. Soc. (London) A198, 226-239 (1949).

49/16 E. S. Perry, W. H. Weber, and B. F. Baubert, J. Am. Chem. Soc. 71, 3720-3726 (1949).

49/17 R. S. Bradley and A. D. Shellard, Trans. Faraday Soc. 45, 501-507 (1949)

49/18 F. R. Buck, K. F. Coles, G. T. Kennedy, and F. Morton, J. Chem. Soc. 2377-2383 (1949)

49/19 J. C. Nicholson, J. Chem. Soc. 1553-1555 (1949).

49/20 N. L. Yarym-Agaev, N. N. Fedos'ev, and K. G. Skorikov, Zh. Fiz. Khim. 11, 1257-1265 (1949).

49/21 A. S. Carson, K. Hartley, and H. A. Skinner, Trans. Faraday Soc. 45, 1159-1167 (1949).

49/22 A. S. Carson, K. Hartley, and H. A. Skinner, Proc Royal Soc. (London) A195, 500-512 (1949).

49/23 D. W. Scott, M. E. Gross, G. D. Oliver, and H. M. Huffman, J. Am. Chem. Soc. 71, 1634-1636 (1949).

49/24 J. R. Partington and A. L. Wynes, J. Phys. Chem. 53, 500-503 (1949).

49/25 L. F. Hatch, G. Sutherland, and W. J. Ross, J. Org. Chem. 14, 1130-1136 (1949); vapor pressure data presented graphically as $\log P$ versus $1 / T$ in paper.

49/26 H. J. Emeleus and H. G. Heal, J. Chem. Soc. 1696-1699 (1949). 49/27 J. Thompson and H. J. Emeleus, J. Chem. Soc. 3080 (1949).

49/28 L. O. Winstrom and L. Kulp, Ind. Eng. Chem. 41, 2584-2586 (1949).

1950

50/1 J. B. Matthews, J. F. Sumner, and E. A. Moellyn-Hughes, Trans. Faraday Soc. 46, 797-803 (1950).

50/2 R. M. Noyes, W. A. Noyes, and H. Steinmetz, J. Am. Chem. Soc. 72, 33-34 (1950).

50/3 A. Michels and T. Wassennar, Physica (Amsterdam) 16, 221-224 (1950).

50/4 K. F. Coles and F. Popper, Ind. Eng. Chem. 42, 1434-1438 (1950). 50/5 D. W. Scott, H. L. Finke, M. E. Gross, G. B. Guthrie, and H. M. Huffman, J. Am. Chem. Soc. 72, 2424-2430 (1950).

50/6 A. F. Forziati, D. L. Camin, and F. D. Rossini, J. Res. Natl. Bur. Standards 45, 406-410 (1950). 
50/7 D. W. Scott, H. L. Finke, W. N. Hubbard, J. P. McCullough, M. E. Gross, K. D. Williamson, G. Waddington, and H. M. Huffman, J. Am. Chem. Soc. 72, 4664-4666 (1950).

50/8 D. W. Scott and G. Waddington, J. Am. Chem. Soc. 72, 4310-4311 (1950).

50/9 H. O. Day and W. A. Felsing, J. Am. Chem. Soc. 72, 1698-1699 (1950).

50/10 J. G. Aston, S. V. R. Mastrangelo, and G. W. Moessen, J. Am. Chem. Soc. 72, 5287-5291 (1950).

50/11 A. Z. Conner, P. J. Elving, J. Benischeck, P. E. Tobias, and S. Steingiser, Ind. Eng. Chem. 42, 106-110 (1950).

50/12 K. Hartley, H. O. Pritchard, and H. A. Skinner, Trans. Faraday Soc. 46, 1019-1025 (1950)

1951

51/1 J. G. Aston, H. L. Fink, G. J. Janz, and K. E. Russell, J. Am. Chem. Soc. 73, 1939-1943 (1951).

51/2 J. P. McCullough, W. B. Person, and R. Spitzer, J. Am. Chem. Soc. 73, 4069-4071 (1951).

51/3 D. G. Flom, N. Alpert, and P. J. Elving, Ind. Eng. Chem. 43,

1178-1181 (1951).

51/4 D. W. Scott, H. L. Finke, J. P. McCullough, M. E. Gross, K. D.

Williamson, G. Waddington, and H. M. Huffman, J. Am. Chem. Soc. 73, 261-265 (1951).

51/5 D. Bryce-Smith and K. E. Howlett, J. Chem. Soc. 1141-1142 (1951).

51/6 G. D. Oliver and J. W. Grisard, J. Am. Chem. Soc. 73, 1688-1690 (1951).

51/7 G. M. Barrow and A. L. McClellan, J. Am. Chem. Soc. 73, 573-575 (1951).

51/8 T. F. Smith and R. F. Bonner, Ind. Eng. Chem. 43, 1169-1173 (1951).

51/9 J. C. Potter and J. H. Saylor, J. Am. Chem. Soc. 73, 90-91 (1951). 51/10 A. C. Egerton, W. Emte, and G. J. Minkoff, Diss. Faraday Soc. 278-282 (1951).

51/11 J. W. Grisard, H. A. Bernhardt, and G. D. Oliver, J. Am. Chem. Soc. 73, 5725-5727 (1951).

51/12 K. Hartley, H. O. Pritchard, and H. A. Skinner, Trans. Faraday

Soc. 47, 254-263 (1951).

51/13 W. H. Johnson, M. V. Kilday, and E. J. Prosen, J. Res. Nat. Bur. Stand. 65A, 215-219 (1961).

51/14 A. B. Burg and C. L. Randolph, J. Am. Chem. Soc. 73, 953-957 (1951).

51/15 J. G. Aston, G. J. Janz, and K. E. Russell, J. Am. Chem. Soc. 73, 1943-1945 (1951).

1952

52/1 V. E. Stiles and G. H. Cady, J. Am. Chem. Soc. 74, 3771-3773 (1952).

52/2 L. C. Leitch and A. T. Morse, Can. J. Chem. 30, 924-932 (1952).

52/3 G. B. Guthrie, D. W. Scott, Jr., and G. Waddington, J. Am. Chem. Soc. 74, 2795-2800 (1952).

52/4 G. E. Coates, F. Glocling, and N. D. Huck, J. Chem. Soc. 44964501 (1952).

$52 / 5$ W. S. Jones and W. S. Tamplin, (glycols) American Chemical

Society's Series of Chemistry monographs (1952).

52/6 J. P. McCullough, D. W. Scott, H. L. Finke, M. E. Gross, K. D.

Williamson, R. E. Pennington, G. Waddington, and H. M. Huffman, J. Am. Chem. Soc. 74, 2801-2804 (1952).

52/7 G. H. Whipple, Ind. Eng. Chem. 44, 1664-1667 (1952).

$52 / 8$ H. L. Finke, D. W. Scott, M. E. Gross, G. Waddington, and H. M. Huffman, J. Am. Chem. Soc. 74, 2804-2806 (1952).

52/9 P. T. White, D. G. Bernard-Smith, and F. A. Fidler, Ind. Eng. Chem. 44, 1430-1438 (1952).

52/10 W. N. Hubbard, H. L. Finke, D. W. Scott, J. P. McCullough, C.

Katz, M. E. Gross, J. F. Messerly, R. E. Pennington, and G. Waddington, J. Am. Chem. Soc. 74, 6025-6030 (1952).

52/11 D. W. Scott, H. L. Finke, W. N. Hubbard, J. P. MuCullough, G. D. Oliver, M. E. Gross, C. Katz, K. D. Williamson, G. Waddington, and H. M. Huffman, J. Am. Chem. Soc. 74, 4656-4662 (1952).
52/12 D. W. Scott, H. L. Finke, J. P. McCullough, M. E. Gross, R. E. Pennington, and G. Waddington, J. Am. Chem. Soc. 74, 2478-2848 (1952).

52/13 T. A. Scott, Jr., D. MacMillan, and E. H. Melvin, Ind. Eng. Chem. 44, 172-175 (1952).

52/14 E. T. G. Fuge, S. T. Bowden, and W. J. Jones, J. Phys. Chem. 56, 1013-1016 (1952).

52/15 G. Edwards, Trans. Faraday Soc. 48, 513-515 (1952).

52/16 J. H. Simons and J. W. Hausteller, J. Chem. Phys. 20, 1516-1519

(1952)

52/17 G. B. Guthrie, D. W. Scott, W. N. Hubbard, C. Katz, J. P.

McCullough, M. E. Gross, K. D. Williamson, and G. Waddington, J. Am. Chem. Soc. 74, 4662-4669 (1952).

52/18 I. Brown, Austr. J. Sci. Research 5, 530-540 (1952).

52/19 G. D. Oliver and J. W. Grisard, J. Am. Chem. Soc. 74, 2705-2707 (1952).

52/20 C. T. Mortimer, H. O. Pritchard, and H. A. Skinner, Trans. Faraday Soc. 48, 220-228 (1952).

52/21 L. Kaplan, W. L. Kester, and J. J. Katz, J. Am. Chem. Soc. 75, 5531-5532 (1952).

52/22 G. A. R. Brandt, H. J. Emeleus, and R. N. Haszeldine, J. Chem. Soc. 2198-2205 (1952).

1953

53/1 J. A. Barker, I. Brown, and F. Smith, Disc. Faraday Soc. 15, 142-150 (1953).

53/2 G. T. Furukawa, R. E. McCoskey, and M. L. Reilly, J. Res. Natl. Bur. Standards 51, 69-72 (1953).

53/3 J. G. Aston, J. L. Wood, and T. P. Zolki, J. Am. Chem. Soc. 75, 6202-6204 (1953).

53/4 E. F. G. Herington and J. F. Martin, Trans. Faraday Soc. 49, $154-162$ (1953).

53/5 V. V. Serpinskii, S. A. Voitkevich, and N. Yu. Lyuboshits, Zh. Fiz. Khim. 27, 1032-1038 (1953); Chem. Abstracts 49, 3594e (1955). 53/6 S. Kardon and J. H. Saylor, J. Am. Chem. Soc. 75, 1997 (1953). 53/7 J. P. McCullough, D. W. Scott, H. L. Finke, W. N. Hubbard, M. E. Gross, C. Katz, R. E. Pennington, J. F. Messerly, and G. Waddington, J. Am. Chem. Soc. 75, 1818-1824 (1953).

53/8 D. W. Scott, H. L. Finke, W. N. Hubbard, J. P. McCullough, C.

Katz, M. E. Gross, J. F. Messerly, R. E. Pennington, and G. Waddington, J. Am. Chem. Soc. 75, 2795-2800 (1953).

53/9 H. Stage, E. Müller, and P. Faldix, Erdöle und Kohle 6, 375-380 (1953). [Enthalpies of vaporization were calculated by fitting published vapor pressure data, taken from various sources to the Antoine equation. Some of the references are to unpublished data measured by chemical companies.]

53/10 R. T. Davis, Jr. and R. W. Schiessler, J. Phys. Chem. 57, 966-968 (1953)

53/11 H. A. Skinner and T. F. S. Tees, J. Chem. Soc. 3378-3383 (1953). 53/12 G. W. Rathjens and W. D. Gwinn, J. Am. Chem. Soc. 75, 5629$5633(1953)$

53/13 H. E. Clements, K. V. Wise, and S. E. Johnson, J. Am. Chem. Soc. 75, 1593-5395 (1953).

53/14 K. A. Walsh, U. S. Atom. Energy Comm. Report LA-1649, 1953, Chem. Abstracts 55, 19391c (1961).

53/15 W. T. Grubb and R. C. Osthoff, J. Am. Chem. Soc. 75, 2230-2232 (1953)

53/16 T. Charnley, C. T. Mortimer, and H. A. Skinner, J. Chem. Soc. 1181-1184 (1953).

53/17 A. Michels, T. Wassenaar, P. Louwerse, R. J. Lunbeck, and G. W. Wolkers, Physica 19, 287-289 (1953).

53/18 G. D. Oliver, H. T. Milton, and J. W. Grisard, J. Am. Chem. Soc. 75, 2827-2829 (1953).

53/19 H. Schaefer and F. Zeppernick, Z. Anorg. Allgem. Chem. 272, 274-278 (1953).

53/20 R. C. Osthoff, W. T. Grubb, and C. A. Burkhard, J. Am. Chem. Soc. 75, 2227-2229 (1953)

1954

54/1 A. C. Jenkins and G. F. Chambers, Ind. Eng. Chem. 46, 2367-2369 (1954) 
54/2 J. P. McCullough, D. W. Scott, R. E. Pennington, I. A. Hossenlopp, and G. Waddington, J. Am. Chem. Soc. 76, 4791-4796 (1954). 54/3 A. E. Potter, Jr. and H. L. Ritter, J. Phys. Chem. 58, 1040-1042 (1954).

54/4 M. Davies and D. G. Jenkin, J. Chem. Soc. 2374-2377 (1954). $54 / 5$ G. T. Furukawa, R. E. McCoskey, and M. L. Reilly, J. Research Natl. Bureau Standards 52, 11-16 (1954).

54/6 T. I. Bowell and G. L. Jones, Jr., J. Phys. Chem. 58, 666-667 (1954).

54/7 D. L. Camin, A. F. Forziati, and F. D. Rossini, J. Phys. Chem. 58, 440-442 (1954).

54/8 G. R. Nicholson, M. Szarc, and J. W. Taylor, J. Chem. Soc. 2767-2769 (1954).

54/9 V. V. Serpinskii, S. A. Voitkevich, and N. Yu. Lyuboshits, Zh. Fiz. Khim. 28, 1969-1974 (1954); Chem. Abstracts 50, 4573i (1956). $54 / 10$ V. V. Serpinskii, S. A. Voitkevich, and N. Yu. Lyuboshits, Zh. Fiz. Khim. 28, 810-813 (1954); Chem. Abstracts 49, 6677c (1955). 54/11 W. H. Hubbard and G. Waddington, Rec. Trav. Chim. 910-913 (1954).

54/12 M. Bukala, J. Majewski, and W. Rodzinski, Przemysl Chem. 10, 6-11 (1954).

54/13 R. C. Osthoff and W. T. Grubb, J. Am. Chem. Soc. 76, 399-401 (1954).

54/14 G. Lord and A. A. Wolf, J. Chem. Soc. 2546-2551 (1954).

54/15 V. Fried, J. Pick, E. Hala, and O. Vilim, Chem. Listy 48, 774-775 (1954).

54/16 K. A. Sense, M. J. Snyder, and J. W. Clegg, J. Phys. Chem. 58, 223-224 (1954)

54/17 K. V. Wise, J. Am. Chem. Soc. 76, 3094-3095 (1954).

54/18 L. H. Long and J. F. Sackman, Trans. Faraday Soc. 50, 1177-1182 (1954).

1955

55/1 J. G. Aston, P. E. Wills, and T. P. Zolki, J. Am. Chem. Soc. 77, 3939-3941 (1955).

55/2 J. J. Jasper and G. B. Miller, J. Phys. Chem. 59, 441-442 (1955).

55/3 G. A. N. Cummings and E. McLaughlin, J. Chem. Soc. 1391-1392 (1955).

55/4 O. R. Foz Gazulla, J. Morcilio, A. Perez-Masia, and A. Mendes, Anales Real Soc. Espan. Fis. Quim. (Madrid) 50B, 23-34 (1955). 55/5 G. H. Kosolapoff, J. Chem. Soc. 2964-2965 (1955).

55/6 J. G. Aston, T. P. Zolki, and J. L. Wood, J. Am. Chem. Soc. 77, 281-284 (1955).

55/7 D. L. Camin and F. D. Rossini, J. Phys. Chem. 59, 1173-1179 (1955).

55/8 V. V. Serpinskii, S. A. Voitkevich, and N. Yu. Lyuboshits, Zh. Fiz. Khim. 29, 653-657 (1955); Chem. Abstracts 50, 16231e (1956). 55/9 E. Von Terres, F. Gebert, H. Hulsemann, H. Petereit, H. Toepsch, and W. Ruppert, Brennst.-Chem. 36, 272-274 (1955).

55/10 O. Ya. Tsypikina, J. Appl. Chem. (U.S.S.R.) 28, 167-172 (1955); Chem. Abstracts 49, 9967h (1955).

55/11 R. W. Schiessler and F. C. Whitmore, Ind. Eng. Chem. 47, 1660-1665 (1955); data are deposited as supplementary material, Document No. 4597.

55/12 J. P. McCullough, H. L. Finke, J. F. Messerly, R. E. Pennington, I. A. Hossenlopp, and G. Waddington, J. Am. Chem. Soc. 77, 6119-6125 (1955).

55/13 D. W. Scott, H. L. Finke, J. P. McCullough, M. E. Gross, J. F. Messerly, R. E. Pennington, and G. Waddington, J. Am. Chem. Soc. 77, 4993-4998 (1955).

55/14 G. T. Furukawa, R. E. McCoskey, M. L. Reilly, and A. W. Herman, J. Res. Natl. Bur. Standards 55, 201-207 (1955).

55/15 L. H. Long and J. F. Sackman, Trans. Faraday Soc. 51, 1062-1064 (1955).

55/16 F. Brown and P. L. Robinson, J. Chem. Soc. 3147-3151 (1955). 55/17 J. E. Spice, L. A. K. Staveley, and G. A. Harrow, J. Chem. Soc. 100-104 (1955).

55/18 C. A. Brown and A. W. Laubengayer, J. Am. Chem. Soc. 77, 3699-3700 (1955).
55/19 R. H. Sherman and W. F. Giauque, J. Am. Chem. Soc. 77, 2154-2160 (1955).

55/20 W. H. Mears, R. F. Stahl, S. R. Orfeo, R. C. Shair, L. F. Kells, W. Thompson, and H. McCann, Ind. Eng. Chem. 47, 1449-1454 (1955). 55/21 A. T. Morse, P. B. Ayscough, and L. C. Leitch, Can. J. Chem. 33, 453-457 (1955).

55/22 R. Dreyer, W. Martin, and U. von Weber, J. Prakt. Chem. 273, 324-328 (1955).

55/23 J. Fischer and J. Bingle, J. Am. Chem. Soc. 77, 6511-6512 (1955).

1956

56/1 D. Ambrose, Trans. Faraday Soc. 52, 772-781 (1956).

56/2 J. C. M. Li and K. S. Pitzer, J. Am. Chem. Soc. 78, 1077-1080 (1956).

56/3 P. Gray, M. W. T. Pratt, and M. J. Larkin, J. Chem. Soc. 210-212

(1956).

56/4 J. C. M. Li, J. Am. Chem. Soc. 78, 1081-1083 (1956).

56/5 G. Milazzo, Ann. Chim. (Rome) 46, 1105-1111 (1956); Chem. Abstracts 51, 7791c (1957).

56/6 J. Pick, V. Fried, E. Hala, and O. Vilim, Collect. Czech. Chem.

Commun. 21, 260-261 (1956).

56/7 R. E. Pennington, D. W. Scott, H. L. Finke, J. P. McCullough, J. F. Messerly, I. A. Hossenlopp, and G. Waddington, J. Am. Chem. Soc. 78, 3266-3272 (1956).

56/8 A. Von Brockhaus and E. Jenckel, Macromol. Chem. 18/19, 262-293 (1956).

56/9 D. L. Camin and F. D. Rossini, J. Phys. Chem. 60, 1446-1451 (1956).

56/10 E. J. Barber and G. H. Cady, J. Phys. Chem. 60, 504-505 (1956). 56/11 F. Fromm and M. C. Loeffler, J. Phys. Chem. 60, 252-253 (1956). 56/12 R. H. Capps and W. M. Jackson, J. Phys. Chem. 60, 811-812 (1956).

56/13 D. W. Scott, J. P. McCullough, W. N. Hubbard, J. F. Messerly, I. A. Hossenlopp, F. R. Frow, and G. Waddington, J. Am. Chem. Soc. 78, 5463-5468 (1956).

56/14 D. N. Glew and L. W. Reeves, J. Phys. Chem. 60, 615 (1956). 56/15 E. M. Toops, Jr., J. Phys. Chem. 60, 304-306 (1956).

56/16 Manufacturing Chemists Association Research Project 1956, Tables 23-9-2(2.0111)-k and 23-9-2(2.0112)-k; Tables 23-10-2(2.0111)-k and 23-10-2(2.0112)-k; Tables 23-11-2(2.0111)-k and 23-11-2(2.0112)-k. The authors suspect that the tabulated Antoine Constants for the various compounds were generated in the manner discussed in Ref. [61/13]. 56/17 Manufacturing Chemists Association Research Project 1956, Tables 23-18-2(1.01111)-k and 23-18-2(1.01112)-k. The authors suspect that the tabulated Antoine Constants for the various compounds were generated in the manner discussed in Ref. [61/13].

56/18 D. Garvin and C. Schubert, J. Phys. Chem. 60, 807-808 (1956). 56/19 D. W. Scott, J. P. McCullough, W. D. Good, J. F. Messerly, R. E. Pennington, T. C. Kincheloe, I. A. Hossenlopp, D. R. Douslin, and G. Waddington, J. Am. Chem. Soc. 78, 5457-5463 (1956).

56/20 H. L. Finke, D. W. Scott, M. E. Gross, J. F. Messerly, and G. Waddington, J. Am. Chem. Soc. 78, 5469-5476 (1956).

56/21 M. T. Rogers and J. L. Speirs, J. Phys. Chem. 60, 1462-1463 (1956).

56/22 W. D. Good, D. W. Scott, and G. Waddington, J. Phys. Chem. 60, 1090-1095 (1956).

56/23 E. Neale, L. T. D. Williams, and V. T. Moores, J. Chem. Soc. 422-427 (1956).

56/24 L. H. Long and J. F. Sackman, Trans. Faraday Soc. 52, 1201-1206 (1956).

56/25 F. W. Kirkbride, J. Appl. Chem. 6, 11-21 (1956).

56/26 D. Brennan and A. R. Ubbelohde, J. Chem. Soc. 3011-3016

(1956).

1957

57/1 F. Call, J. Sci. Food Agric. 8, 81-85 (1957).

57/2 P. Gray and M. W. T. Pratt, J. Chem. Soc. 2163-2168 (1957).

57/3 I. Brown and F. Smith, Aust. J. Chem. 10, 423-428 (1957).

57/4 T. C. Bissot, R. W. Parry, and D. H. Campbell, J. Am. Chem. Soc.

79, 796-800 (1957). 
57/5 W. K. Hall and L. Alexander, J. Phys. Chem. 61, 240-242 (1957).

57/6 J. Dykyj, M. Seprakova, and J. Paulech, Chem. Zvesti 11, 461-466 (1957).

57/7 D. W. Scott, H. L. Finke, J. P. McCullough, J. F. Messerly, R. E. Pennington, I. A. Hossenlopp, and G. Waddington, J. Am. Chem. Soc. 79, 1062-1068 (1957).

57/8 A. Dobry and R. Keller, J. Phys. Chem. 61, 1448-1449 (1957). $57 / 9$ V. V. Serpinskii, S. A. Voitkevich, and N. Yu. Lyuboshits, Zh. Fiz. Khim. 31, 1278-1284 (1957); Chem. Abstracts 52, 3270f (1958). $57 / 10$ J. P. McCullough, W. N. Hubbard, F. R. Frow, I. A. Hossenlopp, and G. Waddington, J. Am. Chem. Soc. 79, 561-566 (1957).

57/11 J. P. McCullough, D. R. Douslin, J. F. Messerly, I. A. Hossenlopp, T. C. Kincheloe, and G. Waddington, J. Am. Chem. Soc. 79, 4289-4295 (1957).

57/12 F. Glaser and H. Ruland, Chemie Ing. Tech. 29, 772 (1957). 57/13 B. C. Easton, M. K. Hargreaves, and R. K. S. Mitchell, J. Appl. Chem. (London) 7, 198-204 (1957).

57/14 J. S. Rowlinson and R. I. Thacker, Trans. Faraday Soc. 53, 1-8 (1957).

57/15 L. Maier, O. Seyforth, F. C. A. Stone, and E. Rochov, J. Am. Chem. Soc. 79, 5884-5889 (1957).

57/16 A. Rose, J. A. Acciari, R. C. Johnson, and W. W. Sanders, Ind. Eng. Chem. 49, 104-109 (1957).

57/17 W. S. Barnhart, R. J. Seffl, R. F. Wade, F. W. West, and J. L. Zollinger, J. Chem. Eng. Data 2, 80-83 (1957).

57/18 E. F. Neilson and D. White, J. Am. Chem. Soc. 79, 5618-5621 (1957).

57/19 J. W. Frazer and R. H. Sanborn, U. S. Atomic Energy Commission, Report UCRL-4978, 1957; Chem. Abstracts 52, 8936a (1958).

57/20 J. Aylett, J. Chem. Soc. 4152-4154 (1957).

57/21 K. D. Williamson and R. H. Harrison, J. Chem. Phys. 26, 14091411 (1957).

57/22 J. B. Pedley, H. A. Skinner, and C. L. Chernick, Trans. Faraday Soc. 53, 1612-1617 (1957).

57/23 L. H. Long and J. F. Sackman, Trans. Faraday Soc. 53, 1606-1611 (1957).

57/24 A. K. Fischer, F. A. Cotton, and J. Wilkinson, J. Am. Chem. Soc. 79, 2044-2046 (1957).

57/25 S. D. Beskov, L. I. Kochetkova, and R. M. Golubeva, Uchenye Zapiski Moskov. Gosudarst. Pedagog. Inst. Im. V. I. Lenina 99, 147-149 (1957); Chem. Abstracts 53, 20993d (1959).

57/26 R. C. Wilhoit, J. Phys. Chem. 61, 114-116 (1957).

57/27 H. D. Kaesz and F. G. A. Stone, J. Chem. Soc. 1433-1435 (1957).

$57 / 28$ J. W. Dale, H. J. Emeleus, R. N. Haszeldine, and J. H. Moss, J. Chem. Soc. 3708-3713 (1957).

1958

58/1 L. Molard, Mem. Poudres 40, 13 (1958); as quoted in [77/6].

58/2 A. Rose, B. T. Paphronis, and E. T. Williams, J. Chem. Eng. Data 3, 216-219 (1958).

58/3 R. E. Frost and E. G. Rochow, J. Inorg. Nucl. Chem. 52, 201-206 (1958); Chem. Abstracts 52, 6999c (1958).

58/4 J. A. Brown and W. H. Mears, J. Phys. Chem. 62, 960-962 (1958). $58 / 5$ J. K. Koehler and W. F. Giauque, J. Am. Chem. Soc. 80, 26592662 (1958).

58/6 D. W. Scott, J. P. McCullough, J. F. Messerly, R. E. Pennington, I. A. Hossenlopp, H. L. Finke, and G. Waddington, J. Am. Chem. Soc. 80, 55-59 (1958).

58/7 J. P. McCullough, H. L. Finke, D. W. Scott, R. E. Pennington, M. E. Gross, J. F. Messerly, and G. Waddington, J. Am. Chem. Soc. 80, 4786-4793 (1958).

58/8 W. N. Hubbard, D. R. Douslin, J. P. McCullough, D. W. Scott, S. S. Todd, J. F. Messerly, I. A. Hossenlopp, A. George, and G. Waddington, J. Am. Chem. Soc. 80, 3547-3554 (1958).

$58 / 9$ V. V. Serpinskii, S. A. Voitkevich, and N. Yu. Lyuboshits, Trudy Vsesoyuz. Nauch.- Issledovatel. Inst. Sintet. I. Natural. Dushistykh Veshchestv 4, 125-130 (1958).

58/10 W. Mahler and A. B. Burg, J. Am. Chem. Soc. 80, 6161-6167 (1958).
58/11 A. B. Burg and F. J. Slota, Jr., J. Am. Chem. Soc. 80, 1107-1109 (1958).

58/12 R. D. Dunlap, C. J. Murphy, Jr., and R. C. Bedford, J. Am. Chem. Soc. 80, 83-85 (1958).

58/13 D. C. Bradley and J. D. Swanwick, J. Chem. Soc. 3207-3214 (1958).

58/14 F. Feher and G. Hitzemann, Z. Anorg. Allgem. Chem. 294, 50-62 (1958).

58/15 C. B. Colburn and A. Kennedy, J. Am. Chem. Soc. 80, 5004

(1958).

58/16 K. A. Sense and R. W. Stone, J. Phys. Chem. 62, 453-457 (1958). 58/17 A. V. Novoselova, K. N. Semenenko, and O. Ya. Turova, Vestnik Moskov. Univ. Ser. Mekh., Astron. Fiz. Khim. 13, 139-147 (1958). 58/18 L. H. Long and J. F. Sackman, Trans. Faraday Soc. 54, 1797-1803 (1958).

58/19 P. Gray and M. W. T. Pratt, J. Chem. Soc. 3403-3412 (1958). 58/20 P. A. Fowell and C. T. Mortimer, J. Chem. Soc. 3734-3736 (1958).

58/21 H. J. Emeleus and J. D. Smith, J. Chem. Soc. 527-528 (1958). 58/22 R. C. Cass, S. E. Fletcher, C. T. Mortimer, P. G. Quincey, and H. D. Springall, J. Chem. Soc. 958-963 (1958).

58/23 R. C. Cass, S. E. Fletcher, C. T. Mortimer, H. D. Springall, and T. R. White, J. Chem. Soc. 1406-1410 (1958).

58/24 E. A. V. Ebsworth and H. J. Emeleus, J. Chem. Soc. 2150-2156 (1958).

58/25 R. C. Cass, S. E. Fletcher, C. T. Mortimer, P. G. Quincey, and H. D. Springall, J. Chem. Soc. 2595-2599 (1958).

58/26 D. C. Bradley, L. J. Kay, J. D. Swanick, and W. Wardlaw, J. Chem. Soc. 3656-3659 (1958).

58/27 C. P. Haber and R. K. Uenishi, Chem. Eng. Data Ser. 3, 323-324 (1958); Chem. Abstracts 53, 16781b (1959).

58/28 H. Bloom and B. J. Welch, J. Phys. Chem. 62, 1594-1595 (1958). 1959

59/1 R. A. McDonald, S. A. Shrader, and D. R. Stull, J. Chem. Eng. Data 4, 311-313 (1959).

59/2 D. L. Hildenbrand and R. A. McDonald, J. Phys. Chem. 68, 1521-1523 (1959).

59/3 Kh. A. Aronovich, L. P. Kastorskii, and K. F. Fedorova, Zh. Fiz. Khim. 41, 20 (1959).

59/4 J. P. McCullough, D. R. Douslin, W. N. Hubbard, S. S. Todd, J. F. Messerly, I. A. Hossenlopp, F. R. Frow, J. P. Dawson, and G. Waddington, J. Am. Chem. Soc. 81, 5884-5890 (1959).

59/5 M. Seprakova, J. Paulech, and J. Dykyj, Chem. Zvesti 313-316 (1959).

59/6 A. B. Burg and L. R. Grant, J. Am. Chem. Soc. 81, 1-5 (1959).

59/7 D. W. Scott, D. R. Douslin, J. F. Messerly, S. S. Todd, I. A.

Hossenlopp, T. C. Kincheloe, and J. P. McCullough, J. Am. Chem. Soc. 81, 1015-1020 (1959).

59/8 J. P. McCullough, R. E. Pennington, J. C. Smith, I. A. Hossenlopp, and G. Waddington, J. Am. Chem. Soc. 81, 5880-5883 (1959).

59/9 M. Usanovich and A. I. Dembitskii, Zh. Obshch. Khim. 29, 1781-1785 (1959).

59/10 D. L. Hildenbrand, G. C. Sinke, R. A. McDonald, and W. R. Kramer, J. Chem. Phys. 31, 650-654 (1959).

59/11 I. Brown and F. Smith, Aust. J. Chem. 12, 407-412 (1959).

59/12 W. D. Good, D. R. Douslin, D. W. Scott, A. George, J. L. Lacina,

J. P. Dawson, and G. Waddington, J. Phys. Chem. 63, 1133-1138 (1959). 59/13 M. Usanovich and A. I. Dembitskii, Zh. Obshch. Khim. 29, 1771-1781 (1959).

59/14 A. J. Leadbetter and J. E. Spice, Can. J. Chem. 37, 1923-1929 (1959).

59/15 E. Terres, L. Brinkmann, D. Fischer, D. Hüllstrung, W. Loz, and G. Weisbrod, Brennstoff-Chem. 40, 279-280 (1959).

59/16 J. B. Pedley and H. A. Skinner, Trans. Faraday Soc. 55, 544-547 (1959).

59/17 W. D. Good, D. W. Scott, J. L. Lacina, and J. P. McCullough, J. Phys. Chem. 63, 1139-1142 (1959).

59/18 F. A. Cotton, A. K. Fischer, and J. Wilkinson, J. Am. Chem. Soc. 81, 800-803 (1959). 
59/19 L. J. Paridon, G. E. MacWood, and J.-H. Hu, J. Phys. Chem. 63, 1998-1999 (1959).

59/20 K. Mödritzer, Chem. Ber. 92, 2637-2640 (1959); Chem. Abstracts 54, 3180e (1960).

59/21 H. J. Emeleus and J. D. Smith, J. Chem. Soc. 375-381 (1959).

59/22 W. R. Cullen and H. J. Emeleus, J. Chem. Soc. 372-375 (1959).

59/23 S. E. Fletcher, C. T. Mortimer, and H. D. Springall, J. Chem. Soc. 580-584 (1959).

59/24 P. A. Folwell and C. T. Mortimer, J. Chem. Soc. 2913-2918

(1959).

59/25 E. Amberger, Angew. Chem. 71, 372-373 (1959); Chem. Abstracts 53, 16780c (1959).

59/26 H. Suga and S. Seki, Bull. Chem. Soc. Jpn. 32, 1088 (1959).

59/27 P. Chaiyavech and M. Van Winkle, J. Chem. Eng. Data 4, 53-57

(1959).

59/28 L. C. Yen and T. M. Reed III, J. Chem. Eng. Data 4, 102-107 (1959).

59/29 F. P. Pike and C. F. Foster, Jr., J. Chem. Eng. Data 4, 305-306

(1959).

1960

60/1 A. Aihara, Bull. Chem. Soc. Jpn. 33, 194-200 (1960).

60/2 R. G. Schmitt and R. C. Hirt, J. Polym. Sci. 45, 35-47 (1960).

60/3 C. R. Mueller and J. Ignatowski, J. Chem. Phys. 32, 1430-1434 (1960).

60/4 R. J. L. Andon, D. P. Biddiscombe, J. D. Cox, R. Handley, D. Harrop, E. F. G. Herington, and J. F. Martin, J. Chem. Soc. 5246-5254 (1960).

60/5 M. L. Klyueva, K. P. Mischenko, and M. K. Fedorov, Zh. Prikl. Khim. 33, 473-475 (1960); Chem. Abstracts 54, 11616g (1960). 60/6 L. G. L. Ward and A. G. MacDiarmid, J. Am. Chem. Soc. 82, 2151-2153 (1960).

60/7 D. E. McLaughlin and M. Tamres, J. Am. Chem. Soc. 82, 5618 5621 (1960).

60/8 D. E. McLaughlin, M. J. Tamres, and S. Searless, Jr., J. Am. Chem. Soc. 82, 5621-5625 (1960).

60/9 H. D. Kaesz and F. G. A. Stone, J. Am. Chem. Soc. 82, 6213-6218 (1960).

60/10 F. E. Brinckman and F. G. A. Stone, J. Am. Chem. Soc. 82 6218-6223 (1960).

60/11 H. D. Kaesz, J. P. Phillips, and F. G. A. Stone, J. Am. Chem. Soc. 82, 6228-6232 (1960).

60/12 S. L. Stafford and F. G. A. Stone, J. Am. Chem. Soc. 82, 6238$6240(1960)$.

60/13 R. J. Brotherton, A. L. McCloskey, J. L. Boone, and H. M. Manasevit, J. Am. Chem. Soc. 82, 6245-6248 (1960).

60/14 R. J. Brotherton, A. L. McCloskey, L. L. Petterson, and H. Steinberg, J. Am. Chem. Soc. 82, 6242-6245 (1960).

60/15 W. P. Van Meter and G. H. Cady, J. Am. Chem. Soc. 82, 60056008 (1960).

60/16 D. N. Glew, Can. J. Chem. 38, 208-211 (1960).

60/17 J. Novak, J. Matous, and J. Pick, Collect. Czech. Chem. Commun. 25, 2405-2413 (1960)

60/18 E. J. Lynch and C. R. Wilke, J. Chem. Eng. Data 5, 300 (1960). 60/19 J. W. Frazer, J. Inorg. Nucl. Chem. 16, 63-66 (1960).

60/20 D. L. Camin and F. D. Rossini, J. Chem. Eng. Data 5, 368-372 (1960).

60/21 R. A. McKay and B. H. Sage, J. Chem. Eng. Data 5, 21-24 (1960).

60/22 F. M. Trent, F. D. Miller, and G. H. Brown, J. Chem. Eng. Data 5, 110-111 (1960)

60/23 A. P. Claydon, P. A. Fowell, and C. T. Mortimer, J. Chem. Soc. 3284-3287 (1960)

60/24 H. J. Emeleus and S. N. Nabi, J. Chem. Soc. 1103-1108 (1960). 60/25 H. J. Emeleus and H. Pugh, J. Chem. Soc. 1108-1112 (1960). 60/26 E. Amberger, Angew. Chem. 72, 494 (1960); Chem. Abstracts 55, 22103f (1961); 60, 4171d (1964).

60/27 R. M. Yarrington and W. B. Kay, J. Chem. Eng. Data 5, 24-29 (1960).

1961
61/1 J. E. Griffiths and M. Onyszchuk, Can. J. Chem. 39, 339-347 (1961)

61/2 E. L. Pace and R. J. Bobka, J. Chem. Phys. 35, 454-457 (1961).

61/3 J. Heinrich, J. Ilavsky, and J. Surovy, Chem. Zvesti 15, 414-418

(1961).

61/4 H. Grosse-Ruyken and R. Kleesaat, Z. Anorg. Allgem. Chem. 308, 122-132 (1961)

61/5 B. Sternback and A. G. MacDiarmid, J. Am. Chem. Soc. 83, 3384-3388 (1961).

61/6 A. Rose and W. R. Supina, J. Chem. Eng. Data 6, 173-179 (1961).

$61 / 7$ W. T. Berg, D. W. Scott, W. N. Hubbard, S. S. Todd, J. F. Messerly,

I. A. Hossenlopp, A. Osborn, D. R. Douslin, and J. P. McCullough, J.

Phys. Chem. 65, 1425-1430 (1961).

61/8 J. P. McCullough, H. L. Finke, W. N. Hubbard, S. S. Todd, J. F.

Messerly, D. R. Douslin, and G. Waddington, J. Phys. Chem. 65,

784-791 (1961).

61/9 R. E. Banks, A. E. Ginsberg, and R. N. Haszeldine, J. Chem. Soc. 1740-1743 (1961).

61/10 S. Malanowski, Bull. Acad. Pol Sci. Ser. Sci. Chim. 9, 71-76

(1961).

61/11 S. Malanowski, Bull. Acad. Pol. Sci. Ser. Sci. Chim. 9, 83 (1961).

61/12 J. Dykvy, M. Seprakova, and J. Paulech, Chem. Zvesti 15,

465-468 (1961); Chem. Abstracts 56, 10930b (1962).

61/13 J. C. M. Li and F. D. Rossini, J. Chem. Eng. Data 6, 268-270

(1961).

61/14 A. B. Burg and J. E. Griffiths, J. Am. Chem. Soc. 83, 4333-4335

(1961)

61/15 D. W. Scott, J. F. Messerly, S. S. Todd, G. B. Gutherie, I. A.

Hosenlopp, R. T. Moore, A. Osborn, W. T. Berg, and J. P. McCullough, J. Phys. Chem. 65, 1320-1326 (1961).

61/16 H. M. Huffman, M. E. Gross, D. W. Scott, and J. P. McCullough,

J. Phys. Chem. 65, 495-503 (1961).

61/17 J. K. Nickerson, K. A. Kobe, and J. J. McKetta, J. Phys. Chem.

65, 1037-1043 (1961).

61/18 J. F. Mathews and J. J. McKetta, J. Phys. Chem. 65, 758-762

(1961).

61/19 A. F. Frolov, M. A. Loginova, and M. M. Kiseleva, Zh. Phys.

Khim. 35, 1784-1788 (1961).

61/20 E. J. Smutny and A. Bondi, J. Phys. Chem. 65, 546-550 (1961).

$61 / 21$ J. R. Case, N. H. Ray, and H. L. Roberts, J. Chem. Soc. 2070-

2075 (1961)

61/22 J. R. Case, N. H. Ray, and H. L. Roberts, J. Chem. Soc. $2066-$ 2070 (1961)

61/23 I. M. Schiopu, O. Bot, and V. Onu, Bul. Inst. Politeh. Iasi. 7, 115-118 (1961); Chem. Abstracts 58, 10793c (1963).

61/24 P. A. Fowell and C. T. Mortimer, J. Chem. Soc. 3793-3796

(1961)

61/25 W. D. Good, J. L. Lacina, and J. P. McCullough, J. Phys. Chem. 65, 2229-2231 (1961).

61/26 H. Zimmerman and H. Greisenfelder, Z. Electrochem. 65, 368-371

(1961)

61/27 E. Amberger and H. Boeters, Angew. Chem. 73, 114 (1961);

Chem. Abstracts 55, 16403h (1961).

61/28 E. Amberger, Chem. Ber. 94, 1447-1452 (1961); Chem. Abstracts 56, 8279e (1962).

61/29 J. F. Ditter, J. C. Perrine, and I. Shapiro, J. Chem. Eng. Data 6, 271 (1961).

61/30 A. Labbauf, J. B. Greenshields, and F. D. Rossini, J. Chem. Eng.

Data 6, 261-263 (1961)

61/31 P. Hestermans and D. White, J. Phys. Chem. 65, 362-365 (1961).

1962

62/1 R. H. Valentini, G. E. Brodale, and W. R. Giauque, J. Phys. Chem. 66, 392-395 (1962).

$62 / 2$ G. Waddington, J. C. Smith, K. D. Williamson, and D. W. Scott, J. Phys. Chem. 66, 1074-1077 (1962).

62/3 A. J. Von Arvia, P. J. Aymoning, and H. J. Schumacher, Z. Anorg. Allgem. Chem. 316, 325-334 (1962).

62/4 A. Kreglewski, Bull. Acad. Pol. Sci., Ser. Sci. Chem. 10, 629-633 (1962) 
62/5 H.-J. Bittrich, E. Kauer, M. Kraft, G. Schoeppe, W. Soell, and A. Ullrich, J. Prakt. Chem. 17, 250-262 (1962).

62/6 A. D. Craig and A. G. MacDiarmid, J. Inorg. Nucl. Chem. 24, 161-164 (1962).

62/7 W. E. Hatton, D. R. Douslin, G. C. Sinke, and D. R. Stull, J. Chem. Eng. Data 7, 229-231 (1962).

62/8 A. D. Craig, J. Chem. Soc. 548-553 (1962).

62/9 D. W. Scott, W. D. Good, S. S. Todd, J. F. Messerly, W. T. Berg, I. A. Hossenlop, J. L. Lucina, A. G. Osborn, and J. P. McCullough, J. Chem. Phys. 36, 406-412 (1962).

62/10 D. W. Scott, D. R. Douslin, H. L. Finke, W. N. Hubbard, J. F. Messerly, I. A. Hossenlopp, and J. P. McCullough, J. Phys. Chem. 66, 1334-1341 (1962)

62/11 M. Mansson and S. Sunner, Acta Chem. Scand. 16, 1863-1869 (1962).

62/13 W. D. Good, D. R. Douslin, and J. P. McCullough, J. Phys. Chem. 66, 958-959 (1962).

62/14 J. H. Stern and F. H. Dorer, J. Phys. Chem. 66, 97-99 (1962).

62/15 N. S. Berman and J. J. McKetta, J. Phys. Chem. 66, 1444-1448

(1962).

62/16 H. Mackle and R. G. Mayrick, Trans. Faraday Soc. 58, 238-243

(1962).

62/17 H. Mackle and R. G. Mayrick, Trans. Faraday Soc. 58, 230-237

(1962).

62/18 G. R. Ross and W. G. Heideger, J. Chem. Eng. Data 7, 505-507

(1962).

62/19 S. M. Williamson and G. H. Cady, Inorg. Chem. 1, 673-677

(1962).

62/20 H. Mackle and R. G. Mayrick, Trans. Faraday Soc. 58, 33-39

(1962).

62/21 J. E. Griffiths and A. B. Burg, J. Am. Chem. Soc. 84, 3442-3450

(1962).

62/22 J. J. Martin, J. Chem. Eng. Data 7, 68-72 (1962).

62/23 H.-J. Bittrich and E. Kauer, Z. Phys. Chem. (Frankfurt/Main) 219, 224-238 (1962).

62/24 G. J. Pasek and G. Thodes, J. Chem. Eng. Data 7, 21-26 (1962).

62/25 A. Bauder and H. Guenthard, Helv. Chim. Acta 45, 1698-1702

(1962).

62/26 P. Sellers and S. Sunner, Acta Chem. Scand. 16, 46-52 (1962).

62/27 J. P. Oliver and L. G. Stevens, J. Inorg. Nucl. Chem. 24, 953-960 (1962).

62/28 G. C. Sinke and D. L. Hildenbrand, J. Chem. Eng. Data 7, 74

(1962).

62/29 S. P. Vohra, T. L. Kang, K. A. Kobe, and J. J. McKetta, J. Chem.

Eng. Data 7, 150-155 (1962).

62/30 G. Geiseler, K. Quitzsch, J. Hoffmann, and W. Moeschler, Z.

Physik. Chem. (Leipzig) 220, 391-396 (1962).

62/31 E. A. V. Ebsworth, H. J. Emeleus, and N. Welcman, J. Chem. Soc. 2290-2293 (1962).

62/32 H. J. Emeleus, K. H. Packer, and N. Welcman, J. Chem. Soc. 2529-2531 (1962).

62/33 A. J. Downs, J. Chem. Soc. 4361-4366 (1962).

62/34 E. Amberger and H. Boeters, Angew. Chem. 74, 293 (1962);

Chem. Abstracts 57, 4276f (1962).

62/35 E. Amberger and H. Boeters, Angew. Chem. 74, 32-33 (1962);

Chem. Abstracts 57, 12522e (1962).

62/36 B. Cohen and A. G. MacDiarmid, Inorg. Chem. 1, 754-756

(1962).

62/37 J. Tjebbes, Acta Chem. Scand. 16, 916-921 (1962).

1963

63/1 R. H. Boyd, J. Chem. Phys. 38, 2529-2535 (1963).

63/2 K. G. McCurdy and K. J. Laidler, Can. J. Chem. 41, 1867-1871

(1963).

63/3 I. Shapiro, B. Keilin, R. E. Williams, and C. D. Good, J. Am.

Chem. Soc. 85, 3167-3171 (1963).

63/4 J. A. Brown, J. Chem. Eng. Data 8, 106-108 (1963).

63/5 J. P. McCullough, J. F. Messerly, R. T. Moore, and S. S. Todd, J.

Phys. Chem. 67, 677-679 (1963).
63/6 H. Hiraoka and J. H. Hildebrand, J. Phys. Chem. 67, 916-918 (1963).

63/7 G. A. Bottomley and G. H. F. Seiflow, J. Appl. Chem. 13, 399-409

(1963).

63/8 D. P. Biddiscombe, R. R. Collerson, R. Handley, E. F. G. Herington, J. F. Martin, and C. H. S. Sprake, J. Chem. Soc. 1954-1957 (1963). 63/9 W. D. Good, D. R. Douslin, and J. P. McCullough, J. Phys. Chem. 67, 1312-1314 (1963).

63/10 D. P. Biddiscombe, R. Handley, D. Harrop, A. J. Head, G. B. Lewis, J. F. Martin, and C. H. S. Sprake, J. Chem. Soc. 5764-5768 (1963).

63/11 D. W. Scott, W. N. Hubbard, J. F. Messerly, S. S. Todd, I. A. Hossenlopp, W. D. Good, D. R. Douslin, and J. P. McCullough, J. Am. Chem. Soc. 67, 680-685 (1963).

63/12 D. W. Scott, W. D. Good, G. B. Gutherie, S. S. Todd, I. A.

Hossenlopp, A. G. Osborn, and J. P. McCullough, J. Am. Chem. Soc. 67, 685-689 (1963).

63/13 M. Kraus, L. Beranek, K. Kochloefl, and V. Bazant, Chem. Prumysl 12, 649-652 (1962); Chem. Abstracts 58, 10746d (1963). 63/14 R. J. Best, J. Chem. Eng. Data 8, 267-270 (1963).

63/15 J. G. Wojtasinski, J. Chem. Eng. Data 8, 381-385 (1963). 63/16 A. Rose and V. N. Schrodt, J. Chem. Eng. Data 8, 9-13 (1963). 63/17 W. P. Gilbreath and G. H. Cady, Inorg. Chem. 2, 496-499 (1963). 63/18 E. T. Benyon, Jr. and J. J. McKetta, J. Phys. Chem. 67, 2761-2765 (1963).

63/19 W. D. Good, S. S. Todd, J. F. Messerly, J. L. Lacina, J. P. Dawson, D. W. Scott, and J. P. McCullough, J. Phys. Chem. 67, 1306-1311 (1963)

63/20 D. Ambrose and R. Townsend, J. Chem. Soc. 3614-3625 (1963).

63/21 A. Capkova and V. Fried, Collect. Czech. Chem. Commun. 28, 2235-2239 (1963).

63/22 C. G. Vinson, Jr. and J. J. Martin, J. Chem. Eng. Data 8, 74-75

(1963)

63/23 D. W. Scott, J. F. Messerly, S. S. Todd, I. A. Hossenlopp, A.

Osborn, and J. P. McCullough, J. Chem. Phys. 38, 532-539 (1963).

63/24 J. A. Dixon, G. R. Yarnell, and J. A. Mountain, J. Chem. Eng.

Data 8, 572-573 (1963).

63/25 V. V. Sokolov, L. P. Zhilina, and K. P. Mischenko, Zh. Prikl Khim. 36, 750-754 (1963).

63/26 M. A. Greenbaum, J. N. Foster, M. L. Arin, and M. Farber, J.

Phys. Chem. 67, 36-40 (1963).

63/27 E. J. Spanier and A. G. MacDiarmid, Inorg. Chem. 2, 214-215

(1963).

63/28 M. Lustig and G. H. Cady, Inorg. Chem. 2, 388-390 (1963).

63/29 R. R. Holmes and R. P. Wagner, Inorg. Chem. 2, 384-388 (1963).

63/30 M. Abedini and A. G. MacDiarmid, Inorg. Chem. 2, 608-613

(1963).

63/31 S. B. Hartley, W. S. Holmes, J. K. Jaques, M. F. Mole, and J. C. McCoubrey, Quat. Rev. Chem. Soc. 17, 204 (1973).

63/32 A. E. Pope and H. A. Skinner, J. Chem. Soc. 3704-3712 (1963).

63/33 G. L. Gal'chenko and R. M. Varushchenko, Zh. Fiz. Khim. 37,

2513-2519 (1963).

63/34 C. T. Mortimer and P. Sellers, J. Chem. Soc. 1978-1981 (1963).

63/35 I. B. Rabinovich, V. I. Tel'noi, N. V. Karyakin, and G. A.

Razuvaev, Dokl. Akad. Nauk. SSSR 149, 324-326 (1963).

63/36 J. V. Davies, A. E. Pope, and H. A. Skinner, Trans. Faraday Soc.

59, 2233-2242 (1963).

63/37 W. F. Lautsch, A. Tröber, W. Zimmer, L. Mehner, W. Linck, H. M.

Lehman, H. Brandenberger, H. Korner, H.-J. Metschker, K. Wagner, and

R. Kaden, Z. Chem. 3, 415-421 (1963).

63/38 J. L. Hales, J. D. Cox, and E. B. Lees, Trans. Faraday Soc. 59,

1544-1554 (1963).

63/39 E. A. V. Ebsworth and S. G. Frankiss, J. Chem. Soc. 661-665

(1963).

63/40 J. V. Urenovitch and A. G. MacDiarmid, J. Chem. Soc. 1091-1095

(1963).

63/41 H. J. Emeleus and N. Welcman, J. Chem. Soc. 1268-1271 (1963).

63/42 H. J. Emeleus and A. Haas, J. Chem. Soc. 1272-1275 (1963). 
63/43 D. C. Abercromby and P. F. Tiley, J. Chem. Soc. 4902-4903 (1963).

63/44 E. Amberger and H. Boeters, Z. Naturforsch. 18B, 157 (1963); Chem. Abstracts 59, 5194g (1963).

63/45 G. Pass and H. L. Roberts, Inorg. Chem. 2, 1016-1019 (1963). 63/46 M. M. Fein, J. Bobinski, N. Mayes, N. Schwartz, and M. S. Cohen, Inorg. Chem. 2, 1111-1115 (1963).

\section{4}

64/1 J. V. Enstein, L. I. Duronina, and A. S. Pashinkin, Zh. Prikl. Khim. 37, 2543-2545 (1964)

64/2 A. V. Kletskii, Inzh. Fiz. Zh. Akad. Nauk. Beldrussk. SSR 7, 40-43 (1964).

64/3 G. Fisher and A. S. Buchanan, Aust. J. Chem. 17, 481 (1964).

64/4 L. K. Peterson and A. B. Burg, J. Am. Chem. Soc. 86, 2587-2591 (1964).

64/5 G. Geiseler and W. Konig, Z. Physik. Chem. (Leipzig) 227, 81-90 (1964).

64/6 K. P. Murphy, J. Chem. Eng. Data 9, 259-260 (1964).

64/7 A. N. Gubkov, N. A. Fermor, and N. I. Smirnov, Zh. Prikl. Khim. 37, 2204-2210 (1964).

64/8 A. Capkova and V. Fried, Coll. Czech. Chem. Commun. 29,

336-340 (1964).

64/9 N. N. Sevrugova, V. A. Sokorskii, and N. M. Zhavoronkov, Zh. Prikl. Khim. (Leningrad) 37, 1989-1993 (1964).

64/10 N. A. Goldberg, V. A. Gorbushchenkov, and Z. G. Teplova, Zh. Prikl. Khim. 37, 745-747 (1964).

64/11 E. G. Mangalin, Khim. Prom. 4, 304-306 (1964).

64/12 D. W. Morecroft, J. Chem. Eng. Data 9, 488-490 (1964).

64/13 A. Rose and V. Schrodt, J. Chem. Eng. Data 9, 12-16 (1964).

64/14 R. Handley, D. Harrop, J. F. Martin, and C. H. S. Sprake, J. Chem. Soc. 4404-4406 (1964).

64/15 D. Ambrose and R. Townsend, Trans. Faraday Soc. 60, 1025-1029 (1964).

64/16 C. R. Patrick and G. S. Prosser, Trans. Faraday Soc. 60, 700-704 (1964).

64/17 H. Mackle and R. T. B. McClean, Trans. Faraday Soc. 60, 817-821 (1964)

64/18 L. C. Duncan and G. H. Cady, Inorg. Chem. 3, 850-852 (1964). 64/19 L. C. Duncan and G. H. Cady, Inorg. Chem. 3, 1045-1047 (1964). 64/20 Yu. Kh. Shaulov, V. S. Tubyanskaya, E. V. Evstegneeva, and G. O. Shmyreva, Russ. J. Phys. Chem. 38, 967-970 (1964).

64/21 L. A. Serafimov, V. S. Timofeev, M. P. Strukova, and S. V. L'vov, Russ. J. Phys. Chem. 38, 1018-1019 (1964).

64/22 C. T. Ratcliffe and J. M. Shreeve, Inorg. Chem. 3, 631-633 (1964).

64/23 N. S. Berman, C. W. Larkam, and J. J. McKetta, J. Chem. Eng.

Data 9, 218-219 (1964).

64/24 V. Kliment, V. Fried, and J. Pick, Collect. Czech. Chem. Commun. 29, 2008-2015 (1964).

64/25 F. S. Fawcett and R. D. Lipscomb, J. Am. Chem. Soc. 86, 2576-2579 (1964)

64/26 W. D. Good, J. L. Lacina, B. L. De Prater, and J. P. McCullough, J. Phys. Chem. 68, 579-586 (1964).

64/27 J. L. Bills and F. A. Cotton, J. Phys. Chem. 68, 806-810 (1964).

64/28 A. E. Pope and H. A. Skinner, Trans. Faraday Soc. 60, 1404-1407 (1964).

64/29 B. J. Aylett and L. K. Peterson, J. Chem. Soc. 3429-3436 (1964). 64/30 R. G. Cavell, J. Chem. Soc. 1992-1995 (1964).

64/31 R. G. Cavell and H. J. Emeleus, J. Chem. Soc. 5825-5832 (1964). 64/32 R. G. Cavell and H. J. Emeleus, J. Chem. Soc. 5896-5897 (1964). 64/33 H. Mackle and P. A. G. O'Hare, Trans. Faraday Soc. 60, 506-509 (1964).

1965

65/1 S. J. Price and M. G. Jacko, Can. J. Chem. 43, 3481-3482 (1965). 65/2 W. E. Putnam, D. M. McEachern, and J. E. Kiplatrick, J. Chem. Phys. 42, 749-755 (1965).

65/3 I. Mertl and J. Polak, Collect. Czech. Chem. Commun. 30, 35263528 (1965)
65/4 I. I. Serebryannaya and S. Sh. Byk, Khim. Prom. 23, 828-829 (1965).

65/5 L. A. McDougall and J. E. Kilpatrick, J. Chem. Phys. 42, $2311-$ 2321 (1965).

65/6 I. V. Kudryashov and R. I. Savechenko, Izv. Vysh. Ucheb. Zav.

Khim. Khim. Tekhnol. 8, 602 (1965).

65/7 R. R. Collerson, J. F. Counsell, R. Handley, J. F. Martin, and C. H. S. Sprake, J. Chem. Soc. 3697-3700 (1965).

65/8 D. R. Douslin and A. Osborn, J. Sci. Instrum. 42, 369-373 (1965).

65/9 A. N. Marinichev and M. P. Susarev, Zh. Prikl. Khim. 38, 378-383

(1965).

65/10 N. V. Lutugina, V. N. Kolbina, and L. I. Reshetova, Zh. Prikl.

Khim. 38, 1541-1546 (1965).

65/11 P. A. Naro, J. Chem. Eng. Data 10, 86 (1965).

65/12 I. Wadso, Acta Chem. Scand. 19, 1079-1087 (1965).

65/13 R. E. Rondeau and L. A. Harrah, J. Chem. Eng. Data 10, 84-85 (1965)

65/14 H. L. Finke, I. A. Hossenlopp, and W. T. Berg, J. Phys. Chem. 69, 3030-3031 (1965).

65/15 M. Davies and B. Kybett, Trans. Faraday Soc. 61, 1608-1617

(1965).

65/16 J. F. Counsell, J. L. Hales, and J. F. Martin, Trans. Faraday Soc. 61, 1869-1875 (1965).

65/17 J. Linek, V. Fried, and J. Pick, Collect. Czech. Chem. Commun. 30, 1358-1365 (1965).

65/18 R. Noftle and G. H. Cady, Inorg. Chem. 4, 1010-1012 (1965). 65/19 J. M. Shreeve, L. C. Duncan, and G. H. Cady, Inorg. Chem. 4, 1516-1517 (1965).

65/20 L. A. Nisel'son and I. I. Lapivus, Russ. J. Phys. Chem. 39, 80-83 (1965).

65/21 I. G. Gakh and E. P. Babin, Russ. J. Phys. Chem. 39, 924 (1965). 65/22 L. A. Nisel'son and I. I. Lapidus, Russ. J. Phys. Chem. 39, 931-932 (1965)

65/23 G. W. Fraser and J. M. Shreeve, Inorg. Chem. 4, 1497-1498 (1965).

65/24 H. Kilian and H. J. Bittrich, Z. Phys. Chem. 230, 383 (1965).

65/25 J. Heinrich, J. Surovy, and J. Dojcansky, Chem. Zvesti 19, 462-464 (1965).

65/26 J. Linek, V. Fried, and J. Pick, Collect. Czech. Chem. Commun. 30, 1358-1365 (1965).

65/27 Yu. Kh. Shaulov, G. O. Schmyreva, and V. S. Tubyanskaya, Zh. Fiz. Khim. 39, 105-109 (1965).

65/28 H. S. Hull, A. F. Reid, and A. G. Turnbull, Austr. J. Chem. 18, 249-252 (1965).

65/29 B. J. Aylett and L. K. Peterson, J. Chem. Soc. 4043-4048 (1965).

\section{6}

66/1 A. W. Laubengayer and C. W. J. Scaife, J. Chem. Eng. Data 11, 172-174 (1966)

66/2 I. Wadso, Acta Chem. Scand. 20, 544-552 (1966).

66/3 T. R. Bott and H. N. Adler, J. Chem. Eng. Data 11, 25-30 (1966).

66/4 W. H. Mears, E. Rosenthal, and J. V. Sinka, J. Chem. Eng. Data 11, $338-342$ (1966).

66/5 A. N. Osborn and D. R. Douslin, J. Chem. Eng. Data 11, 502-509

(1966).

66/6 N. E. Miller, M. D. Murphy, and D. L. Reznicek, Inorg. Chem. 5, 1832-1834 (1966).

66/7 G. Geiseler, J. Fruwert, and R. Huetting, Chem. Ber. 99, 1594-1601 (1966)

66/8 A. P. Kudchadker and B. J. Zwolinski, J. Chem. Eng. Data 11, 253-255 (1966).

66/9 H. L. Morris, M. Tamres, and S. Searles, Inorg. Chem. 5, 21562160 (1966)

66/10 W. D. Good and B. L. DePrater, J. Phys. Chem. 70, 3606-3609 (1966)

66/11 F. D. Evans and P. F. Tiley, J. Chem. Soc. B 134-136 (1966).

66/12 E. F. Meyer and R. E. Wagner, J. Phys. Chem. 70, 3162-3168 (1966).

66/13 M. F. Zimmer, R. A. Robb, and G. A. Carpenter, J. Chem. Eng. Data 11, 577-579 (1966). 
66/14 V. A. Branzhan, S. V. Semenenko, and O. G. Kirillova, Zh. Prikl. Khim. 39, 1399-1403 (1966); Chem. Abstracts 65, 9820f (1966). 66/15 D. D. DesMarteau and G. H. Cady, Inorg. Chem. 5, 169-171 (1966).

66/16 M. Abedini and A. G. MacDiarmid, Inorg. Chem. 5, 2040-2042 (1966).

66/17 J. J. Delfino and J. M. Shreeve, Inorg. Chem. 5, 308-309 (1966). 66/18 B. L. Earl, B. K. Hill, and J. M. Shreeve, Inorg. Chem. 5, 2184-2186 (1966).

66/19 B. Börjesson, Y. Nakase, and S. Sunner, Acta Chem. Scand. 20, 803-810 (1966).

66/20 D. C. Bradley and M. J. Hillyer, Trans. Faraday Soc. 62, 2374 $2381(1966)$

66/21 R. C. Dobbie, J. Chem. Soc. A 1555-1556 (1966).

66/22 A. E. Beezer and C. T. Mortimer, J. Chem. Soc. A 514-516

(1966).

66/23 M. A. Ring, H. E. O'Neal, A. H. Kadhim, and F. Jappe, J. Organomet. Chem. 5, 124-129 (1966).

66/24 D. J. Coleman and H. A. Skinner, Trans. Faraday Soc. 62, 1721-1755 (1966).

66/25 V. I. Tel'noi and I. B. Rabinovich, Russ. Zh. Fiz Khim. 40, $1556-1563$ (1966)

66/26 G. Gattow and M. Dräger, Z. Anorg. Allg. Chem. 343, 232-243 (1966).

66/27 A. Finch, P. J. Gardner, and E. J. Pearn, Trans. Faraday Soc. 62, 1072-1081 (1966).

66/28 A. A. Zalykin and Yu. A. Strepikheev, J. Appl. Chem. USSR 39, 2448 (1966).

66/29 G. P. Luchinskii, Zh. Fiz. Khim. 40, 593-598 (1966).

66/30 I. Holeci, Chem. Prumysl 16, 267-270 (1966).

$66 / 31$ D. H. Brown, G. W. Fraser, and D. W. A. Sharp, J. Chem. Soc. A 171-174 (1966).

66/32 R. C. Dobbie and H. J. Emeleus, J. Chem. Soc. A 933-936 (1966). 66/33 H. J. Emeleus and T. Onak, J. Chem. Soc. A 1291-1292 (1966).

66/34 J. L. Carson, R. C. Stewart, and A. G. Williamson, J. Chem. Eng. Data 11, 231-233 (1966).

66/35 M. Mansson and S. Sunner, Acta Chem. Scand. 20, 845-848 (1966).

1967

67/1 A. Finch, P. J. Gardner, E. J. Pearn, and G. B. Watts, Trans. Faraday Soc. 63, 1880-1888 (1967).

67/2 P. M. Christopher and A. Shilman, J. Chem. Eng. Data 12, 333-335 (1967).

67/3 J. Murto and A. Kivinen, Suomen Kemistil. B 40, 258-264 (1967). 67/4 A. C. Meeks and I. J. Goldfarb, J. Chem. Eng. Data 12, 196 (1967). $67 / 5$ E. L. Pace and A. C. Plaush, J. Chem. Phys. 47, 38-43 (1967). 67/6 K. H. Goebel, J. Schaffenger, and G. Opel, Chem. Tech. 19, 307-308 (1967).

67/7 R. L. Shak, J. Chem. Eng. Data 12, 474-480 (1967).

67/8 A. Finch, P. J. Gardner, and G. B. Watts, Trans. Faraday Soc. 63, 1603-1607 (1967).

67/9 A. C. Plaush and E. L. Pace, J. Chem. Phys. 47, 44-48 (1967) 67/10 H. C. Van Ness, C. A. Soczek, G. L. Peloquin, and R. L. Machado, J. Chem. Eng. Data 12, 217-223 (1967).

67/11 M. Haccuria and M. P. Mathieu, Ind. Chim. Belg. 32, 165-167 (1967).

67/12 H. A. Berman and E. D. West, J. Chem. Eng. Data 12, 197-199 (1966).

67/13 W. A. Van Hook, J. Chem. Phys. 46, 1907-1918 (1967).

67/14 A. B. Kletshii and L. E. Petric, Zh. Fiz. Khim. 41, 1183-1184

(1967).

67/15 G. A. Crowder, Z. H. Taylor, T. M. Reed, and J. A. Young, J. Chem. Eng. Data 12, 481-485 (1967).

67/16 E. F. Westrum and A. Ribner, J. Phys. Chem. 71, 1216-1224 (1967).

67/17 I. I. Konstantinov, E. Z. Zhuravlev, and Z. G. Teplova, Zh. Prikl. Khim. 40, 1084-1087 (1967).

67/18 N. V. Ermakov and V. P. Skripov, Zh. Fiz. Khim. 41, 77-81 (1967).
67/19 D. W. Scott, W. T. Berg, I. A. Hossenlopp, W. N. Hubbard, J. F. Messerly, S. S. Todd, D. R. Douslin, J. P. McCullough, and G.

Waddington, J. Phys. Chem. 71, 2263-2270 (1967).

67/20 A. Ribner and E. F. Westrum, Jr., J. Phys. Chem. 71, 1208-1215

(1967).

67/21 A. J. B. Cruickshank and A. J. B. Cutler, J. Chem. Eng. Data 12, 326-329 (1967).

67/22 M. M. Omar, J. Chem. Soc. C 2038-2040 (1967).

67/23 D. Reedy and G. Urry, Inorg. Chem. 6, 2117-2118 (1967).

67/24 L. L. Lapidus, L. A. Nisel'son, and A. A. Karateeva, Russ. J. Phys. Chem. 41, 241-243 (1967).

67/25 E. A. Miroshnichenko, Yu. A. Lebedev, S. A. Shevelev, V. I. Gulevskaya, A. A. Fainzil'berg, and A. Ya. Apin, Russ. J. Phys. Chem. 41, 783-785 (1967)

67/26 Yu. V. Golubkov, I. I. Lapidus, and L. A. Nisel'son, Russ. J. Phys. Chem. 41, 1122-1123 (1967)

67/27 T. M. Lesteva, S. K. Ogorodnikov, and A. I. Morozova, Zh. Prik1. Khim. (Leningrad) 40, 891-894 (1967).

67/28 M. Lustig, Inorg. Chem. 6, 1064-1065 (1967).

67/29 M. L. Anderson and R. N. Hammer, J. Chem. Eng. Data 12, 442-447 (1967).

67/30 V. Fried, P. Gallant, and G. B. Schneier, J. Chem. Eng. Data 12, 504-508 (1967).

67/31 O. Glemser, U. Biermann, and M. Fild, Chem. Ber. 100, 1082-

1086 (1967)

67/32 N. K. Smith and W. D. Good, J. Chem. Eng. Data 12, 570-572 (1967)

67/33 S. Pawlenko, Chem. Ber. 100, 3591-3598 (1967).

67/34 J. C. Baldwin, M. F. Lappert, J. B. Pedley, and J. A. Treverton, J. Chem. Soc. A 1980-1984 (1967).

67/35 S. M. Danov and Yu. D. Golubev, Tr. Khim. Khim. Tekhnol. 52-55 (1967); Chem. Abstracts 68, 95253a (1968).

67/36 T. L. Charlton and R. G. Cavell, Inorg. Chem. 6, 2204-2208 (1967)

67/37 K. Pihlaja and J. Heikkila, Acta Chem. Scand. 21, 2430-2434

(1967)

67/38 M. Hirata and S. Suda, Kagaku Kogaku 31, 339-342 (1967).

$67 / 39$ J. L. Hales, E. B. Lees, and D. Ruxton, J. Trans. Faraday Soc. 63, 1876-1879 (1967).

$67 / 40$ B. J. Aylett and J. M. Campbell, Inorg. Nucl. Chem. Letters 3, 137-139 (1967).

67/41 V. R. Paetzold, R. Kurze, and G. Engelhardt, Z. Anorg. Allgem.

Chem. 353, 62-71 (1967).

67/42 M. P. Edejer and G. Thodos, J. Chem. Eng. Data 12, 206-209

(1967)

1968

68/1 I. Wadso, Acta Chem. Scand. 22, 2438-2444 (1968).

68/2 E. L. Cherkasskaya, A. M. Tur, Z. F. Petrenkova, and V. I.

Lyubomilov, Zh. Prikl. Khim. 41, 2553-2554 (1968).

68/3 F. Gopal and S. A. Rizvi, J. Indian Chem. Soc. 45, 13-16 (1968).

68/4 A. G. Osborn and D. R. Douslin, J. Chem. Eng. Data 13, 534-537 (1968).

68/5 P. F. Malbrunot, P. A. Meunier, G. M. Scatena, W. H. Mears, K. P. Murphy, and J. V. Sinka, J. Chem. Eng. Data 13, 16-21 (1968).

68/6 W. J. Gaw and F. L. Swinton, Trans. Faraday Soc. 64, 637-647 (1968)

68/7 E. Z. Zhuravlev and I. I. Konstantinov, Zh. Prikl. Khim. 41, 1170-1172 (1968).

68/8 J. O. Hill and I. Wadso, Acta Chem. Scand. 22, 1590-1594 (1968). 68/9 E. Morawetz, Acta Chem. Scand. 22, 1509-1531 (1968).

68/10 D. Ambrose, J. Chem. Soc. 1381-1383 (1968).

68/11 L. Fowler, W. N. Trump, and C. E. Vogler, J. Chem. Eng. Data 13, 209-210 (1968)

68/12 E. E. Baroody, G. A. Carpenter, R. A. Robb, and M. F. Zimmer, J. Chem. Eng. Data 13, 215-217 (1968).

68/13 I. I. Lapidus and L. A. Nisel'son, Russ. J. Phys. Chem. 42, 733-734 (1968).

68/14 Yu. Ya. Maksimov, Russ. J. Phys. Chem. 42, 1550-1552 (1968). 
68/15 C. Ratcliffe and J. M. Shreeve, J. Am. Chem. Soc. 90, 5403-5408 (1968).

68/16 R. V. Kachalova and M. S. Nemcov, Zh. Prikl. Khim. (Leningrad) 41, 2315-2319 (1968).

68/17 R. D. Place and S. M. Williamson, J. Am. Chem. Soc. 90, 2550-3556 (1968).

68/18 N. Welcman and M. Wulf, Israel J. Chem. 6, 37-41 (1968). 68/19 M. Mansson, Y. Nakase, and S. Sunner, Acta Chem. Scand. 22, 171-174 (1968).

68/20 D. P. Baccanari, J. A. Novinski, Y.-C. Pan, M. M. Yevitz, and H. A. Swain, Jr., Trans. Faraday Soc. 64, 1201-1205 (1968).

68/21 O. Glemser and S. P. von Halasz, Z. Naturforsch. 23B, 743-745 (1968).

68/22 O. Glemser, S. P. von Halasz, and U. Biermann, Z. Naturforsch. 23B, 1381-1382 (1968).

68/23 W. J. Gaw and F. L. Swinton, Trans. Faraday. Soc. 64, 2023-2034 (1968).

68/24 V. V. Kushchenko and K. Mishchenko, P. Zh. Prikl. Khim. 41, 646-648 (1968); Chem. Abstracts 68, 117314a (1968).

68/25 B. M. Kuadzhe, G. P. Ustyugov, and A. A. Kudryavtsev, Tr. Mosk. Khim. Tekhnol. Inst. 58, 27-28 (1968); Chem. Abstracts 71, 6728 (1969).

68/26 R. J. L. Andon, J. F. Counsell, J. L. Hales, E. B. Lees, and J. F. Martin, J. Chem. Soc. 2357-2361 (1968).

68/27 L. A. Nisel'son, R. K. Nikolaev, T. D. Sokolova, V. I. Stolyarov, and Yu. M. Korolev, Izv. Sib. Otd. Akad. Nauk. SSSR, Ser. Khim. Nauk 109-114 (1968); Chem. Abstracts 69, 69834s (1969).

68/28 G. Geiseler and J. Hoffmann, Z. Phys. Chem. (Frankfurt am Main) 57, 318-330 (1968)

68/29 M. Cihova, J. Vojtko, and M. Hrusovsky, Zb. Pr. Chemickotechnol. Fak. SVST (Slov. Vys. SK. Tech.) 135-138 (1968); Chem. Abstracts 72, 136676u (1970).

68/30 W. B. Fox, J. S. MacKenzie, E. R. McCarthy, J. R. Holmes, R. F. Stahl, and R. Juurik, Inorg. Chem. 7, 2064-2067 (1968).

68/31 A. S. Khorevskaya and S. Sh. Byk, Zh. Prikl. Khim. (Leningrad)

41, 2566-2568 (1968); Chem. Abstracts 70, 61317a (1969).

1969

69/1 Zh. V. Davydova, I. A. Makolkin, and P. S. Bataev, Zh. Obshch.

Khim. 39, 1668-1669 (1969).

69/2 I. Wadso, Acta Chem. Scand. 23, 2061-2064 (1969).

69/3 J. A. Devore and H. E. O’Neal, J. Phys. Chem. 73, 2644-2648

(1969).

69/4 M. A. Soulie, P. Goursot, A. Peneloux, and J. Metzger, J. Chim.

Phys. Physicochim. Biol. 66, 603-606 (1969).

69/5 R. H. Kemme and S. I. Kreps, J. Chem. Eng. Data 14, 98-102 (1969).

69/6 J. H. Smith and E. L. Pace, J. Phys. Chem. 73, 4232-4236 (1969). 69/7 B. G. Rueben, J. Chem. Eng. Data 14, 235-236 (1969).

69/8 T. M. Lesteva, A. I. Morozova, V. I. Morozova, S. K. Ogorodnikov, and T. M. Tyvina, Zh. Prikl. Khim. 42, 533-538 (1969).

69/9 T. J. V. Findlay, J. Chem. Eng. Data 14, 229-231 (1969).

69/10 G. C. Calero, M. M. Valle, and C. G. Losa, Rev. Acad. Cienc. Fis. Quim. Nat. Zaragoza 24, 137-158 (1969).

69/11 A. L. Woodman and A. Adicoff, J. Chem. Eng. Data 14, 479-480 (1969).

69/12 A. G. Osborn and D. R. Douslin, J. Chem. Eng. Data 14, 208-209 (1969).

69/13 R. J. Sheehan and S. H. Langer, J. Chem. Eng. Data 14, 248-250

(1969).

69/14 J. Konicek, M. Prochazka, V. Krestanova, and M. Smisek, Collect. Czech. Chem. Commun. 34, 2249-2257 (1969).

69/15 J. Cidlinsky and J. Polak, Collect. Czech. Chem. Commun. 34, 1317-1321 (1969).

69/16 F. Franks and B. Watson, Trans. Faraday Soc. 65, 2339-2349 (1969).

69/17 N. G. Krokhin, Russ. J. Phys. Chem. 43, 1342-1343 (1969). 69/18 M. Mansson, J. Chem. Thermodyn. 1, 141-151 (1969). 69/19 I. Brown, W. Fock, and F. Smith, J. Chem. Thermodyn. 1, 273-291 (1969).
69/20 S. M. Danov, V. B. Matin, P. V. Jefremov, and N. K. Slashtshina, Zh. Fiz. Khim. 43, 733-736 (1969); Chem. Abstracts 71, 6729g (1969). 69/21 J. Vojtko, M. Cihova, and M. Hrusovsky, Zb. Pr. Chem.-Tech. Fak. Svst. 179 (1969/1970); Chem. Abstracts 76, 76492b (1972).

69/22 O. Glemser and S. P. Von Halasz, Inorg. Nucl. Chem. Lett. 5, 393-398 (1969)

69/23 R. Lamantine and R. Perrin, Bull. Soc. Chim. France 443-445 (1969).

69/24 H. Mackle, D. V. McNally, and W. V. Steele, Trans. Faraday Soc. 65, 2060-2068 (1969).

69/25 S. Meszaros, Period. Poltech. Chem. Eng. (Budapest) 13, 79-88

(1969); Chem. Abstracts 72, 66308z (1970).

69/26 O. Glemser, R. Mews, and H. W. Roesky, J. Chem. Soc., Chem. Commun. 914, (1969).

69/27 Y. K. Shaulov, A. K. Federov, and V. G. Genchel, Zh. Fiz Khim. 185, 1336-1337 (1969).

69/28 J. E. Sicre, J. T. Dubois, K. J. Eisentraut, and R. E. Sievers, J. Am. Chem. Soc. 91, 3476-3481 (1969).

69/29 M. A. Soulie, P. Goursot, A. Peneloux, and J. Metzger, J. Chim.

Phys. Physicochim. Biol. 66, 607-610 (1969).

69/30 M. Ya. Agarunov and S. N. Hajiev, Dokl. Akad. Nauk. SSSR 185, 221 (1969).

69/31 T. Melia and R. Merrifield, J. Appl. Chem. (London) 19, 79-82 (1969).

69/32 J. Mason, J. Chem. Soc. A 1587-1592 (1969).

69/33 B. J. Aylett and M. J. Hakim, J. Chem. Soc. A 800-803 (1969). 69/34 B. J. Aylett and M. J. Hakim, J. Chem. Soc. A 636-638 (1969).

69/35 B. J. Aylett and M. J. Hakim, J. Chem. Soc. A 639-642 (1969).

69/36 B. J. Aylett and J. M. Campbell, J. Chem. Soc. A 1910-1916 (1969).

69/37 B. J. Aylett and J. M. Campbell, J. Chem. Soc. A 1916-1920

(1969).

69/38 B. J. Aylett, J. M. Campbell, and A. Walton, J. Chem. Soc. A 2110-2113 (1969).

69/39 R. D. George and K. M. Mackay, J. Chem. Soc. A 2122-2126

(1969).

1970

70/1 A. J. Valerga, Dissertation Abstracts 31, 3316-B (1970-1971).

70/2 D. Ambrose and C. H. S. Sprake, J. Chem. Thermodyn. 2, 631-645 (1970).

70/3 J. Polak, S. Murakami, V. T. Lam, and G. C. Benson, J. Chem. Eng. Data 15, 323-328 (1970).

70/4 D. Ambrose, J. F. Counsell, and A. J. Davenport, J. Chem.

Thermodyn. 2, 283-294 (1970).

70/5 E. D. Smith and W. L. Thornsberry, J. Chem. Eng. Data 15,

296-297 (1970).

70/6 R. M. Varushchenko, N. A. Belikova, S. M. Skuratov, and A. F.

Plate, Zh. Fiz. Khim. 44, 3022-3025 (1970).

70/7 D. N. Andreevskii and M. M. Brazhnikov, Vestn. Beloruss. Univ. 2, 14-18 (1970); Chem. Abstracts 76, 158651s (1972).

70/8 J. Konicek and I. Wadso, Acta Chem. Scand. 24, 2612-2616

(1970).

70/9 R. J. Irving and I. Wadso, Acta Chem. Scand. 24, 589-593 (1970). 70/10 G. A. Carpenter, M. F. Zimmer, E. E. Baroody, and R. A. Robb, J. Chem. Eng. Data 15, 150-153 (1970).

70/11 W. D. Good and R. T. Moore, J. Chem. Eng. Data 15, 150-153 (1970).

70/12 A. K. Goncharov, M. Kh. Karapet'yants, and G. M. Kol'yakova, Russ. J. Phys. Chem. 44, 466-467 (1970).

70/13 J. E. Smith and G. H. Cady, Inorg. Chem. 9, 1293-1294 (1970).

70/14 J. Dykyj and A. Vanko, Petrochemica 10, 3-22 (1970).

70/15 L. M. Zaborowski and J. M. Shreeve, J. Am. Chem. Soc. 92, 3665-3668 (1970).

70/16 J. Dykyj, Petrochemica 10, 51-59 (1970); 123-146 (1970); 10 187-206 (1970)

70/17 K. Kusano and I. Wadso, Acta Chem. Scand. 24, 2037-2042 (1970).

70/18 P. Sellers, J. Chem. Thermodyn. 2, 211-219 (1970). 
70/19 D. Harrop, A. J. Head, and G. B. Lewis, J. Chem. Thermodyn. 2, 203-210 (1970).

70/20 J. F. Counsell, J. O. Fenwick, and E. B. Lees, J. Chem.

Thermodyn. 2, 367-372 (1970).

70/21 M. Prochazka, V. Krestanova, J. Konicek, and M. Smisek, Collect. Czech. Chem. Commun. 35, 727-732 (1970).

70/22 E. Koizumi and S. Ouchi, Nippon Kagaku Zasshi 91, 501-503 (1970).

70/23 D. W. Scott, J. Chem. Thermodyn. 2, 833-837 (1970).

70/24 O. Eisen and A. Orav, Eesti Tead. Akad. Toim., Keem. Geol. 19, 202-205 (1970).

70/25 A. J. Valerja and J. E. Kilpatrick, J. Chem. Phys. 52, 4545-4549 (1970).

70/26 A. B. Burg and D.-K. Kang, J. Am. Chem. Soc. 92, 1901-1908 (1970).

70/27 S. P. Von Halasz and O. Glemser, Chem. Ber. 103, 553-560 (1970).

70/28 J. Vojtko, M. Cihova, and M. Hrusovsky, Chem. Zvesti 24, 173-176; Chem. Abstracts 74, 46115x (1971).

70/29 A. Finch, P. J. Gardner, P. McNamara, and G. R. Wellum, J. Chem. Soc. A 3339-3345 (1970)

70/30 S.-J. Chang, D. McNally, S. Sharry-Tehrany, M. J. Hickey, and R. H. Boyd, J. Am. Chem. Soc. 92, 3109-3118 (1970).

70/31 J. D. Cox and G. Pilcher, Thermochemistry of Organic and Organometallic Compounds (Academic, London, 1970).

70/32 Y. K. Shaulov, A. K. Federov, G. Y. Zueva, G. V. Borisyuk, and V. G. Genchen, Russ. J. Phys. Chem. 44, 1181-1182 (1970); Zh. Fiz. Khim. 44, 2081-2083; Chem. Abstracts 74, 7230m (1971).

70/33 M. G. Barlow, R. N. Haszeldine, and R. Hubbard, J. Chem. Soc. C 1232-1237 (1970).

70/34 F. I. Mullayanov, G. V. Galagan, M. F. Buz'ko, Yu. V. Churkin, and A. A. Shiryaeva, Tr. Nauch-Issled. Inst. Neftekhim. Proizvod. 95-97 (1970); Chem. Abstracts 74, 91930v (1971).

70/35 A. K. Baev, Obshch. Prikl. Khim. 146-160 (1970); Chem. Abstracts 74, 91934z (1971).

70/36 V. D. Moisev and N. D. Antonova, Zh. Fiz. Khim. 44, 2912-2913 (1970); Chem. Abstracts 69, 80487e (1971).

70/37 V. B. Sokolov, M. Kh. Karapet'yants, and A. K. Goncharov, Tr Mosk. Khim.-Tekhnol. Inst. 67, 37-39 (1970); Chem. Abstracts 75, 122149z (1971).

70/38 P. Sellers, Acta Chem. Scand. 24, 2453-2458 (1970).

1971

71/1 J. K. Choi and M. J. Joncich, J. Chem. Eng. Data 16, 87-90 (1971). 71/2 H. K. Hall, Jr. and J. H. Baldt, J. Am. Chem. Soc. 93, 140-145 (1971).

71/3 C. Eon, C. Pommier, and G. Guiochon, J. Chem. Eng. Data 16, 408-410 (1971)

71/4 E. F. Meyer, T. A. Renner, and K. S. Stec, J. Phys. Chem. 76, 642-648 (1971).

71/5 K. Kusano and I. Wadso, Acta Chem. Scand. 25, 219-224 (1971).

$71 / 6$ Yu. N. Matyushin, V. I. Pepekin, S. P. Golova, T. I. Godovikova,

and L. I. Khmelnitskii, Bull Acad. Sci. USSR, Div. Chem. Sci. 162-164 (1971).

71/7 E. Morawetz, Chem. Scripta 1, 103-111 (1971).

71/8 P. Sellers, Acta Chem. Scand. 25, 2189-2193 (1971).

71/9 K. Kusano and I. Wadso, Bull. Chem. Soc. Jpn. 44, 1705-1707 (1971).

71/10 N. D. Lebedeva, Yu. A. Katin, and G. Ya. Akhmedova, Russ. J. Phys. Chem. 45, 1192-1193 (1971).

71/11 V. B. Sokolov, M. Kh. Karapet'yants, N. D. Rumyantseva, and V. A. Drozdov, Russ. J. Phys. Chem. 45, 1332-1333 (1971).

71/12 A. K. Goncharov, M. Kh. Karapet'yants, and T. K. Gar, Russ. J. Phys. Chem. 45, 104-105 (1971).

71/13 N. D. Lebedeva, Yu. A. Katin, and G. Ya. Akhmedova, Russ. J. Phys. Chem. 45, 771-772 (1971).

71/14 A. K. Baev, V. V. Dem'yanchuk, G. Girzoev, G. I. Novikov, and N. E. Kolobova, Russ. J. Phys. Chem. 45, 777-778 (1971).

$71 / 15$ V. E. Ditsent, I. I. Skorokhodov, N. A. Terent'eva, and M. N. Zolotareva, Russ. J. Phys. Chem. 45, 901-902 (1971).
71/16 I. I. Skorokhodov, V. E. Ditsent, N. A. Teret'eva, and M. N. Zolotarev, Russ. J. Phys. Chem. 45, 902 (1971).

71/17 F. Swindell, L. M. Zaborowski, and J. M. Shreeve, Inorg. Chem. 10, 1635-1638 (1971).

71/18 R. A. De Marco and J. M. Shreeve, Inorg. Chem. 10, 911-913 (1971)

71/19 L. M. Zaborowski and J. M. Shreeve, Inorg. Chem. 10, 407-409 (1971)

71/20 D. T. Sauer and J. M. Shreeve, Inorg. Chem. 10, 358-362 (1971).

71/21 J. Dykyj, Petrochemia 11, 27-45 (1971).

71/22 Z. I. Geller, V. D. Simonov, M. V. Lyubarskii, E. A. Brakhfogel, L. M. Meizler, and I. A. Paramonov, Izv. Uyssh. Ucheb. Zaved., Nept. Gaz 7, 71-74 (1971).

71/23 I. Mita, I. Imai, and I. Kambe, Thermochimica Acta 2, 337-344 (1971)

71/24 L. A. Nisel'son, K. V. Tret'yakova, E. P. Paremuzov, and E. N. Torbina, Izv. Akad. Nauk SSSR, Neorg. Mater. 7, $792-794$ (1971); Chem. Abstracts 75, 67746q (1971).

71/25 B. Ringner, S. Sunner, and H. Watanabe, Acta Chem. Scand. 25, 141-146 (1971)

71/26 K. Pihlaja and M.-L. Tuomi, Acta Chem. Scand. 25, 465-469 (1971)

71/27 J. Polak and G. C. Benson, J. Chem. Thermodyn. 3, 235-242

(1971).

71/28 R. C. Wihoit and B. J. Zwolinski, Handbook of Vapor Pressures and Heats of Vaporization of Hydrocarbons and Related Compounds, API 44-TRC (Publications in Science and Engineering, College Station, TX, 1971).

71/29 I. B. Rabinovich, E. G. Kiparisov, and Y. A. Aleksandrov, Dok1. Akad. Nauk. SSSR 200, 1116-1118 (1971).

71/30 G. M. Kol'yakova, I. B. Rabinovich, and N. S. Vyazankin, Dok1. Akad. Nauk. SSSR 200, 111-113 (1971)

71/31 P. Sellers, Acta Chem. Scand. 25, 2099-2102 (1971).

71/32 I. E. Nakhutin, N. M. Smirnova, V. I. Krivenko, and G. A.

Loshakov, Zh. Obshch. Khim. 41, 940-943 (1971); Chem. Abstracts 75, 122154x (1971)

71/33 S. A. Voitkevich, M. M. Shchedrina, N. P. Solov'eva, and T. A. Rudol'fi, Maslo-Zhir. Prom. 37, 27-30 (1971).

71/34 D. T. Sauer and J. M. Shreeve, J. Fluorine Chem. 1, 1-11 (1971).

$71 / 35$ D. W. Osborne, F. Schreiner, and H. Selig, J. Chem. Phys. 54, 3790-3798 (1971).

71/36 W. D. Good and R. T. Moore, J. Chem. Thermodyn. 3, 701-705 (1971).

71/37 F. E. Rodgers, J. Phys. Chem. 75, 1734-1737 (1971).

1972

72/1 M. Colomina, J. L. Laynez, R. Perez-Ossorio, and C. Turrion, J. Chem. Thermodyn. 4, 499-506 (1972).

72/2 E. L. Pace and P. E. Hodge, J. Chem. Thermodyn. 4, 441-447 (1972)

72/3 M. Mansson, J. Chem. Thermodyn. 4, 865-871 (1972).

72/4 J. F. Counsell and D. A. Lee, J. Chem. Thermodyn. 4, 915-917 (1972).

72/5 R. Prydz and R. D. Goodwin, J. Chem. Thermodyn. 4, 123-127 (1972).

72/6 T. Boublik and K. Aim, Collect. Czech. Chem. Commun. 37, 3513 (1972)

72/7 R. M. Varuschenko and A. I. Druzhinina, Zh. Fiz. Khim. 46, 1313

(1972)

72/8 G. Jakli and W. A. Van Hook, J. Chem. Thermodyn. 4, 857-864

(1972)

72/9 D. Ambrose, C. H. S. Sprake, and R. Townsend, J. Chem.

Thermodyn. 4, 247-254 (1972).

72/10 A. K. Goncharov and M. Kh. Karapet'yants, Zh. Fiz. Khim. 46, 228-230 (1972)

72/11 W. D. Good, J. Chem. Eng. Data 17, 158-162 (1972).

72/12 W. C. Neely and T. D. Hall, J. Chem. Eng. Data 17, 294-295

(1972). 
72/13 V. B. Sokolov, M. Kh. Karapet'yants, S. P. Kolesnikov, B. L. Perl'mutter, and O. M. Nefedov, Tr. Mosk. Khim.-Tekhnol. Inst. 71, 6-7 (1972); Chem. Abstracts 80, 100456y (1974).

$72 / 14$ V. B. Sokolov, G. P. Bragin, and M. Kh. Karapet'yants, Tr. Mosk. Khim.-Tekhnol. Inst. 71, 8-9 (1972); Chem. Abstracts 80, 100457z (1974).

72/15 G. P. Brajin, M. Kh. Karapet'yants, A. K. Goncharov, and G. M. Kol'yakova, Tr. Mosk. Khim.-Tekhnol. Inst. 71, 10-12 (1972); Chem. Abstracts 80, 100458a (1974).

72/16 V. E. Ditsent, I. I. Skorokhodov, N. A. Terent'eva, and M. N. Zolotareva, Russ. J. Phys. Chem. 46, 317-318 (1972); Zh. Fiz. Khim. 46, 544 (1972); Chem. Abstracts 77, 9812p (1972).

72/17 E. A. Volchkova, D. D. Smol'yaminova, V. G. Genchel, I. L. Lopatkina, and Yu. Kh. Shaulova, Russ. J. Phys. Chem. 46, 1053-1054 (1972).

72/18 R. H. T. Bleyerveld and W. Fieggen, Recl. Trav. Chim. Pays-Bas 91, 477-482 (1972); Chem. Abstracts 76, 145167p (1972).

$72 / 19$ V. E. Ditsent, I. I. Skorokhodov, N. A. Terent'eva, and M. N. Zolotareva, Russ. J. Phys. Chem. 46, 1088 (1972); Zh. Fiz. Khim. 46, 1887-1888 (1972); Chem. Abstracts 77, 106168r (1972); 77, 106166p (1972).

72/20 J. Dykyj, Petrochemia 12, 13-31 (1972); 12, 34-63 (1972). 72/21 R. F. Swindell and J. M. Shreeve, J. Am. Chem. Soc. 94, 57135718 (1972).

72/22 S. G. Metcalf and J. M. Shreeve, Inorg. Chem. 11, 1631-1634 (1972).

72/23 S. R. O'Neill and J. M. Shreeve, Inorg. Chem. 11, 1629-1631 (1972).

72/24 R. F. Swindell, D. P. Babb, T. J. Ouellette, and J. M. Shreeve, Inorg. Chem. 11, 242-245 (1972).

72/25 D. T. Sauer and J. M. Shreeve, Inorg. Chem. 11, 238-242 (1972). 72/26 Ya. A. Strepikheev, O. P. Novikova, and A. L. Chimishkyan, Khim. Prom. (Moscow) 48, 230-231 (1972); Chem. Abstracts 69, 145717t (1972).

72/27 G. Geiseler and J. Sawitowsky, Z. Phys. Chem. (Leipzig) 250, 43-48 (1972).

72/28 E. Morawetz, J. Chem. Thermodyn. 4, 455-460 (1972).

72/29 E. Morawetz, J. Chem. Thermodyn. 4, 139-144 (1972).

$72 / 30$ J. B. Pedley and B. S. Iseard, CATCH Tables, Silicon Compounds (University of Sussex Press, Brighton, 1972).

72/31 G. M. Kol'yakova, I. B. Rabinovich, E. N. Gladyshev, and N. S. Vyazankin, Dokl. Akad. Nauk. SSSR 204, 356-358 (1972).

72/32 A. Finch, P. J. Gardner, and A. F. Webb, J. Chem. Thermodyn. 4, 495-498 (1972).

72/33 R. Sabbah, R. Chastel, and M. Laffitte, Thermochimica Acta 5, 117-127 (1972).

72/34 L. A. Nisel'son, T. D. Sokolova, and R. K. Nikolaev, Vestn. Mosk. Univ. Khim. 13, 432-434 (1972); Chem. Abstracts 78, 8069k (1973). 72/35 M. Nishimura, M. Hakayama, and T. Yano, J. Chem. Eng. Jpn. 5, 223-226 (1972); Chem. Abstracts 78, 48659j (1973).

72/36 G. K. Johnson, P. A. G. O’Hare, and E. H. Appelman, Inorg.

Chem. 11, 800-802 (1972).

$72 / 37$ V. A. Granzhan and E. A. Salganikov, Tr. Nauch.-Issled. Proekt. Inst. Azotn. Prom. Prod. Org. Sin. 85-87 (1972); Chem. Abstracts 79, 118908m (1973).

72/38 K. Nakanishi and O. Toyama, Bull. Chem. Soc. Jpn. 45, 32103211 (1972)

$72 / 39$ D. E. J. Arnold, E. A. V. Ebsworth, H. F. Jessep, and D. W. H. Rankin, J. Chem. Soc., Dalton Trans. 1681-1687 (1972).

72/40 R. A. De Marco and J. M. Shreve, J. Fluorine Chem. 1, 269-276 (1972).

72/41 J. Laynez and I. Wadso, Acta Chem. Scand. 26, 3148-3152 (1972).

72/42 M. Mansson, Acta Chem. Scand. 26, 1707-1708 (1972).

72/43 F. E. Rogers, J. Phys. Chem. 75, 1734-1737 (1971).

1973

73/1 H. K. Hall, Jr., C. D. Smith, and J. H. Bald, J. Am. Chem. Soc. 95, 3197-3201 (1973).

73/2 J. W. Wilson and J. T. F. Fenurck, J. Chem. Thermodyn. 5, 341-345 (1973).
73/3 J. F. Counsell and D. A. Lee, J. Chem. Thermodyn. 5, 583-589 (1973).

73/4 G. R. De Mare, T. Lehman, and M. Termonia, J. Chem. Thermodyn. 5, 829-832 (1973).

73/5 D. H. Rochester and J. R. Symonds, J. Chem. Soc., Faraday Trans. 1, 1267-1273 (1973).

73/6 J. Konicek, Acta Chem. Scand. 27, 1496-1502 (1973).

73/7 D. Ambrose, C. H. S. Sprake, and R. Townsend, J. Chem. Soc.,

Faraday Trans. 1 69, 839-841 (1975).

73/8 H.-J. Rauh, W. Geyer, H. Schmidt, and G. Z. Geiseler, Phys. Chem. (Leipzig) 253, 43-48 (1973).

73/9 F. A. Gothard, D. C. Mentianu, D. G. Breban, and C. I. Cristea, J. Chem. Eng. Data 18, 381-384 (1973).

73/10 E. A. Dietz and D. R. Martin, J. Inorg. Nucl. Chem. 35, 36813684 (1973)

73/11 G. F. Carruth and R. Kobayashi, J. Chem. Eng. Data 18, 115-126 (1973)

73/12 E. F. Meyer and R. D. Hotz, J. Chem. Eng. Data 18, 359-362

(1973).

73/13 V. Svoboda, F. Vesely, R. Holub, and J. Pick, Collect. Czech. Chem. Commun. 38, 3539-3543 (1973).

73/14 J. Linek and I. Wichterle, Collect. Czech. Chem. Commun. 38, 1846-1852 (1973).

73/15 I. N. Fel'dman, V. V. Savko, U. I. Mamai, and M. F. Finkel'shtein, Russ. J. Phys. Chem. 47, 1531 (1973).

73/16 N. N. D'yachkova, E. N. Vigdorovich, and L. A. Ivanyutin, Russ. J. Phys. Chem. 47, 258-259 (1973).

73/17 G. L. Gal'chenko, E. P. Brykina, R. M. Varushchenko, L. S. Vasil'ev, and B. N. Mikhailov, Russ. J. Phys. Chem. 47, 914 (1973). 73/18 J. Kkykj and M. Repas, Petrochemia 13, 179-198 (1973). 73/19 C. J. Schack, D. Pilipovich, and J. F. Hon, Inorg. Chem. 12, 897-900 (1973).

73/20 A. Majid and J. M. Shreeve, J. Org. Chem. 38, 4028-4031 (1973). $73 / 21$ G. H. Sprenger, K. J. Wright, and J. M. Shreeve, Inorg. Chem. 12, 2890-2893 (1973).

73/22 R. A. De Marco and J. M. Shreeve, Inorg. Chem. 12, 1896-1899 (1973).

73/23 K. J. Wright and J. M. Shreeve, Inorg. Chem. 12, 77-80 (1973).

73/24 C. S.-C. Wang and J. M. Shreeve, Inorg. Chem. 12, 81-83 (1973).

$73 / 25$ V. I. Pepekin, Yu. A. Lebedev, and A. Ya. Apin, Dokl. Phys. Chem. 208, 35-37 (1973).

73/26 R. C. Wilhoit and B. J. Zwolinski, J. Phys. Chem. Ref. Data (Suppl. 1) 2, 1-1-1-420 (1973). [Note: The authors report Antoine constants for numerous alcohols that were calculated from vapor pressure data taken from the published literature. For several of the alcohols, the authors had only a very limited number of experimental data points that were pooled from several sources.]

73/27 W. Waradzin and P. Skubla, Chem. Prum. 23, 556-557 (1973); Chem. Abstracts 80, 112780n (1974).

73/28 P. D. Dawson, Jr., I. M. Silberberg, and J. J. McKetta, J. Chem.

Eng. Data 18, 7-15 (1973).

73/29 D. V. S. Jain and O. P. Yadav, Indian J. Chem. 11, 28 -30 (1973).

73/30 H.-D. Beckhaus and C. Rüchardt, Tetrahedron Lett. 1971-1974

(1973).

73/31 G. M. Kol'yakova, I. B. Rabinovich, and E. N. Zorina, Dokl.

Akad. Nauk. SSSR 209, 616-617 (1973).

73/32 V. I. Tel'noi, I. B. Rabinovich, and V. A. Umilin, Dokl. Akad. Nauk. SSSR 209, 127-129 (1973).

73/33 D. S. Barnes and C. T. Mortimer, J. Chem. Thermodyn. 5, 371-377 (1973).

73/34 B. J. Aylett, I. A. Ellis, and C. J. Porritt, J. Chem. Soc., Dalton Trans. 83-87 (1973).

73/35 B. J. Aylett, I. A. Ellis, and J. R. Richmond, J. Chem. Soc., Dalton Trans. 981-987 (1973).

73/36 A. Blair and H. Ihle, J. Inorg. Nucl. Chem. 35, 3795-3803 (1973). 73/37 P. A. Bernstein and D. D. Desmarteau, J. Fluorine Chem. 2, 315-321 (1973).

73/38 T. Abe and J. M. Shreeve, J. Fluorine Chem. 3, 17-26 (1973/ 1974). 


\section{4}

74/1 R. A. Murogova, G. L. Tudorovskaya, V. V. Laufer, V. D. Koxlova, and L. A. Seraphimov, Zh. Prikl. Khim. 47, 2024-2027 (1974).

74/2 S. Sunner and C. A. Wulff, J. Chem. Thermodyn. 6, 287-292 (1974).

74/3 W. D. Good, R. T. Moore, A. G. Osborn, and D. R. Douslin, J. Chem. Thermodyn. 6, 303-310 (1974).

74/4 T. M. Letcher and F. Marsicano, J. Chem. Thermodyn. 6, 509-514 (1974).

74/5 R. M. Varushchenko and L. L. Bulgakova, Tr. Khim. Khim. Teknol. 1, 69-74 (1974).

74/6 D. Ambrose, C. H. S. Sprake, and R. Townsend, J. Chem.

Thermodyn. 6, 693-700 (1974)

74/7 L. F. Myasinkova, V. A. Schmelev, I. L. Vaisman, V. I. Bushinskii, and D. A. Novokhatka, Zh. Prikl. Khim. (Leningrad) 47, 2604-2606 (1974).

74/8 D. Ambrose and C. H. S. Sprake, J. Chem. Thermodyn. 6, 453-456 (1974).

74/9 H. F. Gibbard and J. L. Creek, J. Chem. Eng. Data 19, 308-310 (1974).

74/10 J. Dojcansky and J. Heinrich, Chem. Zvesti 28, 157-159 (1974); Chem. Abstracts 82, 64723p (1975).

74/11 A. G. Osborn and D. R. Douslin, J. Chem. Eng. Data 19, 114-117 (1974).

74/12 P. H. Mogul, M. C. Hochberg, R. Michiel, G. K. Nestel, B. L. Wamsley, and S. D. Coren, J. Chem. Eng. Data 19, 4-6 (1974).

74/13 D. Ambrose and J. H. Ellender, J. Chem. Thermodyn. 6, 909-914 (1974).

74/14 K. Kusano and Y. Saito, Preprints 10th Conference Chemical Thermodyn. Thermal Analysis, Japan 1974, p. 155; as quoted in [85/6]. 74/15 V. N. Kiva, S. I. Nadutkina, L. V. Ivanova, and V. S. Bogdanov, Russ. J. Phys. Chem. 48, 297 (1974).

74/16 G. O. Shmyreva, R. M. Golosova, G. B. Sakharovskaya, A. F. Popov, N. N. Korneev, and A. A. Smolyaninova, Russ. J. Phys. Chem. 48, 447 (1974).

74/17 V. E. Ditsent, I. I. Skorokhodov, N. A. Terent'eva, and M. N. Zolotareva, Russ. J. Phys. Chem. 48, 447 (1974); Zh. Fiz. Khim. 48, 2152-2153 (1974); Chem. Abstracts 81, 158939v (1974).

74/18 G. P. Bragin and M. Kh. Karapet'yants, Russ. J. Phys. Chem. 48, 612 (1974)

74/19 I. L. Gaidym, A. K. Vaev, V. G. Syrkin, A. A. Uel'skii, and A. E. Medvedeva, Russ. J. Phys. Chem. 48, 1111 (1974); Chem. Abstracts 69, 160019p (1974)

74/20 R. M. Varushchenko, A. I. Druzhimina, O. Ya. Kovner, E. M.

Mil'vitskaya, A. A. Bobyleva, N. A. Belikova, and G. L. Gal'chenko, Russ. J. Phys. Chem. 48, 1121 (1974).

74/21 V. E. Ditsent, I. I. Skorokhodov, N. A. Terent'eva, M. N. Zolotareva, and M. B. Lotarev, Russ. J. Phys. Chem. 48, 1279 (1974); Zh. Fiz. Khim. 48, 2152 (1974); Chem. Abstracts 81, 158938u (1974). $74 / 22$ N. A. Bessareb and Yu. M. Martynov, Russ. J. Phys. Chem. 48, 1547-1548 (1974).

74/23 G. O. Shmyreva, V. G. Shlyakova, R. M. Golosova, G. G. Filippov, E. A. Volnina, E. N. Burdasov, and L. E. Gusel'nikov, Russ. J. Phys. Chem. 48, 1699-1700 (1974).

74/24 K. E. Peterman and J. M. Shreeve, Inorg. Chem. 13, 2705-2709 (1974).

74/25 A. Majid and J. M. Shreeve, Inorg. Chem. 13, 2710-2714 (1974).

74/26 L. V. Mozhginskay and L. E. Kolysko, Zh. Fiz. Khim. 48,

1506-1507 (1974).

74/27 A. G. Gilbert and K. G. P. Sulzmann, J. Electrochem. Soc. 121, 832-834 (1974)

74/28 R. Sabbah, R. Chastel, and M. Laffitte, Thermochimica Acta 10, 353-358 (1974).

74/29 L. Malaspina, G. Bardi, and R. Gigli, J. Chem. Thermodyn. 6, 1053-1064 (1974)

74/30 P. Beak, D. S. Mueller, and J. Lee, J. Am. Chem. Soc. 96, 3867-3874 (1974).
74/31 V. E. Ditsent, I. I. Skorokhodov, N. A. Terent'eva, M. N.

Zolotareva, B. N. Parfenov, and O. I. Minosyan, Zh. Fiz. Khim. 48, 2151 (1974); Chem. Abstracts 81, 158937t (1974).

74/32 P. S. Engel, J. L. Wood, J. A. Sweet, and J. L. Margrave, J. Am. Chem. Soc. 96, 2381-2387 (1974).

74/33 M. P. Kozina, L. V. Bychikhina, G. L. Gal'chenko, A. N. Kalinichenko, A. A. Bobyleva, N. A. Belikova, and A. F. Plate, Zh. Fiz. Khim. 48, 2075-2077 (1974); Chem. Abstracts 81, 159978u (1974).

74/34 V. E. Ditsent, I. I. Skorokhodov, N. A. Terent'eva, M. N.

Zolotareva, N. E. Rodzecich, and O. I. Minosyan, Zh. Fiz. Khim. 48,

2153 (1974); Chem. Abstracts 81, 158940p (1974).

74/35 C.-F. Shieh and N. W. Gregory, J. Chem. Eng. Data 19, 11-14 (1974)

74/36 Y. Sassa, R. Konishi, and T. Katayama, J. Chem. Eng. Data 19, 44-48 (1974).

74/37 M. Mansson, Acta Chem. Scand. 28B, 905-908 (1974).

74/38 G. H. Spenger and J. M. Shreeve, J. Fluorine Chem. 4, 201-205

(1974)

1975

75/1 S. Cabani, G. Conti, V. Mollica, and L. Lepori, J. Chem. Soc.,

Faraday Trans. 1 71, 1943-1945 (1975).

75/2 R. J. Irving and M. A. V. Riebeiro da Silva, J. Chem. Soc., Dalton Trans. 798-800 (1975).

75/3 J. O. Fenwick, D. Harrop, and A. J. Head, J. Chem. Thermodyn. 7, 943-954 (1975).

75/4 J. F. Messerly, H. L. Finke, A. G. Osborn, and D. R. Douslin, J. Chem. Thermodyn. 7, 1029-1046 (1975).

75/5 D. Ambrose, J. E. Connett, J. H. S. Green, J. L. Hales, A. J. Head, and J. F. Martin, J. Chem. Thermodyn. 7, 1143-1157 (1975).

75/6 G. Stridh and S. Sunner, J. Chem. Thermodyn. 7, 161-168 (1975).

75/7 W. D. Good, J. F. Messerly, A. G. Osborn, and D. R. Douslin, J.

Chem. Thermodyn. 7, 285-291 (1975).

75/8 D. Ambrose, J. H. Ellender, E. B. Lees, C. H. S. Sprake, and R.

Townsend, J. Chem. Thermodyn. 7, 453-472 (1975).

75/9 S. Bergstrom and G. Olofsson, J. Solution Chem. 4, 535-555

(1975).

75/10 H. Iwasaki and K. Date, Koatsu Gasu 12, 374-380 (1975).

75/11 A. G. Osborn and D. R. Douslin, J. Chem. Eng. Data 20, 229-231

(1975)

75/12 K. Kusano and Y. Saito, Preprints 33rd Annical Meeting Chemical Society Japan, 1975, pp. 1, 123; as quoted in [85/6].

75/13 H. K. Frensdorff and R. K. Adams, J. Chem. Eng. Data 20, 13-15 (1975).

75/14 D. J. Subach and B. J. Zwolinski, J. Chem. Eng. Data 20, 232-234 (1975)

75/15 V. V. Pisarev, A. M. Rozhnov, R. M. Varushchenko, and A. G. Sarkisov, Russ. J. Phys. Chem. 49, 1450-1451 (1975).

75/16 V. V. Pisarev, H. M. Rozhnov, R. M. Varushchenko, and A. G.

Sarkisov, Russ. J. Phys. Chem. 49, 1605 (1975).

75/17 J. Arro, L. Melder, and H. Tamvelius, Trudy Tallinskogo Instit.

Toimetict, 390, 37-43 (1975).

75/18 V. I. Pepekin, Yu. N. Matyushin, A. D. Nikolaeva, A. P. Kirsanov, L. V. Platonova, and Yu. A. Lebedev, Izv. Akad. Nauk SSSR, Ser. Khim. 1870-1871 (1975).

75/19 K. E. Peterman and J. M. Shreeve, Inorg. Chem. 14, 1223-1228 (1975).

75/20 K. E. Peterman and J. M. Shreeve, Inorg. Chem. 14, 1106-1110 (1975)

75/21 R. L. Kirchmeier, U. I. Lasouris, and J. M. Shreeve, Inorg. Chem. 14, 592-596 (1975)

75/22 N. S. Walker and D. D. DesMarteau, J. Fluorine Chem. 5,

135-139 (1975)

75/23 M. M. Brazhnikov, D. N. Andreevskii, A. I. Sachek, and A. D.

Peshchenko, Zh. Prikl. Khim. (Leningrad) 48, 2181-2185 (1975)

75/24 H. Kundel, U. Lille, and N. Kaidas, Tr. Tallin. Politekh. Inst. 390,

$107-115$ (1975)

75/25 Z. A. Guseinov, A. A. Kuliev, K. A. Karasharli, and O. I.

Dzhafarov, Azerb. Khim. Zh. $72-74$ (1975); Chem. Abstracts 84, 65359c (1976) 
75/26 A. Hoepfner, N. Parekh, Ch. Hoerner, and A. Abdel-Hamid, Ber. Bunsenges Phys. Chem. 79, 216-222 (1975).

75/27 D. Ambrose, J. W. Ellender, C. H. S. Sprake, and R. I. Townsend, J. Chem. Soc., Faraday Trans. 71, 35-41 (1975).

75/28 Kh. I. Geidarov, O. I. Dzhafarov, and K. A. Karasharli, Russ. J. Phys. Chem. 49, 197-198 (1975).

$75 / 29$ V. B. Sokolov, M. Kh. Karapet'yants, T. N. Sergeeva, S. P. Kolesnikov, and B. L. Perl'mutter, Izv. Vyssh. Uchebn. Zaved., Khim. Khim. Teknol. 18, 1319-1321 (1975); Chem. Abstracts 84, 22433e (1976).

75/30 V. B. Sokolov, M. Kh. Karapet'yants, T. N. Sergeeva, S. P. Kolesnikov, and B. L. Perl'mutter, Izv. Vyssh. Uchebn. Zaved., Khim. Khim. Teknol. 18, 1234-1237 (1975); Chem. Abstracts 84, 9167b (1976).

75/31 Z. A. Guseinov, K. A. Karasharli, O. I. Dzharfarov, G. G. Nurullaev, N. S. Nametkin, L. E. Gusel'nikov, F. A. Volinina, E. N. Burdasov, and V. N. Vdovin, Dokl. Akad. Nauk (SSSR) 222, 1369-1372 (1975).

75/32 Ch. Hoerner, A. Hoepfner, and B. Schmeiser, Ber. Bunsenges Phys. Chem. 79, 222-225 (1975).

75/33 F. Rivenq, Bull. Soc. Chim. France (Pt. 1) 2433-2434 (1975). 75/34 G. P. Bragin and M. Kh. Karapet'yants, Tr. Khim. Khim. Tekhnol. 78-79 (1975); Chem. Abstracts 85, 83381d (1976).

75/35 K. W. Chun, J. C. Drummond, W. H. Smith, and R. R. Davison, J. Chem. Eng. Data 20, 58-61 (1975).

75/36 M. E. Anthoney, A. S. Carson, and P. G. Laye, 4th Conference International Thermodynamics Chim., [C.R.], Vol. 1, pp. 99-103; Chem. Abstracts 84, 163961n (1976).

75/37 J. F. Masi and R. B. Scott, J. Res. National Bureau Stand., Sect. A 79A, 619-628 (1975).

75/38 D. E. J. Arnold and D. W. H. Rankin, J. Chem. Soc., Dalton Trans. 889-894 (1975).

75/39 R. Vilcu, S. Perisanu, and I. Ciocazanu, 4th Conference International Thermodynamics, Chim. [C.R.] Vol. 1, pp. 105-112. 75/40 M. Gambaruto, J. E. Sicre, and H. J. Schumacher, J. Fluorine Chem. 5, 175-179 (1975).

75/41 R. A. De Marco, T. A. Kovacina, and W. B. Fox, J. Fluorine Chem. 6, 93-104 (1975).

75/42 K. E. Peterman and J. M. Shreeve, J. Fluorine Chem. 6, 83-92 (1975).

75/43 R. A. De Marco, T. A. Kovacina, and W. B. Fox, J. Fluorine Chem. 5, 221-230 (1975).

1976

76/1 G. Stridh, J. Chem. Thermodyn. 8, 193-194 (1976).

76/2 D. Ambrose, J. H. Ellender, C. H. S. Sprake, and R. Townsend, J. Chem. Thermodyn. 8, 165-178 (1976).

76/3 P. S. Engel, R. A. Melaugh, M. Mansson, J. W. Timberlake, A. W. Garner, and F. D. Rossini, J. Chem. Thermodyn. 8, 607-621 (1976).

76/4 R. A. Melaugh, M. Mansson, and F. D. Rossini, J. Chem.

Thermodyn. 8, 623-626 (1976).

76/5 G. Stridh, J. Chem. Thermodyn. 8, 895-899 (1976).

76/6 G. Stridh, J. Chem. Thermodyn. 8, 901-906 (1976).

76/7 V. P. Kolesov, G. M. Slavutskaya, and L. N. Dityat'eva, J. Chem.

Thermodyn. 8, 907-914 (1976).

76/8 J. E. Connett, J. F. Counsell, and D. A. Lee, J. Chem. Thermodyn. 8, 1199-1203 (1976).

76/9 M. E. Anthoney, A. S. Carson, and P. G. Laye, J. Chem. Soc., Perkin Trans. 2 1032-1036 (1976).

76/10 E. F. Meyer and C. A. Hotz, J. Chem. Eng. Data 21, 274-279 (1976).

76/11 S. J. Ascroft, J. Chem. Eng. Data 21, 397-398 (1976).

76/12 K. V. Rao and C. Chiranjivi, J. Chem. Eng. Data 21, 393-394 (1976).

76/13 H. C. Hon, R. P. Singh, and A. P. Kudchadker, J. Chem. Eng. Data 21, 430-431 (1976)

76/14 J. Cihlar, V. Hynek, V. Svoboda, and R. Holub, Collect. Czech. Chem. Commun. 41, 1-6 (1976).

76/15 M. M. Ammar, L. L. Bulgakova, and R. M. Varushchenko, Russ. J. Phys. Chem. 50, 1466-1467 (1976).
76/16 G. K. Borisov and S. G. Chugunova, Russ. J. Phys. Chem. 50, 1791 (1976)

76/17 G. O. Shmyreva, V. G. Shlyakova, R. M. Golosova, A. B.

Petrunin, D. B. Bekker, and A. F. Zhigach, Russ. J. Phys. Chem. 50, 478 (1976).

76/18 C. A. Burton and J. M. Shreeve, J. Am. Chem. Soc. 98, 65456547 (1976).

76/19 K. Steele, B. E. Poling, and D. B. Manley, J. Chem. Eng. Data 21, 399-403 (1976).

76/20 M. M. Brazhnikov, A. D. Peshchenko, and O. V. Ral'ko, Zh. Prikl. Khim. (Leningrad) 49, 1041-1044 (1976).

76/21 V. P. Kuznetsova, E. A. Miroshnichenko, A. N. Zelenetskii, G. V. Rakova, Yu. A. Lebedev, and N. S. Enikolopyan, Dokl. Phys. Chem. 226, 147-150 (1976)

76/22 E. G. Kiparisova, L. Ya. Tsvetkova, and B. V. Lebedev, Russ. J. Phys. Chem. 50, 638 (1976).

76/23 D. L. S. Brown, J. A. Connor, M. L. Leung, M. I. Paz-Andrade, and H. A. Skinner, J. Organomet. Chem. 110, 79-89 (1976).

76/24 V. E. Ditsent, I. I. Skorokhodov, N. A. Terent'eva, M. N.

Zolotareva, Z. V. Belyakova, and Z. V. Belikova, Zh. Fiz. Khim. 50, 1905-1906 (1976); Chem. Abstracts 85, 166806x (1976).

76/25 Z. A. Guseimov, A. A. Kuliev, K. A. Karasharli, and O. I. Dzhafarov, Azerb. Khim. Zh. 72-74 (1975); Chem. Abstracts 84, 65359c (1976)

76/26 D. Ambrose and C. H. S. Sprake, J. Chem. Thermodyn. 8, 601-602 (1976).

76/27 W. S. Hamilton, P. Thompson, and S. Pustejovsky, J. Chem. Eng. Data 21, 428-429 (1976).

76/28 E. R. Falardeau and D. D. Desmarteau, J. Fluorine Chem. 7, 185-195 (1976).

76/29 I. Stahl, R. Mews, and O. Glemser, J. Fluorine Chem. 7, 55-64 (1976).

76/30 E. R. Falardeau and D. D. Desmarteau, J. Fluorine Chem. 7, 409-414 (1976).

76/31 M. J. Hopkinson and D. D. Desmarteau, J. Fluorine Chem. 7, 501-510 (1976)

1977

77/1 M. Mansson, P. Sellers, G. Stridh, and S. Sunner, J. Chem.

Thermodyn. 9, 91-97 (1976).

77/2 P. Sellers, J. Chem. Thermodyn. 9, 139-142 (1977).

$77 / 3$ A. S. Carson, P. G. Laye, and M. Yurkeli, J. Chem. Thermodyn. 9 , 827-829 (1977).

77/4 W. V. Steele and I. Watt, J. Chem. Thermodyn. 9, 843-849 (1977). $77 / 5$ G. Stridh, S. Sunner, and Ch. Svenson, J. Chem. Thermodyn. 9, 1005-1010 (1977).

77/6 P. A. Pella, J. Chem. Thermodyn. 9, 301-305 (1977).

77/7 L. A. Peacock and R. Fuchs, J. Am. Chem. Soc. 99, 5524-5525 (1977).

77/8 V. Svoboda, V. Majer, F. Vesely, and J. Pick, Collect. Czech. Chem. Commun. 42, 1755-1760 (1977).

77/9 N. I. Boldenkov and Yu. Ya Maksimov, Zh. Fiz. Khim. 51, 1502 1504 (1977)

77/10 E. F. Meyer and T. H. Gens, J. Chem. Eng. Data 22, 30-31 (1977).

77/11 H. K. Cammenga, F. W. Schulze, and W. Theuerl, J. Chem. Eng. Data 22, 131-134 (1977).

77/12 V. Svoboda, F. Vesely, R. Holub, and J. Pick, Collect. Czech. Chem. Commun. 42, 943-951 (1977).

77/13 R. M. Varushchenko, M. M. Ammar, and L. L. Bulgakova, Russ. J. Phys. Chem. 51, 167-168 (1977).

77/14 V. V. Pisarev, A. M. Rozhnov, and A. G. Sarkisov, Russ. J. Phys. Chem. 51, 323-324 (1977).

77/15 T. Kitazume and J. M. Shreeve, J. Am. Chem. Soc. 99, 3690-3695 (1977)

77/16 T. Kitazume and J. M. Shreeve, J. Am. Chem. Soc. 99, 4194-4196 (1977).

77/17 C. A. Burton and J. M. Shreeve, Inorg. Chem. 16, 1039-1042 (1977). 
77/18 C. A. Burton and J. M. Shreeve, Inorg. Chem. 16, 1408-1411 (1977).

77/19 T. Kitazume and J. M. Shreeve, Inorg. Chem. 16, 1818-1819

(1977).

77/20 R. M. Varushchenko, M. M. Ammar, and L. L. Bulgakova, Zh. Fiz. Khim. 51, 278-279 (1977).

77/21 E. Z. Zhuravlev, T. L. Melent'eva, P. V. Mulyanov, A. I. Kormushechkina, T. M. Bogdanova, and I. I. Konstantinov, Zh. Prikl Khim. (Leningrad) 50, 2032-2035 (1977).

77/22 H. L. Finke, J. F. Messerly, S. H. Lee, A. G. Osborn, and D. R. Douslin, J. Chem. Thermodyn. 9, 937-956 (1977).

77/23 J. B. Pedley and J. Rylance, N. P. L. Computer Analyzed Thermochemical Data: Organic and Organometallic Compounds (School of Molecular Sciences, University of Sussex Press, Brighton, UK, 1977). 77/24 B. C. Kim and D. H. Kim, Hwahak Kwa Hwahak Kongop 20, 232-234 (1977); Chem. Abstracts 88, 95565s (1978).

77/25 N. D. Lebedeva, V. L. Ryadnenko, N. N. Kiseleva, and L. F. Nazarova, Vses. Konf. Kalorim. [Rasshir. Tezisy Dokl.] 7th 1, 91-95 (1977); Chem. Abstracts 92, 75617q (1980).

77/26 M. V. Lyubarskii and R. I. Smolyanets, Vses. Konf. Kalorim. [Rasshir. Tezisy Dok1.] 7th 1, 119-121 (1977); Chem. Abstracts 92, 75619s (1980).

77/27 V. A. Aleshina, M. P. Kozina, and G. L. Gal'chenko, Vses. Konf. Kalorim. [Rasshir. Tezisy Dokl.] 7th 1, 85-91 (1977); Chem. Abstracts 92, 94582r (1980).

77/28 L. N. Dityat'eva and V. P. Kolesov, Vses. Konf. Kalorim. [Rasshir. Tezisy Dokl.] 7th 1, 95-97 (1977); Chem. Abstracts 92, 58009a (1980). 77/29 T. F. Vasil'eva and V. I. Kotov, Vses. Konf. Kalorim. [Rasshir. Tezisy Dokl.] 7th 1, 102-106 (1977); Chem. Abstracts 92, 58010u (1980).

77/30 J. J. Rao and D. S. Viuswanath, J. Chem. Eng. Data 22, 36-38 (1977).

77/31 T. R. Das, C. O. Reed, Jr., and P. T. Eubank, J. Chem. Eng. Data 22, 3-9 (1977).

77/32 T. R. Das, C. O. Reed, Jr., and P. T. Eubank, J. Chem. Eng. Data 22, 9-15 (1977).

77/33 T. R. Das, C. O. Reed, Jr., and P. T. Eubank, J. Chem. Eng. Data 22, 16-21 (1977).

77/34 N. Novakova and J. Novak, J. Chromatogr. 135, 13-24 (1977).

1978

78/1 W. V. Steele, J. Chem. Thermodyn. 10, 919-927 (1978).

78/2 R. B. Cundall, T. F. Palmer, and C. E. C. Wood, J. Chem. Soc.,

Faraday Trans. 1 74, 1339-1345 (1978).

78/3 P. S. Engel, R. L. Mongomery, M. Mansson, R. A. Leckonby, H. L. Foyt, and F. D. Rossini, J. Chem. Thermodyn. 10, 205-211 (1978).

$78 / 4$ D. G. McCormick and W. S. Hamilton, J. Chem. Thermodyn. 10, 275-278 (1978).

78/5 B. V. Lebedev, L. Ya. Tsvetkova, and I. B. Rabinovich, J. Chem. Thermodyn. 10, 809-815 (1978).

78/6 D. Indritz, J. Stone, and F. Williams, J. Chem. Eng. Data 23, 6-7 (1978).

78/7 R. L. Montgomery, F. D. Rossini, and M. Mansson, J. Chem. Eng. Data 23, 125-129 (1978).

78/8 A. H. N. Mousa, J. Chem. Eng. Data 23, 133-134 (1978).

78/9 W. S. Hamilton, S. Benton, J. French, D. McCormick, S.

Pustejovsky, and P. Thomspon, J. Chem. Eng. Data 23, 201-203 (1978) 78/10 I. K. Igumenov, Yu. V. Chumanchenko, and S. V. Zemskov, Russ. J. Phys. Chem. 52, 1531-1532 (1978).

78/11 Yu. V. Chumachenko, I. K. Igumenov, and S. V. Zemskov, Russ. J. Phys. Chem. 52, 1393-1394 (1978).

78/12 G. O. Shmyreva, V. G. Shlyakova, and R. M. Golosova, Russ. J. Phys. Chem. 52, 135-136 (1978).

78/13 T. D. Grabik, S. G. Konstantinov, G. P. Dudchik, and O. G.

Polyachenok, Russ. J. Phys. Chem. 52, 894-895 (1978).

78/14 T. Kitazume and J. M. Shreeve, Inorg. Chem. 17, 2173-2176 (1978).

78/15 R. P. Corbally, M. J. Perkins, A. S. Carson, P. G. Laye, and W. V. Steele, J. Chem. Soc., Chem. Commun. $778-779$ (1978).

78/16 R. Fuch and L. A. Peacock, Can J. Chem. 56, 2493-2498 (1978).
78/17 B. V. Lebedev, L. Ya. Tsvetkova, I. B. Rabinovich, E. Sh. Finkel'shtein, and B. S. Strel'chick, Termodin. Org. Soedin. 7, 3-7 (1978); Chem. Abstracts 92, 83507u (1980).

78/18 M. A. V. Ribeiro da Silva and R. G. Irving, Rev. Port. Quim. 20, 36-46 (1978); Chem. Abstracts 90, 211054s (1979).

78/19 V. P. Kolesov and L. N. Dityat'eva, Termodin. Org. Soedin. 7, 44-47 (1978); Chem. Abstracts 92, 75657c (1980).

78/20 S. D. Cave, A. Chainese, and A. Prantera, J. Chem. Eng. Data 23, 279-281 (1978).

78/21 S. Sundaram and D. S. Viswanath, J. Chem. Eng. Data 23, 63-64 (1978).

78/22 I. Stahl, R. Mews, and O. Glemser, J. Fluorine Chem. 11, 455-465 (1978).

78/23 R. A. De Marco and W. B. Fox, J. Fluorine Chem. 12, 137-151

(1978).

1979

79/1 S. Sunner, Ch. Svensson, and A. S. Zelepuga, J. Chem. Thermodyn. 11, 491-495 (1979).

79/2 S. Sunner and Ch. Svensson, J. Chem. Soc., Faraday Trans. 1 75, 2359-2365 (1979).

79/3 T. M. Letcher, S. W. Orchard, and M. Albers, J. Chem. Thermodyn. 11, 173-175 (1979).

79/4 C. G. De Kruif and H. A. Oonk, J. Chem. Thermodyn. 11, 287-290 (1979).

79/5 S. H. Lee-Bechtold, I. A. Hossenlop, D. W. Scott, A. G. Osborn, and W. D. J. Good, Chem. Thermodyn. 11, 469-482 (1979).

79/6 Ch. Sevensson, J. Chem. Thermodyn. 11, 593-596 (1979).

79/7 P. P. S. Saluja, L. A. Peacock, and R. Fuchs, J. Am. Chem. Soc.

101, 1958-1962 (1979).

79/8 L. Petros, V. Majer, J. Koubek, V. Svoboda, and J. Pick, Collect.

Czech. Chem. Commun. 44, 3533-3540 (1979).

$79 / 9$ V. Majer, V. Svoboda, J. Koubek, and J. Pick, Collect. Czech.

Chem. Commun. 44, 3521-3528 (1979).

79/10 M. Balish and V. Fried, J. Chem. Eng. Data 24, 91-92 (1979).

79/11 A. B. Macknick and J. M. Prausnitz, J. Chem. Eng. Data 24,

175-178 (1979).

79/12 A. P. Kudchadker, S. A. Kudchadker, R. P. Shukla, and P. R.

Patnaik, J. Phys. Chem. Ref. Data 8, 499-513 (1979).

79/13 V. Majer, V. Svoboda, S. Hala, and J. Pick, Collect. Czech. Chem.

Commun. 44, 637-651 (1979).

79/14 G. Berthon, V. Angot, V. Beden, and O. Enea, J. Chem.

Thermodyn. 11, 539-546 (1979).

79/15 V. S. Markovnik, A. I. Sachek, A. D. Peshchenko, O. V. Shvaro, D.

N. Andreevskii, and N. M. Olizarevich, Termodin. Org. Soedin. 107-110

(1979).

79/16 L. H. Thomas, R. Meatyard, H. Smith, and G. H. Davies, J. Chem. Eng. Data 24, 159-161 (1979).

79/17 R. Fuchs and L. A. Peacock, Can. J. Chem. 57, 2302-2304 (1979). 79/18 S. I. Sukhova, O. N. Vlasov, and L. V. Li, Nov. Khim. Sredstva Azsch Rast. 51-52 (1979); Chem. Abstracts 92, 83359x (1980).

79/19 W. F. Spencer, T. D. Shoup, M. M. Cliath, W. J. Farmer, and R.

Haque, J. Agric. Food Chem. 27, 273-278 (1979).

79/20 I. K. Igumenov, Yu. V. Chumachenko, and S. V. Zemskov, Khim.

Termodin. Termokhim. 65-66 (1979); Chem. Abstracts 91, 217803c

(1979).

79/21 V. I. Bludilina, A. K. Baev, V. K. Matveev, I. L. Gaidym, and E. I. Shcherbina, Zh. Fiz. Khim. 53, 1052-1053 (1979); Chem. Abstracts 91, 9585f (1979).

79/22 D. Das, S. R. Dhawadkar, and M. S. Chandraekharaiah,

Thermochimica Acta 30, 371-376 (1979).

79/23 P. J. Maher and B. D. Smith, J. Chem. Eng. Data 24, 16-22

(1979).

79/24 M. Zander and W. Thomas, J. Chem. Eng. Data 24, 1-2 (1979).

79/25 R. M. Golosova, G. O. Shmyreva, V. G. Shlyakova, A. B.

Petrunin, D. B. Bekker, and A. F. Zhigach, Zh. Fiz. Khim. 53, 766-767

(1979).

79/26 P. Shul'tse, R. M. Varushchenko, G. L. Gal'chenko, T. V. Klimova, and V. I. Stanko, J. Gen. Chem. USSR 50, 1482-1488 (1979); Zh. Obshch. Khim. 50, 1818-1825 (1979). 
79/27 J. Carre, P. Claudy, J. M. Letoffe, M. Kollmannsberger, and J. Bousquet, J. Fluorine Chem. 14, 139-152 (1979). 79/28 A. Sekiya and D. D. Desmarteau, J. Fluorine Chem. 14, 289-297 (1979).

1980

80/1 V. Majer, L. Svab, and V. Svoboda, J. Chem. Thermodyn. 12, 843-847 (1980).

80/2 R. H. Davies, A. Finch, and P. G. Gardner, J. Chem. Thermodyn. 12, 291-296 (1980).

$80 / 3$ V. Majer, Z. Wanger, V. Svoboda, and V. Cadek, J. Chem.

Thermodyn. 12, 387-391 (1980).

80/4 M. H. Abraham and R. J. Irving, J. Chem. Thermodyn. 12, 539-544 (1980).

80/5 R. Fuchs and L. A. Peacock, Can. J. Chem. 58, 2796-2799 (1980). 80/6 C. Van de Rostyne and J. M. Prausnitz, J. Chem. Eng. Data 25, 1-3 (1980).

80/7 M. Urbani, R. Gigli, and V. Picente, J. Chem. Eng. Data 25, 97-100 (1980).

80/8 E. F. Meyer, M. J. Awe, and R. E. Wagner, J. Chem. Eng. Data 25, 371-374 (1980).

80/9 V. Mayer, V. Svoboda, A. Posta, and J. Pick, Collect. Czech. Chem. Commun. 45, 3063-3069 (1980).

80/10 T. Abe and J. M. Shreeve, Inorg. Chem. 19, 3063-3067 (1980).

80/11 A. G. Osborn and D. W. Scott, J. Chem. Thermodyn. 12, 429-438 (1980).

80/12 N. K. Smith, R. C. Stewart, Jr., A. G. Osborn, and D. W. Scott, J. Chem. Thermodyn. 12, 919-926 (1980).

80/13 V. Svoboda, V. Uchtyilova, V. Majer, and J. Pick, Collect. Czech. Chem. Commun. 45, 3233-3240 (1980).

$80 / 14$ J. A. Jonsson, L. Mathiasson, and A.-M. Olsson, Acta Chem. Scand. 34A, 147-150 (1980).

80/15 L. H. Thomas, H. Smith, and G. H. Davis, J. Chem. Tech. Biotechnol. 30, 476-480 (1980).

80/16 H.-D. Beckhaus, G. Kratt, K. Lay, J. Greiselmann, C. Rüchardt, B. Kitschke, and H. J. Lindner, Chem. Ber. 113, 3441-3455 (1980).

80/17 P. Nasir, S. C. Hwang, and R. Kobayashi, J. Chem. Eng. Data 25 298-301 (1980).

80/18 R. Francesconi, C. Castellari, A. Arcelli, and F. Comelli, Can. J. Chem. Eng. 58, 113-115 (1980).

80/19 J. A. Roth and M. Orchin, J. Organometal. Chem. 187, 103-105 (1980).

80/20 N. D. Lebedeva, L. F. Nazarov, and Yu. A. Katin, Zh. Prikl. Khim. (Leningrad) 53, 1394-1395 (1980).

80/21 A. Aihara, Denki Tsushin. Daigaku Gakuho 31, 65-68 (1980); Chem. Abstracts 94, 174045z (1981).

80/22 W. Gombler and H.-U. Weiler, J. Fluorine Chem. 15, 279-288

(1980).

80/23 C. R. Patrick and F. Tomes, J. Fluorine Chem. 15, 267-278

(1980).

80/24 V. S. Pityugin, T. L. Kislitsyna, R. F. Shakirov, L. N.

Sharif'yanova, and E. N. Maslennikov, Khim. Prom-st., Ser.: Khlornaya Prom-st. 11-14 (1980).

$80 / 25$ L. N. Sharif'yanova, F. M. Sadykova, and S. G. Akhmerova, Khim. Prom-st., Ser.: Khlornaya Prom-st. 16-18 (1980).

\section{1}

81/1 S. A. Wieczorek and P. Kobayashi, J. Chem. Eng. Data 26, 8-11, (1981); 26, 11-13 (1981).

81/2 D. R. Edwards and J. M. Prausnitz, J. Chem. Eng. Data 26, 121-124 (1981).

81/3 K. G. De Kruif, J. M. Herman, and P. J. Van den Berg, J. Chem. Eng. Data 26, 359-362 (1981).

$81 / 4$ V. Tekac, V. Majer, V. Svoboda, and V. Hynek, J. Chem.

Thermodyn. 13, 659-662 (1981).

$81 / 5$ T. S. Papina, P. A. Erastov, and V. P. Kolevsov, J. Chem.

Thermodyn. 13, 683-689 (1981).

81/6 C. G. De Kruif, T. Kuipers, J. C. van Miltenburg, R. C. F. Schaake, and G. Stevens, J. Chem. Thermodyn. 13, 1081-1086 (1981).

81/7 K. Bystrom, J. Chem. Thermodyn. 13, 139-145 (1981).
81/8 I. A. Hossenlopp and D. W. Scott, J. Chem. Thermodyn. 13, 405-414 (1981).

81/9 I. A. Hossenlopp and D. W. Scott, J. Chem. Thermodyn. 13, 415-421 (1981).

$81 / 10$ I. A. Hossenlopp and D. W. Scott, J. Chem. Thermodyn. 13, 423-428 (1981).

81/11 D. Ambrose, J. H. Ellender, H. A. Gundry, D. A. Lee, and R. Townsend, J. Chem. Thermodyn. 13, 795-802 (1981). $81 / 12$ M. Shimizu, Y. Saito, and K. Kusano, Preprints 17th Conference on Chemical Thermodynic Thermal Analysis, Japan 1981, p. 50; as quoted in [85/6].

$81 / 13$ V. Majer, V. Svoboda, A. Posta, and J. Pick, Collect. Czech. Chem. Commun. 46, 817-822 (1981).

$81 / 14$ V. Svoboda, V. Charvatova, V. Majer, and J. Pick, Collect. Czech. Chem. Commun. 46, 2983-2988 (1981).

81/15 T. Abe and J. M. Shreeve, Inorg. Chem. 20, 2894-2899 (1981). 81/16 T. Abe and J. M. Shreeve, Inorg. Chem. 20, 2432-2434 (1981). 81/17 D. Ambrose and D. J. Hall, J. Chem. Thermodyn. 13, 61-66 (1981)

81/18 G. N. D'yakova, G. L. Korichev, A. D. Korkhova, T. F. Vasil'eva, and I. A. Vasil'ev, Zh. Prikl. Khim. (Leningrad) 54, 1644-1646 (1981). 81/19 J. S. Chickos, A. S. Hyman, L. H. Ladon, and J. F. Liebman, J. Org. Chem. 46, 4294-4296 (1981).

$81 / 20$ S. N. Guseinova, V. V. Kas'yanov, and Yu. V. Politanskii, Khim. Prom-st. Ser., Khlornaya Prom-st. 28-30 (1981); Chem. Abstracts 95, 225804b (1981).

81/21 G. M. Wilson, R. H. Johnston, S.-C. Hwang, and C. Tsonopoulos, Ind. Eng. Chem., Process Des. Dev. 20, 94-104 (1981).

81/22 D. Ferro, V. Piacente, and M. Pelino, Rev. Roum. Chim. 26, 9-15 (1981)

81/23 R. M. Varushchenko, L. L. Bulgakova, P. S. Minzabekyants, and K. N. Makarov, Russ. J. Phys. Chem. 55, 1480-1482 (1981).

$81 / 24$ V. E. Ditsent, I. I. Skorokhodov, M. N. Zolotareva, V. I. Savuskina, and B. N. Tabenko, Zh. Prikl. Khim. (Leningrad) 54, 1617-1619 (1981). 1982

82/1 B. T. Grayson and L. A. Fosbraey, Pestic. Sci. 13, 269-278 (1982). 82/2 F. L. Rook, J. Chem. Eng. Data 27, 72-73 (1982).

$82 / 3$ C. Castellari, R. Francesconi, and F. Comelli, J. Chem. Eng. Data 27, 156-158 (1982)

82/4 G. C. De Kruif, R. C. F. Schaake, J. C. Van Meltenburg, K. Van der Klauw, and J. G. Blok, J. Chem. Thermodyn. 14, 791-798 (1982).

$82 / 5$ R. Fuchs, J. H. Hallman, and M. O. Perlman, Can. J. Chem. 60, 1832-1835 (1982).

$82 / 6$ R. Fuchs, L. A. Peacock, and W. K. Stephenson, Can. J. Chem. 60, 1953-1958 (1982).

82/7 B. Gutsche and H. Knapp, Fluid Phase Equilib. 8, 285-300 (1982). $82 / 8$ G. S. Hong, R. Wakslak, H. Finston, and V. Fried, J. Chem. Eng.

Data 27, 146-148 (1982).

$82 / 9$ K. Bystrom and M. J. Mansson, J. Chem. Soc., Perkin Trans. II 565-569 (1982).

$82 / 10$ V. Svoboda, V. Charvatova, V. Majer, and V. Hynek, Collect.

Czech. Chem. Commun. 47, 543-549 (1982).

82/11 C. Invernizzi, Termotecnica 4, 78-85 (1982).

$82 / 12$ R. M. Varushchenko, S. S. Puchkov, and A. I. Druzinina, Zh. Fiz. Khim. 56, 2934-2937 (1982)

82/13 A. I. Sachek, A. D. Peshenenko, V. S. Markovnik, O. V. Ral'ko, D. N. Andreevskii, and A. A. Leont'eva, Termodin. Org. Soedin. 94-98 (1982).

82/14 P. Reche and M.-F. Grenier-Loustalot, J. Chromatogr. 238, 317-334 (1982).

82/15 G. Pilcher and H. A. Skinner, in The Chemistry of the Metal-Carbon Bond, edited by F. R. Hartley and S. Patai (Wiley, New York, 1982), Chap. 2.

82/16 B. E. Gammon, J. E. Callanan, I. A. Hossenlopp, A. G. Osborn, and W. D. Good, Proc. Symp. Thermophys. Prop. 8, 402-408 (1982). 82/17 D. J. Wren and A. C. Vikis, J. Chem. Thermodyn. 14, 435-437 (1982).

82/18 J. Furukawa, M. Sakiyama, S. Seki, Y. Saito, and K. Kusano, Bull. Chem. Soc. Jpn. 55, 3329-3330 (1982). 
82/19 R. A. De Marco and W. B. Fox, J. Org. Chem. 47, 3772-3773 (1982).

1983

83/1 J. E. House, Jr., Thermochimica Acta 71, 215-218 (1983).

83/2 V. Machat, thesis, Utzcht, Prague, 1983.

$83 / 3$ V. Uchytilova, V. Majer, V. Svoboda, and V. Hynek, J. Chem.

Thermodyn. 15, 853-858 (1983).

83/4 A. Sivaraman and R. Kobayashi, J. Chem. Thermodyn. 15, 11271135 (1983)

83/5 X.-W. An and M. Mansson, J. Chem. Thermodyn. 15, 287-293 (1983).

83/6 R. Fuchs and J. H. Hallman, Can. J. Chem. 61, 503-505 (1983). 83/7 J. H. Hallman, W. K. Stepenson, and R. Fuchs, Can. J. Chem. 61, 20-44-2047 (1983).

83/8 C. Tsonopoulos and G. M. W. Wilson, AIChE J. 29, 990-999 (1983).

83/9 D. Ferro, V. Piacente, and P. Scardala, Thermochimica Acta 68, 329-339 (1983).

83/10 A.-M. Olsson, J. A. Jonsson, B. Thelin, and T. Liljefors, J. Chem. Ecol. 9, 375-385 (1983).

83/11 Yu. Ya. Van-Chin-Syan, N. S. Kachurina, G. A. Petrovskaya, and S. K. Chuchmarev, Russ. J. Phys. Chem. 57, 1751-1752 (1983).

83/12 G. Natarajan and D. S. Viswanath, Rev. Sci. Instrum. 54, 11751179 (1983).

83/13 A. Tamir, C. Dragoescu, A. Apelblat, and J. Wisniak, Fluid Phase Equilib. 10, 9-42 (1983).

$83 / 14$ T. Schmeling and R. Strey, Ber. Bunsenges. Phys. Chem. 87, 871-874 (1983).

83/15 J. E. House, Jr., J. Fluorine Chem. 22, 299-303 (1983).

83/16 R. M. Clay, S. Corr, G. Keenan, and W. V. Steele, J. Am. Chem. Soc. 105, 2070-2071 (1983).

83/17 A. Sivaraman, R. J. Martin, and R. Kobayashi, Fluid Phase Equilib. 12, 175-188 (1983).

\section{4}

84/1 E. Surova, Chem. Zvesti 38, 83-99 (1984).

$84 / 2$ V. Majer, V. Uchytilova, V. Svoboda, and A. Posta, J. Chem.

Thermodyn. 16, 761-766 (1984).

$84 / 3$ S. C. Mraw and C. F. Keweshan, J. Chem. Thermodyn. 16, 873-883 (1984).

$84 / 4$ V. Majer, V. Svoboda, and M. Lencka, J. Chem. Thermodyn. 16, 1019-1024 (1984)

$84 / 5$ V. Majer, V. Svoboda, and V. Hynek, J. Chem. Thermodyn. 16, 1059-1066 (1984).

84/6 P. Starzewski, I. Wadso, and W. Zielenkiewicz, J. Chem.

Thermodyn. 16, 331-334 (1984).

$84 / 7$ V. Uchytilova, V. Majer, V. Svoboda, and I. Hemer, J. Chem.

Thermodyn. 16, 475-479 (1984).

$84 / 8$ V. Majer, V. Svoboda, J. Pechacek, and S. Hala, J. Chem.

Thermodyn. 16, 567-572 (1984).

84/9 T. Boublik, V. Fried, and E. Hala, The Vapor Pressures of Pure Substances: Selected Values of the Temperature Dependence of the Vapor Pressures of Some Pure Substances in the Normal and Low Pressure Region, 2nd revised edition (Elsevier, Amsterdan, The Netherlands, 1984). The vaporization enthalpies were calculated from the vapor pressures obtained from the Antoine constants reported in this compendium. In cases where the Antoine constant $C=0$, the Antoine Equation $\left(\log _{10} P=A-B /(C+T)\right)$ reduces to the integrated form of the Clausius Clapeyron equation directly. This was the case for many vaporization enthalpies. In those cases where $C \neq 0$, the vaporization enthalpy was calculated as $\Delta H_{V}=2.303 R B[T /(T+C)]^{2}$.

$84 / 10$ A. I. Sachek, V. S. Markovnik, A. D. Peshchenko, A. V. Shvaro, and D. N. Andreevskii, Khim. Prom.-St. (Moscow) 337-340 (1984). 84/11 A. K. Baev and A. A. Barkatin, Russ. J. Phys. 58, 195-198 (1984). 84/12 H.-D. Beckhaus, C. Rüchardt, and M. Smisek, Thermochimica Acta 79, 149-159 (1984).

84/13 A. Sokolovskii and A. K. Baev, Russ. J. Phys. Chem. 58, 195-198 (1984).

84/14 G. G. Nurullaev, S. A. Mekhtiev, K. A. Karasharli, N. F. Musaeva, and M. S. Salakhov, Azerb. Khim. Zh. 132-135 (1984).
84/15 M.-A. Michou-Saucet, J. Jose, C. Michou-Saucet, and J. C. Merlin, Thermochimica Acta 75, 85-106 (1984).

84/16 A. H. N. Mousa, J. Fluorine Chem. 25, 165-168 (1984).

84/17 M. P. Kozina, L. P. Timofeeva, G. L. Gal'chenko, E. S. Balenkova, and M. D. Ordubadi, Vestn. Mosk. Univ., Ser. 2: Khim. 25, 364-368 (1984); Chem. Abstracts 101, 190955q (1984).

84/18 A. E. Shcherbina, L. M. Kaporovskii, and E. A. Shcherbina, Zh. Prikl. Khim. (Leningrad) 57, 1910-1912 (1984); Chem. Abstracts 101, 177895a (1984).

84/19 C. Castellari, R. Francesconi, and F. Comelli, J. Chem. Eng. Data 29, 90-93 (1984).

84/20 C. Castellari, R. Francesconi, and F. Comelli, J. Chem. Eng. Data 29, 126-128 (1984).

$84 / 21$ S. Putcha, R. V. Ivanturi, and R. Machiraju, J. Chem. Eng. Data 29, 135-136 (1984).

84/22 S. R. Bosco and A. G. Turner, J. Chem. Eng. Data 29, 242-243

(1984).

84/23 C. Castellari, R. Francesconi, F. Comelli, and S. Ottani, J. Chem. Eng. Data 29, 283-284 (1984).

84/24 H. Katayama and Y. Harada, J. Chem. Eng. Data 29, 373-375

(1984).

84/25 A. Sivaraman and R. Kobayashi, J. Chem. Eng. Data 29, 375-379

(1984).

84/26 I. Cervenkova and T. Boublik, J. Chem. Eng. Data 29, 425-427

(1984).

$84 / 27$ J. T. Sipowska and S. A. Wieczorek, J. Chem. Thermodyn. 16, 693-699 (1984).

$84 / 28$ N. D. Lebedeva, N. M. Gutner, Yu. A. Katin, N. M. Kozlova, N. N. Kiseleva, E. F. Makhina, and S. L. Dobychin, J. Appl. Chem. (USSR) 57, 2118-2122 (1984).

84/29 P. T. Eubank, L. E. Cediel, J. C. Holste, and K. R. Hall, J. Chem. Eng. Data 29, 389-393 (1984).

84/30 R. Eng and S. I. Sandler, J. Chem. Eng. Data 29, 156-161 (1984). 84/31 D. M. Hull and B. C.-Y. Lu, J. Chem. Eng. Data 29, 417-419 (1984).

84/32 A. K. Baev, A. A. Barkatin, and L. M. Dyagileva, Vestsi Akad. Navuk BSSR, Ser. Khim. 60-62 (1984).

84/33 A. K. Baev, V. E. Mikhailov, and A. A. Baev, Vestsi Akad. Navuk BSSR, Ser. Khim. Navuk 75-77 (1984).

84/34 A. E. Sokolovskii and A. K. Baev, Vestsi Akad. Navuk BSSR, Ser. Khim. Navuk 115-117 (1984).

1985

85/1 V. Majer, V. Svoboda, and M. Lencka, J. Chem. Thermodyn. 17, 365-370 (1985).

85/2 K. Kusano, Thermochimica Acta 88, 109-120 (1985).

$85 / 3$ V. Majer, V. Svoboda, V. Uchytilova, and M. Finke, Fluid Phase Equilibr. 20, 111-118 (1985).

85/4 A. Raviprasad and K. V. Rao, J. Chem. Thermodyn. 17, 117-121 (1985)

85/5 S. A. Wieczorek and J. T. Sipowska, J. Chem. Thermodyn. 17, 255-261 (1985)

85/6 V. Majer and V. Svoboda, Enthalpies of Vaporization of Organic Compounds: A Critical Review and Data Compilation (Blackwell Scientific, Oxford, U.K., 1985).

85/7 G. Barone, G. Castronuovo, G. Della Gatta, V. Elia, and A. Iannone, Fluid Phase Equilib. 21, 157-164 (1985).

85/8 A. E. Sokolovski and A. K. Baev, Vestsi Akad. G. Emii Navuk BSSR, Ser. Khim. Navuk 112-114 (1985).

85/9 A. G. R. Scholz and E. Brunner, J. Chem. Eng. Data 30, 72-74 (1985)

85/10 G. Natarajan and D. S. Viswanath, J. Chem. Eng. Data 30,

137-140 (1985)

85/11 V. Piacente, P. Scardala, D. Ferro, and R. Gigli, J. Chem. Eng. Data 30, 372-376 (1985).

$85 / 12$ K. D. Reddy, M. V. P. Rao, and M. Ramakrishna, J. Chem. Eng. Data 30, 394-397 (1985).

85/13 V. A. Klyuchnikov, T. F. Danilova, M. G. Voronkov, and V. M. D’yakov, Dokl. Phys. Chem. 281, 373-377 (1985). 
85/14 S. N. Kondrat'ev and B. V. Strizhov, Zh. Fiz. Khim. 59, 29332938 (1985).

85/15 A. H. N. Mousa, J. Fluorine Chem. 30, 29-35 (1985).

85/16 E. H. Appelman, M. H. Mendelsohn, and H. Kim. J. Am. Chem. Soc. 107, 6515-6518 (1985).

85/17 C. J. Wormald and T. K. Yerlett, J. Chem. Thermodyn. 17, 1171-1186 (1985)

\section{6}

86/1 E. F. Meyer and J. J. Meyer, J. Chem. Eng. Data 31, 273-274 (1986).

86/2 B. Wisniewska, M. Lencka, and M. Rogalski, J. Chem. Thermodyn. 18, 703-708 (1986).

$86 / 3$ P. J. Achorn, W. G. Haseltine, and J. K. Miller, J. Chem. Eng. Data 31, 385-387 (1986).

86/4 O. L. Flaningan, J. Chem. Eng. Data 31, 266-277 (1986).

86/5 R. M. Varushchenko, Zh. Fiz. Khim. 60, 291-294 (1986).

$86 / 6$ N. Allemand, J. Jose, and J. C. Merlin, Thermochimica Acta 105, 79-90 (1986).

86/7 V. S. Tsvetkov, A. G. Nazmutdinov, K. G. Sharonov, and A. M. Rozhnov, Termodin. Org. Soedin. 71-73 (1986).

86/8 Yu. G. Dmitriev, N. S. Kachurina, C. H. Wang, and V. V.

Kouchukei, Vestn. L'vov. Politekh. Inst. 201, 29-30 (1986).

86/9 M.-A. Michou-Saucet, J. Jose, and C. Michou-Saucet,

Thermochimica Acta 102, 271-279 (1986).

86/10 V. Baliah and K. Gnanasekaran, Indian J. Chem. 25A, 673-674

(1986).

86/11 Ya. Kh. Grinberg, V. B. Lazarev, A. Yu. Zavernyaev, V. A.

Shreider, and S. D. Chepik, Zh. Fiz. Khim. 60, 1386-1389 (1986); Chem. Abstracts 105, 86041k (1986).

86/12 S. Takagi, M. Ishikawa, M. Kumada, T. Kimura, and R. Fujishiro, Thermochimica Acta 109, 55-61 (1986).

86/13 H.-I. Paul, J. Krug, and H. Knapp, Thermochimica Acta 108, 9-27 (1986).

86/14 V. M. Vorotyntsev, V. V. Balabanov, and D. A. Shamrakov, Zh. Fiz. Khim. 60, 234-235 (1986); Chem. Abstracts 104, 96047k (1986).

86/16 C. M. White, J. Chem. Eng. Data 31, 198-203 (1986).

$86 / 17$ T. K. Yerlett and C. J. Wormald, J. Chem. Thermodyn. 18, 719-726 (1986).

$86 / 18$ T. K. Yerlett and C. J. Wormald, J. Chem. Thermodyn. 18, 371-379 (1986).

\section{7}

87/1 X.-W. An, H.-P. Zhu, and R.-H. Hu, Thermochimica Acta 121, 473-477 (1987).

87/2 P. Kneisi and J. W. Zondio, J. Chem. Eng. Data 21, 11-13 (1987). $87 / 3$ P. L. Mills, R. L. Fenton, and F. G. Schaefer, J. Chem. Eng. Data 32, 251-265 (1987).

$87 / 4$ R. Fuchs, E. J. Chambers, and W. K. Stepenson, Can. J. Chem. 65, 2624-2627 (1987).

$87 / 5$ R. M. Stephenson and S. Malanowski, Handbook of the

Thermodynamics of Organic Compounds (Elsevier: New York, 1987).

The data in this listing were obtained directly from this compendium which does not contain literature references. The vaporization enthalpies were calculated from the vapor pressures obtained from the Antoine constants reported in this compendium. Vapor pressures were calculated over a $30 \mathrm{~K}$ temperature range closest to $298 \mathrm{~K}$ and the temperature dependence was reformulated in terms of the integrated form of the Clausius Clapeyron equation.

87/6 D. Ambrose and N. B. Ghiassee, J. Chem. Thermodyn. 19, 911-913 (1987).

87/7 D. Ambrose and N. B. Ghiassee, J. Chem. Thermodyn. 19, 903-909 (1987).

87/8 Z. Atik, Y. Saito, and K. Kusano, J. Chem. Thermodyn. 19, 99-102 (1987).

87/9 D. Ambrose and N. B. Ghiassee, J. Chem. Thermodyn. 19, 505-519 (1987).

87/10 P. L. Mills and R. L. Fenton, J. Chem. Eng. Data 32, 266-273 (1987).

87/11 R. M. Varushchenko, O. L. Loseva, and A. I. Druzhinina, Zh. Fiz. Khim. 61, 31-35 (1987).
87/12 R. M. Varushchenko, O. L. Loseva, and A. I. Druzhinina, Zh. Fiz. Khim. 61, 638-642 (1987).

87/13 H. Yan, J. Gu, X. An, and R.-H. Hu, Huaxue Xuebao 45, 11841187 (1987).

87/14 Yu. Ya. Van-Chin-Syan and N. S. Kachurina, Russ. J. Phys. Chem. 61, 622-624 (1987).

87/15 F. Garcia-Sanchez and A. Trejo, J. Chem. Thermodyn. 19, 359-361 (1987).

87/16 T. Treszczanowicz and B. C.-Y. Lu, J. Chem. Thermodyn. 19 , 391-394 (1987).

87/17 E. C. Azandegbe, Analusis 15, 370-373 (1987).

87/18 H. Al-Najjar and D. Al-Sammerrai, J. Chem. Tech. Biotechnol. 37, 145-152 (1987).

$87 / 19$ O. An, R. Hu, H. Wang, M. Wu, and Y. Zou, Acta Physico-Chim. Sinica 3, 668-671 (1987).

1988

88/1 V. I. Tel'noi, V. N. Larina, E. N. Karataev, and E. N. Deryagina, Zh. Fiz. Khim. 62, 3108-3109 (1988).

88/2 H. Katayama, J. Chem. Eng. Data 33, 75-77 (1988).

88/3 C. F. Askonas and T. E. Daubert, J. Chem. Eng. Data 33, 225-229

(1988).

88/4 A. K. Baglay, L. L. Gurarly, and G. G. Kuleshov, J. Chem. Eng. Data 33, 512-518 (1988).

$88 / 5$ D. Ambrose and N. B. Ghiassee, J. Chem. Thermodyn. 20, 765-766 (1988).

88/6 D. Ambrose, N. B. Ghiassee, and R. Tuckerman, J. Chem.

Thermodyn. 20, 767-768 (1988).

88/7 I. A. Hossenlopp and D. G. Archer, J. Chem. Thermodyn. 20,

1061-1068 (1988).

88/8 D. Ambrose and N. B. Ghiassee, J. Chem. Thermodyn. 20, 12311232 (1988).

$88 / 9$ T. M. Letcher, J. Sewry, and S. W. Orchard, J. Chem. Thermodyn. 20, 1231-1232 (1988).

88/10 M. Bastos, S. O. Nilsson, M. D. M. C. Ribeiro da Silva, M. A. V. Ribeiro da Silva, and I. Wadso, J. Chem. Thermodyn. 20, 1353-1359 (1988).

88/11 R. M. Varushchenko, O. L. Loseva, A. I. Druzhinina, and E. F. Zorina, Zh. Fiz. Khim. 62, 1776-1780 (1988).

88/12 K. Sasse, J. Jose, and J.-C. Merlin, Fluid Phase Equilib. 42, 287-304 (1988).

88/13 R. H. Shay, B. N. Diel, D. M. Schubert, and A. D. Norman, Inorg. Chem. 27, 2378-2382 (1988).

88/14 P. Knauth and R. Sabbah, Bull. Soc. Chem. Fr. 834-836 (1988).

$88 / 15$ M. G. Voronkov, V. P. Baryshok, V. A. Klyuchnikov, T. F.

Danilova, V. I. Pepekin, A. N. Korchagina, and Yu. I. Khudobin, J.

Organomet. Chem. 345, 27-38 (1988).

$88 / 16$ J. F. Messerly, H. L. Finke, W. D. Good, and B. Gammon, J.

Chem. Thermodyn. 20, 485-501 (1988).

88/17 H. Katayama, Bull. Chem. Soc. Jpn. 61, 3326-3328 (1988).

88/18 V. B. Lazarev, Ya. Kh. Grinberg, Z. P. Ozerova, and G. A.

Sharpataya, J. Thermal Anal. 33, 797-799 (1988).

88/19 J.-Q. Dong, R.-S. Lin, and W.-H. Yen, Can. J. Chem. 66, 783-790 (1988).

88/20 D. R. Davies, M. B. Ewing, J. A. Hugill, and M. L. McGlashan, Can. J. Chem. 66, 760-762 (1988).

1989

89/1 R. D. Chirico, A. Nguyen, W. V. Steele, M. M. Strube, and C. J. Tsonopoulos, Chem. Eng. Data 34, 149-156 (1989).

89/2 B. Varughese and J. T. Sommerfeld, J. Chem. Eng. Data 34, 25-26 (1989).

$89 / 3$ H. S. Wu and S. I. Sandler, J. Chem. Eng. Data 34, 209-213

(1989).

89/4 R. D. Chirico, S. E. Knipmeyer, A. Nguyen, and W. V. Steele, J.

Chem. Thermodyn. 21, 1307-1331 (1989).

89/5 M. Kato and M. Tanaka, J. Chem. Eng. Data 34, 206-209 (1989).

89/6 E. Cepeda, C. Gonzalez, and J. M. Resa, J. Chem. Eng. Data 34,

270-273 (1989).

89/7 S. J. Park and J. Gmehling, J. Chem. Eng. Data 34, 399-401

(1989). 
89/8 K. Wang, X.-G. Ying, M.-S. Xia, J.-Z. Xia, and Y. Hu, J. Chem. Eng. Data 34, 126-130 (1989).

89/9 A. Sakoguichi, Y. Iwai, and J. Takenaka, Kagaku Kogaku Ronbunshu 15, 166 (1989).

89/10 X. An and H. Hu, Wuli Huaxue Xuebao 5, 565-571 (1989); Chem. Abstracts 112, 118206f (1990).

89/11 M. G. Voronkov, M. S. Sorokin, V. A. Klyuchnikov, G. N. Shvetz, and V. I. Pepekin, J. Organomet. Chem. 359, 301-313 (1989).

$89 / 12$ M. G. Voronkov, V. A. Klyuchnikov, S. N. Kolabin, G. N. Shvets, P. I. Varushin, E. N. Deryagina, N. A. Korchevin, and S. I. Tsvetnitskaya, Dokl. Phys. Chem. 307, 650-653 (1989).

89/13 R. M. Varushchenko and L. L. Pashchenko, Russ. J. Phys. Chem. 63, 964-967 (1989)

89/14 K. Sasse, J. N'Guimbi, J. Jose, and J. C. Merlin, Thermochimica Acta 146, 53-61 (1989).

89/15 M. D. Vine and C. J. Wormald, J. Chem. Thermodyn. 21, 11511157 (1989).

89/16 E. C. Azandegbe, Analusis 17, 285-289 (1989).

1990

90/1 M. L. P. Leitao, G. Pilcher, Y. Meng-Yan, J. M. Brown, and A. D. Conn, J. Chem. Thermodyn. 22, 885-891 (1990).

90/2 D. A. Hinckley, T. F. Bidleman, W. T. Foreman, and J. R. Tuschall, J. Chem. Eng. Data 35, 232-237 (1990).

90/3 N. O. Smith, J. Chem. Eng. Data 35, 387-389 (1990).

90/4 H.-D. Beckhaus, B. Dogan, J. Pakusch, S. Verevkin, and C.

Rüchardt, Chem. Ber. 123, 2153-2159 (1990).

90/5 T. N. Nesterova, A. G. Nazmutdinov, V. S. Tsvetkov, A. M.

Rozhnov, and I. Yu. Roshchupkina, J. Chem. Thermodyn. 22, 365-377 (1990).

90/6 M. Lenka, J. Chem. Thermodyn. 22, 473-480 (1990).

90/7 D. Ambrose, M. B. Ewing, N. B. Ghiassee, and J. C. S. Ochoa, J. Chem. Thermodyn. 22, 589-605 (1990).

90/8 M. Sohda, M. Okazaki, Y. Iwai, Y. Arai, A. Sakoguchi, R. Ueoka, and Y. Kato, J. Chem. Thermodyn. 22, 607-608 (1990).

90/9 N. V. K. Dutt, A. P. Kahol, P. J. Reddy, and K. S. Raghunandanan, J. Chem. Eng. Data 35, 114-117 (1990).

90/10 J. Ortego, P. Susial, and C. de Alfonso, J. Chem. Eng. Data 35, 216-218 (1990).

90/11 X. An, J. He, and R.-H. Hu, Thermochimica Acta 169, 331-337 (1990).

90/12 R. H. Davies, A. Finch, and K. Salem, Thermochimica Acta 170, 213-216 (1990).

90/13 T. Pompili and V. Piacente, Thermochimica Acta 170, 289-291

(1990).

90/14 V. Piacente and P. Scardala, Thermochimica Acta 159, 193-200 (1990).

90/15 A. M. Rozhnov, V. I. Barkov, K. G. Sharonov, and V. S. Tsvetkov, J. Chem. Thermodyn. 22, 327-333 (1990).

90/16 G. Barone, G. Della Gatta, D. Ferro, and V. Piacente, J. Chem. Soc. Faraday Trans. 86, 75-79 (1990).

90/17 B. Dogan, H.-D. Beckhaus, J. Schaetzer, S. Hellmann, and C. Rüchardt, Chem. Ber. 123, 137-144 (1990).

90/18 M. I. Lopez and J. E. Sicre, J. Phys. Chem. 94, 3860-3863 (1990). 90/19 A. Sprozynski and J. Gregorowicz, Main Group Met. Chem. 13, 1-6 (1990).

1991

91/1 N. I. Buchan, R. M. Potemski, and T. F. Kuech, J. Chem. Eng. Data 36, 372-374 (1991)

91/2 P. Basarova and V. Svoboda, Fluid Phase Equilib. 68, 13-34 (1992). 91/3 H. S. Wu, K. A. Pividal, and S. I. Sandler, J. Chem. Eng. Data 36, 418-421 (1991).

91/4 M. G. Voronkov, V. A. Klyuchnikov, E. V. Sokolova, T. F. Danilova, G. N. Shvets, A. N. Korchagina, L. E. Gussel'nikov, and V. V. Volkova, J. Organomet. Chem. 401, 245-248 (1991).

91/5 M. G. Voronkov, V. A. Klyuchnikov, E. V. Mironenko, G. N. Shvets, T. F. Danilova, and Yu. I. Khudobin, J. Organomet. Chem. 406, 91-97 (1991).
91/6 M. G. Voronkov, V. A. Klyuchnikov, L. I. Marenkova, T. F. Danilova, G. N. Shvets, S. I. Tsvetnitskaya, and Yu. I. Khudobin, J. Organomet. Chem. 406, 99-104 (1991).

91/7 K. B. Wiberg and R. F. Waldron, J. Am. Chem. Soc. 113, $7697-$ 7705 (1991)

91/8 K. B. Wiberg and S. Hao, J. Org. Chem. 56, 5108-5110 (1991). 91/9 V. Piacente, T. Pompili, P. Scardala, and D. Ferro, J. Chem.

Thermodyn. 23, 379-396 (1991).

91/10 R. D. Chirico, S. E. Knipmeyer, A. Nguyen, and W. V. Steele, J. Chem. Thermodyn. 23, 431-450 (1991).

91/11 A. M. Rozhnov, V. V. Safronov, S. P. Verevkin, K. G. Sharonov, and V. I. Alenin, J. Chem. Thermodyn. 23, 629-635 (1991).

91/12 K. G. Sharonov, Y. B. Mishentseva, A. M. Rozhnov, E. A. Miroshnichenko, and L. I. Korchatova, J. Chem. Thermodyn. 23, 637-642 (1991).

91/13 R. D. Chirico, S. E. Knipmeyer, A. Nguyen, and W. V. Steele, J. Chem. Thermodyn. 23, 759-779 (1991).

91/14 W. V. Steele, R. D. Chirico, S. E. Knipmeyer, and A. Nguyen, J. Chem. Thermodyn. 23, 957-977 (1991).

91/15 M. B. Ewing and A. R. H. Goodwin, J. Chem. Thermodyn. 23, 1163-1168 (1991)

91/16 R. Koelliker and G. Bor, Inorg. Chem. 30, 2236-2237 (1991). 91/17 V. V. Dikii and G. Ya. Kabo, Dokl. Akad. Nauk BSSR 35, 158-162 (1991); Chem. Abstracts 114, 206233j (1981).

\section{2}

92/1 C.-H. Lee, Q. Chen, R. S. Mohamed, and G. D. Holder, J. Chem. Eng. Data 37, 179-183 (1992).

92/2 C.-H. Lee, D. M. Dempsey, R. S. Mohamed, and G. D. Holder, J. Chem. Eng. Data 37, 183-186 (1992).

92/3 R. Jadot and M. Fraiha, J. Chem. Eng. Data 37, 509-511 (1992).

92/4 J. S. Chickos, D. G. Hesse, S. Y. Panshin, D. W. Rogers, M.

Saunders, P. M. Uffer, and J. F. Liebman, J. Org. Chem. 57, 1897-1899 (1992)

92/5 M. Gazicki, H. Schalko, P. Svasek, F. Olcaytug, and F. Kohl, J. Vac. Sci. Technol. A 10, 51-58 (1992)

92/6 A. A. Kozyro, G. Ya. Kabo, V. S. Kruck, M. S. Sheiman, I. A.

Yursa, V. V. Simirskii, A. P. Krasulin, V. M. Sevruk, and V. I.

Gogolinskii, J. Chem. Thermodyn. 24, 883-895 (1992).

92/7 J. He, X.-W. An, and R.-H. Hu, Acta Chim. Sinica 50, 943-947 (1992)

92/8 V. Svoboda, V. Kubes, and P. Basarova, J. Chem. Thermodyn. 24, 333-336 (1992)

92/9 M. Gracia, F. Sanchez, P. Perez, J. Valero, and C. Gutierrez Losa, J. Chem. Thermodyn. 24, 463-471 (1992).

92/10 W. V. Steele, R. D. Chirico, S. E. Knipmeyer, and A. Nguyen, J.

Chem. Thermodyn. 24, 499-529 (1992).

92/11 V. Svoboda, V. Kubes, and P. Basarova, J. Chem. Thermodyn. 24, 555-558 (1992)

92/12 M. Salvi-Narkhede, B.-H. Wang, J. L. Adcock, and W. H. Van Hook, J. Chem. Thermodyn. 24, 1065-1075 (1992).

92/13 S. P. Verevkin, H.-D. Beckhaus, and C. Rüchardt, Thermochimica Acta 197, 27-39 (1992)

92/14 J. N'Guimbi, H. Kasehgari, I. Mokbel, and J. Jose, Thermochimica Acta 196, 367-377 (1992).

92/15 W. V. Steele, R. D. Chirico, S. E. Knipmeyer, and A. Nguyen, J. Chem. Thermodyn. 24, 245-271 (1992).

92/16 M.-S. Zhu, J. Wu, and Y.-D. Fu, Fluid Phase Equilib. 80, 99-105 (1992)

92/17 L. A. Weber, Fluid Phase Equilib. 80, 141-148 (1992).

92/18 W. Kimizuka and J. Szydlowski, Fluid Phase Equilib. 77, 261-267 (1992)

92/19 K. Oguchi, M. Yamagishi, and A. Murano, Fluid Phase Equilib. 80 131-140 (1992)

92/20 T. S. Papina, S. M. Pimenova, V. Yu. Zakharov, and V. P. Kolesov, Zh. Khim. Termodin. Termodin. 1, 207-211 (1992).

92/21 D. C. Hawker, Chemosphere 25, 427-436 (1992).

1993

93/1 X.-W. An and Y.-D. Xie, Thermochimica Acta 220, 17-25 (1993). 
93/2 A. Aucejo, J. B. Monton, R. Munoz, and M. Sanchotello, J. Chem. Eng. Data 38, 160-162 (1993).

93/3 C.-H. Lee and G. D. Holder, J. Chem. Eng. Data 38, 320-323 (1993).

93/4 R. Reich and V. Sanhueza, J. Chem. Eng. Data 38, 341-343 (1993). 93/5 P. Susial and J. Ortego, J. Chem. Eng. Data 38, 434-436 (1993). 93/6 A. M. Silva and L. A. Weber, J. Chem. Eng. Data 38, 644-646 (1993).

93/7 P. Susial and J. Ortego, J. Chem. Eng. Data 38, 647-649 (1993).

93/8 J. Farkova and I. Wichterle, Fluid Phase Equilib. 90, 143-148 (1993).

93/9 S. Husain, P. N. Sarma, G. Y. S. K. Swamy, and K. S. Devi, J. Am. Oil Chem. Soc. 70, 149-155 (1993).

93/10 H. Kasehgari, I. Mokbel, C. Viton, and J. Jose, Fluid Phase Equilib. 87, 133-152 (1993).

93/11 R. D. Chirico, S. E. Knipmeyer, A. Nguyen, and W. V. Steele, J. Chem. Thermodyn. 25, 1461-1494 (1993).

93/12 R. D. Chirico, S. E. Knipmeyer, A. Nguyen, N. K. Smith, and W. V. Steele, J. Chem. Thermodyn. 25, 729-761 (1993).

93/13 W. V. Steele, R. D. Chirico, S. E. Knipmeyer, and A. Nguyen, J. Chem. Thermodyn. 25, 965-992 (1993).

93/14 S. V. Syoev, A. N. Golubenko, L. D. Nikulina, and T. N.

Martynova, Thermochimica Acta 225, 137-139 (1993).

93/15 R. Schulze, H.-D. Beckhaus, and C. Rüchardt, Chem. Ber. 126, 1031-1038 (1993).

93/16 P. S. Engel, C. Wang, Y. Chen, C. Rüchardt, and H.-D. Beckhaus, J. Am. Chem. Soc. 115, 65-74 (1993).

93/17 V. V. Diky, G. J. Kabo, A. A. Kozyro, A. P. Krasulin, and V. M.

Sevruk, J. Chem. Thermodyn. 25 1169-1181 (1993).

93/18 J. He, X. An, and R. Hu, Huaxue Xuebao 51, 1059-1065 (1993); Chem. Abstracts 120, 87930w (1994).

93/19 J.-L. M. Abboud, P. Jimenez, M. V. Roux, C. Turrion, and C. Lopez-Mardomingo, Calor. Anal. Therm. 24, 181-183 (1993).

93/20 V. V. Ovchinnikov, A. A. Sobanov, and A. N. Pudovik, Dokl. Phys. Chem. 333, 483-485 (1993).

93/21 N. C. Ferreira, C. A. Moreira dos Santos, and I. C. C. Calegao, Ecl. Quim., Sao Paulo 18, 49-54 (1993).

93/22 H. Wolff, W. Kimizuka, and J. Szydlowski, Fluid Phase Equilib. 90, 163-175 (1993).

\section{4}

94/1 R. Sabbah, D. Tabet, and S. Belaadi, Thermochimica Acta 247, 193-199 (1994).

94/2 V. Piacente, D. Fontana, and P. Scardala, J. Chem. Eng. Data 39, 231-237 (1994).

94/3 F. Welle, S. P. Verevkin, M. Keller, H.-D. Beckhaus, and C. Rüchardt, Chem. Ber. 127, 697-710 (1994).

94/4 K. Rakus, S. P. Verevkin, J. Schatzer, H.-D. Beckhaus, and C. Rüchardt, Chem. Ber. 127, 1095-1103 (1994).

94/5 K. Rakus, S. P. Verevkin, H.-D. Beckhaus, and C. Rüchardt, Chem. Ber. 127, 2225-2234 (1994).

94/6 R. L. Falconer and T. F. Bidleman, Atmosph. Environ. 28, 547-554 (1994).

94/7 A. Aucejo, M. C. Burguet, J. B. Monton, R. Munoz, M. Sanchotello, and M. I. Vazquez, J. Chem. Eng. Data 39, 578-580 (1994).

94/8 K. Aim, J. Chem. Eng. Data 39, 591-594 (1994).

94/9 U. Karlson, W. T. Frankenberger, Jr., and W. F. Spencer, J. Chem.

Eng. Data 39, 608-610 (1994).

94/10 M. A. Krähenbühl and J. Gmehling, J. Chem. Eng. Data 39, 759-762 (1994).

94/11 V. Ruzicka, Jr., M. Zabransky, K. Ruzicka, and V. Majer,

Thermochimica Acta 245, 121-144 (1994).

94/12 K. Ruzicka and V. Majer, J. Phys. Chem. Ref. Data 23, 1-39 (1994).

94/13 B. Koutek, M. Hoskovec, P. Vrkova, K. Konecny, and L. Feltl, J. Chromatog. A 679, 307-317 (1994).

94/14 D. L. Morgan and R. Kobayashi, Fluid Phase Equilib. 97, 211-242 (1994).

94/15 J. Ortega and S. Galvan, J. Chem. Eng. Data 39, 907-910 (1994).
94/16 K. B. Wiberg, K. M. Morgan, and H. Maltz, J. Am. Chem. Soc. 116, 11067-11077 (1994).

94/17 W. V. Steele, R. D. Chirico, A. Nguyen, and S. E. Knipmeyer, J. Chem. Thermodyn. 26, 515-544 (1994).

94/18 K. Aim, J. Chem. Thermodyn. 26, 977-986 (1994).

94/19 A. S. Carson, P. G. Laye, J. B. Pedley, A. M. Welsby, J. S.

Chickos, and S. Hosseini, J. Chem. Thermodyn. 26, 1103-1109 (1994).

$94 / 20$ V. V. Diky, G. J. Kabo, A. A. Kozyro, A. P. Krasulin, and V. M.

Sevruk, J. Chem. Thermodyn. 26, 1001-1013 (1994).

94/21 L. Terenzi and V. Piacenti, Thermochimica Acta 235, 61-66 (1994).

94/22 W. Spieksma, R. Luijk, and H. A. J. Govers, J. Chromatog. A 672, 141-148 (1994).

94/23 K. Liu and R. M. Dickhut, Chemosphere 29, 581-589 (1994).

1995

95/1 M. A. V. Ribeiro da Silva, M. A. R. Matos, and L. M. P. F. Amaral, J. Chem. Thermodyn. 27, 565-574 (1995).

95/2 H. M. A. Al-Maydama, A. Finch, P. J. Gardner, and A. J. Head, J. Chem. Thermodyn. 27, 173-279 (1995).

95/3 J.-L. M. Abboud, P. Jimenez, M. V. Roux, C. Turrion, C.

Lopez-Mardomingo, A. Podosenin, D. W. Rogers, and J. F. Liebman, J.

Phys. Org. 8, 15-20 (1995).

95/4 A. Sakoguchi, R. Ueoka, Y. Kato, and Y. Arai, Kagaku Kogaku

Ronbunshu, 21, 219-222 (1995).

95/5 A. K. Baev, V. E. Mikhailov, and I. N. Chernyak, Russ. J. Gen. Chem. 65, 977-980 (1995).

95/6 M. A. V. Ribeiro da Silva, M. L. C. C. H. Ferrao, and F. Jiye, J. Chem. Eng. Data 40, 426-428 (1995).

95/7 K. Rakus, S. P. Verevkin, W.-H. Peng, H.-D. Beckhaus, and C.

Rüchardt, Liebigs Ann. 2059-2067 (1995).

95/8 K. Rakus, S. P. Verevkin, M. Keller, H.-D. Beckhaus, and C.

Rüchardt, Liebigs Ann. 1483-1493 (1995).

95/9 H. P. Diogo, R. C. Santos, P. M. Nunes, and M. E. Minas da

Piedade, Thermochimica Acta 249, 113-120 (1995).

95/10 M. G. M. Van der Vis and E. H. P. Cordfunke, Thermochimica Acta 265, 129-134 (1995).

95/11 S. P. Verevkin, H.-D. Beckhaus, and C. Rüchardt, Thermochimica Acta 249, 1-11 (1995).

95/12 C. Gabaldon, P. Marzal, and J. B. Monton, J. Chem. Eng. Data 40, 190-193 (1995).

95/13 A. Dejoz, M. C. Burguet, R. Munoz, and M. Sanchotello, J. Chem. Eng. Data 40, 290-292 (1995).

95/14 A. Aucejo, V. Gonzalez-Alfaro, J. B. Monton, and M. I. Vazquez,

J. Chem. Eng. Data 40, 332-335 (1995).

95/15 G.-H. Chen, Q. Wang, Z.-M. Ma, X.-H. Yan, and S.-J. Han, J.

Chem. Eng. Data 40, 361-366 (1995).

95/16 A. Arce, A. Blanco, J. Martinez-Ageitos, and A. Soto, J. Chem.

Eng. Data 40, 515-518 (1995).

95/17 J. Ortego and S. Galvan, J. Chem. Eng. Data 40, 699-703 (1995).

95/18 A. Xu-Wu and G. Da-Jun, Thermochimica Acta 253, 235-242

(1995).

95/19 V. A. Luk'yanova and M. P. Kozina, Russ. J. Phys. Chem. 69, 1906-1907 (1995).

95/20 R. M. Varushchenko and A. I. Droujinina, J. Chem. Thermodyn. 27, 355-368 (1995).

95/21 J. S. Chickos, S. Hosseini, and D. G. Hesse, Thermochimica Acta 249, 41-62 (1995).

95/22 W. V. Steele, R. D. Chirico, A. Nguyen, and S. E. Knipmeyer, J. Chem. Thermodyn 27, 311-334 (1995).

95/23 H. P. Diogo, M. E. Minas de Piedade, J. A. Martinho Simoes, and Y. Nagana, J. Chem. Thermodyn. 27, 597-604 (1995).

95/24 W. V. Steele, R. D. Chirico, A. B. Cowell, A. Nguyen, and S. E. Knipmeyer, J. Chem. Thermodyn. 27, 1407-1428 (1995).

95/25 S. P. Verevkin, B. Dogan, J. Hadrich, H.-D. Beckhaus, and C.

Rüchardt, J. Prakt. Chem. 337, $93-98$ (1995).

95/26 E. F. S. Vieira, J. C. de Queiroz, and F. S. Dias, Thermochimica Acta 256, 249-260 (1995). 
95/27 J. S. Chickos, D. G. Hesse, S. Hosseini, J. F. Liebman, G. D. Mendenhall, S. P. Verevkin, K. Rakus, H.-D. Beckhaus, and C. Rüchardt, J. Chem. Thermodyn. 27, 693-705 (1995).

95/28 M. Nolke, S. P. Verevkin, H.-D. Beckhaus, and C. Rüchardt, Liebigs Ann. 41-51 (1995).

95/29 J. P. Guthrie and Z. Liu, Can. J. Chem. 73, 1395-1398 (1995). 95/30 T. S. Papina, S. M. Pimenova, V. A. Luk'yanova, and V. P. Kolesov, Zh. Fiz. Khim. 69, 2148-2151 (1995); Chem. Abstracts 124, 68148p. (1996).

95/31 V. A. Luk'yanova and M. P. Kozina, Zh. Fiz. Khim. 69, $2094-$ 2095 (1995); Chem. Abstracts 124, 68127f (1996).

\section{6}

96/1 M. A. V. Ribeiro da Silva, M. D. M. C. Ribeiro da Silva, M. F. B. M. Monteiro, M. L. A. C. N. Gomes, J. S. Chickos, A. P. Smith, and J. F. Liebman, Struct. Chem. 7, 367-373 (1996).

96/2 M. A. V. Ribeiro da Silva, V. M. F. Morais, M. A. R. Matos, C. M. A. Rio, and C. M. G. S. Piedade, Struct. Chem. 7, 329-336 (1996).

96/3 W. V. Steele, R. D. Chirico, S. E. Knipmeyer, and A. Nguyen, J. Chem. Eng. Data 41, 1255-1268 (1996).

96/4 W. V. Steele, R. D. Chirico, S. E. Knipmeyer, A. Nguyen, N. K. Smith, and I. R. Tasker, J. Chem. Eng. Data 41, 1269-1284 (1996).

96/5 W. V. Steele, R. D. Chirico, S. E. Knipmeyer, A. Nguyen, and N. K. Smith, J. Chem. Eng. Data 41, 1285-1302 (1996).

96/6 L. A. Weber and D. R. Defibaugh, J. Chem. Eng. Data 41, 382-385 (1996).

96/7 A. G. de Souza, M. G. A. Brasilino, and C. Airoldi, J. Chem.

Thermodyn. 28, 1359-1367 (1996).

96/8 Yu. Ya. Van-Chin-Syan, V. V. Kochubei, V. V. Sergeev, Yu. A. Raevskii, S. I. Gerasimchuk, and Kh. Z. Kotovich, Sov. J. Phys. Chem. (Eng. Trans.) 70, 1789-1794 (1996).

96/9 P. Ulbig, M. Klüppel, and S. Schulz, Thermochimica Acta 271, 9-21 (1996).

96/10 D. D. Back, L. R. Grzyll, and M. Corrigan, Thermochimica Acta 272, 53-63 (1996).

96/11 S. P. Verevkin, H.-D. Beckhaus, R. S. Belen'kaya, K. Rakus, and C. Rüchardt, Thermochimica Acta 279, 47-64 (1996).

96/12 J. Linek, I. Wichterle, and K. Marsh, J. Chem. Eng. Data 41, 1212-1218 (1996).

96/13 N. F. Giles, H. L. Wilson, and W. V. Wilding, J. Chem. Eng. Data 41, 1223-1238 (1996).

96/14 L. A. Weber and D. R. Defibaugh, J. Chem. Eng. Data 41, 1477-1480 (1996).

96/15 M. F. Rodrigues and M. G. Bernardo-Gil, J. Chem. Eng. Data 41, 581-585 (1996).

96/16 S. R. Susay, M. A. Smith, and G. G. Lockwood, Anesth. Analg. 83, 864-866 (1996).

96/17 B. Koutek, M. Hoskovec, P. Vrkocova, K. Konecny, L. Feltl, and J. Vrkoc, J. Chromatogr. A 719, 391-400 (1996).

96/18 R. M. Varushchenko, L. L. Pashchenko, and A. I. Druzhinina, Russ. J. Phys. Chem. 70, 208-211 (1996).

96/19 M. Polednicek, T. Guetachew, J. Jose, V. Ruzicka, V. Rohac, and M. Zabransky, ELDATA: Intl. Electron. J. Phys.-Chem. Data 2, 41-50 (1996).

96/20 S. P. Verevkin, S. Züffle, H.-D. Beckhaus, and C. Rüchardt, Thermochimica Acta 285, 1-10 (1996).

96/21 E. A. Miroshinchenko, Yu. D. Orlov, L. I. Korchatova, V. P. Vorob'eva, and Yu. A. Lebedev, Zh. Fiz. Khim. 70, 1583-1586 (1996). 96/22 C. Viton, M. Chavret, and J. Jose, ELDATA: Int. Electron. J. Phys.-Chem. Data 2, 103-110 (1996).

96/23 V. V. Ovchinnikov, T. B. Makeeva, L. I. Lapteva, and A. I.

Konovalov, Thermochim. Acta 277, 145-150 (1996).

96/24 A. G. van Haelst, F. W. M. van der Wielen, and H. A. J. Govers, J. Chromatog. A 727, 265-273 (1996).

96/25 R. D. Chirico, W. V. Steele, A. Nguyen, T. D. Klots, and S. E. Knipmeyer, J. Chem. Thermodyn. 28, 797-818 (1996).

96/26 R. M. Varushchenko, A. I. Druzhinina, and L. L. Pashchenko, Fluid Phase Equilibria 126, 93-104 (1996).

96/27 O. Dahmani, I. Wichterle, and A. Ait-Kaci, Fluid Phase Equilib.

124, 135-146 (1996).
96/28 V. S. Ushakov, S. M. Sedov, B. A. Knyazev, and B. I. Kuchkaev, Zh. Fiz. Khim. 70, 1573-1577 (1996).

96/29 A. K. Baev, A. I. Podkovirov, and V. I. Kosirkin, Izv. Vyssh.

Uchebn. Zaved., Khim. Khim. Tekhnol. 39, 10-14 (1996).

96/30 M. G. M. Van der Vis, E. H. P. Cordfunke, R. J. M. Konings, and A. Oskam, J. Chem. Soc., Faraday Trans. 92, 973-978 (1996).

96/31 I. B. Sladkov and N. Yu. Novikova, Zh. Prikl. Khim.

(S.-Peterburg) 96, 1840-1844 (1996).

1997

97/1 S. P. Verevkin, J. Chem. Thermodyn. 29, 1495-1501 (1997).

97/2 M. A. V. Ribeiro da Silva, J. M. Goncalves, and G. Pilcher, J.

Chem. Thermodyn. 29, 253-260 (1997).

97/3 M. A. V. Ribeiro da Silva, M. A. R. Matos, and L. M. P. F. Amaral,

J. Chem. Thermodyn. 29, 1545-1551 (1997).

97/4 M. A. V. Ribeiro da Silva, M. A. R. Matos, and L. M. P. F. Amaral, J. Chem. Thermodyn. 29, 1535-1543 (1997).

97/5 S. P. Verevkin, Thermochimica Acta 307, 17-25 (1997).

97/6 W. V. Steele, R. D. Chirico, S. E. Knipmeyer, and A. Nguyen, J.

Chem. Eng. Data 42, 1008-1020 (1997).

97/7 W. V. Steele, R. D. Chiricio, S. E. Knipmeyer, A. Nguyen, and N. K. Smith, J. Chem. Eng. Data 42, 1037-1052 (1997).

97/8 W. V. Steele, R. D. Chirico, S. E. Knipmeyer, and A. Nguyen, J.

Chem. Eng. Data 42, 1021-1036 (1997).

97/9 S. P. Verekin, J. Mogenthaler, and C. Rüchardt, J. Chem.

Thermodyn. 29, 1175-1183 (1997).

97/10 F. W. Welle, S. P. Verevkin, H.-D. Beckhaus, and C. Rüchardt,

Liebigs Ann./Recueil 155-163 (1997).

97/11 P. Hernández and J. Ortega, J. Chem. Eng. Data 42, 1090-1100

(1997).

97/12 L. M. G. Minier and R. Behrens, Jr., Propellants, Explos. Pyrotech. 22, 23-33 (1997).

97/13 B. Koutek, M. Hoskovec, P. Vrkocova, and L. Feltl, J. Chromatogr. A 759, 93-109 (1997).

97/14 F. Schaffer, S. P. Verevkin, H.-J. Rieger, H.-D. Beckhaus, and C. Rüchardt, Liebigs Ann./Recueil 1333-1344 (1997).

97/15 H. Artigas, C. Lafuente, P. Cea, F. M. Royo, and J. S. Urieta, J. Chem. Eng. Data 42, 132-136 (1997).

97/16 T. Sako, S. Horiguchi, H. Ichimaru, and S. Nakagawa, J. Chem.

Eng. Data 42, 169-717 (1997).

97/17 J. S. Chickos and J. A. Wilson, J. Chem. Eng. Data 42, 190-197

(1997).

97/18 W. V. Steele, R. D. Chirico, A. B. Cowell, S. E. Knipmeyer, and A. Nguyen, J. Chem. Eng. Data 42, 1053-1066 (1997).

97/19 J. C. G. Calado, E. J. M. Filipe, and J. N. C. Lopes, J. Chem.

Thermodyn. 29, 1435-1438 (1997).

97/20 A. V. Blokhin, G. J. Kabo, A. A. Kozyro, L. S. Ivashkevich, A. P.

Krasulin, V. V. Diky, and Yu. V. Maksimuk, Thermochimica Acta 292,

19-29 (1997).

97/21 S. P. Verevkin, J. Chem. Thermodyn. 29, 891-899 (1997).

97/22 D. R. Defibaugh, E. Carrillo-Nava, J. J. Hurly, M. R. Moldover, J.

W. Schmidt, and L. A. Weber, J. Chem. Eng. Data 42, 488-496 (1997).

97/23 Yu. V. Maksimuk, G. J. Kabo, A. A. Kozyro, V. V. Simirsky, A. P.

Krasulin, and V. M. Sevruk, J. Chem. Thermodyn. 29, 687-699 (1997).

97/24 M. Hallquist, I. Wangberg, and E. Ljungstrom, Environ. Sci.

Technol. 31, 3166-3172 (1997).

97/25 H. A. Duarte-Garza, C.-A. Hwang, S. A. Kellerman, R. C. Miller,

K. R. Hall, J. C. Holste, K. N. Marsh, and G. E. Gammon, J. Chem.

Eng. Data 42, 497-501 (1997).

97/26 B. L. Korsounskii and T. A. Apina, Int. Annu. Conf. ICT 46.146.7 (1997); Chem. Abstracts 127, 163997k (1997).

97/27 L.-T. Lim and M. A. Tung, J. Food Sci. 62, 1061-1066 (1997).

97/28 H. B. Krop, M. J. M. Velzen, J. R. Parsons, and H. A. J. Govers, J. Am. Oil Chem. Soc. 74, 309-315 (1997).

97/29 S. P. Verevkin, M. Nölke, H.-D. Beckhaus, and C. Rüchardt, J. Org. Chem. 62, 4683-4686 (1997).

97/30 A. K. Baev, Russ. J. Inorg. Chem. 42, 587-596 (1997).

1998

98/1 S. P. Verevkin, H.-D. Beckhaus, U. Schüle, and C. Rüchardt, Struct. Chem. 9, 1-7 (1998). 
98/2 S. P. Verevkin and F. M. Welle, Struct. Chem. 9, 215-221 (1998). 98/3 R. Gudino, L. A. Torres, R. L. Santillan, and N. Farfan, J. Chem. Thermodyn. 30, 671-679 (1998).

98/4 S. P. Verevkin, Thermochimica Acta 310, 229-235 (1998).

98/5 M. A. George, K. M. Young, E. A. Robertson III, S. E. Beck, and G. Voloshin, J. Chem. Eng. Data 43, 60-64 (1998).

98/6 Y. V. Maksimuk, G. J. Kabo, V. V. Simirsky, A. A. Kozyro, and V. M. Sevruk, J. Chem. Eng. Data 43, 293-398 (1998).

98/7 M. A. Abdi and A. Meisen, J. Chem. Eng. Data 43, 133-137 (1998).

98/8 V. Rohac, V. Ruzicka, K. Ruzicka, and K. Aim, J. Chem. Eng. Data 43, 770-775 (1998).

98/9 A. Aucejo, S. Loras, R. Munoz, P. Reich, and H. Segura, J. Chem. Eng. Data 43, 973-977 (1998).

98/10 S. P. Verevkin, Thermochimica Acta 316, 131-136 (1998).

98/11 J. S. Chickos, D. Hesse, S. Hosseini, G. Nichols, and P. Webb, Thermochimica Acta 313, 101-110 (1998).

98/12 S. P. Verevkin, Struct. Chem. 9, 113-119 (1998).

98/13 S. P. Verevkin, J. Chem. Thermodyn. 30, 1069-1079 (1998). 98/14 K. M. Morgan and D. A. Kopp, J. Chem. Soc., Perkin Trans. 2 2759-2763 (1998).

98/15 J. D. Olson, Fluid Phase Equilib. 150/151, 713-720 (1998). 98/16 V. Svoboda, V. Hynek, and B. Koutek, J. Chem. Thermodyn. 30, 1411-1417 (1998)

98/17 V. P. Kolesov, S. M. Pimenova, V. A. Lukyanova, T. S.

Kuznetsova, and M. P. Kozina, J. Chem. Thermodyn. 30, 1455-1464 (1998).

98/18 M. B. Ewing and J. C. Sanchez Ochoa, J. Chem. Thermodyn. 30, 189-198 (1998).

98/19 S. P. Verevkin, J. Chem. Thermodyn. 30, 1029-1040 (1998). 98/20 K. G. Drouillard, G. T. Tomy, D. C. G. Muir, and K. J. Friesen, Environ. Toxicol. Chem. 17, 1252-1260 (1998).

98/21 S. P. Verevkin, W.-H. Peng, H.-D. Beckhaus, and C. Rüchardt, Eur. J. Org. Chem. 2323-2332 (1998).

98/22 S. P. Verevkin, M. Kümmerlin, H.-D. Beckhaus, C. Galli, and C. Rüchardt, Eur. J. Org. Chem. 579-584 (1998).

98/23 X. Zhang, H. Mo, K. Yang, and F. An, Huanjing Huaxue 17, 50-54 (1998); Chem. Abstracts 128, 318311d (1998) (value is referred to as a vaporization enthalpy).

98/24 W. Blanke, G. Klingenberg, and F. Weber, Int. J. Thermophys. 19, 653-662 (1998).

98/25 L. D. Stockton, T. L. Ng, N. Maung, I. B. Poole, J. O. Williams, A. C. Wright, D. F. Foster, and D. J. Cole-Hamilton, J. Cryst. Growth 183, 95-98 (1998).

\section{9}

99/1 M. A. V. Ribeiro da Silva, M. D. M. C. Ribeiro da Silva, M. A. R. Matos, P. Jimenez, M. V. Roux, J. Elguero, R. Claramunt, P. Cabildo, and A. Sanchez-Migallon, J. Chem. Thermodyn. 31, 129-138 (1999). 99/2 S. P. Verevkin, Thermochimica Acta 332, 27-32 (1999). 99/3 S. P. Verevkin, J. Chem. Eng. Data 44, 1245-1251 (1999). 99/4 S. P. Verevkin and A. Heintz, J. Chem. Eng. Data 44, 1240-1244 (1999).

99/5 O. Mo, M. Yanez, M. V. Roux, P. Jimenez, J. Z. Davalos, M. A. V. Ribeiro da Silva, M. D. M. C. Ribeiro da Silva, M. A. R. Matos, L. M. P. F. Amaral, A. Sanchez-Migallon, P. Cabildo, R. Claramunt, J. Elguero, and J. F. Liebman, J. Phys. Chem. 103, 9336-9344 (1999).

99/6 F. S. Costa, M. E. Eusebio, J. S. Redinha, and M. L. P. Leitao, J. Chem. Thermodyn. 31, 895-903 (1999).

99/7 M. A. V. Ribeiro da Silva, M. A. R. Matos, V. M. F. Morais, and M. S. Miranda, J. Org. Chem. 64, 8816-8820 (1999).

99/8 S. P. Verevkin, J. Chem. Eng. Data 44, 175-179 (1999). 99/9 Y. D. Lei, F. Wania, and W. Y. Shiu, J. Chem. Eng. Data 44, 577-582 (1999).

99/10 J. B. Monton, J. de la Torre, M. C. Burguet, R. Munoz, and S. Loras, J. Chem. Eng. Data 44, 1158-1162 (1999).

99/11 J. N'Guimbi, C. Berro, I. Mokbel, E. Rauzy, and J. Jose, Fluid Phase Equilib. 162, 143-158 (1999).
99/12 D. E. G. Jones, H. T. Feng, R. A. Austen, and R. C. Fouchard, J. Therm. Anal. Calorim. 55, 9-19 (1999); Chem. Abstracts 132, 182729u (2000).

99/13 Y. D. Lei, F. Wania, W. Y. Shiu, and D. G. B. Boocock, J. Chem. Eng. Data 44, 200-202 (1999).

99/14 R. Garriga, A. C. Andres, P. Perez, and M. Gracia, J. Chem. Eng. Data 44, 296-302 (1999).

99/15 G. N. Escobedo-Alvarado and S. I. Sandler, J. Chem. Eng. Data 44, 319-322 (1999).

99/16 J. Dykyj, J. Svoboda, R. C. Wilhoit, M. L. Frenkel, and K. R. Hall, Vapor Pressure of Chemicals: Part A. Vapor Pressure and Antoine Constants for Hydrocarbons and Sulfur, Selenium, Tellurium and Hydrogen Containing Organic Compounds (Springer, Berlin, 1999). The vaporization enthalpies were calculated from the vapor pressures obtained from the Antoine constants reported in this compendium. In cases where the Antoine constant $C=0$, the Antoine Equation $\left(\log _{10} P=A-B /(C\right.$

$+T)$ ) reduces to the integrated form of the Clausius Clapeyron equation directly. This was the case for many vaporization enthalpies. In those cases where this condition was not met the vaporization enthalpy was calculated as $\Delta H_{V}=2.303 R B[T /(T+C)]^{2}$.

99/17 V. N. Dibrivnyl, Z. E. Pistun, Yu. Ya. Van-Chin-Syan, A. P. Yuvchenko, and T. D. Zvereva, Russ. J. Phys. Chem. 73, 2043-2045 (1999).

99/18 S. P. Verevkin, J. Chem. Thermodyn. 31, 559-585 (1999). 99/19 S. P. Verevkin, J. Chem. Thermodyn. 31, 1397-1416 (1999). 99/20 V. Rohac, V. Ruzicka, K. Ruzicka, M. Polednicek, K. Aim, J. Jose, and M. Zabransky, Fluid Phase Equilibra 157, 121-142 (1999). 99/21 S. P. Verevkin, Thermochimica Acta 326, 17-25 (1999). 99/22 V. A. Titov, L. N. Zelenina, and T. P. Chusova, Russ. J. Phys. Chem. 73, 835-837 (1999).

99/23 V. N. Dibrivnyl, G. V. Lusiv, Yu. Ya. Van-Chin-Syan, A. P. Yuvchenko, and E. A. Dikusar, Russ. J. Phys. Chem. 73, 2040-3042 (1999).

99/24 R. D. Chirico, S. E. Knipmeyer, A. Nguyen, and W. V. Steele, J. Chem. Thermodyn. 31, 339-378 (1999).

99/25 V. Rohac, J. E. Musgrove, K. Ruzicka, V. Ruzicka, M. Zabransky, and K. Aim. J. Chem. Thermodyn. 31, 971-986 (1999).

99/26 F. Weber, PTB-Mitteilungen 109, 469-471 (1999).

99/27 M. A. E. Diaz, T. Guetachew, P. Landy, J. Jose, and A. Voilley, Fluid Phase Equilib. 109, 469-471 (1999).

99/28 S. Loras, A. Aucejo, and R. Munoz, Fluid Phase Equilib. 156, 185-195 (1999).

99/29 J. S. Lim, J.-Y. Park, B.-G. Lee, Y.-W. Lee, and J.-D. Kim, J. Chem. Eng. Data 44, 1226-1230 (1999).

99/30 A. Aucejo, S. Loras, R. Munoz, and L. M. Ordonez, Fluid Phase Equilib. 156, 173-178 (1999).

99/31 A. Keys, S. G. Bott, and A. R. Barron, Chem. Mater. 11, 35783587 (1999).

99/32 D. Fatu, J. Thermal. Anal. Calor. 56, 739-747 (1999).

99/33 M. I. Zaretskii, E. M. Chartov, L. A. Pushkina, and V. V. Elkin, Russ. J. Appl. Chem. 72, 1702-1705 (1999).

2000

00/1 M. A. V. Ribeiro da Silva, M. D. M. C. Ribeiro da Silva, M. A. R. Matos, P. Jimenez, M. V. Roux, M. A. Martin-Luengo, J. Elguero, R. Claramut, and P. Cabildo, J. Chem. Thermodyn. 32, 237-245 (2000). 00/2 S. P. Verevkin, J. Chem. Thermodyn. 32, 207-216 (2000).

00/3 M. A. V. Ribeiro da Silva, M. A. R. Matos, C. A. Rio, V. M. F. Morais, J. Wang, G. Nichols, and J. S. Chickos, J. Phys. Chem. A 104, $1774-1778$ (2000).

00/4 I. Mokbel, K. Ruzicka, V. Majer, V. Ruzicka, M. Ribeiro, J. Jose, and M. Zabransky, Fluid Phase Equilib. 169, 191-207 (2000).

00/5 S. P. Verevkin and C. Schick, J. Chem. Eng. Data 45, 946-952 (2000).

00/6 S. P. Verevkin, J. Chem. Eng. Data 45, 953-960 (2000).

00/7 S. P. Verevkin, D. Wandschneder, and A. Heintz, J. Chem. Eng. Data 45, 618-625 (2000).

00/8 J. M. Resa, C. Gonzalez, S. Ortiz de Landaluce, and J. Lanz, J. Chem. Eng. Data 45, 867-871 (2000). 
00/9 G. Nichols, J. Orf, S. M. Reiter, J. S. Chickos, and G. W. Gokel, Thermochimica Acta 346, 15-28 (2000).

00/10 P. V. Ova, B. Koultek, and M. Hoskovec, in Practice Oriented Results on Use and Production of Neem Ingredients and Pheromones VI; edited by H. Kleeberg and C. P. W. Zebitz (Druck and Graphic, Giessen, 2000), pp. 211-218.

00/11 Y.-H. Liang and P.-S. Ma, Shiyou Huagong 29, 939-941 (2000). 00/12 S. Rodriguez, H. Artigas, C. Lafuente, A. M. Mainar, and F. M.

Royo, Thermochimica Acta 362, 153-160 (2000).

00/13 R. Reich, M. Cartes, H. Segura, and J. Wisniak, Phys. Chem. Liq. 38, 217-232 (2000).

00/14 S. P. Verevkin, J. Chem. Thermodyn. 32, 247-259 (2000).

00/15 S. P. Verevkin and A. Heintz, J. Chem. Thermodyn. 32, 1169-1182 (2000).

00/16 V. Rohac, M. Censky, D. Zala, V. Ruzicka, K. Ruzicka, K. Sporka, and K. Aim, J. Chem. Eng. Data 45, 1205-1210 (2000).

00/17 M. Palczewska-Tulinska and A. M. Szafranski, J. Chem. Eng. Data 45, 988-990 (2000).

00/18 E. Bourret-Courchesne, Q. Ye, D. W. Peters, J. Arnold, M. Ahmed, S. J. C. Irvine, R. Kanjolia, L. M. Smith, and S. A. Rushworth, J. Cryst. Growth 217, 47-54 (2000).

00/19 B. Sztaray and T. Baer, J. Am. Chem. Soc. 122, 9219-9226

(2000).

00/20 C. J. Wormald and D. P. Fennell, Int. J. Thermophys. 21, 767-779 (2000).

00/21 A. I. Druzhinina, R. M. Varushchenko, V. S. Sarisova, and A. A. Pimerzin, Zh. Fiz. Khim. 74, 404-411 (2000).

00/22 S. V. Melkhanova, S. M. Pimenova, V. P. Kolesov, A. A. Pimerzin, and V. S. Sarkisova, J. Chem. Thermodyn. 32, 1311-1317 (2000).

00/23 S. Bobbo, R. Camporese, and R. Stryjek, J. Chem. Thermodyn. 32, 1647-1656 (2000).

00/24 C. J. Wormald and M. D. Vine, J. Chem. Thermodyn. 32, 329-339 (2000).

00/25 C. J. Wormald and M. D. Vine, J. Chem. Thermodyn. 32, 659-669 (2000).

00/26 C. J. Wormald and G. F. James, J. Chem. Eng. Data 45, 348-352 (2000).

2001

01/1 S. Puri, J. S. Chickos, and W. J. Welsh, Anal. Chem. 73, 1480-1484 (2001).

01/2 A. Wong, Y. D. Lei, M. Alaee, and F. Wania, J. Chem. Eng. Data 46, 239-242 (2001).

01/3 D. Kilikov, S. P. Verevkin, and A. Heintz, Fluid Phase Equilib. 192, 187-206 (2001)

01/4 R. M. Varushchenko, L. L. Paschenko, A. I. Druzhinina, A. V. Abramenkov, and A. A. Pimersin, J. Chem. Thermodyn. 33, 733-744 (2001).

01/5 A. K. Baev, Russ. J. Appl. Chem. 74, 162-163 (2001).

01/6 S. Bernatova and I. Wichterle, Fluid Phase Equilib. 189, 111-118

(2001).

01/7 D. Kulikov, S. P. Verevkin, and A. Heintz, J. Chem. Eng. Data 46, 1593-1600 (2001).

01/8 J. G. Blok, A. C. G. van Genderen, P. R. van der Linde, and H. A. G. Oonk, J. Chem. Thermodyn. 33, 1097-1106 (2001).

01/9 A. K. Baev, Izv. Vyssh. Uchebn. Zaved., Khim. Khim. Tekhnol. 44, $3-13(2001)$

01/10 N. Bureau, J. Jose, I. Mokbel, and J.-C. de Hemptinne, J. Chem.

Thermodyn. 33, 1485-1498 (2001).

01/11 S. A. Tittlemier and G. T. Tomy, Environ. Toxicol. Chem. 20,

146-148 (2001)

01/12 H. Segura, E. Lam, R. Reich, and J. Wisniak, Phys. Chem. Liq. 39, 43-54 (2001).

01/13 L. Zhu, H. Li, C. Wang, and S. Han, J. Chem. Eng. Data 46, 1231-1234 (2001).

01/14 P. Uusi-Kyyny, J.-P. Pokki, J. Aittaman, and S. Liukkonen, J. Chem. Eng. Data 46, 1244-1248 (2001).

01/15 L. A. L. Munoz and M. A. Krahenbuhl, J. Chem. Eng. Data 46 , $120-124$ (2001).
01/16 S. P. Verevkin and A. Heintz, J. Chem. Eng. Data 46, 984-990 (2001).

01/17 K. Chylinski, Z. Fras, and S. K. Malanowski, J. Chem. Eng. Data 46, 29-33 (2001).

01/18 S. P. Verevkin and A. Heintz, J. Chem. Eng. Data 46, 41-46 (2001).

01/19 S. Horstmann, H. Gardeler, K. Fischer, F. Köster, and J. Gmehling, J. Chem. Eng. Data 46, 337-345 (2001).

01/20 K. Chatterjee, D. Dollimore, and K. Alexander, Instrum. Sci.

Technol. 29, 133-144 (2001); Chem. Abstracts 135, 16629t (2001) (reported values are referred to as enthalpies of vaporization).

01/21 J. O. Lalah, K.-W. Schramm, D. Lenoir, B. Henkelmann, N.

Hertkorn, G. Matuschek, A. Kettrup, and K. Günther, Chem. Eur. J. 7, 4790-4795 (2001).

01/22 I. Kul, D. D. DesMarteau, and A. L. Beyerlein, Fluid Phase

Equilib. 185, 241-253 (2001).

01/23 A. K. Baev and V. E. Mikhailov, Russ. J. Appl. Chem. 74,

1435-1438 (2001).

01/24 A. K. Baev and I. N. Chernyak, Russ. J. Inorg. Chem. 46, 310-316; (2001) Zh. Neorg. Khim. 46, 361-367 (2001); enthalpies of vaporization are presented graphically in paper for binary mixtures.

2002

02/1 R. M. Varushchenko and A. I. Druzhinina, Fluid Phase Equilib. 199, 109-119 (2002).

02/2 D. Deng, H. Li, and S. Han, J. Chem. Thermodyn. 34, 1431-1437 (2002)

02/3 T. V. Sorokina, D. Dollimore, and K. S. Alexander, Thermochimica Acta 392/393, 315-321 (2002).

02/4 A. Valtz, C. Coquelet, A. Baba-Ahmed, and D. Richon, Fluid Phase Equilib. 202, 29-47 (2002).

02/5 J.-P. Pokki, P. Uusi-Kyyny, J. Aittamaa, and S. Liukkonen, J. Chem. Eng. Data 47, 371-375 (2002).

02/6 S. Bobbo, L. Fedele, M. Scattolini, and R. Camporese, J. Chem.

Eng. Data 47, 179-182 (2002).

02/7 C.-H. Su, S. Zhu, N. Ramachandran, and A. Burger, J. Cryst.

Growth 235, 313-319 (2002).

02/8 H. Segura, J. Wisniak, G. Galindo, and R. Reich, Phys. Chem. Liq. 40, 67-81 (2002).

02/9 B. E. Swiatek and S. K. Malanowski, J. Chem. Eng. Data 47,

478-481 (2002)

02/10 R. M. Varuschenko, L. L. Pashchenko, A. I. Druzhinina, A. Yu. Churkina, I. I. Vorob'ev, and S. N. Kravhun, Russ. J. Phys. Chem. 76, 546-551 (2002).

02/11 R. M. Varuschenko, C. A. Aitkeeva, A. I. Druzhinina, Y. A.

Myshenseva, and L. L. Pashchenko, Russ. J. Phys. Chem. 76, 724-728

(2002)

02/12 I. M. Marrucho, N. S. Oliveira, and R. Dohrn, J. Chem. Eng. Data 47, 554-558 (2002).

02/13 I. Batiu, Fluid Phase Equilib. 198, 111-121 (2002).

02/14 W. V. Steele, R. D. Chirico, S. E. Knipmeyer, and A. Nguyen, J. Chem. Eng. Data 47, 648-666 (2002).

02/15 W. V. Steele, R. D. Chirico, A. B. Cowell, S. E. Knipmeyer, and A. Nguyen, J. Chem. Eng. Data 47, 667-688 (2002).

02/16 W. V. Steele, R. D. Chirico, A. B. Cowell, S. E. Knipmeyer, and A. Nguyen, J. Chem. Eng. Data 47, 700-714 (2002).

02/17 W. V. Steele, R. D. Chirico, S. E. Knipmeyer, and A. Nguyen, J. Chem. Eng. Data 47, 689-699 (2002).

02/18 Y. D. Lei, R. Chankalal, A. Chan, and F. Wania, J. Chem. Eng. Data 47, 801-806 (2002)

02/19 J. Murata, S. Yamashita, M. Akiyama, S. Katayama, T. Hiaki, and A. Sekiya, J. Chem. Eng. Data 47, 911-915 (2002).

02/20 W. V. Steele, R. D. Chirico, A. B. Cowell, S. E. Knipmeyer, and A. Nguyen, J. Chem. Eng. Data 47, 725-739 (2002).

02/21 W. V. Steele, R. D. Chirico, S. E. Knipmeyer, and A. Nguyen, J. Chem. Eng. Data 47, 715-724 (2002).

02/22 R. M. Varushchenko, L. L. Pashchenko, A. Y. Churkina, and A. V. Shabanova, Russ. J. Phys. Chem. 76, 915-921 (2002).

02/23 A. Rodriquez, J. Canosa, A. Dominquez, and J. Tojo, Fluid Phase Equilib. 198, 95-109 (2002). 
02/24 S. Bobbo, G. Artico, L. Fedele, M. Scattolini, and R. Camporese, J. Chem. Eng. Data 47, 839-842 (2002).

02/25 G. Di Nicola and G. Passerini, J. Chem. Eng. Data 47, 882-886 (2002).

02/26 H. Segura, G. Galindo, R. Reich, J. Wisniak, and S. Loras, Phys. Chem. Liq. 40, 277-294 (2002).

02/27 A. C. G. van Genderen, J. C. van Miltenburg, J. G. Blok, M. J. van Bommel, P. J. van Ekeren, G. J. K. van den Berg, and H. A. J. Oonk, Fluid Phase Equilib. 202, 109-120 (2002).

02/28 S. P. Verevkin, J. Chem. Thermodyn. 34, 263-275 (2002).

02/29 E. L. Krasnykh, T. V. Vasiltsova, S. P. Verevkin, and A. Heintz, J. Chem. Eng. Data 47, 1372-1378 (2002).

02/30 S. Loras, A. Aucejo, J. B. Monton, J. Wisniak, and H. Segura, J. Chem. Eng. Data 47, 1256-1262 (2002).

02/31 A. Rodriquez, J. Canosa, A. Dominquez, and A. Tojo, J. Chem. Eng. Data 47, 1098-1102 (2002).

02/32 S. P. Verevkin, J. Chem. Eng. Data 47, 1071-1097 (2002).

02/33 J. W. Goodrum and D. P. Geller, Bioresource Technol. 84, 75-80

(2002). [Note: The calculated values in the article had to be multiplied by 1,000 (personal communication).]

02/34 O. Pfohl, C. Riebesell, and R. Dohrn, Fluid Phase Equilib. 202, 289-306 (2002).

02/35 M. D. M. C. Ribeiro da Silva, L. M. N. B. F. Santos, A. L. R.

Silva, O. Fernandes, and W. E. Acree, Jr., J. Chem. Thermodyn.

(submitted for publication).

02/36 K. Clou, J. F. Janssens, N. Blaton, A. T. H. Lenstra, and H. O.

Desseyn, Thermochimica Acta (in press).

02/37 A. van Roon, J. R. Parsons, and H. A. J. Govers, J. Chromatogr. A 955, 105-115 (2002).

02/38 S. P. Verevkin and A. Heintz, J. Chem. Soc., Perkin Trans. 2 $728-733$ (2002).

02/39 A. K. Baev and M. A. Shiskho, Russ. J. Appl. Chem. 75, 156-158 (2002).

02/40 A. Hazra, D. Dollimore, and K. Alexander, Thermochimica Acta 392/393, 221-229 (2002).

02/41 C. Wang, H. Li, L. Ma, and S. Han (unpublished data).

02/42 A. Dahmani, I. Mokbel, and J. Jose, Fluid Phase Equilib. 203,

193-204 (2002).

02/43 A. K. Baev and M. A. Shishko, Russ. J. Appl. Chem. 75, 25-28 (2002).

02/44 J. S. Chickos, P. Webb, G. Nichols, T. Kiyobayashi, P.-C. Cheng, and L. Scott, J. Chem. Thermodyn. 34, 1195-1206 (2002).

02/45 M. Lubomska, A. Banas, and S. K. Malanowski, J. Chem. Eng.

Data 47, 1466-1471 (2002).

Unpublished Data

U/1 R. H. Fuchs (unpublished data).

U/2 S. P. Verevkin, E. Krasnykh, T. Vasiltsova, and A. Heintz

(unpublished data as quoted in [02/32]).

\section{Acknowledgment}

J.S.C. would like to thank the past and present students in Chemistry 202, Introduction to the Chemical Literature, who have contributed to this compilation through their term papers.

\section{References for Introductory Material}

${ }^{1}$ J. B. Pedley, R. D. Naylor, and S. P. Kirby, Thermochemical Data of Organic Compounds, 2nd ed. (Chapman and Hall, London, 1986).

${ }^{2}$ W.-Y. Shiu and K.-C. Ma, J. Phys. Chem. Ref. Data 29, 1-130 (2000); 29, 387-462 (2000); A. J. Delle Site, ibid. 26, 157-193 (1997).

${ }^{3}$ S. Puri, J. S. Chickos, and W. Welsh, Anal. Chem. 73, 1480-1484 (2001);
G. Nichols, J. Orf, S. Reiter, J. S. Chickos, and G. W. Gokel, Thermochim. Acta 346, 15-28 (2000); J. S. Chickos and J. Wilson, J. Chem. Eng. Data 42, 190-197 (1997), and references cited therein.

${ }^{4}$ J. S. Chickos, W. E. Acree, Jr., and J. F. Liebman, Estimating Phase Change Entropies and Enthalpies, edited by D. Frurip and K. Irikura, ACS Symposium Series 677 Computational Thermochemistry, Prediction and Estimation of Molecular Thermodynamics (American Chemical Society, Washington, DC, 1998), pp. 63-93, and references cited therein.

${ }^{5}$ R. Sabbah, A. Xu-wu, J. S. Chickos, M. L. Planas Leitao, M. V. Roux, and L. A. Torres, Thermochim. Acta 331, 93-204 (1999).

${ }^{6}$ J. S. Chickos, W. Acree, Jr., and J. F. Liebman, J. Chem. Phys. Ref. Data 28, 1535-1673 (1999).

${ }^{7}$ J. S. Chickos and W. Acree, Jr., J. Phys. Chem. Ref. Data 31, 537-698 (2002).

${ }^{8}$ M. Zabransky, V. Ruzicka, Jr., V. Majer, and E. S. Domalski, J. Phys. Chem. Ref. Data, Monograph No. 6, 1-1596 (1996); E. S. Domalski and E. D. Hearing, J. Phys. Chem. Ref. Data 25, 1-533 (1996).

${ }^{9}$ S. W. Benson, Thermochemical Kinetics, 2nd ed. (Wiley-Interscience, New York, 1976).

${ }^{10}$ W. J. Lyman, W. F. Reehl, and D. H. Rosenblatt, Handbook of Chemical Property Estimation Methods (American Chemical Society, Washington, DC, 1982), Chap. 23.

${ }^{11}$ J. S. Chickos, S. Hosseini, D. G. Hesse, and J. F. Liebman, Struct. Chem. 4, 261-269 (1993); 4, 271-278 (1993).

${ }^{12}$ V. Majer and V. Svoboda, Enthalpies of Vaporization of Organic Compounds: A Critical Review and Data Compilation, IUPAC Chemical Data Series No. 32 (Blackwell, Oxford, 1985).

${ }^{13}$ R. Ruzika and V. Majer, J. Phys. Chem. Ref. Data 23, 1-39 (1994).

${ }^{14}$ E. S. Domalski and E. D. Hearing, J. Phys. Chem. Ref. Data 25, 1-525 (1996).

${ }^{15}$ C. E. Rechsteiner, Jr., in Ref. 10, Chap. 13.

${ }^{16}$ V. Svoboda and H. Smolova, Fluid Phase Equilibria 97, 1-11 (1994); P. Basarova and V. Svoboda, ibid. 105, 27-47 (1995).

${ }^{17}$ A. Vetere, Fluid Phase Equilibria 106, 1-10 (1995).

${ }^{18}$ W. F. An, J. Y. Li, W. W. Cheng, and J. D. Gao, Chem. Eng. J. Biochem. Eng. J. 59, 101-109 (1995).

${ }^{19}$ C.-H. Tu and C. P. Liu, Fluid Phase Equilibria 121, 45-65 (1996).

${ }^{20}$ P, Ulbig, M. Kluppel, and S. Schulz, Thermochim. Acta 271, 9-21 (1996).

${ }^{21}$ L. Coniglio, L. Trassy, and E. Rauzy, Ind. Eng. Chem. Res. 39, 50375048 (2000).

${ }^{22}$ Z. Y. Liu, Chem. Eng. Commun. 184, 221-228 (2001).

${ }^{23}$ N. Gopinathan and D. N. Saraf, Fluid Phase Equilibria 179, 277-284 (2001).

${ }^{24}$ K. Laidler, Can. J. Chem. 34, 626-48 (1956).

${ }^{25}$ M. Ducros, J. F. Greison, and H. Sannier, Thermochim. Acta 36, 39-65 (1980); M. Ducros, J. F. Greison, H. Sannier, and I. Velasco, ibid. 44, 131-40 (1981); M. Ducros and H. Sannier, ibid. 54, 153-157 (1982); M. Ducros and H. Sannier, ibid. 75, 329-340 (1984).

${ }^{26}$ J. P. Guthrie and K. F. Taylor, Can. J. Chem. 61, 602-607 (1983).

${ }^{27}$ J. S. Chickos, A. S. Hyman, L. H. Ladon, and J. F. Liebman, J. Org. Chem. 46, 4294-4296 (1981); J. S. Chickos, D. G. Hesse, J. F. Liebman, and S. Y. Panshin, ibid. 53, 3424-3429 (1988); J. S. Chickos, D. G. Hesse, and J. F. Liebman, ibid. 54, 5250-5256 (1989).

${ }^{28}$ K. H. Myers and R. P. Danner, J. Chem. Eng. Data 38, 175-200 (1993).

${ }^{29}$ L. Constantinou, S. E. Prickett, and M. L. Mavrovouniotis, Ind. Eng. Chem. Res. 32, 1734-1746 (1993); 33, 395-402 (1994).

${ }^{30}$ L. Constantinou and R. Gani, AIChE J. 40, 1697-1710 (1994).

${ }^{31}$ C. H. Tu and C. P. Liu, Fluid Phase Equilibria 121, 45-65 (1996).

${ }^{32}$ K. Sivaji and D. H. L Prasad, Trans I. Chem. E. A76, 970-972 (1998).

${ }^{33}$ K. U. Goss and R. P. Schwarzenbach, Environ. Sci. Techn. 33, 3390-3393 (1999).

${ }^{34}$ I. B. Golovanov and I. G. Tsygankova, Russ. J. Gen. Chem. 69, 12271233 (1999).

${ }^{35}$ J. Marrero and R. Gani, Fluid Phase Equilibria 183 (Special Issue), 183208, (2001).

${ }^{36}$ A. P. Kudchadker and B. J. Zwolinski, J. Chem. Eng. Data 11, 253-255 (1966).

${ }^{37}$ J. C. M. Li and F. D. Rossini, J. Chem. Eng. Data 6, 268-270 (1961). 